




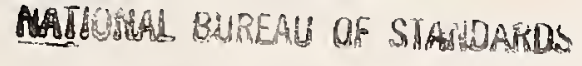

Mas 1979

Index of International Standards

Sophie J. Chumas, Editor

Engineering and Product Standards Division

Institute for Applied Technology

National Bureau of Standards

Washington, D.C. 20234

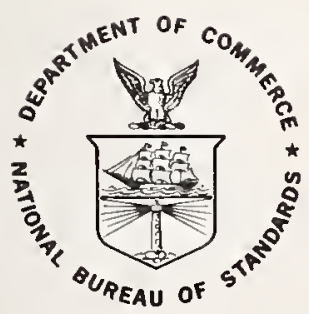

U.S. DEPARTMENT OF COMMERCE, Frederick B. Dent, Secretary NATIONAL BUREAU OF STANDARDS, Richard W. Roberts, Director

Issued March 1974 
Library of Congress Catalog Number: 73-600358

National Bureau of Standards Special Publication 390

Nat. Bur. Stand. (U.S.), Spec. Publ. 390,222 pages (Mar. 1974)

CODEN: XNBSAV 


\section{Foreword}

The National Bureau of Standards, Standards Information Services (NBS-SIS) maintains a reference collection of more than 200,000 engineering and related standards covering the activities of more than 380 U.S. technical societies, professional organizations, and trade associations; U.S. Federal and State governments; and the standards and specifications of the foreign national and international standardizing bodies.

For some time now the National Bureau of Standards has recognized a need for a one source listing of existing standards within the international community. Such a reference source, which provides easy accessibility, is open to a wide range of uses. This Index contains the standards of five international organizations, most of which are nongovernmental. However, future plans are to expand the Index to include many other international governmental organizations such as the Economic Commission for Europe (ECE), the Organization for Economic Cooperation and Development (OECD), the World Health Organization (WHO), and the International Labor Organiza. tion (ILO).

It is particularly important to world trade that better communication be developed in the standards area. Serious efforts are underway among nations and among international organizations to harmonize standards so as to provide a consistently competent body of technical specifications that will enhance world trade and not impede its flow by creating non-tariff barriers.

W. E. ANDRUS, JR.

Program Manager

Engineering and Information

Processing Standards 



\section{Contents}

Page

Foreword

III

1. Introduction

2. How to use the KWIC Index

2.1. Index Entries

2.2. Reading the KWIC Index

3. List of Organizational Names and Addresses

4. Availability of Standards

5. Stop List 5

6. KWIC Index 



\title{
Index of International Standards
}

\author{
Sophie J. Chumas
}

\begin{abstract}
This computer-produced Index, based on the Key-Word-In-Context (KWIC) system, contains over 2,700 standard titles of the International Organization for Standardization (ISO), the International Electrotechnical Commission (IEC), the International Commission on Rules for the Approval of Electrical Equipment (CEE), the International Special Committee on Radio Interference (CISPR), and the International Organization of Legal Metrology (OIML).
\end{abstract}

Key Words: Analyses; International Commission on Rules for the Approval of Electrical Equipment; International Electrotechnical Commission; International Organization for Standardization; International Organization of Legal Metrology; International Special Committee on Radio Interference; recommendations; specifications; standards; test methods.

\section{Introduction}

This Index of international standards, recommen. dations, test methods, analyses, and specifications, contains over 2,700 titles. It is a computer-produced Index based on the Key-Word-In-Context (KWIC) system. The standards-writing organizations represented are: the International Organization for Standardization (ISO), the International Electrotechnical Commission (IEC), the International Commission on Rules for the Approval of Electrical Equipment (CEE), the International Special Committee on Radio Interference (CISPR), and the International Organization of Legal Metrology (OIML). The standards of these international organizations have worldwide application and contribute to world progress.

The purpose of this Index is to identify existing international standards for a particular product, the characteristics or parts of products, the safety or performance of products, any related materials, vocabularies, symbols, test methods, analyses, and other aspects of standards. Criteria for inclusion are that an organization be international in scope and that it have standards published in the English language.

Many titles were editorially changed either to include another descriptive term (key word) for better subject identification or to prevent repetitious titles in the text of the Index. However, none of the titles were edited to cause any change in their meaning. Since standards titles as found in this Index are not always exact, for ordering purposes, the designation number should accompany a request. Bibliographic information is available through the Standards Information Services (NBS-SIS) of the National Bureau of Standards, the American National Standards Institute (ANSI), or the issuing organization. Ordering information for the indexed standards may be found under Section 5.

The following indexing guidelines were established for the standards in this Index:

a. Supplements to standards were indexed only when they carried separate titles in addition to the main title.

b. Amendments to standards were not indexed.

c. Date of the original document was used.

d. When the title contained a word for which there are two spellings, both versions were usually included, e.g., aluminum, aluminium.

This source of reference, which will be updated periodically, will hopefully be of assistance to users concerned with world trade, metrication, and certification; to developing countries; to U.S. and foreign standardizers; to foreign national, regional, international, and U.S. standards bodies; to manufacturers, engineers, builders and architects; to environmentalists; to consumer-oriented organizations; and to students of the standardization discipline.

\section{How to Use the KWIC Index}

\subsection{Index Entries}

An index entry contains four items of information.

Sample Entry (Exact title of standard)

(1) Std. for Rolling Bearings: Tolerances; Metric Series Chamfer Dimension Limits and Maximum Shaft and Housing Fillet Radius

The first item given is the title of the standard, specification, or recommendation. Generally, the key words found in the title are sufficient to identify the product or article covered; however, in some instances additional descriptive terms have been added to the title to aid in identifying the contents. Over 600 words, which are meaningless for searching purposes, do not appear as key words. Examples are: "and," "for," "general," "method," and "practice." A list of these words appears in Section 5, "Stop List." The second item denotes the year of publication. The third item is the issuing organization, identified by an acronym consisting of five letters or less. The fourth item is the standard number assigned by the organization. Items three and four make up the standard's designation number. This alphanumeric designation is always found in a column next to the right-hand margin of the Index. 


\subsection{Reading the $\mathrm{KWIC}$ Index}

The title of each standard can be found under all the significant key words which it contains. These key words are arranged alphabetically down the center of each page together with their surrounding context. Each such permuted title is assigned only one line per key word entry in the Index; therefore, titles longer than one line have been cut by the computer. This truncation is indicated by a virgule (/) at the point where the title was cut.

Sample Entry (The same title in Section 2.1., as it appears in KWIC form, edited and truncated) :

imits and Maximum Shaft and Housing Fil/ Std. for Rolling Bearings: Tolerances; Metric Series Chamfer Dimension L ISO 582

Occasionally both ends of a title will be truncated. When this condition occurs, the virgule will be omitted. Missing portions of a title can be found by locating in the Index one or more of the title's other key words.

An entry line is read from left to right, beginning after the longest blank space in the line and ending at the designation number. If the longest blank space is not at the beginning of the line, the entry will be continued at the left hand margin of the same line.

\section{List of Organizational Names and Addresses}

International Commission on Rules for the Approval of Electrical Equipment (CEE)

310 Utrechtseweg

Arnhem, Netherlands

International Electrotechnical Commission (IEC)

1 , rue de Varembe

1211 Geneve 20

Switzerland/Suisse

International Organization of Legal Metrology (OIML)

11 rue Turgot

75-Paris 9e, France

International Organization for Standardization (ISO)

1 , rue de Varembe

1211 Geneve 20

Switzerland/Suisse

International Special Committee on Radio Interference (CISPR)

1 , rue de Varembe

1211 Geneve 20

Switzerland/Suisse
Secretariat: British Standards Institution 2 Park Street

London W1Y 4AA

England

\section{Availability of Standards}

Standards indexed in this publication are available in the United States from the:

American National Standards Institute (ANSI)

1430 Broadway

New York, New York 10018

ANSI, as a National ISO member body, serves as the sole sales agent in the United States for foreign and international standards.

The following National standardizing bodies serve as national representatives for one another. Each acts within its own country as sole sales agent and information center for the standards of the other National standardizing bodies.

Albania: STASH, Bureau de Standardisation aupres de la Commission du Plan d'Etat de la Republique Populaire, D'Albania, Tirana

Algeria: Office National de la Propriete Industrielle, 5, rue Abou Hamou Moussa, B.P. 1021

Argentina: IRAM, Instituto Argentino de Materiales, Chile 1192, Buenos Aires

Australia: SAA, AS, Standards Association of Australia, 80-86 Arthur Street, North Sidney NSW 2060

Austria: ON, ONORM, Oesterreichisches Normungsinstitut Leopoldsgasse 4A-1021 Wien 2

Barbados, W.I.: Barbados National Standards Institution, Old Bay Street Boys' School, Bay Street, St Michael, Barbados W.I.

Belgium: IBN, Institut Belge de Normalisation, 29 Avenue de la Brabanconne B-1040 Bruxelles 4

Brazil: ABNT;NB,EB, Associacao Brasileira de Normas Tecnicas, Caixa Postal 1680, Rio de Janeiro

Bulgaria: KKCM, Comite de la Qualite de la Normalisation et de la Metrologie, 21, rue 6 Septembre Sofia

Burma: UBARI, UBS, Union of Burma Applied Research Institute, Junction of Kaba Aye PagodaKanbe Roads, Rangoon

Canada: CSA, Canadian Standards Association, 178 Rexdale Blyd, Rexdale 603 Ontario 
Ceylon: BCS, Bureau of Ceylon Standards, 53 Dharmapala Mawatha, Colombo 3

Chile: INDITECNOR, Instituto Nacional de Investigaciones Tecnologias y Normalizacion, Plaza Bulnes 1302, OF 62, Casilla de correo 995, Santiago

China: CNS, National Bureau of Standards, Ministry of Economic Affairs, 5th Floor, Hsin Kuang Life Insurance Building, Bldg 61-1 Sung-Chiang Rd Taipei, Taiwan, 104 Republic of China

Colombia: ICONTEC, Instituto Colombiano de Normas Tecnicas, Apartado Aereo, 14237 Bogota D.E.

Costa Rica: Instituto Centroamericano de Investigaciones y Tecnologia Industrial, 4a Calle y Avenida la Reforma, Zona 10, Guatenala City, Guatemala

Cyprus: The Ministry of Commerce and Industry of the Republic of Cyprus, Nicosia

Czechoslovakia: CSN, Urad pro normalisaci a mereni, Vaclavske namesti c. 19, 11347 praha 1, Nove Mesto

Denmark: DS, Dansk Standardiseringsraad, Aurehojvej 12, DK-2900, Hellerup

Egypt: EOS, Arab Republic of Egypt, Egyptian Organization for Standardization, 2 Latin America Street, Garden City, Cairo

Ethiopia: ESI, Ethiopian Standards Institution, P.O. Box 2310, Addis Ababa

El Salvador: Instituto Centroamericano de Investigaciones y Technologia Industrial, 4a Calle y Avenida la Reforma, Zona 10, Guatemala City, Guatemala

Finland: SFS, Suomen Standardisoimisliitto Box 205 $\mathrm{SF}-00121$ Helsinki 12

France: AFNOR, NF, Association Francaise de Normalisation, Tour Europe, Cedex 7, 92080 Paris-La Defense

Germany: DNA, DIN, Deutscher Normenausschus, 4-7 Burggrafenstrasse, 1 Berlin 30

Ghana: ISIG, National Standards Board, P.O. Box M245 Accra

Greece: NHS, Ministry of National Economy, Industry Sector, Standardization Division, 13 Xerofon Str., Athens 118

Guatemala: ICAITI, Instituto Centroamericano de Investigaciones y Tecnologia Industrial, 4a, Calle y Avenida la Reforma, Zona 10, Apartado Postal 1552

Honduras: Instituto Centroamericano de Investigaciones y Tecnologia Industrial, 4a Calle y Avenida la Reforma, Zona 10, Guatemala City, Guatemala
Hong Kong: Federation of Hong Kong Industries, Eldex Industrial Bldg, 21 Ma Tau Wei Road, Unit A, Munghom Kowloon

Hungary: MSZH Magyar Szabvanyugyi Hivatal Postafiok 24 1450 Budapest 9

Iceland: Industrial Development Institute, Skipholt 37, Reykjavik

India: ISI, IS, Indian Standards Institution, "Manak Bhavan," 9 Bahadur Shah Zafer Merg, New Delhi 1

Indonesia: DNI, NI, Jajasan "Dana Normalisasi Indonesia," Bjalan Braga 38, Bandung

Iran: ISIRI, Institute of Standards and Industrial Research of Iran, Ministry of Economy, P.O. Box 2937, Teheran

Iraq: IOS, Iraqi Organization for Standards Planning Board, P.O. Box 11185, Baghdad

Ireland: IIRS, I.S., Institute for Industrial Research and Standards, Glasnevin House, Ballymun Road, Dublin.9

Israel: SII, Standards Institution of Israel, 42 University Street, Tel Aviv

Italy: UNI, Ente Nazionale Italiano de Unificazione, Piazza Armando Diaz 2, 120123 Milano

Japan: JISC, JIS, Japanese Industrial Standards Committee, Agency of Ind. Science and Techonolgy, Ministry of International Trade and Industry, 3-1 Kasumigaseki Chiyoda-Ku, Tokyo

Kenya: Ministry of Works, P.O. Box 30043, Nairobi, Kenya

Korea: KBS, KS, Korean Bureau of Standards, Ministry of Commerce \& Industry, P.O. Box Kwanghwamoon 282, Seoul

Kuwait: KSS, Ministry of Commerce and Industry, P.O. Box 2944

Lebanon: LIBNOR, Lebanese Standards Institution, P.O. Box 2806, Beirut

Libya: Standards \& Specifications Section, Department of Industrial Organization, Ministry of Industry, Tripoli

Madagascar: Ministere des mines, de I'Industrie, du Commerce et du Ravitaillement, Service du Conditionnement, B.P. 1316 Tananarive 
Malaysia: SIM, Standards Institution of Malaysia, 5 Jalan Semantan, P.O. Box 544, Kuala Lumpur

Malta: Department of Industry, Standards Laboratory, Industrial Estate, Marsa-Malta

Mexico: DGN, Direccion General de Normas, Av. Cuauhtemac No. 80, Mexico 7, D.F.

Morocco: SNIMA, Service de Normalisation Industrielle Marocaine, Direction de l'Industrie, SousSecretaire d'Etat a l'Industrie et aux Mines, Rabat

Netherlands: NNI, Nederlands Normalisatie-instituut, Polakweg, 5 Rijswijk (ZH) -2106

New Zealand: SANZ, Standards Association of New Zealand, Private Bag, Wellington

Nicaragua: Instituto Centroamericano de Investigaciones y Techologia Industrial, 4a Calle y Avanida la Reforma, Zona 10, GuatemaIa City, Guatemala

Nigeria: Federal Ministry of Industries, Nigerian Standards Organization, 11 Kofo Abayomi Road, Victoria Island, Lagos, Nigeria

Norway: NSF, NS, Norges Standardiseringsforbund, Haakon VII's gt. 2, Oslo 1

Pakistan: PSI, Pakistan Standards Institution, 39 Garden Road, Saddar, Karachi 3

Panama: Instituto Centroamericano de Investigaciones y Tecnologia IndustriaI, 4a Calle y Avenida la Reforma, Zona 10, Guatemala City, Guatemala

Paraguay: INTECNOR, Instituto Nacional de Tecnologia y Normalizacion, Brasil y Jose Berges, Annuncion

Peru: ITINTEC, Instituto de Investigacion Tecnologica Industrial y de Normas Tecnicas, Apartado No. 145, Av. Republica de Chile 698, Lima

Philippine Islands: Bureau of Standards of the Philippines, Plaza Sta. Cruz, P.0. Box 3719 Manila

Poland: PKNIM, Polski Komitet Normalizacji i Miar UI. Electoraina 2, Warszawa 51

Portugal: IGPAI, NP, Reparticao de Normalizaco, Avenida de Berna 1, Lisbon

Republic of South Africa: SABS, South African Bureau of Standards, Private Bag 191, Pretoria

Rhodesia: Standards Association of Central Africa, Coventry Road Workington, P.O. Box 2259, Salisbury 4
Romania: IRS; Institutul Roman de Standardizare, Casuta Postala 10 Bucarest 1

Saudi Arabia: The Ministry of Commerce and Industry, Department of Metrology and Standards, Riyadh

Singapore: SIRU, Singapore Institute of Standards and Industrial Research, 179 River Valley Road, P.O. Box 2611, Singapore

Spain: IRANOR, Instituto Nacional de Racionalizacion y Normalizacion, Serrano 150 Madrid 6

Sudan: Sudanese Organization for Standard Specifications, Ministry of Industry and Mining, P.O. Box 2184, Khartoum, Sudan

Sweden: SIS, Sveriges Standardiseringskommission, Box 3295 S-103 66 Stockholm 3

Switzerland: SNV, Association Suisse de Normalisation, 4, Kirchenweg, 8032 Zurich

Syria: Industrial Testing and Research Centre, P.O. Box 845, Damascus

Thailand: CTNNS, Centre for Thai National Standard Specifications, Applied Scientific Research Corporation of Thailand, 196 Phahonythin Road, Bangkhen, Bangkok

Tunisia: Ministere de l'Economie Nationale, 195 rue de la Kasbah Tunis

Turkey: TSE, TS, Turk Standardiari Enstitusu, Necatibey Caddesi 112, Bakanliklar, Ankara

.United Kingdom: BSI, BS, British Standards Institution, 2 Park Street, London W.1 A 2BS, England

Uruguay: UNIT, Instituto Uruguayo de Normas Tecnicas, Agraciadà 1464, Piso 9, Montevideo

U.S.S.R.: GOST, Gosudarstvennyj Komitet Standartov Soveta Ministrov S.S.S.R., Leninsky Prospekt 9b, Moskva M-49

Venezuela: COVENIN, Comision Venezolana de Normas Industriales, Piso 6 Torre Sur, C.S.B. OF 653, Caracas 101

Yugoslavia: JZS, JUS, Jugoslavenski zavod za Standardizaciju, Cara Urosa ul 54, Post pregr 933, 11001 Beograd

Zambia: Zambia Standards Association, P.O. Box R.W. 259, Lusaka, Zambia 


\section{Stop List}

ABILITY

ABOVE

ABSENCE

ACCEPTING

ACCOMMODATE

ACCORDANCE

ACCORDING

ACCUMULATE

ACQUIRED

ACROSS

ACT

$\mathrm{AC} / \mathrm{DC}$

ADD

ADDENDA

ADJACENT

ADJUST

AFFECTING

AFFORD

AFTER

AFTER-FINISHED

AGAINST

AGENCIES

ALL

ALONG

ALPHA

ALSO

ALUMINIUM

AMOUNT

AND/OR

ANNEX

ANSWER

ANSWER-BACK

ANY

APPENDICES

APPENDIX

APPLICABLE

APPLIED

APPLYING

APPRAISE

APPROXIMATE

APPROXIMATELY

APPROXIMATION

ARE

AROUND

ASSIGNMENT

ASSIGNMENTS

ASSOCIATED

ASSURE

AVAILABLE

AW.AY
BACKWARDLY

BASED

BEFORE

BEHIND

BEING

BELOW

BENEATH

BETWEEN

BLENDED

BODIED

BOTH

BROKEN

BTU

BURNED

BUT

CANNOT

CAPABILITIES

CAPABILITY

CAUSED

CENT

CENTIGRADE

CENTIMETER

CENTIMETERS

CENTRALLY

CENTRE

CENTRES

CENT

CERTAIN

CHANGED

CHIEFLY

CHOICE

CHOOSING

CLASS

CLASSES

CLEANED

COINCIDENT

COLOUR

COLOURS

COMBINED

COMMITTEE

COMMONLY

COMPARABLE

COMPARING

COMPARISON

COMPLEMENT

COMPLIANCE

COMPOUNDED

CONFORMING

CONJUNCTION

CONSIDERED

$\begin{array}{ll}\text { CONSISTING } & \text { EVACUATE } \\ \text { CONTENT } & \text { EVIDENCE } \\ \text { CONTENTS } & \text { EXAMINE } \\ \text { CONVENTIONALLY } & \text { EXCEEDING } \\ \text { COUPLED } & \text { EXCEPT }\end{array}$

COVERAGE

CRE

CRES

CS

CUBIC

EXCLUDING

EXISTENT

EXPENDABLE

EXPERIENCE

EXPERIENCES

DEFINED

DEFINITE

DEGREE

DEGREES

DELAYED

DEPLETED

DERIVED

DESIGNATE

DESIGNATED

DESIRABLE

DESIRED

DETAILED

DETERMINE

DETERMINING

DIFFERENT

DIFFICULT

DILUTE

DIRECTLY

DISCUSSION

DISSIMILAR

DIVIDED

DUE

DUPLICATE

DURING

DVA

EASILY
EDGED
EDGEWISE
EFFECT
EFFECTIVENESS

EFFECTS

EIGHTHS

EIGHTS

EITHER

ENTERING

ENTRY

EQUIPPED

EQUIVALENT

ESTABLISHMENT

ESTABLISHMENTS
EXPLANATION

EXPOSED

EXTANT

EXTENDED

EXTENDING

EXTERNALLY

EXTRA

EXTRACTABLE

EXTRACTED

EXTRACTING

EXTREME

FABRICATED

FACED

FALSE

FASTENED

FED

FEEBLY

FIBRE

FIBRES

FIFTY

FINER

FLATWISE

FOR

FORKED

FORMERLY

FORTY

FORWARD

FOUR

FOURTH

FULLY

GAL

GALLON

GENERAL

GENERAL-PURPOSE

GENERATED

GIVEN

GIVING

GRAM

GRAMME

GREAT 
GREATER

GROUPING

GROUPS

GROWTH

HALF

HANDLED

HARD

HAVE

HAVING

HEAVILY

HEAVY

HEIGHT

HEIGHTS

HELD

HIGHLY

HOUR

HOURS

HOW

HUNDRED

IDENTICAL

IMMEDIATE

IMPERMEABLE

IMPERVIOUS

IMPORTANCE

IMPROVED

IMPROVEMENT

IMPROVING

IN

INCH

INCLUDE

INCLUDED

INCLUDES

INCLUDING

INCLUSION

INCLUSIONS

INCLUSIVE

INCORPORATE

INCREASE

INDIVIDUAL

INFLUENCE

INHIBITED

INNER

INSIDE

INSPECT

INTERIM

INTRINSICALLY

INVOLVING

IN.

ITS

$\mathrm{KGF}$
$\mathrm{KHZ}$

KILOCYCLE

KILOCYCLES

KILOHERTZ

KILOVOLT

KILOVOLTS

KILOWATT

KILOWATTS

KIND

KVA

\section{LADEN \\ LAID \\ LARGE \\ LARGER \\ LAY}

LAYING

LAY.IN

LBS.

LEASED

LEAVE

LESS

LICENSED

LIKE

LOCATED

LOWER

MADE

MAKE

MATTERS

MAXIMUM

MAY

MEDIUM

MEGAHERTZ

METH.

METHOD

METHODS

MEV

MHZ

MIL

MILLED

MILLIAMPERE

MILLIMETER

MILS

MINIMIZATION

MINIMIZE

MINIMIZING

MINIMUM

MODE

MODERATE

MORE

MOST
NATURE

NEAR

NEEDED

NOS.

NOT

NOTE

NOTIFICATION

NSF

OBSOLETE

OBTAIN

OBTAINED

OBTAINING

$\mathrm{OFF}$

OF

OHMS

ONE

ONE-STORY

ONE-WAY

ONE-WEAR

ONLY

OPTIONAL

ORDER

ORDERS

ORGANIZED

ORIGIN

OTHER

OUNCE

OUT

OVER

OWN

OWNERS

PACIFIC

PAR

PART

PARTICULAR

PARTICULARLY

PAST

PAYABLE

PENALTIES

PER

PERCEIVED

PERCENT

PERCENTAGE

PERIOD

PERMANENTLY

PERMIT

PERTAINING

PLEASURE

PLUS

POUND
POUNDS

PRACT.

PRACTICE

PRACTICES

PRECAUTIONS

PRECISE

PREMIUM

PREPARED

PRESENCE

PRESENT

PRESENTING

PREVAILING

PREVENT

PREVIOUS

PRICE

PRIMARILY

PRIMARY

PRINCIPAL

PRIOR

PROGRESS

PROMOTE

PROPER

PROPERLY

PROPOSAL

PROPOSALS

PROPOSED

PROVIDE

PROVIDED

PSI

PULL

PURPOSE

PURPOSES

QUADDED

QUADRUPLE

QUARTERING

QUICK

QUICKLY

QUOTATION

REACH

REBOUND

REBUTTED

RECEIVABLE

RECEIVE

RECEIVED

RECOMMENDED

RECOMMENDING

REGARD

REGARDING

RELATED

RELATING 


\begin{tabular}{|c|c|c|c|}
\hline $\begin{array}{l}\text { RELATION } \\
\text { REMAINING } \\
\text { REMOTELY } \\
\text { REMOVABILITY } \\
\text { REPEATED }\end{array}$ & $\begin{array}{l}\text { SPECIFICATION } \\
\text { SPECIFICATIONS } \\
\text { SPECIFIED } \\
\text { SPEC. } \\
\text { SPENT }\end{array}$ & $\begin{array}{l}\text { THAT } \\
\text { THEIR } \\
\text { THEREAFTER } \\
\text { THEREFROM } \\
\text { THEREIN }\end{array}$ & $\begin{array}{l}\text { USE } \\
\text { USED } \\
\text { USING } \\
\text { USUAL } \\
\text { VAPOUR }\end{array}$ \\
\hline $\begin{array}{l}\text { REPRESENTATION } \\
\text { REQUIRE } \\
\text { REQUIRED } \\
\text { RESIST } \\
\text { RESPECT }\end{array}$ & $\begin{array}{l}\text { STAND } \\
\text { STANDARD } \\
\text { STANDARDIZE } \\
\text { STANDARDS } \\
\text { STATE }\end{array}$ & $\begin{array}{l}\text { THESE } \\
\text { THIRD } \\
\text { THIS } \\
\text { THOSE } \\
\text { THREE }\end{array}$ & $\begin{array}{l}\text { VARIATION } \\
\text { VARIATIONS } \\
\text { VARIOUS } \\
\text { VERSA } \\
\text { VERY }\end{array}$ \\
\hline $\begin{array}{l}\text { RESPONSIBILITIES } \\
\text { RESPONSIBILITY } \\
\text { RESTRAINED } \\
\text { RESULTANT } \\
\text { RESULTS }\end{array}$ & $\begin{array}{l}\text { STEPPED } \\
\text { STORY } \\
\text { STRENGTHENED } \\
\text { STUDY } \\
\text { STYLE }\end{array}$ & $\begin{array}{l}\text { THROUGH } \\
\text { THRU } \\
\text { TIGHTLY } \\
\text { TOGETHER } \\
\text { TONS }\end{array}$ & $\begin{array}{l}\text { VICE } \\
\text { WATT } \\
\text { WAY } \\
\text { WHEN } \\
\text { WHERE }\end{array}$ \\
\hline $\begin{array}{l}\text { RETAINED } \\
\text { RPM } \\
\text { SALE } \\
\text { SAME } \\
\text { SELF }\end{array}$ & $\begin{array}{l}\text { STYLES } \\
\text { SUBJECTED } \\
\text { SUCH } \\
\text { SUFFICIENTLY } \\
\text { SUGGESTED }\end{array}$ & $\begin{array}{l}\text { TOTAL } \\
\text { TOTALLY } \\
\text { TOWARD } \\
\text { TRIPLE } \\
\text { TWO }\end{array}$ & $\begin{array}{l}\text { WHICH } \\
\text { WHO } \\
\text { WHOLESALE } \\
\text { WHO'S } \\
\text { WITH }\end{array}$ \\
\hline $\begin{array}{l}\text { SEPARATED } \\
\text { SEVENTY } \\
\text { SEVERAL } \\
\text { SHOWN } \\
\text { SIMILAR }\end{array}$ & $\begin{array}{l}\text { SUITABILITY } \\
\text { SUITABLE } \\
\text { SUPPLEMENT } \\
\text { SUPPLIED } \\
\text { TAKEN }\end{array}$ & $\begin{array}{l}\text { TYPE } \\
\text { TYPES } \\
\text { TYPICAL } \\
\text { UNATTENDED } \\
\text { UNCERTAINTY }\end{array}$ & $\begin{array}{l}\text { WITHIN } \\
\text { WITHOUT } \\
\text { WORKED } \\
\text { ZERO }\end{array}$ \\
\hline $\begin{array}{l}\text { SINGLY } \\
\text { SMALL } \\
\text { SMALLER } \\
\text { SPECIALLY } \\
\text { SPECIFIC }\end{array}$ & $\begin{array}{l}\text { TAKING } \\
\text { TECH } \\
\text { TENDENCIES } \\
\text { TENTATIVE } \\
\text { THAN }\end{array}$ & $\begin{array}{l}\text { UNDER } \\
\text { UNDERSTANDING } \\
\text { UNIT } \\
\text { UNWANTED } \\
\text { UPON }\end{array}$ & \\
\hline
\end{tabular}



(1968) Std. for Documentation-International Code for the

ences $(1968)$

(1970)

(1969)

f Dimensions-Profiles (1966)

1967)

Rec. for

Rec. for

Rec. for

Rec. for

Rec. for Bonded

Rec. for Bonded

Rec. for Bonded Std. for

72)

1972)

rily Used for the Production of Alumin or Fibre (Fiber) Building Boards: Determination of Water Rec. for Measurement of e Production of Aluminium-Determination of Zinc-Atomic n of Aluminium-Determination of Calcium Content-Atomic g (1970) Rec. for Plastics-Determination of Light Rec. for Determination of the Water Std. for Leather: Determination of Rec for Plastics-Determination of Water Rec. for Plastics-Determination of Boiling Water nium (Aluminum) and Its Alloys-Estimation of the Loss of Rec. for R Coated with Rubber or Plastics (1970)
Rec for Vulcanized Rubbers. for Flexible Cellular Materials 66,610 and 6 (1969) Rec. for Plastics

e / Rec. for a Meth. for the Evaluation of the Results of rocedures for Electronic Components and Equipment: Test D: Rec. for an Appraisal of the Problems of ocedures for Electronic Components and Equipment: Test Ga: Rec. for Rolling Bearings-For Railway Axle Boxes: Spec./S and

Rec. for International Code for the Field Rec. for International Code for Model Rec. for International Code for the Field Rec. for Steam Turbines: Rules for Std. for Gas Turbines rt Link Chain for Lifting Purposes-General Conditions of inning-Dimensions (1971/

Usable Width (1971)

Doubling Frames for C / de (Left or Right) (1960) ubling Frames for $\mathrm{C}$ And/ ling Frames (1972) s (1971) of Side (Left or Right) / (1971) Rec. for Textile Machinery and Rec. for Textile Machinery and Std. for Textile Machinery and Std. for Textile Machinery and Rec. for Textile Machinery and Std. for Textile Machinery and Std. for Textile Machinery and Rec. for Textile Machinery and Rec. for Textile Machinery and Rec. for Textile Machinery and raphical Symbols for Hydraulic and Pneumatic Equipment and for Indicating Electrical Measuring Instruments and Their Rec. for Impulse Tests on Cables and Their irect Recording Electrical Measuring Instruments and Their illing Machine Arbors with 7/24 Tapers and Milling Machine Rec. for Rolling Bearings:

Rec. Graphical Symbols: Telecommunication Lines and Heating An/

Rec. for Electrical Installations in Ships: for Tests on Oil Filled and Gas Pressure Cables and Their for Tests on Oil Filled and Gas Pressure Cables and Their for Tests on Oil Filled and Gas Pressure Cables and Their indirect Reading Capacitor Type Pocket Exposure Meters and Rec. for Camera

Electrical Installations in Ships: Accessories, Lighting, aphical Symbols: Machines, Transformers, Primary Cells and aphical Symbols: Machines, Transformers, Primary Cells and aphical Symbols: Machines, Trans'sformers, Primary Cells and

cal Weights from 1 Gramme (Gram) to 10 Kilogrammes (Medium for Rectangular Weights from 5 to 50 Kilogrammes (Medium ditions for General Purpose Parallel Lathes-Test. of the height, with Horizontal or Vertical Spindle-Test. of the ixed Height with Horizontal or Vertical Spindle-Test. of Grinding Wheel Spindle and Reciprocating Table-Test. of Grinding Wheel Spindle and Reciprocating Table-Test. of Determination of Sodium-Gravimetric Meth. Using Uranyl

Plastics-Determination of Light Absorption of Cellulose ation of Viscosity Number and Viscosity Ratio of Cellulose Rec. for Plastics-Determination of Vinyl

nation of the Moisture Content of Nonplasticized Cellulose plastics-Determination of Ash of Unplasticized Cellulose a.c. Machine During Operation at Alternative Voltage (1 a.c. Motor Capacitors (1967)

a.c. Systems (1970)

a.c. $(1971)$

a.c.: Star Delta (1970)

Abbreviation of Titles of Periodicals (1972)

Abbreviations of Generic Names in Titles of Periodicals

Abbreviations of Typical Words in Bibliographical Refer

Abbreviations (Symbols) for Plastics (1969)

Abrasive Belts-Designation-Dimensions-Tolerances

Abrasive Products-Grinding Wheel Dimensions-Part 2

Abrasive Products General Features Designation Ranges $\mathrm{O}$

Abrasive Products: Grinding Wheel Dimensions (Part 1) (

Abrasive Sheets and Discs-Dimensions (1972)

Abrasive Sleeves: Designation, Dimension, Tolerance (19

Abrasive Sleeves: Designation, Dimensions, Tolerances

Absolute Density; Pyknometer Meth. (1968)

ISO

Absorption and of Swelling in Thickness After Immersion

Absorption Coefficients in a Reverberation Room (1963) ISO

Absorption Meth. (1971) /M Oxide Primarily Used for Th ISO

Absorption Meth. (1971) /Marily Used for the Productio ISO

Absorption of Cellulose Acetate Before and After Heatin ISO

Absorption of Paper or Board (Cobb Meth.) (1967) ISO

Absorption of Water (1972)

Absorption (1958)

Absorption (1959)

Absorptive Power by Colorant Drop Test with Prior Acid

Abstracts and Synopses (1961)

Accelerated Ageing and Simulated Service Test of Fabric

Accelerated Ageing or Heat Resistance Tests (1971)

Accelerated Ageing Tests (1972)

Accelerated Conditioning of Test Specimens of Polyamide

Accelerated Corrosion Tests on Coatings Other Than Thos

Accelerated Corrosion Tests on Coatings Other Than Thos
Accelerated Damp Heat (1960)

Accelerated Test. for Atmospheric Corrosion (1971) IEC

Acceleration, Steady State (1968) /Ironmental Test. Pr IEC

Acceptance Inspection (1970)

Acceptance of New Sulphur Hexafluoride (1971)

Acceptance Tests of Hydraulic Turbines (1963)

Acceptance Tests of Hydraulic Turbines (1965)

Acceptance Tests of Storage Pumps (1968)

Acceptance Tests (1962)

Acceptance Tests (1973)

Acceptance (1971)

Accessories-Cylindrical Tubes for Tape Yarns (1972)

Accessories-Nonreversible Rings for Ring Spinning and

Accessories-Pirn and Cross Winders-Definition of SI

Accessories-Reversible Rings for Ring Spinning and Do

Accessories-Spindle Gauges for Ring Spinning and Doub

Accessories-Tubes for Draw Winders for Man Made Fibre

Accessories-Weaving Preparatory Machines-Definition

Accessories-Worsted and Woollen Cards-Working Width

Accessories for Fluid Power Transmission (1970)

Accessories (1960)

Accessories (1966)

Accessories (1968)

Accessories (1968)

Accessories (1969)

Accessories (1971)

Accessories, Lighting, Accumulator (Storage), Batteries

Accessories: External, for Alternating Voltages Up to 2

Accessories: Internal, for Alternating Voltages Up to 2

Accessories: Paper Insulated, Metal Sheathed, for Alter

Accessory Electrometers (1971)

Accessory Shoes (1966)

Accumulator (Storage), Batteries, Heating and Cooking a

Accumulators (1960)

Accumulators: Aerials (Antennas) and Radio Stations (19

Accumulators: Microwave Technology (1968)

Accuracy Class) (1970)

Accuracy Class) (1972)

Accuracy (1970)

Accuracy (1970)

Accuracy (1971)

Accuracy (1971)

Accuracy (1971)

Niernational

IEC

Rec. for Cylindri OIML

International Rec OIML

Rec. for Test Con ISO

IG Machines with Table of of Variable ISO

/ for Milling Machines with Table of F ISO

/Ace Grinding Machines with Horizontal ISO

/Rface Grinding Machines with Vertical ISO

Acetate and Magnesium Acetate (1970) / Industrial Use ISO

Rec. for ISO
/Plastics-Determin ISO

Acetate Before and After Heating (1970)

Acetate in Dilute Solution (1970)

Acetate in Vinyl Chloride-Vinyl Acetate Copolymers (197 ISO

Acetate (1967)

Acetate (1968) 
Determination of Free Acidity of Unplasticized Cellulose astics-Determination of Insoluble Particles in Cellulose Determination of Viscosity Loss on Moulding of Cellulose ermination of Acetic Acid Yield of Unplasticized Cellulose ium-Gravimetric Meth. Using Uranyl Acetate and Magnesium ion of Ethyl Ether Soluble Matter in Plasticized Cellulose

8)

9)
68 )

ec. for Plastics-Determination for Rec. for Rec. for

cids Other Than Formic (Less Than $0.5 \%(\mathrm{~m} / \mathrm{m}$ ) Calculated as

e Extract from Brown Coals and Lignites-Determination of Rec. for Plastics-Determination of the Percentage of

e Unmoulded Stal Rec for Plastics-Determination of the ree Alcohols Content by Determination of Ester Value After

n Content; 2,2'-Bipyridyl Spect/

idue on Ignition; Gravimetric Meth. (/

al Acidity and Calculation of Free So/

al Nitrogen Content: Volumetric Meth./

phur Dioxide Content: Barium Sulphate/

ides of Nitrogen-2,4-Xylenol Spec/

ithiocarbamate Photometric Meth. (1972)

I Use-Determination of Manganese Conten/ Insoluble in Water or in nol: Ortho, Meta, and Para Cresol, Cresylic for Phenol: Ortha, Meta, and Para Cresol, Cresylic

Densit/ Rec for Phenol: Ortha, Meta, and Para Cresol, Cresylic

Distillatio Rec. for Liquefied Phenol: Meta Cresol, Cresylic Residue on Range (1971)

Ortha Cresol Content (1971)

olour (Color) (1971)

of Hydrogen Sulphide (1971) Rec. for Acetic

Rec. for Sulphuric

Rec. for Sulphuric

Rec. for Sulphuric

Rec. for Sulphuric

Rec. for Sulphuric

td. for Sulphuric

Std. for Boric

Std for Boric

Acetate (1969)

Acetate (1970)

Rec. for Cres.

Rec. for Cresylic

Rec. for Cresyli

Rec. for Cresylic

on of the Resistance of Glass to Attack by $6 \mathrm{~N}$ Hydrochloric sulphuric Acid for Industrial Use: Evaluation of Sulphuric or Hydrochloric Acid for Industrial Use: Evaluation of Its lumetric Meth. (1971)

2, 2'-Bipyridyl Photometric Meth/

: 2, 2'-Bipyridyl Photometric Meth/

$-2,2$ '-Bipyridyl Spectrophot/

- Volumetric Meth. (1972)

of Volatile Acids Other Than Formic (Le/ content (1971)

ounds-Volumetric Meth. (1971)

ontent-Volumetric Meth. (1968)

tent-Volumetric Meth. (1968)

sidue on Ignition-Gravimetric Meth. (1/

2,2'-Bipyridyl Spectrophoto/

reducing Substances Volumetric Met/

ent; Barium Sulphate Gravimetric M/

Gravimetric Meth. (1968)

Volumetric Meth. (1968)

ration by Measurement of Density (/

oncentration by Meaaurement of Densit/ Rec. for Nitri

Rec. for Formic Rec. for Nitric

Rec. for Phosphoric Std. for Boric Rec. for Formic

Rec. for Cresylic Rec. for Nitric

Rec. for Phosphoric

Rec. for Phosphoric Rec. for Nitric

Rec. for Hydrochloric Rec. for Hydrochloric Rec. for Hydrochloric

Rec. for Hydrochloric Rec. for Hydrochloric Rec. for Hydrochloric

Rec. for Sulphuric Rec for Formi

Rec. for Determination of Boric Rec. for Pulps: Determination of Rec. for Spices and Condiments: Determination of Rec. for Tea-Determination o

Rec for Dimensions of Lead nals (1963) Rec. for Determination of Volatile Fatty Rec. for Lead Rec. for Lead Rec. for Lead of Test (1972)

Loss of Absorptive Power by Colorant Drop Test with Prior e Solution for Analysis by Means of Attack by Hydrochloric Rec. for Butter-Determination of the Rec. for Essential Oils-Determination of Rec. for Plastics-Determination of Acetic

d Residues: Determination of Ash Insoluble in Hydrochloric or Industrial Use-Test for Colour (Color) with Sulphuric

Products-Determination of Ash Insoluble in Hydrochloric ion of Sodium and Potassium Soluble in Dilute Hydrochloric

ontent-Spectrophotometric Meth. Using Naphthalhydroxamic ion of Titanium Spectrophotometric Meth. with Chromotropic um Borates for Industrial Use: Determinat/ Std. for Boric

cerine for Industrial Use-Determination of Alkalinity or rec. for Nitric Acid for Industrial Use-Determination of

ammonium Nitrate for Industrial Use: Determination of Free Rec for Oleaginous Seeds: Determination of Rec, for Plastics: Determination of Free or Potassium Sulphate for Industrial Use: Determination of
Acetate (1970)

Acetate (1970)

Acetate (1970)

Acetate (1971)

Acetates for Industrial Use-Meth. of Test (1970)

Acetic Acid and for Industrial Use-Meth. of Test (196

Acetic Acid Yield of Unplasticized Cellulose Acetate (1

Acetic Anhydride for Industrial Use-Meth. of Test (19

ISO

Acetic)-Volumetric Meth. (1971)

Acetone for Industrial Use-Meth. of Test (1968)

Acetone Soluble Material (Resinous Substances) (1969)

Acetone Soluble Matter in Phenolic Mouldings (1958)

Acetone Soluble Matter (Resin Content of Material in Th

Acetylation (1971)

/R Essential Oil

Acid and for Industrial Use-Meth. of Test (1968)

Acid and Oleum for Industrial Use: Determination of Iro

Acid and Oleum for Industrial Use: Determination of Res

Acid and Oleum for Industrial Use: Determination of Tot

Acid and Oleum for Industrial Use: Determination of Tot

Acid and Oleum for Industrial Use; Determination of Sul

Acid and Oleums for Industrial Use: Determination of $O x$

Acid and Oxide, and Disodium Tetraborates for Dibenzyld

Acid and Oxide, and Disodium Tetraborates for Industria

Acid and Preparation of Principal Solutions for Other D

Acid and Xylenols for Industrial Use-Determination of

Acid and Xylenols for Industrial Use-Determination of

Acid and Xylenols for Industrial Use-Determination of

Acid and Xylenols for Industrial Use-Determination of

Acid and Xylenols for Industrial Use-Determination of

Acid and Xylenols for Industrial Use-Determination of

Acid and Xylenols for Industrial Use-Determination of

Acid and Xylenols for Industrial Use-Measurement of $\mathrm{C}$

Xylenols for Industrial Use-Test for Absence

Acid at $100{ }^{\circ} \mathrm{C}(1970) \quad$ Rec. for Determinati ISO

Acid Concentration by Measurement of Density (1968)

Acid Concentration by Measurement of Density (1968)

Acid for Industrial Use-Determination of Acidity-Vo

Acid for Industrial Use-Determination of Iron Content

Acid for Industrial Use-Determination of Iron Content

Acid for Industrial Use-Determination of Iron Content

Acid for Industrial Use-Determination of Its Content

Acid for Industrial Use-Determination of Low Contents

Acid for Industrial Use-Determination of Meta Cresol

Acid for Industrial Use-Determination of Nitrous Comp

Acid for Industrial Use-Determination of of Calcium C

Acid for Industrial Use-Determination of Sulphate Con

Acid for Industrial Use-Determination of Sulphated Re

Acid for Industrial Use: Determination of Iron Content:

Acid for Industrial Use: Determination of Oxidizing or

Acid for Industrial Use: Determination of Sulphate Cont

Acid for Industrial Use: Determination of Sulphated Ash

Acid for Industrial Use: Determination of Total Acidity

Acid for Industrial Use: Evaluation of Its Acid Concent

Acid for Industrial Use: Evaluation of Sulphuric Acid C

Acid for Industrial Use: Meth. of Test (1968)

Acid in Latex (1970)

Acid Insoluble Ash (1968)

Acid Insoluble Ash (1969)

Acid Insoluble Ash (1970)

Acid Motor Scooter Batteries (1965)

Acid Number of Latex (1966)

Acid Starter Batteries: Dimensions and Marking of Termi

Acid Starter Batteries: Dimensions of Batteries (1965)

Acid Starter Batteries: General Requirements and Meth.

Acid Traction Batteries (1967)

Acid Treatment (1971)

Acid Under Pressure (1971)

Acid Value of the

Acid Value (1971)

Acid Yield of Unplasticized Cellulose Acetate (1970)

Acid (1968)

Acid (1970)

Acid (1971)

Acid (1971)

Acid (1971)

Acid) (1969) /Uminum and Aluminium Alloys-Determinat

Acidity-Volumetric Meth. (1970)

/ Its Alloys-Estimation of the ISO

/Ium-Preparation of SampI ISO

Acidity-Volumetric Meth. (1971)

Acidity-Volumetric Meth. (1972)

Acidity and Calculation of Free $\mathrm{SO}_{3}$ Content of Oleum;

Acidity of Oils (1968)

Acidity of Unplasticized Cellulose Acetate (1969)

Acidity to Methyl Orange (1973)

Rec. for Oilsee ISO

Rec. for Higher Alcohols F ISO
Rec. for Fruit and Vegetable ISO

ISO

ISO

1776

911

905

1980

1707

1982

849

1914

1913

1911

1981

848 
for Iligher Alcohols for Industrial Use-Determination of Rec. for Crude Vegetable Oils and Fats: Determination of Fruit and Vegetable Products: Determination of Titratable ted Hydrocarbons for Industrial Use-Determination of the ormaldehyde Solutions for Industrial Use: Detcrmination of drochloric Acid for Industrial Use: Determination of Total industrial Use-Determination of Low Contents of Volatile nimal Fats: Determination of Solidification Point of Fatty for Analysis of Soap: Determination of Total Crude Fatty alves: Meth. of Measurement of Noises Due to Mechanical or Rec. for Sound Signalling Devices on Motor Vehicles Rec. for Preferred Frequencies for

ty Response (1971)

or Hearing Conservation Purposes (1971)

erties of Sonic Booms (I973) Rec. for Hardness (1972)

h. of Analysis (1969)

sample (1967)

of the Cloud Point Temperature of Nonionic Surface Glycols and Nonionic Active Matter (Ad/ Contents of Free Glycerol-Spectropho/ tive Matter (Direct Two Phase Titration/ tes on Filling (1969)

hing Powders Before and After Compactio (1969)

\section{erfacial Tension (1963)}

ture of Nonionic Surface Active Agents

rmination of Oxyethylene Groups (1972)

\section{of Analysis (1968)}

Meth. of Analysis (1968)

s: Meth. of Analysis (I968)

ering-Preparation and Use of Unsoiled/

power Sources for Portable Prospecting Equipment for Radio

$r$ and Land Vehicle Mounted Prospecting Equipment for Radio ionic): Determination of Polyethylene Glycols and Nonionic of Chemical Analysis of Manganese Ores-Determination of Rec. for Numeric Section of Ten Key Keyboards for Rec. for Keytop and Printed or Displayed Symbols for

Rec. for Picture Postcards: Area Reserved for the s (1972) Std. for Surface Active Agents: Ethylene Oxide ec. for Preparation of Test Piece and Meth. of Test of the ec. for Preparation of Test Piece and Meth. of Test of the (Quadruple-Shear Test) (I971) ics (1969) s (1960)

Rec. for Determination of the Rec. for Limiting Values for the 1964)

Rec. for Principles of Construction and Rec. for Principles of Construction and Rec. for Principles of Construction and f Electronic Tubes and Valves: Equivalent Input and Output r Radio Frequency Connectors: Coaxial Unmatched Television Frequency Connectors: Two Pin Connector for Twin Balanced

easurement of Essential Electrical Properties of Receiving s: Machines, Transformers, Primary Cells and Accumulators: s Mechanical Handling Equipment for Loose Bulk Materials

h Rubber or Plastics (1970) Rec for Vulcanized Rubbers-Accelerated

ss (1972)

nalysis (1969)

(I967) $s$ and Nonionic Active Matter (Ad/ ts of Free Glycerol-Spectropho/ tter (Direct Two Phase Titration/ filling (I969)

wders Before and After Compactio/

l Tension (I963)

Nonionic Surface Active Agents / on of Oxyethylene Groups (1972) Rec. for Classification of Rec. for Ply

Rec. for Determination of Rec. for Dimensions of

Rec. for Accelerat

Acidity to Phenolphthalein (1970)

Acidity (1968)

Acidity (1968)

Acidity (1970)

Acidity (1972)

Acidity: Volumetric Meth. (1968)

Acids Other Than Formic (Less Than $0.5 \%(\mathrm{~m} / \mathrm{m})$ Calculate Acids (Titre) (1969)

Acids (1965)

Acoustic Excitations (1968)

Acoustic Std. and Technical Spec. (1966)

Acoustical Measurements (1962)

Acoustics-Assessment of Noise with Respect to Communi

Acoustics-Assessment of Occupational Noise Exposure F

Acoustics (1965)

Acoustics: Description and Measurement of Physical Prop

Active Agents-Technical Sodium Alkylsulphonates-Met

Active Agents-Vocabulary (1969)

Active Agents in Powder Form: Preparation of a Reduced

Active Agents Obtained from Ethylene Oxide (1969)

Active Agents (Nonionic): Determination of Polyethylene

Active Agents: Analysis of Soaps-Determination of Low

Active Agents: Detergents-Determination of Anionic-Ac

Active Agents: Determination of Apparent Density of Pas

Active Agents: Determination of Apparent Density of Was

Active Agents: Determination of Stability in Hard Water

Active Agents: Determination of Surface Tension and Int

Active Agents: Determination of the Cloud Point Tempera

Active Agents: Ethylene Oxide Adducts-Iodometric Dete

Active Agents: Glossary (1971)

Active Agents: Measurement of Foaming Power (1968)

Active Agents: Scientific Classification (1968)

Active Agents: Simplified Classification (I972)

Active Agents: Technical Sodium Alkylsulphonates: Meth.

Active Agents: Technical Sodium Primary Alkylsulphates

Active Agents: Tcchnical Sodium Secondary Alkylsulphate

Active Agents: Verification of Certain Effects of Laund

Active Materials (1965)

Active Materials (1967)

Active Matter (Adducts) - Weibull Meth (197?)

Rec. for IEC
Rec, or Power Supply for Ai IEC

Adding and Calculating Machines (I969)

Adding and Calculating Machines (1969)

Adding and Calculating Machines (1969)

Address (1965)

Adducts-Iodometric Determination of Oxyethylene Group

(Adducts)-Weibull Meth. (1972)

Adhesion of Conveyor Belis (1962)

Adhesion of Vulcanized Rubber to Metal Where the Rubber

Adhesion of Vulcanized Rubber to Metal Where the Rubber

Adhesion of Vulcanized Rubbers to Rigid Plates in Shear

Adhesion Strength of Vulcanized Rubbers to Textile Fabr

Adjustment of Centres (Centers) for Transmission Pulley

Adjustment of Hydrometers (1964)

Adjustment of Liquid in Glass Laboratory Thermometers

Adjustment of Volumetric Glassware (1964)

Admittances (1963) -

Admittances (1963)

Aerial Feeders (1965) Rec. for Radio IEC

Aerial Rods and Slabs of Ferromagnetic Oxides (1966) IEC

Aerials in the Frequency Range Form $30 \mathrm{MHz}$ to $1,000 \mathrm{MHz}$ IEC

Aerials (Antennas) and Radio Stations (1968)

Aeroslides (1972)

IL. Symbol IEC

Agar Impression Material (1970)

Std. for Continuou ISO

Ageing and Simulated Service Test of Fabrics Coated Wit ISO

Ageing or Heat Resistance Tests (1971)

Ageing Tests (1972)

Agents-Preparation of Water with Known Calcium Hardne

Agents-Technical Sodium Alkylsulphonates-Meth. of a

Agents-Vocabulary (1969)

Agents in Powder Form: Preparation of a Reduced Sample

Agents Obtained from Ethylene Oxide (1969) /Er

Agents (Nonionic): Determination of Polyethylene Glycol

Agents: Detergents-Determination of Anionic-Active Ma

Agents: Determination of Apparent Density of Pastes on

Agents: Determination of Apparent Density of Washing Po

Agents: Determination of Stability in Hard Water (1969)

Agents: Determination of Surface Tension and Interfacia

ISO

ISO

ISO

ISO

ISO

ISO

ISO

ISO

ISO

ISO

ISO

ISO

ISO

Rec. Rec. for Surface Active Std. for Surface Active Rec. for Surface Active Rec. for Surface Active
Agents: Determination of the Cloud Point Temperature of

Agents: Ethylene Oxide Adducts-Iodometric Determinati

Agents: Glossary (1971)

Agents: Measurement of Foaming Power (1968)

ISO

1846

660

750
1393

2225

904.

1913

455

151-18

512

266

1999

31/VII

2249

176

2174

1104

$862 /$ I

607

1065

2268

2272

2271

1064

697

1063

304

1065

2270

862

696

896

2131

893

894

895

2267

201

253

2268

312

1092

1093

1094

416

2270

2268

252

813

814

1747

36

155

387

386

384

151-3

169.2

169-3

223

I38

117-10

2326

1564.

1419

188

2440

2174

1 I04.

$862 / I$

607

1065

2268

2272

2271

1064

697

1063

304

1065
2270

862

696

896 
Iysis (1968)

of Analysis (1968)

of Analysis (1968)

Preparation and Use of Unsoiled/ 2)

nd Packing (1972)

Determination of Nitrogen by the Kjeldahl Meth/

Rec. for General Purpose Rec. for Power Take Off and Draw Bar for Rec. for Test Code for

Implements (1968)

Rec. for 3 Point Linkage of

for IEC Reference Coupler for the Measurement of Hearing Rec. for the Dimensions of Polarized Plugs for Hearing ements of the Electroacoustical Characteristics of Hearing pment Safety) (1961). Rec. for Marking of Control Settings on Hearing

radio Active Materials (1967)

Rec. or Power Supply for Rec. for High Pressure Rec. for Test. and Rating Room

r Safety Requirements for the Electrical Equipment of Room Rec. for Aircraft Connections for Ground irements for Tests and Measuring Meth. (1970) Rec. for

of Wool Fibres (Fibers) (1969) Rec. for Industrial

Rec. for Rec. for Creepage Distances and Clearances in
Ric Components and Equipment: Test M: Low Flight Dynamics-Part I: Aircraft Motion Relative to the Rec. for Field and Laboratory Measurements of tended for Outdoor Use (1972) Std. for Measurement of (1970) Rec. for Test Code for the Measurement of the
Rer nd Fuse Combinat/ Rec. for Low Voltage Airbreak Switches,

for Low Voltage Airbreak Switches, Airbreak Disconnectors, tch Disconnectors and Fuse Combinat/ Rec. for Low Voltage Std. for Sealed Beam Landing Lamps for Rec. for Precision Fuse Links for

Rec. for Liquid Oxygen Replenishment Couplings for Rec. for Elastomeric Sealing Rings for of Elastomeric Toroidal Sealing Rings for Pipe Fittings in for Dimensions of Elastomeric Toroidal Sealing Rings for Std. for Aluminium Terminal Ends for Crimping to for Symbols for Flight Dynamics-Part II: Motions of the Rec. for 5) Rec. for Breaking Strengths of Preformed Stranded Steel Cables for Std. for Flexible Steel Wire Rope for Std. for Crimped Joints for Rec. for Std. Form of Declaration of Performance of Std. for Tests for Rec. for Voltages for Rec. for

ers, and Direction of Rotation of / Rec. for Numbering of

Rec. for Marking of Rec. for Connections for Rec. for Rubber Hoses for (1971) Rec. for Sizes and Mounting Dimensions of Rec. for Figures for Rec. for Rec. for Rec. for Rec. for Heads of Std. for Symbols for Flight Dynamics-Part I: Rec. for Monitoring Rec. for Procedure for Describing Rec. for Identification of Rec. for Rec. for Rec. for Rec. for Rec. for

Rec. for Safety Features for Ground Power Units for d.c. Rec. for Rec. for Rec. for Std. for

for Dimensions of Elastomeric Toroidal Sealing Rings for of Elastomeric Toroidal Sealing Rings for Pipe Fittings in Rec. for Precision Fuse Links for

Rec. for Directions of Operation of Toggle Switches on Rec. for Gravity Filling Orifices for

(Color) Identification of Mechanical Control Circuits for ions for Single Hole Mounting, Lever Operated Switches for general Purpose Push Pull Single Pole Circuit Breakers for
Agents: Simplified Classification (1972)

Agents: Technical Sodium Alkylsulphonates: Meth. of Ana Agents: Technical Sodium Primary Alkylsulphates: Meth.

Agents: Technical Sodium Secondary Alkylsulphates: Meth Agents: Verification of Certain Effects of Laundering-

Agglomerated-Deformation Under Constant Pressure (197 Agglomerated-Determination of Bulk Density (1972)

Agglomerated Thermal Cork-Characteristics, Sampling a

Agricultural Food Products-General Directions for the

Agricultural Rubber Spray Hose (1970)

Agricultural Tractors (1966)

Agricultural Tractors (1968)

Agricultural Wheeled Tractors for Attachment of Mounted

Aids Using Earphones Coupled to the Ear by Means of Ins

Aids (1957)

Aids (1959)

Aids (1966)

Aim, Structure, and Results of the CEE (Electrical Equi

Air and Land Vehicle Mounted Prospecting Equipment for Air Charging Valves for Aircraft (1969)

Air Conditioners (1968)

Air Conditioners (1972)

Air Conditioning (1969)

Air Dielectric Rotary Variable Capacitors: General Requ Air Hose (1970)

Air Permeability Meth. for Measuring the Mean Diameter

Air Pressure (1966)

Air (1964)

Air (1972)

Airborne and Impact Sound Transmission (1960)

IR Basic Environmental Test. Proce IEC

Airborne Noise Emitted by Compressor Primeover Units in

Airborne Noise Emitted by Rotating Electrical Machinery

Airbreak Disconnectors, Airbreak Switch Disconnectors a

Airbreak Switch Disconnectors and Fuse Combination Unit

Airbreak Switches, Airbreak Disconnectors, Airbreak Swi

Aircraft-Dimensions (1972)

Aircraft-General Requirements (1971)

Aircraft-Interchangeability Dimensions (1971)

Aircraft-Packaging and Identification (1971)

Aircraft-(In. Series-Class 2 Tolerances) (1971)

Aircraft-(In. Series-Class 2 Tolerances) (1971)

Aircraft Aluminum Electrical Cables (1973)

Aircraft and the Atmosphere Relative to the Earth (1969

Aircraft Connection for Water of Drinkable Quality (196

Aircraft Connections for Ground Air Conditioning (1969)

Aircraft Controls (1967) / Designations, Diameters and

Aircraft Controls: Technical Spec. (1973)

Aircraft Electrical Cables (1973)

Aircraft Electrical Equipment (1961)

Aircraft Electrical Plug and Socket Connectors (1972)

Aircraft Electrical Systems (1961)

Aircraft Engine Nacelle Fire Extinguisher Doors (1969)

Aircraft Engines, Engine Cylinders and Combustion Chamb

Aircraft Fuel Nozzle Grounding Plugs and Sockets (1970)

Aircraft Gas Cylinders (1965)

Aircraft Ground Electrical Supplies (1965)

Aircraft Ground Fueling Without Static Conducting Wire

Aircraft Instrument Cases (Rear Mounting Type) (1959)

Aircraft Instrument Dials and Number Plates (1962)

Aircraft Instrument Dials and Pointers (1962)

Aircraft Jacking Pads (1957)

Aircraft Lockwire (1962)

Aircraft Lubricating Nipples (1965)

Aircraft Motion Relative to the Air (1972)

Aircraft Noise Around an Airport (1970)

Aircraft Noise Around an Airport (1970)

Aircraft Pipelines (1970)

Aircraft Pressure Cabin Ground Test Connection (1954)

Aircraft Pressure Refueling Connections (1957)

Aircraft Pressure Reoiling Connection (1965)

Aircraft Sealing Wire (1962)

Aircraft Seat Rails and Pins (1968)

Aircraft Servicing and Engine Starting (1961)

Aircraft Toilet Flushing and Draining Connections (1957

Aircraft Tow Bar Connections to Tractors (1964)

Aircraft Tyre (Tire) Valves (1965)

Aircraft Water Methanol Pressure Connections (1973)

Aircraft (In. Series-Class 1 Tolerances) (1969)

Aircraft (In. Series-Class 1 Tolerances) (1969)

Aircraft (Type A) (1971)

Aircraft (Type B) (1971)

Aircraft (1957)

Aircraft (1959)

Aircraft (1959)

Aircraft (1966)

Aircraft (1966)
Rec. Meth. for Measur

Std. for Symbols for ISO

ISO

ISO

ISO

ISO

ISO

ISO

ISO

ISO

ISO

ISO

IEC

IEC

IEC

CEE

IEC

ISO

ISO

IEC

(5O

IEC

ISO

ISO

ISO

IEC

IEC

IEC

ISO

ISO

ISO

ISO

I ISO

/C ISO

ISO

ISO

ISO

ISO

ISO

ISO

ISO

ISO

ISO

ISO

ISO

ISO

ISO

ISO

ISO

ISO

ISO

ISO

ISO

ISO

ISO

ISO

ISO

ISO

ISO

ISO

ISO

ISO

ISO

ISO

ISO

ISO

ISO

ISO

ISO

ISO

Rec ISO

/Ns ISO

ISO

ISO

ISO

ISO

Rec for Colour ISO

Rec. for Colour ISO
Rec. for Dimens ISO
Rec. Fo IEC

Rec. for Dimensions for ISO

2131

893

894

2267
$219]$

2189

2219

1871

1401

500

789

126

90
118

224

253

1023

859

78

034

334-1

1404

136

68-2-13

REC

1151

140

2151

1680 
Index of International Standards

neral Purpose Electrical Cables with Copper Conductors for $\left(190^{\circ} \mathrm{C}\right)$ Electrical Cables with Copper Conductors, for eral Purpose Electrical Cables with Copper Conductors, for neral Purpose Electrical Cables with Copper Conductors for $\left(260^{\circ} \mathrm{C}\right)$ Electrical Cables with Copper Conductors for ec. for Requirements for 28 Volt d.c. Flat Strip Fuses for Rec. for Gaseous Oxygen Replenishment Couplings for Rec. for High Pressure Air Charging Valves for

hape, Size and Direction of Operation of Lever Controls on for General Purpose Push Pull 3 Pole Circuit Breakers for cal Cables with Aluminium or Aluminum Alloy Conductors for $\left(190{ }^{\circ} \mathrm{C}\right)$ Electrical Cables with Copper Conductors for Rec, for Clearance Dimensions for Tripod Jacks for rmance Requirements for Lever Operated Manual Switches for

for General Purpose Push Pull 3 Pole Circuit Breakers for $\left(260{ }^{\circ} \mathrm{C}\right.$ ) Electrical Cables with Copper Conductors for

cal Cables with Aluminum or Aluminium Alloy Conductors for ng (190 Deg.) Electrical Cables with Copper Conductors for general Purpose Push Pull Single Pole Circuit Breakers for $\left(260{ }^{\circ} \mathrm{C}\right)$ Electrical Cables with Copper Conductors for Std. for Heat Resisting Equipment Wires for Std. for Static Invertors for Std. for ectors for Automatic Coupling (1973) Std. for Heat Resisting Equipment Wires for for ISO Identification Code for Rolling Bearings: Group V olerances, Permissible Load (/ Rec. for Rolling Bearings: Rec. for Monitoring Aircraft Noise Around an rec. for Procedure for Describing Aircraft Noise Around an Rec. for Isopropyl

Rec for Spices and Condiments: Determination of of Alkalinity or Acidity / of Sulphated Ash in Produ/ est Meth.-General (1970)

acetylation/ Rec. for Aliphatic Polyhydric Rec. for Aliphatic Polyhydric Rec. for Aliphatic Polyhydric igher Alcohols for Industrial Use-Determination of Total t $20^{\circ} \mathrm{C}$ (1970)

to Phenolphthalein (I970)

vimetric Meth.) (1970)

index in the Presence of Mercury (II) Ch/

Compounds (Hydroxylammonium Chloride Po/ tion Yield (1970)

cohols Content (Volumetric Meth.) (1970)

ntent by the Karl Fischer Meth. (I970)

lor) in Hazen Units (I970)

ith Sulphuric Acid (1970)

Rec. for Higher Rec. for Higher Rec. for Higher Rec. for Higher Rec. for Higher Rec. for Higher Rec, for Higher Rec. for Higher Rec, for Higher Rec. for Higher Rec. for

Std. for Programming Language: Rec. for Rolling Bearings-Double Row Self I Use-Samples and Test Meth.-General (I970) Rec. for I Use-Determination of Alkalinity or Acidity / Rec. for 1 Use-Determination of Sulphated Ash in Produ/ Rec, for sium and Its Alloys-Determination of Insoluble Zirconium esium and Its Alloys-Determination of Soluble Zirconium Rec. for Pulps: Determination of Rec. for Pulps: Determination of Rec. for Analysis of Soaps: Determination of Total Free Rec. for Analysis of Soaps: Determination of Total of Glass to Attack by a Boiling Aqueous Solution of Mixed Std. for Analysis of Soap: Determination of Free Caustic ustrial Use (Including Foodstuffs): Determination of Total Rec. for Determination of Rec. for Tea-Determination of alcohols-Clycerine for Industrial Use-Determination of Rec. for Urea for Industrial Use-Determination of sium Silicates for Industrial Use-Determination of Total for Sodium Hydroxide for Industrial Use: Determination of

rbonate for Industrial Use: Determination of Total Soluble r Potassium Hydroxide for Industrial Use: Determination of Rec. for Surface Active Agents: Technical Sodium Primary rec. for Surface Active Agents: Technical Sodium Secondary Rec. for Surface Active Agents-Technical Sodium Rec. for Surface Active Agents: Technical Sodium

ment (1973) and Tempered Types with I\% Chromi/ Rec. for Heat Treated, n Type (1959) Rec. for Heat Treated Aluminium (Aluminum) Std. for Magnesium Zinc Zirconium Rec. Cor Comesium Aluminium (Aluminum) Zinc Rec. for Composition of Aluminium (Aluminum) Sid for Aluminium (Aluminum)

Rec. for an International Spec. for Aluminium (Aluminum) neral Purpose Electrical Cables with Aluminium or Aluminum neral Purpose Electrical Cables with Aluminum or Aluminium Rec. for Aluminium (Aluminum)

Rec. for

Rec. for Mechanical Property Limits for Aluminium
Aircraft (1966)

Aircraft (1966)

Aircraft (1966)

Aircraft (1967)

Aircraft (1967)

Aircraft (1968)

Aircraft (1969)

Aircraft (1969)

Aircraft (1969)

Aircraft (1969)

Aircraft (1969)

Aircraft (1969)

Aircraft (1970)

Aircraft (1970)

Aircraft (1970)

Aircraft (1970)

Aircraft (1970)

Aircraft (1970)

Aircraft (1970)

Aircraft (1970)

Aircraft (1973)

Aircraft (1973)

Aircraft: Interchangeability Dimensions of Battery Conn

Aircraft: Meth. of Test (1973)

Airframe Type (1968)

Airframe: Introduction. General, Boundary Dimensions, T

Airport (1970)

Airport (1970)

Alcohol for Industrial Use-Meth. of Test (1968)

Alcohol Soluble Extract (1969)

Alcohols-Glycerine for Industrial Use-Determination

Alcohols-Glycerine for Industrial Use-Determination

Alcohols-Glycerine for Industrial Use-Samples and T

Alcohols Content by Determination of Ester Value After

Alcohols Content (Volumetric Meth ) (1970)

Alcohols for Industrial Use-Determinaion of Density a

Alcohols for Industrial Use-Determination of Acidity

Alcohols for Industrial Use-Determination of Ash (Gra

Alcohols for Industrial Use-Determination of Bromine

Alcohols for Industrial Use-Determination of Carbonyl

Alcohols for Industrial Use-Determination of Distilla

Alcohols for Industrial Use-Determination of Total Al

Alcohols for lndustrial Use-Determination of Water Co

Alcohols for Industrial Use-Measurement of Colour (Co

Alcohols for Industrial Use-Test for Colour (Color) W

Alginate Impression Material (1970)

ALGOL (1972)

Aligning Ball-Radial Internal Clearance (1970)

Aliphatic Polyhydric Alcohols-Glycerine for Industria

Aliphatic Polyhydric Alcohols-Glycerine for Industria

Aliphatic Polyhydric Alcohols-Glycerine for Industria

Alizarin Sulphonate Photometric Meth. (1972)

(Alizarin Sulphonate Photometric Meth.) (1970)

Alkali Resistance (I968)

Alkali Solubility (I968)

Alkali (I968)

Alkali (1968)

Alkali (1968)

Alkali (1973)

Alkalinity-Volumetric Meth. (I973)

Alkalinity of Latex (1966)

Alkalinity of Water Soluble Ash (1970)

Alkalinity or Acidity-Volumetric Meth. (1970)

Alkalinity Volumetric Meth. (1970)

Alkalinity, Expressed in $\mathrm{Na}_{2} \mathrm{O}$ or in $\mathrm{K}_{2} \mathrm{O}-\mathrm{Volumetr}$

Alkalinity, Volumetric Meth. (1969)

Alkalinity: Volumetric Meth. (1968)

Alkalinity: Volumetric Meth. (1969)

Alkylsulphates: Meth. of Analysis (1968)

Alkylsulphates: Meth. of Analysis (1968)

Alkylsulphonates-Meth. of Analysis (1969)

Alkylsulphonates: Meth. of Analysis (1968)

Allocations for Use with High Speed Data Terminal Equip

Alloy and Free Cutting Steels, Pt. 4: Wrought Guenched

Rec. ISO

ISO

ISO

ISO

ISO

ISO

Alloy Busbar Material of the Aluminium Magnesium Silico

Alloy Castings-Chemical Composition (1972)

Alloy Castings and Mechanical Properties of Sand Cast R

Alloy Castings (Complement to R 164) (1961)

Alloy Castings (1960)

Alloy Chill Castings-Reference Test Bar (1972)

Alloy Conductor Wire of the Aluminium Magnesium Silico

Alloy Conductors for Aircraft (I969)

Alloy Conductors for Aircraft (1970)

Alloy Conductors, Steel Reinforced (1966)

Alloy for Dental Amalgam (1970)

Alloy Forgings (1968)
474

470

469

634

539

854

1023

440

1033

1076

1075

1464

1466

1509

1491

1468

1220

1467

1490

2032

2277

I 963

2436

300/III

1002

1761

507

756

940

1615

1616

I614

124

1850

1844

1846

1851

1848

1847

1845

1850

1849

1843

1852

1563

1538

1646

1614

1615

1616

2354

1178

699

692

684

685

695

456

2516

125

1578

I6 I5

1593

1692

979

740

990

894

895

1104

893
2593

$683 / \mathrm{IV}$

114

2119

121

208

164

2378

104

1076

1468

210

1559

829 
rances of Electrodes for the Welding of Mild Stcel and Low ual Ar. Welds Made with Covered Electrodes of Mild and Low ols for Filler Rods for Gas Welding of Mild Steels and Low Covered Electrodes for Arc Welding of Mild Steels and Low posited by Fillcr Rods for Gas Welding Mild Steels and Low ec. for Composition of Magnesium Aluminium (Aluminum) Zinc 0.1 In.)-Tensile Test (1971) Rec. for Zinc

Rec, for Copper and Std for Magnesium Std. for Aluminium (Aluminum) imensions and Sectional Properties of Aluminium (Aluminum) Rec. for Simple Bend Covered Electrodes for Manual Arc Welding of Mild and Low уре) (1966) Rec. for Aluminium Rec. for Copper and Its Rec. for Tensile Test, of Copper and Its Rec for Drift Expanding Test on Copper and Its Rec, for Tensile Test, of Light Metal Ad Its Rec. for Drift Expanding Test on Light Metal and Its Rec. for Flattening Test on Aluminum and Aluminium Rec. for Wrapping Test for Copper and Its Rec. for Tensile Test of Copper and Its Rec. for Simple Torsion Test for Aluminum and Aluminium Rec. for Wrapping Test for Aluminum and Aluminium Rec. for Tensile Test for Light Metal and Its Alloys: Electrolytic Determination of Copper in Aluminium Rec. for Dental Casting Gold

\section{ding Type (1970)}

hardening Type (1970)

uction Hardening Types (1972)

empered Unalloyed Types (1968)

hed and Tempered Types with $1 \% \mathrm{Ch} /$

ed and Tempered Unalloyed with Co/

ed and Tempered Manganese (1970)

ed and Tempered, with $3 \%$ Chromium/

ed and Tempered Chromium Nickel M/

utting Type (1970)

$$
\text { for Sirip Wound Cut Cores of G }
$$

Rec. for Wrought
Rec. for Light Metals and Their

nt of Metals-Anodisation of Aluminium (Aluminum) and Its Composition of Wrought Products of Aluminium and Aluminum 1) Rec, for Copper and Its

Rec. for Light Metals and Their

std for Chemical Analysis of Aluminium (Aluminum) and Its earths, Thorium and Silver -1

Meth. Using Diphenylcarbazid/ Std. for Magnesium and Its Std. for Aluminium and Its Rec. for Chemical Analysis of Zine sulphonate Photometric Meth. / Std. for Magnesium and Its
h. (1972/ Std. for Chemical Analysis of Magnesium and Its h. (1972/ Std. for Chemical Analysis of Magnesium and Its
meth. Wi/ ulphonat/ Rec. for Chemical Analysis of Magnesium and Its ment of Metals-Coloured Anodisation of Aluminium and Its eth/ Rec. for Chemical Analysis of Aluminum and Aluminium Rec. for Chemical Analysis of Magnesium and Its
rec. for Chemical Analysis of Aluminium (Aluminum) and Its ontaining N/ Rec. for Chemical Analysis of Copper and Its -Copper All/ Rec. for Chemical Analysis of Copper and Its nt of Metals-Anodisation of Aluminium (Aluminum) and Its ization (Anodic Oxidation) of Aluminium (Aluminum) and Its isation (Anodic Oxidation) of Aluminium (Aluminum) and Its isation (Anodic Oxidation) of Aluminium (Aluminum) and Its (197I) Rec. for Chemical Analysis of Copper and Its
Rec. for Aluminium (Aluminum) Rec. for Wrought Aluminum and Aluminium Rec. for Aluminium and Aluminum e Alloys (1/ Rec. for Chemical Analysis of Copper and Its w Contents)/ Rec. for Chemical Analysis of Copper and Its Rec. for Chemical Analysis of Zinc and Its Rec. for Light Metals and Their ) (1971) m) (1970) Rec for Light Metals and Their fusion Welded Butt Joints for Aluminium (Aluminum) and Its Rec, for Shipbuilding Details-W rought Aluminum Aluminium (Aluminum) and Its Alloys and Magnesium and Its lloys: Gravimetric Determination of Aluminium in Magnesium Rec. for Vickers Hardness Test for Copper and Its Its Alloys: Gravimetric Determination of Zinc in Aluminum d, Drawn and Extruded Products in Light Metals or in Light Rec for Tensile Test. of Light Metals and Their ec. for Meth. of Mercurous Nitrate Test for Copper and Its Rec. for Bend Test for Copper and Its Rec, for Tensile Test. of Copper and Its Rec. for Brinell Hardness Test for Copper and Its
Alloy High Tensile Steel (1967) Alloy High Tensilc Steels (1967) Alloy High Tensile Types (I967) Alloy High Tensile Types (1967) Alloy High Tensile Types (1967) Alloy Ingots for Casting Purposes (1959)

/ for lengths and Tole ISO /Ons for Straight Man ISO Rec. for Code of Symb ISO /R Code of Symbols for ISO /Ties of Weld Metal De ISO

Alloy Ingots (1963)

Alloy Rolled Flat Products (Thickness Less Than $2.5 \mathrm{~mm}$

Alloy Sand Castings-Reference Test Bar (1972)

Alloy Sand Castings: Reference Test Bar (1972)

Alloy Sections for Marine Use (1970)

Alloy Sheet and Strip of Thickness Between $0.2 \mathrm{~mm}(0.00$

Alloy Stranded Conductors (Aluminum Magnesium Silicon T

Alloy Tubes of Circular Section-Flattening Test (1971

Alloy Tubes of Circular Section (1964)

Alloy 'Tubes (I961)

Alloy Tubes (1969)

Alloy Tubes (I969)

Alloy Tubes (1969)

Alloy Wire (1964)

Alloy Wire (1964)

Alloy Wire (1969)

Alloy Wire (1969)

Alloy Wires (1969)

Alloy (Content Greater Than or Equal to 0.50\%) (1968)

Alloy $(1970)$

Alloy (1972)

Alloy, and Free Cutting Steels-Part 10: Wrought Nitri

Alloy, and Free Cutting Steels-Part 1I: Wrought Case

Alloy, and Free Cutting Steels-Part 12: Flame and Ind

Alloy, and Free Cutting Steels-Part 1: Quenched and T

Alloy, and Free Cutting Steels-Part 2: Wrought, Quenc

Alloy, and Free Cutting Steels-Part 3: Wrought Quench

Alloy, and Free Cutting Steels-Part 5: Wrought Quench

Alloy, and Free Cutting Steels-Part 6: Wrought Quench

Alloy, and Free Cutting Steels-Part 8: Wrought Quench

Alloy, and Free Cutting Steels-Part 9: Wrought Free C

Alloy, Used for Electronic and Telecommunication Equipm

Alloyed Coppers (1971)

Alloys-Brinell Hardness Test (1971)

Alloys-Check of Continuity of Thin Coatings-Copper

Alloys-Chemical Composition (Per Cent) (197I)

Alloys-Code of Designation-Part II: of Tempers (197

Alloys-Code of Designation-Part I: of Materials (19

Alloys-Code of Designation (1971)

Alloys-Complexometric Determination of Magnesium (197

Alloys-Determination of -Containing Zirconium, Rare

Alloys-Determination of Chromium-Spectrophotometric

Alloys-Determination of Copper by Electrolysis (I97 I)

Alloys-Determination of Insoluble Zirconium Alizarin

Alloys-Determination of Rare Earths-Gravimetric Met

Alloys-Determination of Silicon-Spectrophotometric

Alloys-Determination of Soluble Zirconium (Alizarin S

Alloys-Determination of the Light Fastness of Colored

Alloys-Determination of Titanium Spectrophotometric M

Alloys-Determination of Zinc-Volumetric Meth. (1970

Alloys-Determination of Zinc-Volumetric Meth. (1970

Alloys-Electrolytic Determination in Unalloyed Type C

Alloys-Electrolytic Determination in Wrought and Cast

Alloys-Estimation of the Loss of Absorptive Power by

Alloys-Insulation Check by Measurement of Breakdown $\mathrm{P}$

Alloys-Measurement of the Mass of the Oxide Coatings

Alloys-Measurement of Thickness of Oxide Coatings-N

Alloys-Sampling of Copper Refinery Shapes (1971)

Alloys-Sand Cast Test Pieces-Mechanical Properties

Alloys-Selection of Specimens and Test Pieces (1971)

Alloys-Shear Test for Rivet Wire and Rivets (197I)

Alloys-Spectrophotometric Determination of Iron in Th

Alloys-Spectrophotometric Determination of Nickel (Lo

Alloys-Spectrophotometric Determination of Tin (1970)

Alloys-Temper Designation (1971)

Alloys-Vickers Hardness Test (Loads from 1 to $100 \mathrm{kgf}$

Alloys-Volumetric Determination of Aluminium (Aluminu

Alloys and Magnesium and Its Alloys 5 to $50 \mathrm{~mm}$ Thick (1

Alloys for Use in Shipbuilding (1969)

Alloys 5 to $50 \mathrm{~mm}$ Thick (1972)

Alloys (Aluminum Content Between 1.5 and 12

Alloys (Test Loads from 2.5 to $50 \mathrm{kgf}$ ) (1964)

Alloys (Zinc Content Between 0.50 and 6.5\%) (1968)

Alloys (1960)

/Shipbuilding Details-Marking of Rolle

Alloys (1961)

Alloys (1961)

Alloys (1964)

Alloys (1964)

Alloys (1964) 
composition of Wrought Magnesium Aluminium (Aluminum) Zinc rty l.imits for Rivet Stork of Aluminium (Aluminum) and Its limits for Rolled Products of Aluminium (Aluminum) and Its Rec. for Special Wrought Copper Rec. for Cast Copper mits for Extruded Products of Aluminium (Aluminum) and Its s Alloys-Spectrophotometric Determination of Iron in the ys-Electrolytic Determination in Wrought and Cast-Copper otometric Determination of Nickel (Low Contents) in Copper um Alloy / Rec. for Chemical Analysis of Aluminum and Its sium All/ Rec. for Chemical Analysis of Magnesium and Its rec. for Chemical Analysis of Aluminium (Aluminum) and Its lloys (Z/ Rec. for Chemical Analysis of Aluminium and Its drazide / Rec. for Chemical Analysis of Magnesium and Its rec. for Chemical Analysis of Aluminium (Aluminum) and Its
Rec. for Chemical Analysis of Magnesium and Its hroline / Rec. for Chemical Analysis of Aluminium and Its $s \mathrm{~B} /$ Rec. for Chemical Analysis of Aluminium and Aluminum te Meth./ Rec. for Chemical Analysis of Magnesium and Its te Meth./ Rec. for Chemical Analysis of Magnesium and Its rec. for Chemical Analysis of Aluminium (Aluminum) and Its in That Containing C/ Std. for Chemical Analysis of Zinc tween 0.1 Rec. for Chemical Analysis of Magnesjum and Its Rec. for Chemical Analysis of Zinc and Its Rec. for Spices and Condiments: Pimento c) Rec. for Glossary of Gears-Geometrical Definitions .
69)

nds (1971)

uction (197I)

itions (1971)

Given with Enquiries Tenders and /

ection of (For Service) (1971)

ing Switches (I961) tests (1972)

Alloys (196.5)

Alloys (1968)

Alloys (1968)

Alloys (1971)

Alloys (1971)

Alloys (1971)

Alloys (1971)

Alloys (1971)

Alloys (1971)

Alloys: Gravimetric Determination of Aluminium in Magn

Alloys: Gravimetric Determination of Silicon (Content G

Alloys: Gravimetric Determination of Zinc in Aluminum a

Alloys: Photometric Determination of Copper (Oxalyldihy

Alloys: Photometric Determination of Copper (Oxalyldihy

Alloys: Photometric Determination of Iron (Orthophenant

Alloys: Photometric Determination of Iron (Orthophenant

Alloys: Photometric Determination of Manganese (Content

Alloys: Photometric Determination of Manganese: Perioda

Alloys: Photometric Determination of Manganese: Perioda

Alloys: Photometric Determination of Silicon (Content B

Alloys: Polarographic Determination of Lead and Cadmium

Alloys: Polarographic Determination of Zinc (Content Be

Alloys: Spectrophotometric Determination of Iron (1969)

Allspice) Whole and Ground: Spec. (1969)

Alpha-Beta Contamination Meters and Monitors (1970)

Alphabetical Indexes of Equivalent Terms (English, Fren

Alphanumeric Section of Keyboards Operated with Both Ha

Alternating Current Circuit Breakers: Design and Constr

Alternating Current Circuit Breakers: General and Defin

Alternating Current Circuit Breakers: Information to Be

Alternating Current Circuit Breakers: Rating (1971)

Alternating Current Circuit Breakers: Rules for the Sel

Alternating Current Circuit Breakers: Type and Routine

Alternating Current Electricity Meters (1972)

Alternating Current Isolators (Disconnectors) and Earth

Alternating Current Watthour Meters (1960)

Alternating Current Watthour Meters (1964)

Alternating Current Watthour Meters (1968)

Alternating Voltages Above $1,000 \mathrm{~V}$ (1962)

Alternating Voltages from $10 \mathrm{kV} \mathrm{Up}$ to and Including 33

Alternating Voltages from $10 \mathrm{kV} \mathrm{Up}$ to and Including 66

Alternating Voltages $U_{p}$ to $275 \mathrm{kV}(1963)$

Alternating Voltages Up to $275 \mathrm{kV}$ (1967)

Alternating Voltages Up to $275 \mathrm{kV}$ (1967)

Alternative Voltage (1969)

/Ec. for Measurement of the

Aluminium: Preparation of Sample Solution for Analysis

Aluminum-Determination of Calcium Content-Spectroph

Aluminum Alloy Conductors for Aircraft (1969)

Aluminum Alloys-Chemical Composition (Per Cent) (1971

Aluminum Alloys-Shear Test for Rivet Wire and Rivets

Aluminum Alloys for Use in Shipbuilding (1969)

Aluminum Alloys (Zinc Content Between 0.50 and $6.5 \%$ )

Aluminum Alloys: Photometric Determination of Manganese

Aluminum and Aluminium Alloy Tubes (1969)

Aluminum and Aluminium Alloy Wire (1969)

Aluminum and Aluminium Alloy Wire (1969)

Aluminum and Aluminium Alloys-Determination of Titani

Aluminum and Aluminium Alloys-Selection of Specimens

Aluminum and Its Alloys: Electrolytic Determination of

(Aluminum Content Between 1.5 and 12.0\%) (1968)

Aluminum Electrical Cables (1973)

Aluminum for the Protection of Iron and Steel Against (

(Aluminum Magnesium Silicon Type) (1966)

Aluminum or Aluminium Alloy Conductors for Aircraft (19

Aluminum Oxide Primarily Used for the Production of Alu

Aluminum Oxide Primarily Used for the Production of Alu

Aluminum Oxide Primarily Used for the Production of Alu

Aluminum Oxide Primarily Used for the Production of Alu

Rec. for Aluminum Oxide Primarily Used for the Production of Alu

Rec. for

minium-Determination of Zinc Content-Pan Ph/

minium-Preparation of Sample Solution for Ana/

Al

Aluminum (1971)

(Aluminm) Alloy Busbar Material of the Aluminium Magnes IEC

Alloy Castings (Complement to Rl64) (1961) ISO

(Aluminum) Alloy Castings (1960)

(Aluminum) Alloy Chill Castings-Reference Test Bar (19

Rec. for Composition of Aluminium

Rec. for Composition of Aluminium

72) Std. for Aluminium

um Silico/ Rec. for an International Spec. for Aluminium Std. for Aluminium ) etails-Dimensions and Sectional Properties of Aluminium roperties (1971) Rec. for Aluminium

or Surface Treatment of Metals-A nodisation of Aluminium of Magnesium (/ Std. for Chemical Analysis of Aluminium
(Aluminum) Alloy Conductor Wire of the Aluminium Magnesi

(Aluminum) Alloy Conductors, Steel Reinforced (1966)

(Aluminum) Alloy Sand Castings: Reference Test Bar (1972

(Aluminum) Alloy Sections for Marine Use (1970)

(Aluminum) Alloys-Sand Cast Test Pieces-Mechanical P

(Aluminum) and Its Alloys-Check of Continuity of Thin

(Aluminum) and Its Alloys-Complexometric Determination 
(Aluminum) and Its Alloys and Magnesium and Its Alloys 5

$r$ Mechanical Property Limits for Rivet Stock of Aluminium (Aluminum) and Its Alloys (1968)

chanical Property Limits for Rolled Products of Aluminium (Aluminum) and Its Alloys (1968)

anical Property Limits for Extruded Products of Aluminium (Aluminum) and Its Alloys (1971)

silicon (Conten/

copper (Oxalyld/

silicon (Conten/

Rec. for Chemical Analysis of Aluminium Rec. for Chemical Analysis of Aluminium Rec. for Chemical Analysis of Aluminium Rec. for Classification of Aluminium

th. (197) Std. for Iron Ores: Determination of Aluminium lite (Natural and Artificial): Determination of Aluminium c. for the Resistivity of Commercial Hard Drawn Aluminium Rec. for Commercial Annealed Aluminium and for General Purpose Applicatio Fluorine Content-Modified Willard/ Iron Content-1,10 Phenanthroline / Silica Content-Spectrophotometric/ uminium-Determination of Vanadium / uminium: Preparation of Sample Solut/ termination of Loss of Mass at $300 \mathrm{D} /$ reparation and Storage of Test Sampl/

of Sand $\mathrm{Ca}$ / Rec. for Composition of Magnesium Aluminium

Rec. for Composition of Magnesium Aluminium Rec. for Composition of Wrought Magnesium Aluminium is of Zinc Alloys-Volumetric Determination of Aluminium
for Aluminium Oxide Primarily Used for the Production of

for Aluminium Oxide Primarily Used for the Production of

Rec. for Aluminium Oxide Primarily Used for Production of

for Aluminium Oxide Primarily Used for the Production of

for Aluminium Oxide Primarily Used for the Production of

for Aluminium Oxide Primarily Used for the Production of Rec. for Alloy for Dental

ng Materials-Annex B: Preparation of Test Specimens from Mouldings (19) Rec. for Plastics-Determination of Free meth. (1961)

Rec. for Plastics-Detection of Free

Rec. for Natural Rubber Latices, Centrifuged and Creamed, 19/ Rec. for Plastics-Determination of Free Ammonia and ng Foodstuffs): Determination of Total Alkalini/ Std. for ree Acidity-Volumetric Meth. (1972)

value-Potentiometric Meth. (1972)

h. of Measuring Electrode Resistance and Transconductance, $s$ for Ionizing Radiation (1/

Rec. for Test Procedures for Rec. for Sound System Equipment:

Rec. Meth. of Measurement of Radiation from Receivers for Rec. Meth of Measurement on Receivers for valve Connections for Small Medical Gas Cylinders Used for ec. for Rating Systems for Electronic Tubes and Valves and ms (1971)

1971)

ated Nuclear Instruments (1970)

Rec. for

Rec. for Rec. for

Use-Determination/ Water Content Using an Electrolyti Rec. for Calculation of Coal and Coke oduction of Aluminium-Preparation of Sample Solution for Rec. for Plastics-Pve Resins-Sieve determination of Manganese (Contents B/ mination of Zinc in Aluminum Alloys $(\mathrm{Z} /$ mination of Iron (Orthophenanthroline / exometric Determination of Magnesium (/ mination of Zinc-Volumetric Meth. (1/ etric Determination of Silicon (Conten/ etric Determination of Copper (Oxalyld/ etric Determination of Silicon (Conten/ on of Titanium Spectrophotometric Meth/ mination of Copper in Aluminium Alloy / de (1968)

ement (1968)

ent (1968)

ination in Unalloyed Type Containing $\mathrm{N} /$ ination in Wrought and Cast.Copper All/ refinery Shapes (1971)

determination of Nickel (Low Contents)/ determination of Iron in the Alloys ( 1 )

Rec. for

Soluble Zirconium (Alizarin Sulphonat

Zinc-Volumetric Meth. (1970)

Silicon-Spectrophotometric Meth. Wi/
Aluminum) and Its Alloys-Measurement of the Mass of T Rec. for Aluminium Rec. for Aluminium Rec. for Aluminium Std. for Aluminium Std. for Aluminium Std. for Aluminium Rec. for Aluminium Rec. for Aluminium Rec. for Aluminium Rec. for Aluminium Rec. for Aluminium

Std. for
Aluminum) and Its Alloys: Gravimetric Determination of

(Aluminum) and Its Alloys: Photometric Determination of

(Aluminum) and Its Alloys: Photometric Determination of

(Aluminum) Bronzes and Special Types (1965)

Aluminum) Busbar Material (1958)

Aluminum) Conductors, Steel Reinforced (1966)

Aluminum) Content-Oxine Gravimetric and Volumetric Me

Aluminum) Content -8 Hydroxyquinoline Gravimetric Meth

(Aluminum) Electrical Conductor Wire (1959)

Aluminum) Electrical Conductor Wire (1960)

Aluminum) Electrolytic Capacitors for Long Life (Type Aluminum) Fluoride for Industrial Use: Determination of (Aluminum) Fluoride for Industrial Use: Determination of

(Aluminum) Fluoride for Industrial Use: Determination of

(Aluminum) Oxide Primarily Used for the Production of Al

(Aluminum) Oxide Primarily Used for the Production of Al (Aluminum) Oxide Primarily Used for the Production of De

Aluminum) Oxide Primarily Used for the Production Of; $\mathrm{P}$

Aluminum) Oxide (1963)

(Aluminum) Pigments (1971)

Aluminum) Stranded Conductors (1966)

(Aluminum) Zinc Alloy Castings and Mechanical Properties

Aluminum) Zinc Alloy Ingots for Casting Purposes (1959)

Aluminum) Zinc Alloys (1966)

Aluminum) (1970)

Aluminum: Determination of Absolute Density; Pyknometer

luminum: Determination of Iron Content-1,10-Phena

Aluminum: Determination of Loss of Mass at $1000^{\circ} \mathrm{C}$

Aluminum: Determination of Titanium Content; Tiron Phot

Aluminum: Measurement of the Angle of Repose (1968)

Aluminum: Measurement of Untamped Density (1968)

Amalgam (1970)

Aminoplastic Moulding Materials (1970)

Amonia and Amonium Compounds in Phenol-Formaldehyde ISO

Ammonia in Phenol Formaldehyde Mouldings-Qualitative

Ammonia Preserved-Spec. (1971)

Ammonium Compounds in Phenol-Formaldehyde Mouldings

Ammonium Hydrogen Carbonate for Industrial Use (Includi

Ammonium Nitrate for Industrial Use: Determination of $F$

Ammonium Nitrate for Industrial Use: Measurement of $\mathrm{pH}$

Amplification Factor, Conversion Resistance and Transdu

Amplifiers and Preamplifiers for Semiconductor Detector Amplifiers (1969)

Amplitude and Frequency Modulations and Television Broa

Amplitude Modulation Broadcast Transmissions (1954)

Anaesthetic and Resuscitation Purposes (1964)

Analogous Semiconductor Devices (1961)

Analogue d.c. Current Signals for Process Control Syste

Analogue Pneumatic Signal for Process Control Systems (

Analogue Voltage Ranges and Logic Levels for Mains Oper

Analyser (1972) Std. for Liquid Chlorine for Industrial

Analyses to Different Bases (1970)

Analysis by Means of Attack by Hydrochloric Acid Under

Analysis in Water (1970)

Analysis of Aluminium and Aluminum Alloys: Photometric

Analysis of Aluminium and Its Alloys: Gravimetric Deter

Analysis of Aluminium and Its Alloys: Photometric Deter

Analysis of Aluminium (Aluminum) and Its Alloys-Compl

Analysis of Aluminium (Aluminum) and Its Alloys-Deter

Analysis of Aluminium (Aluminum) and Its Alloys: Gravim

Analysis of Aluminium (Aluminum) and Its Alloys: Photom

Analysis of Aluminium (Aluminum) and Its Alloys: Photom

Analysis of Aluminum and Aluminium Alloys-Determinati

Analysis of Aluminum and lts Alloys: Electrolytic Deter

Analysis of Cements: Determination of Sulphur as Sulphi

Analysis of Cements: Main Constituents of of Portland C

Analysis of Cements: Minor Constituents of Portland Cem

Analysis of Copper and Its Alloys-Electrolytic Determ

Analysis of Copper and Its Alloys-Electrolytic Determ

Analysis of Copper and Its Alloys-Sampling of Copper

Analysis of Copper and Its Alloys-Spectrophotometric

Analysis of Copper and Its Alloys-Spectrophotometric

Analysis of Essential Oils (1962)

Analysis of Magnesium and lts Alloys-Determination of

Analysis of Magnesium and Its Alloys-Determination of

Analysis of Magnesium and Its Alloys-Determination of
Analysis of Coke (1968) 
Kare Earths-Gravimetric Meth. (1972) mination of Aluminium in Magnesium All/ mination of lron (Orthophenanthroline mination of Copper (Oxalyldihydrazide / mination of Manganese: Periodate Meth./ mination of Manganese: Periodate Meth./ ermination of Zinc (Content Between 0.1 ygen-Conventionally Express/ (Aluminum) Oxide (1963) 1963)

oxide (1963)

963)

963)

ic Moisture (I963)

963)

s (1963)

ioxide (1963)

1963)

n Content (I963)

ganese Content (1963)

de Content (1966)

ide Content and Magnesium Oxi/ ontent (I967)

ater Content (I966)

ron Content: (Photometric Met/ ontent (1966)

ontent (1966)

nt (I966)

nt (Polarographic Meth., for / (1972)

ee Glycerol-Spectropho/

ow Solubility in Ethanol (1968)

le Matter (1968)

1968)

unsaponified Matter (1969)

(I973)

ids (1965)

half-Octave and Third.Octave Band Filters Intended for the ur (Meth. After Combustion in a Curren/

Silicon (Gravimetric Meth.) (1969)

photometric Meth.) (I967)

imetric Meth. After Combustion in a St/

ectrolysis (197I)

luminium (Aluminum) (I970)

Lead and Cadmium in That Containing $C$

termination of Tin (I970)

ermination of Iron (1969)

68)

$m$ in Zinc (1969)

nd Cadmium (1968)

I968)

opper (I969)

(I963)

Rec. for Determination of Moisture in the Rec. for Determination of Moisture in the Rec. for Determination of Moisture in the ginous Seeds: Reduction of Contract Samples to Samples for egetable Oils and Fats: Preparation of Contract Sample for active Agents: Technical Sodium Alkylsulphonates: Meth. of Agents: Technical Sodium Primary Alkylsulphates: Meth. of gents: Technical Sodium Secondary Alkylsulphates: Meth. of roduction of Aluminium: Preparation of Sample Solution for tive Agents-Technical Sodium Alkylsulphonates-Meth. of m for Std. for Chemical Products and for Meth. of Chemical es-Binary Fibre (Fiber) Mixtures-Quantitative Chemical lating to Solid Mineral Fuels-Part II: Coal Sampling and Std. for Hard Coals-Size d. for Iron Ores: Determination of Hygroscopic Moisture in Rec. for Shipbuilding Details. 1957) Rec. for Shipbuilding Details for Sea Navigation

pbuilding Details for Sea Navigation and Inland Navigation pes, and Joining Shackle/ Std. for Shipbuilding: Rec. for Shipbuilding Details
Rec. for Transfer Cones for Dyeing Purposes: Hal reasing of a Horizontally Folded Specimen by Measuring the

ly Used for the Production of Aluminum: Measurement of the Rec. for Wood Cones for Cross Winding: Half rec. for Cones for Cross Winding for Dyeing Purposes: Half Rec. for Wood Cones for Cross Winding: Half

Rec. for Wood Cones for Cross Winding: Nominal Half Rec. for Lathe Centres (Centers): Centre c. for Dimensions of Hot Rolled Steel Sections-Equal Leg
for Dimensions of Hot Rolled Steel Sections-Unequal Leg
Analysis of Magnesium and Its Alloys-Determination of ISO Analysis of Magnesium and Its Alloys: Gravimetric Deter Analysis of Magnesium and Its Alloys: Photometric Deter Analysis of Magnesium and Its Alloys: Photometric Deter Analysis of Magnesium and Its Alloys: Photometric Deter Analysis of Magnesium and Its Alloys: Photometric Deter Analysis of Magnesium and Its Alloys: Polarographic Det Analysis of Manganese Ores-Determination of Active $O x$ Analysis of Manganese Ores-Determination of Aluminium Analysis of Manganese Ores-Determination of Arsenic ( Analysis of Manganese Ores-Determination of Carbon Di Analysis of Manganese Ores-Determination of Cobalt (1 Analysis of Manganese Ores-Determination of Copper (1 Analysis of Manganese Ores-Determination of Hygroscop Analysis of Manganese Ores-Determination of Lead (196 Analysis of Manganese Ores-Determination of Nickel (1 Analysis of Manganese Ores-Determination of Phosphoru Analysis of Manganese Ores-Determination of Silicon D Analysis of Manganese Ores-Determination of Sulphur Analysis of Manganese Ores-Determination of Total Iro Analysis of Manganese Ores-Determination of Total Man Analysis of Manganese Ores: Determination of Barium Oxi Analysis of Manganese Ores: Determination of Calcium Ox Analysis of Manganese Ores: Determination of Chromium C Analysis of Manganese Ores: Determination of Combined W Analysis of Manganese Ores: Determination of Metallic I Analysis of Manganese Ores: Determination of Titanium C Analysis of Manganese Ores: Determination of Vanadium C Analysis of Manganese Ores: Determination of Zinc Conte Analysis of Manganese Ores: Determination of Zinc Conte Analysis of Small Coke (Nominal Top Size $20 \mathrm{~mm}$ or Less) Analysis of Soaps-Determination of Low Contents of Fr Analysis of Soaps: Determination of Foreign Matter of $L$ Analysis of Soaps: Determination of Glycerol (1969) Analysis of Soaps: Determination of Moisture and Volati Analysis of Soaps: Determination of Total Alkali (1968) Analysis of Soaps: Determination of TotaI Free Alkali ( Analysis of Soaps: Determination of Unsaponifiable and Analysis of Soap: Determination of Chlorides (1965)

Analysis of Soap: Determination of Free Caustic Alkali

Analysis of Soap: Determination of Total Crude Fatty Ac

Analysis of Sounds and Vibrations (1966)

Analysis of Steel and Cast Iron: Determination of Sulph

Analysis of Steel and Cast Iron: Determination of Total

Analysis of Steels: Determination of Manganese (Spectro

Analysis of Steels: Determination of Total Carbon (Gray Analysis of Zinc Alloys-Determination of Copper by EL Analysis of Zinc Alloys-Volumetric Determination of a

Analysis of Zinc Alloys: Polarographic Determination of Analysis of Zinc and Its Alloys-Spectrophotometric De Analysis of Zinc and Its Alloys: Spectrophotometric Det Analysis of Zinc: Photometric Determination of Iron (19

Analysis of Zinc: Polarographic Determination of Cadmiu

Analysis of Zinc: Polarographic Determination of Lead a

Analysis of Zinc: Polarographic Determination of Lead (

Analysis of Zinc: Spectrophotometric Determination of C

Analysis Sample of Coal by the Direct Gravimetric Meth.

Analysis Sample of Coal by the Direct Volumetric Meth.

Analysis Sample of Coke (1968)

Analysis (I968)

Analy sis (1968)

Analysis (1968)

Analysis (1968)

Analysis (1968)

Analysis (1968)

Analysis (1969)

Analysis (1969)

Analysis (1971)

Analysis (1971)

Analysis (1972)

Analytical Samples (1973)

Anchor Chains-End Shackles (1963)

Anchor Chains-Lugless Joining Shac

Anchor Chains-Studles

Anchor Chains, Stud Links (Common, Enlarged, and End Ty ISO

Angle at Top of Cone $4^{\circ} 20^{\prime}(1967)$

Angle of Recovery (1972)

Angle of Repose (1968)

Angle of the Cone $3^{\circ} 30^{\prime}(1963)$

Angle of the Cone $4^{\circ} 20^{\prime}(1963$

IAtion of the Recovery from C ISO Rec for Crude V ISO Rec for Surface ISO Rec. for Surface Active ISO Rec. for Surface Active a ISO

Minum) Oxide Primarily Used for the P ISO Rec. for Surface Ac ISO
Rec. for Guide on the for ISO Rec. for Textil ISO

Rec. for Vocabulary of Terms Re ISO

ISO

ISO

ISO

Angle of the Cone $4^{\circ} 20^{\prime}$ (1963)

C. for Aluminium Oxide Primari ISO

ISO

ISO

ISO

Angle (1969)

Angles-In. Series-Dimensions and Sectional Properti

ISO

Angles-In. Series-Dimensions and Sectional Propert

235 
imensions of lIot Rolled Steel Scctions-Part I: Equal Leg ensions of llot Rolled Steel Seetions-Part 2: Unequal l.eg straight Manual Arc: Welds Made Wi/ Rec. for I.imitation of Rec. for Series of Conical Tapers and Taper Rec. for Inscription of Linear and Rec. for Acetic Rec. for Phthalic Rec. for Maleic

Rec. for Spec. for Photographic Grade Sodium Carbonate, 9) Rec. for ter (1969)

atty Acids (Titre) (1969)

lation Meth. (I969)

Rec. for

Rec. for

Rec. for

Rec. for Definitions of Living

for Surface Active Agents: Detergents-Determination of

(I960) Rec. for Commercial

coatings-Measurement of Thickness-Coulometric Meth. by $\begin{array}{ll}\text { s-1 } & \text { Rec. for Surface Treatment of Metals-Anodisation } \\ \text { s-1 } & \text { Rec. for Surface Treatment of Metals-Anodisation }\end{array}$ s-Insulation Check by Measuremen/ Std. for Anodization f Accelerated Corrosion Tests on Coatings Other Than Those of the / Rec. for Surface Treatment of Metals-Coloured eck of Continuity/ Rec. for Surface Treatment of Metals timation of the L/ Rec. for Surface Treatment of Metals and Its Alloys-1 Rec. for Surface Treatment of Metals and Its Alloy

Rec. for Surface Treatment of Metals ts Alloys-Determination of the Light Fastness of Colored and Its Alloys-Insulation Check by Measuremen/ Std. for es, Transformers, Primary Cells and Accumulators: Aerials eaths (1966)

Rec. for Tests on Rec. for

C, D, E)-Electrical Conductivity-Characte/ Rec. for

$70 \mathrm{~mm}$ Motion Picture Film-Image Area Produced by Camera Rec. for Cinematography-Image Produced by Camera

ec. for Cinematography-Maximum Aspect Ratio of Projector Rec. for Cinematography-Image Produced by Camera

Rec. for Lens

$35 \mathrm{~mm}$ Motion Picture Film-Image Area Produced by Camera d Plate Screens for Industrial Purposes-Nominal Sizes of oth and Perforated Plates in Test Sieves: Nominal Sizes of Rec for Porcelain Laboratory

nternational Electrotechnical Vocabulary: Switchboards and of Hazardous Areas (1972)

of Maximum Surface Temperatures (I9/

nd Test of Flameproof Enclosures of /

nd Test of Electrical Apparatus, Typ/

uction (1971)

for Ignition Tem perature (I966)

pparatus (I968)

closures (1962)

paratus (1967)

paratus: Use of a Screen (I967)

aratus for Intrinsically Safe Circui/

Rec. for Electrical Rec. for Electrical Rec. for Electrical Rec. for Electrical Rec. for Electrical Rec. for Electrical Rec. for Electrical Rec. for Electrical Rec, for Electrical Rec. for Electrical Rec. for Electrical Rec. for Electrical trical Apparatus for Explosive Gas Atmospheres: Spark Test Spec. for Radio Interference Measuring Spec. for Radio Interference Measuring Spec. for Radio Interference Measuring f Materials for the Insulation of Electrical Machinery and Rec. for Index of Electrical Measuring Rec. for Terms Relating to Microcopy

964)

for Essential Characteristics of $35 \mathrm{~mm}$ Microfilm Reading rical Apparatus for Explosive Gas Atmospheres: Sand Filled for Documentation to Be Supplied with Electronic Measuring ical Apparatus for Explosive Gas Atmospheres: Oil Immersed o Micromire (ISO Micro Test Object) for Checking a Reading

Rec. for Safety Requirements for Electronic Measuring nstruction and Test of Flameproof Enclosures of Electrical osive Gas Atmospheres: Construction and Test of Electrical rical Apparatus for Explosive Gas Atmospheres: Sand Filled

for Plastics-Pvc Resins: Determination of the Compacted d from a Specified /
ured from a Specifil

Rec. for Plastics-Determination of

Rec. for Plastics-Determination of Rec. for Plastics-Determination of Surface Active Agents: Determination of mpactio/ Rec. for Surface Active Agents: Determination of Rec. for Flexible Cellular Materials-Determination of Std. for Leather: Determination of

of Coke (1969)

Rec. for Determination of the True and Std. for Minimum Markings to Rec. for Definitions of Terms

nd Inductor $\mathrm{Co} /$ Rec. for Information on Ferrite Materials Rec. for Symbols for Indications
Angles-Metric Series-Dimensions and Sectional Prope Angles-Metric Series-Dimensions and Sectional Prope Angles of Slope and Rotation for Welding Positions for Angles (1969)

Angular Tolerances (1964)

Anhydride for Industrial Use-Meth. of Test (1968)

Anhydride for Industrial Use-Meth. of Test (1970)

Anhydride for Industrial Use-Meth. of Test (1970)

Anhydrous (1965)

Animal Fats: Determination of Insoluble Impurities (196

Animal Fats: Determination of Moisture and Volatile Mat

Animal Fats: Determination of Solidification Point of $\mathrm{F}$

Animal Fats: Determination of Water (Entrainment Distil

Animals for Slaughter-Porcines (1970)

Anionic-Active Matter (Direct Two Phase Titration Proce

Annealed Aluminium (Aluminum) Electrical Conductor Wire

Anodic Dissolution (1972) IEC

Anodic Dissolution (1972)

(Anodic Oxidation) of Aluminium (Aluminum) and Its Alloy

(Anodic Oxidation) of Aluminium (Aluminum) and Its Alloy

Anodisation of Aluminium and Its Alloys-Determination

Anodisation of Aluminium (Aluminum) and Its Alloys-Ch

Anodisation of Aluminium (Aluminum) and Its Alloys-Es

Anodisation (Anodic Oxidation) of Aluminium (Aluminum)

Anodisation (Anodic Oxidation) of Aluminium (Aluminum)

Anodised Aluminum (1971)

ISO

Anodization (Anodic Oxidation) of Aluminium (Aluminum)

IL. Symbols: Machin IFC

Anticorrosiond ations (1968) Statio Cable Sh

Antimagnetic Watches for General Purpose Use (1968)

Antistatic Endless V-Belts (Belts) (Sections Y, Z, A, B

Aperture and Projected Image Area for $35 \mathrm{~mm}$ Films (1958

Aperture and Projected Image Area for $8 \mathrm{~mm}$ Films (1958)

Aperture for Projection of $35 \mathrm{Mm}$ Nonanamorphotic Motion

Aperture for $16 \mathrm{~mm}$ Films (I965)

A perture Markings for Still Cameras (1966)

Aperture (I972)

Apertures (I972)

Apertures (1972)

A pparatus-Quality and Meth. of Test (I970)

Apparatus for Connection and Regulation (1957)

Apparatus for Explosive Gas Atmospheres: Classification

Apparatus for Explosive Gas Atmospheres: Classification

Apparatus for Explosive Gas Atmospheres: Construction a

Apparatus for Explosive Gas Atmospheres: Construction a

Apparatus for Explosive Gas Atmospheres: General Introd

Apparatus for Explosive Gas Atmospheres: Marking (1970)

A pparatus for Explosive Gas Atmospheres: Meth. of Test

Apparatus for Explosive Gas Atmospheres: Oil Immersed

A pparatus for Explosive Gas Atmospheres: Pressurized En

Apparatus for Explosive Gas Atmospheres: Sand Filled Ap

Apparatus for Explosive Gas Atmospheres: Sand Filled Ap

Apparatus for Explosive Gas Atmospheres: Spark Test App

Apparatus for Intrinsically Safe Circuits (1972)

Apparatus for Railways (1959)

Rec. for I

Apparatus for the Frequency Range $0.15 \mathrm{MHz}$ to $30 \mathrm{MHz}$ (1

Apparatus for the Frequency Range $25 \mathrm{Mc} / \mathrm{S}$ to $300 \mathrm{Mc} / \mathrm{S}$ (

Apparatus Having Detectors Other Than Quasi Peak (1967)

Apparatus in Relation to Their Thermal Stability in Ser

Apparatus Used in Connection with Ionizing Radiation (1

Apparatus (1964)

A pparatus (1965)

A pparatus (1967)

Apparatus (1968)

Apparatus (1968)

Apparatus (I968)

Apparatus (1971)

Apparatus (1971)

/Us for Explosive Gas Atmospheres: Co IEC

/Tus for Expl IEC

(I967)

Apparent Bulk Density (1969)

Apparent Density of Cellular Plastic (1968)

Apparent Density of Moulding Material That Can Be Poure

Apparent Density of Moulding Material That Cannot Be Po

Apparent Density of Pastes on Filling (1969)

Apparent Density of $W$ ashing Powders Before and After Co

Apparent Density (197I)

Apparent Density (1972)

Apparent Relative Densities (Density) and the Porosity

Appear on Containers Used for Printing Ribbons (I972)

Appearing in ISO Rec. for Oils and Pigments (1963)

Appearing in Manufacturers Catalogues of Transformer a

Appearing on Machine Tools (1964)

Apples-Guide to Cold Storage (1970)
$657 / 1$

657/1I

598

1119

406

754

1389

I390

424

932

933

935

934

1840

2271

121

2177

2106

2128

2376

1462

2135

2085

2143

2106

2I 28

2135

2376

I 17.10

229

764

1813

2467

73

74

358

466

517

2906

2194

565

1775

50(15)

$79-10$

79-8

79-1

79-7

79-0

79-9

79-4

79.6

9.6

$79-5$

$79-5 \mathrm{~A}$

79-3

79-3

$50(3 I)$

2

5

85
I81

371

452

278

79-6

689

348

79-1

79-7

79-5A

1068

845

60

61

1064

697

1855

2420

1014

2258

339

401

369

1212 
poses (1962)

rpose (1970)

(6)

967)

ryers and Towel D/

anges, /

irons, /

ng Liq/

ppliances (1967)

immers/

ing $\mathrm{An} /$

967)

Particular Spec. for Electric Cooking and Heating
Particular Spec. for Electric Cooking and Heating

Particular Spec. for Electric Cooking and Heating Spec. for Electric Motor Operated

Particular Spec. for Electric Cooking and Heating hair Clippers and Simi/ Spec. for Electric Motor Operated actors (1968) y Noni/ Particular Spec. for Electric Cooking and Heating y Noni/ Particular Spec. for Electric Cooking and Heating ons, Grills, Warm/ achines (I968)

Spec. for Electric Cooking and Heating Spec. for Electric Motor Operated Std. for Pneumatic Handling Spec. for Switches for

milar Purposes: Vacuum Cleaners and Water Suction Cleaning c and Similar Purposes: Shavers, Hair Clippers and Similar ated Appliances for Domestic and Similar Purposes: Massage waffle Irons, Grills, Warming Plates and Other Dry Cooking e 40 Calibrated Load Type for Pulley Blocks and Other Lift Rec. Switches for

Rec for General Test Conditions for Electro Heating ing, Accumulator (Storage), Batteries, Heating and Cooking Rec. for Safety of Household and Similar Electrical 7I) Rec. for Safety of Household and Similar Electrical (197) Rec. for Safety of Household and Similar Electrical $(197 /$ And/ Rec. for Safety of Household and Similar Electrica Rec. for Safety of Household and Similar Electrical Rec. for Safety of Household and Similar Electrical (I9) Rec. for Safety of Household and Similar Electrica Rec. for Safety of Household and Similar Electrical Rec. for Safety of Household and Similar Electrical Rec. for Safety of Household and Similar Electrical Rec. for Concrete Tests-Dimensions, Tolerances and

1970) Rec. for Pipe Connections for Industrial

a Nominal Selection Ratio Of/ Rec. for Insulation Coordination: designation of Main Groups of (1966) Rec. for Magnetic Cores for

trical Properties of Electronic Tubes and Valves: Rec. for Capacitors for Long Life (Type 1) and for General Purpose $r$ Use in Equipment for Telecommunication and in Electronic $r$ Use in Equipment for Telecommunication and in Electronic Unrecorded Magnetic Tapes for Interchange Instrumentation Rec. for Electrotechnical Vocabulary: Electromechanical International Electrotechnical Vocabulary: Electroheating

Symbols: Block Symbols for Transmission and Miscellaneous

eels for Magnetic Tape Used in Interchange Instrumentation

le), for Magnetic Tape Used in Interchange Instrumentation Std. for Unrecorded Magnetic Tapes for Instrumentation spheric Corrosion (I97I) Rec. for an

s Mechanical Handling Equipment for Loose Bulk Materials

s Mechanical Handling Equipment for Loose Bulk Materials ination (1969)

ination of Dry Solids Content at 105/

Rec. for Plastics

Rec, for Plastics

Thaw Cycle Stability Test (1969)

Rec. for Plastics.

Rec. for Plastics-Pvc Resins-Determination of $\mathrm{pH}$ of mination of the Resistance of Glass to Attack by a Boiling constant Relative Humidity in Small Enclosures by Means of

Rec. for International System for the Transliteration of ith Tenon Drive: Interchangeability Dimensions with Cutter ers with Taper Bore $(1: 30$ (Included) ) with Slot Drive and
nterchangeability Dimensions for Milling Cutters and Their (I968) Rec. for Milling Machine Rec. for Mercury

h Threaded Sockets and Connecting Pins for Use in Electric ec. for Diameters and Tolerances for Electrodes for Manual Rec. for Rating of Manual or Code of Symbols for Covered Electrodes for Manual Metal ols for Identific/ Std. for Covered Electrodes for Manual ypes/ Rec. for Code of Symbols for Covered Electrodes for ope and Rotation for Welding Positions for Straight Manual olor) Change Upon Exposure to Light of the Enclosed Carbon

menclature (I969)

awings: Scales (1969) Rec. for Dimensions of Refractory Rec. for

e Calibration of Bone Ribrat Std. for Cinematography-Location of the Printed Image Rec. for Cinematography-Projected Image hy-Image Produced by Camera Aperture and Projected Image
Appliance Couplers for Domestic and Similar General Pur Appliance Couplers for Household and Similar General Pu Appliances for Domestic and Similar Purposes $(1959+19$ Appliances for Domestic and Similar Purposes (1964) Appliances for Domestic and Similar Purposes: Clocks (1 Appliances for Domestic and Similar Purposes: Clothes D Appliances for Domestic and Similar Purposes: Cooking $\mathrm{R}$ Appliances for Domestic and Similar Purposes: Electric Appliances for Domestic and Similar Purposes: for Heati Appliances for Domestic and Similar Purposes: Massage a Appliances for Domestic and Similar Purposes: Portable Appliances for Domestic and Similar Purposes: Room Heat Appliances for Domestic and Similar Purposes: Shavers, Appliances for Domestic and Similar Purposes: Spin Extr Appliances for Domestic and Similar Purposes: Stationar Appliances for Domestic and Similar Purposes: Vacuum Cl Appliances for Domestic and Similar Purposes: Waffle Ir Appliances for Domestic and Similar Purposes: Washing $M$ Appliances for Loose Bulk Materials-Piping (1972)

Appliances (1962)

A ppliances (1965)

Appliances (1966)

Appliances (1967)

Appliances (1967)

Appliances (1971)

Appliances (1972)

Appliances (1972)

Appliances, Internal Communications, Lightning Conducto

Appliances: General Requirements (1970)

Appliances: Particular Requirements for Dishwashers (19

Appliances: Particular Requirements for Electric Irons

Appliances: Particular Requirements for Electric Shaver

Appliances: Particular Requirements for Floor Treatment

Appliances: Particular Requirements for Ranges (1971)

Appliances: Particular Requirements for Spin Extractor

Appliances: Particular Requirements for Toasters, Grill

Appliances: Particular Requirements for Vacuum Cleaners

Appliances: Particular Requirements for Washing Machine

Applicability of Test Pieces (I971)

Application for Plain End Steel and Other Metal Tubes (

Application Guide (1962)

Application in Coincident Current Matrix Stores Having

Application of Carbides for Machining by Chip Removal:

Application of Mechanical Shock (Impulse) Excitation to

Application (Type 2) (1969)

M (Aluminum) Electrolytic

Applications Employing Similar Techniques: General Requ

Applications Employing Similar Techniques: General Requ

Applications General Dimensional Requirements (197I)

Applications (1958)

Applications (1960)

Applications (I969)

Applications (I971)

Applications (197 I)

Rec. for IEC

Rec for Precision $R$ ISO

Applications: Physical Properties and Test Meth. (1973) IS

Appraisal of the Problems of Accelerated Test. for Atmo IEC

Apron Conveyors-Safety Code (I972)

Apron Conveyors (197 I)

D. for Continuou ISO

Aqueous Dispersions of Polymers and Copolymers-Determ

Aqueous Dispersions of Polymers and Copolymers-Determ ISO

Aqueous Dispersions of Polymers and Copolymers-Freeze ISO

Aqueous Extract (1970)

Aqueous Solution of Mixed Alkali (1968) /Ec. for Deter ISO

Aqueous Solutions (1966) /Ics-Meth. for Maintaining IS

Arabic Characters (196I)

Arbors-Metric Series (1973)

Arbors for Shell Reamers (1972)

Arbors or Mandrels-Metric and In. Series (1969)

for Milling Cutters W ISO Std. for Shell Ream ISO Arbors with 7/24 Tapers and Milling Machine Accessories

Arc Convertors (1957) IEC

Arc Furnaces (I967) / Machined Graphite Electrodes Wit IEC Arc Welding and Filler Metals for Gas Welding (1967) ISO Arc Welding Equipment (1968)

Arc Welding of Cast Iron (1969)

Arc Welding of Mild and Low Alloy Steels-Code of Symb

Arc Welding of Mild Steels and Low Alloy High Tensile T

elding of Mild Steel: Dimer

Arc (1968)

Arch Bricks (1969)

IIon of Resistance of Plastics to Colour (C

Architectural and Building Drawings: Definitions and No

Architectural and Building Drawings: Presentation of Dr

Architectural Diagrams (1967)

Area and Being Applied with a Specified Static Force (1

Area for Printing to $8 \mathrm{~mm}$ Type $S$ on $16 \mathrm{~mm}$ Motion Pictur

Area for $16 \mathrm{~mm}$ Film (1963)

Area for 35 mm Films (1958)

Rec. for Cinematograp
10910 -

$10-2 \mathrm{E}$

11-2A

$11-2 \mathrm{G}$

$10-2 \mathrm{O}$

$11 \cdot 2 \mathrm{C}$

$11-2 \mathrm{~F}$

$10.2 \mathrm{~N}$

$10-2 \mathrm{~K}$

$11-2 \mathrm{E}$

$10-2 \mathrm{~A}$

11.2M

I0-2J

2327

24.

10-2A

$10-2 \mathrm{~N}$

$10-20$

$11.2 \mathrm{M}$

1836

328

92-6

335- 1

335-5

335-3

335.8

335- 10

335- 6

$335-4$

$335-9$

335-2

335- 7

1920

II 79

7 I A

281

151.6

388. 1

$389-1$

I 859

$50(35)$

$50(40)$

117.13

1860

1858

2690
355

2390

2140

I I 48

1625

1147

I 264

695

483

233

2780

2402

240

839

84

239

544
700

107 I

2560

635

864
598

878

1145

I046

1047

117-8

373

1785

73 
hy-Image Produced hy Camera Aperture and Projected Image for l.iquid Flow Measurement in Open Channels by Velocity rec. for L,iquid Flow Measurement in Open Channela by Slope nation of Errora in Measurement of Liquid Flow by Velocity a by Chemi/ Rec. for Determination of the Weight Per Unit I A/ Rec. for Cinematography-Sound Records and Scanning r Cinematography: 65 and $70 \mathrm{~mm}$ Motion Picture Film-Image std. for Cinematography: $35 \mathrm{~mm}$ Motion Picture Film-Image Rec. for Picture Postcards:

Rec. for Carpets-Determination of Mass of Pile Per Unit or Envelopes, Postcards and Similar Articles: Cancellation e Textile Floor Coverings-Determination of Mass Per Unit arpets-Determination of Mass of Total Pile Yarn Per Unit nation of Number of Tufts And/or Loops Per Unit Length and ematography: $35 \mathrm{~mm}$ Motion Picture Film-Projectable Image (1966) Rec. for Nominal Cross Sectional Meth. of Test-Part I: Calculation of Linear Dimensions, Rec. for Cinematography-Picture for Explosive Gas Atmospheres: Classification of Hazardous perated with Both Hands (1971) Rec. for Audibert Rec. for Basic

for Shipbuilding-Indications of Details on the General

1 Machines: Symbols for Types of Construction and Mounting 70) Rec. for Lightning Rec. for Lightning Rec. for Determination of of Chemical Analysis of Manganese Ores-Determination of

ational Rec. for Pressure Gauges for Instruments Measuring
Rec. for Paper: Overall Trimmed Sizes of 1967) Rec. for Envelopes, Postcards and Similar ion of Earphones Used in Audiometry (197/ Rec. for an Iec ed Willard-Winter Meth. anthroline Photometric $\mathrm{Me} /$ photometric Meth. Using T/ 1970

ent-8 Hydroxyquinoline

Rec. for Cryolite (Natural and Rec. for Cryolite (Natural and Rec. for Cryolite (Natural and Rec. for Cryolite (Natural and Std for Cryolite (Natural and Rec. for Thermal Insulating Rec. for ing (1964) Rec. for Purposes (1964) Rec. for Asymmetrical Section Corrugated Sheets in and Drainage (1968) Rec. for Rec. for Rec. for

Rec. for Sampling and Inspection of ide to the Use of ISO Rec. 390, Sampling and Inspection of Rec. for Rec. for

Rec, for Rubber Sealing Rings for Joints in Rec. for Building and Sanitary Pipes in hloric Acid for Industrial Use: Determination of Sulphated mm Nonanamor photic Mot/ Rec. for Cinematography-Maximum

for Serrated Bars and Di adhesion of Vulcanized Rubber to Metal Where the Rubber Is adhesion of Vulcanized Rubber to Metal Where the Rubber Is

for Monocrystalline Semiconductor Rectifier Cells, Stacks,

rec. for Sealing Test for Pressurized Waveguide Tubing and or Power and Hand Socket Wrenches (1970) 8 for Power Types (1970) ular Socket Wrenches-Metric Series-Maximum /

Rec. for Rec. for Std. for 963) (197I) onservation Purposes (1971) nt for Roofing and Cladding (1964) Rec. for Rec. for Meth. for Rec. for Acoustics Rec. for Acoustics Rec. for flight Dynamics-Part II: Motions of the Aircraft and the r Std. Atmospheres for Conditioning And/or Test. Reference Rec. for Std. oaphere Spec. (1967) at Pieces (1966)

Rec. for Pla Rec. for Std. ures (19/ tus, Typ/ ures of $/$ e Circui/
Area for $8 \mathrm{~mm}$ Films (1958)

Area Meth (1968)

Area Meth. (1969)

for Collection of Data for Determi ISO

vanized Coatings on Ferrous Material ISO

IEC

Area of $35 \mathrm{~mm}$ Double Width Push Pull Sound Prints Norma ISO

Area Produced by Camera Aperture and Projectable Image-

Area Produced by Camera Aperture (1972)

Area Reserved for the Address (1965)

Area That Can Be Shorn Away from the Backing (1971)

Area (1965)

Area (1970)

Area (1971)

Area (1971)

Area (1972)

Areas and Composition of Conductors of Insulated Cables

Rec. F ISO Rec. for Machine Mad ISO
Rec. for C ISO

Rec, for Carpets $\rightarrow$ Determi ISO Std. for Cin ISO Cial Refrigerated Cabinets - ISO

Areas for Films and Slides for Television (1971) ISO

Areas (1972) Rec. for Electrical Apparatus IEC

Arnu Dilatometer Test for Coal (1963)

Arrangement for the Alphanumeric Section of Keyboards O

Arrangement Plans of Ships (1971)

Arrangements of (1972)

Arresters: Expulsion Type (1962)

Arresters: Nonlinear Resistor Type

Arsenic in Coal and Coke (1967)

Arsenic (1963)

Arterial Blood Pressure (1970)

Articles of Stationery That Include Detachable Sheets (
Articles: Cancellation Area (1965)

Artificial Ear, of the Wide Band Type, for the Calibrat

Artificial)-Determination of Fluorine Content: Modifi

Artificial)-Determination of Iron Content-1,10 Phen

Artificial)-Determination of Silica Content-Spectro

Artificial)-Preparation and Storage of Test Samples (

Artificial): Determination of Aluminium (Aluminum) Cont

Asbestos Boards (1971)

Asbestos Cement Corrugated Sheets for Roofing and Cladd

ISO

Rec ISO

Asbestos Cement Flat Sheets (1964)

Asbestos Cement for Roofing and Cladding (1964)

Asbestos Cement Pipe Fittings for Building and Sanitary

Asbestos Cement Pipes, Joints and Fittings for Sewerage

Asbestos Cement Pressure Pipes (1971)

Asbestos Cement Products (1964)

Asbestos Cement Products (1970)

Asbestos Cement Siding Shingles (1968)

Asbestos Cement Slates for Roofing and Cladding (1964)

Rec for

Asbestos Cement Water Piping (1970)

Asbestos Cement (1964)

Ash: Gravimetric Meth. (1968)

Aspect Ratio of Projector A

Aspects of Reliability (1969)

Assembled to One Metal Plate (1968)

Assembled to 2 Metal Plates (1968)

Assemblies and Equipment (1963)

Assemblies for Motorists (1970)

Assemblies (1968)

Assembly Tools for Bolts and Screws-Driving Squares F

Assembly Tools for Bolts and Screws-Hexagon Drive End

Assembly Tools for Screws and Nuts-Forged and and Tub

Assembly Tools for Screws and Nuts-Nomenclature (1971

Assessing the Oxidation Stability of Insulating Oils (1

Assessment of Noise with Respect to Community Response

Assessment of Occupational Noise Exposure for Hearing C

I Symbols for ISO

Atmosphere Relative to the Earth (1969)

Atmosphere Spec. (1967)

Atmospheres for Conditioning and Test. Textiles (1967)

Rec. Fo ISO

Atmospheres for Conditioning and Test. (1963)

Atmospheres for Conditioning And/or Test. Reference Atm

Atmospheres for the Conditioning and Test. of Rubber Te

Atmospheres: Classification of Hazardous Areas (1972)

Atmospheres: Classification of Maximum Surface Temperat

Atmospheres: Construction and Test of Electrical Appara

Atmospheres: Construction and Test of Flameproof Enclos

Atmospheres: General Introduction (197I)

Atmospheres: Marking (1970)

Atmospheres: Meth. of Test for Ignition Temperature (19

Atmospheres: Oil Immersed Apparatus (1968)

Atmospheres: Pressurized Enclosures (1962)

Atmospheres: Sand Filled Apparatus (1967)

Atmospheres: Sand Filled Apparatus: Use of a Screen (19

Atmospheres: Spark Test Apparatua for Intrinsically Saf

Atmosphere: Test, Reference, and Definitions (1967) 
Rec. for Std.

for an Appraisal of the Problems of Accelerated Test. for for the Production of Aluminium-Determination of Zinc oduction of Aluminium-Determination of Calcium Content Std. for Offset Duplicators

for 3 Point Linkage of Agricultural Wheeled Tractors for eries) (1971) Rec. for Conveyor Chains, 9681 Rec. for Determination of the Resistance of Glass to -Preparation of Sample Solution for Analysis by Means of Rec. for Determination of the Resistance of Glass to Rec. for

pation Factor of Electrical Insulating Materials at Power, ors for Frequencies Below $3 \mathrm{MHz}$ : Concentric Connectors for Rec. for es of Electronic Tubes and Valves: Meth. of Measurement of sses of Emission: Measurements Particularly Related to the Rec. for Pure Tone

for Std. Reference Zero for the Calibration of Pure Tone Rec for Pure Tone Screenin reference Coupler for the Calibration of Earphones Used in e Wide Band Type, for the Calibration of Earphones Used in Rec. for Micrographic Determination of the

Rec for Symbols for Languages, Countries and Rec. for International Electrotechnical Vocabulary: - $t$ : Interchangeability Dimensions of Battery Connectors for for Closed End Drop Wires (Electrical and Mechanical) for Rec. for Weft Pirns for Box Loaders for Rec. for Weft Pirns for Rec. for Single Box Pickers for

1970) Rec. for Shuttles for Pirn Changing Rec. for Rec. for Std. for Std. for Sid. for Sid. for Std. for Std. for Std. for

Spec. for Fluorescent Lamp s Up to $100 \mathrm{~mm}$-Dimensions Important to the Connection of ol Switches (Low Voltage Switching Devices for Control and

(I)

ol Switches (Low Voltage Switching Devices for Control and rmance/ Rec. for Meth. for Specif ying the Characteristics of Rec. for Sound System Equipment: s Filament/ Rec. for Guide for the Collection of Reliability, Rec. for Textile Glass-Determination of the

nt of the Dimensions of a Cylindrical Component Havine Machines (1968) Rec. for (I967)

Rec. for Rolling Bearings-For Railway Rec for Railway Rolling Stock Material Rec. for Magnetic Compasses, Binnacles and Rec. for Roll Film Cameras. Rec. for Dimensions for I27, I20 and 620 Roll Film, for Carpets-Determination of Thickness of Pile Above the mass of Pile Per Unit Area That Can Be Shorn Away from the

$f$ the Electrical Properties of Microwave Tubes and Valves: for Radio Frequency Connectors: Two Pin Connector for Twin Rec. for Gymnastic Equipment Rec. for Quality, Machining and Rec. for Amount of determination (I971) or Pulps: Determination of Saleable Mass, in Lots, of Pulp Rec. for Amount of Bale Coating on Natural Rubber Rec. for Sampling of Raw Rubber in ad Ratings (I958) Rec. for Rolling Bearings-Double Row Self-Aligning Rec. for
entification Code for Roller Bearings: Groups-(I) Radial 60) mensions and General Requir/ Rec. for Locking Devices for Radial Internal Clearance in Unloaded Radial Groove Type ) lerance Classes 6, 5 Rec. for Rolling Bearings-Thrust Rec. for Rolling Bearings-Thrust

Rec. for Rec. for Rec. for

(I969)

Rec. for Miscellaneous Lamps and Rec. for Paints and Varnishes-Surface Drying Test Rec. for Green

brations (/ Rec. for Octave, Half-Octave and Third.Octave - of Measurement for Radio Transmitters: Bandwidth, Out of e for the Drafting of Performance Spec. for Cores of Broad iometry (I97/ Rec. for an IEC Artificial Ear, of the Wide Rec. for Relation Between Sound Pressure Levels of Narrow oscill/ Rec. Meth. of Measurement for Radio Transmitters: bustion Engines-Definition of Right and Left Hand Single
Atmospheric Conditions for Test Purposes (1963)

IEC Atmospheric Corrosion (1971) Atomic Absorption Meth. (1971) Atomic Absorption Meth. (1971) Attachment Features of Plates (1972)

IM Oxide Primarily Used ISO /Marily Used for the Pr ISO

Attachment of Mounted Implements (1968)

Attachments and Chain Wheels-Part I: Chains (Metric S

Attack by a Boiling Aqueous Solution of Mixed Alkali (I

Attack by Hydrochloric Acid Under Pressure (1971)

Attack by $6 \mathrm{~N}$ Hydrochloric Acid at $100{ }^{\circ} \mathrm{C}(1970)$

Audibert Arnu Dilatometer Test for Coal (1963)

Audio and Radio Frequencies Including Metre (Meter) Wav

Audio Circuits in Radio Receivers (1969)

Audio Frequency Generators (1972)

Audio Frequency Output Power and Distortion (I966)

Audio Frequency Part of a Receiver (I971)

Audiometers for General Diagnostic Purposes (1965) Narious Cla IEC

Audiometers (1964)

Audiometers (1965)

Audiometry (1970)

Audiometry (1970)

Austenitic Grain

Size

Automatic Controlling and Regulating Systems (1966)

Automatic Coupling (1973)

Automatic Drawing-in Machines (1969)

Automatic Looms-Dimensions of Pirn Tip (1969)

Automatic Looms (1960)

Automatic Looms (1961)

Automatic Looms (1967)

Automobile-Spark Plug M 14 X 1.25 with Flat Seating (

Automobiles-Engine Test Code-Net Power (197 I)

Automobiles-Load Distribution for Private Cars (1972)

Automobiles : Compact Spark Plugs M 14 X 1,25 with Coni

Rec ISO

ISO

ISO

I Ium ISO

ISO

ISO

IEC

Rec. for IEC Provisional IEC

Automobiles: Compact Spark Plugs M 14 X 1,25 with Flat

Automobiles: Spark Plugs M 10 X 1 with Flat Seating (19

Automobiles: Spark Plugs M 12 X 1,25 with Flat Seating

Automobiles: Spark Plugs M $14 \times 1,25$ with Conical Seat

Automobiles: Spark Plugs M 18 X 1,5 with Conical Seatin

Auxiliaries (1968)

Auxiliaries (1971)

Auxiliary Circuits, Including Contactor Relays) Section

Auxiliary Circuits, Including Contactor Relays) : Gener

Auxiliary Equipment for Shock and Vibration Measurement

Auxiliary Passive Elements (I971)

Availability, and Maintainability Data from Field Perfo

Average Diameter of Staple Fibres (Fibers) or Continuou

Axial Load Fatigue Test. (1969)

Axial Terminations (1969)

Rec. for Measureme

Axis and Motion Nomenclature for Numerically Controlled

Axle Boxes: Acceptance Inspection (I970)

Axles for Trailer (1969)

Azimuth Reading Devices, Class B-General Requirements

Back Window Location and Picture Sizes (1971)

Backing Paper and Spools (197I)

Backing (1970)

Backing (I97I)

Balanced Aerial Feeders (1965)

Balancing Beam (I964)

Balancing of Transmission Pulleys (1962)

Bale Coating on Natural Rubber Bales-Requirement and

Baled in Sheet Form (1968)

Bales-Requirement and Determination (1971)

Bales (1971)

Ball-Radial Internal Clearance (1970)

Ball and Roller Bearings: Meth. of Evaluating Static Lo

Ball and Roller (II) Thrust (III) Tapered Type-Metric

Ball and Socket Couplings of String Insulator Units (19

Ball and Socket Couplings of String Insulator Units: Di

Ball Bearings with Cylindrical Bore-Values (1961)

Ball Bearings with Flat Seats-Normal Tolerances (1961

Ball Bearings with Flat Seats-Tolerances: Part 2-To

Rec. IEC

ISO

ISO

ISO

Rec. F ISO

ISO

ISO

ISO

ISO

ISO

IEC

IEC

ISO

ISO

Ball Couplings for Caravans and Light Trailers (I969)

ISO

Ballasts for Fluorescent Lamps (1962)

Ballasts for High Pressure Mercury Vapour (Vapor) Lamps

ISO

Ballotini Meth.) (I970)

Bananas: Guide to Storage and Transport (1969)

Band Filters Intended for the Analysis of Sounds and VI

Band Power and Power of Nonessential Oscillations (1969

Band Transformers of Ferromagnetic Oxides for Telecommu

Band Type, for the Calibration of Earphones Used in Aud

Bands of Noise in a Diffuse Field and in a Frontally in

Bandwidth, Out of Band Power and Power of Nonessential

Bank Engines (1972) 
of Chemical Analysis of Manganese Ores: Determination o cid for Industrial Use: Determination of Sulphate Content; Industrial Use; Determination of Sulphur Dioxide Content: ate for Industrial Use: Determination of Sulphate Content ide for Industrial Use: Determination of Sulphate Content: ide for Industrial Use: Determination of Sulphate Content: de for Industrial Use: Determination of Sulphate Content Ider Ring (1972)

Sulphate for Industrial Use: Determination of Content

ection of Auxiliaries / Rec. for Photography-Front Len ars for / Rec. for Textile Machinery-Terms for Serrated ated Bars and Dimensions of the Cross Section of Assembled Rec. for Gymnastic Equipment-Paralle Rec. for Knife Sections of Mower Cutter lastics-Determination of Flammability Of: in the Form of ings and Mechanical Properties of Sand Cast Reference Test Rec. for Dimensions of Hot Rolled Steel Rec. for Dimensions of Hot Rolled Steel Rec. for Dimensions of Hot Rolled Stee Rec. for Metal Clad Rec. for Metal Clad Rec for Denture

Rec. for Microcopies on Transparen Rec. for Microcopies on Transparent Bases Sizes of Rec. Rec. for Terms Relating to Microcopies and Their ec. for Calculation of Coal and Coke Analyses to Different ndustrial Use-Determination of Neutral Oils and Pyridine ards Operated with Both Hands (1971)

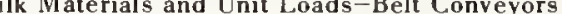

Purpose Metric Screw Threads: Tolerances, Principles and ors for Round Winding Wires (1965)

ors for Round Resistance Wires (1971)

ors of Insulated Rectangular Copper Winding Wir/

meters of Enamelled Round Winding Wires (1964) Rec. for WarperS

Rec. for ISO General Purpose Metric Screw Threads: Rec. for ISO In. Screw Threads:

ics Materials-Socket Fittings for Pipes Under Pressure s with Spigot Ends for Domestic and Industrial Waste Pipe Cyclic (12+12 Hr. Cycle) (1969) topple, Primarily for Equipment Type Specimens / (1969)

ponents and Equipment: General (1968)

ponents and Equipment: Pt. 2 Test (1968)

ponents and Equipment: Test A: Cold (1966)

ponents and Equipment: Test J: Mould Growth (19/

ponents and Equipment: Test Ka: Salt Mist (1964/

ponents and Equipment: Test M: Low Air Pressure/

ponents and Equipment: Test N: Change of Temper/ ponents and Equipment: Test Q: Sealing (1968)

ponents and Equipment: Test B: Dry Heat (1966)

ponents and Equipment: Test T: Soldering (1968)

ponents and Equipment: Test U: Robustness of Te/

ponents and Equipment: Test Ea: Shock (1967)

ponents and Equipment: Test Eb: Bump (1968)

ponents and Equipment: Test Ca: Damp and Steady/

ponents and Equipment: Test D: Accelerated Damp/

ponents and Equipment: Test Ga: Acceleration, S/

ponents and Equipment: Test $\mathrm{H}$ : Storage (1960)

Heat Tests (1968)

e of Temperature Tests (1971)

n (Sinusoidal) (1970)

ation Transfer (1972)

stems (1971)

on Message Transfer (1973)

Rec. for

Rec. for

Rec. for

Rec. for

Rec. for

Rec. for

Rec. for

Rec. fo

Rec. for

Rec. fo

Rec. for

Rec. for

Rec. for

Rec. for

Rec. for

Rec. for

Rec. for

Rec. for

Rec. for

Rec. for

Rec. for

Rec for

Rec. for

Rec. for

Rec. for

Std. for

Rec. for Modular Coordination

Std. for Dictation Equipment: Rec for ISO General Purpose Screw Threads

of Space and Time (1965)

(1957)

ing and Heavy Engineering (1968)

Rec for Measurement of Photosensitive Devices:

ls and Cereal Products. Determination of Moisture Content
Bar (1972)

Barb) and Hauteur Using a Comb Sorter (1969)

Barium Chromate Pigments for Paints (1972)

Barium Oxide Content (1966)

Barium Sulphate Gravimetric Meth. (1968)

Barium Sulphate Gravimetric Meth. (1968)

Barium Sulphate Gravimetric Meth. (1968)

Barium Sulphate Gravimetric Meth. (1969)

Barium Sulphate Gravimetric Meth. (1969)

Barium Sulphate Gravimetric Meth. (1972)

Barium Sulphate Gravimetric Meth. (1973)

Barrels Up to $100 \mathrm{~mm}$-Dimensions Important to the Conn

Bars and Dimensions of the Cross Section of Assembled B ISO

Bars for Mechanical Warp Stop Motions (1971) /for Serr ISO

Bars (1964)

Bars (1967)

Bars (1970)

Bars (1971)

ISO

Rec. for P ISO

Bars: Flat, Metric Series (1969)

Bars: Round, Metric Series (1969)

Bars: Square, Metric Series (1969)

Base Materials for Printed Circuits: Spec. (1970)

Base Materials for Printed Circuits: Test Meth. (1968)

Base Polymer (1970)

Bases Sizes of Rec. Bases (1961)

Bases (1961)

Bases (1962)

Bases (1970)

Bases (1971)

/Cresol, Cresylic Acid and Xylenols for I ISO

Basic Arrangement for the Alphanumeric Section of Keybo ISO

Basic Characteristics of Motorized Driving Pulleys (197 ISO

Basic Data (1969) Rec for ISO Genera ISO

Basic Dimensions of Winding Wires: Diameters of Conduct IEC

Basic Dimensions of Winding Wires: Diameters of Conduct IEC

Basic Dimensions of Winding Wires: Dimension of Conduct IEC

Basic Dimensions of Winding Wires: Maximum Over All Dia IEC

Basic Dimensions (1966)

Basic Dimensions (1968)

Basic Dimensions (1968)

Basic Dimensions: Metric Series (1972)

Basic Dimensions; Metric Series (1962)

Basic Environmental Test Procedures: Test Db: /Socket

Basic Environmental Test Procedures: Test Ec: Drop and

Basic Environmental Test Procedures: Test Ed: Free Fall

Basic Environmental Test. Procedures for Electronic Com

Basic Environmental Test. Procedures for Electronic Com

Basic Environmental Test. Procedures for Electronic Com

Basic Environmental Test. Procedures for Electronic Com

Basic Environmental Test. Procedures for Electronic Com

Basic Environmental Test. Procedures for Electronic Com

Basic Environmental Test. Procedures for Electronic Com

Basic Environmental Test. Procedures for Electronic Com

Basic Environmental Test. Procedures for Electronic Com

Basic Environmental Test. Procedures for Electronic Com

Basic Environmental Test. Procedures for Electronic Com

Basic Environmental Test. Procedures for Electronic Com

Basic Environmental Test. Procedures for Electronic Com

Basic Environmental Test. Procedures for Electronic Com

Basic Environmental Test. Procedures for Electronic Com

Basic Environmental Test. Procedures for Electronic Com

Basic Environmental Test. Procedures for Electronic Com

Basic Environmental Test. Procedures: Guidance for Damp

Basic Environmental Test. Procedures: Guidance on Chan

Basic Environmental Test. Procedures: Test Fc: Vibratio

Basic Ionizing Radiation Symbol (1963)

Basic Mode Control Procedures-Code Independent Inform

Basic Mode Control Procedures for Data Communication Sy

Basic Mode Control Procedures: Conversational Informati

Basic Module (1969)

Basic Operating Requirements (1973)

Basic Profile (1969)

Basic Quantities and Units of the SI (Metric Unit) and

Basic Rack of Cylindrical Gears for General Engineering

Basic Rack of Straight Bevel Gears for General Engineer

Basic Rec. (1969)

Rec for Cerea ISO
1684 
or (ireen Coffee Beans-Determination of Moisture Content (Basic Reference Meth.) (1970)

ng Freight Containers of Up to $\mathbf{3 0}$ Tonnes (Tons) Capacity - Basic Requirements (1972)

tronic Equipment and the Col Rec. for Preliminary L.ist of Rec. for Pirn Winders: Terminology;

Rec. for Cone Winders or Cheese Winders: Terminology; Rec. for Netting for Fishing Rec. for Hanging of Netting
Std Rec. for Stability of Counterbalanced Lift Trucks Rec. for ISO Metric Series for

mate $(1970)$

n Thermosetting Resins (1971) Rec. for Zine Chromate Pigments

Rec. for Plastics. Std. for Woven Glass Fabrics corrosion Tests on Coatings Other Than Those Anodic to the current M/ Std. for Nonconductive Coatings on Nonmagnetic Ilic and Vitreous or Porcelain Enamel Coatings on Magnetic Rec. for Perforated Metal Cheese Centres (Center) for

taple Fi/ Rec. for Textile Glass-Procedure for Sampling for the Determination of Residue on Evaporation on a Water Rec. for Industrial Trucks: Voltages of Traction Rec. for Dimensions of Lead Acid Motor Scooter Rec. for Lead Acid Starter Batteries: Dimensions of Rec. for Lead Acid Traction Rec. for Primary Cells and ns in Ships: Accessories, Lighting, Accumulator (Storage), Rec. for Lead Acid Starter Rec. for Lead Acid Starter Rec. for Lead Acid Starter Rec. for Primary Cells and Rec. for Primary Cells and Rec. for Primary Cells and Std. for Aircraft: Interchangeability Dimensions of Rec. for Connectors for Frequencies Below $3 \mathrm{MHz}$ : Spec. for Rec. for Metal Reeds with Plate
Rec. for Metal Reeds with Double Spring requencies Below $3 \mathrm{MHz}$ : Circular Multipole Connectors with
Rec. for Spindle Noses and Face Plates: es (1968)

$$
\begin{aligned}
& \text { Rec. for Spindle Noses and Face Plates: } \\
& \text { Rec. for }
\end{aligned}
$$

Std. for Sealed

Rec. for Gymnastic Equipment-Bac. for imensions of Hot Rolled Steel Sections-Parallel Flange I sions (1969) Rec. for Sectional Rec. for Shipbuilding Details-Marking of Hatchway Rec. for WarperS

nce Meth.) (1970)

h.) $(1970)$

) (1972)

-Boundary Dimensions (1962) rec. for Rolling Bearings-Internal Clearance in Unloaded bore 1:12-Tolerance Class 5-Special/ Rec. for Rolling ernal Clearance (1970)

earance $(1965)$

n $(1970)$

finitions (1961)

62)

Series-Dimension-Series 48 and 49 (1/ - Diameter Series 8,9,0,1,2,3 and 4, (1/

- Part II-Diameter Series 7 (1970)

Groove Type Ball Bearings with Cylindr/

Diameter Tolerances-Normal and Toler/ c-Diameter Series 9 and 0 (1967)

c Series-Dimensions Series 3 I and $32 /$ its-In. Series (1968)

its-Metric Series (1969)

its-Metric Series-Dimension Series /

ion Limits (1969)

rances (1967)

1 Tolerances (1961)

ances: Part 2-Tolerance Classes 6, 5 /

imensions (1966) Rec. for Cocoa Rec. for Green Coffee Rec. for Green Coffee Std. for Cocoa Rec. for Rolling
Rec. for Rolling Rec. for Rolling
Rec. for Rolling Rec. for Rolling Rec. for Rolling Rec. for Rolling Rec. for Rolling Rec. for Rolling Rec. for Rolling Rec. for Rolling Rec. for Rolling Rec. for Rolling Rec. for Rolling Rec. for Rolling Rec. for Rolling Rec. for Rolling Rec. for Rolling Rec. for Rolling Rec. for Rolling Rec. for Rolling Rec. for Rolling Rec. for Rolling Rec. for Rolling Rec. for Rolling Rec. for Rolling

ial Internal Clearance in Unloaded Radial Groove Type Ball Rec. for Rolling Bearings-Thrust ce Classes 6, 5 /
Basic Terms and Definitions for

Basic Terms and Definitions (1966)

Basic Terms and Definitions (1966)

Basic Terms and Definitions (1969)

Basic Terms (1970)

Basic Terms (1972)

Basic Tests (1969)

Basic Thicknesses of Sheet and Diameters of Wire (1964)

Basic Zinc Potassium Chromate and Zinc Tetrahydroxychro

Basis for Spec. for Industrial Laminated Sheets Based O

Basis for Spec. for Phenolic Moulding Materials (1968)

Basis for Spec. (1972)

Basis Metal (1970)

Basis Metals-Measurement of Coating Thickness-Eddy

Basis Metals-Measurement of Coating Thickness-Magne

Bast Fibre (Fiber) Yarns (1966)

Batch Furnaces with Metallic Heating Resistors (1972)

Batches or Consignments Of, Continuous Filament Yarn, S

Bath (1968)

Batteries for Electric Trucks (1969)

Batteries (1965)

Batteries (1965)

Batteries (1967)

Batteries (1971)

Batteries, Heating and Cooking Appliances, Internal Com

Batteries: Dimensions and Marking of Terminals (1963)

Batteries: Dimensions of Batteries (1965)

Batteries: General Requirements and Meth. of Test (1972

Batteries: General (1971)

Batteries: Spec. Sheets (1972)

Batteries: Terminals (1965)

Bactery Connectors for Automatic Coupling (1973)

Battery Connectors (1965)

Battery Operated Electric Fence Controllers (1956)

Baulk (1967)

Baulk (1967)

Bayonet or Push Pull Coupling (1971)

Rec. for Meth. ISO

ISO

IEC

lEC

lEC

IEC

IEC

IEC

IEC

IEC

IEC

ISO

IEC

CEE

ISO

ISO

Bayonet Type; Sizes for Interchangeability: Metric Seri

Beam Impact Test (V.Notch) for Steel (1960)

Beam Landing Lamps for Aircraft-Dimensions (1972)

Beam Unnotched lmpact Test for Grey Cast Iron (1969) Beam (1964)

Beams-In. Series-Dimensions and Sectional Propertie

Beams for Dyeing Fibres (Fibers) and Yarn (1969)

Beams for Warp Knitting Machines: Terminology and Dimen Beams (1960)

Beams: Basic Dimensions (1966)

Beans-Cut Test (1969)

Beans-Determination of Moisture Content (Basic Refere

Beans-Determination of Moisture Content, (Routine Met

Beans: Determination of Moisture Content (Routine Meth.

Beans: Sampling (1973)

Bearings-CylindricaI Roller-Separate Thrust Collars

Bearings-Definitions (1961)

Bearings-Double Row Cylindrical Type RD with Tapered

Bearings-Double Row Self-Aligning Ball-RadiaI Int

Bearings-Double Row Selfaligning-Radial Internal Cl

Bearings-For Railway Axle Boxes: Acceptance Inspectio

Bearings-Instrument Precision Types (1971)

Bearings-Internal Clearance in Unloaded Bearings-De

Bearings-Meth. of Evaluating Dynamic Load Ratings (19

Bearings-Needle Roller-Boundary Dimensions-Metric

Bearings-Radial-Boundary Dimensions-General PIan

Bearings-Radial-Boundary Dimensions-General Plan

Bearings-Radial Internal Clearance in Unloaded Radial

Bearings-Radial Types with Shields or Seals-Outside

Bearings-Tapered Roller-Boundary Dimensions-Metri

Bearings-Tapered Roller-Boundary Dimensions-Metri

Bearings-Tapered Roller-Boundary Dimensions-Subun

Bearings-Tapered Roller-Boundary Dimensions-Subun

Bearings-Tapered Roller-Boundary Dimensions-Subun

Bearings-Tapered Roller-Boundary Dimensions (1963)

Bearings-Tapered Roller-Boundary Dimensions (1965)

Bearings-Tapered Roller-In. Series-Chamfer Dimens

Bearings-Tapered; Metric Series-Part I: Normal Tole

Bearings-Thrust Ball Bearings with Flat Seats-Norma

Bearings-Thrust Ball Bearings with Flat Seats-Toler

Bearings-Thrust Bearings with Flat Seats-Boundary D

Bearings-Tolerances-Definitions (1969)

Bearings-With Locating Snap Ring-Dimensions (1965)

Bearings with Cylindrical Bore-Values (1961)

Bearings with Flat Seats-Boundary Dimensions (1966)

Bearings with Flat Seats-Normal Tolerances (1961)

Bearings with Flat Seats-Tolerances: Part 2-Toleran

1446

2308

27

476

477

1107

1531

1942

1074
388

1249

1642

800

2113

1462

2360

2178

480

397

1886

759

1044

199

86

92-6

95-3

95-2

95-1

86.1

86-2

$86 \cdot 3$

1963

130-3

367

571

130-7

867

148

1198

946

382

657/VII

1037

1025

151

481

1114

1446

1447

2291

2292

246

200

533

1646

465

1160

1224

200

281

1206

$15 / 1$

$15 / 1$

201

1648

355/III

355/V

355/VII

355/I

355/I1

1123

$577 / 1$

199/I

$199 / 1 \mathrm{I}$

104

1132

464

201

104 
-nuions, Toleranres, Permissible l,oad (/ $1969)$ 6.5) Rec. for Rolling Rec, for Rolling (lil) Tap/ Rec. for ISO ldentification Code for Rolling Rec. for ISO ldentification Code for Rolling Straight Inner Tab (1972) Std. for Rolling )

and 5 (I968)

ts-Metric Series-Outer Rings with F/

ts-Metric Series-Dimension Series 2/ ses 4 (Normal), 3,0 and 00 (1973) Class 4 (1973)

imits and Maximum Shaft and Housing Fil/ 1972)

nner Ring Width (1972)

with Electronic Equipment / Rec for Rules of Behaviour for Plastics-Meth. of Test for the Determination of the Rec. for Seat ndling Equipment for Loose Bulk Materials and Unit Loads nical Handling Equipment for Loose Bulk Materials-Mobile for Continuous Mechanical Handling Equipment-Light Duty uous Mechanical Handling Equipment for Unit Loads-Mobile cal Handling Equipment for Loose Bulk Materials-Troughed cal Handling Equipment for Loose Bulk Materials-Troughed cal Handling Equipment for Loose Bulk Materials-Troughed s Mechanical Handling Equipment for Loose Bulk Materials

Materials-Troughed Belt Conveyors (Other Than Portable) Rec. for Abrasive

List of Characteristics Which May Be Required of Conveyor Rec. for Widths of Flat Transmission Rec. for Tolerances on the Total Thickness of Conveyor Rec. for Motorists Seat Rec. for Troughability of Conveyor

for Tear Propagation Resistance of the Carcass of Conveyor Rec. for Strength of Mechanical Fastenings for Conveyor Rec. for Lengths of Flat Transmission Rec. for Crowns of Pulleys for Flat Transmission Rec. for Diameters of Pulleys for Flat Transmission Rec. for Ply Adhesion of Conveyor Rec. for Sampling of Conveyor Rec. for Marking of Conveyor

for Characteristics of Construction of Ply Type Conveyor Rec. for Widths and Lengths of Conveyor Materials-Troughed Belt Conveyors (Other Than Portable) uctivity-Characte/

Rec. for Antistatic Endless V-Belts

Electrical Conductivity of Conveyor Rec. for Flame Resistance of Conveyor
Strength and Elongation of Conveyor Rec. for

of Thickness Between $0.2 \mathrm{~mm}$ (0.008 In.) Rec. for Simple Rec. for Rec. for

k (1959)

k (1959)

Rec. for Paints and Varnishes Rec. for Simple Rec. for Reverse Rec. for Rotating Bar Rec. for Fibre (Fiber) Building Boards: Determination of Rec. for Plastics. Rec. for Rubber Hoses.

ded Corkboard-Determination of the Modulus of Rupture by Rec. for Steel Tubes-Butt Welding Rec. for Steel Tubes: Butt Welding nation of Acetone Soluble Material (Resinous Su/ Rec. for 69) Rec. for Determination of the Yield of Rec. for Nomenclature of Terms Used in the Rec. for Definitions of Terms Used in the 1970)

mination of Vanadium Content-Photometric Meth. Using $\mathbf{N}$ Rec. for Alpha,
Rec. for Modules and Diametral Pitches of Straight
Rec. for Basic Rack of Straight ng (1968) Rec. for Basic Rack of Straight $r$ by the Purchaser in Order to Obtain / Ryations of Typical Words in Elements (1968) Rec. for Abbreviations of Typical Words in
Rec. for Rec. for Rec. for

-Storage Equipment: Bins and Bunkers, Silos and Hoppers, nalysis (197I)

Rec. for Textiles. Rec. for Graphical Symbols: fication and Nomenclature (1971) 1 Requirements (1967)
Rec. for Graphical Symbols:
Rec. for Gypsum Rock for the Manufacture of

Rec. for Magnetic Compasses,
Bearings: Accessories (1969)

Bearings: Airframe: Introduction. General, Boundary Dim Bearings: Cylindrical Type, Radial Internal Clearance ( Bearings: Group IV Tapered Roller Type-ln. Series (19 Bearings: Group V Airframe Type (1968)

Bearings: Groups-(l) Radial Ball and Roller (11) Thru

Bearings: Locknuts, Narrow Series, and Lockwashers with

Bearings: Meth. of Evaluating Static Load Ratings (1958

Bearings: Radial-Tolerances (1966)

Bearings: Tapered-Metric Series Tolerances Classes 6

Bearings: Tapered Roller-Boundary Dimensions-Subuni

Bearings: Tapered Roller-Boundary Dimensions-Subuni

Bearings: Tapered Roller-Inch Series-Tolerance Clas

Bearings: Tapered Roller-Metric Series-Tolerances-

Bearings: Tolerances; Metric Series Chamfer Dimension L

Bearings: with Locating Snap Ring-Inner Diameter of (

Bearings: with Spherical Outside Surface and Extended I

Beech (Wood) Parquet Strips (1972)

(Behavior) with Respect to Possible Hazards When Dealing

Behaviour of Plastics in a Ventilated Tubular Oven (196

Belt Assemblies for Motorists (1970)

Belt Conveyors-Basic Characteristics of Motorized Dri

Belt Conveyors-Safety Code (1972

Belt Conveyors for Loose Bulk Materials (1971)

Continuous Mecha ISO

Belt Conveyors (Canvas, Rubber, Plastic, Etc.) Safety C

Belt Conveyors (Other Than Portable) Belt Pulleys (1970

Belt Conveyors (Other Than Portable) Belts (1970)

Belt Conveyors (Other Than Portable) Idlers (1970)

Belt Feeders and Conveyors-Safety Code (1970)

Belt Pulleys (1970)

/Handling Equipment for Loose Bulk ISO

Belts-Designation-Dimensions-Tolerances (1970)

Belts According to Their Use (1969)

Belts and Corresponding Pulleys (1956)

Belts and on the Thicknesses of Covers (1967)

Belts with Retractors (1970)

Belts (Characteristic and Meth. of Test) (1968)

Belts (Meth. of Test) (1966)

Belts (Static Test Meth.) (1969)

Belts (1958)

Belts (1959)

Belts (1959)

Belts (1962)

Belts (1962)

Belts (1965)

Belts (1965)

Belts (1970)

Belts (1970)

(Belts) (Sections Y, Z, A, B, C, D, E)-Electrical Cond

Belts: Spec. and Meth. of Test (1962)

Belts: Spec. and Meth. of Test (1962)

Belts: Spec./S and Meth. of Test (1963)

Bend Test for Copper and Its Alloys (1964)

Bend Test for Light Metal and Its Alloy Sheet and Strip

Bend Test for Steel (1959)

Bend Test on Steel Tubes (1960)

Bend Test (Cylindrical Mandrel) (1970)

Bend Test. of Steel Sheet and Strip Less Than $3 \mathrm{~mm}$ Thic

Bend Test. of Steel Sheet and Strip Less Than $3 \mathrm{~mm}$ Thic

Bend Test. of Steel Wire (1973)

Bending Fatigue Test. (1969)

Bending Strength (1972)

Bending Test for Rigid Cellular (1970)

Bending Test (1970)

Bending (1971)

Bends 5 D (90 ${ }^{\circ}$ And 180 Deg.) (1969)

Bends (90 And 180 Deg.) (1962)

Benzene Extract from Brown Coals and Lignites-Determi

Benzene Soluble Extract in Brown Coals and Lignites (19

Benzole Industry-Part I (1971)

Benzole Ind ustry-Part I (1971)

Benzoyl-N-Phenylhydroxylamine (1970)

Beta and Alpha.Beta Contamination Meters and Monitors

Bevel Gears for General and Heavy Engineering (1968)

Bevel Gears for General Engineering and Heavy Engineeri

Bevel Gears (Information to Be Given to the Manufacture

Bibliographical References (1968)

Bibliographical References: Essential and Supplementary

Bibliographical References: Essential Elements (1958)

Bibliographical Strip (1956)

Bin Gates-Safety Code (1970)

Binary Fibre (Fiber) Mixtures-Quantitative Chemical a

Binary Logic Elements (1972)

Binders-Classification, Characteristics and Test Meth

Binders Based on Calcium Sulphate-Definitions, Classi

Binnacles and Azimuth Reading Devices, Class B-Genera
113

1002

1038

$300 / \mathrm{Il}$

$300 / 11$

$300 / \mathrm{I}$

2982

76

492

577/II

2316

355/VIII

578

$577 /$ III

582

2265

2264

2457

1137

1231

1816

2387

2109

2388

2388
1536

1535

1537

1821

1536

1929

1121

22

583

1534

703

05

1120

63

100

99

252

282

433

432

251

1535

1813

284

283

340

398

954

85

167

1519

87

88

144

1143

768

1209

1746

1746
2077

2077
1128

285

1017

975

1542

1543

1618

325

325

678

677

1341

832

690

77

30

833

(17.

117.15

1587

1588

613 
Rec. for Magnetic Compasses and Rec. for Magnetic Compasses and ack for Use in the Rubber Industry Delivered in Bulk or in ng Equipment for Loose Bulk Materials-Storage Equipment: Industrial Use: Determination of Iron Content; $2,2^{\prime}$ nd ustrial Use-Determination of Iron Content: 2, 2' ndustrial Use-Determination of Iron Content-2,2' nd ustrial Use-Determination of Iron Content: 2, $2^{\prime}$ r Industrial Use: Determination of Iron Content-2,2 dustrial Use-Determination of Iron Content-2, $2^{\prime}$ Ind ustrial Use: Determination of Iron Content; $2,2^{\prime}$ dustrial Use-Determination of Iron Content-2, ${ }^{\prime}$ Industrial Use: Determination of Iron Content: 2,2'

for Industrial Use: Determination of Iron Content: 2,2 dustrial Use: Determination of of Iron Content: $2,2^{\prime}$

r Numerical Control of Machines (Compatible with the ISO racter Set for In/ Rec. for Guide for the Definition of 4 Definition of 4 Bit Character Sets Derived from the ISO 7 ation Processing) (I970/ gnetic Tape (I969) erchange (1967) Rec. for Representation of ISO Rec. for Implementation of the Rec. for 6 and agnetic Tape (I96) Rec. for Representation of 6 and 7 Rec. for Implementation of the 6 and Rec. for Rock Drilling-Rotary Drill Rods and Rec. for Rock Drilling-Drill Rods and Detachable Std. for Screwdriver (1972)

Rec. for Vulcanized Rubber-Determination of Carbo h. for Indicating the Stability of the Images of Processed d Sizes of Rolls (I969) of Sheet Material (I969) Rec. for Photographi Rec. for Photographi Rec. for Spices and Condiments:

or Determining Thiosulphate and Tetrathionate in Processed Rec. for Sampling Bulk Shipments of Carbon Rec. for Determination of Ash Content of Carbon Rec. for Determination of Loss on Heating of Carbon

Rec for Determination of Total Sulphur Content of Carbo Fines Content (1970) r Sieve Residue (I970) d Shipments (I970) residue (1970)

Loss on Heating (1970) or in Bins-Spec. for Maximu/ pour Density (1970) Rec. for Pelletized Carbon Rec. for Carbon Rec. for Carbon Rec. for Carbon Rec for Carbon Rec. for Pelletized Carbon Rec. for Pelletized Carbon Rec. for

Rec. for Cold Reduced Tinplate and Cold Reduced low $3 \mathrm{MHz}$ : Rectangular Miniature Multipole Connectors with uencies Below $3 \mathrm{MHz}$ : Rectangular Multipole Connectors with Up to $6.3 \mathrm{~mm}$ (1972) Std. for Hand and Machine Hacksaw measurement of the Performance Characteristics of Electric Rec. for Dimensions of Seam Welding Wheel Rec. for Spec. for Rec. for Plastics-Determination of the xamination (1972) Std. for Welds in Steel-Reference ly Controlled Machines (1/ Rec. for Punched Tape Variable ly Controlled Machines (I969/ Rec. for Punched Tape Fixed ontrolled/ Rec. for Interchangeable Punched Tape Variable ding of Preparatory Functions G An/ ations (I969)

Rec. for Punched Tape Rec. Graphical Symbols ifting Purposes-Grade 40 Calibrated Load Type for Pulle ec. for Verification and Calibration of Hardness Reference ec. for Verification and Calibration of Hardness Reference

1968) ec. for Verification and Calibration of Hardness Reference

\section{est. Machines (1968/}

e Hardness Test. Ma/

Rec. for Calibration of Standardized

Rec. for Calibration of Standardized

Rec. for Calibration of Standardized ec. for Verification and Calibration of Hardness Reference Std. for Muscovite Mica Rec. for Muscovite Mica ec. for Pressure Gauges for Instruments Measuring Arterial Rec. for Graduated Pipettes (Excludin Std. for Iron

Rec. for Paper and Rec. for Paper and oard Mounted Connectors or Printed Wiring Boards with Edge

Rec. for Meth. of Sampling Paper and

a Contact Spacing of $2.54 \mathrm{~mm}$ (0.1 In.) Mating Either with n Liquid or Gaseous Condition in Land Installations and on Rec. for Meth. of the Conditioning of Paper and

rec. for Determination of the Water Absorption of Paper or ing Either with Board Mounted Connectors or Printed Wirin the Design and Use of Components Intended for Mounting on or Fundamental Parameters of Connectors for Printed Wirin
Binnacles for Sea Navigation: Vocabulary (1969) Binnacles, Class A, for Use in Sea Navigation (1965)

Bins and Bunkers, Silos and Hoppers, Bin Gates-Safet

Bipyridyl Photometric Meth. (1968)

Bipyridyl Photometric Meth. (1970)

Bipyridyl Photometric Meth. (1970)

/Dium Carbonate for ISO / Formic Acid for I ISO Ec. for Urea for I ISO Nitric Acid for I ISO

Bipyridyl Photometric Meth. (1971)

Bipyridyl Photometric Meth. (1972)

Bipyridyl Spectrophotometric Meth. (1968)

Bipyridyl Spectrophotometric Meth. (1968)

Bipyridyl Spectrophotometric Meth. (1968)

Ehyde Solutions Fo ISO /Acid for in ISO ID Oleum for ISO /Hate for in ISO IIc Acid for ISO Bipyridyl Spectrophotometric Meth. (1968) Bipyridyl Spectrophotometric Meth. (1969)
Bipyridyl Spectrophotometric Meth. (1969) Bit Character Set) (1968) M Hydroxide ISO /Xide for in ISO Rec for Code Fo ISO

ISO

Bit Coded Character Set for Information Processing Inte

Bit Coded Character Set on 12 Row Punched Cards (Inform

Bit Coded Character Set on 9 Track $12.7 \mathrm{~mm}$ (1/2 In.) $\mathrm{Ma}$

Bit Coded Character Sets for Information Processing Int

Bit Coded Character Sets on Punched Tape (1969)

Bit Coded Character Sets on 7 Track $12.7 \mathrm{~mm}$ (1/2 In.) M

Bit Patterns on 12 Row Punched Cards (1971)

Bits for Dry Drilling-Connecting Dimensions (1970)

Bits for Percussive Drilling (I970)

Bits, for Slotted Head Screws, with Male Hexagon Drive

Black-Pyrolytic Meth. (I97I)

Black and White Films, Plates and Papers (I965)

Black and White Paper for Roll Paper Printers: Preferre

Black and White Papers for General Use: Preferred Sizes

Black and White Pepper, Whole and Ground: Spec. (1969)

White Photographic Film, Plates and Papers (I

Black for the Rubber Industry (I969)

Black for the Rubber Industry (1969)

Black for the Rubber Industry (1969)

Black for the Rubber Industry (1969)

Black for Use in the Rubber Industry-Determination of

Black for Use in the Rubber Industry-Meth. of Test Fo

Black for Use in the Rubber Industry-Sampling Package

Black for Use in the Rubber Industry-Spec. for Sieve

Black for Use in the Rubber Industry-Spec. Limits for

Black for Use in the Rubber Industry Delivered in Bulk

Black for Use in the Rubber Industry: Determination of

Blackhear Malleable Cast Iron (1969)

Blackplate-Part 1: Sheet (I969)

Blade Contacts (1965)

Blade Contacts (1966)

Blades for Slotted Head Screws (1972)

Connectors for Frequencies Be IEC Rec. for Connectors for Freq IEC

作

Blankets (1969)

Blanks (I968)

Bleached Lac (Shellac) (1957)

Bleeding of Colourants (Colorants) (196I)

Block for the Calibration of Equipment for Ultrasonic E

Block Format for Positioning and Straight Cut Numerica

Block Format for Positioning and Straight Cut Numerical

Block Format Positioning and Straight Cut Numerically $C$ Rec. for IEC

Block Formats for the Numerical Control of Machines: C

Block Symbols for Transmission and Miscellaneous Applic

Blocks Rockwell B (1970)

International R OIML

Blocks Rockwell C (I970) International $\mathbf{R}$ OIML

Blocks to Be Used for Brinell Hardness Test. Machines (

Blocks to Be Used for RockwelI B and C Hardness Scale T

Blocks to Be Used for Rockwell Superficial N and T Scal

Blocks to Be Used for Vickers Hardness Test. Machines

Blocks, Thins and Films Meth. for Grading by Size (I958

Blocks, Thins and Splittings: Meth. for Grading by Size ISO

Blood Pressure (1970) International R OIM

Blue Pigments for Paints (1972)

Board-Determination of Ash (1971)

Board-Determination of Tensile Strength (I97I)

Board Mounted Connectors or Printed Wiring Boards with IEC

Board Ships (1966)

Board Test Samples (196I)

(Lors) for Pipes Conveying Fluids I ISO

(Cobb Meth.) (1967)

Boards with Edge Board Contacts (1971)

Boards with Printed Wiring and Circuits (1970)

(0.1 In. ) $\mathrm{ISO}$ ICe for IEC Rec. F IEC

1069 449

1866

1820

744

1707

1982

2226

849

915

852

909

994

983

840

963

963

962

646

1113

961

1717

1718

2351

1408

1008 
eneral Requirements and Measuring Meth. for Printed Wiring Rec. for Thermal Insulating Asbestos Rec. for Fibre (Fiber) Building Rec. for Particle Rec. for Fibre (Fiber) Building Rec. for Fibre (Fiber) Building Rec. for Particle Rec. for Particle Std. for Fibre (Fiber) Building
Rec. for Fibre (Fiber) Building Rec. for Particle

ng in Thickness After Im/ $\mathrm{s}$ of $300 \mathrm{~mm}$ and Over (1969) to $300 \mathrm{~mm}(1963)$ Fiber) Building Rec. for Paper and Rec. for Flyer
Rec. for Flyer Rec. for Textile Machinery and Accessories-Condenser masses Per Unit Length (1971) Rec. for Rec. for Rules for Construction of Stationary or Determination of the Resistance of Glass to Attack by A Rec. for Laboratory mination of Soluble Matter of Crystalline Polypropylene by Change in Woven Fabrics Subjected to Laundering Near the Rec. for Plastics-Determination of

Preparation of Solution of Products Not Easily Soluble in Screw Threads: Tolerances, Limits of Sizes for Commercial imensions of Top Plates-Part I: Oblong Top Plates with 4 ln. Screw Threads: General Plan and Selection for Screws, Purpose Metric Screw Threads-Selected Sizes for Screws,

oss Flats and Corners (1968) ocket Wrenches (1970) Rec. for Hexagon 1970) ose Bolts and Screws: Metric Series (1968) Rec, for Assembly Tools for Rec. for Rec. for Clearance Holes for Metric 62)

Rec. for Machine Tool Tables-T Slots and Corresponding Rec. for Mechanical Properties of Fasteners-Part 1: Rec. for pose Bolts (1968) Rec. for Nominal Lengths for 968)

Rec. for Mechanical Properties of Fasteners: Marking of Rec for Clearance Holes for Metric determination of Gross Calorific Value by the Calorimetric ze Welding: Meth. of Test for Determining the Conventional nce of Electrical Insulating Varnishes by the Helical Coil part 2 (1969)

anges of Dimensions-Profiles (1966)

Rec. for Rec, for Rec. for rt 1) (1967)

r Determination of Modulus in Shear of Vulcanized Rubbers ics-Preparation of Glass Fibre (Fiber) Reinforced, Resin ec. for Particular Types of Winding Wires: Heat or Solvent ec. for Particular Types of Winding Wires: Heat or Solvent Rec. for an IEC Mechanical Coupler for the Calibration of Std. for Documentation-International Standard Rec. for Title Leaves of A Std. for Shipbuilding: Cargo Gear Particulars
d Measurement of Physical Properties of Sonic escription and Measurement of Physical Properties of Sonic
Std. for Lined Industrial Rubber ombustion in a Current of Oxygen and Titration with Sodium ass After Heating at $900{ }^{\circ} \mathrm{C}(1 /$ Std. for Crude Sodium
Std. for Crude Sodium d Boric Oxide Contents-Volumetri/ Std. for Crude Sodium

Acid, Boric Oxide, Disodium Tetraborates and Crude Sodium Std. for Cylindrical Cutters with Plain Std. for Slotting Cutters with Plain Rec. for Side and Face Milling Cutters with Plain Std. for Shell End Mills with Plain

70) Rec for Rubber Hose

ing Bearings-Double Row Cylindrical Type RD with Tapered shell Reamers (1972) Std. for Shell Reamers with Taper Rec. for Pneumatic Cylinders-Cylinder enzyldithiocarbamate Photometric ustrial Use-Determination of Manganese Conten/ ntent-Volumetric Meth. (1972)

\section{Rec. for}

Std. for Std. for

e Sodium Borates for Industrial Use: Determinat m Borates for Industrial Use-Determination of Sodium and ontent-Volumetric Meth. (1972)

ates for Industrial Use: Determinat/ Rec. for Cotter Slots with 5\% Taper Keys for Rec. for Pitch

Boards (1970)

Boards (1971)

Boards: Definition; Classification (1968)

Boards: Definition; Classification (1968)

Boards: Determination of Bending Strength (1972)

Boards: Determination of Density (1968)

Boards: Determination of Density (1968)

Boards: Determination of Dimensions of Test Pieces $(196$

Boards: Determination of Dimensions of Test Pieces (197

Boards: Determination of Moisture Content (1968)

Boards: Determination of Moisture Content (1968)

Boards: Determination of Water Absorption and of Swelli

Board: Sizes of Folders and Files (1967)

Bobbins for Spindle Diameters of $25 \mathrm{~mm}$ or More and Lift

Bobbins for Spindle Diameters Up to $22 \mathrm{~mm}$ and Lifts Up

Bobbins for Woollen (Woolen) Spinning-Dimensions (197

Boiled Linseed Oil (1960)

Boiler Tubes-Dimensions, Tolerances and Conventional

Boilers (1968)

Boiling Aqueous Solution of Mixed Alkali (1968)

Boiling Flasks (1970)

Boiling N-Heptane (1969)

Boiling Point (1968)

Boiling Water Absorption (1959)

Rec. for Plastics-Deter ISO / for Determination of Dimensiona ISO

1so

Bolt and Nut Threads: Medium Quality (1969) /Se Metric ISO

Bolt Holes (1972) Std. for Industrial Castors-D ISO

Bolts and Nuts (Diameter Range 0.06 to 6 In.) (1973) ISO

Bolts and Nuts (1973) Std. for ISO General ISO

Bolts and Nuts: Metric Series (1968)

Std. for ISO General ISO

Bolts and Nuts: Metric Series, Tolerances on Widths Acr

Bolts and Nuts: Widths Across Flats, Heights of Heads,

Bolts and Screws-Driving Squares for Power and Hand S

Bolts and Screws-Hexagon Drive Ends for Power Types (

Bolts and Screws: Radii Under the Head for General Purp

Bolts 1,6 Up to and lncluding $39 \mathrm{~mm}$ Thread Diameter (19

Bolts (1971)

Bolts, Screws and Studs (1968)

Bolts, Screws and Studs: Dimensioning (1961)

Bolts, Screws and Studs: Thread Lengths for General Pur

Bolts, Screws, Studs and Nuts (1969)

Bolts, $42 \mathrm{Up}$ to and Including $150 \mathrm{~mm}$ Thread Diameter (1

Bomb Meth. and Calculation of the Net (1971)

Bond Strength on Steel, Cast Iron and Other Metals (196

Bond Test (1969) /f or Evaluation of the Thermal

onded Abrasive Products-Grinding Wheel Dimensions-

Bonded Abrasive Products General Features Designation $\mathbf{R}$

Bonded Abrasive Products: Grinding Wheel Dimensions (Pa

(Bonded Quadruple Shear Test Piece) (1971)

Bonded, Low Pressure Laminated Plates or Panels for Tes

Bonding Enamelled Round Copper, with High Mechanical Pr

Bonding Self Fluxing Enamelled Round Copper (1970)

Bone Vibrators Having a Specified Contact Area and Bein

Book Numbering (ISBN) (1972)

Book (1969)

Book (1972)

Booms (1973)

Boots with Gene

Borate) (1968)

/Etermination of Sulphur

Borates for Industrial Use-Determination of Loss in $M$

Borates for Industrial Use-Determination of Sodium an

Borates for Industrial Use: Determination of Sulphur Co -Radial Internal Clearance in ISO

Bore-Values (1961)

Bore and Key Drive: Metric Series (1972)

Bore and Key Drive: Metric Series (1972)

Bore and Key Drive: Metric Series (1972)

Bore and Tenon Drive: Metric Series (1973)

Bore Sizes, Test Pressures and Tolerances on Length (19

Bore 1:12-Tolerance Class 5-Special Requirement (19

Bore (1:30 (Included) ) with Slot Drive and Arbors for

Bores and Port Sizes (1970)

Boric Acid and Oxide, and Disodium Tetraborates for Dib

Boric Acid and Oxide, and Disodium Tetraborates for Ind

Boric Acid for Industrial Use-Determination of lts Co

Boric Acid in Latex (1970)

Boric Acid, Boric Oxide, Disodium Tetraborates and Crud

Boric Oxide and Available Oxygen Contents-Volumetric

Boric Oxide Content and Loss on Ignition (1972)

Boric Oxide Contents-Volumetric Meth. (1972)

Bound Reeds (1967) 
Seripm 7 (1970)

(1967)

th and 49 (1/

31 and 321

ion Series 1

ion Series 21

rings with $\mathrm{F} /$

BearingsRec. for Rolling Bearings-Tapered Roller Rec. for Rolling Bearings-Needle Roller Rec. for Rolling Bearings-Tapered Roller Rec. for Rolling Bearings-Tapered Roller Rec. for Rolling Bearings-Tapered Roller Std. for Rolling Bearings: Tapered Roller Std. for Rolling Bearings: Tapered Roller Rec. for Rolling Bearings-Tapered Roller

-Cylindrical Roller-Separate Thrust Collars Rec. for Rolling Bearings-Tapered Roller for Rolling Bearings-Thrust Bearings with Flat Seats p (1969) ec. for Rolling Bearings: Airframe: Introduction. General Rec. for Weft Pirns for Rec. for Rolling Bearings-For Railway Axle Rec. for Pressures in Std. for

ogy (1972)

teristics of the Deposited Meta/ tional Bond Strength on Steel,

Rec. for Classification of Rec. for Filler Rods for Rec. for Filler Rods for Rec. for Oil of

for Determination of Breaking Strength and Elongation a or Lever Switches: Requirements for Type 2, Quick Make and itches: Requirements for Switches of Type 1, Slow Make and

ials-Determination of Tensile Strength and Elongation a rmining the Relative Resistance of Insulating Materials to minum) and Its Alloys-Insulation Check by Measurement of mensions for General Purpose Push Pull Single Pole Circui or Dimensions for General Purpose Push Pull 3 Pole Circuit Requirements for General Purpose Push Pull 3 Pole Circuit irements for General Purpose Push Pull Single Pole Circuit Spec. for Voltage Operated Earth Leakage Circuit Spec. for Minialure Circuit

valuation with Special Regard to Rated Capacity of Circuit Rec. for Guide to the Test. of Circuit

of Operating Devices and for Indicating Lamps for Circuit

Rec. for Low Voltage Distribution Switchgear: Circuit Rec. for High Voltage Alternating Current Circuit Rec. for High Voltage Alternating Current Circui Rec. for High Voltage Alternating Current Circui Rec. for High Voltage Alternating Current Circui Rec. for High Voltage Alternating Current Circuit Rec. for High Voltage Alternating Current Circuit
general Requirement/ Rec. for Low Voltage Fuses with High Textiles: Yarn from Packages-Meth. for Determination of or Fish/

Rec. for Fishing Nets-Meth. of Determining the Rec. for Fishing Nets-Meth. of Determining the Mesh ated with Rubber or Plastics (/ Rec. for Determination of Std. for Ebonite: Determination of Cross or Aircraft Control/ Rec. for Dimensions of Rectangular Refractory Rec. for Dimensions of Refractory Arch Std. for Machine

Rec. for Rec. for Rec. for

Rec. for Light Metals and Their Alloys

Rec for Verification of rec. for Calibration of Standardized Blocks to Be Used for lat Surfaces (1964) Rec. for Tables of Verification and Calibration of Hardness Reference Blocks Rec. for Meth. of Test for Temperature Limit of Rec. for Plastics-Meth. of Determining the r Guide for the Drafting of Performance Spec. for Cores of meth. of Measurement on Receivers for Amplitude Modulation meth. of Measurement on Receivers for Frequency Modulation

ers for Amplitude and Frequency Modulations and Television

Rec. Meth. of Measurement on Receivers for Television Rec. for Spec. for Photographic Grade Potassium

ol for Industrial Use-Determination of Phenols Content

for Higher Alcohols for Industrial Use-Determination of Rec. for Meth. for the Determination of Rec. for Classification of Aluminium (Aluminum) Rec. for Classification of Tin and Special Tin

r. Gas and Coke Residue by Low Temperature Distillation of Material (Resinous Su/ Rec. for Benzene Extract from determination of Sodium and $P$ Potassium Soluble I/

Rec. for Rec. for Determination of Moisture in (1969)

$r$ Determination of the Yield of Benzene Soluble Extract in for Test Procedures for Determining Physical Properties of tary Dimensions and Terminations (/ Rec. for Dimension of
Boundary Dimensions-General Plan-Part II-Diameter Boundary Dimensions-Metric-Diameter Series 9 and 0 Boundary Dimensions-Metric Series-Dimension-Series Boundary Dimensions-Metric Series-Dimensions Series Boundary Dimensions-Subunits-In. Series (1968)

Boundary Dimensions-Subunits-Metric Series-Dimens Boundary Dimensions-Subunits-Metric Series-Dimens

Boundary Dimensions-Subunits-Metric Series-Outer

Boundary Dimensions-Subunits-Metric Series (1969)

Boundary Dimensions (1962)

Boundary Dimensions (1963)

Boundary Dimensions (1965)

Boundary Dimensions (1966)

Boundary Dimensions, Tolerances, Permissible Load (1969

Box Loaders for Automatic Looms-Dimensions of Pirn T

Box Pickers for Automatic Looms (1961)

Boxes: Acceptance Inspection (1970)

Brake Lines and Braking Efficiency (1970)

Braking of Motor Vehicles and Their Trailers-Terminol

Brasses, Leaded, Special and High Tensile Types (1965)

Braze Welding: Meth. of Test for Determining the Charac

Braze Welding: Meth. of Test for Determining the Conven

Brazilian Sassafras (1967)

Break of Fabrics Coated with Rubber or Plastics (1971)

Break (Toggle) (1969)

Break (1963)

Rec. F IEC

Break (1971)

Rec. for Lever $S$ w IEC

/Meth. for Dete IEC

Breakdown Potential (1972)

Breakers for Aircraft (1966)

Breakers for Aircraft (1969)

Idation) of Aluminium (Alu ISO Rec. for Di ISO

Rec. F ISO

Breakers for Aircraft $(1970)$

Rec for Performance ISO

Breakers for Aircraft (1970)

Rec. for Performance ISO
for Performance Requ ISO

Breakers for Domestic and Similar Purposes (1959)

Breakers for Domestic and Similar Purposes (1968)

Breakers in Installations in Ships (1972)

Breakers with Respect to Out of Phase Switching (1968)

Breakers (1936)

Breakers $(1964)$

Breakers: Design and Construction (1971)

Breakers: General and Definitions (1971)

Breakers: Information to Be Given with Enquiries Tender Breakers: Rating (1971)

Breakers: Rules for the Selection of (For Service) (197

Breakers: Type and Routine Tests (1972)

Breaking Capacity for Industrial and Similar Purposes:

Breaking Load and Elongation of Same (Of Single Strands

Breaking Load and Knot Breaking Load of Netting Yarns F

Breaking Load of Netting (1970)

Breaking Strength and Elongation at Break of Fabrics Co Breaking Strength (1972)

Breaking Strengths of Preformed Stranded Steel Cables F

Bricks (1966)

Bricks (1969)

Bridge Reamers (1972)

Brinell Hardness Test for Copper and Its Alloys (1964)

Brinell Hardness Test for Grey Cast Iron (1961)

Brinell Hardness Test for Steel (1968)

Brinell Hardness Test (1971)

Brinell Hardness Test. Machines (1967)

Brinell Hardness Test. Machines (1968)

Brinell Hardness Values (HB) for Use in Tests Made on F

Brinell (1970)

Brittleness for Vulcanized Rubbers (1968)

International Rec. for OIMI

Brittleness Temperature by Impact (1969)

Broad Band Transformers of Ferromagnetic Oxides for Tel

Broadcast Transmissions (1954)

Broadcast Transmissions (1958)

Broadcast Transmissions (1959)

Broadcast Transmissions (1960)

Bromide (1965)

Bromination Meth. (1972)

Rec. IEC

Rec. IEC

/ Radiation from Receiv IEC

IEC

ISO

Std. for Liquefied Phen ISO

Bromine Index (1968)

Bronzes and Special Types (1965)

ISO

Bronzes (1965)

Brown Coal and Lignite (1968)

the Yields of Tar, Wate ISO

Brown Coals and Lignites-Determination of Acetone Sol Wa

Brown Coals and Lignites-Meth. of Extraction for the ISO

Brown Coals and Lignites by the Direct Volumetric Meth. ISO

Brown Coals and Lignites (1969)

Brush Holders, Commutators and Slip Rings (1968)

Brush Materials for Electrical Machines (1972)

Brushes and Holders for Electrical Machinery: Complemen

Rec. Fo ISO

Rec. IEC
$15 /$ II

$355 /$ II

1206

$355 / \mathrm{VI}$

$355 / \mathrm{IV}$

2316

$355 / \mathrm{V}$

246

$355 / 1$

$355 /$ II

104

1002

227

1160
1186

611

426

688

698

590

1421

131-3

131.2

1798

343

2376

530

1033

1509

1467

18

19

363

267

54

157-1

56-3

56-1

56-6

56-2

56.5

56-4

$269-1$

2062

1805

1806

142 ?

2473

564

475

1 I 45

2238

403

184

79

191

156

726

410

812

974.

219

69

91

106

107
420

1904

1848

$76]$

428

427

647

1017

1952

1015

975

276

413 
bimenkions and Tolerances (1962) 8) Rec for Definitions and Nomenclature for Carbon Rec. for Tolerances for Rec. for

Rec. for Asbestos Cement Pipe Fittings for Rec. for Fibre (Fiber) Rec. for Fibre (Fiber) Rec. for Fibre (Fiber) Std. for Fibre (Fiber) Rec. for Fibre (Fiber) Std. for Fibre (Fiber) of Swelling in Thickness After Im/

69) al (1971) Rec. for Architectural and Rec. for Architectural and Std. for Rec. for Rec. for Noncombustibility Test for lar Coordination-Storey and Room Heights for Residential Rec. for Electrical Installations of Rec. for Electrical Installations of Std. for Joints in

d General R/ Rec. Spec. for Insulating Materials Based on Rec. for Determination of the Rec. for Determination of the Std for Granulated Cork tics-Pve Resins: Determination of the Compacted Apparent for Cork-Expanded Pure Agglomerated-Determination of Rec. for Plastics-Determination of td. for Continuous Mechanical Handling Equipment for Loose td. for Continuous Mechanical Handling Equipment for Loose ec. for Continuous Mechanical Handling Equipment for Loose ec. for Continuous Mechanical Handling Equipment for Loose td. for Continuous Mechanical Handling Equipment for Loose td. for Continuous Mechanical Handling Equipment for Loose ec. for Continuous Mechanical Handling Equipment for Loose ec. for Continuous Mechanical Handling Equipment for Loose td. for Continuous Mechanical Handling Equipment for Loose Std. for Pneumatic Handling Appliances for Loose td. for Continuous Mechanical Handling Equipment for Loose ec. for Continuous Mechanical Handling Equipment for Loose c. for Continuous Mechanical Handling Equipment for Loose ec. for Continuous Mechanical Handling Equipment for Loose ec. for Continuous Mechanical Handling Equipment for Loose ec. for Continuous Mechanical Handling Equipment for Loose ec. for Continuous Mechanical Handling Equipment for Loose c. for Continuous Mechanical Handling Equipment for Loose

1 Handling Equipment-Light Duty Belt Conveyors for Loose ec. for Continuous Mechanical Handling Equipment for Loose ec. for Continuous Mechanical Handling Equipment for Loose

$r$ Meth. for the Determination of the Bulking Thickness and (1969)

d Carbon Black for Use in the Rubber Industry Delivered in

$$
\begin{array}{r}
\text { Rec. for Sampling } \\
\text { Rec. for Meth. for the Determination of the }
\end{array}
$$
ocedures for Electronic Components and Equipment: Test Eb: Rec. for Particular Types of Winding Wires:

ent for Loose Bulk Materials-Storage Equipment: Bins and the Supply of Gaseous Fuels: Metric Series-S/ Std for Rec. for the Determination of Flammability and Rec. for Dental
Std. for Dental king Parts (1972) luminum) Alloy

(1959) Rec. for Heat Treated Aluminium (Aluminum) Alloy Rec. for Commercial Purity Aluminium (Aluminum) shanks for Pneumatic Tools and Fitting Dimensions of Chuck shanks for Pneumatic Tools and Fitting Dimensions of Chuck

Rec. for Rock Drilling: Forged Collared Shanks and Chuck Rec. for Preparation of Dry Polymer from saturates and Residual Styrene (1972) Std. for Styrene

thec Pract for the X.Ray Inspection of Fusion Welded Rec. Pract. for Radiographic Inspection of Fusion Welded r Rec. Pract. for Radiographic Inspection of Fusion Welded

r Radiographic Inspection of Circumferential Fusion Welded Rec. for Strength Calculation of Rec. for Steel Tubes Rec. for Steel Tubes: Rec. for Rec. for Rec. fo ference Meth.) (1971)

at (Reference Meth.) (1971)

h. (1970)

Rec. for Push iary Circuits, Including Contactor Relays) Section 1: Push Rec. Regarding the Colour (Color) of Push

teel Equipment for Percussive Long Hole Drilling-Reverse teel Equipment for Percussive Long Hole Drilling-Reverse Fat in Milk by the Gerber Meth. (1966)
Brushes and Holders for Electrical Machinery: Principal

Brushes, Brush Holders, Commutators and Slip Rings (196

Building-Vocabulary (1970)

Building and Sanitary Pipes in Asbestos Cement (1964)

Building and Sanitary Purposes (1964)

Building Boards: Definiticn; Classification (1968)

Building Boards: Determination of Bending Strength (197

Building Boards: Determination of Density (1968)

Building Boards: Determination of Dimensions of Test P

Building Boards: Determination of Moisture Content (I96

Building Boards: Determination of Water Absorption and

Building Drawings: Definitions and Nomenclature (1969)

Building Drawings: Presentation of Drawings: Scales (19

Building Drawings: Projection Meth. (1972)

Building Materials-Determination of Calorific Potenti

Building Materials (1970)

Buildings (1970)

Buildings: Fundamental Principles (1970)

Buildings: General and Definitions (1970)

Building: Fundamental Principles for Design (1972)

Built Up or Treated Mica Paper-Part 1: Definitions an

Bulk Density of Coke in a Large Container (1969)

Bulk Density of Coke in a Small Container (1967)

Bulk Density Test (I972)

Bulk Density (1969)

Bulk Density (1972)

Bulk Factor of Moulding Materials (1961)

Bulk Materials-Aeroslides (1972)

Bulk Materials-Apron Conveyors-Safety Code (I972)

Bulk Materials-Apron Conveyors (1971)

Bulk Materials-Belt Feeders and Conveyors-Safety Co

Bulk Materials-Hydraulic Conveyors-Safety Code (197

Bulk Materials-Mobile Belt Conveyors-Safety Code (1

Bulk Materials-Oscillating Conveyors and Shaking on $R$

Bulk Materials-Oscillating Conveyors and Shaking or $\mathbf{R}$

Bulk Materials-Picking Table Conveyors-Safety Code

Bulk Materials-Piping (1972)

Bulk Materials-Scraper and En Masse Conveyors-Saf

Bulk Materials-Storage Equipment: Bins and Bunkers, S

Bulk Materials-Troughed Belt Conveyors (Other Than Po

Bulk Materials-Troughed Belt Conveyors (Other Than Po

Bulk Materials-Troughed Belt Conveyors (Other Than Po

Bulk Materials-Vibrating Feeders and Conveyors with T

Bulk Materials-Vibrating Feeders and Conveyors, Shaki

Bulk Materials and Unit Loads-Belt Conveyors-Basic

Rec. for Continuous Mechanica

Bulk Materials (1971)

Bulk Materials: Screw Conveyors (1969)

Bulk Materials: Vibrating Conveyors and Feeders with Re

Bulk of Paper (1965)

Bulk or in Bins-Spec. for Maximum Fines Content (1970

Bulk Shipments of Carbon Black for the Rubber Industry ISO

ISO

Bulking Thickness and Bulk of Paper (1965)

Bunched Enamelled Copper, with Silk Covering (1972)
Bunkers, Silos and Hoppers, Bin Gates-Safety Code (19

Burettes (1964)

Buried Unplasticized Polyvinyl Chloride (PVC) Pipes for

Burning Rate of Plastics in the Form of Film (1970)

Burs and Cutters-Fitting Dimensions (1970)

Burs and Cutters-Nominal Sizes and Designation of Wor

Busbar Material of the Aluminium Magnesium Silicon Type

Busbar Material (1958)

Bush Chains and Chain Wheels (1970)

Bushings-Part I (1970)

Bushings-Part II (1970)

Bushings for Alternating Voltages Above 1,000 V (I962)

Bushings for Hollow Hexagonal Drill Steels (1968)

Butadiene Homopolymer and Copolymer Latices (1971)

Butadiene Rubber Latices-Determination of Volatile Un

Butanol for Industrial Use-Meth. of Test (1968)

Butt Joints for Aluminium (Aluminum) and Its Alloys and

Butt Joints for Steel Plates Up to $50 \mathrm{~mm}$ (2 In.) Thick

Butt Joints for Steel Plates 50 to $200 \mathrm{~mm}$ Thick (1972)

Butt Joints in Steel Pipes Up to $50 \mathrm{~mm}$ (2 In.) Wall Thi

Butt Welded Joints (1967)

Butt Welding Bends 5 D $\left(90^{\circ}\right.$ And 180 Deg.) (1969)

Butt Welding Bends (90 And I80 Deg.) (1962)

Butter-Determination of Salt Content (Reference Meth.

Butter-Determination of the Acid Value of the Fat (Re

Butter-Determination of the Refractive Index of the F

Button Switches: General Requirements and Measuring Met

Buttons and Related Control Switches (1972)

Buttons (1955)

Buttress Threaded Equipments $11 / 2$ to $21 / 2$ In. (38 to

Buttress Threaded Equipments $11 / 6$ and $11 / 4$. In. (27 an

Butyrometers for the Determination of the Percentage of 
r Jimennions, Areas And/ Rec. for Commercial Refrigerated or Testy on Anticorrosion Protective Coverings of Metallic Rec. for Radio Frequency Cables: Relevant oltage No/ Rec. for Polyvinyl Chloride Insulated Flexible oltage Not Exceeding / Rec. for Rubber lnsulated Flexible Rec. for Colours (Colors) of the Cores of Flexible Spec. for Polyvinyl Chloride Insulated Spec. for Rubber Insulated Voltages / Rec. for Tests on Oil Filled and Gas Pressure Voltages / Rec. for Tests on Oil Filled and Gas Pressure eathed, Fo/ Rec. for Tests on Oil Filled and Gas Pressure on and Sheath: General Test and M/ Rec. for Low Frequency on and Sheath: Cables in Pairs, T/ Rec. for Low Frequency on and Sheath: Cables in Pairs, T/ 4 Rec. for Low Frequency on and Sheath: Distribution Wires/ Rec. for Low Frequency on and Sheath: Equipment Wires An/ Rec. for Low Frequency on and Sheath: Signalling Cables / Rec. for Low Frequency on and Sheath: Distribution Wires/ Rec. for Low Frequency for Pvc (Polyvinyl Chloride) Insulation for Low Frequency

nce of Plain and Tinned Copper Conductors of Low Frequency

ameters and Breaking Strengths of Preformed Stranded Stee wires with Pve (Polyvinyl Chloride) Insulation and Sheath: pve (Polyvinyl Chloride) Insulation and Sheath: Signalling aircraft (1969) Rec. for General Purpose Electrical ai) Rec. for Meth. of Test for General Purpose Electrical or Performance Requirements for General Purpose Electrical Rec. for Meth. of Test for General Purpose Electrical uctor Resistance of Heat Resisting $\left(260^{\circ} \mathrm{C}\right)$ Electrica ce Requirements for Heat Resisting $\left(190^{\circ} \mathrm{C}\right.$ ) Electrical r Meth. of Test for Heat Resisting $\left(260^{\circ} \mathrm{C}\right)$ Electrical for Meth. of Test for Heat Resisting (190 Deg.) Electrical ce Requirements for Heat Resisting $\left(260^{\circ} \mathrm{C}\right)$ Electrical uctor Resistance of Heat Resisting $\left(190^{\circ} \mathrm{C}\right)$ Electrical ons and Conductor Resistance of General Purpose Electrical vinyl Chloride) Insulation and Sheath: Equipment Wires and

Rec. for Radio Frequency Connectors: R. F. Coaxial, for

Rec. for Radio Frequency Connectors: R. F. Coaxial, for

Rec. for Radio Frequency Connectors: R. F. Coaxial, for Rec. for Electrical Installations in Ships:

Rec. for Calculation of the Continuous Current Rating of

Rec. for Guide to the Selection of High Voltage sectional Areas and Composition of Conductors of Insulated istic Impedances and Dimensions of Radio Frequency Coaxial Rec. for Fire Resisting Characteristics of Electric Rec. for Flame Retardant Characteristics of Electri pvc (Polyvinyl Chloride) Insulation and Sheath of Electric Std. for Crimped Joints for Aircraft Electrical terminal Ends for Crimping to Aircraft A luminum Electrical c. for Tests on Impregnated Paper Insulated Metal Sheathed Rec. for Radio Frequency Rec. for Radio Frequency

c. for Tests on Impregnated Paper Insulated Metal Sheathed ns (1972)

Rec. for Radio Frequenc Rec. for Sealed Nickel
Rec. for Sealed Nickel requirements and Test Meth. (1971/ Rec. for Sealed Nickel is of Zinc Alloys: Polarographic Determination of Lead and Chemical Analysis of Zinc: Polarographic Determination of Analysis of Zinc: Polarographic Determination of Lead and Rec. for Determination of

. for Sodium Chloride for Industrial Use: Determination of

ly Used for the Production of Aluminium-Determination of ily Used for the Production of Aluminum-Determination of $r$ Phosphoric Acid for Industrial Use-Determination of of Rec. for Pulps: Determination of

or Surface Active Agents-Preparation of Water with Known of Chemical Analysis of Manganese Ores: Determination of nclature (1971) Rec. for Binders Based on

$r$ Potassium Hydroxide for Industrial Use: Determination of

for Sodium Hydroxide for Industrial Use: Determination of of Volatile Acids Other Than Formic (Less Than $0.5 \%(\mathrm{~m} / \mathrm{m})$ Rec. for Meth. for Rec, for Classification of Adding and c. for Numeric Section of Ten Key Keyboards for Adding and for Keytop and Printed or Displayed Symbols for Adding and (1970) Rec. for Strength Rec. for Determination of Ester Value and eum for Industrial Use: Determination of Total Acidity an commercial Refrigerated Cabinets-Meth. of Test-Part I tically Loaded in Such a Way That the Transvers/ Rec. for nductors of Low Frequency Cables A/ Rec. for Guide to the (100\% Load Factor) (1971).
Cabin Ground Test Connection (1954)

Cabinets-Meth. of Test-Part I: Calculation of Linea

Cable Connectors Used in Nuclear Instrumentation (1969)

Cable Sheaths (1966)

Cable Spec. (1961)

Cables and Cords with Circular Conductors and a Rated V

Cables and Cords with Circular Conductors and a Rated V

Cables and Cords (1964)

Cables and Flexible Cords (1967)

Cables and Flexible Cords (1968)

Cables and Their Accessories (1966)

Cables and Their Accessories: External, for Alternating

Cables and Their Accessories: Internal, for Alternating

Cables and Their Accessories: Paper lnsulated, Metal Sh

Cables and Wires with Pvc (Polyvinyl Chloride) Insulati

Cables and Wires with Pvc (Polyvinyl Chloride) Insulati

Cables and Wires with Pvc (Polyvinyl Chloride) Insulati

Cables and Wires with Pve (Polyvinyl Chloride) Insulati

Cables and Wires with Pve (Polyvinyl Chloride) Insulat

Cables and Wires with Pyc (Polyvinyl Chloride) Insulat

Cables and Wires with Pve (Polyvinyl Chloride) Insulati

Cables and Wires (1969)

Cables and Wires (1971)

Cables for Aircraft Controls (1967)

to the Calculation of Resista IEC / Designations, Di $1 \mathrm{SO}$

Cables in Pairs, Triples, Quads and Quintuples for Insi

Cables in Singles for Telecommunication Equipment and I

Cables with Aluminium or Aluminum Alloy Conductors for

Cables with Aluminum or Aluminium Alloy Conductors for

Cables with Copper Conductors for Aircraft (1966)

Cables with Copper Conductors for Aircraft (1967)

Cables with Copper Conductors for Aircraft (1967)

Cables with Copper Conductors for Aircraft (1969)

Cables with Copper Conductors for Aircraft (1970)

Cables with Copper Conductors for Aircraft (1970)

Cables with Copper Conductors for Aircraft (1970)

Cables with Copper Conductors, for Aircraft (1966)

Cables with Copper Conductors, for Aircraft (1966)

Cables with Solid or Stranded Conductors, Insulated, Sc

Cables 96 IEC 50-12 (1967)

Cables 96 IEC 50-17 and Larger (1970)

Cables 96 IEC 75-17 and Larger (1971)

Cables (Construction, Test. and Installations) (1965)

Cables (100\% Load Factor) (1971)

Cables (1965)

Cables (1966)

Cables (1967)

Cables (1970)

Cables (1970)

Cables (1970)

Cables (1973)

Cables (1973)

Cables: for Alternating Voltages from $10 \mathrm{kV}$ Up to and 1

Cables: Guide to the Design of Detailed Spec. (1970)

Cables: Nondraining Types for Alternating Voltages from

Cables: Relevant Cable Spec. (1961)

Cadmium Cylindrical Rechargeable Single Cells: Dimensio

Cadmium Cylindrical Rechargeable Single Cells: General

Cadmium in That Containing Copper (1972)

Cadmium in Zinc (1969)

Cadmium on Iron and Steel (1971)

Rec. for

Cadmium (1968)

Caking Power of Coal by the Roga Meth. (1963)

$$
\text { ISO }
$$

Rec. for Chemical ISO

Calcium and Magnesium Contents-EDTA Complexometric Me ISO

Calcium Content-Atomic Absorption Meth. (1971) /Mari ISO

Calcium Content-Spectrophotometric Meth. Using Naphth ISO

Calcium Content-Volumetric Meth. (1968) Rec. Fo ISO

Calcium Content (1968)

Calcium Hardness (1972)

Calcium Sulphate-Definitions, Classification and Nome ISO

Calcium: EDTA Complexometric Meth. (1969)

Calcium; EDTA Complexometric Meth. (1969)

Calculated as Acetic)-Volumetric Meth. (1971)

Calculating Loudness Level (1966)

Calculating Machines (1969)

Calculating Machines (1969)

Calculating Machines (1969)

Calculation of Butt Welded Joints (1967)

Calculation of Coal and Coke Analyses to Different Base

Rec. Fo 1SO

Rec. ISO

TTents ISO

ISO

150

Re ISO

Rec. ISO

ISO

ISO

Calculation of Content of Essential Oils (1968)

Calculation of Free $\mathrm{SO}_{3}$ Content of Oleum; Volumetric

Calculation of Linear Dimensions, Areas and Volumes (19

Calculation of Rectangular Symmetrical Fillet Welds Sta

Calculation of Resistance of Plain and Tinned Copper Co

Calculation of the Continuous Current Rating of Cables
11

1992

313

229

96-2

227

245

17

13

230

141.3

141-2

141 -

189 -

189-2

189-3

189.4

189-5

189.6

189.7

304

344

564

189.2

189.6

1076

1468

474

634

539

1075

1491

1220

1490

470

469

189.5

$169-4$

169-5

$169-6$

92-3

287

183

228

78

331

332

330

1966

1965

55-1

96- 1

96-0

$55-2$
$96-2$

285-2

285 -

2576

1054

2082

713

335

2482

2069

2070

848

777

2174

552
1588

997

986

1913

532

1094

1092

1093

626

1170

709

910

1992
617

617
344

287

Engineering and Product Standards Division 

Rer. for Short l.ink Chain for Lifting Purposes-Grade 40 t Area and Be/ Rec. for an IEC Mechanical Coupler for the Rec. for IEC Provisional Reference Coupler for the for an IEC Artificial Ear, of the Wide Band Type, for the Rec. for

72) Std. for Welds in Steel-Reference Block for the International Rec. for Verification and International Rec. for Verification and International Rec. for Verification and International Rec. for Verification and Rec. for Simplified Meth. for Pressure Rec. for Std. Reference Zero for the

ciprocity Techniq/ (1968)

ll Hardness Test. Machines (1968)

ell Superficial N and T Scale Hardness Test. Ma/
ell B and C Hardness Scale Test. Machines (1968/

rs Hardness Test. Machines (1967)

1 (1960)

Rec. for

Rec. for

Rec. for

Rec. for

Rec. for Load Rec. for Test. and

reciprocity Techni/

Rec. for Precision Meth. for Pressure

ul/ Rec. for Building Materials-Determination of Rec. for Solid Mineral Fuels-Determination of Gros Rec. for Solid Stem Rec. for Enclosed Scale eral Fuels-Determination of Gross Calorific Value by the Rec. for

65 and $70 \mathrm{~mm}$ Motion Picture Film-Image Area Produced by s (1958) Rec. for Cinematography-Image Produced by (1958) Rec. for Cinematography-Image Produced by Rec. for Cinematography-Image Produced by graphy: $35 \mathrm{~mm}$ Motion Picture Film-Image Area Produced by Rec. for Emulsion Position in Rec. for Emulsion and Sound Record Positions in Rec. for Emulsion and Sound Record Positions in Rec. for Emulsion Position in 1 Properties of Electronic Tubes: Meth. of Measurement for e S (1972) Std. for Cinematography

s of Focal Spots of Diagnostic X-Ray Tubes Using a Pinhole

71) Rec. for Roll Film

rec. for Exposure Time Markings for Shutters Used in Still Rec. for Lens Aperture Markings for Stil c. for Dimensions of Small Flash Connections for Hand Held e Emulsion Side of Edge Marked Roll Film for Still Picture Rec. for Spindle Noses and Face Plates Types a and Rec. for Envelopes, Postcards and Similar Articles: Rec. for Cylindrical Slive Rec. for Cylindrical Sliver sealed Metal Food Containers-Internal Diameters of Round Rec. for Cylindrical Sliver

handling Equipment for Unit Loads-Mobile Belt Conveyor Rec. for Characteristics of String Insulator Units of the Rec. for Hexagon Socket Head Std. Meth. of Measurement of Lamp

Rec. Meth. for the Measurement of Direct Interelectrode Std for Industrial Wheels-Dimensions and Nominal Load

ctrometers (1971) Rec. for Coupling Capacitors and Rec. for Indirect Reading Rec. for Coupling

Rec for Preferred Diameters of Wire Terminations of r Paper / Plastic Film Dielectric (1964) Rec. for Fixed Rec. for Fixed Metallized Paper Dielectric Rec. for Polyester Film Dielectric
(S) (1959)

se Applicatio/ Rec. for Power Rec, for Aluminium (Aluminum) Electrolytic Rec. Series Rec. for and Meth, of Test (1972) Rec. for Receiver Type Mec for Fixed Rec. for Dimensions of Ceramic Dielectric Rec. for Ceramic Dielectric Rec. for Ceramic Dielectric

Rec. for Fixed Tantalum Rec. for Preferred Number Series for Resistors and Rec. for a.c. Motor Rec. for a.c. Motor
Rec. for Polystyrene Film Dielectric
Rec. for Power

r Marking Codes for Values and Tolerances of Resistors and Rec. Graphical Symbols: Semiconductor Devices, Rec. for Ceramic Dielectric g Meth. (1970) Rec. for Air Dielectric Rotary Variable $\mathbf{s}$ for Lifting Freight Containers of Up to 30 Tonn Breaking equirement/ Rec. for Low Voltage Fuses with High Breaking
rt Circuit Current Evaluation with Special Regard to Rated
Calculation of the Effective Parameters of Magnetic Pie Calculation of the Net (1971)

Calendar Dates in All Numeric Form (1971)

Calibrated Load Type for Pulley Blocks and Other Lift a Calibration of Bone Vibrators Having a Specified Contac Calibration of Earphones Used in Audiometry (1970) Calibration of Earphones Used in Audiometry (1970)

Calibration of Elastic Proving Devices (1964)

Calibration of Equipment for Ultrasonic Examination (19 Calibration of Hardness Reference Blocks Brinell (1970) Calibration of Hardness Reference Blocks Rockwell B (19 Calibration of Hardness Reference Blocks Rockwell C (19 Calibration of Hardness Reference Blocks Vickers (1970) Calibration of One Inch Condenser Microphones by the Re Calibration of Pure Tone Audiometers (1964)

Calibration of Standardized Blocks to Be Used for Brine

Calibration of Standardized Blocks to Be Used for Rockw Calibration of Standardized Blocks to Be Used for Rockw Calibration of Standardized Blocks to Be Used for Vicke Calibration of Test. Machines for Tensile Test. of Stee

Calibration of Ultrasonic Therapeutic Equipment (1963) Calibration of 1 In. Std. Condenser Microphones by the Calorific Potential (1971)

Calorific Value by the Calorimetric Bomb Meth. and Calc

Calorimeter Thermometers (1968)

Calorimeter Thermometers (1968)

Calorimetric Bomb Meth. and Calculation of the Net (197

Camera Accessory Shoes (1966)

Camera Aperture and Projectable Image-Area (1972)

Camera Aperture and Projected Image Area for $35 \mathrm{~mm}$ Film

Camera Aperture and Projected Image Area for $8 \mathrm{~mm}$ Films

Camera Aperture for $16 \mathrm{~mm}$ Films (1965)

Camera Aperture (1972)

Camera for $16 \mathrm{~mm}$ Silent Motion Picture Film (1956)

Camera for $16 \mathrm{~mm}$ Sound Motion Picture Film (1956)

Camera for $35 \mathrm{~mm}$ Sound Motion Picture Film (1956)

Camera for $8 \mathrm{~mm}$ Silent Motion Picture Film (1956)

Camera Types (197I)

f for Measurements of the Electrica

Camera Usage of $8 \mathrm{~mm}$ Motion Picture Film Perforated Typ

Camera (1970) Rec. for Measurement of the Dim

Cameras-Back Window Location and Picture Sizes (1971)

Cameras Using Films $35 \mathrm{~mm}$ and Smaller Picture Sizes (19

Cameras (1966)

Cameras (1966)

Cameras (1966)

Cameras (1968)

Camlock: Sizes for Interchangeability (1968)

Re ISO

Cancellation Area (1965)

Cans on Castors (1969)

Cans (1963)

Cans (1972)

Cans: Heights Over $1000 \mathrm{~mm}$ (1969)

(Canvas, Rubber, Plastic, Etc.) Safety Code (1972)

Cap and Pin Type (1969)

Cap Screws: Metric Series (1968)

Cap Temperature Rise (1971)

Capacitances of Electronic Tubes and Valves (1962)

Capacities (1972)

Capacitor Dividers (1971)

Capacitor Type Pocket Exposure Meters and Accessory Ele

Capacitors and Capacitor Dividers (1971)

Capacitors and Resistors (1971)

Capacitors for Direct Current Using Impregnated Paper O

Capacitors for Direct Current (1965)

Capacitors for Direct Current (1965)

Capacitors for Frequencies Between 100 and $20000 \mathrm{~Hz}(\mathrm{C}$

Capacitors for Long Life (Type 1) and for General Purpo

Capacitors for Power Systems (1972)

Capacitors for Radio Interference Suppression (1965)

Capacitors for Use in Electronic Equipment (1959)

Capacitors for Use in Electronic Equipment: Terminology

Capacitors of the Plate Type (1967)

Capacitors Type 2 (1965)

Capacitors Type 3 (1970)

Capacitors with Liquid or Solid Electrolyte (1971)

Capacitors (1963)

Capacitors (1967)

Capacitors (1968)

Capacitors (1968)

Capacitors (1968)

Capacitors (1971)

Capacitors, Type 1 (1967)

Capacitors: General Requirements for Tests and Measurin

Capacity-Basic Requirements (1972)

Capacity for Industrial and Similar Purposes: General R

Capacity of Circuit Breakers in Installations in Ships

IEC 
Rer. for Determination of the Moisture llolding Rec. for Counterhalanced Fork L.ift Trucks-Rated of Sorkets and Male Ends (1971) f Interchangeability and Safety of Lampcap/ f Interchangeability and Safety: I.ampholde/ f Interchangeability and Safety: Gauges (1/ Rec. for Rec. for I a mp Rec. for Lamp Rec. for Lamp Rec. for Ball Couplings for Particularly for Private Motor Cars and Light Trailers or Rec. for Throwaway Rec. for Rec. for Turning Tools with Rec. for Turning Tools with Rec. for Turning Tools with Rec. for Application of Rec. for Determination of Rec. for Determination of

main Groups of (1966) ature Combustion Meth. (1967) . (1967)

lour (Color) Change Upon Exposure to Light of the Enclosed Rec. for Vulcanized Rubber-Determination of Rec. for Sampling Bulk Shipments of Rec. for Determination of Ash Content of Rec. for Determination of Loss on Heating of Rec. for Determination of Total Sulphur Content of tion of Fines Content (1970) test for Sieve Residue (1970)

packaged Shipments (1970)

Sieve Residue (1970)

its for Loss on Heating (1970)

n Bulk or in Bins-Spec. for Maximu

ion of Pour Density (1970)

gs (1968) Rec. for Definitions and Nomenclature for Potassium Silicates for Industrial Use-Determination of tassium Hydroxide for Industrial Use: Determination of the Sodium Hydroxide for Industrial Use: Determination of the Rec. for Determination of of Chemical Analysis of Manganese Ores-Determination of of the Loss of Plasticizers from Plastics by the Activated (1972)

c. for Chemical Analysis of Steels: Determination of Total

of Carbon Dioxide Content Expressed as Sodium or Potassium tent-Mercurimetric Meth. (1972) Std. for Sodium Hydrogen e Content-Gravimetric Meth. (/ Std. for Sodium Hlydrogen carbonate-Titrimetric Meth. (/ hydrogen Carbonate Content-Ti/ d Preparation of the Sample (19/ termination of Total Alkalini/

Content: Volhard Volumetric Meth. (1973/ tent; 2,2'-Bipyridyl Photometric M/ mass and of Nonvolatile Matter at $250 \mathrm{De} /$ nsoluble in Water at $50{ }^{\circ} \mathrm{C}(1968)$

ydrogen Carbonate; Volumetric Meth. (196/

Content; Barium Sulphate Gravimetric Mel

luble Alkalinity: Volumetric Meth. (1968)

s (1968)

f Test Samples (1968)

Std. for Sodium Hydrogen

Std. for Sodium Hydrogen

Std. for Sodium Hydrogen

for Ammonium Hydrogen Std. for Sodium Rec. for Sodium Rec. for Sodium Rec. for Sodium Rec. for Sodium Rec. for Sodium Rec. for Sodium Rec. for Sodium Rec. for Sodium Rec. for Spec. for Photographic Grade Sodium ination of the Carbon Dioxide Content, Expressed as Sodium tion of the Carbon Dioxide Content, Expressed as Potassium onate for Industrial Use: Determination of Sodium Hydrogen 1972) Std. for Essential Oils-Determination of for Higher Alcohols for Industrial Use-Determination of Determination and Verification of the Effective Depth of Rec. for Tear Propagation Resistance of the Rec. for Spices and Condiments or Textile Machinery and Accessories-Worsted and Woollen ntation of ISO 7 Bit Coded Character Set on 12 Row Punched

$\mathrm{n}$ of Rectangular Punched Holes in 80 Columns Punched Pape ec. for Information Processing-Spec. for Unpunched Pape ssing-Representation of 8 Bit Patterns on 12 Row Punched Std. for Shipbuilding. hat Can Be Shorn Away from the Backing (1971) unit Area (1971)

$y$ and Measured Pile Fibre (Fiber) Volume Ratio /

Per Unit Length and Area (1971)

backing (1970)

en Ground (1972)

Rec. for

Rec. for

Rec. for

Rec. for

Rec. for

Sid. for Hand Knotted Std. for Hand Made Rec. for Stretchers, Stretcher pling-Test Sample-Preparation of the Main Solution for h End Loops of Healds (1967) Rec. for Heald r 12 V Electrical Equipment Particularly for Private Motor Std. for Automobiles-Load Distribution for Private

Spec. for

Rec, for Fuse Holders for Miniature
Capacity of lIard Coals (1969)

Capacity (1971)

Capillary Solder Fittings for Copper Tubes-Dimensions

Caps and Holders Together with Gauges for the Control O

Caps and Holders Together with Gauges for the Control O

Caps and Holders Together with Gauges for the Control O Caravans and Light Trailers (1969)

Caravans (1970) IS with 6 or 12 V Electrical Equipment ISO

Carbide Indexable Inserts: Dimensions (1969)

Carbide Tips for Turning Tools: Metric Series (1961) ISO

Carbide Tips: Designation and Marking (1966)

Carbide Tips: Internal Tools (Metric Series) (1966)

Carbide Tips: Metric Series (1961)

Carbides for Machining by Chip Removal: Designation of

Carbon and Hydrogen in Coal and Coke by the High Temper

Carbon and Hydrogen in Coal and Coke by the Liebig Meth

Carbon Arc (1968) Ion of Resistance of Plastics to Co ISO

Carbon Black-Pyrolytic Meth. (1971)

Carbon Black for the Rubber Industry (1969)

Carbon Black for the Rubber lndustry (1969)

Carbon Black for the Rubber lndustry (1969)

Carbon Black for the Rubber Industry (1969)

Carbon Black for Use in the Rubber Industry-Determina
Carbon Black for Use in the Rubber lndustry-Meth. of

Carbon Black for Use in the Rubber lndustry-Sampling

Carbon Black for Use in the Rubber lndustry-Spec. for

Carbon Black for Use in the Rubber Industry-Spec. Lim

Carbon Black for Use in the Rubber Industry Delivered I

Carbon Black for Use in the Rubber Industry: Determinat

Carbon Brushes, Brush Holders, Commutators and Slip Rin

Carbon Dioxide Content Expressed as Sodium or Potassium

Carbon Dioxide Content, Expressed as Potassium Carbonat

Carbon Dioxide Content, Expressed as Sodium Carbonate,

Carbon Dioxide in Coal by the Gravimetric Meth. (1969)

Carbon Dioxide (1963)

Carbon Meth. (1961)

Rec. for Meth. ISO
/Ec. for Plastics-Determination ISO

Carbon Tetrachloride for Industrial Use-Meth. of Test

Carbon (Gravimetric Meth. After Combustion in a Stream

Carbonate-Gas Volumetric Meth. (1970)

Eteri

Carbonate for Industrial Use-

/Nation of Chloride Con ISO

Cor Industrial Use-Determination of Moistur 1SO

Carbonate for Industrial Use-Determination of Sodium ISO

Carbonate for Industrial Use-Determination of Sodium

Carbonate for lndustrial Use-List of Meth. of Test an

Carbonate for Industrial Use (Including Foodstuffs): De

Carbonate for Industrial Use: Determination of Chloride

Carbonate for lndustrial Use: Determination of Iron Con

Carbonate for Industrial Use: Determination of Loss of

Carbonate for Industrial Use: Determination of Matter

Carbonate for Industrial Use: Determination of Sodium $\mathrm{H}$

Carbonate for lndustrial Use: Determination of Sulphate

Carbonate for lndustrial Use: Determination of Total So

Carbonate for Industrial Use: Expression of Test Result

Carbonate for Industrial Use: Preparation and Storage $\mathrm{O}$

Carbonate, Anhydrous (1965)

Carbonate, Gas-Volumetric Meth. (1969)

Carbonate: Gas-Volumetric Meth. (1969)

Carbonate: Volumetric Meth. (1968)

E: Determina ISO

C. for Sodium Carb ISO

Carbonyl Compounds Content-Free Hydroxylamine Meth. ( ISO

Carbonyl Compounds (Hydroxylammonium Chloride Potentiom ISO

Carburized and Hardened Cases (1973)

Carcass of Conveyor Belts (Meth. of Test) (1966)

Std. for Steel ISO

ISO

Cardamoms-Spec. (1968)

Cards-Working Width (1971)

Cards (Information Processing) (1970)

Cards (Information Processing) (1971)

Cards (1970)

Cards (1971)

/Ec. for Represe ISO

Ons and Locatio ISO

ISO

Cargo Gear Particulars Book (1972)

Rec for Information Proce isO

Carpets-Determination of Mass of Piie Per Unit Area T

Carpets-Determination of Mass of Total Pile Yarn Per

Carpets-Determination of Measured Surface Pile Densit

Carpets-Determination of Number of Tufts And/or Loops

Carpets-Determination of Thickness of Pile Above the

arpets: Determination of Tuft Leg Length Above the Wov

Carpets: Determination of Types of Knots (1972)

Carriers and Hospital Trolleys: Dimensions (1960)

Carrying Out Certain Determinations (1973)

and Wit ISO

Cars and Ligh

IS with 60 ISO

Cartridge Fuse Links for Miniature Fuses (1962)

Cartridge Fuse Links for Miniature Fuses (1962)

Cartridge Fuse Links (1968)

1018

1214

2016

$61-1$

$61-2$

61.3

1103

883

242

504

514

243

513

609

625

878

1408

1124

1125

1126

1138

1435

1437

1310

1867

1868

1866

1306

276

169

991

980

925

314

2312

1691

2201

2200

2198

2199

2197

2516

742

744

745

746

741

743

740

747

424

980

991

741

1271

1847

2639

882

342

1682

1681

2021

2333

2095

1958

1959

1763

1766

2549

2550

168

2466

570

1724

2416 
Irrated, Alloy, and Free Cutting Steels-Part 11: Wrought for Sizes and Mounting Dimensions of Aircraft Instrument Irawing Dies: Interehangeability Dimensions of Pellets and fication of the Effertive Depth of Carburized and Ilardened Rec. for Radiographic Rec. for

t for Determining the Conventional Bond Strength on Steel, rec. R7 (1957)

e Main Lines (1955)

ntilations-Part IV: Fittings (1966)

nd Ventilation (1966)

Rec. for Brinell Hardness Test for Grey Rec. for Malleable Rec. for Rec. for Rec. for Rec. for Classification of Grey

Rec. for Speroidal or Nodular Graphite Rec. for Whiteheart Malleable Rec. for Blackheart Malleable Rec. for Pearlitic Malleable

Rec. for Designation of the Microstructure of Graphite in Rec. for Beam Unnotched Impact Test for Grey ols for Covered Electrodes for Manual Metal Arc Welding of

$\begin{array}{ll}\text { tion in a Curren/ } & \text { Rec. for Chemical Analysis of Steel and } \\ \text { meth.) (1969) } & \text { Rec. for Chemical Analysis of Steel and }\end{array}$ meth.) (1969)

num) Zinc Alloy Castings and Mechanical Properties of Sand Rec. for Shipbuilding Details-Multipurpose Chocks o Rec. for Aluminium (Aluminum) Alloys-Sand d (Tired) Whee/ Rec. for Railway Rolling Stock Material

and Its Alloys-Electrolytic Determination in Wrought and

etails: Ship Screw Propellers, Manufacturing Tolerance for Rec. for Dental

tion of Specimens for Optical Tests on Plastics Materials: on of Magnesium Aluminium (Aluminum) Zinc Alloy Ingots for

Rec. for Dental Inlay Std. for Magnesium Zinc Zirconium Alloy Std. for Magnesium Alloy Sand Std. for Aluminium (Aluminum) Alloy Chill
anesium Aluminium (Aluminum) Zinc Alloy r Composition of Magnesium Aluminium (Aluminum) Zinc Alloy
Rec. for Cast Iron Pipes: Special
Rec. or Composition of Aluminium (Aluminum) Alloy Rec. for Composition of Aluminium (Aluminum) AlIoy Rec. for Composition of Aluminium (Aluminum) Alloy Sand Std. for Aluminium (Aluminum) Alloy Sand
Rec. for Slotted and

$100 \mathrm{~mm}$ Thread Diameter (1969)

Plates with 4 Bolt Holes (1972) Trucks - Wheels and

formation on Ferrite Materials Appearing in Manufacturers directly by Means of Stylus and Diagram (Working Equipment Means of a Pointer and Graduated Scale (Working Equipment Std. for Electrolytic perties of Electronic Tubes: Meth. of Measurement for Cold Properties of Electronic Tubes and Valves: Measurement of ies of Electronic Tubes and Valves: Meth. of Measuring the Rec. for Electronic Tubes: Meth. of Measurement of Rec. for Expression of the Properties of the Designation of Electrostatic Deflecting Electrodes of and Valves: Meth. of Measurement of Radar and Oscilloscope Valves: Meth. of Measurement of Emission Current from Hot Rec. for Std. for Analysis of Soap: Determination of Free Procedures for Electronic Components and Equipment: Test Rec. Graphical Symbols: Machines, Transformers, Primary ec. for Graphical Symbols: Machines, Transformers, Primary ec. for Graphical Symbols: Machines, Transformers, Primary Rec. for Primary Rec. for Primary Rec. for Primary Rec. for Primary Rec. Test

ds and Meth. of Cleaning (1967)

for Measurement of Photosensitive Devices: Photoconductive Rec, for Monocrystalline Semiconductor Rectifier for Sealed Nickel Cadmium Cylindrical Rechargeable Single for Sealed Nickel Cadmium Cylindrical Rechargeable Single (1971) Std. for Flexible Rec. for Flexible Std. for Flexible Rec. for Flexible Rec. for Flexible Std, for Flexible and Elongation at Break (1971) pieces (1971) iques (1972)

Rec. for Plastics-Compression Test of Rigid Rec. for Plastics-Determination of Apparent Density of

(1972) 72) (1972)

ination of Water Vapour (Vapor) Transmission Rate of Rigid Relative-Density (Specific Gravity) of Plastic, Excluding Rec. for Plastics-Bending Test for Rigid
Case Ilardening Type (1970)

Cases (Rear Mounting Type) (1959)

Rec, for Heat ISO Rec ISO

Cases (1966)

Cases (1973)

Std. for Steel:

Rec. for Hard Metal Wire ISO
el: Determination and Veri ISO

Cast Copper Alloys (197i)

IEC

Cast Iron and Other Metals (1968)

Pipe Fittings Screwed in Accordance with ISO ISO

Iron Pipes: Special Castings and Parts for Pressur ISO

ast Iron Sanitary Pipe Fittings for Waste Water and Ve ISO

Cast lron Sanitary Pipes and Fittings for Waste Water a ISO

Cast Iron (1961)

Cast Iron (1961)

Cast Iron (1969)

Cast Iron (1969)

Cast Iron (1969)

Cast Iron (1969)

Cast Iron (1969)

Cast Iron (1969)

Cast Iron (1969)

Cast Iron: Determination of Sulphur (Meth. After Combus

Cast Iron: Determination of Total Silicon (Gravimetric

Cast Reference Test Bars (1971) Sium Aluminium (Alumi ISO

Cast Steel (1971)

Cast Test Pieces-Mechanical Properties (1971)

ISO

Cast Wheel Centres (Centers) in Nonalloy Steel for Tyre ISO

Cast-Copper Alloys (1971) /Hemical Analysis of Copper ISO

Casting and Finishing (1966)

Casting Gold Alloy (1970)

Casting Meth. (1968)

Casting Purposes (1959)

Rec. for Shipbuilding D

ISO

ISO

Rec. for Plastics-Prepara ISO

Rec. for Compositi ISO

Casting Wax (1970)

Castings-Chemical Composition (1972)

Castings-Reference Test Bar (1972)

Castings-Reference Test Bar (1972)

Castings and Mechanical Properties of Sand Cast Referen

Castings and Parts for Pressure Main Lines (1955)

Castings (Complement to R 164) (1961)

Castings (1960)

Castings: Reference Test Bar (1972)

Castle Nuts with Metric Thread (1963)

Castle Nuts with Metric Thread, $42 \mathrm{Up}$ to and Including

Castors-Dimensions of Top Plates-Part I: Oblong Top ISO

Castors-Vocabulary (1971)

Castors (1969)

Catalogues of Transformer and Inductor Cores (1972)

Category) (1970)

Category) (1970)

Responsive Elements and Recordin

Cathode Copper (1972)

/Lements Giving Direct Indications by OIM

ISO

ISO

ISO

ISO

ISO

ISO

ISO

ISO

ISO

ISO

Cathode Counting and Indicator Types (1970)

Cathode Heating Time and Heater

Cathode Ray Charge Storage Types (1971)

Cathode Ray Oscilloscopes (1971)

Cathode Ray Tubes (1967)

Cathode Ray Tubes (1969)

Cathodes for High Vacuum Electronic Types (1966)

ISO

Cauliflowers: Guide to
Caustic Alkali (1973)

Ca: Damp and Steady Heat (1969)

Cells and Accumulators (1960)

Cells and Accumulators: Aerials (Antennas) and Radio St
Cells and Accumulators: Microwave Technology (1968)

Cells and Batteries (1971)

Cells and Batteries: General (1971)

Cells and Batteries: Spec. Sheets (1972)

Cells and Batteries: Terminals (1965)

Cells for Measuring the Resistivity of Insulating Liqui

Cells for Use in the Visible Spectrum, Meth. of (1970)

Cells, Stacks, Assemblies and Equipment (1963)

Cells: Dimensions (1972)

Cells: General Requirements and Test Meth. (1971)

Cellular Materials-Accelerated Ageing Tests (1972)

Cellular Materials-Determination of Apparent Density

Cellular Materials-Determination of Compression (1972

Cellular Materials-Determination of Tensile Strength

Cellular Materials-Measurement of Dimensions of Test

$\begin{array}{lll}\text { /Rical Pro } & \text { IEC } \\ 6) / L & \text { IEC }\end{array}$

IEC

IEC

Rec, for Meth. for IEC

Cellular Materials: Hardness Test. by lndentation Techn

Cellular Plastic (1968)

Cellular Plastic (1968)

Cellular Plastics-Determination of Linear Dimensions

Cellular Plastics-Determination of Shear Strength (19

Cellular Plastics-Determination of Tensile Properties

Rec for Plastics-Determ ISO

Cellular Plastics (1970)

Cellular Types (1970)

Cellular (1970)

$683 / X 1$ 
Rer. for Plastirs-Determination of Light Absorption of Determination of Viscosity Number and Viscosity Ratio of - Determination of the Moisture Content of Nonplasticized Rec. for Plastics-Determination of Ash of Unplasticized r Plastics: Determination of Free Acidity of Unplasticized ec. for Plastics-Determination of Insoluble Particles in Plastics-Determination of Viscosity Loss on Moulding of tics-Determination of Acetic Acid Yield of Unplasticized determination of Ethyl Ether Soluble Matter in Plasticized ompressive and Flexural Strengths of Plastic Mortar (Rilem Rec. for Asbesto Rec. for Asbesto (1964)

ec. for Asymmetrical Section Corrugated Sheets in Asbesto nage (1968)

Rec. for Asbesto Rec. for Asbestos Rec. for Asbestos Rec. for Sampling and Inspection of Aabestos e Use of ISO Rec. 390, Sampling and Inspection of Asbestos Rec. for Asbesto Rec. for Asbestos

Rec. for Rubber Sealing Rings for Joints in Asbesto Rec. for Building and Sanitary Pipes in Asbestos emical Analysis of Cements: Minor Constituents of Portland ical Analysis of Cements: Main Constituents of of Portland Rec. for Dental Silicate Rec. for Dental Zinc Phosphate Mortar (Rilem Cembu/ Rec. for Meth. of Test. Strength of Rec. for Definitions and Terminology of Rec. for Pozzolanicity Test for Pozzolanic Rec. for Chemical Analysis of Rec. for Chemical Analysis of Rec. for Chemical Analysis of Std. for Centre Drills for Rec. for Centre

s-Type a (1968) Cheese Centres

$3 \mathrm{MHz}$ : Edge Socket Connectors with Open Ends, Off Centre c. for Location and Width of the Recoding Head for Centre ral Purpose Hubs and ReeIs, with $76 \mathrm{~mm}$ (3 In.) Centrehole

$5 \mathrm{~mm}$ Double Width Push Pull Sound Prints Normal and Offset Rec. for Limiting Values for the Adjustment of Centres y Rolling Stock Material-Rolled on Forged Wheel Centres for Railway Rolling Stock Material-Cast Wheel Centres ories of Libraries, Information and Documentation Centres Rec. for Lathe Centre Rec. for Lathe Centre

y Wafer Switches (Low Current Rating): Wafer Switches with mm (1966) Rec. for Rotary Wafer Switches with Rec. for Std. Frequencies for

ec. for General Purpose Hubs and Reels, with $76 \mathrm{~mm}$ ( $3 \mathrm{In}$.) Rec. for Natural Rubber Latices, Rec. for Dimensions of Rec. for Rec. for Rec for

inal Voltage Greater Tha/

Rec. for Tests on Insulators of ation of Instruments for Measuring the ${ }^{\circ} \mathrm{Of}$ Humidity of ic Reference Meth.) (1968)

tine Meth.) (1968)

-Ray Examination (1970)

rains (1966) ash $(1972)$

for Instruments for Measuring the Mass Per Hect Rec. for Rec. for Std. for Std. for Rec. for

Unit Loads-Single Strand Floor Mounted Truck Conveyor ment for Unit Loads-Single Strand Floor Truck Conveyors ical Handling Equipment for Unit Loads-Overhead Monorai Rec. for High Tensile Steel Chains (Round Link) for

cal Handling Equipment for Unit Loads-Overhead Twin Rail

$t$ Link Chain for Lifting Purposes-Grade 40 Noncalibrated c. for Shackle Type Connector Units for High Tensile Stee ptance (1971)

ype for Pulley Blocks and Other Lift Rec. for Short Link

Rec. for Short Link Rec. for Short Link in for Lifting Purposes-Grade 40 Noncalibrated Chain for Rec. for Conveyor Chains, Attachments and Std. for Extended Pitch Precision Roller Chains an Steel Roller Chains Type S 32 to 88 with Their Associated - for Short Pitch Transmission Precision Roller Chains and ec. for Short Pitch Transmission Precision Bush Chains and Rec. for Shipbuilding Details-Anchor

Rec. for Shipbuilding Details for Sea Navigation-Anchor ng Details for Sea Navigation and Inland Navigation Anchor
Cellulose Acetate Before and After Heating (1970) Cellulose Acetate in Dilute Solution (1970)

Cellulose Acetate (1967)

Cellulose Acetate (1968)

Cellulose Acetate (1969)

Cellulose Acetate (1970)

Cellulose Acetate (1970)

Cellulose Acetate (1970)

Cellulose Acetate (1971)

Cembureau Meth.) (1968)

Cement Corrugated Sheet

Cement Flat Sheets (1964)

Cement for Roofing and Cladding (1964)

Cement Pipes, Joints and Fittings for Sewerage and Drai

Cement Pressure Pipes (1971)

Cement Products (1964)

Cement Products (1970)

Cement Siding Shingles (1968)

Cement Slates for Roofing and Cladding (1964)

ISO

Cement

Cement (1964)

Cement (1968)

Cement (1968)

Cement (1970)

stics ISO

Rec. for Plastics ISO

Rec. Fo ISO

Rec for ISO

Rec for Plas ISO

Rec. for Plastics- ISO

TTest. Strength of Cements-C ISO

Cement (1970)

Rec. for Chem ISO

Cements-Compressive and Flexural Strengths of Plastic

Cements (1967)

Cements (1968)

Cements: Determination of Sulphur as Sulphide (1968)

Cements: Main Constituents of of Portland Cement (1968)

Cements: Minor Constituents of Portland Cement (1968)

Center Holes with Radius Form: Type R (1972)

(Center) Drills for the Holes Without Protecting Chamfer

(Center) for Bast Fibre (Fiber) Yarns (1966)

(Center) Guide, Having a Contact Spacing of $2.54 \mathrm{~mm}(0.1$

Center) Sound Records on $16 \mathrm{~mm}$ Perforated Magnetíc Film

(Centerhole), for Magnetic Tape Used in Interchange Inst

/Ecords and Scanning Area of 3 ISO

(Centers) for Transmission Pullevs (1960)

(Centers) for Tyred (Tired) Wheels for Trailer (1969)

(Centers) in Nonalloy Steel for Tyred (Tired) Wheels for

(Centers) (1972)

(Centers): Centre Angle (1969)

Centers): Sizes for Interchangeability (1963)

Central Mounting (1963)

Centralized Network Control Installations (1967) IEC

Centrehole (Centerhole), for Magnetic Tape Used in Inte

Centrifuged and Creamed, Ammonia Preserved-Spec. (197

Ceramic Dielectric Capacitors of the Plate Type (1967)

Ceramic Dielectric Capacitors Type 2 (1965)

Ceramic Dielectric Capacitors Type 3 (1970)

Ceramic Dielectric Capacitors, Type 1 (1967)

Ceramic Material or Glass for Overhead Lines with a Nom

/Rking Std. Meth. for the Verific

Coreal Products: Determination of Moisture Content (Bas

Cereal Products: Determination of Moisture Content (Rou

Cereals and Pulses-Meth. of Test for Irfestation by X

Cereals and Pulses: Determination of the Mass of $1000 \mathrm{G}$

Cereals Pulses-Sampling of Milled Products (1972)

Cereals $(1970)$

International Rec

Cereals, Pulses and Derived Products: Determination of

Cereals: Sampling (As Grain) (1969)

Certification Scheme (1961)

Chain Above Floor)-Safety Code (1971)

Chain Below Floor) Safety Code (1972)

Chain Conveyors-Safety Code (1971)

Chain Conveyors and Coal Ploughs (1967)

Chain Conveyors (Power and Free) Safety Code (1971)

Chain for Conveyors (1969)

Chain for Lifuing Purposes-Crade 40 Calibrated Load T

Chain for Lifting Purposes-Grade 40 Noncalibrated Cha ISO

Chain Slings, Etc. (1971) Rec. for Short Link Cha ISO

Chain Wheels-Part I: Chains (Metric Series) (1971)

Chain Wheels for Transmission and Conveyors (1972)

Chain Wheels (1966)

Chain Wheels (1967)

Chain Wheels (1970)

Chains-End Shackles (1963)

Equipment

Handling Equip ISO Ontinuous Mechan ISO

680

1565

1565

679

597

863

682

680

681

2541

866

480

130-13

890

1858

72

$1005 / \mathrm{IV}$

2146

$298 / 1$

298

$132 \cdot 2$

$132-4$

242

1858

2004

234

187

324

108

108
383

8

711

712

1162

520

2170

15

2171

950

21

2196

2381

610

2150

1835

1082

1834

1836

1835

1835

1977

1275

487

606

1395

Chains-Lugless Joining Shackles, Kenter Type (1957) ISO

Chains-Studless Links (1957) Rec. for Shipbuildi ISO
39

40 
Std. for Extended Pitch Precision Roller Rer. for Short Pitch Transmission Precision Roller Rec. for Short Pitch 'Transmission Precision Bush

Is $(1966)$ Rec. for Steel Roller (1967) Conveyor Chains, Attachments and Chain Wheels-Part

metric Series) (1971) Rec. for High Tensile Steel Std. for Shipbuilding: Anchor Rec. for Conve yor

d Joining Shacklel for Meters for Liquids (Other Than Water) with Measuring ering of Aircraft Engines, Engine Cylinders and Combustion

Rec for Rolling Bearings-Tapered Roller-In. Series or Centre (Center) Drills for the Holes Without Protecting the M/ Rec. for Plastics-Meth. of Test to Determine the e Boiling Point (1) Rec. for Determination of Dimensional Rec. for Plastics-Rec. Pract. for the Determination of foc for Basic Environmental Test. Procedures: Guidance on rocedures for Electronic Components and Equipment: Test $\mathrm{N}$ Determination of Resistance of Plastics to Colour (Color) Determination of Resistance of Plastics to Colour (Color) Determination of Resistance of Plastics to Colour (Color)

$(1970 /$ Rec. for Endless Wide V-Belts for Industrial Speed Rec. for on Load Tap

made Textile Floor Coverings: Determination of Dimensional Rec. for Shuttles for Pirn tion and Determ/ Rec. for Liquid Flow Measurement in Open Rec. for Liquid Flow Measurement in Open Rec. for Liquid Flow Measurement in Open

Rec, for Test Meth for Induction Furnaces with Submerged tant Rate Injec/ Rec. for Liquid Flow Measurement in Open Rec. for ISO Conventional Typographical Rec. for Print Spec. for Magnetic Ink Rec. for Printing Spec. for Optical n of 4 Bit Character Sets Derived from the ISO 7 Bit Coded essing) (1970/ Rec. for Representation of ISO 7 Bit Coded e (1969) Rec. for Implementation of the 7 Bit Coded merical Control of Machines (Compatible with the ISO $7 \mathrm{Bit}$ Set for In/ Rec. for Guide for the Definition of $4 \mathrm{Bit}$ 1967) Rec. for 6 and 7 Bit Coded Std. for Coding of Rec. for Alphanumeric pe (196) Rec. for Implementation of the 6 and $7 \mathrm{Bit}$ Coded ansmission (1970) Rec. for
Rer (Sections Y, Z, A, B, C, D, E)-Electrical Conductivity Rec. for Troughability of Conveyor Belts orated Film (1971) Rec. for Cinematography-Recorded ency Coaxial Cables (1967) Rec. for Rec. for d.c. Periodmeters: ecting and Applying Resilient Devices) (1972)

es and Other Medical Equipment: Definition and Dimensional bration Measurement (1/ Rec. for Meth. for Specifying the Its (1965) Rec. for

Rec. for Measurement of the Performance Rec. for Fire Resisting Rec. for Flame Retardan Rec. for

ing Purposes (1967) Rec. for Meth. for Specifying the hock and Vibration Mea/ Rec. Meth. for Measurements of the Electroacoustical ose Bulk Materials and Unit Loads-Belt Conveyors-Basic 6) Rec. for Genera Par I/ Rec for Meth of Measurement of the Performance inciples of Measuring Met/ inciples of Measuring Met/ inciples of Measuring Met inciples of Measuring Met/ ngular Face (1969)

dPin Type (1969) Rods for Braze Welding: Meth. of Test for Determining the Volum/ Rec. for Meth. of Measurement of the Performance (196) Rec. for Rotating Electrical Machines: Ratings and Rec for Essential According to Their Use (1969) Rec. for List of Rec. for Scales and Sizes for Plotting Frequency tic Tape Recording and Reproducing Systems: Dimensions and Std. for Lifting Hooks-Genera ics Coated with Rubber or Plastics-Determination of Roll Std. for Steel Wire Ropes for General Purposes:

Std. for Cork-Expanded Pure Agglomerated Thermal Cork vacuum and a Subs/ Rec. for Designation of the Quantities for International System for the Transliteration of Arabic national System for the Transliteration of Slavic Cyrillic ational System for the Transliteration of Greek into Latin

for Electronic Tubes: Meth. of Measurement of Cathode Ray
Chains and Chain Wheels for Transmission and Conveyors Chains and Chain Wheels (1967)

Chains and Chain Wheels (1970)

Chains Type S 32 to 88 with Their Associated Chain Whee

Chains (Metric Series) (1971)

Chains (Round Link) for Chain Conveyors and Coal Plough Chains (1973)

Chains, Attachments and Chain Wheels-Part I: Chains (

Chains, Stud Links (Common, Enlarged, and End Types, an

Chambers (1970) International Rec

Chambers, and Direction of Rotation of Engines and Prop

Chamfer Dimension Limits and Maximum Shaft and Housing

Chamfer Dimension Limits (1969)

Chamfers-Type a (1968)

Change in Electrical Properties of Polyethylene Due to

Change in Woven Fabrics Subjected to Laundering Near Th

Change of Mechanical Properties After Contact with Chem

Change of Temperature Tests (1971)

Change of Temperature (1969)

Change Upon Exposure to Daylight (1968)

Change Upon Exposure to Light of the Enclosed Carbon Ar

Changers and Groove Profiles for Corresponding Pulleys

Changers (1966)

Changes in Varying Moisture Conditions (1972)

Changing Automatic Looms (1967)

Channels-Establishment and Operation of a Gauging Sta

Channels by Slope Area Meth. (1969)

Channels by Velocity Area Meth. (1968)

Channels (1972)

Channels: Dilution Meth. for Steady Flow-Part 1: Cons

Character for Legibility Tests (1965)

Character Recognition (1969)

Character Recognition (1971)

Character Set for Information Processing Interchange (1

Character Set on 12 Row Punched Cards (Information Proc

Character Set on 9 Track $12.7 \mathrm{~mm}$ (1/2 In.) Magnetic Tap

Character Set) (1968) Rec. for Code

Character Sets Derived from the ISO 7 Bit Coded Charact
Character Sets for Information Processing Interchange (

Character Sets for Information Processing
Character Sets for MICR and OCR (1972)

Character Sets for Optical Recognition (1969)

Character Sets on Punched Tape (1969)

Character Sets on 7 Track $12.7 \mathrm{~mm}$ (1/2 In.) Magnetic Ta

Character Structure for Start / Stop and Synchronous Tr

Characteristic and Meth. of Test (1971)

Characteristic and Meth, of Test) (1968)

Characteristic for Magnetic Sound Records on $35 \mathrm{~mm}$ Perf

Characteristic Imped ances and Dimensions of Radio Frequ

Characteristics and Test Meth. (1969)

Characteristics and Test Meth. (1972)

Characteristics for Mechanical Isolation (Guide for Sel

Characteristics for Those with a $6 \%$ and a $10 \%$ Taper (19

Characteristics of Auxiliary Equipment for Shock and VI

Characteristics of Construction of Ply Type Conveyor Be

Characteristics of Electric Blankets (1969)

Characteristics of Electric Cables (1970)

Characteristics of Electric Cables (1970)

Characteristics of Electric Infra Red Emitters for Heat

Characteristics of Electro Mechanical Transducers for S

Characteristics of Hearing Aids (1959)

Characteristics of Motorized Driving Pulleys (1970)

Characteristics of Nuclear Reactor Instrumentation (196

Characteristics of Positive Displacement Vacuum Pumps-

Characteristics of Semiconductor Devices and General Pr

Characteristics of Semiconductor Devices and General Pr

Characteristics of Semiconductor Devices and General Pr

Characteristics of Semiconductor Devices and General Pr

Characteristics of Solid Wood Parquet Strips with Recta

Characteristics of String Insulator Units of the Cap an

Characteristics of the Deposited Metal (1968)

Characteristics of Vapour (Vapor) Vacuum Pumps-Part I

Characteristics of 3 Phase, $50 \mathrm{~Hz}$ Turbine Type Machines

Characteristics of $35 \mathrm{~mm}$ Microfilm Reading Apparatus (1

Characteristics Which May Be Required of Conveyor Belts

Characteristics (1968)

Characteristics (1968)

Characteristics (1972)

Characteristics (1972)

Characteristics (1973)

Characteristics, Sampling and Packing (1972)

Characterizing the Magnetic and Electric Properties of

Characters (1961)

Characters (1968)

Characters (1968)

Charge Storage Types (1971) 
41) $(1961)$

Rec. for High Pressure Air Rec. for Plastics-Determination of the

Rec. for

nd Classification (1971)

Rec. for Diagrams,

Rec. for Diagrams,

Rec. for Diagrams,

nd Equipment (1971)

ation) of Aluminium (Aluminum) and Its Alloys-Insulation als-Anodisation of Aluminiun (Aluminum) and Its Alloys $\mathrm{n}$ and Use of the ISO Micromire (ISO Micro Test Object) for Rec. for Section Rec. for t Content (Reference Meth.) (1970) 966)

Rec. for Perforated Metal Rec. for Perforated Parallel Tubes for Rec. for Cone Winders or Rec. for Slotted s (1966) Std. for Whey

(1972) tometric Determination of Manganese (Contents B ric Determination of Zinc in Aluminum Alloys ( $Z /$ ric Determination of Iron (Orthophenanthroline / s-Determination of Zinc-Volumetric Meth. (1) s-Complexometric Determination of Magnesium (/ s: Photometric Determination of Copper (Oxalyld/ s: Gravimetric Determination of Silicon (Conten/ s: Photometric Determination of Silicon (Conten/ termination of Titanium Spectrophotometric Meth/ tic Determination of Copper in Aluminium Alloy / as Sulphide (1968)

ortland Cement (1968)

tland Cement (I968)

ic Determination in Unalloyed Type Containing $\mathrm{N} /$ ic Determination in Wrought and Cast-Copper All/ f Copper Refinery Shapes (1971)

cometric Determination of Nickel (Low Contents)) tometric Determination of Iron in the Alloys (1/ nation of Soluble Zirconium (Alizarin Sulphonat/ nation of Zinc-Volumetric Meth. (1970)

nation of Silicon-Spectrophotometric Meth. Wi/ nation of Rare Earths-Gravimetric Meth. (I972) ric Determination of Aluminium in Magnesium All/ ric Determination of Iron (Orthophenanthroline / ric Determination of Copper (Oxalyldihydrazide / ric Determination of Manganese: Periodate Meth./ ric Determination of Manganese: Periodate Meth. aphic Determination of Zinc (Content Between 0. .) hygroscopic Moisture (1963)

silicon Dioxide (1963)

active Oxygen-Conventionally Express/ total Iron Content (1963)

carbon Dioxide (1963)

nickel (1963)

cobalt (1963)

arsenic (1963)

aluminium (Aluminum) Oxide (I 963)

total Manganese Content (1963)

sulphur (1963)

phosphorus (1963)

copper (I963)

lead (1963)

arium Oxide Content (1966)

ombined Water Content (1966)

alcium Oxide Content and Magnesium Oxi/

hromium Content (I967)

etsllic Iron Content: (Photometric Met)

itanium Content (1966)

anadium Content (1966)

inc Content (I 966)

inc Content (Polarographic Meth., for /

of Total Silicon (Gravimetric Meth.) (1969)

of Sulphur (Meth. After Combustion in a Curren/ (Spectrophotometric Meth.) (1967)

bon (Gravimetric Meth. After Combustion in a St/ per by Electrolysis (197I)

tion of Aluminium (Aluminum) (I970)

nation of Lead and Cadmium in That Containing C

metric Determination of Tin (1970)

etric Determination of Iron (1969)

Iron (I968)

of Cadmium in Zinc (I969)

of Lead and Cadmium (I968)

of Lead (1968)

tion of Copper (I969)

$n$ the Form for Std. for Chemical Products and for Meth. of or Textiles-Binary Fibre (Fiber) Mixtures-Ouantitative

ion of Wrought Products of Aluminium and Aluminum Alloys Std. for Magnesium Zinc Zirconium Alloy Castings

rea of Hot Dip Galvanized Coatings on Ferrous Materials by
Charging Valves for Aircraft (1969)

Charpy Impact Resistance of Rigid Plastics (Flexural Te

ISO

ISO

Charts, Tables Used in Electrotechnology: Definitions a

Charts, Tables (Electrotechnology-Electrical) (I959)

Charts, Tables: Item Designation for Electrical Parts a

Check of Continuity of Thin Coatings-Coper Sulphate

Checking a Reading Apparatus (1968)

Checking of V-Belts (1962)

Cheese and Its Processed Products-Determination of Fa

Cheese Centres (Center) for Bast Fibre (Fiber) Yarns (1

Cheese Dyeing (1967)

Cheese Winders: Terminology; Basic Terms and Definition

Cheese (Fillister) Head Screws-Metric Series (1970)

Rec Cheese: Determination of Fat Content (Reference Meth.)

Rec. for Chemical Analysis of Aluminium and Its Alloys: Gravimet

Rec. for Chemical Analysis of Aluminium and Its Alloys: Photomet

Rec. for Chemical Analysis of Aluminium (Aluminum) and Its Alloy

Std. for Chemical Analysis of Aluminium (Aluminum) and Its Alloy

Rec. for Chemical Analysis of Aluminium (Aluminum) and Its Alloy

Rec. for Chemical Analysis of Aluminium (Aluminum) and Its Alloy

Rec. for Chemical Analysis of Aluminium (Aluminum) and Its Alloy

Rec. for Chemical Analysis of Aluminum and Aluminium Alloys-D

Rec. for Chemical Analysis of Cements: Determination of Sulphur

Rec. for Chemical Analysis of Cements: Main Constituents of of $P$

Rec. for Chemical Analysis of Cements: Minor Constituents of Por

Rec. for Chemical Analysis of Copper and Its Alloys-Electrolyt

Rec. for Chemical Analysis of Copper and Its Alloys-Electrolyt

Rec. for Chemical Analysis of Copper and Its Alloys-Sampling 0

Rec. for Chemical Analysis of Copper and Its Alloys-Spectropho

Rec. for Chemical Analysis of Copper and Its Alloys-Spectropho

Rec. for Chemical Analysis of Magnesium and Its Alloys-Determi

Rec. for Chemical Analysis of Magnesium and Its Alloys-Determi

Rec. for Chemical Analysis of Magnesium and Its Alloys-Determi

Std. for Chemical Analysis of Magnesium and Its Alloys-Determi

Rec. for Chemical Analysis of Magnesium and Its Alloys: Gravimet

Rec. for Chemical Analysis of Magnesium and Its Alloys: Photomet

Rec. for Chemical Analysis of Magnesium and Its Alloys: Photomet

Rec. for Chemical Analysis of Magnesium and Its Alloys: Photomet

Rec. for Chemical Analysis of Magnesium and Its Alloys: Photomet

Rec. for Chemical Analysis of Magnesium and Its Alloys: Polarogr

Rec. for Meth. of Chemical Analysis of Manganese Ores-Determination of

Rec. for Meth. of Chemical Analysis of Manganese Ores-Determination of

Rec. for Meth. of Chemical Analysis of Manganese Ores-Determination of

Rec. for Meth. of Chemical Analysis of Manganese Ores-Determination of

Rec. for Meth. of Chemical Analysis of Manganese Ores-Determination of

Rec. for Meth. of Chemical Analysis of Manganese Ores-Determination of

Rec. for Meth. of Chemical Analysis of Manganese Ores-Determination of

Rec. for Meth. of Chemical Analysis of Manganese Ores-Determination of

Rec. for Meth. of Chemical Analysis of Manganese Ores-Determination of

Rec. for Meth. of Chemical Analysis of Manganese Ores-Determination of

Rec. for Meth. of Chemical Analysis of Manganese Ores-Determination of

Rec. for Meth. of Chemical Analysis of Manganese Ores-Determination of

Rec. For Meth. of Chemical Analysis of Manganese Ores: Determination of C

Meth. of Chemical Analysis of Manganese Ores: Determination of $\mathbf{C}$

c. for Meth. 06 Chemical Analysis of Manganese Ores: Determination of $\mathrm{C}$

Rec. for Meth. of Chemical Analysis of Manganese Ores: Determination of $T$
Rec. for Meth. of Chemical Analysis of Manganese Ores: Determination of $V$

Rec. for Meth. of Chemical Analysis of Manganese Ores: Determination of Z

Rec. for Meth. of Chemical Analysis of Manganese Ores: Determination of $Z$

Rec. for Chemical Analysis of Steel and Cast Iron: Determination

Rec. for Chemical Analysis of Steel and Cast Iron: Determination

Rec. for Chemical Analysis of Steels: Determination of Manganese

Rec. for Chemical Analysis of Steels: Determination of Total Car

Rec. for Chemical Analysis of Zinc Alloys-Determination of Cop

Rec. for Chemical Analysis of Zinc Alloys-Volumetric Determina

Std. for Chemical Analysis of Zinc Alloys: Polarographic Determi

Rec. for Chemical Analysis of Zinc and Its Alloys-Spectrophoto

Rec. for Chemical Analysis of Zinc and Its Alloys: Spectrophotom

Rec. for Chemical Analysis of Zinc: Photometric Determination of

Rec. for Chemical Analysis of Zinc: Polarographic Determination

Rec. for Chemical Analysis of Zinc: Polarographic Determination

Rec. for Chemical Analysis of Zinc: Polarographic Determination

Chemical Analysis of Zinc: Spectrophotometric Determina

Chemical Analysis (1969)

Chemical Analysis (1971)

Chemical Composition (Per Cent) (1971)

Rec. for Guide O ISO

Rec. F ISO

1. for Composit ISO

Chemical Composition (1972)

Chemical Dissolution of the Coating-Gravimetric Meth.
1023

179

$113-1$

113

113-2

2376

2085

689

256

480

574

477

1207

1854

886

798

793

1784

229

795

797

808

1118

796

682

680

$68 \mathrm{I}$

1553

I554

1811

1810

1812

1178

I 783

1975

2355

$79 I$

792

794

809

810

807

310

$3 I 1$

3 I 2

313

$3 I 4$

3 I5

316

317

318

319

320

321

322

323

548

549

552

619

621

550

553

551

620

439

671

629

437

1976

1169

2576

1570

I055

714

1054

713

715

1053

78
1833

209

209

2119
1460 
Rer. for Guide on the Form for Std. for Plastic-Determination of the Resistance of Plastics to tion of Change of Mechanical Properties After Contact with Rec. for Common Names for Pest Control nciples for the Selection of Common Names for Pest Control Std. For Quantities and Units of Physical Rec. for Restraining Devices for

Std. for Aluminium (Aluminum) Alloy Rec. for Spices and Condiments: Rec. for Application of Carbides for Machining by Std. for Woodworking Tools: Std. for Sodium Std. for Sodium Std. for Sodium
ility of Polyvinyl content-Mercurimetric

content-Gravimetric Meth. (1973) tics-Determination of the Thermal Stability of Polyvinyl
Determination of sodium Hydrogen Carbonate for Industria/ Determination of or Potassium Sulo for Rec. for Meat and Its Products-Determination of $r$ Potassium Hydroxide for Industrial Use: Determination of for Sodium Carbonate for Industrial Use: Determination of for Sodium Hydroxide for Industrial Use: Determination of s (1968) Spec. for Rigid Conduits of Polyvinyl Gravimetric Meth. as Potassium Tetr/ Sodium Tatraphenylborate Volumetric/ Rec. for Potassium Content-Gravimetric Meth. (1971)

Rec. for Potassium Content-Flame Emission Photometri/

Rec. for Potassium nd Magnesium Contents-EDTA Complexomet/ Std. for Sodium Expressed as Chlorine-Mercurimetric M/ soluble in Water or in Acid and Preparat/ content-Barium Sulphate Gravimetric $\mathrm{Me}$ of Mass at $110^{\circ} \mathrm{C}(1973)$

lar Conductors and a Rated Voltage $\mathrm{No}$ Std. for Sodium Std. for Sodium Std. for Sodium Std. for Sodium Spec. for Polyvinyl Rec for Plastics-Determination of Chlorine in Vinyl se-Determination of Carbonyl Compounds (Hydroxylammonium Plastics-Determination of Viscosity Number of Polyvinyl

f Test $(1970)$ Rec. for Plastics: Designation of Polyvinyl Rec. for Methylene

fittings for Pipes Under Pressure: Unplasticized Polyvinyl

Oven Test for Moulded Fittings in Unplasticized Polyvinyl ipes for the Transport of Fluids-Unplasticized Polyvinyl ipes for the Transport of Fluids-Unplasticized Polyvinyl etric Series-S/ Std. for Buried Unplasticized Polyvinyl Typ/ Std. for Single Sockets for Unplasticized Polyvinyl td. for Double Socket Fittings for Unplasticized Poly vinyl

of Unplasticized Compounds of Homo and Copolymers of Vinyl rmination of Bromine Index in the Presence of Mercury (II)

opolymers and Their Compounds by Splitting Off of Hydrogen Rec. for Meth. of Test for Pve (Polyvinyl ec. for Low Frequency Cables and Wires with Pve (Polyvinyl ec. for Low Frequency Cables and Wires with Pvc (Polyvinyl ec. for Low Frequency Cables and Wires with Pve (Polyvinyl ec. for Low Frequency Cables and Wires with Pvc (Polyvinyl ec. for Low Frequency Cables and Wires with Pyc (Polyvinyl ec. for Low Frequency Cables and Wires with Pvc (Polyvinyl $(1969 /$ Rec. for Std. Colours (Colors) for Pve (Polyvinyl ec. for Plastics-Determination of Vinyl Acetate in Vinyl Rec for Analysis of Soap: Determination of yde Solutions for Industrial Use: Limit Test for Inorganic or Indust rial Use: Determination of Halogens, Expressed as Rec. for Determination of Nitrogen, Total Sulphur,

tent Using an Electrolytic Analyser (1972) Std. for Liquid ent by Volume in the Vaporized Product (/ ntent-Gravimetric Meth. (1972) termining Only the Volumetric Content) (

Std. for Liquid Std. for Liquid Rec. for Liquid h. (1963) Rec. for Determination of Rec. for Determination of Rec. for
Rec. for Rec. for Rec. for Ortho Rec. for Para Rec. for Shipbuilding Details-Multipurpose Rec. for Zinc Chromate Pigments-Basic Zinc Potassium inc Tetrahydroxychromate $(1970)$ Rec. for Zinc

Std. for Strontium Std. for Barium

zid/ Std. for Aluminium and Its Alloys-Determination of steels, Pt. 4: Wrought Quenched and Tempered Types with $1 \%$ els-Part 2: Wrought, Quenched and Tempered Types with 1\% ng Steels-Part 6: Wrought Quenched and Tempered, with 3\% O6 Chemical Analysis of Manganese Ores: Determination of ree Cutting Steels-Part 8: Wrought Quenched and Tempered Rec. for Electroplated Coatings of Copper Plus Nickel and

Chemical Products and for Meth. of Chemical Analysis (1 150 Chemical Substances (1961) Chemical Substances (1965) Chemicals and Plant Growth Regulators (1971)

Chemicals (Pesticides) and Plant Growth Regulators (197

Chemistry and Molecular Physics (1973)

Children in Motor Vehicles (1970)

Chill Castings-Reference Test Bar (1972)

Chillies, Whole and Ground, Spec. (1969)

Chip Removal: Designation of Main Groups of (1966)

Chisels and Gouges (1973)

Chlorate for Industrial Use: Determination of Chloride

Chlorate for Industrial Use: Determination of Matter in

Chlorate for Industrial Use: Determination of Moisture

Chloride and Related Copolymers and Their Compounds by

Chloride Content-Mercurimetric Meth. (1972) Std. for

Chloride Content-Mercurimetric Meth. (1973)

Chloride Content-Mercurimetric Meth. (1973)

Chloride Content (1970)

Chloride Content: Volhard Volumetric Meth (1969)

Chloride Content: Volhard Volumetric Meth. (1973)

Chloride Content: Volhard Volumetric Meth. (1973)

Chloride for Electrical Installations and Their Fitting

Chloride for Industrial Use-Determination of Content

Chloride for Industrial Use-Determination of Content

Chloride for Industrial Use-Determination of Moisture

Chloride for Industrial Use-Determination of Potassiu

Chloride for Industrial Use: Determination of Calcium a

Chloride for Industrial Use: Determination of Halogens,

Chloride for Industrial Use: Determination of Matter in

Chloride for Industrial Use: Determination of Sulphate

Chloride for Industrial Use: Determination of the Loss

Chloride Insulated Cables and Flexible Cords (1967)

Chloride Insulated Flexible Cables and Cords witlı Circu

Chloride Polymers and Copolymers (1970)

Chloride Potentiometric Meth.) (1970)

Chloride Resin in Solution (1961)

Chloride Resins (1969)

Chloride (Dichloromethane) for Industrial Use-Meth. $\mathrm{O}$

Chloride (PVC) Fittings with Plain Sockets; Metric Seri

Chloride (PVC) for Use Under Pressure (1967)

Chloride (PVC) Pipes-Tolerances on Outside Diameters

Chloride (PVC) Pipes-Tolerances on Wall Thicknesses $U$ Rec. for ISO

Chloride (PVC) Pipes for the Supply of Gaseous Fuels: M

Chloride (PVC) Pressure Pipes with Elastic Sealing Ring

Chloride (PVC) Pressure Pipes with Elastic Sealing Ring

Chloride) Insulation and Sheath of Electric Cables (197

Chloride) Insulation and Sheath: Cables in Pairs, Tripl

Chloride) Insulation and Sheath: Distribution Wires Wit

Chloride) Insulation and Sheath: Distribution Wires Wit

Chloride) Insulation and Sheath: Equipment Wires and Ca

Chloride) Insulation and Sheath: Equipment Wires, Type

Chloride) Insulation and Sheath: General Test and Measu

Chloride) Insulation and Sheath: Signalling Cables in $S$

Chloride) Insulation for Low Frequency Cables and Wires

Chloride.Vinyl Acetate Copolymers (1970)

Chlorides (1965)

Chlorides (1972)

Chlorine-Mercurimetric Meth. (1973)

Chlorine and Phosphorus in Coke (1969)

Chlorine for lndustrial Use-Determination of /Er Con ISO

Std for Formal /Dium Chloride F ISO

Chlorine for Industrial Use-Meth. of Sampling (For De

Chlorine in Coal and Coke Using Eschka Mixture (1967)

Chlorine in Coal by the High Temperature Combustion Met

Chlorobenzene for Industrial Use-Meth. of Test (1970)

Chloroform for Industrial Use-Meth. of Test (1970)

Chlorotoluene for Industrial Use-Meth. of Test (1970)

Chlorotoluene for Industrial Use-Meth. of Test (1970)

Chocks of Cast Steel (1971)

Chromate and Zinc Tetrahydroxychromate (1970)

Chromate Pigments-Basic Zinc Potassium Chromate and Z

Chromate Pigments for Paints (1972)

Chromate Pigments for Paints (1972)

Chromium-Spectrophotometric Meth. Using Diphenylcarba 
epls: Free Cutting-Part 7: Wrought Quenched and Tempered Rec. for Electroplated Coatings of Nickel Plus - Determination of Titanium Spectrophotometric Meth. with for Shanks for Pneumatic Tools and Fitting Dimensions of for Shanks for Pneumatic Tools and Fitting Dimensions of Rec. for Rock Drilling: Forged Collared Shanks and

Rec. for Drill

966)

Rec. for Machine

Im Perforated Type S (1972)

Im with Perforations Along One and Two Edges (1/

for $16 \mathrm{~mm}$ Motion Picture Film (1969)

for Double $8 \mathrm{~mm}$ Motion Picture Film (1969)

projected Image Area for $35 \mathrm{~mm}$ Films (1958)

projected Image Area for $8 \mathrm{~mm}$ Films (1958)

$16 \mathrm{~mm}$ Films (1965)

agnetic Sound Records on $35 \mathrm{~mm}$ Film (1963)

Printing to $8 \mathrm{~mm}$ Type S on $16 \mathrm{~mm}$ Motion Pictur/

ture for Projection of $35 \mathrm{Mm}$ Nonanamorphotic Mot/ nts (1958)

nts (I958)

Television (I971)

963)

ound Records on $35 \mathrm{~mm}$ Perforated Film (1971)

mm Double Width Push Pull Sound Prints Normal A

ilm-Cutting and Perforating Dimensions (1972)

icture Film for Direct Front Projection (1973)

produced by Camera Aperture (1972)

Image Area (1972)

e Area Produced by Camera A perture and Projecta/

Location and Width of Magnetic-Striping and $\mathrm{Ga}$

for Dimensions for General Purpose Push Pull Single Pole

Rec. for Dimensions for General Purpose Push Pull 3 Pole formance Requirements for General Purpose Push Pull 3 Pole nce Requirements for General Purpose Push Pull Single Pole

9) Spec. for Voltage Operated Earth Leakage (I968) urrent Evaluation with Special Regard to Rated Capacity of Rec. for Guide to the Test. of
f Motion of Operating Devices and for Indicating Lamps for Rec. for Low Voltage Distribution Switchgear: Rec. for High Voltage Alternating Current Rec. for High Voltage Alternating Current

$s$ Tenders and / Rec. for High Voltage Alternating Current Rec. for High Voltage Alternating Current Rec. for High Voltage Alternating Current Rec. for High Voltage Alternating Current ce) (1971) Rec. for Shor

Capacity of Circuit Breakers in Installa/

of Current, Distribution Systems, Meth of Connection and

-Data Terminal and Communication Equipment-Interchange c. for Colour (Color) Identification of Mechanical Control r Frequencies Below $3 \mathrm{MHz}$ : Concentric Connectors for Audio Rec. for Rules for Ohmic Resistors Used in the Powe Rec. for Terms and Definitions for Printed Rec. for Grid System for Printed ts Intended for Mounting on Boards with Printed Wiring and

Rec. for Conventions Concerning Electric and Magnetic s Atmospheres: Spark Test Apparatus for Intrinsically Safe s (Low Voltage Switching Devices for Control and Auxiliary $s$ (Low Voltage Switching Devices for Control and Auxiliary Rec. for Metal Clad Base Materials for Printed Rec. for Metal Clad Base Materials for Printed olyvinyl Chloride Insulated Flexible Cables and Cords with Rec. for Rubber Insulated Flexible Cables and Cords with pment (/ Rec. for Connectors for Frequencies Below $3 \mathrm{MHz}$ : Coupli / Rec. for Connectors for Frequencies Below $3 \mathrm{MHz}$ : 966) Rec. for Connectors for Frequencies Below $3 \mathrm{MHz}$ : 73) Std. for Hand and Machine Operated Rec. for Tensile Test. of Copper and Its Alloy Tubes of Rec. for Copper Tubes of Rec. for Hollow Metallic Waveguides: Relevant Spec. for for Flanges for Waveguides: Relevant Spec. for Flanges for s Up to $50 \mathrm{Mm} / \quad$ Rec. Pract. for Radiographic Inspection of 968) Rec. for Metal rec. for Asbestos Cement Corrugated Sheets for Roofing and Rec. for Asbestos Cement Slates for Roofing and ction Corrugated Sheets in Asbestos Cement for Roofing and Rec. for Terminals with Screw for Measurement of Fluid Flow by Means of Venturi Tubes Rec. for Lengths of ngots for Remelting (1968) Rec. for

for Textile Machinery-Dyeing and Finishing Machinery Rec. for Binders Based on Calcium Sulphate-Definitions,
Chromium Types (1970)

Chromium (1970)

Chromotropic Acid) (1969)

Chuck Bushings-Part I (1970)

Chuck Bushings-Part II (1970)

Chuck Bushings for Hollow Hexagonal Drill Steels (1968)

Chuck Tapers (1961)

Chucking Reamers with Parallel or Morse Taper Shanks (1

Cinematography-Camera Usage of $8 \mathrm{~mm}$ Motion Picture Fi

Cinematography-Dimensions for $16 \mathrm{~mm}$ Motion Picture Fi

Cinematography-Dimensions of Daylight Loading Spools

Cinematography-Dimensions of Daylight Loading Spools

Cinematography-Inage Produced by Camera Aperture and

Cinematography-Image Produced by Camera Aperture and

Cinematography-Image Produced by Camera Aperture for

Cinematography-Location of Recording Heads for Four M

Cinematography-Location of the Printed Image Area for

Cinematography-Maximum Aspect Ratio of Projector Aper

Cinematography-Photographic Sound Record on $16 \mathrm{~mm}$ Pri

Cinematography-Photographic Sound Record on $35 \mathrm{~mm}$ Pri

Cinematography-Picture Areas for Films and Slides for

Cinematography-Projected lmage Area for $16 \mathrm{~mm}$ Film (I

Cinematography-Recorded Characteristic for Magnetic S

Cinematography-Sound Records and Scanning Area of 35

Cinem atography $-8 \mathrm{~mm}$ Type S Motion Picture Raw Stock F

Cinemaiography: Projector Usage of $8 \mathrm{~mm}$ Type S Motion P

Cinematography: $35 \mathrm{~mm}$ Motion Picture Film-Image Area

Cinematography: $35 \mathrm{~mm}$ Motion Picture Film-Projectable

Cinematography: 65 and $70 \mathrm{~mm}$ Motion Picture Film-Imag

Cinematography: $8 \mathrm{~mm}$ Motion-Picture Film with Picture-

Cineole Content (1970)

Circuit Breakers for Aircraft (1966)

Circuit Breakers for Aircraft (1969)

Circuit Breakers for Aircraft (1970)

Circuit Breakers for Aircraft (1970)

Circuit Breakers for Domestic and Similar Purposes (195

Circuit Breakers in Installations in Ships (1972)

Circuit Breakers with Respect to Out of Phase Switching

Circuit Breakers (I936)

Circuit Breakers (1964)

Circuit Breakers: Design and Construction (197I)

Circuit Breakers: General and Definitions (1971)

Circuit Breakers: Information to Be Given with Enquirie

Circuit Breakers: Rating (1971)

Circuit Breakers: Rules for the Selection of (For Servi

Circuit Breakers: Type and Routine Tests (1972)

Circuit Current Evaluation with Special Regard to Rated

Circuit Elements (1960) Wind

Circuits-Assignment of Connector Pin Numbers (I972) ISO

Circuits for Aircraft (1959)

Circuits in Radio Receivers (1969)

Circuits of Electrically Powered Vehicles (1970)

Circuits (1965)

Circuits (1970)

Circuits (1970)

Circuits (1972)

Circuits (1972)

Circuits, Including Contactor Relays) Section l: Push B
Circuits, Including Contactor Relays) : General Require

Circuits: Spec. (1970)

Circuits: Test Meth. (1968)

Circular Conductors and a Rated Voltage Not Exceeding 7

Circular Conductors and a Rated Voltage Not Exceeding 7

Circular Connectors for Radio and Associated Sound Equi

Circular Multipole Connectors with Bayonet or Push Pull

Circular Multipole Connectors with Threaded Coupling (1

Circular Screwing Dies and Hand-Operated Die Stocks (19

Circular Section-Flattening Test (1971)

Circular Section (1964)

Circular Section: Dimensions; Metric Series (1962)

Circular Types (1964)

Circular Types (1969)

Circumferential Fusion Welded Butt Joints in Steel Pipe

Clad Base Materials for Printed Circuits: Spec. (1970)

Clad Base Materials ior Printed Circuits: Test Meth. (1

Cladding (1964)

Cladding (1964)

Cladding (1964)

Clamping for External Conductors (1964)

Classical Tubes Used Outside the (1964) Covered by ISO

Classical V.Belts (Sections Z, A, B, C, D, E) (I967)

Classification and Composition of Unalloyed Aluminium I

Classification and Nomenclature (1970)

Classification and Nomenclature (197I)

Classification and Packaging (1970)
$683 / \mathrm{VIl}$

1456

1118

1180

157

723

239

521

178

1019

1020

73

74

1785

71

70

1223

359

1189

1700

1781

2907

2467

120 I

1202

530

1033

1509

1467

18

363

267

54

157. I

56-3

56-1

56.6

56-2

56-5

56-4

363

17.1

130

I $30-8$

322

I94

97

321

375

79-3

$337-2$

337-1

249.2

249-1

227

245

I30-9

130-7

130-4

2568

1556

401

274.

I53-4

I54-4

947

249.2

249-1

393

395

394

REC 2

$781 / \mathrm{I}$

608

115

1506

1588

1216 
Std. for Textile Floor Coverings: er. for Paints and Varnishes-Determination of the Danger ial Types (196.5) sile Types (1965)

ec. for Rec. for Rec. for Rec. for Rec. for Rec. for Rec. for initions of Terms (1961) Rec. for General Rec. for

c. for Electrical Apparatus for Explosive Gas Atmospheres: nt and Evaluation of the Geometrical Parameters/ rical Machinery and Apparatus in Relation T/ Rec. for c. for Electrical Apparatus for Explosive Gas Atmospheres: Rec. for

d Insulating) (1971)

Rec. for Refractory Materials . 72) rec. for Surface Treatment and Metallic Coatings-General Rec. for Rec. for Fibre (Fiber) Building Boards: Definition; Rec. for Particle Boards: Definition; Rec. for Surface Active Agents: Scientific Rec. for Coniferous Sawn (Sawed) Timber-Defects Rec. for Plywood. Rec. for Dictation Equipment Charts, Tables Used in Electrotechnology: Definitions and Std. for Surface Active Agents: Simplified Std. for Muscovite Mica Blocks, Thins and Films-Visual Std for Shaped Insulating Refractory Products

Rec. for Gypsum Rock for the Manufacture of Binders Rec. for Radioisotope All or Nothing Relays (Terminology, Rec. for Guide for the Preparation of Rec. for Layout of Multilingual rated Appliances for Domestic and Similar Purposes: Vacuum

Rec. for Meth. of Measurement of Performance of Vacuum Electrical Appliances: Particular Requirements for Vacuum ic and Similar Purposes: Vacuum Cleaners and Water Suction Rec. for Expression and Presentation of Results of Coal 0)

asuring the Resistivity of Insulating Liquids and Meth. of g $39 \mathrm{~mm}$ Thread Diameter (1962)

Rec. for g $150 \mathrm{~mm}$ Thread Diameter (1968) Rec. for Rolling Bearings-Internal with Cylindr/ Rec. for Rolling Bearings-Radial Internal lling Bearings-Double Row Selfaligning-Radial lnternal c. for Rolling Bearings: Cylindrical Type, Radial Internal arings-Double Row Self-Aligning Ball-Radial Internal Rec. for Creepage Distances and Std. for Std. for device) (1970) International Rec. for ppliances for Domestic and Similar Purposes: Shavers, Hair iances: Particular Requirements for Electric Shavers, Hair rec. Graphical Symbols: Measuring Instruments and Electric tor Operated Appliances for Domestic and Similar Purposes: Determination of the Danger Classification by Flashpoint c. for Paints and Varnishes-Determination of Flashpoint tomatic Drawing-in Machines (1969)

Rec. for s for Frequencies Below $3 \mathrm{MHz}$ : Edge Socket Connectors with I (1970) Rec. for Symbolic Designation of Direction of es of Apertures (1972) Containers and Gauges for the Inspection of Std. for Woven Wire aundering-Preparation and Use of Unsoiled Cotton Control and Heating Appliances for Domestic and Similar Purposes:

ts / Rec. for Surface Active Agents: Determination of the ted Hydrocarbons for Industrial Use-Determination of the Rec. for Determination of Rec. for Hard Rec. for Calculation of Rec. for Determination of Carbon and Hydrogen in Rec. for Determination of Carbon and Hydrogen in Rec. for Determination of Chlorine in Rec. for Determination of Arsenic in and Coke Residue by Low Temperature Distillation of Brown Rec. for Determination of the Volatile Matter of Hard c. for Determination of Moisture in the Analysis Sample of c. for Determination of Moisture in the Analysis Sample of Rec. for Determination of Total Sulphur in Rec. for Determination of Carbon Dioxide in Rec. for Determination of Total Sulphur in Rec. for Determination of Chlorine in Rec. for Determination of Nitrogen in Rec. for Determination of Caking Power of Rec. for Determination of Nitrogen in Rec. for Expression and Presentation of Results of
Classification and Terminology (1972)

Classification by Flashpoint (Closed Cup Meth.) (1970)

Classification of Adding and Calculating Machines (1969

Classification of Aluminium (Aluminum) Bronzes and Spec

Classification of Brasses, Leaded, Special and High Ten

Classification of Copper Nickel Alloys (1965)

Classification of Copper Nickel Zinc Alloys (1965)

Classification of Coppers (1961)

Classification of Dense Refractory Products (1969)

Classification of Ferromagnetic Oxide Materials and Def

Classification of Grey Cast Iron (1961)

Classification of Hazardous Areas (1972)

Classification of Instruments and Devices for Measureme

Classification of Materials for the Insulation of Elect

Classification of Maximum Surface Temperatures (1969)

Classification of Parquet Strips of Solid Oak (1970)

Classification of Prepared Unshaped Materials (Dense an

Classification of Solid Beech (Wood) Parquet Strips (19

Classification of Terms (1971)

Classification of Tin and Special Tin Bronzes (1965)

Classification (1968)

Classification (1968)

Classification (1968)

Classification (1969)

Classification (1969)

Classification (1971)

Classific ation (1971)

Classification (1972)

Classification (1972)

Classific ation (1972)

Classification, Characteristics and Test Meth. (1972)

Classification, Test Meth.) (1971)

Classified Vocabularies (Example of Meth.) (1969)

Classified Vocabularies (1969)

Cleaners and Water Suction Cleaning Appliances (1965)

Cleaners for Household and Similar Use (1971)

Cleaners (1970)

1. for Safety of Household and Similar

Cleaning Appliances (1965)

Ated Appliances for Domest

Cleaning Tests (1969)

Cleaning (1967)

Rec. Test Cells for Me IEC

(197

Clearance Holes for Metric Bolts 1,6 Up to and Includin

Clearance Holes for Metric Bolts, 42 Up to and Includin

Clearance in Unloaded Bearings-Definitions (1961)

Clearance in Unloaded Radial Groove Type Ball Bearings

Rec for Ro ISO

Re ISO

Clearance (1965)

Clearance $(1970)$

Clearances in Air (1964)

Rec. for Rolling Be ISO

Clevis Pins-Metric Series (1972)

Clevis Pins with Heads-Metric Series (1972)

Clinical Thermometers (Mercury, in Glass, with Maximum OIML

Clippers and Similar Appliances (1966)

Clippers and Similar Items (1972)

Clocks (1963)

Clocks (1967)

(Closed Cup Meth.) (1970)

(Closed Cup Meth.) (1971)

Closed End Drop Wires (Electrical and Mechanical) for a

Closed Ends and Having a Contact Spacing of $2.54 \mathrm{~mm}(0$.

Closing and Faces of Doors, Windows and Shutters-Part

1. for Finishes with External Screw Th

Cloth and Perforated Plates in Test Sieves: Nominal Siz

Ts: Verification of Certain Effects of

Clothes Dryers and Towel Dryers (1967) /Ectric Cooking CEE

Cloud Point Temperature of Nonionic Surface Active Agen ISO

Cloud Point (1970)

Coagulum Content of Latex (1968)

Rec. for Liquid Halogena

Coal-Determination of Oxygen (1971)

Coal and Coke Analyses to Different Bases (1970)

Coal and Coke by the High Temperature Combustion Meth.

Coal and Coke by the Liebig Meth. (1967)

Coal and Coke Using Eschka Mixture (1967)

Coal and Coke (1967)

Coal and Lignite (1968)

Coal and of Coke (1967)

Coal by the Direct Gravimetric Meth. (1963)

Coal by the Direct Volumetric Meth. (1963)

Coal by the Eschka Meth. (1963)

Coal by the Gravimetric Meth. (1969)

Coal by the High Temperature Combustion Meth. (1963)

Coal by the High Temperature Combustion Meth. (1963)

Coal by the Kjeldahl Meth. (1963)

Coal by the Roga Meth. (1963)

Coal by the Semi Micro Kjeldahl Meth. (1963)

Coal Cleaning Tests (1969) 
Tensile Steel Chains (Round l.ink) for Chain Conveyors and Rec. for Graphical Symbols for Rec. for Principles and Conventions for Flowsheets for abulary of Terms Relating to Solid Mineral Fuels-Part I: bulary of Terms Relating to Solid Mineral Fuels-Part II: Rec. for Determination of Forms of Sulphur in Rec. for Audibert Arnu Dilatometer Test for Rec. for Determination of the Crucible Swelling Number of Rec. for Determination of the Gray King Coke Type of Rec. for Determination of Total Moisture in Hard Rec. for Determination of Mineral Matter in Rec. for Determination of Phosphorus in Ash from Std. for Hard aterial (Resinous Su/ Rec. for Benzene Extract from Brown ination of Sodium and Potassium Soluble I/ Rec. for Brown ) Rec. for Determination of Moisture in Brown rmination of the Yield of Benzene Soluble Extract in Brown for Determination of the Moisture Holding Capacity of Hard Std. for Metal Slitting Saws with Fine and

characteristics (1972) or Determination of the Impermeability to Water of Fabrics r Accelerated Ageing and Simulated Service Test of Fabrics on of Breaking Strength and Elongation at Break of Fabrics ribution Wires with Solid Conductors, Insulated, Polyamide atings on Ferrous Materials by Chemical Dissolution of the mination (1971) Rec. for Amount of Bale tive Coatings on Nonmagnetic Basis Metals-Measurement of

gs on Magnetic and Nonmagnetic Substrates-Measurement of Enamel Coatings on Magnetic Basis Metals-Measurement of ss Sections (197/ Rec. for Measurement of Metal and Oxide um (Aluminum) and Its Alloys-Check of Continuity of Thin nt of the Thickne/ Rec. for Metallic and Other Nonorganic Rec. for Surface Treatment and Metallic num) and Its Alloys-Measurement of the Mass of the Oxide by Anodic Dissolution (1972) Std. for Metallic uminum) and Its Alloys-Measurement of Thickness of Oxide r Iron) (1970) Rec. for Electroplated Rec. for Electroplated Rec. for Electroplated Rec. for Electroplated Std. for Electroplated Rec. for Electroplated Rec. for Electroplated

Rec. for Requirements for Hot Dip Galvanized mination of the Weight Per Unit Area of Hot Dip Galvanized rement of Coating Thick/ Std. for Electrodeposited Nickel for Nonmagnetic Metallic and Vitreous or Porcelain Enamel oating Thickness-Eddy Current M/ Std. for Nonconductive valuation of the Results of Accelerated Corrosion Tests on n (1969)

haracteristic Impedances and Dimensions of Radio Frequency connectors: General Requi/ Rec. for General Purpose Rigid connectors: Detail Spec. / Rec. for General Purpose Rigid 965) Rec. for Radio Frequency Connectors: Rec. for Radio Frequency Connectors: R. F. Rec. for Radio Frequency Connectors: R. F. Rec. for Radio Frequency Connectors: R. F. of Chemical Analysis of Manganese Ores-Determination of $r$ Determination of the $\mathbb{W}$ ater Absorption of Paper or Board Std. for Programming Language:

Std. for Rotary Drilling Equipment: Upper and Lower Kelly Meth.) (1972)

rec. for Continuous Mechanical Handling Equipment-Safety Rec. for Automobiles-Engine Test Rec. for Test

1965)

the ISO 7 Bit Character Set) (1968) roller (II) Thrust (III) Tap/

-In. Series (1965)

on Type (1968)

on Type $(1968)$
Turbines $(1970)$

2)

es (1963)

968)

by Rotating Electrical Machinery (1970) Rec for International Rec. for International Rec. for International Rec. for Test for Data Communication-Basic Mode Control Procedures Rec. for Copper and Its Alloys . Rec. for Copper and Its Alloys Rec. for Light Metals and Their Alloys Rec for Indexable (Throwaway) Inserts-Designation of Mild Steels and Low Alloy High Tensile Types/ Rec. for Arc Welding of Cast Iron (1969) Fabrics Rec. for

Rec. for Std. for
Std. for

Coal Ploughs (1967)

Coal Preparation Plant (1967)

Coal Preparation Plant (1969)

Coal Preparation (1970)

Coal Sampling and Analysis (1971)

Coal (1960)

Coal (1963)

Coal (1966)

Coal (1966)

Coal (1967)

Coal (1967)

Coal (1967)

Coals-Size Analysis (1972)

Coals and Lignites-Determination of Acetone Soluble M

Rec. for High 150

ISO

ISO

Rec. for Voc ISO

Rec. for Voca ISO

ISO

ISO

ISO

ISO

ISO

ISO

ISO

ISO

ISO

Coals and Lignites-Meth. of Extraction for the Determ

Coals and Lignites by the Direct Volumetric Meth. (1969

Coals and Lignites (1969)

Coals (1969)

Coarse Teeth-Metric Series (1972)

Coated with Rubber or Plastics-Determination of Roll

Coated with Rubber or Plastics (1970)

Coated with Rubber or Plastics (1970)
Coated with Rubber or Plastics (1971)

Coated, in Singles, Pairs, Triples,

Coating-Gravimetric Meth. (1970)

Coating Thickness-Eddy Current Meth (1972) and Deter

Coating Thickness-Magnetic Meth. (1972) /Ckel Coatin ISO

Coating Thickness-Magnetic Meth. (1972)

Coating Thick nesses by Microscopical Examination of $\mathrm{Cr}$

R Porcelain ISO

Coatings-Definition of Terms Concerning the Measureme ISO

Coatings-General Classification of Terms (1971) ISO

Coatings-Gravimetric Meth. (1971) / Aluminium (Alumi ISO

Coatings-Measurement of Thickness-Coulometric Meth. ISO

Coatings-Nondestructive Measurement by Light Section ISO

Coatings of Cadmium on Iron and Steel (1971) ISO

Coatings of Copper Plus Nickel and Chromium on Steel (O ISO

Coatings of Nickel Plus Chromium (1970)

Coatings of Nickel (1970)

Coatings of Tin Nickel Alloy (1972)

Coatings of Tin (1971)

Coatings of Zinc on Iron and Steel (1971)

Coatings on Fabricated Ferrous Products (1970)

Coatings on Ferrous Materials by Chemical Dissolution O

Coatings on Magnetic and Nonmagnetic Substrates-Measu

Coatings on Magnetic Basis Metals-Measurement of Coat

Coatings on Nonmagnetic Basis Metals-Measurement of $C$

Coatings Other Than Those Anodic to the Basis Metal (19

Coaxial Cable Connectors Used in Nuclear Instrumentatio

Coaxial Cables (1967)

Coaxial Transmission Lines and Their Associated Flange

Coaxial Transmission Lines and Their Associated Flange

Coaxial Unmatched Television Aerial Feeder Connector (1

Coaxial, for Cables 96 IEC 50-12 (1967)

Coaxial, for Cables 96 IEC 50-17 and Larger (1970)

Coaxial, for Cables 96 IEC 75.17 and Larger (1971)

Cobalt (1963)

(Cobb Meth.) (1967)

COBOL (1972)

Cocks (1972)

Cocoa Beans-Cut Test (1969)

Cocoa Beans: Determination of Moisture Content (Routine

Cocoa Beans: Sampling (1973)

Code-General Rules (1970)

Code-Net Power (1971)

Code for Agricultural Tractors (1968)

Code for Freight Containers (1972)

Code for Model Acceptance Tests of Hydraulic Turbines

Code for Numerical Control of Machines (Compatible with

Code for Roller Bearings: Groups-(I) Radial Ball and

Code for Rolling Bearings: Group IV Tapered Roller Type

Code for Rolling Bearings: Group V Airframe Type (1968)

Code for Stationary Steam Generators of the Power Stati

Code for Test. of Speed Governing Systems for Hydraulic

Code for the Abbreviation of Titles of Periodicals (197

Code for the Field Acceptance Tests of Hydraulic Turbin

Code for the Field Acceptance Tests of Storage Pumps (1

Code for the Measurement of the Airborne Noise Emitted

Code Independent Information Transfer (1972)

Code of Designation-Part II: of Tempers (1971)

Code of Designation-Part I: of Materials (1971)

Code of Designation (1971)

Code of Symbolization (1971)

Code of Symbols for Covered Electrodes for Arc Welding Rec. for Code of Symbols for Covered Electrodes for Manual Metal
602

622

1953

1952

1015

975

1018

2296

2286

1420

1419

1421

189-7

1460

1434

2360

2361

2178

1463

2085

2064

2079

2106

2177

2128

2082

1457

1456

1458

2179

2093

2081

1461

1460

2361

2178

2360

1462

313

78

339.2

339.2
$169-2$

169-4

169.5

$169-6$

316

535

1989

2290

1114

2291

2292

1819

1585

789

2716

193

840

$300 / I$

$300 /$ II

$300 / I I I$

889

308

4

41

1680

168

2111

$1190 / I$

2092

1832

635

1071 
Stpels and Iow Alloy High Trensile Types (1967)

Rec. for odes for Manual Are Welding of Mild and Iow Alloy Steels ies of Wood Utilized in the Manufacture of Wood Flooring Rec. for Machine Tool Test loose Bulk Materials-Belt Feeders and Conveyors-Safety t: Bins and Bunkers, Silos and Hoppers, Bin Gates-Safety floor Mounted Truck Conveyors (Chain Above Floor)-Safety r Reciprocating Feeders and Oscillating Conveyors-Safety overhead Twin Rail Chain Conveyors (Power and Free) Safety or Unit Loads-Overhead Monorail Chain Conveyors-Safety for Loose Bulk Materials-Mobile Belt Conveyors-Safet

le Strand Floor Truck Conveyors (Chain Below Floor) Safet bulk Materials-Scraper and En Masse Conveyors-Safety bile Belt Conveyors (Canvas, Rubber, Plastic, Etc.) Safety ipment for Loose Bulk Materials-Apron Conveyors-Safety or Loose Bulk Materials-Picking Table Conveyors-Safety nt for Loose Bulk Materials-Hydraulic Conveyors-Safet inition of 4 Bit Character Sets Derived from the ISO 7 Bit n Processing) (I970/ Rec. for Representation of ISO 7 Bit ic Tape (1969) ange (I967)

Rec. for Implementation of the $7 \mathrm{Bit}$ Rec. for 6 and $7 \mathrm{Bi}$

tic Tape (I96) Rec. for Representation of 6 and $7 \mathrm{Bit}$ Rec. for Implementation of the 6 and 7 Bit tors (1968) Rec. for Marking

ec. for Special Meth. of Mechanical Test. to Determine the Rec. for Colour (Color) Sid. for

Tape Block Formats for the Numerical Control of Machines: hydrometers-Conventional Value for the Thermal Expansion rmination of the Efficiency, Metal Recovery and Deposition Rec. for Measurement of Absorption amics-Part III: Derivatives of Forces, Moments and Their Reference Meth.) (I970) ine Meth.) (I970)

Rec. for Green Rec. for Green

ndurance of Electrical Insulating Varnishes by the Helical Iding of Mild Steel: Dimensions of Wires, Spools, Rims and Rec. for CaIculation of Coal and

Rec. for Determination of Carbon and Hydrogen in Coal and Rec. for Determination of Carbon and Hydrogen in Coal and Rec. for Determination of the Bulk Density of Rec. for Determination of the Bulk Density of ec. for Determination of the Yields of Tar, Water, Gas and Rec. for Determination of the Gray King Rec. for Determination of ChIorine in Coal and Std. for Size Analysis of Small Rec. for Determination of the Micum Indices of Rec. for Determination of Total Moisture in

Rec. for Determination of Arsenic in Coal and Rec for Determination of the Shatter Indices of $r$ Determination of the Volatile Matter of Hard Coal and of Rec. for Size Analysis of

c. for Determination of Moisture in the Analysis Sample of ion of Nitrogen, Total Sulphur, Chlorine and Phosphorus in

Apparent Relative Densities (Density) and the Porosity of

ulary of Terms Relating to Solid Mineral Fuels-Part III:

I Properties of Electronic Tubes: Meth. of Measurement for ions, Tolerances and Masses Per Metre (Meter) $U$ Rec. for Rec. for Cold Reduced Tinplate and Rec. for Peaches: Guide to Rec. for Pears-Guide to

Rec. for Cauliflowers: Guide to Rec. for Apples-Guide to Rec. for Spices and Condiments: Determination of ocedures for Electronic Components and Equipment: Test A: Std, for Tool Shanks and Equipment with 7/24 Tapers: Drill Steels (1968) Rec. for Rock Drilling: Forged or Rolling Bearings-Cylindrical Roller-Separate Thrust rement of Liquid Flow by Velocity Area Meth. (I/ Rec. for ility Data from Field Performance/ Rec. for Guide for the stics-Determination of Resistance of Plastics to Colour stics-Determination of Resistance of Plastics to Colour stics-Determination of Resistance of Plastics to Colour
Rec. for Colour Rec, for Test for Colour

aterial (I969)

r Aircraft (1959)

igher A

material (1969)

f Rolls (I969)

Rec. for Photographic Colou

d. For Photography: Determination of ISO Speed of Colou
Code of Symbols for Filler Rods for Gas Welding of Mild

Fred Electr

Code of Symbols for Identification (1973)

Code of Symbols (1971) / Marking of the Different Spec ISO

Code (1961)

Code (1970)

Code (1970)

Code (I971)

Code (197I)

Code (1971)

Code (1971)

Code (I972

Code (I972)

Code (1972)

Code (1972)

Code (1972)

Code (I972)

Code (I972)

/ Marking of the Different Spec 1SO

/lnuous Mechanical Handling Equipment for 1SO /R Loose Bulk Materials-Storage Equipmen $1 S O$ Equipment for Unit Loads-Single Strand ISO

/Ibrating Feeders and Conveyors, Shaking O ISO

/Ical Handling Equipment for Unit Loads- ISO /Ontinuous Mechanical Handling Equipment F 150

/R Continuous Mechanical Handling Equipment ISO

/ Handling Equipment for Unit Loads-Sing 1SO

/ Mechanical Handling Equipment for Loose ISO

/Al Handling Equipment for Unit Loads-Mo ISO

/D. for Continuous Mechanical Handling Equ ISO

Ontinuous Mechanical Handling Equipment F ISO

/or Continuous Mechanical Handling Equipme ISO

Coded Character Set for Information Processing Intercha ISO

Coded Character Set on 12 Row Punched Cards (Informatio ISO

Coded Character Set on 9 Track $12.7 \mathrm{~mm}$ (1/2 In.) Magnet 150

Coded Character Sets for Information Processing Interch ISO

Coded Character Sets on Punched Tape (1969)

Coded Character Sets on 7 Track I2.7 mm (1/2 In.) Magne

Codes for Measuring the Noise Emitted by Machines (1966

Codes for Values and Tolerances of Resistors and Capaci

Codification (1972)

Coding for Deep Penetration Electrodes (1968)

Coding for Pipettes (I970)

Coding of Character Sets for MICR and OCR (I972)

Coding of Preparatory Functions $G$ and Miscellaneous Fun

Coefficient (For Use in the Preparation of Measurement

Coefficient (1972)

Std. for Covered Electrodes-Dete

Coefficients in a Reverberation Room (1963)

Std. for Symbols for Flight Dyn

Coffee Beans-Determination of Moisture Content (Basic

Coffee Beans-Determination of Moisture Content, (Rout ISO

Coil Bond Test (1969) Ifor Evaluation of the Thermal E IEC

Coils (1968) JOlid Wires for Gas Shielded Metal Arc We 1SO

Coke Analyses to Different Bases (I970)

Coke by the High Temperature Combustion Meth. (1967)

Coke by the Liebig Meth. (1967)

Coke in a Large Container (1969)

Coke in a Small Container (1967)

Coke Residue by Low Temperature Distillation of Brown C

Coke Type of Coal (1966)

Coke Using Eschka Mixture (1967)

Coke (Nominal Top Size $20 \mathrm{~mm}$ or Less) (1972)

Coke (1967)

Coke (1967)

Coke (1967)

Coke (1967)

Coke (1967)

Coke (I968)

Coke (1968)

Coke (1969)

Coke (I969)

Coke (197I)

Cold Cathode Counting and Indicator Types (I970)

ISO

Cold Reduced Blackplate-Part 1: Sheet (I969)

Cold Storage (I968)

Cold Storage (I969)

Cold Storage (I969)

Cold Storage (1970)

Cold Water Soluble Extract (1969)

Cold (I966)

Collar Dimensions (1972)

Rec. for Basic Environmental Test. P

Collared Shanks and Chuck Bushings for Hollow Hexagonal

Collars-Boundary Dimensions (I962)

Collection of Data for Determination of Errors in Measu

Collection of Reliability, Availability, and Maintainab

(Color) Change Upon Exposure to Daylight (I968)

(Color) Change Upon Exposure to Light of a Xenon Lamp (1

(CoIor) Change Upon Exposure to Light of the Enclosed Ca

(Color) Coding for Pipettes (1970)

(Color) Fastness of Textiles (1959)

(Color) Film for General Use: Preferred Sizes of Sheet M

(Color) Identification of Mechanical Control Circuits Fo (Color) in Hazen Units (I970)

(Color) of Push Buttons (I955)

(Color) Paper for General Use: Preferred Sizes of Sheet

(Color) Paper for Roll Paper Printers: Preferred Sizes O

(Color) Reversal Films for Still Photography-Sensitome

(Color) Roll Film (I969)

Radio Transmitters: Measure 
for lligher Alrohols for Industrial Use-Test for Colour (Color) with Sulphuric Acid (I970)

d and Xylenols for Industrial Use-Measurement of Colour (Color) (1971)

Rec. ISO

Std. for Petroleum Products-Determination of Colour (Color) (1972)
Alloys-Estimation of the Loss of Absorptive Power by Colorant Drop Test with Prior Acid Treatment (1971)

Rec. for Cresylic Aci ISO or Plastics-Determination of the Bleeding of Colourants (Colorants) (1961)

um and Its Alloys-Determination of the Light Fastness of

Condition in Land Inst/ Rec. for Identification Colours (Colors) for Pipes Conveying Fluids in Liquid or Gaseou
Frequency Cables and Wires (1969/ Rec. for Std. Colours (Colors) for Pve (Polyvinyl Chloride) Insulation for Low

$\begin{array}{ll}\text { Colored Anodised Aluminum (1971) /Odisation of Alumini ISO } & \text { ISO } \\ \text { (Colors) for Pipes Conveying Fluids in Liquid or Gaseous } & \text { ISO }\end{array}$

Rec. F ISO

ISO

Colours (Colors) of Signal Lights on Ships (1972)

Rec. for Colours (Colors) of the Cores of Flexible Cables and Cords (1964. Rec. for Safety Colours (Colors) (1964)

Rec for Surface Treatment of Metals

Colourants (Colorants) (1961)

Coloured Anodisation of Aluminium and Its Alloys-Dete Dimensions of Hot Rolled Steel Sections-Parallel Flange dimensions and Location of Rectangular Punched Holes in 80 wool Fibre (Fiber) Length Barbe (Barb) and Hauteur Using A reak Disconnectors, Airbreak Switch Disconnectors and Fuse

ections (1970)

Rec. for

Is for Inland Navigation-Pipe Lines for the Transport of

Column Sections-In. Series-Dimensions and Sectional

Columns Punched Paper Cards (Information Processing) (1

Comb Sorter (I969) Rec. for Meth. of Test for ISO

Combination Units (1972)

Tage Airbreak

ec. for Textile Glass-Determination of the Percentage of

c. for Numbering of Aircraft Engines, Engine Cylinders and single Bank Engines (197)

) Std. for Reciprocating InternaI Std. for Reciprocating InternaI Std. for Reciprocating Internal Std. for Reciprocating Internal

d. Direction of Motion (/ Std. for Internal

1972) steel and Cast Iron: Determination of Sulphur (Meth. After ls: Determination of Total Carbon (Gravimetric Meth. After Dination of Total Sulphur in Coal by the High Temperature

ron and Hydrogen in Coal and Coke by the High Temperature ductor Wire (I960) Rec. for pose Metric Screw Threads: Tolerances, Limits of Sizes for onductor Wire (1959) Rec. for the Resistivity of (1958)

vers and Towed Vehicles with 24.V Electrical Equipment for
val

I: Calculation of Linear Dimensions, Areas And/ Rec. for Rec. for Rec. for kwood Refuse and Corkwaste-Definitions and $\mathrm{Pa} /$ Rec. for h Regulators (1971) Rec. for Pesticides Considered Not to Require

d Plant Growth /

Rec. for Principles for the Selection of Rec. for Pesticides Considered Not to Require rec. for Shipbuilding Details-Anchor Chains, Stud Links dependent Information Transfer (I972)

ment of / Std. for Data Communication-Data Terminal and Rec. for Basic Mode Control Procedures for Data rage), Batteries, Heating and Cooking $A$ ppliances, Internal

Rec. for Acoustics-Assessment of Noise with Respect to itions and Nomenclature for Carbon Brushes, Brush Holders,

973)

Rec. for Dimensions for Std. for Automobiles : Std. for Automobiles: Rec. for Plastics-Pve Resins: Determination of the on of Apparent Density of Washing Powders Before and After $s$ Under Moist Conditions / Rec. Meth. for Determining the (1969) tion (I965)

Rec.

B-General Requirements (1967) Rec. for Positioning of Magnetic Rec. for Magnetic

ec. for Filler Rods for Cas Welding: Test to Determine the Rec. for Code for Numerical Control of Machines dimension of Brushes and Holders for Electrical Machinery: g Test (1972)

ning for Test. (1972)

Impact Test by Dropping (1972)

n Test (1972)

I Impact Tests (Inclined Plane and P/

ation of Parts When Test. (1972)

Rec. for Rules for the Test. of Electric Rolling Stock on ric Meth. Sy Precipitation of the Quinoline Silicomolybdic ric Moth. by Precipitation of the Quinoline Silicomolybdic

Spectroph :sinenetric Meth. Using the Reduced Silicomolybdic

-Spectrophotometric Meth. as the Reduced Silicomolybdate - Spectrophotometric Meth. with the Reduced Silicomolybdic Spectrophotometric Meth. Using the Reduced Silicomolybdic chemical Analysis of Aluminium (Aluminum) and Its Alloys droxide for Industrial Use: Determination of Calcium; Edta droxide for Industrial Use: Determination of Calcium: Edta

se: Determination of Calcium and Magnesium Contents-Edta Rec. for Measurement of the Dimensions of a Cylindrical c. for Basic Environmental Test. Prosedures for Electronic c. for Basic Environmental Test. Procedures for Electronic
Combinations of Double Ended Wrench Gaps (I969)

Combustible Liquids-Nominal Diameters (1957) / Detai ISO

Combustible Matter of Its Products (1971)

Combustion Chambers, and Direction of Rotation of Engin

Combustion Engines-Definition of Right and Left Hand

Combustion Engines-Designation of the Cylinders (1972

Combustion Engines-Designation of the Direction of Ro

Combustion Engines-Hand Operated Control Devices-St

Combustion Engines: Spark Plug Ignition-Terminology (

Combustion in a Current of Oxygen and Titration with So

Combustion Meth. (1963)

Combustion Meth. (1963)

Combustion Meth. (1967)

Y Ysis of Stee

Rec. for ISO

Rec for Dete ISO

Commercial Annealed Aluminium (Aluminum) Electrical Con Ca ISO

Commercial Bolt and Nut Threads: Medium Quality (I969) ISO

Commercial Hard Drawn Aluminium (Aluminum) Electrical C IEC

Commercial International Traffic (1970) /Ween Prime Mo ISO

Commercial Purity Aluminium (Aluminum) Busbar Material IEC

Commercial Refrigerated Cabinets-Meth. of Test-Part ISO

Commercially Dry Corkwood in Planks-Definitions, Clas

Commercially Dry Virgin Cork, Ramassage, Gleanings, Cor

Common Names-Second List (1969)

Common Names for Pest Control Chemicals and Plant Growt

Common Names for Pest Control Chemicals (Pesticides) an

Common Names: First List (1968)

(Common, Enlarged, and End Types, and Joining Shackles)

Communication-Basic Mode Control Procedures-Code in

Communication Equipment-Interchange Circuits-Assign

Communication Systems (197I)

Communications, Lightning Conductors (1965)

Community Response (197I)

Commutators and Slip Rings (1968)

Commutators and Slip Rings (I97I)

Compact Spark Plugs M 14 X 1,25 with Conical Seating (1

Compact Spark Plugs M 14 X 1,25 with Flat Seating (1973

Compacted Apparent Bulk Density (1969)

Compaction (I 968 )

Comparative Tracking Index of Solid Insulating Material

Compartments for the Storage of Frozen Foodstuffs (1968

Compasses and Binnacles for Sea Navigation: Vocabulary

Compasses and Binnacles, Class A, for Use in Sea Naviga

Compasses in Ships (I968)

Compasses, Binnacles and Azimuth Reading Devices, Class

Compatibility of Steel Filler Rods and the Parent Metal

Compatible with the ISO 7 Bit Character Set) (1968)

Complementary Dimensions and Terminations (1967)

Complete, Filled Transport Packages-Part III: Stackin

Complete, Filled Transport Packages-Part II: Conditio

Complete, Filled Transport Packages-Part IV: Vertical

Complete, Filled Transport Packages-Part Vi: Vibratio

Complete, Filled Transport Packages-Part V: Horizonta

Complete, Filled Transport Packages-Part l: Identific

Completion of Construction and Before Entry into Servic

\section{Ator (Sto IEC}

ISO
ISO
ISO

ISO

ISO

ISO

ISO

ISO

SO

ISO

IEC

ISO

ISO

ISO

IEC

ISO

ISO

ISO

ISO

ISO

ISO

/for IEC

ISO

ISO

ISO

ISO

ISO

Complex (1969)

/Ermination of Silica Content, Gravimet ISO
/Ination of of Silica Content; Gravimet ISO

(L)-Determination of Silica Content- ISO

Complex $(1970)$

Nium-Determination of Silica Content ISO

Complex (I97I)

Complex (I972)

Its Alloys-Determination of Silicon ISO

Complexometric Determination of Magnesium (1973) /for ISO

$\begin{array}{lr}\text { Complexometric Meth. (1969) } & \text { Rec. for Sodium Hy ISO } \\ \text { Complexometric Meth. (1969) } & \text { Rec. for Potassium Hy ISO }\end{array}$

Complexometric Meth. (1973)

Component Having Two Axial Terminations (1969)

Components and Equipment: General (1968) Re IEC

Re IEC

1852

1909

183

2135

508

24.12

173

408

183

2135

657/IX

I 682

920

I085

1168

51

482
2276
1205

1204

2261

2542

67I

437

352

351

609

I1I

1185

105

992

1216

1215

1105

1750

257

765

I70

2111

2110

1745

92.6

1996

276

356

2347

2346

1068

697

I 12

1069

449

694

613

708

840

136.2

2234

2248

2247 
for Hasic Environmental Test. Procedures for Electronic c. for Basic Environmental Test. Procedures for Electronic c. for Basic Environmental Test. Procedures for Electronic c. for Basic Environmental Test. Procedures for Electronic c. for Basic Environmental Test. Procedures for Electronic c. for Basic Environmental Test. Procedures for Electronic c. for Basic Environmental Test. Procedures for Electronic c. for Basic Environmental Test. Procedures for Electronic c. for Basic Environmental Test. Procedures for Electronic c. for Basic Environmental Test. Procedures for Electronic c. for Basic Environmental Test. Procedures for Electronic

c. for Basic Environmental Test. Procedures for Electronic

c. for Basic Environmental Test. Procedures for Electronic

c. for Basic Environmental Test. Procedures for Electronic Wiring and Circ/ Rec. Guidance for the Design and Use of Rec. for Packaging of

itions for the Reliability of Electronic Equipment and the ec. for the Presentation of Reliability Data on Electronic c. for Guide for Choosing Modular Dimensions for Waveguide imensions of Spindle Ends for Manually Operated Electronic plement to R164) (1961)

Rec. for Rec. for Nominal Cross Sectional Areas and Rec. for Nominal Cross Sectional Areas and
y Castings and Mechanical Properties of Sand Ca/ Rec. for y Ingots for Casting Purposes (1959) (1968) inc Alloys (1966)

um Alloys-Chemical Composition (Рer Cent) (19/ (- for Hasic Environmental Test. Procedures for Electronic

Components and Equipment: Test A: Cold (1966)

Components and Equipment: Test B: Dry Hcat (1966)

Components and Equipmcnt: Test Ca: Damp and Steady Heat

Components and Equipment: Test D: Accelerated Damp Heat

Components and Equipment: Test Ea: Shock (1967)

Components and Equipment: Test Eb: Bump (1968)

Components and Equipment: Test Ga: Acceleration, Steady

Components and Equipment: Test H: Storage (1960)

Components and Equipment: Test J: Mould Grow th (1968)

Components and Equipment: Test Ka: Salt Mist (1964)

Components and Equipment: Test M: Low Air Pressure (196

Components and Equipment: Test N: Change of Temperature

Components and Equipment: Test Q: Sealing (1968)

Components and Equipment: Test T: Soldering (1968)

Components and Equipment: Test U: Robustness of Termina

Components Intended for Mounting on Boards with Printed

Components on Continuous Tapes (1968)

Components (Ог Parts) Used Therein (1969)

IS and Defin IFC

Components (Or Parts) (1970)

Components (1971)
Components (1972)

Composition of Aluminium (Aluminum) Alloy Castings (Com

Composition of Aluminium (Aluminum) Alloy Castings (196

Composition of Conductors of Insulated Cables (1966)

Composition of Magnesium Aluminium (Aluminum) Zinc Allo

Composition of Magnesium Aluminium (Aluminum) Zinc Allo

Composition of Unalloyed Aluminium Ingots for Remelting

Composition of Wrought Magnesium Aluminium (Aluminum) Z

Composition of Wrought Products of Aluminium and Alumin

Composition of 99.8 Unalloyed Magnesium Ingots (1959)

Composition of 99.95 Unalloyed Magnesium Ingots (1961)

ought Products of Aluminium and Aluminum Alloys-Chemica td. for Magnesium Zinc Zirconium Alloy Castings-Chemica

Nitric Acid for Industrial Use-Determination of Nitrous odium Borates for Industrial Use: Determination of Sulphur lity of Polyvinylchloride and Related Copolymers and Their ity of Polyvinyl Chloride and Related Copolymers and Thei Rec. for Determination of Rate of Cure of Rubbe Std for Essential Oils-Determination of Carbonyl

for Plastics-Determination of Free Ammonia and Ammonium Rec. for Plastics-Designation of Unplasticized

Determination of the Melt Flow Index of Polyethylene and er Alcohols for Industrial Use-Determination of Carbonyl terials (1963)

terials (1963)

terials-Annex A: Preparation of Te/

terials-Annex B: Preparation of Te/

terials-Annex $C$ : Preparation of $\mathrm{Te} /$

flection at Low Temperatures (/

Rec. for Plastics Rec. for Plastics

Rec. for Plastics

Rec. for Plastics

Rec. for Plastics

High Temp/ Rec. for Vulcanized Rubbers: Determination of Rec. for Plestics

Std. for Flexible Cellular Materials-Determination of ilem Cembu/ Rec. for Meth. of Test. Strength of Cements Rec. for Plastics-Determination of Std. for Measurement of Airborne Noise Emitted by Rec. for Test. of Refrigerant Rec. for Preparation of Dry Films from Determination of the Viscosity Ratio of of Polyamides in uric Acid for Industrial Use: Evaluation of Sulphuric Acid drochloric Acid for Industrial Use: Evaluation of Its Acid vers (1/ Rec. for Connectors for Frequencies Below $3 \mathrm{MHz}$ Rec. for International Unification of Rec. for Conventions

tallic and Other Nonorganic Coatings-Definition of Terms ance of the Principal Radiographic Terms Used in ISO Rec. ty of Test Pieces (1971) Rec. for e Meth.) (1970)

sions (197I/

Rec. for Evaporated Milk and Sweetened

for Simplified Meth. for Pressure Calibration of One Inch Rec. for Spices and Rec. for Spices and Rec. for Spices and Rec. for Spices and Rec. for Spices and Rec. for Spices and Rec. for Spices and Rec. for Spices and Rec. for Spices and Rec. for Spices and Rec. for Spices and Rec. for Spices and Rec. for Spices and Rec. for Spices and Rec. for Spices and

(Colors) for Pipes Conveying Fluids in Liquid or Gaseous
Composition (1972)

Compounds-Volumetric Meth. (1971)

Compounds-Volumetric Meth. (1972)

Compounds by Splitting Off of Hydrogen Chloride (1970)

Compounds by the Shearing Disk Viscometer (1968)

Compounds Content-Free Hydroxylamine Meth. (1972)

Compounds in Phenol-Formaldehyde Mouldings (1959)

Compounds of Homo and Copolymers of Vinyl Chloride (197

Compounds of (1967)

Rec. for Plastics

Compounds (Hydroxylammonium Chloride Potentiometric Me

Compression Moulding Test Specimens of Thermoplastic Ma

Compression Moulding Test Specimens of Thermosetting Ma

Compression Moulding Test Specimens of Thermosetting Ma

Compression Moulding Test Specimens of Thermosetting Ma

Compression Moulding Test Specimens of Thermosetting Ma

Compression Set of Vulcanized Rubbers Under Constant De

Compression Set Under Constant Deflection at Normal and

Compression Test of Rigid Cellular Plastic (1968)

Compression (1972)

Compressive and Flexural Strengths of Plastic Mortar ( $R$

Compressive Properties of Plastics (1967)

Compressor Primeover Units Intended for Outdoor Use (19

Compressors (I968)

Concentrated Natural Rubber Latex (1966)

Concentrated Solution (1967)

Concentric Connectors for Audio Circuits in Radio Recei

Concepts and Terms (1968)

Concerning Electric and Magnetic Circuits (1972)

Concerning the Measurement of the Thickness (1971)

Concerning Welding (1971) ISO 1027, 1 I06

Concrete Tests-Dimensions, Tolerances and Applicabil

Condensed Milk-Determination of Fat Content (Referenc

Condenser Bobbins for Woollen (Woolen) Spinning-Dimen

Condenser Microphones by the Reciprocity Technique (197

Condenser Microphones by the Reciprocity Technique (197

Condiments-Cardamoms-Spec. (1968)

Condiments: Black and White Pepper, Whole and Ground: S

Condiments: Chillies, Whole and Ground, Spec. (1969)

Condiments: Determination of Acid Insoluble Ash (1969)

Condiments: Determination of AIcohol Soluble Extract (1

Condiments: Determination of Cold Water Soluble Extract

Condiments: Determination of Extraneous Matter (1969)

Condiments: Determination of Moisture Content (Entrainm

Condiments: Determination of Nonvolatile Ether Extract

Condiments: Determination of Total Ash (1969)

Condiments: Determination of Water Insoluble Ash (1969)

Condiments: Ginger, Whole, in Pieces and Ground: Spec.

Condiments: Nomenclature (1968)

Condiments: Pimento (Allspice) Whole and Ground; Spec.

Condition in Land Installations and on Board Ships (196
Composition (Per Cent) (1971)

Condiments: Sampling (1969)
Re IEC

IEC.

Re IEC

Re IEC

IEC

IEC

/E IEC

IEC

Re IEC

Re IEC

IEC

IEC

IEC

IEC
IEC

D IEC

ISO

ISO

IEC

ISO

ISO

ISO

ISO

ISO

ISO

ec. for ISO

1SO

ISO
ISO

ISO

ISO

1. ISO

ISO

ISO

ISO

ISO

ISO

ISO

ISO

ISO

ISO

ISO

Sulph ISO

/for Hy ISO

IEC

ISO

/Me ISO

ISO

ISO

ISO

IEC

IEC

ISO

ISO

ISO

ISO

ISO

ISO

ISO

ISO

ISO

ISO

ISO

ISO

68-2-1

$68-2-2$

$68-2-3$

$68.2 \cdot 27$

8-2-29

68-2.7

68.2.8

- $2-10$

$68.2 \cdot 1]$

68-2-13

68-2-14

$68-2 \cdot 17$

68-2.20

68-2-21

321 
Rer. for Test. and Rating Room Air fety Requirements for the Electrical Equipment of Room Air

Rec. for Std. Atmospheres for the Rec. for Std. Atmospheres for Rec. for Plastics-Std. Atmospheres for Rec. for Std. Atmospheres for

ns (1967) Rec. for packaging-Complete, Filled Transport Packages-Part II: Rec. for Meth. of the
Rec. for Sampling and Std. for Leather $6(1969)$ Rec. for Plastics-Accelerated n Radio Receivers for Various Classes of Emission: General Rec. for General Test mage Quality I/ Std. for Radiography of Welds and Viewing mage Quality I/
of the Accuracy (1970) Std. for Radiography of Welds and Rec. for Test
Recting ght with Horizontal or Vertical Spindle-l le Height, with Horizontal or Vertical Spi/ 1 Grinding Wheel Spindle and Reciprocating/ grinding Wheel Spindle and Reciprocating $\mathrm{T} /$ Rec. for Test Rec. for Test Rec. for Test Rec. for Test id Electrical Insulating Materials (1971) Rec. for Std. Atmospheric Rec. for Std

Rec. for Short Link Chain for Lifting Purposes-General Rec. Meth. of Measurement for Radio Transmitters: General Rec. for Resistance to Humid and Normal Threshold of Hearing Under Free Field Listening - Section 1-Std Valves and Conditions: Section 2-Test d Round Copper with Good Dielectric Properties Under Humid e Tracking Index of Solid Insulating Materials Under Moist determination of Resistance to Ozone Cracking Under Static

- Determination of Dimensional Changes in Varying Moisture crystal Units for Oscillators: Section I-Std. Valves and Std. for Lined

or Rubber Hoses for Aircraft Ground Fueling Without Static -Belts (Belts) (Sections Y, Z, A, B, C, D, E)-Electrical

(1962)

es with Copper Conductors, for A/

ctrical Cables with Copper Condu/ ctrical Cables with Copper Condu/ y of Commercial Hard Drawn Aluminium (Aluminum) Electrical c. for Commercial Annealed Aluminium (Aluminum) Electrical nd Sheath: Equipment Wires, Type I, with Solid or Stranded chloride Insulated Flexible Cables and Cords with Circular r Rubber Insulated Flexible Cables and Cords with Circular

irements for General Purpose Electrical Cables with Copper

of Test for General Purpose Electrical Cables with Copper Heat Resisting $\left(260^{\circ} \mathrm{C}\right)$ Electrical Cables with Copper purpose Electrical Cables with Aluminium or Aluminum Alloy Heat Resisting $\left(190^{\circ} \mathrm{C}\right)$ Electrical Cables with Copper Heat Resisting $\left(260^{\circ} \mathrm{C}\right)$ Electrical Cables with Copper purpose Electrical Cables with Aluminum or Aluminium Alloy or Heat Resisting (190 Deg.) Electrical Cables with Copper Heat Resisting $\left(260^{\circ} \mathrm{C}\right)$ Electrical Cables with Copper

Rec. for Basic Dimensions of Winding Wires: Diameters of

Rec. for Basic Dimensions of Winding Wires: Diameters of Rec. for Nominal Cross Sectional Areas and Composition of

Rec. for Basic Dimensions of Winding Wires: Dimension of o the Calculation of Resistance of Plain and Tinned Copper Rec. for Identification by Hour Numbers of the Phase Rec. for Aluminium Alloy Stranded Rec. for Terminals with Screw Clamping for External and Cooking Appliances, Internal Communications, Lightning Rec. for Aluminium (Aluminum) Stranded Rec. for Marking of Insulated

Heat Resisting $\left(190^{\circ} \mathrm{C}\right)$ Electrical Cables with Copper esistance of General Purpose Electrical Cables with Copper ride) Insulation and Sheath: Distribution Wires with Solid

ride) Insulation and Sheath: Distribution Wires with Solid

Sheath: Equipment Wires and Cables with Solid or Stranded Rec for Aluminium (Aluminum) Rec. for Aluminium (Aluminum) Alloy

2) ions and Their Fittings (1968) ces (1972)

Spec. for Stee Spec. for Rigid Std for Truncated

Rec. for Refractory Products Determination of Pyrometric (1971)

Petroleum Products: Lubricating Grease-Determination of Definitions (1966) Rec. for Textile Machinery and Accessories

Rec. for Wood Cones for Cross Winding: Half Anec. for Rec. for Wood Cones for Cross Winding: Half Angle of the 8 for Cross Winding for Dyeing Purposes: Half Angle of the r Transfer Cones for Dyeing Purposes: Half Angle at Top of or Wood Cones for Cross Winding: Nominal Half Angle of the

Conditioners (1968)

Conditioners (1972)

Conditioning and Test. of Rubber Test Pieces (1966)

$R$

Conditioning and Test. Textiles (1967)

Conditioning and Test. (1963)

Conditioning And/or Test. Reference Atmosphere Spec. (1

Conditioning Atmosphere: Test, Reference, and Definitio

Conditioning for Test. (1972)

Conditioning of Paper and Board Test Samples (1961)

Conditioning of Ropes for Test. (I969)

Conditioning of Test Pieces for Physical Tests (1972)

Conditioning of Test Specimens of Polyamide 66, 610 and

Conditioning (1969)

Conditions and Measuring Meth. Applying to Several Type

Conditions for Electro Heating Appliances (1972)

Conditions for Films: Utilization of Rec. Patterns of I

Conditions for General Purpose Parallel Lathes-Test.

Conditions for Milling Machines with Table of Fixed Hei

Conditions for Milling Machines with Table of of Variab

Conditions for Surface Grinding Machines with Horizonta

Conditions for Surface Grinding Machines with Vertical

Conditions for Test Purposes (1963)

Conditions for Use Prior to and During the Test. of Sol

Conditions of Acceptance (1971)

Conditions Of, Frequency, Output Power and Consumption

Conditions (1958)

Conditions (1961)

Conditions (I962)

IL Loudness Contours for Pure Tones

Uartz Crystal Units for Oscillators IEC

Conditions (1970)

Conditions ([971)

Conditions (I972) Ar Types of Winding Wires: Enamelle IEC

/Eth. for Determining the Comparativ IEC Std. for Vulcanized Rubbers- ISO

CAchine Made Textile Floor Coverings ISO

Conditions: Section 2-Test Conditions (1962) /Uartz IEC

Conducting Rubber Footwear (1972)

Conducting Wire (1971)

Conductivity-Characteristic and Meth. of Test (1971)

ISO

ISO

Conductivity of Conveyor Belts: Spec. and Meth. of Test

ISO

Conductivity Tough Pitch Copper Refinery Shapes (1971)

Conductor Resistance of Heat Resisting $\left(190^{\circ} \mathrm{C}\right)$ Ele

$\begin{array}{ll}\text { Conductor Resistance of Heat Resisting }\left(260^{\circ} \mathrm{C}\right) \text { Ele } & \text { ISO } \\ \text { Conductor Wire of the Aluminium Magnesium Silicon Type } & \text { IEC }\end{array}$

Conductor Wire (1959) Rec. for the Resistivit IEC

Conductor Wire (1960)

Re IEC

Conductor, Insulated, Single (1967) /Ide) Insulation a IEC

Conductors and a Rated Voltage Not Exceeding 750 V (196 IEC

Conductors and a Rated Voltage Not Exceeding $750 \mathrm{~V}$ (196 IEC

Conductors for Aircraft (1966) \% for Performance Requ ISO

Conductors for Aircraft (1967) Rec. for Meth. ISO

Conductors for Aircraft (1967) Rec. for Meth. ISO

/Onductor Resistance of ISO
Rec. for General ISO

Conductors for Aircraft $(1969)$

Mance Requirements for ISO

/ for Meth. of Test for ISO

1. of Test for General ISO

Conductors for Aircraft (I970)

Conductors for Aircraft (1970)

Conductors for Aircraft (I970)

Conductors for Aircraft (I970)

Conductors for Round Resistance Wires (1971)

Conductors for Round Winding Wires (I965)

Conductors of Insulated Cables (1966)

Conductors of Insulated Rectangular Copper Winding Wire

Conductors of Low Frequency Cables and Wires (1971)

Conductors of 3 Phase Electric Systems (1963)

Conductors (Aluminum Magnesium Silicon Type) (1966)

Conductors (1964)

Conductors (1965)

Conductors (1966)

Conductors (1972)

Conductors, for Aircraft (1966)

/Ator (Storage), Batteries, Heating

Conductors, for Aircraft (1966)

Conductors, Insulated, in Pairs, Triples, Quadruples an

Conductors, Insulated, Polyamide Coated, in Singles, Pa

Conductors, Steel Reinforced (1966)

Conductors, Steel Reinforced (1966)

Conduits and Fittings for Electrical Installations (196

Conduits of Polyvinyl Chloride for Electrical Installat

Cone Abrasive Sleeves: Designation, Dimensions, Toleran

Cone Equivalent (Refractoriness) (1966)

Cone Penetration (1972)

Cone Winders or Cheese Winders: Terminology; Basic Term

Cone $3^{\circ} 30^{\prime}(1963)$

Cone $4^{\circ} 20^{\prime}$ (1963)

Cone $4^{\circ} 20^{\prime}(1963)$

Cone $4^{\circ} 20^{\prime}(1967)$

Cone $5^{\circ} 57^{\prime}(1963)$ 
of the cine $4^{\circ} 20^{\circ}(196.3)$

$30)^{\prime}(196.3)$

$20^{\prime}(1963)$

$5^{\circ} 57^{\prime}(196,3)$

deg. $20^{\prime}(1967)$

in. (1959)

in. (1959)

in. (1959)

Interpretation of Test Results: Estimation of the Mean
Rec. for Significance to Purchasers of Marks Indicating

I Equipment: Definition and Dimensional Charact/ Rec. for

Rec. for Interchangeable

Std. for Automobiles: Spark Plugs M 14 X 1,25 with

Std. for Automobiles: Spark Plugs M 18 X 1,5 with

td. for Automobiles : Compact Spark Plugs M 14 X 1,25 with Rec. for Cylindrical and $1 / 10$

968)

e (1968)

on $(1969)$

(1969)

finitions (1969)

nitions (1969)

k Drilling-Rotary Drill Rods and Bits for Dry Drilling

cal Machined Graphite Electrodes with Threaded Sockets and in Television Receivers (1965) Rec. for High Voltage
$\mathrm{kV}$ d.c. and a Maximum Working Temperature of $1 /$ Rec. for I Symbols: Kind of Current, Distribution Systems, Meth. of lectrotechnicaI Vocabulary: Switchboards and Apparatus for Rec. for Industrial Trucks-Dimensions of Stillages nt Lens Barrels Up to $100 \mathrm{~mm}$-Dimensions Important to the

Rec. for Index of Electrical Measuring Apparatus Used in ree Sur/ Rec. for Vocabulary of Terms and Symbols Used in Rec. for Aircraft Pressure Cabin Ground Test Rec. for Aircraft Pressure Reoiling tical Guidance (1971)

h 24-V Electrical Equipment for Comm/ rchangeability (1969) 65)

Rec. for Electrical Rec. for Mechanical Rec. for Rec. for Aircraft Rec for Dimensions of Small Flash eel and Other Metal Tubes (1970) aesthetic and Resuscitation Pur/ pment Particularly for Private Motor/

Rec. for Pipe Rec. for Yoke Type Valve Rec. for Electrical

Rec. for Aircraft Tow Bar Rec. for Aircraft Pressure Refueling Rec. for Aircraft Toilet Flushing and Draining Rec. for Photographic Equipment-Tripod Std. for Aircraft Water Methanol Pressure

Rec. for Radio Frequency Connectors: Two Pin terminal Equipment (1973) Std. for ors (1969) unication Equipment-Interchange Circuits-Assignment of ncy Connectors: Coaxial Unmatched Television Aerial Feeder

ec. for Connectors for Frequencies Below $3 \mathrm{MHz}$ : Concentric td. for Aircraft: Interchangeability Dimensions of Battery upply T/ Rec. for Connectors for Frequencies Below $3 \mathrm{MHz}$ : ors (1965)

tors for Radio and Associated Sound Equipment (/ ole Connectors with Threaded Coupling (1966) ole Connectors with Bayonet or Push Pull Coupli/ ectors for Audio Circuits in Radio Receivers (1/ coupling an External Low Voltage Power Supply T/ radio Receivers and Associated Sound Equipment / nectors with Closed Ends and Having a Contact S/ nectors with Open Ends, Off Centre (Center) Gui/ g Information in Detail Spec. (1970)

iature Multipole Connectors with Blade Contacts/ tipole Connectors with Blade Contacts (1966) requirements and Measuring Meth. (1962)

Rec. for Fundamental Parameters of Rec for Connectors for Frequencies Below $3 \mathrm{MHz}$. Circular ipment / Rec. for Connectors for Frequencies Below $3 \mathrm{MHz}$ : cing of $2.54 \mathrm{~mm}(0.1 \mathrm{In}$.) Mating Either with Board Mounted Rec. for Coaxial Cable

connectors for Frequencies Below $3 \mathrm{MHz}$ : Circular Multipole r Frequencies Below $3 \mathrm{MHz}$ : Rectangular Miniature Multipole nectors for Frequencies Below $3 \mathrm{MHz}$ : Rectangular Multipole c. for Connectors for Frequencies Below $3 \mathrm{MHz}$ : Edge Socket c. for Connectors for Frequencies Below $3 \mathrm{MHz}$ : Edge Socket connectors for Frequencies Below $3 \mathrm{MHz}$ : Circular Multipole rec. for Dimensions of the Mating Parts of Radio Frequency Rec. for Connectors for Frequencies Below $3 \mathrm{MHz}$ : Battery Std. for Tests for Aircraft ElectricaI Plug and Socke
Cones for Cross Winding for Dyeing Purposes: Half Angle Cones for Cross Winding: Half Angle of the Cone $3^{\circ}$

Cones for Cross Winding: Nominal Half Angle of the Cone

Cones for Dyeing Purposes: Half Angle at Top of Cone 4

Cones for Yarn Winding (Cross Wound): Taper $3^{\circ} 30 \mathrm{M}$

Cones for Yarn Winding (Cross Wound): Taper $4{ }^{\circ} 20 \mathrm{M}$

Cones for Yarn Winding (Cross Wound): Taper $9{ }^{\circ} 15 \mathrm{M}$ Cones (1969)

Confidence Interval (1973)

Std. for Statistica

Conformity with Standards (1966)

Conical Fittings for Syringes, Needles and Other Medica

Conical Ground Glass Joints (1964)

Conical Seating (1972)

Conical Seating (1972)

Conical Seating (1973)

Conical Shaft Ends (1969)

Conical Tapers and Taper Angles (1969)

Coniferous Sawn Timber: Sizes-Meth. of Measurement (1

Coniferous Sawn Timber: Sizes-Tolerances and Shrinkag

Coniferous Sawn (Sawed) Timber-Defects-Classificati

Coniferous Sawn (Sawed) Timber-Defects-Measurement

Coniferous Sawn (Sawed) Timber-Defects-Terms and De

Coniferous Sawn (Sawed) Timber-Sizes-Terms and Defi

Connecting Dimensions (1970)

Connecting Pins for Use in Electric Arc Furnaces (1967)

Connecting Pin for Use in Electric

Connecting Wires Having a Rated Voltage of $20 \mathrm{kV}$ and 25

Connection and Circuit Elements (1960)

Connection and Regulation (1957)

Connection for Water of

Connection Gauge (1970)

Connection of Auxiliaries (1971)

Connection with Ionizing Radiation (1964) 65)

Rec. Graphica IEC

for International $\mathrm{E}$ IEC

ISO

ISO

for Photography-Fro ISO

Clow with a IEC

Connection (1954)

Connection (1965)

Connections-General Requirements, Test Meth. and Prac

Connections Between Prime Movers and Towed Vehicles Wit

Connections Between Towing Vehicles and Trailers-Inte

Connections for Aircraft Ground Electrical Supplies (19

Connections for Ground Air Conditioning (1969)

Connections for Hand Held Cameras (1966)

Connections for Industrial Application for Plain End St

Connections for Small Medical Gas Cylinders Used for an

Connections for Vehicles with 6 or $12 \mathrm{~V}$ Electrical Equi

Connections to Tractors (1964)

Connections (1957)

Connections (1957)

Connections (1971)

Connections (1973)

Connector for Twin Balanced Aerial Feeders (1965)

Connector Pin Allocations for Use with High Speed Data

Connector Pin Numbers (1972) /- Data Terminal and

$\begin{array}{ccc}\text { Connector Pin Numbers (1972) Data Terminal and Comm IS } & \text { IS } \\ \text { Connector Units for High Tensile Steel Chain for Convey } & \text { IS } \\ \text { Connector (1965) } & \text { Rec. for Radio Freque } & \text { IE }\end{array}$

Connectors for Audio Circuits in Radio Receivers (1969)

Connectors for Automatic Coupling (1973)

Connectors for Coupling an External Low Voltage Power S

Connectors for Frequencies Below $3 \mathrm{MHz}$ : Battery Connect

Rec. for Connectors for Frequencies Below $3 \mathrm{MHz}$ : Circular Connec

Rec. for Connectors for Frequencies Below $3 \mathrm{MHz}$ : Circular Multip

Rec. for Connectors for Frequencies Below $3 \mathrm{MHz}$ : Circular Multip

Rec. for Connectors for Frequencies Below $3 \mathrm{MHz}$ : Concentric Conn

Connectors for Frequencies Below $3 \mathrm{MHz}$ : Connectors for

Rec. for Connectors for Frequencies Below $3 \mathrm{MHz}$ : Edge Socket Con

Connectors for Frequencies Below $3 \mathrm{MHz}$. Guide to Drawin

Connectors for Frequencies Below $3 \mathrm{MHz}$ : Rectangular Min

Connectors for Frequencies Below $3 \mathrm{MHz}$ : Rectangular Mul

Connectors for Frequencies Below $3 \mathrm{MHz}$ : (Mc/S) General

Connectors for Printed Wiring Boards (1964)

Connectors for Radio and Associated Sound Equipment (19

Connectors for Radio Receivers and Associated Sound Equ

Connectors or Printed Wiring Boards with Edge Board Con

Connectors Used in Nuclear Instrumentation (1969)

Connectors with Bayonet or Push Pull Coupling (1971)

Connectors with Blade Contacts (1965)

R Connectors Fo

Connectors with Blade Contacts (1966)

Rec for Con

Connectors with Closed Ends and Having a Contact Spacin
Connectors with Open Ends, Off Centre (Center) Guide, H

Connectors with Threaded Coupling (1966)

Connectors (1964)

Connectors (1965)

Connectors (1972) 
connector (196.5)

Rec. for Radio Frequency id Coaxial Transmission lines and Their Associated Flange (K.5) Rec. for Radio Frequency
gid Coaxial Transmission lines and Their Associated Flange arger $(1970)$ arger (1971) arger (1971) Rec. for Radio Frequency Rec. for Radio Frequency Rec. for Radio Frequency Rec. for Radio Frequency cs-Assessment of Occupational Noise Exposure for Hearing Rec. for Preliminary Reliability

rec. for Textile Glass-Procedure for Sampling Batches or termination of Compression Set of Vulcanized Rubbers Under vulcanized Rubbers: Determination of Compression Set Under for Cork-Expanded Pure Agglomerated-Deformation Under

in Open Channels: Dilution Meth. for Steady Flow-Part 1: of Aqueous So/ Rec. for Plastics-Meth. for Maintaining Rec. for Test Enclosures of Noninjection Type for nation of the Permeability of Vulcanized Rubbers to Gases Rec. for Chemical Analysis of Cements: Main Rec. for Chemical Analysis of Cements: Minor diameter of Staple Fibres (Fibers) or Continuous Filaments y Thermometers (1964) Rec. for Principles of Rec. for Principles of Rec. for Principles of $\mathrm{s}$ for the Test. of Electric Rolling Stock on Completion of ec. for Rotating Electrical Machines: Symbols for Types of c. for Electrical Apparatus for Explosive Gas Atmospheres: c. for Electrical Apparatus for Explosive Gas Atmospheres: or Fundamental Principles for Protection in the Design and Rec. for Characteristics of Rec. for Rules for

h Voltage Alternating Current Circuit Breakers: Design and Rec. for Electrical Installations in Ships: Cables Rec. for Nuclear Instruments: on Against Ionizing Radiat/

1 Purpose Metric Screw Threads: Tolerances, Deviations for Std. for Modular Units for Machine Tool Rec. for Telemetering for

itters: General Conditions Of, Frequency, Output Power and $r$ for the Calibration of Bone Vibrators Having a Specified

MHz: Edge Socket Connectors with Closed Ends and Having A ectors with Open Ends, Off Centre (Center) Guide, Having A the Determination of Change of Mechanical Properties After he Measurement of Surface Roughness by the Profile Meth.

hing Devices for Control and Auxiliary Circuits, Including

hing Devices for Control and Auxiliary Circuits, Including mhz: Rectangular Miniature Multipole Connectors with Blade c. for Dimensions of the Crimp Area of Machined Crimp Type

s Below $3 \mathrm{MHz}$ : Rectangular Multipole Connectors with Blade ounted Connectors or Printed Wiring Boards with Edge Board Elements of Electromechanic/ Rec. for Graphical Symbols: for Determination of the Bulk Density of Coke in a Small for Determination of the Bulk Density of Coke in a Large Std. for Hermetically Sealed Metal Food Rec. for General Purpose Series 1 Freight 9) Rec. for Finishes with External Screw Thread for Glass equirements (1972) Rec, for Packaging of Winding Wires: Std. for Hooks for Lifting Freight Std. for Minimum Markings to Appear on Rec. for Hermetically Sealed Metal Food Rec. for Essential Oils: Labelling and Marking Rec. for Marking of Series 1 and Series 2 Freight Rec. for Terminology Relating to Freight Rec. for Spec. for Corner Fittings for Series 1 Freight Rec. for Spec. and Test. of Series 1 Freight Rec for Tea Sampling-Part I: from Large Rec. for Dimensions and Ratings of Freight Std. for Identification Marking Code for Freight s: Polarographic Determination of Lead and Cadmium in That ec. for External Diameters of Cylindrical Radiation Probes ide to the Choice of Series of Preferred Numbers and Those trial Use-Determination of Sulphated A sh in Products Not Its Alloys-Electrolytic Determination in Unalloyed Type energy (1/ Rec. for Pictorial Marking of Transit Packages Std. for Magnesium and Its Alloys-Determination of . Rec. for Alpha, Beta and Alpha-Beta oleum for Industrial Use; Determination of Sulphur Dioxide um Hydroxide for Industrial Use: Determination of Sulphate um Hydroxide for Industrial Use: Determination of Sulphate dium Hydroxide for Industrial Use: Determination of Silica sium Hydroxide for Industrial Use: Determination of Silica olite (Natural and Artificial)-Determination of Fluorine um Hydroxide for Industrial Use: Determination of Chloride um Carbonate for Industrial Use: Determination of Chloride um Hydroxide for Industrial Use: Determination of Chloride Oleum for Industrial Use: Determination of Total Nitrogen for Formic Acid for Industrial Use-Determination of Iron
Connectors: Coaxial Unmatched Television Aerial Feeder IEC

Connectors: Detail Spec. (1972) Jor General Purpose Ri lEC

Connectors: General Requirements and Measuring Meth. (1 IEC

Connectors: General Requirements and Measuring Meth. (1) lEC

Connectors: R. F. Coaxial, for Cables 96 IEC 50-12 (1967 IEC

Connectors: R. F. Coaxial, for Cables 96 lEC 50-17 and $L$ lEC

Connectors: R. F Coaxial, for Cables 96 IEC 75-17 and L IEC

Connectors: Two Pin Connector for Twin Balanced Aerial IEC

Conservation Purposes (1971) Rec. for Acousti ISO

Considerations (1968)

Consignments Of, Continuous Filament Yarn, Staple Fibre lSO

Constant Deflection at Low Temperatures (1971) /for De 1SO

Constant Deflection at Normal and High Temperatures (19 ISO

Constant Pressure (1972) Std. 1SO

Constant Rate Injection Meth. (1966) /Low Measurement ISO

Constant Relative Humidity in Small Enclosures by Means ISO

Constant Relative Humidity (1968) IEC

Rec. for Determi ISO

Constituents of of Portland Cement (1968) ISO

Constituents of Portland Cement (1968)

Constituting a Textile Glass Yarn-Cross Section Meth.

Construction and Adjustment of Hydrometers (1964)

Construction and Adjustment of Liquid in Glass Laborato

Construction and Adjustment of Volumetric Glassware (19

Construction and Before Entry into Service (1963)

Construction and Mounting Arrangements of (1972)

Construction and Test of Electrical Apparatus, Type of

Construction and Test of Flameproof Enclosures of Elect

Construction of Installations for Work on Unsealed Radi

Construction of Ply Type- Conveyor Belts (1965)

Construction of Stationary Boilers (1968)

Construction (1971)

Construction, Test. and Installations) (1965)

Constructional Types (1969)

Construction: Slide Units (1973)

Consumption and Demand (1970)

Rec. for ISO Genera ISO

ISO

Consumption (1968) /H. of Measurement for Radio Transm

Contact Area and Being Applied with a Specified Static

Contact Spacing of $2.54 \mathrm{~mm}$ (0.1 In.) Mating Either with

Contact Spacing of $2.54 \mathrm{~mm}$ (0.1 In ) (197I)

Contact with Chemical Substances (1965)

Contact (Stylus) Instruments of Progressive Profile Tra

Contactor Relays) Section 1: Push Buttons and Related C

Contactor Relays) : General Requirements (1970)

Contacts (1965) /R Connectors for Frequencies Below 3 IEC

Contacts (1966)

Contacts (1966)

Re IEC

$\begin{array}{ll}\text { Contacts, Switchgear, Mechanical Controls, Starters and } & \text { IEC } \\ \text { Container (1967) } & \text { Rec ISO }\end{array}$

Container (1969)

Containers-Internal Diameters of Round Cans (1972)

Rec ISO

Containers-Minimum Internal Dimensions (1970)

Containers and Gauges for the Inspection of Closures (1

Containers for Round Types (1968)

Containers of Up to 30 Tonnes (Tons) Capacity-Basic R

Containers Used for Printing Ribbons (1972)

Containers (1959)

Containers (1961)

Containers (1968)

Containers (1968)

Containers (1970)

Containers (1970)

Containers (1970)

Containers (1970)

Containers (1972)

Containing Copper (1972)

Mical A nalysis of Zinc Alloy

Containing More Rounded Values (1966)

Containing More Than $0.5 \%$ of Such, by

Containing Not Less Than $99.90 \%$ (1971)

Copper and

Containing Zirconium, Rare Earths, Thorium and Silver-

Contamination Meters and Monitors (1970)

Content: Barium Sulphate Gravimetric Meth. (1968)

Content: Barium Sulphate Gravimetric Meth. (1969)

Content: Barium Sulphate Gravimetric Meth. (1969)

Content: Gravimetric Meth. by Insolubilization (1969)

Content: Gravimetric Meth. by Insolubilization (1969)

Content: Modified Willard-Winter Meth. (1970)

Content: Volhard Volumetric Meth (1969)

Content: Volhard Volumetric Meth. (1973)

Content: Volhard Volumetric Meth. (1973)

Content: Volumetric Meth. (1968)

Content: 2, 2'-Bipyridyl Photometric Meth. (1970) 
for Nitric Arid for Industrial Use-Determination of Iron ydrochloric Arid for Industrial Use: Determination of Iron ium IIydroxide for Industrial Use: Determination of of Iron assium Hydroxide for Industrial Use: Determination of Iron analysis of Manganese Ores: Determination of Metallic Iron chloric Acid for Industrial Use: Determination of Sulphate um Carbonate for Industrial Use: Determination of Sulphate $\mathrm{m}$ Hydroxide for lndustrial Use: Determination of of Silica for the Production of Aluminum: Determination of Titanium sodium Carbonate for Industrial Use: Determination of lron c Acid and Oleum for Industrial Use: Determination of Iron disation of Aluminium (Aluminum) and Its Alloys-Check of

(1971) Rec for Calculation of the

textile Glass-Determination of the Linear Density of lts rec. for Textile Glass-Determination of the Twist in lts glass-Procedure for Sampling Batches or Consignments Of, ation of the Average Diameter of Staple Fibres (Fibers) or elt Conveyors for Loose Bulk Materials (1971)

- General Rules (1970)

Materials: Vibrating Conveyors and Feeders Wit/

Materials: Screw Conveyors (1969)

Materials-Troughed Belt Conveyors (Other Tha

Materials-Troughed Belt Conveyors (Other Tha)

Materials-Troughed Belt Conveyors (Other Tha

Materials-Oscillating Conveyors and Shaking /

Materials-Vibrating Feeders and Conveyors Wi/

Materials and Unit Loads-Belt Conveyors-Ba/

Materials-Storage Equipment: Bins and Bunker

Materials-Belt Feeders and Conveyors-Safet/

Materials-Vibrating Feeders and Conveyors, S/

Materials-Oscillating Conveyors and Shaking /

Materials-Apron Conveyors (1971)

Materials-Aeroslides (1972)

Materials-Mobile Belt Conveyors-Safety Cod/

Materials-Picking Table Conveyors-Safety C/

Materials-Apron Conveyors-Safety Code (197)

Materials-Scraper and En Masse Conveyors -/

Materials-Hydraulic Conveyors-Safety Code

-Overhead Monorail Chain Conveyors-Safety C/

- Overhead Twin Rail Chain Conveyors (Power An/

-Single Strand Floor Mounted Truck Conveyors /

- Single Strand Floor Truck Conveyors (Chain B

-Mobile Belt Conveyors (Canvas, Rubber, Plast/

Rec. for

Rec. for

Rec. for

Rec. for

Rec. for

Rec. for

Rec. for

Rec. for

Rec. for

Rec. for

Rec. for

Rec. for

Rec. for

Rec. for

Rec. for

Std. for

Std. for

Std. for

Std. for

Std. for

Sid. for

Rec. for

Rec. for

Rec. for

Std. for

Std. for

Under Free Field Listeni/ Rec. for Packaging of Components on
Rec. for Normal Equal Loudness

Rec. for Crude Vegetable Oils and Fats: Preparation of Rec for Oleaginous Seeds: Reduction of

Rec. for Low Voltage Controlgear for Industrial Use: Rec. for Presentation of

c. for Control Switches (Low Voltage Switching Devices for c. for Control Switches (Low Voltage Switching Devices for Rec. for Common Names for Pest for Principles for the Selection of Common Names for Pest Rec. for Colour (Color) Identification of Mechanical cts of Laundering-Preparation and Use of Unsoiled Cotton

Reciprocating Internal Combustion Engines-Hand Operated Rec. for Temperature

Rec. for Std. Frequencies for Centralized Network

ec. for Lamp Caps and Holders Together with Gauges for the

ec. for Lamp Caps and Holders Together with Gauges for the

ec. for Lamp Caps and Holders Together with Gauges for the acter Set) (1968) Rec. for Code for Numerical
an/ Rec. for Punched Tape Block Formats for the Numerical fer (1972) Std. for Data Communication-Basic Mode Std. for Data Communication-Basic Mode
Rec. for Basic Mode transfer (1973) Std. for Basic Mode Rec. for Marking of

trol and Auxiliary Circuits, Including Contacto/ Rec. for trol and Auxiliary Circuits, Including Contacto/ Rec, for ding Contactor Relays) Section 1: Push Buttons and Related Rec. for Analogue d.c. Current Signals for Process Rec. for Analogue Pneumatic Signal for Process Rec. for Ignitrons to Be Used in Welding Machine Rec. for Low Voltage

of Protection of Enclosures for Low Voltage Switchgear and ships: Switchgear, Electrical Protection, Distribution and Rec for Definitions for Switchgear and

Rec. for High Voltage Metal Enclosed Switchgear and

Rec. for Axis and Motion Nomenclature for Numerically Block Format for Positioning and Straight Cut Numerically

able Block Format Positioning and Straight Cut Numerically

Block Format for Positioning and Straight Cut Numerically nd Tempered Types with $1 \%$ Chromium and $0.2 \%$ Molybdenum els-Part 3: Wrought Quenched and Tempered Unalloyed with Spec. for Mains Operated Electric Fence Spec. for Battery Operated Electric Fence
Content: 2, 2'-Bipyridyl Photometric Meth. (1971) Content: 2,2'-Bipyridyl Spectrophotometric Meth. Content: 2,2'-Bipyridyl Spectrophotometric Meth. Content: 2,2-Bipyridyl Spectrophotometric Meth. (196 Content: (Photometric Meth. for Metallic Iron Content U Content; Barium Sulphate Gravimetric Meth. (1968) Content; Barium Sulphate Gravimetric Meth. (1968) Content; Gravimetric Meth. by Precipitation of the Quin

Content: Tiron Photometric Meth (1968)

Content; 2,2'-Bipyridyl Photometric Meth. (1968)

Content; 2,2'-Bipyridyl Spectrophotometric Meth.

Continuity of Thin Coatings-Copper Sulphate Test (197

Continuous Current Rating of Cables (100\% Load Factor)

Continuous Filament and Staple Fibre (Fiber) Yarns and

Continuous Filament and Staple Fibre (Fiber) Yarns (197

Continuous Filament Yarn, Staple Fibre (Fiber) Type and

Continuous Filaments Constituting a Textile Glass Yarn

Continuous Mechanical Handling Equipment-Light Duty B

Continuous Mechanical Handling Equipment-Safety Code

Continuous Mechanical Handling Equipment for Loose Bulk

Continuous Mechanical Handling Equipment for Loose Bulk

Continuous Mechanical Handling Equipment for Loose Bulk

Continuous Mechanical Handling Equipment for Loose Bulk

Continuous Mechanical Handling Equipment for Loose Bulk

Continuous Mechanical Handling Equipment for Loose Bulk

Continuous Mechanical Handling Equipment for Loose Bulk

Continuous Mechanical Handling Equipment for Loose Bulk

Continuous Mechanical Handling Equipment for Loose Bulk

ontinuous Mechanical Handling Equipment for Loose Bulk

Continuous Mechanical Handling Equipment for Loose Bulk

Continuous Mechanical Handling Equipment for Loose Bulk

Continuous Mechanical Handling Equipment for Loose Bulk

Continuous Mechanical Handling Equipment for Loose Bulk

Continuous Mechanical Handling Equipment for Loose Bulk

Continuous Mechanical Handling Equipment for Loose Bulk

Continuous Mechanical Handling Equipment for Loose Bulk

Continuous Mechanical Handling Equipment for Loose Bulk

ontinuous Mechanical Handling Equipment for Loose Bulk

Continuous Mechanical Handling Equipment for Unit Loads

Continuous Mechanical Handling Equipment for Unit Loads

Continuous Mechanical Handling Equipment for Unit Loads

Continuous Mechanical Handling Equipment for Unit Loads

Continuous Mechanical Handling Equipment for Unit Loads

Continuous Tapes (1968)

Contours for Pure Tones and Normal Threshold of Hearing

Contract Sample for Analysis (1968)

Contract Samples to Samples for Analysis (1968)

Contractors (1971)

Contributions to Periodicals (1961)

Control and Auxiliary Circuits, Including Contactor Re

Control and Auxiliary Circuits. Including Contactor Rel

Control Chemicals and Plant Growth Regulators (1971)

Control Chemicals (Pesticides) and Plant Growth Regulat

Control Circuits for Aircraft (1959)

Control Cloth (1972)

Ts: Verification of Certain Effe

Control Devices-Std. Direction of Motion (1972)

Control Devices for Quartz Crystal Units (1970)

Control Installations (1967)

Control of Interchangeability and Safety of Lampcaps (1

Control of Interchangeability and Safety: Gauges (1969)

Control of Interchangeability and Safety: Lampholders (

Control of Machines (Compatible with the ISO 7 Bit Char

Control of Machines: Coding of Preparatory Functions G

Control Procedures-Code Independent Information Trans

Control Procedures for Data Communication Systems (1971

Control Procedures: Conversational Information Message

Control Settings on Hearing Aids (1966)

Control Switches (Low Voltage Switching Devices for Con

Control Switches (Low Voltage Switching Devices for Con

Control Switches (1972)

Control Systems (1971)

Control Systems (1971)

Control (1967)

Controlgear for Industrial Use: Contractors (197I)

Controlgear (1963)

Controlgear (1965)

Controlgear (1968)

Controlgear (1969)

Controlled Machines (1968)

Controlled Machines (1969)

Controlled Machines (1969)

Controlled Machines (1969)

Controlled Sulphur Content (1970)

Controlled Sulphur Content (1970)

Controllers (1956)

Controllers (1956)

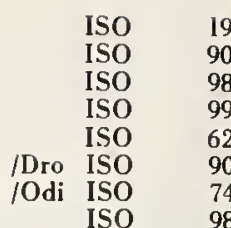

1982

994

621 
for International Flectrotechnical Vocabulary: Automatic for Modular Coorlination-Keference l.ines of Horizontal Rec. for Shape, Size and Direction of Operation of Lever Rec. for Direction of Operation of Machine Too Strengths of Preformed Stranded Steel Cables for Aircraft c. for Graphical Symbols: Contacts, Switchgear, Mechanical Std. for Flexible Steel Wire Rope for Aircraft Rods for Braze Welding: Meth. of Test for Determining the ec. for Stainless Steel Tubes-Dimensions, Tolerances and Rec. for Boiler Tubes-Dimensions, Tolerances and production of Determination of Loss of Mass at $300^{\circ} \mathrm{C}$ Gears (1973) Std. for Technical Drawings: lations of Ventilation Systems in Ships (1967) lations of Sanitary Systems in Ships (1968)

llations of Pipe Line Systems in Ships (1967) ts (1965)

t (For Use in the Preparati/ 1972) 1969)

Rec. for Glass Hydrometers

Rec, for Principles for limetres (Millimeters) and Vice Versa (1964) rec. for International Electrotechnical Vocabulary: Static

Rec for Identification $\mathrm{Rec}$. for Mercury Arc Rec. for List of Characteristics Which May Be Required of Rec. for Tolerances on the Total Thickness of Rec, for Troughability of Rec. for Tear Propagation Resistance of the Carcass of Rec. for Strength of Mechanical Fastenings for Rec. for Ply Adhesion of Rec. for Sampling of Rec. for Characteristics of Construction of Ply Type Rec. for Marking of Rec. for Widths and Lengths of rec. for Full Thickness Tensile Strength and Elongation of Rec. for Electrical Conductivity of Rec. for Flame Resistance of Rec. for

Chains (Metric Series) (1971) g Equipment for Loose Bulk Materials and Unit Loads-Belt onveyors, Shaking or Reciprocating Feeders and Oscillating andling Equipment for Unit Loads-Overhead Monorail Chair Handling Equipment for Loose Bulk Materials-Mobilc Belt quipment for Loose Bulk Materials-Scraper and En Masse anical Handling Equipment for Loose Bulk Materials-Apron andling Equipment for Loose Bulk Materials-Picking Table al Handling Equipment for Loose Bulk Materials-Hydraulic Rec. for High Tensile Steel Chains (Round Link) for Chain cal Handling Equipment for Loose Bulk Materials: Vibrating Handling Equipment for Loose Bulk Materials-Oscillating Handling Equipment for Loose Bulk Materials-Oscillating continuous Mechanical Handling Equipment-Light Duty Belt equipment for Loose Bulk Materials-Vibrating Feeders and mechanical Handling Equipment for Unit Loads-Mobile Belt uipment for Unit Loads-Single Strand Floor Mounted Truck dling Equipment for Unit Loads-Single Strand Floor Truck andling Equipment for Loose Bulk Maierials-Troughed Belt andling Equipment for Loose Bulk Materials-Troughed Belt andling Equipment for Loose Bulk Materials-Troughed Belt ndling Equipment for Unii Loads-Overhead Twin Rail Chain ckle Type Connector Units for High Tensile Steel Chain for hanical Handling Equipment for Loose Bulk Materials: Screw anical Handling Equipment for Loose Bulk Materials-Apron cision Roller Chains and Chain Wheels for Transmission and equipment for Loose Bulk Materials-Vibrating Feeders and Purposes: Cooking Ranges,/ Particular Spec. for Electric Purposes: for Heating Liq/

Purposes: Portable Immers

Purposes: Stationary Noni/

Purposes: Room Heating An/

Purposes: Electric Irons, /

Purposes: Waffle Irons, Grills, Warm

Purposes: Clothes Dryers and Towel D/

Purposes $(1959+1964)$

Particular Spec. for Electric

Particular Spcc. for Electric

Particular Spec. for Electric

Particular Spec. for Electric Particular Spec. for Electric rposes: Waffle Irons, Grills, Warming Plates and Other Dry s, Lighting, Accumulator (Storage), Batteries, Heating and and Heating Appliances for Domestic and Similar Purposes: liances for Domestic and Similar Purposes: Cooking Ranges, Rec. for Rotating Electrical Machines: Meth. of e Harness (1967) Rec. for Heald Frames: r Coordination-Reference Lines of Horizontal Controlling g Coordinating Dimensions (1970)

buildings (1970)

Rec. for Mocular Rec. for Modular Rec. for Modular
Controlling and Regulating Systems (1966)

Controlling Coordinating Dimensions (1970)

Controls on Aireraft (1969)

Controls (1965)

Controls (1967)

Controls, Starters and Elements of Electromechanical Re

Conventional Bond Strength on Steel, Cast lron and Othe

Conventional Masses Per Unit Length (1969)
Conventional Masses Per Unit Length (1971)

(Conventional Moisture) (1968)

Conventional Representation of

Primarily Used for the $1 \mathrm{SO}$

Conventional Signs to Be Used in Schemes for the Instal

Conventional Signs to Be Used in Schemes for the Instal

Conventional Signs: to Be Used in Schemes for the Insta

Conventional Typographical Character for Legibility Tes

Conventional Value for the Thermal Expansion Coefficien

Conventions Concerning Electric and Magnetic Circuits (

Conventions for Flow sheets for Coal Preparation Plant (

Conversational Information Message Transfer (1973)

Conversion of Toleranced Dimessions from In./S into Mil

Conversion Resistance and Transductance (1966)

Convertors (1956)

Conveying Fluids in Liquid or Gaseous Condition in Land

Conveyor Belts According to Their Use (1969)

Conveyor Belts and on the Thicknesses of Covers (1967)

Conveyor Belts (Characteristic and Meth. of Test) (1968

Rec IEC

Rec ISO

ISO

ISO

ISO

ISO

$1 \mathrm{SO}$

150

ISO

ISO

ISO

ISO

ISO

ISO

IEC

ISO

ISO

/Electr IEC

IEC

IEC

ISO

ISO

ISO

Conveyor Belts (Meth. of Test) (1966)

Conveyor Belts (Static Test Meth.) (1969)

Conveyor Belts (1962)

Conveyor Belts (1962)

Conveyor Belts (1965)

Conveyor Belts (1965)

Conveyor Belts (1970)

Conveyor Belts: Spec. and Meth. of Test (1962)

Conveyor Belts: Spec, and Meth. of Test (1962)

Conveyor Belts: Spec./S and Meth. of Test (1963)

Conveyor Chains, Atiachments and Chain Wheels-Part I

Conveyors-Basic Characteristics of Motorized Driving

Inuous Mechanical Hand ISO

Conveyors-Safety Code (1970)

Conveyors-Safety Code (1971)

Conveyors-Safety Code (1971)

Conveyors-Safety Code (1972

/Ibrating Feeders and C ISO

/Ontinuous Mechanical $\mathrm{H}$ ISO

/R Continuous Mechanical ISO

Mechanical Handling E ISO

/D. for Continuous Mech ISO

/Ontinuous Mechanical H ISO

Ior Continuous Mechanic ISO

Conveyors-Safety Code (1972)

Conveyors-Safety Code (1972)

Conveyors and Coal Ploughs (1967)

Conveyors and Feeders with Rectangular or Trapezoidal T

Conveyors and Shaking on Reciprocating Feeders with Tub

Conveyors and Shaking or Reciprocating Feeders with Rec

Conveyors for Loose Bulk Materials (1971)

Conveyors (Canvas, Rubber, Plastic, Etc.) Safety Code ( ISO

Conveyors (Chain Above Floor)-Safety Code (1971) /Eq ISO

Conveyors (Chain Below Floor) Safety Code (1972)

Conveyors (Other Than Portable) Belt Pulleys (1970)

Conveyors (Other Than Portable) Belts (1970)

Conveyors (Other Than Portable) Idlers (1970)

Conveyors (Power and Free) Safety Code (1971)

Han ISO

/H ISO

Anical H ISO

iNical H ISO

IIcal Ha ISO

Conveyors (1969)

Conveyors (1969)

Conveyors (1971)

Rec. for Continuous Mec ISO

Conveyors (1972)

Rec for Continuous Mech ISO

Std for Extended Pitch Pre ISO

Conveilla

Cooking and Heating Appliances for Domestic and Similar

Cooking and Heating Appliances for Domestic and Similar

Cooking and Heating Appliances for Domestic and Similar

Cooking and Hicating Appliances for Domestic and Similar

Cooking and Heating Appliances for Domestic and Similar

Cooking and Heating Appliances for Domestic and Similar

Cooking and Heating Appliances for Domestic and Similar

Cooking and Heating Appliances for Domestic and Similar

Cooking and Heating Appliances for Domestic and Similar

Cooking Appliances (1967) ffor Domestic and Similar Pu CEE

Cooking Appliances, Internal Communications, Lightning IEC

Cooking Ranges, Cooking Tables and Similar (1968) /Ing CEE

Cooking Tables and Similar (1968) /Ing and Heating App CEE

Cooling (1969)

Coordinated Dimensions in Relationship with Pitch of Th

Coordinating Dimensions (1

Coordination-Reference Lines of Horizontal Controllin

Coordination-Storey and Room Heights for Residential

Coordination-Vocabulary (1971)

Coordination Of, and with End Loops of Healds (1967) 
Rec. for Insulation

Rec. for Insulation

Rec. for Modular

Rec. for Modular

Preparation of Dry Polymer from Butadiene Homopolymer and Rec. for Plastics-Aqueous Dispersions of Polymers and Rec. for Plastics-Aqueous Dispersions of Polymers and Rec. for Plastics-Aqueous Dispersions of Polymers and of the Thermal Stability of Polyvinylchloride and Related of the Thermal Stability of Polyvinyl Chloride and Related stics-Designation of Unplasticized Compounds of Homo and Determination of Chlorine in Vinyl Chloride Polymers and rmination of Vinyl Acetate in Vinyl Chloride.Vinyl Acetate Rec for Phosphorus Deoxidized Std for Petroleum Products Corrosiveness to Rec. for Special Wrought Rec. for Cast

ectrophotometric Determination of Nickel (Low Contents) in han $2.5 \mathrm{~mm}(0.1 \mathrm{ln})$-Tensile Test (1971)

ning Test (1971)

Rec. for Rec. for Rec. for Tensile Test. of Rec. for Drift Expanding Test on Rec. for Wrapping Test for Rec for Tensile Test of Rec. for Rec. for

of Tempers (1971) f Materials (1971) nalloyed Type Containing $\mathrm{N} /$ rought and Cast-Copper All/ pes (1971) n of Nickel (Low Contents)/ $\mathrm{n}$ of Iron in the Alloys (1/ 1964) Rec. for Vickers Hardness Test for
Rec. for Meth. of Mercurous Nitrate Test for Rec. for Bend Test for Rec. for Tensile Test. of

Rec for Brinell Hardness Test for c. for Chemical Analysis of Zinc Alloys-Determination of ce Requirements for General Purpose Electrical Cables with $r$ Meth. of Test for General Purpose Electrical Cables with ance of Heat Resisting $\left(260^{\circ} \mathrm{C}\right)$ Electrical Cables with $n t s$ for Heat Resisting $\left(190^{\circ} \mathrm{C}\right.$ ) Electrical Cables with est for Heat Resisting $\left(260^{\circ} \mathrm{C}\right)$ Electrical Cables with Test for Heat Resisting (190 Deg.) Electrical Cables with $\mathrm{nts}$ for Heat Resisting $\left(260{ }^{\circ} \mathrm{C}\right)$ Electrical Cables with guide ta the Calculation of Resistance of Plain and Tinned ance of Heat Resisting $\left(190^{\circ} \mathrm{C}\right)$ Electrical Cables with uctor Resistance of General Purpose Electrical Cables with rmination of Copper (Oxalyldihydrazide Meth. Applicable to d) $(1970)$

of Aluminum and Its Alloys: Electrolytic Determination of Rec. for Determination of Rec. for Classification of Rec. for Classification of

0) Rec for Electroplated Coatings of Rec. for Fire Refined High Conductivity Tough Pitch Rec. for Fire Refined Tough Pitch r Chemical Analysis of Copper and Its Alloys-Sampling of Std. for Electrolytic Tough Pitch m) and lts Alloys-Check of Continuity of Thin Coatings 1) Rec. for Capillary Solder Fittings for
Rec. for ries (1962)

ng Wires: Dimension of Conductors of Insulated Rectangular ec. for Particular Types of Winding Wires: Enamelled Round of Magnesium and lts Ailoys: Photometric Determination of um (Aluminum) and Its Alloys: Photometric Determination of International Sid for Resistance for of Chemical Analysis of Manganese Ores-Determination of Rec. for Pulps: Determination of ical Analysis of Zinc: Spectrophotometric Determination of cular Types of Winding Wires: Self Fluxing Enamelled Round ires: Heat or Solvent Bonding Self Fluxing Enamelled Round

Rec. for Raw-Rubber and Rubber Latex-Determination of Std. for Electrolytic Cathode aphic Determination of Lead and Cadmium in That Containing ec. for Particular Types of Winding Wires: Enamelled Round ec. for Particular Types of Winding Wires: Enamelled Round ec. for Particular Types of Winding Wires: Enamelled Round ec. for Particular Types of Winding Wires: Enamelled Round ec. for Particular Types of Winding Wires: Enamelled Round of Winding Wires: Heat or Solvent Bonding Enamelled Round ec. for Particular Types of Winding Wires: Enamelled Round

- for Particular Types of Winding Wires: Bunched Enamelled Rec. for Classification of Rec. for Wrought Alloyed Rec. for Wrought Rec. for Ropes and

Rec. for Polyvinyl Chloride Insulated Flexible Cables and
Coordination (1972)

Coordination: Application Guide (1962)

Coordination: Basic Module (1969)

Coordination: llorizontal Multimodules $(1969+1970)$

Copolymer Latices (1971)

Copolymers-Determination of Dry Solids Content at 105

Copolymers-Determination (1969)

Copolymers-Freeze Thaw Cycle Stability Test (1969)

Copolymers and Their Compounds by Splitting Off of $\mathrm{Hydr}$

Copolymers and Their Compounds by the Discoloration Met

Copolymers of Vinyl Chloride (1970)

Copolymers (1970)

Copolymers (1970)

Copper-Refinery Shapes (1971)

Rec. for Plastics

Copper-Strip Test (1972)

Copper Alloys (1971)

Copper Alloys (1971)

Copper Alloys (1971)

Copper and lts Alloy Tubes of Circular Section-Flatte

Copper and Its Alloy Tubes of Circular Section (1964)

Copper and lts Alloy Tubes (1961)

Copper and lts Alloy Wire (1964)

Copper and lis Alloy Wire (1964)

Copper and Its Alloys-Code of Designation-Part II:

Copper and Its Alloys-Code of Designation-Part I: O

Copper and Its Alloys-Electrolytic Determination in U

Copper and lts Alloys-Electrolytic Determination in W

Copper and lts Alloys-Sampling of Copper Refinery Sha

Copper and Its Alloys-Spectrophotometric Determinatio

Couper and Its Alloys-Spectrophotometric Determinatio

Copper and lts Alloys (Test Loads from 2.5 to $50 \mathrm{kgf}$ ) (

Copper and lts Alloys (1961)

Copper and Its Alloys (1964)

Copper and Its Alloys (1964)

Copper and Its Alloys (1964)

Copper by Electrolysis (1971)

Copper Conductors for Aircraft (1966)

Copper Conductors for Aircraft (1967)

Copper Conductors for Aircraft (1967)

Copper Conductors for Aircraft (1969)

Copper Conductors for Aircraft (1970)

Copper Conductors for Aircraft (1970)

Copper Conductors for Aircraft (1970)

Copper Conductors of Low Frequency

Copper Conductors, for Aircraft (1966)

Copper Conductors, for Aircraft (1966)

Copper Content Between 0.002 and 0.4\%) (1968)

Copper Nickel Alloys (1965)

Copper Nickel Zinc Alloys (1965)

Copper Plus Nickel and Chromium on Steel (Or lron) (197

Copper Refinery Shapes (1971)

Copper Refinery Shapes (1971)

Copper Refinery Shapes (1971)

Copper Refinery Shapes (1972)

Copper Sulphate Test (1971)

Ion of Aluminium (Aluminu ISO

Copper Tubes of Circular Section: Dimensions: Metric Se

Time IEC

Copper with Good Dielectric Properties Under Humid Cond IEC

Copper (Oxalyldihydrazide Meth. Applicable to Copper Co ISO

Copper (Oxalyldihydrazide Meth. Applicable to Its Conte

Copper (1925)

Copper (1963)

Copper (1968)

Copper (1969)

Rec. for Meth. $1 S O$

Rec. for Meth. 1SO

Rec. for Chem 1SO

Copper (1970)

Copper (1970)

Copper (1971)

Copper (1972)

Copper (1972)

Rec, Spec. for Parti

ISpec, for Particular Types of Winding W IEC

ISO

Copper, with a Temperature Index of 180 for Use in Refr

Copper, with a Temperature lndex of $180(1972)$

Copper, with a Temperature lndex of 220 (1972)

Copper, with High Mechanical Properties for Use in Ref

Copper, with High Mechanical Properties (1970)

Copper, with High Mechanical Properties (1970)

Copper, with Silk Covering (1972)

Coppers (1961)

Coppers (1971)

Coppers (1971)

Cordage-Vocabulary (1971)

Cords with Circular Conductors and a Rated Voltage Not
71

$71 \mathrm{~A}$

1006
$1040 / I 1$

2028

1625

1148

1147

182

1163

1158

1159

1430

2160

1187

1338

1810

1555

1556

401

195

397

402

$1190 / 11$

1553

1554

1811

1810

1812

399

196

398

400

403

1976

474

634

539

1075

1491

1220

1490

344

470

469

794

796

1396

429

430

1457

1428

1429

1811

431

2085
2016

274

182-3

$317-6$

794

795

322

778

1053

317-4

$317-2$

1654

2311

2576
$317-3$

317.10

317.8

317.7

317.9

317.5

317-1

317.11

197

1336

1337

1968

227 
exceding / Rer. for Rubber Insulated Flexible Cables and - for Colours (Colors) of the Cores of Flexible Cables and spec. for Polyvinyl Chloride Insulated Cables and Flexible Stocked Sizes (1972) Spec. for Rubber Insulated Cables and Flexible Std. for

Rec for Graphical Symbols: Ferrite es Having a Nominal Selection Ratio Of/ Rec. for Magnetic ions: Measuring Meth. (1971) Rec. for Rec. for Dimensions of

Rec. for Guide for the Dra Rec. for Dimensions of Screw Rec. fi Golours (Colors) of the ectronic and Telecommunication / Rec. for Strip Wound Cu Rec. for Paper: Internal Diameters of

Rec. for Guide for the Drafting of Perforamnce Spec. for ated Parts (1967) Rec. for Dimensions of Cross $\mathrm{g}$ in Manufacturers Catalogues of Transformer and Inductor Std. for Granulated

Std. for Cork-Expanded Pure Agglon erated Thermal onstant Pressure (1972)

lk Density (1972)

teristics, Sampling and Packing (1972)

1972)

Std. for

Std. for

Std. for

Std. fo

Rec. for Cranulated

Rec. for Granulated

te-Definitions and $\mathrm{Pa} /$

rmination of Moistur/

ling (1972)

Rec. for Commercially $\mathrm{n}$ " irgin Std. for Corkwood in Planks, Virgin Std. for Corkwood in Planks, Virgin Rec. for Pure Expanded

bending (1971) Rec. for Pure Expanded

dry Virgin Cork, Ramassage, Gleanings, Corkwood Refuse a..

d in Planks, Virgin Cork, Ramassage, Gleanings, Refuse and d in Planks, Virgin Cork, Ramassage, Gleanings, Refuse and ckaging (1970)

refuse and Corkwaste-Sampling (1972) Rec. for Commercially Dry

refuse and Corkwaste-Sampling (1972)

c. for Commercially Dry Virgin Cork, Ramassage, Gleanings, Rec. for Std. for Rec. for Spec. for nuts: Metric Series, Tolerances on Widths Across Flats and es of Electronic Tubes and Valves: Meth. of Measurement on Rec. for Sizes of International Rec. for Rec. for Machine Tool Tables-T Slots and $\mathrm{d}$ in the Plastics Industry (English, French, Russian) (Cs: $d$ in the Plastics Industry (English, French, Russian) (NL: ed in the Plastics Industry (English, French, Russian) (D: i) Rec. for Rectangular or Square Parallel Keys and Their $d$ in the Plastics Industry (English, French, Russian) (Pl: Rec, for Widths of Flat Transmission Belts and elts for Industrial Speed Changers and Groove Profiles fo Rec. for Guiding Principles for Protection Against $r$ a Meth for the Evaluation of the Results of Accelerated aisal of the Problems of Accelerated Test. for Atmospheric and Aluminum for the Protection of Iron and Steel Agains Std. for Petroleum Products Rec. for Asbestos Cemen adding (1964)

Rec. for Asymmetrical Section rs (1971) in in Rec. for lue (1972) Effects of Laundering-Preparation and Use of Unsoiled Rec. for Meth. of Sampling Raw Rec. for Rec. for Diameters of Drafting Rollers for Std. for Metallic Coatings-Measurement of Thickness adiation Probes Containing Geiger-Muller or Proportiona Electronic Tubes: Meth. of Measurement of Geiger-Muller )

Rec. for Stability of Rec. for Slotted

millimetres (Millimeters) and In./S (19/ millimetres (Millimeters) and In/

Rec. for Slotted Raised of Electronic Tubes: Meth. of Measurement for Cold Cathode td. for Textiles: Determination of Twist in Yarns-Direc Rec. for Symbols for Languages specified Contact Area and Be/ Rec, for an IEC Mechanical etry (1970) Rec. for IEC Provisional Reference ones Coupled to the Ear by Means/ Rec. for IEC Reference 2)

Spec. for Appliance Rec. Appliance

Rec, for Plugs, Socket Outlets and for Connectors for Frequencies Below $3 \mathrm{MHz}$ : Connectors for ability (1971)
Cords with Circular Conductors and a Rated Voltage Not Cords (1964)

Cords (1967)

Cords (1968)

Core Drills with Parallel, and Morse Taper Shanks-Rec

Cores and Magnetic Storage Matrices (1972)

Cores for Application in Coincident Current Matrix Stor

Cores for Inductors and Transformers for Telecommunicat

Cores for Motion Picture and Magnetic Films (1969)

Cores Made of Ferromagnetic Oxides and Associated Parts

Cores Made of Ferromagnetic Oxides (1966)

Cores of Broad Band Transformers of Ferromagnetic Oxide

Cores of Flexible Cables and Cords (1964)

Cores of Grain Oriented Silicon Iron Alloy, Used for EL

Cores of Reels (1966)

Cores of Tuned Transformers and Inductors of Ferromagne

Cores (X-Cores) Made of Ferromagnetic Oxides and Associ

Cores (1972)

Cork-Bulk Density Test (1972)

Cork-Characteristics, Sampling and Packing (1972)

Cork-Expanded Pure Agglomerated-Deformation Under C

Cork-Expanded Pure Agglomerated-Determination of $\mathrm{Bu}$

Cork-Expanded Pure Agglomerated Thermal Cork-Charac

Cork-Granulated-Determination of Moisture Content

Cork-Granule Size Test (1971)

Cork-Sampling (1971)

Cork, Ramassage, Gleanings, Corkwood Refuse and Corkwas

Cork, Ramassage, Gleanings, Refuse and Corkwaste-Dete

Cork, Ramassage, Gleanings, Refuse and Corkwaste-Samp

Corkboard-Determination of Moisture Content (1971)

Corkboard-Determination of the Modulus of Rupture by

rkwaste-Definitions and Packaging (1970)

o. ' waste-Determination of Moisture Content (1972)

Cor aste-Sampling (1972) Std. for Corkwoo

Cori- $\mathrm{c}$ - in Planks-Definitions, Classification and $\mathrm{Pa}$

Corkwosd in Planks, Virgin Cork, Ramassage, Gleanings,

Corkwood ? Planks, Virgin Cork, Ramassage, Gleanings,

Corkwood efuse and Corkwaste-Definitions and Packagi

Cork: Glc's:ry (1967)

Cork. Grar sla ed and Powder-Spec. (1972)

Corner Fitti ws for Series 1 Freight Containers (1970)

Rec. for Hexagon Bolts and ISO

Corners (1968)

Corona Stabilizers (1969)

Correspondence Envelopes and Pockets (1962)

Correspondence Symbol (1970)

Corresponding Bolts (1971)

Corresponding Czech Terms) (1964)

Corresponding Dutch Terms) (1968)

Corresponding German Terms) (1964)

Corresponding Keyways (Dimensions in Millimetres) (Mill

Quivalent Terms Use

Quivalent Terms Use ISO

Quivalent Terms Us ISO

Corresponding Keyways (Dimensions in Millimetres) (Mill ISO

Corresponding Polish Terms) (1963)

Corresponding Pulleys (1956)

Corresponding Pulleys (1970)

Corrosion by Hot Dip Galvanizing (1970)

Corrosion Tests on Coatings Other Than T

Corrosion (1971)

Rec. for an Appr
Rec. for Metal Spraying of Zinc

Corrosiveness to Copper-Strip Test (1972)

Corrugated Sheets for Roofing and Cladding (1964)

Corrugated Sheets in Asbestos Cement for Roofing and Cl

Cotter Slots with 5\% Taper Keys for Boring Machine Tape

Cotton Control Cloth (1972)

Ts: Verification of Certa

Cotton Fibres (Fibers)

Cotton Spinning Machinery: Working Width (1963)

Cotton, Wool, Spun Silk and Staple Fibre (Fiber) (1959)

Coulometric Meth. by Anodic Dissolution (1972)

Counter Tubes or Scintillation Detectors (1967)

Counter Types (1971)

Counterbalanced Fork Lift Trucks-Rated Capacity (1971

Counterbalanced Lift Trucks-Basic Tests (1969)

Countersunk (Flat) Head Tapping Screws-Dimensions in

Countersunk (Oval) Head Tapping Screws-Dimensions in

Counting and Indicator Types (1970)

Counting Meth. (1972)

Countries and Authorities (1967)

Coupler for the Calibration of Bone Vibrators Having a

Coupler for the Calibration of Earphones Used in Audiom

Coupler for the Measurement of Hearing Aids Using Earph

Couplers for Domestic and Similar General Purposes (196

Couplers for Household and Similar General Purpose (197

Couplers for Industrial Purposes (1969)

Coupling an External Low Voltage Power Supply to Portab

Coupling Between Tractors and Semi Trailers Interchange

Coupling Capacitors and Capacitor Dividers (1971) 
Helow 3 MHz: Circular Multipole Connectors with Threaded z: Circular Multipole Connectors with Bayonet or Push Pull angeability Dimensions of Battery Connectors for Automatic (1971) Rec. for Pneumatic Rec. for Gaseous Oxygen Replenishmen Rec. for Ball Rec. for Pipe

Rec. for Ball and Socket eral Requir/ Rec. for Locking Devices for Ball and Socke Rec. for Flat Seal for Hydraulic of Venturi Tubes-Classical Tubes Used Outside the Scope etal Recovery and Deposition Coefficient (1972) ow Alloy High Tensile Types/ Rec. for Code of Symbols for ow Alloy Steels-Code of Symbols for Identific/ Std. for Iron (1969) Rec. for Code of Symbols for

Welding Positions for Straight Manual Arc Welds Made with Std. for Wire Reinforced, Rubbe ypes of Winding Wires: Bunched Enamelled Copper, with Silk ic Loading (1971) Rec. for Machine Made Textile Floor Rec. for Textile Floor Tests (1971) Rec. for Machine Made Textile Floor Rec. for Machine Made Textile Floo Rec. for Tests on Anticorrosion Protective Std. for Textile Floor ing Moisture Conditi/ Std. for Machine Made Textile Floor
Rec. for Shipbuilding Details for Inland Navigation rec. for Shipbuilding Details-Marking of Wooden Hatchway otal Thickness of Conveyor Belts and on the Thicknesses of Rec. for Meth. of Milk Recording of (De Mattia Type / Rec. for Determination of Resistance to mattia Type/ Rec. for Determination of Resistance to Flex Vulcanized Rubbers-Determination of Resistance to Ozone 1972) Rec. for Natural Rubber Latices, Centrifuged and for Textile Fabrics-Determination of the Recovery from Rec. for Determination of Tensil

tures (1961)

Temperature Interrupted) (1961)

Rec. fo

Rec, for Interrupted Rec. for Noninterrupted Rec. for

tear Strength of Vulcanized Natural and Synthetic Rubbers r Cresylic Acid for Industrial Use-Determination of Meta $\mathrm{d}$ and Xylenols for Industrial Use-Determination of Ortha ing Point (1971)

Evaporation (197)

le in Sodium Hydroxide So

Determination Of/

Determination Of/

Determination of/

Determination of Densit/

Rec. for Phenol: Ortha, Meta, and Para

Rec. for Phenol, $O, M$, and $P$ Rec. for Phenol: Ortho, Meta, and Para Rec. for Phenol: Ortha, Meta, and Para Rec. for Phenol: Ortha, Meta, and Para Rec. for Liquefied Phenol: Meta nation Of/ Rec. for Phenol: Ortha, Meta, and Para Cresol, nation Of/ Rec. for Phenol: Ortha, Meta, and Para Cresol, nation of Densit/ Rec. for Liquefied Phenol: Meta Cresol, nation of Distillation Range (1971)

nation of Residue on Distillation (1971) nation of Ortha Cresol Content (1971)

ment of Colour (Color) (1971)

r Absence of Hydrogen Sulphide (1971)

a Cresol Content (1971)

Rec. for Dimensions of the Rec. for Dimensions of the Crimp Area of Machined Sid, for aterials (1970) Std. for Aluminium Terminal Ends for Rec. for Principles of

associated Parts (1967) Std. for Ebonite: Determination of Rec. for Dimensions o es of Electronic Tubes and Valves: Meth. of Measurement of r Continuous Filaments Constituting a Textile Gless Yasn Machinery-Terms for Serrated Bars and Dimensions of the insulated Cables (1966) Rec. for Nomina

cal Symbols for Use on Detailed Maps, Plans and Geologica cal Symbols for Use on Detailed Maps, Plans and Geological cal Symbols for Use on Detailed Maps, Plans and Geological Rec. for Files and Rasps: Lengths and

Oxide Coating Thicknesses by Microscopical Examination of 0) Rec. for Textile Machinery and Accessories-Pirn and ne $4^{\circ} 20^{\prime}(1963)$

963)

' (1963) Rec. for Wood Cones for Rec. for Wood Cones for Rec. for Wood Cones for Rec for Paper Cones for Yarn Windin Rec. for Paper Cones for Yarn Winding Rec. for Paper Cones for Yarn Winding
Coupling Threads for Hydraulic or Pneumatic Piping (Pip

ISO

Coupling (1966)

Coupling (1971)

Coupling (1973)

Couplings Between Tractors and Trailers (1971)

Rec. for Connectors for Frequencie IEC

/Connectors for Frequencies Below 3 Mh IEC Std. for Aircraft: Interch ISO

Couplings for Aircraft-Interchangeability Dimensions

Couplings for Aircraft (1969)

Couplings for Caravans and Light Trailers (1969)

Couplings for Hydraulic Piping (Pipe Threads) (1970)

Couplings of String Insulator Units (1960)

Couplings of String Insulator Units: Dimensions and Gen

Couplings (1970)

Covered by ISO Rec. R781 (1969)

F Fluid Flow by Means

Covered Electrodes-Determination of the Efficiency, M

Covered Electrodes for Arc Welding of Mild Steels and L

Covered Electrodes for Manual Arc Welding of Mild and L

Covered Electrodes for Manual Metal Arc Welding of Cast

Covered Electrodes of Mild and Low Alloy High Tensile S

Covered Hydraulic Hose (1972)

Covering (1972)

Rec. for Particular T

Coverings-Determination of Mass Per Unit Area (1970)

Coverings-Determination of Thickness Loss Under Dynam

Coverings-Determination of Thickness (1971)

Coverings-Sampling and Cutting Specimens for Physical

Coverings of Metallic Cable Sheaths (1966)

Coverings: Classification and Terminology (1972)

Coverings: Determination of Dimensional Changes in Vary

Covers for Deck Openings for 220 min Pumps (1957)

Covers (1960)

Covers (1967)

Rec. for Tolerances on the T

Cows (1970)

Crack Growth of Vulcanized Natural or Synthetic Rubber

Cracking of Vulcanized Natural or Synthetic Rubber (De

Cracking Under Static Conditions (1972)

Creamed, Ammonia Preserved-Spec. (1971)

Cream: Determination of F at Content (Reference Meth.) (

Creasing of a Horizontally Folded Specimen by Measuring Creep of Plastics (1968)

Creep Stress Rupture Test. of Steel at Elevated Tempera

Creep Test. of Steel at Elevated Temperatures (Load and

Creep Test. of Steel at Elevated Temperatures (1961)

Creepage Distances and Clearances in Air (1964)

ISO

ISO

ISO

ISO

ISO

EC

IEC

ISO

ISO

ISO

ISO

ISO

ISO

ISO

ISO

ISO

ISO

ISO

IEC

ISO

ISO

ISO

ISO

ISO

ISO

ISO

Std. for ISO

ISO

ISO

ISO

ISO

ISO

ISO

CEE

(Crescent Test Piece) (1957)

Cresol Content (1971)

Cresol Content (1971)

Ec. for Determination of ISO

Rec. Fo ISO

Rec. for Cresylic Aci ISO

Cresol for Industrial Use-Determination of Crystalliz

Cresol for Industrial Use-Determination of Residue on

Cresol for Industrial Use-Test for Impurities Insolub

Cresol, Cresylic Acid and Xylenols for Industrial Use-

Cresol, Cresylic Acid and Xylenols for Industrial Use-

Cresol, Cresylic Acid and Xylenols for Industrial Use-

Cresol, Cresylic Acid and Xylenols for Industrial Use-

Cresylic Acid and Xylenols for Industrial Use-Determi

Cresylic Acid and Xylenols for Industrial Use-Determi

Cresylic Acid and Xylenols for Industrial Use-Determi

Cresylic Acid and Xylenols for Industrial Use-Determi

Cresylic Acid and Xylenols for Indust rial Use-Determi

Cresylic Acid and Xylenols for Industrial Use-Determi

Cresylic Acid and Xylenols for Industrial Use-Determi

Cresylic Acid and Xylenols for Industrial Use-Measure

Cresylic Acid and Xylenols for Industrial Use-Test Fo

Cresylic Acid for Industrial Use-Determination of Met

Crimp Area of Machined Crimp Type Contacts (1966)

Crimp Type Cont acts (1966)

Crimped Joints for Aircraft Electrical Cables (1973)

Crimping to Aircraft Aluminum Electrical Cables (1973)

Criticality Safety in Handling and Processing Fissile M

Cross Breaking Strength (1972)

Cross Cores ( $X$-Cores) Made of Ferromagnetic Oxides and

Cross Moduletion in (1969)

Cross Section Meth. (1971)

of the Electrical Properti IEC

(F Staple Fibres (Fibers) 0

Cros Sectional Areas and Composition of Conductors of

Cross Scctions-Part II: Representation of Sedimentary

Cross Sections-Part I: General Rules of Representatio

Cross Sections (1961)

Cross Sections (1970)

Cross Winders-Definition of Sic

Cross Winding for Dyeing Purposes: Half Angle of the Co

Cross Winding: Half Angle of the Cone $3^{\circ} 30^{\prime}$ (1

Cross Winding: Half Anglc of the Cone $4^{\circ} 20^{\prime}(1$

Cross Winding: Nominal Half Angle of the Cone 5 57

Cross Wound): Taper $3^{\circ} 30^{\prime}(1959)$

(Cross Wound): Taper $4^{\circ} 20^{\prime}$ (1959)

(Cross Wound): Taper $9^{\circ} 15^{\prime}$ (1959)

1943

$130-4$

130-7

1963

1728

1465

1022

1103

120

$372-1$
1941

$781 / \mathrm{I}$

2401

635

2560

598

1436

317-11

1764

2094

1765

1957

2424

2551

41
152

583

1546

133

132

1431

2004

2450

2313

206

203

REC

34

1911

1910

1901

1900

1902

1897

1898

1899

1903

1897

1898

1899

1903

1906

1907

1910 
Rec. for Crowns of Pulleys for Flat Transmission Belts (1959)

of Sodium and Boric Oxide Contents-Volumetri/

Sid. for$$
\text { td. }
$$

$(1968)$ Id for Boric Acid, Boric Oxide, Disodium Tetraborates and

le Impurities (1968)

e and Volatile Matter (1968)

sample for Analysis (1968)

Rec. for Rec. for

uorine Content: Modified Willard-Winter Meth on Content-1,10 Phenanthroline Photometric Me/ lica Content-Spectrophotometric Meth. Using T/ rage of Test Samples (1970)

minium (Alumin um) Content-8 Hydroxyquinoline ! and Conditions: Section 2-Test Conditi/ Use of (1962) (1962) (Dilter Rec. for Temperature Control Devices for Quartz Rec. for Plastics-Determination of Soluble Matter of Std. Spec. for Photographic Grade Sodium Thiosulphate, Rec. for Determination of eta, and Para Cresol for Industrial Use-Determination of ination of the Danger Classification by Flashpoint (Closed paints and Varnishes-Determination of Flashpoint (Closed and Varnishes: Determination of Flow Time by Use of a Flow Rec. for Modified Erichsen r (1968) Rec. for Paints and Varnishes enquiries Tenders and / Rec. For Determination of Rate of Rec. for High Voltage Alternating Rec. for High Voltage Alternating Rec. for High Voltage Alternating Rec. for High Voltage Alternating Rec. for High Voltage Alternating or Service) (1971) Rec. for Symbols for Alternating y of Circuit Breakers in Installa/ Rec. for Short Circuit (1961)

tronic Tubes and Valves: Meth. of Measurement of Emission Rec. for High Voltage Fuses: of/

Rec. for Magnetic Cores for Application in Coincident tic Basis Metals-Measurement of Coating Thickness-Eddy Rec. for Meth. of Measurement of ron: Determination of Sulphur (Meth. After Combustion in A Rec. for Calculation of the Continuous Rec. for Rotary Wafer Switches (Low ole Mounting; Maximum 26 Positions and Diameter $60 \mathrm{~mm}$ (Low h. (1962) 1963) Rec. for Rotary Wafer Switches (Low Rec. for Rotary Wafer Switches (Low Rec. for Std. Rec. for Analogue d.c

Dielectric (1964) Rec. for Rec. for Fixed Capacitors for Direct Rec. for Alternating Rec. for Class 1.0 Alternating Rec. for Class 0.5 Alternating $s$ of Electronic Tubes and Valves: Measurement of Electrode perties of Electronic Tubes and Valves: Heater or Filament

Rec. for Polyester Film Dielectric Capacitors for Direct or Fixed Metallized Paper Dielectric Capacitors for Direct circuit Elements (I960)

Rec. Graphical Symbols: Kind of (1957)

Rec. for Std. Rated

r Electronic and Telecommunication / Rec. for Strip Wound ed Tape Variable Block Format for Positioning and Straight unched Tape Variable Block Format Positioning and Straight nched Tape Fixed Block Format for Positioning and Straight Rec. for Cocoa Beans tters with Tenon Drive: Interchangeability Dimensions with Rec. for Knife Sections of Mower Rec. for Dental Burs and s (1972) eries/ 972) Std. for Dental Burs and . for Cylindrical Std. for Slotting Rec. for Side and Face Milling Cutter Arbors-Metric Series (19/ Std. for Milling $m$ Types (1970) Rec. for Heat Treated Steels: Free on Picture Raw Stock Film (1966) ture Raw Stock Film (1966)
Crude Fatty Acids (1965)

Crude Sodium Borates for Industrial Use-Determination Crude Sodium Borates for Industrial Use-Determination Crude Sodium Borates for Ind ustrial Use: Determination Crude Vegetable Oils and Fats: Determination of Acidity Crude Vegetable Oils and Fats: Determination of Insolub Crude Vegetable Oils and Fats: Determination of Moistur Crude Vegetable Oils and Fats: Preparation of Contract

Crushing Strength (1972)

Cryolite (Natural and Artificial)-Determination of Fl

Cryolite (Natural and Artificial)-Determination of Ir Cryolite (Natural and Artificial)-Determination of SI Cryolite (Natural and Artificial)-Preparation and Sto Cryolite (Natural and Artificial): Determination of Alu Crystal Units for Oscillators: Section 1-Std. Valves Crystal Units for Oscillators: Section 3-Guide to the

Crystal Units (1968) IE the Measurement of Frequency IEC

Crystal Units (1970)

Crystalline Polypropylene by Boiling N-Heptane (1969) Crystalline (1972)

Crystallizing Point-General Meth. (1970)

Crystallizing Point (1971) Rec. for Phenol: Ortha, M ISO

Cup Meth.) (1970)

Cup Meth.) (1971)

1. for Paints and Varnishes-Determ ISO Rec. for ISO

Cup (1972)

Std for Paints ISO

Cupping Test for Steel Sheet and Strip (1960)

Std. for Paints ISO

Cupping Test (1970)

Cure of Rubber Compounds by the Shearing Disk Viscomete

Current Circuit Breakers: Design and Construction (1971

Current Circuit Breakers: General and Definitions (1971

Current Circuit Breakers: Information to Be Given with

Current Circuit Breakers: Rating (1971)

Current Circuit Breakers: Rules for the Selection of $(\mathrm{F}$

Current Circuit Breakers: Type and Routine Tests (1972)

Current Electricity Meters (1972)

Current Evaluation with Special Regard to Rated Capacit

Current from Hot Cathodes for High Vacuum Electronic Ty

Current Isolators (Disconnectors) and Earthing Switches

Current Limiting (1965)

Current Matrix Stores Having a Nominal Selection Ratio

Current Meth. (1972) Aconductive Coatings on Nonmagne ISO

Current Noise Generated in Fixed Resistors (1965) IEC

Current of Oxygen and Titration with Sodium Borate) (19 ISO

Current Rating of Cables (100\% Load Factor) (1971) IEC

Current Rating) with 2 Hole Mounting (1963) IEC

Current Rating) (1966) /Rotary Wafer Switches with $2 \mathrm{H}$ IEC

Current Rating): General Requirements and Measuring Met IEC

Current Rating): Wafer Switches with Central Mounting ( IEC

Current Ratings (1938)

Current Signals for Process Control Systems (1971)

Current Transformers (1966)

Current Using Impregnated Paper or Paper / Plastic Film

Current Watthour Meters (1960)

Current Watthour Meters (1964)

Current Watthour Meters (1968)

Current (1963)

Current (1963)

Current (1965)

Current (1965)

/Asurements of the Electrical Propertie for Measurements of the Electrical Pro

Current, Distribut

Rec. F IEC

/Perties of Electronic Tubes and Valve
/Ed

Currents (2 to 63A) of Fuse Links for Low Voltage Fuses IEC

Cut and Number of Knife Sections (1970) ISO

Cut Cores of Grain Oriented Silicon Iron Alloy, Used Fo IEC

Cut Numerically Controlled Machines (1969) / for Punch ISO

Cut Numerically Controlled Machines (1969) /Angeable P ISO

Ee. for Pu ISO

Cut Test (1969)

Cutter Arbors-Metric Series (1973)

Cutter Bars (1967)

Cutters-Fitting Dimensions (1970)

Cutters-Nominal Sizes and Designation of Working Part

Cutters and Their Arbors or Mandrels-Metric and In. S

Cutters with Plain Bore and Key Drive: Metric Series (1

Cutters with Plain Bore and Key Drive: Metric Series (1

Cutters with Plain Bore and Key Drive: Metric Series (1

Cutters with Tenon Drive: Interchangeability Dimensions Cutters (1966)

Cutting-Part 7: Wrought Quenched and Tempered Chromiu

Cutting and Perforating Dimensions for Double $-8 \mathrm{~mm}$ Moti

Rec. for Cutting and Perforating Dimensions for Double-8 $\mathrm{mm}$ Moti
Rec. for Cutting and Perforating Dimensions for $35 \mathrm{~mm}$ Motion Pic
2409

100

501

1772

455

2216

2218

660

663

662

661

2474

1693

1694

1620

1619

122-2

122-3

283

314

922

419

1392

1901

1516

2431

149

1520

667

56-3

56-1

56-6

56-2

56.5

$56-4$

387

363

151-13

129

282-1

281

2360

195

671

287

132-3

$132-5$

132-1

132-2

59

381

185

80

43

170

280

151-1

$151-2$

202

166

117-1

151-15

1168

329

1058

1057

1059

1114

2780

563

1797

2157

240

2584

2585

2587

2780

523

$683 / \mathrm{VII}$

$\begin{array}{ll}\text { ISO } & 486 \\ \text { ISO } & 491\end{array}$ 
nematography $-8 \mathrm{~mm}$ Type S Motion Picture Raw Stock Film Spec. for Welding: Repulators for Gas Cylinders Used in Rec. for 972) Std. for

c. for Machine Made Textile Floor Coverings-Sampling and Rec. for Heat Treated, Alloy, and Free Rec. for Heat Treated, Alloy, and Free Std. for Heat Treated, Alloy, and Free Rec. for Heat Treated, Alloy, and Free Rec. for Heat Treated, Alloy, and Free Rec. for Heat Treated, Alloy, and Free Rec. for Heat Treated, Alloy, and Free Rec. for Heat Treated, Alloy, and Free Rec. for Heat Treated, Alloy, and Free Rec. for Heat Treated, Alloy, and Free Rec. for Heat Treated, Alloy and Free Types (1972)

d Types (1968) Types with $1 \% \mathrm{Ch}$ manganese (1970) with $3 \%$ Chromium chromium Nickel M/ 0) pes with $1 \%$ Chromi/ ted, Alloy, and Free Cutting Steels-Part 9: Wrought Free ueous Dispersions of Polymers and Copolymers-Freeze Thaw tal Test Procedures: Test Db: Damp Heat, Cyclic $(12+12 \mathrm{Hr}$ r Basic Environmental Test Procedures: Test Db: Damp Heat, Rec. for Pneumatic Cylinders

$$
\text { Rec. for Pneumatic }
$$

-Metric Series (1971)

Rec for Hydraulic

ation of / Rec. for Numbering of Aircraft Engines, Engine Rec. for Marking of Industrial Gas

rec. for Yoke Type Valve Connections for Small Medical Gas Spec. for Welding: Regulators for Gas Rec. for Identification of the Contents of Medical Gas Rec. for Marking of Aircraft Gas procating Internal Combustion Engines-Designation of the Rec. for Rec. for olerance (1972)

learance in Unloaded Radial Groove Type Ball Bearings with 69) ic Series (1972) Rec. for Measurement of the Dimensions of $A$ 66) Rec. for Modules and Diametral Pitches of
Rec. for Basic Rack of Rec. for Preferred Modules and Diametral Pitches of acturer by the Purchaser in Order to Obtain The/ Rec. for sockets and Connecting $\mathrm{Pi}$ Rec. for Nominal Dimensions of Rec. for Paints and Varnishes-Bend Test Rec. for External Diameters of or Proportional Counter /

Rec. for Sealed Nickel Cadmium ents and Test Meth. (1971/ Dimensions (1962) millimeters) (1955)

Rec. for Sealed Nickel Cadmium Rec. for Rolling Bearings Rec. for Straight Sided Splines (For Rec. for Rec. for Rec. for

Rec. for Textile Machinery

class 5-Special/ Std. for Textile Machinery and Accessories Rec. for Rolling Bearings-Double Row Rec. for Rolling Bearings International Rec. for mes (Medium Accuracy Class) (1970/ Tansliteration of Slavic for International System for the Tran (Cs: Corresponding Rec. for Safety Features for Ground Power Units for connecting Wires Having a Rated Voltage of $20 \mathrm{kV}$ and $25 \mathrm{Kv}$ Rec. for Analogue (1968) Rec. for Requirements for 28 Vol Rec. for ocedures for Electronic Components and Equipment: Test $\mathrm{Ca}$ : ec. for Basic Environmental Test. Procedures: Guidance for r Electronic Components and Equipment: Test D: Accelerated Rec. for Basic Environmental Test Procedures: Test Db: Rec. for Paints and Varnishes-Pendulum (19) Rec. for Paints and Varnishes-Determination of the de Independent Information Transfer (1972) Std. for uipment-Interchange Circuits-Assignment of I Std. for Rec. for Basic Mode Control Procedures for id Flow by Velocity Area Meth. (1/ Rec. for Collection of llection of Reliability, Availability, and Maintainability al Requirements (1972) Std. for Rec. for Dimensions for Punched Paper Tape for Rec. for the Presentation of Reliability Std. for Data Communication.

Circuits-Assignment of / std. for Connector Pin Allocations for Use with High Speed for International Gear Notation: Symbols for Geometrical ose Metric Screw Threads: Tolerances, Principles and Basic Rec. for Writing of Calendar film (1969) 1969)

Rec. for Cinematomions of ance of Plastics to Colour (Color) Change Upon Exposure to tric Exposure-Simulation of the Spectral Distribution of
Cutting and Perforating Dimensions (1972)

Cutting and Related Processes (1972)

Cutting Knotted Netting to Shape (Tapering) (1970)

Cutting Netting to Shape-Determination of the Rate (1

Cutting Specimens for Physical Tests (1971)

Cutting Steels-Part 10: Wrought Nitriding Type (1970)

Cutting Steels-Part 1I: Wrought Case Hardening Type (

Cutting Steels-Part 12: Flame and Induction Hardening

Cutting Steels-Part 1: Quenched and Tempered Unalloye

Cutting Steels-Part 2: Wrought, Quenched and Tempered

Cutting Steels-Part 3: Wrought Quenched and Tempered

Cutting Steels-Part 5: Wrought Quenched and Tempered

Cutting Steels-Part 6: Wrought Quenched and Tempered,

Cutting Steels-Part 8: Wrought Quenched and Tempered

Cutting Steels-Part 9: Wrought Free Cutting Type (197

Std. for Ci ISO

ISO

ISO

ISO

Re ISO

ISO

ISO

ISO

ISO

ISO

ISO

ISO

ISO

ISO

ISO

Cutting Steels, Pt. 4: Wrought Quenched and Tempered Ty

Rec. for Heat Trea ISO

Cycle Stability Test (1969)

Rec, for Plastics-Aq ISO

Cycle) (1969)

Cyclic (12+12 Hr. Cycle) (1969)

Cylinder Bores and Port Sizes (1970)

Rec. for Basic Environmen IEC

Cylinders-Cylinder Bores and Port Sizes (1970)

Rec. Fo

Cylinders-Internal Diameters and Piston Rod Diameters

Cylinders and Combustion Chambers, and Direction of Rot

Cylinders for the ldentification of the Content (1965)

Cylinders Used for Anaesthetic and Resuscitation Purpos

Cylinders Used in Cutting and Related Processes (1972)

Cylinders (1957)

Cylinders (1965)

Cylinders (1972)

Cylindrical Abrasive Sleeves. Designation, Dimension, T

Cylindrical and 1/10 Conical Shaft Ends (1969)

Cylindrical Bore-Values (I961)

Cylindrical Component Having Two Axial Terminations (19

Cylindrical Cutters with Plain Bore and Key Drive: Metr

Cylindrical Gears for General and Heavy Engineering (19

Cylindrical Gears for General Engineering (1957)

Cylindrical Gears for General Engineering (1966)

Cylindrical Gears (Information to Be Given to the Manuf

Cylindrical Machined Graphite Electrodes with Threaded

(Cylindrical Mandrel) (1970)

Cylindrical Radiation Probes Containing Geiger-Muller

Cylindrical Rechargeable Single Cells: Dimensions (1972

Cylindrical Rechargeable Single Cells: General Requirem

Cylindrical Roller-Separate Thrust Collars-Boundary

Cylindrical Shafts) Nominal Dimensions in Millimetres (

Cylindrical Sliver Cans on Castors (1969)

Cylindrical Sliver Cans (1963)

Cylindrical Sliver Cans: Heights Over $1000 \mathrm{~mm}$ (1969)

Cylindrical Tubes for Draw Twisters (1970)

Cylindrical Tubes for Tape Yarns (1972)

Cylindrical Type RD with Tapered Bore 1:12-Tolerance

Cylindrical Type, Radial Internal Clearance (1969)

Cylindrical Weights from 1 Gramme (Gram) to 10 Kilogram

Cyrillic Characters (1968)

Czech Terms) (1964)

Quivalent Terms Used in the Plast

d.c. Aircraft Servicing and Engine Starting (1961)

d.c. and a Maximum Working Temperature of $105^{\circ} \mathrm{C}$ Fo

d.c. Current Signals for Process Control Systems (1971)

d.c. Flat Strip Fuses for Aircraft (1968)

d.c. Periodmeters: Characteristics and Test Meth. (1969

D-Type Fuses for Domestic and Similar General Purposes

Damp and Steady Heat (1969)

/Ic Environmental Test. P

Damp Heat Tests (1968)

(1960)

Damp Heat, Cyclic (

Damping Test (1971)

Danger Classification by Flashpoint (Closed Cup Meth.)

Data Communication-Basic Mode Control Procedures-Co

Data Communication-Data Terminal and Communication $\mathrm{E}$

Data Communication Systems (1971)

Data for Determination of Errors in Measurement of Liqu

Data from Field Performance of Electronic Items (1971)

Data Interchange on Rolled Up Punched Paper Tape-Gene

Data Interchange (1969)

Data on Electronic Components (Or Parts) (1970)

Data Terminal and Communication Equipment-Interchange

Data Terminal Equipment (1973)

Data (1968)

Data (1969)

Dates in All Numeric Form (1971)

Dates (1973)

Daylight Loading Spools for Double $8 \mathrm{~mm}$ Motion Picture

Rec $1 S O$

ISO

ISO

ISO

ISO

ISO

ISO

ISO

ISO

ISO

ISO

ISO

ISO

ISO

ISO

IEC

ISO

IEC

IEC

IEC

ISO

ISO

ISO

SO

ISO

ISO

ISO

ISO

ISO

OIML

ISO

ISO

IEC

IEC

IEC

CEE

Potion Picture Film (

/or Plastics-Determination of Resist ISO

Daylight (1972)

1700

1532

$683 / \mathrm{X}$

$683 / X I$

$683 / X I I$

$683 / 1$

$683 / \mathrm{II}$

$683 /$ III

$683 / \mathrm{V}$

$683 / \mathrm{V} 1$

$683 / \mathrm{V} 1 \mathrm{II}$

$683 / I X$

$683 / \mathrm{IV}$

$683 / \mathrm{IX}$

1147

$68-2-30$

$68-2-30$

1939

1939

091

482

448

407

2503

443

1205

2421

775

201

294

2584 
Rec. for Basic Environmental Test Procedures: Test hehaviour (Behavior) with Respect to Possible Hazards When ylenols for Industrial Use-Determination of Water by the f the International System of Units and a Selection of the Rec. for Double or Shipbuilding Details for Inland Navigation-Covers fo ment (I961) Rec. for Std. Form of (oial Meth. of Mechanical Test. to Determine the Coding for or Meth. of Test for Determining Whether an Electrode Is A Rec. for Coniferous Sawn (Sawed) Timber Rec. for Coniferous Sawn (Sawed) Timber Rec, for Coniferous Sawn (Sawed) Timber ittings for Syringes, Needles and Other Medical Equipment: e Uses (1966) Rec. for Rec. for Industrial Tractors

$197 /$ y (1959) Rec. for Dyeing and Finishing Machines: Rec. for Rec. for Weaving Looms: xtile Machinery and Accessories-Pirn and Cross Winders. machinery and Accessories-Weaving Preparatory Machines hickne/ Rec. for Metallic and Other Nonorganic Coatings 7 Bit Coded Character Set for In/ Rec for Guide for the (English, Frenc/ Rec. for Glossary of Gears-Geometrica Rec. for Glossary of Gears-Geometrical c. for Diagrams, Charts, Tables Used in Electrotechnology: Rec. for Varnish Fabrics for Electrical Purposes: aterials Based on Built Up or Treated Mica Paper-Part I vibrators Operating Over the Frequency Ran/ Rec. for Std. holders, Commutators and Slip Rings (1968) Rec. for Rec. for Architectural and Building Drawings: ork, Ramassage, Gleanings, Corkwood Refuse and Corkwaste Rec. for Rec. for Terms and Rec. for

and the $\mathrm{Co} /$

(I970)

\section{7)}

Pigments (1963)

t I (1971)

7I) Rec. for Rials and Rec, for Plastics Rec. for

d Pulleys (1969) Rec. for Terms and for International Electrotechnical Vocabulary: Fundamenta lling Bearings-Internal Clearance in Unloaded Bearings Rec. for Pirn Winders: Terminology; Basic Terms and ne Winders or Cheese Winders: Terminology; Basic Terms and Rec. for Conditioning Atmosphere: Test, Reference, and ons of Motor Vehicles and Their Trailers: Designations and Rec. for Fuse ec. for Coniferous Sawn (Sawed) Timber-Sizes-Terms and Rec. for Netting for Fishing-Basic Terms and Rec. for Glossary of Gears-Geometrica Rec. for Rolling Bearings-Tolerances for Coniferous Sawn (Sawed) Timber-Defects-Terms and ec. for Electrical Installations of Buildings: General and Rec. for Vehicle Weights-Denominations and ness by the Profile Meth.-Generai Statements-Terms and Voltage Alternating Current Circuit Breakers: General and Rec. for Binders Based on Calcium Sulphate Rec. for Commercially Dry Corkwood in Planks Rec. for Fibre (Fiber) Building Boards: Rec. for Particle Boards:

Rec. for Meth. for the Designation of Electrostatic on of Compression Set of Vulcanized Rubbers Under Constant d Rubbers: Determination of Compression Set Under Constant Rec. for Plastics-Determination of Temperature of Std. for Cork-Expanded Pure Agglomerated. on and in Electronic Applications / Rec. for Thermal Time

Tear Strength of Small Test Pieces of Vulcanized Rubbers ec. for Determination of the Kappa Number of Pulp (Deg. of for Pelletized Carbon Black for Use in the Rubber Industry Rec. for General Technical Rec. for Packaging of Winding Wires

Rec. for Low Voltage Motor Starters: Reduced, a.c.: Star Rec. for Maximum Rec. for Telemetering for Consumption and Rec. for Vehicle Weights

materials-Classification of Prepared Unshaped Materials Rec. for Classification of

facturing Processes (I972) Std. for
Db: Damp Heat, Cyclic $(12+12$ Hr. Cycle) (1969)

Dealing with Electronic Equipment and Other Employing $S$

Dean and Stark Meth (1971)

Esol, Cresylic Acid and X

Decimal Multiples and Submultiples of the SI Units (Met

Deck Flat Pallets for Through Transit of Goods (1961)

Deck Openings for $220 \mathrm{~mm}$ Pumps (1957)

Declaration of Performance of Aircraft Electrical Equip

Deep Penetration Electrodes (1968)

Deep Penetration Type (1967)

Defects-Classification (1969)

Defects-Measurement (1969)

Defects-Terms and Definitions (1969)

Definition and Dimensional Characteristics for Those Wi

Definition and Marking of Safety Film for Motion Pictur

Definition and Nominal Rating (1969)

Definition of Left and Right Sides (1969)

Definition of Right and Left Hand Single Bank Engines

Definition of Side (Left or Right) of Spinning Machiner

Definition of Side (Left or Right) (1959)

Definition of Side (Left or Right) (I960)

Definition of Side (Left or Right) (I960)

Definition of Terms Concerning the Meas

of 4 Bit Character Sets Derived from the ISO

Definitions-Alphabetical Indexes of Equivalent Term

Definitions-Worm Gear (1972)

Definitions and Classification (1971)

Definitions and General Requirements (1972)

Definitions and General Requirements (I972)

Definitions and Meth. of Measurement for Piezoelectric

Definitions and Nomenclature for Carbon Brushes, Brush

Definitions and Nomenclature (1969)

Definitions and Packaging (1970)

Definitions and Terminology of Cements (1967)

Definitions for Printed Circuits (1965)

Definitions for Switchgear and Controlgear (I968)

Rcially Dry Virgin C

Rec. for Te ISO

lor Textile ISO

ISO

ISO

ISO

ISO

Re IEC

IEC

Ulating M IEC

IEC

IEC

ISO

IEC

IEC

Definitions for the Reliability of Electronic Equipment

Definitions of Living Animals for Slaughter-Porcines

ISO

(Definitions of Optical Properties) (1971)

Definitions of Rotation and Slope for Straight Welds (

Definitions of Terms Appearing in ISO Rec. for Oils and

Definitions of Terms Used in the Benzole Industry-Par

Definitions of Terms Used in the Petroleum Industry (19

Definitions of Terms (196I)

Definitions of Terms (1969)

Definitions of Welding Processes (1968)

Definitions Relating to Drives Using V-Belts and Groove

Definitions (1956)

Definitions (I96I)

Definitions (1966)

Definitions (1966)

Definitions (1967)

Definitions (1967)

Definitions (1969)

Definitions (1969)

Definitions (1969)

Definitions (I969)

Definitions (1969)

Definitions (1969)

Definitions (1970)

Definitions (1970)

Definitions (1970)

Definitions (197I)

Definitions, Classification and Nomenclature (1971)
Definitions, Classification and Packaging (1970)

Definition; Classification (1968)

Definition: Classification (1968)

Deflecting Electrodes of Cathode Ray Tubes (1967)

Deflection at Low Temperatures (I97I)

Deflection at Normal and High Temperatures (1972)

Deflection Under Load (1958)

Deformation Under Constant Pressure (1972)

est Piece) (1968)

Delivered in Bulk or in Bins-Spec. for Maximum Fines

Delivery Requirements for Steel (1964)

Delivery Spools (1968)

Delta (1970)

Demand Indicators, Class 1.0 (1966)

Demand (1970)

Denominations and Definitions (1970)

(Dense and Insulating) (1971)

Dense Refractory Products (1969)

Dense Shaped Refractory Products-Nomenclature of Manu

ISO

ISO

150

ISO

ISO

ISO

$\mathrm{SO}$

SO

ISO

ISO

ISO

ISO

Rec ISO

ISO

ce Rough ISO Rec. for High IEC

ISO

ISO

ISO

ISO

IEC for Determinati ISO Ize ISO

IEC

ISO

150

$15 O$

ISO

IEC

IEC

IEC

IEC

ISO

Rec. for Refractory ISO

ISO

ISO

ISO

68-2-30

1898

1000

198

41

224

876

632

1029

1030

1031

594

543

1084

1036

2276

92

108

141

I42

2064

581

$1122 / \mathrm{I}$

$1122 / 2$

113-1

371 .

302

276

1046

1215

597

194

277

1840

1912

627

339

1543

I998

125

472

857

1081

$50(05)$

200 
r. for Textiles Fihres (Fihers)-Determination of l.inear Rec. for Carpety-Determination of Measured Surface Pile Rec for Determination of the ic, Exclu/ Rec, for Plastics-Meth. for Determining the for Iligher Alcohols for Industrial Use-Determinaion of ic Acid and Xylenols for Industrial Use-Determination of or Purified Glycerol for Industrial Use-Determination of Rec for Plastics-Liquid Resins-Measurement of ermination of Density of Samples in Solution-Meth. Using Rec. for

Rec. for Plastics-Determination of Apparen Rec. for Determination of the Bul Rec. for Determination of the Bulk ber/ Rec. for Textile Glass-Determination of the Linea Rec. for Meth. for the Determination of Rec. for Meth. for the Determination of specified / Rec. for Plastics $\rightarrow$ Determination of Apparen a Specifi/ Rec. for Plastics-Determination of Apparen Rec. for Surface Active Agents: Determination of Apparen Potassium Silicates for Industrial Use-Determination of Rec. for Surface Active Agents: Determination of Apparent Std. for Granulated Cork-Bulk Rec. for Relative 1968)

for Textiles: Yarn from Packages-Determination of Linea Rec. for Diffuse Transmission ec. for Textiles-Universal System for Designating Linea Rec. for Fibre (Fiber) Building Boards: Determination of Rec. for Particle Boards: Determination of

ed for the Production of Aluminum: Measurement of Untamped aluation of Sulphuric Acid Concentration by Measurement of se: Evaluation of Its Acid Concentration by Measurement of Pvc Resins: Determination of the Compacted Apparent Bulk lack for Use in the Rubber Industry: Determination of Pour or Flexible Cellular Materials-Determination of Apparent Std. for Leather: Determination of Apparen Cork-Expanded Pure Agglomerated-Determination of Bulk determination of the True and Apparent Relative Densities for the Production of Aluminum: Determination of Absolute Rec. for Alloy for Rec. for Std. for Rec. for Rec. for Rec. for Rec. for Std. for Rec. for Rec. for Rec. for Phosphorus or Determining the Mechanical Properties of the Weld Metal st for Determining the Mechanical Properties of Weld Metal Meth. of Test for Determining the Characteristics of the odes-Determination of the Efficiency, Metal Recovery and

for Steel: Determination and Verification of the Effective sure Pipes with Elastic Sealing Ring Type Joints: Minimum ssure Pipes with Elastic Sealing Ring Type Joints: Minimum 1972) Std. for Starch, Includin Std. for Symbols for Flight Dynamics-Part III
Rec. for Procedure fo
Rec. for Requirements fo
Std. for Acoustics
Rec. for Microcopies: Legibility Tests

nets (1970) Std. for Symbols for Flight Dynamics-Part III
Rec. for Procedure fo
Rec. for Requirements fo
Std. for Acoustics
Rec. for Microcopies: Legibility Tests onic Booms (1973) t Object) for Ch/ se in Photograph/ Std. for Symbols for Flight Dynamics-Part III
Rec. for Procedure for
Rec. for Requirements for
Std. for Acoustics
Rec. for Microcopies: Legibility Tests Rec. for Microcopies: Legibility Tests e/ $\quad$ for Fundamental Principles for Protection in the ec. for High Voltage Alternating Current Circuit Breakers: oards with Printed Wiring and Circ/ Rec. Guidance for th Rec. for Sound System Equipment: Mechanical Rec. for Radio Frequency Cables: Guide to the Std. for Joints in Building: Fundamental Principles for Rec. for Requirements for Describing and Rec. for Textiles-Universal System for Rec. for Indexable (Throwaway) Inserts Rec for Abrasive Belts

Rec. for Wire, Bar and Tube Drawing Dies Rec. for Copper and Its Alloys-Code of Rec. for Copper and Its Alloys-Code of

Rec. for Turning Tools with Carbide Tips: Rec. for Diagrams, Charts, Tables: Item Rec. for Plastics

rials $(1970)$ eye $(1970)$ Rec, for Shuttles-Terms and Rec, for Netting Yarns for Fighing Nets: Windows and Shutters-Part I (1970) Rec. for Symbolic athode Ray Tubes (1967) for Application of Carbides for Machining by Chip Removal Rec. for Number Rec. for Plastics:
Density-Gravimetric Meth. (1971)

Density and Measured Pile Fibre (Fiber) Volume Ratio (l

Density and Relative-Density of Essential Oils (1962)

Density and Relative-Density (Specific Gravity) of Plas

Density at $20^{\circ} \mathrm{C}(1970)$

Density at $20^{\circ} \mathrm{C}(1971)$

Density at $20^{\circ} \mathrm{C}(1972)$

/Enol: Meta Cresol, Cresyl ISO

Std. F ISO

Density by the Pyknometer Meth. (1970)

Density Hydrometer-Meth. Using Pyknometer (1970)

Density Hydrometers for General Purposes (1968)

Density of Cellular Plastic (1968)

Density of Coke in a Large Container (1969)

Density of Coke in a Small Container (1967)

Density of Its Continuous Filament and Staple Fibre (Fi

Density of Latex (1968)

Density of Liquids at $20^{\circ} \mathrm{C}(1968)$

Density of Moulding Material That Can Be Poured from a

Density of Moulding Material That Cannot Be Poured from

Density of Pastes on Filling (1969)

Density of Samples in Solution-Meth. Using Density $\mathrm{Hy}$

Density of Washing Powders Before and After Compaction

Density Test (1972)

Density $60 / 60^{\circ} \mathrm{F}$ Hydrometers for General Purposes (

Density (Mass Per Unit Length) Skein Meth. (1972)

Density (Photography) (1954)

Density (Tex System) (1969)

Density (1968)

Density (1968)

Density $(1968)$

Density (1968)

Density (1968)

Density (1969)

Density $(1970)$

Density (1971)

Density (1972)

/Et ISO

ISO

ISO

$\mathrm{SO}$

Rec. for Aluminium Oxide Primarily Us ISO I Sulphuric Acid for Industrial Use: Ev ISO /for Hydrochloric Acid for Industrial U ISO Rec. for Plastics ISO Rec. for Pelletized Carbon B ISO

Density (1972)

Std. for ISO

Density) and the Porosity of Coke (1969)

Density; Pyknometer Meth. (1968)

Rec. for ISO

Oxide Primarily Used ISO

Dental Amalgam (1970)

Dental Burs and Cutters-Fitting Dimensions (1970)

Dental Burs and Cutters-Nominal Sizes and Designation

Dental Casting Gold Alloy (1970)

Dental Inlay Casting Wax (1970)

Dental Mercury (1970)

Dental Silicate Cement (1970)

Dental Vocabulary-List I-Basic Terms (1972)

Dental Zinc Phosphate Cement (1970)

Denture Base Polymer (1970)

Deoxidized Copper-Refinery Shapes (1971)

Deposited by Electrodes $3.15 \mathrm{~mm}$ or More in Diameter (19

Deposited by Filler Rods for Gas Welding Mild Steels an

/ Filler Rods for Braze Weldin Std. for Covered Electr ISO

Deposited Metal (1968)

Deposition Coefficient (1972)

Depth of Carburized and Hardened Cases (1973)

Y vinyl Chloride (PVC)

Derivatives and by -Products: Vocabulary (1973)

Derivatives of Forces, Moments and Their Coefficients ( ISO

Describing Aircraft Noise Around an Airport (1970)

Describing and Designating Knotted Netting for Fishing

Description and Measurement of Physical Properties of S
Description and Use of the ISO Micromire (ISO Micro Tes

Description of the ISO Mire (ISO Test Object) and Its U

Design and Construction of Installations for Work on Un

Design and Construction (1971)

Design and Use of Components Intended for Mounting on B

Design Features (1971)

Design of Detailed Spec. (1970)

Design (1972)

Designating Knotted Netting for Fishing Nets (1970)

Designating Linear Density (Tex System) (1969)

Designation-Code of Symbolization (1971)

Designation-Dimensions-Tolerances (1970)

Designation-Marking-Dimensions (1970)

Designation-Part II: of Tempers (1971)

Designation-Part I: of Materials (1971)

Degignation and Marking (1966)

Designation for Electrical Parts and Equipment (1971)

Designation for Polystyrene Moulding and Extrusion Mate

Designation in Relation to the Position of the Shuttle

Designation in the Tex System (1968)

Designation of Direction of Closing and Faces of Doors,

Designation of Electrostatic Deflecting Electrodes of $\mathrm{C}$

Designation of Main Groups of (1966)

Designation of Organic Refrigerants (1968)

Designation of Polyvinyl Chloride Resins (1969)

1973

1844

1903

2099

1675

649

845

1013

567

1889

758

1064

1687

697

2031

1144

819

903

911

905

1068

1306

2420

2189

1014

1559

1797

2157

1562

1561

1560

1565

1942

1566

1567

1430

615

637

688

2401

2639

2045

2048

1227

507

(530

1530
2249

689

446

1710

56-3

321

268-14

96-0

2445

1530

1144

1832

1684 .

$1190 / \mathrm{I}$

504

113-2

1622

1586 
Std. for Reriprocating Internal Combustion Engines

Sitl. for Reciprocating Internal Combustion Engines and Kelated Products (1966)

ron $(1969)$

Rec. for is and Electric Properties of Vacuum and a Subs/ Rec. for 61) Rec. for Numbering of Electrodes and lymers of Vinyl Chloride (1970) Rec. for Plastics Sid. for Dental Burs and Cutiers-Nominal Sizes and
Rcc. for Textiles Rec. for Bonded Abrasive Products General Feature Rec. for Light Metals and Their Alloys-Code of Rec. for Light Metals and Their Alloys-Tempe Std. for Polyethylene Thermoplastic Materials Std. for Textile Glass Yarns

Rec. for Cylindrical Abrasive Sleeves. Std. for Truncated Cone Abrasive Sleeves: Rec. for Paper: Untrimmed Sizes, Rec for Dimensions of Motor Vehicles and Their Trailers: rmed Stranded Steel Cables for Aircraft Control/

Rec, for Rec. for Rock Drilling-Drill Rods and erall Trimmed Sizes of Articles of Stationery That Include r Frequencies Below $3 \mathrm{MHz}$ : Guide to Drawing Information in Rec. for Fixed Resistors: Wirewound Resistors, Type 1 . transinission Lines and Their Associated Flange Connectors: Rec. for Shipbuilding Rec. for Shipbuilding Rec. for Shipbuilding Rec. for Shipbuilding Rec. for Shipbuilding Rec. for Shipbuilding Rec. for Shipbuilding Rec. for Shipbuilding Rec. for Shipbuilding Rec. for Shipbuilding Rec. for Shipbuilding Rec. for Shipbuilding Rec. for Shipbuilding Rec. for Shipbuilding Rec. for Shipbuilding Rec. for Shipbuilding Rec. for Shipbuilding Rec. for Shipbuilding

d Fixed Lights (1969) ng (1969) $\mathrm{s}$ for $220 \mathrm{~mm}$ Pumps (1957) $957)$

sport of Combustible Liquids-Nom/

ining Shackles, Kenter Type (1957)

Chains-Studless Links (1957)

ps (1969)

Rec. for Shipbuilding-Indications of

Rec. for Shipbuilding

Rec. for Shipbuilding

Rec. for Shipbuildin

ttles and Fixed Lights by the Punct

Rec. for the Use of Longitudinal Parity $t$

tric/ Rec. for International Electrotechnical Vocabulary: ngs-Qualitative Meth. (1961) Rec. for Plastics

Rec. for Test Procedures for Semiconducto

cedures for Amplifiers and Preamplifiers for Semiconductor

Spec. for Radio Interference Measuring Apparatus Having er-Muller or Proportional Counter Tubes or Scintillation rect Two Phase Titration/ Std for Surface Active Agents: Rec. for Higher Alcohols for Industrial Use f Carburized and Hardened Cases (1973) Std. for Steel: Chemical Analysis of Copper and Its Alloys-Electrolytic

Chemical Analysis of Copper and Its Alloys-Electrolytic lyser (1972) Std. for Liquid Chlorine for Industrial Use horium and Silver-l Std. for Magnesium and Its Alloys inium Oxide Primarily Used for the Production of Aluminum: Std. for Leather:

lulose Acetate (1970)

Rec. for Benzene Extract from Brown Coals and Lignites
Rec. for Pulps: Rec. for Spices and Condiments: Rec. for Tea Rec for Essential Oils Rec. for Nitric Acid for Industrial Use Rec. for Oleaginous Seeds: Std. for Potassium Sulphate for Industrial Use: Rec. for Higher Alcohols for Industrial Use Rec. for Crude Vegetable Oils and Fats: Sid. for Formald ehyde Solutions for Ind ustrial Use: Rec. for Meth. of Chemical Analysis of Manganese Ores d Plates in Shear (Quadruple-Shear Test) (1971)

Rec. for Rec. for Spices and Condiments: Rec. for Pulps: Rec. for Pulps: Rec. for

Rec for Tea hatic Polyhydric Alcohols-Glycerine for Industrial Use Rec. for Urea for Industrial Use Rec. for Sodium Hydroxide for Industrial Use:

Rec for Potassium Hydroxide for Industrial Use: chemical Analysis of Magnesium and Its Alloys: Gravimetric
Designation of the Cylinders (1972)

Designation of the Direction of Rotation (1972)

Designation of the Direction of Twist in Textile Yarns

Designation of the Microstructure of Graphite in Cast I

Designation of the Quantities Characterizing the Magnet

Designation of Units in Electronic Tubes and Valves (19

Designation of Unplasticized Compounds of Homo and Copo

Designation of Working Parts (1972)

Designation of Yarns (1969)

Designation Ranges of Dimensions-Profiles (1966)

Designation (1971)

Designation (1971)

Designation (1972)

Designation (1972)

Designation, Dimension, Tolerance (1972)

Designation, Dimensions, Tolerances (1972)

Designation, Tolerances (1966)

Designations and Definitions (1967)

Designations, Diameters and Breaking Strengths of Prefo

Detachable Bits for Percussive Drilling (1970)

Detachablc Sheets (1967)

Detail Spec. (1970)

Detail Spec. (1971)

Detail Spec. (1972) for General P

Details-Anchor Chains, Stud Links (Common, Enlarged,

Details-Dimensions and Sectional Properties of Alumin

Details-Galvanized Steel Wire Ropes (1963)

Details-Marking of Hatchway Beams (1960)

Details-Marking of Rolled, Drawn and Extruded Product

Details-Marking of Wooden Hatchway Covers (1960)

Details-Multipurpose Chocks of Cast Steel (1971)

Details-Ships Scuttles (1971)

Details-Tests on Galvanized Steel Wire for Ropes (196

Details-Toughened Glasses for Ships Sidc Scuttles an

Details-Wrought Aluminum Alloys for Use in Shipbuildi

Details for Inland Navigation-Covers for Deck Opening

Details for Inland Navigation-Mushroom Ventilators (1

Details for Inland Navigation-Pipe Lines for the Tran

Details for Inland Navigation Sprocket Wheels (1956)

Details for Sea Navigation-Anchor Chains-Lugless Jo

Details for Sea Navigation and Inland Navigation Anchor

Details on the General Arrangement Plans of Ships (1971

Details: Mating Dimensions for Pipeline Flanges for Shi

Details: Ship Screw Propellers, Manufacturing Tolerance

Details: Test. of Toughened Glasses for Ships Side Scu

Detect Errors in Information Messages (1969)

Detection and Measurement of Ionizing Radiation by Elec

Detection of Free Ammonia in Phenol Formaldehyde Mouldi

Detectors for Ionizing Radiation (1970)

Detectors for Ionizing Radiation (1970)

Detectors Other Than Quasi Peak (1967)

IEC

Detectors (1967)

Detergents-Determination of Anionic. Active

Determinaion of Density at $20^{\circ} \mathrm{C}(1970)$

Determination and Verification of the Effective Depth $O$

Determination in Unalloyed Type Containing Not Less Tha

Determination in Wrought and Cast-Copper Alloys (1971)

Determination of

Determination of - Containing Zirconium, Rare Earths, T

Determination of Absolute Density; Pyknometer Meth. (19

Determination of Absorption of Water (1972)

Determination of Acetic Acid Yield of Unplasticized Cel

Determination of Acetone Soluble Material (Resinous Sub

Determination of Acid Insoluble Ash (1968)

Determination of Acid Insoluble Ash (1969)

Determination of Acid Insoluble Ash (1970)

Determination of Acid Value (1971)

Determination of Acidity-Volumetric Meth. (1971)

Determination of Acidity of Oils (1968)

Determination of Acidity to Methyl Orange (1973)

Determination of Acidity to Phenolphthalein (1970)

Determination of Acidity (1968)

Determination of Acidity (1972)

Determination of Active Oxygen-Conventionally Express

Determination of Adhesion of Vulcanized Rubbers to Rigi

Determination of Alcohol Soluble Extract (1969)

Determination of Alkali Resistance (1968)

Determination of Alkali Solubility (1968)

Determination of Alkalinity of Latex (1966)

Determination of Alkalinity of Water Soluble Ash (1970)

Determination of Alkalinity or Acidity-Volumetric Met

Determination of Alkalinity Volumetric Meth. (1970)

Determination of Alkalinity, Volumetric Meth. (1969)

Determination of Alkalinity: Volumetric Meth. (1969)

Determination of Aluminium in Magnesium Alloys (Aluminu

IEC
ISO

ISO

ISO

IEC

ISC

ISO

ISO

ISO

ISO

ISO

ISO

ISO

ISO

ISO

ISO

ISO

IEC

IEC

ISO

ISO

ISO

ISO

ISO

ISO

ISO

ISO

ISO

ISO

ISO

ISO

ISO

ISO

ISO

ISO

ISO

ISO

IEC

ISO

CISPR

ISO

ISO

ISO

$\mathrm{SO}$

ISO

ISO

ISO

ISO

ISO

ISO

ISO

ISO

ISO

ISO

ISO

ISO

ISO

ISO

ISO

ISO
ISO

ISO

ISO

ISO

ISO

ISO

ISO

ISO

ISO

ISO

1205 1204 945 206 135 1163 2157 1139 525
2092 2107

1872

2078

2421
2422

479

612

564.

1718
618

130-0

115-3-1

339-2

347

170

1175

346

151

154

152

1685
1751 
ravimetrir and Volumetric Meth. (197)

for lron Ores:

xycquinoline /

kec for Meth of Kec. for Chemical A nalysis of Zine Alloys-Volumetric e 'Titration/ Std. for Surface Active Agents: Detergents 1968)

Rec. for Plastics that Can Be Poured from a Specified / that Cannot Be Poured from a Specifi/ Rec. for Plastics Rec. for Plastics (1969)

fore and After Compactio/

Rec. for Surface Active Agents: Rec, for Flexible Cellular Materials Sid. for Leather Rec. for

Rec. for Meth. of Chemical Analysis of Manganese Ores Rec for Urea for Industrial Use Std. for Glycerols for Industrial Use Rec. for Plastics-Pve Resins Rec. for bber lndustry (1969) Rec. for 68) Rec. for Oilseed Residues: 71) (1968) Rec. for Fruit and Vegetable Products Rec. for Rec. for Plastics Rec. for Higher Alcohols for Industrial Use Rec. for Meat and lts Products: Rec. for Pulps

Rec. for Paper and Board

Std for Cereals, Pulses and Derived Products: Std. for Formaldehyde Solutions for Industrial Use: Rec. for Meth. of Chemical Analysis of Manganese Ores: Rec. for Fibre (Fiber) Building Boards Rec. for Plastics Rec. for of Sin/ Std. for Textiles: Yarn from Packages-Meth. for eak of Fabrics Coated with Rubber or Plastics (/ Rec. for ry (11) Ch/ Rec. for Higher Alcohols for Industrial Use Rec, for Meth, for the Std. for Cork-Expanded Pure Agglomerated Rec. for Plastics

Rec. for Chemical Analysis of Zinc: Polarographic

(1963) Rec for

complexomet/ Std. for Sodium Chloride for lndustrial Use num Oxide Primarily Used for the Production of Aluminium nium Oxide Primarily Used for the Production of Aluminum -

Rec. for Pulps:

i) Rec. for Meth. of Chemical Analysis of Manganese Ores:

69)

69)

Rec. for Potassium Hydroxide for Industrial Use:

Rec. for Sodium Hydroxide for lndustrial Use: Rec. for Building Materials y the High Temperature Combustion Meth. (1967) Rec. for y the Liebig Meth. (1967)

Rec. for

Rec. for Vulcanized Rubber

c. for Sodium and Potassium Silicates for lndustrial Use ric Meth. (1969)

Rec. for

Rec. for Meth. of Chemical Analysis of Manganese Ores oxylamine Meth. (1972)

hloride Po/

ties (1972)
contact with Che/

id. for Essential Oils

Rec. for Higher Alcohols for Industrial Use id. for Ropes

(1973)

(1972) Sid. for Sodium Hydrogen Carbonate for Industria/

(1973)

Std. for Sodium Chlorate for Industrial Use:

Std. for Potassium Sulphate for Industrial Use: Rec. for Meat and Its Products

eth. $(1973 /$

eth. $(1973)$

eth $(19 /$

Std. for Sodium Carbonate for Industrial Use

Std. for Sodium Hydroxide for Industrial Use: Rec. for Potassium Hydroxide for Industrial Use: Rec. for Analysis of Soap:

Rec. for

Mixture (1967)

re Combustion Meth. (1963)

d Copolymers (1970)

ing Diphenylcarbazid/ Rec. for

Sed, for Aluminium and Its Alloys -

Rec. for Meth. O6 Chemical Analysis of Manganese Ores: Rec. for

Rec. for Meth of Chemical Analysis of Manganese Ores

Rec. for Spices and Condiments: Std. for Petroleum Products

Rec. for Meth. of Chemical Analysis of Manganese Ores: under Constant Deflection at Low Temperatures (/ Rec. for ion at Normal and High Temp/ Rec. for Vulcanized Rubbers: 67)

meth. (1/ um Tetr/ um Tetra/ Std. for Flexible Cellular Materials
Rec. for Plastics Std. for Petroleum Products: Lubricating Grease Std. for Potassium Sulphate for Industrial Use Rec. for Potassium Chloride for Industrial Use Std. for Potassium Sulphate for Industrial Use:
Determination of Aluminium (Aluminum) Content-Oxine $G$ Determination of Aluminium (Aluminum) Content-8 Hydro Determination of Aluminium (Aluminum) Oxide (1963) Determination of Aluminium (Aluminum) (1970)

Determination of Anionic-Active Matter (Direct Two Phas Determination of Apparent Density of Cellular Plastic ( Determination of Apparent Density of Moulding Material Determination of Apparent Density of Moulding Material Determination of Apparent Density of Pastes on Filling Determination of Apparent Density of Washing Powders Be Determination of A pparent Density (1971)

Determination of Apparent Density (1972)

Determination of Arsenic in Coal and Coke (1967)

Determination of Arsenic (1963)

Determination of Ash-Gravimetric Meth. (1970)

Determination of Ash-Gravimetric Meth. (1972)

Determination of Ash and Sulphated-Ash (1970)

Determination of Ash Content of Carbon Black for the Ru

Determination of Ash in Raw Natural Rubber (1962)

Determination of Ash lnsoluble in Hydrochloric Acid (19

Determination of Ash Insoluble in Hydrochloric Acid (19

Determination of Ash of Solid Mineral Fuels (1970)

Determination of Ash of Unplasticized Cellulose Acetate

Determination of Ash (Gravimetric Meth.) (1970)

Determination of Ash (1969)

Determination of Ash (1970)

Determination of Ash (1971)

Determination of Ash (1972)

Determination of Ash (1972)

Determination of Barium Oxide Content (1966)

Determination of Bending Strength (1972)

Determination of Boiling Water Absorption (1959)

Determination of Boric Acid in Latex (1970)

Determination of Breaking Load and Elongation of Same Determination of Breaking Strength and Elongation at $\mathrm{Br}$

Determination of Bromine lndex in the Presence of Mercu Determination of Bromine Index (1968)

Determination of Bulk Density (1972)

Determination of Bulk Factor of Moulding Materials (196

Determination of Cadmium in Zinc (1969)

Determination of Caking Power of Coal by the Roga Meth.

Determination of Calcium and Magnesium Contents-EDTA Determination of Calcium Content-Atomic Absorption Me Determination of Calcium Content-Spectrophotometric M Determination of Calcium Content (1968)

Determination of Calcium Oxide Content and Magnesium $\mathrm{Ox}$ Determination of Calcium: EDTA Complexometric Meth. (19 Determination of Calcium; EDTA Complexometric Meth. (19 Determination of Calorific Potential (1971)

Determination of Carbon and Hydrogen in Coal and Coke B Determination of Carbon and Hydrogen in Coal and Coke B Determination of Carbon Black-Pyrolytic Meth. (1971)

Determination of Carbon Dioxide Content Expressed as So

Determination of Carbon Dioxide in Coal by the Gravimet

Determination of Carbon Dioxide (1963)

Determination of Carbonyl Compounds Content-Free $\mathrm{Hydr}$

Determination of Carbonyl Compounds (Hydroxylammonium C

Determination of Certain Physical and Mechanical Proper

Determination of Change of Mechanical Properties After

Determination of Chloride Content-Mercurimetric Meth.

Determination of Chloride Content-Mercurimetric Meth.

Determination of Chloride Content-Mercurimetric Meth.

Determination of Chloride Content (1970)

Determination of Chloride Content: Volhard Volumetric M

Determination of Chloride Content: Volhard Volumetric $M$

Determination of Chloride Content: Volhard Volumetric

Determination of Chlorides (1965)

Determination of Chlorine in Coal and Coke Using Eschka

Determination of Chlorine in Coal by the High Temperatu

Determination of Chlorine in Vinyl Chloride Polymers an

Determination of Chromium-Spectrophotometric Meth. Us

Determination of Chromium Content (1967)

Determination of Cineole Content (1970)

Determination of Coagulum Content of Latex (1968)

Determination of Cobalt (1963)

Determination of Cold Water Soluble Extract (1969)

Determination of Colour (Color) (1972)

Determination of Combined Water Content (1966)

Determination of Compression Set of Vulcanized Rubbers

Determination of Compression Set Under Constant Deflect

Determination of Compression (1972)

Determination of Compressive Properties of Plastics (19

Determination of Cone Penetration (1972)

Determination of Content-Barium Sulphate Gravimetric

Determination of Content-Gravimetric Meth. as Potassi

Determination of Content-Gravimetric Meth. as Potassi 
umetric/

Rec. for Potassium Chloride for Industrial Use Sid. for Potassium Sulphate for Industrial Use: Rec. for Chemical Analysis of Zine Alloys chemical Analysis of Aluminum and Its Alloys: Electrolytic

d and Unvulcanized) (1970) Rec. for chemical Analysis of Magnesium and Its Alloys: Photometric alysis of Aluminium (Aluminum) and Its Alloys: Photometric

Rec. for Meth. of Chemical Analysis of Manganese Ores Rec. for Pulps:

Rec. for Chemical Analysis of Zinc: Spectrophotometric Rec. for Raw-Rubber and Rubber Latex. Std. for Ebonite: Std. for Ebonite: Rec. for

970)

Phenol: Ortha, Meta, and Para Cresol for Industrial Use ta Cresol, Cresylic Acid and Xylenols for Industrial Use Std. for Purified Glycerol for Industrial Use . Rec. for Meth. for the Rec. for Meth. for the

c. for Sodium and Potassium Silicates for Industrial Use Rec. for Fibre (Fiber) Building Boards: Rec. for Particle Boards: Rec. for Oilseed Residues: bjected to Laundering Near the Boiling Point (1/ Rec. for e Conditi/ Std. for Machine Made Textile Floor Coverings: Rec. for Particle Boards: Std. for Fibre (Fiber) Building Boards: Rec. for Raw Natural Rubber

Rec. for Cresylic Acid and Xylenols for Industrial Use Rec. for Higher Alcohols for Industrial Use Std. for Petroleum Products-Lubricating Grease . c. for Sodium and Potassium Silicates for Industrial Use

973)

th.) (1970) Std. for Gluce Rec. for Pulps: Rec. for Glucose Syrup. Std. for Natural Rubber Latex .

lastics-Aqueous Dispersions of Polymers and Copolymers d. for Sodium and Potassium Silicates for Industrial Use y Velocity Area Meth. (1/ Rec. for Collection of Data for or Essential Oils-Estimation of Free Alcohols Content by of Essential Oils (1968) zed Cellulose Acetate (1971)

Rec. for Plastics Rec. for Spices and Condiments: Rec. for Milk

Rec. for Cheese and Its Processed Products Rec. for Dried Milk Rec. for Evaporated Milk and Sweetened Condensed Milk Std. for Whey Cheese: Std. for Cream: Rec. for f Individual Fibres (Fibers) (1962) meametric Meth. (1972) Std. for Pelletized Carbon Black for Use in the Rubber Industry ics in the Form of Film (1970) 1970)

Rec for (1972) Rec. for Plastics Rec. for Paints and Varnishes Std. for Plastics

Std for Paints and Varnishes: std. for Aluminium (Aluminum) Fluoride for Industrial Use: inter Meth./ hanol (1968)

Rec for Analysis of Soaps: Std for Formaldehyde Solutions for Industrial Use: Rec. for Std. for Ammonium Nitrate for Industrial Use: e Acetate (1969) Rec. for Plastics: Phenol-Formaldehyde Mouldings (19) Rec. for Plastics Std. for Analysis of Soap: mouldings (1959) Std. for Meat and Its Products. Rec. for Plastics Rec. for Rec. for ric Bomb Meth. and Calcul/ Rec. for the Analysis of Soaps: urimetric M/ Std. for Sodium Chloride for Industrial Use: Hardness (85 to 100 IRHD) (1971) hardness (10 to 35 IRHD) (1971)

Rec. for Rec. for Rec. for Rec. for Synthetic Rubber Latices

ples (1973) Id for Iron Ores: Rec. for Meth. of Chemical Analysis of Manganese Ores Rec. for Plastics-Pvc Resins -
Rec. for Oleaginous Seeds:

Extinguishing Thermosetting of (196/ Rec. for Plastics. ans of a Durometer (Shore Hardness) ।

Rec, for Plastics Rec. for Crude Vegetable Oils and Fats:
Determination of Content-Sodium Tatraphenylborate Vol Determination of Content-Sodium Tetraphenylborate Vol Determination of Copper by Electrolysis (1971)

Determination of Copper in Aluminium Alloy (Content Gre Determination of Copper in Compounded Rubber (Vulcanize Determination of Copper (Oxalyldihydrazide Meth. Applic Determination of Copper (Oxalyldihydrazide Meth. Applic Determination of Copper (1963)

Determination of Copper (1968)

Determination of Copper (1969)

Determination of Copper (1971)

Determination of Cross Breaking Strength (1972)

Determination of Crushing Strength (1972)

Determination of Crystallizing Point-General Meth. (1 Determination of Crystallizing Point (1971)

Determination of Density at $20^{\circ} \mathrm{C}(1971)$

Determination of Density at $20^{\circ} \mathrm{C}(1972)$

Determination of Density of Latex (1968)

Determination of Density of Liquids at $20^{\circ} \mathrm{C}$ (1968)

Determination of Density of Samples in Solution-Meth.

Determination of Density (1968)

Determination of Density (1968)

Determination of Diethyl Ether Extract (1968)

Determination of Dimensional Change in Woven Fabrics Su

Determination of Dimensional Changes in Varying Moistur

Determination of Dimensions of Test Pieces (1968)

Determination of Dimensions of Test Pieces (1972)

Determination of Dirt (1971)

Determination of Distillation Range (1971)

Determination of Distillation Yield (1970)

Determination of Dropping Point (1972)

Determination of Dry Matter-Gravimetric Meth. (1970)

Determination of Dry Matter-Refractive Index Meth. (1

Determination of Dry Matter Content (1967)

Determination of Dry Matter of Moisture (Vacuum Oven Me Determination of Dry Rubber Content (1972)

Determination of Dry Solids Content at $105^{\circ} \mathrm{C}(1970$

Determination of Dynamic Viscosity (1972)

Determination of Errors in Measurement of Liquid Flow B

Determination of Ester Value After Acetylation (1971)

Determination of Ester Value and Calculation of Content

Determination of Ethyl Ether Soluble Matter in Plastici

Determination of Extraneous Matter (1969)

Determination of Fat Content (Reference Meth.) (1970)

Determination of Fat Content (Reference Meth.) (1970)

Determination of Fat Content (Reference Meth.) (1970)

Determination of Fat Content (Reference Meth.) (1970)

Determination of Fat Content (Reference Meth.) (1972)

Determination of Fat Content (Reference Meth.) (1972)

Determination of Fibre Length by Measuring the Length $\mathrm{O}$

Determination of Fineness of Flax Fibres (Fibers)-Per

Determination of Fineness of Grind (1971)

Determination of Fines Content (1970)

Determination of Flammability and Burning Rate of Plast

Determination of Flammability Of: in the Form of Bars (

Determination of Flashpoint (Closed Cup Meth.) (1971)

Determination of Flexural Properties of Rigid Plastics

Determination of Flow Time by Use of a Flow Cup (1972)

Determination of Fluorine Content-Modified Willard-

Determination of Fluorine Content: Modified Willard-W

Determination of Foreign Matter of Low Solubility in Et

Determination of Formaldehyde Content (1972)

Determination of Forms of Sulphur in Coal (1960)

Determination of Free Acidity-Volumetric Meth. (1972)

Determination of Free Acidity of Unplasticized Cellulos

Determination of Free Ammonia and Ammonium Compounds in

Determination of Free Caustic Alkali (1973)

Determination of Free Fat Content (1973)

Determination of Free Phenols in Phenol-Formaldehyde

Determination of Freezing Point of Essential Oils (1969

Determination of Fusibility of Fuel Ash (1967)

Determination of Glycerol (1969)

Determination of Gross Calorific Value by the Calorime

Determination of Halogens, Expressed as Chlorine-Merc

Determination of Hardness of Vulcanized Rubbers of High

Determination of Hardness of Vulcanized Rubbers of Low

Determination of Hardness of Vulcanized Rubbers (1968)

Determination of High Speed Mechanical Stability (1971)

Determination of Hygroscopic Moisture in Analytical Sam

Determination of Hygroscopic Moisture (1963)

Determination of Impurities and Foreign Matter (1970)

Determination of Impurities (1968)

Determination of Incandescence Resistance of Rigid Self

Determination of Indentation Hardness of Plastics by Me

Determination of Insoluble Impurities (1968)

Determination of Insoluble Impurities (1969)
ISO

ISO

ISO

ISO

ISO

ISO

ISO

ISO

ISO

ISO

ISO

ISO

Rec. for ISO

Enol: Me ISO

ISO

ISO

ISO

ISO

ISO

ISO

ISO

ISO

ISO

ISO

ISO

ISO

ISO

ISO

ISO

ISO

ISO

St ISO

ISO

ISO

ISO

ISO

ISO

ISO

ISO

ISO

ISO

ISO

ISO

ISO

ISO

Rec. Fo ISO

ISO

ISO

ISO

ISO

ISO

ISO

ISO

ISO

ISO

ISO

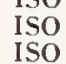

ISO

ISO

ISO

ISO

ISO

ISO

ISO

ISO

ISO

ISO

ISO

ISO

ISO

ISO

ISO

ISO

ISO

ISO

ISO

ISO

2052 
te $(1970)$

- Photometric Meth.

Rec. for Plastics Std. for Magnesium and Its Alloys tometric Me/ std. for Aluminium (Alıminum) Fluoride for Industrial Use: pectrophot/ Rec. for Phosphoric Acid for lndustrial Use olyphosphate and Sodium Pyrophosphate for Industrial Use otometric Meth. (1970/ omet/ Std. for Formaldehyde Solutions for Industrial Use Rec. for Urea for Industrial Use otometric Meth/ otometric Meth/ ctrophoto/ photome/ tometric M/ Rec. for Formic Acid for Industrial Use Rec. for Nitric Acid for lndustrial Use Rec. for Hydrochloric Acid for Industrial Use:

Rec. for Potassium Hydroxide for Industrial Use: Rec. for Sodium Carbonate for Industrial Use: Rec. for Sulphuric Acid and Oleum for Industrial Use: cal Analysis of Copper and Its Alloys-Spectrophotometric chemical Analysis of Magnesium and Its Alloys: Photometric chemical Analysis of Aluminium and Its Alloys: Photometric Rec. for Chemical Analysis of Zinc: Photometric Rec. for Pulps:

emical Analysis of Zinc and Its Alloys: Spectrophotometric ilms for Still Photography-Sensi/ Std. for Photography
Std. for Boric Acid for Industrial Use Std. for Boric Oxide for Industrial Use Std. for Natural Rubbcr Latex

Std. for Chemical Analysis of Zinc Alloys: Polarographic Rec. for Chemical Analysis of Zinc: Polarographic

Rec. for Meth. of Chemical Analysis of Manganese Ores Rec. for Chemical Analysis of Zinc: Polarographic before and After Heating (1970)

71)

skein Meth. (197) Rec. for Plastics
Rec. for Textiles Fibres (Fibers) Std. for Textiles: Yarn from Packages Std. for Rigid Cellular Plastics at $250 \mathrm{De} /$ Rec. for Sodium Carbonate for Industrial Use aluminium Oxide Primarily Used for Production of Aluminum: nium (Aluminum) Oxide Primarily Used for the Production of e Rubber Industry (1969) Rec. for opho/ Std. for Surface Active Agents: Analysis of Soaps olyphosphate and Sodium Pyrophosphate for Industrial Use c. for Mechanical Test. of Steel at Elevated Temperatures: is of Aluminium (Aluminum) and Its Alloys-Complexometric and $O x i d e$, and Disodium Tetraborates for Industrial Use

Rec. for 1967)

al Analysis of Aluminium and Aluminum Alloys: Photometric Rec. for Chemical Analysis of Steels
Rec. for Pulps

Rec. for Raw-Rubber and Rubber Latex

chemical Analysis of Magnesium and Its Alloys: Photometric chemical A nalysis of Magnesium and Its Alloys: Photometric Shorn Away from the Backing (1971) Rec. for Carpets (1971)

Rec. for Machine Made Textile Floor Coverings Rec. for Sodium Carbonate for Industrial Use: (1968) nd Preparat/ Sid. for Sodium Chloride for Industrial Use:
Rec. for Sodium Tripolyphosphate for Industrial Use lution of Products Not Easily Soluble in Boiling Water and Std. for Sodium Chlorate for Industrial Use: ured Pile Fibre (Fiber) Volume Ratio / Rec. for Carpets Std. for Natural Rubber Latex Rec for Cresylic Acid for Industrial Use 1/ Rec. for Meth. of Chemical Analysis of Manganese Ores: (1959) Std. for Formaldehyde Solutions for Industrial Use: Rec. for Plastics

s (1961) Sid. for Textiles-Cotton Fibres (Fibers) Rec. for Plastics Rec. for Fruit and Vegetable Products:
Rec. for Rec. for
Rec. for

(Bonded Quadruple Shear Test Piece) (1971) Rec. for Dextrose Rec. for Crude Vegetable Oils and Fats:
Rec. for Oleaginous Seeds: Rec. for Analysis of Soaps: Rec. for Oilseed Residues: Rec for Animal Fats: 1971) Rec. for Potassium Chloride for Industrial Use

\section{3)} .) $(1970)$ (1968) 1969) 1963) ) Rec. for Potassium Chloride for Industrial Use
Sodium Hydrogen Carbonate for Industrial Use Std. for Sodium Hydrogen Carbonate for Industrial Use -
Std. for Sodium Chlorate for Industrial Use: Rec. for Green Coffee Beans

Rec. for Cereals and Cereal Products: Rec. for Spices and Condiments: Rec. for Paper-Meth. for the Rec. for Cereals and Cereal Products: Std. for Cocoa Beans:
Determination of Insoluble Particles in Cellulose Aceta Determination of Insoluble Zirconium Alizarin Sulphonat Determination of Iron Content-1,10-Phenanthroline P Determination of Iron Content - 1,10 Phenanthroline Pho Determination of Iron Content-1,10 Phenanthroline Pho Determination of Iron Content-2, 2'-Bipyridyl S Determination of Iron Content-2, $2^{\prime}$-Bipyridyl S Determination of lron Content-2,2'-Bipyridyl $\mathrm{pH}$ Determination of Iron Content-2,2' Bipyridyl Phot Determination of Iron Content: 2, 2'-Bipyridyl pH Determination of Iron Content: 2, 2'-Bipyridyl $\mathrm{pH}$ Determination of Iron Content: 2,2'-Bipyridyl Spe Determination of Iron Content: 2,2-Bipyridyl Spectro Determination of Iron Content; 2,2'-Bipyridyl Pho Determination of lron Content; $2,2^{\prime}$-Bipyridyl Spe Determination of Iron in the Alloys (1971)

Determination of Iron (Orthophenanthroline Meth. Appli

Determination of Iron (Orthophenanthroline Meth. Applic

Determination of Iron (1968)

Determination of Iron (1968)

Determination of Iron (1969)

Determination of ISO Speed of Colour (Color) Reversal

Determination of lits Content-Volumetric Meth. (1972)

Determination of Its Content-Volumetric Meth. (1972)

Determination of $\mathrm{KOH}$ Number (1972)

Determination of Lead and Cadmium in That Containing Co

Determination of Lead and Cadmium (1968)

Determination of Lead (1963)

Determination of Lead (1968)

Determination of Light Absorption of Cellulose Acetate

Determination of Linear Density-Gravimetric Meth. (19

Determination of Linear Density (Mass Per Unit Length)

Determination of Linear Dimensions (1972)

Determination of Loss in Mass After Heating at $900^{\circ}$

Determination of Loss in Mass at $103^{\circ} \mathrm{C}(1970)$

Determination of Loss of Mass and of Nonvolatile Matter

Determination of Loss of Mass at $1000^{\circ} \mathrm{C}$ and $1200 \mathrm{D}$

Determination of Loss of Mass at $300^{\circ} \mathrm{C}$ (Convention

Determination of Loss on Heating of Carbon Black for Th

Determination of Loss on Ignition (1968)

Determination of Low Contents of Free Glycerol-Spectr

Determination of Low Contents of Volatile Acids Other T

Determination of Lower Yield and Proof Stresses (Stress

Determination of Magnesium (1973)

Determination of Manganese Content-Formaldehyde Oxim

Determination of Manganese in Compounded Rubber (1970)

Dctermination of Manganese (Contents Between 0.005 and

Determination of Manganese (Spectrophotometric Meth.) (

Determination of Manganese (1970)

Determination of Manganese (1971)

Determination of Manganese: Periodate Meth. (Content Be

Determination of Manganese: Periodate Meth. (Content Le

Determination of Mass of Pile Per Unit Area That Can Be

Determination of Mass of Total Pile Yarn Per Unit Area

Determination of Mass Per Unit Area (1970)

Determination of Matter Insoluble in Water at $50^{\circ} \mathrm{C}$

Determination of Matter Insoluble in Water or in Acid a

Determination of Matter Insoluble in Water (1968)

Determination of Matter Insoluble in Water (1972)

Determination of Matter Insoluble in Water (1973)

Determination of Measured Surface Pile Density and Meas

Determination of Mechanical Stability (1972)

Determination of Metallic Iron Content: (Photometric Me

Determination of Methanol Content (1972)

Determination of Methanol Soluble Matter in Polystyrene

Determination of Micronaire Value (1972)

Determination of Migration of Plasticizers from Plastic

Determination of Mineral Impurities (1968)

Determination of Mineral Matter in Coal (1967)

Determination of Modulus in Shear of Vulcanized Rubbers

Determination of Moisture-Vacuum Oven Meth. (1970)

Determination of Moisture and Volatile Matter (1968)

Determination of Moisture and Volatile Matter (1968)

Determination of Moisture and Volatile Matter (1968)

Determination of Moisture and Volatile Matter (1968)

Determination of Moisture and Volatile Matter (1969)

Determination of Moisture Content-Gravimetric Meth. (

Determination of Moisture Content-Gravimetric Meth.

Determination of Moisture Content-Gravimetric Meth.

Determination of Moisture Content (Basic Reference Meth

Determination of Moisture Content (Basic Reference Meth

Determination of Moisture Content (Entrainment Meth.) (

Determination of Moisture Content (Oven Drying Meth.) (

Deternination of Moisture Content (Routine Meth.) (1968

Determination of Moisture Content (Routine Meth.) (1972 
Rer. for Fihre (Fiber) Building Boards Rec. for Particle Boards:

Rec. for Pure Expanded Corkboard . Std. for Cork-Granulated

Virgin Cork, Ramassage, Gleanings, Refuse and Corkwaste Std. for Meat and Its Products 0) Rec. for Green Coffee Beans

the Direct Volumetric Meth. (1969)

I by the Direct Gravimetric Meth. (1963)

I by the Direct Volumetric Meth. (1963)

e (1968)

Rec. for

Rec. for

Rec. for

Rec. for

Rec for Starch

ra Cresol, Cresylic Acid and Xylenols for Industrial Use cal Analysis of Copper and Its Alloys-Spectrophotometric Rec. for Meth. of Chemical Analysis of Manganese Ores Rec, for Urea for Industrial Use or Agricultural Food Products-General Directions for the (1963) Rec. for Meat and Its Products: Rec. for Idahl Meth. (1963)

Rec. for Rec. for Raw Natural Rubber and Its Latex
Rec. for 1971)

Rec. for Nitric Acid for Industrial Use Rec. for Spices and Condiments:

length and Area (1971) Rec. for Carpets (1968) spectropho/ Rec. for Phosphoric Acid for Industrial Use y Precipit/

Rec for Sodium Hydroxide for Industrial Use: Rec. for Oleaginous Seeds: Rec. for Oilseed Residues: Rec. for Cresylic Acid and Xylenols for Industrial Use c/ Std. for Sulphuric Acid and Oleums for Industrial Use: etric Met/ Rec. for Hydrochloric Acid for Industrial Use: surface Active Agents: Ethylene Oxide Adducts-Iodometric Rec. for Hard Coal Rec. for

s) by the Projection Microscope (1973)

Std. for Wool Rec. for Plastics-Pvc Resins Rec. for Meth. for the

972)

(1973)

Rec, for Fruit and Vegetable Products Std. for Liquefied Phenol for Industrial Use Std. for Iron Ores: Rec. for ve Matter (Ad/ Std. for Surface Active Agents (Nonionic): tometri/ Rec. for Potassium Chloride for Industrial Use or Pelletized Carbon Black for Use in the Rubber Industry: I at Elevated Temperatures (1961) ness) (1966)

Rec for Refractory Pro Std. for Chemical Analysis of Magnesium and Its Alloys e Shearing Disk Viscometer (1968) th. (1971)

1-With Rising

Temperatu/

Rec. for Vulcanized Rubbers Rec. for

(1968) Rec. for Cresylic Acid and Xylenols for Industrial Use Phenol: Ortha Meta, and Para Cresol for Industrial Use for Liquid Halogenated Hydrocarbons for Industrial Use (1) Rec. for Sulphuric Acid and Oleum for Industrial Use: r) Change Upon Exposure to Daylight r) Change Upon Exposure to Light of / r) Change Upon Exposure to Light of Rec. for Plastics Rec. for Plastics ed Natural or Synthetic Rubber (De Mattia Type / Rec. for zed Natural or Synthetic Rubber (De Mattia Type/ Rec. for tic Conditions (1972) (1971)

or Vulcanized Rubbers

Rec. for Paints and Varnishes.

in Sheet Form (1968) Fabrics Coated with Rubber or Plastics Rec. for Pulps: Rec. for Butter

Std for Rigid Cellular Plastics c. for Sodium and Potassium Silicates for Industrial Use num Oxide Primarily Used for the Production of Aluminium th. Using $\mathrm{T} /$ Rec. for Cryolite (Natural and Artificial)

std for Aluminium (Aluminum) Fluoride for Industrial Use: d. for Sodium and Potassium Silicates for Industrial Use

recipit/ nsolubiliz nsolubi/ Rec. for Potassium Hydroxide for Industrial Use: Rec. for Sodium Hydroxide for Industrial Use: Rec. for Potassium Hydroxide for Industrial Use: Rec. for Chemical Analysis of Magnesium and Its Alloys Rec. for Meth. of Chemical Analysis of Manganese Ores alysis of Aluminium (Aluminum) and Its Alloys: Photometric alysis of Aluminium (Aluminum) and Its Alloys: Gravimetric Rec. for Natural Rubber Latices

h. (19) Rec. for Potassium Hydroxide for Industrial Use 1 Acet/ Rec. for Potassium Hydroxide for Industrial Use
Determination of Moisture Content (1968) Determination of Moisture Content (1968)

Determination of Moisture Content (1971)

Determination of Moisture Content (1972)

Determination of Moisture Content (1972)

Determination of Moisture Content (1973)

Determination of Moisture Content, (Routine Meth.) (197

Determination of Moisture in Brown Coals and Lignites B

Determination of Moisture in the Analysis Sample of Coa

Determination of Moisture in the Analysis Sample of Coa Determination of Moisture in the Analysis Sample of Cok Determination of Moisture (1970)

Determination of Neutral Oils and Pyridine Bases (1971)

Determination of Nickel (Low Contents) in Copper Alloys Determination of Nickel (1963)

Determination of Nitrogen-Volumetric Meth. (1970)

Determination of Nitrogen by the Kjeldahl Meth. (1971)

Determination of Nitrogen Content (1969)

Determination of Nitrogen in Coal by the Kjeldahl Meth.

Determination of Nitrogen in Coal by the Semi Micro Kje

Determination of Nitrogen (1971)

Determination of Nitrogen, Total Sulphur, Chlorine and

Determination of Nitrous Compounds-Volumetric Meth.

Determination of Nonvolatile Ether Extract (1969)

Determination of Number of Tufts And/or Loops Per Unit

Determination of of Calcium Content-Volumetric Meth.

Determination of of Iron Content: 2,2'-Bipyridyl

Determination of of Silica Content; Gravimetric Meth. B

Determination of Oil Content (1968)

Determination of Oil Content (1968)

Determination of Ortha Cresol Content (1971)

Determination of Oxides of Nitrogen-2,4-Xylenol Spe

Determination of Oxidizing or Reducing Substances Volum

Determination of Oxyethylene Groups (1972)

Determination of Oxygen (1971)

Determination of Paper Substance (1967)

Determination of Percentage of Medullated Fibres (Fiber

Determination of $\mathrm{pH}$ of Aqueous Extract (1970)

Determination of $\mathrm{pH}$ of Latex (1969)

Determination of $\mathrm{pH}(1970)$

Determination of Phenols Content-Bromination Meth. (1

Determination of Phosphorus Content-Volumetric Meth.

Determination of Phosphorus in Ash from Coal (1967)

Determination of Phosphorus (1963)

Determination of Polyethylene Glycols and Nonionic Acti

Determination of Potassium Content-Flame Emission Pho

Determination of Pour Density (1970)

Determination of Proof Stress and Proving Test for Stee

Determination of Pyrometric Cone Equivalent (Refractori

Determination of Rare Earths-Gravimetric Meth. (1972)

Determination of Rate of Cure of Rubber Compounds by Th

Determination of Rebound Resilience-Lupke Pendulum Me

Determination of Refractoriness Under Load (Differentia

Determination of Residue on Distillation (1971)

Determination of Residue on Evaporation on a Water Bath

Determination of Residue on Evaporation (1971)

Determination of Residue on Evaporation (1972)

Determination of Residue on Ignition; Gravimetric Meth

Determination of Resistance of Plastics to Colour (Colo

Determination of Resistance of Plastics to Colour (Colo

Determination of Resistance of Plastics to Colour (Colo

Determination of Resistance to Crack Growth of Vulcaniz

Determination of Resistance to Flex Cracking of Vulcani

Determination of Resistance to Ozone Cracking Under Sta

Determination of Resistance to Water-Immersion Meth.

Determination of Roll Characteristics (1972)

Determination of Saleable Mass, in Lots, of Pulp Baled

Determination of Salt Content (Reference Meth.) (1971)

Determination of Shear Strength (1972)

Determination of Silica Content-Gravimetric Meth. by

Determination of Silica Content-Spectrophotometric Me Determination of Silica Content-Spectrophotometric Me Determination of Silica Content-Spectrophotometric Me Determination of Silica Content-Titrimetric Meth (19

Determination of Silica Content, Gravimetric Meth. by P Determination of Silica Content: Gravimetric Meth. by I Determination of Silica Content: Gravimetric Meth. by I Determination of Silicon-Spectrophotometric Meth. Wit Determination of Silicon Dioxide (1963)

Determination of Silicon (Content Between 0.02 and $0.4 \%$ Determination of Silicon (Content Greater Than or Equal Determination of Sludge Content (1971)

Determination of Sodium-Flame Emission Photometry Met Determination of Sodium-Gravimetric Meth. Using Urany Determination of Sodium and Boric Oxide and Available $O$ Determination of Sodium and Boric Oxide Content and Los 
metri/ Sid. for Crude Sodium Borates for lndustrial Use for Hrown Coals and I.ignites-Meth. of Extraction for the Std. for Sodium IIydrogen Carbonate for Industrial Use num Oxide Primarily Used for the Production of Aluminium Std. for Sodium IIydrogen Carbonate for Industrial Use meth. (196/

itre) (1969)

ylene by Boiling $\mathrm{N}-\mathrm{Heptane}$ (1969) Rec. for Animal Fats: Rec. for Rec. for Plastics Rec. for Rubber Rec. for Surface Active Agents: Rec. for Plastics.

temperature (1965)

vimetric Mel

968)

imetric Me/

imetric/

imetric $\mathrm{Me}$

imetric $\mathrm{M} /$ hatic Polyhydric Alcohols-Glycerine for Industrial Use -
Rec. for Hydrochloric Acid for Industrial Use: etric Meth. (1/

Std. for Sodium Chloride for Industrial Use: Rec for Phosphoric Acid for Industrial Use. Rec. for Sodium Hydroxide for Industrial Use: Rec. for Potassium Hydroxide for Industrial Use: Rec. for Sodium Carbonate for Industrial Use: Rec. for Hydrochloric Acid for Industrial Use: Rec. for Nitric Acid for Industrial Use. Std. for Petroleum Products: Tetraborates and Crude Sodium Borates for Industrial Use: (e) Rec. for Sulphuric Acid and Oleum for Industrial Use; urren/ Rec. for Chemical Analysis of Steel and Cast Iron: Rec. for Meth. of Chemical Analysis of Manganese Ores n (1963) Rec. for Surface Active Agents: 0) vulcanized Rubbers (Delft Test Piece) (1968) d Synthetic Rubbers (Crescent Test Piece) (1957) 1958)

Rec. for Rec for Rec. for Paper
Rect Rec. for Plastics Rec. for Rec for Plastics. Rec. for Plastics Std. for Rigid Cellular Plastics ak (1971) Rec. for Flexible Cellular Materials Rec. for Paper and Board Std. for Ebonite: Rec. for

lcanized Rubbers (1968) ent of Material in the Unmoulded Sta/ eth.) (1971) Rec. for Butter bbers to Tor Liquid Halogenated Hydrocarbons for Industrial Use 967) Rec. for
rographic (Fibers) or Continuous Filament ted Tubular Ov/

(1961)

ntainer (1969)

ntainer (1967)

r (I965)

as Sodium /

as Pota/ plastics (Flexural Test) (1961)

c Surface Active Agents Rec. for Micrographis -Meth. of Test for the Rec. for Plastics Rec. for

Rec. for Meth for the 969) for Liquid Halog Product ( 1966)

t (Closed Cup Meth.) (19/ sential Oils (1962) osition Coefficient (1972) Is (1963)

for Industrial Use

ins at $98^{\circ} \mathrm{C}(1968)$

ins at $121^{\circ} \mathrm{C}(1968)$

Coated with Rubber or Plastics (1970)

ulating Materials (1964)

astics (Flexural Tests) (1961)

gnification) (1963)

Rec. for Meth of Test for Rec. for Plastics. Rec. for

(18-Coloured Anodisation of Aluminium and Its Alloys Std for Photography-Expendable Photoflash Lamps ilament and Staple Fibre (Fiber/ Rec. for Textile Glass Std. for Sodium Chloride for Industrial Use: by the Activated Carbon Meth. (1961/ reinforced Plastics (1970) Rec. for Plastics Rec. for Plastics Rec. for Cereals and Pulses: d Compounds of (1967) 1969) Rec. for Plastics
Rec. for Plastics Rec. for Plastics. Rec. for Plastics Rec. for
Detcrmination of Sodium and Boric Oxide Contents-Volu Determination of Sodium and Potassium Soluble in Dilute Determination of Sodium Carbonate-Titrimetric Meth. ( Determination of Sodium Content-Flame (Emission) Spec Determination of Sodium Ilydrogen Carbonate Content-T Determination of Sodium Hydrogen Carbonate; Volumetric Determination of Solidification Point of Fatty Acids (T Determination of Solubility of Essential Oils in Ethano Determination of Soluble Matter of Crystalline Polyprop Determination of Soluble Zirconium (Alizarin Sulphonate Determination of Solvent Extract (1971)

Determination of Stability in Hard Water (1969)

Determination of Stiffness in Torsion as a Function of Determination of Sulphate Content-Barium Sulphate Gra Determination of Sulphate Content-Volumetric Meth. (1 Determination of Sulphate Content: Barium Sulphate Grav Determination of Sulphate Content: Barium Sulphate Grav Determination of Sulphate Content; Barium Sulphate Grav Determination of Sulphate Content; Barium Sulphate Grav Determination of Sulphated Ash in Products Not Containi Determination of Sulphated Ash: Gravimetric Meth. (1968 Determination of Sulphated Residue on Ignition-Gravim Determination of Sulphur-Lamp Meth. (1973)

Determination of Sulphur as Sulphide (1968)

Determination of Sulphur Compounds-Volumetric Meth. ( Determination of Sulphur Dioxide Content: Barium Sulpha Determination of Sulphur (Meth. After Combustion in a C Determination of Sulphur (1963)

Determination of Surface Tension and Interfacial Tensio Determination of Surface Tension of Rubber Latices (197 Determination of Tear Strength of Small Test Pieces of Determination of Tear Strength of Vulcanized Natural an Determination of Tearing Resistance (1971)

Determination of Temperature of Deflection Under Load ( Determination of Tensile Creep of Plastics (1968)

Determination of Tensile Properties of Films (1970)

Determination of Tensile Properties (1966)

Determination of Tensile Properties (1972)

Determination of Tensile Strength and Elongation at Bre Determination of Tensile Strength (1971)

Determination of Tensile Strength (1972)

Determination of Tensile Stress Strain Properties of Vu Determination of the Acetone Soluble Matter (Resin Cont Determination of the Acid Value of the Fat (Reference M Determination of the Acidity (1970)

Determination of the Adhesion Strength of Vulcanized Ru Determination of the Austenitic Grain Size of Steels (1 Determination of the Average Diameter of Staple Fibres

Determination of the Behaviour of Plastics in a Ventila Determination of the Bleeding of Colourants (Colorants) Determination of the Bulk Density of Coke in a Large Co Determination of the Bulking Thickness and Bulk of Pape Determination of the Carbon Dioxide Content, Expressed Determination of the Carbon Dioxide Content, Expressed Determination of the Charpy Impact Resistance of Rigid Determination of the Cloud Point Temperature of Nonioni Determination of the Cloud Point (1970)

Determination of the Compacted Apparent Bulk Density (1 Determination of the Content by Volume in the Vaporized Determination of the Crucible Swelling Number of Coal ( Determination of the Danger Classification by Flashpoin Determination of the Density and Relative-Density of Es Determination of the Dilute Solution Viscosity of Polym Determination of the Efficiency, Metal Recovery and Dep Determination of the Electric Strength of Insulating Oi

Determination of the Gray King Coke Type of Coal (1966) Determination of the Hydrolytic Resistance of Glass Gra Determination of the Hydrolytic Resistance of Glass Gra Determination of the Impermeability to Water of Fabrics Determination of the Insulation Resistance of Solid Ins Determination of the Izod Impact Resistance of Rigid Pl Determination of the Kappa Number of Pulp (Deg. of Deli Determination of the Light Fastness of Colored Anodised Determination of the Light Output (1972)

Determination of the Linear Density of Its Continuous F Determination of the Loss of Mass at $110^{\circ} \mathrm{C}$ (1973) Determination of the Loss of Plasticizers from Plastics Determination of the Loss on Ignition of Textile Glass Determination of the Mass of 1000 Grains (1966) Determination of the Maximum Temperature and the Rate $O$ Determination of the Melt Flow Index of Polyethylene an Determination of the Melt Flow Rate of Thermoplastics ( Determination of the Melting Point of Polyamides (1970) Determination of the Micum Indices of Coke (1967) Determination of the Modulus of Rupture by Bending (197 
(iellulose Acetate (1967)

coals (1969)

(1967)

er in Phenolic Mouldings (1958)

f Its Products (1971)

$\mathrm{s}$ in Polyamides (1967)

erber Meth. (1966)

ne with Wijs Solution (1961)

ation Factor of Electrical Insulating/ Rec. Meth. for the ntally Folded Specimen by Mea/ (1962) ence Meth.) (1971)

astics (1966)

Boiling Aqueous Solution of Mixed Alkali (1968/
n Hydrochloric Acid at $100{ }^{\circ} \mathrm{C}(1970)$

Substances (1961) 971

Rec. for Plastics Pipes for the Transport of Fluids

els-Establishment and Operation of a Gauging Station and Low Temperature (Gehman Test) (1971) ble Gases from Plastics (1968)

oride and Related Copolymers and The/ ride and Related Copolymers and Thei/ r (1967)

es (Density) and the Porosity of Coke (1969)

nd Staple Fibre (Fiber) Yarns (/ rmoplastics (1968)

ins in Dilute Solution (1963)

n Concentrated Solution (1967)

f Coke (1967)

(Cobb Meth.) (1967)

Rec. for

Rec. for Plastics

Rec. for Plastics

Rec. for Rec. for

Rec. for Textile Glass

Rec. for Plastics

Rec. for Plastics

Rec. for Plastics. Rec. fo Rec. for

rate of Films and Thin Sheets-Dish/

lvanized Coatings on Ferrous Materials by $\mathrm{Che} \mathrm{mi}$

n Brown Coals and Lignites (1969)

Residue by Low Temperature Distillation of Bro/ 1971)

970)

$$
\text { Rec. for Textile }
$$

Rec. for Carpets

Rec. for Machine Made Textile Floor Coverings mical Analysis of Zinc and Its Alloys-Spectrophotometric Rec. for Meth. of Chemical Analysis of Manganese Ores: inium Oxide Primarily Used for the Production of Aluminum for Chemical Analysis of Aluminum and Aluminium Alloys sol Rec. for Fruit and Vegetable Products: Rec. for Sulphuric Acid and Oleum for Industrial Use: h.) (1970) Rec. for Hydrochloric Acid for Industrial Use: Rec. for Higher Alcohols for Industrial Use Rec. for Analysis of Soaps: rogen Carbonate for Industrial Use (Including Foodstuffs) c. for Sodium and Potassium Silicates for Industrial Use Rec. for Oilseed Residues: Rec. for Spices and Condiments: Rec. for Tea

combustion in a St/

$$
\text { Rec. for Chemical Analysis of Steels: }
$$
Rec, for Analysis of Soap: Std. for Meat and Meat Products Rec. for Analysis of Soaps:

Rec. for Meth. of Chemical Analysis of Manganese Ores Rec. for Meth. of Chemical Analysis of Manganese Ores

Rec. for Rec. for h./ Rec. for Sulphuric Acid and Oleum for Industrial Use: 9) Rec. for Chemical Analysis of Steel and Cast Iron Rec. for Fruit and Vegetable Products eth. (1968/ Rec. for Sodium Carbonate for Ind ustrial Use: for the Rubber Industry (1969) th. (1963) erature Combustion Meth. (I963) (1972)

Std. for Hand Knotted Carpets: Std. for Textiles:

Std. for Hand Made Carpets: (1969) Rec. for Analysis of Soaps: um) Oxide Primarily Used for the Production of Aluminium Rec. for Meth. of Chemical Analysis of Manganese Ores: acetate Copolymers (1970)

e Acetate (1970)

f Cellulose Acetate in Dilute Soluti/ ephthalate) in Dilute Solution (1970/

Resin in Solution (1961)

bers by the Shearing Disk Viscometer (1963)

Rec. for Plastics

Rec. for Plastics

Rec. for Plastics

Rec. for Plastics

Rec. for Plastics

Rec. for

of Polyethylenes and Polypropylenes /

Rec. for Plastics
Rec. for Plastics

Determination of the Moisture Content of Nonplasticized

Determination of the Moisture Ilolding Capacity of Hard

Determination of the Optical Rotation of Essential Oils

Determination of the Percentage of Acetone Soluble Matt Determination of the Percentage of Combustible Matter $O$ Determination of the Percentage of Extractable Material Determination of the Percentage of Fat in Milk by the G Determination of the Percentage of Styrene in Polystyre Determination of the Permeability of Vulcanized Rubbers Determination of the Permittivity and Dielectric Dissip Determination of the Rate (1972)

Determination of the Recovery from Creasing of a Horizo Determination of the Refractive Index of Essential Oils Determination of the Refractive Index of the Fat (Refer Determination of the Refractive Index of Transparent Pl Determination of the Resistance of Glass to Attack by a Determination of the Resistance of Glass to Attack by 6 Determination of the Resistance of Plastics to Chemical Determination of the Resistance to Internal Pressure (1 Determination of the Shatter Indices of Coke (1967)

Determination of the Stage Discharge Relation (1969) Determination of the Stiffness of Vulcanized Rubbers at Determination of the Temperature of Evolution of Flamma Determination of the Thermal Stability of Polyvinyl Chl Determination of the Thermal Stability of Polyvinylchlo Determination of the Thickness of Single Sheets of Pape Determination of the True and Apparent Relative Densiti Determination of the Twist in Its Continuous Filament a Determination of the Vicat Softening Temperature of the Determination of the Viscosity Number of Polyamides Res Determination of the Viscosity Ratio of of Polyamides I Determination of the Volatile Matter of Hard Coal and O Determination of the Water Absorption of Paper or Board Determination of the Water Content in Polyamides (1969) Determination of the Water Vapour (Vapor) Transmission Determination of the Weight Per Unit Area of Hot Dip Ga Determination of the Yield of Benzene Soluble Extract I Determination of the Yields of Tar, Water, Gas and Coke Determination of Thickness Loss Under Dynamic Loading ( Determination of Thickness of Pile Above the Backing (1 Determination of Thickness (1971)

Determination of Tin (1970)

Determination of Titanium Content (1966)

Determination of Titanium Content; Tiron Photometric Me Determination of Titanium Spectrophotometric Meth. with Determination of Titratable Acidity (1968)

Determination of Total Acidity and Calculation of Free Determination of Total Acidity: Volumetric Meth. (1968) Determination of Total Alcohols Content (Volumetric Met Determination of Total Alkali (1968) Determination of Total Alkalinity-Volumetric $\mathrm{Meth}$. (1
Determination of Total Alkalinity, Expressed in $\mathrm{Na}_{2} \mathrm{O}$ Determination of Total Ash (1968)

Determination of Total Ash (1969)

Determination of Total Ash (1970)

Determination of Total Carbon (Gravimetric Meth. After

Determination of Total Crude Fatty Acids (1965)

Determination of Total Fat Content (1973)

Determination of Total Free Alkali (1968)

Determination of Total Iron Content (1963)

Determination of Total Manganese Content (1963)

Determination of Total Moisture in Coke (1967)

Determination of Total Moisture in Hard Coal (1967)

Determination of Total Nitrogen Content: Volumetric Met

Determination of Total Silicon (Gravimetric Meth.) (196

Determination of Total Solids of Latex (1966)

Determination of Total Solids (1969)

Determination of Total Soluble Alkalinity: Volumetric M

Determination of Total Sulphur Content of Carbon Black

Determination of Total Sulphur in Coal by the Eschka Me

Determination of Total Sulphur in Coal by the High Temp

Determination of Tuft Leg Length Above the Woven Ground

Determination of Twist in Yarns-Direct Counting Meth

Determination of Types of Knots (1972)

Determination of Unsaponifiable and Unsaponified Matter

Determination of Vanadium Content-Photometric Meth. U

Determination of Vanadium Content (1966)

Determination of Vinyl Acetate in Vinyl Chloride.Vinyl

Determination of Viscosity Loss on Moulding of Cellulos

Determination of Viscosity Number and Viscosity Ratio 0

Determination of Viscosity Number of Poly (Ethylene Ter

Determination of Viscosity Number of Polyvinyl Chloride

Determination of Viscosity of Natural and Synthetic Rub

Determination of Viscosity of Rubber Latices (1970)

ISO

ISO

ISO

ISO

ISO

ISO

ISO

1SO

ISO

IEC

ISO

ISO

ISO

ISO

ISO

ISO

ISO

ISO

ISO

ISO

IsO

ISO

ISO

ISO

ISO

SO

ISO

ISO

ISO

ISO

ISO

ISO

ISO

ISO

ISO

ISO

ISO

ISO

ISO

ISO

ISO

ISO

ISO

ISO

ISO

ISO

ISO

ISO

ISO

ISO

ISO

ISO

ISO

ISO

ISO

ISO

ISO

ISO

ISO

ISO

ISO

ISO

ISO 
$(x,(3))$
$(1(x, 2)$
$(0)$
ane $(1972)$

ene (1972)

irkness After Im/

Plastics-Pve Resins

Rec. for
Resins.

Std. for Styrene Butadiene Rubber Latices. Std. for Fibre (Fiber) Building Boards:

Rec. for Plastics
ra Cresol, Cresylic Acid and Xylenols for Industrial Use

Rec. for

2)

ra Cresol, Cresylic Acid and Xylenols for Industrial Use (1970) Std. for Liquid Chlorine for Industrial Use Std. for Gylcerols for Industrial Use Rec. for Higher Alcohols for Industrial Use Rec. for Tea

Rec. for Spices and Condiments: Rec, for Sodium Hydroxide for Industrial Use: Rec. for Potassium Hydroxide for Industrial Use: Rec. for Fruit and Vegetable Products: Rec. for Tea.

of Rigid Cellular Plastics (1970)

(1969)

n Microscope Meth. (1960)

Rec. for Plastics.

Rec. for Animal Fats: Rec. for

um Oxide Primarily Used for the Production of Aluminium Rec. for Chemical Analysis of Magnesium and Its Alloys chemical Analysis of Aluminium (Aluminum) and Its Alloys num Oxide Primarily Used for the Production of Aluminium

Rec. for Meth. of Chemical Analysis of Manganese Ores:

Rec. for Meth. of Chemical Analysis of Manganese Ores chemical Analysis of Aluminium and Its Alloys: Gravimetric emical Analysis of Magnesium and Its Alloys: Polarographic lastics-Aqueous Dispersions of Polymers and Copolymers of Bale Coating on Natural Rubber Bales-Requirement and

$r$ in Acid and Preparation of Principal Solutions for Other Preparation of the Main Solution for Carrying Out Certain for ISO General Purpose Metric Screw Threads: Tolerances, system of Limits and Fits-Part I: General Tolerances and

for Clinical Thermometers (Mercury, in Glass, with Maximum cating Internal Combustion Engines-Hand Operated Control 1936) Rec. for Std. Direction of Motion of Operating Spec. Sheets for Tubes and Valves and Dimensions of Test. for Essential Ratings and Characteristics of Semiconductor for Essential Ratings and Characteristics of Semiconductor for Essential Ratings and Characteristics of Semiconductor for Essential Ratings and Characteristics of Semiconductor Rec. for Letter Symbols for Semiconductor or Units: Dimensions and General Requir/ Rec. for Locking
Rec. for Restraining ontacto/ Rec. for Control Switches (Low Voltage Switching ontacto/ Rec. for Control Switches (Low Voltage Switching al Parameters/ Rec. for Classification of Instruments and pec. (1966) $\quad$ Rec. for Sound Signalling
Rec. Temperature Control r Sizes of Photocopies (On Paper) Readable Without Optical or Electronic Tubes and Valves and Analogous Semiconductor Rec. for Calibration of Elastic Proving dization of Semiconductor Devices: Preparation of Drawings microwave Tubes and Valves: Gas Filled Microwave Switching ical Isolation (Guide for Selecting and Applying Resilient Rec. Graphical Symbols: Semiconductor

rec. for Magnetic Compasses, Binnacles and Azimuth Reading Rec. for Measurement of Photosensitive

Rec. for Mechanical Standardization of Semiconductor Rec. for Measurement of Photosensitive pectrum, Meth. Of/ Rec. for Measurement of Photosensitive
Rec. for Measurement of Photosensitive
Rec. for Meth. of Measurement of Photosensitive pectrum, Meth. Of/ Rec. for Measurement of Photosensitive
Rec. for Measurement of Photosensitive
Rec. for Meth. of Measurement of Photosensitive (1970) Rec. for Mechanical Standardization of Semiconductor Rec. for Pure Tone Audiometers for General Rec. for Measurement of the Dimensions of Focal Spots of ive Elements and Recording Directly by Means of Stylus and Rec. Graphical Symbols: Symbols for Architectural Rec. Graphical Symbols: Frequency Spectrum initions and Classification (1971)

l) (1959)

al Parts and Equipment (1971)

(1965)

Rec. for

Rec. for

Rec. for Figures for Aircraft Instrument Rec. for Aircraft Instrument

ments (1967) Rec. for External

nt/ Rec. for Textile Glass-Determination of the Average Rec. for Air Permeability Meth. for Measuring the Mean std. for Rolling Bearings: with Locating Snap Ring-Inner ds: General Plan and Selection for Screws, Bolts and Nuts Rec. for Rivet Shank Diameters -Radial-Boundary Dimensions-General Plan-Part II . g Bearings-Radial-Boundary Dimensions-General Plan bearings-Tapered Roller-Boundary Dimensions-Metric .
Determination of Volatile Fatty Acid Number of I.atex (1

Determination of Volatile Matter in Raw Natural Rubber Determination of Volatile Matter (Including Water) (197 Determination of Volatile Unsaturates and Residual Styr Determination of Water Absorption and of Swelling in Th Determination of Water Absorption (1958)

Determination of Water by the Dean and Stark Meth. (197 Determination of Water by the Karl Fischer Meth. (1968) Determination of Water by the Karl Fischer Meth. (1971) Determination of Water Content-Gravimetric Meth. (197 Determination of Water Content-Karl Fischer Meth. (19 Determination of Water Content by the Karl Fischer Meth Determination of Water Extract (1970)

Determination of Water Insoluble Ash (1969)

Determination of Water Insoluble Matter (1969)

Determination of Water Insoluble Matter (1969)

Determination of Water Insoluble Solids (1968)

Determination of Water Soluble and Insoluble Ash (1970)

Determination of Water Vapour (Vapor) Transmission Rate

Determination of Water (Entrainment Distillation Meth.

Determination of Wool Fibre (Fiber) Diameter: Projectio

Determination of Zinc-Atomic Absorption Meth. (1971)

Determination of Zinc-Volumetric Meth. (1970)

Determination of Zinc-Volumetric Meth. (1970)

Determination of Zinc Content-Pan Photometric Meth. (

Determination of Zinc Content (Polarographic Meth., for

Determination of Zinc Content (1966)

Determination of Zinc in Aluminum Alloys (Zinc Content

Determination of Zinc (Content Between 0.1 and 4\%) (196

Determination (1969)

Determination (1971)

Determinations (1972)

Determinations (1973)

Deviations for Con

Device) (1970)

Devices-Std. Direction of Motion (1972)

of Matter Insoluble in Water O ISO

/L Use: Sampling-Test Sample- ISO

Devices and for Indicating Lamps for Circt

Devices and Gauges (1969)

Treakers

Rec. for ISO ISO

International Rec. OIML /for Recipro ISO

Tube and Valve Shields: IEC

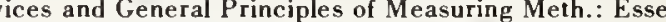

Devices and General Principles of Measuring Meth.: Gene

Devices and General Principles of Measuring Meth.: Gene

Devices and General Principles of Measuring Meth.: Refe

Devices and Integrated Microcircuits (1969)

Devices for Ball and Socket Couplings of String Insulat

Devices for Children in Motor Vehicles (1970)

Devices for Control and Auxiliary Circuits, Including $\mathrm{C}$

Devices for Control and Auxiliary Circuits, Including C

Devices for Measurement and Evaluation of the Geometric

Devices for Quartz Crystal Units (1970)

Devices on Motor Vehicles Acoustic Std. and Technical S

Devices (1960)

Devices (1961)

Devices (1964)

Devices (1969)

Devices (1972)

Devices) (1972)

Devices, Capacitors (1971)

Devices, Class B-General Requirements (1967)

Rec. for Rating Systems F IEC

Rec. for Mechanical Standar IEC

Devices: Basic Rec. (1969)

Devices: Dimensions (1970)

Devices: Photoconductive Cells for Use in the Visible S

Devices: Photomultipliers (1971)

Devices: Phototubes (1969)

Devices: Preparation of Drawings Devices (1969)

Dextrose-Determination of Moisture-Vacuum Oven Meth

Diagnostic Purposes (1965)

Diagnostic X-Ray Tubes Using a Pinhole Camera (1970)

Diagram (Working Equipment Category) (1970)

Diagrams (1967)

Diagrams (1968)

Diagrams, Charts, Tables Used in Electrotechnology: Def

Diagrams, Charts, Tables (Electrotechnology-Electrica

Diagrams, Charts, Tables: Item Designation for Electric

Dial Gauges Reading in $0.01 \mathrm{Mm}, 0.001 \mathrm{In}$. and $0.0001 \mathrm{in}$

Dials and Number Plates (1962)

Dials and Pointers (1962)

Diameter of Planchets Used in Nuclear Electronic Instru

Diameter of Staple Fibres (Fibers) or Continuous Filame

Diameter of WooI Fibres (Fibers) (1969)

Diameter of (1972)

(Diameter Range 0.06 to 6 In.) (1973)

(Diameter Range 1 to $36 \mathrm{Mm}$ ) (1969)

Diameter Series 7 (1970)

Diameter Series $8,9,0,1,2,3$ and 4, (1968)

Diameter Series 9 and 0 (1967) 
ng Brarings-Rarlial Ty pes with Shields or Seals-Outside r Switches with Central Mounting; Maximum 12 Positions and er Switches with 2 llole Mounting: Maximum 26 Positions and oles for Metric Bolts 1,6 Up to and Including $39 \mathrm{~mm}$ Thread the Weld Metal Deposited by Electrodes $3.15 \mathrm{~mm}$ or More in les for Metric Bolts, $42 \mathrm{Up}$ to and Including $150 \mathrm{~mm}$ Thread $s$ with Metric Thread, $42 \mathrm{Up}$ to and Including $100 \mathrm{~mm}$ Thread or Hydraulic Cylinders-Internal Diameters and Piston Rod steel Cables for Aircraft Control/ Rec. for Designations, of Plastics Materials for the Transport of Fluids (Outside of Plastics Materials for the Transport of Fluids (Outside 1) Rec. for Hydraulic Cylinders-Internal welding and Filler Metals for Gas Welding (1967) Rec. for Rec. for Recommended Range of Outside 1) Std. for Long Shank Machine Taps with Nominal Rec. for Basic Dimensions of Winding Wires: Rec. for Basic Dimensions of Winding Wires: Rec. for Paper: Internal Rec. for External iger-Muller or Proportional Counter / Rec. for lk and Staple Fibre (Fiber) (1959) c. for Basic Dimensions of Winding Wires: Maximum Over All for Hermetically Sealed Metal Food Containers-Internal tating Tools with Parallel Shanks (1961) Rec. for (1971)

Rec. for Preferred (1969)

for ISO Metric Series for Basic Thicknesses of Sheet and Rec. for Flyer Bobbins for Spindle Rec. for Flyer Bobbins for Spindle Rec. for Rivet Shank

e Lines for the Transport of Combustible Liquids-Nominal port of Fluids-Polyethylene (PE)-Tolerances on Outside zed Polyvinyl Chloride (PVC) Pipes-Tolerances on Outside Std. for Steel Tubes-Outside Rec. for Determination of Wool Fibre (Fiber) heavy Engineering (1966) Rec. for Modules and neering (1966)

nd Heavy Engineering (1968)

Rec. for Preferred Modules and Rec. for Modules and
0)

d. for Boric Acid and Oxide, and Disodium Tetraborates for

70) Rec. for Pulps: Extraction of Materials Sor Para Rec. for Para
Diameter Tolerances-Normal and Tolerance Class 6 (197 Diameter $40 \mathrm{~mm}$ (1966) Diameter $60 \mathrm{~mm}$ Diameter (1962)

Diameter (1967)

Diameter (1968)

Diameter (1969)

Diameters-Metric Series (1971)

Diameters and Breaking Strengths of Preformed Stranded

Diameters and Nominal Pressures) Part II: In. Series (1
Diameters and Nominal Pressures): Part I: Metric Series

Diameters and Piston Rod Diameters-Metric Series (197

Diameters and Tolerances for Electrodes for Manual Arc

Diameters for Milling Cutters (1966)

Diameters from $324 \mathrm{~mm}$ and $1 / 8$ to $1 \mathrm{In}$. (1972)

Diameters of Conductors for Round Resistance Wires (197

Diameters of Conductors for Round Winding Wires (1965)

Diameters of Cores of Reels (1966)

Diameters of Cylindrical Radiation Probes Containing Ge

Diameters of Drafting Rollers for Cotton, Wool, Spun SI

Diameters of Enamelled Round Winding Wires (1964)

Diameters of Pulleys for Flat Transmission Belts (1959)

Diameters of Round Cans (1972)

Diameters of Shanks and Sizes of Driving Squares for Ro Diameters of Wire Terminations of Capacitors and Resist

Diameters of Wire (1964)

Diameters of $25 \mathrm{~mm}$ or More and Lifts of $300 \mathrm{~mm}$ and Over

Diameters Up to $22 \mathrm{~mm}$ and Lifts Up to $300 \mathrm{~mm}$ (1963)

Diameters (Diameter Range 1 to $36 \mathrm{Mm}$ ) (1969)

Details for Inland Navigation-Pip - IS

Rec. for Plastic Pipes for the Trans

Diameters (1957)

Diameters $(1970)$

Diameters (1970)

Diameters (1971)

/the Transport of Fluids-Unplastici

Diameter: Projection Microscope Meth. (1960)

Diametral Pitches of Cylindrical Gears for General and

Diametral Pitches of Cylindrical Gears for General Engi

Diametral Pitches of Straight Bevel Gears for General a

Dibenzyldithiocarbamate Photometric Meth. (1972)

Dichlorobenzene for Industrial Use-Meth. of Test (197

Dichlorobenzene for Industrial Use-Meth. of Test (197 Dichloromethane (1967)

(Dichloromethane) for Industrial Use-Meth. of Test (19

Machine Operated Circular Screwing Dies and Hand-Operated Rec. for Fixed Metallized Paper Rec. for Polyester Film Rec. for Dimensions of Ceramic Rec. for Ceramic Rec. for Ceramic

Rec. for Polystyrene Film Rec. for Ceramic

Rec. Meth. for the Determination of the Permittivity and $r$ Types of Winding Wires: Enamelled Round Copper with Good ents for Tests and Measuring Meth. (1970)

Rec. for Air ct Current Using Impregnated Paper or Paper / Plastic Film Rec. for Wire, Bar and Tube Drawing (1966)

Std. for Hand and Machine Operated Circular Screwing Rec. for Hard Metal Wire Drawing Rec. for Oilseed Residues: Determination of ory Products-Determination of Refractoriness Under Load etween Sound Pressure Levels of Narrow Bands of Noise in A Rec. for Rec. for Audibert Arnu

injec/ Rec. for Liquid Flow Measurement in Open Channels: Std. for Rolling Bearings: Tolerances; Metric Series Chamfer r Rolling Bearings-Tapered Roller-In. Series-Chamfer ry: Complementary Dimensions and Terminations (/ Rec. for Winding Wir/ Rec. for Basic Dimensions of Winding Wires: Roller-Boundary Dimensions-Subunits-Metric Series Roller-Boundary Dimensions-Subunits-Metric Series Rec. for Cylindrical Abrasive Sleeves: Designation, gs-Needle Roller-Boundary Dimensions-Metric Series ring Near the Boiling Point (1) Rec. for Determination of for Machine Made Textile Floor Coverings: Determination of inges, Needles and Other Medical Equipment: Definition and tapes for Interchange Instrumentation Applications General Drawings-Tolerances of Form and of Position-Part III: Rec. for Engineering Drawing: Rec. for Bolts, Screws and Studs:
and 4, (I/ Rec. for Rolling Bearings-Radial-Boundary (1970) Rec. for Rolling Bearings-Radial-Boundary Std. for Prevailing Torque Type Steel Hexagon Locknuts: Rec. for Rolling Bearings-Tapered Roller-Boundary (I) Rec. for Rolling Bearings-Needle Roller-Boundary 21 Rec. for Rolling Bearing-Tapered Roller-Boundary
Dictation Equipment-Classification (1971)

Dictation Equipment: Basic Operating Requirements (1973 Std. for Hand and ISO

Diecks (1973)

Dielectric Capacitors for Direct Current (1965)

Dielectric Capacitors for Direct Current (1965)

Dielectric Capacitors of the Plate Type (1967)

Dielectric Capacitors Type 2 (1965)

Dielectric Capacitors Type 3 (1970)

Dielectric Capacitors (1968)

Dielectric Capacitors, Type 1 (1967)

Dielectric Dissipation Factor of Electrical Insulating

Dielectric Properties Under Humid Conditions (1970)

Dielectric Rotary Variable Capacitors: General Requirem

Dielectric (1964)

IEC

Dies-Designation-Marking-Dimensions (1970)

Dies and Hand.Operated Die Stocks (1973)

Dies: Interchangeability Dimensions of Pellets and Case

Diethyl Ether Extract (1968)

Differential-With Rising Temperature) (1970)

Diffuse Field and in a Frontally Incident Free Field Fo

Diffuse Transmission Density (Photography) (1954)

Dilatometer Test for Coal (1963)

Dilution Meth. for Steady Flow-Part 1: Constant Rate

Dimension Limits and Maximum Shaft and Housing Fillet

Dimension Limits (1969)

Dimension of Brushes and Holders for Electrical Machin

Dimension of Conductors of Insulated Rectangular Copper

Dimension Series 29 and 13 (1973)

Dimension Series 30,31 and 32 (1970) /Rings-Tapered ISO

Dimension-Series 48 and 49 (1971)

for Rolling Bearin ISO

Dimensional Change in Woven Fabrics Subjected to Launde ISO Dimensional Changes in Varying Moisture Conditions (197 ISO

Dimensional Characteristics for Those with a $6 \%$ and a 1 ISO

Dimensional Requirements (1971) / Unrecorded Magnetic ISO

Dimensioning and Tolerancing of Profiles (1971) /Nical ISO

Dimensioning (1961)

Dimensions-General Plan-Diameter Series 8,9,0,1,2,3

Dimensions-General Plan-Part II-Diameter Series 7

Dimensions-In. Series (1972)

Dimensions-Metric Series-Dimension.Series 48 and 49

Dimensions-Metric Series-Dimensions Series 31 and 3
Dimension. Tolerance (1972)

Dimensioning (1959)

Dimensions-Metric-Diameter Series 9 and 0 (1967)
1648

$132 \cdot 4$

132.5

$273 / 1$

615

$273 / \mathrm{II}$

$288 / \mathrm{II}$

2091

564

330

161

209]

544

523

2283

182.4

182 -

499

256

98

] 82.2

99

1361

237

301

388

$344 /$ II

$344 / \mathrm{I}$

1051

51

1164

1330

64
137

54

467

678

2215

1698

1699

624

1869

1731

1730

2568

166

202

234

187

324

275

108
250

317-6

334- 1

80

1684

2568

524

736

1893

454

349

555

582

1123

136.2

182.3

$355 / V I I$

355/VII

2421

1206

675

2551

594.

1859

1660

129
225

$15 / \mathrm{I}$

$15 / 1$

2359

$355 / I I I$

1206

$355 / V I$ 
Std. for l'revailing Torque Type Hexagon locknuts: Kec. for Bunded Abrasive Products-Grinding Whee

I Abrasive l'roducts (;eneral Features Designation Ranges of Rec. for Rolling Bearings-Tapered Roller-Boundary $\begin{array}{ll}* 1 & \text { Rec. for Rolling Bearings-Tapered Roller-Boundary } \\ \text { s } 2 / & \text { Std. for Rolling Bearings: Tapered Roller-Boundary }\end{array}$ h F/ Std. for Rolling Bearings: Tapered Roller-Boundary Rec. for Rolling Bearings-Tapered Roller-Boundary Rec. for Abrasive Belts-Designation

Rec. for Magnetic Tape Recording and Reproducing Systems electrical Cables with Copper Conductors, for A/ Rec. for $190^{\circ} \mathrm{C}$ ) Electrical Cables with Copper Condu

$260{ }^{\circ} \mathrm{C}$ ) Electrical Cables with Copper Condu

Rec. for

ealds (1967)

writing and Printing Pap/

Rec. for Heald Carrying Rods Rec. for Meth, of Expression of Rec. for $35 \mathrm{~mm}$ Filmstrips: $s$ for Ball and Sockel Couplings of String Insulator Units: Rec. for Symbols, 80 Columns Punched Paper Cards (Information Pr/ Rec. for Rec. for Lead Acid Starter Batteries:

for Pipe Threads for Parallel and Taper-Threads-General ain End Steel Tubes, Welded or Seamless-General Table of Std. for Industrial Wheels al Machines with Frame Numbers 355 to 1000 (197/ Rec. for achines .. Frame Numbers 56 to 400 and Flange N/ Rec. for Rec. for ion Welding Machines (1968)

Rec. fo

num) Alloy Sections for /

Rec. for Shipbuilding Details el Sections-Part 2: Unequal Leg Angles-Metric Series teel Sections-Part I: EquaI Leg Angles-Metric Series ed Steel Sections-Parallel Flange I Beams-In. Series Sections-Parallel Flange Column Sections-In. Series Rolled Steel Sections-Unequal Leg Angles-In. Series ot Rolled Steel Sections-Equal Leg Angles-In. Series

rushes and Holders for Electrical Machinery: Complementary

of Brushes and Holders for Electrical Machinery: Principal

ng Stock Material-Rough Tyres (Tires) for Trailer Stock:

m (1966)

rcuit Breakers for Aircraft (1966)

Breakers for Aircraft (1969)

$3 \mathrm{~mm}$ (1972) Std. for Hand and Machine Hacksaw Blades:

rels-Metric and In. Sewies/ Rec. for Interchangeability

nd Associated Parts (1971)

Rec. for Shipbuilding Details: Mating

(1969)

Rec. for

tches for Aircraft (1966)

ectives on $35 \mathrm{~mm}$ Film, 5-Perforation Format (/

Rec. for Rec. for Rec. for Clearance

Rec. for Guide for Choosing Modular

r and Spools (1971)

Rec. for

ons Along One and Two Edges (1/ Std. for Cinematography 6) Rec. for

d Vice Versa (1964)

Rec. for Cutting and Perforatin Rec. for Photography-Front Lens Barrels $U_{p}$ to $100 \mathrm{~mm}$ Rec. for Straight Sided Splines and Gauges Rec. for Tapping Screw Thread

Rec. for Slotted Pan Head Tapping Screws Rec. for Slotted Countersunk (Flat) Head Tapping Screws or Slotted Raised Countersunk (Oval) Head Tapping Screws or Straight Sided Splines (For Cylindrical Shafts) Nominal with or Without Gib Head and Their Corresponding Keyways r or Square Parallel Keys and Their Corresponding Keywayz

967) Rec. for Heald Frames: Coordinated terminations (1969)

ides (1966)

Rec. for Measurement of the Rec. for

type) (1959)

Rec for Sizes and Mounting Rec. for Lead Acid Starter Batteries:
Std. for Aircraft: Interchangeability

(1973) ery: Principal Dimensions and Tolerances (1962) e Type (1967)

Rec. for Shanks for Pneumatic Tools and Fitting Rec. for Shanks for Pneumatic Tools and Fitting Rec. for Rec. for

ms (1969)

ic Oxides and Associated Parts (1967)

with Threaded Sockets and Connecting $\mathrm{Pi}$

Rec. for Nominal

otion Picture Film (1969)

picture Film (1969)

reraft (In. Series-Class 1 Tolerar

Rec. for Cinematography Rec. for Cinematography rcraft-(In Series-Class 2 Tolerances) (197/

pe Fittings in Aircraft (In. Series-Class 1 T/

pe Fittings in Aircraft-(In. Series-Class 2)

Rec. for

Rec. for

Rec. for

Rec. for

Rec. for

ng a Pinhole Camera (1970)

Rec. for Measurement of the
Dimensions-Metric Series (1972)

Dimensions-Part 2 (1969)

imensions-P'rofiles $(1966)$

Dimensions-Subunits-In. Series (1968)

Dimensions-Subunits-Metric Series-Dimension Serie

Dimensions-Subunits-Metric Series-Dimension Serie

Dimensions-Subunits-Metric Series-Outer Rings Wit

Dimensions-Subunits-Metric Series (1969)

Dimensions-Tolerances (1970)

Dimensions and Characteristics (1968)

Dimensions and Conductor Resistance of General Purpose

Dimensions and Conductor Resistance of Heat Resisting (

Dimensions and Conductor Resistance of Heat Resisting

Dimensions and Coordination Of, and with End Loops of $H$

Dimensions and Direction of Manufacture of Unprocessed

Dimensions and Formats (1968)

Dimensions and General Requirements (1971)

Ing Device

Dimensions and Layout for Safety Signs (1967)

Dimensions and Location of Rectangular Punch

Dimensions and Marking (1972)

Dimensions and Masses Per Unit Length (1971)

Dimensions and Nominal Load Capacities (1972)

Dimensions and Output Ratings for Foot Mounted Electric

IEC

f. for Pl ISO

ISO

IEC

Dimensions and Output Ratings for Rotating Electrical

Dimensions and Pitching of Slots on Platens for Project

Dimensions and Ratings of Freight Containers (1970)

IEC

ISO

ISO

imensions and Sectional Properties of Aluminium (Alumi

Rolled Ste ISO

TT Rolled S ISO

/F Hot Roll ISO

/Lled Steel ISO

/Ons of Hot ISO

/Sions of $\mathrm{H}$ ISO

Dimensions and Sectional Properties (1969)

Dimensions and Sectional Properties (1969)

Dimensions and Sectional Properties (1969)

Dimensions and Terminations (1967)

Dimensions and Tolerances (1962)

Dimensions and Tolerances (1969)

Dimensions for Commutators and Slip Rings (197

for Dimension of B IEC

Rec. for Dimensions IEC

C. for Railway Rolli ISO

ISO

ISO

Dimensions for General Purpose Push Pull 3 Pole Circuit

Dimensions for Lengths Up to $450 \mathrm{~mm}$ and Pitches Up to 6

Dimensions for Milling Cutters and Their Arbors or Mand

Dimensions for Pipeline Flanges for Ships (1969)

Dimensions for Pot Cores Made of Ferromagnetic Oxides a

Dimensions for Punched Paper Tape for Data Interchange

Dimensions for Single Hole Mounting, Lever Operated Swi

Dimensions for Stereo Still Photography Using $35 \mathrm{~mm}$ Obj

Dimensions for Tripod Jacks for Aircraft (1970)

Daveguide Components (1971)

Dimensions for 127, 120 and 620 Roll Film, Backing Pape

Dimensions for $16 \mathrm{~mm}$ Motion Picture Film with Perforati

Dimensions for $35 \mathrm{~mm}$ Film Magazines for Still Picture U

Dimensions for 35 mm Motion Picture Raw Stock Film (196

Dimensions from In./S into Millimetres (Millimeters) an

Dimensions Important to the Connection of Auxiliaries (

Dimensions in In./S (1961)

Dimensions in Millimetres (Millimeters) and In./S (1970

Dimensions in Millimetres (Millimeters) and In./S (1970

Dimensions in Millimetres (Millimeters) and In./S (1970

Dimensions in Millimetres (Millimeters) and In./S (1970

Dimensions in Millimetres (Millimeters) (1955)

(Dimensions in Millimetres) (Millimeters) (1969)

Dimensions in Millimetres) (Millimeters) (1969)

Dimensions in Relationship with Pitch of the Harness (1

Dimensions of a Cylindrical Component Having Two Axial

Dimensions of Aerial Rods and Slabs of Ferromagnetic Ox

Dimensions of Aircraft Instrument Cases (Rear Mounting

Dimensions of Batteries (1965)

Dimensions of Battery Connectors for Automatic Coupling

Dimensions of Brushes and Holders for Electrical Machin

Dimensions of Ceramic Dielectric Capacitors of the Plat

Dimensions of Chuck Bushings-Part I (1970)

Dimensions of Chuck Bushings-Part II (1970)

Dimensions of Cores for Motion Picture and Magnetic Fi]

Dimensions of Cross Cores (X-Cores) Made of Ferromagne

Dimensions of CylindricaI Machined Graphite Electrodes

Dimensions of Daylight Loading Spools for Double $8 \mathrm{~mm} \mathrm{M}$

Dimensions of Daylight Loading Spools for $16 \mathrm{~mm}$ Motion

Dimensions of Elastomeric Toroidal Sealing Rings for Ai

Dimensions of Elastomeric Toroidal Sealing Rings for $\mathrm{Ai}$

Dimensions of Elastomeric Toroidal Sealing Rings for Pi

Dimensions of Elastomeric Toroidal Sealing Rings for Pi

Dimensions of Electronic Tubes and Valves (1966)

Dimensions of Focal Spots of Diagnostic X-Ray Tubes Usi

Dimensions of Hot Rolled Steel Bars: Flat, Metric Serie

2358

1117

$355 / \mathrm{IV}$

$355 /$ VII

$355 / V I I l$

2316

$355 / \mathrm{V}$

1929

94

469

470

539

570

217

372.1

557

1682

95-3

2284

336

2175

72

865

668

1175

$657 / \mathrm{II}$

657/I

657/VII

$657 / \mathrm{IX}$

$657 / \mathrm{IV}$

657/III

136-2

136-1

1005/II

356

486

530

1033

2336

240

964

133

1154

493

515

1464

374

732

1007

491

370

1948

232

1478

1481

1482

1483

14

774

773

569

294

223

103

95-2

1963

136-1

234

1180

1571

1039

226

239

1020

1019 
les-In. Series-Dimensions and Sectional Pro/ ge Column Sections-ln. Series-Dimensions An/ ge I Beams-In. Series-Dimensions and Sectio/ leg Angles-Metric Series-Dimensions and Se/ al l.eg Angles-Metric Series-Dimensions and / ngles-In. Series-Dimensions and Sectional P/ its for Systems with Nominal Voltages Greater T/ Rec. for Dimensions of Hot Rolled Steel Sections-Equal Leg Ang Rec. for Dimensions of Hot Rolled Steel Sections-Parallel Flan Rec. for Dimensions of Hot Rolled Steel Sections-Parallel Flan Rec. for Dimensions of Hot Rolled Steel Sections-Part I: Equal Rec. for Dimensions of Hot Rolled Steel Sections-Part 2: Unequ Rec. for Dimensions of Hot Rolled Steel Sections-Unequal Leg a Rec. for Dimensions of Indoor and Outdoor Post Insulators and Un Rec. for Dimensions of Lead Acid Motor Scooter Batteries (1965) Rec. for the Rated Impedances and ations and Definitions (1967) Rec. for instruments) (1968)

$$
\text { Rec. for Principal }
$$
Rec. for

Rec. for Hard Metal Wire Drawing Dies: Interchangeability Rec. for Weft Pirns for Box Loaders for Automatic Looms

Rec. for the es of Printed Matter (19/ Rec. for Meth. of Expression of film (Other Than Type S) (1968) Rec. for Rec. for Characteristic Impedances and Rec. Ior Rec. for Rec. for Rec. for Rec. for

(1966) eras (1966) onic Components (1972) Tubes Rec. for es (For Loads Greater Than 1500 kgf) (I969) Rec. for Industrial Trucks. es (1968) Rec. for Rec. Ior Rec. for Flexible Cellular Materials-Measurement of Std. for Fibre (Fiber) Building Boards: Determination of e and Valve Shields: Spec. Sheets for Tubes and Valves and tacts (I966) Rec. for Rec. for Textile Machinery-Terms for Serrated Bars and ctors (I964)

th 4 Bolt Holes (I972) Rec. Ior des (1966)

g Machines (1969)

$r$ Round Winding Wires (I965)

r Round Resistance Wires (197I)

Std. for Industrial Castors.

Rec. for Rec. for Rec. for Basic Rec. for Basic Rec. for Basic Rec. Ior Basic

Insulated Rectangular Copper Winding Wir

of Enamelled Round Winding Wires (1964) g of Mild Steel: Sockets for Electronic Tubes and Valves: Spec. Sheets and s-Tapered Roller-Boundary Dimensions-Metric Series

for Milling Cutters with Tenon Drive: Interchangeability Rec. for Bonded Abrasive Products: Grinding Wheel for Stretchers, Stretcher Carriers and Hospital Trolleys: -Cylindrical Roller-Separate Thrust Collars-Boundary Rec. for Rolling Bearings-Tapered Roller-Boundary Rec. for Rolling Bearings-Tapered Roller-Boundary Rec. for Rolling Bearings-With Locating Snap Ring Rec. for WarperS Beams: Basic

ling Bearings-Thrust Bearings with Flat Seats-Boundary Rec. for ISO General Purpose Metric Screw Threads: Basic Rec. for ISO In. Screw Threads: Basic Rec for Throwaway Carbide Indexable Inserts:

Rec. Ior Hand Operated Stillage Trucks: Principal ectional Beams for Warp Knitting Machines: Terminology and Rec. for Dental Burs and Cutters-Fitting

for Mechanical Standardization of Semiconductor Devices:

Wire, Bar and Tube Drawing Dies-Designation-Marking . ral Purpose Series I Freight Containers-Minimum Internal

- Rotary Drill Rods and Bits for Dry Drilling-Connecting

n-Reference Lines of Horizontal Controlling Coordinating Rec. for Photography-Projector Slides

Rec. for Pipeline Flanges for General Use-Mating

Replenishment Couplings for Aircraft-Interchangeability

Track Magnetic Sound Records on $35 \mathrm{~mm}$ Film-Location and sories-Condenser Bobbins for Woollen (Woolen) Spinning Std. for Sealed Beam Landing Lamps for Aircraft std. for Rigid Cellular Plastics-Determination of Linear Std. for Hand Finishing Sticks and Oil Stones Std. for Abrasive Sheets and Dises

td. for Tool Shanks and Equipment with 7/24 Tapers: Collar aled NickeI Cadmium Cylindrical Rechargeable Single Cells:

S Motion Picture Raw Stock Film-Cutting and Perforating nd Doubling Frames for C and El Travellers-Principal nd Doubling Frames for C and El Travellers-Principal

Tubular Socket Wrenches-Metric Series-Maximum Outside

d Cabinets-Meth. of Test-Part I: Calculation of Linear
Dimensions of Loudspeakers (1960)

Dimensions of Motor Vehicles and Their Trailers: Design Dimensions of Pallet Trucks (1966)

Dimensions of Panels and Racks (For Nuclear Electronic

Dimensions of Panels (1969)

Dimensions of Pellets and Cases (1966)

Dimensions of Pirn Tip (1969)

Dimensions of Polarized Plugs for Hearing Aids (1957)

Dimensions of Processed Writing Paper and Certain Class

Dimensions of Projection Reels for $8 \mathrm{~mm}$ Motion Picture

Dimensions of Radio Frequency Coaxial Cables (1967)

Dimensions of Rectangular Refractory Bricks (1966)

Dimensions of Refractory Arch Bricks (1969)

Dimensions of Screw Cores Made of Ferromagnetic Oxides

Dimensions of Seam Welding Wheel Blanks (1968)

Dimensions of Small Flash Connections for Hand Held Cam

Dimensions of Sockets and Male Ends (1971)

Dimensions of Spindle Ends for Manually Operated Electr

Dimensions of Stillages-Connection Gauge (I970)

Dimensions of Straight Resistance Spot Welding Electrod

Dimensions of Test Pieces (1968)

Dimensions of Test Pieces (197I)

Dimensions of Test Pieces (1972)

Dimensions of Test. Devices and Gauges (1969)

Dimensions of the Crimp Area of Machined Crimp Type Co

Dimensions of the Cross Section of Assembled Bars for $M$

Dimensions of the Mating Parts of Radio Frequency Conne

Dimensions of Top Plates-Part I: Oblong Top Plates Wi

Dimensions of Tubes, Pins and Rods of Ferromagnetic Oxi

Dimensions of Tubular Electrode Holders for Spot Weldin

Dimensions of Winding Wires: Diameters of Conductors Fo

Dimensions of Winding Wires: Diameters of Conductors Fo

Dimensions of Winding Wires: Dimension of Conductors of

Dimensions of Winding Wires: Maximum Over All Diameters

Dimensions of Wires, Spools, Rims and Coils (1968)

Dimensions of Wiring Jigs and Pin Straighteners (I965)

Dimensions Series 31 and $32(1970)$

lor Rolling Bearing ISO

Dimensions with Cutter Arbors-Metric Series (1973) ISO

Dimensions (Part 1) (1967)

Dimensions (1960)

Dimensions (1962)

Dimensions (1963)

Dimensions (1965)

Dimensions (I965)

Dimensions (I966)

Dimensions (1966)

Dimensions (1968)

Dimensions (I969)

Dimensions (1969)

Dimensions (1969)

Dimensions (1970)

Dimensions (1970)

Dimensions (1970)

Dimensions (1970)

Dimensions (1970)

Dimensions (1970)

Dimensions (1971)

Dimensions (197I)

Dimensions (1971)

Dimensions (1971)

Dimensions (197 I)

Dimensions (1972)

Dimensions (1972)

Dimensions (1972)

Dimensions (1972)

Dimensions (1972)

Dimensions (1972)

Dimensions (1972)

Dimensions (1972)

Dimensions (1972)

Dimensions (1972)

ons, Areas and Volumes (1971)

Dimensions, Tolerances and Applicability of Test Pieces

Rec. ISO

Rec. for Rolling Bearings ISO

ISO

ISO

ISO

Rec. for Rol ISO

ISO

ISO

ISO

ISO

Rec. for S ISO

Rec ISO

Rec. for Gene ISO

Rec. for Rock Drilling ISO

Rec. for Modular Coordinatio ISO

ISO

Rec. for Liquid Oxygen 1SO

Rec for Recording Heads for 6 ISO

Ec. for Textile Machinery and Acces ISO

ISO

ISO

ISO

ISO

Rec. for Se IEC

Std. for Cinematography $-8 \mathrm{~mm}$ Type ISO

/Eversible Rings for Ring Spinning a ISO

Eversible Rings for Ring Spinning a ISO

or Screws and Nuts-Forged and and ISO

ISO

ISO
Dimensions of Straight Resistance Spot Welding Electrod

Dimensions (I968)

$1035 / 1$

$1035 / 11$

$657 / 1 \mathrm{II}$

$657 / I X$

$657 / \mathrm{VII}$

$657 / \mathrm{I}$

$657 /$ II

$657 /$ IV

273

199

124

612

509

297

1097

524

1131

90

353

892

78

475

1 I 45

221

693

519

2016

390

1756

1045

670

821

1794

766

288.2

203

1865

159

2184

220

1089

182-1

$182-4$

I82.3

182-2

864

I 49.2

$355 / V$

2780

603

46

$355 / \mathrm{I}$
$355 / \mathrm{Il}$

464

104

724

725

883

938
I025

1797

19I-2

1684

1894

I717

1790

1755

2084

1465 
Rec for Boiler Tuhes.

rn Tubes for Ring Spinning and Ring Doubling Spindles: In. Tuhes for Ring Spinning and Ring Doubling Spindles Metric Rec. for Cold Drawn Precision Steel Tuhes: Metric Series Sid, for Truncated Cone Abrasive Sleeves: Designation, olling Bearings: Airframe: Introdurtion. General, Boundary terials-Socket Fittings for Pipes Under Pressure-Basic Rec. for Copper Tubes of Circular Section

Spigot Ends for Domestic and Industrial Waste Pipe: Basic ium Silicates for Industrial Use-Determination of Carbon

Hydroxide for Industrial Use: Determination of the Carbon

Hydroxide for Industrial Use: Determination of the Carbon cid and Oleum for Industrial Use; Determination of Sulphur Rec. for Titanium Rec. for Determination of Carbon mical Analysis of Manganese Ores-Determination of Carbon ical Analysis of Manganese Ores-Determination of Silicon n of Active Oxygen-Conventionally Expressed as Manganese (1970) Rec. for Requirements for Hot Rec. for Determination of the Weight Per Unit Area of Hot guiding Principles for Protection Against Corrosion by Hot determination of Chromium-Spectrophotometric Meth. Using Std. for Textiles: Determination of Twist in Yarns ic Film Dielectric (1964) Rec. for Fixed Capacitors for Rec. for Fixed Metallized Paper Dielectric Capacitors for ilm (1956) Rec. for Emulsion Position in Projector for

hy: Projector Usage of $8 \mathrm{~mm}$ Type S Motion Picture Film for mination of Moisture in the Analysis Sample of Coal by the nd Vacuum Gauges, with Pressure Responsive Elements Giving and Valves (1962)

Rec. Meth. for the Measurement of Rec. for Low Voltage Motor Starters: Rec. for

heir Accessories (1968) ents: Detergents-Determination of Anionic-Active Matter
mination of Moisture in the Analysis Sample of Coal by the termination of Moisture in Brown Coals and Lignites by the utters-Part I (1970)

Rec. for Symbolic Designation of nting Pap/ Rec. for Meth. of Expression of Dimensions and ting Lamps for Circuit Breakers (1936) Rec. for Std. 969)

Combustion Engines-Hand Operated Control Devices-Std.

Rec. for Shape, Size and

Rec. for

c. for Rotating Electrical Machines: Terminal Markings and

aft Engines, Engine Cylinders and Combustion Chambers, and

procating Internal Combustion Engines Designation of the Rec. for Motion Picture Film Perforated Along One Edge Idahl Meth/ Rec. for A gricultural Food Products-General (1957)

ermination of the Dilute Solution Vi/

Rec, for Plastics.

Centres (Centers) (1972)

Std. for

Rec. for Raw Natural Rubber Determination of International Rec. for Optical Pyrometers with Rec. for Partial ration of a Gauging Station and Determination of the Stage Rec. for Rubber Hoses for Oil Suction and resistance of Insulating Materials to Breakdown by Surface

chloride and Related Copolymers and Their Compounds by the airbreak Switches, Airbreak Disconnectors, Airbreak Switch Rec. for Alternating Current Isolators
ombinat/ Rec. for Low Voltage Airbreak Switches, Airbreak Std. for Abrasive Sheets and

apour (Vapor) Transmission Rate of Films and Thin Sheets Rec. for Processed

asurement of the Electrical Properties of Microwave Tubes: viscosity of Natural and Synthetic Rubbers hy the Shearing nation of Rate of Cure of Rubber Compounds by the Shearing strial Use: Determinat/ Std. for Boric Acid, Boric Oxide, metric Meth. (1972) Std. for Boric Acid and Oxide, and n of Sodium and Boric Oxide Content and Loss On/ Std. for n of Manganese Conten/ Std. for Boric Acid and Oxide, and (1969)

of Dry Solids Content at 105/ Rec. for Plastics-Aqueous cle Stability Test (1969) Rec. for Plastics-Aqueous 1969) measurement of the Performance Chacteristics of Positive for the Determination of the Permitivity and Dielectric t Dip Galvanized Coatings on Ferrous Materials by Chemical s-Measurement of Thickness-Coulometric Meth. by Anodic Rec. for Creet,age

Rec. for A nimal Fats: Determination of Water (Entrainment lds of Tar, Water, Gas and Coke Residue by Low Temperature
Dimensions, Toleranccs and Conventional Masses Per Unit

Dimensions, Tolerances and Gauges (1963)

ISO

Dimensions, Tolerances and Gauges (1964)

Rec. for Wa ISO
/Ec. for Warp isO

Dimensions, Tolerances and Masses Per Metre (Meter) (19 ISO

Dimensions, Tole rances (1972)

Dimensions, Tolerances, Permissible Load (1969) /for R ISO

Dimensions: Metric Series (1972) /Tings of Plastics Ma ISO

Dimensions; Metric Series (1962)

Dimensions; Metric Series (1962)

ISO

ISO

Dioxide Content Expressed as Sodium or Potassium Carbon

Dioxide Content, Expressed as Potassium Carbonate: Gas

Dioxide Content, Expressed as Sodium Carbonate, Gas-V

Dioxide Content: Barium Sulphate Gravimetric Meth. (196

Dioxide for Paints (1967)

Dioxide in Coal by the Gravimetric Meth. (1969)

Dioxide (1963)

Dioxide (1963)

Dioxide (1963)

Rec. for Meth. of Chem

L ysis of Manganese Ores-Determinatio ISO

Dip Galvanized Coatings on Fabricated Ferrous Products ISO

Dip GaIvanized Coatings on Ferrous Materials by Chemica ISO

Dip Galvanizing (1970)

Diphenylcarbazide (1972)

I Aluminium and Its Alloys ISO

Direct Counting Meth. (1972)

Direct Current Using Impregnated Paper or Paper / Plast

ISO

Direct Current (1965)

Direct Current (1965)

Direct Front Projection of $16 \mathrm{~mm}$ Silent Motion Picture

Direct Front Projection of $8 \mathrm{~mm}$ Silent Motion Picture F

Std. for Cinematograp ISO Rec. for Deter ISO

Direct Gravimetric Meth. (1963)

Direct Indications by Means of a Pointer and Graduated

Direct Interelectrode Capacitances of Electronic Tubes

Direct on Line, a.c. (1971)

Direct Reading Pocket Type Electroscope Exposure Meters

Direct Recording Electrical Measuring Instruments and T

Direct Two Phase Titration Procedure) (1972)

/Ctive Ag ISO

Rec. for Deter ISO

Direct Volumetric Meth. (1963)

Direct Volumetric Meth. (1969)

Direction of Closing and Faces of Doors, Windows and Sh Rec. for De ISO

ISO

IEC

Direction of Motion (1972)

/for Reciprocating

Direction of Operation of Lever Controls on Aircraft (1

Direction of Operation of Machine Tool Controls (1965)

Direction of Rotation (1972)

Direction of Rotation of Engines and Propellers (1966)

Direction of Rotation (1972)

Direction of Twist in Textile Yarns and Related Product

Direction of Winding (1971)

Directions for the Determination of Nitrogen by the Kje

Directions of Operation of Toggle Switches on Aircraft

Directives for the Standardization of Meth. for the Det

Directories of Libraries, Information and Documentation

Dirt (1971)

Disappearing Filaments (1970)

Discharge Measurements (1968)

Discharge Relation (1969)

Discharge (1971)

Discharges (1970)

Discoloration Meth. (1963)

Meth. for Determining the Relative IEC IAl Stability of Polyvinyl ISO

/Tage IEC

(Disconnectors) and Earthing Switches (1961)

Disconnectors, Airbreak Switch Disconnectors and Fuse C IEC

Discs-Dimensions (1972)

Dish Meth. (1970)

Dishwashers (1971)

Disk Records and Rep

Disk Seal Tubes (1972)

Disk Viscometer (1963) Rec. for Safety of Household and

ISO

Disk Viscometer (1968)

Disodium Tetraborates and Crude Sodium Borates for Indu ISO

Disodium Tetraborates for Dibenzyldithiocarbamate Photo ISO

Disodium Tetraborates for Ind ustrial Use-Determinatio ISO

Disodium Tetraborates for Ind ustrial Use-Determinatio

Dispersions of Polymers and Copolymers-Determination

Dispersions of Polymers and Copolymers-Determination

Dispersions of Polymers and Copolymers-Freeze Thaw Cy

Displacement Vacuum Pumps-Part I: Volume Rate of Flow

Displayed Symbols for Adding and Calculating Machines (

Dissipation Factor of Elect rical Insulating Materials a

Dissolution of the Coating-Gravimetric Meth. (1970)

Dissolution (1972)

Distances and Clearances in Air (1964)

Std. for Metallic Coating

Distillation Meth. (1969)

Distillation of Brown Coal and Lignite (1968)
1129

343

368 
ic Acid and Xylenols for Industrial Use-Determination of for lligher Alcohols for Industrial Use-Determination of Rec. for Test Meth. for Xylenols for Industrial Use-Determination of Residue on Meth. of Measurement of Audio Frequency Output Power and installations in Ships: Switchgear, Electrical Protection, Std. for Automobiles-Load use in Sensitometric Exposure-Simulation of the Spectral Electrotechnical Vocabulary: Generation, Transmission and use in Sensitometric Exposure-Simulation of the Spectral use in Sensitometric Exposure-Simulation of the Spectral Rec. for Low Voltage lements (1960) Rec. Graphical Symbols: Kind of Current, wires with Pvc (Polyvinyl Chloride) Insulation and Sheath: wires with Pvc (Polyvinyl Chloride) Insulation and Sheath: ating Stations and Substations, Lines for Transmission and Rec. for Coupling Capacitors and Capacitor Std. for Numbering of

terial (1967) Rec. for Paper Patterns for Rec. for

the ISO Mire (ISO Test Object) and Its Use in Photographic of Titles of Periodicals (1972) isbn) (1972) Std. for apparatus (1968) Std. for Directories of Libraries, Information and Rec. for

Rec. for Short Contents List of Periodicals or Other td. for Numbering of Divisions and Subdivisions in Written f Plastics Materials: Socket Fittings with Spigot Ends for Spec. for Appliance Couplers for Spec. for D-Type Fuses for Rec for Std for Plugs and Socket Outlets for ts for Mains Operated Electronic and Related Equipment for ts for Mains Operated Electronic and Related Equipment for ment for Splash Proof Mains Operated Electronic Equipment Spec. for Switches (Electrical) for

Spec. for Electric Cooking and Heating Appliances for c. for Voltage Operated Earth Leakage Circuit Breakers for Spec. for Plugs and Socket Outlets for

Spec. for Electric Motor Operated Appliances for Spec. for Miniature Circuit Breakers for

Spec. for Lighting Fittings for Incandescent Lamps for Rec, for Fuses for

Spec, for Electric Motor Operated Appliances for D/ Spec. for Electric Cooking and Heating Appliances for ular Spec. for Electric Cooking and Heating Appliances for ular Spec. for Electric Cooking and Heating Appliances for ular Spec. for Electric Cooking and Heating Appliances for Spec. for Electric Motor Opcrated Appliances for ) ular Spec. for Electric Cooking and Heating Appliances for ular Spec. for Electric Cooking and Heating Appliances for nd Simi/ Spec. for Electric Motor Operated Appliances for Spec. for Electric Motor Operated Appliances for ular Spec. for Electric Cooking and Heating Appliances for Sucti/ Spec. for Electric Motor Operated Appliances for $\mathrm{rm} / \quad$ Spec. for Electric Cooking and Heating Appliances for Spec. for Electric Motor Operated Appliances for

Rec. for Aircraft Engine Nacelle Fire Extinguisher Symbolic Designation of Direction of Closing and Faces of 1961) Rec. for Personal Photographic

Tolerance Class 5-Special/ Rec. for Rec. for Combinations of Rec. for Rolling Bearings Rec. for Frames for Single or Rec. for Rolling Bearings Rec. for Rolling Bearings ance (1970) 65) Sealing / Std. for Rec for Metal Reeds with

Cinematography-Sound Records and Scanning Area of $35 \mathrm{Mm}$ cinematography-Dimensions of Daylight Loading Spools for Rec. for Cutting and Perforating Dimensions for $y$ and Accessories-Reversible Rings for Ring Spinning and nd Accessories-Nonreversible Rings for Ring Spinning and Rec. for Rings for Ring Spinning and ery and Accessories-Spindle Gauges for Ring Spinning and ges (1964/ Rec. for Warp Tubes for Ring Spinning and Ring 8 (1963) Rec. for Warp Tubes for Ring Spinning and Ring ormers and Inductors of Ferromagn/ Rec. for Guide for the ransformers of Ferromagnetic Oxid/ Rec. for Guide for the Rec. Guide for the

Fibre (Fiber) (1959) sbestos Cement Pipes, Joints and Fittings for Sewerage and Rec. for Aircraft Toilet Flushing and Rec. for Power Take Off and Rec for Textile Machinery-Cylindrical Tubes for Rec. for Textile Machinery and Accessories-Tubes for
Distillation Range (1971)

Rec. for Cresyl ISO Rec. ISO

Distillation (Yield and Range) (1969) Distillation (1971)

Distortion (1966) Distribution and Controlgear (1965)

Rec. for Cresylic Acid and ISO

Distribution for Private Cars (1972)

Distribution of Daylight (1972)

Distribution of Electrical Energy (1965)

Distribution of Photoflood lllumination (1972) 1. for Electrical IEC ISO

Distribution of Tungsten Illumination (1972)

1-Light Sources for 150 Nternational IEC iS for 150

Distribution Switchgear: Circuit Breakers (1964) /Ces for ISO

Distribution Systems, Meth. of Connection and Circuit E IEC

Distribution Wires with Solid Conductors, Insulated, in IEC

Distribution Wires with Solid Conductors, lnsulated, Po IEC

Distribution (1963) Rec. Graphical Symbols: Gener

Dividers (1971)

Divisions and Subdivisions in Written Documents (1972)

Dobbies (1967)

Document Reproduction ((1965) / Tests; Description of 1SO

Documentation-International Code for the Abbreviation ISO

Documentation-International Standard Book Numbering ( ISO

Documentation Centres (Centers) (1972) ISO

Documentation to Be Supplied with Electronic Measuring IEC

Documents (1955)

Documents (1972)

Domestic and Industrial Waste Pipe: Basic Dimensions; $M$

Domestic and Similar General Purposes (1962)

Domestic and Similar General Purposes (1968)

Domestic and Similar General Use (1957)

Domestic and Similar General Use (1965)

Domestic and Similar General Use (1965) CEE 1

Domestic and Similar General Use) (1969)

Domestic and Similar Purposes (1955)

Domestic and Similar Purposes $(1959+1964)$

Domestic and Similar Purposes (1959)

Domestic and Similar Purposes (1963)

Domestic and Similar Purposes (1964)

Domestic and Similar Purposes (1968)

Domestic and Similar Purposes (1968)

Domestic and Similar Purposes (1968)

Domestic and Similar Purposes: Clocks (1967)

Domestic and Similar Purposes: Clothes Dryers and Towel

Domestic and Similar Purposes: Cooking Ranges, Cooking

Domestic and Similar Purposes: Electric Irons, Ironers

Domestic and Similar Purposes: for Heating Liquids (196

Domestic and Similar Purposes: Massage Appliances (1967

Domestic and Similar Purposes: Portable Immersion Heate

Domestic and Similar Purposes: Room Heating and Similar

Domestic and Similar Purposes: Shavers, Hair Clippers a

Domestic and Similar Purposes: Spin Extractors (1968)

Domestic and Similar Purposes: Stationary Noninstantane

Domestic and Similar Purposes: Vacuum Cleaners and Wate

Domestic and Similar Purposes: Waffle Irons, Grills, W a

Domestic and Similar Purposes: Washing Machines (1968)

Doors-Terminology (1972)

Doors (1969)

Doors, Windows and Shutters-Part I (1970)

Dosimeters (1971)

Double Deck Flat Pallets for Through Transit of Goods (

Double Ended Wrench Gaps (1969)

Double Row Cylindrical Type RD with Tapered Bore 1:12-

Double Row of Healds (1967)

Double Row Self-Aligning Ball-Radial Internal Clear

Double Row Selfaligning-Radial Internal Clearance (19

Double Socket Fittings for Unplasticized Polyvinyl Chlo

Double Spring Baulk (1967)

Double Width Push Pull Sound Prints Normal and Offset C

Double $8 \mathrm{~mm}$ Motion Picture Film (1969)

Double-8 mm Motion Picture Raw Stock Film (1966)

Doubling Frames for $\mathrm{C}$ and El Travellers-Principal

Doubling Frames for $\mathrm{C}$ and El Travellers-Principal

Doubling Frames for Ear Shaped Travellers (1959)

Doubling Frames (1972) Std. for Textile Machin ISO

Doubling Spindles Metric Dimensions, Tolerances and Gau ISO

Doubling Spindles: In. Dimensions, Tolerances and Gauge ISO

Drafting of Perforamnce Spec. for Cores of Tuned Transf

Drafting of Performance Spec. for Cores of Broad Band T

Drafting of Spec. for Microwave Ferrites (1972)

Drafting Rollers for Cotton, Wool, Spun Silk and Staple

Drainage (1968)

Draining Connections (1957)

Draw Bar for Agricultural Tractors (1966)

Draw Twisters (1970)

Draw Winders for Man Made Fibres (1971)
1906

1845

918

1907

151-10

92-4.

2416

2239
$50(25)$

2242

2241

$157-$

117-1

$189-4$

189-7

$117-5$

358

2145

576

573

446

2108

2108
2146

2146
278

18

2145

265

22

16

83

1

$65 \mathrm{~A}$

14

11911.1

18

10910-1

19

25

241

10-2E

$11-2 \mathrm{~N}$

11-2A

$11.2 \mathrm{G}$

11-2B

$10-20$

11-2C

11-2F

$10-2 \mathrm{~N}$

$10-2 \mathrm{~K}$

11-2E

10.2A

$11-2 \mathrm{M}$

$10-2 \mathrm{~J}$

1804

1021

1226

1757

198

1085

533

568

1646

465

2048

57

72

1020

486

95

96

97

368

343

218

219

392

98

881

47

500

1472

2105 
and Ciasen $(1966)$

Rec. for Wire, Bar and Tuhe Rec. for Hard Metal Wire

Rec, for Connectors for Frequencies Below $3 \mathrm{MHz}$ : Guide to Rec. for Tube d End Drop Wires (Electrical and Mechanical) for Automatic ngs (1971)

Rec. for Technical

Rec. for Technical

Rec. for Technical

Practical Examples of Indications $\mathrm{O} /$

I Standardization of Semiconductor Devices: Preparation of
Rec. for Preparation of Outline f Position-Part I: Generalities, Symbols, Indications on echnical Drawings-Meth. of Indicating Surface Texture on f Position-Part IV: Practical Examples of Indications on Gears (1973) Std. for Technical Rec. for Architectural and Building Rec. for Architectural and Building Std. for Building Std. for Technical Rec. for Engineering Rec. for Engineering

959)

Allo/

straight $\mathrm{Re}$

Rec for the Resistivity of Commercial Hard hent Lengths (19/ Rec. for Lengths and Tolerances for
Rer ec. for Lengths and Tolerances for Filler Rods, Other Than Tolerances and Masses Per Metre (Meter) (/ s-Spec. (1971) th.) (1970)

1)

(1969)

sions (1970)

(1970)

-Rope Threaded Equi/

-Rope Threaded Equi/

-Reverse Buttress T/

- Reverse Buttress T/

Rec for Rock Drilling. Hollow Hexagonal

ed Collared Shanks and Chuck Bushings for Hollow Hexagonal ec. for Rock Drilling-Rotary Drill Rods and Bits for Dry

$$
\text { e Drilling (1970) }
$$

ve Long Hole Drilling-Rope Threaded Equi/

ve Long Hole Drilling-Rope Threaded Equi/

ve Long Hole Drilling-Reverse Buttress T/

ve Long Hole Drilling-Reverse Buttress T/

Extension Drill Steel Equipment for Percussive Long Hole

Extension Drill Steel Equipment for Percussive Long Hole

Extension Drill Steel Equipment for Percussive Long Hole

Extension Drill Steel Equipment for Percussive Long Hole

Connecting Dimensions (1970)

k Drilling-Drill Rods and Detachable Bits for Percussive Hollow Hexagonal Drill Steels (1968) 68) Rec. for Rock Rec. for Rock Rec. for Rock Rec. for Parallel Shank Twist a (1968) Std. for Centre

cked Sizes (1972)

Taper Shank (1961) Rec. for Centre (Center) Std. for Std. for Core Rec. for Twist

shell Reame with Slot rewdriver Bits, for Slotted Head Screws, with Male Hexagon Rec. for Shaft Heights for Driving and

Rec. for Terms and Definitions Relating to - Metric Series (19/ Std. for Milling Cutters with Tenon Std. for Cylindrical Cutters with Plain Bore and Key Std. for Slotting Cutters with Plain Bore and Key

for Side and Face Milling Cutters with Plain Bore and Key Std. for Shell End Mills with Plain Bore and Tenon Rec. for Shaft Heights for oads-Belt Conveyors-Basic Characteristics of Motorized 0) Rec. for Assembly Tools for Bolts and Screws (1961) Rec. for Diameters of Shanks and Sizes of Rec. Environmental Test Procedures: Test Ec: ons (1965) s-Estimation of the Loss of Absorptive Power by Colorant awing.in Machines (1969) Rec. for Closed End petroleum Products-Lubricating Grease-Determination of

lled Transport Packages-Part IV: Vertical Impact Test by

r Purposes: Waffle Irons, Grills, Warming Plates and Other d Packaging (1970)
Drawing Dies-Designation-Marking-Dimensions (1970 Drawing Dies: Interchangeability Dimensions of Pellets Drawing Information in Detail Spec. (1970)

Drawing Mandrels (1970)

Drawing-in Machines (1969)

Drawings-Meth. of Indicating Surface Texture on Drawi for Close

Drawings-Tolerances of Form and of Position-Part II

Drawings-Tolerances of Form and of Position-Part IV

Drawings Devices (1969)

Drawings of Oscilloscope and Picture Tubes (1962)

Drawings (1969)

Drawings (1971)

Drawings (1971)

Rec. for Tolerances

Drawings-Tolera

IL Drawings-To
Representation of

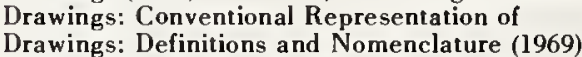

Drawings: Presentation of Drawings: Scales (1969)

Drawings: Projection Meth. (1972)

Drawings: Representation of Springs (1973)

Drawing: Dimensioning (1959)

Drawing: Principles of Presentation (1959)

Drawn Aluminium (Aluminum) Electrical Conductor Wire (1

Drawn and Extruded Products in Light Metals or in Light

Drawn or Extruded Filler Rods for Welding, Supplied in

Drawn or Extruded, for Welding (1967)

Drawn Precision Steel Tubes: Metric Series, Dimensions,

Drawn Wire for General Purpose Nonalloy Steel Wire Rope

Dried Milk-Determination of Fat Content (Reference Me

Drift Expanding Test on Copper and Its Alloy Tubes (196

Drift Expanding Test on Light Metal and Its Alloy Tubes

Drift Expanding Test on Steel Tubes (1960)

Drill Chuck Tapers (1961)

Drill Rods and Bits for Dry Drilling-Connecting Dimen

Drill Rods and Detachable Bits for Percussive Drilling

Drill Steel Equipment for Percussive Long Hole Drilling

Drill Steel Equipment for Percussive Long Hole Drilling

Drill Steel Equipment for Percussive Long Hole Drilling

Drill Steel Equipment for Percussive Long Hole Drilling

Drill Steels in Bar Form (1968)

Drill Steels (1968)

Drilling-Connecting Dimensions (1970)

for Rock Drilling: Fol

Drilling-Drill Rods and Detachable Bits for Percussiv

Drilling-Extension Drill Steel Equipment for Percussi

Drilling-Extension Drill Steel Equipment for Percussi

Drilling-Extension Drill Steel Equipment for Percussi

Drilling-Extension Drill Steel Equipment for Percussi

Drilling-Reverse Buttress Threaded Equipments 1 1/2 T

Drilling-Reverse Buttress Threaded Equipments 1 l/6 a

Drilling-Rope Threaded Equipments $11 / 2$ to 2 In. ( 38

Drilling-Rope Threaded Equipments $7 / 8$ to $11 / 4$ In. (2

Drilling-Rotary Drill Rods and Bits for Dry Drilling

Drilling Equipment: Kellys (1972)

Drilling Equipment: Upper and Lower Kelly Cocks (1972)

Drilling (1970)

Drilling: Forged Collared Shanks and Chuck Bushings for

Drilling: Hollow Hexagonal Drill Steels in Bar Form (19

Drilling: Integral Stems (1968)

Drills-Long Series (1966)

Drills for Center Holes with Radius Form: Type R (1972)

Drills for the Holes Without Protecting Chamfers-Type

Drills for Use Prior to Tapping Screw Threads (1972)

Drills with Parallel, and Morse Taper Shanks-Rec. Sto

Drills: Parallel Shank Jobber and Stub Series and Morse

Drinkable Ouality (1965)

Drive and Arbors for Shell Reamers (1972)

Drive Ends for Power Types (1970)

Drive (1972)

Driven Machines (1966)

Drives Using V-Belts and Grooved Pulleys (1969)

Drive: Interchangeability Dimensions with Cutter Arbors

Drive: Metric Series (1972)

Drive: Metric Series (1972)

Drive: Metric Series (1972)

Drive: Metric Series (1973)

Driving and Driven Machines (1966)

Driving Pulleys (1970)

/Oose Bulk Materials and Unit L

Driving Squares for Power and Hand Socket Wrenches (197

Driving Squares for Rotating Tools with Parallel Shanks

Drop and Topple, Primarily for Equipment Type Specimens

Drop Test with Prior Acid Treatment (1971)
Drop Wires for Mechanical and Electrical Warp Stop Moti

Drop Wires (Electrical and Mechanical) for Automatic Dr

Dropping Point (1972)

Dropping (1972)

Dry Cooking Appliances (1967)

Std. for Packaging-Complete, Fi /for Domestic and Simila CEE Dry Corkwood in Planks-Definitions, Classification an

\section{Iso}

IEC

ISO

ISO

ISO

ISO

IEC

O ISO

ec. for T ISO

ISO

ISO

ISO

ISO

ISO

ISO

ISO

IEC

ISO

ISO

ISO

ISO

ISO

ISO

ISO

ISO

ISO

ISO

ISO

ISO

ISO

ISO

ISO

ISO

ISO

ISO

ISO

ISO

ISO

ISO

ISO

ISO

ISO

ISO

ISO

ISO

ISO

ISO

ISO

ISO

ISO

ISO

ISO

ISO

ISO

ISO

ISO

ISO

ISO

ISO

ISO

ISO

ISO

ISO

ISO

Rec. ISO

ISO

ISO

ISO

ISO

ISO

IEC

ISO

ISO

SO

CEE

ISO 
Rec. for Rock Drilling-Rotary Drill Rods and Bits for Rec. for Preparation of rocedures for Electronic Components and Equipment: Test B: Potassium Silicates for Industrial Use-Determination of Std. for Glucose Syrup: Determination of Rec. for Pulps: Determination of Rec. for Tea-Preparation of Ground Sample of Known Rec. for Glucose Syrup-Determination of
Rec. for Preparation of

tices (1971)

Std for Natural Rubber latex-Determination of Dispersions of Polymers and Copolymers-Determination of and Corkwaste-Definitions and $\mathrm{Pa} /$ Rec. for Commercially ting Appliances for Domestic and Similar Purposes: Clothes er-Meth. for the Determination of Moisture Content (Oven Rec. for Paints and Varnishes-Surface

(1972) Std. for Offset Std. for mination of Indentation Hardness of Plastics by Means of A ics Industry (English, French, Russian) (NL: Corresponding Rec. for Continuous Mechanical Handling Equipment-Light

enclature (1970)

ight Sides (1969)

Rec. for Rating of Sound Insulation for Rec. for Textile Machinery -

Rec. for Beams for Rec. for Transfer Cones for n. (1967) (1963) Rec. for Cones for Cross Winding for Rec. for Perforated Parallel Tubes for Cheese Rec. for Rolling Bearings-Meth. of Evaluating le Floor Coverings-Determination of Thickness Loss Under Potassium Silicates for Industrial Use-Determination of Their Coefficients (1972) osphere Relative to the Eart/ Std. for Symbols for Flight Rec. for Symbols for Flight Std. for Symbols for Flight (I972) Earphones Coupled to the

Roubling Frames for hones Used in Audiometry (197/ Rec. for an IEC Artificial eference Coupler for the Measurement of Hearing Aids Using $r$ IEC Provisional Reference Coupler for the Calibration of ificial Ear, of the Wide Band Type, for the Calibration of Purposes (I959) Spec. for Voltage Operated motions of the Aircraft and the Atmosphere Relative to the

for Voltage Measurement by Means of Sphere Gaps (1 Sphere rec. for Alternating Current Isolators (Disconnectors) and alysis of Magnesium and Its Alloys-Determination of Rare its Alloys-Determination of -Containing Zirconium, Rare )

Procedures for Electronic Components and Equipment: Test Std. for Std. for Std. for

Procedures for Electronic Components and Equipment: Test mens /

Rec. for Basic Environmental Test Procedures: Test magnetic Basis Metals-Measurement of Coating Thickness Rec. for Motion Picture Film Perforated Along One ith Board Mounted Connectors or Printed Wiring Boards with Rec. for Identification of the Emulsion Side of ntact S/ Rec. for Connectors for Frequencies Below $3 \mathrm{MHz}$ : er) Guil Rec. for Connectors for Frequencies Below $3 \mathrm{MHz}$ : 96/ Rec. for Location and Width of the Recording Head for und Stripe on $16 \mathrm{~mm}$ Motion Picture Film Perforated Along for Magnetic Striping of $16 \mathrm{~mm}$ Film Perforated Along Both mm Motion Picture Film with Perforations Along One and Two Rec. for
Spec. for

um Hydroxide for Industrial Use: Determination of Calcium: um Hydroxide for Industrial Use: Determination of Calcium:

ial Use: Determination of Calcium and Magnesium Contents Rec. for Basic Environmental Test Procedures: Tesi Std. for Steel: Determination and Verification of th rties of Fasteners: Nuts with Specified Proof Load Values, Rec. for Calculation of the ting Electrical Machines: Meth. for Determining Losses and Rec. for Pressures in Brake Lines and Brakin 1972) Std. for Covered Electrodes-Determination of the Rec. for Calibration of unplasticized Polyvinyl Chloride (PVC) Pressure Pipes with unplasticized Polyvinyl Chloride (PVC) Pressure Pipes with identification (1971)

series-Class 2 Tolerances) (197/

ries-Class 1 Tolerances) (1969)

Aircraft (In. Series-Class 1 T/

Aircraft-(In. Series-Class 2)

Rec. for

Rec. for Dimensions of Rec. for Dimensions of Rec. for Dimensions of

Rec. for Dimensions of Rec. for Conventions Concernin rodes with Threaded Sockets and Connecting Pins for Use in rec. for Measurement of the Performance Characteristics of

Dry Drilling-Connecting Dimensions (1970)

Dry Films from Concentrated Natural Rubber Latex (1966)

Dry Heat (1966)

Rec. for Basic Environmental Test. P IEC

Dry Matter-Gravimetric Meth. (1970)

/ for Sodium and ISO

Dry Matter-Refractive Index Meth. (1973)

ISO

ISO

Dry Matter Content (1967)

Dry Matter Content (1970)

Dry Matter of Moisture (Vacuum Oven Meth.) (1970)

Dry Polymer from Butadiene Homopolymer and Copolymer La ISO

Dry Rubber Content (1972)

Dry Solids Content at $105^{\circ} \mathrm{C}(1970)$

Dry Virgin Cork, Ramassage, Glean

Dryers and Towel Drye

Drying Test (Ballotini Meth.) (1970)

Duplicators-Attachment Features of Plates (1972)

ISO

ISO

(1) Duplicating Stencils

Durometer (Shore Hardness) (1968) for Plastics-Deter ISO

Dutch Terms) (1968) /Quivalent Terms Used in the Plast ISO

Duty Belt Conveyors for Loose Bulk Materials (1971) ISO

Dwellings (1968)

Dyeing and Finishing Machinery-Classification and Nom

ISO

ISO

Dyeing and Finishing Machines: Definition of Left and $R$

ISO

Dyeing Fibres (Fibers) and Yarn (1969)

Dyeing Purposes: Half Angle at Top of Cone $4{ }^{\circ} 20 \mathrm{Mi}$

Dyeing Purposes: Half Angle of the Cone $4^{\circ} 20^{\prime}$

ISO

Dyeing (1967)

Dynamic Load Ratings (1962)

Dynamic Loading (1971)

Dynamic Viscosity (1972) Std. for Sodium and ISO

Dynamics-Part Il: Motions of the Aircraft and the Atm ISO

Dynamics-Part I: Aircraft Motion Relative to the Air ISO

Ear by Means of Inserts (1961) /Rence Coupler for the IEC

Ear Shaped Travellers (1959)

Ear, of the Wide Band Type, for the Calibration of Earp

Earphones Coupled to the Ear by Means of Inserts (1961)

Earphones Used in Audiometry (1970)

Earphones Used in Audiometry (1970)

/C. for an

Earth (1969)

Earthed) (1960)

Earthing Switches (1961)

Earths-Gravimetric Meth. (1972)

Earths, Thorium and Silver-Periodate Photometric Meth ISO

Ea: Shock (1967) Rec. for Basic Environmental Test IEC

Ebonite: Determination of Cross Breaking Strength (1972 ISO

Ebonite: Determination of Crushing Strength (1972) ISO

Ebonite: Determination of Tensile Strength (1972) ISO

Eb: Bump (1968) Rec. for Basic Environmental Test IEC

Ec: Drop and Topple, Primarily for Equipment Type Speci IEC

Eddy Current Meth. (1972) /Nconductive Coatings on Non ISO

Edge-Direction of Winding (1971)

Edge Board Contacts (197I)

Nconductive Coatings on Non ISO

/ (0.1 In.) Mating Either W IEC

Eod Rilm for Still Picture Cameras (1968)

Edge Socket Connectors with Closed Ends and Having a Co

Edge Socket Connectors with Open Ends, Off Centre (Cent

Edge Sound Records on $16 \mathrm{~mm}$ Perforated Magnetic Film (1

1717

498

$68-2-2$

1688

1743

638

1572

1742

126
1625

1215

$11.2 \mathrm{~N}$

287

1517

2 I 32

2133
868

$194 / N 1$

2109

717

1036

1037

575

324

574

281
2094

2123

1153

1152

1151

126

318

318
126

303

318

18

1152

52

129

2355
2353

68-2-27

2473

2474

2472

68-2-29

68-2-3I

2360

I 200

130-11

130-11

I30-13

891

490

163

69

238

986

986

2482

68.2-32

2639

$898 / \mathrm{IV}$

205

34-2

1186

2401

376

2045

2048

1749

1800

1078

1077

375

239
299 
Rec. for Fire Resisting Characteristics of Electric Cables (1970) Rec. for Flame Retardant Characteristics of Electric Cables (1970) lest for P've (Polyvinyl Chloride) Insulation and Sheath of Electric Cables (1970) Rec. Graphical Symbols: Measuring Instruments and Electric Clocks (1963)

Electric Cooking and Heating Appliances for Domestic an

d Similar Purposes: Cooking Ranges,

d Similar Purposes: for Heating liq/

d Similar Purposes: Portable lmmers/

d Similar Purposes: Stationary Noni/

d Similar Purposes: Room Heating An/

d Similar Purposes: Electric Irons, /

Particular Spec. for

Particular Spec. for

Particular Spec. for

Particular Spec. for

Particular Spec. for

Pariicular Spec. for

d Similar Purposes: Waffle Irons, Grills, Warm/

d Similar Purposes: Clothes Dryers and Towel D/

d Similar Purposes (1959 + 1964)

onal Electrotechnical Vocabulary: Nuclear Power Plants for

Rec. for Safety Requirements for

Rec. for a.c

Spec. for Mains Operated

Spec. for Battery Operated

Rec. for Characteristics of

Rec. for Meth. of Measurement of Performance of

similar Electrical Appliances: Particular Requirements for and Heating Appliances for Domestic and Similar Purposes

bulary: Detection and Measurement of Ionizing Radiation by ilar Purposes: Vacuum Cleaners and Water Sucti/ Spec. for ilar Purposes: Clocks (1967)

ilar Purposes: W ashing Machines (1968)

ilar Purposes: Spin Extractors (1968)

ilar Purposes: Shavers, Hair Clippers and Simi/

ilar Purposes: Massage Appliances (1967)

ilar Purposes (1964)

Spec. for

Spec. for

Spec. for

Spec. for

Spec. for

Spec. for

ignation of the Quantities Characterizing the Magnetic and

ers, Generators (With Associated Prime Movers) and Motors,

Rec. Meth. for Measuring Performance of Household

d Before Entry into Serv/

Rec. for Rules for the Test. of

similar Electrical Appliances: Particular Requirements for

thermal Endurance of Enamelled Wire by the Lowering of the

Procedure for Thermal Endurance of Insulating Varnishes

Rec. for Meth. for the Determination of the

r Frequencies (1967) Rec. Meth. of Test for ication by Hour Numbers of the Phase Conductors of 3 Phase Rec. for Rules for

Rec. for International Electrotechnical Vocabulary: for Industrial Trucks: Voltages of Traction Batteries for
Rec. for Closed End Drop Wires hines (I969) ssification of Hazardous Areas (1972)

ssification of Maximum Surface Temperatures (19/

struction and Test of Flameproof Enclosures of /

struction and Test of Electrical Apparatus, Typ/

eral Introduction (197I)

king (I970)

h. of Test for Ignition Temperature (1966)

Immersed Apparatus (1968)

ssurized Enclosures (1962)

d Filled Apparatus (1967)

d Filled Apparatus: Use of a Screen (I967)

rk Test Apparatus for Intrinsically Safe Circui/

Rec. for

Rec. for

Rec. for

Rec. for

Rec. for

Rec. for

Rec. for

Rec. for

Rec. for

Rec. for

Rec. for

spheres: Construction and Test of Flameproof Enclosures of us for Explosive Gas Atmospheres: Construction and Test of

washers (I97I)

tric Irons (197)

tric Shavers, H/

$r$ Treatment And

es (1971)

Extractors (I9/

ters, Grills, W/

um Cleaners (I9/

ing Machines ( $\mathrm{I} /$

uctors for Aircraft (1969)

uctors for $\mathrm{Ai}$ / Rec. for Safety of Household and Similar Rec. for Safety of Household and Similar Rec. for Safety of Household and Similar Rec. for Safety of Household and Similar Rec. for Safety of Household and Similar Rec. for Safety of Household and Similar Rec. for Safety of Household and Similar Rec. for Safety of Household and Similar Rec. for Safety of Household and Similar Rec. for Safety of Household and Similar Rec. for General Purpose
Rec. for Meth. of Test for General Purpose $r$ Performance Requircments for Heat Resisting (I90 ${ }^{\circ} \mathrm{C}$ ) Rec. for Meth. of Test for Heat Resisting (190 Deg.)

I97/ $r$ Performance Requirements for Heat Resisting $\left(260^{\circ} \mathrm{C}\right)$

1)

Rec. for Meth. of Test for Heat Resisting $\left(260^{\circ} \mathrm{C}\right.$ )

I91

I967) Rec. for Performance Requirements for General Purpose $n$ s and Conductor Resistance of Heat Resisting $\left(260^{\circ} \mathrm{C}\right)$ for Dimensions and Conductor Resistance of General Purpose ns and Conductor Resistance of Heat Resisting $\left(190^{\circ} \mathrm{C}\right.$ ) Std. for Crimped Joints for Aircraf

Aluminium Terminal Ends for Crimping to Aircraft Aluminum c Endless V.Belts (Belts) (Sections Y, Z, A, B, C, D, E)

th. of Test (1962) Rec. for
Electric Cooking and Heating Appliances for Domestic an

Electric Cooking and Heating Appliances for Domestic an

Electric Cooking and Heating Appliances for Domestic an

Electric Cooking and Heating Appliances for Domestic an

Electric Cooking and Heating Appliances for Domestic an

Electric Cooking and Heating Appliances for Domestic an

Electric Cooking and Heating Appliances for Domestic an

Electric Cooking and Heating Appliances for Domestic an

Electric Energy Generation (1968)

Electric Fans and Regulators for Use in Ships (1969)

Electric Fans and Regulators (1971)

Electric Fans and Regulators (1972)

Electric Fence Controllers (1956)

Electric Fence Controllers (1956)

Electric Infra Red Emitters for Heating Purposes (1967)

IEC

CEE

CEE

Electric Irons for Household or Similar Use (1970)

IC for Safety of Household and IEC

lectric Irons (I97I)

Electric Irons, Ironers

C. Machines (1965)

CEE

CEE

Electric Means (1968)

Elor Operated Appliances for Domestic and Sim CEE

Electric Motor Operated Applias

Electric Motor Operated Appliances for Domestic and Sim

Electric Motor Operated Appliances for Donestic and Sim

lectric Motor Operated Appliances for Domestic and Sim

Electric Motor Operated Appliances for Domestic and Sim

Electric Properties of Vacuum and a Substance (1966)

Electric Propulsion Plant, Tankers (1965)

Electric Ranges and Ovens (1971)

Electric Rolling Stock on Completion of Construction an

Electric Shavers, Hair Clippers and Similar Items (I972

Electric Strength Between Twisted Wires (1966)

Electric Strength Meth. (1971)

Electric Strength of Insulating Oils (1963)

TF the IE

Electric Strength of Solid Insulating Materials at Powe IEC

Electric Systems (1963)

Electric Traction Equipment (1968)

Electric Traction (I957)

Electric Trucks (1969)

(Electrical and Meclianical) for Automatic Drawing-in Mac

Electrical Apparatus for Explosive Gas Atmospheres: Cla

Electrical Apparatus for Explosive Gas Atmospheres: Cla

Electrical Apparatus for Explosive Gas Atmospheres: Con

Electrical Apparatus for Explosive Gas Atmospheres: Con

Electrical Apparatus for Explosive Gas Atmospheres: Gen

Electrical Apparatus for Explosive Gas Atmospheres:

Electrical A pparatus for Explosive Gas Atmospheres: Oil

Electrical Apparatus for Explosive Gas Atmospheres: Pre

Electrical Apparatus for Explosive Gas Atmospheres: San

Electrical Apparatus for Explosive Gas Atmospheres: San

Electrical Apparatus for Explosive Gas Atmospheres: Spa

Electrical Apparatus (1971) /Us for Exp
Electrical Apparatus, Type of Protection E (1969)

Electrical Appliances: General Requirements (1970)

Electrical Appliances: Particular Requirements for Dish

Electrical Appliances: Particular Requirements for Elec

Electrical Appliances: Particular Requirements for Elec

Electrical Appliances: Particular Requirements for Floo

Electrical Appliances: Particular Rcquirements for Rang

Electrical Appliances: Particular Requirements for Spin

Electrical Appliances: Particular Requirements for Toas

Electrical Appliances: Particular Requirements for Vacu

Electrical Appliances: Particular Requirements for Wash

Electrical Cables with Aluminium or Aluminum Alloy Cond

Electrical Cables with Aluminum or Aluminium Alloy Cond

Electrical Cables with Copper Conductors for Aircraft (

Electrical Cables with Copper Conductors ior Aircraft (

Electrical Cables with Copper Conductors for Aircraft (

Electrical Cables with Copper Conductors for Aircraft (

Electrical Cables with Copper Conductors for Aircraft (

Electrical Cables with Copper Conductors for Aircraft (

Electrical Cables with Copner Conductors for Aircraft (

Electrical Cables with Copper Conductors, for Aircraft

Electrical Cables with Copper Conductors, for Aircraft

Electricai Cables (1973)

Electrical Cables (1973)

Electrical Conductivity-Characteristic and Meth. of T

Electrical Conductivity of Conveyor Belts: Spec. and Me

Electrical Conductor Wirc (1959)

Electrical Conductor Wire (1960)

Rec. Cor Commercial Annealed Aluminium (Aluminum) 
hiclew with 24-V Electrical Equipment for Comm/ irical fiquipment Particularly for Private Motor/ l Vorahulary: Generation, Transmission and Distribution of nections Between Prime Movers and Towed Vehicles with 24.V eral Use ( 1965$)$

ries Production I,ines Lines (1967)

Rec. for for Houaehold and

Std. for Safety Requirements for th Rec. for Safety Requirements for the ec. for Electrical Connections for Vehicles with 6 or 12 Aim, Structure, and Results of the CEE c. for Std. Form of Declaration of Performance of Aircraft Spec. for Rigid Conduits of Polyvinyl Chloride for Accumulator (Storage), Batteries, Heating An/

Rec. for Rec. for Rec. for Rec. for Rec. fo Rec. for er and Lighting, Semiconductor Rectifiers, Gene/ ciples (1970) nitions $(1970)$

Spec. for Steel Conduits and Fittings for Rec. for Tegts on Large Hollow Porcelains for Use in $\mathbf{n}$ of the Permittivity and Dielectric Dissipation Factor of rec. Meth. of Test for Volume and Surface Resistivities of of Test Procedures for Evaluating the Thermal Endurance of Conditions for Use Prior to and During the Test. of Solid d Test (/ Rec. for Evaluation of the Thermal Endurance of for the Classification of Materials for the Insulation of the Measurement of the Airborne Noise Emitted by Rotating Rec. for Dimension of Brushes and Holders for es (1962) Rec. for Dimensions of Brushes and Holders for ge N/ Rec. for Dimensions and Output Ratings for Rotating Rec. for Rules for Rotatin

Rec. for Dimensions and Output Ratings for Foot Mounted 8 (1968)

for Determining Physical Properties of Brush Materials for fficiency from Tests (Excluding Machin/ machine Quantities from Tests (1967) Rec. for Rotatin Rec. for Rotating Rec. for Rotating Rec. for Rotating Rec. for Rotating Rec. for Rotating Rec. for Rotating Rec. for Rotating Rec. for Rotating Rec. for Index of

hase, $50 \mathrm{~Hz}$ Turbine Type Machines (196/ and Mounting Arrangements of (1972) Rotation (1972) ionizing Radiation (1964) (I968)

Rec. for Direct Recording Rec. for Indicating Rec. for Diagrams, Charts, Tables: Item Designation for Std. for Tests for Aircraf quivalent Input and Output / eater or Filament Current (/ easurement of Electrode Cur/ eth. of Measurement of Audi/ eth. of Measurement of Radi/ eth. of Measuring Electrode/ eth. of Meaaurement of Emis/ eth. of Measurement of Rada/ eth. of Measurement of Spur eth. of Measurement of $\mathrm{Gaaf} /$ eth. of Meaaurement of Nois/ eth. of Meaaurement on Coro/ eth. of Meaaurement of Thyr eth. of Measurement of Cros/ eth. of Meaaurement of Vacu/ eth. of Measuring Hiss and / eth. of Application of Mech/ eaaurement of Equivalent $\mathrm{No}$ easurement of Cathode Heati eth. of Meaauring the Catho/ oiae Factor (I963)

recautiona Relating to Meth/ aurement for Television Pic/ aurement for Cold Cathode C/ surement of Geiger-Muller/ surement for Camera Types (/) ckward Wave Oscillator Tubes/ a Filled Microwave Switching/ neral (1972)

gh Power Klyatrons (1972) w Power Oscillator Klystrona/ gnetrons (I972)

rminology (I972) ea (1972) c. for Plastica-Meth of Teat to Determine the Change i ency Range Form 3 / Rec. For Measurements Rec. for Measurements of th Rec. for Measurements of the Rec. for Measurements of the Rec. for Measurements of the Rec. for Meaaurements of the Rec. for Measurements of the Rec. for Measurementa of the Rec. for Measurements of the Rec. for Measurements of the Rec. for Measurements of the Rec. for Measurements of the Rec. for Measurements of the Rec. for Measurements of the Rec. for Measurements of th Rec, for Measurements of the Rec. for Measurements of the Rec. for Measurements of the Rec. for Measurements of th Rec. for Measurements of the Rec. for Measurements of the Rec. for Measurements of the Rec. for Measurements of the Rec. for Meaaurements of the Rec. for Mea aurement of the Rec. for Mea aurement of the Rec. for Meaaurement of the Rec. for Mea aurement of the Rec. for Mea aurement of the Rec. for Meaaurement of the Rec. for Measurement of the Rec. for Measurement of the
Electrical Connections Between Prime Movers and Towed V ISO

Electrical Connections for Vehicles with 6 or $12 \mathrm{~V}$ Elec Nternational Electrotechnica IE Electrical Energy (1965) /Nternational Electrot
Electrical Equipment for Commercial International Traff Electrical Equipment of Machine Tools: Electronic Types Electrical Equipment of Machine Tools: Machines for Gen Electrical Equipment of Machine Tools: Used in Large Se Electrical Equipment of Refrigerators and Food Freezers Electrical Equipment of Room Air Conditioners (1972) Electrical Equipment Particularly for Private Motor Car (Electrical Equipment Safety) (196I)

Electrical Equipment (I961)

Electrical Installations and Their Fittings (1968)

Electrical Installations in Ships: Accessories, Lightin

Electrical Installations in Ships: Cables (Construction

Electrical Installations in Ships: General Requirements Electrical Installations in Ships: Graphical Symbols (1 Electrical Installations in Ships: Switchgear, Electric

Electrical Installations in Ships: Transformers for Pow Electrical Installations of Buildings: Fundamental Prin Electrical Installations of Buildings: General and Defi Electrical Installations (1962)

Electrical Installations (1967)

Electrical Insulating Materials at Power, Audio and Rad Electrical Insulating Materials (1958)

Electrical Insulating Materials (1966)

Electrical Insulating Materials (1971)

Electrical Insulating Varnishes by the Helical Coil Bon

Electrical Machinery and Apparatus in Relation to Their Electrical Machinery (1970) Rec, for Test Code for

ectrical Machinery: Complementary Dimensions and Term

Electrical Machinery: Principal Dimensions and Toleranc

Electrical Machines .- Frame Numbers 56 to 400 and Flan

Electrical Machines for Rail and Road Vehicles (1971)

Electrical Machines with Frame Numbers 355 to 1000 (197

Electrical Machines (1972)

Rec. for Test Proc

Electrical Machines: Degrees of Protection by Enclosure

Electrical Machines: Meth for Determining Synchronous

Electrical Machines: Meth. of Cooling (1969)

Electrical Machines: Noise Limits (1972)

Electrical Machines: Rating and Performance (1969)

Electrical Machines: Ratings and Characteristics of $3 \mathrm{P}$

Electrical Machines: Symbols for Types of Construction

Electrical Machines: Terminal Markings and Direction of

Electrical Measuring Apparatus Used in Connection with

Electrical Measuring Instruments and Their Accessories

Electrical Measuring Instruments and Their Accessorie

Electrical Parts and Equipment (I97I)

Electrical Plug and Socket Connectors (1972)

Electrical Properties of Electronic Tubes and Valves: E Rec. for Measurements of the
Electrical Properties of Electronic Tubes and Valves: $H$

Electrical Properties of Electronic Tubes and Valves:

Electrical Properties of Electronic Tubes and Valves: $M$ Electrical Properties of Electronic Tubes and Valves: M Electrical Properties of Electronic Tubes and Valves: $M$ Electrical Properties of Electronic Tubes and Valves: $M$ Electrical Properties of Electronic Tubes and Valves: $M$ Electrical Properties of Electronic Tubes and Valves: $M$ Electrical Properties of Electronic Tubes and Valves: $M$ Electrical Properties of Electronic Tubea and Valves: $M$ Electrical Properties of Electronic Tubes and Valves: M Electrical Properties of Electronic Tubes and Valves: $M$ Electrical Properties of Electronic Tubes and Valves: $M$ Electrical Properties of Electronic Tubes and Valves: $M$ Electrical Properties of Electronic Tubes and Valves: $M$ Electrical Properties of Electronic Tubes and Valves: M Electrical Properties of Electronic Tubes and Valves: $M$ Electrical Properties of Electronic Tubes and Valves: $M$ Electrical Properties of Electronic Tubes and Valves: $M$ Electrical Properties of Electronic Tubes and Valves: $N$ Electrical Properties of Electronic Tubes and Valves: $P$ Electrical Properties of Electronic Tubes: Meth. of Mea Electrical Properties of Electronic Tubes: Meth. of Mea Electrical Properties of Electronic Tubes: Meth. of Mea Electrical Properties of Electronic Tubes: Meth. of Mea Electrical Properties of Microwave Tubes and Valves: Ba Electrical Propertiea of Microwave Tubea and Valvea: $\mathrm{Ga}$ Electrical Properties of Microwave Tubea and Valves: Ge Electrical Propertiea of Microwave Tubes and Valves: $\mathrm{H}$ Electrical Propertiea of Microwave Tubes and Valvea: Lo Electrical Propertiea of Microwave Tubes and Valves: $\mathrm{Ma}$ Electrical Propertiea of Microwave Tubes and Valves: Te Electrical Propertiea of Polyethylene Due to the Migrat Electrical Propertiea of Receiving Aerials in the Frequ Electrical Properties of Microwave Tubea: Diak Seal Tub 
Rere. for Filectrical Installations in Ships: Switchgear, Kec. for Varnish Fabrics for
Rec. for Varnish Fabrics for in (1972)

(1967)

suring, with Nonspecified or Independent Specif/ Rec. for erials at Elevated Temperatur/ Rec. for Meth. of Test for

Rec for Connections for Aircraft Rec for

Rec. for Drop Wires for Mechanical and Spec. for Switches

Rec. for Diagrams, Charts, Tables (Electrotechnology or Rules for Ohmic Resistors Used in the Power Circuits of Rec. for Quantities and Units of Rec. for Symbols for Alternating Current Rec. for the Field of Quantities and Units Used in Rec. for General Test Conditions for mea/ Rec. for Meth. for Specifying the Characteristics of (a) Rec. Meth. for Measurements of the

Rec. for International Electrotechnical Vocabulary: Rec. for International Electrotechnical Vocabulary: Rec. for International Electrotechnical Vocabulary: Properties of Electronic Tubes and Valves: Measurement of and Valves: Meth. of Measurement of Spurious and Unwanted

Rec. for Dimensions of Tubula Determining Whether an perties of Electronic Tubes and Valves: Meth. of Measuring netic Substrates-Measurement of Coating Thick

Std. fo overy and Deposition Coefficient (1972) and Valves (1961)

High Tensile Types/ Gas Welding (1967) Rec. for Diameters and Tolerances for Steels-Code of Symbols for Identific/ Rec. for Numbering of 969)

high Tensile Steel (1/ or Straight Manual Arc Welds Made with Covered the Mechanical Properties of the Weld Metal Deposited by

Rec. for Dimensions of Straight Resistance Spot Welding

Rec. for Dimensions of Straight Resistance Spot Welding

hanical Test. to Determine the Coding for Deep Penetration Rec. for International Electrotechnical Vocabulary: mical Analysis of Zinc Alloys-Determination of Copper by

Rec. for Fixed Tantalum Capacitors with Liquid or Solid r Industrial Use-Determination/Water Content Using an general Purpose Applicatio/ Rec. for Aluminium (Aluminum $\begin{array}{ll}\text { N/ } & \text { Rec. for Chemical Analysis of Copper and Its Alloys } \\ \text { II/ } & \text { Rec. for Chemical Analysis of Copper and Its Alloys }\end{array}$ Rec. for Chemical Analysis of Aluminum and Its Alloys:

Rec. for Electrotechnical Vocabulary:

Switchgear, Mechanical Controls, Starters and Elements of national Electrotechnical Vocabulary: Electrochemistry and

eading Capacitor Type Pocket Exposure Meters and Accessory ar Gene/ Spec. for Safety Requirements for Mains Operated ar Gener/ Rec. for Safety Requirements for Mains Operated d Cut Cores of Grain Oriented Silicon Iron Alloy, Used for witches for Use in Equipment for Telecommunication and in

rimarily for Use in Equipment for Telecommunication and in Rec. for Basic Environmental Test. Procedures for Rec. for Basic Environmental Test. Procedures for Rec. for Basic Environmental Test. Procedures for 1966) steady/ d Damp/ 67) Rec. for Basic Environmental Test. Procedures for Rec. for Basic Environmental Test. Procedures for Rec. for Basic Environmental Test. Procedures for Rec. for Basic Environmental Test. Procedures for $\begin{array}{ll}\text { 8) } & \text { Rec. for Basic Environmental Test. Procedures for } \\ \text { ion, S/ } & \text { Rec. for Basic Environmental Test. Procedures for }\end{array}$ 960) Rec. for Basic Environmental Test. Procedures for th (19) Rec. for Basic Environmental Test. Procedures for (1964/ Rec. for Basic Environmental Test. Procedures for essure/ Rec. for Basic Environmental Test. Procedures for temper/ Rec. for Basic Environmental Test. Procedures for 968 )

$1968)$

Rec. for Basic Environmental Test. Procedures for Rec. for Basic Environmental Test. Procedures for Rec. for Basic Environmental Test. Procedures for of $\mathrm{Te} /$ Rec. for Dimensions of Spindle Ends for Manually Operated havior) with Respect to Possible Hazards When Dealing with list of Basic Terms and Definitions for the Reliability of Rec. for Safety Equipment for Splash Proof Mains Operated c. for Receiver Type Metallized Mica Capacitors for Use in
Electrical Protection, Distrihution and Controlgear (19

Electrical Purposes: Definitions and General Requiremen Electrical Purposes: Meth. of Test (1972)

Electrical Relays: Instantaneous All or Nothing Relays

Electrical Relays: Single Input Energizing Quantity Mea

Electrical Relays: Specified Time All or Nothing (1969)

Electrical Resistance and Resistivity of Insulating Mat

Electrical Safety of Office Machines (1972)

Electrical Supplies (1965)

Electrical Systems (1961)

Electrical Warp Stop Motions (1965)

(Electrical) for Domestic and Similar Purposes (1955)

Electrical) (1959)

Electrically Powered Vehicles (1970)

Electricity and Magnetism (1965)

Electricity Meters (1972)

Electricity (1964)

Electro Heating Appliances (1972)

Electro Mechanical Transducers for Shock and Vibration

Electroacoustical Characteristics of Hearing Aids (1959

Electroacoustics (1960)

Electrobiology (1959)

Electrochemistry and Electrometallurgy (1960)

/Asurements of the Electrical IEC

Electrode Current (1963)

-Perties of Electronic Tubes

Electrode Holders for Spot Welding Machines (1969)

Electrode Is a Deep Penetration Type (1967)

Electrode Resistance and Transconductance, Amplificatio

Electrodeposited Nickel Coatings on Magnetic and Nonmag

Electrodes-Determination of the Efficiency, Metal Rec

Electrodes and Designation of Units in Electronic Tubes

Electrodes for Arc Welding of Mild Steels and Low Alloy

Electrodes for Manual Arc Welding and Filler Metals for

Electrodes for Manual Arc Welding of Mild and Low Alloy

Electrodes for Manual Metal Arc Welding of Cast Iron (1

Electrodes for the Welding of Mild Steel and Low Alloy

Electrodes of Cathode Ray Tubes (1967)

Electrodes of Mild and Low Alloy High Tensile Steels (1

Electrodes with Threaded Sockets and Connecting Pins Fo

Electrodes $3.15 \mathrm{~mm}$ or More in Diameter (1967)

Electrodes (For Loads Greater Than 1500 kgf) (1969)

Electrodes (1968)

Electrodes (1968)

Electroheating Applications (1960)

ISO

Electrolysis (1971)

Rec. for Special Meth. of Mec

Electrolyte (1971)

Electrolytic Analyser (1972) Std. for Liquid Chlorine F

Electrolytic Capacitors for Long Life (Type 1) and for

Electrolytic Cathode Copper (1972)

Electrolytic Determination in Unalloyed Type Containing

Electrolytic Determination in Wrought and Cast-Copper a

Electrolytic Determination of Copper in Aluminium Alloy

Electrolytic Tough Pitch Copper Refinery Shapes (1972) Rec. for Che ISO

ISO

Electromechanical Applications (1958)

Electromechanical Relays (1963)

Electrometallurgy (1960)

Electrometers (1971)

Electronic and Related

Electronic and Related Equipment for Domestic and Simil

Electronic and Telecommunication Equipment (1971)

Electronic Applications Employing Similar Techniques: G

Electronic Applications Employing Similar Techniques: G

Electronic Components and Equipment: General (I968)

Electronic Components and Equipment: Pt. 2 Test (1968)

Electronic Components and Equipment: Test A: Cold (1966

Electronic Components and Equipment: Test B: Dry Heat (

Electronic Components and Equipment: Test Ca: Damp and

Electronic Components and Equipment: Test D: Accelerate

Electronic Components and Equipment: Test Ea: Shock (19

Electronic Components and Equipment: Test Eb: Bump (196

Electronic Components and Equipment: Test Ga: Accelerat

Electronic Components and Equipment: Test H: Storage (1

Electronic Components and Equipment: Test J: Mould Grow

Electronic Components and Equipment: Test Ka: Salt Mist

Electronic Components and Equipment: Test M: Low Air Pr

Electronic Components and Equipment: Test N: Change of

Electronic Components and Equipment: Test Q: Sealing (1

Electronic Components and Equipment: Test T: Soldering

Electronic Components and Equipment: Test U: Robustness

Electronic Components (Or Parts) (1970)

Electronic Components (1972)

Electronic Equipment and Other Employing Similar Techni

Electronic Equipment and the Components (Or Parts) Used

Electronic Equipment (Domestic and Similar General Use)

Electronic Equipment (1959)

Electronic Equipment: Terminology and Meth. of Test (19

IEC

IEC

IEC

IEC

IEC

ISO

ISO

CEE

IEC

ISO

IEC

IEC

IEC

IEC

IEC

EC

IEC

ISO

IEC 
Rec. for External Diameter of Planchets Used in Nuclear

Rec for Dimensions of Panels and Racks (For Nuclear bility, and Maintainability Data from Field Performance of Rec. for Documentation to Be Supplied with Rec. for Safet y Requirements for

Rec. for Expression of the Functional Performance of c. for Numbering of Electrodes and Designation of Units in $r$ the Measurement of Direct Interelectrode Capacitances of Rec. for Dimensions of t / Rec. for Measurements of the Electrical Properties of eth. of Test (1963)
(/ Rec. for Measurements of the Electrical Properties of $\begin{array}{ll}\text { (I) } & \text { Rec. for Measurements of the Electrical Properties of } \\ \text { ti) } & \text { Rec. for Measurements of the Electrical Properties of }\end{array}$ ur/ Rec. for Measurements of the Electrical Properties of no/ Rec. for Measurements of the Electrical Properties of ch/ Rec. for Measurements of the Electrical Properties of di/ Rec. for Measurements of the Electrical Properties of os/ Rec. for Measurements of the Electrical Properties of is/ Rec. for Measurements of the Electrical Properties of sf/ Rec. for Measurements of the Electrical Properties of is/ Rec. for Measurements of the Electrical Properties of di) Rec. for Measurements of the Electrical Properties of da/ Rec. for Measurements of the Electrical Properties of ur/ Rec. for Measurements of the Electrical Properties of yr/ Rec. for Measurements of the Electrical Properties of cu/ Rec. for Measurements of the Electrical Properties of ro/ Rec. for Measurements of the Electrical Properties of de/ Rec. for Measurements of the Electrical Properties of d / Rec. for Measurements of the Electrical Properties of ho/ Rec. for Measurements of the Electrical Properties of Rec. for Measurements of the Electrical Properties of th/ Rec. for Measurements of the Electrical Properties of s of Wiring Jigs and Pin Straighten/ Rec. for Sockets for 1 Symbols: Variability, Examples of Resistors, Elements of C/ $\quad$ Rec. for Measurements of the Electrical Properties of ic/ Rec. for Measurements of the Electrical Properties of harge Storage Types (1971) Rec. for

er/ Rec. for Measurements of the Electrical Properties of of Application of Mechanical Shock (Impulse) Excitation to ment of Emission Current from Hot Cathodes for High Vacuum Rec. for Electrical Equipment of Machine Tools: Is to Be Used in Electrotechnology: Telecommunications and 7I) um on Steel (Or Iron) (1970)

)

B (1967)

regulating Systems (1966)

Rec. for Direct Reading Pocket Type of Ionizing Radiation by Electric/

rometallurgy (1960)

s (1960)

ions (1958)

956)

nd Distribution of Electrical Ene/

(1956)

lectric Energy Generation (1968)

Physics (1964)

measuring Instruments (1958)

paratus for Railways (1959)

for Connection and Regulation (1/ 1970) Rec. for Rec. for Rec. for Rec. for Std. for Rec. for Rec. for Rec. for Rec. for Meth for the Designation of Rec. for International Rec. for International Rec. for International Rec. for International Rec. for International Rec. for International Rec. for International Rec. for

Rec. for International Rec. for International Rec. for International Rec. for International Rec. for International Rec. for International Rec. for International Rec. for International Rec. for International Rec. for International Rec. for International Rec. for International Rec. for International Rec. for International Rec. for International Rec. for International Rec. for International

Rec. for Diagrams, Charts, Tables Rec. for Diagrams, Charts, Tables Used in Rec. for Letter Symbols to Be Used in 1972) Rec. for Letter Symbols to Be Used in
Electronic lnstruments (1967)

Electronic Instruments) (1968)

Electronic ltems (1971)

Electronic Measuring Apparatus (1968)

Electronic Measuring Apparatus (1971)

Electronic Measuring Equipment (1971)

Electronic Tubes and Valves and Analogous Semiconductor

Electronic Tubes and Valves (1961)

Electronic Tubes and Valves (1962)

Electronic Tubes and Valves (1966)

IE

IEC

IEC

IEC

IEC.

IEC

Re IEC

Rec. Meth. Fo IEC

Electronic Tubes and Valves: Equivalent Input and Outpu

Electronic Tubes and Valves: General Requirements and $M$

Electronic Tubes and Valves: Heater or Filament Current

Electronic Tubes and Valves: Measurement of Cathode Hea

Electronic Tubes and Valves: Measurement of Electrode C

Electronic Tubes and Valves: Measurement of Equivalen

Electronic Tubes and Valves: Meth. of Application of Me

Electronic Tubes and Valves: Meth. of Measurement of Au

Electronic Tubes and Valves: Meth. of Measurement of $\mathrm{Cr}$

Electronic Tubes and Valves: Meth. of Measurement of Em

Electronic Tubes and Valves: Meth. of Measurement of $\mathrm{Ga}$

Electronic Tubes and Valves: Meth. of Measurement of No

Electronic Tubes and Valves: Meth. of Measurement of Ra

Electronic Tubes and Valves: Meth. of Measurement of $\mathrm{Ra}$

Electronic Tubes and Valves: Meth. of Measurement of $S p$

Electronic Tubes and Valves: Meth. of Measurement of Th

Electronic Tubes and Valves: Meth. of Measurement of Va

Electronic Tubes and Valves: Meth of Measurement on Co

Electronic Tubes and Valves: Meth. of Measuring Electro

Electronic Tubes and Yalves: Meth. of Measuring Hiss an

Electronic Tubes and Valves: Meth. of Measuring the Cat

Electronic Tubes and Valves: Noise Factor (1963)

Electronic Tubes and Valves: Precautions Relating to $\mathrm{Me}$

Electronic Tubes and Valves: Spec. Sheets and Dimension

Electronic Tubes, Valves and Rectifiers (1964)

Electronic Tubes: Meth. of Measurement for Camera Type

Electronic Tubes: Meth. of Measurement for Cold Cathode

Electronic Tubes: Meth. of Measurement for Television P

Electronic Tubes: Meth. of Measurement of Cathode Ray $C$

IEC

IEC

IEC

IEC

IEC

IEC

IEC

IEC

IEC

IEC

IEC

IEC

IEC

IEC

IEC

IEC

IEC

IEC

IEC

IEC

ectronic Tubes: Meth. of Measurement of Geiger-Mull

Electronic Types (1965)

Electronic Types (1966)

Electronic Types (1968)

Onic Tubes and Valves: Meth.

Electronic Voltmeters (1967)

and Valves: Meth. of Measure

Electronics (1956)

Electronics (1972)

Electroplated Coatings of Cadmium on Iron and Steel (19

Electroplated Coatings of Nickel Plus Chromium (1970)

Electroplated Coatings of Nickel (1970)

Electroplated Coatings of Tin Nickel Alloy (1972)

Electroplated Coatings of Tin (1971)

Electroplated Coatings of Zinc on Iron and Steel (1971)

Electroplating and Related Processes-Vocabulary (1971

Electroscope Exposure Meters (1971)

Electrostatic Deflecting Electrodes of Cathode Ray Tube

Electrotechnical Vocabulary: Automatic Controlling and

Electrotechnical Vocabulary: Detection and Measurement

Electrotechnical Vocabulary: Electric Traction (1957)

Electrotechnical Vocabulary: Electroacoustics (1960)

Electrotechnical Vocabulary: Electrobiology (1959)

Electrotechnical Vocabulary: Electrochemistry and Elect

Electrotechnical Vocabulary: Electroheating Application

Electrotechnical Vocabulary: Electromechanical Applicat

Electrotechnical Vocabulary: Electronics (1956)

Electrotechnical Vocabulary: Fundamental Definitions (1

Electrotechnical Vocabulary: General Index (1970)

Electrotechnical Vocabulary: Generation, Transmission a

Electrotechnical Vocabulary: Lighting (1970)

Electrotechnical Vocabulary: Machines and Transformers

Electrotechnical Vocabulary: Nuclear Power Plants for E

Electrotechnical Vocabulary: Protective Relays (1956)

Electrotechnical Vocabulary: Radiocommunications (1970)

Electrotechnical Vocabulary: Radiology and Radiological

Electrotechnical Vocabulary: Scientific and Industrial

Electrotechnical Vocabulary: Signalling and Security Ap

Electrotechnical Vocabulary: Static Convertors (1956)

Electrotechnical Vocabulary: Switchboards and Apparatus

Electrotechnical Vocabulary: Telegraphy and Telephony (

Electrotechnical Vocabulary: Transducers (1955)

Electrotechnical Vocabulary: Waveguides (1961)

Electrotechnology-Electrical) (1959)

Electrotechnology: Definitions and Classification (1971

Electrotechnology: General (1971)

Electrotechnology: Telecommunications and Electronics

Elements and Recording Directly by Means of Stylus and

149-1

151-2

151.8

151-1

151-7

151-6

151-10

151-21

151-13

$151-17$

$151-18$

151-11

151-14

151-15

$151-20$

151-23

151-19

151-12

151-5

151.9

$151-4$

151-0

149-2

117-6

$151-26$

151-22

151-16

151.24

151.25

151-6

151-13

204-3

217

$50(07)$

27-2

2082

1457

1456

1458

2179

2081
2080

1758

236

$50(37)$

$50(66)$

$50(30)$

$50(08)$

$50(70)$

$50(50)$

$50(40)$

$50(35)$

$50(07)$

$50(05)$

$50(00)$

$50(25)$

$50(45)$

$50(10)$

$50(26)$

$50(16)$

$50(60)$

$50(65)$

$50(20)$

$50(31)$ 
re and Vacuum, and Vacuum Gauges, with Pressure Responsive 4. Contacts, Switchgear, Mechanical Controls, Starters and ec. Graphical Symbols: Variability, Examples of Resistors, Rec. for Bibliographical References: Essential ent, Distribution Systems, Meth. of Connection and Circuit or Bibliographical References: Essential and Supplementary Rec. for Sound System Equipment: Auxiliary Passive Rec. for Graphical Symbols: Binary Logic Rec. for Interrupted Creep Test. of Steel at Rec. for Noninterrupted Creep Test. of Steel at Rec. for Creep Stress Rupture Test. of Steel at etermination of Proof Stress and Proving Test for Steel at ical Resistance and Resistivity of Insulating Materials at Proof Stresses (S/ Rec. for Mechanical Test. of Steel at astics (/ Rec. for Determination of Breaking Strength and cellular Materials-Determination of Tensile Strength and Rec. for Full Thickness Tensile Strength and 1962) om Packages-Meth. for Determination of Breaking Load and es of Electronic Tubes and Valves: Meth. of Measurement of ndustrial Use-Determination of Potassium Content-Flame oxide for Industrial Use-Determination of Sodium-Flame on of Aluminium-Determination of Sodium Content-Flame of Measurement on Radio Receivers for Various Classes of of Measurement on Radio Receivers for Various Classes of of Measurement on Radio Receivers for Various Classes of oor Use (1972) Std. for Measurement of Airborne Noise

for the Preparation of Test Codes for Measuring the Noise c. for Test Code for the Measurement of the Airborne Noise Rec. for Measurement of Noise Rec. for Characteristics of Electric Infra Red

e Hazards When Dealing with Electronic Equipment and Other pment for Telecommunication and in Electronic Applications pment for Telecommunication and in Electronic Applications Sound Motion Picture Film (1956)

Sound Motion Picture Film (1956)

mm Sound Motion Picture Film (1956)

ure Film (1956)

ure Film (1956)

ion of $16 \mathrm{~mm}$ Silent Motion Picture Film (1956)

ion of $8 \mathrm{~mm}$ Silent Motion Picture Film (1956)

Rec. for Identification of the

e Cameras (1968)

Std. for Nonmagnetic Metallic and Vitreous or Porcelain

Rec. for Particular Types of Winding Wires: Bunched

under / Rec. Spec. for Particular Types of Winding Wires: Spec. for Particular Types of Winding Wires: Self Fluxing pes of Winding Wires: Heat or Solvent Bonding Self Fluxing (1970/ Rec. Spec. for Particular Types of Winding Wires: for Use in / Rec. for Particular Types of Winding Wires: (1972) Rec. for Particular Types of Winding Wires: (1972) Rec. for Particular Types of Winding Wires: (1970/ Rec. Spec. for Particular Types of Winding Wires:

particular Types of Winding Wires: Heat or Solvent Bonding for Use in / Rec. for Particular Types of Winding Wires: dimensions of Winding Wires: Maximum Over All Diameters of Rec. Meth. of Test for Winding Wires:

t Procedure for the Evaluation of the Thermal Endurance of ics to Colour (Color) Change Upon Exposure to Light of the Rec. for Rec. for

Rec. for Long Rec. for Short

Rec. for High Voltage Metal 1963)

Meth. for Maintaining Constant Relative Humidity in Small Rec. for ${ }^{\circ} \mathrm{Of}$ Protection of

osive Gas Atmospheres: Construction and Test of Flameproof umidit $y(1968)$

Rec. for Test

rical Apparatus for Explosive Gas Atmospheres: Pressurized

for Rotating Electrical Machines: Degrees of Protection by pmm (200 and 800 RPI), NRZI, and 63 RPmm (1600 RPI), Phase Drawing.in Machines (1969)

Rec. for Closed

Carrying Rods-Dimensions and Coordination Of, and with Std. and Long Series (1970) s (1973) Rec. for (1972)

Std. for Shell Std. for

Rec. for Hardenability Test by Rec. for Shipbuilding Details-Anchor Chains for Pipe Connections for Industrial A pplication for Plain imensions and Masses Per Unit Length (19) Rec. for Plain

details-Anchor Chains, Stud Links (Common, Enlarged, and Rec. for Combinations of Double

Electrical Conductivity-Characte/ Rec. for Antistatic groove Profiles for Corresponding Pulleys (1970/ Rec. for (1973)

td for Aluminium als: Socket Fittings with Spigot Rec. for Dimensions of Spindle
Elements Giving Direct Indications by Means of a Pointe

Elements of Electromechanical Relays (1963) /Al Symbol IE

Elements of Electronic Tubes, Valves and Rectifiers (19

Elements (1958)

Elements (1960)

Elements (1968)

Rec. Graphical Symbols: Kind of Curr IEC

Rec. F ISO

Elements (1971)

Elements (1972)

Elevated Temperatures (Load and Temperature Interrupted

\section{IEC}

Elevated Temperatures (1961)

ISO

Elevated Temperatures (1961)

Elevated Temperatures (1971)

Rec. for D ISO

Elevated Temperatures: Determination of Lower Yield and Electr IEC

Elongation at Break of Fabrics Coated with Rubber or PI ISO

Elongation at Break (1971) Rec. for Flexible ISO

Elongation of Conveyor Belts: Spec. and Meth. of Test ( ISO

Elongation of Same (Of Single Strands) (1972) /Yarn Fr ISO

Emission Current from Hot Cathodes for High Vacuum Elec IEC

Emission Photometric Meth. (1971) /Sium Chloride for I ISO

Emission Photometry Meth. (1970)

Emission) Spectrophotometric Meth. (1970)

1. for Potassium Hydr ISO He Producti ISO

mission: General Conditions and Measuring Meth. Applyi

Emission: Measurements Particularly Related to the Audi

Emission: Specialized Frequency Measurements on Fm Rece

Emitted by Compressor Primeover Units Intended for Outd

for General Requirements ISO

Emitted by Rotating Electrical Machinery (1970) Re ISO

Emitted by Vehicles (1964)

Emitters for Heating Purposes (1967)

Employing Similar Techniques (1968)

Employing Similar Techniques: General Requirements to

Em ploying Similar Techniques: General Requirements and

Emulsion and Sound Record Positions in Camera for $16 \mathrm{~mm}$

Emulsion and Sound Record Positions in Camera for $35 \mathrm{~mm}$

Emulsion and Sound Record Positions in Projector for 35

Emulsion Position in Camera for $16 \mathrm{~mm}$ Silent Motion Pic

Emulsion Position in Camera for $8 \mathrm{~mm}$ Silent Motion Pict

Emulsion Position in Projector for Direct Front Project

Emulsion Position in Projector for Direct Front Project

Emulsion Side of Edge Marked Roll Film for Still Pictur

Enamel Coatings on Magnetic Basis Metals-Measurement

Enamelled Copper, with Silk Covering (1972)

Enamelled Round Copper with Good Dielectric Properties

Enamelled Round Copper (1970)

Enamelled Round Copper (1970)

Enamelled Round Copper (1970) ISpec. for Particular Ty
Enamelled Round Copper, with a Temperature Index of 155

Enamelled Round Copper, with a Temperature Index of 180

Enamelled Round Copper, with a Temperature Index of 220

Enamelled Round Copper, with High Mechanical Properties

Enamelled Round Copper, with High Mechanical Properties

Enamelled Round Copper, with High Mechanical Properties

Enamelled Round Winding Wires (1964)

Rec. for Basic

Enamelled Round (1968)

Enamelled Wire by the Lowering of the Electric Strength

Enclosed Carbon Arc (1968)

Ion of Resistance of Plast

Enclosed Scale Calorimeter Thermometers (1968)

Enclosed Scale General Purpose Thermometers (1970)

Enclosed Scale Thermometers for Precision Use (1968)

Enclosed Scale Thermometers for Precision Use (1968)

Enclosed Switchgear and Controlgear (1969)

Enclosures by Means of Aqueous Solutions (1966)

Enclosures for Low Voltage Switchgear and Controlgear (

Enclosures of Electrical Apparatus (1971)

Enclosion

Enclosures (1962)

Enclosures (1968)

Encoded (1971)

Rec. for Elect IEC
Rec. IEC

Rec. IEC

Wires (Electrical and Mechanical) for Automati ISO

End Loops of Healds (1967) Rec. for Heal ISO

End Mills with Parallel Shanks with Morse Taper, Shanks ISO

End Mills with Plain Bore and Tenon Drive: Metric Serie ISO

End Mills with 7/24 Taper Shanks-Std, and Long Series ISO

End Quenching Steel (Jominy Test) (1967)

End Shackles (1963)

End Steel and Other Metal Tubes (1970)

End Steel Tubes, Welded or Seamless-

End Types, and Joining Sh

Endless V-Belts (Belts) (Sections Y, Z, A, B, C, D, E)

Endless Wide V-Belts for Industrial Speed Changers and

Ends and Having a Contact Spacing of $2.54 \mathrm{~mm}$ (0.1 In.)

Ends for Crimping to Aircraft Aluminum Electrical Cable

Ends for Domestic and Industrial Waste Pipe: Basic Dime

Ends for Manually Operated Electronic Components (1972) OIML

IEC

IEC

ISO

ISO

IEC

IEC

IEC

17

117-3

117-6

77

117-1

268-6

117-15

203

205

345

783

1421

1798

283

2062

151-13

2050

1550

1617

315-1

$315-2$

315-5

2151

495

1680

362

240

284

388-1

389-1

27

23

24

$\begin{array}{ll}\text { ISO } & 265 \\ \text { IEC } & 390\end{array}$ 
c. for Assemhly Tools for Bolts and Screws-Hexagon Drive Rec. for Cylindrical and 1/10 Conical Shaft fittings for Copper Tubes-Dimensions of Sockets and Male Std. for Spiral Ratchet Screwdriver

Frequencies Below 3 MHz: Edge Socket Connectors with Open Preparation of Test Procedures for Evaluating the Thermal ical Coil Bond Test (/) Rec. for Evaluation of the Thermal eth. (1971) Rec. for Test Procedure for the Evaluation of the Thermal ependent Specif/ Rec. for Test Procedure for Thermal trotechnical Vocabulary: Nuclear Power Plants for Electric Std. for Nuclear y: Generation, Transmission and Distribution of Electrical ges Containing Photographic Materials Sensitive to Radiant Rec. for Var Hour (Reactive $s$ with Elastic Sealing Ring Type Joints: Minimum Depths of $s$ with Elastic Sealing Ring Type Joints: Minimum Depths of of Rotation of 1 Rec. for Numbering of Aircraft Engines, res for Ground Power Units for d.c. Aircraft Servicing and Rec. for Automobiles

Rec. for Basic Rack of Straight Bevel Gears for General Rec. for Rec. for Rec. for Steels for General Rec. for Basic Rack of Cylindrical Gears for General ametral Pitches of Cylindrical Gears for General and Heavy les and Diametral Pitches of Cylindrical Gears for General of Straight Bevel Gears for General Engineering and Heavy tral Pitches of Straight Bevel Gears for General and Heavy Engines (197) Std. for Reciprocating Internal Combustion 2) Std. for Reciprocating Internal Combustion Std. for Reciprocating Internal Combustion Std. For Reciprocating Internal Combustion
ders and Combustion Chambers, and Direction of Rotation of on Engines-Definition of Right and Left Hand Single Bank direction of Rotation of / Rec. for Numbering of Aircraft Sid. for Internal Combustion or List of Equivalent Terms Used in the Plastics Industry or List of Equivalent Terms Used in the Plastics Industry or List of Equivalent Terms Used in the Plastics Industry or List of Equivalent Terms Used in the Plastics Industry or List of Equivalent Terms Used in the Plastics Industry al Definitions-Alphabetical Indexes of Equivalent Terms Shipbuilding Details-Anchor Chains, Stud Links (Common, ing Current Circuit Breakers: Information to Be Given with Coupling an External low Voltage Power Supply to Portable Rec. for Animal Fats: Determination of Water Spices and Condiments: Determination of Moisture Content Rec. for Sizes of Correspondence Area (1965)

ic $(12+12$ Hr. Cycle) $(1969)$

; Primarily for Equipment Type Specimens /

Rec. for Basic Rec. for Basic Rec. for Basic Rec. for Basic Rec. for Basic Rec. for Basic Rec. for Basic Rec. for Basic Rec. for Basic Rec. for Basic Rec. for Basic Rec. for Basic Rec. for Basic Rec. for Basic Rec. for Basic Rec. for Basic Rec. for Basic Rec. for Basic Rec. for Basic Rec. for Basic Rec. for Basic Rec. for Basic Rec. for Basic $s$ and Equipment: Test U: Robustness of

$s$ and Equipment: Test Ea: Shock (1967)

8 and Equipment: Test Eb: Bump (1968)

$s$ and Equipment: Test D: Accelerated Damp/

$s$ and Equipment: Test Ga: Acceleration, S/

8 and Equip
tests (1968)

emperature Tests (1971)

usoidal) (1970)

Annex C: Preparation of Test Specimens from Polyester and I Prol Rec. for Dimensions of Hot Rolled Steel Sections rec. for Dimensions of Hot Rolled Steel Sections-Part I: hold of Hearing Under Free Field Listeni/ Rec. for Normal a Diffuse Field and in a Frontally Incident Free Field for vimetric Determination of Silicon (Content Greater Than or tion of Copper in Aluminium Alloy (Content Greater Than or Rec. for Gymnastic Rec. for Dictation Rec. for Gymnastic

for Data Communication-Data Terminal and Communication terials (1971)
Ends for Power Types (1970) Ends (1969)

Ends (1971)

Ends (1972)

Ends, Off Centre (Center) Guide, Having a Contact Spaci

Endurance of Electrical Insulating Materials (1966)

Endurance of Electrical Insulating Varnishes by the Hel

Endurance of Enamelled Wire by the Lowering of the Elec

Endurance of Insulating Varnishes-Electric Strength M

Energy Generation (1968) Rec, for International Elec IEC

Energy Glossary: First Series of Terms (1972) ISO

Energy (1965)

Energy (1968)

Energy) Meters (1963)

Engagement (1973)

Engagement (1973)

INternational Electrotechnical Vocabular IEC

IEC

/Yvinyl Chloride (PVC) Pressure Pipe ISO /Yvinyl Chloride (PVC) Pressure Pipe ISO

ISO

Engine Starting (1961)

Engine Test Code-Net Power (1971)

Engineering and Heavy Engineering (1968)

Engineering Drawing: Dimensioning (1959)

Engineering Drawing: Principles of Presentation (1959)

Rec. for Safety Featu ISO

iso

ISO

ISO

ISO

Engineering Purposes (1969)

Engineering (1957)

Engineering (1966)

Engineering (1966)

Engineering (1968)

Engineering (1968)

Rec. for Modules and Diame isO

Rec. for Modules and Di ISO

Rec. for Preferred Modu ISO

Rec. for Basic Rack ISO

Engines-Designation of the Cylinders (1972)

Engines-Designation of the Direction of Rotation (197

Engines and Propellers (1966) /T Engines, Engine Cylin ISO

Engines (1972) /D. for Reciprocating Internal Combusti ISO

Engines, Engine Cylinders and Combustion Chambers, and ISO

Engincs: Spark Plug Ignition-Terminology (1972)

(English, French, Russian) (Cs: Corresponding Czech Term

(English, French, Russian) (D: Corresponding German Term

(English, French, Russian) (NL: Corresponding Dutch Term

(English, French, Russian) (PI: Corresponding Polish Ter

(English, French, Russian) (1969)

English, French, Russian) (1970)

Enlar

Enquiries Tenders and Orders and Rules for Transport. E

Entertainment Equipment (1971)

Entrainment Distillation Meth. (1969)

Entraiument Meth.) (1969)

Envelopes and Pockets (1962)

Envelopes, Postcards and Similar Articles: Cancellation

Environmental Test Procedures: Test Db: Damp Heat, Cyc

Environmental Test Procedures: Test Ec: Drop and Topple

Environmental Test Procedures: Test Ed: Free Fail (1969

Environmental Test. Procedures for Electronic Component

Environmental Test. Procedures for Electronic Component

Environmental Test. Procedures for Electronic Component

Environmental Test. Procedures for Electronic Component

Environmental Test. Procedures for Electronic Component

Environmental Tcst. Procedures for Electronic Component

Environmental Test. Procedures for Electronic Component

Environmental Test. Procedures for Electronic Component

Environmental Test. Procedures for Electronic Component

Environmental Test. Procedures for Electronic Component

Environmental Test. Procedures for Electronic Component

Environmental Test. Procedures for Electronic Component

Environmental Test. Procedures for Electronic Component

Environmental Test. Procedures for Electronic Component

Environmental Test. Procedures for Electronic Component

Environmental Test. Procedures for Electronic Component

Environmental Test. Procedures for Electronic Componcnt

Environmental Test. Procedures: Guidance for Damp Heat

Environmental Test. Procedures: Guidance on Change of T

Environmental Test. Procedures: Test Fc: Vibration (Sin

Equal Resin Moulding Materials (1970)

Equal Leg Angles-Metric Series-Dimensions and Secti ISO

Equal Loudness Contours for Pure Tones and Normal Thres ISO

Equal Loudness (1965) / / Equal to $0.30 \%$ ) (1968)

Equal to $0.50 \%)(1968)$

Equipment-Balancing Beam (1964)

(Aluminum) and lis Alloys: Gra

Alloys: Electrolytic Determina ISO

Equipment-Classification (1971)

Equipment-Horizontal Bar (1964)

Equipment-Interchange Circuits-Assignment of Connec

Equipment-Light Duty Belt Conveyors for Loose Bulk Ma
1173

775

2016

2352
$130-13$

216

290

172

370

(50)-3

921

$50(25)$

884
145

145

2045

2048

482

1021

223

677

129

128

1052

54

54
467

677

678
2276

1205

1204

2261

482

2276

2542

194/CS

194/D

$194 / \mathrm{NL}$

194/PL

194

$1122 / \mathrm{I}$

170

56.6

130. 10

934

939

269

415

$68-2 \cdot 30$

68-2-31

68.2.32

68-1

$68-2$

68-2-1

68-2-10

68-2.11

68-2-13

68-2-14

68.2.17

68-2-2

$68-2.20$

68-2-21

68-2.27

68-2.29

68-2-3

68-2.4

68-2.7

68.2-8

68.2.28

68-2-33

68-2. 6

295/C

657/III

$657 / \mathrm{I}$

226

454

797
796

386

1731

379

2110

SO

2109 
Rec. for Gymnastic
Rec. for Gymnastic
Rec. for Continuous Mechanical Handling
Rec. for Photographic

Rec. for Graphical Symbols for Hydraulic and Pneumatic sheath: Signalling Cables in Singles for Telecommunication h Respect to Possible Hazards When Dealing with Electronic

ic Terms and Definitions for the Reliability of Electronic recording Directly by Means of Stylus and Diagram (Working cations by Means of a Pointer and Graduated Scale (Working tween Prime Movers and Towed Vehicles with 24-V Electrical ety Requirements for Mains Operated Electronic and Related ety Requirements for Mains Operated Electronic and Related

afety Code (197) 971) onveyors-Safet/ s-Safety Code / ors-Safety Cod/ ors and Shaking / ors and Shaking / eyors-Safety C/ sse Conveyors -/ Bins and Bunker/ eyors (Other Tha/ eyors (Other Tha/ eyors (Other Tha/ and Conveyors $\mathrm{Wi}$ and Conveyors, S/ ( Conveyors-Ba/ 69) Std. for Continuous Mechanical Handling Std. for Continuous Mechanical Handling Rec. for Continuous Mechanical Handling Rec. for Continuous Mechanical Handling Std. for Continuous Mechanical Handling Std. for Continuous Mechanical Handling Rec. for Continuous Mechanical Handling Rec. for Continuous Mechanical Handling Std. for Continuous Mechanical Handling Std. for Continuous Mechanical Handling Rec. for Continuous Mechanical Handling Rec. for Continuous Mechanical Handling Rec. for Continuous Mechanical Handling Rec. for Continuous Mechanical Handling Rec. for Continuous Mechanical Handling Rec. for Continuous Mechanical Handling Rec. for Continuous Mechanical Handling Rec. for Continuous Mechanical Handling Rec. for Continuous Mechanical Handling Rec. for Transfusion

uttress T/

uttress $\mathrm{T} /$

aded Equi/

aded Equi/

Rec. for Rock Drilling-Extension Drill Steel Rec. for Rock Drilling-Extension Drill Steel Rec. for Rock Drilling-Extension Drill Steel Rec. for Rock Drilling-Extension Drill Steel Rec. for Power Sources for Portable Prospecting

Power Supply for Air and Land Vehicle Mounted Prospecting for Meth. for Specifying the Characteristics of Auxiliary uipment (Domestic and Similar General Us/ Rec. for Safety cations / Rec. for Thermal Time Delay Switches for Use in cati/ Rec. for Thermostatic Switches Primarily for Use in or Welds in Steel-Reference Block for the Calibration of s, Rubber, Plast/ eyors-Safety C/ veyors (Power An) truck Conveyors / nveyors (Chain B/

Std. for Continuous Mechanical Handling Rec. for Continuous Mechanical Handling Rec. for Continuous Mechanical Handling Std. for Continuous Mechanical Handling Rec. for Electrical 965) tion Lines Lines (1967) Rec. for Electrical old and / Std. for Safety Requirements for the Electrical Rec. for Safety Requirements for the Electrical ctrical Connections for Vehicles with 6 or $12 \mathrm{~V}$ Electrical Aim, Structure, and Results of the CEE (Electrical 1 Test Procedures: Test Ec: Drop and Topple, Primarily for wires with Pvc (Polyvinyl Chloride) Insulation and Sheath: Std. for Heat Resisting Std. for Heat Resistin

wires with Pve (Polyvinyl Chloride) Insulation and Sheath: Std. for Tool Shanks and

afety Equipment for Splash Proof Mains Operated Electronic iver Type Metallized Mica Capacitors for Use in Electronic

Form of Declaration of Performance of Aircraft Electrical

Rec. for Test. and Calibration of Ultrasonic Therapeutic

line Semiconductor Rectifier Cells, Stacks, Assemblies and

Rec. for Processed Disk Records and Reproducin $3 \mathrm{MHz}$ : Connectors for Radio Receivers and Associated Sound Rec. for Safety Requirements for Radio Transmitting Rec. for Rules for Electric Traction Rec. for Rating of Resistance Welding Rec. for Rating of Manual Arc Welding sion of the Functional Performance of Electronic Measuring Charts, Tables: Item Designation for Electrical Parts and xternal Low Voltage Power Supply to Portable Entertainment $3 \mathrm{MHz}$ : Circular Connectors for Radio and Associated Sound icon Iron Alloy, Used for Electronic and Telecommunication $t$ of Speed Fluctuations in Sound Recording and Reproducing ctor Pin Allocations for Use with High Speed Data Terminal quipment for Percussive Long Hole Drilling-Rope Threaded

Percussive Long Hole Drilling-Reverse Buttress Threaded Percussive Long Hole Drilling-Reverse Buttress Threaded quipment for Percussive Long Hole Drilling-Rope Threaded ec. for Polycristalline Semiconductor Rectifier Stacks and
Equipment-Parallel Bars (1964) Equipment-Rings (1964)

Equipment-Safety Code-General Rules (1970)

Equipment-Tripod Connections (1971)

Equipment-Vaulting and Pommelled Horses (1964)

Equipment and Accessories for Fluid Power Transmission Equipment and Installation (1969)

Equipment and Other Employing Similar Techniques (1968)

Equipment and the Components (Or Parts) Used Therein (1

Equipment Category) (1970)

Equipment Category) (1970)

/E Responsive Elements and /Lements Giving Direct Indi

quipment for Commercial International Traffic (1970)

Equipment for Domestic and Similar General Use (1965)

Equipment for Domestic and Similar General Use (1965) C

Equipment for Loose Bulk Materials-Aeroslides (1972)

Equipment for Loose Bulk Materials-Apron Conveyors-

Equipment for Loose Bulk Materials-Apron Conveyors (1

Equipment for Loose Bulk Materials-Belt Feeders and C

Equipment for Loose Bulk Materials-Hydraulic Conveyor

Equipment for Loose Bulk Materials-Mobile Belt Convey

Equipment for Loose Bulk Materials-Oscillating Convey

Equipment for Loose Bulk Materials-Oscillating Convey

Equipment for Loose Bulk Materials-Picking Table Conv

Equipment for Loose Bulk Materials-Scraper and En Ma

Equipment for Loose Bulk Materials-Storage Equipment:

Equipment for Loose Bulk Materials-Troughed Belt Conv

Equipment for Loose Bulk Materials-Troughed Belt Conv

Equipment for Loose Bulk Materials-Troughed Belt Conv

Equipment for Loose Bulk Materials-Vibrating Feeders

Equipment for Loose Bulk Materials-Vibrating Feeders

Equipment for Loose Bulk Materials and Unit Loads-Bel

Equipment for Loose Bulk Materials: Screw Conveyors (19

Equipment for Loose Bulk Materials: Vibrating Conveyors

Equipment for Medical Use (1969)

Equipment for Percussive Long Hole Drilling-Reverse B

Equipment for Percussive Long Hole Drilling-Reverse B

Equipment for Percussive Long Hole Drilling-Rope Thre

Equipment for Percussive Long Hole Drilling-Rope Thre

Equipment for Radio Active Materials (1965)

Equipment for Radio Active Materials (1967)

Equipment for Shock and Vibration Measurement (1966)

Equipment for Splash Proof Mains Operated Electronic Eq

Equipment for Telecommunication and in Electronic Appli

Equipment for Telecommunication and in Electronic Appli

Equipment for Ultrasonic Examination (1972)

Equipment for Unit Loads-Mobile Belt Conveyors (Canva

Equipment for Unit Loads-Overhead Monorail Chain Conv

Equipment for Unit Loads-Overhead Twin Rail Chain Con

Equipment for Unit Loads-Single Strand Floor Mounted

Equipment for Unit Loads-Single Strand Floor Truck Co

Equipment of Machine Tools: Electronic Types (1968)

Equipment of Machine Tools: Machines for General Use (1

Equipment of Machine Tools: Used in Large Series Produc

Equipment of Refrigerators and Food Freezers for Househ

Equipment of Room Air Conditioners (1972)

Equipment Particularly for Private Motor Cars and Light

Equipment Safety) (1961)

Equipment Type Specimens (1969)

/or Basic Environmenta

Equipment Wires for Aircraft (1973)

Equipment Wires for Aircraft: Meth. of Test (1973)

Equipment Wires, Type I, with Solid or Stranded Conduct

Equipment with 7/24 Tapers: Collar Dimensions (1972)

Equipment (Domestic and Similar General Use) (1969)

Equipment (1959)

Equipment (1961)

Equipment (1963)

Equipment (1963)

Equipment (1964)

Equipment (1965)

Equipment (1966)

Equipment (1968)

Equipment (1968)

Equipment (1968)

Equipment (1971)

Equipment (1971)

Equipment (1971)

Equipment (1971)

Equipment (1971)

Equipment (1972)

Equipment (1973)

Equipment $1 \mathrm{l} / 2$ to $2 \mathrm{In}$. (38 to $51 \mathrm{Mm}$ ) (1970)

Equipment $11 / 2$ to $21 / 2 \mathrm{In}$. (38 to $64 \mathrm{Mm}$ ) (1970)

Equipment $1 \mathrm{l} / 6$ and $1 \mathrm{l} / 4 \mathrm{In}$. (27 and $32 \mathrm{Mm}$ ) (1970)

Equipment $7 / 8$ to $11 / 4$ In. (22 to $32 \mathrm{Mm})(1970)$

Equipment (1960) 
Rec. for Sound System Rec. for Sound System Std. for Dictation ical Handling Equipment for Loose Bulk Materials-Storage r Conical Fittings for Syringes, Needles and Orher Medical Rec. for Sound System vironmental Test. Procedures for Electronic Components and Rec. for Sound System Std. for Rotary Drilling Rec. for Sound System Rec. for Sound System Rec. for Sound System vironmental Test. Procedures for Electronic Components and Rec. for Fixed Capacitors for Use in Electronic vironmental Test. Procedures for Electronic Components and vironmental Test. Procedures for Electronic Components and vironmental Test. Procedures for Electronic Components and vironmental Test. Procedures for Electronic Components and vironmental Test. Procedures for Electronic Components and vironmental Test. Procedures for Electronic Components and vironmental Test. Procedures for Electronic Components and vironmental Test. Procedures for Electronic Components and vironmental Test. Procedures for Electronic Components and vironmental Test. Procedures for Electronic Components and

Rec. for Safety Requirements for Radio Transmitting vironmental Test. Procedures for Electronic Components and vironmental Test. Procedures for Electronic Components and vironmental Test. Procedures for Electronic Components and vironmental Test. Procedures for Electronic Components and vironmental Test. Procedures for Electronic Components and Std. for Rotary Drilling with Enquiries Tenders and Orders and Rules for Transport, Rec, for Modified

eth. (1)

Rec. for the Use of Longitudinal Parity to Detect Rec. for Collection of Data for Determination of Rec. for Determination of Total Sulphur in Coal by the Rec. for Determination of Chlorine in Coal and Coke Using Rec. for Bibliographical References: Rec. for paratus (1965)

the Frequency Range Form 3/ Rec. Meth. of Measurement of Rec. for Bibliographical References:

ntent-Free Hydroxylamine Meth. (1972)

Determination of Ester Value After Acetylation/ sample (1963) Rec. for Determination of Solubility of Rec for Std. for Rec. for Rec. for Rec. for Sid. Layout for Meth. of Analysis of Rec. for Determination of the Refractive Index of for Determination of the Density and Relative-Density of Rec. for Determination of the Optical Rotation of determination of Ester Value and Calculation of Content of Rec. for Determination of Freezing Point of

Rec. for Rec. for Rec. for Rec. for Rec. for Rec. for Rec. for

devices and General Principles of Measuring Met/ devices and General Principles of Measuring Met/ devices and General Principles of Measuring Met/ nductor Devices and General Principles of Measuring Meth.: $s$ (1968)

-Estimation of Free Alcohols Content by Determination of

Ester Value After Acetylation/ Rec. for Determination of Rec. for Phthalate Rec. for Essential Oils als-Anodisation of Aluminium (Aluminum) and Its Alloys Std. for Statistical Interpretation of Test Results: Rec. for

Rec. for Determination of Solubility of Essential Oils in oaps: Determination of Foreign Matter of Low Solubility in

Rec. for Oilseed Residues: Determination of Diethyl c. for Spices and Condiments: Determination of Nonvolatile 1971) Rec. for Plastics-Determination of Ethyl

yethylene Groups (1972)

Rec. for Plastics-Determination of emperature of Nonionic Surface Active Agents Obtained from for Plastics-Determination of Viscosity Number of Poly Rec. for Oil of Rec for Rolling Bearings-Meth of Rec. for Ball and Roller Bearings: Meth of Rec. for Guide for the Preparation of Test Procedures for 1 Films for Still Photography-Sensitometric Exposure and density (/) nt of Densit/

Rec. for Hydrochloric Acid for Industrial Use: Rec. for Sulphuric Acid for Industrial Use: ssification of Instruments and Devices for Measurement and ual Examination / Rec. for Plastics-Rec. Pract. for the
Equipment: Amplifiers (1969)

Equipment: Auxiliary Passive Elements (1971)

IEC
ISO

Equipment: Basic Operating Requirements (1973)

Equipment: Bins and Bunkers, Silos and Hoppers, Bin Ga

ISO

Equipment: Definition and Dimensional Characteristics F

Equipment: Explanation of General Terms (1971)

Equipment: General (1968)

Equipment: General (1970)

Equipment: Kellys (1972)

Equipment: Loudspeakers (1972)

Equipment: Mechanical Design Features (1971)

Equipment: Microphones (1972)

Equipment: Pt. 2 Test (1968)

Equipment: Terminology and Meth. of Test (1972)

Equipment: Test A: Cold (1966)

Equipment: Test B: Dry Heat (1966)

Equipment: Test Ca: Damp and Steady Heat (1969)

Equipment: Test D: Accelerated Damp Heat (1960)

Equipment: Test Eb: Bump (1968)

Equipment: Test Ga: Acceleration

Equipment: Test J: Mould Growth (1968)

Equipment: Test Ka: Salt Mist (1964)

Equipment: Test Meth. (1967)

Equipment: Test M: Low Air Pressure (1966)

Equipment: Test N: Change of Temperature (1969)

Rec. for Basic En

IEC

IEC

IEC

ISO

IEC

IEC

Rec. for Basic En IEC

IEC

Rec. for Basic En IEC

Rec. for Basic En IEC

quipment: Test Q: Sealing (1968)

Equipment: Test T: Soldering (1968)

Equipment: Test U: Robustness of Terminations (1960)

Equipment: Upper and Lower Kelly Cocks (1972) ISO

Erection and Maintenance (1971) Jormation to Be Given IEC

Erichsen Cupping Test for Steel Sheet and Strip (1960)

IIc En IEC
IIc En IEC

Rec. for Basic En IEC

Rec. for Basic En IEC

Rec. for Basic En IEC

1. for Basic En IEC

Ec. for Basic En IE

IEC

Errors in Information Messages (1969)

Errors in Measurement of Liquid Flow by Velocity Area M

Eschka Meth. (1963)

Eschka Mixture (1967)

Essential and Supplementary Elements (1968)

Essential Characteristics of $35 \mathrm{~mm}$ Microfilm Reading Ap

Essential Electrical Propertic

Essential Oils-Determination of Acid Value (1971)

Essential Oils-Determination of Carbonyl Compounds Co Ic En IEC

Estial Oils-Determination of Cineole Content (1970

Essential Oils-Meth. of Test-Preparation of Sample

Essential Oils in Ethanol (1968)

Essential Oils (1962)

Essential Oils (1962)

Essential Oils (1962)

Essential Oils (1967)

Essential Oils (1968)

Essential Oils: Labelling and Marking Containers (1961)

Essential Oils: Packing (1961)

Essential Oils: Sampling (1961)

Essential Ratings and Characteristics of Semiconductor

Essential Ratings and Characteristics of Semiconductor

Essential Ratings and Characteristics of Semiconductor

Essential Ratings and Characteristics of Semiconductor

Atings and Characteristics of Semico IEC

/R Essential Oils ISO

Ester Value and Calculation of Content of Essential Oil

Esters for Industrial Use-Meth. of Test (1970)

Estimation of Free Alcohols Content by Determination of

Estimation of the Loss of Absorptive Power by Color

Ethanol for Industrial Use-Meth. of Test (1970)

Ethanol (1968)

Ethanol (1968)

Ether Extract (1968)

Rec. for Analysis of $S$

Ether Extract (1969)

Ether Soluble Matter in Plasticized Cellulose Acetate

Ethyl Ether Soluble Matter in Plasticized Cellulose Ace

Ethylene Oxide Adducts-Iodometric Determination of Ox

Ethylene Oxide (1969) /Ermination of the Cloud Point T ISO

Ethylene Terephthalate) in Dilute Solution (1970) /Ec. ISO

Eucalyptus Globulus (1968)

Evaluating Dynamic Load Ratings (1962)

Evaluating Static Load Ratings (1958)

Evaluating the Thermal Endurance of Electrical Insulati

Evaluation Meth. (1972)

/Eed of Colour (Color) Reversa

Evaluation of Its Acid Concentration by Measurement of

Evaluation of Sulphuric Acid Concentration by Measureme

Evaluation of the Geometrical Parameters of Surface Fin

Evaluation of the Resistance of Plastic to Fungi by Vis
268.3

594

$68-1$

2289

$268-5$

268-14

$268-4$

$68-2$

384-1

68-2-1

$68-2-2$

$68-2-3$

68-2-4

68-2.27

$68-2.29$

68-2.7

68-2-8

$68 \cdot 2 \cdot 10$

68-2-11

215-2

68.2.13

68-2.14

68-2-17

68-2-20

68-2-21

2290

56-6

149

1155

1088

334

587

690

452

138

1242

271

1202

124

356

875

278

280

279

592

709

104

211

210

212

4.

147-1

$147-2$

147-1

709

1385

1241

2143

2602

1388

875

673

736

1108

1875

1875

2270

1065

1228

770

281

76

216

905

911

1878

846 
* on Coating Other Than Thosc/ Rec. for a Meth. for the ating Varnishen by the IJelical Coil Bund Test (/ Rec. for $y$ the lowering of the El/ Rec. for Test Procedure for the cuit Breakers in Installa/ Rec. for Test Procedure for the
Rec. for Short Circuit Current Meth.) (1970) Rec. for Rec. for Natural Rubber Latices, Rec for Meth for the Determination of Residue on ra Cresol for Industrial Use-Determination of Residue on rocarbons for Industrial Use-Determination of Residue on Rec. for Plastics-Determination of the Temperature of Rec. for Paints and Varnishes nt of Metal and Oxide Coating Thicknesses by Microscopical evaluation of the Resistance of Plastic to Fungi by Visual ereals and Pulses-Meth. of Test for Infestation by X-Ray ence Block for the Calibration of Equipment for Ultrasonic for Guide for the Preparation of Classified Vocabularies -Tolerances of Form and of Position-Part IV: Practical lves and Rectifiers/ Rec. Graphical Symbols: Variability, r Microcopies: Scale of $35 \mathrm{~mm}$ Microfilms for lnternational valves: Meth. of Application of Mechanical Shock (Impulse) (1971)

th. of Measurement of Noises Due to Mechanical or Acoustic

pture by Bending (1971)

Pressure (1972)

ity (1972)

cs, Sampling and Packing (1972)

Rec. for Pure Rec. for Pure Std. for Cork Std. for Cork Std. for Cork. Rec. for Drift Rec. for Drift Rec. for Drift Rec. for Ring

for Glass Hydrometers-Conventional Value for the Thermal graphic Terms Used in ISO Rec. Concerning Weldi/ Rec. for areas (1972)

rface Temperatures (19/ ctrical Apparatus, Typ/ meproof Enclosures of $/$

emperature (1966)

f a Screen (1967)

rinsically Safe Circui/ Rec. for Electrical Apparatus for Rec. for Electrical Apparatus for Rec. for Electrical Apparatus for Rec. for Electrical Apparatus for Rec. for Electrical Apparatus for Rec. for Electrical Apparatus for Rec. for Electrical Apparatus for Rec. for Electrical Apparatus for Rec. for Electrical Apparatus for Rec. for Electrical $\Lambda$ pparatus for Rec. for Electrical Apparatus for Rec. for Electrical Apparatus for for Photography-Light Sources for Use in Sensitometric . for Photography-Light Sources for Use in Sensitometric

d. for Photography: Light Sources for Use in Sensitometric olor) Reversal Films for Still Photography-Sensitometric

Rec. for Acoustics-Assessment of Occupational Noise

Rec. for Meth. Determining Photographic Speed and Rec. for Indirect Reading Capacitor Type Pocket Rec. for Direct Reading Pocket Type Electroscope al Protection (/ Rec. for Portable X.- or Gamma Radiation as (1966)

Rec, for on of Resistance of Plastics to Colour (Color) Change Upon on of Resistance of Plastics to Colour (Color) Change Upon on of Resistance of Plastics to Colour (Color) Change Upon um Chloride for Industrial Use: Determination of Halogens, ese Ores-Determination of Active Oxygen-Conventionally dustrial Use: Determination of the Carbon Dioxide Content, dustrial Use: Determination of the Carbon Dioxide Content,

r Industrial Use-Determination of Carbon Dioxide Content es for Industrial Use-Determination of Total Alkalinity,

Tests (1969)

f Unprocessed Writing and Printing Pap/

Certain Classes of Printed Matter (19/

Rec for Sodium Carbonate for measuring Equipment (1971)

Sound or Noise (1959)

I Noise (1963)

es (1971)

e Drilling-Rope Threaded Equi/ e Drilling-Rope Threaded Equi/ e Drilling-Reverse Buttress T/ e Drilling-Reverse Buttress T/ Rec. for Oil of Lemon Rec. for Rec. for Meth. of Rec. for Meth. of Rec. for Rec. for Rec. for Rec. for High Voltage Fuses: Rec. for Lightning Arresters: Rec. for Rock Drilling . Rec. for Rock Drilling Rec. for Rock Drilling Rec. for Rock Drilling Rec. for Reduction Sleeves and Rec. for Terminals with Screw Clamping for ic Instruments (1967) aining Geiger-Muller or Proportional Counter / Rec. for rs for Frequencies Below $3 \mathrm{MHz}$ : Connectors for Coupling an or the Inspection of Closures (I9/ Rec. for Finishes with Oil Filled and Gas Pressure Cables and Their Accessories: Rec. for Aircraft Engine Nacelle Fire
Evaluation of the Results of Accelerated Corrosion Test Evaluation of the Thermal Endurance of Electrical Insul Evaluation of the Thermal Endurance of Enamelled Wire B Evaluation with Special Regard to Rated Capacity of Cir Evaporated Milk and Sweetened Condensed Milk-Determin Evaporated Preserved-Spec. (1971)

Evaporation on a Water Bath (1968)

Evaporation (1971)

/C. for Phenol: Ortha, Meta, and Pa ISO

Evaporation (1972) Evolution of Flammable Gases from Plastics (1968)

Examination and Preparation of Samples for Test. (1970)

Examination of Cross Sections (1970) IC. for Measureme 1SO

Examination (1968)

Examination (1970)

Examination (1972)

lor Plastics-Rec. Pract. for the ISO Rec. for C ISO

Std. for Welds in Steel-Refer 1SO

Rec. 1SO

Examples of Indications on Drawings (1971) /L Drawings 1SO

Examples of Resistors, Elements of Electronic Tubes, Va IEC

Exchange (1961) J Onic Tubes and IEC

Excitation to Electronic Types (1965) Tubes and IEC
Excitations (1968) /of Electronic Tubes and Valves: Me IEC

Expanded Corkboard-Determination of Moisture Content ISO

Expanded Corkboard-Determination of the Modulus of $\mathrm{Ru}$

Expanded Pure Agglomerated-Deformation Under Constant

Expanded Pure Agglomerated-Determination of Bulk Dens

Expanded Pure Agglomerated Thermal Cork-Characteristi

Expanding Test on Copper and Its Alloy Tubes (1961)

Expanding Test on Light Metal and Its Alloy Tubes (1969

Expanding Test on Steel Tubes (1960)

Expanding Test on Steel Tubes (1964)

Expansion Coefficient (For Use in the Preparation of Me

Explanations on the Significance of the Principal Radio

Explosive Gas Atmospheres: Classification of Hazardous

Explosive Gas Atmospheres: Classification of Maximum Su

Explosive Gas Atmospheres: Construction and Test of Ele

Explosive Gas Atmospheres: Construction and Test of Fla

Explosive Gas Atmospheres: General Introduction (1971)

Explosive Gas Atmospheres: Marking (1970)

Explosive Gas Atmospheres: Meth. of Test for Ignition T

Explosive Gas Atmospheres: Oil Immersed Apparatus (1968

Explosive Gas Atmospheres: Pressurized Enclosures (1962

Explosive Gas Atmospheres: Sand Filled Apparatus (1967)

Explosive Gas Atmospheres: Sand Filled Apparatus: Use O
Explosive Gas Atmospheres: Spark Test Apparatus for Int

Exposure-Simulation of the Spectral Distribution of D

Exposure-Simulation of the Spectral Distribution of $\mathrm{P}$

Exposure-Simulation of the Spectral Distribution of $\mathrm{T}$

Exposure and Evaluation Meth. (1972) /E

Exposure Index (1954)

Exposure Meters and Accessory Electrometers (1971)

Exposure Meters (1971)

Exposure Rate Meters and Monitors for Use in Radiologic

Exposure Time Markings for Shutters Used in Still Camer

Exposure to Daylight (1968) Jor Plastics-Determinati ISO

Exposure to Light of a Xenon Lamp (1968) / Determinati ISO

Exposure to Light of the Enclosed Carbon Arc (1968) /I ISO

Expressed as Chlorine-Mercurimetric Meth. (1973) /Di ISO

Expressed as Manganese Dioxide (1963) /Lysis of Mangan ISO

Expressed as Potassium Carbonate: Gas-Volumetric Meth

Expressed aa Sodium Carbonate, Gas-Volumetric Meth. (

Expressed as Sodium or Potassium Carbonate-Gas Volume

Expressed in $\mathrm{Na}_{2} \mathrm{O}$ or in $\mathrm{K}_{2} \mathrm{O}-$ Volumetric Meth. (197

Expressed, Italy (1968)

Expression and Presentation of Results of Coal Cleaning

Expression of Dimensions and Direction of Manufacture $O$

Expression of Dimensions of Processed Writing Paper and

Expression of Test Results (1968)

Expression of the Functional Performance of Electronic

Expression of the Physical and Subjective Magnitudes of

Expression of the Power and Intensity Levels of Sound

Expression of the Properties of Cathode Ray Oscilloscop

Expulsion and Similar Types (1970)

Expulsion Type (1962)

Extension Drill Steel Equipment for Percussive Long Hol

Extension Drill Steel Equipment for Percussive Long Hol

Extension Drill Steel Equipment for Percussive Long Hol

Extension Drill Steel Equipment for Percussive Long Hol

Extension Sockets for Tools with Morse Taper Shanks (19

External Conductors (1964)

External Diameter of Planchets Used in Nuclear Electron

External Diameters of Cylindrical Radiation Probes Cont

External Low Voltage Power Supply to Portable Entertain

External Screw Thread for Glass Containers and Gauges F

External, for Alternating Voltages Up to $275 \mathrm{kV}$ (1967)

Extinguisher Doors (1969) 
Determination of Incandescence Resistance of Rigid Self Aretone Soluble Material (Resinous Su/ Rer. for Benzene

Rec. for Determination of the Yield of Benzene Soluble Rer. for Oilseed Residues: Determination of Diethyl Ethe or Spices and Condiments: Determination of Alcohol Soluble Spices and Condiments: Determination of Nonvolatile Ether spices and Condiments: Determination of Cold Water Soluble Rec for Tea-Determination of Water for Plastics-Pvc Resins-Determination of $\mathrm{pH}$ of Aqueous Rec. for Rubber-Determination of Solvent Rec. for Brown Coals and Lignites-Meth. of perated Appliances for Domestic and Similar Purposes: Spin ar Electrical Appliances: Particular Requirements for Spin Rec. for Spices and Condiments: Determination of lengths (19/ Rec. for Lengths and Tolerances for Drawn or c. for Shipbuilding Details-Marking of Rolled, Drawn and s (1971) Rec. for Mechanical Property Limits for engths and Tolerances for Filler Rods, Other Than Drawn or c. for Plastics-Designation for Polystyrene Moulding and and Designation in Relation to the Position of the Shuttle nting Machines Used for Information Processing-Widths of

a Horizontally Folded Specimen by Mea/ Std. for Woven Glass Std. for Textile of Roll Characteristics (1972)

Rec. for Accelerated Ageing and Simulated Service Test of Rec. for Determination of the Impermeability to Water of ermination of Breaking Strength and Elongation at Break of I Requirements (1972) Rec. for Varnish Rec. for Varnish (1) Rec. for Determination of Dimensional Change in Woven (1968) Rec. for Meth. of Test for Resistance of of the Adhesion Strength of Vulcanized Rubbers to Textile ric Series (1972)

bility (1968)

Metric Series (1968) Rec, for Side and Rec. for Spindle Noses and Rec. for Spindle Noses and racteristics of Solid Wood Parquet Strips with Rectangular Rec. for Symbolic Designation of Direction of Closing and termination of the Permittivity and Dielectric Dissipation Rec. for Plastics-Determination of Bulk

lectrical Properties of Electronic Tubes and Valves: Noise tion of the Continuous Current Rating of Cibles (100\% Load g Electrode Resistance and Transconductance, Amplification ec. for Basic Environmental Test Procedures: Test Ed: Free Rec. for Electric Rec. for Safety Requirements for Electric Rec. for a.c. Electric

69) tive Heights of Thread/

Extinguishing Thermosetting of (1961) /C. for Plastics ISO Extract from Brown Coals and lignites-Determination $O$ ISO Extract in Brown Coals and lignites (1969)

Extract (1968)

Extract (1969)

Extract (1969)

Extract (1969)

Extract (1970)

Extract (1970)

Extract (1971)

Extraction for the Determination of Sodium and Potassiu

Extraction of Materials Soluble in Dichloromethane (196

Rec. ISO

ISO

Extractors (1968)

Spec, for Electric Motor O CEE

Extraneous Matter (1969)

1. for Safety of Household and Simil IEC

Extruded Filler Rods for Welding, Supplied in Straight ISO

Extruded Products in Light Metals or in Light Alloys (1 ISO

Extruded Products of Aluminium (Aluminum) and Its Alloy ISO

Extruded, for Welding (1967) Rec. for L ISO
Extrus

Extrusion Materials (1970)

Re ISO

Eye (1970)

Fabric Ribbons on Spools (1972)

Rec for Shuttles-Terms ISO

Fabrics-Basis for Spec. (1972)

D. for Office and Pri ISO

Fabrics-Determination of the Recovery from Creasing 0

Fabrics Coated with Rubber or Plastics-Determination ISO

Fabrics Coated with Rubber or Plastics (1970) ISO

Fabrics Coated with Rubber or Plastics (1970)

Fabrics Coated with Rubber or Plastics (1971)

Fabrics for Electrical Purposes: Definitions and Genera

Fabrics for Electrical Purposes: Meth. of Test (1972)

/for Det ISO

Fabrics Subjected to Laundering Near the Boiling Point

Fabrics to Penetration by Water (Hydrostatic Head Test)

Face Milling Cutters with Plain Bore and Key Drive: Met ISO

Face Plates Types a and Camlock: Sizes for Interchangea ISO

Face Plates: Bayonet Type; Sizes for Interchangeability ISO

Face (1969) Rec. for General Manufacturing Cha ISO

$\begin{array}{ll}\text { Faces of Doors, Windows and Shutters-Part I (1970) } & \text { ISO } \\ \text { Factor of Electrical Insulating Materials at Power, Aud } & \text { IEC }\end{array}$

$\begin{array}{ll}\text { Factor of Electrical Insulating Materials at Power, Aud } & \text { IEC } \\ \text { Factor of Moulding Materials (1961) } & \text { ISO }\end{array}$

Factor (1963) Rec. for Measurements of the E IEC

Factor) (1971) Rec. for Calcula IEC

Factor, Conversion Resistance and Transductance (1966) IEC

$\begin{array}{ll}\text { Fall (1969) } & \text { IEC } \\ \text { Fans and Regulators for Use in Ships (1969) } & \text { IEC }\end{array}$

Fans and Regulators (1971)

Fans and Regulators (1972)

Fasteners-Part 1: Bolts, Serews and Studs (1968)

Rec. for Mechanical Properties of

Rec. for Mechanical Properties of

Rec. for Mechanical Properties of

Std. for Mechanical Properties of

Fasteners: Marking of Bolts, Screws, Studs and Nuts (19

Fasteners: Nuts with Specified Proof Load Values (1969)

Fasteners: Nuts with Specified Proof Load Values, Effec

Fastenings for Conver (1969-

Fastness of Colored Anodised Aluminum (1971) /Odisatio is

Fastness of Textiles (1959)

(heys-Determination of the Light

Rec. for Test for Colour (Color)

Rec. for Milk-Determination of

Rec. for Dried Milk-Determination of

Fat Content (Reference Meth.) (1970)

Fat Content (Reference Meth.) (1970)

for Cheese and lts Processed Products-Determination of Fat Content (Reference Meth.) (1970)

rated Milk and Sweetened Condensed Milk-Determination of Fat Content (Reference Meth.) (1970)

Std. for Whey Cheese: Determination of Fat Content (Reference Meth.) (1972)

Std. for Cream: Determination of

Fat Content (Reference Meth.) (1972)

Std. for Meat and Meat Products-Determination of Total

Std. for Meat and Its Products-Determination of Free

or Butyrometers for the Determination of the Percentage of

Rec. for Butter-Determination of the Acid Value of the

for Butter-Determination of the Refractive Index of the

Rec. for General Principles for Rec for Axial Load

Rec. for Rotating Bar Bending

Rec. for Crude Vegetable Oils and

Rec. for Crude Vegetable Oils and Rec. for Anima

68)

ids (Titre) (1969)

meth. (1969) )

Rec. for Crude Vegetable Oils and Rec. for A nimal Rec. for Anima Rec. for Anima

Rec. for Crude Vegetable Oils and Rec. for Determination of Volatile for Animal Fats: Determination of Solidification Point of

Rec. for Analysis of Soap: Determination of Total Crude Rec. for Basic Environmental Test. Procedures: Test 966) Rec. for Bonded Abrasive Products Genera cing and Engine Starting (1961) Rec. for Safety Std. for Offset Duplicators-Attachmen

Rec. for Sound System Equipment: Mechanical Design Frequency Connectors: Coaxial Unmatched Television Aerial
hanical Handling Equipment for Loose Bulk Materials-Belt
Fat Content (1973)

Fat in Milk by the Gerber Meth. (1966)

Fat (Reference Meth.) (1971)

Fat (Reference Meth.) (1971)

Fatigue Test. of Metals (1964)

Fatigue Test. (1969)

Fatigue Test. (1969)

Fats: Determination of Acidity (1968)

Fats: Determination of Insoluble Impurities (1968)

Fats: Determination of Insoluble Impurities (1969)

Fats: Determination of Moisture and Volatile Matter (19

Fats: Determination of Moisture and Volatile Matter (19

Fats: Determination of Solidification Point of Fatty Ac

Fats: Determination of Water (Entrainment Distillation

Fats: Preparation of Contract Sample for Analysis (1968

Fatty Acid Number of Latex (1966)

Fatty Acids (Titre) (1969)

Fatty Acids (1965)

Fc: Vibration (Sinusoidal) (1970)

Features Designation Ranges of Dimensions-Profiles (1

Features for Ground Power Units for d.c. Aircraft Servi Features of Plates (1972)

Features (1971)

Feeder Connector (1965)

Feeders and Conveyors-Safety Code (1970) /Inuous Mec ISO
181

1017

975

736

940

1108

1574

1264
1407

1952

624

$10-2 \mathrm{~K}$

$335-4$

927

546

154

827

545
1622

1622

2257

2113

2313

2286

1419

1420

1421

394-1

394-2

675

811

2587

702
867
1072

1072

1226

250

151.4

287

151.12

68-2-32

307

342

385

$898 / \mathrm{I}$

898/III

$898 /$ II

$898 / \mathrm{IV}$

1120

2135

105/1-6

1211

1736

1735

1737

1854

2450

1443

1444

488

1740

1739

373

1099

II 43

660

663

932

662

933

935

934

661

506

935

455

$68 \cdot 2 \cdot 6$

525

223

2132

268.14

169-2

1821 
al llandling Fquipment for Loose Bulk Materials-Vibrating Vibrating Feeders and Conveyors, Shaking or Reciprocating quipment for l.oose Bulk Matcrials: Vibrating Conveyors and

rials-Oscillating Conveyors and Shaking or Reciprocating rials-Oscillating Conveyors and Shaking on Reciprocating ncy Connectors: Two Pin Connector for Twin Balanced Aeria Rec. for Machine Tool Speeds and Spec. for Mains Operated Electric Spec. for Battery Operated Electric Rec. for Graphical Symbols: Rec, for Information on

$s$ of Transformer and Inductor $\mathrm{Col}$ Rec. Guide for the Drafting of Spec for Microwave Rec. for General Classification of (1961) Rec. for Dimensions of Cross Cores (X-Cores) Made of Rec. for Dimensions for Pot Cores Made of Performance Spec. for Cores of Broad Band Transformers of nce Spec. for Cores of Tuned Transformers and Inductors of Rec. for Dimensions of Tubes, Pins and Rods of Rec. for Dimensions of Screw Cores Made of Rec. for Dimensions of Aerial Rods and Slabs of the Weight Per Unit Area of Hot Dip Galvanized Coatings on requirements for Hot Dip Galvanized Coatings on Fabricated ec. for Meth. of Test for Wool Fibre Length Using a Single

8)

th (1972)

test Pieces (1972)

nt (1968)

on and of Swelling in Thickness After Im/ of Wool Fibre Rec. for Meth. of Test for Wool Fibre Rec for Textiles-Binary Fibre

ter $(1969)$

Plates / Rec. for Plastics-Preparation of Glass Fibre

$r$ Consignments Of, Continuous Filament Yarn, Staple Fibre of Measured Surface Pile Density and Measured Pile Fibre inear Density of Its Continuous Filament and Staple Fibre r Perforated Metal Cheese Centres (Center) for Bast Fibre of the Twist in Its Continuous Filament and Staple Fibre ting Rollers for Cotton, Wool, Spun Silk and Staple Fibre Meth. (1971)

Rec. for Textiles Fibre Std. for Textiles-Cotton Fibres Std. for Determination of Fineness of Flax Fibres Rec. for Beams for Dyeing Fibres

or Wool: Determination of Percentage of Medullated Fibres

- Determination of the Average Diameter of Staple Fibres fibre Length by Measuring the Length of Individual Fibres lity Meth. for Measuring the Mean Diameter of Wool Fibres Rec. for Generic Names for Man Made Fibre Rec. for International Code for the Rec. for International Code for the

ound Pressure Levels of Narrow Bands of Noise in a Diffuse t Sound Transmission (1960)

Noise in a Diffuse Field and in a Frontally Incident Free for Pure Tones and Normal Threshold of Hearing Under Free (1962)

f Reliability, Availability, and Maintainability Data from Rec. for

ss-Determination of the Linear Density of Its Continuou xtile Glass-Determination of the Twist in Its Continuou rical Properties of Electronic Tubes and Valves: Heater or Rec. for Tungsten

cedure for Sampling Batches or Consignments Of, Continuou e Average Diameter of Staple Fibres (Fibers) or Continuou nternational Rec. for Optical Pyrometers with Disappearing Rec. for Magnetic Tape Labelling and

Rec for Paper and Board: Sizes of Folders and Rec. for Paper: Holes for Genera

xternal, for Alternating Voltages / nternal, for Alternating Voltages / aper Insulated, Metal Sheathed, Fo/ Rec. for Tests on Oil Rec. for Tests on Oil Rec. for Tests on Oil

$\mathrm{kV} \mathrm{Up}$ to and Including $66 \mathrm{kV}$ (Excluding Gas Pressure, Oil

$r$ Electrical Apparatus for Explosive Gas Atmospheres: Sand

r Electrical Apparatus for Explosive Gas Atmospheres: Sand

e Electrical Properties of Microwave Tubes and Valves: Gas

72)

est. (1972)

st by Dropping (1972)

72)

ests (Inclined Plane and P/

arts When Test. (1972)

Std. for Packaging-Complete

Gas Welding. Ter Electrodes for Manual Arc Welding and ining the Characteristics of the Deposite
Feeders and Conveyors with Tubular Trough (1970)

Feeders and Oscillating Conveyors-Safety Code (1971)

IAnic ISO

ISO

ISO

Feeds with Rectangular or Trapezoidal Trough (1969)

Feeders with Rectangular or Trapezoidal Trough (1970)

ISO

Feeders with Tubular Trough (1971)

for Loose Bulk Mate ISO

eders (1965)

Rec. for Radio Freque IEC

Fence Controllers (1956)

Fence Controllers (1956)

Ferrite Cores and Magnetic Storage Matrices (1972)

Ferrite Materials Appearing in Manufacturers Catalogue

Ferrites (1972)

Ferromagnetic Oxide Materials and Definitions of Terms

Ferromagnetic Oxides and Associated Parts (1967)

Ferromagnetic Oxides and Associated Parts (1971)

Ferromagnetic Oxides for Telecommunication (1966)

Ferromagnetic Oxides for Telecommunication (1966)

Ferromagnetic Oxides (1966)

Ferromagnetic Oxides (1966)

Ferromagnetic Oxides (1966)

Ferrous Materials by Chemical Dissolution of the Coatin

Ferrous Products (1970)

Fiber Length Measuring Machine (1970)

Fiber Sampling for Test. (1969)

(Fiber) Building Boards: Definition; Classification (196

(Fiber) Building Boards: Determination of Bending Streng

ISO

CEE

CEE

IEC

IEC

IEC

IEC

IEC

I of IEC

/Ram IEC

IEC

IEC

IEC

ISO

Rec. for ISO

ISO

ISO

ISO

ISO

ISO

(Fiber) Building Boards: Determination of Dimensions of

ISO

ISO

(Fiber) Building Boards: Determination of Water Absorpti

ISO

(Fiber) Length Barbe (Barb) and Hauteur Using a Comb Sor

ISO

ISO

Fiber) Mixtures-Quantitative Chemical Analysis (1971)

ISO

(Fiber) Type and Roving Package (1971) /Pling Batches O ISO

(Fiber) Volume Ratio (1971) Jor Carpets-Determination ISO

(Fiber) Yarns and Rovings in the Form of Packages (1971) ISO

(Fiber) Yarns (1966) Rec. Fo ISO

(Fiber) Yarns (1971)

/for Textile Glass-Determination ISO

Rec. for Diameters of Draf ISO

(Fibers)-Determination of Linear Density-Gravimetric

ISO

$\begin{array}{ll}\text { (Fibers)-Determination of Micronaire Value (1972) } & \text { ISO } \\ \text { (Fibers)-Permeametric Meth. (1972) } & \text { ISO }\end{array}$

(Fibers) and Yarn (1969)

ISO

(Fibers) by the Projection Microscope (1973)

Fibers) or Continuous Filaments Constituting a Textile

Std. F ISO

ISO

(Fibers) (1962)

(Fibers) (1969)

Rec. for Determination of ISO Rec. for Air Permeabi ISO

Field Acceptance Tests of Hydraulic Turbines (1963)

Field Acceptance Tests of Storage Pumps (1968)

Field and in a Frontally Incident Free Field for Equal

ISO

IEC

IEC

Field and Laboratory Measurements of Airborne and Impac

Field Listening Conditions (1961) /L Loudness Contours ISO

Field of Quantities and Units Used in Electricity (1964 IEC

Field Performance of Electronic Items (1971) /Ection O IEC

Figures for Aircraft Instrument Dials and Number PIates ISO

Filament and Staple Fibre (Fiber) Yarns and Rovings in

Filament and Staple Fibre (Fiber) Yarns (1971) /for Te ISO

Filament Current (1963) /for Measurements of the Elect IEC

Filament Lamps for General Service (1961)

Filament Yarn, Staple Fibre (Fiber) Type and Roving Pac

Filaments Constituting a Textile Glass Yarn-Cross Sec ISO

Filaments (1970)

File Structure for Information Interchange (1969)

Files and Rasps: Lengths and Cross Sections (1961)

Files (1967)

Filing Purposes (1968)

Filled and Gas Pressure Cables and Their Accessories: E

Filled and Gas Pressure Cables and Their Accessories: I

Filled and Gas Pressure Cables and Their Accessories: P

Filled and Nondraining Types) (1965)

Filled Apparatus (1967)

Filled Apparatus: Use of a Screen (1967)

Voltages from 10 IEC

ISO

ISO

ISO

ISO

ISO

IEC

Filled Microwave Switching Devices (1972) /Ement of Th IEC

Rec. Fo IEC

Rec. Fo IEC

Filled Transport Packages-Part III: Stacking Test (19

Filled Transport Packages-Part II: Conditioning for T

Filled Transport Packages-Part IV: Vertical Impact Te

Filled Transport Packages-Part Vi: Vibration Test (19

Filled Transport Packages-Part V: Horizontal Impact T

Filled Transport Packages-Part 1: Identification of $P$

Filler Metals for Gas Welding (1967)

Ec for Diametr

ill Rods and the Parent Metal in the Welding of Stee

1815

2125

1049

1807

2139

$117 \cdot 16$

01

392
125

226

133

219

218

220

223

1460

461

1822

1130

818

768

819

767

769

137

920

1833

1268

1886

1959

1889

480

1890

98

973

2403

2370

1037

647

1888

270
1136

2076

41

198

454 
ining the Conventional Bond Strength on Steel, / Rec. for ining the Mechanical Properties of Weld Metal Deposited by y High Tensile Types (1967) Rec. for Code of Symbols for atibility of Steel Filler Rods and the Parent M/ Rec. for 19/ Rec. for Lengths and Tolerances for Drawn or Extruded (1967) Rec. for Lengths and Tolerances for

ies Chamfer Dimension Limits and Maximum Shaft and Housin ransvers/ Rec. for Calculation of Rectangular Symmetrical ive Agents: Determination of Apparent Density of Pastes on Rec, for Slotted Cheese for Cinematography-8 mm Type S Motion Picture Raw Stock cta/ Std. for Cinematography: 65 and $70 \mathrm{~mm}$ Motion Picture Std. for Cinematography: $35 \mathrm{~mm}$ Motion Pictur ecording Heads for 6 Track Magnetic Sound Records on $35 \mathrm{Mm}$ Std for Cinematography: $35 \mathrm{~mm}$ Motion Picture Recording Heads for Three Magnetic Sound Records on $35 \mathrm{Mm}$ 1971) Rec. for Rol Rec. for Polyeste Rec, for Polystyren

Direct Current Using Impregnated Paper or Paper / Plastic (1969)

ematography: Projector Usage of $8 \mathrm{~mm}$ Type $S$ Motion Picture Rec. for Photographic Colour (Color)

or Identification of the pullout Force (I969)

1971) Rec. for Dimensions for $35 \mathrm{Mm}$ Rec. for Magnetic Striping of $16 \mathrm{Mm}$ Rec. for Motion Picture for Single Magnetic Sound Stripe on $16 \mathrm{~mm}$ Motion Picture - for Cinematography-Camera Usage of $8 \mathrm{~mm}$ Motion Picture e Area for Printing to $8 \mathrm{~mm}$ Type $S$ on $16 \mathrm{~mm}$ Motion Picture or $35 \mathrm{~mm}$ Film Magazines for Still Picture Use and Test for laid) and 8 Strand (Plaited) Polypropylene Monofilament or for Cinematography-Dimensions for $16 \mathrm{~mm}$ Motion Picture ping and $\mathrm{Ca} /$ Std. for Cinematography: $8 \mathrm{~mm}$ Motion-Picture for Dimensions of Projection Reels for $\mathbf{8} \mathrm{mm}$ Motion Picture emulsion Position in Camera for $8 \mathrm{~mm}$ Silent Motion Picture mulsion Position in Camera for $16 \mathrm{~mm}$ Silent Motion Picture Record Positions in Camera for $35 \mathrm{~mm}$ Sound Motion Picture Record Positions in Camera for $16 \mathrm{~mm}$ Sound Motion Pictur cord Positions in Projector for $35 \mathrm{~mm}$ Sound Motion Picture

for Direct Front Projection of $8 \mathrm{~mm}$ Silent Motion Picture

for Direct Front Projection of $16 \mathrm{~mm}$ Silent Motion Picture cords on $35 \mathrm{~mm}$ Film and 1 Magnetic Sound Record on $17.5 \mathrm{Mm}$

Rec. for Cinematography-Projected Image Area for $16 \mathrm{Mm}$ f Recording Heads for Four Magnetic Sound Records on $35 \mathrm{Mm}$ Perforating Dimensions for $35 \mathrm{~mm}$ Motion Picture Raw Stock rating Dimensions for Double-8 $\mathrm{mm}$ Motion Picture Raw Stock centre (Center) Sound Records on $16 \mathrm{~mm}$ Perforated Magnetic

g Head for Edge Sound Records on $16 \mathrm{~mm}$ Perforated Magnetic Rec. for Identification of Exposed Colour (Color) Rol nsions of Daylight Loading Spools for $16 \mathrm{~mm}$ Motion Picture of Daylight Loading Spools for Double $8 \mathrm{~mm}$ Motion Picture

f Flammability and Burning Rate of Plastics in the Form of racteristic for Magnetic Sound Records on $35 \mathrm{~mm}$ Perforated Rec. for Dimensions for 127, I20 and 620 Roll

nd Tetrathionate in Processed Black and White Photographic r Stereo Still Photography Using $35 \mathrm{~mm}$ Objectives on $35 \mathrm{Mm}$ Std. for Muscovite Mica Blocks, Thins and Rec. for Cinematography-Picture Areas for rmination of the Water Vapour (Vapor) Transmission Rate of phy: Determination of ISO Speed of Colour (Color) Reversa Rec. for Preparation of Dry

Rec. for Muscovite Mica Blocks, Thins and Rec. for Photography-Cameras Usin duced by Camera Aperture and Projected Image Area for $8 \mathrm{Mm}$ uced by Camera Aperture and Projected Image Area for $35 \mathrm{Mm}$ ture for Projection of $35 \mathrm{Mm}$ Nonanamorphotic Motion Picture nematography-Image Produced by Camera Aperture for $16 \mathrm{Mm}$

c. for Dimensions of Cores for Motion Picture and Magnetic rec. for Plastics-Determination of Tensile Properties of

$g$ the Stability of the Images of Processed Black and White Rec. for $35 \mathrm{Mm}$

Std. for Radiography of Welds and Viewing Conditions for quency and Equivalent Resistance of Unwanted Resonances of ons (/ Rec. for Octave, Half-Octave and Third-Octave Band Rec. for Piezoelectric

1972)

Std. for Metal Slitting Saws with Std. for Determination of Rec. for Paints and Varnishes-Determination of on Black for Use in the Rubber Industry-Determination of Industry Delivered in Bulk or in Bins-Spec. for Maximum

nt and Evaluation of the Geometrical Parameters of Surface $s$ and Gauges for the Inspection of Closures (19)

Rec. for 1970)

Rec. for Dyeing and Rec. for Gravity

Filler Rods for Braze Welding: Meth. of Test for Determ Filler Rods for Cas Welding Mild Steels and Low Alloy $\mathrm{H}$

Filler Rods for Gas Welding of Mild Steels and Low Allo

Filler Rods for Gas Welding: Test to Determine the Comp

Filler Rods for Welding, Supplied in Straight Lengths (

Filler Rods, Other Than Drawn or Extruded, for Welding

Fillet Radius (1972) /Bearings: Tolerances; M
Fillet Welds Statically Loaded in Such a Way That the T

Fillet Welds Statically Loaded in
Filling Orifices for Aircraft (1959)

Filling (1969)

(Fillister) Head Screws-Metric Series (1970)

Rec. for Surface Act ISO

ISO

Film-Cutting and Perforating Dimensions (1972) Std. ISO

Film-Image Area Produced by Camera Aperture and Proje ISO

Film-Image Area Produced by Camera Aperture (1972) ISO

Film-Location and Dimensions (1971)

Film-Projectable Image Area (1972)

Film and l Magnetic Sound Record on $17.5 \mathrm{~mm}$ Film (1960)

Film Cameras-Back Window Location and Picture Sizes (

Film Dielectric Capacitors for Direct Current (1965)

Film Dielectric Capacitors (1968)

Film Dielectric (1964)

IEC

Film for Direct Front Projection (1973) Std. for Cin ISO

Film for General Use: Preferred Sizes of Sheet Material ISO

Film for Motion Picture Uses (1966)

Film for Still Picture Cameras (1968)

Rec. F ISO

Film Magazines for Still Picture Use and Test for Film ISO

Film Perforated Along Both Edges (1960)

Film Perforated Along One Edge-Direction of Winding ( ISO

Film Perforated Along l Edge (1966)

Film Perforated Type S (1972)

Film Perforated $8 \mathrm{~mm}$ Type S, 1-4 (1972)

Film Pullout Force (1969)

Film Ropes (1970)

Rec. for Dimensions F

Rec ISO

Std ISO

Film with Picture-Location and Width of Magnetic-Stri ISO

Film (Other Than Type S) (1968)

Film (1956)

Film (1956)

Film (1956)

Film (1956)

Film (I956)

Film (1956)

Film (1956)

Film (1960)

Film (I963)

Film (1963)

Film (I966)

Film (1966)

Film (1968)

Film (I968)

Film (I969)

Film (1969)

Film (I969)

Film (1970)

Film (1971)

Film, Backing Paper and Spools (1971)

Film, 5-Perforation Format (I966)

Films-Visual Classification (1972)

Films and Slides for Television (1971)

Rec. ISO

Rec. for ISO

Rec. for E ISO

Rec. for Emulsion and Sound ISO

Rec. for Emulsion and Sound ISO

Rec for Emulsion and Sound Re ISO

Rec. for Emulsion Position in Projector ISO

Rec. for Emulsion Position in Projector ISO

/Cording Heads for Three Magnetic Sound Re ISO

Rec. for Cinematography-Location O ISO

Rec. for Cutting and ISO

Rec for Cutting and Perfo ISO

Ation and Width of the Recoding Head for ISO

C. for Location and Width of the Recordin ISO

Rec, for Cinematography-Dime ISO

Rec for Cinematography-Dimensions ISO

Rec. for the Determination O ISO
inematography-Recorded Cha ISO

Rmining Thiosulphate a ISO

(for Dimensions Fo ISO

ISO

ISO

Filn and Thin Sheets-Dish Meth. (I970)

Films for Still Photography-Sensitometric Exposure an ISO

Films from Concentrated Natural Rubber Latex (I966) ISO

Films Meth. for Grading by Size (1958)

Films $35 \mathrm{~mm}$ and Smaller Picture Sizes (1971)

Films (1958)

Films (1958)

Films (1963)

Films (1965)

1. Maximum Aspect Ratio of Projector Aper ISO Rec for

Films $(1969)$

Re ISO

Films (1970)

Films, Plates and Papers (1965)

Filmstrips: Dimensions and Formats (1968)

R Meth. for Indicatin ISO

Filitin

Filter Crystal Units (1968) Jor the Measurement of Fre IEC

Filters Intended for the Analysis of Sounds and Vibrati IEC

Filters (197I)

Fine and Coarse Teeth-Metric Series (1972)

Fineness of Flax Fibres (Fibers)-Permeametric Meth. (- ISO

Fineness of Grind (1971)

Fines Content (1970)

Fines Content (1970)

Rec. for Pelletized Carb ISO

Finish (1970)

TRbon Black for Use in the Rubber

Finishes with External Screw Thread for Class Container

Finishing Machinery-Classification and Nomenclature (

Finishing Machines: Definition of Left and Right Sides 
Std. for Sid. for Hand Screw Propellers, Manufacturing Tolerance for Casting and Rec. for Aircraft Engine Nacelle Rec. for Rec. for Rec. for Rec. for ec. for Pesticides Considered Not to Require Common Names: Vocabulary/ Rec. for Statistical Vocabulary and Symbols: Rec. for Paper Vocabulary: Std. for Nuclear Energy Glossary: Rec. for Determination of Water by the Karl ndustrial Use-Determination of Water Content by the Karl Is for Industrial Use-Determination of Water by the Karl for Industrial Use-Determination of Water Content-Karl Rec. for Netting for
Breaking Load of Netting Yarns for Fish/ ror Rec. for nd Knot Breaking Lo rements for Describing and Designating Knotted Netting for
Rec. for Netting Yarns for rinciples of Criticality Safety in Handling and Processing Rec. for ISO System of Limits and Rec. for ISO System of Limits and Rec. for Shanks for Pneumatic Tools and Rec. for Shanks for Pneumatic Tools and Rec. for Dental Burs and Cutters.

(197I) Rec. for Pipes and ale Ends (197I) Rec. for Asbestos Cement Pipe Rec. for Capillary Solder Spec. for Steel Conduits and Spec. for Lighting $r$ Purposes (1968) rec. for Pipes and Fittings of Plastics Materials-Socket
Rec. for Socket nyl Chloride (PVC) Fittings with Plain S/ Rec. for Spec. for Corner Rec for Asbestos Cement Pipes, Joints and ent: Definition and Dimensional Charact/ Rec. for Conical Rec. for Lighting ssure Pipes with Elastic Sealing / Std. for Double Socket Rec for Cast Iron Sanitary Pipes and Rec. for Cast Iron Sanitary Pipe ttings (I966) Dimensions of Elastomeric Toroidal Sealing Rings for Pipe
Dimensions of Elastomeric Toroidal Sealing Rings for Pipe Dimensions of Elastomeric Toroidal Sealing Rings for Pipe
Rec. for Oven Test for Moulded use Under Pressure-Basic Dimensions/ Rec. for Pipes and
pes Under Pressure$\begin{array}{ll}\text { pes Under Pressure-Basic Dimensions/ } & \text { Rec. for Pipes and } \\ \text { igot Ends for Domestic and Industrial/ } & \text { Rec. for Pipes and }\end{array}$ Rec. for Malleable Cast Iron Pipe

rea/ Rec. for Pipe Threads for Gas List Tubes and Screwed Under Pressure: Unplasticized Polyvinyl Chloride (PVC)

Rec. for Pipes and Fittings of Plastics Materials: Socket

Pipe Fittings for Waste Water and Ventilations-Part IV:

Polyvinyl Chloride for Electrical Installations and Their erically Controlled Machines (1969/ Rec. for Punched Tape aper or Paper / Plastic Film Dielectric (1964) Rec. for nology and Meth. of Test (I972) Rec. for ec. for Test Conditions for Milling Machines with Table of s: Test. of Toughened Glasses for Ships Side Scuttles and getails-Toughened Glasses for Ships Side Scuttles and Current (1965) Rec. for Rec. for

ec. for Meth. of Measurement of Current Noise Generated in Requirements for Low Power Nonwirewound Types / Rec. for pec. (1971)

$\mathrm{n}$ of Meth. of Test and General Requirements (I9/ yte (I97I)

Rec. for Rec. for Rec. for Rec, for Rec. for

or Heat Treated, Alloy, and Free Cutting Steels-Part 12: for Industrial Use-Determination of Potassium Content . (Test (1963)

$m$ Hydroxide for Industrial Use-Determination of Sodium .

0)

Rec. for

ers (I965)

Rec, for High Voltage Connecting Wire with

roduction of Aluminium-Determination of Sodium Content us for Explosive Gas Atmospheres: Construction and Test of f Film (1970) Rec for the Determ Rec. for Plastics-Determination of lastics-Determination of the Temperature of Evolution of ec. for Dimensions of Hot Rolled Steel Sections-Parallel pose Rigid Coaxial Transmission Lines and Their Associated pose Rigid Coaxial Transmission Lines and Their Associated ec. for Dimensions of Hot Rolled Steel Sections-Parallel otating Electrical Machines -- Frame Numbers 56 to 400 and

y Dimensions-Subunits-Metric Series-Outer Rings with Rec. for Flanges for Waveguides: Relevant Spec. for
Finishing Reamers for Morse and Metric Tapers, with Par

Finishing Sticks and Oil Stones-Dimensions (1972) ISO Finishing (1966) Rec. for Shipbuilding Details: Ship ISO Fire Extinguisher Doors (1969)

Fire Refined High Conductivity Tough Pitch Copper Refin

Fire Refined Tough Pitch Copper Refinery Shapes (1971)

\section{ISO}

Fire Resistance Tests of Structures (1968)

Fire Resisting Characteristics of Electric Cables (1970

First List (1968)

First Series of Terms and Symbols-Part 1: Statistical

First Series of Terms (1958)

First Series of Terms (I972)

Fischer Meth. (1968)

Fischer Meth. (I970)

Fischer Meth. (1971)

Fischer Meth. (1972)

Fishing-Basic Terms and Definitions (1969)

ISO

Fishing Nets-Meth. of Determining the Breaking Load a ISO

Fishing Nets-Meth. of Determining the Mesh Breaking L ISO

Fishing Nets (1970) Rec. for Requi ISO

Fishing Nets: Designation in the Tex System (1968)

Fissile Materials (1970)

Fits-Part II: Inspection of PIain Workpieces (I971)

Fits-Part I: General Tolerances and Deviations (1962)

Fitting Dimensions of Chuck Bushings-Part I (1970)

Fitting Dimensions of Chuck Bushings-Part II (1970)

Fitting Dimensions (1970)

Fittings-Stainless Steel Tubes for the Food Industry

Fittings for Building and Sanitary Purposes (1964)

Fittings for Copper Tubes-Dimensions of Sockets and M ISO

Rec. for P ISO

ISO

ISO

ISO

ISO

ISO

Fittings for Electrical Installations (I962)

Fittings for Incandescent Lamps for Domestic and SimiIa

Fittings for Pipes Under Pressure-Basic Dimensions: M

Fittings for Pipes Under Pressure: Unplasticized Polyvi

Fittings for Series 1 Freight Containers (1970)

Fittings for Sewerage and Drainage (1968)

Fittings for Syringes, Needles and Other Medical Equipm

Fittings for Tubular Fluorescent Lamps (1965)

Fittings for Unplasticized Polyvinyl Chloride (PVC) Pre

Fittings for Waste Water and Ventilation (1966)

Fittings for Waste Water and Ventilations-Part IV: Fi

Fittings in Aircraft-(In. Series-Class 2 Tolerances

Fittings in Aircraft (In. Series-Class 1 Tolerances)

Fittings in Unplasticized Polyvinyl Chloride (PVC) for

Fittings of Plastics Materials-Socket Fittings for Pi

Fittings of Plastics Materials: Socket Fittings with $S_{p}$

Fittings Screwed in Accordance with ISO Rec. R7 (1957)

Fittings Where Pressure Tight Joints Are Made on the Th

Fittings with Plain Sockets; Metric Series (I968)

Fittings with Spigot Ends for Domestic and Industrial W

Fittings (1966)

Rec. for Cast Iron Sanitary ISO

Spec. for Rigid Conduits of

Fixed Block Format for Positioning and Straight Cut Num

Fixed Capacitors for Direct Current Using Impregnated P

Fixed Capacitors for Use in Electronic Equipment: Termi

Fixed Height with Horizontal or Vertical Spindle-Test

IUilding Detail ISO

Fixed Lights (1969) Rec. for Shipbuildin

Fixed Metallized Paper Dielectric Capacitors for Direct

Fixed Nonwirewound Resistors, Type II (I959)

Fixed Resistors (1965)

Fixed Resistors: Selection of Meth. of Test and General

Fixed Resistors: Terms and Meth. of Test (1971)

Fixed Resistors: Wirewound Resistors, Type 1-Detail S

Fixed Resistors: Wirewound Resistors, Type 1-Selectio

Fixed Tantalum Capacitors with Liquid or Solid Electrol

Fixed Wirewound Resistors Type 2 (1969)

Flame and Induction Hardening Types (I972)

Flame Emission Photometric Meth. (1971)

Flame Emission Photometry Meth. (1970)

Flame Resistance of Conveyor Belts: Spec./S

Use in Television Receiv

Flame (Emission) Spectrophotometric Meth. (I970)

Flameproof Enclosures of Electrical Apparatus (1971)

Flammability and Burning Rate of Plastics in the Form 0

\section{ISO}

CEE

CEE

ISO

ISO

ISO

ISO

IEC

ISO

ISO

ISO

ISO

ISO

ISO

ISO ISO

ISO

ISO

IEC

ISO

Flammability Of: in the Form of Bars (1970)

Flammable Gases from Plastics (I968)

Flange Column Sections-In. Series-Dimensions and Se

Flange Connectors: Detail Spec. (1972) lor General Pur IEC

Flange Connectors: General Requirements and Measuring $M$ IFC

Flange I Beams-In. Series-Dimensions and Sectional ISO

Flange Numbers F55 to F1080 (1971) /Tput Ratings for R IEC

Flange (1973) /Ling Bearings: Tapered Roller-Boundar ISO

Flanges for Circular Types (1969) 
Rec. for Flanges for Waveguides: Relevant Spec. for Rec. for Pipeline Rer. for Flanges for Waveguides: Relevant Spec. for for Shipbuilding Details: Mating Dimensions for Pipeline ing Meth. (1964)

ordinary Rectangular Types (1968)

circular Types (1969)

rec. for Mounting of Plain Grinding Wheels by Means of Hub

Rec. for

Rec. for

Rec. for

Rec. for Dimensions of Smal

Varnishes-Determination of the Danger Classification by Rec. for Paints and Varnishes-Determination of
Rec. for Laboratory Boiling

International Rec. for One Mark Glass Measurin Rec. for 1 Mark Volumetric Rec. for Double Deck

ensile Test (1971) Rec, for Copper and Alloy Rolled Rec. for Hollow Metallic Waveguides: Relevant Spec. for

for Hollow Metallic Wayeguides: Relevant Spec for Medium for Flanges for Waveguides: Relevant Spec. for Flanges for for Hollow Metallic Waveguides: Relevant Spec. for Medium Rec. fo

Rec. for Automobile-Spark Plug M 14 X 1.25 with std. for Automobiles: Compact Spark Plugs M 14 X 1,25 with Std. for Automobiles: Spark Plugs M 10 X 1 with Std. for Automobiles: Spark Plugs M 12 X 1,25 with Rec. for Rolling Bearings-Thrust Bearings with Rec. for Rolling Bearings-Thrust Ball Bearings with $5 /$ Rec. for Rolling Bearings-Thrust Ball Bearings with Rec. for Asbestos Cement Rec. for

Rec. for Requirements for 28 Volt d.c. $s$ of Brinell Hardness Values (HB) for Use in Tests Made on Rec. for Widths of Rec for Lengths of Rec. for Crowns of Pulleys for

millimeters) and In./S (19/

Rec. for Diameters of Pulleys for Rec. for Slottcd Countersunk Rec for Dimensions of Hot Rolled Steel Bars: bolts and Nuts: Metric Series, Tolerances on Widths Across ries (196/ Rec. for Hexagon Bolts and Nuts: Widths Across 1969)

Rec. for Copper and Its Alloy Tubes of Circular Section Rec. for Std. for Determination of Fineness of (De Mattia Type/ a Rated Voltage No/ Rec, for Determination of Resistance to Rec. for Polyvinyl Chloride Insulated Rated Voltage Not Exceeding / Rec. for Rubber Insulated

(1972)

Density (1971)

ion (1972)

strength and Elongation at Break (1971)

of Test Pieces (1971)

ion Techniques (1972)

Spec for Polyvinyl Chloride Insulater Spec. for Rubber Insulated Cables and

al Spec. (1973) Std. for Plastics-Determination of for Meth. of Test. Strength of Cements-Compressive and ination of the Charpy Impact Resistance of Rigid Plastics rmination of the lzod Impact Resistance of Rigid Plastics nts and Their Coefficients (1972)

the Atmosphere Relative to the Eart/ he Air (1972)

Std. for Symbols for Sid. for Symbols for Rec. for Projector and

1970)

Dynamic Loading (1971)

ysical Tests (1971)

n Varying Moisture Conditi/ 1) chanical Handling Equipment for Unit Loads-Single Strand Rec. Meth. for Measuring Performance o similar Electrical Appliances: Particular Requirements for chanical Handling Equipment for Unit Loads-Single Strand Single Strand Floor Mounted Truck Conveyors (Chain Above t Loads-Single Strand Floor Truck Conveyors (Chain Below ferent Species of Wood Utilized in the Manufacture of Wood ow Measurement in Open Channels: Dilution Meth. for Steady utside the Scope Covered B/

Rec. for Measurement of Fluid Rec. for Measurement of Fluid Rec. for Measurement of Fluid Data for Determination of Errors in Measurement of Liquid ints and Varnishes: Determination of Flow Time by Use of A
Flanges for Flat Rectangular Types (1968)

Flanges for General Use-Mating Dimensions (1971)

Flanges for Ordinary Rectangular Types (1968)

Flanges for Ships (1969)

Flanges for Waveguides: General Requirements and Measur

Flanges for Waveguides: Relevant Spec. for Flanges for

Flanges for Waveguides: Relevant Spec. for Flanges for

Flanges (1968)

Flanging Test on Steel Tubes (1960)

Flash Connections for Hand Held Cameras (1966)

Flashpoint (Closed Cup Meth.) (1970)

Flashpoint (Closed Cup Meth.) (1971)

Flasks (1970)

Flasks (1970)

Flasks (1971)

Flat Pallets for Through Transit of Goods (1961)

Flat Products (Thickness Less Than $2.5 \mathrm{~mm}(0.1 \mathrm{In})-.\mathrm{T}$

Flat Rectangular Types (1964)

Flat Rectangular Types (1967)

Flat Rectangular Types (1968)

Flat Rectangular (1968)

Flat Seal for Hydraulic Couplings (1970)

Flat Seating (1970)

Flat Seating (1973)

Flat Seating (1973)

Flat Seating (1973)

Flat Seats-Boundary Dimensions (1966)

FIat Seats-Normal Tolerances (1961)

Flat Seats-Tolerances: Part 2-Tolerance Classes 6,

Flat Sheets (1964)

Flat Steel Healds for General Use (1964)

Flat Strip Fuses for Aircraft (1968)

Flat Surfaces (1964)

Flat Transmission Belts and Corresponding Pulleys (1956 ISO

Flat Transmission Belts (1958)

Flat Transmission Belts (1959)

Flat Transmission Belts (1959)

(Flat) Head Tapping Screws-Dimensions in Millimetres (

Flat, Metric Series (1969)

Flats and Corners (1968)

Flats, Heights of Heads, Thicknesses of Nuts: Metric Se

Flattening Test on Aluminum and Aluminium Alloy Tubes (

Flattening Test on Steel Tubes (1961)

Flattening Test (1971)

Flax Fibres (Fibers)-Permeametric Meth. (1972)

Flex Cracking of Vulcanized Natural or Synthetic Rubber

Flexible Cables and Cords with Circular Conductors and

Flexible Cables and Cords with Circular Conductors and

Flexible Cables and Cords (1964)

Flexible Cellular Materials-Accelerated Ageing Tests

Flexible Cellular Materials-Determination of Apparent

Flexible Cellular Materials-Determination of Compress

Flexible Cellular Materials-Determination of Tensile

Flexible Cellular Materials-Measurement of Dimensions

Flexible Cellular Materials: Hardness Test. by Indentat

Flexible Cords (1967)

Flexible Cords (1968)

Flexible Steel Wire Rope for Aircraft Controls: Technic

Flexural Properties of Rigid Plastics (1972)

Flexural Strengths of Plastic Mortar (Rilem Cembureau M

Rec, for Plastic

(Flexural Test) (1961)

(Flexural Tests) (1961)

Flight Dynamics-Part III: Derivatives of Forces, Mome

Flight Dynamies-Part II: Motions of the Aircraft and ISO

Flight Dynamics-Part I: Aircraft Motion Relative to T ISO

Floodlighting Lamps (1971)

Floor Coverings-Determination of Mass Per Unit Area (

Floor Coverings-Determination of Thickness Loss Under

Floor Coverings-Determination of Thickness (1971)

Floor Coverings-Sampling and Cutting Specimens for $\mathrm{pH}$

Floor Coverings: Classification and Terminology (1972)

Floor Coverings: Determination of Dimensional Changes

Floor Mounted Truck Conveyors (Chain Above Floor)-Sa

Floor Polishers for Household and Similar Purposes (197

Floor Treatment and Wet Scrubbing Machines for Househol

Floor Truck Conveyors (Chain Below Floor) Safety Code (

(Equipment for Unit Loads ISO

Floor)-Safety Code (1971)

Floor) Safety Code (1972)

looring-Code of Symbols (1971) / Marking of the Dif ISO

Flow-Part 1: Constant Rate Injection Meth. (1966) /L ISO

Flow by Means of Orifice Plates and Nozzles (1967)

Flow by Means of Venturi Tubes-Classical Tubes Used $O$

ISO

ISO

Flow by Means of Venturi Tubes (1968)

Flow by Velocity Area Meth. (1969)

Flow Cup (1972)
/ for Collection of ISO $\mathrm{Std}$, for $\mathrm{Pa}$ ISO 
Rec. for Plastics-Determination of the Melt Flow Index of Polyethylene and Compounds of (1967) peration of a Gauging Station and Determ/ 1969) Rec. for Liquid (1968)

Rec. for Liquid teady Flow-Part 1: Constant Rate Injec/

Rec. for Liquid Rec. for Liquid Rec. for Plastics-Determination of the Mel Std. for Paints and Varnishes: Determination of Symbols Used in Connection with the Measurement of Liquid ositive Displacement Vacuum Pumps-Part I: Volume Rate of cs of Vapour (Vapor) Vacuum Pumps-Part I: Volume Rate of Rec. for Principles and Conventions for
Rec. for Meth. of Measurement of Speed
Rec. for Measurement of used Outside the Scope Covered B/ Rec. for Measurement of Rec. for Measurement of for Hydraulic and Pneumatic Equipment and Accessories for essure (197) eters (1970) sses Up to / Tolerances/ Tolerances/ Rec. for Plastics Pipes for the Transport of Rec. for Plastic Pipes for the Transport of Rec. for Plastics Pipes for the Transport of Rec. for Plastics Pipes for the Transport of Rec. for Plastics Pipes for the Transport of c. for Identification Colours (Colors) for Pipes Conveying Rec. for Pipes of Plastics Materials for the Transport of Rec. for Pipes of Plastics Materials for the Transport of Rec. for Tubular Rec. for Ballasts for Rec. for Glow Starters for Tubular Rec. for Lighting Fittings for Tubular Rec. for Lampholders and Starterholders for Tubular content-Modified Willard ent-1,10 Phenanthroline / Std. for Aluminium (Aluminum) Std. for Aluminium (Aluminum) ntent-Spect rophotometric/ Std. for Aluminium (Aluminum) m (Aluminum) Fluoride for Industrial Use: Determination of for Cryolite (Natural and Artificial)-Determination of Rec. for Aircraft Toilet Rec. for Hand and Long

Rec. Spec. for Particular Types of Winding Wires: Self

cular Types of Winding Wires: Heat or Solvent Bonding Self d Lifts of $300 \mathrm{~mm}$ and Over (1969)

ts $U p$ to $300 \mathrm{~mm}(1963)$ amera $(1970)$ Rec. for Surface Active Agents: Measurement of ermination of the Recovery from Creasing of a Horizontally Rec. for Paper and Board: Sizes of Std. for Hermetically Sealed Metal Rec. for Hermetically Sealed Metal irements for the Electrical Fquipment of Refrigerators and ec. for Pipes and Fittings-Stainless Steel Tubes for the n of Nitrogen by the Kjeldahl Meth/ Rec. for Agricultural ial Low Temperature Compartments for the Storage of Frozen Ammonium Hydrogen Carbonate for Industrial Use (Including to 1000 (197) Rec. for Dimensions and Output Ratings for Std. for Lined Industrial Rubber Std. for Lined Conducting Rubber

Magazines for Still Picture Use and Test for Film Pullout ied Contact Area and Being Applied with a Specified Static for Symbols for Flight Dynamics-Part III: Derivatives of Rec. for Analysis of Soaps: Determination of or Plastics-Pve Resins-Determination of Impurities and Maximum / Std. for Assembly Tools for Screws and Nuts xagonal Drill Steels (1968) Rec. for Rock Drilling: For/ Rec. for Railway Rolling Stock Material-Rolled on Rec. for Mechanical Property Limits for Aluminium Alloy

ancing of $\mathrm{Pr} /$ $\begin{array}{ll}\text { ancing of } \mathrm{Pr} / & \text { Rec. for Technical Drawings-Tolerances of } \\ \text { ndications } \mathrm{O} / & \text { Rec. for Technical Drawings-Tolerances of }\end{array}$ ndications on Drawings (1969)

emical Analysis (1969) al Equipment (1961) Rec. for Tolerances of Rec. for Guide on the Rec. for Std. nation of Flammability and Burning Rate of Plastics in the filament and Staple Fibre (Fiber) Yarns and Rovings in the cal Properties of Receiving Aerials in the Frequency Range c. for Rock Drilling: Hollow Hexagonal Drill Steels in Bar mination of Saleable Mass, in Lots, of Pulp Baled in Sheet Rec. for Writing of Calendar Dates in All Numeric ormaldehyde Solutions for Industrial Use: Determination of

Rec for Plastics-Detection of Free Ammonia in Phenol for Plastics-Determination of Free Phenols in Phenol ination of Free Ammonia and Ammonium Compounds in Phenol of Ash (1972)

for Industrial Use-Determination of Manganese Content .

of Acidity (1972)

$\mathrm{n}$ of Iron Content-2,2' Bipyridyl Photomet/

Std. for

Std. for
Rec. for Spec. for

Rec. for

Flow Measurement in Open Channels-Establishment and $O$

Flow Measurement in Open Channels by Slope Area Meth. (

Flow Measurement in Open Channels by Velocity Area Meth

Flow Measurement in Open Channels: Dilution Meth. for S

Flow Rate of Thermoplastics (1969)

Flow Time by Use of a Flow Cup (1972)

Flow with a Free Surface (1968)

Flow (Pumping Speed) (1970)

Flow (Pumping Speed) (1970)

(1969)

Flowsheets for Coal Preparation Plant (1969)

Fluctuations in Sound Recording and Reproducing Equipme

Fluid Flow by Means of Orifice Plates and Nozzles (1967

Fluid Flow by Means of Venturi Tubes-Classical Tubes

Fluid Flow by Means of Venturi Tubes (1968)

Fluid Power Transmission (1970)

ffor Graphical Symbol

Fluids-Determination of the Resistance to Internal Pr

Fluids-Polyethylene (PE)-Tolerances on Outside Diam

Fluids-Polyethylene (PE)-Tolerances on Wall Thickne

Fluids-Unplasticized Polyvinyl Chloride (PVC) Pipes-

Fluids-Unplasticized Polyvinyl Chloride (PVC) Pipes-

Fluids in Liquid or Gaseous Condition in Land lnstallat

luids (Outside Diameters and Nominal Pressures) Part

Fluids (Outside Diameters and Nominal Pressures): Part

Fluorescent Lamp Auxiliaries (1968)

Fluorescent Lamps for Genc

Fluorescent Lamps (1963)

Fluorescent Lamps (1965)

Fluorescent Lamps (1972)

Fluoride for Industrial Use: Determination of Fluorine

Fluoride for Industrial Use: Determination of Iron Cont

Fluoride for Industrial Use: Determination of Silica Co

Fluorine Content-Modified Willard-Winter Meth. (197

Fluorine Content: Modified Willard-Winter Meth. (1970

Flushing and Draining Connections (1957)

Fluted Machine Reamers, Morse Taper Shank (1961)

Fluxing Enamelled Round Copper (1970)

Fluxing Enamelled Round Copper (1970) ISpec.
Flyer Bobbins for Spindle Diameters of $25 \mathrm{~mm}$ or More an
Flyer Bobbins for Spindle Diameters Up to $22 \mathrm{~mm}$ and Lif

Flyer Bobbins for Spin

Focal Spots of Diagnostic X-Ray Tubes Using a Pinhole C

Folded Specimen by Measuring the Angle of Recovery (197

Folders and Files (1967)

Food Containers-Internal Diameters of Round Cans (197

Food Containers (1959)

Food Freezers for Household and Similar Purposes (1970)

Food Industry (1971)

Food Products-General Directions for the Determinatio

Foodstuffs (1968)

/Ehold Refrigerators-Part II: Spec

Foodstuffs): Determination of Total Alkalinity-Volume

Foot Mounted Electrical Machines with Frame Numbers 355

Footwear for Use at Low Temperatures (1972)

Footwear (1972)

Force (1969)

Force (1971)

Rec. for Dimensions for $35 \mathrm{~mm}$ Film

Forces, Moments and Their Coefficients (1972)

Foreign Matter of Low Solubility in Ethanol (1968)

Foreign Matter (1970)

Forged and and Tubular Socket Wrenches-Metric Serie

Forged Collared Shanks and Chuck Bushings for Hollow He

Forged Wheel Centres (Centers) for Tyred (Tired) Wheels Forgings (1968)

Fork Lift Trucks-Rated Capacity (1971)

Form and of Position-Part III: Dimensioning and Toler

Form and of Position-Part IV: Practical Examples of 1

Form and of Position-Part 1: Generalities, Symbols, I

Form for Std. for Chemical Products and for Meth. of $\mathrm{Ch}$

Form of Bars (1970)

Form of Declaration of Performance of Aircraft Electric

Form of Film (1970)

Form of Packages (1971)

Form $30 \mathrm{MHz}$ to $1,000 \mathrm{MHz}(1962)$

Form (1968)

Form (1968)

Form (1971)

Formaldehyde Content (1972)

Formaldehyde Mouldings-Quali

Formaldehyde Mouldings (1959)

Formaldehyde Mouldings (1959)

Formaldehyde Oxime Photometric Meth. (1972) Rec. for the Determi ISO
IAr Density of Its Continuous ISO

I of Essential Electri IEC

Re ISO

Rec. for Pulps: Deter ISO Std. ISO

Rec. F ISO

ISO

ISO

ISO

ISO

ISO

ISO

Formaldehyde Solutions for Industrial Use: Determinatio

Std. for F $\begin{array}{ll}\text { ISO } \\ \text { ISO }\end{array}$

Rec ISO

Formaldehyde Solutions for Industrial Use: Determinatio for Plastics-Determ ISO /Raborates ISO

ISO

ISO

ISO 
n of Firmaldehyde Content (1972) n of Methanol Content (1972) or lnorganic Chlorides (1972) or Inorganic Chlorides (1972) or Heavy Metals (Excluding Iron) (1972 irolled Machines (1/ Rec. for Punched Tape Variable Block irolled Machines (1969/ Rec. for Punched Tape Fixed Block led/Rec. for Interchangeable Punched Tape Variable Block aphy Using $35 \mathrm{~mm}$ Objectives on $35 \mathrm{~mm}$ Fiim, 5-Perforation Rec. for Index of a Publication (Standardization of

f Preparatory Functions G An/ Rec. for Punched Tape Block Rec. for $35 \mathrm{~mm}$ Filmstrips: Dimensions and Rec. for Textile Machinery-Types of content: 2, 2'-Bipyridyl Photometric Meth/ ontents of Volatile Acids Other Than Formic (Le/

Rec. for

Rec, for

determination of Low Contents of Volatile Acids Other Than Rec. for Determination of Rec. for General Principles for the Rec. for Surface Active Agents in Powde Std. for Centre Drills for Center Holes with Radius Std. for Programming Language: Rec. for Paper Vocabulary:

d Output Ratings for Foot Mounted Electrical Machines with ons and Output Ratings for Rotating Electrical Machines Rec. for "lwin Wire Healds for essories-Reversible Rings for Ring Spinning and Doubling ories-Nonreversible Rings for Ring Spinning and Doubling Rec. for Rings for Ring Spinning and Doubling Rec. for ccessories-Spindle Gauges for Ring Spinning and Doubling ch of the Harness (1967) Rec. for Heald Rec. for Oil of Peppermint: for Ammonium Nitrate for Industrial Use: Determination of Rec. for Plastics: Determination of fter Acetylation/

Rec for Essential Oils-Estimation of Rec. for Analysis of Soaps: Determination of Total ehyde Mouldings (19) tive Meth. (1961)

Rec. for Plastics-Determination of Rec. for Plastics-Detection of romium Types (1970) 1970) ype (1970)

ening Types (1972)

lloyed Types (1968) pered Types with $1 \% \mathrm{Ch} /$ ered Unalloyed with $\mathrm{Co} /$ ered Manganese (1970) ered, with $3 \%$ Chromium/ ered Chromium Nickel M/ (1970)

ed Types with $1 \%$ Chromi/ Rec. for Basic Environmental Test Procedures: Test Ed: Std. for Meat and Its Products-Determination of ds of Noise in a Diffuse Field and in a Frontally Incident

tours for Pure Tones and Normal Threshold of Hearing Under ents: Analysis of Soaps-Determination of Low Contents of ntial Oils-Determination of Carbonyl Compounds Content Rec. for Plastics-Determination of ial Use: Determination of Total Acidity and Calculation of d in Connection with the Measurement of Liquid Flow with A unit Loads-Overhead Twin Rail Chain Conveyors (Power and lastics-Aqueous Dispersions of Polymers and Copolymers nts for the Electrical Equipment of Refrigerators and Food Rec. for Determination of Basic Requirements (1972) Std. for Hooks for Lifting Rec. for Marking of Series 1 and Series 2 Rec. for Spec. for Corner Fittings for Series Rec. for Spec. and Test. of Series 1 Rec. for Dimensions and Ratings of Std. for Identification Marking Code for or Meth. of Sampling Manganese Ores; Part I: Ore Loaded in $f$ Equivalent Terms Used in the Plastics Industry (English, f Equivalent Terms Used in the Plastics Industry (English, f Equivalent Terms Used in the Plastics Industry (English, f Equivalent Terms Used in the Plastics Industry (English, $f$ Equivalent Terms Used in the Plastics Industry (English, tions-Alphabetical Indexes of Equivalent Terms (English, Rec. for Connectors for

and Associated Sound Equipment ( with Threaded Coupling (1966) with Bayonet or Push Pull Coupli/ o Circuits in Radio Receivers (1/ Rec. for Connectors for Rec. for Connectors for Rec. for Connectors for Rec. for Connectors for
Formaldehyde Solutions for Industrial Use: Determinatio Formaldehyde Solutions for Industrial Use: Determinatio Formaldehyde Solutions for Industrial Use: Limit Test F Formaldehyde Solutions for Industrial Use: Limit Test F Formaldehyde Solutions for Industrial Use: Limit Test F Format for Positioning and Straight Cut Numerically Con Format for Positioning and Straight Cut Numerically Con Format Positioning and Straight Cut Numerically Control Format (1966)

Formats for the Numerical Control of Machines: Coding $O$ Formats (1968)

Formers for Yarn Packages (1970)

Formic Acid for Industrial Use-Determination of Iron

Formic Acid for Industrial Use-Determination of Low C

Fornic Acid for Industrial Use: Meth. of Test (1968)

Formic (Less Than $0.5 \%(\mathrm{~m} / \mathrm{m})$ Calculated as Acetic)-Vo

Forms of Sulphur in Coal (1960)

Formulation of Graphical Symbols (1972)

Form: Preparation of a Reduced Sample (1967)

Form: Type R (1972)

FORTRAN (1972)

Fourth Series of Terms (1964)

rame Numbers 355 to 1000 (1970)

Frame Numbers 56 to 400 and Flange Numbers

Frame Weaving (1967)

Frames for C and El Travellers-Principal Dimensio

Frames for $C$ and El Travellers-Principal Dimensio

Frames for Ear Shaped Travellers (1959)

Frames for Single or Double Row of Healds (1967) tor Textile Machinery and a 150

Frames: Coordinated Dimensions in Relationship with Pit ISO

France, Italy, United Kingdom and U. S. A. (1968)

Free Acidity-Volumetric Meth. (1972)

Free Acidity of Unplasticized Cellulose Acetate (1969)

Free Alcohols Content by Determination of Ester Value a

Free Alkali (1968)

Free Ammonia and Ammonium Compounds in Phenol-Formald

Free Ammonia in Phenol Formaldehyde Mouldings-Qualita

Free Caustic Alkali (1973)

Free Cutting-Part 7: Wrought Quenched and Tempered $\mathrm{Ch}$

Free Cutting Steels-Part 10: Wrought Nitriding Type (

Free Cutting Steels-Part 11: Wrought Case Hardening T

Free Cutting Steels-Part 12: Flame and Induction Hard

Free Cutting Steels-Part 1: Quenched and Tempered Una

Free Cutting Steels-Part 2: Wrought, Quenched and Tem

Free Cutting Steels-Part 3: Wrought Quenched and Temp

Free Cutting Steels-Part 5: Wrought Quenched and Temp

Free Cutting Steels-Part 6: Wrought Ouenched and Temp

Free Cutting Steels-Part 8: Wrought Quenched and Temp

Free Cutting Steels-Part 9: Wrought Free Cutting Type

Free Cutting Steels, Pt. 4: Wrought Quenched and Temper

Free Fall (1969)

Free Fat Content (1973)

Free Field for Equal Loudness (1965)

Free Field Listening Conditions (1961)

Free Hydroxylamine Meth. (1972)

Free Phenols in Phenol-Formaldehyde Mouldings (1959)

Free $\mathrm{SO}_{3}$ Content of Oleum; Volumetric Meth. (1968) ISO

Free Surface (1968) /Cabulary of Terms and Symbols Use ISO

Free) Safety Code (1971) /Ical Handling Equipment for 1SO

Freeze Thaw Cycle Stability Test (1969) Rec. for P ISO

Freezers for Household and Similar Purposes (1970) /Me IEC

Freezing Point of Essential Oils (1969)

Freight Containers-Minimum Internal Dimensions (1970)

Freight Containers of $U_{p}$ to 30 Tonnes (Tons) Capacity-

Freight Containers (1968)

Freight Containers (1968)

Freight Containers (1970)

Freight Containers (1970)

Freight Containers (1970)

Freight Wagons (1963)

French, Russian) (Cs: Corresponding Czech Terms) (1964)

French, Russian) (D: Corresponding German Terms) (1964)

French, Russian) (NL: Corresponding Dutch Terms) (1968)

French, Russian) (Pl: Corresponding Polish Terms) (1963

French, Russian) (1969)

French, Russian) (1970)

Rec. for List $\mathrm{O}$

Frequencies Below $3 \mathrm{MHz}$ : Battery Connectors (1965) lof Gears-Geometrical Defini

requencies Below $3 \mathrm{MHz}$ : Circular Connectors for Radio

Fequencies Below $3 \mathrm{MHz}$ : Circular Multipole Connectors

Frequencies Below $3 \mathrm{MHz}$ : Circular Multipole Connectors

Frequencies Below $3 \mathrm{MHz}$ : Concentric Connectors for Audi

0


-rnal Jow Voltage Power Supply T/ and Associated Sound Equipment / osed Finds and llaving a Contact S/ en Ends, Off Centre (Center) Gui/ n Detail Spec. (1970)

e Connectors with Blade Contacts/ rs with Blade Contacts (1966)

d Measuring Meth. (1962)

ns (1967)

Electrical Insulating Materials at Power, Audio and Radi Rec for IEC Std.

r Electric Strength of Solid Insulating Materials at Power ces of Filter Crystal / Rec. Meth. for the Measurement of ) Insulation and Sheath: General Test and M/ Insulation and Sheath: Cables in Pairs, T/ Insulation and Sheath: Equipment Wires, T/ Insulation and Sheath: Distribution Wires/ Insulation and Sheath: Equipment Wires An/ Insulation and Sheath: Signalling Cables / Insulation and Sheath: Distribution Wires/

Rec. for Connectors for Rec. for Connectors for Rcc. for Connectors for Rec. for Connectors for Rec: for Connectors for Rec. for Connectors for Rec for Connectors for Power Capacitors for ec. for Preferred Rec. for Low Rec. for Low Rec. for Low Rec. for Low Rec. for Low Rec. for Low Rec. for Low $s$ (Colors) for Pve (Polyvinyl Chloride) Insulation for Low Resistance of Plain and Tinned Copper Conductors of Low th. (1971) (1970)

Rec. for Radio Rec. for Radio Rec. for Radio

Rec. for Scales and Sizes for Plotting

ec. for Characteristic lmpedances and Dimensions of Radio Rec. for Dimensions of the Mating Parts of Radio al Feeder Connector (1965) g Meth. (1965)

0-12 (1967)

$0-17$ and Larger (1970)

5-17 and Larger (1971)

ed Aerial Feeders (1965)

dio Receivers for Various Classes of En Rec. Meth. of Measurement on Receivers for Measurement of Radiation from Receivers for Amplitude and electronic Tubes and Valves: Meth. of Measurement of Audio electronic Tubes and Valves: Meth, of Measurement of Radio

f Emission: Measurements Particularly Related to the Audio ssential Electrical Properties of Receiving Aerials in the measurement for Piezoelectric Vibrators Operating Over the Spec. for Radio Interference Measuring Apparatus for the Spec. for Radio Interference Measuring Apparatus for the Measuring Set Spec. for the Rec. Graphical Symbols: Frequency (Std. Musical Pitch)/ Rec. for Standard Tuning
ng Frequency (Standard Musical Pitch) Rec. for Std. Tuning ng Frequency (Standard Musical Pitch) Rec. for Std. Tuning measurement for Radio Transmitters: General Conditions Of Rec. for to the Connection of Auxiliaries / Rec. for Photography 956) Rec. for Emulsion Position in Projector for Direct 56) Rec. for Emulsion Position in Projector for Direct jector Usage of $8 \mathrm{~mm}$ Type S Motion Picture Film for Direct evels of Narrow Bands of Noise in a Diffuse Field and in $A$ i: Special Low Temperature Compartments for the Storage of oluble in Hydrochloric Acid (1971)

0)

impurities (1968)

le Acidity (1968)

lids (1969)

soluble Solids (1968)

Rec. for

Rec. for

Rec. for

Rec. for

Rec. for

Rec. for

Rec. for Fresh

Rec. for Determination of Fusibility of

Rec. for Aircraf

Rec. for Rubber Hoses for Aircraft Ground

alorimetric Bomb Meth. and Calcul/

Rec. for Solid Mineral

Rec. for Vocabulary of Terms Relating to Solid Minera

Rec. for Vocabulary of Terms Relating to Solid Mineral

Rec. for Vocabulary of Terms Relating to Solid Mineral Rec. for Determination of Ash of Solid Minera

d Polyvinyl Chloride (PVC) Pipes for the Supply of Gaseous yor Belts: Spec. and Meth. of Test (1962)

Rec. for Rec. for

Rec. for Layout of Printing and

for Plastics-Determination of Stiffness in Torsion as A Rec, for Horology

t (1971) Rec. for Expression of the

hines: Coding of Preparatory Functions $G$ and Miscellaneous Rec. for International Electrotechnical Vocabulary:
Frequencies Below $3 \mathrm{MHz}$ : Connectors for Coupling an Ext requencies Below 3 Mllz: Connectors for Radio Receivers Frequencies Below $3 \mathrm{MHz}$ : Ed ge Socket Connectors with $\mathrm{Cl}$ Frequencies Below $3 \mathrm{MHz}$ : Edge Socket Connectors with Op Frequencies Below $3 \mathrm{MHz}$ : Guide to Drawing lnformation I Frequencies Below $3 \mathrm{MHz}$ : Rectangular Miniature Multipol Frequencies Below $3 \mathrm{MHz}$ : Rectangular Multipole Connecto Frequencies Below $3 \mathrm{MHz}$ : (Mc/S) General Requirements an Frequencies Between 100 and $20000 \mathrm{~Hz}(\mathrm{c} / \mathrm{s})(1959)$ Frequencies for Acoustical Measurements (1962)

Frequencies for Centralized Network Control Installatio

Frequencies lncluding Metre (Meter) Wavelengths (1969)

Frequencies (1965)

Frequencies (1967)

Rec. Meth. of Test Fo

Frequency and Equivalent Resistance of Unwanted Resonan

requency Cables and Wires with Pve (Polyvinyl Chloride

Frequency Cables and Wires with Pvc (Polyvinyl Chloride

Frequency Cables and Wires with Pvc (Polyvinyl Chloride

Frequency Cables and Wires with Pvc (Polyvinyl Chloride

Frequency Cables and Wires with Pve (Polyvinyl Chloride

Frequency Cables and Wires with Pvc (Polyvinyl Chloride

Frequency Cables and Wires with Pvc (Polyvinyl Chloride

Frequency Cables and Wires (1969)

Frequency Cables and Wires (1971)

/Ec. for Std. Colour

Frequency Cables: General Requirements and Measuring Me

Frequency Cables: Guide to the Design of Detailed Spec.

Frequency Cables: Relevant Cable Spec. (1961)

Frequency Characteristics (1968)

Frequency Coaxial Cables (1967)

Frequency Connectors (1964)

Frequency Connectors: Coaxial Unmatched Television Aeri

Frequency Connectors: General Requirements and Measurin

Frequency Connectors: R. F. Coaxial, for Cables 96 IEC 5

Frequency Connectors: R. F. Coaxial, for Cables 96 IEC 5

Frequency Connectors: R. F. Coaxial, for Cables 96 IEC 7

Frequency Connectors: Two Pin Connector for Twin Balanc

Frequency Generators (1972)

Frequency Measurements on Fm Receivers of the Response

Frequency Modulation Broadcast Transmissions (1958)

Frequency Modulations and Television Broadcast Transmis

Frequency Output Power and Distortion (1966)

Frequency Output Power (1966)

(Ectrical Properties of IEC

Frequency Part of a Receiver (1971)

/Various Classes O IEC

Frequency Range Form $30 \mathrm{MHz}$ to $1,000 \mathrm{MHz}(1962)$ / of E IEC

Frequency Range Up to $30 \mathrm{MHz}$ (1969) /Ons and Meth. of lEC

Frequency Range $0.15 \mathrm{MHz}$ to $30 \mathrm{MHz}$ (1972)

Frequency Range $25 \mathrm{Mc} / \mathrm{S}$ to $300 \mathrm{Mc} / \mathrm{S}$ (1961)

Frequency Range $300 \mathrm{MHz}$ to $1,000 \mathrm{MHz}$ (1967)

Frequency Speetrum Diagrams (1968)

Frequency (Standard Musical Pitch) Rec. for Std. Tuning

Frequency (Std. Musical Pitch) (1955)

Frequency, Output Power and Consumption (1968)

ISC

Fresh Fruits and Vegetables: Sampling (1968)

resh Pineapples-Guide to Storage and Transport (1970

Front Lens Barrels $U_{p}$ to $100 \mathrm{~mm}$-Dimensions lmportant

Front Projection of $16 \mathrm{~mm}$ Silent Motion Picture Film (1

Front Projection of $8 \mathrm{~mm}$ Silent Motion Picture Film (19 Tuni ISO

Picture Fin (19): Pro

Frontally Incident Free Field for Equal Loudness (1965)

Frozen Foodstuffs (1968)

(Ehold Refrigerat

and Vetable Products-Determination of Ash Ins

and Vegetable Products-Determination of $\mathrm{pH}$ (197

Fruit and Vegetable Products: Determination of Minera

Fruit and Vegetable Products: Determination of Total So

Fruit and Vegetable Products: Determination of Water in

Fruits and Vegetables: Sampling (1968)

Fuel Ash (1967)

Fuel Nozzle Grounding Plugs and Sockets (1970)

Fueling Without Static Conducting Wire (1971)

Fuels-Determination of Gross Calorific Value by the C

Fuels-Part IIl: Coke (1971)

Fuels-Part II: Coal Sampling and Analysis (1971)

Fuels-Part 1: Coal Preparation (1970)

Fuels (1970)

Fuels: Metric Series-Spec (1973)

Full Thickness Tensile Strength and Elongation of Conve

Function Key Symbols on Typewriters (1969)

Function Keys on Typewriters (1969)

Function of Temperature (1965)

Functional and Nonfunctional Jewels (1969)

Functional Performance of Electronic Measuring Equipmen is for the Numerical Control of Mac ns $M$ (1969)

Fundamental Definitions (1956)

Fundamental Parameters of Connectors for Printed Wiring
CISPR

CISPR

CISPR

$\mathrm{SO}$

IEC $\quad 130-10$

$30-11$

$130-13$

130.0

$130-6$

$130-5$

130-1

110

266

242

250

196

24.3

283

189-1

189.2

$189-3$

$189-4$

189.5

$189-6$

189.7

304.

344

96-1

96-0

96.2

263

159

169.2

169-1

169-4.

169-5

169.6

169-3

403

315-5

91

106

151.10

151.11

$315-2$

138

302

4

16

16

244-1

874

1838

1948

26

29

1781

454

825

763

1842

762

750

1026

751

874

540

46

1825

1928

1213/III

$1213 / \mathrm{II}$

$1213 / \mathrm{I}$

1171

2703

283

1090

1091

458

1112

359

1056

$50(05)$

171 
Std. for Joints in Building: Construction of Installations for Work on Unse/ Rec. for Rec. for Electrical Installations of Buildings: International Rec. for Vocabulary of L.egal Metrology on and Slope for Straight Welds (1967) Rec. for

Pract. for the Evaluation of the Resistance of Plastic to

f Moulding Material That Cannot Be Poured from a Specified $y$ of Moulding Material That Can Be Poured from a Specified Rec. for Test Meth. for Batch Rec. for Test Meth. for Induction readed Sockets and Connecting Pins for Use in Electric Arc Airbreak Disconnectors, Airbreak Switch Disconnectors and

Rec. for Rec. for Rec. for Precision Rec. for Precision Rec. for Precision Rec. for Std. Rated Currents (2 to 63A) of Spec. for Cartridge Rec. for Cartridge

Rec for Fuse Holders for Miniature Cartridge Rec. for Requirements for 28 Volt d.c. Flat Strip Spec. for D.Type Rec. for

milar Purposes: General Requirement/ Rec. for Low Voltage d. Rated Currents (2 to 63A) of Fuse Links for Low Voltage Spec. for Cartridge Fuse Links for Miniature Rec. for Cartridge Fuse Links for Miniature Rec. for High Voltage Rec. for High Voltage Rec. for Determination of its Alloys /

Rec. Pract. for Radiographic Inspection of Thi/ Std. for Rec. Pract. for Radiographic Inspection of 0) rec. Pract. for Radiographic Inspection of Circumferential Rec. for Requirements for Hot Dip

for Determination of the Weight Per Unit Area of Hot Dip Rec, for Shipbuilding Details-Tests on Rec. for Shipbuilding Details ing Principles for Protection Against Corrosion by Hot Dip se in Radiological Protection (/

Rec. for Portable X... or Rec. for Tolerances on Spanner with Picture-Location and Width of Magnetic-Striping and Rec. for Voltage Measurement by Means of Sphere Rec. for Combinations of Double Ended Wrench the Carbon Dioxide Content, Expressed as Sodium Carbonate, Carbon Dioxide Content, Expressed as Potassium Carbonate: Bro/ Rec. for Determination of the Yields of Tar, Water, eratures (19/ paratus, Typ/ closures of $/$ Rec. for Electrical Apparatus for Explosive Rec. for Electrical Apparatus for Explosive Rec. for Electrical Apparatus for Explosive Rec. for Electrical Apparatus for Explosive Rec. for Electrical Apparatus for Explosive Rec. for Electrical Apparatus for Explosive (1966) Rec. for Electrical Apparatus for Explosive Rec. for Electrical Apparatus for Explosive Rec. for Electrical Apparatus for Explosive Rec. for Electrical Apparatus for Explosive (1967) Rec. for Electrical Apparatus for Explosive Safe Circui/ Rec. for Electrical Apparatus for Explosive
Rec. for Marking of Ind ustrial 65)

r) Rec. for Yoke Type Valve Connections for Small Medical 72) Spec. for Welding: Regulators for Rec. for Identification of the Contents of Medical Rec. for Marking of Aircraft $f$ the Electrical Properties of Microwave Tubes and Valves: Rec, for Steel Tubes for

t Joints Are Made on the Threa/ Rec. for Pipe Threads for nternational Rec. for General Prescriptions for Volumetric r Alternating Voltages /

\section{r Alternating Voltages /}

ted, Metal Sheathed, Fo/ Rec. for Tests on Oil Filled and Rec. for Tests on Oil Filled and g Voltages from $10 \mathrm{kV}$ Up to and Including $33 \mathrm{kV}$ (Excluding g Voltages from $10 \mathrm{kV}$ Up to and Including $66 \mathrm{kV}$ (Excluding s of Wires, Spools, Rims and Co/

Rec. for Solid Wires for Std. for

oxide Content Expressed as Sodium or Potassium Carbonate ical Properties of Weld Metal Deposited by Filler Rods for ypes (1967) Rec. for Code of Symbols for Filler Rods for or Electrodes for Manual Arc Welding and Filler Metals for el Filler Rods and the Parent M/ Rec. for Filler Rods for Rec. for Steel Pipes for n Colours (Colors) for Pipes Conveying Fluids in Liquid or asticized Polyvinyl Chloride (PVC) Pipes for the Supply of

69)

determination of the Temperature of Evolution of Flammable
Fundamental Principles for Design (1972)

ISO

ISO

Fundamental Principles (1970)

Fundamental Terms) (1969)

Fundamental Welding Positions and Definitions of Rotati

1710

ISC $\quad 364.2$ Fungi by Visual Examination (1968) or Plastics-Rec. ISS

Funnel (1958) /S-Determination of Apparent Density 0 ISO

Funnel (1958) Tics-Determination of Apparent Densit

Furnaces with Metallic Heating Resistors (1972)

Furnaces with Submerged Channels (1972)

Furnaces (1967) / Mach
Fuse Combination Units (1972)

Fuse Definitions (1969)

Fuse Holders for Miniature Cartridge Fuse Links (1968)
Fuse Links for Aircraft-Gencral Requirements (1971)

Clectrodes with Th IEC

Fuse Links for Aircraft (Type A) (1971)

Fuse Links for Aircraft (Type B) (1971)

Fuse Links for Low Voltage Fuses (1957)

Fuse Links for Miniature Fuses (1962)

Fuse Links for Miniature Fuses (1962)

Fuse Links (1968)

Fuses for Aircraft (1968)

Fuses for Domestic and Similar General Purposes (1968)

Fuses for Domestic and Similar Purposes (1968)

Fuses with High Breaking Capacity for Industrial and SI

Fuses (1957)

Fuses (1962)

Fuses (1962)

Fuses: Current Limiting (1965)

Fuses: Expulsion and Similar Types (1970)

Fusibility of Fuel Ash (1967)

Fusion Welded Butt Joints for Aluminium (Aluminum) and

Fusion Welded Butt Joints for Steel Plates Up to $50 \mathrm{~mm}$

Fusion Welded Butt Joints for Steel Plates 50 to $200 \mathrm{~mm}$

Fusion Welded Butt Joints in Steel Pipes Up to $50 \mathrm{~mm}$ (2

Galvanized Coatings on Fabricated Ferrous Products (197

Galvanized Coatings on Ferrous Materials by Chemical D

Galvanized Steel Wire for Ropes (1963)

Galvanized Steel Wire Ropes (1963)

Galvanizing (1970) Rec. for Guid ISO

ISO

Gaps and Sockets (Metric Values for General Use) (1968)

Gaps of Recording and Reproducing Magnetic Heads for Ma

Gaps (1 Sphere Earthed) (1960)

Gaps (1969)

Gas-Volumetric Meth. (1969)

Gas-Volumetric Meth. (1969)

Cas Atmospheres. Clas by Low Temperature Distillation of

Gas Atmospheres: Classification of Maximum Surface Temp

Gas Atmospheres: Construction and Test of Electrical Ap

Gas Atmospheres: Construction and Test of Flameproof En

Gas Atmospheres: General Introduction (1971)

Gas Atmospheres: Marking (1970)

Gas Atmospheres: Meth. of Test for Ignition Temperature

Gas Atmospheres: Oil Immersed Apparatus (1968)

Gas Atmospheres: Pressurized Enclosures (1962)

Gas Atmospheres: Sand Filled Apparatus (1967)

Gas Atmospheres: Sand Filled Apparatus: Use of a Screen

Gas Atmospheres: Spark Test Apparatus for Intrinsically

Gas Cylinders for the Identification of the Content (19

Gas Cylinders Used for Anaesthetic and Resuscitation Pu

Gas Cylinders Used in Cutting and Related Processes (19

Gas Cylinders (1957)

Gas Cylinders (1965)

Gas Filled Microwave Switching Devices (1972)

Gas List Threading (1971)

Gas List Tubes and Screwed Fittings Where Pressure Tigh

Gas Meters (1970)

Gas Pressure Cables and Their Accessories: External, Fo

Gas Pressure Cables and Their Accessories: Internal, Fo

Gas Pressure Cables and Their Accessories: Paper Insula

Gas Pressure Types (1965)

/Aining Types for Alternatin

Gas Pressure, Oil Filled and Nondraining Types) (1965)

Gas Shielded Metal Arc Welding of Mild Steel: Dimension

Gas Turbines-Acceptance Tests (1973)

Gas Volumetric Meth. (1970)

/Etermination of Carbon Di

Gas Welding Mild Steels and Low Alloy High Tensile Type

as Welding of Mild Steels an

Gas Welding (1967) JEc, for Diameters and Tolerances F ISO

Gas Welding: Test to Determine the Compatibility of Ste

Gas, Water and Sewage, Welded or Seamless (1967) ISO

Gaseous Condition in Land Installations and on Board Sh ISO

Gaseous Fuels: Metric Series-Spec. (1973) /Ried Unpl ISO

Gaseous Oxygen Replenishment Couplings for Aircraft (19 ISO

Gases from Plastics (1968)
Rec. for Plastics- ISO
627

60

396

239

408

291

257
1547

1548

1549

88

4 
determination of the Permeability of Vulcanized Rubhers to en of Filectronic Tubes and Valves: Meth. of Measurement of torage Equipment: Bins and Bunkers, Silos and lloppers, Bin r Industrial Trucks-Dimensions of Stillages-Connection Rec. for Straight Sided Splines and International Rec. for Pressure

e $(1970)$

of Lampcap

Lampholde

Gauges (1/ Rec. for Lamp Caps and Holders Together with

65)

and Ring Doubling Spindles: In. Dimensions, Tolc d Ring Doubling Spindles Metric Dimensions, Tolerances and $h$ Gauges for the Control of Interchangeability and Safety: $s$ for Tubes and Valves and Dimensions of Test. Devices and cording Pressure, Combined Pressure and Vacuum, and Vacuum icating Pressure, Combined Pressure and Vacuum, and Vacuum rement in Open Channels-Establishment and Operation of $\mathrm{A}$ Rec. for ISO General Purpose Metric Screw Threads.

Procedures for Electronic Components and Equipment: Test Rec. for International Std. for Shipbuilding: Cargo

o the Manufacturer by the Purchaser in Order to Obtain the

o the Manufacturer by the Purchaser in Order to Obtain the of Equivalent Terms (English, Frenc/ Rec. for Glossary of Rec. for Glossary of Rec. for Glossary of

Rec. for Modules and Diametral Pitches of Cylindrica Rec. for Modules and Diametral Pitches of Siraight Bevel Rec. for Basic Rack of Straight Bevel Rec. for Basic Rack of Cylindrical

for Preferred Modules and Diametral Pitches of Cylindrical he Purchaser in Order to Obtain The/ he Purchaser in Order to Obtain / representation of

Rec. for Cylindrical

of the Stiffness of Vulcanized Rubbers at Low Temperature al Properties of Electronic Tubes: Meth. of Measurement of ernal Diameters of Cylindrical Radiation Probes Containing Rec. for Tolerances of Form and of Position-Part I: sion and Distribution (1963) Rec. Graphical Symbols: nical Vocabulary: Nuclear Power Plants for Electric Energy Ene/ Rec. for InternationaI Electrotechnical Vocabulary: Rec. for Test Code for Stationary Steam

sformers for Power and Lighting, Semiconductor Rectifiers, Rec. for Audio Frequency Rec. for Rec. for Abbreviations of for Graphical Symbols for Use on Detailed Maps, Plans and for Graphical Symbols for Use on Detailed Maps, Plans and for Graphical Symbols for Use on Detailed Maps, Plans and Rec. for International Gear Notation: Symbols for alent Terms (English, Frenc/ Rec. for Clossary of Gears. Rec. for Glossary of Gears Rec. for Glossary of Gears. Rec. for (1962)

truments and Devices for Measurement and Evaluation of the
the Determination of the Percentage of Fat in Milk by the tics Industry (English, French, Russian) (D: Corresponding Millimetres) (Milli/ Rec. for Taper Keys with or Without Rec. for Spices and Condiments: Fibres (Fibers) or Continuous Filament/ inuous Filament and Staple Fibre (Fiber/ matter of Its Products (1971) lament and Staple Fibre (Fiber) Yarns (/ of, Continuous Filament Yarn, Staple Fi/ Std. for Woven ure Laminated Plates / Rec. for Plastics-Preparation of Tha/ Rec. for Tests on Insulators of Ceramic Material or Rec. for Determination of the Hydrolytic Resistance of Rec for Determination of the Hydrolytic Resistance of expansion Coefficient (For Use in the Preparati/ Rec. for Rec. for Interchangeable Conical Ground Rec. for Interchangeable Spherical Ground

for Principles of Construction and Adjustment of Liquid in International Rec. for One Mark lastics-Determination of the Loss on Ignition of Textile
alkali (I968) Rec. for Determination of the Resistance of es (Fibers) or Continuous Filaments Constituting a Textile Std. for Textile International Rec. for Clinical Thermometers (Mercury, in e Punc/ Rec. for Shipbuilding Details: Test. of Toughened Rec for Shipbuilding Details-Toughened
Gases (Constant Volume Meth.) (1971)

Rec. for ISO

Gasfilled Types (1969)

ates-Safety Code (1970)

Gauge (1970)

Gauges Dimensions in $\ln . / \mathrm{S}$ (1961)

Nts of the Electrical Properti IEC R L Loose Bulk Materials $\rightarrow S$ ISO

Rec. Fo $1 S O$

Gauges for Instruments Measuring Arterial Blood Pressur

Gauges for Ring Spinning and Doubling Frames (1972)

Gauges for the Control of Interchangeability and Safety

Gauges for the Control of Interchangeability and Safety

Gauges for the Control of lnterchangeability and Safety

Gauges for the Inspection of Closures (1969)

Gauges Reading in $0.01 \mathrm{Mm}, 0.001 \mathrm{ln}$ and $0.0001 \mathrm{ln}$. (19

Gauges (1963)

Gauges (1964)

Gauges (1969)

Gauges (1969)

IEc. for Warp Tubes for Ring Spinning an ISO

for Lamp Caps and Holders Together Wit IEC

for Tube and Valve Shields: Spec. Sheet IEC

Responsive Flements and Reco

Gauges, with Pressure Responsive Elements Giving Direct

Gauging Station and Determination of the Stage Discharg

Gauging (1970)

- Acceleration, Steady State (1968)

OIML
OIML

ISO

ISO

Gear Notation: Symbols for Geometrical Data (1968)

Gear Particulars Book (1972)

Gear Required) (1971)

Gear Required) (1971)

/Ears (Information to Be Given T ISO

Ears (Information to Be Civen T ISO

Gears-Geometrical Definitions-Alphabetical Indexes

Gears-Geometrical Definitions-Worm Gear (1972)

Gears-Geometrical Definitions (1969)

Gears for General and Heavy Engineering (1966)

Gears for General and Heavy Engineering (1968)

Gears for General Engineering and Heavy Engineering (19

Gears for General Engineering (1957)

Gears for General Engineering (1966)

Gears (Information to Be Given to the Manufacturer by $\mathrm{T}$

Gears (Information to Be Given to the Manufacturer by $\mathrm{T}$

Gears (1973) Std. for Technical Drawings: Conventional

Geiger-Muller Counter Types (1971)

Rec. for Determination of the Electric

Geiger-Muller or Proportional Counter Tubes or Scinti

Generalities, Symbols, Indications on Drawings (1969)

Generating Stations and Substations, Lines for Transmis

Generation (1968)

Rec. for International F

Generation, Transmission and Distribution of
Generators of the Power Station Type (1968)

Generators (With Associated Prime Movers) and Motors, E

Generators (1972)

Generic Names for Man Made Fibres (Fibers) (1971)

Generic Names in Titles of Periodicals (1968)

Geological Cross Sections-Part III: Representation of

Geological Cross Sections-Part 1I: Representation of

Geological Cross Sections-Part I: General Rules of Re

Geometrical Data (1968)

Geometrical Definitions-Alphabetical Indexes of Equiv

Geometrical Definitions-Worm Gear (1972)

Geometrical Definitions (1969)

Geometrical Inspection of Grooves of Pulleys for V-Belt

Geometrical Parameters of Surface Finish (1970)

Gerber Meth. (1966)

Rec. for Butyrometers for ISO

German Terms) (1964)

Quivalent Terms Used in the Plo

Gib Head and Their Corresponding Keyways (Dimensions in

Ginger, Whole, in Pieces and Ground: Spec. (1969)

Glass-Determination of the Average Diameter of Staple

Glass-Determination of the Linear Density of lts Cont

Glass-Determination of the Percentage of Combustible

Glass-Determination of the Twist in Its Continuous Fi

Glass-Procedure for Sampling Batches or Consignments

Glass Containers and Gauges for the Inspection of Closu

Glass Fabrics-Basis for Spec. (1972)

Glass Fibre (Fiber) Reinforced, Resin Bonded, Low Press

Glass for Overhead Lines with a Nominal Voltage Greater

Glass Grains at I2I ${ }^{\circ} \mathrm{C}$ (I968)

Glass Grains at $98^{\circ} \mathrm{C}(1968)$

Glass Hydrometers-Conventional Value for the Thermal

Glass Joints (1964)

Glass Joints (I968)

Glass Laboratory Thermometers (1964)

Glass Messuring Flasks (I970)

Glass Reinforced Plastics (I970)

Glass to Attack by a Boiling Aqueous Solution of Mixed

Glass to Attack by $6 \mathrm{~N}$ Hydrochloric Acid at $\mathrm{I} 00^{\circ} \mathrm{C}$ (

Glass Yarn-Cross Section Meth. (1971)

Glass Yarns-Designation (1972)

Glass, with Maximum Device) (1970)

Glasses for Ships Side Scuttles and Fixed Lights by Th

Glasses for Ships Side Scuttles and Fixed Lights (1969 
or Principles of Construction and Adjust ment of Volumetric Rec. for Meth. for Thermal Shock Tests on Laboratory and $\mathrm{Pa}$ Rec. for Commercially Dry Virgin Cork, Ramassage, tur/ Std. for Corkwood in Planks, Virgin Cork, Ramassage, Std. for Corkwood in Planks, Virgin Cork, Ramassage, Rec. for Oil of Eucalyptus cal Indexes of Equivalent Terms (English, Frenc/ $\quad$ Rec. for (1972) $\quad$ Rec. for Rec. for Cork: Rec. for Surface Active Agents: Std. for Nuclear Energy Rec. for Rec. for (Vacuum Oven Meth.) (1970) Index Meth. (1973) ity or Acidity / ed Ash in Produ/ General (1970) Std. for Analysis of Soaps-Determination of Low Contents of Free at $20^{\circ} \mathrm{C}(1972)$ Rec. for the Analysis of Soaps: Determination of Std. for ravimetric Meth. (1972)

ce Active Agents (Nonionic): Determination of Polyethylene Rec. for Dental Casting

icular Types of Winding Wires: Enamelled Round Copper with Rec. for Pictorial Markings for Handling of Rec. for Double Deck Flat Pallets for Through Transit of Rec. for Large Pallets for Through Transit of Std. for Woodworking Tools: Chisels and Rec. for International Code for Test. of Speed Rec. for Spec, for Photographic Rec. for Spec. for Photographic Rec. for Spec. for Photographic Rec. for Spec. for Photographic Rec, for Spec, for Photographic Std. Spec. for Photographic $\begin{array}{ll}\text { er Lift/ } & \text { Rec. for Short Link Chain for Lifting Purposes - } \\ \text { 71) } & \text { Rec. for Short Link Chain for Lifting Purposes }\end{array}$ Rec. for Muscovite Mica Blocks, Thins and Films Meth. for or Phlogopite Mica Blocks, Thins and Splittings: Meth. for Rec. for ements Giving Direct Indications by Means of a Pointer and and Telecommunication / Rec. for Strip Wound Cut Cores of Rec. for Micrographic Determination of the Austenitic Rec. for Cereals: Sampling (As c. for Determination of the Hydrolytic Resistance of Glass c. For Determination of the Hydrolytic Resistance of Glass for Cereals and Pulses: Determination of the Mass of 1000 f Instruments for Measuring the ${ }^{\circ}$ Of Humidity of Cereal Std. for Cork Std. for Cork: Std. for Rec. for Rec. for

Rec. for Granulated Cork. Rec. for Rec. for Rec. for Rec. for Rec. for eological Cross Sections-Part I: General Rule eological Cross Sections-Part III: Representa/

Rec. for Electrical Installations in Ships: Rec. for General Principles for the Formulation of Rec. for

iscellaneous Applications (1969)

trols, Starters and Elements of Electromechanic/ atrices (1972)

Lines for Transmission and Distribution (1963) s, Meth. of Connection and Circuit Elements (1960) 8 and Accumulators: Aerials (Antennas) and Radi/ 8 and Accumulators: Microwave Technology (1968) 8 and Accumulators (1960)

locks (1963)

971)

1967)

ies (1971)

8 (1968) elements of Electronic Tubes, Valves and Rectifiers/

g Pi/ Rec. for Nominal Dimensions of Cylindrical Machined Rec for Designation of the Microstructure of res: Determination of Aluminium (Aluminum) Content-Oxine Rec. for Chemical Analysis of Magnesium and Its Alloys: Chemical Analysis of Aluminium (Aluminum) and Its Alloys: Rec. for Chemical Analysis of Aluminium and Its Alloys:
Glassware (1964)

Glassware (1968)

Gleanings, Corkwood Refuse and Corkwaste-Definitions

Gleanings, Refuse and Corkwaste-Determination of Mois

Gleanings, Refuse and Corkwaste-Sampling (1972)

Globulus (1968)

Glossary of Gears-Geometrical Definitions-Alphabeti

Glossary of Gears-Geometrical Definitions-Worm Gear

Glossary of Gears-Geometrical Definitions (1969)

Glossary (1967)

Glossary (1971)

Glossary: First Series of Terms (1972)

Glow Starters for Tubular Fluorescent Lamps (1963)

Glucose Syrup-Determination of Dry Matter of Moisture

Glucose Syrup: Determination of Dry Matter-Refractive

Glycerine for Industrial Use-Determination of Alkalin

Glycerine for Industrial Use-Determination of Sulphat

Glycerine for Industrial Use-Samples and Test Meth.-

Glycerol-Spectrophotometric Meth. (1972)

Glycerol for lndustrial Use-Determination of Density

Glycerol (1969)

Glycerols for Industrial Use-Determination of Ash-G

Glycols and Nonionic Active Matter (Adducts)-Weibull

Gold Alloy (1970)

Good Dielectric Properties Under Humid Conditions (1970

Goods (General Symbols) (1968)

Goods (1961)

Goods (1963)

Gouges (1973)

Governing Systems for Hydraulic Turbines (1970)

Grade Hydroquinone (1965)

Grade P Methylaminophenol Sulphate (1965)

Grade Potassium Bromide (1965)

Grade Sodium Carbonate, Anhydrous (1965)

Grade Sodium Sulphite (1965)

Grade Sodium Thiosulphate, Crystalline (1972)

Grade 40 Calibrated Load Type for Pulley Blocks and Oth

Grade 40 Noncalibratcd Chain for Chain Slings, Etc. (19

Grading by Size (1958)

Grading by Size (1965)

Graduated Pipettes (Excluding Blowout Types) (1968)

Graduated Scale (Working Equipment Category) (1970)

Grain Oriented Silicon Iron Alloy, Used for Electronic

Grain Size of Steels (1967)

Grain) (1969)

Grains at $121{ }^{\circ} \mathrm{C}(1968)$

Grains at $98{ }^{\circ} \mathrm{C}(1968)$

Grains (1966)

Grains (1970)

Granulated-Determination of Moisture Content (1972)

Granulated Cork-Bulk Density Test (1972)

Granulated Cork-Granule Size Test (1971)

Granulated Cork-Sampling (1971)

Granule Size Test (1971)

Graphical Symbols for Coal Preparation Plant (1967)

Graphical Symbols for Hydraulic and Pneumatic Equipment

Graphical Symbols for Use on Detailed Maps, Plans and G

Graphical Symbols for Use on Detailed Maps, Plans and G

Graphical Symbols for Use on Detailed Maps, Plans and C

Graphical Symbols (1965)

Graphical Symbols (1972)

Rec Graphical Symbols: Binary Logic Elements (1972)

s: Block Symbols for Transmission and $M$

Rec. for Graphical Symbols: Contacts, Switchgear, Mechanical Con

Rec. for Graphical Symbols: Ferrite Cores and Magnetic Storage M

Rec. Graphical Symbols: Frequency Spectrum Diagrams (1968)

Rec. for Graphical Symbols: General Index (1972)

Rec. Graphical Symbols: Generating Stations and Substations,

Rec. Graphical Symbols: Kind of Current, Distribution System

Rec. for Graphical Symbols: Machines, Transformers, Primary Cell

Rec. for Graphical Symbols: Machines, Transformers, Primary Cell

Rec. Graphical Symbols: Machines, Transformers, Primary Cell

Rec. Graphical Symbols: Measuring Instruments and Electric C

Rec. Graphical Symbols: Semiconductor Devices, Capacitors (1

Rec. Graphical Symbols: Symbols for Architectural Diagrams (

Rec. Graphical Symbols: Telecommunication Lines and Accessor

Rec. Graphical Symbols: Telephony, Telegraphy and Transducer

Rec. Graphical Symbols: Variability, Examples of Resistors,

Graphite Cast Iron (1969)

Graphite Electrodes with Threaded Sockets and Connectin Graphite in Cast Iron (1969)

Gravimetric and Volumetric Meth. (1973)

Gravimetric Determination of Aluminium in Magnesium Al

Iron

Gravimetric Determination of Silicon (Content Greater T

Gravimetric Determination of Zinc in Aluminum Alloys ( $\mathrm{Z}$
Rec. F ISO

ISO

ISO

ISO

ISO

ISO

ISO

ISO

ISO

ISO

ISO

ISO

ISO

ISO

150

ISO

ISO

ISO

150

ISO

ISO

IEC

ISO

ISO

ISO

ISO

IEC

ISO

ISO

ISO

ISO

ISO

ISO

ISO

ISO

Rec. F ISO

ISO

/L OIML

IEC

ISO

ISO

Re ISO

$\operatorname{Re}$ ISO

Rec. ISO

OlML

ISO

ISO

ISO

ISO

ISO

ISO

ISO

ISO

ISO

ISO

ISO

IEC

IEC

IEC

IEC

IEC

IEC

IEC

IEC

IEC

IEC

IEC

IEC

IEC

IEC

IEC

ISO

IEC

ISO

ISO

ISO

ISO

384

718
1215
2386

770

$1122 / 1$

$1122 / 2$

1122

633

862

921

921
155
1742

1743

1615

1616

1614

2272

2099

1066

2098

2268

1562

317-6

780

198

329
2729
308

423

422

420

424

418

419

1836

1835 
hemiral A nalysis of Steels: Determination of Total Carbon m ('hloride for Industrial Use-Determination of Content um Sulphate for Industrial Use: Determination of Content oxide for Industrial Use: Determination of Silica Content: oxide for Industrial Use: Determination of Silica Content: tes for Industrial Use-Determination of Silica Content . de for Industrial Use: Determination of of Silica Content: oxide for Industrial Use: Determination of Silica Content, m Hydroxide for lndustrial Use-Determination of Sodium $\mathrm{n}$ of Moisture in the Analysis Sample of Coal by the Direct c Acid for Industrial Use: Determination of Sulphated Ash: al Use: Determination of Sulphate Content; Barium Sulphate for Industrial Use: Determination of Residue on Ignition; Determination of Sulphur Dioxide Content: Barium Sulphate al Use: Determination of Sulphate Content; Barium Sulphate Rec. for Determination of Carbon Dioxide in Coal by the al Use: Determination of Sulphate Content: Barium Sulphate al Use: Determination of Su'phate Content: Barium Sulphate Rec. for Urea for Ind sstrial Use-Determination of Ash . ferrous Materials by Chemical Dissolution of the Coating licates for Industrial Use-Determination of Dry Matter -

Products Not Containing More Than $0.5 \%$ of Such, by Mass xtiles Fibres (Fibers)-Determination of Linear Density

e for Industrial Use-Determination of Moisture Content

s Alloys-Measurement of the Mass of the Oxide Coatings -

ial Use-Determination of Sulphated Residue on Ignition

for Glycerols for Industrial Use-Determination of Ash

rine for Industrial Use-Determination of Water Content ation of Aluminium (Aluminum) Content-8 Hydroxyquinoline e for Industrial Use-Determination of Moisture Content . 1 Use: Determination of Sulphate Content-Barium Sulphate Magnesium and Its Alloys-Determination of Rare Earths te for Industrial Use: Determination of Moisture Content industrial Use: Determination of Content-Barium Sulphate is of Steel and Cast Iron: Determination of Total Silicon higher Alcohols for Industrial Use-Determination of Ash Rec. for

for Determining the Density and Relative-Density (Specific Rec. for Determination of the Std. for Petroleum Products: Lubricating

Std, for Petroleum Products-Lubricatin Rec. for International System for the Transliteration of Rec. for (Basic Reference Meth.) (1970) Rec. for Rec. for

(Routine Meth.) (1970)

Rec. for Brinell Hardness Test for Rec. for Classification of Rec. for Beam Unnotched Impact Test for Rec. for

ectrical Appliances: Particular Requirements for Toasters, ppliances for Domestic and Similar Purposes: Waffle lrons, c. for Paints and Varnishes-Determination of Fineness of and Reciprocating/ and Reciprocating $\mathrm{T} /$

Rec. for Test Conditions for Surface Rec. for Test Conditions for Surface Rec. for Bonded Abrasive Products Rec. for Bonded Abrasive Products: est Conditions for Surface Grinding Machines with Vertical t Conditions for Surface Grinding Machines with Horizontal Rec. for Mounting of Plain

for Endless Wide V.Belts for Industrial Speed Changers and Rec. for Grooved Pulleys for V-Belts:

Rec. for Grooved Pulleys for Narrow V-Belts: Rec. for Grooved Pulleys for V-Belts:

ng Bearings-Radial Internal Clearance in Unloaded Radial SPA, SPB (1965) 962)

Rec. for Rec. for terms and Definitions Relating to Drives Using V-Belts and Rec. for Geometrical Inspection of d Calcul/ Rec. for Solid Mineral Fuels-Determination of Rec. for Aircraft Connections for Rec. for Connections for Aircraft Rec. for Rubber Hoses for Aircraft Rec. for Interchangeable Conical Rec. for Interchangeable Spherical

ne Starting (1961)

Rec. for Safety Features for Rec. for Tea-Preparation of Rec. for Aircraft Pressure Cabin Carpets: Determination of Tuft Leg Length Above the Woven Rec. for Spices and Condiments: Chillies, Whole and Rec. for Aircraft Fuel Nozzle

c. for Spices and Condiments: Ginger, Whole, in Pieces and r Spices and Condiments: Black and White Pepper, Whole and

c. for Spices and Condiments: Pimento (Allspice) Whole and Rec. for ISO Identification Code for Rolling Bearings: Rec. for ISO Identification Code for Rolling Bearings:
(Gravimetric Meth. After Combustion in a Stream of Oxyge Gravimetric Meth. as Potassium Tetraphenylborate (1971)

Gravimetric Meth. as Potassium Tetraphenylborate (1973)

Gravimetric Meth. by Insolubilization (1969)

Gravimetric Meth. by Insolubilization (1969)

Gravimetric Meth. by Insolubilization (1970)

Gravimetric Meth. by Precipitation of the Quinoline Sil

llum Hydr iSO

M Silica ISO

Geth by Precipitation of the Quinoline Sil ISO

Gravimetric Meth. Using Uranyl Acetate and Magnesium Ac ISO

Gravimetric Meth. (1963)

Gravimetric Meth. (1968)

Gravimetric Meth. (1968)

Gravimetric Meth. (1968)

Gravimetric Meth. (1968)

Gravimetric Meth. (1968)

Gravimetric Meth. (1969)

Gravimetric Mcth. (1969)

Gravimetric Meth. (1969)

Gravimetric Meth. (1970)

Gravimetric Meth. (1970)

Gravimetric Meth. (1970)

Gravimetric Meth. (1970)

Gravimetric Meth. (1971)

Gravimetric Meth. (1971)

Gravimetric Meth. (1971)

Gravimetric Meth. (1971)

Gravimetric Meth. (1972)

Gravimetric Meth. (1972)

Gravimetric Meth. (1972)

Gravimetric Meth. (1972)

Gravimetric Meth. (1972)

Gravimetric Meth. (1972)

Gravimetric Meth. (1973)

Gravimetric Meth. (1973)

(Gravimetric Meth.) (1969)

Rec. for Determinatio ISO

Rec. for Hydrochlori ISO

Drochloric Acid for Industri ISO

/for Sulphuric Acid and Oleum ISO

/Nd Oleum for Industrial Use; ISO

/Odium Carbonate for Industri ISO

/Odium Hydroxide for Industri ISO

/Ssium Hydroxide for Industri ISO

Dip Galvanized Coatings on ISO

I for Sodium and Potassium SI ISO

/Mination of Sulphated Ash in ISO Rec. for Te ISO

Rec. for Potassium Chlorid ISO

/ Aluminium (Aluminum) and It ISO

/ for Nitric Acid for lndustr ISO Std. ISO

Std for Liquid Chlo ISO

(Al and Artificial): Determin ISO

(for Sodium Hydrogen Carbonat ISO

/Odium Chloride for Industria ISO

/Td. for Chemical Analysis of ISO Sid for Sodium Chlora ISO

I for Potassium Sulphate for ISO

Rec. for Chemical Analys ISO

Gravimetric Meth.) (1970) Rec. for ISO

Gravity Filling Orifices for Aircraft (1959)

Gravity) of Plastic, Excluding Cellular Types (1970)

Gray King Coke Type of Coal (1966)

Grease-Determination of Cone Penetration (1972)

Grease-Determination of Dropping Point (1972)

Greek into Latin Characters (1968)

Green Bananas: Guide to Storage and Transport (1969)

Green Coffee Beans-Determination of Moisture Content

Green Coffee Beans-Determination of Moisture Content,

Grey Cast Iron (1961)

Grey Cast Iron (1961)

Grey Cast lron (1969)

Grid System for Printed Circuits (1970)

Grills, Waffle Irons and Roasters (1972)

Grills, Warming Plates and Other Dry Cooking Appliances Grind (1971)

Grinding Machines with Horizontal Grinding Wheel Spindl

Grinding Machines with Vertical Grinding Wheel Spindle

Grinding Wheel Dimensions-Part 2 (1969)

Grinding Wheel Dimensions (Part 1) (1967)

Grinding Wheel Spindle and Reciprocating Table-Test.

Grinding Wheel Spindle and Reciprocating Table-Test.

Grinding Wheels by Means of Hub Flanges (1968)

Groove Profiles for Corresponding Pulleys (1970)

Groove Sections A, B, C, D, E (1957)

Groove Sections SPZ, SPA, SPB (1965)

Groove Sections Y and Z (1962)

Groove Type Ball Bearings with Cylindrical Bore-Value

Grooved Pulleys for Narrow V-Belts: Groove Sections Spz

Grooved Pulleys for V-Belts: Groove Sections A, B, C, D

Grooved Pulleys for V-Belts: Groove Sections Y and Z (1

Grooved Pulleys (1969)

Grooves of Pulleys for V-Belts (1962)

Gross Calorific Value by the Calorimetric Bomb Meth. an

Ground Air Conditioning (1969)

Ground Electrical Supplies (1965)

Ground Fueling Without Static Conducting Wire (1971)

Ground Glass Joints (1964)

Ground Glass Joints (1968)

Ground Power Units for d.c. Aircraft Servicing and Engi

Ground Sample of Known Dry Matter Content (1970)

Ground Test Connection (1954)

Ground (1972)

Ground, Spec. (1969)

Grounding Plugs and Sockets (1970)

Ground: Spec. (1969)

Ground: Spec. (1969)

Ground; Spec. (1969)

Group IV Tapered Roller Type-In. Series (1965)

Group V Airframe Type (1968) 
Rer. for Basir Environmental Test. Procedures: Guidance for Damp Heat Tests (1968)

for Mounting on Boards with Printed Wiring and Circ/

Rec.

Rec. for Basic Environmental Test. Procedures:

nnections-General Requirements, Test Meth. and Practical ponents (1971) Rec. for
Rec. for Loading
echanical Isolation

72) Characteristics for Mechanical Isolation and Maintainability Data from Field Performance/ d from the ISO 7 Bit Coded Character Sct for In/ $f$ Tuned Transformers and Inductors of Ferromagn/ $f$ Broad Band Transformers of Ferromagnetic Oxid/ (1972)

xample of Meth.) (1969)

ting the Thermal Endurance of Electrical Insula/ $r$ Meth. of Chemical Analysis (1969)

Guidance for the Design and Use of Components Intended

Guidance on Change of Temperature Tests (1971)

IEC

IEC

Rec. for Solderless Wrapped Co IEC

Guide for Choosing Modular Dimensions for Waveguide Com IEC

Guide for Oil Immersed Transformers (1972)

Guide for Selecting and Applying Resilient Devices) (19

for Guide for the Collection of Reliability, Availability,

Rec. for Guide for the Definition of 4 Bit Character Sets Derive

Rec. for Guide for the Drafting of Performance Spec. for Cores O

Rec. Guide for the Drafting of Spec. for Microwave Ferrites

Rec. for Guide for the Preparation of Classified Vocabularies (E

Rec. for Guide for the Preparation of Test Procedures for Evalua

Rec. for Guide on the Form for Std. for Chemical Products and Fo

Rec. for Peaches:

Rec. for Pears

Rec. for Cauliflowers:

Rec. for Apples

Rec. for Connectors for Frequencies Below $3 \mathrm{MHz}$ :

Rec. for Green Bananas:

Rec. for Fresh Pineapples .

ned Copper Conductors of Low Frequency Cables A/ Rec. for those Containing More Rounded Values (1966)

Rec for Radio Frequency Cabl Cables:

Rec. for

Rec. for

Rec. for Std. for

on of Asbestos Cement Products (1970)

73)

rec. for Quartz Crystal Units for Oscillators: Section 3.
Rec. for Insulation Coordination: Application Rec. for Insulation Coordination: Application
Connectors with Open Ends, Off Centre (Center) edge Socket Connectors with Open Ends, Off Centre (Center)
hec. Dip Galvanizing (1970)
Rec.

Rec. for

Std. for

Std. for

Rec. for

Rec. for

Rec. for

Rec. for

Rec. for

Rec. for

tion, Characteristics and Test Meth. (1972)

Std. for Hand and Machine

Pitches Up to $6.3 \mathrm{~mm}$ (1972)

Appliances: Particular Requirements for Electric Shavers, the Analysis of Sounds and Vibrations (/

tion of the Acidity (1970)

tion of the Cloud Point (1970)

tion of Residue on Evaporation (1972)

Rec. for Octave

Rec. for Liquid

Rec. for Liquid

for Sodium Chloride for Industrial Use: Determination of

(1961)

Up to $450 \mathrm{~mm}$ and Pitches $U_{p}$ to $6.3 \mathrm{~mm}$ (1972)

nd.Operated Die Stocks (1973)

Rec. for

Std. for

Std. for

Std. for

Rec. for Dimensions of Small Flash Connections for above the Woven Ground (1972)

2)

n (

Std. for

Std. for Reciprocating Internal Combustion Engines

970)

Rec. for

internal Combustion $E_{12}$ gines-Definition of Right and Left tools for Bolts and Screws-Driving Squares for Power and ds-General Dimensions and Marking (1972) Rec. for Short Machine and

. for Hand and Machine Operated Circular Screwing Dies and Rec. for Principles of Criticality Safety in 1972)

e Bulk Materials (1971)

s (1972)

veyors (I971)

veyors-Safety Code (197)

ers and Conveyors-Safet

Conveyors-Safety Code /

It Conveyors-Safety Cod/

ng Conveyors and Shaking

ng Conveyors and Shaking / able Conveyors-Safety C/ nd En Masse Conveyors quipment: Bins and Bunker/ belt Conveyors (Other Tha/ belt Conveyors (Other Tha) belt Conveyors (Other Tha/

Feeders and Conveyors Wi/

Feeders and Conveyors, S/
Guide to Cold Storage (1968)

Guide to Cold Storage (1969)

Guide to Cold Storage (1969)

Guide to Drawing Information in Detail Spec. (1970)

uide to Storage and Transport (1969)

Guide to Storage and Transport (1970)

Guide to the Calculation of Resistance of Plain and Tin

Guide to the Choice of Series of Preferred Numbers and

Guide to the Design of Detailed Spec. (1970)

Guide to the Selection of High Voltage Cables (1965)

Guide to the Use of ISO Rec. 390, Sampling and Inspecti

Guide to the Use of Preferred Numbers and Series of (19

Guide to the Use of (1962)

Guide (1962)

Guide, Having a Contact Spacing of $2.54 \mathrm{~mm}(0.1 \mathrm{In}$.) (1

Guiding Principles for Protection Against Corrosion by

Gum Spirit of Turpentine and Wood Turpentines (1965)

Gylcerols for Industrial Use-Determination of Water C

Gylcerols for Industrial Use-Meth. of Sampling (1972)

ymnastic Equipment-Balancing Beam (1964)

Gymnastic Equipment-Horizontal Bar (1964)

Gymnastic Equipment-Parallel Bars (1964)

Gymnastic Equipment-Rings (1964)

ymnastic Equipment-Vaulting and Pommelled Horses (19

Gypsum Rock for the Manufacture of Binders-Classifica

Hacksaw Blades: Dimensions for Lengths Up to $450 \mathrm{~mm}$ and

Hair Clippers and Similar Appliances (1966)

Hair Clippers and Similar Items (1972)

Half-Octave and Third-Octave Band Filters Intended

Halogenated Hydrocarbons for Industrial Use-Determin

Halogenated Hydrocarbons for Industrial Use-Determina

Halogenated Hydrocarbons for Ind ustrial Use-Determina

Halogens, Expressed as Chlorine-Mercurimetric Meth.

Hand and Long Fluted Machine Reamers, Morse Taper Shank

Hand and Machine Hacksaw Blades: Dimensions for Lengths

Hand and Machine Operated Circular Screwing Dies and $\mathrm{Ha}$

Hand Finishing Sticks and Oil Stones-Dimensions (1972

Hand Held Cameras (1966)

Hand Knotted Carpets: Determination of Tuft Leg Length

Hand Made Carpets: Determination of Types of Knots (197

Hand Operated Control Devices-Std. Direction of Motio

Hand Operated Stillage Trucks: Principal Dimensions (19

Hand Operated Wrenches and Sockets-Technical Spec. (1

/D. for Reciprocating

Hand Single Bank Engines (1972)

Rec, for Assembly

Hand Taps for Pipe Threads for Parallel and Taper-Threa

Hand Taps (1966)

Hand-Operated Die Stocks (1973)

Handling and Processing Fissile Materials (1970)

Handling Appliances for Loose Bulk Materials-Piping (

Handling Equipment-Light Duty Belt Conveyors for Loos

Handling Equipment for Loose Bulk Materials-Aeroslide

Hand ling Equipment for Loose Bulk Materials-Apron Con

Handling Equipment for Loose Bulk Materials-Apron Con

Handling Equipment for Loose Bulk Materials-Belt Feed

Handling Equipment for Loose Bulk Materials-Hydraulic

Handling Equipment for Loose Bulk Materials-Mobile Be

Handling Equipment for Loose Bulk Materials-Oscillati

Handling Equipment for Loose Bulk Materials-Oscillati

Handling Equipment for Loose Bulk Materials-Picking T

Handling Equipment for Loose Bulk Materials-Scraper a

Handling Equipment for Loose Bulk Materials-Storage $E$

Handling Equipment for Loose Bulk Materials-Troughed

Handling Equipment for Loose Bulk Materials-Troughed

Handling Equipment for Loose Bulk Materials-Troughed

Handling Equipment for Loose Bulk Materials-Vibrating

Handling Equipment for Loose Bulk Materials-Vibrating
Guide to Cold Storage (1970)

Guide to the Test. of Circuit Breakers with Respect to

Handling Equipment-Safety Code-General Rules (1970)
$68-2 \cdot 28$

$68-2-33$

352

374

354

2017

362

963

218

219

392

9 i 9

216

873

1134

949

1212

130.0

931

1838

344

497

96.0

183

267

1260

17

$122-2$

$71 \mathrm{~A}$

130-13

1459

2097

2096

382

379

378

380

381

1587

2336

$10-2 \mathrm{~N}$

335-8

225

1393

1394

2210

2481

236

2336

2568

2220

519

2549

2550

2261

938

1711

2276

1174

2284

529

2568

1709

2327

2109

1819

2326

2140

2390

1821

2392

2387

1807

2139

2389
2391

1820

1535

1536

1537

1815

2125 
adn-Belt Conveyors-Ba/ ayorn $(1969)$

conveyorm and Feeders Wit/ rs (Canvas, Rubber, Plast/ hain Conveyors-Safety C/ chain Conveyors (Power An/ Mounted Truck Conveyors / Truck Conveyors (Chain B/

Rec. for Pictorial Markings for (1967) Rec. for and Verification of the Effective Depth of Carburized and ed, Alloy, and Free Cutting Steels-Part 11: Wrought Case oy, and Free Cutting Steels-Part 12: Flame and Induction dness) / 100 IRHD) (1971) 5 IRHD) (1971) Rec. for Plastics-Determination of Indentation Rec. for Determination of Rec. for Determination of Rec. for Determination of

International Rec, for Verification and Calibration of International Rec. for Verification and Calibration of International Rec. for Verification and Calibration of International Rec. for Verification and Calibration of ion of Standardized Blocks to Be Used for Rockwell B and C m 2.5 to $50 \mathrm{kgf}$ (1964)

Rec. for Rec. for Brinell Rec. for Brinell Rec. for Vickers Rec. for Brinell Rec. for Rockwell Rec. for Light Metals and Their Alloys-Vickers Rec. for Rockwell Superficial Rec. for Light Metals and Their Alloys-Brinell Std. for Flexible Cellular Materials: Rec. for Verification of Brinell

Calibration of Standardized Blocks to Be Used for Vickers Rec. for Verification of Vickers Rec. for Verification of Rock well B and C Scale Calibration of Standardized Blocks to Be Used for Brinell ec. for Verification of Rockwell Superficial N and T Scale d Blocks to Be Used for Rockwell Superficial N and T Scale aces (1964)

Rec. for Tables of Brinell ce Active Anation of Hardness of Vulcanized Rubbers of Low or Determination of Hardness of Vulcanized Rubbers of High tation Hardness of Plastics by Means of a Durometer (Shore (1970)

: Coordinated Dimensions in Relationship with Pitch of the Details-Marking of . th. of Test for Wool Fibre (Fiber) I.ength Barbe (Barb) and ilament or Film Ropes (1970) Rec. for 3 Strand apparatus for Explosive Gas Atmospheres: Classification of

for Rules of Behaviour (Behavior) with Respect to Possible hols for Industrial Use-Measurement of Colour (Color) in limetres) (Milli/ Rec. for Taper Keys with or Without Gib ted Magnetic/ Rec. for Hexagon Socket Rec. for Location and Width of the Recoding $\begin{array}{ll}\text { s (1968) } & \text { Rec. for Location and Width of the Recording } \\ \text { Rec. for Bolts and Screws: Radii Under the }\end{array}$ Rec. for Slotted Cheese (Fillister) Rec. for Slotted Pan Std. for Screwdriver Blades for Slotted Std. for Screwdriver Bits, for Slotted Rec. for Slotted Pan

eters) and $\ln . / \mathrm{S}(1970)$ eters) and In./S (19/ eters) and $\mathrm{In} /$ Rec. for Slotted Countersunk (Flat)
Rec. for Slotted Raised Countersunk (Oval) Rec. for Hexagon Rec. for Hexagon resistance of Fabrics to Penetration by Water (Hydrostatic Std. for Slotted 63) Std. for Clevis Pins with Rec. for Cinematography-Location of Recording ic-Striping and Gaps of Recording and Reproducing Magnetic d 1 Magnetic Sound Record/ Location and Dimensions (1971)

$$
\text { Rec. for Location of Recording }
$$
Rec. for Recording

or Hexagon Bolts and Nuts: Widths Across Flats, Heights of nd with End Loops of Healds (1967) th Pitch of the Harness (1967)

Heights of Rec. for Rec. for Twin Wire Rec. for Flat Steel Rec. for Twin Wire

Rec, for Frames for Single or Double Row of ds-Dimensions and Coordination Of, and with End Loops of
Handling Equipment for Loose Bulk Materials and Unit Lo

llandling Equipment for loose Bulk Materials: Screw Conv IIandling Equipment for Loose Bulk Materials: Vibrating Handling Equipment for Unit Loads-Mobile Belt Conveyo Handling Equipment for Unit Loads-Overhead Monorail C IIandling Equipment for Unit Loads-Overhead Twin Rail Handling Equipment for Unit Loads-Single Strand Floor Handling Equipment for Unit Loads-Single Strand Floor Handling of Goods (General Symbols) (1968) Hands (1971) Hanging of Netting-Basic Terms (1970)

Hardenability Test by End Quenching Ste

Hardened Cases (1973)

Hardening Type (1970)

Hardening Types (1972)

Steel (Jominy Test)

Steel: Determination ISO
Rec. for Heat Treat ISO

Std. for Heat Treated, All ISO

(Shore Har

Hardness of Vulcanized Rubbers of High Hardness (85 to

Hardness of Vulcanized Rubbers of Low Hardness (10 to 3

Hardness of Vulcanized Rubbers (1968)

(1970)

Hardness Reference Blocks Rockwell B (1970)

Hardness Reference Blocks Rockwell C (1970)

Hardness Reference Blocks Vickers (1970)

Hardness Scale Test. Machines (1968)

Hardness Test for Grey Cast lron (1961)

Hardness Test for Steel (Load 5 to $100 \mathrm{kgf}$ ) (1967)

Hardness Test for Steel (1968)

Hardness Test (B and C Scales) for Steel (1968)

Hardness Test (Loads from 1 to $100 \mathrm{kgf}$ ) (1971)

Hardness Test (N and T Scales) for Steel (1969)

Hardness Test (1971)

Hardness Test. by Indentation Techniques (1972)

Hardness Test. Machines (1967)

Hardness Test. Machines (1967)

Hardness Test. Machines (1968)

Hardness Test. Machines (1968)

Hardness Test. Machines (1968)

Hardness Test. Machines (1969)

Hardness Test. Machines (1970)

Hardness Values (HB) for Use in Test Maderion of Stand

Hardness Values (HV) for Metallic Materials (1964)

Hardness (10 to 35 IRHD) (1971)

Hardness (1972)

Hardness (85 to 100 IRHD) (1971)

Hardness) (1968)

Harness (1967)

(or

Std. For Surfa ISO

Rec. ISO

atchway Beams (1960)

Hatchway Covers (1960)

Hauteur Using a Comb Sorter (1969)

Rec. for Heald Frames iSO

ISO

ISO

Rec. for Me $1 \mathrm{SO}$

Hawser Laid) and 8 Strand (Plaited) Polypropylene Monof ISO

Hazardous Areas (1972) Rec. for Electrical IEC

Hazards When Dealing with Electronic Equipment and Othe IEC

Hazen Units (1970)

Rec. for Higher Alco ISO

Head Cap Screws: Metric Series (1968)

Head for Centre (Center) Sound Records on $16 \mathrm{~mm}$ Perfora

Head for Edge Sound Records on $16 \mathrm{~mm}$ Perforated Magneti

Head for General Purpose Bolts and Screws: Metric Serie

Head Screws-Metric Series (1970)

Head Screws-Metric Series (1970)

Head Screws (1972)

Head Screws, with Male Hexagon Drive (1972)

Head Tapping Screws-Dimensions in Millimetres (Millim

Head Tapping Screws-Dimensions in Millimetres (Millim

Head Tapping Screws-Dimensions in Millimetres (Millim

Head Tapping Screws-In. Serics (1970)

Head Tapping Screws-Metric Series (1970)

Head Test) (1968)

Headless Screws-Metric Series (1972)

Rec for Meth of Test for ISO

Heads-Metric Series (1972)

Heads for Four Magnetic Sound Records on $35 \mathrm{~mm}$ Film (19

Heads for Magnetic Sound Record (1972) /Idth of Magnet

Heads for Three Magnetic Sound Records on $35 \mathrm{~mm}$ Film an ISO

Heads for 6 Track Magnetic Sound Records on $35 \mathrm{~mm}$ Film ISO

Heads of Aircraft Lubricating Nipples (1965)

Heads, Thicknesses of Nuts: Metric Series (1968)

Heald Carrying Rods-Dimensions and Coordination Of, a

Heald Frames: Coordinated Dimensions in Relationship Wi

Healds for Frame Weaving (1967)

Healds for General Use (1964)

Healds for Jacquard Weaving (1967)

Healds (1967)

Healds (1967)
1816

1050

1049

2388

2149

2150

2196

2381

780

642

2639

683/XI

868

.

1400
1818

48

9

11

12

10
674

674

403

184

81 
of the Aluminium Magnesium Silicon Type (1959)

nched and Tempered Chromium Types (1970)

ught Quenched and Tempered Types with $1 \%$ Chromi/

Rec. for
Rec. for

Rec. for

Wrought Nitriding Type (1970)

Wrought Case Hardening Type (1970)

Flame and Induction Hardening Types (1972)

quenched and Tempered Unalloyed Types (1968)

wrought, Quenched and Tempered Types with $1 \% \mathrm{Ch} /$

wrought Quenched and Tempered Unalloyed with $\mathrm{Co} /$

wrought Quenched and Tempered Manganese (1970)

wrought Quenched and Tempered, with 3\% Chromium/

wrought Quenched and Tempered Chromium Nickel M

wrought Free Cutting Type (1970)

Rec. for Quantities and Units of

ctronic Components and Equipment: Test D: Accelerated Damp

dures for Electronic Components and Equipment: Test B: Dry

ctronic Components and Equipment: Test Ca: Damp and Steady

ec. for Basic Environmental Test Procedures: Test Db: Damp

the Electrical Properties of Electronic Tubes and Valves:

Tubes and Valves: Measurement of Cathode Heating Time and Rec. for Meth. for Measuring Performance of Water

ic and Similar Purposes: Stationary Noninstantaneous $W$ ater nces for Domestic and Similar Purposes: Portable Immersion

: Accessories, Lighting, Accumulator (Storage), Batteries,

heating Appliances for Domestic and Similar Purposes: Room $959+1964)$

ooking Ranges, / Particular Spec. for Electric Cooking and Spec. for Electric Cooking and lectric Irons,/ or Heating Liq/

ortable Immers/

oom Heating An/

tationary Noni/

affle Irons, Grills, Warm Particular Spec. for Electric Cooking and Particular Spec. for Electric Cooking and Particular Spec. for Electric Cooking and Particular Spec. for Electric Cooking and Rec for General Test Conditions for Electro 8 for Industrial Use-Determination of Loss in Mass After Heating Appliances for Domestic and Similar Purposes: for Rec. for Determination of Loss on

ec. for Characteristics of Electric Infra Red Emitters for

Rec for Test Meth for Batch Furnaces with Metallic ies of Electronic Tubes and Valves: Measurement of Cathode for Use in the Rubber Industry-Spec. Limits for Loss on of Light Absorption of Cellulose Acetate Before and After Rec, for Transliteration of ernational Rec. for Instruments for Measuring the Mass Pe hermal Endurance of Electrical Insulating Varnishes by the Rec. for Protective

soluble Matter of Crystalline Polypropylene by Boiling $\mathrm{N}$ ameters of Round Cans (1972)

Std. for

Spec./S and Acceptance of New Sulphur

Rec for Washers for

dths Across Flats and Corners (1968)

Rec. for

Heads, Thicknesses of Nuts: Metric Series (196/

Rec. fo

Rec. for Assembly Tools for Bolts and Screws

for Screwdriver Bits, for Slotted Head Screws, with Male

Rec. for

\& (1972)

Std. for Prevailing Torque Type Stee Std. for Prevailing Torque Type Stee Std. for Prevailing Torque Type

Rec. for
Std. for

Rec. for Rock Drilling: Hollow

ling: Forged Collared Shanks and Chuck Bushings for Hollow ses: General Requirement/ Rec. for Low Voltage Fuses with 971) Rec. for Fire Refined ec. for Determination of Hardness of Vulcanized Rubbers of

Hearing Aids Using Earphones Coupled to the Ear by Mean Hearing Aids (1957)

Hearing Aids (1959)

Hearing Aids (1966)

Rec. Meth. Fo IEC

Hearing Conservation Purposes (1971)

Rec. for 1SO

ISO

Heat or Solvent Bonding Enamelled Round Copper, with Hi IEC

Heat or Solvent Bonding Self Fluxing Enamelled Round Co

Heat Resistance Tests (1971)

Heat Resisting Equipment Wires for Aircraft (1973)

Heat Resisting Equipment Wires for Aircraft: Meth. of T

IEC

$1 \mathrm{SO}$

ISO

eat Resisting $\left(190^{\circ} \mathrm{C}\right)$ Electrical Cables with Copp

Heat Resisting $\left(190^{\circ} \mathrm{C}\right)$ Electrical Cables with Copp

Heat Resisting (190 Deg.) Electrical Cables with Copper

Heat Resisting $\left(260^{\circ} \mathrm{C}\right)$ Electrical Cables with Copp

Heat Resisting $\left(260^{\circ} \mathrm{C}\right)$ Electrical Cables with Copp

Heat Resisting $\left(260^{\circ} \mathrm{C}\right)$ Electrical Cables with Copp

Heat Tests (1968)

Heat Treated Aluminium (Aluminum) Alloy Busbar Material

Heat Treated Steels: Free Cutting-Part 7: Wrought Que

Heat Treated, Alloy and Free Cutting Steels, Pt. 4: Wro

Heat Treated, Alloy, and Free Cutting Steels-Part 10:

Heat Treated, Alloy, and Free Cutting Steels-Part 11:

Heat Treated, Alloy, and Free Cutting Steels-Part 12:

Heat Treated, Alloy, and Free Cutting Steels-Part 1:

Heat Treated, Alloy, and Free Cutting Steels-Part 2:

Heat Treated, Alloy, and Free Cutting Steels-Part 3:

Heat Treated, Alloy, and Free Cutting Steels-Part 5

Heat Treated, Alloy, and Free Cutting Steels-Part 6 :

Heat Treated, Alloy, and Free Cutting Steels-Part 8:

Heat Treated, Alloy, and Free Cutting Steels-Part 9:

Heat (1966)

Heat (1969)

/Ic Environmental Test. Procedures for Ele IEC

Heat, Cyclic (12+12 Hr. Cycle) (1969)

(1)

Heaters for Household Purposes (1972)

for Measurements of

Heaters (1966)

Heaters (1968)

/King and Heating Appliances for Domest Jor Electric Cooking and Heating Applia Heating and Cooking Appliances, Internal Communications

Heating and Similar (1968)

I for Electric Cooking and

Heating Appliances for Domestic and Similar Purposes (1

Heating Appliances for Domestic and Similar Purposes: C

Heating Appliances for Domestic and Similar Purposes: C

Heating Appliances for Domestic and Similar Purposes: E

Heating Appliances for Domestic and Similar Purposes: F

Heating Appliances for Domestic and Similar Purposes: P

Heating Appliances for Domestic and Similar Purposes: R

Heating Appliances for Domestic and Similar Purposes: S

Heating Appliances for Domestic and Similar Purposes: W

Heating Appliances (1972)

Heating at $900{ }^{\circ} \mathrm{C}(1972)$

Heating Liquids (1966)

I for Crude Sodium Borate

Heating of Carbon Black for the Rubber Industry (1969)

Spec. for Electric Cooking and

Heating Purposes (1967)

Heating Resistors (1972)

Heating (1970)

Heating (1970)

Hebrew (1962)

Hectolitre of Cereals (1970)

Helical Coil Bond Test (1969)

Helmets for Road Users (1970)

Heptane (1969)

Hermetically Sealed Metal Food Containers-Internal Di

Rec. for Carbon Black ISO

IEC

Hexafluoride (1971)

Hexagon Bolts and Nuts: Metric Series (1968)

Hexagon Bolts and Nuts: Metric Series, Tolerances on W/

Hexagon Bolts and Nuts: Widths Across Flats, Heights of

Hexagon Drive Ends for Power Types (1970)

Hexagon Drive (1972)

Hexagon Head Tapping Screws-In. Series (1970)

Hexagon Head Tapping Screws-Metric Series (1970)

Hexagon Locknuts-Mechanical and Performance Propertie

Hexagon Locknuts: Dimensions-In. Series (1972)

Hexagon Locknuts: Dimensions-Metric Series (1972)

Hexagon Socket Head Cap Screws: Metric Series (1968)

Hexagon Socket Set Screws-Metric Series (1972)

Hexagonal Drill Steels in Bar Form (1968)

Hexagonal Drill Steels (1968)

High Breaking Capacity for Industrial and Similac. for

High Conductivity Tough Pitch Copper Refinery Shapes (1

Rec. for Roc
lar Purpo

126 
cular Typen of Winding Wires: Enamelled Round Copper, with rular Types of Winding Wires: Enamelled Round Copper, with $f$ the Electrical Properties of Microwave Tubes and Valves: Rec. for Schedule for Rec. for Ballasts for Std. for Connector Pin Allocations for Use with Rec. for Synthetic Rubber Latices-Determination of Rec. for Determination of Total Sulphur in Coal by the Rec. for Determination of Chlorine in Coal by the termination of Carbon and Hydrogen in Coal and Coke by the of Compression Set Under Constant Deflection at Normal and Rec, for Shackle Type Connector Units for ors and Coal Ploughs (1967) of Electrodes for the Welding of Mild Steel and Low Alloy
elds Made with Covered Electrodes of Mild and Low Alloy

c Welds Made with Covered Electrodes of Mild and Low Alloy
Rec. for Classification of Brasses, Leaded, Special and r Filler Rods for Gas Welding of Mild Steels and Low Alloy ed Electrodes for Arc Welding of Mild Steels and Low Alloy d by Filler Rods for Gas Welding Mild Steels and Low Alloy of Measurement of Emission Current from Hot Cathodes fo gn and Construction (1971)

ral and Definitions (197I)

rmation to Be Given with Enquiries Tenders and / ng (1971)

$s$ for the Selection of (For Service) (1971)

and Routine Tests (1972)

Rec for Guide to the Sel

ation for Use in Television Receivers (1965)

(1969)

nsity at $20{ }^{\circ} \mathrm{C}(1970)$

cidity to Phenolphthalein (1970)

sh (Gravimetric Meth.) (1970)

romine Index in the Presence of Mercury (Il) Ch/

arbonyl Compounds (Hydroxylammonium Chloride Po/ istillation Yield (1970)

otal Alcohols Content (Volumetric Meth.) (1970)

ater Content by the Karl Fischer Meth. (1970)

our (Color) in Hazen Units (1970)

olor) with Sulphuric Acid (1970)

perties of Electronic

$s$ for Barrel Thread for E14 and E27 Lampholders with Shade ons and Terminations (/ Rec. for Dimension of Brushes and and Tolerances (1962) Rec. for Dimensions of Brushes and

Rec. for Dimensions of Tubular Electrode Rec. for Fuse Rec. for Lamp Caps and Rec. for Lamp Caps and

Rec. for Lamp Caps and angeability and Safety of Lampop/ angeability and Safety: Gauges (1) for Definitions and Nomenclature for Carbon Brushes, Brush
Rec. for Determination of the Moisture Rec. for Determination of the Moistur
rill Steel Equipment for Percussive Lon ling-Extension Drill Steel Equipment for Percussive Lon ling-Extension Drill Steel Equipment for Percussive Long ling-Extension Drill Steel Equipment for Percussive Long rec. for Rotary Wafer Switches (Low Current Rating) with

(Low Current Ratin/

read Diameter (I962)

hread Diameter (1968)

Rec. for Dimensions and Location of Rectangular Punched Std. for Centre Drills for Center Rec. for Centre (Center) Drills for the ions of Top Plates-Part I: Oblong Top Plates with 4 Bolt Rec for Rock Drilling:

ck Drilling: Forged Collared Shanks and Chuck Bushings for Types (I964) tangular Types (1964) asuring Meth. (I964)

lat Rectangular (I968)

lat Rectangular Types (1967)

Rectangular Types (1964)

1967)

for Plastics-Designation of Unplasticized Compounds of Rec. for Preparation of Dry Polymer from Butadien Rec. for Plastics-Spec. for Polyamide

Std. for Lifting

(Tons) Capacity-Basic Requirements (1972)

Std. for Nomenclature of Liftin materials-Storage Equipment: Bins and Bunkers, Silos and Rec. for Rec. for

High Mechanical Properties for Use in Refrigerant Syste

(1970)

High Mechanical Properties (1970)

High Power Klystrons (1972)

High Pressure Air Charging Valves for Aircraft (1969)

igh Pressure Mercury Vapour (Vapor) Lamps (1965)

High Pressure Mercury Vapour (Vapor) Lamps (1969)

High Speed Data Terminal Equipment (1973)

High Speed Mechanical Stability (1971)

High Temperature Combustion Meth. (1963)

High Temperature Combustion Meth. (1963)

High Temperature Combustion Meth. (1967)

High Temperatures (1972) /lzed Rubbers: Determination

High Tensile Steel Chain for Conveyors (1969)

High Tensile Steel Chains (Round Link) for Chain Convey

/ for Lengths and Tol

High Tensile Steel (1967)

High Tensile Steels (1967)

High Tensile Types (1965)

IOns for Straight Manual Ar

igh Tensile Types (1967)

High Tensile Types (1967)

High Tensile Types (1967)

High Vacuum Electronic Types (1966)

Rec for Code of Symbols Fo ISO

IR Code of Symbols for Cover ISO

/Ties of Weld Metal Deposite ISO I and Valves: Meth IEC

High Voltage Alternating Current Circuit Breakers: Gene

High Voltage Alternating Current Circuit Breakers: Info

High Voltage Alternating Current Circuit Breakers: Rati

High Voltage Alternating Current Circuit Breakers: Rule

High Voltage Alternating Current Circuit Breakers: Type

High Voltage Cables (1965)

High Voltage Connecting Wire with Flame Retarding Insul

High Voltage Fuses: Current Limiting (1965)

High Voltage Fuses: Expulsion and Similar Types (1970)

High Voltage Metal Enclosed Switchgear and Controlgear

High Voltage Switches (1968)

High Voltage Test Techniques (1962)

Higher Alcohols for Industrial Use-Determinaion of De

Higher Alcohols for Industrial Use-Determination of a

Higher Alcohols for Industrial Use-Determination of a

Higher Alcohols for Industrial Use-Determination of B

Higher Alcohols for Industrial Use-Determination of C

Higher Alcohols for lndustrial Use-Determination of D

Higher Alcohols for Industrial Use-Determination of T

Higher Alcohols for Industrial Use-Determination of W

Higher Alcohols for Industrial Use-Measurement of Col

Higher Alcohols for Industrial Use-Test for Colour (C

/Easurements of the Electrical Pro

Hiss and Hum (1964)

Holder Ring (1972) Rec. for Sheet IEC

Holders for Electrical Machinery: Principal Dimensions

Holders for Miniature Cartridge Fuse Links (1968)

Holders for Spot Welding Machines (1969)

Holders Together with Gauges for the Control of Interch

Holders Together with Gauges for the Control of Interch

Holders Together with Gauges for the Control of Interch

Holders, Commutators and Slip Rings (1968)

Holding Capacity of Hard Coals (1969)

Hole Drilling-Reverse Buttress Threaded Equipments 1

Hole Drilling-Reverse Buttress Threaded Equipments 1

Hole Drilling-Rope Threaded Equipments $11 / 2$ to 2 In.

Hole Drilling-Rope Threaded Equipments $7 / 8$ to $11 / 4$ I

Hole Mounting (1963)

Hole Mounting, Lever Operated Switches for Aircraft (19

Hole Mounting; Maximum 26 Positions and Diameter $60 \mathrm{~mm}$

Holes for General Filing Purposes (1968)

Holes for Metric Bolts 1,6 Up to and Including $39 \mathrm{~mm} \mathrm{Th}$

Holes for Metric Bolts, 42 Up to and Including $150 \mathrm{~mm} \mathrm{~T}$

Holes in 80 Columns Punched Paper Cards (Information $\mathrm{Pr}$

Holes with Radius Form: Type R (1972)

Holes Without Protecting Chamfers-Type a (1968)

Holes (1972)

Std. for Industrial Castors-Dimens

Hollow Hexagonal Drill Steels in Bar Form (1968)

Hollow Hexagonal Drill Steels (1968)

Hollow Metallic Waveguides: Relevant Spec. for Circula

Rec. for Hollow Metallic Waveguides: Relevant Spec. for Flat Rec

Rec. for Hollow Metallic Waveguides: General Requirements and Me

Rec. for Hollow Metallic Waveguides: Relevant Spec. for Medium F

Rec. for Hollow Metallic Waveguides: Relevant Spec. for Medium F

Hollow Metallic Waveguides: Relevant Spec. for Ordinary

Hollow Porcelains for Use in Electrical Installations (

Homo and Copolymers of Vinyl Chloride (1970)

Homopolymer and Copolymer Latices (1971)

Homopolymers (1971)

Hooks-General Characteristics (1972)

Hooks for Lif

Hoppers, Bin Gates-Safety Code (1970)
$317-9$

$317-5$

317.1

235-6

1023

188

262

2593

2006

351

352

609

815

1082

610

547

598

426

636

635

637

151.13

$56-6$

56.2

56-5

$56-4$

183

197

282-1

$282 \cdot 2$

298

265

60

1844

1846

1851

1848

1847

1845

1850

1849

1843

1852

151-5

399

136-2

136-1

257

1089

61-1

61-2

61-3

276

1018

1721

1722

1720

1719

132.3

493

$132-5$

838
$273 / I$

273/II

1682

2541

866

2184

722

723

153.4

153-3

153.1

153.5

153-6

153-2

233

1163

2028

1874

2141

2308

1837

1820 
Rec. for Gymnastic Equipment - Horizontal Bar (1964)

Rec. for Modular Coordination-Reference Lines of ec. for Test Conditions for Surface Grinding Machines with Rec. for Modular Coordination: tions for Milling Machines with Table of Fixed Height with or Milling Machines with Table of of Variable Height, with fabrics-Determination of the Recovery from Creasing of A gth (I970)

Rec. for Gymnastic Equipment-Vaulting and Pommelled Rec. for General Purpose Agricultural Rubber Spray Rec. for Hydrostatic Test. of Rubbe Rec. for General Purpose Rubber Water Rec. for Industrial Air

Std. for Wire Reinforced, Rubber Covered Hydraulic Rec. for Rubber ting Wire (197I)

Rec. for Rubber Rec. for Stretchers, Stretcher Carriers and and Valves: Meth. of Measurement of Emission Current from Rec. for Requirements for mil Rec. for Determination of the Weight Per Unit Area of for Guiding Principles for Protection Against Corrosion by Rec. for Dimensions of Rec. for Dimensions of Rec. for Dimensions of Rec. for Dimensions of Rec. for Dimensions of Rec. for Dimensions of Rec. for Dimensions of Rec. for Dimensions of Rec. for Dimensions of Rec. for Safety of Rec. for Safety of Rec. for Safety of Rec. for Safety of Rec. for Safety of Rec. for Safety of Rec. for Safety of Rec. for Safely of Rec. for Safety of Rec. for Safety of

Rec. Appliance Couplers for

quirements (1970) Requirements for Vacuum Cleaners (19) Requirements for Electric Irons (197)

Requirements for Spin Extractors (I9/

Requirements for Dishwashers (197I)

Requirements for Ranges (1971)

Requirements for Electric Shavers, H

Requirements for Toasters, Grills, W/ lectrical Equipment of Refrigerators and Food Freezers for ec. Meth. for Measuring Performance of Floor Polishers for meth. of Measurement of Performance of Vacuum Cleaners for Rec. Meth. for Measuring Performance of

Meth. of Measurement of Performance of Electric Irons for for Meth. for Measuring Performance of Water Heaters for ure Compartments for the Storage of Frozen Food/ Rec. for nts (1968)

Rec. for

tric Series Chamfer Dimension Limits and Maximum Shaft and Rec. for Mounting of Plain Grinding Wheels by Means of le), for Magnetic Tape Used in / Rec. for General Purpose f Electronic Tubes and Valves: Mcth. of Measuring Hiss and Rec. for Resistance to amelled Round Copper with Good Dielectric Properties Under ec. for Plastics-Meth. for Maintaining Constant Relative the Verification of Instrumcnts for Measuring the ${ }^{\circ} \mathrm{O}$ test Enclosures of Noninjection Type for Constant Relative Rec. for Lif eboats for Less Than One nation of Sodium and Boric Oxide and Available / Std. for lnid Power Transmission (/ Rec. for Graphical Symbols for s Mechanical Handling Equipment for Loose Bulk Materials

Diameters-Metric Series (1971) Rec. for Flat Seal for Std. for Wire Reinforced, Rubber Covered Rec. for Coupling Threads for Rec. for Pipe Couplings for for International Code for the Field Acceptance Tests of Rec. for International Code for Model Acceptance Tests of nternational Code for Test. of Speed Governing Systems for for Mineral Solvents for Paints: White Spirits and Related due on Evaporation (I972)

acidity (I970)

cloud Point (I970)

Std. for Liquid Halogenated

Rec. for Liquid Halogenated

Rec for Liquid Halogenated total Acidity: Volumetric Meth. (1968)

sulphate Content; Barium Sulphate Gravimetric M/

sulphated Ash: Gravimetric Meth. (I968)

oxidizing or Reducing Substances Volumetric Met/

iron Content: 2,2'-Bipyridyl Spectrophoto/

Acid Concentration by Measurement of Density tion of Sample Solution for Analysis by Means of Attack by Rec. for Rec. for Rec. for
Rec. for

Horizontal Controlling Coordinating Dimensions (1970)

Horizontal Grinding Wheel Spindle and Reciprocating Tab

Horizontal Impact Tests (Inclined Plane and Pendulum Te

Horizontal Multimodules (I969 + 1970)

Horizontal or Vertical Spindle-Test. of Accuracy (197

Horizontal or Vertical Spindle-Test. of the Accuracy

Horizontally Folded Specimen by Measuring the Angle of

Horology-Functional and Nonfunctional Jewels (1969)

Horses (1964)

Hose-Bore Sizes, Test Pressures and Tolerances on Len Hose (1970)

Hose (1970)

Hose (1970)

Hose $(1970)$

Hose (1972)

Hoses-Bending Test (1970)

Hoses for Aircraft Ground Fueling Without Static Conduc

Hoses for Oil Suction and Discharge (1971)

Hospital Trolleys: Dimensions (1960)

Hot Cathodes for High Vacuum Electronic Types (1966)

Hot Dip Galvanized Coatings on Fabricated Ferrous Produ

Hot Dip Galvanized Coatings on Ferrous Materials by Che Hot Dip Galvanizing (1970)

Hot Rolled Steel Bars: Flat, Metric Series (1969)

Hot Rolled Steel Bars: Round, Metric Series (I969)

Hot Rolled Steel Bars: Square, Metric Series (1969)

Hot Rolled Steel Sections-Equal Leg Angles-In. Scti

Hot Rolled Steel Sections-Parallel Flange Column sect

Hot Rolled Steel Sections-Parallel Flange I Beams-I

Hot Rolled Steel Sections-Part I: Equal Leg Angles-

Hot Rolled Steel Sections-Part 2: Unequal Leg Angles

Hot Rolled Steel Sections-Unequal Leg Angles-In. Se

Household and Similar Electrical Appliances: General Re

Household and Similar Electrical Appliances: Particular

Household and Similar Electrical Appliances: Particular

Household and Similar Electrical Appliances: Particular

Household and Similar Electrical Appliances: Particular

Household and Similar Electrical Appliances: Particular

Household and Similar Electrical Appliances: Particular

Household and Similar Electrical Appliances: Particular

Household and Similar Electrical Appliances: Particular

Household and Similar Electrical Appliances: Particular

Household and Similar General Purpose (1970)

Household and Similar Purposes (1970)

Household and Similar Purposes (1971)

Household and Similar Use (I971)

Household Electric Ranges and Ovens (1971)

Household or Similar Use (1970)

Household Purposes (1972)

Household Refrigerators-Part II: Special Low Temperat

Ments for the E IEC

Household Refrigerators-Part I: Performance Requireme

Hubs and Reels, with $76 \mathrm{~mm}$ (3 In.) Centrehole (Centerho

Hum (1964) JEasurements of the Electrical Properties O IEC

Humid Conditions (1958)

Humid Conditions (1970)

Humidity in Small Enclosures by Means of Aqueous Soluti

Humidity (1968)

Hundred People (1963)

Hydrated Sodium Perborates for Industrial Use-Determi

Hydraulic and Pneumatic Equipment and Accessories for F

Hydraulic Conveyors-Safely Code (1972)

Hydraulic Couplings (1970)

Hydraulic Cylinders-Internal Diameters and Piston Rod

Hydraulic Hose (1972)

Hydraulic or Pneumatic Piping (Pipe Threads) (1970)

Hydraulic Piping (Pipe Threads) (1970)

Hydraulic Turbines (I963)

Hydraulic Turbines (1965)

Hydraulic Turbines (1970)

Hydrocarbon Solvents (1972)

Hydrocarbons for Industrial Use-Determination of Resi

Hydrocarbons for Industrial Use-Determination of the

Hydrocarbons for Industrial Use-Determination of the

Hydrochloric Acid at $100^{\circ} \mathrm{C}$ (I970)

Hydrochloric Acid for Industrial Use: Determination of Rec. for Hydrochloric Acid for Industrial Use: Determination of Rec. for Hydrochloric Acid for Industrial Use: Determination of Rec. for Hydrochloric Acid for Industrial Use: Evaluation of Its Hydrochloric Acid Under Pressure (1971)

379

1790

2244

1040/19II

1984

2313

1112

381

1307

I 401

1402

1403

1436

1746

1825

1823

168

15I-13

1461

1460

1459

$1035 /$ III

$1035 / \mathrm{I}$

1035/II

657/III

657/IX

657/VII

657/I

657/II

$657 / \mathrm{IV}$

335-I

335-10

335-2

335-3

$335-4$

335-5

335-6

335.7

335.8

335.9

320

3 I 6

369

312

350

311

379
825

824

582

666

1858

15 I-5

REC 1

317-6

483

8

260

338

1917

1219

2392

1941

209I 
•. for ()ilseed Residues: Determination of Ash lnsoluble in Hydrochloric Acid (I968) and Vegetalile P'roducts-Determination of Ash Insoluble in he Determination of Sodium and Potassium Soluble in Dilute gen Carbonate for Industrial Use-Determination of Sodium oride Content-Mercurimetric Meth. (I972) Std. for Sodium f Sodium Carbonate-Titrimetric Meth. (/ f Sodium Hydrogen Carbonate Content-Ti/ f Moisture Content-Gravimetric Meth. (/ $f$ Test and Preparation of the Sample (19/ uffs): Determination of Total Alkalini/ Std. for Sodium Std. for Sodium Std. for Sodium Std. for Sodium dium Carbonate for Industrial Use: Determination of Sodium related Copolymers and Their Compounds by Splitting Off of stion Meth. (1967) Rec. for Determination of Carbon and Rec. for Determination of Carbon and 68) acid and XylenoIs for Industrial Use-Test for Absence of on of Density of Samples in Solution-Meth. Using Density ion Coefficient (For Use in the Preparati) Rec. for Glass Rec. for ReIative Rec. Ior Density Rec. for Principles of Construction and Adjustment of Rec. for Spec. for Photographic Grade of Test for Resistance of Fabrics to Penetration by Water Rec. for

Flame Emission Photometry Meth. (19) Gravimetric Meth. Using Uranyl Acel/ ty, Volumetric Meth. (1969)

ty: Volumetric Meth. (1969)

EDTA Complexometric Meth. (1969)

EDTA Complexometric Meth. (1969) Content: Volhard Volumetric Meth. (1973/

Content: Volhard Volumetric Meth (19/ tent: 2,2-Bipyridyl Spectrophotome/ content: 2,2'-Bipyridyl Spectropho/ a Content; Gravimetric Meth. by Precipit/ ontent: Gravimetric Meth. by Insolubiliz/ ontent: Gravimetric Meth. by Insolubi/ ontent, Gravimetric Meth. by Precipit/ Content: Barium Sulphate Gravimetric Me/ Content: Barium Sulphate Gravimetric/ on Dioxide Content, Expressed as Sodium on Dioxide Content, Expressed as Pota/ soluble Matter (1969)

soluble Matter (1969)

f Test Sample (1969)

f Test Sample (1969)

ution (1969)

ution (1969)

preparation of the Main Solution for $r$ Industrial Use-Test for Impurities Insoluble in Sodium Oils-Determination of Carbonyl Compounds Content-Free for Industrial Use-Determination of Carbonyl Compounds ficial): Determination of Aluminium (Aluminum) Content-8 Std. for lron Ores: Determination of of Chemical Analysis of Manganese Ores-Determination of Rec. for Rec. for

of 3 Phase Electric Systems (1963) adial Ball and Roller (II) Thrust (III) Tap/ red Roller Type-In. Series (1965) ame Type (1968)

ids in Liquid or Gaseous Condition in Land Inst/ 2)

aft (1959)

Rec. for Potassium
Rec. for Potassium Rec. for Sodium Rec. for Potassium Rec. for Sodium
Rec. for Solum Std. for Sodium Rec. for Potassium Rec. for Potassium Rec. for Sodium Rec. for Sodium Rec. for Sodium Rec. for Potassium Rec. for Sodium ec. for Potassium Rec. for Sodium Rec. for Potassium
Rec. for Sodium Rec. for Potassium Rec. for Sodium Rec. for Potassium Rec. for Sodium Rec. for Potassium Hydrochloric Acid (I971) lydrochloric Acid (1971) lydrogen Carbonate Content-Titrimetric Meth. (1972) ISO Hydrogen Carbonate for Industrial Use- /Nation of Chl ISO Hydrogen Carbonate for Industrial Use-Determination $O$ ISO Hydrogen Carbonate for IndustriaI Use-Determination O ISO Hydrogen Carbonate for Industrial Use-Determination O ISO Hydrogen Carbonate for Industrial Use-List of Meth. $O$ ISO Hydrogen Carbonate for Industrial Use (Including Foodst ISO Hydrogen Carbonate: Volumetric Meth. (1968) /C. for So ISO Hydrogen Chloride (1970) /Ty of PolyvinylchIoride and ISO Hydrogen in Coal and Coke by the High Temperature Combu ISO Hydrogen in Coal and Coke by the Liebig Meth. (1967) Hydrogen Sulphide (1971) Rec. for Cresylic ISO Hydrolytic Resistance of Glass Grains at $121{ }^{\circ} \mathrm{C}(19$ Hydrolytic Resistance of Glass Grains at $98^{\circ} \mathrm{C}(196$ Hydrometer-Meth. Using Pyknometer (1970) Hydrometers-Conventional Value for the Thermal Expan

Hydrometers for General Purposes (1968)

Hydrometers for General Purposes (1968)

Hydrometers (1964)

Hydroquinone (1965)

Hydrostatic Head Test) (1968)

Hydrostatic Test. of Rubber Hose (1970) $\begin{array}{ll} & \text { ISO } \\ \text { /Eterminati } & \text { ISO }\end{array}$ ISO

Hydroxide for Industrial Use-Determination of Sodium

Rec. for Meth. ISO Hydroxide for Industrial Use-Determination of Sodium Hydroxide for Industrial Use: Determination of Alkalini Hydroxide for Industrial Use: Determination of Alkalini Hydroxide for Industrial Use: Determination of Calcium: Hydroxide for Industrial Use: Determination of Calcium; Hydroxide for Industrial Use: Determination of Chloride Hydroxide for IndustriaI Use: Determination of Chloride Hydroxide for Industrial Use: Determination of lron Con Hydroxide for Industrial Use: Determination of of Iron Hydroxide for Industrial Use: Determination of of Silic Hydroxide for Industrial Use: Determination of Silica C Hydroxide for Industrial Use: Determination of Silica C Hydroxide for Industrial Use: Determination of Silica C Hydroxide for Industrial Use: Determination of Sulphate Hydroxide for Industrial Use: Determination of Sulphate Hydroxide for Industrial Use: Determination of the Carb Hydroxide for Industrial Use: Determination of the Carb Hydroxide for Industrial Use: Determination of Water in Hydroxide for Industrial Use: Determination of Water in Hydroxide for Industrial Use: Preparation and Storage $\mathrm{O}$ Hydroxide for Industrial Use: Preparation and Storage $O$ Hydroxide for Industrial Use: Preparation of Sample SoI Hydroxide for Industrial Use: Preparation of Sample Sol Hydroxide for Industrial Use: Sampling-Test Sample-

(P-Cresol Fo ISO Hydroxylamine Meth. (1972) Std. for Essential ISO Hydroxylammonium Chloride Potcntiometric Meth.) (1970) ISO Hydroxyquinoline Gravimetric Meth. (1972) /Al and Arti ISO Hygroscopic Moisture in Analytical Samples (1973) ISO Hygroscopic Moisture (1963) Hypodermic Needles (1967)

Rec. for Iso Rec. for Iso Rec. for Iso Rec. for Std. for Rec. for Rec. for Packaging-Complete, Filled Transport Packages-Part 1.

(1957) Rec. for Marking of Industrial Gas Cylinders for the

Film for Still Picture Cameras (1968) Rec. for for Radiographic Image Quality Indicators: Principles and for Elastomeric Sealing Rings for Aircraft-Packaging and Std. for Leather: Laboratory Samples-Location and welding of Mild and Low Alloy Steels-Code of Symbols for Materials-Troughed Belt Conveyors (Other Than Portable) for Industrial Use-Determination of Sulphated Residue on Std. for Internal Combustion Engines: Spark Plug Rec. for Plastics-Determination of the Loss on apparatus for Explosive Gas Atmospheres: Meth. of Test for yrophosphate for Industrial Use-Determination of Loss on etermination of Sodium and Boric Oxide Content and Loss on and Oleum for Industrial Use: Determination of Residue on Rec. for
Identification by Hour Numbers of the Phase Conductors

Identification Code for Roller Bearings: Groups-(I) R

Identification Code for Rolling Bearings: Group IV Tape

Identification Code for Rolling Bearings: Group V Airfr

ldentification Colours (Colors) for Pipes Conveying Flu

dentification Marking Code for Freight Containers (197

Identification of Aircraft Pipelines (1970)

Identification of Exposed Colour (Color) Roll Film (196

Identification of Mechanical Control Circuits for Aircr

Identification of Parts When Test. (1972)

Identification of the Content (1965)

Identification of the Contents of Medical Gas Cylinders

Identification of the Emulsion Side of Edge Marked Roll

Identification (1969)

Identification (1971)

Identification (1972)

Identification (1973)

Idlers (1970) Ignition-Gravimetric Meth. (1971) / for Nitric Acid ISO

/Ered Electrodes for Manual Arc ISO

gnition-Terminology (1972)

Ignition of Textile GIass Reinforced Plastics (1970) ISO

Ignition Temperature (1966) Rec. for Electrical IEC Ignition (1968) / Sodium Tripolyphosphate and Sodium P ISO Ignition (1972) $M$ Tetraborates for Industrial Use-D ISO /for Sulphuric Acid ISO Ignitrons to Be Used in Welding Machine Control (1967) llumination for Comparison Viewing of Photographic Tra ISO Illumination (1972) /Ces for Use in Sensitometric Expo ISO Illumination (1972) IS for Use in Sensitometric Exposu ISO 
pictur/

Std. for Cinematography-l,ocation of the Printed

Rec. for Cinematography-Projected

tography-Image Produced by Camera Aperture and Projected tography-lmage Produced by Camera Aperture and Projected td. for Cinematography: 65 and $70 \mathrm{~mm}$ Motion Picture Film

Std. for Cinematography: $35 \mathrm{~mm}$ Motion Picture Film or Cinematography: $35 \mathrm{~mm}$ Motion Picture Film-Projectable rea for $35 \mathrm{~mm}$ Films (I958) rea for $8 \mathrm{~mm}$ Films (1958) ) Rec. for Cinematography Rec. for Cinematography (1969) wing Conditions for Films: Utilization of Rec. Patterns of m-Image Area Produced by Camera Aperture and Projectable apers/ Rec. for Meth. for Indicating the Stability of the or Electrical Apparatus for Explosive Gas Atmospheres: Oi Rec. for Loading Guide for Oil ing Appliances for Domestic and Similar Purposes: Portable ion of Water Absorption and of Swelling in Thickness Afte $n$ ts and Varnishes-Determination of Resistance to W/ater

6I)
$961)$ Rec. for Plastics-Determination of the Charpy rec. for Field and Laboratory Measurements of Airborne and -Complete, Filled Transport Packages-Part IV: Vertica Rec. for Beam Unnotched
Rec. for Izod

Rec. for Charpy

Rec. for Beam

Rec. for Verification of Pendulum : Complete, Filled Transport Packages-Part V: Horizontal tics-Meth. of Determining the Brittleness Temperature by tubes and Valves: Meth. of Measuring the Cathode Interface

bles (I967)

r Plastics (I970) Rec. for the Rated (I/2 In.) Magnetic Tape (196)

ack I2.7 mm (I/2 In.) Magnetic Tape (I969)

of Agricultural Wheeled Tractors for Attachment of Mounted photography-Front Lens Barrels Up to $I 00 \mathrm{~mm}$-Dimension alternating Voltages from $10 \mathrm{kV}$ Up to / Rec. for Tests on raining Types for Alternating Voltages/

Rec. for Tests on 964) Rec. for Fixed Capacitors for Direct Current Using Rec. for Alginat Rec. for Agar Rec. for

ubes and Valves: Meth. of Application of Mechanical Shock Frequency Measurements on $\mathrm{Fm}$ Receivers of the Response to Rec. for Plastics-Pvc Resins-Determination of phenol, $\mathrm{O}, \mathrm{M}$, and $\mathrm{P}-\mathrm{Cresol}$ for Industrial Use-Test for Rec. for Phenol for Industrial Use-Test for Rec. for Oleaginous Seeds: Determination of for Fruit and Vegetable Products: Determination of Minera

Crude Vegetable Oils and Fats: Determination of Insoluble Rec. for Animal Fats: Determination of Insoluble ermosetting of (I96) Rec. for Plastics-Determination of 968) Spec. for Lighting Fittings for arrow Bands of Noise in a Diffuse Field and in a Frontally lled Transport Packages-Part V: Horizontal Impact Tests r (Shore Hardness) / Rec. for Plastics-Determination of Std. for Flexible Cellular Materials: Hardness Test. by

Data Communication-Basic Mode Control Procedures-Code

Input Energizing Quantity Measuring, with Nonspecified o her Alcohols for Industrial Use-Determination of Bromine or Glucose Syrup: Determination of Dry Matter-Refractive

9) ons / Rec. for Plastics-Determination of the Melt Flow eth. for Determining the Comparative Trackin Rec. for Butter-Determination of the Refractiv Rec. for Plastics-Determination of the Refractive

Winding Wires: Enamelled Round Copper, with a Temperature Winding Wires: Enamelled Round Copper, with a Temperature Winding Wires: Enamelled Round Copper, with a Temperature Winding Wires: Enamelled Round Copper, with a Temperature rec. for Meth. Determining Photographic Speed and Exposure Rec. for Meth. for the Determination of Bromin ec. for International Electrotechnical Vocabulary: Genera Rec. for Graphical Symbols: General Rec. for Throwaway Carbide Rec. for

ymbolization (1971) glossary of Gears-Geometrical Definitions-Alphabetica Rec. for Significance to Purchasers of Mark Rec. for for Std. Direction of Motion of Operating Devices and for vacuum Gauges, with Pressure Resp/ International Rec. for Rec. for Technical Drawings-Meth. of
Image Area for Printing to $8 \mathrm{~mm}$ Type $S$ on $16 \mathrm{~mm}$ Motion

Image Area Produced by Camera Aperture (1972)

Image Area (1972)

Image Produced by Camera Aperture and Projected Image a

Image Produced by Camera Aperture and Projected Image a

Image Produced by Camera Aperture for $16 \mathrm{~mm}$ Films (1965 ISO

Image Quality Indicators (1973) /Aphy of Welds and Vie ISO

Image Quality Indicators: Principles and Identification

$\begin{array}{lll}\text { Image-Area (1972) } & \text { / Hy: } 65 \text { and } 70 \mathrm{~mm} \text { Motion Picture Fil ISO } \\ \text { Images of Processed Black and White FiIms, Plates and P } & \text { ISO }\end{array}$

Immersed Apparatus (1968)

Immersed Transformers (1972)

Immersion Heaters (1968)

Immersion in Water (1972)

Rec. F IEC

Immersion Meth. (197I)

lor Electric Cooking and Heat CEE

Building Boards: Determinat ISO

Rec. for Pai ISO

Impact Resistance of Rigid Plastics (Flexural Test) (19 ISO

Impact Resistance of Rigid Plastics (Flexural Tests) (1 ISO

Impact Sound Transmission (1960)

Impact Test by Dropping (1972)

Impact Test for Grey Cast Iron (I969)

Std. for Packaging ISO

Impact Test for Steel (1959)

Impact Test (U.Notch) for Steel (1959)

Impact Test (V-Notch) for Steel (1960)

Impact Test. Machines for Test. Steels (1965)

ISO
ISO

ISO

ISO

Impact Tests (Inclined Plane and Pendulum Tests (1972) ISO

Impact (1969)

Rec. for Plas ISO

Impedance (1966)

Electrical Properties of Electronic IEC

Impedances and Dimensions of Loudspeakers $(1960)$

Impedances and Dimensions of Radio Frequency Coaxial Ca

Impermeability to Water of Fabrics Coated with Rubber $\mathrm{O}$

Implementation of the 6 and 7 Bit Coded Character Sets

Implementation of the 7 Bit Coded Character Set on $9 \mathrm{Tr}$

Implements (1968)

Rec. for 3 Point Linkage ISO

Important to the Connection of Auxiliaries (I971)

Impregnated Paper Insulated Metal Sheathed Cables: for

Impregnated Paper Insulated Metal Sheathed Cables: Nond

Impregnated Paper or Paper / Plastic Film Dielectric (I

Impression Material (1970)

Impression Material (1970)

Impulse Tests on Cables and Their Accessories (1966)

(Impulse) Excitation to Electronic Types (1965)

Impulsive Interference (1971)

Impurities and Foreign Matter (1970)

Impurities Insoluble in Sodium Hydroxide Solution-Vis

Impurities Insoluble in Water-Visual Test (1971)

Impurities (1968)

Impurities (I968)

Impurities (1968)

Impurities (1969)

Index of a Publication (Standardization of Format) (196

Index of Electrical Measuring Apparatus Used in Connect

Index of Essential Oils (I962)

Index of Polyethylene and Compounds of (1967)

Index of Solid Insulating Materials Under Moist Conditi

Index of the Fat (Reference Meth.) (1971)

Index of Transparent Plastics (I966)

Index of 155 (I 970)

/Ec. Spec. for Particular Types of IEC

Index of I80 for Use in Refrigerant Systems (1972) lof IEC

Index of 180 (1972) Rec. for Particular Types of IEC

Index of 220 (1972) Rec. for Particular Types of IEC

Index (1954)

Index (1968)

Index (1970)

Index (1972)

Indexable Inserts: Dimensions (1969)

Rec. for Particular Types of IEC

Indexable (Throwaway) Inserts-Designation-Code of S

Indicating Conformity with Standards (1966)

Indicating Electrical Measuring Instruments and Their a

Indicating Lamps for Circuit Breakers (1936)

Indicating Pressure, Combined Pressure and Vacuum, and

Indicating Surface Texture on Drawings (1971)
IEC

762

932

181 
rk and White Films, Plates and Papers/ of Ships (1971)

$s$ of Form and of Position-Part I: Generalities, Symbols,

$s$ of Form and of Position-Part IV: Practical Examples of

Tubes: Meth. of Measurement for Cold Cathode Counting and s for Films: Utilization of Rec. Patterns of Image Quality

Rec. for Maximum Demand

Rec. for Radiographic Image Quality

Rec. for Determination of the Micum

Rec. for Determination of the Shatte

and Accessory Electrometers (1971)

$s$ with Nominal Voltages Greater T/

inal Voltages Greater Than 1,000 V (19)

\section{Rec. for Dimensions of} Rec. for Tests on Rec. for Test Meth. for

eated, Alloy, and Free Cutting Steels-Part 12: Flame and Appearing in Manufacturers Catalogues of Transformer and uring Meth. (1971) Rec. for Cores for

$\mathrm{g}$ of Perforamnce Spec. for Cores of Tuned Transformers and

rec. for Low Voltage Fuses with High Breaking Capacity for tal Tubes (1970) Rec. for Pipe Connections for Oblong Top Plates with \& Bolt Holes (1972) Std. for
S ns (1971)

International Electrotechnical Vocabulary: Scientific and Std. for Wire and Plate Screens for

Spec. for Plugs and Socket Outlets for

ance (1972)

\section{(1972)}

sponding Pulleys (1970/

69)

n Gauge (1970)

71)

lectric Trucks (1969)

Rec. for Plugs, Socket Outlets and Couplers for

Std. for Lined

Rec for Endless Wide V-Belts for

Rec. for

Rec. for

Rec. for

Rec. for

Rec. for Sodium and Potassium Silicates for 1970) metric Meth. (1972) Std. for Sodium Hydrogen Carbonate for Electrolytic Analyser (1972) Std. for Liquid Chlorine fo meth. (1971) alein (1970) Meth. (1970)

\section{h. $($ I 970$)$}

h. (1972)

.) $(1970)$

presence of Mercury (II) $\mathrm{Ch} /$

t Expressed / Rec. for Sor

Meth. as Potassium Tetr/

aphenylborate Volumetric/

Rec for Nitric Acid for

Rec. for Higher Alcohols for

Rec. for Aliphatic Polyhydric Alcohols-Glycerine for

Rec. for Urea for

Std. for Glycerols for

Rec. for Higher Alcohols for

Rec. for Higher Alcohols for

and Potassium Silicates for

Rec. for Higher Alcohols for
Rec. for Potassium Chloride for Rec. for Potassium Chloride fo

1971) Rec. for Phenol: Ortha, Meta, and Para Cresol for uefied Phenol: Meta Cresol, Cresylic Acid and Xylenols for

(1972)

Solution-1

971)

ric Meth. (1/

72)

Bipyridyl Spectrophot

Rec. for Phosphoric Acid for

n.-Bipyridyl Photometric Meth. (1970/

n.-Bipyridyl Photometric Meth/

n.-Bipyridyl Photometric Meth/

ric Meth. (1972)

ric Meth. (1972)

ating at $900^{\circ} \mathrm{C}(1 /$ . for Sodium Tripolyphosphate and Sodium Pyrophosphate for Acids Other Than Formic (Le/ Rec. for Formic Acid for d. for Boric Acid and Oxide, and Disodium Tetraborates fo ater (1968)

1971)

avimetric Meth. (1971)

avimetric Meth. (/

Rec. for Sodium Tripolyphosphate for

Rec. for Cresylic Acid for

Rec. for Potassium Chloride for for Sodium Hydrogen Carbonate fo Meth. (1970)

olumetric Meth. (1971)

volumetric Meth. (1968)

(1971)

mination Meth. (1972)

lame Emission Photometri

on (1971)

n (197)

Rec for Pheci. ndicating the Stability of the Images of Processed Bla

Indications Appearing on Machine Tools (1964)

lndications by Means of a Pointer and Graduated Scale (

Indications of Details on the General Arrangement Plans

Rec. for Tolerance

L Drawings-Tolerance

Rical Properties of Electronic IFC

Indications on Drawings (1971)

Indicator Types (1970)

Indicators (1973)

ndicators, Class 1.0 (1966)

Indicators: Principles and Identification (1969)

Aphy of Welds and Viewing Condition

Indices of Coke (1967)

Indices of Coke (1967)

Indirect Reading Capacitor Type Pocket Exposure Meters

Indoor and Outdoor Posi Insulators and Units for System

Indoor and Outdoor Post Insulators for Systems with Nom

Induction Furnaces with Submerged Channels (1972)

Induction Hardening Types (1972) $\quad$ Std. for Heat Tr IS

Inductors and Transformers for Telecommunications: Meas

Inductors of Ferromagnetic Oxides for Telecommunication

Industrial Air Hose (1970)

Industrial and Similar Purposes: General Requirements (

Industrial Application for Plain End Steel and Other Me

Industrial Castors-Dimensions of Top Plates-Part I:

Industrial Gas Cylinders for the Identification of the

Industrial Laminated Sheets Based on Thermosetting Resi

Industrial Length Measurements (1951)

Industrial Measuring Insiruments (1958)

Industrial Purposes-Nominal Sizes of Apertures (1972)

Industrial Purposes (1966)

Industrial Purposes (1969)

Industrial Rubber Boots with General Purpose Oil Resist

Industrial Rubber Footwear for Use at Low Temperatures

Industrial Speed Changers and Groove Profiles for Corre

Industrial Tractors-Definition and Nominal Rating (19

Industrial Trucks-Dimensions of Stillages-Connectio

Industrial Trucks-Wheels and Castors-Vocabulary (19

Industrial Trucks: Voltages of Traction Batteries for E

Industrial Use

Industrial Use-

/Nation of Chloride Content-Mercuri

Industrial Use-Determinaion of Density at $20^{\circ} \mathrm{C}$ (

Industrial Use-Determination of Using an

Industrial Use-Determination of Acidity-Volumetric

Industrial Use-Determination of Acidity to Phenolphth

Industrial Use-Determination of Alkalinity or Acidity

Industrial Use-Determination of Alkalinity Volumetric

Industrial Use-Determination of Ash-Gravimetric Met

Industrial Use-Determination of Ash-Gravimetric Met

Industrial Use-Determination of Ash (Gravimetric Meth

Industrial Use-Determination of Carbon Dioxide Conten

Industrial Use-Determination of Carbonyl Compounds ( $\mathrm{H}$

Industrial Use-Determination of Content-Gravimetric

industrial Use-Determination of Content-Sodium Tatr

Industrial Use-Determination of Crystallizing Poini (

Industrial Use-Determination of Density at $20^{\circ} \mathrm{C}$

Industrial Use-Determination of Density at $20^{\circ} \mathrm{C}$

Industrial Use-Determination of Density of Samples in

Industrial Use-Determination of Distillation Range (1

Industrial Use-Determination of Distillation Yield (1

Industrial Use-Determination of Dry Matter-Gravimet

Industrial Use-Determination of Dynamic Viscosity (19

Industrial Use-Determination of Iron Content-2, $2 \mathrm{M}$

Industrial Use-Determination of Iron Content-2, $2 \mathrm{M}$

Industrial Use-Determination of Iron Content-2,2 Mi

Industrial Use-Determination of Iron Content: $2,2 \mathrm{M}$

Industrial Use-Determination of Iron Content: $2,2 \mathrm{Mi}$

Industrial Use-Determination of Its Content-Volumet

Industrial Use-Determination of Its Content-Volumet

Industrial Use-Determination of Loss in Mass After He

Industrial Use-Determination of Loss on Ignition (196

Industrial Use-Determination of Low Contents of Volat

Industrial Use-Determination of Manganese Content-F

Inoustrial Use-Determination of Matter Insoluble in W

Industrial Use-Determination of Mcta Cresol Content (

Industrial Use-Determination of Moisture Content- $\mathbf{G r}$

Industrial Use-Determination of Moisture Content-Gr

Industria! Use-Determination of Neutral Oils and Pyri

Industrial Use-Determination of Nitrogen-Volumetric

Industrial Use-Determination of Nitrous Compounds-V

Industrial Use-Determination of of Calcium Content-

Industrial Use-Deternination of Ortha Cresol Content

Industrial Use-Determination of Phenols Content-Bro

Industrial Use-Determination of Potassium Content-F

Industrial Use--Determination of Residue on Distillati

Industrial Use-Determination of Residue on Evaporatio
ISO

ISO

OIML

ISO

SO

ISC

IEC

ISO

ISO

ISO

IEC

IEC

ISO

IEC

IEC

ISO

IEC

ISO

ISO

ISO

ISO

Rec. for IEC

ISO

IEC

ISO

ISO

ISO

ISO

ISO

ISO

ISO

ISO

ISO

ISO

Iso

ISO

ISO

ISO

ISO

ISO

ISO

ISO

ISO

ISO

ISO

ISO

ISO

ISO

ISO

ISO

ISO

ISO

ISO

ISO

ISO

ISO

ISO

ISO

ISO

ISO

ISO

ISO

ISO

ISO

ISO

ISO

ISO

ISO

ISO

421 
n (1972) imetric: Meth/ imetrir Meth/ on Photometry Meth. (19/ meth. Using Uranyl Acet/ e Content and Loss On/ $\mathrm{e}$ and Available / e Contents-Volumetri/ trimetric Meth. (/ nate Content $-\mathrm{Ti} /$ lumetric Meth. (1968) u/ Rec. for Aliphatic Polyhydric Alcohols-Glycerine for

Std. for liquid Halogenated Hydrocarbons for Rer. for Sodium and Polassium Silicates for Std. for Sodium and Potassium Silicales for Rec. for Potassium Hydroxide for Rec. for Potassium Hydroxide for Std. for Disodium Tetraborates for Std. for Hydrated Sodium Perborates for Std. for Crude Sodium Borates for Std. for Sodium Hydrogen Carbonate for Std. for Sodium Hydrogen Carbonate for Rec. for Phosphoric Acid for ignition-Gravimetric Meth. (1) Rec. for Nitric Acid for Rec. for Liquid Halogenated Hydrocarbons for ) Rec. for Liquid Halogenated Hydrocarbons for in the Vaporized Product (I t (Volumetric Meth.) (1970) Sid. for Liquid Chlorine for ressed in $\mathrm{Na}$ Rec. for Sodium and Potassium Silicates for tha, Meta, and Para Cresol, Cresylic Acid and Xylenois for tho, Meta, and Para Cresol, Cresylic Acid and Xylenols for metric Meth. (1972)

fischer Meth. (1972)

karl Fischer Meth. (1970)

of the Sample (19/

Units (1970)

Sid. for Liquid Chlorine for Std. for Gylcerols for Rec. for Higher Alcohols for Std. for Sodium Hydrogen Carbonate for Rec. for Higher Alcohols for y the Volumetric Content) (/ Rec. for Sodium Tripolyphosphate for
Rec. for Liquid Chlorine for

Rec. for Cresylic Acid and Xylenols for Std. for Gylcerols for

Rec. for Acetic Acid and for Rec, for Acetic Anhydride for Rec. for N-Butanol for Rec. for Isopropyl Alcohol for Rec, for Acetone for Rec. for Phthalate Esters for Rec. for Solvent Acetates for Rec. for Methanol for Rec. for Ethanol for Rec. for Phthalic Anhydride for Rec. for Maleic Anhydride for Rec. for Paraformaldehyde for

Rec. for Ortho Chlorotoluene for

Rec. for Para Chlorotoluene for Rec. for Chlorobenzene for Rec. for Ortho Dichlorobenzene for

Rec for Para Dichlorobenzene for Rec. for Methylene Chloride (Dichloromethane) for Rec. for Chloroform for

Std. for Trichloroethylene for

Std. for Perchloroethylene for Std. for Carbon Tetrachloride for $\begin{array}{ll}\text { t Easily Sol/ } & \text { Std. for Sodium and Potassium Silicates for } \\ 970 & \text { Rec. for Sodium and Potassium Silicates for }\end{array}$ Rec for Aliphatic Polyhydric Alcohols-Clycerine for (1971) Rec. for Cresylic Acid and Xylenols for Acid (1970) m Hydroxide So/ -Visual Test (1971)

Total Alkalini/

Rec. for Higher Alcohols for Rec. for Phenol, O, M, and P-Cresol for Rec. for Phenol for ge (1973)

Std. for Ammonium Hydrogen Carbonate for Rec. for Low Voltage Controlgear for Std. for Potassium Sulphate for

\section{Meth. (1969)}

Meth. (1969) contents-EDTA Complexomet/
metric Meth. (1969)

metric Meth. (1969)

curimetric Meth. (1973)

curimetric Meth. (1973)

ard Volumetric Meth. (1973/

ard Volumetric Meth. (1973)

ard Volumetric Meth (19)

ate Gravimetric Meth. (l)

meth. as Potassium Tetra/

phenylborate Volumetric

ified Willard/

1972)

ric Meth. (1972)

Std. for

Std. for Formaldehyde Solutions for Rec. for Sodium Hydroxide for

Rec. for Potassium Hydroxide for Std. for Formaldehyde Solutions for Std. for Sodium Chloride for

Rec. for Pot assium Hydroxide for Rec. for Sodium Hydroxide for Std. for Sodium Chlorate for Std. for Potassium Sulphate for Std. for Sodium Carbonate for Std. for Sodium Hydroxide for Rec. for Potassium Hydroxide for Std. for Potassium Sulphate for Std. for Potassium Sulphate for Std. for Potassium Sulphate for Aluminium (Aluminum) Fluoride for Std. for Formaldehyde Solutions for Std. for Ammonium Nitrate for Chlorine-Mercurimetric M/ Std. for Sodium Chloride for
enanthroline / Std. for Aluminium (Aluminum) Fluoride for Bipyridyl Photomet/ -Bipyridyl Spectrophoto/ ipyridyl Spectrophotome/ -Bipyridyl Photometric M/ -Bipyridyl Spect/

Rec. for Hydrochloric Ac

Rec for Sodium Carbonate for Rec. for Sulphuric Acid and Oleum for
Industrial Use-Determination of Residue on Evaporatio

Industrial Use-Determination of Silica Content-Grav

Industrial Use-Determination of Silic Content-Titr

Industrial Use-Determination of Sodium-Flame Emissi

Industrial Use-Determination of Sodium-Gravimetric

Industrial Use-Determination of Sodium and Boric Oxid

Industrial Use-Determination of Sodium and Boric Oxid Industrial Use-Determination of Sodium and Boric Oxid Industrial Use-Determination of Sodium Carbonate-Ti Ind ustrial Use-Determination of Sodium Hydrogen Carbo Industrial Use-Determination of Sulphate Content-Vo Industrial Use-Determination of Sulphated Ash in Prod Industrial Use-Determination of Sulphated Residue on Industrial Use-Determination of the Acidity (1970)

Industrial Use-Determination of the Cloud Point (1970

Industrial Use-Determination of the Content by Volume Industrial Use-Determination of Total Alcohols Conten

Industrial Use-Determination of Total Alkalinity, Exp

Industrial Use-Determination of Water by the Dean and Industrial Use-Determination of Water by the Karl Fis Industrial Use-Determination of Water Content-Gravi Industrial Use-Determination of Water Content-Karl Industrial Use-Determination of Water Content by the Industrial Use-List of Meth. of Test and Preparation Industrial Use-Measurement of Colour (Color) in Hazen Industrial Use-Measurement of Colour (Color) (1971)

Industrial Use-Measurement of $\mathrm{pH}$ Potentiometric Meth Industrial Use-Meth. of Sampling (For Determining Onl Industrial Use-Meth. of Sampling (1972)

Industrial Use-Meth. of Test (1968)

Industrial Use-Meth. of Test (1968)

Industrial Use-Meth. of Test (1968)

Industrial Use-Meth. of Test (1968)

Industrial Use-Meth. of Test (1968)

Industrial Use-Meth. of Test (1970)

Industrial Use-Meth. of Test (1970)

Industrial Use-Meth. of Test (1970)

Industrial Use-Meth. of Test (1970)

Industrial Use-Meth. of Test (1970)

Industrial Use-Meth. of Test (1970)

Industrial Use-Meth. of Test (1970)

Ind ustrial Use-Meth. of Test (1970)

Industrial Use-Meth. of Test (1970)

Industrial Use-Meth. of Test (1970)

Industrial Use-Meth. of Test (1970)

Industrial Use-Meth. of Test (1970)

Industrial Use-Meth. of Test (1970)

Industrial Use-Meth. of Test (1970)

Industrial Use-Meth. of Test (1972)

Industrial Use-Meth. of Test (1972)

Industrial Use-Meth. of Test (1972)

Industrial Use-Preparation of Solution of Products No Industrial Use-Samples and Meth. of Test-General (1

Industrial Use-Samples and Test Meth.-General (1970

Industrial Use-Test for Absence of Hydrogen Sulphide

Industrial Use-Test for Colour (Color) with Sulphuric

Industrial Use-Test for Impurities Insoluble in Sodiu

Industrial Use-Test for Impurities Insoluble in Water

Industrial Use (Including Foodstuffs): Determination of

Industrial Use: Contractors (1971)

Industrial Use: Determination of Acidity to Methyl Oran

Industrial Use: Determination of Acidity (1972)

Industrial Use: Determination of Alkalinity, Volumetric

Industrial Use: Determination of Alkalinity: Volumetric

Industrial Use: Determination of Ash (1972)

Industrial Use: Determination of Calcium and Magnesium

Industrial Use: Determination of Calcium: EDTA Complexo

Industrial Use: Determination of Calcium; EDTA Complexo

Industrial Use: Determination of Chloride Content-Mer Industrial Use: Determination of Chloride Content-Mer Industrial Use: Determination of Chloride Content: Volh Industrial Use: Determination of Chloride Content: Volh Industrial Use: Determination of Chloride Content: Volh Industrial Use: Determination of Content-Barium Sulph Industrial Use: Determination of Content-Gravimetric Industrial Use: Determination of Content-Sodium Tetra Industrial Use: Determination of Fluorine Content-Mod Industrial Use: Determination of Formaldehyde Content ( Industrial Use: Determination of Free Acidity-Volumet Industrial Use: Determination of Halogens, Expressed as Industrial Use: Determination of Iron Content-1,10 pH Industrial Use: Determination of Iron Content-2,2 Min Industrial Use: Determination of Iron Content: 2,2

Industrial Use: Determination of Iron Content: 2,2-B

Industrial Use: Determination of Iron Content: 2,2

ec. for Potassium Hydroxide for 
nvolatile Matter at $250 \mathrm{De} /$ ler (1973)

ter or in Acid and Preparat/ ter at $50^{\circ} \mathrm{C}(1968)$

vimetric Meth. (1973) in.-Bipyridyl Spectropho/ vimetric Meth. by Precipit/ 4-Xylenol Spec/ substances Volumetric Met/ ravimetric Meth. (/ rophotometric/ etric Meth. by Precipit/ etric Meth. by Insolubiliz etric Meth. by Insolubi/ ate; Volumetric Meth. (196) ium Sulphate Gravimetric $\mathrm{Me} /$ um Sulphate Gravimetric Me/ um Sulphate Gravimetric/ um Sulphate Gravimetric Me/ um Sulphate Gravimetric M/ tric Meth. (1968) Oxide, Disodium Tetraborates and Crude Sodium Borates for tent, Expressed as Sodium / tent, Expressed as Pota/ $0{ }^{\circ} \mathrm{C}$ (1973)

ulation of Free So/ ric Meth. (1968)

Volumetric Meth./ ty: Volumetric Meth. (1968) (1969)

\section{(1969)}

Measurement of Density ( ion by Measurement of Densit/

iron) (1972)

2)

c Meth. (1972)

\section{(1969)}

(1969)

(1968)

the Main Solution for

- Barium Sulphate terials: Socket Fittings with Spigot Ends for Domestic and ies (1972) Rec. for Pelletized Carbon Black for Use in the Rubber Rec. for Carbon Black for Use in the Rubber Rec. for Nomenclature of Terms Used in the Benzole Rec. for Definitions of Terms Used in the Benzole Rec. for Carbon Black for Use in the Rubber Rec. for Carbon Black for Use in the Rubber Rec. for Carbon Black for Use in the Rubber u/ Rec. for Pelletized Carbon Black for Use in the Rubber c/ Rec. for List of Equivalent Terms Used in the Plastics e/ Rec. for List of Equivalent Terms Used in the Plastics p/ Rec. for List of Equivalent Terms Used in the Plastics Rec. for List of Equivalent Terms Used in the Plastics Rec. for Vocabulary for the Refractories for Sampling Bulk Shipments of Carbon Black for the Rubber etermination of Ash Content of Carbon Black for the Rubber mination of Loss on Heating of Carbon Black for the Rubber on of Total Sulphur Content of Carbon Black for the Rubber

Rec for Definitions of Terms Used in the Petroleum or Pipes and Fittings-Stainless Steel Tubes for the Food

Rec. for Pelletized Carbon Black for Use in the Rubber Rec. for Cereals and Pulses-Meth. of Test fo Std. for Directories of Libraries.

r Connectors for Frequencies Below $3 \mathrm{MHz}$ : Guide to Drawing i), NRZI, and $63 \mathrm{R} /$ Rec. for Unrecorded Magnetic Tape for Rec. for Magnetic Tape Labelling and File Structure fo Rec. for 7 Track $8 \mathrm{RPmm}$ (200 RPI) Magnetic Tape for Rec. for 9 Track 8 RPmm (200 RPI) Magnetic Tape for Rec for 9 Track 32 Pmm (800 RPI) Magnetic Tape for

Std. for Basic Mode Control Procedures: Conversationa ec. for the Use of Longitudinal Parity to Detect Errors in rers Catalogues of Transformer and Inductor $\mathrm{Co} /$ tape (1971)

ns on 12 Row Punched Cards (1971)

Rec. for

Rec. for

Rec. for

$\mathrm{s}(1970)$

ools (197)
Industrial Use: Determination of Loss of Mass and of No Industrial Use: Determination of Matter Insoluble in Wa lndustrial Use: Determination of Matter Insoluble in $W_{a}$ Industrial Use: Determination of Matter Insoluble in W/ lndustrial Use: Determination of Methanol Content (1972 Industrial Use: Determination of Moisture Content-Gra Industrial Use: Determination of of Iron Content: $2,2 \mathrm{M}$ Industrial Use: Determination of of Silica Content; Gra Industrial Use: Determination of Oxides of Nitrogen-2 lndustrial Use: Determination of Oxidizing or Reducing Industrial Use: Determination of Residue on Ignition; $\mathrm{G}$ Industrial Use: Determination of Silica Content-Spect

Industrial Use: Determination of Silica Content, Gravim Industrial Use: Determination of Silica Content: Gravim Industrial Use: Determination of Silica Content: Gravim Industrial Use: Determination of Sodium Hydrogen Carbon Industrial Use: Determination of Sulphate Content-Bar Industrial Use: Determination of Sulphate Content: Bari Industrial Use: Determination of Sulphate Content: Bari Industrial Use: Determination of Sulphate Content; Bari Industrial Use: Determination of Sulphate Content; Bari Industrial Use: Determination of Sulphated Ash: Gravime Industrial Use: Determination of Sulphur Compounds-Vo Industrial Use: Determination of the Carbon Dioxide Con Industrial Use: Determination of the Carbon Dioxide Con Industrial Use: Determination of the Loss of Mass at 11 Industrial Use: Determination of Total Acidity and Calc Industrial Use: Determination of Total Acidity: Volumet Industrial Use: Determination of Total Nitrogen Content Industrial Use: Determination of Total Soluble Alkalini Industrial Use: Determination of Water Insoluble Matter Industrial Use: Determination of Water Insoluble Matter Industrial Use: Evaluation of Its Acid Concentration by Industrial Use: Evaluation of Sulphuric Acid Concentrat Industrial Use: Expression of Test Results (1968) Industrial Use: Limit Test for Heavy Metals (Excluding Industrial Use: Limit Test for Inorganic Chlorides (197 Ind ustrial Use: Limit Test for Inorganic Sulphates (197 Industrial Use: Measurement of $\mathrm{pHI}$ Value-Potentiometri Industrial Use: Meth. of Test (1968)

Industrial Use: Preparation and Storage of Test Sample Industrial Use: Preparation and Storage of Test Sample Industrial Use: Preparation and Storage of Test Samples Industrial Use: Preparation of Sample Solution (1969) Industrial Use: Preparation of Sample Solution (1969) Industrial Use: Sampling-Test Sample-Preparation of Industrial Use; Determination of Sulphur Dioxide Conten Industrial Waste Pipe: Basic Dimensions; Metric Series Ind ustrial Wheels-Dimensions and Nominal Load Capacit Industry-Determination of Fines Content (1970)

Industry-Meth. of Test for Sieve Residue (1970)

lndustry-Part I (1971)

Industry-Part I (1971)

Industry-Sampling Packaged Shipments (1970)

Ind ustry-Spec. for Sieve Residue (1970)

Industry-Spec. Limits for Loss on Heating (1970)

Industry Delivered in Bulk or in Bins-Spec. for Maxim Industry (English, French, Russian) (Cs: Corresponding Industry (English, French, Russian) (D: Corresponding G Industry (English, French, Russian) (NL: Corresponding Industry (English, French, Russian) (Pl: Corresponding Ind ustry (English, French, Russian) (1969)

Industry (1968)

Indust ry (1969)

Industry (1969)

Industry (1969)

Ind ustry (1969)

Industry (1971)

Industry (1971)

Industry: Determination of Pour Density (1970)

Infestation by X-Ray Examination (1970)

Information and Documentation Centres (Centers) (1972)

Information in Detail Spec. (1970)

Information Interchange-8 and $32 \mathrm{RPmm}$ (200 and $800 \mathrm{Rp}$

Information Interchange (1969)

Information Interchange (1971)

Information Interchange (1971)

Information Interchange (1971)

Information Message Transfer (1973)

Information Messages (1969)

Information on Ferrite Materials Appearing in Manufactu

Information Processing-Properties of Unpunched Paper

Information Processing-Representation of 8 Bit Patter

Information Processing-Spec. for Unpunched Paper Card

Information Processing-Widths of Fabric Ribbons on $\mathrm{Sp}_{p}$

Information Processing Interchange (1967)
ISO

745 
er Sets Derived from the ISO 7 Bit Coded Character Set for rdinal Dates (1973)

Rec. for Flowchart Symbols for

作 12 Row Punched Cards haser in Order to Obtain The/

haser in Order to Obtain I

Rec. for Cylindrical Gears Rec. for Straight Bevel Gears ec. for High Voltage AIternating Current Circuit Breakers: ication-Basic Mode Control Procedures-Code Independent Rec. for

Rec. for Characteristics of Electric r Composition of Magnesium Aluminium (Aluminum) Zinc Alloy for Classification and Composition of Unalloyed Aluminium Rec. for Composition of 99.8 Unalloyed Magnesium Rec. for Composition of 99.95 Unalloyed Magnesium Rec. for Zinc Alloy Rec. for Zinc

1s: Dilution Meth. for Steady Flow-Part 1: Constant Rate rials $(1963)$ Rec. for Plastics -

Pumps (1957)

Rec. for Print Spec. for Magnetic Rec. for Shipbuilding Details for Rec. for Shipbuilding Details for

bustible Liquids-Nom/ Rec. for Shipbuilding Details for Rec. for Shipbuilding Details for Sea Navigation and Rec. for Shipbuilding Details for Rec. for Dental

Formaldehyde Solutions for Industrial Use: Limit Test for Formaldehyde Solutions for Industrial Use: Limit Test for ical Properties of Electronic Tubes and Valves: Equivalent or Independent Specif/ Rec. for Electrical Relays: Single Rec. for Indexable (Throwaway) earing Aids Using Earphones Coupled to the Ear by Means of Rec. for Throwaway Carbide Indexable use: Determination of Silica Content: Gravimetric Meth. by use: Determination of Silica Content: Gravimetric Meth. by e-Determination of Silica Content-Gravimetric Meth. by Rec. for Pulps: Determination of Acid

Rec. for Spices and Condiments: Determination of Water

Rec. for Spices and Condiments: Determination of Acid Rec. for Tea-Determination of Water Soluble and Rec, for Tea-Determination of Acid

Rec. for Crude Vegetable Oils and Fats: Determination of Rec. for Animal Fats: Determination of Rec. for Oilseed Residues: Determination of Ash c. for Fruit and Vegetable Products-Determination of Ash $\mathrm{m}$, and $\mathrm{P}-\mathrm{Cresol}$ for Industrial Use-Test for Impurities Rec. for Phenol for Industrial Use-Test for Impurities dium Carbonate for Industrial Use: Determination of Matter odium Chloride for Industrial Use: Determination of Matter polyphosphate for Industrial Use-Determination of Matter asily Soluble in Boiling Water and Determination of Matter odium Chlorate for Industrial Use: Determination of Matter odium Hydroxide for Industrial Use: Determination of Water ssium Hydroxide for Industrial Use: Determination of Water Rec. for Plastics-Determination of h. 1

for Fruit and Vegetable Products: Determination of Water Std. for Magnesium and Its Alloys-Determination of Rec. for Sampling and

Rec. for Guide to the Use of ISO Rec. 390, Sampling and in Steel Pipes Up to $50 \mathrm{Mm} /$ aluminum) and Its Alloys (

s Up to $50 \mathrm{~mm}$ (2 In.) Thick/

50 to $200 \mathrm{~mm}$ Thi/ Rec. Pract. for Radiographic
Containers and Gauges for the Std. Rec. Pract. for the X-Ray Rec. Pract. for Radiographic Std. for Rec. Pract. for Radiographic Rec. for Geometrical Rec. for ISO System of Limits and Fits-Part II: for Rolling Bearings-For Railway Axle Boxes: Acceptance ling Cables in Singles for Telecommunication Equipment and es Conveying Fluids in Liquid or Gaseous Condition in Land c. for Rigid Conduits of Polyvinyl Chloride for Electrical rinciples for Protection in the Design and Construction of th Special Regard to Rated Capacity of Circuit Breakers in tor (Storage), Batteries, Heating An/ Installations) (1965)

Rec. for Electrical Rec. for Electrical Rec. for Electrical Rec. for Electrical Rec. for Electrical Rec. for Electrical Rec. for Electrical Rec. for Electrical on, Distribution and Controlgear (19/
ting. Semiconductor Rectifiers, Gene/

70)

rec. for Conventional Signs: to Be Used in Schemes for the Rec. for Conventional Signs to Be Used in Schemes for the Rec. for Conventional Signs to Be Used in Schemes for the Spec. for Steel Conduits and Fittings for Electrical Rec. for Std. Frequencies for Centralized Network Control
Information Processing Interchange (1969)

Information Processing Interchange: Representation of $\mathrm{O}$

Bit Charact ISO

Information Processing (1969)

Information Processing) (1970)

(Information Processing) (1971)

/Ons and Lecation of $\mathrm{Re}$ ISO

(o

Information to Be Given to the Manufacturer by the Purc

Information to Be Given with Enquiries Tenders and Orde

Information Transfer (1972)

Std. for Data Commun

Informative Labelling (1965)

Infra Red Emitters for Heating Purposes (1967)

Ingots for Casting Purposes (1959)

Ingots for Remelting (1968)

Ingots (1959)

Ingots (1961)

Ingots (1963)

Ingots (1968)

Injection Meth. (1966) /Low Measurement in Open Channe

Injection Moulding Test Specimens of Thermoplastic Mate

Ink Character Recognition (1969)

Inland Navigation-Covers for Deck Openings for $220 \mathrm{~mm}$

Inland Navigation-Mushroom Ventilators (1957)

Inland Navigation-Pipe Lines for the Transport of Com

Inland Navigation Anchor Chains-Studless Links (1957)

Inland Navigation Sprocket Wheels (1956)

Inlay Casting Wax (1970)

Inorganic ChIorides (1972)

Inorganic Sulphates (1972)

Input and Output Admittances (1963)

Input Energizing Quantity Measuring, with Nonspecified

Inserts-Designation-Code of Symbolization (1971)

Inserts (1961)

Inserts: Dimensions (1969)

Insolubilization (1969)

Insolubilization (1969)

Insolubilization (1970)

Insoluble Ash (1968)

Insoluble Ash (1969)

Insoluble Ash (1969)

Insoluble Ash (1970)

Insoluble Ash (1970)

Insoluble Impurities (1968)

Insoluble Impurities (1969)

Insoluble in Hydrochloric Acid (1968)

Insoluble in Hydrochloric Acid (1971)

Insoluble in Sodium Hydroxide Solution-Visual Test (1

Insoluble in Water-Visual Test (1971)

Insoluble in Water at $50^{\circ} \mathrm{C}(1968)$

Insoluble in Water or in Acid and Preparation of Princi

Ium I Ium Hydroxide for Industrial ISO /M Silicates for Industrial Us ISO

ISO

ISO

ISO

ISO

ISO

ISO

ISO

ISO

Re ISO

ISO

ISO

Insoluble in Water (1968)

Insoluble in Water (1972)

Insoluble in Water (1973)

Insoluble Matter (1969)

Insoluble Matter (1969)

Insoluble Particles in Cellulose Acetate (1970)

Rec. for Sodium Tri ISO

I Solution of Products Not E ISO Std. for S ISO Rec. for S ISO

Insoluble Solids (1968)

Insoluble Zirconium Alizarin Sulphonate Photometric Met

Rec. for Pota ISO

ISO

Inspection of Asbestos Cement Products (1964)

Rec ISO

ISO

ISO

Inspection of Asbestos Cement Products (1970)

ISO

Inspection of Circumferential Fusion Welded Butt Joints

ISO

列

Inspection of Fusion Welded Butt Joints for Steel Plate ISO

Inspection of Fusion Welded Butt Joints for Steel Plate ISO

Inspection of Grooves of Pulleys for V-Belts (1962) ISO

Inspection of Plain Workpieces (1971)

ISO

Inspection (1970)

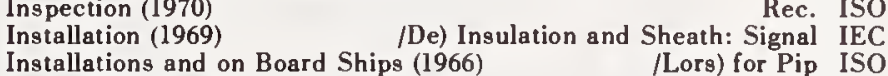

Installations and Their Fittings (1968) Spe CEE

Installations for Work on Unsealed Radioactive Material ISO

Installations in Ships (1972) /T Current Evaluation Wi IEC

Installations in Ships: Accessories, Lighting. Accumula

Installations in Ships: Cables (Construction, Test. and

Installations in Ships: General Requirements (1964)

Installations in Ships: Graphical Symbols (1965)

Installations in Ships: Switchgear, Electrical Protecti

Installations in Ships: Transformers for Power and Ligh

Installations of Buildings: Fundamental Principles (197

Installations of Buildings: General and Definitions (19

Installations of Pipe Line Systems in Ships (1967)

Installations of Sanitary Systems in Ships (1968)

Installations of Ventilation Systems in Ships (1967)

Installations (1962)

Installations (1967)

963

2711

1028

1679

1682

1340

1341

56-6

211

436

240

122

115

114

207

301

555

294

1004

41

42

51

40 
for Tests on large Hollow Porcelains for Use in Electrical Cahles in Pairs, Triples, Ouads and Quintuples for Inside al Installations in Ships: Cahles (Construction, Test. and Rec. for Electrical Relays: Rec. for Sizes' and Mounting Dimensions of Aircraft Rec. for Figures for Aircraft Rec. for Aircraft
Rec. for Rolling Bearings. ement/ Rec. for Unrecorded Magnetic Tapes for Interchange for Precision Reels for Magnetic Tape Used in Interchange rehole (Centerhole), for Magnetic Tape Used in Interchange est Meth. (1973) Std. for Unrecorded Magnetic Tapes for Rec. for General Characteristics of Nuclear Reactor Rec. for General Principles of Nuclear Reactor

Rec. for Coaxial Cable Connectors Used in Nuclear Rec. for Classification of Rec. Graphical Symbols: Measuring Rec. for Indicating Electrical Measuring

Rec. for Direct Recording Electrical Measuring tional Rec. for Working Std. Meth. for the Verification of reals (1970) the Profile Meth.-General Statements-Terms/ International Rec. for the Profile Meth.-Contact (Stylus) Instrumen/

Rec. for International Rec for Pressure Gauges for otechnical Vocabulary: Scientific and Industrial Measuring External Diameter of Planchets Used in Nuclear Electronic Rec. for Supply Voltages for Transistorized Nuclear

voltage Ranges and Logic Levels for Mains Operated Nuclear of Progressive Profile Transformation-Profile Recording for Dimensions of Panels and Racks (For Nuclear Electronic onal Protection Against Ionizing Radiat/ Rec. for Nuclear Spec. for Polyvinyl Chloride Spec. for Rubber

nal Cross Sectional Areas and Composition of Conductors of Rec. for Marking of ctors and a Rated Voltage No/ Rec. for Polyvinyl Chloride ctors and a Rated Voltage Not Exceeding / Rec. for Rubher es from $10 \mathrm{kV} \mathrm{Up} \mathrm{to} \mathrm{/} \mathrm{Rec.} \mathrm{for} \mathrm{Tests} \mathrm{on} \mathrm{Impregnated} \mathrm{Paper}$ alternating Voltages/ Rec. for Tests on Impregnated Paper

ic Dimensions of Winding Wires: Dimension of Conductors of tion and Sheath: Distribution Wires with Solid Conductors,

illed and Gas Pressure Cables and Their Accessories: Paper tion and Sheath: Distribution Wires with Solid Conductors, ipment Wires and Cables with Solid or Stranded Conductors, equipment Wires, Type I, with Solid or Stranded Conductor,

Rec. for Thermal Rec. Test Cells for Measuring the Resistivity of meth. of Test for Electrical Resistance and Resistivity of Rec. Meth. of Test for Electric Strength of Solid rmittivity and Dielectric Dissipation Factor of Electrical paper-Part I: Definitions and General R/ Rec. Spec. for rec. Test Meth. for Determining the Relative Resistance of

h. for Determining the Comparative Tracking Index of Solid of Test for Volume and Surface Resistivities of Electrical or the Determination of the Insulation Resistance of Solid cedures for Evaluating the Thermal Endurance of Electrical

for Use Prior to and During the Test. of Solid Electrical

Rec. Spec. for New or Meth. for the Determination of the Electric Strength of Std. for Shaped

Rec for Teat Procedure for Thermal Endurance of rec. for Evaluation of the Thermal Endurance of Electrical - Classification of Prepared Unshaped Materials (Dense and Rec. for Meth. of Test for Pvc (Polyvinyl Chloride)

w Frequency Cables and Wires with Pvc (Polyvinyl Chloride) w Frequency Cables and Wires with Pvc (Polyvinyl Chloride) w Frequency Cables and Wires with Pve (Polyvinyl Chloride) w Frequency Cables and Wires with Pvc (Polyvinyl Chloride) w Frequency Cables and Wires with Pvc (Polyvinyl Chloride) w Frequency Cables and Wires with Pvc (Polyvinyl Chloride) w Frequency Cables and Wires with Pvc (Polyvinyl Chloride) anodic Oxidation) of Aluminium (Aluminum) and Its Alloys Rec. for Rec. for Rec for Rating of Sound ec. for Std. Colours (Colors) for Pve (Polyvinyl Chloride) rec. for High Voltage Connecting Wire with Flame Retarding ation T/ Rec. for the Classification of Materials for the Rec. for Characteristics of String Rec. for Ball and Socket Couplings of String or Locking Devices for Ball and Socket Couplings of String greater $\mathrm{T} /$ an $1,000 \mathrm{~V}(19)$
Installations (1967) nstallations (1972)

Installations) (1965)

Instantaneous All or Nothing Relays (1967)

Rec. IEC

(Chloride) Insulation and Sheath: IEC Rec. for Electric IEC

IEC

Instrument Cases (Rear Mounting Type) (1959)

Instrument Dials and Number Plates (1962)

Instrument Dials and Pointers (1962)

Instrument Precision Types (1971)

Instrumentation Applications Gener
Instrumentation Applications (1971)

Instrumentation Applications (1971)

ISO

Rec. ISO

Instrumentation (1966)

Instrumentation (1967)

Instrumentation (1969)

Instruments and Devices for Measurement and Evaluation

Instruments and Electric Clocks (1963)

Instruments and Their Accessories (1960)

Instruments and Their Accessories (1968)

Instruments for Measuring the ${ }^{\circ} \mathrm{Of}$ Humidity of Cerea

Instruments for Measuring the Mass Per Hectolitre of Ce

Instruments for the Measurement of Surface Roughness by

Instruments for the Measurement of Surface Roughness by

Instruments Measuring Arterial Blood Pressure (1970)

Instruments (1958)

Instruments (1967)

Instruments (1968)

Rec. for In

Instruments (1970)

Instruments (1970)

Instruments) (1968)

lnstruments: Constructional Requirements to Afford Pers

Rec. for IEC

Rec for Analogue IEC

nsulated Cables and Flexible Cords (1967)

Insulated Cables and Flexible Cords (1968)

Insulated Cables (1966)

Insulated Conductors (1972)

Insulated Flexible Cables and Cords with Circular Condu

Insulated Flexible Cables and Cords with Circular Condu

Insulated Metal Sheathed Cables: for Alternating Voltag

Insulated Metal Sheathed Cables: Nondraining Types for

Insulated Rectangular Copper Winding Wire (1972)

Insulated, in Pairs, Triples, Quadruples and Quintuples

Insulated, Metal Sheathed, for Alternating Voltages Up

Insulated, Polyamide Coated, in Singles, Pairs, Triples

Insulated, Screened, Single or One Pair (1969)

: Equ IEC

Insulated, Single (1967)

Insulating Liquids and Meth. of Cleaning (1967)

Insulating Materials at Elevated Temperatures (1971)

Insulating Materials at Power Frequencies (1967)

Insulating Materials at Power, Audio and Radio Frequenc

Insulating Materials Based on Built Up or Treated Mica

Insulating Materials to Breakdown by Surface Discharges

Insulating Materials Under Moist Conditions (1971)

Insulating Materials (1958)

Insulating Materials (1964)

Insulating Materials (1966)

Rec. Meth. IEC

Insulating Materials (1971)

Rec. for Meth. of Test F IEC

Rec. for Std. Conditions

chgear (1972)

Insulating Oils (1963)

lnsulating Oils (1963)

Insulating Refractory Products-Classification (1972)

Insulating Varnishes-Electric Strength Meth. (1971)

Insulating Varnishes by the Helical Coil Bond Test (196

Insulating) (1971)

Rec. for Refractory Materials

(I970)

Insulation and Sheath: Cables in Pairs, Triples, Quads

Insulation and Sheath: Distribution Wires with Solid Co

Insulation and Sheath: Distribution Wires with Solid Co

Insulation and Sheath: Equipment Wires and Cables with

Insulation and Sheath: Equipment Wires, Type I, with So

Insulation and Sheath: General Test and Measuring Meth.

Insulation and Sheath: Signalling Cables in Singles for

Insulation Check by Measurement of Breakdown Potential

Insulation Coordination (1972)

Insulation Coordination: Application Guide (1962)

Insulation for Dwellings (I968)

Insulation for Low Frequency Cables and Wires (1969)

Insulation for Use in Television Receivers (1965)

Insulation of Electrical Machinery and Apparatus in Rel

Insulation Resistance of Solid Insulating Materials (19

Insulator Units of the Cap and Pin Type (1969)

Insulator Units (1960)

Insulator Units: Dimensions and General Requirements (1

Insulators and Units for Systems with Nominal Voltages

Insulators for Systems with Nominal Voltages Greater Th 
nes with a Nominal Voltage Greater Tha/ Rec. for Tests on Rec. for Schedule for Sodium Rer. for Letter Symbols for Semiconductor Devices and Circ/ Rec. Guidance íor the Design and Use of Components nt of Airborne Noise Emitied by Compressor Primeover Units rec. for Octave, Half-Octave and Third-Octave Band Filters Rec. for Expression of the Power and d 63 R/ Rec. for Unrecorded Marnetic. Tape for Information ommunication-Data Terminal and Communication Equinrsent ional Requirement/ Rec. for Urarecorded Magnetic Tapcs for Rec. for Precision Reels for Magnetic Tape Used in (3 In.) Centrchole (Centerhole), for Magnetic Tape Used in equirements (1972) Std. for Data

and 7 Bit Coded Character Sets for Information Processing Rec. for Dimensions for Punched Paper Tape for Data magnetic Tape Labelling and File Structure for Information e ISO 7 Bit Coded Character Set for Information Processing for 7 Track 8 RPmm (200 RPl) Magnetic Tape for Information for 9 Track 8 RPmm (200 RPl) Magnetic Tape for lnformation for 9 Track 32 Pmm (800 RPl) Magnetic Tape for lnformation p Caps and Holders Together with Gauges for the Control of

p Caps and Holders Together with Gauges for the Control of

p Caps and Holders Together with Gauges for the Control of heir Arbors or Mandrels-Metric and In. Series/

Rec. for Automatic Coupling (1973

Std. for Aircraft: 6) Std. for Milling Cuiters with Tenon Dive:
. for Liquid Oxygen Replenishment Couplings ícr Aircraft ygen Replenishment Couplings ír Aircraft -
Rec. for Lathe Centres (Centers): Sizes fox Rec. for Lathe Centres (Centers): Sizes fox
indle Noses and Face Plates Types a and Camlock: Sizes for hanical Connections Between Towing Vehicles and Trailers.

for Mechanical Coupling Between Tractors and Semi Trailers for Spindle Noses and Face Plates: Bayonet Type; Sizes for $\begin{array}{ll}\text { tioning and Straight Cut Numerically Controlled/ } & \text { Rec. for }\end{array}$ Std. for Information Processing ves (1962) Rec. Meth. for the Measurement of Direct Iectronic Tubes and Valves: Meth. of Measuring the Cathode urface Active Agents: Determination of Surface Tension and Rec. for Matters Relating to Radio Spec. for Radio Spec. for Radio Spec. for Radio e $0.15 \mathrm{MHz}$ to $30 \mathrm{MHz}$ (1972) e $25 \mathrm{Mc} / \mathrm{S}$ to $300 \mathrm{Mc} / \mathrm{S}$ (196l) Than Quasi Peak (1967)

Rec. for Capacitors for Radio 961

Measurements on Fm Receivers of the Response to Impulsive 1961)

Rec. for Rolling Bearings

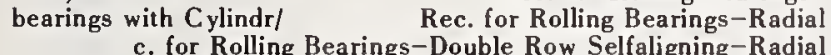
c. for Rolling Bearings-Double Row Selfaligning-Radial olling Bearings-Double Row Self-Aligning Ball-Radial eft Hand Single Bank Engines (197) ers (1972)

ion of Rotation (1972)

ices-Std. Direction of Motion (/ inology (1972) ries $(1971)$ ator (Storage), Batteries, Heating and Cooking Appliances, Rec. for Hydraulic Cylinders. Rec. for Paper:

Std. for Hermetically Sealed Metal Food Containers. for General Purpose Series l Freight Containers-Minimum Rec. for Lathe Tool Posts (Overall

e Transport of Fluids-Determination of the Resistance to Rec. for Turning Tools with Carbide Tips: Oil Filled and Gas Pressure Cables and Their Accessories: lic Turbines (1965) for Hydraulic Turbines (1970) riodicals (1972)

draulic Turbines (1963)

orage Pumps (1968)

ntrolling and Regulating Systems (1966)

d Measurement of Ionizing Radiation by Electric/ ction (1957)

tics (1960)

gy (1959)

stry and Electrometallurgy (1960)

ng Applications (1960)

(1956)

definitions (1956)

x (1970)

transmission and Distribution of Electrical Ene/

70)

Transformers (1956)

r Plants for Electric Energy Generation (1968)

Sid, for Documente Limits of Radio

Insulators of Ceramic Material or Glass for Overhead L.i

lutended for Mounting on Boards with Printed Wiring and

Intended for the Analysis of Sounds and

lnterchange -8 and 32 RPmm (200 and 800 RPl), NRZl, an

Inierchange Circuits-Assignment of Connector Pin Numb

Interchange Instrumentation Applications General Dimens

Interchange Instrumentation Applications (1971)

Interchange listrumentation Applications (1971)

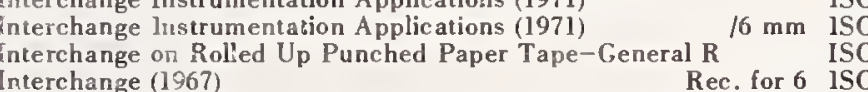

Interchange (1969)

Interchange (1969)

interchange (1969)

Interchange (1971)

Interchange (1971)

Interchinge (!97!)

Interchangeability and Safety of Lampcaps (1970)

Intcrchangeability and Safety: Gauges (1969)

Interchangeability and Safety: Lampholders (1969)

Interchangeability Dimensions for Milling Cutters and T

Interchangeability Dimensions of Battery Connectors for

Interchangeability Dimensions of Pellets and Cases (196

Interchangeability Dimensions with Cutter Arbors-Metr

lnterchangeability Dimensions (1971)

Intercliangeability (1963)

lntcrchangeability (1968)

Interchangeability (1969)

Interchangeability (1971)

Interchangeability: Metric Series (1968)

Interchangeable Conical Ground Glass Joints (1964)

lnterchangeable Punched Tape Variable Block Format Posi

Rec. for 6 iSO

Rec. for ISO

Interchangeable Spherical Ground Glass Joints (1968)

Interchange: Representation of Ordinal Dates (1973)

Interelectrode Capacitances of Electronic Tubes and Val

Interface Impedance (1966)

lnterfacial Tension (1963)

Interference and Its Suppression (1969)

Interference and Report of National Limits (1967)

Interference Measuring Apparatus for the Frequency Rang

lnterference Measuring Apparatus for the Frequency Rang

Interference Measuring Apparatus Having Detectors Other Interference Suppression (1965)

Interference (1971)

Internal Clearance in Unloaded Bearings-Definitions (

Internal Clearance in Unloaded Radial Groove Type Ball

Internal Clearance (1965)

Internal Clearance (1969)

Internal Clearance (1970)

Internal Combustion Engines-Definition of Right and L

lnternal Combustion Engines-Designation of the Cylind

Internal Combustion Engines-Designation of the Direct

Internal Combustion Engines-Hand Operated Controì Dev

Internal Combustion Engines: Spark Plug lgnition-Term

Internal Communications, Lightning Conductors (1965)

Internal Diameters and Piston Rod Diameters-Metric Se

Internal Diameters of Cores of Reels (1966)

Internal Diameters of Round Cans (1972)

lnternal Dimensions (1970)

Internal Height) (1961)

Internal Pressure (1970)

Internal Tools (Metric Series) (1966)

C. for Plastics Pipes for T

lnternal, for Alternating Voltages Up to $275 \mathrm{kV}$ (1967)

International Code for Model Acceptance Tests of Hydrau

Rec. for International Code for Test. of Speed Governing Systems

International Code for the Abbreviation of Titles of $\mathrm{Pe}$

Rec. for International Code for the Field Acceptance Tests of $\mathrm{Hy}$

Rec. for International Code for the Field Acceptance Tests of St

Rec. for International Electrotechnical Vocabulary: Automatic Co

Rec. for International Electrotechnical Vocabulary: Detection an

Rec. for International Electrotechnical Vocabulary: Electric Tra

Rec. for International Electrotechnical Vocabulary: Electroacous

Rec. for International Electrotechnical Vocabulary: EIectrobiolo

Rec. for International Electrotechnical Vocabulary: Electrochemi

Rec. for International Electrotechnical Vocabulary: EIectroheat

Rec. for International Electrotechnical Vocabulary: Electronics

Rec. for International Electrotechnical Vocabulary: Fundamental

Rec. for International Electrotechnical Vocabulary: General Inde

Rec. for International EIectrotechnical Vocabulary: Generation,

Rec. for International Electrotechnical Vocabulary: Lighting (19

Rec. for International Electrotechnical Vocabulary: Machines and

Rec. for International Electrotechnical Vocabulary: Nuclear Powe 
- . lays $(1950)$

rations (1970)

d Radiological Physics (J964)

nd Industrial Measuring lnstruments (1958)

nd Security Apparatus for Railways (1959)

rtors (1956)

and Apparatus for Connection and Regulation (1/ nd Telephony (1970)

(1955)

1961)

ta (1968)

Rec. for Microcopies: Scale of $35 \mathrm{~mm}$ Microfilms for

in Glass, with Maximum Device) (1970)

e (Gram) to 10 Kilogrammes (Medium Accuracy Class) (1970/ tric Gas Meters (1970)

essure and Vacuum, and Vacuum Gauges, with Pressure Resp/ ss Per Hectolitre of Cereals (1970)

ater) with Measuring Chambers (1970)

automatic Weighing Machines (1972)

(1970)

ring Filaments (1970)

0)

measuring Arterial Blood Pressure (1970)

ssure and Vacuum, and Vacuum Gauges, with Pressure Respo/

Kilogrammes (Medium Accuracy Class) (1972)

hardness Reference Blocks Vickers (1970)

hardness Reference Blocks Rockwell B (1970)

hardness Reference Blocks Rockwell C (1970)

hardness Reference Blocks Brinell (1970)

undamental Terms) (1969)

ication of Instruments for Measuring the ${ }^{\circ}$ Of Humidit/

uctor Wire of the Aluminium Magnesium Silico/

Rec. for an

characters (1961)

nto Latin Characters (1968)

cyrillic Characters (1968)

cimal Multipl/

Std for Rules for the Use of Unit wed Vehicles with 24-V Electrical Equipment for Commercial

- Confidence Interval (1973)

es (Load and Temperature Interrupted) (1961)

Std. for Statistical

Rec for

ation of Test Results: Estimation of the Mean-Confidence

lectrical Apparatus for Explosive Gas Atmospheres: General

Permissible Load (/ Rec. for Rolling Bearings: Airframe:

Std. for Surface Active Agents: Ethylene Oxide Adducts

Electrotechnical Vocabulary: Detection and Measurement of Rec. for Basic

of Electrical Measuring A pparatus Used in Connection with

Rec. for Test Procedures for Semiconductor Detectors for

plifiers and Preamplifiers for Semiconductor Detectors for

ctional Requirements to Afford Personal Protection Against

$f$ Hardness of Vulcanized Rubbers of Low Hardness (10 to 35

hardness of Vulcanized Rubbers of High Hardness (85 to 100

Rec. for Strip Wound Cut Cores of Grain Oriented Silicon

Determining the Conventional Bond Strength on Steel, Cast

Metal Spraying of Zinc and Aluminum for the Protection of Rec. for Electroplated Coatings of Zinc on

Rec. for Electroplated Coatings of Cadmium on

Std. for

rily Used for the Production of Aluminum: Determination of for Cryolite (Natural and Artificial)-Determination of $\mathrm{m}$ (Aluminum) Fluoride for Industrial Use: Determination of

for Phosphoric Acid for Industrial Use-Determination of sodium Pyrophosphate for Industrial Use-Determination of

1970/ Rec. for Urea for Industrial Use-Determination of ormaldehyde Solutions for Industrial Use: Determination of rmination of Iron (Orthophenanthroline Meth. Applicable to rmination of Iron (Orthophenanthroline Meth. Applicable to of Metallic Iron Content: (Photometric Meth. for Metallic

emical Analysis of Manganese Ores-Determination of Total rec. for Formic Acid for Industrial Use-Determination of rec. for Nitric Acid for Industrial Use-Determination of for Hydrochloric Acid for Industrial Use: Determination of $r$ Sodium Hydroxide for Industrial Use: Determination of of r Potassium Hydroxide for Industrial Use: Determination of ical Analysis of Manganese Ores: Determination of Metallic for Sodium Carbonate for lndustrial Use: Determination of phuric Acid and Oleum for Industrial Use: Determination of

opper and Its Alloys-Spectrophotometric Determination of

$t$-Oxine Gravimetric and Volumetric Meth. (197/

lytical Samples (1973)

tric Meth. (1973) nternational Electrotechnical Vocabulary: Protective $\mathbf{R}$ International Electrotechnical Vocabulary: Radiocommuni International Electrotechnical Vocabulary: Radiology an lnternational Electrotechnical Vocabulary: Scientific a lnternational Electrotechnical Vocabulary: Signalling a lnternational Electrotechnical Vocabulary: Static Conve lnternational Electrotechnical Vocabulary: Static Conve
lnternational Electrotechnical Vocabulary: Switchboards lnternational Electrotechnical Vocabulary: Telegraphy a International Electrotechnical Vocabulary: Transducers International Electrotechnical Vocabulary: Waveguides ( lnternational Exchange (1961)

International Gear Notation: Symbols for Geometrical Da International Rec. for Clinical Thermometers (Mercury, International Rec. for Correspondence Symbol (1970)

International Rec. for Cylindrical Weights from 1 Gramm International Rec. for General Prescriptions for Volume International Rec. for Indicating Pressure, Combined Pr International Rec. for lnstruments for Measuring the Ma International Rec. for Meters for Liquids (Other Than W International Rec. for Metrological Regulations for Non International Rec. for One Mark Glass Measuring Flasks International Rec. for Optical Pyrometers with Disappea International Rec. for Polarimetric Saccharimeters (197 International Rec. for Pressure Gauges for Instruments International Rec. for Recording Pressure, Combined Pre International Rec. for Rectangular Weights from 5 to $\mathbf{5 0}$ International Rec. for Verification and Calibration of International Rec. for Verification and Calibration of International Rec. for Verification and Calibration of International Rec. for Verification and Calibration of International Rec. for Vocabulary of Legal Metrology (F International Rec. for Working Std. Meth. for the Verif International Spec. for Aluminium (Aluminum) Alloy Cond International Standard Book Numbering (ISBN) (1972) International Std. for Resistance for Copper (1925)

International System for the Transliteration of Arabic International System for the Transliteration of Greek I International System for the Transliteration of Slavic International System of Units and a Selection of the De

International Traffic (1970) ISO International Unification of Concepts and Terms (1968) Interpretation of Test Results: Estimation of the Mean Interrupted Creep Test. of Steel at Elevated Temperatur Interval (1973) $\begin{array}{rr}\text { Std. for Statistical lnterpret } & \text { ISO } \\ \text { Rec. for E } & \text { IEC }\end{array}$ Introduction (1971)

Introduction. General, Boundary Dimensions, Tolerances, ISO Invertors for Aircraft (1973)

lodometric Determination of Oxyethylene Groups (1972)

Ionizing Radiation by Electric Means (1968)

lonizing Radiation Symbol (1963)

lonizing Radiation (1964)

Ionizing Radiation (1970)

lonizing Radiation (1970)

Ionizing Radiation (1972)

IRHD) (1971)

IRHD) (1971)

Iron and Other Metals (1968)

Iron and Steel Against Corrosion (1971)

lron and Steel (1971)

Iron and Steel (1971)

Iron Blue Pigments for Paints (1972)

Iron Content-1,10-Phenanthroline Photometric Meth.

Iron Content-1,10 Phenanthroline Photometric Meth. (1

Iron Content-1,10 Phenanthroline Photometric Meth. (1

Iron Content-2, 2'-Bipyridyl Spectrophotometric

Iron Content-2, 2'-Bipyridyl Spectrophotometric

Iron Content-2,2'-Bipyridyl Photometric Meth. (

Iron Content-2,2' Bipyridyl Photometric Meth. (19

Iron Content Between 0.002 and $0.05 \%$ ) (1968)

Iron Content Between 0.05 and 2.50\%) (1968)

Iron Content Up to and Including $2 \%$ ) (1967)

Iron Content (1963)

Iron Content: 2, 2'-Bipyridyl Photometric Meth.

Iron Content: 2, 2'-Bipyridyl Photometric Meth. (

lron Content: 2,2'-Bipyridyl Spectrophotometric M

Iron Content: 2,2'-Bipyridyl Spectrophotometric M

Iron Content: 2,2-Bipyridyl Spectrophotometric Meth.

Iron Content: (Photometric Meth. for Metallic Iron Cont

Iron Content; 2,2'-Bipyridyl Photometric Meth. (1

Iron Content; $2,2^{\prime}$-Bipyridyl Spectrophotometric $M$

for Chemical Analysis of C ISO

Iron Ores: Determination of Aluminium (Aluminum) Conten

Std. for lron Ores: Determination of Hygroscopic Moisture in Ana

Std. for lron Ores: Determination of Phosphorus Content-Volume
IEC

IEC

IEC

IEC

IEC

IEC

IEC

IEC

ISO

O1ML

OIML

OIML

OIML

OIML 15

OIML 5

OIML 3

OIML 18

OIML $\quad 14$

OIML $\quad 16$

OIML

OIML 10

OIML 11

OIML 12

OIML 9

OIML -1

IEC 104

ISO 2108

IEC 28

SO 233

ISO 843

SO $\quad 1000$

1185

860

2602

203

2602

79.0

1002

2277

2270
$50(66)$

361

181

333

340

405

1818

1400

329

698

2063

2081

2082

2495

805

1694

2368

849

852

1595

2226

792

793

$62 I$

313

1707

1982

909

983

994

621

744

915

1812

2771

2596

2599 
r7 (1957)

n lines (1955)

tions-Part IV: Fittings (1966)

ntilation (1966)

of Aluminium and Its Alloys. Photometric Determination of Rec. for Brinell Hardness Test for Grey Cast Rec. for Classification of Grey Cast Rec. for Pulps: Determinat, in of

or Chemical Analysis of Zinc: Photometric Determination of Rec. for Speroidal or Nodular Graphite Cas Rec. for Whiteheart Malleable Cast Rec. for Blackheart Malleable Cas Rec. for Pearlitic Malleable Cas

Rec. for Beam Unnotched Impact Test for Grey Cas for Designation of the Microstructure of Graphite in Cast or Covered Electrodes for Manual Metal Arc Welding of Cast $f$ Zinc and Its Alloys: Spectrophotometric Determination of

d Coatings of Copper Plus Nickel and Chromium on Steel (Or

for Industrial Use: Limit Test for Heavy Metals (Excluding

liances for Domestic and Similar Purposes: Electric Irons,

nces: Particular Requirements for Toasters, Grills, W affle

Rec. for Meth. of Measurement of Performance of Electric

lectrical Appliances: Particular Requirements for Electric

ating Appliances for Domestic and Similar Purposes: Waffle

ing Appliances for Domestic and Similar Purposes: Electric in a Curren/ ) (1969)

Rec. for Chemical Analysis of Steel and Cast Rec. for Chemical Analysis of Steel and Cas Rec. for Paper: Untrimmed Stock Sizes for the Rec. for Paper: Untrimmed Stock Sizes for the Spec. for Safety

evices) (1972)

Characteristics for Mechanica Rec. for Alternating Current Rec. for 968)

Rec. for Oil of Lemon, Expressed,

Rec. for Diagrams, Charts, Tables:

71)

Rec. for Diagrams, Charts, Tables:
rom Field Performance of Electronic Maintainability Data from Field Performance of Electronic
quirements for Electric Shavers, Hair Clippers and Simila s) (1961)

Rec. for Plastics-Determination of the Rec. for Aircraf Rec. for Clearance Dimensions for Tripod Rec. for Twin Wire Healds for Rec. for Horology-Functional and Nonfunctional ic Tubes and Valves: Spec. Sheets and Dimensions of Wiring Rec. for Twist Drills: Parallel Shank

r Chains, Stud Links (Common, Enlarged, and End Types, and lding Details for Sea Navigation-Anchor Chains-Lugless Rec. for Asbestos Cement Pipes, (1961)

r Gas List Tubes and Screwed Fittings Where Pressure Tigh Rec. for Pipe Threads Where Pressure Tight Std. for Crimped rec. Pract. for the X.Ray Inspection of Fusion Welded But . Pract. for Radiographic Inspection of Fusion Welded But

Pract. for Radiographic Inspection of Fusion Welded But Rec. for Rubber Sealing Rings for

1972) Std. for

iographic Inspection of Circumferential Fusion Welded But Rec. for Interchangeable Conical Ground Glass Rec for Strength Calculation of Butt Welded Rec. for Interchangeable Spherical Ground Glass loride (PVC) Pressure Pipes with Elastic Sealing Ring Type loride (PVC) Pressure Pipes with Elastic Sealing Ring Type Rec. for Hardenability Test by End Quenching Steel Rec. for Determination of the Rec. for Determination of Water by the for Industrial Use-Determination of Water Content by the ylenols for Industrial Use-Determination of Water by the rols for Industrial Use-Determination of Water Content

Procedures for Electronic Components and Equipment: Tcst Std. for Rotary Drilling Equipment: Upper and Lower Std. for Rotary Drilling Equipment: sea Navigation-Anchor Chains-Lugless Joining Shackles, Std. for Cylindrical Cutters with Plain Bore and Std. for Slotting Cutters with Plain Bore and rec. for Side and Face Milling Cutters with Plain Bore and Rec. for Numeric Section of Ten Rec. for Function
Rec, for Numeric Section of Ten Key rec. for Basic Arrangement for the Alphanumeric Section of limetres) (Milli/

Rec. for Rectangular or Square Paralle Rec. for Cotter Slots with $5 \%$ Taper Rec. for Layout of Printing and Function eyways (Dimensions in Millimetres) (Milli/
Iron Oxide Pigments (1970)

Iron Pipe Fittings Screwed in Accordance with ISO Rec

on Pipes: Special Castings and Parts for Pressure Mai

ISO

Iron Sanitary Pipe Fittings for Waste Water and Ventila
Iron Sanitary Pipes and Fittings for Waste Water and Ve Iron (Orthophenanthroline Meth. Applicable to Iron Cont Iron (Orthophenanthroline Meth. Applicable to Iron Cont Iron (1961)

Iron (1961)

lron (1968)

lron (1968)

Iron (1969)

Iron (1969)

Iron (1969)

Iron (1969)

Iron (1969)

Iron (1969)

Iron (1969)

Iron (1969)

Iron) (1970)

Iron) (1972)

Ironers and Pressing Machines (1965)

Irons and Roasters (1972)

Irons for Household or Similar Use (1970)

Irons (1971)

IEC

Iron, Crills, Warming Plates and Other Dry Cooking App

rons, Ironers and Pressing Machines (1965)

Iron: Determination of Sulphur (Meth. After Combustion ISO

lron: Determination of Total Silicon (Gravimetric Meth. ISO

ISO-A Series, ISO Primary Range (1966)

ISO-A Series: ISO Supplementary Range (1967)

Isolating Transformers (1964)

Isolation (Guide for Selecting and Applying Resilient D

Isolators (Disconnectors) and Earthing Switches (1961)

Isopropyl Alcohol for Industrial Use-Meth. of Test (1

Italy (1968)

Italy, United Kingdom and U. S. A. (1968)

Item Designation for Electrical Parts and Equipment (19 /Ection of Reliability, Availability, and

Items (1971)

Izod Impact Resistance of Rigid Plastics (Flexural Test

lzod Impact Test for Steel (1959)

Jacking Pads (1957)

Jacks for Aircraft (1970)

Jacquard Weaving (1967)

Jewels (1969)

Jigs and Pin Straighteners (1965)

Jobber and Stub Series and Morse Taper Shank (1961)

Joining Shackles) (1960)

Joining Shackles, Kenter Type (1957) /Shipbuilding Details-Ancho

Joints and Fittings for Sewerage and Drainage (1968)

Joints Are Made on the Threads (1/8 In. to 6 In./S) (19

Joints Are Not Made on the Threads (1/8 In. to 6 In./S)

Joints for Aircraft Electrical Cables (1973)

Joints for Aluminium (Aluminum) and Its Alloys and Magn

Joints for Steel Plates Up to $50 \mathrm{~mm}$ (2 ln.) Thick (1969

Joints for Steel Plates 50 to $200 \mathrm{~mm}$ Thick (1972)

Joints in Asbestos Cement Water Piping (1970)

Joints in Building: Fundamental Principles for Design (

Joints in Steel Pipes Up to $50 \mathrm{~mm}$ (2 In.) Wall Thicknes

Joints (1964)

Joints (1967)

Joints (1968)

Joints: Minimum Depths of Engagement (1973)

Joints: Minimum Depths of Engagement (1973)

(Jominy Test) (1967)

Kappa Number of Pulp (Deg. of Delignification) (1963)

Karl Fischer Meth. (1968)

Karl Fischer Meth. (1970)

Karl Fischer Meth. (1971)

Karl Fischer Meth. (1972)

Ka: Salt Mist (1964)

Kelly Cocks (1972)

Kellys (1972)

Kenter 'Type (1957)

Key Drive: Metric Series (1972)

Key Drive: Metric Series (1972)

Key Drive: Metric Series (1972)

Key Keyboards for Adding and Calculating Machines (1969

Key Symbols on Typewriters (1969)

Keyboards for Adding and Calculating Machines (1969)

Keyboards Operated with Both Hands (1971)

Keys and Their Corresponding Keyways (Dimensions in Mil

Keys for Boring Machine Tapers (1971)

Keys on Typewriters (1969)

Keys with or Without Gib Head and Their Corresponding $K$
1248

49

$531 / \mathrm{I}$

531

792

793

184

185

779

714

1083

942

943

944

946

945

1071

1055

1457

2223

II-2C

335.9

311

335-3

$11-2 \mathrm{M}$

$11.2 \mathrm{G}$

671

439

478

593

15

2017

129

756

855

856

113.2

362

335-8

180

84

43

1464

365

1112

149-2

235

170

39

881

228

1966

2437

1106

2405

1398

2445

947

383

626

641

2045

2048

642

302

760

1849

1897

2097

68-2-11

2290

2289

2584

2585

2587

1092

1090

1092

2126

773

1080

1091 
calculating Mar.hines (1969)

Rer. for ectangular or Square Parallel Keys and Their Corresponding aper Keys with or Without Gib Ilead and Their Corresponding al Rec. for Cylindrical Weights from l Gramme (Gram) to 10 International Rec. for Rectangular Weights from 5 to 50 Rec. for Determination of the Gray Rec. for Oil of Peppermint: France, Italy, United Rec. for Semitrailer (Trailer) 5Th Whee Rec. for Determination of Nitrogen in Coal by the c. for Determination of Nitrogen in Coal by the Semi Micro eneral Directions for the Determination of Nitrogen by the rical Properties of Microwave Tubes and Valves: High Power erties of Microwave Tubes and Valves: Low Power Oscillator Rec. for

Rec. for Combine Harvesters - Width of Cut and Number of Rec. for Sectional Beams for Warp Fishing Nets-Meth. of Determining the Breaking Load and Std for Hand Made Carpets: Determination of Types of Wheven Ground (1972) Std. for Hand Rec. for Requirements for Describing and Designating Rer. for Cuttin

std. for Surface Active Agents-Preparation of Water with Rec for Tea-Preparation of Ground Sample of Std. for Natural Rubber Latex-Determination of e (1969) Rec. for Magnetic Tape Rec. for Essential Oils: Rec. for Informative Rec. for Porcelain Rec. for Rec. for

ansmission (1960)

Rec. for Meth. for Thermal Shock Tests on Rec. for Field and
Std. for Leather inciples of Construction and Adjustment of I,iquid in Glass Rec. for Spec. for Bleached Rec. for Pilot (1967) Rec. for Dobby glass Fibre (Fiber) Reinforced, Resin Bonded, Low Pressure Rec. for Plastics-Basis for Spec. for Industrial Spec. for Fluorescen Std. Meth. of Measurement of rol of Interchangeability and Safety of Lampcap/ rol of Interchangeability and Safety: Lampholde/ rol of Interchangeability and Safety: Gauges (1/ Rec. for Rec. for Rec. for Std. for Petroleum Products: Determination of Sulphur

to Colour (Color) Change Upon Exposure to Light of a Xenon gauges for the Control of Interchangeability and Safety o lamps (I972)

Rec. for Sheets for Barrel Thread for E14 and E2? Rec. for Edison Screw Spec. for Edison Screw

h Gauges for the Control of Interchangeability and Safety: Std. for Photography-Expendable Photoflas Rec. for Miscellaneou Std. for Sealed Beam Landin

irection of Motion of Operaling Devices and for Indicating Spec. for Lighting Fittings for Incandescent Rec. for Tubular Fluorescen Rec. for Tungsten Filamen Rec. for Schedule for Sodium Rec, for Ballasts for Fluorescen Rec. for Glow Starters for Tubular Fluorescent Rec. for Lighting. Fittings for Tubular Fluorescent rec. for Schedule for High Pressure Mercury Vapour (Vapor) rec. for Ballasts for High Pressure Mercury Vapour (Vapor) Rec. for Projector and Floodlighting for Lampholders and Starterholders for Tubular Fluorescen $r$ Pipes Conveying Fluids in Liquid or Gaseous Condition in tive Materials (1967)

Rec. or Power Supply for Air and Std. for Sealed Beam Rec. for Symbols for Std. for Programming Std. for Programming Std. for Programming Rec. for Time

Rec. for Raw-Rubber and Rubber Std. for Natural Rubber Std. for Natural Rubber

Rec. for Raw-Rubber and Rubber Std. for Natural Rubber Rec. for Raw Natural Rubber and Its Rec. for Detcrminstion of Total Solids of

Rec. for Detcrmination of Alkalinity of Rec for Determination of Velatile Fatty Acid Number of Preparation of Dry Films from Concentrated Natural Rubber
Keytop and Printed or Displayed Symbuls for Adding and Keyways (Dimensions in Millimetres) (Millimeters) (1969 Keyways (Dimensions in Millimetres) (Millimeters) (1969 Kilogrammes (Medium Accuracy Class) (1970)

Kilogrammes (Medium Accuracy Class) (1972)

King Coke Type of Coal (1966)

Kingdom and U. S. A. (1968)

Kingpin (1963)

Kjeldahl Meth. (1963)

KjeIdahl Meth. (1963)

Kjeldahl Meth. (1971)

Klystrons (1972)

Klystrons (1972)

Knife Sections of Mow

Knife Sections (1970)

Knitting Machines: Terminology and Dimensions (1969)

Knot Breaking Load of Netting Yarns for Fishing Nets (1

Knots (1972)

Knotted Carpcts: Determination of Tuft Leg Length Above

Knotted Netting for Fishing Nets (1970)

Knotted Netting to Shape (Tapering) (1970)

Known Calcium Hardness (1972)

Known Dry Matter Content (1970)

KOH Number (1972)

Labelling and File Structure for Information Interchang

Labelling and Marking Containers (1961)

Labelling (1965)

Laboratory Apparatus-Quality and Meth. of Test (1970)

Laboratory Boiling FIasks (1970)

Laboratory Crucibles (Porcelain and Silica) (1970)

Laboratory Glassware (1968)

Laboratory Measurements of Airborne and Impact Sound Tr

Laboratory Samples-Location and Identification (1972)

Laboratory Thermometers (1964)

Lac (Shellac) (1957)

Ladders (1968)

Lags and Pegs in Wood, Metal or Other Suitable Material

Laminated Plates or Panels for Test Purposes (1970)

Laminated Sheets Based on Thermosetting Resins (1971)

Lamp Auxiliaries (1968)

Lamp Cap Temperature Rise (1971)

Lamp Caps and Holders Together with Gauges for the Cont

Lamp Caps and Holders Together with Gauges for the Cont

Lamp Caps and Holders Together with Gauges for the Cont

Lamp Meth. (1973)

Lamp (1968)

/ Determination of Resistance of Plastics / Lamp Caps and Holders Together with

Lampholders and Starterholders for Tubular Fluorescent

Lampholders with Shade Holder Ring (1972)

Lam pholders (1967)

Lampholders (1969)

Lampholders (1969)

Lamps-Determination Caps and Hold

Lamps and Ballasts (1968)

Lamps for Aircraft-Dimensions (1972)

Lamps for Circuit Breakers (1936)

Lamps for Domestic and Similar Purposes (1968)

Lamps for General Lighting Service (1961)

Lamps for General Service (1961)

Lamps (Integral Type) (1965)

Lamps (1962)

Lamps (1963)

Lamps (1965)

Lamps (1965)

Lamps (1969)

Lamps (1971)

Lamps (1972)

Land Installations and on Board Ships (1966)

IEC

Landing Lamps for Aircraft-Dimensions (1972)

Languages, Countries and Authorities (1967)

Language: ALGOL (1972)

Language: COBOL (1972)

Language: FORTRAN (1972)

Lapse Between Vulcanization and Test. of Rubbers (1970)

Latex-Determination of Copper (1971)

Latex-Determination of Dry Rubber Content (1972)

Latex-Determination of KOH Number (1972)

Latex-Determination of Manganese (1971)

Latex-Determination of Mechanical Stability (1972)

Latex-Determination of Nitrogen (1971)

Latex (1966)

Latex (1966)

Latex (1966)

Latex (1966)

Latex (1968)
ISO

ISO

ISO

OIML

ISO $\quad 502$

ISO $\quad 856$

337

Re ISO

333

1871

$235 \cdot 6$

$235-5$

563

1168

1025

1805

2550

2549

1530

1532

2174

1572

127

1001

211

436

1775

1773

1772

718

140

2418

386

799

573

1268

1642

12

360

61.1

61-2

61.3

2192

879

61.1

400 
Rec. for Meth. for the Determination of Density of Latex (1968)

Rec. for Determination of Coagulum Content of I,atex (1968)

Rec. for Meth. for the Determination of $\mathrm{pH}$ of Latex (1969)

Rec. for Determination of Boric Acid in Latex (1970)

Rec. for Lathe Centres (Centers): Centre Angle (1969)

1963)

Rec. for Lathe Centres (Centers): Sizes for Interchangeability (

Rec. for Lathe Tool Posts (Overall Internal Height) (1961)

Rec. for Test Conditions for General Purpose Parallel Lathes-Test. of the Accuracy (1970)

ity (1971)

idual Styrene (1972)

Std. for Synthetic Rubber

Rec. for Synthetic Rubbe

Rec. for Natural Rubber

Std. for Styrene Butadiene Rubber Rec. for Rubbers an

Rec for Detion of Surface Tension of Rubber

on of Dry Polymer from Butadiene Homopolymer and Copolymer

pec. (1971)

Rec. for Natural Rubber

Rec. for Natural Rubber

international System for the Transliteration of Greek into

Surface Active Agents: Verification of Certain Effects of nation of Dimensional Change in Woven Fabrics Subjected to Rec. for Std.

Rec. for Symbols, Dimensions and

$\begin{array}{ll}\text { Rec. for Layout of Multilingual Classified Vocabularies (1969) } & \text { ISO } \\ \text { Rens and } & \text { ISO }\end{array}$

Rec. ior Layour of Periodicals (1954)

69)

terminals (1963)

965)

eth. of Test (1972)

Rec. for Layout of Printing and Function Keys on Typewriters (19 ISO

Latices-Determination of High Speed Mechanical Stabil ISO Latices-Determination of Sludge Content (1971) ISO

Uetermination of Volatile Unsaturates and Res

Latices (1970)

Latices (1970)

Rec. for Preparati ISO

Latices, Centrifuged and Creamed, Ammonia Preserved-S ISO

Latices, Evaporated Preserved-Spec. (1971)

Latin Characters (1968)

Latering-Preparation and Use of Unsoiled Cotton Con

Laundering Near the Boiling Point (1968) I for Determi ISO

ayout for Meth. of Analysis of Essential Oils (1962) ISO

ISO

Rec. for Lead Acid Starter Batteries: Dimensions and Marking of

Rec. for Lead Acid Starter Batteries: Dimensions of Batteries (1

Rec. for Lead Acid Starter Batieries: General Requirements and M

Rec. for Lead Acid Traction Batteries (1967)

al Analysis of Zinc Alloys: Polarographic Determination of Lead and Cadmium in That Containing Copper (1972)

ChemicaI Analysis of Zinc: Polarographic Determination of Lead and Cadmium (1968)

Lead (1963)

Rec. for Red Lead (1966)
Rec. for White Lead (1966)

Rec. for Red Lead (1966)
Rec. for White Lead (1966)

Chemical Analysis of Zinc: Polarographic Determination of Lead (1968)

(1965)

Leakage Circuit Breakers for Domestic and Similar Purpo

Rec for ISO

Rec. for ISO

Rec. for Meth. ISO

ISO

Rec, for ISO

ISO

Spect for $\mathrm{V}(\mathrm{a})$

ses $(1959)$

$s$ (1972)

on (1972)

Std. for Leather-Conditioning of Test Pieces for Physical Test

Std for Leather: Determination of Absorption of Water (1972)

Std. for Leather: Determination of Apparent Density (1972)

Std. for Leather: Laboratory Samples-Location and Identificati

Std. for Leather: Physical Test.-Measurement of Thickness (197

Rec for Title Leaves of a Book (1969)

Rec. for Dyeing and Finishing Machines: Definition of Left and Right Sides (1969)

ting Internal Combustion Engines-Definition of Right and Left Hand Single Bank Engines (1972)

Rec. for Definition of Side (Left or Right) of Spinning Machinery (1959)

Rec for Weaving Looms: Definition of Side (Left or Right) (1959)

accessories-Pirn and Cross Winders-Definition of Side (Left or Right) (1960)

ories-Weaving Preparatory Machines-Definition of Side (Left or Right) (1960

Rec. for Dimensions of Hot Rolled Steel Sections-Equal

rec. for Dimensions of Hot Rolled Steel Sections-Unequal

or Dimensions of Hot Rolled Steel Sections-Part I: Equal

Dimensions of Hot Rolled Steel Sections-Part 2: Unequal

Std. for Hand Knotted Carpets: Determination of Tuft International Rec for Vocabulary of

Rec. for ISO Conventional Typographical Character for ire (ISO Micro Test Object) for Ch/

Object) and Its Use in Photograph/

Rec. for Microcopies: Rec. for Microcopies: Rec. for Oil of

Std. for Hand Knotted Carpets: Determination of Tuft Leg

s-Determination of Number of Tufts And/or Loops Per Unit

69)

bers) (1962) Rec. for Meth. of Test for Wool Fibre (Fiber) Rec. for Determination of Fibre

Rec. for Std. Reference Temperature for Industrial

$r$ Meth. of Test for Wool Fibre Length Using a Single Fiber

Rec. for Determination of Fibre Length by Measuring the Rec for Meth. of Test for Wool Fibre

970)

-Dimensions, Tolerances and Conventional Masses Per Unit rubber Hose-Bore Sizes, Test Pressures and Tolerances on -Dimensions, Tolerances and Conventional Masses Per Unit seamless-General Table of Dimensions and Masses Per Unit

Packages-Determination of Linear Density (Mass Per Unit Rec. for Files and Rasps:

s for Welding, Supplied in Straight Lengths (19/ $n$ or Extruded, for Welding (1967)

Mild Steel and Low Alloy High Tensile Steel (1/

General Purpose Bolts (1968)

)(1967)

Rec. for

Rec. for

Rec. for

Rec. for Nominal Rec. for

Rec. for Widths and

Rec. for

Rec. for

Leg Angles-In. Series-Dimensions and Sectional Prop

Rec. for Textile Machinery and ISO
or Textile Machinery and Access ISO

Leg Angles-Metric Series-Dimensions and Sectional P

Leg Angles-Metric Series-Dimensions and Sectional P

Leg Length Above the Woven Ground (1972)

Legal Metrology (Fundamental Terms) (1969)

Legibility Tests (1965)

Legibility Tests, Description and Use of the ISO Microm

Legibility Tests; Description of the 1SO Mire (ISO Test

Exon, Expressed, Italy (1968)

Length Above the Woven Ground (1972)

Length and Area (1971)

Length Barbe (Barb) and Hautcur Using a Comb Sorter (19

政

Length Measurements (1951)

Length Measuring Machine (1970)

Rec. Fo ISO

Length of Individual Fibres (Fibers) (1962)

Length Using a Single Fiber Length Measuring Machine (1

Length (1969)

Length (1970)

Length (1971)

uring Machine (1 $\quad$ ISO

Length (1971)

Length) Skein Meth. (1972) Rec. for Boiler Tubes ISO
for Plain End Steel Tubes, Welded or ISO

Lengths and Cross Sections (1961)

/D. for Textiles: Yarn from ISO

Lengths and Tolerances for Drawn or Extruded Filler Rod

Lengths and Tolerances of Electrodes for the Welding of

Lengths for Bolts, Screws and Studs: Thread Lengths for

Lengths of Classical V-Belts (Sections Z, A, B, C, D, E

Lengths of Conveyor BeIts (1970)

Lengths of Flat Transmission Belts (1958)

Std. for Hand and Machine Hacksaw Blades: Dimensions for Lengths Up to $450 \mathrm{~mm}$ and Pitches Up to $6.3 \mathrm{~mm}$ (1972)

Lengths of Narrow V-Belts: Sections SPZ, SPA, SPB (1965

Lengths of Y.. Section V-Belts 
I Sockets Screwed in Accordance with ISO Rec R7. Minimum Lengths (1957) or Fixtruded Filler Rods for Welding, Supplied in Straight Lengths (1967) Rec. for

Microcircuits (1969)

(1971)

munications and Electronics (1972)
Connertion of Auxiliaries /

Rec. for Photography-Front Rec. for Rec. for Rec. for ostcards and of Rec. for Sound Rec for Precision Sound Rec. for Meth. for Calculating Loudness Rec. for Analogue Voltage Ranges and Logic

Rec. for Relation Between Sound Pressure in a Frontally / Rec. for Expression of the Power and Intensity Rec. for Shape, Size and Direction of Operation of Rec. for Performance Requirements for Rec. for Dimensions for Single Hole Mounting,

(1962)

Rec. for w Make and Break (1963)

Break (Toggle) (1969)

Rec. for Rec. for rs) (1972)

Std. for Directories of

ermination of Carbon and Hydrogen in Coal and Coke by the

for Aluminium (Aluminum) Electrolytic Capacitors for Long Rec. for

Grade 40 Calibrated Load Type for Pulley Blocks and Other Rec. for Stability of Counterbalanced Rec. for Counterbalanced Fork pacity-Basic Requirements (1972) Std. for Hooks for Std. for Std. for Nomenclature of

71)

lley Blocks and Other Lift/

in Slings, Etc. (1971) Rec. for Short Link Chain for Fyer Bobbins for Spindle Diameters of $25 \mathrm{~mm}$ or More and c. for Flyer Bobbins for Spindle Diameters Up to $22 \mathrm{~mm}$ and heating (1970) Rec. for Plastics-Determination of Rolled, Drawn and Extruded Products in Light Metals or in Rec. for Continuous Mechanical Handling Equipment isation of Aluminium and Its Alloys-Determination of the Rec, for Tensile Test, of

between $0.2 \mathrm{~mm}(0.008 \mathrm{In}$. Rec. for Simple Bend Test for Rec. for Drift Expanding Test on Rec. for Tensile Test for Rec. for Rec. for Rec. for Rec. for

1971)

1)

loads from 1 to $100 \mathrm{kgf})(1971)$

Rec for Tensile Test. of

etails-Marking of Rolled, Drawn and Extruded Products in ance of Plastics to Colour (Color) Change Upon Exposure to ance of Plastics to Colour (Color) Change Upon Exposure to raphy-Expendable Photoflash Lamps-Determination of the

hickness of Oxide Coatings-Nondestructive Measurement by ation of the Spectral Distributio/ ation of the Spectral Distribution/

ation of the Spectral Distributio/ Std. for Photography Std. for Photography: ectrical Equipment Particularly for Private Motor Cars and (1963) Rec. for Ball Couplings for Caravans and nd Similar Purposes (1968) Rec. for Spec. for Rec. for

Rec. for Tubular Fluorescent Lamps for General Rec. for International Electrotechnical Vocabulary: Rec. for Electrical Installations in Ships: Accessories, ctrical Installations in Ships: Transformers for Power and Rec. for ystems (1970) Rec. for

, Heating and Cooking Appliances, Internal Communications, t. of Toughened Glasses for Ships Side Scuttles and Fixed Colours (Colors) of Signal

ils-Toughened Glasses for Ships Side Scuttles and Fixed Residue by Low Temperature Distillation of Brown Coal and esinous Su/ Rec. for Benzene Extract from Brown Coals and Sodium and Potassium Soluble I/ Rec. for Brown Coals and Rec. for Determination of Moisture in Brown Coals and of the Yield of Benzene Soluble Extract in Brown Coals and Rec. for Meth. of Test for Temperature

Std for Formaldehyde Solutions for Industrial Use: Std for Formaldehyde Solutions for Industrial Use: Std. for Formaldehyde Solutions for Industrial Use: positions for Straight Manual Arc Welds Made Wi/ Rec. for Rec. for

Rec. for High Voltage Fuses: Current rec. for Plastics-Determination of Viscosity: Number and es (1971) ions $(1962)$
Rec for Ste ISO ISO

Lens Barrels Up to $100 \mathrm{~mm}$-Dimensions Important to the Letter Symbols for Semiconductor Devices and Integrated Letter Symbols to Be Used in Electrotechnology: General Letter Symbols to Be Used in Electrotechnology: Telecom Lettercards (1963)

Level Meters (1961)

Level Meters (1965)

Level (1966)

Levels for Mains Operated Nuclear Instruments (1970)

Levels of Narrow Bands of Noise in a Diffuse Field and

Levels of Sound or Noise (1963)

Lever Controls on Aircraft (1969)

Lever Operated Manual Switches for Aircraft (1970)

Lever Operated Switches for Aircraft (1966)

Lever Switches: General Requirements and Measuring Meth

Lever Switches: Requirements for Switches of Type 1, Sl

Lever Switches: Requirements for Type 2, Quick Make and

Libraries, Information and Documentation Centres (Cente

Liebig Meth. (1967)

Life (Type 1) and for General Purpose Application (Type

Lifeboats for Less Than One Hundred People (1963)

Lift Appliances (1971)

/K Chain for Lifting Purposes-

Lift Trucks-Basic Tests (1969)

Lift Trucks-Rated Capacity (1971)

Lifting Freight Containers of Up to 30 Tonnes (Tons) Ca

Lifting Hooks-General Characteristics (1972)

Lifting Hooks (1973)

Lifting Purposes-General Conditions of Acceptance (19

Lifting Purposes-Grade 40 Calibrated Load Type for Pu

Lifting Purposes-Grade 40 Noncalibrated Chain for Cha

Lifts of $300 \mathrm{~mm}$ and Over (1969)

Lifts Up to $300 \mathrm{~mm}$ (1963)

Light Absorption of Cellulose Acetate Before and After

Light Alloys (1960)

Light Duty Belt Conveyors for Loose Bulk Materials (197

Light Fastness of Colored Anodised Aluminum (1971)

Light Metal Ad Its Alloy Tubes (1969)

Light Metal and Its Alloy Sheet and Strip of Thickness

Light Metal and Its Alloy Tubes (1969)

Light Metal and Its Alloy Wires (1969)

Light Metals and Their Alloys-Brinell Hardness Test (

Light Metals and Their Alloys-Code of Designation (19

Light Metals and Their Alloys-Temper Designation (197

Light Metals and Their Alloys-Vickers Hardness Test (

Light Metals and Their Alloys (1961)

Light Metals or in Light Alloys (1960)

Light of a Xenon Lamp (1968)

Light of the Enclosed Carbon Arc (1968)

Light Output (1972)

Light Section Microscope (1971)

/Shipbuilding D ISO

/ Determination of Resist ISO /Ion of Resist ISO

Std. for Photog ISO

Ys-Measurement of T ISO

Light Sources for Use in Sensitometric Exposure-Simul ISO

Light Sources for Use in Sensitometric Exposure-Simul ISO

Light Sources for Use in Sensitometric Exposure-Simul ISO

Light Trailers or Caravans (1970) IS with 6 or $12 \mathrm{~V}$ EL ISO

Light Trailers (1969)

Lighting and Signalling for Motor Vehicles and Trailers

Lighting Fittings for Incandescent Lamps for Domestic a

Lighting Fittings for Tubular Fluorescent Lamps (1965)

Lighting Service (1961)

Lighting (1970)

Lighting, Accumulator (Storage), Batteries, Heating and

Lighting, Semiconductor Rectifiers, Generators (With as

Lightning Arresters: Expulsion Type (1962)

Lightning Arresters: Nonlinear Resistor Type for a.c. S

Lightning Conductors (1965)

Lights by the Punch Meth. (1967)

(a) , Batteries IEC

Lights on Ships (1972)

Lights (1969)

Lignite (1968)

Uilding Details: Tes 1SO

Rec. for Shipbuilding Deta ISO the Yields of Tar, Water, Gas and Coke ISO

ef Acetone Soluble Material ( $R$

eth. of Extraction for the Determination of

Lignites by the Direct Volumetric Meth. (1969) ISO

Lignites (1969)

Limit of Brittleness for Vulcanized Rubbers (1968)

Limit Test for Heavy Metals (Excluding Iron) (1972)

Limit Test for Inorganic Chlorides (1972)

Limit Test for Inorganic Sulphates (1972)

Limitation of Angles of Slope and Rotation for Welding

Limiting Values for the Adjustment of Centres (Centers)

Limiting (1965)

Limiting-Number of Polyethylenes and Polypropylenes in

Limits and Fits-Part II: Inspection of Plain Workpiec

Limits and Fits-Part I: General Tolerances and Deviat 
ling Bearings: Tolerances; Metric Series Chamfer Dimension Rec. for Mechanical Property Rec. for Mechanical Property d Its Alloys (1971) c. for Carbon Black for Use in the Rubber Industry-Spec. alloys (1968) its Alloys (1968) Rec. for Mechanical Property Rec. for Mechanical Property its $(1967)$

for ISO General Purpose Metric Screw Threads: Tolerances, Limits of Radio Interference and Report of National bearings-Tapered Roller-In. Series-Chamfer Dimension Rec. for Rotating Electrical Machines: Noise signs: to Be Used in Schemes for the Installations of Pipe Rec. for

Rec. for Low Voltage Motor Starters: Direct on Rec. for Inscription of Rec. for Textiles Fibres (Fibers)-Determination of bre (Fiber/ Rec. for Textile Glass-Determination of the Std. for Textiles: Yarn from Packages-Determination of Rec. for Textiles-Universal System for Designating Std. for Rigid Cellular Plastics-Determination of igerated Cabinets-Meth. of Test-Part I: Calculation of sistance $(1972)$ Std. for tures (1972) Std. for Std. for Rec. Graphical Symbols: Telecommunication Rec. for Pressures in Brake ec. I Rec. for General Purpose Rigid Coaxial Transmission equi/ Rec. for General Purpose Rigid Coaxial Transmission rec. for Shipbuilding Details for Inland Navigation-Pipe c. Graphical Symbols: Generating Stations and Substations, (1970) quipment of Machine Tools: Used in Large Series Production ts on Insulators of Ceramic Material or Glass for Overhead I Iron Pipes: Special Castings and Parts for Pressure Main

nt of Machine Tools: Used in Large Series Production Lines Acceptance (1971)

oad Type for Pulley Blocks and Other Lift/ Rec. for Short Rec. for Short Rec. for Short Rec. for High Tensile Steel Chains (Round of Mounted Implements (1968)

Rec. for 3 Point Rec. for Precision Fuse Rec. for Precision Fuse Rec. for Precision Fuse Rec. for Std. Rated Currents (2 to 63A) of Fuse Spec. for Cartridge Fuse Rec. for Cartridge Fuse ckle/ Rec. for Shipbuilding Details-Anchor Chains, Stud Navigation and Inland Navigation Anchor Chains-Studless Rec. for Fuse Holders for Miniature Cartridge Fuse Rec. for Raw, Refined and Boiled Rec. for

phenols Content-Bromination Meth. (1972) Std. for ls for Industrial Use-Determination of Densit/ Rec. for ter Content Using an Electrolytic Analyser (1972) Std. for he Content by Volume in the Vaporized Product (/ ater Content-Gravimetric Meth. (1972)

(For Determining Only the Volumetric Content) (/ tion of Data for Determination of Errors in Measurement of $t$ and Operation of a Gauging Station and Determ/ meth. (1969)

ea Meth. (1968)

for Steady Flow-Part 1: Constant Rate Injec/ termination of the Acidity (1970)

termination of the Cloud Point (1970)

termination of Residue on Evaporation (1972) ntification Colours (Colors) for Pipes Conveying Fluids in Rec. for Fixed Tantalum Capacitors with terchangeability Dimensions (1971) r Meth. (1970) Rec. for d Navigation-Pipe Lines for the Transport of Combustible ec. Test Cells for Measuring the Resistivity of Insulating Rec. for Meth. for the Determination of Density of International Rec. for Meters for 0)

Appliances for Domestic and Similar Purposes: for Heating for Vulcanized Rubbers-Meth. of Test for Resistance to ient (For Use in the Preparation of Measurement Tables for of Electronic Equipment and the Co/ Std. for Dental Vocabulary or Belts According to Their Use (1969) (English, French, Russian) (1969)

(English, French, Russian) (Cs: Corresponding C/ (English, French, Russian) (D: Corresponding Ge/ (English, French, Russian) (NL: Corresponding D/ (English, French, Russian) (Pl: Corresponding P/ imits and Maximum Shaft and Housing Fillet Radius (197 Limits for Aluminium Alloy Forgings (1968)

Limits for Extruded Products of Aluminium (Aluminum) an Limits for Loss on Heating (1970)

Limits for Rivet Stock of Aluminium (Aluminum) and Its

Limits for Rolled Products of Aluminium (Aluminum) and

Limits of Radio Interference and Report of National Lim

Limits of Sizes for Commercial Bolt and Nut Threads: Me

Limits (1967)

Limits (1969)

Limits (1972)

Line Systems in Ships (1967)

Rec. for Rolling

Line Traps (1971)

Line, a.c. (1971)

Linear and Angular Tolerances (1964)

Rec. for Conventional ISO

Linear Density-Gravimetric Meth. (1971)

Linear Density of Its Continuous Filament and Staple Fi

Linear Density (Mass Per Unit Length) Skein Meth. (1972

Linear Density (Tex System) (1969)

Linear Dimensions (1972)

Linear Dimensions, A reas and Volumes (1971)

Lined Conducting Rubber Footwear (1972)

Lined Industrial Rubber Boots with General Purpose Oil

Lined Industrial Rubber Footwear for Use at Low Tempera

Lines and Accessories (1971)

Lines and Braking Efficiency (1970)

Lines and Their Associated Flange Connectors: Detail Sp

Lines and Their Associated Flange Connectors: General R

Lines for the Transport of Combustible Liquids-Nomina

Lines for Transmission and Distribution (1963)

Lines Lines (1967)

Lines with a Nominal Voltage Greater Than $1000 \mathrm{~V}(1972)$

Lines (1955)

Lines (1967)

Rec. for Electrical Equipme

Link Chain for Lifting Purposes-General Conditions of

Link Chain for Lifting Purposes-Grade 40 Calibrated L

Link Chain for Lifting Purposes-Grade 40 Noncalibrate

Link) for Chain Conveyors and Coal Ploughs (1967)

Linkage of Agricultural Wheeled Tractors for Attachment

Links for Aircraft-General Requirements (1971)

Links for Aircraft (Type A) (1971)

Links for Aircraft (Type B) (1971)

Links for Low Voltage Fuses (1957)

Links for Miniature Fuses (1962)

Links for Miniature Fuses (1962)

Links (Common, Enlarged, and End Types, and Joining Sha

Links (1957)

Links (1968)

Linseed Oil (1960)

Linseed Stand Oils (1962)

Liquefied Phenol for Industrial Use-Determination of

Liquefied Phenol: Meta Cresol, Cresylic Acid and Xyleno

Liquid Chlorine for Industrial Use-Determination of

Liquid Chlorine for Industrial Use-Determination of T

Liquid Chlorine for Industrial Use-Determination of W

Liquid Chlorine for Industrial Use-Meth. of Sampling

Liquid Flow by Velocity Area Meth. (1969)

$1 \mathrm{SO}$

列

Liquid Flow Measurement in Open Channels by Slope Area ISO

Liquid Flow Measurement in Open Channels by Velocity Ar

Liquid Flow Measurement in Open Channels: Dilution Meth ISO

Liquid Flow with a Free Surface (1968) /Cabulary of Te ISO

Liquid Halogenated Hydrocarbons for Industrial Use-De

Liquid Halogenated Hydrocarbons for Industrial Use-Dc ISO

Liquid Halogenated Hydrocarbons for Industrial Use-De

Liquid in Glass Laboratory Thermometers (1964)

Liquid or Gaseous Condition in Land Installations and $\mathrm{O}$

Liquid or Solid Electrolyte (1971)

Liquid Oxygen Replenishment Couplings for Aircraft-In

Liquid Resins-Measurement of Density by the Pyknomete

Liquids-Nominal Diameters (1957)

Liquids and Meth. of Cleaning (1967)

/ Details for Inlan

Liquids at $20^{\circ} \mathrm{C}(1968)$

Liquids (Other Than Water) with Measuring Chambers (197 OIM

Liquids (1966) /Spec. for Electric Cooking and Heating CEE

Liquids (1971)

Liquids)

List I-Basic Terms (1972)

$$
\text { Rec }
$$
/Lue for the Thermal Expansion Coeffic

List of Basic Terms and Definitions for the Reliability Rec. for List of Characteristics Which May Be Required of Convey Rec. for List of Equivalent Terms Used in the Plastics Industry Rec. for List of Equivalent Terms Used in the Plastics Industry Rec. for List of Equivalent Terms Used in the Plastics Industry Rec. for List of Equivalent Terms Used in the Plastics lndustry Rec. for List of Equivalent Terms Used in the Plastics Industry 
Sid. for Sodium llydrogen Carbonate for Industrial Use Rec. for Short Contents Rec. for Steel Tubes for Gas ints Are Made on the Threa/ Rec. for Pipe Threads for Gas r Pesticides Considered Not to Require Common Names: First pesticides Considered Not to Require Common Names-Second ure Tones and Normal Threshold of Hearing Under Free Field

Rec. for

Rec for Definitions of

Yarn from Packages-Meth. for Determination of Breaking Rec. for Fishing Nets-Meth. of Determining the Breaking interrupted Creep Test. of Steel at Elevated Temperatures Steel $(1960)$

Std. for Industrial Wheels-Dimensions and Nominal Std. for Automobiles

Iculation of the Continuous Current Rating of Cables (100\% Rec. for Axial

Meth. of Determining the Breaking Load and Knot Breaking for Fishing Nets-Meth. of Determining the Mesh Breaking for Ball and Roller Bearings: Meth. of Evaluating Static

Rec. for Rolling Bearings-Meth. of Evaluating Dynamic Rec for on

hort Link Chain for Lifting Purposes-Grade 40 Calibrated hanical Properties of Fasteners: Nuts with Specified Proof hanical Properties of Fasteners: Nuts with Specified Proof Rec. for Vickers Hardness Test for Steel efractory Products-Determination of Refractoriness Under lastics-Determination of Temperature of Deflection Under ion. General, Boundary Dimensions, Tolerances, Permissible Rec. for Meth. of Sampling Manganese Ores; Part I: Ore ulation of Rectangular Symmetrical Fillet Welds Statically Rec. for Weft Pirns for Box Rec. for Daylight

Rec, for Cinematography-Dimensions of Day Rec. for Cinematography-Dimensions of Daylight Coverings-Determination of Thickness Loss Under Dynamic nical Handling Equipment for Loose Bulk Materials and Unit std. for Continuous Mechanical Handling Equipment for Unit rec. for Continuous Mechanical Handling Equipment for Unit rec. for Continuous Mechanical Handling Equipment for Unit rec. for Continuous Mechanical Handling Equipment for Unit std. for Continuous Mechanical Handling Equipment for Unit for Light Metals and Their Alloys-Vickers Hardness Test for Vickers Hardness Test for Copper and Its Alloys (Test nsions of Straight Resistance Spot Welding Electrodes (For Rec. for Rolling Bearings -With Std, for Rolling Bearings: with g Heads for 6 Track Magnetic Sound Records on $35 \mathrm{~mm}$ Film Std. for Leather: Laboratory Samples Rec. for Roll Film Cameras-Back Window or Cinematography: $8 \mathrm{~mm}$ Motion-Picture Film with Picture ter) Sound Records on $16 \mathrm{~mm}$ Perforated Magnetic/ Rec. for Records on $16 \mathrm{~mm}$ Perforated Magnetic Film (196/ Rec. for ords on 35 mm Film (1963) Rec. for Cinematography cords on $35 \mathrm{~mm}$ Film and 1 Magnetic Sound Record/ Rec. for ched Paper Cards (Information Pr/ Rec. for Dimensions and Type S on $16 \mathrm{~mm}$ Motion Pictur/ Rec. for Dimensions and Std. for Cinematography -
Rec. for Insulator Units: Dimensions and General Requir Type Steel Hexagon inner Tab (1972) Std. for Rolling Bearings: Std. for Prevailing Torque Type Steel Hexagon Std. for Prevailing Torque Type Hexagon

Std. for Rolling Bearings: Locknuts, Narrow Series, and Rec. for Aircraft

70) Rec. for Graphical Symbols: Binary Rec. for Analogue Voltage Ranges and Rec, for Hand and Drilling-Extension Drill Steel Equipment for Percussive Drilling-Extension Drill Steel Equipment for Percussive Drilling-Extension Drill Steel Equipment for Percussive

Drilling-Extension Drill Steel Equipment for Percussive Rec. for Aluminium (Aluminum) Electrolytic Capacitors for Rec. for Parallel Shank Twist Drills

a with Parallel Shanka with Morse Taper, Shanks-Std. and Std. for End Mills with 7/24 Taper Shanks-Std. and $4 \mathrm{~mm}$ and $1 / 8$ to $1 \mathrm{In}$. (1972) Std. for Rec. for

sagea (1969) Rec. for the Use of Rec. for Weft Pirns for Box Loaders for Automatic Rec. for Weft Pirns for Automatic Rec, for Single Box Pickers for Automatic Rec. for Shuttles for Pirn Changing Automatic Rec. for Std. Working Widths of Weaving Rec. for Weavin trying Roda-Dimensions and Coordination Of, and with End
List of Meth. of Test and Preparation of the Sample (19

list of Periodicals or Other Documents (1955)

list Threading (1971)

List Tubes and Screwed Fittings Where Pressure Tight Jo

List (1968)

List (1969)

Listening Conditions (1961)

\section{Rec. Fo \\ IL Loudness Contours for P}

ISO

Lithopone (1966)

Living Animals for Slaughter-Porcines (1970)

Load and Elongation of Same (Of Single Strands) (1972)

Load and Knot Breaking Load of Netting Yarns for Fishin

Load and Temperature Interrupted) (1961)

Load Calibration of Test. Machines for Tensile Test. of

Load Distribution for Private Cars (1972)

Load Factor) (1971)

Load Fatigue Test. (1969)

Load of Netting Yarns for Fishing Nets (1970)

Load of Netting (1970)

Load Ratings (1958)

Load Ratings (1962)

Load Tap Changers (1966)

Load Type for Pulley Blocks and Other Lift Appliances (

Load Values (1969) Rec. for Mec ISO

Load Values, Effective Heights of Thread $0.4 \mathrm{D}$ to Less ISO

Load 5 to $100 \mathrm{kgf}$ ) (1967)

Load (Differential-With Rising Temperature) (1970)

Load (1958)

Load (1969)

Lagons in Freight $\mathbb{W}$ agon

Loaded in Such a Way That the Transverse Section Is Not

Loaders for Automatic Looms-Dimensions of Pirn Tip (1

Loading Guide for Oil Immersed Transformers (1972)

Loading Spools for Double $8 \mathrm{~mm}$ Motion Picture Film (196

Loading Spools for $16 \mathrm{~mm}$ Motion Picture Film (1969)

Loading (1971)

Rec. for T

Loads-Belt Conveyors-Basic Characteristics of Motor

Loads-Mobile Belt Conveyors (Canvas, Rubber, Plastic,

Loads-Overhead Monorail Chain Conveyors-Safety Code

Loads-Overhead Twin Rail Chain Conveyors (Power and F

Loads-Single Strand Floor Mounted Truck Conveyors (Ch

Loads-Single Strand Floor Truck Conveyors (Chain Belo

Loads from 1 to $100 \mathrm{k} \mathrm{of}$ (1971)

Loads from 2.5 to $50 \mathrm{kgf}$ (1964)

Loads Greater Than 1500 kgf (1969)

Locating Snap Ring-Dimensions (1965)

Locating Snap Ring-Inner Diameter of (1972)

Location and Dimensions (1971)

Location and Identification (1972)

ISO

ec. for $\mathrm{P}$ ISO

ISO

Iso

ISO

ISO

ISO

ISO

ISO

ISO

ISO

ISO

ISO

Rec. ISO

Rec. ISO

Rec. for Dime ISO

ISO

ISO

Location and Picture Sizes (1971)

Location and Width of Magnetic-Striping and Gaps of Rec

Location and Width of the Recoding Head for Centre (Cen

Location and Width of the Recording Head for Edge Sound

Location of Recording Heads for Four Magnetic Sound Rec

Location of Recording Heads for Three Magnetic Sound Re

Location of Rectangular Punched Holes in 80 Columns Pun

Location of the Printed Image Area for Printing to $8 \mathrm{~mm}$

Locking Devices for Ball and Socket Couplings of String

Locknuts-Mechanical and Performance Properties (1972)

Locknuts, Narrow Seriea, and Lockwashers with Straight

Locknuts: Dimensions-In. Series (1972)

Locknuts: Dimensions-Metric Series (1972)

Lockwashers with Straight Inner Tab (1972)

Lock wire (1962)

Logic Elements (1972)

Logic Levels for Mains Operated Nuclear Instruments (19

Long Enclosed Scale Thermometers for Precision Use (196

Long Fluted Machine Reamers, Morse Taper Shank (1961)

Long Hole Drilling-Reverse Buttress Threaded Equipmen

Long Hole Drilling-Reverse Buttress Threaded Equipmen

Long Hole Drilling-Rope Threaded Equipments $11 / 2$ to

Long Hole Drilling-Rope Threaded Equipments $7 / 8$ to 1

Long Life (Type 1) and for General Purpose Application

Long Series (1966)

Long Series (1970)

Rec. for End Mill

Long Shank Machine Taps with Nominal Diameters from 32

Long Solid Stem Thermometers for Precision Uae (1968)

Longitudinal Parity to Detect Errors in Information Mes

Looms-Dimensions of Pirn Tip (1969)

Looms (1960)

Looms (1961)

Looms (1967)

Looms (1968)

Looms: Definition of Side (Left or Right) (1959)

Rec. for Heald $\mathrm{Ca}$ ISO
2197

7

765
1105

226

1840

2062

1805

203

147

2175

2416

287

1099

1805

1806

76

281

214.

1836

898/II

81

1893

75

1002

617

1131

354

1020

1019

2094

1816

2388

2149
2150

2196

2381

192

399

1045

2265

1753

2418

1203

1201

890

891

360

162

1682

1785

372-1

2982

2359
2358

2982

245

117.15

323

655

236

1721

1722

1720

1719

103

494

1641

2324

2283

653

1155

1131

143

227

572

109

108
570 
rer. for C.arpets-Determination of Number of Tufts And/or Sid. for Continuous Mechanical Handling Equipment for Std. for Continuous Mechanical llandling Equipment for Rec. for Continuous Mechanical Handling Equipment for Rec. for Continuous Mechanical Handling Equipment for Std. for Continuous Mechanical Handling Equipment for Std. for Continuous Mechanical Handling Equipment for Rec. for Continuous Mechanical Handling Equipment for Rec. for Continuous Mechanical Handling Equipment for Std. for Continuous Mechanical Handling Equipment for Std. for Pneumatic Handling Appliances for Std. for Continuous MechanicaI Handling Equipment for Rec. for Continuous Mechanical Handling Equipment for Rec. for Continuous Mechanical Handling Equipment for Rec. for Continuous Mechanical Handling Equipment for Rec. for Continuous Mechanical Handling Equipment for Rec. for Continuous MechanicaI Handling Equipment for Rec. for Continuous Mechanical Handling Equipment for Rec. for Continuous Mechanical Handling Equipment for

hanical Handling Equipment-Light Duty Belt Conveyors for Rec. for Continuous Mechanical Handling Equipment for Rec. for Continuous Mechanical Handling Equipment for crude Sodium Borates for Industrial Use-Determination of Rec. for Tea-Determination of of Aluminium (Aluminum) and Its Alloys-Estimation of the for Sodium Carbonate for Industrial Use: Determination of rimarily Used for Production of Aluminum: Determination of

r Sodium Chloride for Industrial Use: Determination of the xide Primarily Used for the Production of Determination of bon Meth. (196I/ Rec. for Plastics-Determination of the (I969)

1970) on Black for Use in the Rubber Industry-Spec. Limits for Rec. for Plastics-Determination of the sodium Pyrophosphate for Industrial Use-Determination of Use-Determination of Sodium and Boric Oxide Content and Rec. for Plastics-Determination of Viscosity

for Textile Floor Coverings-Determination of Thickness

c. for Rotating Electrical Machines: Meth. for Determining Rec. for Pulps: Determination of Saleable Mass, in Rec. for Normal Equal Rec. for Meth. for Calculating f Hearing Under Free Field Listeni/ use Field and in a Frontally Incident Free Field for Equal
Rec. for the Rated Impedances and Dimensions of Rec. for the Rated Impedances and Dimensions of Rec. for Sound System Equipment: rocedures for Electronic Components and Equipment: Test M: tolerances of Electrodes for the Welding of Mild Steel and

Manual Arc Welds Made with Covered Electrodes of Mild and symbols for Filler Rods for Gas Welding of Mild Steels and for Covered Electrodes for Arc Welding of Mild Steels and 1 Deposited by Filler Rods for Gas Welding Mild Steels and for Covered Electrodes for Manual Arc Welding of Mild and urface Active Agents: Analysis of Soaps-Determination of rec. for Formic Acid for Industrial Use-Determination of d Its Alloys-Spectrophotometric Determination of Nickel Rec. for Rotary Wafer Switches

2 Hole Mounting; Maximum 26 Positions and Diameter $60 \mathrm{~mm}$ Meth. (I962) ng (I963) Rec. for Rotary Wafer Switches Rec. for Rotary Wafer Switches ride) Insulation and Sheath: General Test and $M$ ride) Insulation and Sheath: Cables in Pairs, T/ ride) Insulation and Sheath: Equipment Wires, T/ ride) Insulation and Sheath: Distribution Wires/ ride) Insulation and Sheath: Equipment Wires An/ ride) Insulation and Sheath: Signalling Cables / ride) Insulation and Sheath: Distribution Wires/ lours (Colors) for Pvc (Polyvinyl Chloride) Insulation for ion of Resistance of Plain and Tinned Copper Conductors of ec. for Determination of Hardness of Vulcanized Rubbers of s: Selection of Meth. of Test and General Requirements for $f$ the Electrical Properties of Microwave Tubes and Valves: eparation of Glass Fibre (Fiber) Reinforced, Resin Bonded, food/

for Analysis of Soaps: Determination of Foreign Matter of Rec. for Household Refrigerators-Part II: Special ation of the Yields of Tar, Water, Gas and Coke Residue by or Determination of the Stiffness of Vulcanized Rubbers at

ion Set of Vulcanized Rubbers Under Constant Deflection at Std for Lined Industrial Rubber Footwear for Use at airbreak Switch Disconnectors and Fuse Combinat/ Rec. for (I971)

I964)

trial and Similar Purposes: General Requirement/ Reo. for Std. Rated Currents (2 to 63A) of Fuse Lir Rec. for Rec. for

Rec. for Std. Rated Currents (2 to 63A) of Fuse Links for Rec. for
Rec. for I970) equencies Below $3 \mathrm{MHz}$ : Connectors for Coupling an External loops Per Unit Length and Area (1971)

cose Bulk Materials-Aeroslides (1972)

Loose Bulk Materials-Apron Conveyors-Safety Code (1

Loose Bulk Materials-Apron Conveyors (1971)

Loose Bulk Materials-Belt Feeders and Conveyors-Saf

Loose Bulk Materials-Hydraulic Conveyors-Safety Cod

Loose Bulk Materials-Mobile BeIt Conveyors-Safety C

Loose Bulk Materials-Oscillating Conveyors and Shakin

Loose Bulk Materials-Oscillating Conveyors and Shakin

Loose Bulk Materials-Picking Table Conveyors-Safety

Loose Bulk Materials-Piping (1972)

Loose Bulk Materials-Scraper and En Masse Conveyors

Loose Bulk Materials-Storage Equipment: Bins and Bunk

Loose Bulk Materials-Troughed Belt Convevors (Other T

Loose Bulk Materials-Troughed Belt Conveyors (Other T

Loose Bulk Materials-Troughed Belt Conveyors (Other T

Loose Bulk Materials-Vibrating Feeders and Conveyors

Loose Bulk Materials-Vibrating Feeders and Conveyors,

Loose Bulk Materials and Unit Loads-Belt Conveyors-

Loose Bulk Materials (197I)

Rec for

Loose Bulk Materials: Screw Conveyors (1969)

Loose Bulk Materials: Vibrating Conveyors and Feeders W

Loss in Mass After Heating at $900{ }^{\circ} \mathrm{C}(1972)$ / for ISO

Loss in Mass at $103^{\circ} \mathrm{C}(1970)$

Loss of Absorptive Power by Colorant Drop Test with Pri

Loss of Mass and of Nonvolatile Matter at $250{ }^{\circ} \mathrm{C}$ (1

Loss of Mass at $1000^{\circ} \mathrm{C}$ and $1200^{\circ} \mathrm{C}(1968)$

Loss of Mass at $110^{\circ} \mathrm{C}(1973)$

Loss of Mass at $300^{\circ} \mathrm{C}$. (Conventional Moisture) (196

Loss of Plasticizers from Plastics by the Activated Car

Loss on Heating of Carbon Black for the Rubber Industry

Loss on Heating (1970)

Loss on Ignition of Textile Glass Reinforced Plastics (

Tetraborates for

Loss on Innition (1972)

IM Tetrabo

Loss on Moulding of Cellulose Acetate

Loss Under Dynamic Loading (I97I)

Losses and Efficiency from Tests (Excluding Machines Fo

Lots, of Pulp Baled in Sheet Form (1968)

Loudness Contours for Pure Tones and Normal Threshold O

Loudness Level (1966)

Loudness (1965)

Loudspeakers (1960)

Loudspeakers (I966)

Loudspeakers (1972)

Low Air Pressure (1966)

LLs of Narrow Bands of Noise in a Diff ISO

IEC

Low Alloy High Tensile Steel (I967)

/R Basic Environmental Test. P IEC

Low Alloy High Tensile Steels (I967)

Low Alloy High Tensile Types (1967)

Low Alloy High Tensile Types (1967)

Low Alloy High Tensile Types (1967)

Low Alloy Steels-Code of Symbols for

Low Contents of Free Glycerol-Spectrophoto

for Lengths and ISO

lOns for Straight ISO

Rec. for Code of ISO

IR Code of Symbols ISO

/Ties of Weld Meta ISO

Low Contents) in Copper Alloys (197I)

Low Current Rating) with 2 Hole Mounting (1963) IEC

(Low Current Rating) (1966) /Rotary Wafer Switches with IEC

Low Current Rating): General Requirements and Measuring IEC

(Low Current Rating): Wafer Switches with Central Mounti IEC

IEC

IEC

IEC

IEC

IEC

Frequence (Polyvinyl Chlo

Low Frequency Cables and Wires with Prc (Polyvinyl Chlo /Ec. for Std. Co IEC to the Calculat IFC

Low Frequency Cables and Wires (197I)

Low Hardness ( 10 to 35 IRHD) (197I)

Low Power Nonwirewound Types (I972)

Low Power Oscillator Klystrons (I972)

lor Fixcd Resistor IEC

Pressure Laminated Plates or Panels for Test Purpos

Low Temperature Compartments for the Storagc of Frozen

ISO

Tew Terature (Gehman Test) (197I)

ow Temperatures (197I)

Low Temperatures (1972)

Low Voltage Airbreak Switches, Airbreak Disconnectors,

Low Voltage Controlgear for Industrial Use: Contractors

Low Voltage Distribution Switchgear: Circuit Breakers

Low Voltage Fuses with High Breaking Capacity for Indus

Low Voltage Fuses (I957)

Low Voltage Motor Starters: Direct on Line, a.c. (197I)

Low Voltage Motor Starters: Reduced, a.c.: Star Delta (

Rec. $F$

ISO

ISO

IEC

IEC

IEC

IEC

IEC

IEC

IEC 
Rec. for ${ }^{\circ}$ Of Protection of Enclosures for low Voltage Switchgear and Controlgear (1963)

(:ircuils, lncluding Contacto/

(ircuits, Including Contacto/

Rec. for Control Switches (1972) uation of the Thermal Endurance of Enamelled Wire by the 972) Std. for Petroleum Products: Sid. for Petroleum Products . Rec. for Heads of Aircraft Shipbuilding Details for Sea Navigation-Anchor Chains . Rec. for Microcopy: Measurement of the Screen vulcanized Rubbers-Determination of Rebound Resilience c. for Milling Machine Arbors with 7/24 Tapers and Milling

essories (1968)

Rec. for Short Rec. for Milling Std. for

Rec. for lgnitrons to Be Used in Welding

hanks (1966)

Rec for Measurement of the Winding Resistance of an a.c. $0 \mathrm{~mm}$ and Pitches Up to $6.3 \mathrm{~mm}$ (1972) Std. for Hand and Mass Per Unit Area (1970)

Thickness (1971)

ting Specimens for Physical Tests (1971)

dimensional Changes in Varying Moisture Conditi/ ed Die Stocks (1973)

Rec. for

Rec. for Std. for Std. for Hand and Determining Synchronous Rec. for Cotter Slots with 5\% Taper Keys for Boring 8 to 1 In. (1972) Sid. for Long Shank Std. for Modular Units for Rec. for Direction of Operation of Rec. for Rec. for Rec. for 1971)

Rec. for Symbols for Indications Appearing on Rec. for Electrical Equipment of Rec. for Electrical Equipment of Rec. for Electrical Equipment of nes (1967) or Wool Fibre Length Using a Single Fiber Length Measuring
of Vulcanized Natural or Synthetic Rubber (De Mattia Type of Vulcanized Natural or Synthetic Rubber (De Mattia Type Rec. for Dimensions of the Crimp Area of connecting $\mathrm{Pi} /$ Rec for Nominal Dimensions of Cylindrical Rec. for Textile Machinery-Dyeing and Finishing

tion and Nomenclature (1970)

he Cross Section of Assembled Bars for / Rec. for Textile
Rec. for Textile Rec. for Textile Rec. for Textile Rec. for Textile Rec. for Textile Std. for Textile Std. for Textile Rec. for Textile Std. for Textile Std. for Textile Rec. for Textile Rec. for Textile Rec. for Textile ines-Maximum Spinning-Dimensions (197 yarns (1972)

g Spinning and Doubling Frames for C finition of Side (Left or Right) (1960) and Doubling Frames for C And nning and Doubling Fram

s-Definition of Side (Left or Right) /

Working Width (1971) assification of Materials for the Insulation of Electrical Rec. for Definition of Side (Left or Right) of Spinning ement of the Airborne Noise Emitted by Rotating Electrical Rec. for Dimension of Brushes and Holders for Electrical Rec. for Dimensions of Brushes and Holders for Electrical Rec. for Cotton Spinning or Textile Machinery and Accessories-Weaving Preparatory textile Machinery and Accessories-Cone Sectional Warping

for Dimensions and Output Ratings for Rotating Electrical Rec. for International Electrotechnical Vocabulary: Rec. for Electrical Equipment of Machine Tools: ticular Requirements for Floor Treatment and Wet Scrubbing Rec. for Rules for Rotating Electrical Rec. for Load Calibration of Test. Rec. for Verification of Pendulum Impact Test. or Determining Losses and Efficiency from Tests (Excluding bric Ribbons on Spools (197) Std. for Office and Printing Dimensions and Output Ratings for Foot Mounted Electrical iprocating/ Rec. for Test Conditions for Surface Grinding vertical Spindle-1 Rec. for Test Conditions for Milling tal or Vertical Spi/ Rec. for Test Conditions for Milling rocating $\mathrm{T} /$ (1968)

Rec. for Test Conditions for Surface Grinding Rec. for Code for Numerical Control of and Similar Purposes: Electric Irons, Ironers and Pressing Rec. for Shait Heights for Div eparation of Test Codes for Measuring the Noise Emitted by Rec. for Verification of Brinell Hardness Test. Standardized Blocks to Be Used for Vickers Hardness Test. Rec. for Verification of Vickers Hardness Test. for Verification of Rockwell B and C Scale Hardness Test. low Voltage Switching Devices for Control and Auxiliary

low Voltege Switching Devices for Control and Auxiliary

Lowering of the Electric Strength Between Twisted Wires

Lubricating Grease-Determination of Cone Penetration

Lubricating Grease-Determination of Dropping Point (1

Lubricating Nipples (1965)

Lugless Joining Shackles, Kenter Type (1957)

Luminance of Microfilm Readers (1968)

Lupke Pendulum Meth. (1971)

Machine Accessories (1968)

Machine and Hand Taps (1966)

Machine Arbors with $7 / 24$ Tapers and Milling Machine Acc

Machine Bridge Reamers (1972)

Machine Chucking Reamers with Parallel or Morse Taper S

Machine Control (1967)

Machine During Operation at Alternative Voltage (1969)

Machine Hacksaw Blades: Dimensions for Lengths Up to 45

Machine Made Textile Floor Coverings-Determination of

Machine Made Textile Floor Coverings-Determination of

Machine Made Textile Floor Coverings-Sampling and Cut

Machine Made Textile Floor Coverings: Determination of

Machine Operated Circular Screwing Dies and Hand-Operat

Machine Quantities from Tests (1967)

Machine Reamers, Morse Taper Shank (1961)

(Sectat

Machine Tapers (1971)

Machine Taps with Nominal Diameters from $324 \mathrm{~mm}$ and $1 /$

Machine Tool Construction: Slide Units (1973)

Machine Tool Controls (1965)

Machine Tool Speeds and Feeds (1961)

Machine Tool Tables-T Slots and Corresponding Bolts (

Machine Tool Test Code (1961)

Machine Tools (1964)

Machine Tools: Electronic Types (1968)

Machine Tools: Machines for General Use (1965)

Machine Tools: Used in Large Series Production Lines Li

Rec. for Meth. of Test F ISO

Machine (1970)

Machine) (1959)

Machine) (1959)
Machined Crimp Type Contacts (1966)

Ination of Resistance to Crack Growth

(Nation of Resistance to Flex Cracking

Machinery-Classification and Nomenclature (1970)

Machinery-Cylindrical Tubes for Draw Twisters (1970)

Machinery-Dyeing and Finishing Machinery-Classifica

Machinery-Terms for Serrated Bars and Dimensions of T

Machinery-Types of Formers for Yarn Packages (1970)

Machinery and Accessories-Condenser Bobbins for Wooll

Machinery and Accessories-Cone Sectional Warping Mach

Machinery and Accessories-Cylindrical Tubes for Tape

Machinery and Accessories-Nonreversible Rings for Rin

Machinery and Accessories-Pirn and Cross Winders-De

Machinery and Accessories-Reversible Rings for Ring S

Machinery and Accessories-Spindle Gauges for Ring Spi

Machinery and Accessories-Tubes for Draw Winders for

Machinery and Accessories-Weaving Preparatory Machine

Machinery and Accessories-Worsted and Woollen Cards-

Machinery and Apparatus in Relation to Their Thermal St

Machinery (1959)

Machinery (1970)

Rec. for Test Code for the $\mathrm{M}$

Machinery: Complementary Dimensions and Terminations (1

Machinery: Principal Dimensions and Tolerances (1962)

Machinery: Working Width (1963)

Machines-Definition of Side (Left or Right) (1960)

Machines-Maximum Usable Width (1971)

Machines.. Frame Numbers 56 to 400 and Flange Number

Machines and Transformers (1956)

Machines for General Use (1965)

Machines for Household and Similar Purpose (1972)

Machines for Rail and Road Vehicles (1971)

Machines for Tensile Test. of Steel (1960)

Machines for Test. Steels (1965)

Machines for Traction Vehicles) (1972)

Machines Used for Information Processing-Widths of F

Machines with Frame Numbers 355 to 1000 (1970)

Machines with Horizontal Grinding Wheel Spindle and Re

Machines with Table of Fixed Height with Horizontal or

Machines with Table of of Variable Height, with Horizon

Machines with Vertical Grinding Wheel Spindle and Recip

Machines (Compatible with the ISO 7 Bit Character Set) I and Heating Appliances for Domestic

(1966)

Machines (1966)

Machines (1967)

Machines (1967)

Machines (1968)

1. for General Requirements for the $\mathrm{Pr}$

Rec. for Calibration of ISO

Rec. 150
144

$337 \cdot 1$

$337-2$

172

2137

2176

413

39

782

1767
839

529

839

2238

521

237

279

2336

1764

1765

1957

2551

2568

34.4

236

1080

2283

2562

447

229

299

230

369

$204 \cdot 3$

204 .

204.2

1822

133

132

203

239

1506

1472

1506

1865

1809

1946

2012

2065

96

141

95

2105

142

342

85

92

1680

136-2

136-1

341

142

2012

72
$50(10)$

204.1

335-10

349

147

442

34.2

2257

$72 \mathrm{~A}$

1986

1984

1701

1985

840

11-2G

496

495

156

640

146 
ur Axis and Motion Nomenclature for Numerically Controlled ns and l'itching of Slots on Platens for Projection Welding Standardized Blorks to Be Used for Brinell II ardness Test. ated Appliances for Domestic and Similar Purposes: Washin ratings and Characteristics of 3 Phase, $50 \mathrm{~Hz}$ Turbine Type locks to Be Used for Rockwell B and C Hardness Scale Test. Rec. for Classification of Adding and Calculatin r Dimensions of Tubular Electrode Holders for Spot Weldin ic Section of Ten Key Keyboards for Adding and Calculatin ation of Rockwell Superficial N and T Scale Hardness Test. nd Printed or Displayed Symbols for Adding and Calculating wires (Electrical and Mechanical) for Automatic Drawing-in at for Positioning and Straight Cut Numerically Controlled format Positioning and Straight Cut Numerically Controlled at for Positioning and Straight Cut Numerically Controlled used for Rockwell Superficial N and T Scale Hardness Test. electrical Appliances: Particular Requirements for Washing Rec. for Electrical Safety of Office

or Terminal Markings and Direction of Rotation of Rotating ec. for Metrological Regulations for Nonautomatic Weighin

(1960)

ning Physical Properties of Brush Materials for Electrical

Aerials (Antennas) and Radi/

Microwave Technology (1968)

or Punched Tape Block Formats for the Numerical Control of Rec. for Dyeing and Finishing Rec. for Rotating Electrical Rec. for Rotating Electrical Rec. for Rotating Electrical Rec. for Rotating Electrical Rec. for Rotating Electrical Rec. for Rotating Electrical Rec. for Rotating Electrical Rec, for Rotating Electrical Rec. for Rotating Electrical Turbine Type Machines (196/ g Arrangements of (1972) 1972)

Rec. for Sectional Beams for Warp Knitting Rec. for Quality,

f (1966)

ut Force (1969)

Rec. for Application of Carbides for nd Geological Cross Sections-Part IlI: Representation of 2) ion of Sodium-Gravimetric Meth. Using Uranyl Acetate and and Its Alloys: Gravimetric Determination of Aluminium

mechanical Properties of Sand $\mathrm{Ca}$ Rec. for Composition of sting Purposes (1959) Rec. for Composition of
Rec. for Composition of Wrought g Zirconium, Rare Earths, Thorium and Silver-I Sid. for irconium Alizarin Sulphonate Photometric Meth / Std for -Gravimetric Meth. (1972/ pectrophotometric Meth. Wi/ conium (Alizarin Sulphonat metric Meth. (1970) ed Butt Joints for Aluminium (Aluminum) and Its Alloys and aluminium in Magnesium All iron (Orthophenanthroline) copper (Oxalyldihydrazide ) manganese: Periodate Meth./ manganese: Periodate Meth./ f Zinc (Content Between 0./ ( Calcium and Rec for Composition of 99.8 Unalloyed Rec. for Composition of 99.95 Unalloyed manganese Ores: Determination of Calcium Oxide Content and aluminium (Aluminum) Alloy Conductor Wire of the Aluminium luminium (Aluminum) Alloy Busbar Material of the Aluminium Rec. for Aluminium Alloy Stranded Conductors (Aluminum osition (1972) Std. for

aluminum) and Its Alloys-Complexometric Determination of Rec for Designation of the Quantities Characterizing the ating Thick/ Std. for Electrodeposited Nickel Coatings on etic Metallic and Vitreous or Porcelain Enamel Coatings on Rec. for Conventions Concerning Electric and cabulary (1969) ea Navigation (1965)

Rec. for Rec. for

es, Class B-General Requirements (1967) Rec. for Positioning of Rec. for

Rec. for
head for Centre (Center) Sound Records on $16 \mathrm{~mm}$ Perforated

Recording Head for Edge Sound Records on $16 \mathrm{~mm}$ Perforated Rec. for Dimensions of Cores for Motion Picture and of Magnetic.Striping and Gaps of Recording and Reproducing Rec. for Print Spec. for onmagnetic Substrates-Measurement of Coating Thickness magnetic Basis Metals-Measurement of Coating Thickness Rec. for Calculation of the Effective Parameters of heads for Three Magnetic Sound Records on $35 \mathrm{~mm}$ Film and
Machines (1968)

Machines (1968)

Machines (1968)

Machines (1968)

Machines (1968)

Machines (1968)

Machines (1969)

Machines (1969)

Machines (1969)

Machines (1969)

Machines (1969)

Machines (1969)

Machines (1969)

Machines (1969)

Machines (1969)

Machines (1970)

Machines (1971)

Machines (1972)

Machines (1972)

Machines (1972)

Machines (1972)

Rec. for Test Procedures for Determi I

Mells and Accumulators IEC

IEC

$\begin{array}{ll}\text { Machines: Coding of Preparatory Functions G and Miscell } & \text { ISO } \\ \text { Machines: Definition of Left and Right Sides (1969) } & \text { ISO }\end{array}$

Machines: Degrees of Protection by Enclosures (1968)

Machines: Meth. for Determining Losses and Efficiency $F$

Machines: Meth. for Determining Synchronous Machine Qua

Machines: Meth. of Cooling (1969)

Machines: Noise Limits (1972)

Machines: Rating and Performance (1969)

Machines: Ratings and Characteristics of 3 Phase, $50 \mathrm{~Hz}$

Machines: Symbols for Types of Construction and Mountin

Machines: Terminal Markings and Direction of Rotation (

Machines: Terminology and Dimensions (1969)

Machining and Balancing of Transmission Pulleys (1962)

Machining by Chip Removal: Designation of Main Groups $\mathrm{O}$

Magazines for Still Picture Use and Test for Film Pullo

Magmatic Rocks (1970)

Magnesium Acetate (1970)

Plans a / Industrial Use-Determinat ISO

Magnesium Alloy Sand Castings-Reference Test Bar (197

Magnesium Alloys (Aluminum Content Between 1.5 and 12.0

Magnesium Aluminium (Aluminum) Zinc Alloy Castings and

Magnesium Aluminium (Aluminum) Zinc Alloy Ingots for $\mathrm{Ca}$

Magnesium Aluminium (Aluminum) Zinc Alloys (1966)

Magnesium and Its Alloys-Determination of -Containin

Magnesium and Its Alloys-Determination of Insoluble Z

Magnesium and Its Alloys-Determination of Rare Earths

Magnesium and Its Alloys-Determination of Silicon-S

Magnesium and Its Alloys-Determination of Soluble Zir

Magnesium and Its Alloys-Determination of Zinc-Volu

Magnesium and Its Alloys 5 to $50 \mathrm{~mm}$ Thick (1972)

Magnesium and Its Alloys: Gravimetric Determination of

Magnesium and Its Alloys: Photometric Determination of

Magnesium and Its Alloys: Photometric Determination of

Magnesium and Its Alloys: Photometric Determination of

Magnesium and Its Alloys: Photometric Determination of

Magnesium and Its Alloys: Polarographic Determination O

Magnesium Contents-EDTA Complexometric Meth. (1973)

Magnesium Ingots (1959)

Magnesium Ingots (1961)

Magnesium Oxide Content (1966)

Magnesium Silicon Type (1958)

Magnesium Silicon Type (1959)

Magnesium Silicon Type) (1966)

Magnesium Zinc Zirconium Alloy Castings-Chemical Comp
Magnesium (1973) /for Chemical Analysis of Alumin

Chemical Analysis of ISO Niternational Spec for IEC Rec for Heat Treated a IEC

Magnetic and Electric Properties of Vacuum and a Substa

Magnetic and Nonmagnetic Substrates-Measurement of Co

Magnetic Basis Metals-Measurement of Coating Thicknes

Magnetic Circuits (1972)

Magnetic Compasses and Binnacles for Sea Navigation: Vo

Magnetic Compasses and Binnacles, Class $\mathrm{A}$, for Use in $\mathrm{S}$

Magnetic Compasses in Ships (1968)

Magnetic Compasses, Binnacles and Azimuth Reading Devic

Magnetic Cores for Application in Coincident Current Ma

/Ation and Width of the Recoding ISO

Magnetic Film (1968)

Magnetic Film (1968)

Magnetic Films (1969)

Magnetic Ink Character Recognition (1969) ISO

Magnetic Meth. (1972) /Ckel Coatings on Magnetic and N ISO

Magnetic Meth. (1972)

/Ckel Coatings on Magnetic and $\mathrm{N}$ ISO
/R Porcelain Enamel Coatings on ISO

(1966)

Cording ISO 
$\mathrm{g}$ and (;aps of Recording and Reproducing Magnetic. Ileads for rnmions (1971) nd Record/ Rer. for Recording lleads for 6 Track . lor location of Recording Heads for Three Rec. for Cinematography-Recorded Characteristic fo orated Along l Edge (1966) Rec. for Single Rec. for Graphical Symbols: Ferrite Cores and dges $(1960)$ $\mathrm{mm}$ (200 and $800 \mathrm{RPI}), \mathrm{NRZI}$, and $63 \mathrm{R} /$ Rec. for Unrecorded Rec. for 7 Track 8 RPmm (200 RPI) Rec. for 9 Track 8 RPmm (200 RPI) Rec. for 9 Track $32 \mathrm{Pmm}$ (800 RPI)

ion Interchange (1969)

Rec. for

ions and Characteristics (1968)

Rec. for and Reels, with $76 \mathrm{~mm}$ ( $3 \mathrm{ln}$.) Centrehole (Centerhole), for cations (1971) Rec. for Precision Reels for

the 7 Bit Coded Character Set on 9 Track $12.7 \mathrm{~mm}$ (1/2 In.) nd 7 Bit Coded Character Sets on 7 Track $12.7 \mathrm{~mm}$ (1/2 In.) al Properties and Test Meth. (1973) Std. for Unrecorded ions General Dimensional Requirement/ Rec. for Unrecorded m Motion-Picture Film with Picture-Location and Width of Rec. for Quantities and Units of Electricity and f the Electrical Properties of Microwave Tubes and Valves: Rec. for Expression of the Physical and Subjective Rec. for Chemical Analysis of Cements:

of Carbides for Machining by Chip Removal: Designation of r Cast Iron Pipes: Special Castings and Parts for Pressure ndustrial Use: Sampling-Test Sample-Preparation of the Spec. for

estic and Similar Genel estic and Similar Gener/

Spec. for Safety Requirements for ar General Us/ Rec. for Safety Equipment for Splash Proof Rec. for Analogue Voltage Ranges and Logic Levels for guide for the Collection of Reliability, Availability, and res by Means of Aqueous So/ Rec. for Plastics-Meth. for $s$ Tenders and Orders and Rules for Transport, Erection and Ider Fittings for Copper Tubes-Dimensions of Sockets and Std. for Screwdriver Bits, for Slotted Head Screws, with

70)

with ISO Rec. R7 (1957)

Rec. for Whiteheart Rec. for Blackheart Rec. for Pearlitic Rec. for Generic Names for ile Machinery and Accessories-Tubes for Draw Winders for Rec. for

Rec for Paints and Varnishes-Bend Test (Cylindrical ability Dimensions for Milling Cutters and Their Arbors or Rec. for Tube Drawing isodium Tetraborates for Industrial Use-Determination of emical Analysis of Manganese Ores-Determination of Total termination of Active Oxygen-Conventionally Expressed as

ntionally Express/ oxide (1963)

(1963)

\section{3)}

nt (1963)

1966)

and Magnesium Oxi/

(1966)

(Photometric Met/

)

aphic Meth., for /

963)

luminium and Aluminum Alloys: Rec. for Chemical Analysis of Steels: Determination of Rec. for Pulps-Determination of ree Cutting Steels-Part 5: Wrought Quenched and Tempered Rec. for Raw.Rubber and Rubber Latex-Determination of of Magnesium and Its Alloys: Photometric Determination of of Magnesium and Its Alloys: Photometric Determination of Rec. for 3 or 4 Strand Rec. for 8 Strand Plaited

of

Magnetic Sound Record (1972)

IIdth of Magnetic-Stripin ISO

Magnetic Sound Records on $35 \mathrm{~mm}$ Film-Location and Dim ISO

Magnetic Sound Records on $35 \mathrm{~mm}$ Film and 1 Magnetic Sou ISO

Magnetic Sound Records on $35 \mathrm{~mm}$ Film (1963) Rec. ISO

Magnetic Sound Records on 35 mm Perforated Film (1971) ISO

Magnetic Sound Stripe on $16 \mathrm{~mm}$ Motion Picture Film Perf

Magnetic Storage Matrices (1972)

Magnetic Striping of $16 \mathrm{~mm}$ Film Perforated Along Eoth E

Magnetic Tape for Information Interchange -8 and $32 \mathrm{Rp}$

Magnetic Tape for Information Intercbange (1971)

Magnetic Tape for Information Interchange (1971)

Magnetic Tape for Information Interchange (1971)

Magnetic Tape Labelling and File Structure for Informat

Magnetic Tape Recording and Reproducing Systems: Dimens

Magnetic Tape Used in Interchange Instrumentation Appli

Magnetic Tape Used in Interchange Instrumentation Appli

Rec. for Implementation of ISO

Magnetic Tape (1969)

C. for Implementation of the 6 a ISO

$\begin{array}{lll}\text { Magnetic Tape (1969) /C. for Implementation of the } 6 \text { a ISO } \\ \text { Magnetic Tapes for Instrumentation Applications: Physic } & \text { ISO }\end{array}$

Magnetic Tapes for Interchange Instrumentation Applicat ISO

Magnetic-Striping and Gaps of Recording and Reproducing ISO

Magnetism (1965)

Magnetrons (1972)

Magnitudes of Sound or Noise (1959)

Rec. for Measurement O IEC

Main Constituents of of Portland Cement (1968)

Main Groups of (1966) Rec for Application ISO

Main Lines (1955)

Main Solution for Carrying Out Certain Determinations ( Rec. Fo ISO

Mains Operated Electric Fence Controllers (1956)

Mains Operated Electronic and Related Equipment for Dom

Mains Operated Electronic and Related Equipment for Dom

Mains Operated Electronic Equipment (Domestic and Simil

Mains Operated Nuclear Instruments (1970)

Maintainability Data from Field Performance of Electron

Maintaining Constant Relative Humidity in Small Enclosu

Maintenance (1971)

Male Ends (1971)

Humidity in Small Enclosu ISO

Male Hexagon Drive (1972)

Rec. for Capillary So ISO

(19

$\begin{array}{ll}\text { Malleable Cast Iron Pipe Fittings Screwed in Accordance } & \text { ISO } \\ \text { Malleable Cast Iron (1969) } & \text { ISO }\end{array}$

Malleable Cast Iron (1969)

Malleable Cast Iron (1969)

Man Made Fibres (Fibers) (1971)

Man Made Fibres (1971)

Managerial Aspects of Reliability (1969)

Mandrel) (1970)

Mandrels-Metric and In. Series (1969)

Mandrels (1970)

Manganese Content-Formal

Manganese Dioxide (1963)

Ma

\section{Ma}

Mar

f

Manganese Ores-Determination of Copper (1963)
Manganese Ores-Determination of Hygroscopic Moisture
Manganese Ores-Determination of Lead (1963)

Manganese Ores-Determination of Nickel (1963)

Manganese Ores-Determination of Phosphorus (1963)

Manganese Ores-Determination of Silicon Dioxide $(1963$

Manganese Ores-Determination of Sulphur (1963)

Manganese Ores-Determination of Total Iron Content (1

Manganese Ores-Determination of Total Manganese Conte

Manganese Ores: Determination of Barium Oxide Content

Manganese Ores: Determination of Calcium Oxide Content

Manganese Ores: Determination of Chromium Content (1967

Manganese Ores: Determination of Combined Water Content

Manganese Ores: Determination of Metallic Iron Content:

Manganese Ores: Determination of Titanium Content (1966

Manganese Ores: Determination of Vanadium Content (1966

Manganese Ores: Determination of Zinc Content (Polarogr

Manganese Ores: Determination of Zinc Content (1966)

Manganese Ores; Part I: Ore Loaded in Freight Wagons (1

Manganese (Contents Between 0.005 and 1.5\%) (1968)

Manganese (Spectrophotometric Meth.) (1967)

Manganese (1970)

Manganese (1970)

Rec. for Heat Treated, Alloy, and F

Manganese (1971)

Manganese: Periodate Meth. (Content Between 0.01 and 0.

Manganese: Periodate Meth. (Content Less Than 0.01\%) (1

Manila and Sisal Ropes (1970)

Manila and Sisal Ropes (1971)
I201

1753

162

360

1189

490

$117-16$

1864

1861
1862

1863

1001

94

1858

1860

962

961

2690

1859

1201

$31 / \mathrm{V}$

235-4

131

680

513

13

2466

5

65

$65 \mathrm{~A}$

323

362

483

56-6

2016

2351

1390

49

942

943

944

2076

2105

300

1519

240

1651

2214

319

312

1397

312

318
317

314

316

322

310

323

315

321

311

320

313

319

548

552

619

549

621

550

553

620

551 
of Symhols for Identific/ Std. for Covered Electrodes for $s$ of Slope and Rotation for Welding Positions for Straight Rec. for Code of Symbols for Covered Electrodes for Rec. for Performance Requirements for Lever Operated Rec. for Dimensions of Spindle Ends for

s and Test Meth. (1972) Rec. for Gypsum Rock for the ec. for Meth. of Expression of Dimensions and Direction of $r$ Marking of the Different Species of Wood Utilized in the rec. for Cylindrical Gears (Information to Be Given to the - for Straight Bevel Gears (Information to Be Given to the o) Rec. for Information on Ferrite Materials Appearing in ips with Rectangular Face (I969) Rec. for General td. for Dense Shaped Refractory Products-Nomenclature of ) Rec. for Shipbuilding Details: Ship Screw Propellers, epresenta/ Rec.for Graphical Symbols for Use on Detailed presentat/ Rec. for Graphical Symbols for Use on Detailed eral Rule/ Rec. for Graphical Symbols for Use on Detailed onal Properties of Aluminium (Aluminum) Alloy Sections for International Rec. for One Rec. for One Rec. for 1 Rec. for Identification of the Emulsion Side of Edge Rec. for Wire, Bar and Tube Drawing Dies-Designation.

d Capacitors (1968) Std. for Identification Rec. for Rec. for Essential Oils: Labelling ard Rec. for Mechanicai Properties of Fasteners: Rec. for Rec. for

ion of the Content (1965)

Metals or in Light Allo/ Details Rec. for
Rec. for 68)

Rec for Lead Acid Starter. for e Manufacture of Wood Flooring-Code of Symbol/ ions and erials Sensitive to Radiant Energy (1/

Rec. for Turning Tools with Carbide Tips: Designation and c. for Electrical Apparatus for Explosive Gas Atmospheres: ds for Parallel and Taper-Threads-General Dirnensions and Rec. for Rotating Electrical Machines: Terminal Rec. for Pictorial Rec. for Exposure Time Rec. for Lens A perture ons (I972) Std for Mlinimum Rec. for Significance to Purchasers of Rec. for Principles of Operation of Standards Ash in Products Not Containing More Than $0.5 \%$ of Such, by dium Borates for Industrial Use-Determination of Loss in ium Carbonate for Industrial Use: Determination of Loss of Used for Production of Aluminum: Determination of Loss of Rec. for Tea-Determination of Loss in

Chloride for Industrial Use: Determination of the Loss of marily Used for the Production of Determination of Loss of the Backing (1971) Rec. for Carpets-Determination of $f$ Aluminium (Aluminum) and Its Alloys-Measurement of the
Rec. for Carpets-Determination of Rec, for Cereals and Pulses: Determination of the International Rec. for Instruments for Measuring the or Machine Made Textile Floor Coverings-Determination of les: Yarn from Packages-Determination of Linear Density Rec. for Pulps: Determination of Saleable tor Operated Appliances for Domestic and Similar Purposes: dling Equipment for Loose Bulk Materials-Scraper and En ion Steel Tubes: Metric Series, Dimensions, Tolerances and less Steel Tubes-Dimensions, Tolerances and Conventional for Boiler Tubes-Dimensions, Tolerances and Conventional (I964) ubes, Welded or Seamless-General Table of Dimensions and r Types (I964)

Rec. for Hollow

el for Tyred (Tired) Wheel

Tyred (Tired) Wheels For

sions and Tolerances (196)

Rec. for Railway Rolling Stock Rec. for Railway Rolling Stock ermination of the Acetone Soluble Matter (Resin Content of Rec. for Heat Treated Aluminium (Aluminum) Alloy Busbar tage Greater Thal

r Plastics-Determination of Apparent Density of Moulding
Manual Arc Welding and Filler Metals for Gas Welding (1 Manual Arc Welding Equipment (1968) Manual Arc Welding of Mild and Low Alloy Steels-Code Manual Arc W'elds Made with Covered Electrodes of Mild a Manual Metal Arc Welding of Cast Iron (1969) Manual Switches for Aircraft (1970)

Manually Operated Electronic Components (1972)

Manufacture of Binders-Classification, Characteristic

Manufacture of Unprocessed Writing and Printing Paper

Manufacture of Wood Flooring-Code of Symbols (1971)

Manufacturer by the Purchaser in Order to Obtain the Ge Manufacturer by the Purchaser in Order to Obtain the Ge

Manufacturers Catalogues of Transformer and Inductor $C$

Manufacturing Characteristics of Solid Wood Parquet Str Manufacturing Processes (1972)

Manufacturing Tolerance for Casting and Finishing (I966

Maps, Plans and Geological Cross Sections-Part III: R

Maps, Plans and Geological Cross Sections-Part II: Re

Maps, Plans and Geological Cross Sections-Part I: Gen

Marine Use (1970) /Ding De

Mark Pipettes

Mark Volumetric Flasks (1971)

Marked Roll Film for Still Picture Cameras (1968)

Marking-Dimensions (I970)

Marking Code for Freight Containers (1972)

Marking Codes for Values and Tolerances of Resistors an

Marking Containers (1961)

Marking of Aircraft Gas Cylinders (1965)

Marking of Bolts, Screws, Studs and Nuts (1969)

Marking of Control Settings on Hearing Aids (1966)

Marking of Conveyor Belts (I965)

Marking of Hatchway Beams (1960)

Marking of Industrial Gas Cylinders for the Identificat

Marking of Irsulated Conductors (1972)

Marking of Rolled, Drawn and Extruded Products in Light

Marking of Safety Film for Motion Picture Uses (1966)

Marking of Series 1 and Series 2 Freight Containers (19

Marking of Terminals (1963)

Marking of the Different Species of Wood Utilized in Th

Marking of Transit Packages Containing Photographic Mat

Marking of Wooden Hatchway Covers (1960)

Marking (1966)

Marking (1970)

Marking (1972)

Std. for Hand

Markirgs for Handling of Goods (General Symbols) (I968)

Markings for Still Cameras (I966)

Markings to Appear on Containers Used for Printing Ribb

Marks Indicating Conformity with Standards (1966)

Marks (1961)

Mass-Gravimetric Meth. (I970)

Mination of Sulphated ISO

Mass After Heating at $900^{\circ} \mathrm{C}(1972)$

Mass and of Nonvolatile Matter at $250^{\circ} \mathrm{C}(1968)$

Mass at $1000^{\circ} \mathrm{C}$ and $1200^{\circ} \mathrm{C}(1968)$

Mass at $103^{\circ} \mathrm{C}(\mathrm{I} 970)$

Mass at $110^{\circ} \mathrm{C}(1973)$

Mass at $300{ }^{\circ} \mathrm{C}$ (Conventional Moisture) (1968)

Mass of Pile Per Unit Area That Can Be Shorn Away from

Mass of the Oxide Coatings-Gravimetric Meth. (197I)

Mass of Total Pile Yarn Per Unit Area (I971)

Mass of 1000 Grains (1966)

Mass Per Hectolitre of Cereals (1970)

Mass Per Unit Area (1970)

(Mass Per Unit Length) Skein Meth. (1972)

Mass, in Lots, of Pulp Baled in Sheet Form (I968)

Massage Appliances (I967)

Masse Conveyors-Safety Code (I972)

Masses Per Metre (Meter) (I967)

Masses Per Unit Length (1969)

Masses Per Unit Length (1971)

Masses Per Unit Length (1971)

Metallic Waveguides: Relevant Spec. for Flat Rectangul

Material-Axles for Trailer (1969)

Material-Cast Wheel Centres (Centers) in Nonalloy Ste

Material-Rolled on Forged Wheel Centres (Centers) for

Material-Rough Tyres (Tires) for Trailer Stock: Dimen

Material-Sampling (1972)

Std for Pots for $\mathrm{Pr}$ ISO

Material-Tyres (Tires) for Trailer Stock (1969)

Material in the Unmoulded State) of Phenolic Moulding M

Material of the Aluminium Magnesium Silicon Type (1959)

Material or Glass for Overhead Lines with a Nominal Vol

Material That Can Be Poured from a Specified Funnel (19 
$r$ Plastics-Determination of Apparent Density of Moulding rown Coals and lignites-Determination of Acetone Soluble Rec. for Commercial Purity Aluminium (Aluminum) Busbar for Dobby l.ags and Pegs in Wood, Metal or Other Suitable our (Color) Film for General Use: Preferred Sizes of Shee ur (Color) Paper for General Use: Preferred Sizes of Shee and White Papers for General Use: Preferred Sizes of Sheet Rec. for Alginate Impression Rec. for Agar Impression Std. for Flexible Cellula or Continuous Mechanical Handling Equipment for Loose Bulk ics-Compression Moulding Test Specimens of Thermosetting ics-Compression Moulding Test Specimens of Thermosetting ics-Compression Moulding Test Specimens of Thermosetting or Continuous Mechanical Handling Equipment for Loose Bulk or Continuous Mechanical Handling Equipment for Loose Bulk or Continuous Mechanical Handling Equipment for Loose Bulk is (Dense and Insulating) (1971)

Rec. for Refractory Std. for Polyethylene Thermoplastic Rec. for Flexible Cellular Rec. for Building Std. for Flexible Cellula Rec. for Flexible Cellula

ation at Break (1971) Equipment for Loose Bulk 971) Rec. for Flexible Cellula or Continuous Mechanical Handling Equipment for Loose Bulk or Continuous Mechanical Handling Equipment for Loose Bulk or Continuous Mechanical Handling Equipment for Loose Bulk or Continuous Mechanical Handling Equipment for Loose Bulk Std. for Pneumatic Handling Appliances for Loose Bulk or Continuous Mechanical Handling Equipment for Loose Bulk asic Dimensions/ Rec. for Pipes and Fittings of Plastics or Continuous Mechanical Handling Equipment for Loose Bulk or Continuous Mechanical Handling Equipment for Loose Bulk or Continuous Mechanical Handling Equipment for Loose Bulk or Continuous Mechanical Handling Equipment for Loose Bulk or Continuous Mechanical Handling Equipment for Loose Bulk or Continuous Mechanical Handling Equipment for Loose Bulk Rec. for General Classification of Ferromagnetic Oxide or Continuous Mechanical Handling Equipment for Loose Bulk former and Inductor $\mathrm{Co} /$ Rec. for Information on Ferrite st for Electrical Resistance and Resistivity of Insulating c. Meth. of Test for Electric Strength of Solid Insulating and Dielectric Dissipation Factor of Electrical Insulating

1: Definitions and General R/ Rec. Spec. for Insulating ht Per Unit Area of Hot Dip Galvanized Coatings on Ferrous st Procedures for Determining Physical Properties of Brush Rec. for Sampling Raw Rec. for Metal Clad Base Rec. for Metal Clad Base

d Apparatus in Relation T/

8 and Nominal Pressures): Par

$s$ and Nominal Pressures) Part/

Plastics-Determination of the Percentage of Extractable torial Marking of Transit Packages Containing Photographic

Rec. for Pulps: Extraction of

eth. for Determining the Relative Resistance of Insulatin rmining the Comparative Tracking Index of Solid Insulating refractory Materials-Classification of Prepared Unshaped

Volume and Surface Resistivities of Electrical Insulating

c. for Plastics-Determination of Bulk Factor of Moulding stics-Injection Moulding Test Specimens of Thermoplastic ics-Compression Moulding Test Specimens of Thermoplastic ics-Compression Moulding Test Specimens of Thermosetting

tof Material in the Unmoulded State) of Phenolic Moulding

c. for Tables of Vickers Hardness Values (HV) for Metallic rmination of the Insulation Resistance of Solid Insulating ources for Portable Prospecting Equipment for Radio Active Evaluating the Thermal Endurance of Elcctrical Insulating and Vehicle Mounted Prospecting Equipment for Radio Active Rec. for Plastics-Basis for Spec. for Phenolic Moulding Rec. for Quantity Packaging of Sensitized Photographic Rec. for Noncombustibility Test for Building

stics-Designation for Polystyrene Moulding and Extrusion $s$ of Criticality Safety in Handling and Processing Fissile ex A: Preparation of Test Specimens from Phenolic Moulding of Test Specimens from Polyester and Epoxy Resin Moulding : Preparation of Test Specimens from Aminoplastic Moulding

truction of Installations for Work on Unsealed Radioactive r Copper and Its Alloys-Code of Designation-Part I: of dling Equipment-Light Duty Belt Conveyors for Loose Bulk ior to and During the Test. of Solid Electrical Insulating s-Preparation of Specimens for Optical Tests on Plastics 72) Std for Flexible Cellular -Preparation of Specimens for Optical Tests on Plastics or Continuous Mechanical Handling Equipment for Loose Bulk
Material That Cannot Be Poured from a Specified Funnel Material (Resinoug Substances) (1969) / Extract from B ISO Material (1958)

Material (1967)

Material (1969)

Material (1969)

Material (1969)

Material (1970)

Material (1970)

Materials-Accelerated Ageing Tests (1972)

Materials-Aeroslides (1972)

Materials-Annex A: Preparation of Test Specimens from

Materials-Annex B: Preparation of Test Specimens from

Materials-Annex C: Preparation of Test Specimens from

Materials-Apron Conveyors-Safety Code (1972)

Materials-Apron Conveyors (1971)

Materials-Belt Feeders and Conveyors-Safety Code (1

Materials-Classification of Prepared Unshaped Materia

Materials-Designation (1972)

Materials-Determination of Apparent Density (1971)

Materials-Determination of Calorific Potential (1971)

Materials-Determination of Compression (1972)

Materials-Determination of Tensile Strength and Elong

Materials-Hydraulic Conveyors-Safety Code (1972)

Materials-Measurement of Dimensions of Test Pieces (1

Materials-Mobile Belt Conveyors-Safety Code (1972

Materials-Oscillating Conveyors and Shaking on Recipr

Materials-Oscillating Conveyors and Shaking or Recipr

Materials-Picking Table Conveyors-Safety Code (1972

Materials-Piping (1972)

Materials-Scraper and En Masse Conveyors-Safety C

Materials-Socket Fittings for Pipes Under Pressure-

Materials-Storage Equipment: Bins and Bunkers, Silos

Materials-Troughed Belt Conveyors (Other Than Portabl

Materials-Troughed Belt Conveyors (Other Than Portabl

Materials-Troughed Belt Conveyors (Other Than Portabl

Materials-Vibrating Feeders and Conveyors with Tubula

Materials-Vibrating Feeders and Conveyors, Shaking or

Materials and Definitions of Terms (1961)

Materials and Unit Loads-Belt Conveyors-Basic Chara

Materials Appearing in Manufacturers Catalogues of Tra

Materials at Elevated Temperatures (1971)

Materials at Power Frequencies (1967)

Materials at Power, Audio and Radio Frequencies Includi

Materials Based on Built Up or Treated Mica Paper-Par

Materials by Chemical Dissolution of the Coating-Grav

Materials for Electrical Machines (1972)

Materials for Paints and Varnishes (1968)

Materials for Printed Circuits: Spec. (1970)

Materials for Printed Circuits: Test Meth. (1968)

Materials for the Insulation of Electrical Machinery an

Materials for the Transport of Fluids (Outside Diameter

Materials for the Transport of Fluids (Outside Diameter

Materials in Polyamides (1967)

Materials Sensitive to Radiant Energy (1968)

Materials Soluble in Dichloromethane (1967)

Materials to Breakdown by Surface Discharges (1970)

Materials Under Moist Conditions (1971)

Materials (Dense and Insulating) (1971)

Materials (1958)

Materials (1961)

Materials (1963)

Materials (1963)

Materials (1963)

Materials (1963)

Materials (1964)

Materials (1964)

Materials (1965)

Materials (1966)

Materials (1967)

Materials (1968)

Materials (1969)

Materials (1970)

Materials (1970)

Materials (1970)

Materials (1970)

Materials (1970)

Materials (1970)

Materials (1970)

Materials (1971)

Materials (1971)

Materials (1971)

Materials: Casting Meth. (1968)

Materials: Hardness Test. by Inde

Materials: Moulding Meth. (1968)

Materials: Screw Conveyors (1969)
ISO

ISO

Rec ISO

ID. F ISO ISO

2140

1821

1927

1872

1855

1716

1856

1798

2392

1794

2387

2139

1807

2389

2327

2391

264

1820

1535

1536

1537

1815

2125

125

1816

401

345

243

250

371-1

1460

413

842

249-2

249-1

85

161

330

599

884

624

343 
$\therefore$ and lndustrial/ Rec. for Pipes and Fittings of Plastics or Continuous Mechanical 1 andling Equipment for Loose Bulk Rec. for Railway Rolling Stock Rec. for Railway Rolling Stock nces and Technology (1961) Rec. for Rec. for Shipbuilding Details: Rec. for Pipeline Flanges for General Use sed Ends and Having a Contact Spacing of $2.54 \mathrm{~mm}(0.1 \mathrm{ln}$.) Rec. for Dimensions of the for Graphical Symbols: Ferrite Cores and Magnctic Storage . for Magnetic Cores for Application in Coincident Current assium Silicates for Industrial Use-Determination of Dry Std. for Glucose Syrup: Determination of Dry rial Use: Determination of Loss of Mass and of Nonvolatile Rec. for Pulps: Determination of Dry

Rec. for Tea-Preparation of Ground Sample of Known Dry Rec. for Determination of Mineral stics-Determination of the Percentage of Acetone Soluble Rec. for Plastics-Determination of Ethyl Ether Soluble Rec. for Plastics-Determination of Methanol Soluble Rec. for Determination of Volatile for Sodium Carbonate for Industrial Use: Determination of . for Sodium Chloride for Industrial Use: Determination of ium Tripolyphosphate for Industrial Use-Determination of

$s$ Not Easily Soluble in Boiling Water and Determination of . for Sodium Chlorate for Industrial Use: Determination of ane (1969) Rec. for Plastics-Determination of Soluble

ile Glass-Determinatioc. for Determination of the Volatile Rec. for Analysis of Soaps: Determination of Foreign Rec. for Glucose Syrup-Determination of Dry Determination of Polyethylene Glycols and Nonionic Active ctive Agents: Detergents-Determination of Anionic-Active rec. for Plastics-Pvc Resins-Determination of Volatile Rec. for Plastics-Determination of the Acetone Soluble mmed Sizes of Writing Paper and Certain Classes of Printed

of Processed Writing Paper and Certain Classes of Printed r Oleaginous Seeds: Determination of Moisture and Volatile r Oilseed Residues: Determination of Moisture and Volatile Analysis of Soaps: Determination of Moisture and Volatile able Oils and Fats: Determination of Moisture and Volatile ec. for Spices and Condiments: Determination of Extraneous c. for Animal Fats: Determination of Moisture and Volatile of Soaps: Determination of Unsaponifiable and Unsaponified oxide for Industrial Use: Determination of Water Insoluble oxide for Industrial Use: Determination of Water Insoluble ics-Pvc Resins-Determination of Impurities and Foreign and Varnishes-Determination of Volatile and Nonvolatile crack Growth of Vulcanized Natural or Synthetic Rubber (De lex Cracking of Vulcanized Natural or Synthetic Rubber (De tistical Interpretation of Test Results: Estimation of the Rec. for Air Permeability Meth. for Measuring the

ics-Determination of Indentation Hardness of Plastics by Pressure Responsive Elements Giving Direct Indications by ntaining Constant Relative Humidity in Small Enclosures by aluminium-Preparation of Sample Solution for Analysis by Rec. for Mounting of Plain Grinding Wheels by ment of Hearing Aids Using Earphones Coupled to the Ear by Rec. for Measurement of Fluid Flow by Rec. for Voltage Measurement by ith Pressure Responsive Elements and Recording Directly by he Scope Covered B/ Rec. for Measurement of Fluid Flow by Rec. for Measurement of Fluid Flow by etection and Measurement of lonizing Radiation by Electric rpets-Determination of Measured Surface Pile Density and fiber) Volume Ratio / Rec. for Carpets-Determination of s/ Rec. for Classification of Instruments and Devices for (1960) easurement of Thickness of Oxide Coatings-Nondestructive
Rec. for Voltage of the Electrical Properties of Electronic Tubes: Meth. of of the Electrical Properties of Electronic Tubes: Meth. of Rec. for Meth. of the Frequency Ran/ Rec. for Std. Definitions and Meth. of and Power and Power of Nonessential Oscill/ Rec. Meth. of of, Frequency, Output Power and Consumptio/ ular to Transposers for Monochrome and Col/ Modulation (1971)

Rec. Meth. of Rec. Meth. of of the Electrical Properties of Electronic Tubes: Meth. of ion of a Gauging Station and Determ/

Rec. for Liquid Flow Rec. for Liquid Flow Rec. for Liquid Flow 68) Flow-Part 1: Constant Rate Injec/ Rec. for Liquid Flow Rec. for on Room (1963)

meover Units lntended for Outdoor Use (1972)

Std. for

trical Properties of Electronic Tubes and Valves: Meth. of Aluminium (Aluminum) and Its Alloys-Insulation Check by
Materials: Socket Fittings with Spigot Ends for Domesti Materials: Vibrating Conveyors and Feeders with Rectang Material: Solid Wheels for Trailer Stock (1969)

Material: Wheel Sets for Trailer Stock (1969)

Mathematical Signs and Symbols for Use in Physical Scie Mating Dimensions for Pipeline Flanges for Ships (1969) Mating Dimensions (1971)

Mating Either with Board Mounted Connectors or Printed Mating Parts of Radio Frequency Connectors (1964) Matrices (1972)

Matrix Stores Having a Nominal Selection Ratio of $2: 1$

ISO

ISO

ISO

ISO

ISO

ISO

ISO

IEC

Rec. IEC

IEC

Matter-Gravimetric Meth. (1970)

Matter-Refractive Index Meth. (1973)

Matter at $250^{\circ} \mathrm{C}$ (1968)

/ for Sodium

ISO

ISO

Matter Content (1967)

Matter Content (1970)

/Dium Carbonate for Indust ISO

ISO

ISO

Rec. for Pla ISO

Matter in Phenolic Mouldings (1958)

Matter in Plasticized Cellulose Acetate (1971)

Matter in Polystyrene (1959)

Matter in Raw Natural Rubber (1962)

Matter Insoluble in Water at $50^{\circ} \mathrm{C}(1968)$

Matter Insoluble in Water or in
Matter Insoluble in Water (1968)

Matter Insoluble in Water (1972)

Matter Insoluble in Water (1973)
Matter of Crystalline Polypropylene by Boiling N-Hept
Matter of Hard Coal and of Coke (1967)

Matter of lts Products (1971)

Matter of Low Solubility in Ethanol (1968)

Matter of Moisture (Vacuum Oven Meth.) (1970)

Matter (Adducts)-Weibull Meth. (1972)

Matter (Direct 'Two Phase Titration Procedure) (1972

Matter (Including Water) (1970)

Matter (Resin Content of Material in the Unmoulded Stat

Matter (1961)

Matter (1963)

Matter (1968)

Matter (1968)

Matter (1968)

Macter (1968)

Matter (1969)

Matter (1969)

Matter (1969)

Matter (1969)

Matter (1969)

Matter (1970)

Matter (1970)

Mattia Type Machine) (1959)

Mattia Type Machine) (1959)

Mean-Confidence Interval (1973)

Rec. for Tri $1 \mathrm{SO}$
Dimensions isO

Rec. Fo ISO

Rec. Fo ISO

Rec. for ISO

Rec. for Crude Veget ISO

Re ISO

Rec. for Analysis ISO

Rec. for Sodium Hydr ISO

Rec. for Potassium Hydr ISO

Rec. for Plast ISO

Rec. for Paints ISO

/Ination of Resistance to ISO

/Nation of Resistance to F ISO

Mean Diameter of Wool Fibres (Fibers) (1969)

Std. for Sta ISO

Ior Plast ISO

Means of a Pointer and Graduated Scale (Working Equipme OlML

Means of Aqueous Solutions (1966) /Ics-Meth. for Mai ISO

Means of Attack by Hydrochloric Acid Under Pressure (19 ISO

Means of Hub Flanges (1968)

Means of Inserts (1961)

/Rence Cour

Means of Orifice Plates and Nozzles (1967)

Means of Sphere Gaps (1 Sphere Earthed) (1960)

Means of Stylus and Diagram (Working Equipment Category

Means of Venturi Tubes-Classical Tubes Used Outside T

Means of Venturi Tubes (1968)

$\begin{array}{lll}\text { Means (1968) /Rnational Electrotechnical Vocabulary: D } & \text { IEC } \\ \text { Measured Pile Fibre (Fiber) Volume Ratio (1971) /or Ca } & \text { ISO }\end{array}$

Measured Surface Pile Density and Measured Pile Fibre ( Tor Ca ISO

Measurement and Evaluation of the Geometrical Parameter ISO

Measurement by Light Section Microscope (1971) /Ys-M ISO

Mcasurement by Means of Sphere Gaps (1 Sphere Earthed) IEC

Measurement for Camera Types (1971) /for Measurements IEC

Measurement for Cold Cathode Counting and Indicator Typ IEC

Measurement for Loudspeakers (1966)

Measurement for Piezoelectric Vibrators Operating Over

Measurement for Radio Transmitters: Bandwidth, Out of B

Measurement for Radio Transmitters: General Conditions

Measurement for Radio Transmitters: Measurements Partic

Measurement for Radio Transmitters: Wanted and Unwanted

Measurement for Television Picture Types (1968)

Measurement in Open Channels-Establishment and Operat

Measurement in Open Channels by Slope Area Meth. (1969)

Measurement in Open Channels by Velocity Area Meth. (19

Measurement in Open Channels: Dilution Meth. for Steady

Measurement of Absorption Coefficients in a Reverberat

Measurement of Airborne Noise Emitted by Compressor Pri

Measurement of Audio Frequency Output Power and Distort

/Idation) of ISO
265

1049

$1005 / \mathrm{VI}$

$1005 / \mathrm{VI}$

$31 / \mathrm{Xl}$

964

130-11

159

117.16

281

1688

1743

745
638

1572

602

59

1875

118

248

746
2479

850

2122

2461

922

562

1887

673

1742

2268

2271

1269

308

216

353

665

771
672

662

927

933
1067

987

998

1265

1515

133

132

2602

1136
868

483

2073

666

126

541

52
19

$781 / 1$

781

$50(66)$

1959

1959

1878

2128

151-26

151.22

200

302

244.2

244. 1

244-5

244.3

151.16

1100

1070

748

555

354

2151

151-10

2376 
The Elcctrical Properties of Electronic Tubes and Valves: Rec. for Electronic Tubes: Meth. of for Nonennductive Coatings on Nonmagnetic Basis Metals us or Porcelain Enamel Coatings on Magnetic Basis Metals d Nickel Coatings on Magnetic and Nonmagnetic Substrates Rec. for Higher Alcohols for Industrial Use Rec. for Cresylic Acid and Xylenols for Industrial Use rs (1965)

rical Properties of Electronic Tubes and Valves: Meth. of Rec. for Plasticsustrial Use: Evaluation of Sulphuric Acid Concentration by or Industrial Use: Evaluation of lts Acid Concentration by Rec. for Plywood

Rec for Flexible Cellular Materials ecironic Tubes and Valves (1962) Rec. Meth. for the the Electrical Properties of Electronic Tubes and Valves: trical Properties of Electronic Tubes and Valves: Meth. of the Electrical Properties of Electronic Tubes and Valves: ving Aerials in the Frequency Range Form 3/ Rec. Meth. of d Nozzles (1967)

lassical Tubes Used Outsioe the Scope Covered B/ Rec. for 68)

Rec. for Surface Active Agents: nwanted Resonances of Filter Crystal / Rec. Meth. for the trical Properties of Electronic Tuhes and Valves: Meth. of of the Electrical Properties of Electronic Tubes: Meth. of the Ear by Means / Rec. for IEC Reference Coupler for the $r$ International Electrotechnical Vocabulary: Detection and
Std. Meth. of

rec. for Collection of Data for Deterinination of Errors in ocabulary of Terms and Symbols Used in Connection with the croscopical Examination of Cross Sections (197/ Rec. for Rec. for

trical Properties of Electronic Tubes and Valves: Meth. of old or Similar Use (1970)

hold and Similar Use (1971)

Rec. for Meth. of Rec. for Sodium Tripolyphosphate for Industrial Use Std. for Ammonium Nitrate for Industrial Use: cells for Use in the Visible Spectrum, Meth. Of/

Rec. for

Rec. for (1971)

Rec. for Meth. of Std. for Acoustics: Description and trical Properties of Electronic Tubes and Valves: Mcth. of nd Frequency Modulations and Television Br/ Rec. Meth. of trical Properties of Electronic Tubes and Valves: Meth. of d Reproducing Equipment (1972)

trical Properties of Electronic Tubes and Valves. Meth. of General Statements-Terms/

Contact (Stylus) Instrumen/ lectrical Machinery (1970) Rec. for Instruments for the Rec. for Instruments for the Rec. for Test Code for the

inium Oxide Primarily Used for the Production of Aluminum: t Having Two Axial Terminations (1969)

tic X-Ray Tubes Using a Pinhole Camera (1970)

ubes and Valves: Terminology (1972)

ubes and Valves: General (1972)

ubes: Disk Seal Tubes (1972)

ubes and Valves: Magnetrons (1972)

ubes and Valves: Low Power Oscillator Klystrons/

ubes and Valves: High Power Klystrons (1972)

ubes and Valves: Gas Filled Microwave Switching/

ubes and Valves: Backward Wave Oscillator Tubes/

anodic Oxidation) of Aluminium (Aluminum) and Irs Alloys ric Blankets (1969)

ive Displacement Vacuum Pumps-Part 1/

r (Vapor) Vacuum Pumps-Part I: Volum/

8 (1968)

Rec. for

Rec. for

Rec. for

Rec. for

Rec. for

Rec. for

Rec. for

Rec. for Rec. for

Rec. for Meth. of Rec. for Meth. of $r$ Nonorganic Coatings-Definition of Terms Concerning the e During Operation at Alternative Voltage (1969/ dissolution (1972)

Std. for Mietaliic Rec. for anodic Oxidation) of Aluminium (Aluminum) and lis Alloys Std. for Leather: Physical Test. trical Properties of Electronic Tubes and Valves: Meth. of

inium Oxide Primarily Used for the Production of Aluminum: trical Properties of Electronic Tubes and Valves: Meth. of trical Properties of Electronic Tubes and Valves: Meth. of mission: General Conditions and Measur/ mission: Measurements Particularly Rel/ mission: Specialized Frequency Measure/ cast Transmissions (1954) cast Transmissions (1958) missions (1960) Rec. for Meth. of Rec. for Meth. of Rec. for Meth. of Rec. Meth. of Rec. Meth. of Rec. Meth. of ermal Expansion Coefficient (For Use in the Preparation of

Rec. for Petroleum acteristics of Auxiliary Equipment for Shock and Vibration ctronic Tubes and Valves: Precautions Relating to Meth. of
Measurement of Cathode Heating Time and Heater Warm Up Measurement of Cathode Ray Charge Storage Types (1971) Measurement of Coating Thickness-Eddy Current Meth. ( Measurement of Coating Thickness-Magnetic Meth. (1972 Measurement of Coating Thickness-Magnetic Meth. (1972 Measurement of Colour (Color) in Hazen Units (1970) Measurement of Colour (Color) (1971) Measurement of Cross Modulation in (1969) Measurement of Current Noise Generated in Fixed Elec Measurement of Density by the Pyknometer Meth. (1970) Measurement of Density (1968) Mcasurement of Density (1968) Measurement of Dimensions of Panels (1969) Sulphuric Acid for Ind ISO Measurement of Direct Interelectrode Capacitances of EL IEC Measurement of Electrode Current (1963) /Asurements of IEC Measurement of Emission Current from Hot Cathodes for $\mathrm{H}$ IEC Measurement of Equivalent Noise Resistance (1964) / of IEC

Measurement of Essential Electrical Properties of Recei Measurement of Fluid Flow by Means of Orifice Plates an Measurement of Fluid Flow by Means of Venturi Tubes-C Measurement of Fluid Flow by Means of Venturi Tubes (19 Measurement of Foaming Power (1968)

Measurement of Frequency and Equivalent Resistance of $U$ Measurement of Gasfilled Types (1969) 971) Measurement of Geiger-Muller Counter Types (1971) Measurement of lonizing Radiation by Electric Means (19 Measurement of Lamp Cap Temperature Rise (1971) Measurement of Liquid Flow by Velocity Area Meth. (1969 Measurement of Liquid Flow with a Free Surface (1968) Measurement of Metal and Oxide Coating Thicknesscs by $M$ Measurement of Noise Emitted by Vehicles (1964) Measurement of Noises Due to Mechanical or Acoustic Exc Measurement of Performance of Electric lrons for Househ Measurement of Performance of Vacuum Cleaners for House Measurement of pH Potentiometric Meth. (1968) Measurement of $\mathrm{pH}$ Value-Potentiometric Meth. (1972) Measurement of Photosensitive Devices: Basic Rec. (1969 Measurement of Photosensitive Devices: Photoconductive Measurement of Photosensitive Devices: Photomultipliers Measurement of Photosensitive Devices: Phototubes (1969 Measurement of Physical Properties of Sonic Booms (1973 Measurement of Radar and Oscilloscope Cathode Ray Tubes Measurement of Radiation from Receivers for Amplitude a Measurement of Radio Frequency Output Power (1966) Measurement of Speed Fluctuations in Sound Recording an Measurement of Spurious and Unwanted Electrode Currents Measurement of Surface Roughness by the Profile Meth.Measurement of Surface Roughness by the Profile Meth.Measurement of tbe Airborne Noise Emitted by Rotating $\mathbf{E}$ Measurement of the Angle of Repose (1968) Alum ISO Measurement of the Electrical Properties of Microwave T Measurement of the Electrical Properties of Microwave T Measurement of the Electrical Properties of Microwave $T$ Measurement of the Electrical Properties of Microwave $\mathbf{T}$ Measurement of the Electrical Properties of Microwave T Measurement of the Electrical Properties of Microwave T Measurement of the Electrical Properties of Microwave T Measurement of the Electrical Properties of Microwave T Measurement of the Mass of the Oxide Coatings-Gravime Measurement of the Performance Characteristics of Elect Measurement of the Performance Characteristics of Posit Measurement of the Performance Characteristics of Vapou Measurement of the Screen Luminance of Microfilm Reader Measurement of the Thickness (1971) /Metallic and Measurement of the Winding Resistance of an a.c. Machin Measurement of Thickness-Coulometric Meth. by Anodic Measurement of Thickness of Oxide Coatings-Nondestruc Measurement of Thickness (1972) Measurement of Untamped Density (1968) (1969) Rec for Alum ISO Measurement of Vacuum Pulse Modulator Types (1970) IEc IEC Measurement on Corona Stabilizers (1969) / of the Elec IEC Measurcment on Radio Receivers for Various Classes of $E$ IEC Measurement on Radio Receivers for Various Classes of $F$ Measurement on Radio Receivers for Various Classes of E Measurement on Receivers for Amplitude Modulation Broad Measurement on Receivers for Frequency Modulation Broad Messurement on Receivers for Television Broadcast Trans Measurement Tables for Liquids) (1970)

Measurcment Tables (1970)

Measurement (1966) Measurement (1966)
/ for Meth. for Specifying the Char IEC If the Electrical Properties of Ele IEC
151.8 
Rec. for Coniferous Sawn Timber: Sizes-Meth. of Measurement (1968)

Kec for Coniferous Sawn (Sawed) Timber-Defects - Measurement (1969)

$(1960)$ (ieneral Principles of Measuring Meth.: Reference Meth. of Rec. for Field and laboratory

Tubes and Valves: Precautions Relating to Meth/

Tubes and Valves: Measurement of Electrode Cur/

Tubes and Valves: Meth. of Measurement of Audi/

Tubes and Valves: Meth. of Measurement of Radi/

Tubes and Valves: Meth. of Measuring Electrode/

Tubes and Valves: Meth. of Measurement of Emis/

Tubes and Valves: Meth. of Measurement of Rada/

Tubes and Valves: Meth. of Measurement of $S_{p u r /}$

Tubes: Meth. of Measurement for Television Pic/

Tubes and Valves: Meth. of Measurement of Gasf/

Tubes and Valves: Meth. of Measurement of Nois/

Tubes and Valves: Meth. of Measurement on Coro/

Tubes and Valves: Heater or Filament Current (/

Tubes and Valves: Meth. of Measurement of Thyr/

Tubes and Valves: Meth. of Measurement of Cros/

Tubes: Meth. of Measurement for Cold Cathode C/

Tubes and Valves: Meth. of Measurement of Vacu/

Tubes: Meth. of Measurement of Geiger-Muller/

Tubes: Meth. of Measurement for Camera Types (/

Rec. for Measurements of the Electrical Properties of Electronic

/Stics of Semiconductor Devices and IEC

Rec. for Measurements of the Electrical Properties of Electronic

Rec. for Measurements of the Electrical Properties of Electronic

Rec. for Measurements of the Electrical Properties of Electronic

Rec. for Measurements of the Electrical Properties of Electronic

Rec for Measurements of the Electrical Properties of Electronic

Rec. for Measurements of the Electrical Properties of Electronic

Rec. for Measurements of the Electrical Properties of Electronic

Rec. for Measurements of the Electrical Properties of Electronic

Rec. for Measurements of the Electrical Properties of Electronic

Rec. for Measurements of the Electrical Properties of Electronic

Rec. for Measurements of the Electrical Properties of Electronic

Rec. for Measurements of the Electrical Properties of Electronic

Rec. for Measurements of the Electrical Properties of Electronic

Rec. for Measurements of the Electrical Properties of Electronic

Rec. for Measurements of the Electrical Properties of Electronic

Rec. for Measurements of the Electrical Properties of Electronic

Rec. for Measurements of the Electrical Properties of Electronic

Rec. for Measurements of the Electrical Properties of Electronic

Rec. for Measurements of the Electrical Properties of Electronic

Rec. for Measurements of the Electrical Properties of Electronic

Rec. for Measurements of the Electrical Properties of Electronic

Rec. for Measurements of the Electrical Properties of Electronic

Rec. for Measurements of the Electrical Properties of Electronic

Rec. for Measurements of the Electrical Properties of Electronic

Rec. Meth. for

Tubes and Valves: Meth. of Measuring the Catho/

f Hearing Aids (1959)

nd Col/ Rec. Meth. of Measurement for Radio Transmitters:

rement on Radio Receivers for Various Classes of Emission:

Rec. for Std. Reference Temperature for Industrial Length Rec. for Preferred Frequencies for Acoustical of Electro Mechanical Transducers for Shock and Vibration
$30 \mathrm{MHz}(1972)$

$300 \mathrm{Mc} / \mathrm{S}(1961)$ eak (1967)

diation (1964) Rec. for Partial Discharge Spec. for Radio Interference Spec. for Radio Interference Spec. for Radio Interference Rec. for Index of Electrica Rec. for Documentation to Be Supplied with Electroni

Rec. for Safety Requirements for Electronic International Rec. for Pressure Gauges for Instruments tional Rec. for Meters for Liquids (Other Than Water) with trical Properties of Electronic Tubes and Valves: Meth. of for Expression of the Functional Performance of Electronic International Rec. for One Mark Glass trical Properties of Electronic Tubes and Valves: Meth. of Rec. Graphical Symbols.

Rec. for Indicating Electrica Rec. for Direct Recording Electrical nal Electrotechnical Vocabulary: Scientific and Industria of Test for Wool Fibre Length Using a Single Fiber Length rs for Various Classes of Emission: General Conditions and Rec. for General Requirements and Rec. for Lever Switches: General Requirements and er Switches (Low Current Rating): General Requirements and $r$ Frequencies Below $3 \mathrm{MHz}$ : $(\mathrm{Mc} / \mathrm{S})$ General Requirements and neral Principles of Measuring Meth.: General Principles of

Rec. for Flanges for Waveguides: General Requirements and for Hollow Metallic Waveguides: General Requirements and for Radio Frequency Connectors: General Requirements and

Rec. for Sensitive Switches: General Requirements and Rec. for Push Button Switches: General Requirements and ry Variable Capacitors: General Requirements for Tests and Rec. for Radio Frequency Cables: General Requirements and res for Inductors and Transformers for Telecommunications eir Associated Flange Connectors: General Requirements and olyvinyl Chloride) Insulation and Sheath: General Test and ristics of Semiconductor Devices and General Principles of ristics of Semiconductor Devices and General Principles of ristics of Semiconductor Devices and General Principles of ristics of Semiconductor Devices and General Principles of ons Employing Similar Techniques: General Requirements and ons Employing Similar Techniques: General Requirements and and Similar Purposes (1971)

ovens (I971)

rposes (1972)

$1,000 \mathrm{MHz}(1967)$

Rec. Meth. for Rec. Meth. for

ecovery from Creasing of a Horizontally Folded Specimen by trical Properties of Electronic Tubes and Valves: Meth. of working Std. Meth. for the Verification of Instruments for Rec. for Determination of Fibre Length by
International Rec. for Instruments for

Measurements (1968)
Measurement (1970)

Measurement (1970) IStics of Semiconductor Devices and
Measurements of Airborne and Impact Sound Transmission Measurements of the Electroacoustical Characteristics O Measurements on Fm Receivers of the Response to lmpuls Measurements Particular to Transposers for Monochrome a Measurements Particularly Related to the Audio Frequenc Measurements (1951)

Measurements (1962)

Measurements (1965)

Measuring Apparatus for the Frequency Range $0.15 \mathrm{MHz}$ to

Measuring Apparatus for the Frequency Range $25 \mathrm{Mc} / \mathrm{S}$ to

Measuring Apparatus Having Detectors Other Than Quasi P

Measuring Apparatus Used in Connection with Ionizing $\mathbf{R a}$

Measuring Apparatus (1968)

Measuring Apparatus (1971)

Measuring Arterial Blood Pressure (1970)

Measuring Chambers (1970)

Measuring Electrode Resista

Measuring Flasks (1970)

Measuring Hiss and Hum (1964)

Measuring Instruments and Electric Clocks (1963)

Measuring Instruments and Their Accessories (1960)

Measuring Instruments (1958)

Measuring Machine (1970)

Several Types of Receivers

Measuring Meth for Printed Wiring Boards (1970)

Measuring Meth. (1962)

Measuring Meth. (1962)

Measuring Meth. (1962)

Measuring Meth. (1963)

Measuring Meth. (1964)

Measuring Meth. (I964)

Measuring Meth. (1965)

Measuring Meth. (1968)

Measuring Meth. (1970)

Measuring Meth. (1970)

Measuring Meth. (1971)

Measuring Meth. (1971)

Measuring Meth. (1971)

Measuring Meth. (1972)

Measuring Meth.: Essential (1966)

Measuring Meth.: Reference Meth. of Measurement (1970) IEC

Measuring Methods (1972)

Measuring Methods (1972)

Measuring Performance of Floor Polishers in Electronic

I and in Electronic Applicati IEC

Measuring Performance of Household Electric Ranges and

Measuring Performance of Water Heaters for Household Pu

Measuring Set Spec. for the Frequency Range $300 \mathrm{MHz}$ to

737

1030

147.3

140

151-0

$151-1$

$151 \cdot 10$

151-11

151.12

151.14

151.15

151.16

151.17

151.18

151.19

151.2

151.20

151.21

151.22

151.23

151-25

151.26

151-3

151.4

151.5

151.6

151.7

151.8

151-9

118

315.5

244-5

315-2

18

270

5

181

278

348

16

151.12

359

4

151-5

117.4

51

258

$50(20)$

1822

315. 1

326

13 I. 1

132.

130-1

147.2

154-1

153.

169.1

163.

341-1

334 -

$96 \cdot 1$

367.1

339.

189.1

147.

147.0

I $47-2$

I 47.3

388 .

389.1

369

350

379

Measuring the Angle of Recovery (1972) (Ation of the R ISO

Measuring the Cathode Interface Impedance (1966)

Measuring the ${ }^{\circ}$ Of Humidity of Cereal Grains (1970)

Measuring the Length of Individual Fibres (Fibers) (196

Measuring the Mass Per Hectolitre of Cereals (1970)
2313

151.9

8

270

Engineering and Product Standards Division 
h. of Cleaning (1967) Rec. Test Cells for

nt (1970)

nt (1973)

nt (1973)

t (1969)

tent (1973)

3)

terchangeability $(1969)$ lers-Interchangeability (1969) Rec. for Colour (Color) Identification of chanic/ Rec. for Graphical Symbols: Contacts, Switchgear, s Having a Specified Contact Area and Be/ Rec. for an lec interchangeability (1971) Rec. for eth.) (1969)

rs for Loose Bulk Materials (1971) ules $(1970)$

Troughed Belt Conveyors (Other Tha/ Troughed Belt Conveyors (Other Tha/ Troughed Belt Conveyors (Other Tha/ Oscillating Conveyors and Shaking /

Vibrating Feeders and Conveyors Wi/ and Unit Loads-Belt Conveyors-Ba/

Storage Equipment: Bins and Bunker/ Belt Feeders and Conveyors-Safet/ Vibrating Feeders and Conveyors, S/ Oscillating Conveyors and Shaking / Apron Conveyors (1971)

Aeroslides (1972)

Mobile Belt Conveyors-Safety Cod/ Picking Table Conveyors-Safety C/ Apron Conveyors-Safety Code (197) Scraper and En Masse Conveyors Hydraulic Conveyors-Safety Code / Vibrating Conveyors and Feeders Wit/ Screw Conveyors (1969)

elt Conveyors (Canvas, Rubber, Plast/ Monorail Chain Conveyors-Safety C/ Twin Rail Chain Conveyors (Power An/ trand Floor Mounted Truck Conveyors/ trand Floor Truck Conveyors (Chain B/ resilient Devices) (1972)

ic Tubes and Valves: Meth. of Measurement of Noises Due to Plastics-Rec. Pract. for the Determination of Change of

Types of Winding Wires: Enamelled Round Copper, with High ews and Studs (1968)

crews, Studs and Nuts (1969)

Proof Load Values (1969) of Mannesium Aluminium (Aluminum) Zinc Alloy Castings ectrodes $3.15 \mathrm{~mm}$ or $\mathrm{M} /$ Rec. for Meth. for Determining the Rods for Gas/ Rec. for Meth. of Test for Determining the Heat or Solvent Bonding Enamelled Round Copper, with High

Types of Winding Wires: Enamelled Round Copper, with High for Aluminium (Aluminum) Alloys-Sand Cast Test Pieces (1968) Std. for Ropes-Determination of Certain Physical and minium (Aluminum) and Its Alloys (1971) (Aluminum) and Its Alloys (1968)

nium (Aluminum) and Its Alloys (1968) Rec. for Rec. for Rec. for Rec. for es of Electronic Tubes and Valves: Meth. of Application of for Synthetic Rubber Latices-Determination of High Speed Std. for Natural Rubber Latex-Determination of

mensions (1970)

eparation of Drawings Devices (1969) ration Electrodes (1968) Rec. for Special Meth. of c. for Meth. for Specifying the Characteristics of Electro and Dimensions of the Cross Section of Assembled Bars for Rec. for Closed End Drop Wires (Electrical and Rec. for Quantities and Units of

Rec. for Conical Fittings for Syringes, Needles and Other ation Pur/ Rec. for Yoke Type Valve Connections for Small Rec. for Identification of the Contents of Rec. for Syringes for Rec. for Transfusion Equipment for

Std. for Wool: Determination of Percentage of Rec. for Plastics-Determination of the Rec. for Plastics-Determination of the Rec. for Plastics-Determination of the
Measuring the Mean Diameter of Wool Fibres (Fibers) (19 Measuring the Noise Emitted by Machines (1966)

Measuring the Resistivity of lnsulating liquids and Met Measuring, with Nonspecified or lndependent Specified T Meat and lts Products-Detcrmination of Chloride Conte Meat and Its Products-Determination of Free Fat Conte Meat and lts Products-Determination of Moisture Conte Meat and Its Products: Determination of Ash (1969) Meat and Its Products: Determination of Nitrogen Conten Meat and Meat Products-Determination of Total Fat Con Meat Products-Determination of Total Fat Content (197 Mechanical and Electrical Warp Stop Motions (1965) Mechanical and Performance Properties (1972)

Mechanical Connections Between Towing Vehicles and Trai Mechanical Control Circuits for Aircraft (1959) Mechanical Controls, Starters and Elements of Electrome Mechanical Coupler for the Calibration of Bone Vibrator Mechanical Coupling Between Tractors and Semi Trailers Mechanical Design Features (1971)

Mechanical Fastenings for Conveyor Belts (Static Test M Mechanical Handling Equipment-Light Duty Belt Conveyo Mechanical Handling Equipment-Safety Code-General R Mechanical Handling Equipment for Loose Bulk Materials Mechanical Handling Equipment for Loose Bulk Materials Mechanical Handling Equipment for Loose Bulk Materials Mechanical Handling Equipment for Loose Bulk Materials Mechanical Handling Equipment for Loose Bulk Materials Mechanical Handling Equipment for Loose Bulk Materials Mechanical Handling Equipment for Loose Bulk Materials Mechanical Handling Equipment for Loose Bulk Materials Mechanical Handling Equipment for Loose Bulk Materials Mechanical Handling Equipment for Loose Bulk Materials
Mechanical Handling Equipment for Loose Bulk Materials Mechanical Handling Equipment for Loose Bulk Materials Mechanical Handling Equipment for Loose Bulk Materials Mechanical Handling Equipment for Loose Bulk Materials Mechanical Handling Equipment for Loose Bulk Materials Mechanical Handling Equipment for Loose Bulk Materials Mechanical Handling Equipment for Loose Bulk Materials Mechanical Handling Equipment for Loose Bulk Materials Mechanical Handling Equipment for Loose Bulk Materials: Mechanical Handling Equipment for Loose Bulk Materials: Mechanical Handling Equipment for Unit Loads-Mobile B Mechanical Handling Equipment for Unit Loads-Overhead Mechanical Handling Equipment for Unit Loads-Overhead Mechanical Handling Equipment for Unit Loads-Single S Mechanical Handling Equipment for Unit Loads-Single S Mechanical Isolation (Guide for Selecting and Applying Mechanical or Acoustic Excitations (1968)

Mechanical Properties After Contact with Chemical Subst Mechanical Properties for Use in Refrigerant Systems (1 Mechanical Properties of Fasteners-Part 1: Bolts, Scr Mechanical Properties of Fasteners: Marking of Bolts, S Mechanical Properties of Fasteners: Nuts with Specified Mechanical Properties of Fasteners: Nuts with Specified Mechanical Properties of Sand Cast Reference Test Bars

Mechanical Properties of the Weld Metal Deposited by EL

Mechanical Properties of Weld Metal Deposited by Filler Mechanical Properties (1970)

Mechanical Properties (1970)

Mechanical Properties (1971)

Mechanical Properties (1972)

Mechanical Property Limits for Aluminium Alloy Forgings

Mechanical Property Limits for Extruded Products of Alu

Mechanical Property Limits for Rivet Stock of Aluminium

Mechanical Property Limits for Rolled Products of Alumi Mechanical Shock (Impulse) Excitation to Electronic Typ Mechanical Stability (1971)

Mechanical Stability (1972)

Mechanical Standardization of Semiconductor Devices: Di

Mechanical Standardization of Semiconductor Devices: $\mathrm{Pr}$ Mechanical Test. of Steel at Elevated Temperatures: Det Mechanical Test. to Determine the Coding for Deep Penet Mechanical Transducers for Shock and Vibration Measurem Mechanical Warp Stop Motions (1971) /for Serrated Bars Mechanical) for Automatic Drawing-in Machines (1969) Mechanics (1960)

Medical Equipment: Definition and DimensionaI Character Medical Gas Cylinders Used for Anaesthetic and Resuscit Medical Gas Cylinders (1957)

Medical Use (1967)

Medical Use (1969)

Medullated Fibres (Fibers) by the Projection Microscope

Melt Flow Index of Polyethylene and Compounds of (1967)

Melt Flow Rate of Thermoplastics (1969)

Melting Point of Polyamides (1970)

Mercurimetric Meth. (1972) Std. for Sodium Hydrogen Car

2. ISO 
te for Industrial Use: Determination of Chloride Content te for Industrial Use: Determination of Chloride Content al Use: Determination of llalogens, Expressed as Chlorine Rec. for Meth. of Rec. for

Rec. for Schedule for High Pressure Rec for Ballasts for High Pressure al Use-Determination of Bromine Index in the Presence of Rec. for Denta International Rec. for Clinical Thermometers Rec. for Fishing Nets-Meth. of Determining the Basic Mode Control Procedures: Conversational Information use of Longitudinal Parity to Detect Errors in Information c. for Cresylic Acid for Industrial Use-Determination of use-Determination of Densit/ $\mathbf{n}$ of Residue on Evaporation (197/ n of Crystallizing Point (1971) ndustrial Use-Determination Of/ ndustrial Use-Determination Of/ ndustrial Use-Determination Of/

Rec. for Liquefied Phenol: Rec. for Phenol: Ortha, Rec. for Phenol: Ortha, Rec. for Phenol: Ortho, Rec. for Phenol: Ortha, Rec. for Phenol: Ortha, n $0.2 \mathrm{~mm}(0.008 \mathrm{In}$.

Rec. for Tensile Test. of Light Rec. for Simple Bend Test for Light Rec. for Drift Expanding Test on Ligh

Rec. for Tensile Test for Ligh Rec. for Measurement of amination of Cross Sections (197/ rec. for Code of Symbols for Covered Electrodes for Manua pools, Rims and $\mathrm{Col}$

Rec. for Solid Wires for Gas Shielded rns (I966)

I970)

th. (1968)

Std. for Woodworking Tools: Rec. for Perforated Rec. for eth. for Determining the Mechanical Properties of the Weld of Test for Determining the Mechanical Properties of Weld s (I972) Rec. for High Voltage Std. for Hermetically Sealed Rec. for Hermetically Sealed mine the Compatibility of Steel Filler Rods and the Parent Rec. for Dobby Lags and Pegs in Wood, nized Rubber to Metal Where the Rubber Is Assembled to One canized Rubber to Metal Where the Rubber Is Assembled to 2 for Covered Electrodes-Determination of the Efficiency, Rec. for Rec. for

kV Up to / Rec. for Tests on Impregnated Paper Insulated g Voltages/ Rec. for Tests on Impregnated Paper Insulated as Pressure Cables and Their Accessories: Paper Insulated, Series (I972)

of Iron and Steel Against Corrosion (1971) Std. for $s$ for Industrial Application for Plain End Steel and Other and Meth. of Test of the Adhesion of Vulcanized Rubber to and Meth. of Test of the Adhesion of Vulcanized Rubber to of Pellets and Cases (I966) Rec. for Hard Test for Determining the Characteristics of the Deposited ion Tests on Coatings Other Than Those Anodic to the Basis terms Concerning the Measurement of the Thickne/ Rec. for agnetic Basis Metals-Measurement / Std. for Nonmagnetic Rec. for Tests on Anticorrosion Protective Coverings of 7I) Rec. for Surface Treatment and ric Meth. by Anodic Dissolution (1972) Std. for Rec. for Test Meth. for Batch Furnaces with rmination of Metallic Iron Content: (Photometric Meth. for of Chemical Analysis of Manganese Ores: Determination of

Meth. (I964) Rec. for Tables of Vickers Hardness Values (HV) for tangular (I968)

tangular Types (1967)

gular Types (I964)

ent (I959)

nt (1965)

loys-Check of Continuity/ loys-Estimation of the $\mathrm{L} /$ luminum) and Its Alloysluminum) and Its Alloysys-Determination of the

t M/ Std. for Nonconductive Coatings on Nonmagnetic Basis nd Vitreous or Porcelain Enamel Coatings on Magnetic Basis Rec, for Ligh Rec. for Light Rec. for Light from 1 to $100 \mathrm{kgf}$ (I97I) Rec. for Light Rec. for Tensile Test. of Light olerances for Electrodes for Manual Arc Welding and Filler - Marking of Rolled, Drawn and Extruded Products in Light ldehyde Solutions for Industrial Use: Limit Test for Heavy Rec. for General Principles for Fatigue Test. of
Mercurimetric Meth. (1973)

Mercurimetric Meth. (1973)

Mercurimetric Meth. (1973)

Mercurous Nitrate Test for Copp

Mercury Arc Convertors (1957)

Mercury Vapour (Vapor) Lamps (1965)

Mercury Vapour (Vapor) Lamps (1969)

Mercury.(II) Chloride (1970)

Mercury (1970)

Mercury, in Glass, with Maximum Device) (1970)

Mesh Breaking Load of Netting (1970)

Message Transfer (1973)

Messages (1969)

Meta Cresol Content (1971)

Std. for Sodium Chlora ISO

Std for Potassium Sulpha ISO

Dium Chloride for Industri ISO

ISO

IEC

IEC

IEr Alcohols for Industri ISO

ISO

ISO

Std. for ISO

resylic Acid and Xulenols for Industrial

Meta, and Para Cresol for Industrial Use-Determinatio

Meta, and Para Cresol for Industrial Use-Determinatio

Meta, and Para Cresol, Cresylic Acid and Xylenols for I

Meta, and Para Cresol, Cresylic Acid and Xylenols for

Meta, and Para Cresol, Cresylic Acid and Xylenols for

Metal Ad Its Alloy Tubes (1969)

Metal and Its Alloy Sheet and Strip of Thickness Betwee

Metal and Its Alloy Tubes (1969)

Metal and Its Alloy Wires (1969)

Metal and Oxide Coating Thicknesses by Microscopical Ex

Metal Arc Welding of Cast Iron (1969)

Metal Arc Welding of Mild Steel: Dimensions of Wires, S

Metal Bodied Planes (1973)

Metal Cheese Centres (Center) for Bast Fibre (Fiber) Ya

Metal Clad Base Materials for Printed Circuits: Spec. (

Metal Clad Base Materials for Printed Circuits: Test Me

Metal Deposited by Electrodes $3.15 \mathrm{~mm}$ or More in Diamet

Metal Deposited by Filler Rods for Gas Welding Mild Ste

Metal Enclosed Switchgear and Controlgear (1969)

Metal Food Containers-Internal Diameters of Round Can

Metal Food Containers (1959)

Metal in the Welding of Steels (I968)

Metal or Other Suitable Material (1967)

Metal Plate (1968)

1. of Test of the Adhesion of Vulca ISO

Metal Plates (1968)

H of Test of the Adhesion of Vul ISO

Metal Recovery and Deposition Coefficient (1972) Std. ISO

Metal Reeds with Double Spring Baulk (I967)

Metal Reeds with Plate Baulk (1967)

Metal Sheathed Cables: for Alternating Voltages from 10

Metal Sheathed Cables: Nondraining Types for Alternatin

Metal Sheathed, for Alternating Voltages Up to $275 \mathrm{kV}$ (

Metal Slitting Saws with Fine and Coarse Teeth-Metric

Metal Spraying of Zinc and Aluminum for the Protection

Metal Tubes (I970)

Metal Where the Rubber Is Assembled to 2 Metal Plates (

Metal Wire Drawing Dies: Interchangeability Dimensions

Metal (1968)

Metal (1970)

/ Filler Rods for Braze Welding: Meth. of

Metallic and Other Nonorganic Coatings-Definition of

Metallic and Vitreous or Porcelain Enamel Coatings on M

Metallic Cable Sheaths (1966)

Metallic Coatings-General Classification of Terms (19

Metallic Coatings-Measurement of Thickness-Coulomet

Metallic Heating Resistors (1972)

Metallic Iron Content Up to and Including 2\%) (1967)

Metallic Iron Content: (Photometric Meth. for Metallic

Metallic Materials (1964)

Metallic Waveguides: General Requirements and Measuring

Metallic Waveguides: Relevant Spec. for Medium Flat Rec

Metallic Waveguides: Relevant Spec for Medium Flat Rec

Metallic Waveguides: Relevant Spec. for Ordinary Rectan

Metallized Mica Capacitors for Use in Electronic Equipm

Metallized Paper Dielectric Capacitors for Direct Curre

Metals-Anodisation of Aluminium (Aluminum) and Its Al

Metals-Anodisation of Aluminium (Aluminum) and Its Al

Metals-Anodisation (Anodic Oxidation) of Aluminium (A

Metals-Anodisation (Anodic Oxidation) of Aluminium (A

Metals-Coloured Anodisation of Aluminium and Its Allo

Metals-Measurement of Coating Thickness-Eddy Curren

Metals-Measurement of Coating Thickness-Magnetic Me

Metals and Their Alloys-Brinell Hardness Test (1971)

Metals and Their Alloys-Code of Designation (1971)

Metals and Their Alloys-Temper Designation (1971)

Metals and Their Alloys-Vickers Hardness Test (Loads

Metals and Their Alloys (1961)

Metals for Gas Welding (1967)

Metals or in Light Alloys (1960)

/Ec. for Diameters and T

Metals (Excluding Iron) (1972)

Shipbuilding Details Std. for Forma ISO

Metals (1968)

/Lding: Meth. of Test for Determining Th 
als at Power, Audio and Radio Frequencies Including Metre (Meter) Wavelengths (1969) -iric Series, Dimensions, Tolerances and Masses Per Metre Rer. for Indirect Reading Capacitor Type Pocket Exposure Rec. for Portable X.. or Gamma Radiation Exposure Rate ambers (1970) Rec. for Alpha, Beta and Alpha.Beta Contamination International Rec. for Rec. for Alternating Current Watthour Rec. for Sound Level Ree. for Var Hour (Reactive Energy) Rec. for Class 1.0 Alternating Current Watthour Rec. for Precision Sound LeveI Rec. for Class 0.5 Alternating Current Watthour national Rec. for General Prescriptions for Volumetric Gas Rec. for Direct Reading Pocket Type Electroscope Exposure Rec. for Symbols for Alternating Current Electricity ormaldehyde Solutions for Industrial Use: Determination of Rec. for Std. for Aircraft Water Rec for Plastics-Determination of m Sulphate for Industrial Use: Determination of Acidity to Rec. for Spec. for Photographic Grade P Rec. for -Meth. of Test (1970)

Materials at Power, Audio and Radio Frequencies Including ubes: Metric Series, Dimensions, Tolerances and Masses Per Rolling Bearings-Tapered Roller-Boundary Dimensions ter $(1962)$ ensions for Milling Cutters and Their Arbors or Mandrels eter $(1968$

Rec. for Clearance Holes for

or Warp Tubes for Ring Spinning and Ring Doubling Spindles Kec. for ISO General Purpose Std. for ISO General Purpose Std. for ISO General Purpose and Nuts (1973) Rec. for ISO General Purpose Rec. for ISO General Purpose Rec. for ISO General Purpose Rec. for ISO General Purpose ommercial Bolt and Nut Thre/ data (1969)

earings: Tapered Roller-Boundary Dimensions-Subunits arings-Tapered Roller-Boundary Dimensions-Subunits r Rolling Bearings-Needle Roller-Boundary Dimensions of Hot Rolled Steel Sections-Part I: Equal Leg Angles f Hot Rolled Steel Sections-Part 2: Unequal Leg Angles Rolling Bearings-Tapered Roller-Boundary Dimensions

screws and Nuts-Forged and and Tubular Socket Wrenches earings: Tapered Roller-Boundary Dimensions-Subunits Rec. for Rolling Bearings-Tapered; inyl Chloride (PVC) Pipes for the Supply of Gaseous Fuels: Std. for Rolling Bearings: Tapered Roller

and Housing Fil/ Std. for Rolling Bearings: Tolerances;

ers of Wire (1964)

$$
\text { Rec. for Rolling Bearings: Tapered . }
$$

Fluids (Outside Diameters and Nominal Pressures): Part I:

Rec for Carbide Tips for Turning Tools:

Rec. for Turning Tools with Carbide Tips:

Rec. for Copper Tubes of Circular Section: Dimensions; for Domestic and Industrial Waste Pipe: Basic Dimensions;

i) Radial Ball and Roller (II) Thrust (III) Tapered Type .

Rec. for Hexagon Socket Head Cap Screws:

Rec. for Washers for Hexagon Bolts and Nuts:

d Face Plates: Bayonet Type; Sizes for Interchangeability:

radii Under the Head for General Purpose Bolts and Screws:

ized Polyvinyl Chloride (PVC) Fittings with Plain Sockets;

idths Across Flats, Heights of Heads, Thicknesses of Nuts:

Rec. for Dimensions of Hot Rolled Steel Bars: Round,

Rec. for Dimensions of Hot Rolled Steel Bars: Square,

Rec. for Dimensions of Hot Rolled Steel Bars: Flat,

arings-Tapered Roller-Boundary Dimensions-Subunits

Rec. for Slotted Cheese (Fillister) Head Screws Rec. for Hexagon Head Tapping Screws Rec. for Slotted Pan Head Screws Rec. for Split Pins

Cylinders-Internal Diameters and Piston Rod Diameters Std. for Metal Slitting Saws with Fine and Coarse Teeth Std for Clevis Pins

Std for Clevis Pins with Heads

Sid. for Slotted Headless Screws Std. for Hexagon Socket Set Screws

td. for Cylindrical Cutters with Plain Bore and Key Drive: Std, for Slotting Cutters with Plain Bore and Key Drive: for Prevailing Torque Type Hexagon Locknuts: Dimensions de and Face Milling Cutters with Plain Bore and Key Drive:

cket Fittings for Pipes Under Pressure-Basic Dimensions:

Std. for Shell End Mills with Plain Bore and Tenon Drive:

Drive: Interchangeability Dimensions with Cutter Arbors

Rec. for Turning Tools with Carbide Tips: Internal Tools

Rec. for Turning Tools with Carbide Tips: Internal Tools (Metric Series) (1966)
yor Chains, Attachments and Chain Wheels-Part I: Chains (Meiric Series) (1971)

Metric Series (1969)

Metric Series (1972)
/Lectrical Insulating Materi IEC

Meter) (1967)

Meters and Accessory Electrometers (1971)

Meters and Monitors for Use in Radiological Protection

Meters and Monitors (1970)

Meters for Liquids (Other Than Water) with Measuring Ch

Meters (1960)

Meters (1961)

Meters (1963)

Meters (1964)

Meters (1965)

Meters (1968)

Meters (1970)

Meters (1971)

Meters (1972)

Methanol Content (1972)

Methanol for Industrial Use-Meth. of Test (1970)

Methanol Pressure Connections (1973)

Methanol Soluble Matter in Polystyrene (1959)

Methyl Orange (1973)

Methylaminophenol Sulphate (1965)

Methylene Chloride (Dichloromethan

ne) for Industrial Use

Metre (Meter) (1967)

/for Cold Drawn Precision Steel T ISO

Metric-Diameter Series 9 and 0 (1967)

Rec. for ISO

ISterchangeability Dim

Metric Bolts 1,6 Up to and lncluding $39 \mathrm{~mm}$ Thread Diame ISO

Metric Bolts, $42 \mathrm{Up}$ to and Including $150 \mathrm{~mm}$ Thread Diam ISO

Metric Dimensions, Tolerances and Gauges (1964) /Ec. F ISO

Metric Screw Threads-Gauging (1970)

Metric Screw Threads-General Plan (1973)

Metric Screw Threads-Selected Sizes for Screws, Bolts

Metric Screw Threads: Basic Dimensions (1968)

Metric Screw Threads: Tolerances, Deviations for Constr

Metric Screw Threads: Tolerances, Limits of Sizes for C

Metric Screw Threads: Tolerances, Principles and Basic

Metric Series-Dimension Series 29 and 13 (1973)

Metric Series-Dimension Series 30, 31 and 32 (1970)

Metric Series-Dimension-Series 48 and 49 (1971)

Metric Series-Dimensions and Sectional Properties (19

Metric Series-Dimensions and Sectional Properties (19

Metric Series-Dimensions Series 31 and 32 (1970)

Metric Series-Maximum Outside Dimensions (1972)

Metric Series-Outer Rings with Flange (1973)

Metric Series-Part I: Normal Tolerances (1967)

/Ried Unplasticized Polyv ISO

Metric Series-Spec. (1973)

Metric Series-Tolerances-Class 4 (1973)

Metric Series Chamfer Dimension Limits and Maximum Shaf ISO

Metric Series for Basic Thicknesses of Sheet and Diamet ISO

Metric Series Tolerances Classes 6 and 5 (1968)

Metric Series (1960)

IS Materials for the Transport of ISO

Metric Series (1961)

Metric Series (1961)

Metric Series (1962)

Metric Series (1962)

Metric Series (1963)

Metric Series (1968)

Metric Series (1968)

Metric Series (1968)

Metric Series (1968)

Metric Series (1968)

Metric Series (1968)

Metric Series (1969)

Mctric Series (1969)

Metric Series (1969)

Metric Series (1970)

Metric Series (1970)

Metric Series (1970)

Metric Series (1971)

Metric Series (1971)

Metric Series (1972)

Metric Series (1972)

Metric Series (1972)

Metric Series (1972)

Metric Series (1972)

Metric Series (1972)

Metric Series (1972)

Metric Series (1972)

Metric Series (1972)

Metric Series (1972)

Metric Series (1973)

Metric Series (1973)

ISO

/Socket Fittings with Spigot Ends ISO

for Roller Bearings: Groups-( ISO

Rec. for Spindle Noses an ISO

Rec. for Bolts and Screws: 150

I Pipes Under Pressure: Unpłastic ISO

/C. for Hexagon Bolts and Nuts: W ISO

ISO

ISO

ISO

ISO

ISO

ISO

Rec. for Hydraulic ISO

ISO

ISO

ISO

ISO

ISO

ISO

ISO

ISO

Std. ISO

Rec. for SI ISO

/Tings of Plastics Materials-So ISO

ISO

1. for Milling Cutters with Tenon ISO

$1 \mathrm{SO}$

Rec. for Conve ISO
Rec. for Rolling Be ISO 
tre $(M+\operatorname{ter})(1968)$
ane

72)

meter (1969)

ines $(1972)$ Rec. for Tolerances on Spanner Gaps and Sockets of the Decimal Multiples and Submultiples of the SI Units

Equipment (

Pull Coupli/

ng (1966)

receivers (1)

wer Supply T/

d Equipment /

a Contact S/

(Center) Guil

ade Contacts/

ts $(1966)$

962)

972)

(1958)

Size (1965)

International Rec for

International Rec, for Vocabulary of Legal Rec. for Connectors for Frequencies Below 3 Rec. for Connectors for Frequencies Below 3 Rec. for Connectors for Frequencies Below 3 Rec. for Connectors for Frequencies Below 3 Rec. for Connectors for Frequencies Below 3 Rec. for Connectors for Frequencies Below 3 Rec. for Connectors for Frequencies Below 3 Rec. for Connectors for Frequencies Below 3 Rec. for Connectors for Frequencies Below 3 Rec. for Connectors for Frequencies Below 3 Rec. for Connectors for Frequencies Below 3 Rec. for Connectors for Frequencies Below 3 Rec. for Connectors for Frequencies Below 3 Std. for Muscovite Rec. for Muscovite Rec. for Phlogopite Rec. for Receiver Type Metallized
pec. for Insulating Materials Based on Built Up or Treated Std for Coding of Character Sets for

Rec. for Determination of Nitrogen in Coal by the Semi ility Tests, Description and Use of the ISO Micromire (Iso or Letter Symbols for Semiconductor Devices and Integrated

he ISO Micromire (ISO Micro Test Object) for $\mathrm{Ch} /$ Rec. for Terms Relating to

ire (ISO Test Object) and Its Use in Photograph/

I Exchange (1961)

film Readers (1968) Rec. for Rec. for Rec. for Rec. for rec. for Microcopy: Measurement of the Screcn Luminance of Rec. for Essential Characteristics of $35 \mathrm{Mm}$ Rec. for Microcopies: Scale of $35 \mathrm{Mm}$ Rec. for $35 \mathrm{~mm}$ and $16 \mathrm{Mm}$

of Steels (1967) Rec. for rocopies: Legibility Tests, Description and Use of the Iso

for Textiles-Cotton Fibres (Fibers)-Determination of ion Meth. for Pressure Calibration of 1 In. Std. Condenser ified Meth. for Pressure Calibration of One Inch Condenser Rec. for Sound System Equipment:

r Determination of Wool Fibre (Fiber) Diameter: Projection ide Coatings-Nondestructive Measurement by Light Section percentage of Medullated Fibres (Fibers) by the Projection for Measurement of Metal and Oxide Coating Thicknesses by Rec. for Designation of the Rec. Guide for the Drafting of Spec. for rical Properties of Microwave Tubes and Valves: Gas Filled s: Machines, Transformers, Primary Cells and Accumulators:

bes/ Rec. for Measurement of the Electrical Properties of

ing/ Rec. for Measurement of the Electrical Properties of Rec. for Measurement of the Electrical Properties of Rec. for Measurement of the Electrical Properties of Rec. for Measurement of the Electrical Properties of Rec. for Measurement of the Electrical Properties of Rec. for Measurement of the Electrical Properties of Rec. for Measurement of the Electrical Properties of Rec. for Determination of the change in Electrical Properties of Polyethylene Due to the Rec. for Plastics-Determination of Straight Manual Arc Welds Made with Covered Electrodes of ic/

Std. for Covered Electrodes for Manual Arc Welding of or Lengths and Tolerances of Electrodes for the Welding of ec. for Code of Symbols for Filler Rods for Gas Welding of Code of Symbols for Covered Electrodes for Arc Welding of ies of Weld Metal Deposited by Filler Rods for Gas Welding rec. for Solid Wires for Gas Shielded Metal Arc Welding of 1970)

1970 Rec. for $1970)$ Rec. for Dried Rec. for Evaporated Milk and Sweetened Condensed

t Content (Reference Meth.) (1970) Rec. for Evaporated rometers for the Determination of the Percentage of Fat in Rec. for Meth. of rec. for Tapping Screw Thread-Dimensions in Millimetres otted Pan Head Tapping Screws-Dimensions in Millimetres nk (Flat) Head Tapping Screws-Dimensions in Millimetres
nk (Oval) Head Tapping Screws-Dimensions in Millimetres
Metric Series, Dimensions, Tolerancec and Masses Per Me Metric Series, Tolerances on Widthe $A$ cross Flats and Co Metric Tapers, with Parallel and Mo:se Taper Shanks (19 Metric Thread (1963)

Metric Thread, $42 \mathrm{Up}$ to and Including $100 \mathrm{~mm}$ Thread Dia (Metric Unit) and of Space and Time (1965)

(Metric Values for General Use) (1968) /Ional System of Units and a Selection

Metrological Regulations for Nonautomatic Weighing Mach Metrology (Fundamental Terms) (1969)

MHz: Battery Connectors (1965)

MHz: Circular Connectors for Radio and Associated Sound MHz: Circular Multipole Connectors with Bayonet or Push MHz: Circular Multipole Connectors with Threaded Coupli $\mathrm{MHz}$ : Concentric Connectors for Audio Circuits in Radio MHz: Connectors for Coupling an External Low Voltage Po MHz: Connectors for Radio Receivers and Associated Soun MHz: Edge Socket Connectors with Closed Ends and Havin MHz: Edge Socket Connectors with Onen Ends, Off Centre MHz: Guide to Drawing Information in Detail Spec. (1970

MHz: Rectangular Miniature Multipole Connectors with Bl MHz: Rectangular Multipole Connectors with Blade Contac MHz: (Mc/S) General Requirements and Measuring Meth. (1 Mica Blocks, Thins and Films-Visual Classification (1

Mica Blocks, Thins and Films Meth. for Grading by Size

Mica Blocks, Thins and Splittings: Meth. for Grading by

Mica Capacitors for Use in Electronic Equipment (1959)

Mica Paper-Part 1: Definitions and General Requiremen

MICR and OCR (1972)

Micro Kjeldahl Meth. (1963)

Micro Test Object) for Checking a Reading Apparaius (19

Microcircuits (1969)

Microcopies and Their Bases (1962)

Microcopies on Transparent Bases Sizes of Rec. Bases (1

Microcopies: Legibility Tests, Description and Use of T

Microcopies: Legibility Tests; Description of the ISO M

Microcopies: Scale of $35 \mathrm{~mm}$ Microfilms for Internationa

Microcopy A pparatus (1964)

Microcopy: Measurement of the Screen Luminance of Micro

Microfilm Readers (1968)

Microfilm Reading Apparatus (1965)

Microfilms for International Exchange (1961)

Microfilms, Spools and Reels (1969)

Micrographic Determination of the Austenitic Grain Size

Micromire (ISO Micro Test Object) for Checking a Readin

Micronaire Value (1972)

Microphones by the Reciprocity Technique (1971)

Microphones by the Reciprocity Technique (1972)

Microphones (1972)

Microscope Meth. (1960)

Microscope (1971)

Microscope (1973)

Rec Fo ISO

Rec. Fo ISO

Ys-Measurement of Thickness of Ox ISO
Sid. for Wool: Determination of ISO

Microscopical Examination of Cross Sections (1970) /C. ISO

Microstructure of Graphite in Cast Iron (1969)

Microwave Ferrites (1972)

Microwave Switching Devices (1972)

Ement of the Elect IEC

Microwave Technology (1968) Rec. for Graphical Symbol IEC

Microwave Tubes and Valves: Backward Wave Oscillator Tu IEC

Microwave Tubes and Valves: Gas Filled Microwave Switch IEC

Microwave Tubes and Valves: General (1972)

Microwave Tubes and Valves: High Power Klystrons (1972)

Microwave Tubes and Valves: Low Power Oscillator Klystr

Microwave Tubes and Valves: Magnetrons (1972)

Microwave Tubes and Valves: Terminology (1972)

Microwave Tubes: Disk Seal Tubes (1972)

Micum Indices of Coke (1967)

Migration of of Plasticizers (1970)

Migration of Plasticizers from Plastics (1961)

Mild and Low Alloy High Tensile Steels (1967)

Mild and Low Alloy Steels-Code of Symbols for Identif

Mild Steel and Low Alloy High Tensile Steel (1967)

Mild Steels and Low Alloy IHigh Tensile Types (1967)

Mild Steels and Low Alloy High Tensile Types (1967)

Mild Steel: Dimensions of Wires, Spools, Rims and Coils

Milk-Determination of Fat Content (Reference Meth.) (

Milk-Determination of Fat Content (Reference Meth.)

Milk-Determination of Fat Content (Reference Meth.) (

Milk and Its Products: Sampling (1969)

Milk and Sweetened Condensed Milk-Determination of Fa

Milk by the Gerber Meth. (1966)

Milk Recording of Cows (1970)

(Millimeters) and In./S (1970)

(Millimeters) and In./S (1970)

(Millimeters) and In./S (1970)

(Millimeters) and In./S (1970)
Iso

ISO

ISO

SO

ISO

ISO

IEC

IEC

IEC

IEC

IEC

IEC

IEC

IEC

IEC

ISO

ISO

ISO

IEC

ISO

ISO

IEC

ISO

ISO

ISO

ISO

ISO

ISO

ISO

ISO

Std ISO

EC

EC

137

2128

2647

1463

945

392

235-7

117-11

$235-8$

235-7

235.2

235-6

235-5

235-4

235-1

235-3

556

1627

177

598

2560

547

636

636
635

637

864

1211

1736

1737

707

1737

1546

1478

1481

1482

1483 
sion of Toleranced Dimensions from $1 \mathrm{n}$./S into Millimetres for (Cylindrical Shafts) Nominal Dimensions in Millimetres d Their Corresponding Keyways (Dimensions in Millimetres) d Their Corresponding Keyways (Dimensions in Millimetres) Rec. for Tapping Screw Thread-Dimensions in

Rec. for Slotted Pan Head Tapping Screws-Dimensions in ted Countersunk (Flat) Head Tapping Screws-Dimensions in sed Countersunk (Oval) Head Tapping Screws-Dimensions in

c. for Conversion of Toleranced Dimensions from In./S into ded Splines (For Cylindrical Shafts) Nominal Dimensions in ut Gib Head and Their Corresponding Keyways (Dimensions in rallel Keys and Their Corresponding Keyways (Dimensions in nd In. Series/ Rec. for Interchangeability Dimensions for eries (1972) mensions with Cutter Arbors-Metric Series (19/ Std. for Rec. for Recommended Range of Outside Diameters for Rec. for Milling Machine Arbors with 7/24 Tapers and ntal or Vertical Spindle-1

Horizontal or Vertical Spi/ td. and Long Series (1970) 973)

72)

by the Calorimetric Bomb Meth. and Calcul/ Rec. for Test Conditions for Rec. for Test Conditions for Rec. for End

Std. for Shell Find Std. for End Rec. for Solid Rec. for Vocabulary of Terms Relating to Solid Rec. for Vocabulary of Terms Relating to Solid Rec. for Vocabulary of Terms Relating to Solid Rec. for Determination of Ash of Solid

Rec. for Fruit and Vegetable Products: Determination of

hydrocarbon Solvents (1972)

Rec. for Determination of Std. for

poses (1968) Rec. for Fuse Holders for Spec. for Spec, for Cartridge Fuse Links for Rec. for Cartridge Fuse Links for c. for Connectors for Frequencies Below $3 \mathrm{MHz}$ : Rectangular Rec. for Iso

Rec. for Chemical Analysis of Cements: for Microcopies: Legibility Tests; Description of the Iso rec. Graphical Symbols: Block Symbols for Transmission and control of Machines: Coding of Preparatory Functions $G$ and Rec. for

res for Electronic Components and Equipment: Test Ka: Salt stance of Glass to Attack by a Boiling Aqueous Solution of or Determination of Chlorine in Coal and Coke Using Eschka Rec. for Textiles-Binary Fibre (Fiber)

s MechanicaI Handling Equipment for Loose Bulk Materials Continuous Mechanical Handling Equipment for Unit Loads p (1960) Rec. for International Code for Rec. for

tural and Artificial)-Determination of Fluorine Conten : de for Industrial Use: Determination of Fluorine Content ntrolling Coordinating Dimensions (1970) Rec. for dential Buildings (1970)

970)

$s(1973)$ Rec. for Choosing Std. for

Rec. Meth of Measurement on Receivers for Amplitude Rec. Meth. of Measurement on Receivers for Frequency electronic Tubes and Valves: Meth. of Measurement of Cross of Measurement for Radio Transmitters: Wanted and Unwanted nt of Radiation from Receivers for Amplitude and Frequency nic Tubes and Valves: Meth. of Measurement of Vacuum Pulse

Tubes and Valves: Meth. of Measurement of Thyratron Pulse Rec. for Modular Coordination: Basic

general Engineering (1966)

Rec. for Preferred general and Heavy Engineering (1966)

or General and Heavy Engineering (1968) Rec. for Rec. for e Shear Test Piece) (1971) Rec. for Determination of Rec. for Pure Expanded Corkboard-Determination of the arative Tracking Index of Solid Insulating Materials Under Rec. for Dextrose-Determination of Rec. for Crude Vegetable Oils and Fats: Determination of Rec. for Oleaginous Seeds: Determination of Rec. for Analysis of Soaps: Determination of Rec, for Oilseed Residues: Determination of Rec. for Animal Fats: Determination of coverings: Determination of Dimensional Changes in Varying $r$ Potassium Chloride for Industrial Use-Determination of m Hydrogen Carbonate for Industrial Use-Determination of for Sodium Chlorate for Industrial Use: Determination of Rec. for Plastics-Determination of the

Rec. for Cereals and Cereal Products: Determination of Rec. for Green Coffee Beans-Determination of
(Millimeters) and Vice Versa (1964) Rec for Straight Sided for Conver ISO (Millimeters) (1955) (Millimeters) $(1969)$ (Millimeters) (1969) Keys with or /Ngular or Square Parallel Keys an ISO Millimetres (Millimeters) and In./S (1970) Millimetres (Millimeters) and ln./S (1970) Millimetres (Millimeters) and In./S (1970) Millimetres (Millimeters) and Vice Versa (1964) for Slot ISO Lotted Rai ISO Re ISO Millimetres (Millimeters) (1955)

Millimetres) (Millimeters) (1969)

Millimetres) (Millimeters) (1969)

Milling Cutters and Their Arbors or Mandrels-Metric a

Milling Cutters with Plain Bore and Key Drive: Metric S ISO

Milling Cutters with Tenon Drive: Interchangeability $\mathrm{Di}$ ISO

Miling Cutters (1966)

Milling Machine Accessories (1968)

Milling Machines with Table of Fixed Height with Horizo

Milling Machines with Table of of Variable Height, with

Mills with Parallel Shanks with Morse Taper, Shanks-S

Mills with Plain Bore and Tenon Drive: Metric Series (1

Mills with 7/24 Taper Shanks-Std. and Long Series (19

Mineral Fuels-Determination of Gross Calorific Value

Mineral Fuels-Part III: Coke (1971)

Mineral Fuels-Part II: Coal Sampling and Analysis (19

Mineral Fuels-Part I: Coal Preparation (1970)

Mineral Fuels (1970)

Mineral Impurities (1968)

Mineral Matter in Coal (1967)

Mineral Solvents for Paints: White Spirits and Related

Miniature Cartridge Fuse Links (1968)

Miniature Circuit Breakers for Domestic and Similar Pur

Miniature Fuses (1962)

Miniature Fuses (1962)

Miniature Multipole Connectors with Blade Contacts (196

Miniature Screw Threads (1970)

Minor Constituents of Portland Cement (1968)

Mire (ISO Test Object) and Its Use in Photographic Docu

Miscellaneous Applications (1969)

Miscellaneous Functions M (1969)

Miscellaneous Lamps and Ballasts (1968)

is for the Numerical ISO

ISO

ISO

ISO

ISO

ISO

ISO

ISO

ISO

ISO

ISO

ISO

ISO

ISO

IEC

CEE

CEE

IEC

IEC

ISO

IEC

Mist (1964)

Mixed Alkali (1968)

Mixt ure (1967)

Mixtures-Quantitative Chemical Analysis (1971)

Mobile Belt Conveyors-Safety Code (1972

Mobile Belt Conveyors (Canvas, Rubber, Plastic, Etc.) S

Model Acceptance Tests of Hydraulic Turbines (1965)

Modified Erichsen Cupping Test for Steel Sheet and Stri

R Cryolite (Na ISO

Modified Willard-Winter Meth. (1970)

Modified Willard-Winter Meth. (1972)

Uminum) Fluori

Modular Coordination-Reference Lines of Horizontal Co

Modular Coordination-Storey and Room Heights for Resi

Modular Coordination-Vocabulary (1971)

Modular Coordination: Basic Module (1969)

Modular Coordination: Horizontal Multimodules $(1969+1$

Modular Dimensions for Waveguide Components (1971)

Modular Units for Machine Tool Construction: Slide Unit

Modulation Broadcast Transmissions (1954)

Modulation Broadcast Transmissions (1958)

Modulation in (1969)

lof the Electrical Properties of Rec. Meth. IEC

Modulation (1971)

Modulations and Television Broadcast Transmissions (195

Modulator Types (1970)

Modulators (1969)

/Ectrical Properties of Electro

Modules and Diametral Pitches of Cylindrical Gears for

Modules and Diametral Pitches of Cylindrical Gears for

Modules and Diametral Pitches of Straight Bevel Gears F

Modulus in Shear of Vulcanized Rubbers (Bonded Quadrupl

Modulus of Rupture by Bending (1971)

Moist Conditions (1971)

Moisture-Vacuum Oven Meth. (1970)

Moisture and Volatile Matter (1968)

Moisture and Volatile Matter (1968)

Moisture and Volatile Matter (1968)

Moisture and Volatile Matter (1968)

Moisture and Volatile Matter (1969)

Moisture Conditions (1972)
Moisture Content-Gravimetric Meth. (1971)

ISO

Moisture Content-Gravimetric Meth. (1972)

Moisture Content-Gravimetric Meth. (1973)

Moisture Content of Nonplasticized Cellulose Acetate (1

Moisture Content (Basic Reference Meth.) (1968)

Moisture Content (Basic Reference Meth.) (1970) 
Rec. for Spices and Condiments: Determination of Rer. for Paper-Meth. for the Determination of Rec. for Cereals and Cereal Products: Determination of Std. for Cocoa Beans: Determination of Rec. for Fibre (Fiber) Building Boards: Determination of Rec. for Particle Boards: Determination of Rec. for Particle Boards: Determination of
Rec. for Pure Expanded Corkboard-Determination of
Std. for Cork-Granulated-Determination of assage, Gleanings, Refuse and Corkwaste-Determination of
Std. for Meat and Its Products-Determination of Std. for Meat and Its Products-Determination of
Rec. for Green Coffee Beans-Determination of Rec. for Determination of the metric Meth. (1969)

Std. for Iron Ores: Determination of Hygroscopic

ravimetric Meth. (1963)

olumetric Meth. (I963)

Rec. for Determination of Rec. for Determination of Total Rec. for Determination of Total Rec. for Determination of Rec. for Determination of Rec. for Determination of Rec. for Glucose Syrup-Determination of Dry Matter of Analysis of Manganese Ores-Determination of Hygroscopic Rec. for Starch-Determination of

Determination of Loss of Mass at $300^{\circ} \mathrm{C}$ (Conventional Std. for Quantities and Units of Physical Chemistry and ught Quenched and Tempered Types with I\% Chromium and ls-Part 8: Wrought Quenched and Tempered Chromium Nicke ght, Quenched and Tempered Types with $1 \%$ Chromium and : Wrought Quenched and Tempered, with 3\% Chromium and ols for Flight Dynamics-Part III: Derivatives of Forces,

r Portable X.. or Gamma Radiation Exposure Rate Meters and c. for Alpha, Beta and Alpha-Beta Contamination Meters and o Transmitters: Measurements Particular to Transposers for assemblies and Equipment (I963)

Strand (Hawser Laid) and 8 Strand (Plaited) Polypropylene Rec. for 3 Strand Polyethylene us Mechanical Handling Equipment for Unit Loads-Overhead shanks (I972)

Std for Finishing Reamers for Rec. for Hand and Long Fluted Machine Reamers, or Twist Drills: Parallel Shank Jobber and Stub Series and Std. for Core Drills with Parallel, and

for Reduction Sleeves and Extension Sockets for Tools with Rec. for Machine Chucking Reamers with Parallel or ing Reamers for Morse and Metric Tapers, with Parallel and Rec. for End Mills with Parallel Shanks with

of Cements-Compressive and Flexural Strengths of Plastic

(1968)

r Circuit Breakers (I936)

rture and Projecta/ rture (1972)

Std for Cinematography: on of Winding (1971)

Rec. for Single Magnetic Sound Stripe Magnetic Sound Stripe on $16 \mathrm{Mm}$ Std. for Cinematography-Camera Usage of $8 \mathrm{Mm}$

he Printed Image Area for Printing to $8 \mathrm{~mm}$ Type $S$ on $16 \mathrm{Mm}$

Edges (I/ Std. for Cinematography-Dimensions for $16 \mathrm{Mm}$ Rec. for Dimensions of Projection Reels for $8 \mathrm{Mm}$ Rec. for Emulsion Position in Camera for $16 \mathrm{~mm}$ Silent Rec. for Emulsion Position in Camera for $8 \mathrm{~mm}$ Silent lsion and Sound Record Positions in Camera for $35 \mathrm{~mm}$ Sound lsion and Sound Record Positions in Camera for $16 \mathrm{~mm}$ Sound on and Sound Record Positions in Projector for $35 \mathrm{~mm}$ Sound on in Projector for Direct Front Projection of $8 \mathrm{~mm}$ Silent

$\mathrm{n}$ in Projector for Direct Front Projection of $16 \mathrm{~mm}$ Silent tography-Dimensions of Daylight Loading Spools for $16 \mathrm{Mm}$ hy-Dimensions of Daylight Loading Spools for Double $8 \mathrm{Mm}$ Projector Aperture for Projection of $35 \mathrm{Mm}$ Nonanamorphotic Dimensions (I972) Std. for Cinematography-8 mm Type S ec. for Cutting and Perforating Dimensions for Double. $8 \mathrm{Mm}$ Rec. for Cutting and Perforating Dimensions for $35 \mathrm{Mm}$ Rec. for Definition and Marking of Safety Film for

Std. for Symbols for Flight Dynamics-Part I: Aircraft ngines-Hand Operated Control Devices-Std. Direction of f Magnetic.Striping and $\mathrm{Ga} /$ Std. for Cinematography: $8 \mathrm{Mm}$ the Eart/ Rec. for Symbols for Flight Dynamics-Part II:

ec. for Drop Wires for Mechanical and Electrical Warp Stop

e Cross Section of Assembled Bars for Mechanical Warp Stop Rec. for a.c.

th 6 or I2 V Electrical Equipment Particularly for Private oses: Vacuum Cleaners and Water Sucti/ oses: Clocks (I967)

oses: Washing Machines (1968)

oses: Spin Extractors (1968)

Spec for Electric

Spec. for Electric

Spec. for Electric Rec. for

Moisture Content (Entrainment Meth.) (1969)

Moisture Content (Oven Drying Meth.) (1963)

ISO

ISO

ISO

ISO

Moisture Content (Routine Meth.) (1972)

Moisture Content (1968)

Moisture Content (1968)

Moisture Content (1971)

Moisture Content (1972)

Moisture Content (1972)

Moisture Content (1973)

Moisture Content, (Routine Meth.) (1970)

Moisture Holding Capacity of Hard Coals (1969)

Moisture in Analytical Samples (1973)

Moisture in Brown Coals and Lignites by the Direct Volu

Moisture in Coke (1967)

Moisture in Hard Coal (I967)

Moisture in the Analysis Sample of Coal by the Direct C

Moisture in the Analysis Sample of Coal by the Direct

Moisture (Vacuum Oven Meth.) (1970)

Moisture (1963)

Moisture (1970)

Moisture) (1968)

Molecular Physics (1973)

Rec, for Meth, of Chemical ISO

ISO

$\mathrm{SO}$

/ Wro ISO

Molybdenum Types (I970)

Molybdenum (1968)

Molybdenum (1970)

(1972)

Monitoring Aircraft Noise Around an Airport (I970) ISO

Monitors for Use in Radiological Protection (1972) /Fo IEC

Monitors (1970)

Monochrome and Colour (Color) Television (1971)

Re IEC

Monofilament or Film Ropes (1970)

Monofilament Ropes (1971)

Morse and Metric Tapers, with Parallel and Morse Taper

Morse Taper Shank (1961)

Morse Taper Shank (1961)

Morse Taper Shanks-Rec. Stocked Sizes (1972)

Morse Taper Shanks (1961)

Morse Taper Shanks (1966)

Morse Taper Shanks (1972)

Morse Taper, Shanks-Std. and Long Series (1970)

Mortar (Rilem Cembureau Meth.) (1968)

Mosaic Parquet Panels (1967)

Motion Nomenclature for Numerically Controlled Machines
Motion of Operating Devices and for Indicating Lamps Fo

Motion Picture and Magnetic Films (1969)

Motion Picture Film-Image Area Produced by Camera Ape

Motion Picture Film-Image Area Produced by Camera Ape

Motion Picture Film-Projectable Image Area (1972)

Motion Picture Film for Direct Front Projection (1973)

Motion Picture Film Perforated Along One Edge-Directi

Motion Picture Film Perforated Along 1 Edge (1966)

Motion Picture Film Perforated Type S (1972)

Motion Picture Film Perforated $8 \mathrm{~mm}$ Type S, 1-4 (1972

Motion Picture Film with Perforations Along One and Two

Motion Picture Film (Other Than Type S) (1968)

Motion Picture Film (1956)

Motion Picture Film (1956)

Motion Picture Film (1956)

Motion Picture Film (1956)

Motion Picture Film (1956)

Motion Picture Film (1956)

Motion Picture Film (I956)

Motion Picture Film (I969)

Motion Picture Film (I969)

Motion Picture Films (I963)

- Maximum Aspect Ratio of IS

Motion Picture Raw Stock Film (I966)

Motion Picture Uses (1966)

Motion Relative to the Air (I972)

Motion (1972) Ifor Reciprocating Internal Combustion E ISO

$\begin{array}{ll}\text { Motion (1972) } & \text { for Reciprocating Internal Combustion E } \\ \text { Motion-Picture Film with Picture-Location and Width O } & \text { ISO }\end{array}$

Motions of the Aircraft and the Atmosphere Relative to

Rec for Emu ISO

Rec. for Emulsi ISO

Emulsion Positi ISO

Rec. for Cinema ISO

ISO

ISO

ISO

$\mathrm{SO}$

ISO

tions (1965)

Motions (1971)

Motor Capacitors (1967)

Ifor Serrated Bars and Dimensions of Th ISO

Motor Cars and Light Trailers or Caravans (1970)

Motor Operated Appliances for Domestic and Similar Purp

Motor Operated Appliances for Domestic and Similar Purp

Motor Operated Appliances for Domestic and Similar Purp

IS Wi ISO

CEE

287

712

767

823

2066

2190

2386

1442

1447

2596

1015

579

589

331

348

687

1742

1666

803

$31 /$ VIII

$683 / \mathrm{IV}$

683/VIII

$683 / \mathrm{II}$

$683 / \mathrm{VI}$

1153

1761

395

325

244.5

146

1346

1969

2149

2250

236

235

235/II

238

521

2250

1641

679

631

841

54

1039

2467

2906

2907

I781

1200

490

1787

I 785

69

892

25

28

23

27
24

29

10 I9

1020

358

1700

486

491

543

II 5 I

2261 
omes: Shavers, Hair (Clippers and Simi/ ones: Massage Appliances (1967) cmes (1964)

initions (1967)

Spec. for Electric Spec. for Electric Spec. for Electric Spec. for Portable Rec. for Dimensions of Lead Acid Rec. for Low Voltage Rec. for Low Voltage Rec. for Sound Signalling Devices on Std. for Braking of Rec. for Dimensions of Rec. for Lighting and Signalling for Rec. for Restraining Devices for Children in Rec. for Seat Belt Assemblies for Rec. for

and Unit Loads-Belt Conveyors-Basic Characteristics of Rectifiers, Generators (With Associated Prime Movers) and rocedures for Electronic Components and Equipment: Test J: vc) for Use Under Pressure (1967) Rec. for Oven Test for Rec. for Plastics-Designation for Polystyrene

Rec. for Plastics-Determination of Apparent Density of

Rec. for Plastics-Determination of Apparent Density of Rec. for Plastics-Determination of Bulk Factor of in Content of Material in the Unmoulded State) of Phenolic Rec. for Plastics-Basis for Spec. for Phenolic als-Annex A: Preparation of Test Specimens from Phenolic eparation of Test Specimens from Polyester and Epoxy Resin

Annex B: Preparation of Test Specimens from Aminoplastic tion of Specimens for Optical Tests on Plastics Materials: Rec. for Plastics-Determination of Viscosity Loss on

3)

nex A: Preparation of $\mathrm{Te} /$ nex B: Preparation of Te/ nex $\mathrm{C}$ : Preparation of $\mathrm{Tel}$ nex

Rec, for Plastics-Compression Rec. for Plastics-Injection Rec. for Plastics-Compression Rec. for Plastics-Compression Rec. for Plastics-Compression Rec. for Plastics-Compression lastics-Detection of Free Ammonia in Phenol Formaldehyde on of the Percentage of Acetone Soluble Matter in Phenolic

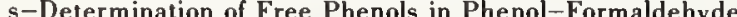
ee Ammonia and Ammonium Compounds in Phenol-Formaldehyde tact Spacing of $2.54 \mathrm{~mm}(0.1 \mathrm{In}$.) Mating Either with Board 000 (197) Rec. for Dimensions and Output Ratings for Foot linkage of Agricultural Wheeled Tractors for Attachment of s (1967) Rec. or Power Supply for Air and Land Vehicle al Handling Equipment for Unit Loads-Single Strand Floor electrical Machines: Symbols for Types of Construction and mounting Type) (1959)

es (1968)

Rec. for Sizes and

guidance for the Design and Use of Components Intended for and Mounting Dimensions of Aircraft Instrument Cases (Rear for Rotary Wafer Switches (Low Current Rating) with 2 Hole switches (Low Current Rating): Wafer Switches with Central Rec. for Dimensions for Single Hole Rec. for Rotary Wafer Switches with Central Rec. for Rotary Wafer Switches with 2 Hole current Ratin/ Rec. for Electrical Connections Between Prime t for Comm/ emiconductor Rectifiers, Generators (With Associated Prime
Rec. for Knife Sections of ties of Electronic Tubes: Meth. of Measurement of Geiger meters of Cylindrical Radiation Probes Containing Geiger Rec. for 3 Strand Polyamide Rec. for 3 Strand Polyester Rec. for Layout of

Rec. for Modular Coordination: Horizontal ternational System of Units and a Selection of the Decimal Rec. for Connectors for Frequencies Below 3 MHz: Circular nectors for Frequencies Below $3 \mathrm{MHz}$ : Rectangular Miniature c. for Connectors for Frequencies Below $3 \mathrm{MHz}$ : Rectangular

Rec, for Connectors for Frequencies Below $3 \mathrm{MHz}$ : Circular

ication (1972)

g by Size (1958) Rec. for Shipbuilding Details . Std. for Rec, for Shipbuilding Details for Inland Navigation andard Musical Pitch) Rec. for Std. Tuning Frequency (Std. Rec. for Aircraft Engine
Rec. for Pesticides Considered Not to Require Common ators (197I) Rec. for Generic Growth / Rec. for Common Rec. for Abbreviations of Generic Common Rec. for nation of Calcium Content-Spectrophotometric Meth. Using
ally / Rec. for Relation Between Sound Pressure Levels of (1972) Std. for Rolling Bearings: Locknuts, Rec. for Grooved Pulleys for
Motor Operated Appliances for Domestic and Similar Purp Motor Operated Appliances for Domestic and Similar Purp Motor Operated Appliances for Domestic and Similar Purp Motor Operated Tools (1967)

Motor Scooter Batteries (1965)

Motor Starters: Direct on Line, a.c. (1971)

Motor Starters: Reduced, a.c.: Star Delta (1970)

Motor Vehicles Acoustic Std. and Technical Spec. (1966)

Motor Vehicles and Their Trailers-Terminology (1972)

Motor Vehicles and Their Trailers: Designations and Def Motor Vehicles and Trailers (1963)

Motor Vehicles (1970)

Motorists (1970)

Motorists Seat Belts with Retractors (1970)

Motorized Driving Pulleys (1970)

Motors, Electric Propulsion Plant, Tankers (1965)

Mould Growth (1968) /Tor IEC

Moulding Material That Can Be Poured from a Specified F

Moulding Material That Cannot Be Poured from a Specifie

Moulding Materials (1961)

Moulding Materials (1963)

Moulding Materials (1968)

Moulding Materials (1970)

Moulding Materials (1970)

Moulding Materials (1970)

Moulding Meth. (1968)

Moulding of Cellulose Acetate (1970)

/Acetone Soluble Matter (Res

ISO

Ing Materials-Annex C: Pr ISO

lof Thermosetting Materials ISO

Rec. for Plastics-Prepara ISO

MouIding Test Specimens of Thermoplastic Materials (196

Moulding Test Specimens of Thermoplastic Materials (196

Moulding Test Specimens of Thermosetting Materials-An

Moulding Test Specimens of Thermosetting Materials-An

Moulding Test Specimens of Thermosetting Materials-An

Moulding Test Specimens of Thermosetting Materials (196

Mouldings-Qualitative Meth. (1961)

Mouldings (1958)

Mouldings (1959)

Mouldings (1959)

Rec. for P

1. for Plastics-Determination of $\mathrm{Fr}$ ISO

Mounted Connectors or Printed Wiring Boards with Edge B IEC

Mounted Electrical Machines with Frame Numbers 355 to 1 IEC

Mounted Implements (1968) Rec. for 3 Point ISO

Mounted Prospecting Equipment for Radio Active Material IEC

Mounted Truck Conveyors (Chain Above Floor)-Safety Co ISO

Mounting Arrangements of (1972) Rec. for Rotating

Mounting Dimensions of Aircraft Instrument Cases (Rear

Mounting of Plain Grinding Wheels by Means of Hub Flang

Mounting on Boards with Printed Wiring and Circuits (19

Mounting Type) (1959)

Mounting (1963)

Mounting (1963)

Rec. for Sizes

Mounting, Lever Operated Switches for Aircraft (1966)

Mounting; Maximum 12 Positions and Diameter $40 \mathrm{~mm}$ (1966

Mounting. Maximum 26 Positions and Diameter $60 \mathrm{~mm}$ (Low

Movers and Towed Vehicles with 24.V Electrical Equipmen

Movers) and Motors, Electric Propulsion Plant, Tankers

Mower Cutter Bars (1967)

Muller Counter Types (1971)

/ of the Electrical Proper

Multifilament Ropes (1969)

Multifilament Ropes (1969)

Multilingual Classified Vocabularies (1969)

Multimodules $(1969+1970)$

Multiples and SubmuItiples of the SI Units (Metric) (19

Multipole Connectors with Bayonet or Push Pull Coupling

Multipole Connectors with Blade Contacts (1965)

Multipole Connectors with Blade Contacts (1966)

Multipole Connectors with Threaded Coupling (1966)

Multipurpose Chocks of Cast Steel (1971)

Muscovite Mica Blocks, Thins and Films-Visual Classif

Muscovite Mica Blocks, Thins and Films Meth. for Gradin

Mushroom Ventilators (1957)

Musical Pitch) Rec. for Std. Tuning Frequency (Std. Mus

Musical Pitch) (1955)

/R Standard Tuning Frequency (St
/

Nacelle Fire Extinguisher Doors (1969)

Names-Second List (1969)

Names for Man Made Fibres (Fibers) (1971)

Names for Pest Control Chemicals and Plant Growth Regul

Names for Pest Control Chemicals (Pesticides) and Plant

Names in Titles of Periodicals (1968)

Names: First List (1968)

Naming Principles (1968)

Naphthalhydroxamic Acid (1971)

Narrow Bands of Noise in a Diffuse Field and in a Front

Narrow Series, and Lockwashers with Straight Inner Tab

Narrow V-Belts: Groove Sections SPZ, SPA, SPB (1965)
EE $\quad 10-2 \mathrm{~N}$

$10+10-1$

20

199

292-1

292.2

512

611

612

303

1713

1231

1534

1816

92.5

68-2-10

580

1622

60

61

171

308

800

295/A

295/C

295/B

869

1599

293

294

295/A

295/B

$295 / C$

295

172

59
119

119

120
130.1

$72 \mathrm{~A}$

730

253

2196

34-7

103

666

321

103

132-3

132.2

493

$132-4$
$132-5$

$132-5$

1185

92.5

563

151.25

1140

1141

1149

$1040 /$ I9II

1000

130.7

130-6

130.5

130.4

1685

2185

67

42

16

16

1021

1105

2076

1750

257

833
765

704

2070

454

2982

459 
Rec. for l,engths of Narrow V-Belts: Sections SPZ, SPA, SPB (1965)

Limits of Radio Interference and Report of National Limits (1967)

tent: Modified Willard-Winter Meth./ - 1, 10 Phenanthroline Photometric Me/ nt-Spectrophotometric Meth. Using T/ st Samples (1970)

uminum) Content-8 Hydroxyquinoline /

Rec. for Cryolite (Natural and Artificial)-Determination of Fluorine Con

Rec. for Cryolite (Natural and Artificial)-Determination of Iron Content

Rec. for Cryolite (Natural and Artificial)-Determination of Silica Conte

Rec. for Cryolite (Natural and Artificial)-Preparation and Storage of Te

Std. for Cryolite (Natural and Artificial): Determination of Aluminium (AI

Rec. for Determination of Viscosity of Natural and Synthetic Rubbers by the Shearing Disk Visc $\begin{array}{ll}\text { ometer (1963) Rec. For Determination of Viscosity of } \\ 571 & \text { Rec. for Determination of Tear Strength of Vulcanized }\end{array}$ 571 Rec. for Determination of Tear Strength of Vulcanized

Determination of Resistance to Crack Growth of Vulcanized n (I971)

971)

Rec. for Raw

ent (1972)

2)

Rec. for Amount of Bale Coating on Rec. for Raw Std. for Std. for Std. for ility (I972)

$t(1971)$

a Preserved-Spec. (1971)

971) entrated Rec. for Rec. for Rec. for Rec. for Determination of Ash in Raw Rec. for Determination of Volatile Matter in Raw Rec. for Sampling of Raw kenter Type (1957) Rec. for Shipbuilding Details for Sea (1957)

Rec. for Shipbuilding Details for Inland Rec. for Shipbuilding Details for Inland e Liquids-Nom/ Rec. for Shipbuilding Details for Inland ec. for Shipbuilding Details for Sea Navigation and Inland ss Links (1957)

Rec. for Shipbuilding Details for Sea Rec. for Shipbuilding Details for Inland

Magnetic Compasses and Binnacles, Class A, for Use in Sea Rec. for Magnetic Compasses and Binnacles for Sea imension-Series 48 and 49 (1) Rec. for Rolling Bearings ensional Charact/ Rec. for Conical Fittings for Syringes, Rec. for Hypodermic Rec. for Automobiles-Engine Test Code alue by the Calorimetric Bomb Meth. and Calculation of the breaking Load of Netting Yarns for Fish/ Rec. for Fishing etting (1970)

Rec. for Fishin

for Describing and Designating Knotted Netting for Fishing Rec. for Netting Yarns for Fishing Rec. for Hanging of

)

Rec. for

c. for Requirements for Describing and Designating Knotted Std. for Cutting Rec. for Cutting Knotted

of Determining the Breaking Load and Knot Breaking Load of system (1968) Rec. for

hing Nets-Meth. of Determining the Mesh Breaking Load of Rec. for Std. Frequencies for Centralized

ic Acid and Xylenols for Industrial Use-Determination of Std. for Electroplated Coatings of Tin Rec. for Classification of Copper Rec. for Electroplated Coatings of Copper Plus imensions (1972) Rec. for Sealed eneral Requirements and Test Meth. (1971/ Rec. for Sealed
- Measurement of Coating Thick/ Rtd. for Electrodeposited - Measurement of Coating Thick/ Std. for Electrodeposited ng Steels-Part 8: Wrought Quenched and Tempered Chromium Rec. for Classification of Copper opper and Its Alloys-Spectrophotometric Determination of of Chemical Analysis of Manganese Ores-Determination of Rec. for Electroplated Coatings of Rec. for Heads of Aircraft Lubricating ty-Volumetric Meth. (1972)

Std. for Ammonium
Std. for Ammonium otentiometric Meth. (I972)

ty-Volumetric Meth. (I97I)

Rec. for Meth. of Mercurous

content: 2, 2'-Bipyridyl Photometric Meth/

Rec. for Rec. for Rec. for Rec. for us Compounds-Volumetric Meth. (1971)

ated Residue on Ignition-Gravimetric Meth. (I/ treated, Alloy, and Free Cutting Steels-Part 10: Wrought and Oleums for Industrial Use: Determination of Oxides of ood Products-General Directions for the Determination of Rec. for Meat and Its Products: Determination of Acid and Oleum for Industrial Use: Determination of Total Rec. for Determination of
Rec. for Determination of

c. for Raw Natural Rubber and Ita Latex-Determination of Rec. for Determination of rec. for Nitric Acid for Industrial Use-Determination of Rec. for Speroidal or Rec. for Monitoring Aircraft
Natural and Synthetic Rubbers (Crescent Test Piece) (19 Natural or Synthetic Rubber (De Mattia Type Machine) (1 Natural or Synthetic Rubber (De Mattia Type Machine) (1 Natural Rubber and Its Latex-Determination of Nitroge Natural Rubber Bales-Requirement and Determination (1 Natural Rubber Determination of Dirt (197I)

Natural Rubber Latex-Determination of Dry Rubber Cont

Natural Rubber Latex-Determination of KOH Number (197

Natural Rubber Latex-Determination of Mechanical Stab

Natural Rubber Latex (1966)

Natural Rubber Latices-Determination of Sludge Conten

Natural Rubber Latices, Centrifuged and Creamed, Ammoni

Natural Rubber Latices, Evaporated Preserved-Spec. (1

Natural Rubber (I962)

Natural Rubber (1962)

Natural Rubber (I962)

Navigation-Anchor Chains-Lugless Joining Shackles,

Navigation-Covers for Deck Openings for $220 \mathrm{~mm}$ Pumps

Navigation-Mushroom Ventilators (1957)

Navigation-Pipe Lines for the Transport of Combustibl

Navigation Anchor Chains-Studless Links (1957)

Navigation and Inland Navigation Anchor Chains-Studle

Navigation Sprocket Wheels (1956)

Navigation (1965)

Navigation: Vocabulary (1969)

Needle Roller-Boundary Dimensions-Metric Series-D

Needles and Other Medical Equipment: Definition and Dim

Needles (1967)

Net Power (1971)

Net (197I)

ISO

Nets-Meth. of Determining the Breaking Load and Knot

Nets-Meth. of Determining the Mesh Breaking Load of N ISO

Nets (1970) Rec. for Requirements ISO

Rec. for Requirements ISO

Netting-Basic Terms (1970)

Netting for Fishing-Basic Terms and Definitions (1969

Netting for Fishing Nets (1970)

Netting to Shape-Determination of the Rate (1972)

Netting to Shape (Tapering) (1970)

Netting Yarns for Fishing Nets (I970)

/G Nets-Meth. ISO

Netting (1970)

Network Control Installations (1967)

Neutral Oils and Pyridine Bases (1971)

Nickel Alloy (1972)

Nickel Alloys (I965)

Nickel and Chromium on Steel (Or Iron) (1970)

Nickel Cadmium Cylindrical Rechargeable Single Cells: D

Nickel Cadmium Cylindrical Rechargeable Single Cells: C Tex ISO Rec. for Fis ISO

/Cresol, Cresyl isO

Nickel Coatings on Magnetic and Nonmagnetic Substrates ISO

Nickel Molybdenum Types (1970) / Alloy, and Free Cutti ISO

Nickel Plus Chromium (1970)

Nickel Zinc Alloys (1965)

Nickel (1963)

Nickel (1970)

Nipples (I965)

Nitrate for Industrial Use: Determination of Free Acidi

Nitrate for Industrial Use: Measurement of $\mathrm{pH}$ Value-P

Nitrate Test for Copper and Its Alloys (1961)

Nitric Acid for Industrial Use-Determination of Acidi

Nitric Acid for Industrial Use-Determination of Iron

Nitric Acid for Industrial Use-Determination of Nitro

Nitric Acid for Industrial Use-Determination of Sulph

Nitriding Type (1970)

Nitrogen-Volumetric Meth. (1970)

Rec. for Heat ISO

Nitrogen-2,4-Xylenol Spectrophotometric Meth. (1972 ISO

Nitrogen by the Kjeldahl Meth. (1971) / Agricultural F ISO

Nitrogen Content (1969)

/R Sulphuric ISO

Nitrogen Content: Volumetric Meth. (1968) /R Sulphuric ISO

Nitrogen in Coal by the Semi Micro Kjeldahl Meth. (1963 ISO

Nitrogen (1971)

Nitrogen, Total Sulphur, Chlorine and Phosphorus in Cok

Re ISO

Nodular Graphite Cast Iron (1969)

Noise Around an Airport (1970)
Nets: Designation in the Tex System (1968)

Nickel (Low Contents) in Copper Alloys (1971)
460

1693

1694

1620

1619

2367

289

34.
132

133

1656

1434

249

126

127

35

498

2005

2004

2027

247

248

250

39

41

42

51

40

21

449

1069

1206

594

596

1585

1928

I 805

I806

I530

858

I53 I

1107

I530

2075

I532

I 805

858

1806

242

1899

2179

429

I 457

285-2

285 .

2361

$683 /$ VII

1456

430

1810

315

1458

4 I 3

2364

2365

196

1980

1982

I981

I 983

$683 / \mathrm{X}$

1592

2363

$187 \mathrm{I}$

937

914

332

333

1656

926

1981

I083

1761 
Rec. for Procedure for Describing Aircraft Std. for Measurement of Airborne

r Outroor Une (1972) ements for the Preparation of Test Codes for Measuring the Rec. for Test Code for the Measurement of the Airborne Rec. for Measurement of Rec. for Acoustics-Assessment of Occupational the Electrical Properties of Electronic Tubes and Valves: Rec. for Meth. of Measurement of Current Relation Between Sound Pressure Levels of Narrow Bands of Rec. for Rotating Electrical Machines: of Electronic Tubes and Valves: Measurement of Equivalent Rec. for Acoustics-Assessment of sion of the Physical and Subjective Magnitudes of Sound or $r$ Expression of the Power and Intensity Levels of Sound or es of Electronic Tubes and Valves: Meth. of Measurement of tors and Slip Rings (1968) Rec. for Definitions and Rec. for Axis and Motion Std. for

rt I (1971) Std. for Dense Shaped Refractory Products Rec. for Rec. for Spices and Condiments: for Architectural and Building Drawings: Definitions and nery-Dyeing and Finishing Machinery-Classification and Rec. for Rubbers and Latices Rec. for Assembly Tools for Screws and Nuts ased on Calcium Sulphate-Definitions, Classification and ors of Insulated Cables (1966) Rec. for Std. for Long Shank Machine Taps with on-Pipe Lines for the Transport of Combustible Liquids

Rec. for Straight Sided Splines (For Cylindrical Shafts) trodes with Threaded Sockets and Connecting Pi/ Rec. for Rec. for Wood Cones for Cross Winding: gths for General Purpose Bolts (1968) Rec. for Std. for Industrial Wheels-Dimensions and terials for the Transport of Fluids (Outside Diameters and terials for the Transport of Fluids (Outside Diameters and Rec. for Industrial Tractors-Definition and

r Application in Coincident Current Matrix Stores Having A Std, for Dental Burs and Cutters.

Std. for Wire and Plate Screens for Industrial Purposes

for Woven Wire Cloth and Perforated Plates in Test Sieves: Std. for Size Analysis of Small Coke

ors of Ceramic Material or Glass for Overhead Lines with A sts on Indoor and Outdoor Post Insulators for Systems with oor and Outdoor Post Insulators and Units for Systems with y Rolling Stock Material-Cast Wheel Centres (Centers) in Rec. for Drawn Wire for General Purpose Aspect Ratio of Projector Aperture for Projection of $35 \mathrm{Mm}$ International Rec. for Metrological Regulations for

Rec. for Short Link Chain for Lifting Purposes-Grade 40 Rec. for asurement of Coating Thickness-Eddy Current M/ Std. for

Its Alloys-Measurement of Thickness of Oxide Coatings ests on Impregnated Paper Insulated Metal Sheathed Cables:

nd Including $66 \mathrm{kV}$ (Excluding Gas Pressure, Oil Filled and

io Transmitters: Bandwidth, Out of Band Power and Power of Rec. for Horology-Functional and Rec. for Test Enclosures of g Appliances for Domestic and Similar Purposes: Stationary g Ap Rec. for

ents (Nonionic): Determination of Polyethylene Glycols and ve Agents: Determination of the Cloud Point Temperature of nionic Active Matter (Ad/ Std. for Surface Active Agents ness-Eddy Current M Std for Nonconductive Coatings on oatings on Magnetic Basis Metals-Measurement Std. for Std. for Electrodeposited Nickel Coatings on Magnetic and e Measurement of the Thickne/ Rec. for Metallic and Other es for $C /$ for Plastics-Determination of the Moisture Content of
Std. for Textile Machinery and Accessories Rec. for

l Relays: Single Input Energizing Quantity Measuring, with Rec. for Spices and Condiments: Determination of e for Ind ustrial Use: Determination of Loss of Mass and of for Paints and Varnishes-Determination of Volatile and Rec. for Rec. for Fixed on of Meth. of Test and General Requirements for Low Power ermination of Compression Set Under Constant Deflection at scanning Area of $35 \mathrm{~mm}$ Double Width Push Pull Sound Prints ypes with Shields or Seals-Outside Diameter Tolerances

1 Threshold of Hearing Under Free Field Listeni/ Rec. for in Such a Way That the Transverse Section Is Not Under Any rec. for Normal Equal Loudness Contours for Pure Tones and Rolling Bearings-Thrust Ball Bearings with Flat Seats -
c. for Rolling Bearings-Tapered; Metric Series-Part I:
Noise Around an Airport (1970)

Noise Emitted by Compressor Primeover Units Intended Fo

Noise Emitted by Machines (1966)

ral Requir

(1970)

Noise Emitted by Vehicles (1964)

Noise Exposure for Hearing Conservation Purposes (1971)

Noise Factor (1963)

Noise Generated in Fixed Resistors (1965)

Noise in a Diffuse Field and in a Frontally Incident Fr

Noise Limits (1972)

Noise Resistance (1964) I of the Electrical Properties

Noise with Respect to Community Response (1971)

Noise (1959)

Rec. for Expres ISO

Noises Due to Mechanical or Acoustic Excitations (1968)

Nomenclature for Carbon Brushes, Brush Holders, Commuta

Nomenclature for Numerically Controlled Machines (1968)

Nomenclature of Lifting Hooks (1973)

Nomenclature of Manufacturing Processes (1972)

Nomenclature of Terms Used in the Benzole Industry-Pa

Nomenclature (1968)

Nomenclature (1969)

Nomenclature (1970)

Nomenclature (1971)

Nomenclature (1971)

Rec. for Textile Machi ISO

ISO

(1971)

Rec for Binders $B$ ISO

Nominal Cross Sectional Areas and Composition of Conduc

Nominal Diameters from $324 \mathrm{~mm}$ and $1 / 8$ to 1 In. (1972)

Nominal Diameters (1957)

NominaI Dimensions in Millimetres (Millimeters) (1955)

Nominal Dimensions of Cylindrical Machined Graphite Ele

ISO

Nominal Half Angle of the Cone $5^{\circ} 57^{\prime}(1963)$

Nominal Lengths for Bolts, Screws and Studs: Thread Len

Nominal Load Capacities (1972)

NominaI Pressures) Part II: In. Series (1963)

Nominal Pressures): Part I: Metric Series (1960)

Nominal Rating (1969)

NominaI Selection Ratio of 2 : 1 (1969)

Nominal Sizes and Designation of Working Parts (1972)

Nominal Sizes of Apertures (1972)

Nominal Sizes of Apertures (1972)

Nominal Top Size $20 \mathrm{~mm}$ or Less) (1972)

Nominal Voltage Greater Than $1000 \mathrm{~V}(1972)$

Nominal Voltages Greater Than 1,000 V (1964)

Nominal Voltages Greater Than 1,000 V (1968)

Nonalloy Steel for Tyred (Tired) Wheels for Trailer Sto

Nonalloy Steel Wire Ropes-Spec. (1971)

Nonanamorphotic Motion Picture Films (1963)

Nonautomatic Weighing Machines (1972)

Noncalibrated Chain for Chain Slings, Etc. (1971)

Noncombustibility Test for Building Materials (1970)

Nonconductive Coatings on Nonmagnetic Basis Metals-Me

Nondestructive Measurement by Light Section Microscope

Nondraining Types for Alternating Voltages from $10 \mathrm{kV} \mathrm{U}$

Nondraining Types) (1965)

Nonessential Oscillations (1969)

Nonfunctional Jewels (1969)

Measurement for Rad IEC

Noninjection Type for Constant Relative Humidity (1968) King and Heatin CEE

Noninterrupted Creep Test, of Steel at Elevated Tempera

Nonionic Active Matter (Adducts)-Weibull Meth. (1972)

Nonionic Surface Active Agents Obtained from Ethylene 0

(Nonionic): Determination of Polyethylene Glycols and No

Nonlinear Resistor Type for a.c. Systems (1970)

Nonmagnetic Basis Metals-Measurement of Coating Thick

Nonmagnetic Metallic and Vitreous or Porcelain Enamel C

Nonmagnetic Substrates-Measurement of Coating Thickne

Nonorganic Coatings-Definition of Terms Concerning Th

Nonplasticized Cellulose Acetate (1967)

Nonreversible Rings for Ring Spinning and Doubling Fram

Nonscrewed Steel Tubes for General Purposes (1962)

Nonspecified or Independent Specified Times (1971)

Nonvolatile Ether Extract (1969)

Nonvolatile Matter at $250^{\circ} \mathrm{C}$ (1968)

Nonvolatile Matter (1970)

Nonwirewound Potentiometers Type 2 (1966)

Nonwirewound Resistors, Type II (1959)

Nonwirewound Types (1972)

Normal and High Temperatures (1972)

Normal and Offset Centerline Types (1958)

Normal and Tolerance Class 6 (1971)

Fixed Resistors: Selecti

IDium Carbor

ISO

ISO

ISO

ISO

ISO

ISO

Re ISO

1SO

$1 \mathrm{SO}$

ICa IEC

/Arings-Radial T ISO
Narmal Equal Loudness Contours for Pure Tones and Norma

Trical Fillet Welds Statically ISO

Normal Threshold of Hearing Under Free Field Listening

Normal Tolerances (1961)

Rec. for ISO

Normal Tolerances (1967)
507 
terchangeability (1968)

ngeability: Metrir Series (1968)

)(1971)

c. for Spindle

Rec, for Internatic

Rec. for Radioisotope All or

Rec. for Electrical Relays: Instantaneous All or

Rec. for Electrical Relays: Specified Time All or

Rec, for Aircraft Fuel

r Measurement of Fluid Flow by Means of Orifice Plates and information lnterchange -8 and $32 \mathrm{RPmm}$ ( 200 and $800 \mathrm{RPI}$ ). Rec. for External Diameter of Planchets Used in

Rec. for Dimensions of Panels and Racks (For Std. for

Rec. for Coaxial Cable Connectors Used in Rec. for Supply Voltages for Transistorized nalogue Voltage Ranges and Logic Levels for Mains Operated ord Personal Protection Against Ionizing Radiat/ Rec. for 68) Rec. for International Electrotechnical Vocabulary: Rec. for General Characteristics of Rec. for General Principles of

pylenes / Rec. for Plastics-Determination of Viscosity: te Soluti/ Rec. for Plastics-Determination of Viscosity Rec. for

Rec. for Determination of the Crucible Swelling Rec for Combine Harvesters-Width of Cut and Rec. for Determination of Volatile Fatty Acid Rec. for Plastics-Determination of Viscosity

ion $(1970 /$

Rec. for Plastics-Determination of the Viscosity Rec. for Plastics-Determination of Viscosity Rec. for Determination of the Kappa 1971) Rec. for Carpets-Determination of Rec. for Figures for Aircraft Instrument Dials and Rec. for Preferred Std. for Natural Rubber Latex-Determination of Koh bustion Chambers, and Direction of Rotation of / Rec. for ments (1972)

ctronic Tubes and Valves (1961)

Std. for

Rec. for Rec, for

Std. for Documentation-International Standard Book Std. for Guide to the Use of Preferred

Rec for Guide to the Choice of Series of Preferred Electrical Machines -. Frame Numbers 56 to 400 and Flange tems (1963) Rec. for Identification by Hour ut Ratings for Foot Mounted Electrical Machines with Frame d Output Ratings for Rotating Electrical Machines .. Frame pment-Interchange Circuits-Assignment of Connector Pin Std. for Series of Preferred Rec. for Writing of Calendar Dates in All

culating Machines (1969)

7 Bit Character Set) (1968)

Rec. for Code for
Rec. for Punched Tape Block Formats for the Rec. for Axis and Motion Nomenclature for ape Variable Block Format for Positioning and Straight Cut ed Tape Variable Block Format Positioning and Straight Cut d Tape Fixed Block Format for Positioning and Straight Cut reads: Tolerances, Limits of Sizes for Commercial Bolt and series-Maximum / Std. for Assembly Tools for Screws and Rec. for Assembly Tools for Screws and Rec. for Slotted and Castle Rec. for Slotted and Castle

thread Diameter (1969) Rec. for Mechanical Properties of Fasteners:

s of Thread/ Std for Mechanical Properties of Fasteners: Threads: General Plan and Selection for Screws, Bolts and operties of Fasteners: Marking of Bolts, Screws, Studs and etric Screw Threads-Selected Sizes for Screws, Bolts and Rec, for Washers for Hexagon Bolts and uts: Widths Across Flats, Heights of Heads, Thicknesses of and Corners (1968) Rec. for Hexagon Bolts and $s$ of Nuts: Metric Series (196/ Rec. for Hexagon Bolts and Rec. for Classification of Parquet Strips of Solid s: Legibility Tests; Description of the ISO Mire (ISO Test

Description and Use of the ISO Micromire (ISO Micro Test

c. for Dimensions for Stereo Still Photography Using $35 \mathrm{Mm}$ or Industrial Castors-Dimensions of Top Plates-Part I: rposes (1971) Rec for Acoustics-Assessment of Std. for Coding of Character Sets for MICR and ded for the Analysis of Sounds and Vibrations (/ Rec. for ssing-Widths of Fabric Ribbons on Spools (197) Std. for Rec for Electrical Safety of ea of $35 \mathrm{~mm}$ Double Width Push Pull Sound Prints Normal and 2) Std. for lly Powered Vehicles (1970) Rec. for Rules for Rec. for Oleaginous Seeds: Determination of Rec. for Oilseed Residues: Determination of s: Paper Insulated, Metal Sheathed, Fo/ Rec. for Tests on

Noses and Face Plates Types a and Camlock: Sizes for in

Noses and Face Plates: Bayonet Type: Sizes for Intercha

Notation: Symbols for Geometrical Data (1968) ISO

Nothing Relays (Terminology, Classification, Test Meth. IEC Nothing Relays (1967)

Nothing (1969)

Nozzle Grounding Plugs and Sockets (1970)

Nozzles (1967)

NRZI, and 63 RPmm (1600 RPI), Phase Encoded (1971)

Nuclear Electronic Instruments (1967)

Nuclear Electronic Instruments) (1968)

Nuclear Energy Glossary: First Series of Terms (1972)

Nuclear Instrumentation (1969)

Nuclear Instruments (1968)

Nuclear Instruments (1970)

Nuclear Instruments: Constructional Requirements to Aff

Nuclear Power Plants for Electric Energy Generation (19

Nuclear Reactor Instrumentation (1966)

Nuclear Reactor Instrumentation (1967)

Number and Limiting-Number of Polyethylenes and Polypro

Number and Viscosity Ratio of Cellulose Acetate in Dilu

Number Designation of Organic Refrigerants (1968)

Number of Coal (1966)

Number of Knife Sections (1970)

Number of Latex (1966)

Number of PoIy (Ethylene Terephthalate) in Dilute Solut

Number of Polyamides Resins in Dilute Solution (1963)

Number of Polyvinyl Chloride Resin in Solution (1961)

Number of Pulp (Deg. of Delignification) (1963)

Number of Tufts And/or Loops Per Unit Length and Area (

Number Series for Resistors and Capacitors (1963)

Number (1972)

Numbering of Aircraft Engines, Engine Cylinders and Com

Numbering of Electrodes and Designation of Units in Ele

Numbering of Weeks (1971)

Numbering (ISBN) (1972)

\section{ISO}

Numbers and Series of (1973)

Numbers and Those Containing More Rounded Values (1966) ISO

Numbers F55 to F1080 (1971)

/Tput Ratings for Rotating IEC

Numbers of the Phase Conductors of 3 Phase Electric Sys IEC Numbers 355 to $1000(1970) \quad / C$. for Dimensions and Outp IEC Numbers 56 to 400 and Flange Numbers F55 to F1080 (1971

Numbers (1972) /- Data Terminal and Communication Equi ISO

Numbers (1973)

Numeric Form (1971)

Numeric Section of 'Ten Key Keyboards for Adding and $\mathrm{Cal}$

Numerical Control of Machines (Compatible with the ISO

Numerical Control of Machines: Coding of Preparatory Fu

Numerically Controlled Machines (1969)

Numerically Controlled Machines (1969)

Nut Threads: Medium Quality (1969)

Nuts with Metric Thread (1963)

Nuts with Metric Thread, $42 \mathrm{Up}$ to and Including $100 \mathrm{~mm}$

Object) and lts Use in Photographic Document Reproducti ISO

Object) for Checking a Reading Apparatus (1968) /Tests ISO

Objectives on $35 \mathrm{~mm}$ Film, 5-Perforation Format (1966) ISO

Oblong Top Plates with 4 Bolt Holes (1972) Std. F ISO

Occupational Noise Exposure for Hearing Conservation Pu ISO

OCR (1972)

Octave, Half-Octave and Third-Octave Band Filters Inten

Office and Printing Machines Used for lnformation Proce

\section{IEC}

ISO

Office Machines (1972)

Ecords and Scanning Ar ISO

Offet Duplicators-Attachment Features of Plates (197 ISO

Ohmic Resistors Used in the Power Circuits of Electrica

Oil Content (1968)

Oil Content (1968)

\section{IEC}

ISO

Oil Filled and Gas Pressure Cables and Their Accessorie IEC

578

702

301

$255-1$

$255-2$

46

1864

248

297

921

313

293
323

405

50 (26)

232

231

1191

1157

817
501

1168

506

1228

307

174

302
1763

267

63

127

482

2145

135

2015

2108

497

497
72

152

$72 \mathrm{~A}$

2110

3

2014

1092

840

1056 
*: Internal, for Alternating Voltages /

s: Foxternal, for Alternating Voltages / m $10 \mathrm{kV} \mathrm{Up} 10$ and lncluding $66 \mathrm{kV}$ (Excluding Gas Pressure, c. for Electrical A pparatus for Explosive Gas Atmospheres: Rec. for Loading Guide for

Rec. for

Rec. for

Rec. for

Rec. for

Rec. for

td. for Lined Industrial Rubber Boots with General Purpose Std. for Hand Finishing Sticks and Rec. for Rubber Hoses for Rec. for Raw, Refined and Boiled Linseed

Rec. for Raw Tung Rec. for Essential Std. for Essential Rec. for Essential Rec. for Essential Rec. for Essential ee Hydroxylamine Meth. (1972)

tion of Ester Value After Acetylation/ 63)

\section{8)}

atter (1968)

sis (1968)

Rec. for Crude Vegetable
Rec. for Definitions of Terms Appearing in ISO Rec. for and $X$ ylenols for Industrial Use-Determination of Neutral

Rec. Spec. for New Insulating

Rec. for Determination of Solubility of Essential Rec. for Linseed Stand

Rec. for Std. Layout for Meth. of Analysis of Essential ec. for Determination of the Refractive Index of EssentiaI rmination of the Density and Relative-Density of Essential

Meth. for Assessing the Oxidation Stability of Insulating $r$ the Determination of the Electric Strength of Insulating ec. for Determination of the Optical Rotation of Essential

Rec. for Oleaginous Seeds: Determination of Acidity of ion of Ester Value and Calculation of Content of Essential

Rec. for Determination of Freezing Point of Essential rochloric Acid (1968)

t (1968)

e Matter (1968)

8)

\section{e Matter (1968)}

les for Analysis (1968)

: 2,2'-Bipyridyl Spect/

gnition; Gravimetric Meth. (/

$y$ and Calculation of Free So/ en Content: Volumetric Meth./ ide Content: Barium Sulphate/ itrogen-2,4-Xylenol Spec/ of Total Acidity

Rec for Sulphuric A

Rec for Sulphuric

Rec. for Sulphuric Acid and

Rec. for Sulphuric Acid and

Rec. for Sulphuric Acid and

Std. for Sulphuric Acid and and Calculation of Free $\mathrm{SO}_{3}$ Content of Rec. for Liquid Flow Measurement in Rec. for Liquid Flow Measurement in Rec. for Liquid Flow Measurement in Constant Rate Injec/ Rec. for liquid Flow Measurement in
\& for Frequencies Below $3 \mathrm{MHz}$ : Edge Socket Connectors with s for Frequencies Below $3 \mathrm{MHz}$ : Edge Socket Connectors with
ipbuilding Details for lnland Navigation-Covers for Deck

\section{4)}

vacuum Cleaners and Water Sucti/

clocks (1967)

washing Machines (1968)

spin Extractors (1968)

shavers, Hair Clippers and Simi/

massage Appliances (1967)

tocks (1973)

or Reciprocating Internal Combustion Engines-Hand

Spec. for Voltage

Spec. for Mains
Spec. for Battery

and Similar Gene/

and Similar Gener/

Spec. for Safety Requirements for Mains
Rec. for Safety Requirements for Mains $\begin{array}{ll} & \text { Rec. for Dimensions of Spindle Ends for Manually } \\ \text { eral Us/ } & \text { Rec. for Safety Equipment for Splash Proof Mains }\end{array}$ Rec. for Performance Requirements for Lever
ec. for Analogue Voltage Ranges and Logic Levels for Mains Rec. for Hand Rec. for Dimensions for Single Hole Mounting, Lever
Oil Filled and Gas Pressure Cables and Their Accessorie

Oil Filled and Gas Pressure Cables and Their Accessorie

Oil Filled and Nondraining Types) (1965)

Oil Immersed Apparatus (1968)

Oil Immersed Transformers (1972)

Oil of Brazilian Sassafras (1967)

Oil of Eucalyptus Globulus (1968)

Oil of Lemon, Expressed, Italy (1968)

Oil of Peppernint: France, Italy, United Kiıgdom and U.

Oil of Rosemary (1971)

il Resistance (1972)

Oil Stones-Dimensions (1972)

Oil Suction and Discharge (1971)

Oil (1960)

Oil (1962)

Oils-Determination of Acid Value (1971)

Oils-Determination of Carbonyl Compounds Content-Fr

Oils-Determination of Cineole Content (1970)

Oils-Estimation of Free Alcohols Content by Determina

Oils-Meth. of Test-Preparation of Sample Sample (19

Oils and Fats: Determination of Acidity (1968)

Oils and Fats: Determination of Insoluble Impurities (1

Oils and Fats: Determination of Moisture and Volatile $M$

Oils and Fats: Preparation of Contract Sample for Analy

Oils and Pigments (1963)

Oils and Pyridine Bases (1971)

Oils in Ethanol (1968)

Oils (1962)

Oils (1962)

Oils (1962)

Oils (1962)

Oils (1963)

Oils (1963)

Oils (1967)

Oils (1968)

Oils (1968)

Oils (1969)

Oilseed Residues: Determination of Ash Insoluble in Hyd /Cresol, Cresylic Acid ISO

IEC

ISO

ISO

ISO

Rec, for Dete ISO

Rec. for IEC

Rec. for Meth. Fo IEC

ISO

ISO

Rec, for Determinat ISO

ISO

ISO

Oilseed Residues: Determination of Moisture and Volatil

Oilseed Residues: Determination of Oil Content (1968)

Oilseed Residues: Determination of Total Ash (1968)

OiIseeds-Sampling (1967)

Oils: Labelling and Marking Containers (1961)

Oils: Packing (1961)

Oils: Sampling (1961)

Oleaginous Seeds: Determination of Acidity of Oils (196

Oleaginous Seeds: Determination of Impurities (1968)

Oleaginous Seeds: Determination of Moisture and Volatil

Oleaginous Seeds: Determination of Oil Content (1968)

Oleaginous Seeds: Reduction of Contract Samples to Samp

Oleum for Industrial Use: Determination of Iron Content

Oleum for Industrial Use: Determination of Residue on I

Oleum for Industrial Use: Determination of Total Acidit

Oleum for Industrial Use: Determination of Total Nitrog

Oleum for Industrial Use; Determination of Sulphur Diox

Oleums for Industrial Use: Determination of Oxides of $\mathrm{N}$

Oleum; Volumetric Meth. (1968)

IIal Use: Deter

Open Channels-Establishment and Operation of a Gaugin

Open Channels by Slope Area Meth. (1969)

Open Channels by Velocity Area Meth. (1968)

Open Channels: Dilution Meth. for Steady Flow-Part 1:

Open Ends, Off Centre (Center) Guide, Having a Contact

Openings for $220 \mathrm{~mm}$ Pumps (1957)

Operated Appliances for Domestic and Similar Purposes (

Operated Appliances for Domestic and Similar Purposes:

Operated Appliances for Domestic and Similar Purposes:

Operated Appliances for Domestic and Similar Purposes:

Operated Appliances for Domestic and Similar Purposes:

Operated Appliances for Domestic and Similar Purposes:

Operated Appliances for Domestic and Similar Purposes:

Operated Circular Screwing Dies and Hand-Operated Die S Operated Control Devices-Std. Direction of Motion (19

Operated Earth Leakage Circuit Breakers for Domestic an

Operated Electric Fence Controllers (1956)

Operated Electric Fence Controllers (1956)

Operated Electronic and Related Equipment for Domestic

Operated Electronic and Related Equipment for Domestic

Operated Electronic Components (1972)

Operated Electronic Equipment (Domestic and Similar Gen

Operated Manual Switches for Aircraft (1970)

Operated Nuclear Instruments (1970)

Operated Stillage Trucks: Principal Dimensions (1969)

Operated Switches for Aircraft (1966)

Operated Tools (1967)
141.2

141.3

$55-\mathrm{I}$

79-6

354

590

770

855

856

1342

2025

1823

150

277
1242

1242
1271

1202

$124]$

356

660

663

662

661

339

1899

296

875

276
278 
avic Arrangement for the Alphanumeric Section of Keyboards hreakers (19.36) Rec. for lland Rec. for Std. Direction of Motion of
tions and Meth. of Measurement for Piezoelectric Vibrators Std. for Dictation Equipment: Basic rement of the Winding Resistance of an a.c. Machine During quid Flow Measurement in Open Channels-Establishment and Rec. for Shape, Size and Direction of Rec. for Direction of Rec. for Principles of Rec. for Directions of Rec. for Printing Spec. for

Rec. for Sizes of Photocopics (On Paper) Readable Without for Paper Vocabulary-6Th Series of Terms (Definitions of International Rec. for Rec. for Alphanumeric Character Sets for Rec. for Determination of the

8) Rec. for Plastics-Preparation of Specinıens for 68) Rec. for Plastics-Preparation of Specimens for ate for Industrial Use: Determination of Acidity to Methyl for Information Processing Interchange: Representation of Rec. for Hollow Metallic Waveguides: Relevant Spec, for for Flanges for Waveguides: Relevant Spec. for Flanges for Rec. for Meth. of Sampling Manganese Ores; Part I: express/ Rec. for Meth. of Chemical Analysis of Manganese 3) Rec. for Meth. of Chemical Analysis of Manganese Rec. for Meth. of Chemical Analysis of Manganese Rec. for Meth. of Chemical Analysis of Manganese Rec. for Meth. of Chemical Analysis of Manganese Rec. for Meth. of Chemical Analysis of Manganese Rec. for Meth. of Chemical Analysis of Manganese Rec. for Meth. of Chemical Analysis of Manganese Rec. for Meth. of Chemical Analysis of Manganese Rec. for Meth. of Chemical Analysis of Manganese Rec. for Meth. of Chemical Analysis of Manganese Rec. for Meth. of Chemical Analysis of Manganese Rec. for Meth. of Chemical Analysis of Manganese Rec. for Meth. of Chemical Analysis of Manganese xine Gravimetric and Volumetric Meth. (197) Std. for Iron Rec. for Meth. of Chemical Analysis of Manganese Rec. for Meth. of Chemical Analysis of Manganese Rec. for Meth. O6 Chemical Analysis of Manganese Rec. for Meth, of Chemical Analysis of Manganese al Samples (I973)
Std. for Iron ric Met/ Rec. for Meth. of Chemical Analysis of Manganese meth. (1973)

Rec. for Meth. of Chemical Analysis of Manganese Rec. for Meth. of Chemical Analysis of Manganese Rec. for Meth. of Chemical Analysis of Manganese Rec. for Meth. of Chemical Analysis of Manganese Rec. for Meth. of Sampling Manganese Rec. for Number Designation of

lecommunication / Rec. for Strip Wound Cut Cores of Grain Rec, for Measurement of Fluid Flow by Means of Rec. for Gravity Filling

ic Acid and Xylenols for Industrial Use-Determination of mination of Residue on Evaporation (197/

mination of Crystallizing Point (1971)

$s$ for Industrial Use-Determination Of/

$s$ for Industrial Use-Determination Of/ (1970)

( $($ I970)

$s$ for Industrial Use-Determination Of/ gnesium and Its Alloys: Photometric Determination of Iron uminium and Its Alloys: Photometric Determination of Iron eeders and Conveyors, Shaking or Reciprocating Feeders and s Mechanical Handling Equipment for Loose Bulk Materials s Mechanical Handling Equipment for Loose Bulk Materials

rs: Bandwidth, Out of Band Power and Power of Nonessential

trical Properties of Microwave Tubes and Valves: Low Power al Properties of Microwave Tubes and Valves: Backward Wave ction 2-Test Conditi/ Rec. for Quartz Crystal Units for Rec. for Quartz Crystal Units for Rec, for Quartz Crystal Units for Rec. for Preparation of Outline Drawings of tronic Tubes and Valves: Meth. of Measurement of Radar and Rec. for Expression of the Properties of Cathode Ray nal Voltages Greater T/ Rec. for Dimensions of Indoor and es Greater Than I,000 V (19/ Rec. for Tests on Indoor and

e Noise Emitted by Compressor Primeover Units Intended for Roller-Boundary Dimensions-Subunits-Metric Series. Rec. for Plugs, Socket

Rec. for Std for Plugs and Socket Spec. for Plugs and Socket Spec. for Plugs and Socket
Operated with Both Hands (1971)

Rec. for B ISO

Operated Wrenches and Sockets-Technical Spec. (1970) ISO

$\begin{array}{ll}\text { Operating Devices and for Indicating I,amps for Circuit } & \text { IEC } \\ \text { Operating Over the Frequency Range Up to } 30 \mathrm{MHz}(1969) & \text { IEC }\end{array}$

IEC

Operating Requirements (1973)

Operation at Alternative Voltage (1969)

Ec. for Measu IEC

Operation of a Gauging Station and Determination of the ISO

Operation of Lever Controls on Aircraft (1969) ISO

Operation of Machine Tool Controls (1965)

Operation of Standards Marks (1961)

Operation of Toggle Switches on Aircraft (1957)

Optical Character Recognition (1971)

OpticaI Devices (I960)

Optical Properties) (1971)

Optical Pyrometers with Disappearing Filaments (1970)

Optical Recognition (1969)

Optical Rotation of Essential Oils (1967)

Optical Tests on Plastics Materials: Casting Meth. (196

Optical Tests on Plastics Materials: Moulding Meth. (19

Orange (I973)

Std. for Potassium Sulph ISO

Ordinal Dates (I973)

Ordinary Rectangular Types (1964)

Ordinary Rectangular Types (1968)

Ore Loaded in Freight Wagons (1963)

Ores-Determination of Active Oxygen-Conventionally

Ores-Determination of Aluminium (Aluminum) Oxide (196

Ores-Determination of Arsenic (1963)

Ores-Determination of Carbon Dioxide (1963)

Ores-Determination of Cobalt (1963)

Ores-Determination of Copper (1963)

Ores-Determination of Hygroscopic Moisture (1963)

Ores-Determination of Lead (1963)

Ores-Determination of Nickel (1963)

Ores-Determination of Phosphorus (1963)

Ores-Determination of Silicon Dioxide (1963)

Ores-Determination of Sulphur (1963)

Ores-Determination of Total Iron Content (1963)

Ores-Determination of Total Manganese Content (1963)

Ores: Determination of Aluminium (Aluminum) Content-O

Ores: Determination of Barium Oxide Content (1966)

Ores: Determination of Calcium Oxide Content and Magnes

Ores: Determination of Chromium Content (I967)

Ores: Determination of Combined Water Content (1966)

Ores: Determination of Hygroscopic Moisture in Analytic

Ores: Determination of Metallic Iron Content: (Photomet

Ores: Determination of Phosphorus Content-Volumetric

Ores: Determination of Titanium Content (1966)

Ores: Detcrmination of Vanadium Content (1966)

Ores: Determination of Zinc Content (Polarographic Meth

Ores: Determination of Zinc Content (I966)

Ores; Part I: Ore Loaded in Freight Wagons (1963)

Organic Refrigerants (I968)

Organization, Rules and Procedures of the CISPR (1971)

Oriented Silicon Iron Alloy, Used for Electronic and Te

Orifice Plates and Nozzles (1967)

Orifices for Aircraft (1959)

Ortha Cresol Content (1971)

Ortha, Meta, and Para Cresol for Industrial Use-Dete

Ortha, Meta, and Para Cresol, Cresylic Acid and Xyleno

Ortha, Meta, and Para Cresol, Cresylic Acid and Xylenol

Ortho Chlorotoluene for Industrial Use-Meth. of Test

Ortho Dichlorobenzene for Industrial Use-Meth. of Tes

Ortho, Meta, and Para Cresol, Cresylic Acid and Xylenol

(Orthophenanthroline Meth. Applicable to Iron Content Be

Orthophenanthroline Meth. Applicable to

Oscillating Conveyors and Shaking or Reciprocating Feed

ISO

Std. ISO

Rec. IEC

ISO

ISO

ISO

ISO

ISO

ISO

ISO

ISO

ISO

ISO

ISO

ISO

ISO

ISO

ISO

ISO

ISO

ISO

ISO

ISO

ISO

ISO

ISO

CISPR

IEC

ISO

ISO

ISO

ISO

ISO

ISO

ISO

ISO

ISO

ISO

ISO

ISO

ISO

7 Measurement for Radio Transmitte IEC

Oscillator Klystrons (1972) /R Measurement of the Elec IEC

Oscillator Tubes-0 Type (1972) /Nt of the Electric IEC

Oscillators: Section 1-Std. Valves and Conditions: Se IEC

Oscillators: Section 3-Guide to the Use of (1962) IEC

Oscillators: Section 4: Std. Outlines (1962)

Oscilloscope and Picture Tubes (1962)

Oscilloscope Cathode Ray Tubes (1969)

Oscilloscopes (1971)

Outdoor Post Insulators and Units for Systems with Nom

Outdoor Post Insulators for Systems with Nominal Voltag

Outdoor Use (1972)

Outer Rings with Flange (1973

Std. for Measurement of Airborn

Outlets and Couplers for Industrial Purposes (1969)

Outlets for Domestic and Similar General Use (1957)

Outlets for Domestic and Similar Purposes (1963)

/Perties of Elec IEC

IEC

IEC

IEC

ISO

ISO

IEC

CEE

Outlets for Industrial Purposes (1966)

2126

1711

34

1730

279

1100

440

447

189

44

1831

169

1912

18

1073

592

870

869

2489

2711

153-2

154-2

309

318

317

314

316

322

310

323

315

311

320

313

(19

548

552

619

549

2596

621

2599

550

553
620

551

309

817

329

54 I

102

1910

1900

1901

1898

I899

I 695

I 698

1897

792 
Rec. for Preparation of for Quartz Crystal Units for Oscillators: Section 4: Std. rties of Electronic Tubes and Valves: Equivalent Input and
for Radio Transmitters: General Conditions Of, Frequency,

Tubes and Valves: Meth. of Measurement of Audio Frequency

Tubes and Valves: Meth. of Measurement of Radio Frequency h Frame Numbers 355 to 1000 (197) Rec. for Dimensions and e Numbers 56 to 400 and Flange N/ Rec. for Dimensions and Expendable Photoflash Lamps-Determination of the Light or Rolling Bearings-Radial Types with Shields or Seals $r$ Pipes of Plastics Materials for the Transport of Fluids r Pipes of Plastics Materials for the Transport of Fluids Rec. for Recommended Range of

he Transport of Fluids-Polyethylene (PE)-Tolerances on plasticized Polyvinyl Chloride (PVC) Pipes-Tolerances on Std. for Steel Tubes and and Tubular Socket Wrenches-Metric Series-Maximum
Std. for Rolling Bearings: with Spherical

luid Flow by Means of Venturi Tubes-Classical Tubes Used millimeters) and In/ Rec. for Slotted Raised Countersunk r Paper-Meth. for the Determination of Moisture Content Rec. for Dextrose-Determination of Moisture-Vacuum se Syrup-Determination of Dry Matter of Moisture (Vacuum yl Chloride (PVC) for Use Under Pressure (1967) Rec. for ation of the Behaviour of Plastics in a Ventilated Tubular for Measuring Performance of Household Electric Ranges and clude Detachable Sheets (1967)

Rec. for Lathe Tool Posts Rec. for Paper:
for Tests on Insulators of Ceramic Material or Glass for Continuous Mechanical Handling Equipment for Unit Loads Continuous Mechanical Handling Equipment for Unit Loads Std. for Duplicators-Minimum esium and Its Alloys: Photometric Determination of Copper inum) and Its Alloys: Photometric Determination of Copper Rec. for Meth. for Assessing the ulation Check by Measuremen/ Std. for Anodization (Anodic rec. for Surface Treatment of Metals-Anodisation (Anodic rec. for Surface Treatment of Metals-A nodisation (Anodic Groups (1972) Std. for Surface Active Agents: Ethylene tes for Industrial Use-Determination of Sodium and Boric of Cross Sections (197/ Rec. for Measurement of Metal and (Aluminum) and Its Alloys-Measurement of the Mass of the um (Aluminum) and Its Alloys-Measurement of Thickness of tes for Industrial Use-Determination of Sodium and Boric mical Analysis of Manganese Ores: Determination of Calcium emical Analysis of Manganese Ores: Determination of Barium ores: Determination of Calcium Oxide Content and Magnesium tes for Industrial Use-Determination of Sodium and Boric -Volumetric Meth. (1972) Std. for Boric Rec. for General Classification of Ferromagnetic Rec. for Iron

ination of Loss of Mass at $1000 \%$

determination of Silica Content-Spec/ $\begin{array}{ll}\text { determination of Silica Content-Spec/ } & \text { Rec. for Aluminum } \\ \text { determination of Sodium Content-Flam/ } & \text { Rec. for Aluminum }\end{array}$ determination of Vanadium / Rec. for Aluminium (Aluminum) determination of Calcium Content-Ato/ determination of Zinc-Atomic Absorpt/ determination of Zinc Content-Pan Ph/ preparation of Sample Solution for Ana/ for Aluminium (Aluminum) etermination of Calcium Content-Spe/ termination of Iron Content-1,10-1 termination of Titanium Content; Tiro/ termination of Absolute Density; Pykn/ asurement of the Angle of Repose (196) asurement of Untamped Density (1968) Rec. for Aluminium (Aluminum) and Storage of Test Sampl/ Rec. for Aluminium (Aluminum)

of Manganese Ores-Determination of Aluminium (Aluminum) e of Nonionic Surface Active Agents Obtained from Ethylene amate Photometric Meth. (1972) etermination of Manganese Conten/ or Industrial Use: Determinat/

Std. for Boric Acid and Std. for Boric Acid and Std. for Boric Acid, Boric

(X.Cores) Made of Ferromagnetic

Rec. for Dimensions for Pot Cores Made of Ferromagnetic pec. for Cores of Broad Band Transformers of Ferromagnetic cores of Tuned Transformers and Inductors of Ferromagnetic

huric Acid and Oleums for Industrial Use: Determination of Rec. for Dimensions of Screw Cores Made of Ferromagnetic c. for Dimensions of Tubes, Pins and Rods of Ferromagnetic for Dimensions of Aerial Rods and Slabs of Ferromagnetic for Hydrochloric Acid for Industrial Use: Determination of al Use-Determination of Manganese Content-Formaldehyde iron Ores: Determination of Aluminium (Aluminum) Content ents: Ethylene Oxide. Adducts-Iodometric Determination of
Outline Drawings of Oscilloscope and Picture Tubes (196

IEC Outlines (1962)

Output Admittances (1963) Rec. IEC
/Nts of the Electrical Prope IEC Output Power and Consumption (1968) /H. of Measurement IEC Output Power and Distortion (1966) /Ties of Electronic IEC Output Power (1966) Ectrical Properties of Electronic IEC Output Ratings for Foot 'Mounted Electrical Machines Wit lEC Output Ratings for Rotating Electrical Machines .. Fram IEC Output (1972) Std. for Photography ISO

utside Diameter Tolerances-Normal and Tolerance Clas (Outside Diameters and Nominal Pressures) Part II: In. S

Outside Diameters and Nominal Pressures): Part I: Metri

Outside Diameters for Milling Cutters (1966)

Uutside Diameters (1970)

Outside Diameters (1971)

the Transport of Fluids-Un ISO

Outside Dimensions (1972) lor Screws and Nuts-Forged ISO

Outside Surface and Extended Inner Ring Width (1972) ISO

Outside the Scope Covered by ISO Rec. R781 (1969) /F F ISO

(Oval) Head Tapping Screws-Dimensions in Millimetres ( ISO

(Oven Drying Meth.) (1963)

Oven Meth. (1970)

Oven Meth.) (1970)

Oven Test for Moulded Fittings in Unplasticized Polyvin ISO

Oven (1969)

Ovens (1971)

/Plastics-Meth. of Test for the Determin ISO

Rec. Meth. IEC

(1961)

Overall Trimmed Sizes of Articles of Stationery That in

Overhead Lines with a Nominal Voltage Greater Than 1000

Overhead Monorail Chain Conveyors-Safety Code (1971)

Overhead Twin Rail Chain Conveyors (Power and Free) Saf

ISO

ISO

IEC

ISO

Overprint on Duplicating Stencils (1972)

Oxalyldihydrazide Meth. A pplicable to Copper Content Be

Oxalyldihydrazide Meth. Applicable to Its Content Betwe

Oxidation Stability of Insulating Oils (1963)

Oxidation) of Aluminium (Aluminum) and Its Alloys-Ins

Oxidation) of Aluminium (Aluminum) and Its Alloys-Mea

Oxidation) of Aluminium (Aluminum) and Its Alloys-Mea

Oxide Adducts-Iodometric Determination of Oxyethylene

Oxide and Available Oxygen Contents-Volumetric Meth.

Oxide Coating Thicknesses by Microscopical Examination

Oxide Coatings-Gravimetric Meth. (1971)

Oxid Coatings-Nondestructive Measurement by Light Se ISO

Oxide Content and Loss on Ignition (1972) M Tetrabora ISO

Oxide Content (1966)

Oxide Content (1966)

Rec. for Meth. of Ch ISO

Oxide Contents-Volumetric Meth. (1972) Analysis of Manganese ISO

Oxide for Industrial Use-Determination of Its Content

Oxide for Paints (1962)

Oxide Materials and Definitions of Terms (1961)

Oxide Pigments (1970)

Oxide Primarily Used for Production of Aluminum: Determ

Oxide Primarily Used for the Production of Aluminium-

Oxide Primarily Used for the Production of Aluminium-

Oxide Primarily Used for the Production of Aluminium

Oxide Primarily Used for the Production of Aluminium-

Oxide Primarily Used for the Production of Aluminium-

Oxide Primarily Used for the Production of Aluminium-

Oxide Primarily Used for the Production of Aluminium-

Oxide Primarily Used for the Production of Aluminium: $P$

Oxide Primarily Used for the Production of Aluminum-D

Oxide Primarily Used for the Production of Aluminum: De

Oxide Primarily Used for the Production of Aluminum: De

Oxide Primarily Used for the Production of Aluminum: De

Oxide Primarily Used for the Production of Aluminum: Me

Oxide Primarily Used for the Production of Aluminum: Me

Oxide Primarily Used for the Production of Determinatio

Oxide Primarily Used for the Production Of; Preparation

Rec. for Meth. of Chemical Analysis

Oxide (1963)

Ermination of the Cloud Point Temperatur

Oxide, and Disodium Tetraborates for Dibenzyldithiocarb

Oxide, and Disodium Tetraborates for Industrial Use-D

Oxide, Disodium Tetraborates and Crude Sodium Borates $F$

Oxides and Associated Parts (1967)

Oxides and Associated Parts (1971)

Oxides for Telecommunication (1966)

Oxides for Telecommunication (1966)

Oxides of Nitrogen-2,4-Xylenol Spectrophotom

Oxides (1966)

Oxides (1966)

Oxides (1966)

Oxidizing or Reducing Substances Volumetric Meth. (1968

I of Performance S IEC /Ramnce Spec. for IEC

Oxime Photometric Meth. (1972)

Oxine Gravimetric and Volumetric Meth. (1973)

ic Meth. (1968

Re IEC

Re IEC

Oxyethylene Groups (1972) Std. for Surface Active Ag 1 SO
139

$122-3$

151.3

244-1

$151-10$

151.11

$72 \mathrm{~A}$

1229

1648

330

161
523

1164

1330

64

2236
2264

$781 / \mathrm{I}$

1483

287

1742

580

1137

350

618

383
2149

2150

2133

794

795

2376

2106

2128

2270

1917

1463

2106

2128

1916

552

548

2216

1915

275

125

1248

806

1232

1617

1618

2069

2071

2072

2073

2070

805

901

902

903

803

802

318

1065

2215
2214

1918

226

133

219
218
2363

221 
miral Analysis of Manganese Ores-Determination of Active ination of Sulphur (Meth. After Combustion in a Current of se-1)etermination of Sodium and Boric Oxide and Available geahility Dimensions (197I) Rec. for liquid Rec. for Gaseous Rec. for Hard Coal-Determination of Carbon (Gravimetric Meth. After Combustion in a Stream of d. for Vulcanized Rubbers-Determination of Resistance to inuous Filament Yarn, Staple Fibre (Fiber) Type and Roving for Carbon Black for Use in the Rubber Ind ustry-Sampling it Length) Skein Meth. (197/

Elongation of Same (OF Sin/ Std. for Textiles: Yarn from Std. for Textiles: Yarn from Std. for Packaging-Complete, Filled Transpor Std. for Packaging-Complete, Filled Transpor Std. for Packaging-Complete, Filled Transport 972) Std for Packaging-Complete, Filled Transport ane and $\mathrm{P}$ Std. for Packaging: Complete, Filled Transport

1972) Std. for Packaging-Complete, Filled Transport Rec. for Pictorial Marking of Transi

Machinery-Types of Formers for Yarn and Staple Fibre (Fiber) Yarns and Rovings in the Form of

1: Identification of Parts When Test. (1972)

ii: Conditioning for Test. (1972)

iii: Stacking Test (1972)

vi: Vibration Test (1972)

iv: Vertical Impact Test by Dropping (1972) Rec. for Elastomeric Sealing Rings for Aircraft

Std. for Std. for

Std. for

Std. for Rec. for

(1968)

Rec. for Quantity
Rec. for Rec. for
Rec. for

y Dry Corkwood in Planks-Definitions, Classification and gleanings, Corkwood Refuse and Corkwaste-Definitions and Horizontal Impact Tests (Inclined Plane and P/ Std. for Rec. For Essential Oils:

Agglomerated Thermal Cork-Characteristics, Sampling and (1970) Rec. for Aircraft Jacking Rec. for Rec. for Rec. for Rec. for Rec. for Rec. for Rec. for Rec. for Rec. for Rec. for Rec. for Rec. for Rec. for eth.) (1970) Rec. for Sampling Raw Materials for
Std. for Std. for

of a Flow Cup (1972) Rec. for Zine Oxide for Rec. for Titanium Dioxide for Std. for Strontium Chromate Pigments for Std. for Barium Chromate Pigments for Std. for Iron Blue Pigments for Std. for Mineral Solvents for

(1972) or Stranded Conductors, Insulated, Screened, Single or One $\mathrm{h}$ : Distribution Wires with Solid Conductors, Insulated, in solid Conductors, Insulated, Polyamide Coated, in Singles,

Pve (Polyvinyl Chloride) Insulation and Sheath: Cables in Rec. for Principal Dimensions of Rec. for Double Deck Flat Rec. for Large

Rec. for Vocabulary of Terms Relating to Rec. for Slotted Rec. for Slotted

llimeters) and In./S (1970)

Production of Aluminium-Determination of Zinc Content 1968) Rec. for Dimensions of reinforced, Resin Bonded, Low Pressure Laminated Plates or
Rec. for Paints and Varnishes-Standard

Rec. for Mosaic Parquet

Rec. for Plywood-Measurement of Dimensions of Rec. for
Rec. for

(Oven Drying Meth.) (1963)

for Insulating Materials Based on Built Up or Treated Mica

Rec. for Meth. of Sampling Rec. for Meth. of the Conditioning of Rec. for

Rec. for Trimmed Sizes of Writing for Meth. of Expression of Dimensions of Processed Writing ec. for Dimensions for 127, 120 and 620 Roll Film, Backing
Oxygen-Conventionally Expressed as Manganese Dioxide

ISO Oxygen and Titration with Sodium Borate) (1968) /Eterm ISO Oxygen Contents-Volumetric Meth. (1972) /Ndustrial U ISO Oxygen Replenishment Couplings for Aircraft-Interchan ISO Oxygen Replenishment Couplings for Aircraft (1969) ISO Oxygen (1971)

Oxygen) (1965)

Ysis of Steels: Determination of Total ISO Orone Cracking Under Static Conditions (1972) St ISO Package (1971) /Pling Batches or Consignments Of, Cont ISO Packaged Shipments (1970)

Packages-Determination of Linear Density (Mass Per Un

Packages-Meth for Determination of Breaking Load and ISO

Packages-Part III: Stacking Test (1972)

Packages-Part II: Conditioning for Test. (1972)

Packages-Part IV: Vertical Impact Test by Dropping (1

Packages-Part Vi: Vibration Test (1972)

Packages-Part V: Horizontal Impact Tests (Inclined PI

Packages-Part 1: Identification of Parts When Test. (

Packages Containing Photographic Materials Sensitive to

Packages (1970)

Packages (1971) /Ar Density of Its Continuous Filament

Packaging-Complete, Filled Transport Packages-Part

ackaging-Complete, Filled Transport Packages-Part

Packaging-Complete, Filled Transport Packages-Part

Packaging-Complete, Filled Transport Packages-Part Packaging and Identification (1971)

Packaging of Components on Continuous Tapes (1968)

Packaging of Sensitized Photographic Materials (1969)

Packaging of Winding Wires: Containers for Round Types

Packaging of Winding Wires: Delivery Spools (1968)

ackaging (1970)

Packaging (1970)

Commerciall IS

Packaging: Complete, Filled Transport Packages-Part $\mathrm{V}$ ISO

Packing (1961) ISO

Packing (1972) Std. for Cork-Expanded Pure ISO

Pads (1957)

Paints and Varnishes-Bend Test (Cylindrical Mandrel)

Paints and Varnishes-Cupping Test (1970)

Paints and Varnishes-Determination of Fineness of Gri

Paints and Varnishes-Determination of Flashpoint (Clo

Paints and Varnishes-Determination of Resistance to $W$

Paints and Varnishes-Determination of the Danger Clas

Paints and Varnishes-Determination of Volatile and No

Paints and Varnishes-Examination and Preparation of S

Paints and Varnishes-Pendulum Damping Test (1971)

Paints and Varnishes-Sampling (1970)

Paints and Varnishes-Scratch Test (1970)

Paints and Varnishes-Standard Panels for Test. (1970)

Paints and Varnishes-Surface Drying Test (Ballotini M

Paints and Varnishes (1968)

Paints and Varnishes: Crosscut Test (1972)

Paints and Varnishes: Determination of Flow Time by Use

Paints (1962)

Paints (1967)

Paints (1972)

Paints (1972)

Paints (1972)

Paints: White Spirits and Related Hydrocarbon Solvents

Pair (1969)

$/ \mathrm{H}$ : Equipment Wires and Cables with Solid IEC

Pairs, Triples, Quadruples and Quintuples (1968)

Pairs, Triples, Quadruples and Quintuples (1971)

Pairs, Triples, Quads and Quintuples for Inside Install

Pallet Trucks (1966)

Pallets for Through Transit of Goods (1961)

Pallets for Through Transit of Goods (1963)

Pallets (1965)

Pan Head Screws-Metric Series (1970)

Pan Head Tapping Screws-Dimensions in Millimetres (Mi ISO

Pan Photometric Meth. (1971)

Panels and Racks (For Nuclear Electronic Instruments) ( IEC

Panels for Test Purposes (1970) / Glass Fibre (Fiber) ISO

Panels for Test. (1970) ISO

Panels (1967)

Panels (1969)

Paper-Determination of Tearing Resistance (1971)

Paper-Meth. for the Determination of Moisture Conten

Paper-Part 1: Definitions and General Requirements (1

Paper and Board-Dctermination of Ash (1971)

Paper and Board-Determination of Tensile Strength (19

Paper and Board for Test. (1968)

Paper and Board Test Samples (1961)

Paper and Board: Sizes of Folders and Files (1967)

Paper and Certain Classes of Printed Matter (1961)

Paper and Certain Classes of Printed Matter (1963)

Paper and Spools (1971) 
weation of Rectangular Punched Holes in 80 Columns Punched 30 (19.59) $20^{\prime}(19.59)$

$15^{\prime}(1959)$

I (1969)

(1969)

(1969)

voltages from $10 \mathrm{kV} \mathrm{Up} \mathrm{to} \mathrm{/}$

$s$ for Alternating Voltages/ Oil Filled and Gas Pressure Cables and Their Accessories: Rec. for Determination of the Water Absorption of for Fixed Capacitors for Direct Current Using Impregnated Rec. for

Rec. for Photographic Black and White Paper for Roll Rec. for Photographic Colour (Color) Paper for Roll Rec for Determination of Std. for Data Interchange on Rolled Up Punched Rec. for Dimensions for Punched Rec. for.Information Processing-Properties of Unpunched Rec. for Rec. for Rec. for Fixed Metallized Rec. for Photographic Colour (Color) Rec. for Photographic Black and White ec. for Photographic Black and White Rec for Tests on Impreated Rec. for Rec. for Rec. for Rec. for Rec. for Rec. for Rec. for Substances of Rec. for Width of Sheets of rection of Manufacture of Unprocessed Writing and Printing for the Determination of the Bulking Thickness and Bulk of ec. for Determination of the Thickness of Single Sheets of d Capacitors for Direct Current Using Impregnated Paper or Rec. for Sizes of Photocopies (On al (1969) Rec. for Photographic Black and White the Images of Processed Black and White Films, Plates and in Processed Black and White Photographic Film, Plates and Rec. for Rec. for Rec. for Rec. for Rec. for Rec. for Rec. for

primary Range (1966)

supplementary Range (1967)

1970)

allizing Point (1971)

ue on Evaporation (197)

use-Determination of

use-Determination Of/

use-Determination Of/

(1970)

70) king (1972) Rec. for Gymnastic Equipment

ns An/

ectio/ Rec. for Dimensions of Hot Rolled Steel Sections Rec. for Rectangular or Square Rec. for Test Conditions for General Purpose Rec. for Machine Chucking Reamers with Std. for

hank (1961) Rec. for Twist Drills: g Series (1970) Rec. for End Mills with hanks and Sizes of Driving Squares for Rotating Tools with Rec. for Perforated

1972)

4) Rec. for Fundamental
Ror Core Drills with Rec. for Calculation of the Effective Devices for Measurement and Evaluation of the Geometrical o Determine the Compatibility of Steel Filler Rods and the Rec. for the Use of Longitudinal Rec. for Mosaic Rec. for Classification of c. for General Manufacturing Characteristics of Solid Wood Std. for Classification of Solid Beech (Wood) Rec. for Rec. for Rec. for Rec. for eces (1968) 8) Rec. for Plastics-Determination of Insoluble Std. for Shipbuilding: Cargo Gear

Diagrams, Charts, Tables: Item Desienation for Electrical Rec. for Cast Iron Pipes: Special Castings and Rec. for Dimensions of the Mating
Paper Cards (Information Processing) (1971)

/Ons and I. ISO

Paper Cards (1970)

Paper Cones for Yarn Winding (Cross Wound): Taper 3 Deg

Paper Cones for Yarn Winding (Cross Wound): Taper 4 Deg

Paper Cones for Yarn Winding (Cross Wound): Taper 9 Deg

Paper Dielectric Capacitors for Direct Current (1965)

Paper for General Use: Preferred Sizes of Sheet Materia

Paper for Roll Paper Printers: Preferred Sizes of Rolls

Paper Insulated Metal Sheathed Cables: for Alternating

Paper Insulated Metal Sheathed Cables: Nondraining Type

Paper Insulated, Metal Sheathed, for Alternating Voltag

Paper or Board (Cobb Meth.) (1967)

Paper or Paper / Plastic Film Dielectric (1964)

Paper Patterns for Dobbies (1967)

Paper Printers: Preferred Sizes of Rolls (1969)

Paper Printers: Preferred Sizes of Rolls (1969)

Paper Substance (1967)

Paper Tape-General Requirements (1972)

Paper Tape for Data Interchange (1969)

Paper Tape (1971)

Paper Vocabulary-5Th Series of Terms (1970)

Paper Vocabulary-6Th Series of Terms (Definitions of

Paper Vocabulary: First Series of Terms (1958)

Paper Vocabulary: Fourth Series of Terms (1964)

Paper Vocabulary: Second Series of Terms (1959)

Paper Vocabulary: Third Series of Terms (1961)

Paper (1958)

Paper (1959)

Paper (1961)

Paper (1965)

Meth of Expression of Dimensions and Di ISO

Rec. for Meth.

Paper (1967)

Paper / Plastic Film Dielectric (1964)

Paper) Readable Without Optical Devices (1960)

Rec. for Fixe IEC

IEC

Papers for General Use: Preferred Sizes of Sheet Materi ISO

$\begin{array}{lll}\text { Papers (1965) } & \text { /R Meth. for Indicating the Stability of ISO } \\ \text { Papers (1965) } & \text { /Rmining Thiosulphate and Tetrathionate ISO }\end{array}$

Papers (1965)

Paper: Holes for General Filing Purposes (1968)

Paper: Internal Diameters of Cores of Reels (1966)

Paper: Overall Trimmed Sizes of Articles of Stationery

Paper: Untrimmed Sizes, Designation, Tolerances (1966)

Paper: Untrimmed Stock Sizes for the ISO-A Series, ISO

Paper: Untrimmed Stock Sizes for the ISO-A Series: ISO

Para Chlorotoluene for Industrial Use-Meth. of Test (

Para Cresol for Industrial Use-Determination of Cryst

Para Cresol for Industrial Use-Determination of Resid

Para Cresol, Cresylic Acid and Xylenols for Industrial

Para Cresol, Cresylic Acid and Xylenols for Industrial

Para Cresol, Cresylic Acid and Xylenols for Industrial

Para Dichlorobenzene for Industrial Use-Meth. of Test

Paraformaldehyde for Industrial Use-Meth. of Test (19

Parallel and Morse Taper Shanks (1972)

Parallel and Taper-Threads-General Dimensions and Mar

Parallel Bars (1964)

Parallel Flange Column Sections-In. Series-Dimensio

Parallel Flange I Beams-In. Series-Dimensions and S

Parallel Keys and Their Corresponding Keyways (Dimensio

Parallel Lathes-Test. of the Accuracy (1970)

Parallel or Morse Taper Shanks (1966)

Parallel Pins, Unhardened-Metric Series (1972)

Parallel Shank Jobber and Stub Series and Morse Taper S

Parallel Shank Twist Drills-Long Series (1966)

Parallel Shanks with Morse Taper, Shanks-Std. and Lon

Parallel Shanks (1961)

Parallel Tubes for Cheese Dyeing (1967)

Rec. for Diameters of $S$

Parallel, and Morse Taper Shanks-Rec. Stocked Sizes (

Parameters of Connectors for Printed Wiring Boards (196

Parameters of Magnetic Piece Parts (1966)

Parameters of Surface Finish (1970)

Parent Metal in the Welding of SteeIs (1968)

Parity to Detect Errors in Information Messages (1969)

Parquet Panels (1967)

Parquet Strips of Solid Oak (1970)

Parquet Strips with Rectangular Face (1969)

Parquet Strips (1972)

Partial Discharge Measurements (1968)

Particle Boards: Definition; Classification (1968)

Particle Boards: Determination of Density (1968)

Particle Boards: Determination of Dimensions of Test Pi

Particle Boards: Determination of Moisture Content (196

Particles in Cellulose Acetate (1970)

Particulars Book (1972)

Parts and Equipment (1971)

Parts for Pressure Main Lines (1955)

Parts of Radio Frequency Connectors (1964)

Std. for Packaging-Compl ISO
1682

1681

112

110

166
1010

1009

1011

55-1

55-2

141-1

535

80

576

I009

1011

536

2195

1154

1729

1295

1912

66

372

135

231

58

101

217

438

534

80
169

1008

421

417

838

499

618

479

478

593

1696

1901

1900

1897

1898

1899

1699

1391

2250

2284

378

657/IX

$657 / \mathrm{V}$

773

1708

521

2338

235

494

1641

237

574

235/II

171

205

708
1155

631

1324

1072

2457

270

820

822

821

823

1598

2333

113-2

13

159

2206 
Calculation of the Effective Parameters of Magnetic Piece Parts (1966) oren (X.(ores) Made of Ferromagnetic Oxides and Associated Parts (1967) for Pot Cores Made of Ferromagnetic Oxides and Associated Parts (1971) urs and Cutters-Nominal Sizes and Designation of Working Parts (1972) reliability of Electronic Equipment and the Components (Or sentation of Reliability Data on Electronic Components (Or Rec. for Sound System Equipment: Auxiliary urface Active Agents: Determination of Apparent Density of Rec. for Paper elds and Viewing Conditions for Films: Utilization of Rec.

Rec. for Information Processing-Representation of $8 \mathrm{Bit}$ Rec. for ence Measuring Apparatus Having Detectors Other Than Quasi Rec. for Rec. for

Std. for Pots for Propagation and Transplantation Made of Rec. for Dobby Lags and

- Determination of Fines Content (1970)

delivered in Bulk or in Bins-Spec. for Maximu/

Determination of Pour Density (1970)

Rec. for Rec. for Rec. for

Metal Wire Drawing Dies: Interchangeability Dimensions of Rec. for Paints and Varnishes Rec. for Verification of ized Rubbers-Determination of Rebound Resilience-Lupke ages-Part V: Horizontal Impact Tests (Inclined Plane and Rec. for Plastics-Test. with the Torsion Rec. for Meth. of Test for Resistance of Fabrics to meth. of Mechanical Test. to Determine the Coding for Deep th. of Test for Determining Whether an Electrode Is a Deep oleum Products: Lubricating Grease-Determination of Cone Rec. for Lifeboats for Less Than One Hundred Rec. for Spices and Condiments: Black and White

968) Rec. for Oil of and Boric Oxide and Available / Std. for Hydrated Sodium Std. for

ec. for Rock Drilling-Drill Rods and Detachable Bits for c. for Rock Drilling-Extension Drill Steel Equipment for

c. for Rock Drilling-Extension Drill Steel Equipment for c. for Rock Drilling-Extension Drill Steel Equipment for c. for Rock Drilling-Extension Drill Steel Equipment for nductors of Ferromagn/ Rec. for Guide for the Drafting of Rec. for Magnetic Striping of $16 \mathrm{~mm}$ Film Rec. for Motion Picture Film

Single Magnetic Sound Stripe on $16 \mathrm{~mm}$ Motion Picture Film ecorded Characteristic for Magnetic Sound Records on $35 \mathrm{Mm}$ e Recoding Head for Centre (Center) Sound Records on $16 \mathrm{Mm}$ idth of the Recording Head for Edge Sound Records on $16 \mathrm{Mm}$ (Fiber) Yarns (1966)

Rec. for Rec. for

tures (1972)

Std. for Woven Wire Cloth and

Cinematography-Camera Usage of $8 \mathrm{~mm}$ Motion Picture Film a for Printing to $8 \mathrm{~mm}$ Type $S$ on $16 \mathrm{~mm}$ Motion Picture Film aw Stock Film (1966)

ck Film (1966)

Rec. for Cutting and

Rec. for Cutting and

till Photography Using $35 \mathrm{~mm}$ Objectives on $35 \mathrm{~mm}$ Film, 5 .

matography-Dimensions for $16 \mathrm{~mm}$ Motion Picture Film with Rec. for Measurement of the

cuum Pumps-Part I/

Rec. for Meth. of Measurement of the mps-Part I: Volum/

Rec. for Meth. of Measurement of the Rec. for Std. Form of Declaration of Rec. for Meth. of Measurement of use (1970) ability, Availability, and Maintainability Data from Field r Purposes (1971)

1) Rec. for Expression of the Functional Rec. Meth. for Measuring Rec. Meth. for Measuring Use (1971)

Rec. for Meth. of Measurement of Rec. for Meth. for Measuring ailing Torque Type Steel Hexagon Locknuts-Mechanical and Cables with Copper Conductors for Aircraft (19) aingle Pole Circuit Breakers for Aircraft (1970/

3 Pole Circuit Breakers for Aircraft (1970)

) Electrical Cables with Copper Conductors for / ) Electrical Cables with Copper Conductors for / ches for Aircraft (1970)

of Ferromagnetic Oxid Rec. for Household Refiger for Rec. for Guide for the Drafting of Rec. for Rotating Electrical Machines: Rating and um and Its Alloys: Photometric Determination of Manganese: um and Its Alloys: Photometric Determination of Manganese:

-Containing Zirconium, Rare Earths, Thorium and Silver . Rec. for Quantities and Units of Rec. for Short Contents List of Rec. for Layout of

Rec for Presentation of Contributions to Rec. for Rec. for Rec. for Rec. for Rec. for Parts) Used Parts) (1970) Pastes on Filling (1969)

Patterns for Dobbies (1967)

Patterns of Image Quality Indicators (1973)

Patterns on 12 Row Punched Cards (1971)

Peaches: Guide to Cold Storage (1968)

Peak (1967)

Pearlitic Malleable Cast Iron (1969)

Pears-Guide to Cold Storage (1969)

Peat and Other Plant Material-Sampling (1972)

Pegs in Wood, Metal or Other Suitable Material (1967)

Pelletized Carbon Black for Use in the Rubber Industry

Rec. for IEC

Rec. for Dimensions of Cross C IEC Rec. for Dimensions IEC Std. for Dental B ISO

IS and Definitions for the IEC Rec. for the Pre IEC

Rec. for S ISO

ISO

Aphy of W ISO

ISO

ISO

Pelletized Carbon Black for Use in the Rubber Industry

Pelletized Carbon Black for Use in the Rubber Industry:

ellets and Cases (1966)

Rec. for Hard

Pendulum Damping Test (1971)

Pendulum Impact Test. Machines for Test. Steels (1965)

Pendulum Meth. (1971)

Pendulum Tests (1972)

Pendulum (1967)

Penetration by Water (Hydrostatic Head Test) (1968)

Penetration Electrodes (1968)

Penetration Type (1967)

Penetration (1972)

People (1963)

Tre. For Vulcan ISO

/Complete, Filled Transport Pack ISO

ISO

Rec. for Special ISO

Rec. for Me ISO

Std, for Petr ISO

ISO

Pepper, Whole and Ground: Spec. (1969)

Peppermint: France, Italy, United Kingdom and U. S. A. (1

Perborates for Industrial Use-Determination of Sodium

Perchloroethylene for Industrial Use-Meth. of Test (1

Percussive Drilling (1970)

Percussive Long Hole Drilling-Reverse Buttress Thread

Percussive Long Hole Drilling-Reverse Buttress Thread

Percussive Long Hole Drilling-Rope Threaded Equipment

Percussive Long Hole Drilling-Rope Threaded Equipment

Perforamnce Spec. for Cores of Tuned Transformers and I

Perforated Along Both Edges (1960)

Perforated Along One Edge-Direction of Winding (1971)

Perforated Along 1 Edge (1966)

Perforated Film (1971)

Perforated Magnetic Film (1968)

Perforated Magnetic Film (1968)

Rec. for ISO

IC. for Location and W ISO

Perforated Parallel Tubes for Cheese Dyeing (1967) ISO

Perforated Plates in Test Sieves: Nominal Sizes of Aper ISO

Perforated Type S (1972)

Perforated $8 \mathrm{~mm}$ Type $\mathrm{S}, 1-4(1972)$

Std for ISO

Perforating Dimensions for Double-8 $\mathrm{mm}$ Motion Picture $\mathrm{R}$ ISO

Perforating Dimensions for $35 \mathrm{~mm}$ Motion Picture Raw Sto

Perforating Dimensions (1972) Std. for Cinematography ISO

Perforation Format (1966)

Perforations Along One and Two Edges (1972)

Performance Characteristics of Electric Blankets (1969)

Performance Characteristics of Positive Displacement Va ISO

Performance Characteristics of Vapour (Vapor) Vacuum Pu ISO

Performance of Aircraft Electrical Equipment (1961) ISO

Performance of Electric Irons for Household or Similar IEC

Performance of Electronic Items (1971) /Ection of Reli IEC

Performance of Electronic Measuring Equipment (1971)

Performance of Floor Polishers for Household and Simila

Performance of Household Electric Ranges and Ovens (197

Performance of Vacuum Cleaners for Household and Simila

Performance of Water Heaters for Household Purposes (19

Performance Properties (1972)

Performance Requirements for General Purpose Electrica

Performance Requirements for General Purpose Push Pul

Performance Requirements for General Purpose Push Pul

Performance Requirements for Heat Resisting $\left(190^{\circ} \mathrm{C}\right.$

Performance Requirements for Heat Resisting $\left(260^{\circ} \mathrm{C}\right.$

Performance Requirements for Lever Operated Manual Swit

Performance Requirements (1968)

Performance Spec. for Cores of Broad Band Transformers

Performance (1969)

Periodate Meth. (Content Between 0.01 and 0.8\%) (1968)

Periodate Meth. (Content Less Than 0.01\%) (1968)

Periodate Photometric Meth. (1972)

Periodic and Related Phenomena (1958)

/Nesi

Periodicals or Other Documents (1955)

Periodicals (1954)

Periodicals (1961)

Periodicals (1968)

205

226

133

2157

271

319

1064

2504

202

873

1134
2259

573

1435

1866

1306

524

1522

442

1767

224

537

811

876

632

2137

338

959

856

1917

2213

1718

1721

1722

1719

1720

218

163

1200

490

1189

890

891

480

574
565

65

1787
1785

486

491

1700

515

69

299

1607

1608

224

311

362

359
369

350

312

379

2320

474

1467

1075

1490

1466

824

219

34- 1

809

810

2353 
Permcability of Vulcanized Rubbers to Gases (Constant V

Permeametric Meth. (1972)

Permissible Load (1969)

Permittivity and Dielectric Dissipation Factor of Elect

nuclear Instruments: Constructional Requirements to Afford

Personal Photographic Dosimeters (1971)

Rec. for Common Names for

Rec. for Principles for the Selection of Common Names for

Personal Protection Against Ionizing Radiation (1972)

Pest Control Chemicals and Plant Growth Regulators (197

Pest Control Chemicals (Pesticides) and Plant Growth Re

Pesticides Considered Not to Require Common Names-Sec

ond List (1969)

Rec. for

the Selection of Common Names for Pest Control Chemicals Rec. for Definitions of Terms Used in the

Pesticides Considered Not to Require Common Names: Firs

(Pesticides) and Plant Growth Regulators (1971)

972)

of Dropping Point ([972)

Std for

Petroleum Measurement Tables (1970)

(1972)

Std for

Std. for

Pelour (Color) (1

Petroleum Products Corrosiveness to Copper-Strip Test

Petroleum Products: Determination of Sulphur-Lamp Met
Petroleum Products: Lubricating Grease-Determination

of Cone Penetration (I972)

Std. for

Rec. for Identification by Hour Numbers of the

dentification by Hour Numbers of the Phase Conductors of 3

d 32 RPmm (200 and 800 RPI), NRZI, and 63 RPmm (I600 RPI) de to the Test. of Circuit Breakers with Respect to Out of gents-Determination of Anionic-Active Matter (Direct Two ting Electrical Machines: Ratings and Characteristics of 3 uction of Aluminum: Determination of Iron Content-1,10

ral and Artificial)-Determination of Iron Content-1,10

e for Industrial Use: Determination of Iron Content-1,10 Rec. for Plastics-Determination of Free Phenols in - Determination of Free Ammonia and Ammonium Compounds in ntent-Bromination Meth. (1972) Std. for Liquefied le in Water-Visual Test (1971)

Rec. for

) Rec. for Plastics-Detection of Free Ammonia in for Impurities Insoluble in Sodium Hydroxide So/ Rec. for Iter (Resin Content of Material in the Unmoulded State) of Rec. for Plastion Test Specimens from ng Materials-Annex A: Preparation of Test Specimens from
termination of the Percentage of Acetone Soluble Matter in Alcohols for Industrial Use-Determination of Acidity to for Liquefied Phenol for Industrial Use-Determination of Rec. for Plastics-Determination of Free

ustrial Use-Determination of Densit/ Rec. for Liquefied

Phase Conductors of 3 Phase Electric Systems (1963)

Phase Electric Systems (1963)

Phase Encoded (1971)

Phase Switching (1968)

Phase Titration Procedure) (1972)

Phenanthroline Photometric Meth. (1968) / for the Prod ISO

Phenanthroline Photometric Meth. (1970)

Phenanthroline Photometric Meth. (I972)

Ryolite (Natu ISO

(Inum) Fluorid ISO

Phenol-Formaldehyde Mouldings (1959)

Phenol-Formaldehyde Mouldings (1959)

Phenol for Industrial Use-Determination of Phenols Co

1. for Plastics ISO

Phenol Formaldehyde Mouldings-Qualitative Meth. (1961

Phenol, $\mathrm{O}, \mathrm{M}$, and $\mathrm{P}$-Cresol for Industrial Use-Test

c Moulding Materials (1963)

Acetone Soluble $\mathrm{Ma}$

Phenolic Moulding Materials (I968)

Ens of Thermosetti ISO

Phenolic Moulding Materials (1970)

Ens of Thermosetti ISO
Rec. for Plastics-De ISO

(1958)

Phenols Content-Bromination Meth. (1972)

Rec. for Higher ISO

Phenols in Phenol-Formaldehyde Mouldings (I959)

Std. ISO

Phenol: Meta Cresol, Cresylic Acid and Xylenols for Ind Rec., for Phenol: Ortha, Meta, and Para Cresol for Industrial Use

Determination of Residue on Evaporation (197/

Determination of Crystallizing Point (1971)

Rec. for

Rec. for

Rec. for

$X$ ylenols for Industrial Use-Determination Of/

$\mathrm{X}$ ylenols for Industrial Use-Determination Of/

Rec. for

Rec. for Quantities and Units of Periodic and Related

nadium Content-Photometric Meth. Using N-Benzoyl-N

Grading by Size (I965)

Rec. for

ron Content-2, 2'-Bipyridyl Spectrophot/

f Calcium Content-Volumetric Meth. (I968)

ulphate Content-Volumetric Meth. (I968)

$$
\begin{array}{r}
\text { Rec. for Dental Zinc } \\
\text { Rec. for }
\end{array}
$$

Rec. for Std. for Iron Ores: Determination of Rec. for

Rec for Determination of for Determination of Nitrogen, Total Sulphur, Chlorine and of Chemical Analysis of Manganese Ores-Determination of meth. Of/ Rec. for Measurement of Photosensitive Devices:
(I960)
Rec. for Sizes of

Rec. for Sizes of 972)

Std. for Photography-Expendable

tric Exposure-Simulation of the Spectral Distribution of ers: Preferred Sizes of Rolls (1969)

eferred Sizes of Sheet Material (1969)

rred Sizes of Sheet Material (I969)

erred Sizes of Sheet Material (I969)

rs: Preferred Sizes of Rolls (1969)

Rec. for

Penol: Ortha, Meta, and Para Cresol for Industrial Use

Phenol: Ortha, Meta, and Para Cresol, Cresylic Acid and

Phenol: Ortha, Meta, and Para Cresol, Cresylic Acid and

Phenol: Ortho, Meta, and Para Cresol, Cresylic Acid and

Phenomena (I958)

Phenylhydroxylamine (1970)

Phlogopite Mica Blocks, Thins and Splittings: Meth. for

Phosphate Cement (1970)

Phosphoric Acid for Industrial Use-Determination of I

Phosphoric Acid for Industrial Use-Determination of $\mathrm{O}$

Phosphoric Acid for Industrial Use-Determination of S

Phosphorus Content-Volumetric Meth. (1973)

Phosphorus Deoxidized Copper-Refinery Shapes (197I)

Phosphorus in Ash from Coal (1967)

Phosphorus in Coke (I969)

Phosphorus (1963)

Photoflash Lamps-Determination of the Light Output (1

Photoflood Illumination (1972) iS for Use in Sensitome

Photographic Black and White Paper for Roll Paper Print

. Photographic Black and White Papers for General Use: Pr

Rec. for Photographic Colour (Color) Film for General Use: Prefe

Rec. for Photographic Colour (Color) Paper for General Use: Pref
Rec. for Photographic Colour (Color) Paper for Roll Paper Printe

scription of the ISO Mire (ISO Test Object) and Its Use in

Rec. for Personal

Rec. for

hiosulphate and Tetrathionate in Processed Black and White

Rec. for Spec. for

Rec. for Spec. for

Rec. for Spec. for

Rec. for Spec. for
Rec. for Spec. for Rec. for Spec. for
Std. Spec. for

Rec. for Pictorial Marking of Transit Packages Containing Rec. for Quantity Packaging of Sensitized Rec. for Cinematography Rec. for Cinematography Rec. for Meth. Determining Photographic Document Reproduction ((I965)

Photographic Dosimeters (1971)

Photographic Equipment-Tripod Connections (197I)

Photographic Film, Plates and Papers (1965)

Photographic Grade Hydroquinone (1965)

Photographic Grade P Methylaminophenol Sulphate (1965)

Photographic Grade Potassium Bromide (I965)

Photographic Grade Sodium Carbonate, Anhydrous (1965)

Photographic Grade Sodium Sulphite (I965)

Photographic Grade Sodium Thiosulphate, Crystalline (19

Photographic Materials Sensitive to Radiant Energy (I96

Photographic Materials (1969)

Photographic Sound Record on $16 \mathrm{~mm}$ Prints (I958)

Photographic Sound Record on $35 \mathrm{~mm}$ Prints (1958)

Photographic Speed and Exposure Index (1954) 
ture Sizes (1971)

on of the light Output (1972)

ons Important to the Connection of Auxiliaries / otographic Transparencies with Reflection Colou/ posure-Simulation of the Spectral Distributio/ posure-Simulation of the Spectral Distributio/

Rec. for Photography-Cameras Using Films $35 \mathrm{~mm}$ and Smaller Pic

Std. for Photography-Expendable Photoflash Lamps-Determinati

Rec. for Photography-Front Lens Barrels Up to $100 \mathrm{~mm}$-Dimensi

Std. for Photography-Illumination for Comparison Viewing of $\mathrm{pH}$

Std. for Photography-Light Sources for Use in Sensitometric Ex

Std. for Photography-Light Sources for Use in Sensitometric Ex

Rec. for Photography-Projector Slides-Dimensions (1971)

on of ISO Speed of Colour (Color) Reversal Films for Still erforation Format (/

Rec. for Dimensions for Stereo Still Rec. for Diffuse Transmission Density

Std. for r) Reversal Films for Still Photography-Sensi/ osure-Simulation of the Spectral Distribution/ Rec. for Chemical Analysis of Magnesium and Its Alloys:

Chemical Analysis of Aluminium (Aluminum) and Its Alloys: Rec. for Chemical Analysis of Magnesium and Its Alloys: Rec. for Chemical Analysis of Aluminium and Its Alloys: Rec. for Chemical Analysis of Zinc:

c. for Chemical Analysis of Aluminium and Aluminum Alloys: Rec. for Chemical Analysis of Magnesium and Its Alloys: Rec. for Chemical Analysis of Magnesium and Its Alloys: Chemical Analysis of Aluminium (Aluminum) and Its Alloys: f Manganese Ores: Determination of Metallic Iron Content: duction of Aluminium-Determination of Vanadium Content num: Determination of Iron Content-1,10-Phenanthroline 1 Use: Determination of Iron Content; 2,2'-Bipyridyl

tion of Aluminum: Determination of Titanium Content; Tiro use-Determination of Iron Content: 2, 2'-Bipyridyl use-Determination of Iron Content-2,2'-Bipyridyl

ial)-Determination of Iron Content $-1,10$ Phenanthroline use-Determination of Iron Content: 2, 2'-Bipyridyl

duction of Aluminium-Determination of Zinc Content-Pan Use-Determination of Potassium Content-Flame Emission ide, and Disodium Tetraborates for Dibenzyldithiocarbamate ing Zirconium, Rare Earths, Thorium and Silver-Periodate al Use: Determination of Iron Content-2 2' Bipyridyl

1 Use: Determination of Iron Content-1,10 Phenanthroline Determination of Insoluble Zirconium Álizarin Sulphonate - Determination of Manganese Content-Formaldehyde Oxime

-Determination of Soluble Zirconium (Alizarin Sulphonate

Industrial Use-Determination of Sodium-Flame Emission Rec. for Measurement of Photosensitive Devices: Rec. for Measurement of $\mathrm{n}$ the Visible Spectrum, Meth. Of/ Rec. for Measurement of Rec. for Measurement of Rec. for Meth. of Measurement of 70) 1970)

Rec. for Meth. of Measurement of Photosensitive Devices: 959) Std. for Ropes-Determination of Certain Rec. for Expression of the Std. for Quantities and Units of nrecorded Magnetic Tapes for Instrumentation Applications: achines (1972) Rec. for Test Procedures for Determining Std. for Acoustics: Description and Measurement of Rec. for Mathematical Signs and Symbols for Use in Sid. for Leather: xtile Floor Coverings-Sampling and Cutting Specimens for Std. for Leather-Conditioning of Test Pieces for al Electrotechnical Vocabulary: Radiology and Radiological $r$ Quantities and Units of Physical Chemistry and Molecular Rec. for Single Box

s Mechanical Handling Equipment for Loose Bulk Materials raphic Materials Sensitive to Radiant Energy (1/ Rec. for ls) (1968)

Std. for Cinematography: $8 \mathrm{~mm}$ Motion-Picture Film with Rec. for Dimensions of Cores for Motion Rec. for Cinematography

on of the Emulsion Side of Edge Marked Roll Film for Still nd Projecta/ Std. for Cinematography: 65 and $70 \mathrm{~mm}$ Motion 1972) Std. for Cine for Cinematography: $35 \mathrm{~mm}$ Motion
Std Std. for Cinematography: $35 \mathrm{~mm}$ Motion

for Cinematography: Projector Usage of $8 \mathrm{~mm}$ Type S Motion inding (1971)

Rec. for Single Magnetic Sound Stripe on $16 \mathrm{~mm}$ Motion Std. for Cinematography-Camera Usage of $8 \mathrm{~mm}$ Motion (1)

ed Image Area for Printing to $8 \mathrm{~mm}$ Type $S$ on $16 \mathrm{~mm}$ Motion

Std. for Cinematography-Dimensions for $16 \mathrm{~mm}$ Motion

Rec. for Dimensions of Projection Reels for $8 \mathrm{~mm}$ Motion ec. for Emulsion Position in Camera for $8 \mathrm{~mm}$ Silent Motion c. for Emulsion Position in Camera for $16 \mathrm{~mm}$ Silent Motion nd Sound Record Positions in Camera for $35 \mathrm{~mm}$ Sound Motion nd Sound Record Positions in Camera for $16 \mathrm{~mm}$ Sound Motion sound Record Positions in Projector for $35 \mathrm{~mm}$ Sound Motion rojector for Direct Front Projection of $8 \mathrm{~mm}$ Silent Motion ojector for Direct Front Projection of $16 \mathrm{~mm}$ Silent Motion y-Dimensions of Daylight Loading Spools for $16 \mathrm{~mm}$ Motion

Photography-Sensitometric Exposure and Evaluation Met

Photography Using $35 \mathrm{~mm}$ Objectives on $35 \mathrm{~mm}$ Film, 5-P (Photography) (1954)

Photography: Determination of ISO Speed of Colour (Colo Photography: Light Sources for Use in Sensitometric Exp Photometric Determination of Copper (Oxalyldihydrazide Photometric Determination of Copper (Oxalyldihydrazide Photometric Determination of Iron (Orthophenanthroline Photometric Determination of Iron (Orthophenanthroline Photometric Determination of Iron (1968)

Photometric Determination of Manganese (Contents Betwee

Photometric Determination of Manganese: Periodate Meth. Photometric Determination of Manganese: Periodate Meth Photometric Determination of Silicon (Content Between 0 (Photometric Meth. for Metallic Iron Content Up to and I

Photometric Meth. Using N-Benzoyl-N-Phen ylhydroxy

Photometric Meth. (1968)

Photometric Meth. (1968)

Photometric Meth. (1968)

Photometric Meth. (1970)

Photometric Meth. (1970)

Photometric Meth. (1970)

Photometric Meth. (1971)

Photometric Meth. (1971)

Photometric Meth. (1971)

Photometric Meth. (1972)

Photometric Meth. (1972)

Photometric Meth. (1972)

Photometric Meth. (1972)

Photometric Meth. (1972)

Photometric Meth. (1972)

Photometric Meth.) (1970)

Photometry Mcth. (1970)

Photomultipliers (1971)

Photosensitive Devices: Basic Rec. (1969)

Photosensitive Devices: Photoconductive Cells for Use I

Photosensitive Devices: Photomultipliers (1971)

Photosensitive Devices: Phototubes (1969)

Phototubes (1969)

Phthalate Esters for Industrial Use-Meth. of Test (19

Phthalic Anhydride for Industrial Use-Meth. of Test (

Physical and Mechanical Properties (1972)

Physical and Subjective Magnitudes of Sound or Noise (1

Physical Chemistry and Molecular Physics (1973)

Physical Properties and Test Meth. (1973)

Physical Properties of Brush Materials for Electrical M

Physical Properties of Sonic Booms (1973)

Physical Sciences and Technology (1961)

Physical Test.-Measurement of Thickness (1972)

Physical Tests (1971)

Physical Tests (1972)

Physics (1964)

Rec. for Machine Made Te

ISO

Physics (1973)

Rec. for Internation IEC

Pickers for Automatic Looms (1961)

Std. Fo ISO

Picking Table Conveyors-Safety Code (1972)

/Ontinuou ISO

Pictorial Marking of Transit Packages Containing Photog

Pictorial Markings for Handling of Goods (General Symbo

Picture-Location and Width of Magnetic-Striping and G

Picture and Magnetic Films (1969)

Picture Areas for Films and Slides for Television (1971

(1)

Picture Film-Image Area Produced by Camera Aperture a

Picture Film-Image Area Produced by Camera Aperture ( ISO

Picture Film-Projectable Image Area (1972)

Picture Film for Direct Front Projection (1973)

Picture Film Perforated Along One Edge-Direction of W

Picture Film Perforated Along l Edge (1966)

Picture Film Perforated Type S (1972)

Picture Film Perforated 8 mm Type S, 1-4 (1972)

Picture Film with Perforations Along One and Two Edges

Picture Film (Other Than Type S) (1968)

Picture Film (1956)

Picture Film (1956)

Picture Film (1956)

Picture Film (1956)

Picture Film (1956)

Picture Film (1956)

Picture Film (1956)

Picture Film (1969) 
mensions of l)aylight loading Spools for Double $8 \mathrm{~mm}$ Motion Picture Film (1969)

tor Aperture for Projection of $35 \mathrm{Mm}$ Nonanamorphotic Motion Picture Films (1963)

Rec. for Cinematography-Di ISO 1- Maximum Aspect Ratio of Projec ISO Rec. for

ions (1972) Std. for Cinematography-8 mm Type S Motion c. for Cutting and Perforating Dimensions for $35 \mathrm{~mm}$ Motion Cutting and Perforating Dimensions for Double-8 $\mathrm{mm}$ Motion Rec. for Roll Film Cameras-Back Window Location and

c. for Photography-Cameras Using Films $35 \mathrm{~mm}$ and Smalle

c. for Preparation of Outline Drawings of Oscilloscope and

$\mathrm{s}$ of Electronic Tubes: Meth. of Measurement for Television

Rec. for Dimensions for $35 \mathrm{~mm}$ Film Magazines for Stil

Rec. for Definition and Marking of Safety Film for Motion ubber to Metal Where the Ru/ Rec. for Preparation of Test ubber to Metal Where the Ru/ Rec. for Preparation of Test c. for Calculation of the Effective Parameters of Magnetic

of Vulcanized Natural and Synthetic Rubbers (Crescent Tes gth of Small Test Pieces of Vulcanized Rubbers (Delft Test n Shear of Vulcanized Rubbers (Bonded Quadruple Shear Test Rec. for Aluminium (Aluminum) Alloys-Sand Cast Test Rec. for Spices and Condiments: Ginger, Whole, in Std for Leather-Conditioning of Test

Rec. for Selection and Preparation of Samples and Test

Rec. for Determination of Tear Strength of Small Test Atmospheres for the Conditioning and Test. of Rubber Test

for Particle Boards: Determination of Dimensions of Test ble Cellular Materials-Measurement of Dimensions of Test

e Tests-Dimensions, Tolerances and Applicability of Test num and Aluminium Alloys-Selection of Specimens and Test

iber) Building Boards: Determination of Dimensions of Test Rec. for n/ Rec. for Std. Definitions and Meth. of Measurement for
hydroxychromate (1970) Rec. for Zinc Chromate Std. for Barium Chromate Std. for Iron Blue or Definitions of Terms Appearing in ISO Rec. for Oils and Rec. for Ultramarine Rec. for Iron Oxide

Rec. for General Meth. of Test for Rec. for Aluminium (Aluminum) Rec. for Carpets-Determination of Thickness of io / Rec for Carpets-Determination of Measured Surface etermination of Measured Surface Pile Density and Measured ing (1971) Rec. for Carpets-Determination of Mass of Rec. for Carpets-Determination of Mass of Total Rec. fo

quipment (1973)

Rec. for Spices and Condiments: Std. for Connector

Rec. for Radio Frequency Connectors: Two equipment-Interchange Circuits-Assignment of Connector and Valves: Spec. Sheets and Dimensions of Wiring Jigs and $r$ Characteristics of String Insulator Units of the Cap and Rec. for Fresh

imensions of Focal Spots of Diagnostic X-Ray Tubes Using A Rec. for Split Sid. for Clevis

Rec. for Dimensions of Tubes,

d Graphite Electrodes with Threaded Sockets and Connecting Std. for Clevis

Rec. for Aircraft Seat Rails and Sid. for Parallel Rec. for nd Steel and Other Metal Tubes-(1970) Rec. for

Rec. for Asbestos Cement v: Fittings (1966) Rec. for Cast Iron Sanitary for Dimensions of Elastomeric Toroidal Sealing Rings for for Dimensions of Elastomeric Toroidal Sealing Rings for

957)

Rec. for Malleable Cast Iron onal Signs: to Be Used in Schemes for the Installations of om/ Rec. for Shipbuilding Details for Inland Navigation -
ere Pressure Tight Joints Are Made on the Threa/ Rec. for imensions and Marking (1972) Std. for Hand Taps for $n$ the Threads (1/8 In. to 6 In./S) (1961)

Rec. for Rec, for Pipe Couplings for Hydraulic Piping c. for Coupling Threads for Hydraulic or Pneumatic Piping Rec. for

Rec. for Shiphuilding Details: Mating Dimensions for Rec. for Identification of Aircraft ansport of Fluids-Unplasticized Polyvinyl Chloride (PVC) ansport of Fluids-Unplasticized Polyvinyl Chloride (PVC) Industry (1971)

Rec. for 6) Rec. for Cast Iron Sanitary
ngs for Pipes Under Pressure-Basic Dimensions/ gs with Spigot Ends for Domestic and Industrial/ Rec. for Rec. for
Picture Postcards and of Lettercards (1963)

Picture Postcards: Area Reserved for the Address (1965)

Picture Raw Stock Film-Cutting and Perforating Dimens ISO

Picture Raw Stock Film (1966)

Picture Raw Stock Film (1966)

Re ISO

Picture Sizes (1971)

Picture Sizes (1971)

Picture Tubes (1962)

Picture Types (1968)

Picture Use and Test

Piece and Meth. of Test of the Adhesion of Vulcanized R

Piece and Meth. of Test of the Adhesion of Vulcanized R

Piece Parts (1966)

Rec. for ISO

ISO

$\operatorname{Re}$ ISO

Re IEC

/Ents of the Elcctrical Propertie IEC

Piece) (1957)

Piece) (1968)

Piece) (1971)

Rec. fo
Piece) (1971)
Pieces and Ground: Spec. (1969)

Ec for Determination of Tear Strength ISO

Rec. for Determination of Tear Stren ISO

Rec. for Determination of Modulus I ISO

Pieces for Physical Tests (1972)

Pieces for Wrought Steel (1964)

Pieces of Vulcanized Rubbers (Delft Test Piece) (1968)

eces $(1966)$

ieces $(1968)$

Pieces (1971)

Pieces (1971)

Pieces (1971)

ieces (1972)

Piezoelectric Filters (1971)

Piezoclectric Vibrators Opcrating Over the Frequency Ra

Pigments-Basic Zinc Potassium Chromate and Zinc Tetra

Pigments for Paints (1972)

Pigments for Paints (1972)

Pigments for Paints (1972)

Pigments (1968)

Pigments (1970)

Pigments (1970)

Pigments (1971)

Pile Above the Backing (1970)

Pile Density and Measured Pile Fibre (Fiber) Volume Rat

Pile Fibre (Fiber) Volume Ratio (1971)

Pile Yarn Per Unit Area (197i)

Pilot Ladders (1968)

Pimento (Allspice) Wholc and Ground; Spec. (1969)

Pin Allocations for Use with High Speed Data Terminal E

Pin Connector for Twin Balanced Aerial Feeders (1965)

for Std. ISO

Rec ISO

Rec. for Flexi ISO

Rec. for Concret ISO

in Numbers (1972)

Pin Type (1969)

Pineapples-Guide to Stor Ockets for Electronic Tubes Rec. Fo IEC

Pins-Metric Series (1971)

Pins-Metric Series (1972)

Pins and Rods of Ferromagnetic Oxides (1966)

Pins for Use in Electric Are Furnaces (1967)

Pins with Heads-Metric Series (1972)

Pins (1968)

Pins, Unhardened-Metric Series (1972)

Pipe Connections for Industrial Application for Plain E

Pouplings for Hydraulic Piping (Pipc Threads) (197

Pipe Fittings for Building and Sanitary Purposes (1964)

Pipe Fittings for Waste Water and Ventilations-Part I

Pipe Fittings in Aircraft-(In. Series-Class 2 Toler

Pipe Fittings in Aircraft (In. Series-Class 1 Toleran

Pipe Fittings Screwed in Accordance with ISO Rec. R7 (1

Pipe Line Systems in Ships (1967)

Rec. for

Pipe Lines for the Transport of Combustible Liquids-N

Pipe Threads for Gas List Tubes and Screwed Fittings Wh

Pipe Threads for Parallel and Taper.Threads-General D

Pipe Threads Where Pressure Tight Joints Are Not Made O

(Pipe Threads) (1970)

(Pipe Tbreads) (1970)

Pipeline Flanges for General Use-Mating Dimensions (1

Pipeline Flanges for Ships (1969)

Pipelines (1970)

Pipes-Tolerances on Outside Diameters (1970)

Pipes-Tolerances on Wall Thicknesses Up to $6 \mathrm{~mm}(1970$

Pipes and Fittings for Waste Water and Ventilation (196

Pipes and Fittings of Plastics Materials-Socket Fitti

Pipes and Fittings of Plastics Materials: Socket Fittin

Pipes Conveying Fluids in Liquid or Gaseous Condition I
Pigments (1963)

Pin Straightencrs (1965)

Pipes and Fittings-Stainless Steel Tubes for the Food
1020

358

1700

491

1203

1754

139

151-16

1007

543

813

814
205

34

816

1827

2147

1003

2419

377

816

471

821

1794

1920

2142

766

368

302

1249

2040

2068

2495

339

788

1248

787

1247

1766

1959

1959

2095

1958

799

973

2593

169-3

2110

149-2

305

1838

336

1234

2340

220

239

2341

837

2338

1179

1944

392

$531 / 1$

1799

1077

49

538

51

2284

228

1944

1943

2084

964

12

1330

1165

2037

531

264

265 
Rec. for Steel Sttl. for Buried Inplasticized Polyvinyl Chloride (PVC) - Resistance to Internal Pressure (197/ Tolerances on Outside Diameters (1970) Tolerances on Wall Thicknesses Up to / inyl Chloride (PVC) Pipes-Tolerances/ inyl Chloride (PVC) Pipes-Tolerances/ Rec. for Building and Sanitary

(Outside Diameters and Nominal Pressures): Par/ Rec. for (Outside Diameters and Nominal Pressures) Part/ Rec. for $s$ and Fittings of Plastics Materials-Socket Fittings for (PVC) Fittings with Plain S/ Rec. for Socket Fittings for tion of Circumferential Fusion Welded Butt Joints in Steel ockets for Unplasticized Polyvinyl Chloride (PVC) Pressure ttings for Unplasticized Polyvinyl Chloride (PVC) Pressure

968) Rec. for Asbestos Cement Pressure Rec. for Asbestos Cement Rec. for Cast Iron Rec. for Graduated Rec. for One Mark Rec. for Colour (Color) Coding for ittings with Spigot Ends for Domestic and Industrial Waste Rec. for Coupling Threads for Hydraulic or Pneumatic Rec. for Pipe Couplings for Hydraulic $r$ Rubber Sealing Rings for Joints in Asbestos Cement Water r Pneumatic Handling Appliances for Loose Bulk Materials ht) (1960) Rec. for Textile Machinery and Accessories 1966)

Pirns for Box Loaders for Automatic Looms-Dimensions of

of Pirn Tip (1969) Rec. for Rec. for Weft Rec. for Weft Rec. for Hydraulic Cylinders-Internal Diameters and Rec. for Rec. for Fire Refined High Conductivity Tough Rec. for Fire Refined Tough Std. for Electrolytic Tough

Heald Frames: Coordinated Dimensions in Relationship with smission and Conveyors (1972)

Is (1970)

eels (I967)

Std. for Extended Rec. for Short Rec. for Shor

Rec. for Standard Tuning Frequency (Standard Musical usical Pitch) Rec. for Std. Tuning Frequency (Std. Musical neering (1966)

966) Rec. for Modules and Diametra ne Hacksaw Blades: Dimensions for Lengths $U_{p}$ to $450 \mathrm{~mm}$ and hines (I968) Rec. for Dimensions and les $A$ /

Rec. for Guide to the Calculation of Resistance of Std. for Cylindrical Cutters with Std for Slotting Cutters with Rec. for Side and Face Milling Cutters with Std. for Shell End Mills with

Rec. for Pipe Connections for Industrial Application for le of Dimensions and Masses Per Unit Length (19/ Rec. for Rec. for Mounting of sure: Unplasticized Polyvinyl Chloride (PVC) Fittings with for ISO System of Limits and Fits-Part II: Inspection of Rec. for 8 Strand )

Rec. for 3 Strand (Hawser L aid) and 8 Strand Rolling Bearings-Radial-Boundary Dimensions-General Rolling Bearings-Radial-Boundary Dimensions-General Range 0.06 to $6 /$ Std. for ISO In. Screw Threads: General td. for ISO General Purpose Metric Screw Threads-General Rec. for External Diameter of sport Packages-Part V: Horizontal Impact Tests (Inclined Std. for Woodworking Tools: Metal Bodied Std for Woodworking Tools: Wooden Bodied Rec. for Shanks for Turning and 0) Rec. for Commercially Dry Corkwood in orkwaste-Sampling (I972) orkwaste-Determination of Moistur/ nta/ Rec. for Graphical Symbols for Use on Detailed Maps, tat/ Rec. for Graphical Symbols for Use on Detailed Maps, ule/ Rec. for Graphical Symbols for Use on Detailed Maps,

ilding-Indications of Details on the General Arrangement Rec. for Common Names for Pest Control Chemicals and f Common Names for Pest Control Chemicals (Pesticides) and for Propagation and Transplantation Made of Peat and Other Rec. for Graphical Symbols for Coal Preparation

ciples and Conventions for Flowsheets for Coal Preparation h Associated Prime Movers) and Motors, Electric Propulsion Rec. for Refrigerating r International Electrotechnical Vocabulary: Nuclear Power tors for Direct Current Using Impregnated Paper or Paper trength of Cements-Compressive and Flexural Strengths of
Pipes for Gas, Water and Sewage, Welded or Seamless (19

Pipes for the Supply of Gaseous Fuels: Metric SeriesPipes for the Transport of Fluids-Determination of Th Pipes for the Transport of Fluids-Polyethylene (PE)Pipes for the Transport of Fluids-Polyethylene (PE)Pipes for the Transport of Fluids-Unplasticized Polyv Pipes for the Transport of Fluids-Unplasticized Polyv Pipes in Asbestos Cement (1964)

Pipes of Plastics Materials for the Transport of Fluids Pipes of Plastics Materials for the Transport of Fluids Pipes Under Pressure-Basic Dimensions: Metric Series Pipes Under Pressure: Unplasticized Polyvinyl Chloride Pipes Up to $50 \mathrm{~mm}$ (2 In.) Wall Thickness (1969)

Pipes with Elastic Sealing Ring Type Joints: Minimum De

Pipes with Elastic Sealing Ring Type Joints: Minimum De Pipes (1971)

Pipes, Joints and Fittings for Sewerage and Drainage (I

Pipes: Special Castings and Parts for Pressure Main Lin

Pipettes (Excluding Blowout Types) (1968)

Pipettes (1968)

Pipettes (1970)

Pipe: Basic Dimensions; Metric Series (1962)

Piping (Pipe Threads) (I970)

Piping (Pipe Threads) (1970)

Piping (1970)

Piping (1972)

Pirn and Cross Winders-Definition of Side (Left or Ri

Pirn Changing Automatic Looms (1967)

Pirn Tip (I969)

Pirns for Automatic Looms (I960)

Pirns for Box Loaders for Automatic Looms-Dimensions

Piston Rod Diameters-Metric Series (1971)

Pitch Bound Reeds (1967)

Pitch Copper Refinery Shapes (1971)

Pitch Copper Refinery Shapes (1971)

Pitch Copper Refinery Shapes (I972)

Pitch of the Harness (I967)

Pitch Precision Roller Chains and Chain Wheels for Tran Rec. for ISO

Pitch Transmission Precision Bush Chains and Chain Whee ISO

Pitch Transmission Precision Roller Chains and Chain Wh ISO

Pitch) Rec. for Std. Tuning Frequency (Std. Musical Pit ISO

Pitch) (1955) /R Standard Tuning Frequency (Standard M ISO

Pitches of Cylindrical Gears for General and Heavy Engi ISO

Pitches of Cylindrical Gears for General Engineering (1

Pitches of Straight Bevel Gears for General and Heavy E ISO

Pitches Up to $6.3 \mathrm{~mm}$ (1972) Std. for Hand and Machi ISO

Pitching of Slots on Platens for Projection Welding Mac

Plain and Tinned Copper Conductors of Low Frequency Cab IEC

Plain Bore and Key Drive: Metric Series (1972)

Plain Bore and Key Drive: Metric Series (1972)

Plain Bore and Tenon Drive: Metric Series (I973)

Plain End Steel and Other Metal Tubes (1970)

Plain End Steel Tubes, Welded or Seamless-Ceneral Tab

Plain Grinding Wheels by Means of Hub Flanges (1968) ISO

Plain Sockets; Metric Series (1968) / Pipes Under Pres ISO

Plain Workpieces (1971)

Rec. ISO

(Plaited) Polypropylene Monofilament or Film Ropes (1970 ISO

Plan-Diameter Series $8,9,0,1,2,3$ and $4,(1968)$ / for ISO

Plan-Part II-Diameter Series 7 (1970) Rec. for ISO

Plan and Selection for Screws, Bolts and Nuts (Diameter ISO

Plan (1973)

Planchets Used in Nuclear Electronic Instruments (1967) IEC

Plane and Pendulum Tests (1972) /Complete, Filled Tran ISO

Planes (1973)

Planes (1973)

Planing Tools: Sections and Tolerances (1961)

ISO

Planks-Definitions, Classification and Packaging (197

Planks, Virgin Cork, Ramassage, Gleanings, Refuse and C

Planks, Virgin Cork, Ramassage, Gleanings, Refuse and C

Plans and Geological Cross Sections-Part III: Represe

Plans and Geological Cross Sections-Part II: Represen

Plans and Geological Cross Sections-Part I: General R

lans of Ships (1971)

Plant Growth Regulators (1971)

Plant Growth Regulators (197I)

Plant Material-Sampling (1972)

Plant (I967)

Plant (I969)

Plant, Tankers (1965)

Plants-Safety Requirements (1971)

Plants for Electric Energy Generation (1968)

Plastic Film Dielectric (1964)

Plastic Mortar (Rilem Cembureau Meth (1968)

Es for the Selection Std. for Pots ISO

Rec. for Prin ISO

ISO

ISO

ISO

ISO

SO

ISO

SO 
Plastic, Excluding Cellular Types (1970) Rec. for Plastics-Determination of the Loss of Plasticity Test (197I)

f Polyamide 66, 6I0 and 6 (1969)

rs-Freeze Thaw Cycle Stability Test (1969)

rs-Determination (I969)

rs-Determination of Dry Solids Content at 105/

ets Based on Thermosetting Resins (197I) als (1968)

\section{oplastic Materials (1963)}

osetting Materials (1963)

osetting Materials-Annex A: Preparation of Te/ osetting Materials-Annex B: Preparation of Te/ osetting Materials-Annex C: Preparation of Te/ I968)

\section{rusion Materials (1970)}

mo and Copolymers of Vinyl Chloride (1970)

hyde Mouldings-Qualitative Meth. (I961)

ticized Cellulose Acetate (1970)

$r$ Plastic (1968)

g Material That Can Be Poured from a Specified / g Material That Cannot Be Poured from a Specifi/ ose Acetate (1968)

959)

erials (196I)

polymers and Copolymers (1970)

lastics (1967)

in Plasticized Cellulose Acetate (197I)

m of Bars (I970)

d Plastics (1972)

ompounds in Phenol-Formaldehyde Mouldings (19/

rmaldehyde Mouldings (1959)

Rigid Self Extinguishing Thermosetting of (196/

stics by Means of a Durometer (Shore Hardness)

ulose Acetate (1970)

se Acetate Before and After Heating (1970) Std. for Rigid

\section{polysty rene (1959)}

Plasticizers (1970) Determine the Change
Plastics-Accelerated Conditioning of Test Specimens $O$

Rec. for Plastics-Aqueous Dispersions of Polymers and Copolyme

Rec. for Plastics-Aqueous Dispersions of Polymers and Copolyme

Rec. for Plastics-Aqueous Dispersions of Polymers and Copolyme

Rec. for Plastics-Basis for Spec. for Industrial Laminated She

Rec. for Plastics-Basis for Spec. for Phenolic Moulding Materi

Rec. for Plastics-Bending Test for Rigid Cellular (1970)

Rec. for Plastics-Compression Moulding Test Specimens of Therm

Rec. for Plastics-Compression Moulding Test Specimens of Therm

Rec. for Plastics-Compression Moulding Test Specimens of Therm

Rec. for Plastics-Compression Moulding Test Specimens of Therm

Rec. for Plastics-Compression Moulding Test Specimens of Therm

Rec. for Plastics-Compression Test of Rigid Cellular Plastic (

Rec. for Plastics-Definitions of Terms (1969)

Rec. for Plastics-Designation for Polystyrene Moulding and Ext

Rec. for Plastics-Designation of Unplasticized Compounds of Ho

Rec. for Plastics-Detection of Free Ammonia in Phenol Formalde

Rec. for Plastics-Determination of Acetic Acid Yield of Unplas

Rec. for Plastics-Determination of Apparent Density of Cellula

Rec. for Plastics-Determination of Apparent Density of Mouldin

Rec. for Plastics-Determination of Apparent Density of Mouldin

Rec. for Plastics-Determination of Ash of Unplasticized Cellul

Rec. for Plastics-Determination of Boiling Water Absorption (1

Rec. for Plastics-Determination of Bulk Factor of Moulding Mat

Rec. for Plastics-Determination of Chlorine in Vinyl Chloride

Rec. for Plastics-Determination of Compressive Properties of $\mathbf{P}$

Rec. for Plastics-Determination of Ethyl Ether Soluble Matter

Rec. for Plastics-Determination of Flammability Of: in the for

Std. for Plastics-Determination of Flexural Properties of Rigi

Rec. for Plastics-Determination of Free Ammonia and Ammonium C

Rec. for Plastics-Determination of Free Phenols in Phenol-Fo

Rec. for Plastics-Determination of Incandescence Resistance of

Rec. for Plastics-Determination of Indentation Hardness of Pla

Rec. for Plastics-Determination of Insoluble Particles in Cell

Rec. for Plastics-Determination of Light Absorption of Cellulo

\section{rom Plastics (1961)}

olour (Color) Change Upon Exposure to Daylight

olour (Color) Change Upon Exposure to Light of /

olour (Color) Change Upon Exposure to Light of /

ne Polypropylene by Boiling N-Heptane (I969) unction of Temperature (I965)

nder Load (1958)

(I970)

\section{ed Stal}

(Resin Content of

e of Rigid Plastics (Flexural Test) (1961)

of Rigid Plastics (Flexural Tests) (196I)

om Plastics by the Activated Carbon Meth. (I96I/

tile Glass Reinforced Plastics (1970)

the Rate of Increase During the Setting of Uns/

ethylene and Compounds of (1967)

oplastics (1969)

ides (I970)

plasticized Cellulose Acetate (1967)

oluble Matter in Phenolic Mouldings (1958)

le Materials in Polyamides (1967)

n Polystyrene with Wijs Solution (1961)

nsparent Plastics (1966)

to Chemical Substances (1961)

n of Flammable Gases from Plastics (I968)

lyvirylchloride and Related Copolymers and Thei/

lyvinyl Chloride and Related Copolymers and The ture of Thermoplastics (1968)

yamides Resins in Dilute Solution (1963)

olyamides in Concentrated Solution (1967)

ides (I969)

ansmission Rate of Films and Thin Sheets-Dish/ ride-Vinyl Acetate Copolymers (I970)

of Cellulose Acetate (1970)
Cellular

Rec. for

Rec. for

Rec. for Rec. for

Rec. for

Rec. for

Rec. for

Rec. for

Rec. for

Rec. for

Rec. for

Rec. for

Rec. fo

Rec. for

Rec. for

Rec. for

Rec. for

Rec. for

Rec. for

Rec. for

Rec. for

Rec. for

Rec. for

Rec. for

Rec. for

Rec. for

Rec. for

Rec. for

Rec. for

Rec. for

Rec. for

Rec. for

Rec. for
Rec. for

Plastics-Determination of Linear Dimensions (1972).

Plastics-Determination of Methanol Soluble Matter in

Plastics-Determination of Resistance of Plastics to $\mathrm{C}$

Plastics-Determination of Resistance of Plastics to $C$

Plastics-Determination of Resistance of Plastics to $\mathrm{C}$

Plastics-Determination of Roll Characteristics (1972)

Plastics-Determination of Shear Strength (1972)

Plastics-Determination of Soluble Matter of Crystalli

Plastics-Determination of Stiffness in Torsion as a F

Plastics-Determination of Temperature of Deflection $U$

Plastics-Determination of Telusile Properties of Film

Plastics-Determination of the Acetone Soluble Matter

Plastics-Determination of the Bleeding of Colourants

Plastics-Determination of the Charpy Impact Resistanc

Petermination of the Izod Impact Resistance

Plastics-Determination of the Loss of Plasticizers Fr

Plastics-Determination of the Maximum Temperature and

Plastics-Determination of the Melt Flow Index of Poly

Plastics-Determination of the Melting Point of Polyam

Plastics-Determination of the Moisture Content of Non

Plastics-Determination of the Percentage of Acetone $S$

Plastics-Determination of the Percentage of Extractab

Plastics-Determination of the Percentage of Styrene I

Plastics-Determination of the Refractive Index of Tra

lastics-Determination of the Resistance of Plastics

Plastics-Determination of the Temperature of Evolutio

Plastics-Determination of the Vicat Softening Tempera

Plastics-Determination of the Viscosity Ratio of of

Plastics-Determination of the Water Content in Polyam

Plastics-Determination of the Water Vapour (Vapor) Tr

Plastics-Determination of Vinyl Acetate in Vinyl Chlo

or Plas ISO

ISO

ISO

Rec. Fo ISO 
ily Katio of (Cellulose Acetate in Dilute Soluti/ thylene Terephthalate) in Dilute Solution (1970/ yl (:hloride Resin in Solution (1961) ing-Number of Polyethylenes and Polypropylenes /

ission Rate of Rigid Cellular Plastics (1970) for the Determination of the Dilute Solution Vi/ lastic Materials (1963) e Pyknometer Meth. (1970)

ve-Density (Specific Gravity) of Plastic, Exclu/ dity in Small Enclosures by Means of Aqueous So/ ture by Impact (1969)

ehaviour of Plastics in a Ventilated Tubular Ov/ ctrical Properties of Polyethylene Due to the M/ d, Resin Bonded, Low Pressure Laminated Plates / n Plastics Materials: Moulding Meth. (1968) n Plastics Materials: Casting Meth. (I968) ted-Ash (1970)

Foreign Matter (1970)

extract (1970)

r (Including Water) (1970)

pparent Bulk Density (1969)

of Mechanical Properties After Contact with Che/ ance of Plastic to Fungi by Visual Examination /

(1963)

c. for Plastics-Determination of Indentation Hardness of Plastics-Determination of the Loss of Plasticizers from -Meth. of Test for the Determination of the Behaviour of for the Determination of Flammability and Burning Rate of sponding C/ Rec. for List of Equivalent Terms Used in the oonding $\mathrm{Ge} /$ sponding D/ sponding $\mathrm{P} /$ Rec. for List of Equivalent Terms Used in the Rec. for List of Equivalent Terms Used in the Rec. for List of Equivalent Terms Used in the Rec. for List of Equivalent Terms Used in the essure-Basic Dimensions/ Rec. for Pipes and Fittings of Diameters and Nominal Pressures): Par/ Rec. for Pipes of Diameters and Nominal Pressures) Part/_Rec. for Pipes of $r$ Plastics-Preparation of Specimens for Optical Tests on $r$ Plastics-Preparation of Specimens for Optical Tests on Domestic and Industrial/ Rec. for Pipes and Fittings of ion of the Resistance to Internal Pressure (197) ne (PE)-Tolerances on Wall Thicknesses Up to / zed Polyvinyl Chloride (PVC) Pipes-Tolerances/ zed Polyvinyl Chloride (PVC) Pipes-Tolerances/

Rec. for

Rec. for

Rec. for Rec. for

Rec. for Plastics-Determination of the Resistance of

ight / Rec. for Plastics-Determination of Resistance of

$t$ of $/$ Rec. for Plastics-Determination of Resistance of Rec. for Plastics-Determination of Resistance o s-Determination of the Charpy Impact Resistance of Rigid ics-Determination of the Izod Impact Resistance of Rigid plastics-Determination of Migration of Plasticizers from ics-Determination of the Refractive Index of Transparen for Plastics-Determination of Compressive Properties of Rec for Determination of Tensile Creep of on of the Temperature of Evolution of Flammable Gases from Rec. for Abbreviations (Symbols) for nation of the Loss on Ignition of Textile Glass Reinforced e Impermeability to Water of Fabrics Coated with Rubber or nd Simulated Service Test of Fabrics Coated with Rubber or f Water Vapour (Vapor) Transmission Rate of Rigid Cellular $h$ and Elongation at Break of Fabrics Coated with Rubber or

$r$ Plastics-Determination of Flexural Properties of Rigid 9) Rec. for d Cellulose Acetate (1969)

f Apertures (1972) Rec. for Metal Reeds with Std. for Wire and ec for Dimensions of Ceramic Dielectric Capacitors of the rubber to Metal Where the Rubber Is Assembled to One Metal Rec. for Dimensions and Pitching of Slots on 972) Std. for Industrial Castors-Dimensions of Top

Rec. for Measurement of Fluid Flow by Means of Orifice mestic and Similar Purposes: Waffle Irons, Grills, Warming tability of the Images of Processed Black and White Films, rathionate in Processed Black and White Photographic Film

$r$ Determination of Adhesion of Vulcanized Rubbers to Rigid Std. for Woven Wire Cloth and Perforated y (1968) (Fiber) Reinforced, Resin Bonded, Low Pressure Laminated Rec. for Spindle Noses and Face
ographic Inspection of Fusion Welded Butt Joints for Steel al Castors-Dimensions of Top Plates-Part I: Oblong Top ographic Inspection of Fusion Welded Butt Joints for Stee

Rec. for Figures for Aircraft Instrument Dials and Number d Rubber to Metal Where the Rubber Is Assembled to 2 Meta
Plastics-Determination of Viscosity Number and Viscos Plastics-Determination of Viscosity Number of Poly (E Plastics-Determination of Viscosity Number of Polyvin Plastics-Determination of Viscosity: Number and Limit Plastics-Determination of Water Absorption (1958)

Plastics-Determination of Water Vapour (Vapor) Transm Plastics-Directives for the Standardization of Meth.

Plastics-Injection Moulding Test Specimens of Thermop Plastics-Liquid Resins-Measurement of Density by Th Plastics-Meth. for Determining the Density and Relati Plastics-Meth. for Maintaining Constant Relative Humi Plastics-Meth. of Determining the Brittleness Tempera Plastics-Meth. of Test for the Determination of the B Plastics-Meth. of Test to Determine the Change in Ele Plastics-Preparation of Glass Fibre (Fiber) Reinforce Plastics-Preparation of Specimens for Optical Tests O Plastics-Preparation of Specimens for Optical Tests O Plastics-Pve Resins-Determination of Ash and Sulpha Plastics-Pvc Resins-Determination of Impurities and Plastics-Pvc Resins-Determination of $\mathrm{pH}$ of Aqueous Plastics-Pvc Resins-Determination of Volatile Matte Plastics-Pve Resins-Sieve Analysis in Water (1970) Plastics-Pve Resins: Determination of the Compacted a

IS

ISO

ISO

ISO

ISO

ISO

ISO

ISO

ISO

ISO

ISO

ISO

ISO

ISO

ISO

ISO

ISO

ISO

ISO

ISO

ISO

ISO

ISO

ISO

ISO

ISO

ISO

Plastics-Rec. Pract. for the Evaluation of the Resist

Plastics-Spec. for Polyamide Homopolymers (1971)

Plastics-Std. Atmospheres for Conditioning and

Plastics-Test. with the Torsion Pendulum (1967)
Plastics by Means of a Durometer (Shore Hardness) (1968

Plastics by the Activated Carbon Meth. (1961)

Plastics in a Ventilated Tubular Oven (1969)

Plastics in the Form of Film (1970)

Plastics Industry (English, French, Russian) (Cs: Corre

Plastics Industry (English, French, Russian) (D: Corres

Plastics Industry (English, French, Russian) (NL: Corre

Plastics Industry (English, French, Russian) (Pl: Corre

Plastics Industry (English, French, Russian) (1969)

Plastics Materials-Socket Fittings for Pipes Under Pr

Plastics Materials for the Transport of Fluids (Outside

Plastics Materials for the Transport of Fluids (Outside

Plastics Materials: Casting Meth. (1968)

Plastics Materials: Moulding Meth. (1968)

Plastics Materials: Socket Fittings with Spigot Ends Fo

Plastics Pipes for the Transport of Fluids-Determinat

Plastics Pipes for the Transport of Fluids-Polyethyle

Plastics Pipes for the Transport of Fluids-Unplastici
Plastics Pipes for the Transport of Fluids-Unplastici

Plastics to Chemical Substances (1961)

Plastics to Colour (Color) Change Upon Exposure to Dayl

Plastics to Colour (Color) Change Upon Exposure to Ligh

Plastics to Colour (Color) Change Upon Exposure to Ligh

Plastics (Flexural Test) (1961)

Plastics (Flexural Tests) (1961)

Plastics (1961)

Plastics (1966)

Plastics (1967)

Rec. for Plast ISO

Rec. for ISO Rec. for Plast ISO

Rec. ISO

Plastics (1968)

Plastics (1968)

Plastics (1969)

Plastics (1970)

Plastics (1970)

Plastics (1970)

Plastics (1970)

Plastics (197I)

Plastics (1972)

Plastics: Designation of Polyvinyl Chloride Resins (196
Plastics: Determination of Free Acidity of Unplasticize

Rec. for Plastics-Determinati ISO

Rec. for Plastics-Determi ISO

Rec for Determination of Th ISO

Rec. for Accelerated Ageing a ISO

Rec. for Plastics-Determination O ISO

/for Determination of Breaking Strengt ISO

Std. Fo ISO

ISO

Plate Baulk (1967)

Plate Screens for Industrial Purposes-Nominal Sizes O

Plate Type (1967)

I. of Test of the Adhesion of Vulcanized

Platens for Projection Welding Machines (1968)

Plates-Part I: Oblong Top Plates with 4 Bolt Holes (1

Plates and Nozzles (1967)

Plates and Other Dry Cooking Appliances (1967)

Plates and Papers (1965)

Plates and Papers (1965)

Plates in Shear (Qumining Thios

/for Do CEE

Plates in Test Sieves: Nominal Sizes of Apertures (1972 ISO

Plates or Panels for Test Purposes (1970) / Glass Fibr ISO

Plates Types a and Camlock: Sizes for Interchangeabilit ISO

Plates Up to $50 \mathrm{~mm}$ (2 In.) Thick (1969) /Act. for Radi ISO

Plates with 4 Bolt Holes (1972)

Plates 50 to $200 \mathrm{~mm}$ Thick (1972)

Std for Industri ISO

Plates (1962)

/Rec. Pract. for Radi ISO

ISO

/H. of Test of the Adhesion of Vulcanize ISO

1157

1228

174

1191

1663

1628

294.

1675
1183

483

974

1137

1627

869

870

1270

1265
1264

1269

1624
1068

846

1874

537

868

176

1137

1326

I94/CS

I94/D

194/NI

194

264

161

330

870

869

265

1167

1166

1165

1330

175

877

878

879

179

180

177

489

604

899

87 I

1043
1172

1419

1663

1421

178

1060

1061

367

2194

234

813

865

2I 84

541 
Sitd. for ()ffet Duplicators-Attachment Features of Plates (1972)

ric Spries (1968) Rec. for Spindle Noses and Face Rec. for Srales and Sizes for ile Steel Chains (Round I,ink) for Chain Conveyors and Coal Std. for Tests for Aircraft Electrical Std. for Internal Combustion Engines: Spark Rec. for Automobile-Spark Rec. for Sid. for Spec. for Spec. for al Use (1957) ses (1963)

Rec. for Aircraft Fuel Nozzle Grounding Rec. for the Dimensions of Polarized Std. for Automobiles: Spark Std. for Automobiles: Spark Std. for Automobiles: Spark Std. for Automobiles : Compact Spark Std. for Automobiles: Compact Spark Std. for Automobiles: Spark

$\operatorname{ses}(1969)$ Rec. for Rec. for Rec. for Characteristics of Construction of Rec. for Rec. for Rec. for Rec. for Used in the Plastics Industry (English, French, Russian)

(1971)

70)

nsmission (/

- Piping (1972)

s-Part I (1970)

s-Part II (1970)

1)

chment of Mounted Implements (1968) Rec. for
Rec. for Rec. for Std. for

Rec for Coupling Threads for Hydraulic or Rec. for Analogue Rec. for Shanks for Rec. for Shanks for

Rec. for Indirect Reading Capacitor Type Rec. for Direct Reading Rec. for Sizes of Correspondence Envelopes and

Rec. for Determination of Crystallizing Rec. for Determination of Freezing

Rec. for Animal Fats: Determination of Solidification Rec. for Plastics-Determination of the Melting rec. for Surface Active Agents: Determination of the Cloud in Woven Fabrics Subjected to Laundering Near the Boiling drocarbons for Industrial Use-Determination of the Cloud cresol for Industrial Use-Determination of Crystallizing Products-Lubricating Grease-Determination of Dropping esponsive Elements Giving Direct Indications by Means of A Rec. for Aircraft Instrument Dials and International Rec. for Rec. for the Dimensions of

Containing C/ Rec. for Chemical Analysis of Zinc: Std. for Chemical Analysis of Zinc Alloys: Rec. for Chemical Analysis of Zinc: Rec. for Chemical Analysis of Zinc:

Rec for Chemical Analysis of Magnesium and Its Alloys: analysis of Manganese Ores: Determination of Zinc Content

Rec. for Dimensions for General Purpose Push Pull Single Rec. for Dimensions for General Purpose Push Pull 3 r Performance Requirements for General Purpose Push Pull 3 formance Requirements for General Purpose Push Pull Single ics Industry (English, French, Russian) (PI: Corresponding Rec. Meth. for Measuring Performance of Floor

Rec. for Plastics-Determination of Viscosity Number of eath: Distribution Wires with Solid Conductors, Insulated, Rec. for Plastics-Spec. for Rec. for 3 Strand

r Plastics-Accelerated Conditioning of Test Specimens of

for Plastics-Determination of the Viscosity Ratio of of c. for Plastics-Determination of the Viscosity Number of etermination of the Percentage of Extractable Materials in Rec. for Plastics-Determination of the Water Content in Rec. for Plastics-Determination of the Melting Point of pments (1960)

ng Materials-Annex C: Preparation of Test Specimens from (1965) Rec. for Rec. for 3 Strand and the Rate of Increase During the Setting of Unsaturated ec. for Plastics-Determination of the Melt Flow Index of $f$ Test to Determine the Change in Electrical Properties of td. for Surface Active Agents (Nonionic): Determination of Rec. for 3 Strand Std. for
Plates: Bayonet Type; Sizes for Interchangeability: Met Rec. for High Tens Ploughs (1967)

Plug and Socket Connectors (1972)

Plug Ignition-Terminology (1972)

Plug M 14 X 1.25 with Flat Seating (1970)

Plugs and Socket Outlets for Domestic and Similar Gener

Plugs and Socket Outlets for Domestic and Similar Purpo

Plugs and Socket Outlets for Industrial Purposes (1966)

Plugs and Sockets (1970)

Plugs for Hearing Aids (1957)

Plugs M 10 X 1 with Flat Seating (1973)

Plugs M 12 X 1,25 with Flat Seating (1973)

Plugs M 14 X 1,25 with Conical Seating (1972)

Plugs M 14 X 1,25 with Conical Seating (1973)

Plugs M 14 X 1,25 with Flat Seating (1973)

Plugs M 18 X 1,5 with Conical Seating (1972)

Plugs, Socket Outlets and Couplers for Industrial Purpo

Ply Adhesion of Conveyor Belts (1962)

Ply Type Conveyor Belts (1965)

Plywood-Classification (1969)

Plywood-Measurement of Dimensions of Panels (1969)

Plywood-Veneer, for General Use-Requirements (1969)

Plywood: Vocabulary (1972)

(PI: Corresponding Polish Terms) (1963)

Uivalent Terms ISO

Pmm (800 RPI) Magnetic Tape for Information Interchange

Pneumatic Couplings Between Tractors and Trailers (1971

Pneumatic Cylinders-Cylinder Bores and Port Sizes (19

Pneumatic Equipment and Accessories for Fluid Power Tra

Pneumatic Handling Appliances for Loose Bulk Materials

Pneumatic Piping (Pipe Threads) (1970)

Pneumatic Signal for Process Control Systems (1971)

Pneumatic Tools and Fitting Dimensions of Chuck Bushing

Pneumatic Tools and Fitting Dimensions of Chuck Bushing

Pocket Exposure Meters and Accessory Electrometers (197

Pocket Type Electroscope Exposure Meters (1971)

Pockets (1962)

Point-General Meth. (1970)

Point Linkage of Agricultural Wheeled Tractors for Atta

Point of Essential Oils (1969)

Point of Fatty Acids (Titre) (1969)

Point of Polyamides (1970)

Point Temperature of Nonionic Surface Active Agents Obt

Point (1968)

Point (1970)

Point (1971)

Point (1972)

/ for Determination of Dimensional Change Rec. for Liquid Halogenated Hy

Rec. for Phenol: Ortha, Meta, and Para

Pointer and Graduated Scale (Working Equipment Category

Pointers (1962)

Polarimetric Saccharimeters (1970)

Polarized Plugs for Hearing Aids (1957)

Polarographic Determination of Cadmium in Zinc (1969)

Polarographic Determination of Lead and Cadmium in That

Polarographic Determination of Lead and Cadmium (1968)

Polarographic Determination of Lead (1968)

Polarographic Determination of Zinc (Content Between 0.

Polarographic Meth., for Zine Content from 0.005 to 0.1

Pole Circuit Breakers for Aircraft (1966)

Pole Circuit Breakers for Aircraft (1969)

Pole Circuit Breakers for Aircraft (1970)

Pole Circuit Breakers for Aircraft (1970)

Pole Circuit Breakers for Aircraft (1970) /Ec. for Per ISO
Polish Terms) (1963) /Uivalent Terms Used in the Plast ISO

Polishers for Household and Similar Purposes (1971)

Poly (Ethylene Terephthalate) in Dilute Solution (1970)

Polyamide Coated, in Singles, Pairs, Triples, Quadruple

Polyamide Homopolymers (1971)

Polyamide Multifilament Ropes (1969)

Polyamide 66, 610 and 6 (1969)

Polyamides in Concentrated Solution (1967)

Polyamides Resins in Dilute Solution (1963)

Polyamides (1967)

Polyamides (1969)

Polyamides (1970)

Polycristalline Semiconductor Rectifier Stacks and Equi

Polyester and Epoxy Resin Moulding Materials (1970)

Polyester Film Dielectric Capacitors for Direct Current

Polyester Multifilament Ropes (1969)

(N of the Maximum Temperature

Polyeter Resins (1967)

Polyethylene Due to the Migration of of Plasticizers (1

Polyethylene Glycols and Nonionic Active Matter (Adduct

Polyethylene Monofilament Ropes (1971)

Polyethylene Thermoplastic Materials-Designation (197

Polyethylene (PE)-Tolerances on Outside Diameters (19

Polyethylene (PE)-Tolerances on Wall Thicknesses Up T

ISO

ISO

IEC

ISO

ISO

ISO

CEE

CEE

ISO

IEC

ISC

ISO

ISO

ISO

ISO

IEC

ISO

ISO

ISO

ISO

ISO

ISO

ISO

ISO

ISO

ISO

IEC

ISO

ISO

ISO

ISO

ISO

ISO

ISO ISO ISO

ISO

OIML

IEC

ISO

ISO

ISO

ISO

ISO

ISO

ISO

ISO

OIML 
Determination of Viscosity: Number and limiting-Number of termination of Alkalinity or Acidity /

termination of Sulphated Ash in Produ/ mplcs and Test Meth.-General (1970) s (1971) Rec. For Aliphatic Rec. for Preparation of Dry Rec. for Denture Base

ontent at 105

est (1969)

Rec. for Plastics-Aqueous Dispersions of Rec. for Plastics-Aqueous Dispersions of Rec. for Plastics-Aqueous Dispersions of for Plastics-Determination of Chlorine in Vinyl Chloride for the Determination of the Dilute Solution Viscosity of

Plastics-Determination of Soluble Matter of Crystalline

Rec. for 3 Strand (Hawser Laid) and 8 Strand (Plaited) viscosity: Number and Limiting-Number of Polyethylenes and Rec. for

Rec. for Plastics-Designation for

r Plastics-Determination of the Percentage of Styrene in

for Plastics-Determination of Methanol Soluble Matter in for Plastics-Determination of the Thermal Stability of ir Fittings (1968)

(1967) Spec. for Rigid Conduits of with Circular Conductors and a Rated Voltage No/ Rec. for Rec. for Plastics-Determination of Viscosity Number of Rec. for Plastics: Designation of or Socket Fittings for Pipes Under Pressure: Unplasticized Rec. for Oven Test for Moulded Fittings in Unplasticized plastics Pipes for the Transport of Fluids-Unplasticized plastics Pipes for the Transport of Fluids-Unplasticized s Fuels: Metric Series-S/ Std. for Buried Unplasticized aling / Std. for Double Socket Fittings for Unplasticized ables (1970) Rec. for Meth. of Test for Pve irs, T/ Rec. for Low Frequency Cables and Wires with Pvc Wires/ Rec. for Low Frequency Cables and Wires with Pyc Wires/ Rec. for Low Frequency Cables and Wires with Pre res, T/ Rec. for Low Frequency Cables and Wires with Pvc res An/ Rec. for Low Frequency Cables and Wires with Pve and M/ Rec. for Low Frequency Cables and Wires with Pvc ables $/$

Rec. for Low Frequency Cables and Wires with Pve Rec. for Std. Colours (Colors) for Pve for Plastics-Determination of the Thermal Stability of Rec. for Gymnastic Equipment-Vaulting and Rec. for Laboratory Crucible asurement / Std. for Nonmagnetic Metallic and Vitreous or est (1970)

Rec. for Tests on Large Hollow Rec. for Definitions of Living Animals for Slaughter the True and Apparent Relative Densities (Density) and the Rec. for Pneumatic Cylinders-Cylinder Bores and ctors for Coupling an External Low Voltage Power Supply to and Heating Appliances for Domestic and Similar Purposes: Is (1965) Rec. for Power Sources fo Rec. fo

d Monitors for Use in Radiological Protection (/ Rec. Fo loose Bulk Materials-Troughed Belt Conveyors (Other Than
loose Bulk Materials-Troughed Belt Conveyors (Other Than loose Bulk Materials-Troughed Belt Conveyors (Other Than c. for Chemical Analysis of Cements: Minor Constituents of for Chemical Analysis of Cements: Main Constituents of of

Rec. for Technical Drawings-Tolerances of Form and of

Rec. for Technical Drawings-Tolerances of Form and of n Drawings (1969) (1956) Rec. for Tolerances of Form and of

Rec. for Emulsion (I956)

mm Silent Motion Picture Film (1956)

Rec for Emulsion mm Silent Motion Picture Film (1956)

Rec. for Emulsio Rec. for Emulsion c. for Shuttes-Terms and Designation in Relation to the ec for Interchangeable Punched Tape Variable Block Forma hines (1/ Rec. for Punched Tape Variable Block Format for hines (1969/ Rec. for Punched Tape Fixed Block Format for aight Welds (1967)

Rec. for Fundamental Weldin or Rotary Wafer Switches with Central Mounting; Maximum 12 for Rotary Wafer Switches with 2 Hole Mounting; Maximum 26

(1956)

or Limitation of Angles of Slope and Rotation for Welding

(1956)

ilm (1956)

Rec. for Emulsion and Sound Record

Rec. for Emulsion and Sound Record meth. of Measurement of the Performance Characteristics of I Rec. for Rules of Behaviour (Behavior) with Respect to ages Greater T/ Rec. for Dimensions of Indoor and Outdoo er Than $1,000 \mathrm{~V}(19)$ Rec. for Tests on Indoor and Outdoor
Polyethylenes and Polypropylenes in Dilute Solution (19 Polyhydric Alcohols-Glycerine for Industrial Use-De

Polyhydric Alcohols-Glycerine for Industrial Use-De

Polyhydric Alcohols-Glycerine for Industrial Use-Sa

Polymer from Butadiene Homopolymer and Copolymer Latice ISO Polymer (1970)

Polymers and Copolymers-Determination of Dry Solids C

Polymers and Copolymers-Determination (1969)

Polymers and Copolymers-Freeze Thaw Cycle Stability T

Polymers and Copolymers (1970)

Rec. ISO

Polymers (1970) /Ives for the Standardization of Meth. ISO

Polypropylene by Boiling N-Heptane (1969)

Polypropylene Monofilament or Film Ropes (1970)

Polypropylenes in Dilute Solution (1970)

Polystyrene Film Dielectric Capacitors (1968)

Polystyrene Moulding and Extrusion Materials (1970)

Polystyrene with Wijs Solution (1961)

Polystyrene (1959)

Polyvinyl Chloride and Related Copolymers and Their Com

Polyvinyl Chloride for Electrical Installations and the

Polyvinyl Chloride Insulated Cables and Flexible Cords

Polyvinyl Chloride Insulated Flexible Cables and Cords

Polyvinyl Chloride Resin in Solution (1961)

Polyvinyl Chloride Resins (1969)

Polyvinyl Chloride (PVC) Fittings with Plain Sockets: M

Polyvinyl Chloride (PVC) for Use Under Pressure (1967)

Polyvinyl Chloride (PVC) Pipes-Tolerances on Outside

Polyvinyl Chloride (PVC) Pipes-Tolerances on Wall Thi

Polyvinyl Chloride (P.VC) Pipes for the Supply of Gaseou

Polyvinyl Chloride (PVC) Pressure Pipes with Elastic Se

Polyvinyl Chloride (PVC) Pressure Pipes with Elastic Se (Polyvinyl Chloride) Insulation and Sheath of Electric C

(Polyvinyl Chloride) Insulation and Sheath: Cables in $\mathrm{Pa}$

Polyvinyl Chloride) Insulation and Sheath: Distribution

(Polyvinyl Chloride) Insulation and Sheath: Distribution

(Polyvinyl Chloride) Insulation and Sheath: Equipment W/

Polyvinyl Chloride) Insulation and Sheath: General Test

(Polyvinyl Chloride) Insulation and Sheath: Signalling C

(Polyvinyl Chloride) Insulation for Low Frequency Cables

Polyvinylchloride and Related Copolymers and Their Comp

Rec. for ISO

ISO

Mination of ISO

IEC

Rec. Fo ISO

Rec. ISO

ISO

CEE

CEE

IEC

ISO

ISO

ISO

ISO

ISO

ISO

ISO

ISO

IEC

IEC

IEC

IEC

IEC

IEC

IEC

ISO

so

(Porcelain and Silica) (1970)

Porcelain Enamel Coatings on Magnetic Basis Metals-Me

Porcelain Laboratory Apparatus-Quality and Meth. of T

Porcelains for Use in Electrical Installations (1967)

Porcines (1970)

Porosity of Coke (1969)

ISO

Port Sizes (1970)

Portable Entertainment Equipment (1971)

Portable Immersion Heaterś (1968)

Portable Motor Operated Tools (1967)

Retermination of ISO

( $3 \mathrm{MHz}$ : Conne

tor Electric Cooking CEE

Portable Prospecting Equipment for Radio Active Materia IEC

Portable X-- or Gamma Radiation Exposure Rate Meters an IEC

Portable) Belt Pulleys (1970) /Handling Equipment for ISO

Port able) Belts (1970)

Portable) Idlers (1970)

Portland Cement (1968)

/Anical Handling Equipment for ISO

/Nical Handling Equipment for ISO

Portland Cement (1968)

Position-Part III: Dimensioning and Tolerancing of $\mathrm{Pr}$

Position-Part IV: Practical Examples of Indications O

Position-Part I: Generalities, Symbols, Indications 0

Position in Camera for $16 \mathrm{~mm}$ Silent Motion Picture Film

Position in Camera for $\mathbf{8} \mathrm{mm}$ Silent Motion Picture Film

Position in Projector for Direct Front Projection of 16

Position in Projector for Direct Front Projection of 8

Position of the Shuttle Eye (1970)

Positioning and Straight Cut Numerically Controlled Mac

Positioning and Straight Cut Numerically Controlled Mac

Positioning and Straight Cut Numerically Controlled Mac

Positioning of Magnetic Compasses in Ships (1968)

Positions and Definitions of Rotation and Slope for Str

Positions and Diameter $40 \mathrm{~mm}$ (1966)

Positions and Diameter $60 \mathrm{~mm}$ (Low Current Rating) (1966

Positions for Straight Manual Arc Welds Made with Cover

Positions in Camera for $16 \mathrm{~mm}$ Sound Motion Picture Film

Positions in Camera for $35 \mathrm{~mm}$ Sound Motion Picture Film

Positions in Projector for $35 \mathrm{~mm}$ Sound Motion Picture F

Positive Displacement Vacuum Pumps-Part I: Volume Rat

Possible Hazards When Dealing with Electronic Equipment

Post Insulators and Units for Systems with Nominal Volt

Post Insulators for Systems with Nominal Voltages Great

Postcards and of Lettercards (1963)

Postcards and Similar Articles: Cancellation Area (1965

Postcards: Area Reserved for the Address (1965)

Posts (Overall Internal Height) (1961)
Re ISO

Rec. ISO

ISO

SO

ISO

SO

ISO

ISO

$\operatorname{Re}$ ISO

ISO

ISO

ISO

Rec. F IEC

IEC

ISO

ISO

ISO

ISO

ISO

EC

IEC

ISC

SO

ISO

ISO

119

1615

1614

2028

1567

1625

148

1147

1628

922

1346

1191

275

1622

118

305

227

174

727

580

1330

1165

2703

2045

2048

189-2

$189-7$

189-3

189-5

$189-1$

189-6

304

182

381

1772

2178

1014 
Potassium Chloride for Industrial Use-Determination 0

f Content-Sodium Tatraphenylborate Volumetric/

Rec. for Potassium Chloride for Industrial Use-Determination 0

Potassium Chloride for Industrial Use-Determination 0

Potassium Chromate and Zinc Tetrahydroxychromate (1970)

Potassium Content-Flame Emission Photometric Meth. (I

f the Carbon Dioxide Content Expressed as Potal

f Chloride Content: Volhard Volumetric Meth (19)

f Sulphate Content: Barium Sulphate Gravimetric/

f Iron Content: 2,2-Bipyridyl Spectrophotome/

f Silica Content: Gravimetric Meth. by Insolubi/

f Silica Content, Gravimetric Meth. by Precipit/

f Calcium: EDTA Complexometric Meth. (1969)

Storage of Test Sample (1969)

sample Solution (1969)

Sample-Preparation of the Main Solution for /

of Density of Samples in Solution-1 of Dry Matter-Gravimetric Meth. (1/ of Silica Content-Gravimetric Meth/ of Carbon Dioxide Content Expressed / of Total Alkalinity, Expressed in $\mathrm{Na} /$ of Dynamic Viscosity (1972)

of Silica Content-Titrimetric Meth/

Solution of Products Not Easily Sol/ th. of Test-General (1970)

Content-Gravimetric Meth. as Potassium Tetra/

Content-Sodium Tetraphenylborate Volumetric /

Content-Barium Sulphate Gravimetric Meth. (1/

Chloride Content-Mercurimetric Meth. (1973)

Acidity to Meth yl Orange (1973) rial Use-Determination of Content-Gravimetric Meth. as trial Use: Determination of Content-Gravimetric Meth. as Rec. for Building Materials-Determination of Calorific Its Alloys-Insulation Check by Measurement of Breakdow Rec. for Nonwirewound

um Tripolyphosphate for Industrial Use-Measurement of $\mathrm{Ph}$ nium Nitrate for Industrial Use: Measurement of $\mathrm{pH}$ Value rmination of Carbonyl Compounds (Hydroxylammonium Chloride nd Other Plant Material-Sampling (1972) Std. for bon Black for Use in the Rubber Industry: Determination of on of Apparent Density of Moulding Material That Cannot Be ation of Apparent Density of Moulding Material That Can Be Std. for Cork: Granulated and Rec. for Surface Active Agents in ctive Agents: Determination of Apparent Density of Washing dio Transmitters: General Conditions Of, Frequency, Output and Valves: Meth. of Measurement of Audio Frequency Output

pment for Unit Loads-Overhead Twin Rail Chain Conveyor Assembly Tools for Bolts and Screws-Driving Squares for Rec. for Expression of the

c. for Electrical Installations in Ships: Transformers for measurement for Radio Transmitters: Band width, Out of Band $\mathrm{Hz}(\mathrm{c} / \mathrm{s})(1959)$

num) and Its Alloys-Estimation of the Loss of Absorptive Rec. for Rec. for

Rec for Rules for Ohmic Resistors Used in the

est for Electric Strength of Solid Insulating Materials at

Electrical Properties of Microwave Tubes and Valves: High election of Meth. of Test and General Requirements for Low Rec. for Determination of Cakin

for Radio Transmitters: Bandwidth, Out of Band Power and

e Electrical Properties of Microwave Tubes and Valves: Low ec. for International Electrotechnical Vocabulary: Nuclea dio Active Materials (1965)

Rec. for Test Code for Stationary Steam Generators of the ng Equipment for Radio Active Materials (1967) Rec. or

low 3 MHz: Connectors for Coupling an External Low Voltage 1966) Rec. Series Capacitors for Rec. fo

ydraulic and Pneumatic Equipment and Accessories for Fluid sembly Tools for Bolts and Screws-Hexagon Drive Ends for ting (1961)

and Valves: Meth of Measuc. for Safety Features for Ground Rec. for Surface Active Agents: Measurement of Foaming Rec. for Automohiles-Engine Test Code-Net otermination

Rec. for Potassium Hydroxide for Industrial Use-Determination

Rec. for Potassium Hydroxide for Industrial Use: Determination 0

Rec. for Potassium Hydroxide for Industrial Use: Determination 0

Rec. for Potassium Hydroxide for Industrial Use: Determination o

Rec. for Potassium Hydroxide for Industrial Use: Determination 0

Rec. for Potassium Hydroxide for Industrial Use: Determination 0

Rec. for Potassium Hydroxide for Industrial Use: Determination 0

Rec. for Potassium Hydroxide for Industrial Use: Determination 0

Rec. for Potassium Hydroxide for Industrial Use: Determination 0

Rec. for Potassium Hydroxide for Industrial Use: Determination 0

Rec. for Potassium Hydroxide for Industrial Use: Preparation and

Rec. for Potassium Hydroxide for Industrial Use: Preparation of

Rec. for Potassium Hydroxide for Industrial Use: Preparation of
Std. for Potassium Hydroxide for Industrial Use: Sampling-Test

Potassium Hydroxide for Industrial U
Potassium Silicates for Industrial Use

Potassium Silicates for Industrial Use-Determination

Potassium Silicates for Industrial Use-Determination

Potassium Silicates for Industrial Use-Determination

Potassium Silicates for Industrial Use-Determination

Potassium Silicates for Industrial Use-Determination

Potassium Silicates for Industrial Use-Determination

Potassium Silicates for Industrial Use-Determination

Potassium Silicates for Industrial Use-Preparation of

Potassium Silicates for Industrial Use-Samples and Me

Potassium Soluble in Dilute Hydrochloric Acid (1971)

Potassium Sulphate for Industrial Use: Determination of

Potassium Sulphate for Industrial Use: Determination of

Potassium Sulphate for Industrial Use: Determination of

Potassium Sulphate for Industrial Use: Determination of

Potassium Sulphate for Industrial Use: Determination of

Potassium Tetraphenylborate (1971)

Potassium Tetraphenylborate (1973)

Potential (1971)

Potential (1972)

Potentiometers Type 2 (1966)

Potentiometric Meth. (1968)

Sulphate for Indus

ISO

ISO

ISO

ISO

ISO

ISO

ISO

ISO

ISO

ISO

ISO

ISO

ISO

ISO

ISO

ISO

ISO

ISO

ISO

ISO

ISO

ISO

ISO

ISO

ISO

ISO

Potentiometric Meth. (1972)

Idation) of Aluminium (Aluminum) and ISO

Potentiometric Meth.) (1970)

Rec. for Sodi ISO

Std. for A mmo ISO

Pour Density (1970)

Poured from a Specified Funnel (1958)

Poured from a Specified Funnel (1958)

Made of Peat a

ISO

Powder-Spec. (1972)

IS-Determinati IS

ITics-Determin ISO

Powder Form: Preparation of a Reduced Sample (1967)

Powders Before and After Compaction (1968) / Surface a ISO

Power and Consumption (1968)

Power and Distortion (1966)

/H. of Measurement for Ra IEC

(Power and Free) Safety Code (1971) /Ical Handling Equi ISO

Power and Hand Socket Wrenches (1970) Rec. for ISO
Pow

Power and Intensity Levels of Sound or Noise (1963)

Power and Lighting, Semiconductor Rectifiers, Generator

ISO

IEC

Power and Power of Nonessential Oscillations (1969)

I IEC

Power by Colorant Drop Test with Prior Acid Treatment (

ISO

IEC

Power Capacitors (I968)

Power Circuits of Electrically Powered Vehicles (1970)

Rec. Meth. of T IEC

Power Frequencies (1967)

Power Klystrons (1972)

IEC

Power of Coal by the Roga Meth (1963)

Power of Nonessential Oscillations (1969)

Power Oscillator Klystrons (1972) /R Meas lor Fixed Resistors: S IEC

ISO

I Measuremen IEC Reasurement of Th IEC

Power Station Type (1968)

Power Supply for Air and Land Vehicle Mounted Prospecti

Power Supply to Portable Entertainment Equipment (1971)

Power Systems (1972)

Power Take Off and Draw Bar for Agricultural Tractors (

Power Transformers (1967)

Power Transmission (1970)

Power Types (1970)

Power Units

/for Graphical Symbols for $\mathrm{H}$

Power (1966)

Power (1968)

Power (1971)

133 
c Dissipation Factor of Electrical Insulating Materials at ohmic: Resistors Used in the Power Circuits of Electrically Rec. for Pozzolanicity Test for

Rec. fo

I Drawings-Tolerances of Form and of Position-Part IV: wrapped Connections-General Requirements, Test Meth. and
Rec. for Test Procedures for Amplifiers and radiation (1)

Determination of of Silica Content; Gravimetric Meth. by use: Determination of Silica Content, Gravimetric Meth. by Rec. for Short Pitch Transmission s (1971) Rec. for Rec. for Rec. for Rec. for
Rec. for condenser Microphones by the Reciprocity Techni/ nstrumentation Applications (1971) on and Conveyors (1972) Std. for Extended Pitch Rec. for Short Pitch Transmission Rec. for ances and Masses Per Metre (Meter) (/) Rec. for Cold Drawn Rec. for Rolling Bearings-Instrumen Rec. for Long Solid Stem Thermometers for Rec. for Short Solid Stem Thermometers for Rec. for Long Enclosed Scale Thermometers for Rec. for Short Enclosed Scale Thermometers for and Resistors (I97I) Rec. for Rec. for Rec. for Rec. for gears for General Engineering (1966) 963)

Std. for Guide to the Use of

ues (1966)

Rec. for Guide to the Choice of Series of Std. for Series of

photographic Colour (Color) Paper for Roll Paper Printers: hotographic Black and White Paper for Roll Paper Printers: ec. for Photographic Colour (Color) Paper for General Use: rec. for Photographic Colour (Color) Film for General Use: for Photographic Black and White Papers for General Use: rec. for Designations, Diameters and Breaking Strengths of Reliability of Electronic Equipment and the Co/ Rec. for Rec. for Rec. for Sodium Hydroxide for Industrial Use: Rec. for Potassium Hydroxide for Industrial Use: Rec. for Sodium Carbonate for Industrial Use: ium (Aluminum) Oxide Primarily Used for the Production Of Rec. for Cryolite (Natural and Artificial) ve Agents: Verification of Certain Effects of Laundering Rec. for Surface Active Agents in Powder Form: .) (1969) Rec. for Guide for the Rec. for Crude Vegetable Oils and Fats: for Mechanical Standardization of Semiconductor Devices er Latex (I966)

nd Copolymer Latices (197I)

nded, Low Pressure Laminated Plates /

Rec. for t (1970)

Rec. for

alue for the Thermal Expansion Coefficient (For Use in the ture Tubes (1962)

Determination of Matter Insoluble in Water or in Acid and Rec. for Essential Oils-Meth. of Test num Oxide Primarily Used for the Production of Aluminium num) Oxide Primarily Used for the Production of Aluminium: Rec. for Sodium Hydroxide for Industrial Use
Rec. for Potassium Hydroxide for Industrial Use

1 (1964) Rec. for Selection and
Rec for Paints and Varnishes-Exantion and d. for Sodium and Potassium Silicates for Industrial Use materials: Moulding Meth. (I968)

materials: Casting Meth. (1968) Rec. for Plastics
Rec. for Plastics ed by Machines (19/ Rec. for General Requirements for the sion of Vulcanized Rubber to Metal Where the Ru/ Rec. for sion of Vulcanized Rubber to Metal Where the Ru/ Rec. for al Endurance of Electrical Insula/ Rec. for Guide for the Iding Test Specimens of Thermosetting Materials-Annex B lding Test Specimens of Thermosetting Materials-Annex A: lding Test Specimens of Thermosetting Materials-Annex C: ium Hydroxide for Industrial Use: Sampling-Test Sample n Carbonate for Industrial Use-List of Meth. of Test and Std. for Surface Active Agents Rec for Graphical Symbols for Coa ec. for Principles and Conventions for Flowsheets for Coal ry of Terms Relating to Solid Mineral Fuels-Part I: Coa Std. for Raw Rubber-Sample

t) /

Formats for the Numerical Control of Machines: Coding of Rec. for Textile Machinery and Accessories-Weaving
International Rec. for General International Rec. for Genera

Rec. for Architectural and Building Drawings:
Power, Audio and Radio Frequencies lncluding Metre (Met

Powered Vehicles (1970)
Pozzolanic Cements (1968)

Pozzolanicity Test for Pozzolanic Cements (1968)

Practical Examples of Indications on Drawings (1971)

Practical Guidance (1971)

Rec. for

mplifiers for Semiconductor Detectors for Ionizing

Precipitation of the Quinoline Silicomolybdic Complex (

Precision Bush Chains and Chain Wheels (1970)

Precision Fuse Links for Aircraft-General Requirement

Precision Fuse Links for Aircraft (Type A) (1971)

Precision Fuse Links for Aircraft (Type B) (1971)

Precision Meth. for Pressure Calibration of 1 In. Std.

Precision Reels for Magnetic Tape Used in Interchange I

Precision Roller Chains and Chain Wheels for Transmissi

Precision Roller Chains and Chain Wheels (1967)

Precision Sound Level Meters (1965)

Precision Steel Tubes: Metric Series, Dimensions, Toler

Precision Types (197I)

Precision Use (I968)

Precision Use (1968)

Precision Use (1968)

Precision Use (1968)

Preferred Diameters of Wire Terminations of Capacitors

Preferred Frequencies for Acoustical Measurements (1962

Preferred Modules and Diametral Pitches of Cylindrical

Preferred Number Series for Resistors and Capacitors (1

Preferred Numbers and Series of (1973)

Preferred Numbers and Those Containing More Rounded Va

Preferred Numbers (1973)

Preferred Sizes of Rolls (1969)

Preferred Sizes of Rolls (I969)

Preferred Sizes of Sheet Material (1969)

Preferred Sizes of Sheet Material (1969)

Preferred Sizes of Sheet Material (1969)

Preformed Stranded Steel Cables for Aircraft Controls

Preliminary List of Basic Terms and Definitions for the

Preliminary Reliability Considerations (1968)

Preparation and Storage of Test Sumple (1969)

Preparation and Storage of Test Sample (1969)

Preparation and Storage of Test Samples (1968)

Preparation and Storage of Test Samples (I968)

Preparation and Storage of Test Samples (I970)

Preparation and Use of Unsoiled Cotton Control Cloth (1

Preparation of a Reduced Sample (1967)

Preparation of Classified Vocabularies (Example of Meth

Preparation of Contract Sample for Analysis (1968)

Preparation of Drawings Devices (1969)

Preparation of Dry Films from Concentrated Natural Rubb

Preparation of Dry Polymer from Butadiene Homopolymer

Preparation of Glass Fibre (Fiber) Reinforced, Resin Bo

Preparation of Ground Sample of Known Dry Matter Conten

Preparation of Measurement Tables for Liquids) (I970)

Preparation of Outline Drawings of Oscilloscope and Pic

Preparation of Principal Solutions for Other Determinat

Preparation of Sample Sample (1963)

Preparation of Sample Solution for Analysis by Means of

Preparation of Sample Solution for Analysis (1968)

Preparation of Sample Solution (1969)

Preparation of Sample Solution (1969)

Preparation of Samples and Test Pieces for Wrought Stee

Preparation of Samples for Test. (1970)

Preparation of Solution of Products Not Easily Soluble

Preparation of Specimens for Optical Tests on Plastics

Preparation of Specimens for Optical Tests on Plastics

Preparation of Test Codes for Measuring the Noise Emitt

Preparation of Test Piece and Meth. of Test of the Adhe

Preparation of Test Piece and Meth. of Test of the Adhe

Preparation of Test Procedures for Evaluating the Therm

Preparation of Test Specimens from Aminoplastic Mouldin

Preparation of Test Specimens from Phenolic Moulding Ma

Preparation of Test Specimens from Polyester and Epoxy

Preparation of the Main Solution for Carrying Out Certa

Preparation of the Sample (1972) Cor for Sodium H

Preparation Plant (1967)

Preparation Plant (1969)

Preparation (1970)

Preparation (1972)

Preparatory Functions G and Miscellaneous Functions M (

Preparatory Machines-Definition of Side (Left or Righ

Prescriptions for Volumetric Gas Meters (1970)

Presentation of Contributions to Periodicals (1961)

Presentation of Drawings: Scales (1969)

Presentation of Reliability Data on Electronic Componen
IEC

IEC

ISO

ISO

IEC

IEC

ISO

ISO

ISO

ISO

ISO

IEC

ISO

ISO

ISO

IEC

ISO

ISO

ISO

ISO

ISO

ISO

IEC

ISO

ISO

IEC

ISO

ISO

ISO

Rec. for ISO

Rec. for P ISO

ISO

ISO

Rec ISO

ISO

IEC

IEC

ISO

ISO

Alumin ISO

ISO

IsO

ISO

ISO

ISO

Rec IEC

ISO

ISO

ISO

ISO

ISO

IEC

ISO

ISO

ISO

/Mi ISO

ISO

ISO

ISO

ISO

ISO

ISO

ISO

ISO

ISO

IEC

ISO

ISO

ISO

ISO

ISO

ISO

ISO

ISO

ISO

SO

ISO

OIML

ISO

ISO

IEC

250

322
863

863

166

352

340

1395

1547

1548

I860

1275

606

179

560

1224

655

656 
Rec. for Expression and Rec. for Fngineering Drawing: Principles of Rec. for Natural Rubber Latices, Evaporated r Natural Rubber latices, Centrifuged and Creamed, Ammonia domestic and Similar Purposes: Electric Irons, Ironers and gs of Plastics Materials-Socket Fittings for Pipes Under Rec. for High esp/ International Rec. for Indicating Pressure, Combined espo/ International Rec. for Recording Pressure, Combined Rec. for Aircraf

ternating Voltages /

ternating Voltages /

Metal Sheathed, Fo/ by the Reciprocity Techniq/

s by the Reciprocity Techni/

\section{d Pressure (1970)} in a Frontally / Rec for Relation Between Sound Rec. for Cast Iron Pipes: Special Castings and Parts for Rec. for Schedule for High Rec. for Ballasts for High Rec. for Tests on Oil Filled and Gas
Rec. for Tests on Oil Filled and Gas Rec. for Tests on Oil Filled and Gas Rec. for Simplified Meth. for Rec. for Precision Meth. for Std. for Aircraft Water Methanol International $R$. for Polyvinyl Chloride (PVC)

Single Sockets for Unplasticized Polyvinyl Chloride (PVC)
socket Fittings for Unplasticized Polyvinyl Chloride (PVC)
Rec. for Asbestos Cement
Rec. for Aircraft
Rec. for Aircraft

Single Sockets for Unplasticized Polyvinyl Chloride (PVC)
socket Fittings for Unplasticized Polyvinyl Chloride (PVC)
Rec. for Asbestos Cement
Rec. for Aircraft
Rec. for Aircraft

Single Sockets for Unplasticized Polyvinyl Chloride (PVC)
socket Fittings for Unplasticized Polyvinyl Chloride (PVC)
Rec. for Asbestos Cemen
Rec. for Aircraf
Rec. for Aircraft ure, Combined Pressure and Vacuum, and Vacuum Gauges, with pipe Threads for Gas List Tubes and Screwed Fittings Where in. $106 \mathrm{In} . / \mathrm{S})(1961)$ Rec. for Pipe Threads Where ltages from $10 \mathrm{kV}$ Up to and Including $33 \mathrm{kV}$ (Excluding Gas

$s$ for Electronic Components and Equipment: Test M: Low Air gs in Unplasticized Polyvinyl Chloride (PVC) for Use Under $r$ Pressure Gauges for Instruments Measuring Arterial Blood $r t$ of Fluids-Determination of the Resistance to Internal

for Analysis by Means of Attack by Hydrochloric Acid Under

-Expanded Pure Agglomerated-Deformation Under Constant es, with Pressure Resp/

es, with Pressure Respo/

International Rec. for Indicating

International Rec. for Recordin to and Including $66 \mathrm{kV}$ (Excluding Gas Rec. for

for the Transport of Fluids (Outside Diameters and Nominal for the Transport of Fluids (Outside Diameters and Nominal gs with Plain S/ Rec. for Socket Fittings for Pipes Under c. for Electrical Apparatus for Explosive Gas Atmospheres: Rec. for Sealing Test fo

uipment for Comm/ Rec. for Electrical Connections Between ing Semiconductor Rectifiers, Generators (With Associated

d. for Measurement of Airborne Noise Emitted by Compressor for ISO General Purpose Metric Screw Threads: Tolerances, aration Plant (1969)

Rec. for Radiographic Image Quality Indicators: Std. for Joints in Building: Fundamental Rec. for General galvanizing (1970)

n of Installations for Work on Unse/

72) Rec. for Guiding ontrol Chemicals (Pesticides) and Plant Growth / Rec. For Gec. for ures (1973)

s (1964)

glass Laboratory Thermometers (1964)

Glassware (1964)

sing Fissile Materials (1970) and General Principles of Measuring Meth.: General and Characteristics of Semiconductor Devices and General $s$ and Characteristics of Semiconductor Devices and General $s$ and Characteristics of Semiconductor Devices and General $s$ and Characteristics of Semiconductor Devices and General Rec. for General Rec. for

Rec. for Engineering Drawing: Rec. for Namin ec. for Electrical Installations of Buildings: Fundamental

9)

Rec. for Terms and Definitions for Rec. for Grid System for Rec. for Metal Clad Base Materials for Rec. for Metal Clad Base Materials for

Motion Pictur Std. for Cinematography-Location of the for Trimmed Sizes of Writing Paper and Certain Classes of mensions of Processed Writing Paper and Certain Classes of Machines (1969) and Use of Components Intended for Mounting on Boards with m (0.1 In.) Mating Either with Board Mounted Connectors or
Presentation of Results of Coal Cleaning Tests (1969)

Preserved-Spec. (1971)

Preserved-Spec. (1971)

Pressure-Basic 7 and Heating Appliances for CEE

Pressure Air Charging Valves for

ITin ISO

Pressure and Vacuum, and Vacuum Gauges, with Pressure $R$

Pressure and Vacuum, and Vacuum Gauges, with Pressure $R$

Pressure Cabin Ground Test Connection (1954)

Pressure Cables and Their Accessories: External, for Al

Pressure Cables and Their Accessories: Internal, for Al

Pressure Cables and Their Accessories: Paper Insulated,

Pressure Calibration of One Inch Condenser Microphones

Pressure Calibration of $1 \mathrm{In}$. Std. Condenser Microphone

Pressure Connections (1973)

Pressure Gauges for Instruments Measuring Arterial Bloo

Pressure Laminated Plates or Panels for Test Purposes (

Pressure Levels of Narrow Bands of Noise in a Diffuse F

Pressure Main Lines (1955)

Pressure Mercury Vapour (Vapor) Lamps (1965)

Pressure Mercury Vapour (Vapor) Lamps (1969)

Pressure Pipes with Elastic Sealing Ring Type Joints: M

Pressure Pipes with Elastic Sealing Ring Type Joints: M

Pressure Pipes (1971)

Pressure Refueling Connections (1957)

Pressure Reoiling Connection (1965)

Pressure Responsive Elements and Recording Directly by

Pressure Responsive Elements Giving Direct Indication

Pressure Tight Joints Are Made on the Threads (1/8 In.

Pressure Tight Joints Are Not Made on the Threads (1/8

/Aining Types for Alternating Vo IEC

ressure Types (1965)

Pressure (1966)

Pressure (1967)

Pressure (1970)

Pressure (1970)

Pressure (1971)

Rec for Oven Test for Moulded Fittin

Pressure (1972)

ISO

OIML

ISO

IEC

IEC

IEC

IEC

ISO

OIM

ISO

ISO

IEC

IEC

ISO

ISO

1SO

ISO

OIML

OIML

ISO

Pressure, Combined Pressure and Vacuum, and Vacuum Gaug

Pressure, Combined Pressure and Vacuum, and Vacuum Gaug

Pressure, Oil Filled and Nondraining Types) (1965)

Pressures and Tolerances on Length (1970)

Pressures in Brake Lines and Braking Efficiency (1970)

/Tics Materials

Pressures): Part I: Metric Series (1960)

IS Materials

Pressure: Unplasticized Polyviny

Pressurized Enclosures (1962)

Pressurized Waveguide Tubing and Assemblies (1968)

Prime Movers and Towed Vehicles with 24-V Electrical Eq

Prime Movers) and Motors, Electric Propulsion Plant, Ta

Primeover Units Intended for Outdoor Use (1972)

Principles and Basic Data (1969)

Principles and Conventions for Flowsheets for Coal Prep

Principles and Identification (1969)

Principles for Design (1972)

Principles for Fatigue Test. of Metals (1964)

Principles for Protection Against Corrosion by Hot Dip

Principles for Protection in the Design and Constructio

Principles for the Formulation of Graphical Symbols (19

Principles for the Selection of Common Names for Pest C

Principles for the Verification of the Safety of Struct

Principles of Construction and Adjustment of Hydrometer

Principles of Construction and Adjustment of Liquid in

Principles of Construction and Adjustment of Volumetric

Principles of Criticality Safety in Handling and Proces

Principles of Measuring Meth. (1963)

/Ating IE

Principles of Measuring Meth.: General and Terminology

Principles of Measuring Meth.: General Principles of Me

Principles of Measuring Meth.: Reference Meth. of Measu

Principles of Nuclear Reactor Instrumentation (1967)

Principles of Operation of Standards Marks (1961)

Principles of Presentation (1959)

Principles (1968)

Principles (1970)

Print Spec. for Magnetic Ink Character Recognition (196

Printed Circuits (1965)

Printed Circuits (1970)

Printed Circuits: Spec. (1970)

Printed Circuits: Test Meth. (1968)

Printed Image Area for Printing to $8 \mathrm{~mm}$ Type $S$ on $16 \mathrm{~mm}$

Printed Matter (1961)

Printed Matter (1963) /. for Meth. of Expression of Di

Printed or Displayed Symbols for Adding and Calculating /Ce for the Design IEC

Printed Wiring Boards with Edge Board Contacts (1971) 
Kec. for Fundamental Parameters of Connectors for Rer. for General Requirements and Measuring Meth. for rec. for Photographic Black and White Paper for Roll Paper Rec. for Photographic Colour (Color) Paper for Roll Paper Rec. for Layout of ths of Fabric Ribbons on Spools (197) Std. For Office and ns and Direction of Manufacture of Unprocessed Writing and std. for Minimum Markings to Appear on Containers Used for

Rec. for

or Cinematography-Location of the Printed Image Area fo ds and Scanning Area of $35 \mathrm{~mm}$ Double Width Push Pull Sound c. for Cinematography-Photographic Sound Record on $35 \mathrm{Mm}$

c. for Cinematography-Photographic Sound Record on $16 \mathrm{Mm}$ wing of Photographic Transparencies with Reflection Colou Std. for Automobiles-Load Distribution for icles with 6 or $12 \mathrm{~V}$ Electrical Equipment Particularly for er / Rec. for External Diameters of Cylindrical Radiation

\section{(1971)}

Rec. for an Appraisal of the

\section{t $(1970)$}

Rec. for

inuous Filament Yarn, Staple Fi/ Rec. for Textile GIass f Enamelled Wire by the Lowering of the El/ Rec. for Test -Electric Strength Meth. (1971) Spec. for Statutes and Rules of ation of Anionic-Active Matter (Direct Two Phase Titration 2) Std. for Data Communication-Basic Mode Contro ductor Detectors for Ionizing Radiation (1/

Rec. for Basic Mode Control

MateriaIs for Electrical Machines (1972) Rec. for Test eral (1968)

2 Test (1968)

t A: Cold (1966)

t J: Mould Growth (19/

t Ka: Salt Mist (1964)

t M: Low Air Pressure/

t N: Change of Temper/

t Q: Sealing (I968)

t B: Dry Heat (I966)

t T: Soldering (1968)

t U: Robustness of Te/

t Ea: Shock (1967)

t Eb: Bump (1968)

t Ca: Damp and Steady/

t D: Accelerated Damp/

t Ga: Acceleration, S/

t H: Storage (1960)

trical Insula/

iation (I970)

Rec. for Basic Environmental Test. Rec. for Basic Environmental Test. Rec. for Basic Environmental Test. Rec. for Basic Environmental Test. Rec. for Basic Environmental Test. Rec. for Basic Environmental Test. Rec. for Basic Environmental Test. Rec. for Basic Environmental Test. Rec. for Basic Environmental Test. Rec. for Basic Environmental Test. Rec. for Basic Environmental Test. Rec. for Basic Environmental Test. Rec. for Basic Environmental Test. Rec. for Basic Environmental Test. Rec. for Basic Environmental Test. Rec. for Basic Environmental Test. Rec. for Basic Environmental Test. Rec. for Guide for the Preparation of Tes Rec. for Test Organization, Rules and (1973)
71)

) (1969)

ipment Type Specimens /
Std. for Basic Mode Control Rec. for Basic Environmental Test. Rec. for Basic Environmental Test. Rec. for Basic Environmental Tes Rec. for Basic Environmental Test Rec. for Basic Environmental Tes Rec. for Basic Environmental Test.

Rec. for Analogue d.c. Current Signals for Rec. for Analogue Pneumatic Signal for c. for Meth. for Indicating the Stability of the Images o c. Meth. for Determining Thiosulphate and Tetrathionate in

rence Meth.) (1970) Rec. for Cheese and It matter (19/ Rec. for Meth. of Expression of Dimensions of Rec. for Electroplating and Related Rec. for Definitions of Weldin ahaped Refractory Products-Nomenclature of Manufacturin : Regulatora for Gas Cylinders Used in Cutting and Related

Punched Cards (197I) Rec. for Information Rec. for Information Rec. for Information std. for Office and Printing Machines Used for Information Rec. for Principles of Criticality Safety in Handling and Rec. for 6 and 7 Bit Coded Character Sets for Information ved from the ISO 7 Bit Coded Character Set for Information (1973) Std. for Information Rec. for Flowchart Symbols for Information t Coded Character Set on 12 Row Punched Cards (Information nched Holes in 80 Columns Punched Paper Cards (Information ematography: 65 and $70 \mathrm{~mm}$ Motion Picture Film-Image Area r $35 \mathrm{~mm}$ Films (1958) r 8 mm Films (I958)

Rec. for Cinematography-Image Rec. for Cinematography-Image Rec. for Cinematography-Image

for Cinematography: $35 \mathrm{~mm}$ Motion Picture Film-Image Area

-Determination of the Content by Volume in the Vaporized ent-Ato/

lectrical Equipment of Machine Tools: Used in Large Series $\mathrm{nt}-\mathrm{Spec/}$
Printed Wiring Boards (1964)

Printed Wiring Boards (1970)

Printers: Preferred Sizes of Rolls (1969)

Printers: Preferred Sizes of Rolls (1969)

Printing and Function Keys on Typewriters (1969)

Printing Machines Used for Information Processing-Wid

Printing Paper (1961)

Printing Ribbons (1972)

/Meth. of Expression of Dimensio

Printing Spec. for Optical Character Recognition (1971)

Printing to $8 \mathrm{~mm}$ Type S on $16 \mathrm{~mm}$ Motion Picture Film Pe

Prints Normal and Offset Centerline Types (1958)

Prints (1958)

Prints (1958)

Prints (1972)

Private Cars (1972)

Re ISO

Re 150

Probes Containing Geiger-Muller or ProportionaI Count

Problems of Accelerated Test. for Atmospheric Corrosion

Procedure for Describing Aircraft Noise Around an Airpo

Procedure for Sampling Batches or Consignments Of, Cont

Procedure for the Evaluation of the Thermal Endurance $O$

Procedure for Thermal Endurance of Insulating Varnishes

Procedure of the CEE (1961)

Procedure) (1972)

/Ctive Agents: Detergents-Determin

Procedures-Code Independent Information Transfer (I97

Procedures for Amplifiers and Preamplifiers for Semicon

Procedures for Data Communication Systems (1971)

Procedures for Determining Physical Properties of Brush

Procedures for Electronic Components and Equipment: Gen

Procedures for Electronic Components and Equipment: $\mathrm{Pt}$.

Procedures for Electronic Components and Equipment: Tes

Procedures for Electronic Components and Equipment: Tes

Procedures for Electronic Components and Equipment: Tes

Procedures for Electronic Components and Equipment: Tes

Procedures for Electronic Components and Equipment: Tes

Procedures for Electronic Components and Equipment: Tes

Procedures for Electronic Components and Equipment: Tes

Procedures for Electronic Components and Equipment: Tes

Procedures for Electronic Components and Equipment: Tes

Procedures for Electronic Components and Equipment: Tes

Procedures for Electronic Components and Equipment: Tes

Procedures for Electronic Components and Equipment: Tes

Procedures for Electronic Components and Equipment: Tes

Procedures for Electronic Components and Equipment: Tes

Procedures for Electronic Components and Equipment: Tes

Procedures for Evaluating the Thermal Endurance of Elec

Procedures for Semiconductor Detectors for'Ionizing Rad

Procedures of the CISPR (1971)

Procedurea: Conversational Information Message Transfer

Procedures: Guidance for Damp Heat Tests (1968)

Procedures: Guidance on Change of Temperature Tests (I9

Procedures: Test Db: Damp Heat, Cyclic $(12+12 \mathrm{Hr}$. Cycle

Procedures: Test Ec: Drop and Topple, Primarily for Equ

Procedures: Test Ed: Free Fall (1969)

Procedures: Test Fc: Vibration (Sinusoidal) (1970)

Process Control Systems (1971)

Process Control Systems (197I)

Processed Black and White Films, Plates and Papers (196

Processed Black and White Photographic Film, Plates and

Processed Disk Records and Reproducing Equipment (I964)

Processed Products-Determination of Fat Content (Refe

Processed Writing Paper and Certain Classes of Printed

Processes-Vocabulary (1971)

Processes (1968)

Processes (1972)

Processes (1972) Spec. for Welding ISO

Processing-Representation of 8 Bit Patterns on 12 Row

Processing-Spec. for Unpunched Paper Cards (1970)

Processing-Widths of Fabric Ribbons on Spools (1972)

Processing Fissile Materials (1970)

Processing Interchange (I967)

Processing Interchange (I969)

Processing Interchange: Representation of Ordinal Date

Processing (1969)

Procesaing) (1970)

Processing) (1971)

Ec. for Representation of ISO $7 \mathrm{Bi}$

Ons and Location of Rectangular Pu

Produced by Camera Aperture and Projectable Image-Area

Produced by Camera Aperture and Projected Image Area Fo

Produced by Camera Aperture and Projected Image A

Produced by Camera Aperture (1972)

(for Liquid Chlorine for Industrial Use
(967)

Product (1972)

Production Lines Lines (1967)

Production of Aluminium-Determination of Calcium Cont

Production of Aluminium-Determination of Silica Conte

17

326

1009

I091

2257

217

2258

1831

70

71

2243

2416

1724

256

355

507

I886

172

370

0

2271

2III

340

1745

413
68.1

68-2

68-2.1

68-2-I0

68-2-I I

68-2-I3

68.2.14

$68 \cdot 2 \cdot 17$

68-2-2

68-2.20

$68 \cdot 2 \cdot 21$

$68 \cdot 2 \cdot 27$

68-2-29

$68 \cdot 2 \cdot 3$

68-2-4

68-2-7

68-2-8

216

333

10

2629

$68 \cdot 2 \cdot 28$

$68 \cdot 2-33$

68-2-30

68.2-31

68-2-32

68-2-6

381

382

421

417

98

I735

353

2080

857

2246

2503

I729

2021

1681

2257

1709

646

963

2711

1028 
nt-Flam/ Rec. for Aluminum Oxide Primarily Used for the rer. for Aluminium (Aluminum) Oxide Primarily Used for the c Absorpt/Rec. for Aluminum Oxide Primarily Used for the -Pan Ph/ Rec. for Aluminum Oxide Primarily Used for the n for Ana/ Rec. for Aluminum Oxide Primarily Used for the rec. for Aluminium (Aluminum) Oxide Primarily Used for the nt-Spe/ Rec. for Aluminium Oxide Primarily Used for the ty; Pykn/ Rec. for Aluminium Oxide Primarily Used for the 1,10-1 Rec. for Aluminium Oxide Primarily Used for the t $1000 \%$ Rec. for Aluminium Oxide Primarily Used for $\mathrm{nt}$; Tiro/ Rec. for Aluminium Oxide Primarily Used for the ose (I96) Rec. for Aluminium Oxide Primarily Used for the (1968) Rec. for Aluminium Oxide Primarily Used for the rec. for Aluminium (Aluminum) Oxide Primarily Used for the rec. for Aluminium (AIuminum) Oxide Primarily Used for the ic Acid (197I) Sid. for Shaped Insulating Refractory Rec. for Fruit and Vegetable Rec. for Meat and Its Std. for Petroleum Rec. for Cheese and Its Processed Std. for Meat and Its Std. for Meat and Its

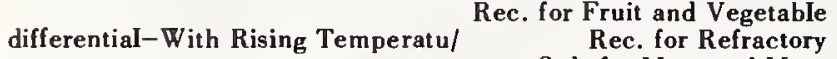
nitrogen by the KjeldahI Meth/ Std. for Meat and Meat Rec. for Agricultural Food Rec. for Bonded Abrasive ng Point (I972) Std. for Petroleum 2) Std. for Dense Shaped Refractory Rec. for Guide on the Form for Std. for Chemical efractoriness) (1966) ons-Profiles (I966) Std. for Petroleum Rec. for Refractory ipbuilding Details-Marking of Rolled, Drawn and Extruded ine for IndustriaI Use-Determination of Sulphated Ash in Silicates for Indust rial Use-Preparation of Solution of mposition (Per Cent) (19/ Rec. for Composition of Wrought Rec. for Mechanical Property Limits for Rolled Rec. for Mechanical Property Limits for Extruded e Test (I97I) Rec. for Copper and Alloy Rolled Flat Rec. for Sampling and Inspection of Asbestos Cement
ion of the Direction of Twist in Textile Yarns and ReIated Rec. for CIassification of Dense Refractory ents for Hot Dip Galvanized Coatings on Fabricated Ferrous

f ISO Rec. 390, Sampling and Inspection of Asbestos Cement termination of the Percentage of Combustible Matter of Its Std. for Cereals PuIses-Sampling of Milled Rec. for Meat and Its Std. for Cereals, Pulses and Derived rence Meth.) (1968)

th.) (I968)

\section{)}

netration (1972) Rec. for Fruit and Vegetable Rec. for Cereals and Cereal Rec. for Cereals and Cereal Rec. for Meat and Its Std. for Petroleum Rec. for Fruit and Vegetable Rec. for Fruit and Vegetable Rec. for Fruit and Vegetable Rec. for Bonded Abrasive Std. for Petroleum Rec. for Milk and Its

Std. for Starch, Including Derivatives and by nstruments for the Measurement of Surface Roughness by the
nstruments for the Measurement of Surface Roughness by the nstruments for the Measurement of Surface Roughness by the
Rec. for ISO General Purpose Screw Threads-Basic less Wide V-Belts for Industrial Speed Changers and Groove oducts General Features Designation Ranges of Dimensions nd of Position-Part III: Dimensioning and Tolerancing of Std. for Std. for Std. for

ess by the Profile Meth.-Contact (Stylus) Instruments of Std. for Cinematography: $35 \mathrm{~mm}$ Motion Picture Film Picture Film-Image Area Produced by Camera Aperture and Rec. for Cinematography for Cinematography-Image Produced by Camera Aperture and for Cinematography-Image Produced by Camera Aperture and Std. for Building Drawings:

Rec. for Determination of Wool Fibre (Fiber) Diameter: ination of Percentage of Medullated Fibres (Fibers) by the Rec. for Emulsion Position in Projector for Direct Front atography-Maximum Aspect Ratio of Projector Aperture for Rec. for Emulsion Position in Projector for Direct Fron an Type S) (1968) Rec. for Dimensions of

Rec. for Dimensions and Pitching of Slots on Platens for
Production of Aluminium-Determination of Sodium Conte Production of Aluminium-Determination of Vanadium Con Production of Aluminium-Determination of Zinc-Atomi Production of Aluminium-Determination of Zinc Content

Production of Aluminium-Preparation of Sample Solutio Production of Aluminium: Preparation of Sample Solution Production of Aluminum-Determination of Calcium Conte Production of Aluminum: Determination of Absolute Densi Production of Aluminum: Determination of Iron ContentProduction of Aluminum: Determination of Loss of Mass a Production of Aluminum: Determination of Titanium Conte Production of Aluminum: Measurement of the Angle of Rep Production of Aluminum: Measurement of Untamped Density Production of Determination of Loss of Mass at $300^{\circ}$

Production Of; Preparation and Storage of Test Samples Products-Classification (I972)

Products-Determination of Ash Insoluble in Hydrochlor Products-Determination of Chloride Content (1970)

Products-Determination of Colour (Color) (1972)

Products-Determination of Fat Content (Reference Meth

Products-Determination of Free Fat Content (1973)

Products-Determination of Moisture Content (1973)

Products-Determination of $\mathrm{pH}$ (I970)

Products-Determination of Refractoriness Under Load (

Products-Determination of Total Fat Content (1973)

Products-General Directions for the Determination of

Products-Grinding Wheel Dimensions-Part 2 (I969)

Products-Lubricating Grease-Determination of Droppi

Products-NomencIature of Manufacturing Processes (197

Products and for Meth. of Chemical Analysis (I969)

Products Corrosiveness to Copper-Strip Test (1972)

Products Determination of Pyrometric Cone Equivalent ( $R$

Products General Features Designation Ranges of Dimensi

Products in Light Metals or in Light Alloys (I960)

Products Not Containing More Than $0.5 \%$ of Such, by Mass

Products Not Easily Soluble in Boiling Water and Determ

Products of Aluminium and Aluminum Alloys-Chemical Co

Products of Aluminium (Aluminum) and Its Alloys (1968)

Products of Aluminium (Aluminum) and Its Alloys (1971)

Products (Thickness Less Than $2.5 \mathrm{~mm}$ (0.1 In.)-Tensil

Products (I964)

Products (1966)

Products (I969)

Rec. for Designat

Products (1970)

Products (1970)

Products (1971)

Products (1972)

Rec. for Guide to the Use O ISO

Rec. for Textile Glass-De ISO

Products: Determination of Ash (1969)

Products: Determination of Ash (I972)

Products: Determination of Mineral Impurities (I968)

Products: Determination of Moisture Content (Basic Refe

Products: Determination of Moisture Content (Routine Me

Products: Determination of Nitrogen Content (1969)

Products: Determination of Sulphur-Lamp Meth. (1973)

Products: Determination of Titratable Acidity (1968)

Products: Determination of Total Solids (1969)

Products: Determination of Water Insoluble Solids (1968

Products: Grinding Wheel Dimensions (Part 1) (1967)

Products: Lubricating Grease-Determination of Cone Pe

Products: Sampling (1969)

Products: Vocabulary (1973)

Profile Meth.-Contact (Stylus) Instruments of Progres

Profile Meth.-General Statements-Terms and Definiti

Profile (1969)

Profiles for Corresponding Pulleys (1970)

Profiles (I966)

Profiles (1971)

(Nical Drawings-Tolerances of Form

Programming Language: ALGOL (1972)

Programming Language: COBOL (1972)

Programming Language: FORTRAN (I972)

Progressive Profile Transformation-Profile Recording

Projectable Image Area (I972)

Projectable Image-Area (1972)

Projected Image Area for $16 \mathrm{~mm}$ Film (1963)

Hy: 65 and $70 \mathrm{~mm}$ Motion

Projected Image Area for $8 \mathrm{~mm}$ Films (1958)

Projection Meth. (1972)

Projection Microscope Meth. (1960)

Projection Microscope (1973)

Projection of $16 \mathrm{~mm}$ Silent Motion Picture Film (1956)

Projection of $35 \mathrm{Mm}$ Nonanamorphotic Motion Picture FiIms

Projection of $8 \mathrm{~mm}$ Silent Motion Picture Film (1956)

Projection Reels for $8 \mathrm{~mm}$ Motion Picture Film (Other Th

Projection WeIding Machines (1968) 
Isage of $8 \mathrm{~mm}$ Type S Motion Picture Film for Direct Front ic Mot/Rec. for Cinematography-Maximum Aspect Ratio of otion Picture Film (1956) Rec. for Emulsion Position in tion Picture Film (1956) Rec. for Emulsion Position in Rec. for Emulsion and Sound Record Positions in Rec. for Photography direct Front Projection (1973) Std. for Cinematography: or Mechanical Properties of Fasteners: Nuts with Specified or Mechanical Properties of Fasteners: Nuts with Specified Similar General Us/ peratures (1961) at Elevated Temperatures: Determination of Lower Yield and plant Material-Sampling (1972)

(Meth. of Test) (1966) Std. for Pots for bustion Chambers, and Direction of Rotation of Engines and ishing (1966) Rec. for Shipbuilding Details: Ship Screw Rec. Pract. for the Determination of Change of Mechanical Magnetic Tapes for Instrumentation Applications: Physical inding Wires: Enamelled Round Copper, with High Mechanical 1972) Rec. for Shipbuilding Details-Dimensions and Sectional for Determining Physical

nput and Output / lament Current $(/$ of Electrode Cur/ of Equivalent No/ of Cathode Heati/ lication of Mech/ surement of Audi/ surement of Radi/ suring Electrode/ surement of Emis/ surement of Rada/ surement of Spur/ surement of Gasf/ surement of Nois/ surement on Coro/ surement of Thyr/ surement of Cros/ surement of Vacu/ suring Hiss and / suring the Catho/ (1963)

relating to Meth/ $r$ Television Pic/ r Cold Cathode $\mathrm{C} /$ r Camera Types $(/$ Geiger-Muller/ ds (1968)

$s$ and Nuts (1969)

Values (1969)

Values, Effective Heights of Thread/ Rec. for Measurements of the Electrical Rec. for Measurements of the Electrical Rec. for Measurements of the Electrical Rec. for Measurements of the Electrical Rec. for Measurements of the Electrical Rec. for Measurements of the Electrical Rec. for Measurements of the Electrical Rec. for Measurements of the Electrical Rec. for Measurements of the Electrical Rec. for Measurements of the Electrical Rec. for Measurements of the Electrical Rec. for Measurements of the Electrical Rec. for Measurements of the Electrical Rec. for Measurements of the Electrical Rec. for Measurements of the Electrical Rec. for Measurements of the Electrical Rec. for Measurements of the Electrical Rec. for Measurements of the Electrical Rec. for Measurements of the Electrical Rec. for Measurements of the Electrical Rec. for Measurements of the Electrical Rec. for Measurements of the Electrical Rec. for Measurements of the Electrical Rec. for Measurements of the Electrical Rec. for Measurements of the Electrical Rec. for Measurements of the Electrical Rec. for Mechanical Rec. for Mechanical Rec for Mechanical Std. for Mechanical Rec. for Plastics-Determination of Tensile Oscillator Tubes/ crowave Switching/

Rec. for Measurement of the Electrical Rec. for Measurement of the Electrical Rec. for Measurement of the Electrical ystrons (1972) illator Klystrons/ 972)

1972)

Rec. for Measurement of the Electrical Rec. for Measurement of the Electrical Rec. for Measurement of the Electrical Rec. for Measurement of the Electrical Rec. for Measurement of the Electrical Rec..for Plastics-Determination of Compressive form 31 tics-Meth. of Test to Determine the Change in Electrical Rec. Meth. of Measurement of Essential Electrical
Std. for Plastics-Determination of Flexural um Aluminium (Aluminum) Zine Alloy Castings and Mechanical td. for Acoustics: Description and Measurement of Physical $15 \mathrm{~mm}$ or M/ Rec. for Meth. for Determining the Mechanical Rec. for Information Processing -
of the Quantities Characterizing the Magnetic and Electric Rec. for Determination of Tensile Stress Strain

as/ Rec. for Meth. of Test for Determining the Mechanical winding Wires: Enamelled Round Copper with Good Dielectric Rec. for Plastics-Determination of Tensile qual Leg Angles-Metric Series-Dimensions and Sectional qual Leg Angles-Metric Series-Dimensions and Sectional lel Flange I Beams-In. Series-Dimensions and Sectional ge Column Sections-In. Series-Dimensions and Sectional unequal Leg Angles-In. Series-Dimensions and Sectional - Equal Leg Angles-In. Series-Dimensions and Sectional Ivent Bonding Enamelled Round Copper, with High Mechanical inding Wires: Enamelled Round Copper, with High Mechanical ium (Aluminum) Alloys-Sand Cast Test Pieces-Mechanical td. for Rigid Cellular Plastics-Determination of Tensile $r$ Ropes-Determination of Certain Physical and Mechanical
Projection (1973)

Std, for Cinematography: Projector ISO Projector and Floodlighting Lamps (1971) IEC

Projector Aperture for Projection of 35Mm Nonanamorphot ISO

Projector for Direct Front Projection of $16 \mathrm{~mm}$ Silent M ISO

Projector for Direct Front Projection of $8 \mathrm{~mm}$ Silent Mo ISO

Projector for $35 \mathrm{~mm}$ Sound Motion Picture Film (1956) ISO

Projector Slides-Dimensions (1971)

Projector Usage of $8 \mathrm{~mm}$ Type S Motion Picture Film for

Proof Load Values (1969)

Proof Load Values, Effective Heights of Thread 0.4 D to

Proof Mains Operated Electronic Equipment (Domestic and

Proof Stress and Proving Test for Steel at Elevated Tem

Proof Stresses (Stress) and Proving Test (1968)

Propagation and Transplantation Made of Peat and Other

Propagation Resistance of the Carcass of Conveyor Belts

Propellers (1966)

the Carcass of Con veyor Belts

Propellers, Manufacturing Tolerance for Casting and Fin

Properties After Contact with Chemical Substances (1965

Properties and Test Meth. (1973)

Properties for Use in Refrigerant Systems (1972)

Properties of Aluminium (Aluminum) Alloy Sections for $M$

Properties of Brush Materials for Electrical Machines (

Properties of Cathode Ray Oscilloscopes (1971)

Properties of Electronic Tubes and Valves: Equivalent I

Properties of Electronic Tubes and Valves: Heater or Fi

Properties of Electronic Tubes and Valves: Measurement

Properties of Electronic Tubes and Valves: Measurement

Properties of Electronic Tubes and Valves: Measurement

Properties of Electronic Tubes and Valves: Meth. of App

Properties of Electronic Tubes and Valves: Meth. of Mea

Properties of Electronic Tubes and Valves: Meth. of Mea

Properties of Electronic Tubes and Valves: Meth. of Mea

Properties of Electronic Tubes and Valves: Meth. of Mea

Properties of Electronic Tubes and Valves: Meth. of Mea

Properties of Electronic Tubes and Valves: Meth. of Mea

Properties of Electronic Tubes and Valves: Meth. of Mea

Properties of Electronic Tubes and Valves: Meth. of Mea

Properties of Electronic Tubes and Valves: Meth. of Mea

Properties of Electronic Tubes and Valves: Meth. of Mea

Properties of Electronic Tubes and Valves: Meth. of Mea

Properties of Electronic Tubes and Valves: Meth. of Mea

Properties of Electronic Tubes and Valves: Meth. of Mea

Properties of Electronic Tubes and Valves: Noise Factor

Properties of Electronic Tubes and Valves: Precautions

Properties of Electronic Tubes: Meth. of Measurement Fo

Properties of Electronic Tubes: Meth. of Measurement Fo

Properties of Electronic Tubes: Meth. of Measurement Fo

Properties of Electronic Tubes: Meth. of Measurement of Properties of Fasteners-Part 1: Bolts, Screws and Stu

Properties of Fasteners: Marking of Bolts, Screws, Stud

Properties of Fasteners: Nuts with Specified Proof Load

Properties of Fasteners: Nuts with Specified Proof Load

Properties of Films (1970)

Properties of Microwave Tubes and Valves: Backward Wave

Properties of Microwave Tubes and Valves: Gas Filled Mi

Properties of Microwave Tubes and Valves: General (1972

Properties of Microwave Tubes and Valves: High Power Kl

Properties of Microwave Tubes and Valves: Low Power Osc

Properties of Microwave Tubes and Valves: Magnetrons (1

Properties of Microwave Tubes and Valves: Terminology (

Properties of Microwave Tubes: Disk Seal Tubes (1972)

Properties of Plastics (1967)

Properties of Polyethylene Due to the Migration of of $\mathbf{P}$

Properties of Receiving Aerials in the Frequency Range

Properties of Rigid Plastics (1972)

Properties of Sand Cast Reference Test Bars (1971)

Properties of Sonic Booms (1973)

Properties of the Weld Metal Deposited by Electrodes 3.

Properties of Unpunched Paper Tape (1971)

Properties of Vacuum and a Substance (1966)

Properties of Vulcanized Rubbers (1968)

Properties of Weld Metal Deposited by Filler Rods for C

Properties Under Humid Conditions (1970)

Properties (1966)

Properties (1968)

Properties (1968)

Properties (1969)

Properties (1969)

Properties (1969)

Properties $(1969)$

Properties (1970)

Properties (1970)

Properties (1971)

Properties (1972)

Properties (1972)
ISO

ISO

Rec. F ISO

ISO

IEC

TTeel ISO

ISO

ISO

ISO

ISO

corded ISO

lof W IEC

ISO

IEC

IEC

IEC

IEC

IEC

IEC

IEC

IEC

IEC

IEC

IEC

IEC

IEC

IEC

IEC

IEC

IEC

IEC

IEC

IEC

IEC

IEC

ISO

ISO

ISO

ISO

ISO

IEC

IEC

IEC

IEC

IEC

IEC

ISO

ISO

IEC

ISO

/SI ISO

ISO

ISO

ISO

IEC

IEC

ISO

/Rolled Steel Sections-Part 2: Une ISO /T Rolled Steel Sections-Part I: E ISO IF Hot Rolled Steel Sections-Paral ISO /Lled Steel Sections-Parallel Flan ISO iOns of Hot Rolled Steel Sections- ISO /Sions of Hot Rolled Steel Sections ISO Types of Winding Wires: Heat or So IEC /Ec. Spec. for Particular Types of W IEC Rec. for Alumin ISO

Std. Fo ISO

178

357

358

26

24

1781 
minum) and Its Alloys (1971)

and Its Alloys (1968)

num) and Its Alloys (1968)

Rec. for Mechanical

Rec. for Mechanical

Rec. for Mechanical

cylindrical Radiation Probes Containing Geiger-Muller or rators (With Associated Prime Movers) and Motors, Electric Rec. for Power Sources for Portable Rec. or Power Supply for Air and Land Vehicle Mounted Rec. for Centre (Center) Drills for the Holes Witbout es: Construction and Test of Electrical Apparatus, Type of

70) Rec. for Guiding Principles for
nstruments: Constructional Requirements to Afford Personal Rec. for Rotating Electrical Machines: Degrees of ons for Work on Unse/ Controlgear (1963) Rec. for Fundamental Principles for Rec. for ${ }^{\circ}$ Of Rec. for Metal Spraying of Zinc and Aluminum for the Exposure Rate Meters and Monitors for Use in Radiological Electrical Installations in Ships: Switchgear, Electrical Rec. for Tests on Anticorrosion

Rec. for International Electrotechnical Vocabulary: Rec. for Calibration of Elastic

Rec. for Determination of Proof Stress and termination of Lower Yield and Proof Stresses (Stress) and rphones Used in Audiometry (1970) Rec. for Iec for Lifting Purposes. for Index of A Rec. for Crowns of pb (1965)

Rec. for Diameters of Rec. for Grooved

c. for Widths of Flat Transmission Belts and Corresponding $s$ for the Adjustment of Centres (Centers) for Transmission Rec. for Quality, Machining and Balancing of Transmission d Definitions Relating to Drives Using V-Belts and Grooved trial Speed Changers and Groove Profiles for Corresponding rials-Troughed Belt Conveyors (Other Than Portable) Belt

elt Conveyors-Basic Characteristics of Motorized Driving $\mathrm{mm}$ Film Magazines for Stil] Picture Use and Test for Film ec. for Pulps: Determination of Saleable Mass, in Lots, of Rec. for Determination of the Kappa Number of

Rec. for Rec. for Rec. for Rec. for Rec. for

Rec. for Rec. for Rec. for Rec. for Rec. for Baled in Sheet Form (1968) ne (1967)

Rec. for

lectronic Tubes and Valves: Meth. of Measurement of Vacuum tronic Tubes and Valves: Meth. of Measurement of Thyratron tion (1970)

Rec. for Cereals and Std. for Cereals Std. for Cereals, Rec. for

Rec. for Cereals and Rec. for

e Displacement Vacuum Pumps-Part I: Volume Rate of Flow vapour (Vapor) Vacuum Pumps-Part I: Volume Rate of Flow erformance Characteristics of Positive Displacement Vacuum f ihe Performance Characteristics of Vapour (Vapor) Vacuum or Inland Navigation-Covers for Deck Openings for $220 \mathrm{Mm}$ ternational Code for the Field Acceptance Tests of Storage d Glasses for Ships Side Scuttles and Fixed Lights by the

Representation of ISO 7 Bit Coded Character Set on 12 Row on Processing-Representation of 8 Bit Patterns on 12 Row ation Pr/ Rec. for Dimensions and Location of Rectangular ns and Location of Rectangular Punched Holes in 80 Columns Std. for Data Interchange on Rolled $U_{p}$ Rec. for Dimensions for

Machines: Coding of Preparatory Functions G An/ aight Cut Numerically Controlled Machines (1969/ straight Cut Numerically Controlled Machines (1/

Rec. for Rec. for Rec. for ight Cut Numerically Controlled/ Rec. for Interchangeable for Representation of 6 and 7 Bit Coded Character Sets on Gears (Information to Be Given to the Manufacturer by the 8 (1966) Gears (Information to Be Given to the Manufacturer by the Rec. for Significance to
Property Limits for Alu minium Alloy Forgings (1968)

Property Limits for Extruded Products of Aluminium (Alu

Property Limits for Rivet Stock of Aluminium (Aluminum)

Property Limits for Rolled Products of Aluminium (Alumi

Proportional Counter Tubes or Scintillation Detectors (

Propulsion Plant, Tankers (1965)

Prospecting Equipment for Radio Active Materials (1965)

Prospecting Equipment for Radio Active Materials (1967)

Protecting Chamfers-Type a (1968)

Protection E (1969) ITus for Explosive Gas Atmospher IEC

Protection Against Corrosion by Hot Dip Galvanizing (19 ISO

Protection Against Ionizing Radiation (1972) /Uclear I IEC

Protection by Enclosures (1968)

Protection in the Design and Construction of Installati

Protection of Enclosures for Low Voltage Switchgear and

Protection of Iron and Steel Against Corrosion (1971)

Protection (1972) /for Portable X-- o
Protection, Distribution and Controlgear (1965)

Protective Coverings of Metallic Cable Sheaths (1966)

Protective Helmets for Road Users (1970)

Protective Relays (1956)

Proving Devices (1964)

Proving Test for Steel at Elevated Temperatures (1961)

Proving Test (1968)

Publication (Standardization of Format) (1969)

Pulley Blocks and Other Lift Appliances (1971)

Pulleys for Flat Transmission Belts (1959)

Pulleys for Flat Transmission Belts (1959)

Pulleys for Narrow V-Belts: Groove Sections SPZ, SPA, S

ISO

ISO

ISO

ISO

Pulleys for V-Belts (1962)

Pulleys for V-Belts: Groove Sections A, B, C, D, E $(195$

Pulleys for V-Belts: Groove Sections $Y$ and $Z$ (1962)

Pulleys (1956)

Pulleys (1960)

Re ISO

Pulleys (1962)

Rec. for Limiting Value ISO

ISO

Rec. for Terms an ISO

Pulleys (1970)

Pulleys (1970)

Pulleys (1970)

Pullout Force (1969)

Pulp Baled in Sheet Form (1968)

Pulp (Deg. of Delignification) (1963)

Pulps-Determination of Ash (1970)
Pulps-Determination of Manganese (1970)

Pulps: Determination of Acid Insoluble Ash (1968)

Pulps: Determination of Alkali Resistance (1968)

Pulps: Determination of Alkali Solubility (1968)

Pulps: Determination of Copper (1968)

Pulps: Determination of Dry Matter Content (1967)

/Ec. for Endless Wide V-Belts for Indus ISO

andling Equipment for LoOse Bulk Mate ISO Rec. for Dimensions for 35 ISO

ISO

ISO

ISO

ISO

ISO

ISO

ISO

ISO

ISO

ISO

Pulps: Determination of Saleable Mass, in Lots, of Pulp

Pulps: Extraction of Materials Soluble in Dichlorometha

/Ectrical Properties of E IEC

Pulse Modulator Types (1970)

Pulse Modulators (1969)

ectrical Properties of Elec

Peth. of Test for Infestation by X-Ray Examina

Pulses-Sampling of Milled Products (1972)

Pulses and Derived Products: Determination of Ash (1972

Pulses Meth. of Test (1967)

Pulses: Determination of the Mass of 1000 Grains (1966)

Pulses: Sampling (1969)

(Pumping Speed) (1970)

/Performance Characteristics of
Pumps-Part I: Volume Rate of Flow (Pumping Speed) (19

Pumps-Part I: Volume Rate of Flow (Pumping Speed) (19

Pumps (1957)

Pumps (1968)

Punch Meth. (1967)

Punched Cards (Inform

Punched Cards (1971)

Punched Holes in 80 Columns Punched Paper Cards (Inform

Punched Paper Cards (Information Processing) (1971)

Punched Paper Tape-General Requirements (1972)

Punched Paper Tape for Data Interchange (1969)

Punched Tape Block Formats for the Numerical Control of

Punched Tape Fixed Block Format for Positioning and Str

Punched Tape Variable Block Format for Positioning and

Punched Tape Variable Block Format Positioning and Stra

Punched Tape (1969)

Purchaser in Order to Obtain the Gear Required) (1971)

Purchaser in Order to Obtain the Gear Required) (1971)

Purchasers of Marks Indicating Conformity with Standard

Pure Agglomerated-Deformation Under Constant Pressure
2320 
Std. for Cork-Expanded Std. for Cork-Expanded Rec. for Rec. for Rec. for of Rupture hy Bending (1971) 1965)

Rec. for Std. Reference Zero for the Calibration of c. for Normal Equal Loudness Contours for

ield Listeni/ Reld Listeni/ $\quad$ Rec
Density at $20^{\circ} \mathrm{C}(1972)$ Std. for Std. for Steel Wire Ropes for General

lectric Motor Operated Appliances for Domestic and Simila ic Cooking and Heating Appliances for Domestic and Similar ic Cooking and Heating Appliances for Domestic and Similar Rec. for Varnish Fabrics for Electrical

ic Cooking and Heating Appliances for Domestic and Similar ic Cooking and Heating Appliances for Domestic and Simila ses with High Breaking Capacity for Industrial and Similar Rec. for Transfer Cones for Dyeing

Rec. for Cones for Cross Winding for Dyein lectric Motor Operated Appliances for Domestic and Similar Rec. for Varnish Fabrics for Electrical ic Cooking and Heating Appliances for Domestic and Similar ic Cooking and Heating Appliances for Domestic and Similar lectric Motor Operated Appliances for Domestic and Similar lectric Motor Operated Appliances for Domestic and Similar ic Cooking and Heating Appliances for Domestic and Similar lectric Motor Operated Appliances for Domestic and Similar ic Cooking and Heating Appliances for Domestic and Similar lectric Motor Operated Appliances for Domestic and Similar g Meth. (1970)

Rec. for auxiliary Circuits, Including Contactor Relays) Section 1: Rec. Regarding the Colour (Color) of below $3 \mathrm{MHz}$ : Circular Multipole Connectors with Bayonet or $70 /$ Rec. for Performance Requirements for General Purpose 66) Rec. for Dimensions for General Purpose

hy-Sound Records and Scanning Area of $35 \mathrm{~mm}$ Double Width Rec. for Dimensions for General Purpose Rec. for Performance Requirements for General Purpose

70) tter (1970)

70) g Water) (1970) Rec. for Plastics

k Density (1969) ric Cables (1970) in Pairs, T/ ution Wires/ ution Wires/ nt Wires, T/ nt Wires An/ Test and $\mathrm{M} /$ ing Cables / ables and Wires (1969/ Rec, for Std. Colours (Colora) for holyvinyl Chlorid he Transport of Fluids-Unplasticized Polyvinyl Chloride he Tranaport of Fluids-Unplasticized Polyvinyl Chloride ea-S/ Std for Buried Unplasticized Polyvinyl Chloride . for Single Sockets for Unplasticized Polyvinyl Chloride uble Socket Fittings for Unplasticized Polyvinyl Chloride production of Aluminum: Determination of Absolute Density; r Plastics-Liquid Resins-Measurement of Density by the in Solution-Meth. Using Density Hydrometer-Meth. Uaing ols for Industrial Uae-Determination of Neutral Oils and c. for Vulcanized Rubber-Determination of Carbon Black International Rec. for Optical Rec. for Refractory Products Determination of Rec. for $n$ Content-1 Rec. for Sodium Tripolyphosphate and Sodium Rec. for Sodium Tripolyphosphate and Sodium

f Adhesion of Vulcanized Rubbers to Rigid Plates in Shear wires with Solid Conductors, Insulated, in Pairs, Triples, , Insulated, Polyamide Coated, in Singles, Pairs, Triples, chloride) Insulation and Sheath: Cables in Pairs, Triples, tection of Free Ammonia in Phenol Formaldehyde Mouldings Rec. for Porcelain Laboratory Apparatus onditiona for Films: Utilization of Rec. Patterns of Image Rec. for Radiographic Image Rec. for Aircraft Connection for Water of Drinkable imits a (1962)

its of Sizes for Commercial Bolt and Nut Threads: Medium

Rec. for Textiles-Binary Fibre (Fiber) Mixtures Rec. for Rec. for Rec. for
Pure Agglomerated-Determination of Bulk Density (1972 Pure Agglomerated Thermal Cork-Characteristics, Sampl Pure Expanded Corkboard-Determination of Moisture Con Pure Expanded Corkboard-Determination of the Modulus Pure Tone Audiometers for General Diagnostic Purposes ( Pure Tone Audiometers (1964)

Pure Tone Screening Audiometers (1965)

Pure Tones and Normal Threshold of Hearing Under Free F

Purified Glycerol for Industrial Use-Determination of

Purity Aluminium (Aluminum) Busbar Material (1958)

Purposes: Characteristics (1973)

Purposes: Clocks (1967)

Purposes: Clothes Dryers and Towel Dryers (1967)

Purposes: Cooking Ranges, Cooking Tables and Similar (1

Purposes: Definitions and General Requirements (1972)

Purposes: Electric Irons, Ironers and Pressing Machines

Purposes: for Heating Liquids (1966)

Purposes: General Requirements (1968)

Purposes: Half Angle at Top of Cone $4^{\circ} 20^{\prime}(196$

Purposes: Half Angle of the Cone $4^{\circ} 20^{\prime}$ (1963)

Purposes: Massage Appliances (1967)

Purposes: Meth. of Test (1972)

Purposes: Portable Immersion Heaters (1968)

Purposes: Room Heating and Similar (1968)

Purposes: Shavers, Hair Clippers and Similar Applianc

Purposes: Spin Extractors (1968)

Purposes: Stationary Noninstantaneol

Purposes: Vacuum Cleaners and Water Suction Cleaning A

Purposes: Waffle Irons, Grills, Warming Plates and Othe

Purposes: Washing Machines (1968)

Push Button Switches: General Requirements and Measuri

Push Buttons and Related Control Switches (1972)

Spec. for Electr Iow Voltage Fu

ISO

ISO

ISO

ISO

IEC

ISO

IEC

ISO

IEC

ISO

for E CEE

/Ectr CEE

CEE

IEC

CEE

IEC

ISO

ISO

Spec. for E CE

IEC

for Electr CEE I for Electr CEE

Push Buttons (1955)

Push Pull Coupling (1971)

/Connectors for

Push Pull Single Pole Circuit Breakers for Aircraft (19

Push Pull Single Pole Circuit Breakers for Aircraft (19

Push Pull Sound Prints Normal and Offset Centerline Typ

Push Pull 3 Pole Circuit Breakers for Aircraft (1969)

Push Pull 3 Pole Circuit Breakers for Aircraft (1970)

Pve Resins-Determination of Ash and Sulphated-Ash (I9

Pvc Resins-Determination of Impurities and Foreign Ma

Pvc Resins-Determination of $\mathrm{pH}$ of Aqueous Extract (19

Pvc Resins-Determination of Volatile Matter (Includin

Pvc Resins-Sieve Analysis in Water (1970)

Pvc Resins: Determination of the Compacted Apparent Bul

CEE

CEE

CEE

ec. for E CEE IEC and IEC IEC

IEC ISO ISO

Pve (Polyvinyl Chloride) Insulation and Sheath of Elec

Pvc (Polyvinyl Chloride) Insulation and Sheath: Cables

Pvc (Polyvinyl Chloride) Insulation and Sheath: Distrib

Pve (Polyvinyl Chloride) Insulation and Sheath: Distrib

Pvc (Polyvinyl Chloride) Insulation and Sheath: Equipme

Pvc (Polyvinyl Chloride) Insulation and Sheath: Equipme

Pve (Polyvinyl Chloride) Insulation and Sheath: General

Pvc (Polyvinyl Chloride) Insulation and Sheath: Signall

Pvc (Polyvinyl Chloride) Insulation for Low Frequency C

(Pve) Fittings with Plain Sockets; Metric Series (1968)

(Pve) for Use Under Pressure (1967)

Rec. for

(Pvc) Pipes-Tolerances on Outside Diameters (1970)

(Pvc) Pipes-Tolerances on Wall Thicknesses Up to $6 \mathrm{~mm}$

Pvc) Pipes for the Supply of Gaseous Fuels: Metric Seri

(Pve) Pressure Pipes with Elastic Sealing Ring Type Join

(Pve) Pressure Pipes with Elastic Sealing Ring Type Join

IT ISO

Pyknometer Meth. (1968)

Pyknometer (1970)

Pyknometer (1970) 10 xide Primarily Used for the ISO

Pyrolytic Meth. (1971)

/Etermination of Density of Samples ISO /Cresol, Cresylic Acid and Xylen ISO

Pyrometers with Disappearing Filaments (1970)

Pyrometric Cone Equivalent (Refractoriness) (I966)

Pyrometric Reference Cones (1969)

Pyrophosphate for Industrial Use-Determination of Iro

Re ISO

OIML

ISO

Pyrophosphate for Industrial Use-Determination of Los

ISO

ISO

Quadruple-Shear Test) (1971)

Quadruples and Quintuples (1968)

Quadruples and Quintuples (1971)

Rec. for Determination O ISO

/Heath: Distribution IEC

Quads and Quintuples for Inside Installations (1972) Conductors IEC

Qualitative Meth. (1961)

Quality and Meth. of Test (1970)

Quality Indicators (I973)

Rec, for Plastics-De ISO

ISO

Quality Indicators: Principles and Identification (1969

Quality (1965)

Quality (1969)

Quality, Machining and Balancing of Transmission Pulley

Quantitative Chemical Analysis (1971)

Quantities and Units of Acoustics (1965)

Quantities and Units of Electricity and Magnetism (1965

Quantities and Units of Heat (1960)

2189

2219

2077

177

389

178

2099

2408

10-2E

$11-2 \mathrm{~N}$

11-2A

394-1

11-2B

269-1

575

324

10-20

394-2

11-2C

$11-2 \mathrm{~F}$

$10-2 \mathrm{~K}$

11-2E

$10-2 \mathrm{~A}$

11-2M

$10-2 \mathrm{~J}$

$341-1$

$337-2$

$130-7$

I $130-7$

530

72

1033

1509

1270 
(1958)

r Physics (1973)

re and Time (1965)

perties of Vacuum and a Subs/ Rec. for Designation of the ecif/ $(1969)$ Rec. for Electrical Relays. Single Input Energizing (1969)

valves and Conditions: Section 2-Test Conditi/ to the Use of (1962) utlines (1962) Treated, Alloy, and Free Cutting Steels-Part 8: Wrought c. for Heat Treated Steels: Free Cutting-Part 7: Wrough Treated, Alloy, and Free Cutting Steels-Part 5: Wrough treated, Alloy, and Free Cutting Steels-Part 2: Wrought, for Heat Treated, Alloy, and Free Cutting Steels-Part 1: Treated, Alloy, and Frec Cutting Steels-Part 3: Wrought Treated, Alloy, and Free Cutting Steels-Part 6: Wrough Rec. for Hardenability Test by End Reports and Study

Silica Content, Gravimetric Meth. by Precipitation of the Silica Content; Gravimetric Meth. by Precipitation of the insulation and Sheath: Cables in Pairs, Triples, Quads and d Conductors, Insulated, in Pairs, Triples, Quadruples and lyamide Coated, in Singles, Pairs, Triples, Quadruples and Rec. for Radio Frequency Connectors: Rec. for Radio Frequency Connectors: Rec. for Radio Frequency Connectors: Rec. for Basic d Heavy Engineering (1968) Rec. for Basic es of Electronic Tubes and Valves: Meth. of Measurement of
Rec. for Rolling Bearings Diameter Series 7 (1970) Rec. for Rolling Bearings Rec. for Rolling Bearings:

ISO Identification Code for Roller Bearings: Groups-(I) r Rolling Bearings-Radial Internal Clearance in Unloaded e Ball Bearings with Cylindr/ Rec. for Rolling Bearings Rec. for Rolling Bearings-Double Row Selfaligning Rec. for Rolling Bearings: Cylindrical Type, . for Rolling Bearings-Double Row Self-Aligning Ball olerances-Normal and Toler/ Rec. for Rolling Bearings

it Packages Containing Photographic Materials Sensitive to

echnical Vocabulary: Detection and Measurement of Ionizing radiological Protection (/ dulations and Television Br/ Rec. Meth. of Measurement of onal Counter / Rec. for External Diameters of Cylindrical Rec. for Basic Ionizin

rical Measuring Apparatus Used in Connection with Ionizin $r$ Test Procedures for Semiconductor Detectors for Ionizing and Preamplifiers for Semiconductor Detectors for Ionizing equirements to Afford Personal Protection Against Ionizing ws: Metric Series (1968) Rec. for Bolts and Screws:

for Power Sources for Portable Prospecting Equipment for for Air and Land Vehicle Mounted Prospecting Equipment for

ctors for Frequencies Below $3 \mathrm{MHz}$ : Circular Connectors for

tor of Electrical Insulating Materials at Power, Audio and ing Meth. (1971) Spec. (1970)

Rec. for Rec. for Rec. for

Rec. for Characteristic Impedances and Dimensions of Rec for Dimensions of the Mating Parts of n Aerial Feeder Connector (1965) asuring Meth. (1965)

IEC 50-12 (1967)

IEC 50.17 and Larger (1970)

IEC 75-17 and Larger (1971)

balanced Aerial Feeders (1965) Rec. for Rec. for Rec. for Rec. for Rec. for Rec. for ef Rec. for Matters Relamits of

Spec. for Spec. for Spec. for

y Range $0.15 \mathrm{MHz}$ to $30 \mathrm{MHz}$ (1972) y Range $25 \mathrm{Mc} / \mathrm{S}$ to $300 \mathrm{Mc} / \mathrm{S}$ (1961) Other Than Quasi Peak (1967)

for Connectors for Frequent Rec. For Meth. of Measurement on ements Particularly Rel/

lized Frequency Measure

Rec. for Meth. of Measurement on cor Meth. of Measurement on Below $3 \mathrm{MHz}$ : Concentric Connectors for Audio Circuits in rs, Primary Cells and Accumulators: Aerials (Antennas) and wer of Nonessential Oscill/ Rec. Meth. of Measurement for
Quantities and Units of Mechanics (1960)

Quantities and Units of Periodic and Related Phenomena

Quantities and Units of Physical Chemistry and Molecula Quantities and Units of the SI (Metric Unit) and of Spa

Quantities and Units Used in Electricity (1964)

Quantities Characterizing the Magnetic and Electric Pro

Quantities from Tests (1967) Rec. for Rotating Elec

Quantity Measuring, with Nonspecified or Independent Sp

Quantity Packaging of Sensitized Photographic Materials

Quartz Crystal Units for Oscillators: Section 1-Std.

Quartz Crystal Units for Oscillators: Section 3-Guide

Quartz Crystal Units for Oscillators: Section 4: Std. O

Quartz Crystal Units (I970)

Spec. for Radio in CISPR
olybdenum Types ISO

Quasi Peak (1967)

Quenched and Tempered Chromium Nickel Molybdenum Types ISO
Quenched and Tempered Chromium Types (1970) Re ISO

Quenched and Tempered Manganese (1970) Rec. for Heat ISO

Quenched and Tempered Types with $1 \%$ Chromium and $0.2 \% \mathrm{M}$ ISO

Quenched and Tempered Unalloyed Types (1968) Rec. ISO

Quenched and Tempered Unalloyed with Controlled Sulphur ISO

Quenched and Tempered, with $3 \%$ Chromium and $0.5 \%$ Molybd ISO

Quenching Steel (Jominy Test) (1967)

Questions of the CISPR (1969)

Quinoline Silicomolybdic Complex (1969)

Quinoline Silicomolybdic Complex (1969)

Quintuples for Inside Installations (1972)

\section{Ermination of ISO \\ Ination of of ISO}

Chloride) IEC

Quintuples (1968)

/Ith Solid Cond
Ruintuples (1971) F. Coaxial, for Cables 96 IEC 50-12 (1967)

/Heath: Distribution Wires with Soli IEC

R. F. Coaxial, for Cables 96 IEC 50-17 and Larger (1970)

R. F. Coaxial, for Cables 96 IEC 75-17 and Larger (1971)

Rack of Cylindrical Gears for General Engineering (1957

Rack of Straight Bevel Gears for General Engineering an

Racks (For Nuclear Electronic Instruments) (1968)

Radar and Oscilloscope Cathode Ray Tubes (1969)

Radial-Boundary Dimensions-General Plan-Diameter

Radial-Boundary Dimensions-General Plan-Part II-

Radial-Tolerances (1966)

Radial Ball and Roller (II) Thrust (III) Tapered Type-

Radial Groove Type Ball Bearings with Cylindrical Bore

Radial Internal Clearance in Unloaded Radial Groove Typ

Radial Internal Clearance (1965)

Radial Internal Clearance (1969)

Radial Internal Clearance (1970)

Radial Types with Shields or Seals-Outside Diameter T

Radiant Energy (1968) / for Pictorial Marking of Trans ISO

Radiation by Electric Means (1968) /Rnational Electrot IEC

Radiation Exposure Rate Meters and Monitors for Use in

Radiation from Receivers for Amplitude and Frequency Mo

Radiation Probes Containing Geiger-Muller or Proporti

Radiation Symbol (1963)

Radiation (1964)

Radiation (1970)

Radiation (1970)

Radiation (1972)

Rec. for Index of Elect

IEC

Rec. Fo IEC

/ for Test Procedures for Amplifiers IEC

Radii Under the Head for General Purpose Bolts and Scre ISO

Radio Active Materials (1965)

Radio Active Materials (1967)

Radio and Associated Sound Equipment (1971) or Power Supply

Radio Frequencies Including Metre (Meter) Wavelengths (

Radio Frequency Cables: General Requirements and Measur

Radio Frequency Cables: Guide to the Design of Detailed

Radio Frequency Cables: Relevant Cable Spec. (1961)

Radio Frequency Coaxial Cables (1967)

Radio Frequency Connectors (1964)

Radio Frequency Connectors: Coaxial Unmatched Televisio

Radio Frequency Connectors: General Requirements and Me

Radio Frequency Connectors: R. F. Coaxial, for Cables 96

Radio Frequency Connectors: R. F. Coaxial, for Cables 96

Radio Frequency Connectors: R. F. Coaxial, for Cables 96

Radio Frequency Connectors: Two Pin Connector for Twin

Radio Frequency Output Power (1966)

/Ectrical Properti

Radio Interference and Its Suppression (1969)

Radio Interference and Report of National Limits (1967)

Radio Interference Measuring Apparatus for the Frequenc

Radio Interference Measuring Apparatus for the Frequenc

Radio Interference Measuring Apparatus Having Detectors

Radio Interference Suppression (1965)

Radio Receivers and Associated Sound Equipment (1965)

Radio Receivers for Various Classes of Emission: Genera

Radio Receivers for Various Classes of Emission: Measur

Radio Receivers for Various Classes of Emission: Specia

Radio Receivers (1969) / for Connectors for Frequencie

Radio Stations (1968)

IL Symbols: Machines, Transforme

Radio Transmitters: Bandwidth, Out of Band Power and Po

Radio Transmitters: General Conditions Of, Frequency, 0
31/III

31/VI

$31 / I$

164

206

255-3

425

122-1

122.2

$122-3$

314

683/VIII

683/VII

$683 / \mathrm{V}$

$683 / \mathrm{II}$

$683 / \mathrm{I}$

683/III

683/VI

642

8

996

189-2

$189-4$

189-7

$169-4$

169.5

$169-6$

53

677

297

151-14

$15 / I$

15/II

492

$300 / 1$

201

201

465

1038

1646

1648

884

$50(66)$

395

106

256

361

181

333

340

405

885

201

253
130.9

250

96-1

$96-0$
$96-2$

78

159

169.2

169.1

169-4

$169-5$

$169-6$

169-3

151-11

IEC

IEC

IEC

IEC

IEC

IEC

IEC

161 
era for Monochrome and $\mathrm{Col} /$ I)

Rec. Meth. of Measurement for Rec. Meth. of Measurement for Rec. for Safety Requirements for Rec. for Safety Requirements for ign and Construction of Installations for Work on Unsealed Rec. for International Electrotechnical Vocabulary:

Rec. for dentification (1969)

d Butt Joints in Steel Pipes Up to $50 \mathrm{Mm} /$

r Steel Plates Up to $50 \mathrm{~mm}$ (2 In.) Thick/

Rec. for

r Steel Plates 50 to $200 \mathrm{~mm}$ Thi/ utilization of Rec. Patterns of Image Quality I/ $\quad$ Std. for fication, Test Meth.) (1971)

Rec. for

r International Electrotechnical Vocabulary: Radiology and

mma Radiation Exposure Rate Meters and Monitors for Use in Rec. for International Electrotechnical Vocabulary: Std. for Centre Drills for Center Holes with mfer Dimension Limits and Maximum Shaft and Housing Fillet Rec. for Rules for Rotating Electrical Machines for chanicaI Handling Equipment for Unit Loads-Overhead Twin Rec. for Aircraft Seat Rec. for Rolling Bearings-For 9) Rec. for Rec. for Rec. for Rec. for Rec. for Rec. for Rec. for nters) in Nonalloy Steel for Tyred (Tired) Whee/ r Trailer Stock: Dimensions and Tolerances (196/ ler Stock (1969)

r Stock (1969)

stock (1969)

echnical Vocabulary: Signalling and Security Apparatus for ons in Millimetres (Millimeters) and In/ Rec. for Slotted efinitions and $\mathrm{Pa}$ / Rec. for Commercially Dry Virgin Cork, ion of Moistur/ Std. for Corkwood in Planks, Virgin Cork, 1972)

Std. for Corkwood in Planks, Virgin Cork, lectrical Properties of Receiving Aerials in the Frequency Rec. for Recommended t for Piezoelectric Vibrators Operating Over the Frequency al Plan and Selection for Screws, Bolts and Nuts (Diameter r Radio Interference Measuring Apparatus for the Frequency Rec. for Rivet Shank Diameters (Diameter r Radio Interference Measuring Apparatus for the Frequency Measuring Set Spec. for the Frequency r: Untrimmed Stock Sizes for the ISO-A Series, ISO Primary rimmed Stock Sizes for the ISO-A Series: ISO Supplementary ylenols for Industrial Use-Determination of Distillation Rec. for Test Meth. for Distillation (Yield and ruments (1970) Rec. for Analogue Voltage

rec. Meth. for Measuring Performance of Household Electric for Bonded Abrasive Products General Features Designation similar Electrical Appliances: Particular Requirements for ting Appliances for Domestic and Similar Purposes: Cooking Rec. for Rubber: Raw and Unvulcanized

al Analysis of Magnesium and Its Alloys-Determination of and Its Alloys-Determination of -Containing Zirconium, Rec. for Files and Std. for Spiral

hannels: Dilution Meth. for Steady Flow-Part 1: Constant tion (/ Rec. for Portable X.. or Gamma Radiation Exposure
iscometer (1968)
Rec. for Determination of iscometer (1968) s-Determination of the Water Vapour (Vapor) Transmission ics of Positive Displacement Vacuum Pumps-Part l: Volume cteristics of Vapour (Vapor) Vacuum Pumps-Part I: Volume lastics-Determination of the Maximum Temperature and the

Rec. for the Determination of Flammability and Burning stics-Determination of Water Vapour (Vapor) Transmission Rec for Plastics-Determination of the Melt Flow

Std. for Cutting Netting to Shape-Determination of the or Short Circuit Current Evaluation with Special Regard to Fuses (1957) Rec. for Counterbalanced Fork Lift Trucks. Rec. for the

d Flexible Cables and Cords with Circular Conductors and A d Flexible Cables and Cords with Circular Conductors and A king Temperature of $1 / \quad$ Rec. for Connecting Wires Having A Rec. for Rotating Electrical Machines: Rec. for Calculation of the Continuous Current Rec. for Rec. for
Rec. for ogous Semiconductor Devices (1961) Rec. for Industrial Tractors Rec. for ec. for High Voltage Alternating Current Circuit Breakers: Rec. for Rotary Wafer Switches (Low Current
Radio Transmitters: Measurements Particular to Transpos Radio Transmitters: Wanted and Unwanted Modulation (197

Radio Transmitting Equipment (1966)

Radio Transmitting Equipment: Test Meth. (1967)

Radioactive Materials (1970)

for Protection in the Des

Racommunications (1970)

Radiographic Image Quality Indicators: Principles and I

Radiographic lnspection of Circumferential Fusion Welde

Radiographic Inspection of Fusion Welded Butt Joints Fo

Radiographic Inspection of Fusion Welded Butt Joints Fo

Radiographic Terms Used in ISO Rec. Concerning Welding

Radiography of Welds and Viewing Conditions for Films:

Radioisotope All or Nothing Relays (Terminology, Classi

Radiological Physics (1964)

Radiological Protection (1972)

Radiology and Radiological Physics (1964)

Radius Form: Type R (1972)

Radius (1972)

/Bearings: Tolerances; Metric Series Cha

Rail and Road Vehicles (1971)

Rail Chain Conveyors (Power and Free) Safety Code (1971

Rails and Pins (1968)

Railway Axle Boxes: Acceptance Inspection (1970)

Railway Rolling Stock Material-Axles for Trailer (196

Railway Rolling Stock Material-Cast Wheel Centres (Ce

Railway Rolling Stock Material-Rolled on Forged Wheel

Railway Rolling Stock Material-Rough Tyres (Tires) Fo

Railway Rolling Stock Material-Tyres (Tires) for Trai

Railway Rolling Stock Material: Solid Wheels for Traile

Railway Rolling Stock Material: Wheel Sets for Trailer

Railways (1959)

Raised Countersunk (Oval) Head Tapping Screws-Dimensi ISO

Ramassage, Gleanings, Corkwood Refuse and Corkwaste-D

Ramassage, Gleanings, Refuse and Corkwaste-Determinat

Ramassage, Gleanings, Refuse and Corkwaste-Sampling (

Range Form $30 \mathrm{MHz}$ to $1,000 \mathrm{MHz}(1962)$ / of Essential E

Range of Outside Diameters for Milling Cutters (1966)

Range $0.15 \mathrm{MHz}$ to $30 \mathrm{MHz}(1972)$

Range 1 to $36 \mathrm{Mm}$ ) (1969)

Range $25 \mathrm{Mc} / \mathrm{S}$ to $300 \mathrm{Mc} / \mathrm{S}$ (1961)

Range $300 \mathrm{MHz}$ to $1,000 \mathrm{MHz}$ (1967)

Range (1966)

Range (1967)

Range (1971)

Range) (1969)

/ In. Screw Threads: Gener ISO
Spec. Fo CISPR

ISO

Spec. Fo CISPR

ISO

Rec. for Pape ISO

Rec. for Paper: Unt ISO

Rec. for Cresylic Acid and X ISO

Ranges and Logic Levels for Mains Operated Nuclear Inst

Ranges and Ovens (1971)

Ranges of Dimensions-Profiles (1966)

Ranges (1971)

Rec. for

Ranges, Cooking Tables and Similar (1968)

Rapid Plasticity Test (1971)

Rare Earths-Gravimetric Meth. (1972)

Rare Earths, Thorium and Silver-Periodate

Rasps: Lengths and Cross Sections (1961)

Ratchet Screwdriver Ends (1972)

Rate Injection Meth. (1966)

ISO

Rate Meters and Monitors for Use in Radiological Protec

Rate of Cure of Rubber Compounds by the Shearing Disk V ISO

Rate of Films and Thin Sheets-Dish Meth. (1970) /Tic ISO

Rate of Flow (Pumping Speed) (1970) IAnce Characterist ISO

Rate of Flow (Pumping Speed) (1970) (Performance Chara ISO

Rate of Increase During the Setting of Unsaturated Poly ISO

Rate of Plastics in the Form of Film (1970) ISO

$\begin{array}{ll}\text { Rate of Rigid Cellular Plastics (1970) Rec. for Pla ISO } & \text { R }\end{array}$

Rate of Thermoplastics (1969)

Rate (1972)

Rated Capacity of Circuit Breakers in Installations in

ISO

Rated Capacity (1971)

Rated Currents (2 to 63A) of Fuse Links for Low Voltage

Rated Impedances and Dimensions of Loudspeakers (1960)

Rated Voltage Not Exceeding 750 V (1967)

Rated Voltage Not Exceeding 750 V (1967)

/Ber Insulate

Rated Voltage of $20 \mathrm{kV}$ and $25 \mathrm{kV}$ d.c. and a Maximum Wor

Rating and Performance (1969)

Rating of Cables (100\% Load Factor) (1971)

Rating of Manual Arc Welding Equipment (1968)

Rating of Resistance Welding Equipment (1968)

Rating of Sound Insulation for Dwellings (1968)

Rating Room Air Conditioners (1968)

Rating Systems for Electronic Tubes and Valves and Anal

Rating (1969)

Rating (1971)

Rating) with 2 Hole Mounting (1963)
244-5

244.3

215.2

1710

$50(60)$

406

1027

947

1106

24.05

947/A

2504

346

$50(65)$

395

$50(65)$

2541

582

349

2150

837

1160

1005/III

$1005 / \mathrm{V}$

1005/IV

1005/II

$1005 / I$

$1005 / V I$

1005/VII

$50(31)$

1483

1215

2386

2385

138

523

302

263

2

478

593

1906

918
323

350

525

335-6

$11.2 \mathrm{~A}$

2007

2355

2353

234

2352

555

395

667

1195

1607

1608

584

1326

1663

1133

2075

363

1214

88

124

245

227

246

34.1

287

700

669

717

859

134

1084

56-2

132-3 
ling; Maximum 26 Positions and Diameter $60 \mathrm{~mm}$ (low Current Rec. for Rotary Wafer Switches (low Current Rec. for Rotary Wafer Switches (Low Current d General Principles of Measuring Met/ d General Principles of Measuring Met/ d General Principles of Measuring Met/ d General Principles of Measuring Met/
d General Principles of Measuring Met/ Rec. for Essential Rec. for Essential Rec. for Essential Rec. for Essential ype Machines (196/

Numbers 355 to 1000 (197) Rec. for Rotating Electrical Machines: rs 56 to 400 and Flange N/

Rec. for Dimensions and Output Rec. for Dimensions and Rec. for Std. Current

Ball and Roller Bearings: Meth. of Evaluating Static Load c. for Rolling Bearings-Meth. of Evaluating Dynamic Load plastics-Determination of Viscosity Number and Viscosity Rec. for Plastics-Determination of the Viscosity namorphotic Mot/ Rec. for Cinematography-Maximum Aspect oincident Current Matrix Stores Having a Nominal Selection urface Pile Density and Measured Pile Fibre (Fiber) Volume Rec. for Rubber: Rec. for Meth. of Sampling Rec. for Sampling Rec. for
Rec. for rogen (1971) Rec. for Determination of Ash in Rec. for Determination of Volatile Matter in Rec. for Sampling of Sid. for Rec. for Sampling of

Std. for Cinematography $-8 \mathrm{~mm}$ Type $\mathrm{S}$ Motion Picture utting and Perforating Dimensions for $35 \mathrm{~mm}$ Motion Picture and Perforating Dimensions for Double $-8 \mathrm{~mm}$ Motion Picture

Rec. for Rec. for Rec. for 1971) e (1971)

Rec. for

rec. for Electronic Tubes: Meth. of Measurement of Cathode Rec. for Expression of the Properties of Cathode ignation of Electrostatic Deflecting Electrodes of Cathode es: Meth. of Measurement of Radar and Oscilloscope Cathode Rec. for Var Hour Rec. for General Characteristics of Nuclear Rec. for General Principles of Nuclear

Rec. for Rules for Traction Transformers and Rec. for Sizes of Photocopies (On Paper) icrocopy: Measurement of the Screen Luminance of Microfilm Rec. for Essential Characteristics of $35 \mathrm{~mm}$ Microfilm f the ISO Micromire (ISO Micro Test Object) for Checking A sory Electrometers (1971) Rec. for Indirect Rec. for Magnetic Compasses, Binnacles and Azimuth Rec. for Dial Gauges Rec. for Direct morse Taper Shanks (1972) Std. for Finishing ive and Arbors for Shell Reamers (1972) Rec. for Machine Chucking Rec. for Special Tolerances for Sid. for Machine Bridge re (1:30 (Included) ) with Slot Drive and Arbors for Shell Rec. for Hand and Long Fluted Machine 69)

izes and Mounting Dimensions of Aircraft Instrument Cases

c Tests (1969)

ctronic Equipment (1959)

Rec. for

ents Particularly Related to the Audio Frequency Part of A nnectors for Frequencies Below $3 \mathrm{MHz}$ : Connectors for Radio elevision $\mathrm{Br} / \quad$ Rec. Meth. of Measurement of Radiation from ons (1954)

ons (1958)

Rec. Meth. of Measurement on

Rec. Meth. of Measurement on

itions and Measur/

Particularly Rel/

frequency Measure/

Rec. for Meth. of Measurement on Radio

Rec. for Meth. of Measurement on Radio

Rec. for Meth. of Measurement on Radio wire with Flame Retarding Insulation for Use in Television um Working Temperature of $105^{\circ} \mathrm{C}$ for Use in Television w $3 \mathrm{MHz}$ : Concentric Connectors for Audio Circuits in Radio onditions and Measuring Meth. Applying to Several Types of meth. of Measurement of Essential Electrical Properties of $\begin{array}{ll}\text { t Meth. (1971/ } & \text { Rec. for Sealed Nickel Cadmium Cylindrical } \\ & \text { Rec. for Sealed Nickel Cadmium Cylindrical }\end{array}$ Ik Materials-Vibrating Feeders and Conveyors, Shaking or oose Bulk Materials-Oscillating Conveyors and Shaking or oose Bulk Materials-Oscillating Conveyors and Shaking on of Right and Left Hand Single Bank Engines (197/ Std. for
Rating) (1966)

/Rotary Wafer Switches with 2 Hole Moun lEC

Rating): General Requirements and Measuring Meth. (1962 Rating): W afer Switches with Central Mounting (1963)

Ratings and Characteristics of Semiconductor Devices an

Ratings and Characteristics of Semiconductor Devices an

Ratings and Characteristics of Semiconductor Devices an

Ratings and Characteristics of Semiconductor Devices an

Ratings and Characteristics of 3 Phase, $50 \mathrm{~Hz}$ Turbine $T$

Ratings for Foot Mounted Electrical Machines with Frame

Ratings for Rotating Electrical Machines .. Frame Numbe

Ratings of Freight Containers (1970)

Ratings (1938)

Ratings (1958)

Ratings (1962)

Ratio of Cellulose Acetate in Dilute Solution (1970)

Ratio of of Polyamides in Concentrated Solution (1967)

Ratio of Projector Aperture for Projection of $35 \mathrm{Mm}$ Nona

Ratio of 2 : 1 (1969)

lor Carpets-Determination of Measured S

Raw and Unvulcanized-Rapid Plasticity Test (1971)

Raw Cotton for Test. (1961)

Raw Materials for Paints and Varnishes (1968)

Raw NaturaI Rubber and Its Latex-Determination of Nit

Raw Natural Rubber Determination of Dirt (1971)

Raw Natural Rubber (1962)

Raw Natural Rubber (1962)

Raw Natural Rubber (1962)

Raw Rubber-Sample Preparation (1972)

Raw Rubber in Bales (1971)

Raw Stock Film-Cutting and Perforating Dimensions (19

Raw Stock Film (1966)

Raw Stock Film (1966)

Raw Tung Oil (1962)

Raw, Refined and Boiled Linseed Oil (1960)

Rec. for C. ISO

Raw-Rubber and Rubber Latex-Determination of Copper (

Raw-Rubber and Rubber Latex-Determination of Manganes

Ray Charge Storage Types (1971)

Ray Oscilloscopes (1971)

Ray Tubes (1967)

Ray Tubes (1969)

Reactive Energy) Meters (1963)

Reactor Instrumentation (1966)

Reactor Instrumentation (1967)

Reactors (1968)

Reactors (1969)

Readable Without Optical Devices (1960)

Readers (1968)

Reading Apparatus (1965)

Reading Apparatus (1968)

Reading Capacitor Type Pocket Exposure Meters and Acces

lEC

IEC

IEC

IEC

IEC

IEC

IEC

ISO

IEC

Re ISO

ISO

ISO

IEC

ISO

ISO

ISO

ISO

ISO

ISO

ISO

ISO

ISO

ISO

ISO

Reading Devices, Class B-General Requirements (1967)

Reading in $0.01 \mathrm{Mm}, 0.001 \mathrm{In}$. and $0.0001 \mathrm{In}$. (1965)

Reading Pocket Type Electroscope Exposure Meters (1971)

Reamers for Morse and Metric Tapers, with Parallel and

Reamers with Parallel or Morse Taper Shanks (1966)

Reamers with Taper Bore (1:30 (Included)) with Slot Dr

Reamers (1966)

Reamers (1972)

Reamers (1972)

Reamers, Morse Taper Shank (1961)

Rear Mounting Type) (1959)

Rec. for Rolling Bearings-Tolerances-Definitions (19
Rec. for Stability of Counterbalanced Lift Trucks-Bas

Rec. for Stability of Counterbalanced Lift Trucks-Basi
Rec. for Transfusion Equipment for Medical Use (1969)

Receiver Type Metallized Mica Capacitors for Use in Ele

Receivers and Associated Sound Equipment (1965)

\section{ISO}

ISO

IEC

IEC

Receivers for Frequency Modulation Broadcast Transmiss

Receivers for Television Broadcast Transmissions (1960)

Receivers for Various Classes of Emission: General Cond

Receivers for Various Classes of Emission: Measurements

Receivers for Various Classes of Emission: Specialized

Receivers (1965)

Receivers (1967)

Receivers (1969)

Receivers (1971)

of $20 \mathrm{kV}$ and $25 \mathrm{kV}$ donnecting IEC

/ for Connectors for Frequencies Belo IEC

/Rious Classes of Emission: General C IEC

Recriving Aerials in the Frequen Range Form $30 \mathrm{MHz}$ to

Rechargeable Single Cells: Dimensions (1972)

Rechargeable Single Cells: General Requirements and Tes

Reciprocating Feeders and Oscillating Conveyors-Safet

Reciprocating Feeders with Rectangular or Trapezoidal T

Reciprocating Feeders with Tubular Trough (1971)

Reciprocating Internal Combustion Engines-Definition

$132-5$

$132-1$

$132-2$

$147-0$

147.1

147.2

$147-3$

34-3

$72 A$

668

76

281

1157

600

358

281

1959

2007

220

42

249
247

248

250

1796

1795

1700

491

486

277

150

1654

655

$151-24$
351

236

151.14

145

232

231

289

310

169

782

452

689

1759

613

463

1758

2250

521

2402

522

2238

2402

236

103

1132

1074

1135

116

$315-2$

130-2

106

69

91

107

315.1 

grinding Machines with Vertical Grinding Wheel Spindle and ure Calibration of 1 In. Std. Condenser Microphones by the ssure Calibration of One Inch Condenser Microphones by the m Perforated Magnetic/ Rec. for Location and Width of the Rec. for Print Spec. for Magnetic Ink Character Rec. for Alphanumeric Character Sets for Optical Rec. for Printing Spec. for Optical Character Rec. for Cinematography-Photographic Sound Magnetic Sound Records on $35 \mathrm{~mm}$ Film and 1 Magnetic Sound Rec. for Cinematography-Photographic Sound

re Film (1956)

re Film (1956)

$\begin{array}{ll}\text { re Film (1956) } & \text { Rec. for Emulsion and Sound } \\ \text { cture Film (1956) } & \text { Rec. for Emulsion and Sound }\end{array}$

and Reproducing Magnetic Heads for Magnetic Sound mm Perforated Film (1971)

Rec. for Cinematography Rec. for Transverse Track

c. for Meth. of Measurement of Speed Fluctuations in Sound ture-Location and Width of Magnetic.Striping and Gaps of cteristics (1968) Rec. for Magnetic Tape

and Vacuum Gauges, with Pressure Responsive Elements and cessories (1968)

ed Magnetic Film (196) Rec. for Direct m Film (1963)

Rec. for Location and Width of the Rec. for Cinematography-Location of $5 \mathrm{~mm}$ Film-Location and Dimensions (1971)

Rec. for Location of Rec. for
nstruments of Progressive Profile Transformation-Profile acuum Gauges, with Pressure Respo/ Rec. for Meth. of Milk International Rec. for Rec. for Processed Disk Il Sound Prints Normal A/ Rec. for Cinematography-Sound $n$ and Width of the Recoding Head for Centre (Center) Sound or Location and Width of the Recording Head for Edge Sound Rec. for Recording Heads for 6 Track Magnetic Sound for Location of Recording Heads for Three Magnetic Sound aphy-Location of Recording Heads for Four Magnetic Sound inematography-Recorded Characteristic for Magnetic Sound overed Electrodes-Determination of the Efficiency, Metal n by Mea/ Std. for Textile Fabrics-Determination of the $f$ a Horizontally Folded Specimen by Measuring the AngIe of ons of Winding Wires: Dimension of Conductors of Insulated acturing Characteristics of Solid Wood Parquet Strips with ontacts/ Rec. for Connectors for Frequencies Below $3 \mathrm{MHz}$ : 966) Rec. for Connectors for Frequencies Below $3 \mathrm{MHz}$ : ding Keyways (Dimensions in Millimetres) (Milli/ Rec. for loose Bulk Materials: Vibrating Conveyors and Feeders with lating Conveyors and Shaking or Reciprocating Feeders with ards (Information $\mathrm{Pr}$ ) Rec. for Dimensions and Location of in Such a Way That the Transvers/ Rec. for Calculation of c. for Hollow Metallic Waveguides: Relevant Spec. for Flat or Hollow Metallic Waveguides: Relevant Spec. for Ordinary hollow Metallic Waveguides: Relevant Spec. for Medium Flat

langes for Waveguides: Relevant Spec. for Flanges for Flat es for Waveguides: Relevant Spec. for Flanges for Ordinary curacy Class) (1972) International Rec. for hollow Metallic Waveguides: Relevant Spec. for Medium Flat Rec. for Monocrystalline Semiconductor Rec, for Polycristalline Semiconductor les of Resistors, Elements of Electronic Tubes, Valves and Ships: Transformers for Power and Lighting, Semiconductor Rec. for Characteristics of Electric Infra

Rec. for Cold Reduced Tinplate and Cold

for Surface Active Agents in Powder Form: Preparation of A nation of Silica Content-Spectrophotometric Meth. as the ion of Silica Content-Spectrophotometric Meth. Using the termination of Silicon-Spectrophotometric Meth. with the sheet (1969) Rec for Cold

Rec for Low Voltage Motor Starters: ric Acid for Industrial Use: Determination of Oxidizing or 1968) Taper Shanks (1961) Rec. for Oleaginous Seeds: Rec. for

Rec. for Meta Rec for Metal Rec. for Pitch Bound tion Applications (1971) (1968) Rec. for Precision Rec. for Dimensions of Projection Rec for Paper: Internal Diameters of Cores of Rec. for $35 \mathrm{~mm}$ and $16 \mathrm{~mm}$ Microfilms, Spools and magnetic Tape Used in / Rec. for General Purpose Hubs and Rec. for Std. Atmospheres for Conditioning And/or Test.
Reciprocating Internal Combustion Engines-Hand Operat

Reciprocating Table-Test. of Accuracy (1971)

Ace

/Rrace ISO

Reciprocity Technique (1971)

Reciprocity Technique (1972)

/Simplified Meth. for Pre IEC
/ 1972$)$

Recoding Head for Centre (Center) Sound Records on $16 \mathrm{M}$

Recognition (1969)

Recognition (1969)

Recognition (1971)

Record on $16 \mathrm{~mm}$ Prints (1958)

Record on $17.5 \mathrm{~mm}$ Film (1960)

Record on $35 \mathrm{~mm}$ Prints (1958)

ISO

ISO

ISO

ISO

/Cording Heads for Three ISO

ISO

Record Positions in Camera for $16 \mathrm{~mm}$ Sound Motion Pictu

Record Positions in Camera for $35 \mathrm{~mm}$ Sound Motion Pictu

ISO

Record Positions in Projector for $35 \mathrm{~mm}$ Sound Motion Pi

ISO

Recorded Characteristic for Magnetic Sound Records on 3 ISO

Recorders (1972)

Recording and Reproducing Equipment (1972)

Recording and Reproducing Magnetic Heads for Magnetic S

IEC

Recording and Reproducing Systems: Dimensions and Chara

Recording Directly by Means of Stylus and Diagram (Work

Recording Electrical Measuring Instruments and Their Ac

Recording Head for Edge Sound Records on $16 \mathrm{~mm}$ Perforat

Recording Heads for Four Magnetic Sound Records on $35 \mathrm{M}$

Recording Heads for Three Magnetic Sound Records on 35

Recording Heads for 6 Track Magnetic Sound Records on 3

Re IEC

ISO

IEC

OIML

IEC

ISO

ISO

ISO

ISO

Recording Instruments (1970)

/Th.-Contact (Stylus) I ISO

Recording of Cows (1970)

Recording Pressure, Combined Pressure and Vacuum, and $\mathrm{V}$

Records and Reproducing Equipment (1964)

Records and Scanning Area of $35 \mathrm{~mm}$ Double Width Push Pu ISO

Records on $16 \mathrm{~mm}$ Perforated Magnetic Film (1968) /Atio ISO

Records on $16 \mathrm{~mm}$ Perforated Magnetic Film (1968)

Records on $35 \mathrm{~mm}$ Film-Location and Dimensions (1971)

C. F ISO

ISO

Records on $35 \mathrm{~mm}$ Film and 1 Magnetic Sound Record on 17 ISO

Records on $35 \mathrm{~mm}$ Film (1963)

Records on $35 \mathrm{~mm}$ Perforated Film (1971)

Rec. for Cinematogr ISO

Rec. for C ISO

Recovery and Deposition Coefficient (1972) Std for C ISO

Recovery from Creasing of a Horizontally Folded Specime ISO

Recovery (1972) /Ation of the Recovery from Creasing O ISO

Rectangular Copper Winding Wire (1972) / Basic Dimensi IEC

Rectangular Face (1969) Rec. for General Manuf ISO

Rectangular Miniature Multipole Connectors with Blade C IEC

Rectangular Multipole Connectors with Blade Contacts ( 1 IEC

Rectangular or Square Parallel Keys and Their Correspon ISO

Rectangular or Trapezoidal Trough (1969) /Uipment for ISO

Rectangular or Trapezoidal Trough (1970) /Ials-Oscil ISO

$\begin{array}{ll}\text { Rectangular Punched Holes in } 80 \text { Columns Punched Paper C } & \text { ISO } \\ \text { Rectangular Refractory Bricks (1966) } & \text { ISO }\end{array}$

Rectangular Symmetrical Fillet Welds Statically Loaded

Rectangular Types (1964)

Rectangular Types (1964)

Rectangular Types (1967)

Rectangular Types (1968)

Rectangular Types (1968)

Rectangular Types (1968) for Flang IEC
Rectangular Weights from 5 to 50 Kilogrammes (Medium Ac
OIM

Rectangular (1968)

Rectifier Cells, Stacks, Assemblies and Equipment (1963 Rec. Ior IEC

Rectifier Stacks and Equipments (1960)

Rectifiers (1964)

mals: Variabity, Examp

Rectiers, Generators (With Associated Prime Movers) a

Red Emitters for Heating Purposes (1967)

Red Lead (1966)

Reduced Blackplate-Part 1: Sheet (1969)

Reduced Sample (1967)

Reduced Silicomolybdate Complex (1970)

Reduced Silicomolybdic Complex (1970)

Reduced Silicomolybdic Complex (1971)

Reduced Silicomolybdic Complex (1972)

Reduced Tinplate and Cold Reduced

Reduced, a.c.: Star Delta (1970)

Reducing Substances Volumetric Meth. (1968)

ISO

Re IEC

Rec. F IEC

Rec. for IEC

Reduction of Contract Samples to Samples for Analysis ( ISO

Reeds with Double Spring Baulk (1967)

Reeds with Plate Baulk (1967)

Reeds (1967)

Reels for Magnetic Tape Used in Interchange Instrumenta

Reels for $8 \mathrm{~mm}$ Motion Picture Film (Other Than Type S)

Reels (1966)

Reels (1969)

Reels, with $76 \mathrm{~mm}$ (3 In.) Centrehole (Centerhole), for

Nium-Dec. ISO

(L)-Determinat ISO

IIts Alloys-De ISO

Use: Determinat ISO

Reference Atmosphere Spec. (1967) 

national Rec. for Verification and Calibration of Hardness national Rec. for Verification and Calibration of Hardness national Rec. for Verification and Calibration of Hardness national Rec. for Verification and Calibration of Hardness Rec. for Pyrometric

in Audiometry (1970) Rec. for IEC Provisiona sing Earphones Coupled to the Ear by Means / Rec. for Iec dimensions (1970) Rec. for Modular Coordination nductor Devices and General Principles of Measuring Meth. Cereal Products: Determination of Moisture Content (Basic Rec. for Milk-Determination of Fat Content Rec for Dried Milk-Determination of Fat Content en Coffee Beans-Determination of Moisture Content (Basic and Its Processed Products-Determination of Fat Content d Sweetened Condensed Milk-Determination of Fat Content Rec. for Butter-Determination of Salt Content for Butter-Determination of the Acid Value of the Fat butter-Determination of the Refractive Index of the Fat Std. for Whey Cheese: Determination of Fat Content Std. for Cream: Determination of Fat Conten Rec. for Std. Std. for Magnesium Alloy Sand Castings Std. for Aluminium (Aluminum) Alloy Chill Castings Std. for Aluminium (Aluminum) Alloy Sand Castings: zinc Alloy Castings and Mechanical Properties of Sand Cas ters (1964) Rec. for Sid. Rec. for Bibliographica Rec. for Bibliographica

hapes (1971) Rec. for Raw Rec. for Fire Rec. for Fire

rec. for Fire Refined High Conductivity Tough Pitch Coppe Rec. for Fire Refined Tough Pitch Coppe Rec. for Phosphorus Deoxidized Copper

cal Analysis of Copper and Its Alloys-Sampling of Copper Std for Electrolytic Tough Pitch Copper for Comparison Viewing of Photographic Transparencies with Std. for Glucose Syrup: Determination of Dry Matter Rec. for Determination of the Rec. for Butter-Determination of the Rec. for Plastics-Determination of the Rec. for Vocabulary for the emperatu/ Rec. for Refractory Products-Determination of

tory Products Determination of Pyrometric Cone Equivalent Rec. for Dimensions of
Rec. for Dimensions of Rectangular ped Materials (Dense and Insulating) (1971) Rec. for cesses (1972) uivalent (Ref ractoriness) (1966)

Rec. for peratu/

Std. for Dense Shaped Rec. for

Rec. for Classification of Dense Rec. for Test. of

d Round Copper, with a Temperature lndex of 180 for Use in d Round Copper, with High Mechanical Properties for Use in Rec. for Number Designation of Organi tion of Linear Dimensions, Areas And/ Rec. for Commercial Rec. for Rec. for Test. of Rec. for Units and Symbols for tments for the Storage of Frozen Food/ Rec. for Household
Rec. for Household tments for the Storage of Frozen Food/ Rec. for Household
Rec. for Household Rec. for Aircraft Pressure for Corkwood in Planks, Virgin Cork, Ramassage, Gleanings nal Electrotechnical Vocabulary: Automatic Controlling and

Vocabulary: Switchboards and Apparatus for Connection and d Processes (1972) International Rec. for Metrologica Spec. for Welding: Rec. for Electric Fans and Rec. for Safety Requirements for Electric Fans and r Common Names for Pest Control Chemicals and Plant Growth $s$ for Pest Control Chemicals (Pesticides) and Plant Growth Rec. for a Electric Fans and s-Determination of the Loss on Ignition of Textile Glass Rec. for Aluminium (Aluminum) Conductors, Steel Rec. for Plastics-Preparation of Glass Fibre (Fiber) Std. for Wire Rec. for Heald Frames: Coordinated Dimensions in Rec. for Heald Frames: Coordinated Dimensions in
Rec. for Determination of the True and Apparent
Reference Block for the Calibration of Equipment for UI Reference Blocks Brinell (1970)

Reference Blocks Rockwell B (1970)

Reference Blocks Rockwell C (1970)

Reference Blocks Vickers (1970)

Reference Cones (1969)

Reference Coupler for the Calibration of Earphones Used

Reference Coupler for the Measurement of Hearing Aids U

Reference Lines of Horizontal Controlling Coordinating

Stics of Semico

Reference Meth. of Measurement (1970)

Reference Meth.) (1968)

(Reference Meth.) (1970)

Rec. for Cereals and

(1970)

Reference Meth.) (1970)

(Reference Meth.) (1970)

(Reference Meth.) (1970)

(Reference Meth.) (1971)

Rec for Gre ISO

Rec. for Cheese ISO

Rec. for Evaporated Milk an ISO

ISO

(Reference Meth.) (1971)

Rec ISO

Rec. for ISO

ISO

(Reference Meth.) (1972)

Reference Temperature for Industrial Length Measurement

ISO

ISO

Reference Test Bar (1972)

Reference Test Bar (1972)

Reference Test Bar (1972)

ISO

ISO

Reference Test Bars (1971)

Sium Aluminium (Aluminum) ISO

Reference Zero for the Calibration of Pure Tone Audiome

Reference, and Definitions (1967)

References (1968)

References: Essential and Supplementary Elements (1968)

References: Essential Elements (1958)

Refined and Boiled Linseed Oil (1960)

Refined High Conductivity Tough Pitch Copper Refinery S

Refined Tough Pitch Copper Refinery Shapes (1971)

Refinery Shapes (1971)

Refinery Shapes (1971)

Refinery Shapes (1971)

Refinery Shapes (1971)

Refinery Shapes (1972)

Reflection Colour Prints (1972)

Refractive Index Meth. (1973)

Refractive Index of Essential Oils (1962)

Refractive Index of the Fat (Reference Meth.) (1971)

Refractive Index of Transparent Plastics (1966)

Refractories Industry (1968)

Refractoriness Under Load (Differential-With Rising T Refractoriness) (1966)

Refractory Arch Bricks (1969)

Refractory Bricks (1966)

Refractory Materials-Classification of Prepared Unsha

Refractory Products-Classification (1972)

Refractory Products-Determination of Refractoriness U

Refractory Products-Nomenclature of Manufacturing Pro

Refractory Products (1969)

Refrigerant Compressors (1968)

Refrigerant Systems (1972)

Refrigerant Systems (1972)

lof Winding Wires: Enamelle

Refrigerants (1968)

Refrigerated Cabinets-Meth. of Test-Part I: Calcula

Refrigerating Plants-Safety Requirements (1971)

Refrigerating Systems (1968)

Ref rigeration (1968)

Refrigerators-Part II: Special Low Temperature Compar

Refrigerators-Part I: Performance Requirements (1968)

Refrigerators and Food Freezers for Household and Simil

Refueling Connections (1957)

Refuse and Corkwaste-Definitions and Packaging (1970)

Refuse and Corkwaste-Determination of Moisture Conten

Refuse and Corkwaste-Sampling (1972)

Std. ISO

Regulating Systems (1966)

Rec for Internatio

Regulation (1957)

Rec. for Internatio
for International Electrotechnical

Regulations for Nonautomatic Weighing Machines (1972)

Regulators for Gas Cylinders Used in Cutting and Relate

Regulators for Use in Ships (1969)

Regulators (1971)

Regulators (1971)

Regulators (1971)

Regulators (1972)

Reinforced Plastics (1970)

Reinforced (1966)

Reinforced (1966)

Reinforced, Resin Bonded, Low Pressure Laminated Plates

Reinforced, Rubber Covered Hydraulic Hose (1972)

Relationship with Pitch of the Harness (1967)

Es for the Selection of Common Rec. Fo

Rec. Fo ISO

Relative Densities (Density) and the Porosity of Coke (

ISO

ISO Rec. for Conditioning Atmosphere: Test,
rec. for Abbreviations of Typical Words in Bibliographical Std. for Shaped Insulating d. for Safety Requirements for the Electrical Equipment of mmercially Dry Virgin Cork, Ramassage, Gleanings, Corkwood Rec. for Aluminium (Aluminum) Alloy Conductors, Steel

Refractory Products Determination of Pyrometric Cone Eq

\section{1}

SO 389

ISO $\quad 558$

632

ISO $\quad 150$

ISO 1428

ISO $\quad 1428$

ISO $\quad 1429$

ISO $\quad 1430$

ISO 1811

431

2243

1743

280

1739

489

836

1893

528

1145

475

1927

2245

893

2246

528

1109

917

$317-10$

317.9

817

1992

1662

916

786

825

824 
Rec. for
urpomes (1968) Rec. for Plastics-Meth. for Maintaining Constan rec. for Test Enclosures of Noninjection Type for Constant n by Surface Dischar/ Rec. Test Meth. for Determining the for Symbols for Flight Dynamics-Part I: Aircraft Motion mics-Part II: Motions of the Aircraft and the Atmosphere Rec. for Determination of the Density and Rec. for Plastics-Meth. for Determining the Density and Rec. for Radioisotope All or Nothing for International Electrotechnical Vocabulary: Protective nical Controls, Starters and Elements of Electromechanical

Rec. for Electrical Relays: Instantaneous All or Nothing es for Control and Auxiliary Circuits, Including Contactor es for Control and Auxiliary Circuits, Including Contactor h Nonspecified or Independent Specif/ $\quad$ Rec. for Electrical Rec for Electrical Rec. for Radio Frequency Cables: Rec. for Hollow Metallic Waveguides: Rec. for Flanges for Waveguides:

1968)

es (1968)

Rec. for Flanges for Waveguides: Rec. for Hollow Metallic Waveguides: Rec. for Hollow Metallic Waveguides: Rec. for Hollow Metallic Waveguides: Rec. for Hollow Metallic Waveguides: Rec. for Preliminary 970) Rec. for the Presentation of or Preliminary List of Basic Terms and Definitions for the Rec. for Managerial Aspects of m Field Performance/ Rec. for Guide for the Collection of fication and Composition of Unalloyed Aluminium Ingots for Rec. for Application of Carbides for Machining by Chip ty Dimensions (1971) Rec. for Aircraft Pressure Rec. for Liquid Oxygen Rec. for Gaseous Oxygen

Limits of Radio Interference and

or the Production of Aluminum: Measurement of the Angle of Rec. for Processed Disk Records and

f Measurement of Speed Fluctuations in Sound Recording and $n$ and Width of Magnetic.Striping and Gaps of Recording and

68) Rec. for Magnetic Tape Recording and ire (ISO Test Object) and Its Use in Photographic Document Rec. for Amount of Bale Coating on Natural Rubber Bales rds (1970)

pe RD with Tapered Bore 1:12-Tolerance Class 5-Special Rec. for General
Rec. for Lever Switches: General c. for Rotary Wafer Switches (Low Current Rating): General

for Connectors for Frequencies Below $3 \mathrm{MHz}$ : (Mc/S) General Rec. for Hollow Metallic Waveguides: General Rec. for Flanges for Waveguides: General Rec. for Radio Frequency Connectors: General Rec. for Sensitive Switches: General Rec. for Push Button Switches: General Rec. for Radio Frequency Cables: General sion Lines and Their Associated Flange Connectors: General ctronic Applications Employing Similar Techniques: General ctronic Applications Employing Similar Techniques: General

Rec. for Sockets for Electronic Tubes and Valves: General Rec. for Tube and Valve Shields: General Rec. for Lead Acid Starter Batteries: General

kel Cadmium Cylindrical Rechargeable Single Cells: General ting for Fishing Nets (1970)

Rec. for of Household and Similar Electrical Appliances: Particular Rec. for Safety

of Household and Similar Electrical Appliances: Particular of Household and Similar Electrical Appliances: Particular Rec. for Safety

of Household and Similar Electrical Appliances: Particular Copper Conductors for Aircraft (19/ circuit Breakers for Aircraft (1970/

it Breakers for A ircraft (1970)

Cables with Copper Conductors for $/$ Cables with Copper Conductors for / ted Ferrous Products (1970) craft (1970)

Fixed Resistors: Selection o equipm or Fixed Resistors: Selection of Meth. of Test and General equipment for Domestic and Similar Gene/ equipment for Domestic and Similar Gener/ Spec. for Safety Rec. for Safety Rec. for Safety h. (1967) Rec. for Safety of Household and Similar Electrical Appliances: Particular of Household and Similar Electrical Appliances: Particular Rec. for General Technical Delivery
Relative Density $60 / 60^{\circ} \mathrm{F}$ Hydrometers for General P Relative Humidity in Small Enclosures by Means of Aqueo Relative Humidity (1968)

Relative Resistance of Insulating Materials to Breakdow

Relative to the Air (1972)

Relative to the Earth (1969)

Relative-Density of Essential Oils (1962)

Relative-Density (Specific Gravity) of Plastic, Excludi

Relays (Terminology, Classification, Test Meth.) (1971)

Relays (1956)

Relays (1963)

Relays (1967)

Rec. IEC

/Al Symbols: Contacts, Switchgear, Mecha IEC

Relays) Section 1: Push Buttons and Related Control Swi IEC

Relays): General Requirements (1970) /Switching Devic IEC

Relays: Instantaneous All or Nothing Relays (1967) IEC

Relays: Single Input Energizing Quantity Measuring, Wit IEC

Relays: Specified Time All or Nothing (1969) IEC

Relevant Cable Spec. (1961)

Relevant Spec. for Circular Types (1964)

Relevant Spec. for Flanges for Circular Types (1969)

Relevant Spec. for Flanges for Flat Rectangular Types (

Relevant Spec. for Flanges for Ordinary Rectangular Typ

Relevant Spec. for Flat Rectangular Types (1964)

Relevant Spec. for Medium Flat Rectangular Types (1967)

Relevant Spec. for Medium Flat Rectangular (1968)

Relevant Spec. for Ordinary Rectangular Types (1964)

Reliability Considerations (1968)

Reliability Data on Electronic Components (Or Parts) (1

Reliability of Electronic Equipment and the Components

Reliability (1969)

Reliability, Availability, and Maintainability Data Fro

Remelting (1968)

Removal: Designation of Main Groups of (1966)

Reoiling Connection (1965)

Replenishment Couplings for Aircraft-Interchangeabili

Replenishment Couplings for Aircraft (1969)

Report of National Limits (1967)

Reports and Study Questions of the CISPR (1969)

Repose (1968)

/C. for Aluminium Oxide Primarily Used F

Reproducing Equipment (1964)

Reproducing Equipment (1972)

(1)

Reproducing Systems: Dimensions and Characteristics (19

Reproduction ((1965)

/ Tests; Description of the ISO M ISO

Requirement and Determination (1971)

Requirement (1966) /Arings-Double Row Cylindrical Ty ISO

Requirements and Measuring Meth. for Printed Wiring Boa IEC

Requirements and Measuring Meth. (1962)

Requirements and Measuring Meth. (1962)

Requirements and Measuring Meth. (1962)

Requirements and Measuring Meth. (1964)

Requirements and Measuring Meth. (1964)

Requirements and Measuring Meth. (1965)

Requirements and Measuring Meth. (1968)

Requirements and Measuring Meth. (1970)

Requirements and Measuring Meth. (1971)

Requirements and Measuring Meth. (1971)

Requirements and Measuring Methods (1972)

Requirements and Measuring Methods (1972)

Requirements and Meth. of Test (1963)

Requirements and Meth. of Test (1969)

Requirements and Meth. of Test (1972)

Requirements and Test Meth. (1971)

Requirements for Describing and Des

Requirements for Dishwashers (1971)

Requirements for Electric Fans and R

Requirements for Electric Shavers, Hair Clippers and S

Requirements for Electronic Measuring Apparatus (1971)

Requirements for General Purpose Electrical Cables with

Requirements for General Purpose Push Pull Single Pole

Requirements for General Purpose Push Pull 3 Pole Circu

Requirements for Heat Resisting $\left(190^{\circ} \mathrm{C}\right)$ Electrical

Requirements for Heat Resisting $\left(260^{\circ} \mathrm{C}\right)$ Electrical

Requirements for Hot Dip Galvanized Coatings on Fabrica

Requirements for Lever Operated Manual Switches for Air

Requirements for Low Power Nonwirewound Types (1972)

Requirements for Mains Operated Electronic and Related

Requirements for Mains Operated Electronic and Related

Requirements for Radio Transmitting Equipment (1966)

Requirements for Radio Transmitting Equipment: Test Met

Rec. for Safety

Requirements for Ranges (1971)

1. for Safety IEC

Requirements for Steel (1964)

Requirements for Switches of Type 1, Slow Make and Brea
650 483 260 343 1151 1152 279

1183 346 50 (I6) $117-3$

255- 1

337.2

$337-1$

255-1

255-3

255-2

96-2

153-4

154-4

$154-3$

154 .

153.3

153-6

153-5

153-2

272

319

271

300

362

115

513

451

1465

1022

9

902

98

386

1201

94

446

1434

533

326

131-1

132-1

130-1

153-1

154-1

169-1

163-1

341.1

96-1

339-1

388-1

389.1

149-1

288-1

95-1

285-1

1530

335-5

342

335-3

$335-8$

348

335- 10

474

1467

1509

1075

1490

1461

1466

115-2

1

65

215-1

215-2

335-6

335-4

404

131-2 
ac. for Air l)ielectric Rotary Variable Capacitors: General rs and Food Freezers for llousehold and / onditioners (1972)

uring the Noise Emitted by Machines (19/

Std. for Safety

Rec. for Safety

Rec. for General

of llousehold and Similar Electrical Appliances: Particular (1969)

of Household and Similar Electrical Appliances: Particular of Household and Similar Electrical Appliances: Particular raft (1968)

zing Radiat/

Rec for Nuclear Instruments. Construction

Rec. for Electrical Installations in Ships: General

Binnacles and Azimuth Reading Devices, Class B-General Rec. for Household Refrigerators-Part I: Performance

king Capacity for Industrial and Similar Purposes: General Rec. for Plywood-Veneer, for General Use

ty of Household and Similar Electrical Appliances: General Auxiliary Circuits, Including Contactor Relays) : General Rec. for Precision Fuse Links for Aircraft-General Rec. for Refrigerating Plants-Safety

terchange Instrumentation Applications General Dimensional resistors, Type 1-Selection of Meth. of Test and General ouplings of String Insulator Units: Dimensions and General

data Interchange on Rolled Up Punched Paper Tape-General

h Fabrics for Electrical Purposes: Definitions and General ight Containers of Up to 30 Tonnes (Tons) Capacity-Basic up or Treated Mica Paper-Part 1: Definitions and General Std. for Dictation Equipment: Basic Operating

Rec. for Solderless Wrapped Connections-General Rec. for Picture Postcards: Area ec. for Modular Coordination-Storey and Room Heights for rubber Latices-Determination of Volatile Unsaturates and or Determination of the Yields of Tar, Water, Gas and Coke ic Acid and Xylenols for Industrial Use-Determination of Rec. for Meth. for the Determination of eta, and Para Cresol for Industrial Use-Determination of genated Hydrocarbons for Industrial Use-Determination of itric Acid for Industrial Use-Determination of Sulphated phuric Acid and Oleum for Industrial Use: Determination of bon Black for Use in the Rubber Industry-Spec. for Sieve

$k$ for Use in the Rubber Industry-Meth. of Test for Sieve c Acid (1968)

Rec. for Oilseed Rec. for Oilseed Rec. for Oilseed Rec. for Oilseed Rec. for Oilseed

Rec. for Vulcanized Rubbers-Determination of Rebound for Mechanical Isolation (Guide for Selecting and Applying Plastics-Preparation of Glass Fibre (Fiber) Reinforced, or Plastics-Determination of the Acetone Soluble Matter - Determination of Viscosity Number of Polyvinyl Chloride

C: Preparation of Test Specimens from Polyester and Epoxy and Lignites-Determination of Acetone Soluble Material

(1970)

ter) (1970)

(1970)

tics-Determination of the Viscosity Number of Polyamides te of Increase During the Setting of Unsaturated Polyester Rec. for Plastics: Designation of Polyvinyl Chloride

ec. for Industrial Laminated Sheets Based on Thermosetting nsity (1969) Rec. for Plastics-Pvc levated Temperatur/ Rec. for Meth. of Test for Electrical Electronic Tubes and Valves: Meth. of Measuring Electrode

nce and Transconductance, Amplification Factor, Conversion International Std. for

for Water Resistant Watches-Part I: Wristwatches-Water native Voltage (1969/ Rec. for Measurement of the Winding (1963)

tic Head Test) (1968)

pper Conductors, for A/

Rec for Meth of Test for Rec. for Dimensions and Conductor Rec. for Determination of the Hydrolytic Rec. for Determination of the Hydrolyti

tion of Mixed Alkali (1968/

$1100{ }^{\circ} \mathrm{C}(1970)$

bles with Copper Condu/ Rec. for Dimensions and Conductor bles with Copper Condu/ Rec. for Dimensions and Conducto ace Dischar/ Rec. Test Meth. for Determining the Relative

Frequency Cables A/ Rec. for Guide to the Calculation of Rec. for Plastics-Rec. Pract. for the Evaluation of the Rec. for Plastics-Determination of the Rec. for Plastics-Determination of Rec. for Plastics-Determination of posure-to Daylight / posure to Light of / posure to Light of /

Rec, for Plastics-Determination of Rec. for Plastics-Determination of the Charpy Impact
Requirements for Tests and Measuring Meth. (1970)

Requirements for the Electrical Equipment of Refrigerat

Requircments for the Electrical Equipment of Room Air C

Requirements for the Preparation of Test Codes for Meas

Requirements for Toasters, Grills, Waffle Irons and Roa

Requirements for Type 2, Quick Make and Break (Toggle)

Requirements for Vacuum Cleaners (1970)

Requirements for Washing Machines (1971)

1. for Safety IEC

I for Safety IEC

Requirements for 28 Volt d.c. Flat Strip Fuses for Airc ISO

Requirements to Afford Personal Protection Against Ioni

Requirements (1964)

Requirements (1967)

Requirements (1968)

Requirements (1968)

Requirements (1969)

Requirements (1970)

Requirements (1970)

Requirements (1971)

Requirements (1971)

Requirements (1971)

Requirements (1971)

Requirements (1971)

Requirements (1972)

Requirements (1972)

Requirements (1972)

Requirements (1972)

Requirements (1973)

Rec. for Magnetic Compasses ISO

ISO

/ Low Voltage Fuses with High Brea IEC

Rec. for Safe IEC

Switching Devices for Control and IEC

ISO

/ Unrecorded Magnetic Tapes for in ISO 1. for Fixed Resistors: Wirewound IEC IIng Devices for Ball and Socket C. IEC Std. for ISO

Rec. for Varnis IEC

Std. for Hooks for Lifting Fre ISO

/Ulating Materials Based on Built IEC

Requirements, Test Meth. and Practical Guidance (1971) ISC

Reserved for the Address (1965)

Residential Buildings (1970)

Residual Styrene (1972)

Std. for Styrene Butadiene

ISO

ISO

Residue by Low Temperature Distillation of Brown Coal a ISO

Residue on Distillation (1971)

Residue on Evaporation on a Water Bath (1968)

Rec. for Cresyl ISO

Residue on Evaporation (1971)

C. for Phenol: Ortha, M ISO

Residue on Evaporation (1972)

Std. for Liquid Halo ISO

Residue on Ignition-Gravimetric Meth. (1971)

Residue on Ignition; Gravimetric Meth. (1968)

Residue (1970)

I for N ISO

/for Sul ISO

Residue (1970)

Rec. for Car ISO

Residues: Determination of Ash Insoluble in Hydrochlori

Residues: Determination of Diethyl Ether Extract (1968)

Residues: Determination of Moisture and Volatile Matter

Residues: Determination of Oil Content (1968)

Residues: Determination of Total Ash (1968)

Resilience-Lupke Pendulum Meth. (1971)

Resilient Devices) (1972)

ISO

Characteristics ISO
Panels F

Resin Bonded, Low Pressure Laminated Plates or Panels

Resin in Solution (1961)

Rec for Plastics ISO

Resin Moulding Materials (1970)

Resinous Substances) (1969)

/Ing Materials-Annex ISO

/ Extract from Brown Coals ISO

Resins-Determination of Impurities and Foreign Matter

Resins-Determination of $\mathrm{pH}$ of Aqueous Extract (1970)

Resins-Determination of Volatile Matter (Including Wa

Resins-Measurement of Density by the Pyknometer Meth.

Resins-Sieve Analysis in Water (1970)

ISO

Resins in Dilute Solution (1963) Rec. for Plas ISO

Resins (1967) /N of the Maximum Temperature and the Ra ISO

Resins (1969)

Rec for Plastics-Basis for $S_{p}$ ISO

Resins (1971) Rec. for Plastics-Basis for Sp ISO

Resins: Determination of the Compacted Apparent Bulk De

Resistance and Transconductance, Amplification Factor, IEC

Resistance and Transductance (1966) /Electrode Resista IEC

Resistance for Copper (1925)

Resistance for General Use (1972)

Resistance of an a.c. Machine During Operation at Alter

Resistance of Conveyor Belts: Spec./S and Meth. of Test

Resistance of Fabrics to Penetration by Water (Hydrosta

Resistance of General Purpose Electrical Cables with Co

Resistance of Glass Grains at $121^{\circ} \mathrm{C}(1968)$

Resistance of Glass Grains at $98^{\circ} \mathrm{C}(1968)$

Resistance of Glass to Attack by a Boiling Aqueous Solu

Resistance of Glass to Attack by $6 \mathrm{~N}$ Hydrochloric Acid a

Resistance of Heat Resisting $\left(190^{\circ} \mathrm{C}\right)$ Electrical Ca

Resistance of Heat Resisting $\left(260^{\circ} \mathrm{C}\right.$ ) Electrical Ca

Resistance of Insulating Materials to Breakdown by Surf

Resistance of Plain and Tinned Copper Conductors of Low

Resistance of Plastic to Fungi by Visual Examination (1

Resistance of Plastics to Chemical Substances (1961)

Resistance of Plastics to Colour (Color) Change Upon Ex

Resistance of Plastics to Colour (Color) Change Upon Ex

Resistance of Plastics to Colour (Color) Change Upon Ex

Resistance of Rigid Plastics (Flexural Test) (1961)
Std ISO

IEC

ISO

ISO

ISO

ISO

ISO

ISO

ISO

ISO

ISO

- IEC

IEC

ISO

ISO

ISO

ISO

ISO

ISO

334-1

316

378

495

335-9

131-3

335-2

335. 7

854

405

92.1

613

824

69.1

1098

335.

337.1

1547

1662

1859

115-3

372-1

2195

394. 1

2308

371.1

352

416
1789

2008

647

1907

759
1900

2210

1983

913

1867

1437

735

736

771

734

749

1767

2017
1268

308

295/C

1017

1270

1265

1264

1269

1675

1624

307

584

1060

1642 
$(196)$

Rec for Plastics-Determination of the Izod Impact Rec. for Plastics-Determination of Incandescence est) $(1966)$ for Meth. of Test for the Determination of the Insulation han $1500 \mathrm{kgf})(1969)$

Rec. for Dimensions of Straigh Rec. for Dimensions of Straight Rec. for Fire

Rec. for Vulcanized Rubbers-Accelerated Ageing or Heat thetic Rubber (De Mattia Type / Rec. for Determination of Rec. for Determination of Rec. for nthetic Rubber (De Mattia Type/ s Pipes for the Transport of Fluids-Determination of the Std for Vulcanized Rubbers-Determination of Rec. for Paints and Varnishes-Determination of Rec. for Rating of

972) nductors for Round nsions of Winding Wires: Diameters of Conductors for Round ectronic Tubes and Valves: Measurement of Equivalent Noise
Rec for Pulps: Determination of Alkali Rec. for Pulps: Determination of Alkali
Rec. for Paper-Determination of Tearing for Lined Industrial Rubber Boots with General Purpose Oil ance for General Use (1972)

Std. for Water Rec. for Fire Std. for Heat Std. for Hea 1973) nductors for / Rec. for Performance Requirements for Heat
ndu/ Rec. for Dimensious and Conductor Resistance of Heat ndu/ Rec. for Dimensious and Conductor Resistance of Heat
uctors for Aircraft (197/ Rec. For Meth. of Test for Heat nductors for / Rec. for Performance Requirements for Heat nductors for Aircraft (1/ Rec. for Meth. of Test for Hea ndu/ Rec. for Dimensions and Conductor Resistance of Heat Rec. Meth. of Test for Volume and Surface
m) Electrical Conductor Wire (1959) (I967) Rec. Test Cells for Measuring the tur/ Rec. for Meth. of Test for Electrical Resistance and Rec. for Lightning Arresters: Nonlinear Rec. for Preferred Number Series for

Rec. for Marking Codes for Values and Tolerances of Rec. for Fixed Wirewound Rec. for Rules for Ohmic wered Vehicles (I970) $r$ Meth. of Measurement of Current Noise Generated in Fixed c. for Test Meth. for Batch Furnaces with Metallic Heating tifiers/ Rec. Graphical Symbols: Variability, Examples of Rec. for Fixed Nonwirewound Rec. for Fixed Resistors: Wirewound ral Requirements (I9) Rec. for Fixed Resistors: Wirewound rements for Low Power Nonwirewound Types / Rec. for Fixed 197I) Rec. for Fixed Rec. for Fixed eth. of Test and General Requirements (I9/ Rec. for Fixed urement of Frequency and Equivalent Resistance of Unwanted Specialized Frequency Measurements on Fm Receivers of the Acoustics-Assessment of Noise with Respect to Community ined Pressure and Vacuum, and Vacuum Gauges, with Pressure ined Pressure and Vacuum, and Vacuum Gauges, with Pressure 0) of Test Std. for Statistical Interpretation of Test
1973) for Small Medical Gas Cylinders Used for A naesthetic and s for Small Medical Gas Cylinders Used for Anaesthetic and 965 Rec. for Motorists Seat Belts with Rec. for Measurement of Absorption Coefficients in A Photography: Determination of ISO Speed of Colour (Color) mm Thick (1959) Rec. for Drill Steel Equipment for Percussive Long Hole Drilling for C And/ Drill Steel Equipment for Percussive Long Hole Drilling achines Used for Information Processing-Widths of Fabric minimum Markings to Appear on Containers Used for Printing Reciprocating Internal Combustion Engines-Definition of for Dyeing and Finishing Machines: Definition of Left and Rec. for Definition of Side (Left or Rec. for Weaving Looms: Definition of Side (Left or ies-Pirn and Cross Winders-Definition of Side (Left or weaving Preparatory Machines-Definition of Side (Left or sions (1972) th (I972) erties (I972) Rec for Plastics-Compression Test of Std. for Std. for

determination of Water Vapour (Vapor) Transmission Rate of ange Connectors: General Rec. for Plastics-Bending Test for lange Connectors: Detail Spec. $\quad$ Rec. for General Purpose
Resistance of Rigid Plastics (Flexural Tests) (1961)

Resistance of Rigid Self Extinguishing Thermosetting of Resistance of Solid Insulating Materials (1964)

Resistance of the Carcass of Conveyor Belts (Meth. of T

Resistance of Unwanted Resonances of Filter Crystal Uni

Resistance Spot Welding Electrodes (For Loads Greater T

Resistance Spot Welding Electrodes (1968)

Resistance Tests of Structures (1968)

Resistance Tests (1971)

Resistance to Crack Growth of Vulcanized Natural or Syn

Resistance to Flex Cracking of Vulcanized Natural or Sy

Resistance to Humid Conditions (1958)

Resistance to Internal Pressure (I970)

Resistance to Liquids (1971)

Resistance to Ozone Cracking Under Static Conditions (1

Resistance Welding Equipment (1968)

Resistance Wires (1971)

Rec. for Basic Dime

Resistance (1964)

Resistance (1968)

Resistance (1971)
Resistance (1972)

Resistant Watches-Part I: Wristwatches-Water Resist

Resisting Characteristics of Electric Cables (1970)

Resisting Equipment Wires for Aircraft (I973)

Resisting Equipment Wires for Aircraft: Meth. of Test

Resisting $\left(190^{\circ} \mathrm{C}\right)$ Electrical Cables with Copper $\mathrm{Co}$

Resisting $\left(190^{\circ} \mathrm{C}\right)$ Electrical Cables with Copper Co

Resisting (190 Deg.) Electrical Cables with Copper Cond

Resisting $\left(260^{\circ} \mathrm{C}\right)$ Electrical Cables with Copper Co

Resisting $\left(260{ }^{\circ} \mathrm{C}\right)$ Electrical Cables with Copper Co

Resisting $\left(260^{\circ} \mathrm{C}\right.$ ) Electrical Cables with Copper Co

Resistivities of Electrical Insulating Materials (1958)

Resistivity of Commercial Hard Drawn Aluminium (Aluminu

Resistivity of Insulating Liquids and Meth. of Cleaning

Resistivity of Insulating Materials at Elevated Tempera

Resistor Type for a.c. Systems (1970)

Resistors and Capacitors (I963)

Resistors and Capacitors (1968)

Resistors Type 2 (1969)

Resistors Used in the Power Circuits of Electrically Po

Resistors (1965)

Resistors (1971)

Resistors (1972)

Resistors, Elements of Electronic Tubes, Valves and Rec

Resistors, Type II (1959)

Resistors, Type I-Detail Spec. (197I)

Resistors, Type 1-Selection of Meth. of Test and Gene

Resistors: Selection of Meth. of Test and General Requi

Resistors: Terms and Meth. of Test (I971)

Resistors: Wirewound Resistors, Type 1-Detail Spec. (

Resistors: Wirewound Resistors, Type 1-Selection of $M$

Resonances of Filter Crystal Únits (I968)

Response to Impulsive Interference (197I)

or the Meas IEC

Response (1971)

Responsive Elements and Recording Directly by Means o

Responsive Elements Giving Direct Indications by Means

Restraining Devices for Children in Motor Vehicles (197

Results: Estimation of the Mean-Confidence Interval

Resuscitation Purposes (1964) /E Type Valv

Retarding Insulation for Use in Television Receivers (1

Retractors (1970)

Reverberation Room (1963)

Reversal Films for Still Photography-Sensitometric Ex

Reverse Bend Test. of Steel Sheet and Strip Less Than 3

Reverse Bend Test. of Steel Wire (1973)

Reverse Buttress Threaded Equipments 1 1/2 to 2 I/2 In.

Reverse Buttress Threaded Equipments I I/6 and $11 / 4$ in

Reversible Rings for Ring Spinning and Doubling Frames

Ribbons on Spools (1972)

Ribbons (I972)

Right and Left Han

Right Sides (1969)

Right) of Spinning Machinery (I959)

/D. for Office and Printing M ISO

Rec. Fo IEC

Rec. for IEC

Re IEC

IEC

IEC

IEC

IEC

IEC

EC

EC Rec. for ISO

OIML

OIMI

ISO

ISO

ISO

IEC

IEC

ISO

ISO

ISO

ISO

ISO

D. for ISO

Rec. ISO

ISO

Right) (1959)

Right) (1960)

Rigid CelluIar Plastic (1968)

Rec. for Textile Machinery and Accessor

68r Textile Machinery and Accessories- ISO

ISO

ISO

ISO

Rigid Cellular Plastics-Determination of Shear Stren

Rigid Cellular Plastics-Determination of Tensile Prop

ISO

Rigid Cellular Plastics (1970)

Rec. for Plastics-

Rigid Cellular (1970)

Rigid Coaxial Transmission Lines and Their Associated F

Rigid Coaxial Transmission Lines and Their Associated F
ISO

IEC

180

181

167

505

1045

670

834

188

REC

1167

1817

1431

152

669

182-4

151.7

699

1974

2025

228

331

2032

2436

1075

470

1220

1490

149

539

93

II 1

345

99 -

62

266

266

322
195

301

397

117-6

109

115-3-1

115-3

115-2

1 I5. 1

1I5-3-I

$115-3$

283

315-5

1996

19

1713

2602

407

332

197

1534

354

2240

I 722 
ec. for Determination of Adhesion of Vulcanized Rubbers to or Plastics-Determination of Incandescence Resistance of is-Compressive and Flexural Strengths of Plastic Mortar al Arc Welding of Mild Steel: Dimensions of Wires, Spools, Rec. for Rolling Bearings - With Locating Snap Std. for Rolling Bearings: with Locating Snap d Gauges (1964/ Rec. for Warp Tubes for Ring Spinning and gauges (1963)

Rec, for

r Textile Machinery and Accessories-Reversible Rings for lers (1959)

Rec. for Rings for

Rigid Plates in Shear (Quadruple.Shear Test) (1971)

Rigid Self Extinguishing Thermosetting of (1961) F ISO
Rilem Cembureau Meth.) (1968) /Test. Strength of Cemen ISO

Rims and Coils (1968)

Ring-Dimensions (1965)

Ring-Inner Diameter of (1972)

Olid Wires for Gas Shielded Met

Ring Doubling Spindles Metric Dimensions, Tolerances an

Ring Doubling Spindles: In. Dimensions, Tolerances and

Ring Expanding Test on Steel Tubes (1964)

Ring Spinning and Doubling Frames for C and El Trav

Ring Spinning and Doubling Frames for $\mathrm{C}$ and El Trav

Ring Spinning and Doubling Frames for Ear Shaped Travel

Ring Spinning and Doubling Frames (1972)

for Textile Machinery and Accessories-Spindle Gauges for ons, Tolerances and Gauges (1964/ Rec. for Warp Tubes for s, Tolerances and Gauges (1963) Rec. for Warp Tubes for lyvinyl Chloride (PVC) Pressure Pipes with Elastic Sealing

lyvinyl Chloride (PVC) Pressure Pipes with Elastic Sealing earings: with Spherical Outside Surface and Extended Inner arrel Thread for E14 and E27 Lampholders with Shade Holder $(197)$ Rec. for Elastomeric Sealing 969) Rec. for Dimensions of Elastomeric Toroidal Sealing Rec. for Dimensions of Elastomeric Toroidal Sealing Rec. for Rubber Sealing $\begin{aligned} \text { ss } 2 / & \text { Rec. for Dimensions of Elastomeric Toroidal Sealing } \\ 1 \mathrm{~T} / & \text { Rec. for Dimensions of Elastomeric Toroidal Sealing }\end{aligned}$ Std. for Textile Machinery and Accessories-Reversible std. for Textile Machinery and Accessories-Nonreversible ped Travellers (1959) Rec. for $\mathrm{r}$-Boundary Dimensions-Subunits-Metric Series-Outer re for Carbon Brushes, Brush Rec. for Dimensions for Commutators and Slip Std. Meth. of Measurement of Lamp Cap Temperature mination of Refractoriness Under Load (Differential-With

8) Rec. for Mechanical Property Limits for minium and Aluminum Alloys-Shear Test for Rivet Wire and Rec. for Protective Helmets for

c. for Rules for Rotating Electrical Machines for Rail and icular Requirements for Toasters, Grills, Waffle Irons and rocedures for Electronic Components and Equipment: Test U: ussive Drilling (1970)

cussive Long Hole Drilling-Rope Threaded Equi/ cussive Long Hole Drilling-Rope Threaded Equi/ cussive Long Hole Drilling-Reverse Buttress T/ cussive Long Hole Drilling-Reverse Buttress T/ ling-Connecting Dimensions (1970) $s$ for Hollow Hexagonal Drill Steels (1968) m (1968)

haracteristics and Test Meth. (1972) al Cross Sections-Part II. ical Cross Sections-Part III: Representation of Magmatic rec. for Calibration of Standardized Blocks to Be Used for Rec. for Verification of

Verification and Calibration of Hardness Reference Blocks Verification and Calibration of Hardness Reference Blocks

Steel (1969) nes (1969)

Rec. for Rec. for Rec. for Verification of ec. for Calibration of Standardized Blocks to Be Used for c. for Hydraulic Cylinders-Internal Diameters and Piston ps of Healds (1967) (1970) Rec. for Heald Carrying Rec. for Rock Drilling-Rotary Drill Rec. for Rock Drilling-Drill Rec. for Dimensions of Aerial

Iding: Test to Determine the Compatibility of Steel Filler he Characteristics of the Deposited Meta/ Rec. for Filler he Conventional Bond Strength on Steel,

he Mechanical Properties of Weld Metal Deposited by Filler tensile Types (1967) ty of Steel Filler Rods and the Parent M/ c. for Lengths and Tolerances for Drawn or Extruded Filler Rec. for Dimensions of Tubes, Pins and Rec. for Lengths and Tolerances for Filler Rec. for Determination of Caking Power of Coal by the Fabrics Coated with Rubber or Plastics-Determination of zes (1971)

ec. for Identification of the Emulsion Side of Edge Marked Rec. for Identification of Exposed Colour (Color) Rec. for Dimensions for 127,120 and 620
Ring Spinning and Ring Doubling Spindles Metric Dimensi

Ring Spinning and Ring Doubling Spindles: In. Dimension

Ring Type Joints: Minimum Depths of Engagement (1973)

Ring Type Joints: Minimum Depths of Engagement (1973)

Std for Rolling B ISO

Ring Width (1972)

Ring (1972)

Rec. for Sheets for B IEC

Rings for Aircraft-Packaging and Identification (1971

Rings for Aircraft-(In. Series-Class 2 Tolerances)

Rings for Aircraft (In. Series-Class 1 Tolerances) (1

Rings for Joints in Asbestos Cement Water Piping (1970)

Rings for Pipe Fittings in Aircraft-(In. Series-Cla

Rings for Pipe Fittings in Aircraft (In. Series-Class

Rings for Ring Spinning and Doubling Frames for $C$ and

Rings for Ring Spinning and Doubling Frames for $C$ and

Rings for Ring Spinning and Doubling Frames for Ear Sha

/Ling Bearings: Tapered Rolle ISO

Rings with Flange (1973)

Rings (1964)

Rings (1968)

Rings (1971)

Rise (1971)

Rising Temperature) (1970)

Rec. for Definitions and Nomenclatu IEC

IEC

7 Efractory Products-Deter ISO

Rivet Stock of Aluminium (Aluminum) and Its Alloys (196 ISO

Rivets (1971)

Road Users (1970)

Rec. for Alu ISO

ISO

Road Vehicles (1971)

Roasters (1972)

Re IEC
/D Similar Electrical Appliances: Part IEC

Vironmental Test. P IEC

Rock Drilling-Drill Rods and Detachable Bits for Perc

Rock Drilling-Extension Drill Steel Equipment for Per

Rock Drilling-Extension Drill Steel Equipment for Per

Rock Drilling-Extension Drill Steel Equipment for Per

Rock Drilling-Extension Drill Steel Equipment for Per

Rock Drilling-Rotary Drill Rods and Bits for Dry Dril

Rock Drilling: Forged Collared Shanks and Chuck Bushing

Rock Drilling: Hollow Hexagonal Drill Steels in Bar for

Rock Drilling: Integral Stems (1968)

Rock for the Manufacture of Binders-Classification, C

(De on Detailed Maps, Plans and Geologic ISO

Rocks (1968)

/R Use on Detailed Maps, Plans and Geolog

Rocks (1970)

/R Use on Detailed Maps, Plans an
ardness Scale Test. Machines (1968)

Rockwell B and C Hardness Scale Test. Machines (1968)
Rockwell B and C Scale Hardness Test. Machines (1968)

Rockwell B (1970)

Rockwell C (1970)

International Rec. for

Rockwell Hardness Test (B and C Scales) for Steel (1968

Rockwell Superficial Hardness Test (N and T Scales) for

Rockwell Superficial N and T Scale Hardness Test. Machi

Rockwell Superficial N and T Scale Hardness Test. Machi

Rod Diameters-Metric Series (1971)

Rods-Dimensions and Coordination Of, and with End Loo

Rods and Bits for Dry Drilling-Connecting Dimensions

Rods and Detachable Bits for Percussive Drilling (1970)

Rods and Slabs of Ferromagnetic Oxides (1966)

Rods and the Parent Metal in the Welding of Steels (196

Rods for Braze Welding: Meth. of Test for Determining T

Rods for Braze Welding: Meth. of Test for Determining T

Rods for Gas Welding Mild Steels and Low Alloy High Ten

Rods for Gas Welding of Mild Steels and Low Alloy High

Rods for Gas Welding: Test to Determine the Compatibili

Rods for Welding, Supplied in Straight Lengths (1967)

Rods of Ferromagnetic Oxides (1966)

Rods, Other Than Drawn or Extruded, for Welding (1967)

Roga Meth. (1963)

Roll Characteristics (1972)

Roll Film Cameras-Back Window Location and Picture SI

Roll Film for Still Picture Cameras (1968)

Roll Film (1969)

Roll Film, Backing Paper and Spools (1971)
26

1747

181

679

864

464

2265

368

343

374

95

96

97

368

2045

2048

2264

399

1749

1800

1078

1398

1799

1077

95

96

2316

380

276

356

360
1893

1051

828

2101

1511

335-9

68-2-21

1718

1719

1720

1721

1722

1717

722

721

1587

$710 / \mathrm{II}$

710/III

674

716

11

12

1024

1079

1355

2091

570

1717

1718

223

708

688

698

637

636

708

546

220

545

335

2286

1203

897

1048

732 
Rec: for l'hotographic Black and White Paper for Rer. for Photographic Colour (Color) Paper for n.)-Tensile lest (1971) ed) Wheels For/ (1968) Rec. for Copper and Alloy Rec. for Railway Rolling Stock Material . Rec. for Mechanical Property I.imits for Rec. for Dimensions of Hot Rec. for Dimensions of Hot Rec. for Dimensions of Hot Rec. for Dimensions of Hot Rec. for Dimensions of Hot Rec. for Dimensions of Hot Rec. for Dimensions of Hot Rec. for Dimensions of Hot Rec. for Dimensions of Hot Std. for Data Interchange on

Dimensions and Sectional Pro -In. Series-Dimensions An/ eries-Dimensions and Sectio/ ic Series-Dimensions and $\mathrm{Se}$ tric Series-Dimensions and / -Dimensions and Sectional P/ 72 in Light Allo/ 9 and 0 (1967) $n$-Series 48 and 49 (1/ ns Series 31 and $32 l$ 968)

-Outer Rings with F (1969)

-Dimension Series /

-Dimension Series 2/

Rec. for Shipbuilding Details-Marking of Rec. for Rolling Bearings-Tapered Rec. for Rolling Bearings-Needle Rec. for Rolling Bearings-Tapered Rec. for Rolling Bearings-Tapered Std. for Rolling Bearings: Tapered Rec. for Rolling Bearings-Tapered Rec. for Rolling Bearings-Tapered Std. for Rolling Bearings: Tapered Rec. for Rolling Bearings-Tapered Rec. for Rolling Bearings-Tapered Rec. for Rolling Bearings-Tapered Std. for Rolling Bearings: Tapered Std. for Rolling Bearings: Tapered 0 and 00 (1973)

(1962)

i) Thrust (III) Tap/ $s$ (1958) veyors (1972)

Rec. for Rolling Bearings-Cylindrical Rec. for ISO Identification Code for Rec. for Ball and Std. for Extended Pitch Precision in Wheels (1966) Rec. for Short Pitch Transmission Precision identification Code for Rolling Bearings: Group IV Tapered iber) (1959) ion Code for Roller Bearings: Groups-(I) Radial Ball and Collars-Boundary Dimensions (1962) Rec. for Diameters of Drafting tapered Bore 1:I2-Tolerance Class 5-Special/ dial Internal Clearance (1970)

ernal Clearance (1965)

nspection (1970)

ngs-Definitions (1961)

ings (I962)

- Metric Series-Dimension-Series 48 and $49(1 /$

al Plan-Diameter Series $8,9,0,1,2,3$ and 4 , (1)

al Plan-Part II-Diameter Series 7 (1970)

d Radial Groove Type Ball Bearings with Cylindr/

Outside Diameter Tolerances-Normal and Toler/

(1963)

(1965)

-Metric-Diameter Series 9 and 0 (1967)

-Subunits-In. Series (1968)

-Subunits-Metric Series (1969)

-Metric Serics-Dimensions Series 31 and $32 /$

-Subunits-Metric Series-Dimension Series /

r Dimension Limits (1969)

mal Tolerances (1967)

-Normal Tolerances (1961)

-Tolerances: Part 2-Tolerance Classes 6, 5 /

undary Dimensions (1966)

Rec. for

Rec. for

Rec. for

Rec. for

Rec. for

Rec. for
Rec. for

Rec. for

Rec. for

Rec. for

Rec. for

Rec. for

Rec. for

Rec. for

Rec. for

Rec. for

Rec. for

Rec. for

Rec. for

Rec. for

Rec. for

Rec. for

Rec. for
Rec. for

Rec. for

Rec. for

Rec. for

Rec. for

Rec. for

dary Dimensions, Tolerances, Permissible Load (/ arance $(1969)$

ries $(1965)$

Rec. for ISO Identification Code for
Rec. for ISO Identification Code for
972) ers with Straight Inner Tab (1972)

Std. for

Rec. for

Rec. for

Std. for

Std. for

Std. for

Std. for

Std. for

Std. for

Std. for

Rec. for Railway

Rec. for Railway

Rec. for Railway

Rec. for Railway

Rec. for Railway

Rec. for Railway
Roll Paper Printers: Preferred Sizes of Rolls (1969)

Roll Paper Printers: Preferred Sizes of Rolls (1969)

Rolled Flat Products (Thickness Less Than $2.5 \mathrm{~mm}(0.1$ I

Rolled on Forged Wheel Centres (Centers) for Tyred (Tir

Rolled Products of Aluminium (Aluminum) and Its Alloys

Rolled Steel Bars: Flat, Metric Series (1969)

Rolled Steel Bars: Round, Metric Series (1969)

Rolled Steel Bars: Square, Metric Series (1969)

Rolled Steel Sections-Equal Leg Angles-In. Series-

Rolled Steel Sections-Parallel Flange Column Sections

Rolled Steel Sections-Parallel Flange I Beams-In. S

Rolled Steel Sections-Part 1: Equal Leg Angles-Metr

Rolled Steel Sections-Part 2: Unequal Leg Angles-Me

Rolled Steel Sections-Unequal Leg Angles-In. Series

Rolled Up Punched Paper Tape-General Requirements (19

Rolled, Drawn and Extruded Products in Light Metals or

Roller-Boundary Dimensions-Metric-Diameter Series

Roller-Boundary Dimensions-Metric Series-Dimensio

Roller-Boundary Dimensions-Metric Series-Dimensio

Roller-Boundary Dimensions-Subunits-In. Series (1

Roller-Boundary Dimensions-Subunits-Metric Series

Roller-Boundary Dimensions-Subunits-Metric Series

Roller-Boundary Dimensions-Subunits-Metric Series

Roller-Boundary Dimensions-Subunits-Metric Series

Roller-Boundary Dimensions (1963)

Roller-Boundary Dimensions (1965)

Roller-In. Series-Chamfer Dimension Limits (1969)

Roller-Inch Series-Tolerance Classes 4 (Normal), 3 ,

Roller-Metric Series-Tolerances-Class 4 (1973)

Roller-Separate Thrust Collars-Boundary Dimension

Roller Bearings: Groups-(I) Radial Ball and Roller (I

Roller Bearings: Meth. of Evaluating Static Load Rating

Roller Chains and Chain Wheels for Transmission and Con

Roller Chains and Chain Wheels (1967)

Roller Chains Type S 32 to 88 with Their Associated Cha

Roller Type-In. Series (1965)

Roller (II) Thrust (III) Tapered Type-Metric Series (

Rollers for Cotton, Wool, Spun Silk and Staple Fibre (

Rolling Bearings-Cylindrical Roller-Separate Thrust

Rolling Bearings-Double Row Cylindrical Type RD with

Rolling Bearings-Double Row Self-Aligning Ball-Ra

olling Bearings-Double Row Selfaligning-Radial Int

olling Bearings-For Railway Axle Boxes: Acceptance

Rolling Bearings-Instrument Precision Types (1971)

Rolling Bearings-Internal Clearance in Unloaded Beari

Rolling Bearings-Meth. of Evaluating Dynamic Load Rat

Rolling Bearings-Needle Roller-Boundary Dimensions

Rolling Bearings-Radial-Boundary Dimensions-Gener

Rolling Bearings-Radial-Boundary Dimensions-Gener

Rolling Bearings-Radial Types with Shields or Seals-

Rolling Bearings-Tapered Roller-Boundary Dimensions

Rolling Bearings-Tapered Roller-Boundary Dimensions

Rolling Bearings-Tapered Roller-Boundary Dimensions

Rolling Bearings-Tapered Roller-Boundary Dimensions

Rolling Bearings-Tapered Roller-Boundary Dimensions

Rolling Bearings-Tapered Roller-Boundary Dimensions

Rolling Bearings-Tapered Roller-Boundary Dimensions

Rolling Bearings-Tapered Roller-In. Series-Chamfe

Rolling Bearings-Tapered; Metric Series-Part I: Nor

Rolling Bearings-Thrust Ball Bearings with Flat Seats

Rolling Bearings-Thrust Ball Bearings with Flat Seats

Rolling Bearings-Tolerances-Definitions (1969)

Rolling Bearings - With Locating Snap Ring-Dimensions

Rolling Bearings: Accessories (1969)

Rolling Bearings: Airframe: Introduction. General, Boun

Rolling Bearings: Cylindrical Type, Radial Internal Cle

Rolling Bearings: Group IV Tapered Roller Type-In. Se

Rolling Bearings: Group V Airframe Type (1968)

Rolling Bearings: Locknuts, Narrow Series, and Lockwash

Rolling Bearings: Radial-Tolerances (1966)

Rolling Bearings: Tapered-Metric Series Tolerances Cl

Rolling Bearings: Tapered Roller-Boundary Dimensions

Rolling Bearings: Tapered Roller-Boundary Dimensions

Rolling Bearings: Tapered Roller-Inch Series-Tolera

Rolling Bearings: Tapered Roller-Metric Series-Tole

Rolling Bearings: Tolerances; Metric Series Chamfer Dim

Rolling Bearings: with Locating Snap Ring-Inner Diame
Rolling Bearings: with Spherical Outside Surface and Ex

Rolling Stock Material-Axles for Trailer (1969)

Rolling Stock Material-Cast Wheel Centres (Centers) I

Rolling Stock Material-Rolled on Forged Wheel Centres

Rolling Stock Material-Rough Tyres (Tires) for Traile

Rolling Stock Material-Tyres (Tires) for Trailer Stoc

Rolling Stock Material: Solid Wheels for Trailer Stock

ISO

150

ISO

ISO

ISO

ISO

ISO

ISO

ISO

ISO

ISO

ISO

ISO

ISO

ISO

ISO

ISO

ISO

ISO

ISO

ISO

ISO

ISO

ISO

ISO

ISO

ISO

ISO

ISO

ISO

ISO

ISO

ISO

ISO

ISO

ISO

ISO

ISO

ISO

ISO

ISO

ISO

ISO

ISO

ISO

ISO

ISO

ISO

ISO

ISO

ISO

ISO

ISO

ISO

ISO

ISO

ISO

ISO

ISO

ISO

ISO

ISO

ISO

ISO

ISO 
(x,9)

entry intu Serv/ (Color) Paper for Roll Paper Printers: Preferred Sizes of nd White Paper for Roll Paper Printers: Preferred Sizes of Rec. for Asbestos Cement Corrugated Sheets for Rec. for Asbestos Cement Slates for mmetrical Section Corrugated Sheets in Asbestos Cement for Rec. for Test. and Ratin

c. for Safety Requirements for the Electrical Equipment of and Heating Appliances for Domestic and Similar Purposes: Rec. for Modular Coordination-Storey and Measurement of Absorption Coefficients in a Reverberation Std. for Flexible Steel Wire Sid. for

Drill Steel Equipment for Percussive Long Hole Drilling Drill Steel Equipment for Percussive Long Hole Drilling I Properties (1972) ec. for Drawn Wire for General Purpose Nonalloy Steel Wire Std. for Steel Wire Rec. for Sampling and Conditioning of Rec for Shipbuilding Details-Galvanized Steel Wire Shipbuilding Details-Tests on Galvanized Steel Wire for Rec. for 3 Strand Polyamide Multifilament Rec. for 3 Strand Polyester Multifilamen Rec. for 3 or 4 Strand Manila and Sisal and 8 Strand (Plaited) Polypropylene Monofilament or Film Rec. for 3 Strand Polyethylene Monofilament Rec. for 8 Strand Plaited Manila and Sisal

\section{g Dimensions (1970)} Rec. for Oil of

(1972)

sts and Measuring Meth. (1970)

Positions and Diameter $40 \mathrm{~mm}$ (1966)

Rec. for Rock Drilling Std. for Std. for positions and Diameter $60 \mathrm{~mm}$ (Low Current Ratin/ Rec. for mounting (1963)

uirements and Measuring Meth. (1962)

hes with Central Mounting (1963)

Rec. for Rec. for Rec. for Rec. for Rec. for

Code for the Measurement of the Airborne Noise Emitted by and Flange N/ Rec. for Dimensions and Output Ratings for (1971) enclosures (1968)

ses and Efficiency from Tests (Excluding Machin/ chronous Machine Quantities from Tests (1967)

969)

cs of 3 Phase, $50 \mathrm{~Hz}$ Turbine Type Machines (196/

truction and Mounting Arrangements of (1972)

ection of Rotation (1972)

for Diameters of Shanks and Sizes of Driving Squares for

Rec. for Fundamental Welding Positions and Definitions of welds Made Wi/ Rec. for Limitation of Angles of Slope and engine Cylinders and Combustion Chambers, and Direction of Rec. for Determination of the Optical Rec. for Terminal Markings and Direction of ernal Combustion Engines-Designation of the Direction of olerances (196/ Rec. for Railway Rolling Stock Material umen/ Rec. for Instruments for the Measurement of Surface erms/ Rec. for Instruments for the Measurement of Surface Rec. for Surface

cally Sealed Metal Food Containers-Internal Diameters of ec. Spec. for Particular Types of Winding Wires: Enamelled Particular Types of Winding Wires: Self Fluxing Enamelled ding Wires: Heat or Solvent Bonding Self Fluxing Enamelled ec. Spec. for Particular Types of Winding Wires: Enamelled n / Rec. for Particular Types of Winding Wires: Enamelled Rec. for Particular Types of Winding Wires: Enamelled Rec. for Particular Types of Winding Wires: Enamelled n / Rec. for Particular Types of Winding Wires: Enamelled ec. Spec. for Particular Types of Winding Wires: Enamelled Types of Winding Wires: Heat or Solvent Bonding Enamelled Rec. for High Tensile Steel Chains c Dimensions of Winding Wires: Diameters of Conductors for Rec. for Packaging of Winding Wires: Containers for of Winding Wires: Maximum Over All Diameters of Enamelled c Dimensions of Winding Wires: Diameters of Conductors for Rec. Meth. of Test for Winding Wires: Enamelled Rec. for Dimensions of Hot Rolled Steel Bars: e of Series of Preferred Numbers and Those Containing More

Is and Cereal Products: Determination of Moisture Content

$r$ Green Coffee Beans-Determination of Moisture Content,

Std. for Cocoa Beans: Determination of Moisture Content
Rolling Stock Material: Wheel Sets for Trailer Stock (1

ISO

$\begin{array}{ll}\text { Rolling Stuck on Cumpletion of Construction and Before } & \text { IEC } \\ \text { Rolls (1969) } & \text { Rec. for Photographic Colour }\end{array}$

Rolls (1969)

Roofing and Cladding (1964)

Roofing and Cladding (1964)

Rec. for Photographic Black a ISO

ISO

Roofing and Cladding (1964)

Room Air Conditioners (1968)

Room Air Conditioners (1972)

Room Heating and Similar (1968)

Room Heights for Residential Buildings (1970)

Room (1963)

Rope for Aircraft Controls: Technical Spec. (1973)

$\begin{array}{ll}\text { ISO } \\ \text { Rec. for Asy } & \text { ISO } \\ \text { ISO }\end{array}$

Re IEC

for Electric Cooking CEE

ISO

Rope Thimhles (1972)

Rope Threaded Equipments 1 1/2 to 2 In. (38 to $51 \mathrm{Mm}$ ) (

Rope Threaded Equipments $7 / 8$ to $1 \mathrm{l} / 4 \mathrm{In}$. (22 to $32 \mathrm{Mm}$ )

Rec. for ISO

ISO

ISO

ISO

ISO

Res-Determination

Ropes and Cordage-Vocabulary (1971)

Ropes for General Purposes: Characteristics (1973)

Ropes for Test. (1969)

Ropes (1963)

Ropes (1963)

Ropes (1969)

Ropes (1969)

Ropes (1970)

Ropes (1970)

Ropes (1971)

Ropes (1971)

ISO

ISO

ISO

Rec. for ISO

ISO

ISO

Rec. for 3 Strand (Hawser Laid) ISO

Rosemary (197 I)

Rotary Drill Rods and Bits for Dry Drilling-Connectin

Rotary Drilling Equipment: Kellys (1972)

Rotary Drilling Equipment: Upper and Lower Kelly Cocks

Rotary Variable Capacitors: General Requirements for Te

Rotary Wafer Switches with Central Mounting; Maximum 12

Rotary Wafer Switches with 2 Hole Mounting; Maximum 26

Rotary Wafer Switches (Low Current Rating) with 2 Hole

Rotary Wafer Switches (Low Current Rating): General Req

Rotary Wafer Switches (Low Current Rating): Wafer Switc

Rotating Bar Bending Fatigue Test. (1969)

Rotating Electrical Machinery (1970)

Rec. for Tes

Rotating Electrical Machines -- Frame Numbers 56 to 400

Rotating Electrical Machines for Rail and Road Vehicles

Rotating Electrical Machines: Degrees of Protection by

Rotating Electrical Machines: Meth. for Determining Los

Rotating Electrical Machines: Meth. for Determining Syn

Rotating Electrical Machines: Meth. of Cooling (1969)

Rotating Electrical Machines: Noise Limits (1972)

Rotating Electrical Machines: Rating and Performance (1

Rotating Electrical Machines: Ratings and Characteristi

Rotating Electrical Machines: Symbols for Types of Cons

Rotating Electrical Machines: Terminal Markings and Dir

Rotating Tools with Parallel Shanks (1961)

Rotation and Slope for Straight Welds (1967)

Rotation for Welding Positions for Straight Manual Arc

Rotation of Engines and Propellers (1966)

Rotation of Essential Oils (1967)

Rotation of Rotating Machines (1972)

Rotation (1972)

Std. for Reciprocating Int

Rough Tyres (Tires) for Trailer Stock: Dimensions and T

Roughness by the Profile Meth.-Contact (Stylus) Instr

Roughness by the Profile Meth.-General Statements-T

Roughness (1966)

Round Cans (1972)

Round Copper with

Round Copper (1970)

Round Copper (1970)
Round Copper, with a Temperature Index of 155 (1970)
Round Copper, with a Temperature Index of 180 for Use I

Std. for Hermeti ISO

ISO

ISO

ISO

ISO

ISO

IEC

IEC

IEC

IEC

IEC

IEC

ISO

ISO

IEC

IEC

IEC

IEC

IEC

IEC

IEC

IEC

IEC

IEC

Rec ISO

ISO

ISO

ISO

ISO

IEC

ISO

ISO

ISO

ISO

IEC

Rec. Spec. for IEC

Round Copper, with a Temperature Index of 180 (1972)

Round Copper, with a Temperature Index of 220 (1972)

Round Copper, with High Mechanical Properties for Use
Round Copper, with High Mechanical Properties (1970)

Round Copper, with High Mechanical Properties (1970)

(Round Link) for Chain Conveyors and Coal Ploughs (1967)

Round Resistance Wires (1971)

Round Types (1968)

Round Winding Wires (1964)

Round Winding Wires (1965)

Round (1968)

Round, Metric Series (1969)

Rounded Values (1966)

(Routine Meth.) (1968)

(Routine Meth.) (1970)

(Routine Meth.) (1972)

$1005 / V I I$

16.5

1011

1009

393

395

859

378

$11.2 \mathrm{~F}$

1789

354

2020

2262

1720

1719

2307

2232

1968

2408

1142

346

345

1140

1141

1181

1346

1969

1970

1342

1717

2289

2290

$334-1$

$132-4$

132.5

$132-3$

132-1

$132-2$

1143

1680

72

$34-5$
34.2

34.4

34-6

$34-9$

34-1

34-3

34-7

237

627

598

482

592
34.8

1204

$1005 / I$

1880

1879

468

1361 
igh Voltage Alternating (iurrent Circuit Breakers: Type and f, (iontinuous Filament Yarn, Staple Pihre (Fiher) Type and its Continuous Filament and Staple Fibre (Fiber) Yarns and nce Class 5-Special/ Rec for Rolling Bearings-Double Rec. for Frames for Single or Double for Representation of ISO 7 Bit Coded Character Set on 12 970) mation Processing-Representation of 8 Bit Patterns on 12 Rec. for Rolling Bearings-Double Rec. for Rolling Bearings-Double Rec. for 7 Track 8 RPmm (200 Rec. for 9 Track $8 \mathrm{RPmm}(200$ Rec. for 9 Track 32 Pmm (800 8 and 32 RPmm (200 and 800 RPI), NRZI, and 63 RPmm (1600 nterchange -8 and 32 RPmm (200 and 800 RPI), NRZI, and 63 e (1971)

e (1971) Rec. for 7 Track 8 (1971)

ec. for 9 Track 8 Rec. for Vulcanized Rec. fo Std. for Raw

Rec. for Raw Natura

Rec. for Amount of Bale Coating on Natural Std. for Lined Industrial Rec. for Determination of Rate of Cure o Std. for Natural Rubber Latex-Determination of Dry Std for Wire Reinforced, Rec. for Raw Natura Std. for Lined Industria Std. for Lined Conductin Rec. for

on Length (1970) Rec. for Hydrostatic Test. of Rec. for Rec. for Rec. for

Conducting Wire (1971)

Rec. for Sampling of Raw Rec. for Pelletized Carbon Black for Use in the Rec. for Carbon Black for Use in the Rec. for Carbon Black for Use in the Rec. for Carbon Black for Use in the Rec. for Carbon Black for Use in the 0) r Maximu/ Rec. for Pelletized Carbon Black for Use in the Rec. for Sampling Bulk Shipments of Carbon Black for the . for Determination of Ash Content of Carbon Black for the $r$ Determination of Loss on Heating of Carbon Black for the rmination of Total Sulphur Content of Carbon Black for the Rec. for Pelletized Carbon Black for Use in the

Spec. for

r Conductors and a Rated Voltage Not Exceeding / Rec. for st of the Adhesion of Vulcanized Rubber to Metal Where the st of the Adhesion of Vulcanized Rubber to Metal Where the

2)

972) ec. for Preparation of Dry Films from Concentrated Natura Stability (1971)

and Residual Styrene (1972) ec. for Synthetic Rec. for Natural Rec. For Determination of Surface Tension of ved-Spec. (1971) ics (1972) Rec. for Natura Rec. for Natural Fabrics Coated with tion of the Impermeability to Water of Fabrics Coated with d Ageing and Simulated Service Test of Fabrics Coated with ng Strength and Elongation at Break of Fabrics Coated with r Piping (1970) Rec. for Rec. for General Purpose Agricultura ec. for Std. Atmospheres for the Conditioning and Test. of test Piece and Meth of Test of the Adhesion of Vulcanized test Piece and Meth. of Test of the Adhesion of Vulcanized Std. for

Rec. for General Purpose istance to Crack Growth of Vulcanized Natural or Synthetic stance to Flex Cracking of Vulcanized Natural or Synthetic Rec. for Determination of Copper in Compounded Rec. for Determination of Ash in Raw Natural

Rec. for Determination of Volatile Matter in Raw Natura Rec. for Sampling of Raw Natura

Rec. for Determination of Manganese in Compounded 1971)

Equipment for Unit Loads-Mobile Belt Conveyors (Canvas, endulum Meth. (1971)
Routine Tests (1972)

Roving Package (1971)

Rovings in the Form of

Rec. for $\mathrm{H}$ IEC

/Pling Batches or Consignments O ISO

/Ar Density of ISO

Row of Healds (1967)

Row Punched Cards (Information Processing) (1970) /Ec. ISO

Row Punched Cards (1971) Rec. for Infor ISO

Row Self-Aligning Ball-Radial Internal Clearance (1 ISO

Row Selfaligning-Radial Internal Clearance (1965)

RPI) Magnetic Tape for Information Interchange (1971)

RPI) Magnetic Tape for Information Interchange (1971)

RPI) Magnetic Tape for Information Interchange (1971)

RPI), Phase Encoded (1971) /R Information Interchange ISO

RPmm (1600 RPI), Phase Encoded (1971) /R Information I ISO

RPmm (200 RPI) Magnetic Tape for Information Interchang ISO

RPmm (200 RPI) Magnetic Tape for Information Interchang ISO

Rubber-Determination of Carbon Black-Pyrolytic Meth ISO

Rubber-Determination of Solvent Extract (1971)

Rubber-Sample Preparation (1972)

Rubber and Its Latex-Determination of Nitrogen (1971)

Rubber Bales-Requirement and Determination (1971)

Rubber Boots with General Purpose Oil Resistance (1972)

Rubber Compounds by the Shearing Disk Viscometer (1968)

Rubber Content (1972)

Rubber Covered Hydraulic Hose (1972)

Rubber Determination of Dirt (1971)

Rubber Footwear for Use at Low Temperatures (1972)

Rubber Footwear (1972)

Rubber Hose-Bore Sizes, Test Pressures and Tolerances

Rubber Hose (1970)

Rubber Hoses-Bending Test (1970)

Rubber Hoses for Aircraft Ground Fueling Without Static

Rubber Hoses for Oil Suction and Discharge (1971)

Rubber in Bales (1971)

Rubber Industry-Determination of Fines Content (1970)

Rubber Industry-Meth. of Test for Sieve Residue (1970

Rubber Industry-Sampling Packaged Shipments (1970)

Rubber Industry-Spec. for Sieve Residue (1970)

Rubber Industry-Spec. Limits for Loss on Heating (197

Rubber Industry Delivered in Bulk or in Bins-Spec. Fo

Rubber Industry (1969)

Rubber Industry (1969)

Rubber Industry (1969)

Rubber Industry (1969)

Rubber Industry: Determination of Pour Density (1970)

Rubber Insulated Cables and Flexible Cords (1968) CEE

Rubber Insulated Flexible Cables and Cords with Circula IEC

Rubber Is Assembled to One Metal Plate (1968) /. of Te ISO

Rubber Is Assembled to 2 Metal Plates (1968) /H. of Te ISO

Rubber Latex-Determination of Dry Rubber Content (197

H. of Te ISO

ISO

Rubber Latex-Determination of KOH Number (1972)

Rubber Latex-Determination of Manganese (1971)

Rubber Latex-Determination of Mechanical Stability (1

Rubber Latex (1966)

Rubber Latices-Codification (1972)

Rubber Latices-Determination of High Speed Mechanical

Rubber Latices-Determination of Sludge Content (1971)

Rubber Latices-Determination of Volatile Unsaturates

Rubber Latices (1970)

Rubber Latices (1970)

Rubber Latices, Centrifuged and Creamed, Ammonia Preser

Rubber Latices, Evaporated Preserved-Spec. (1971)

Rubber or Plastics-Determination of Roll Characterist

Rubber or Plastics (1970)

Rec. for Determina ISO

Rubber or Plastics (1970)

Rec for Accelerate

Rubber or Plastics (1971)

ffor Determination of Breaki ISO

Rubber Sealing Rings for Joints in Asbestos Cement Wate ISO

Rubber Spray Hose (1970)

Rubber Test Pieces (1966)

Rubber to Metal Where the Rubber Is Assembled to One Me

Rubber to Metal Where the Rubber Is Assembled to 2 Meta

Rubber Vocabulary (1972)

Rubber Water Hose (1970)

Rubber (De Mattia Type Machine) (1959)

Rubber (De Mattia Type Machine) (1959)

Rubber (Vulcanized and Unvulcanized) (1970)

Rubber (1962)

Rubber (1962)

Rubber (1962)

Rubber (1970)

Rubber, Plastic, Etc.) Safety Code (1972)

Rubbers-Accelerated Ageing or Heat Resistance Tests

Rubbers-Determination of Rebound Resilience-Lupke P
$56-4$

1886

1889

568
1679

2021

1646

465

1861
1862

1863

1864

1864

1861

1862

1408
1407

1796

1656

1434

2025

667

126

1436

249

2252
2024

1307

1402

1746

1825

1823

1795

1435

1437

1310

1867

1868

1866

1124

1125

1126

1138

1306

2

245
813

814

1654

126

127

1655

35

498
2438

2006

2005

2008

1409

1652

2004

2286

1420

1419

1421

1398

1401

471

813

814

1382

1403

133

132

1396

247

248

250

1397

2388
188

1767 
Rec, for Determination of the Stiffness of Vulcanized c. for Betermination of Viscosity of Natural and Synthetic

Rec. for Determination of Hardness of Vulcanized Rec. for Determination of Hardness of Vulcanized Rec. for Determination of the Permeability of Vulcanized

Rec. for Determination of Adhesion of Vulcanized

Rec. for Determination of Adhesion of Vulcanized Rec. for Determination of Compression Set of Vulcanized Rec. for Determination of Modulus in Shear of Vulcanized ation of Tear Strength of Vulcanized Natural and Synthetic nation of Tear Strength of Small Test Pieces of Vulcanized Rec. for Determination of Hardness of Vulcanized mination of Tensile Stress Strain Properties of Vulcanized f Test for Temperature Limit of Brittleness for Vulcanized

Rec. for Time Lapse Between Vulcanization and Test. of Deflection at Normal and High Temp/ Rec. for Vulcanized 971) Organization Rec. for Steam Turbines:

Electrically Powered Vehicles (1970) d Vehicles (1971)

ec. for High Voltage Altemating Current Circuit Breakers: tion of Construction and Before Entry into Serv/ of Units and a Selection of the Decimal Multipl/ hazards When Dealing with Electronic Equipment Spec. for Statutes and

aps, Plans and Geological Cross Sections-Part I: Genera uous Mechanical Handling Equipment-Safety Code-Genera Pure Expanded Corkboard-Determination of the Modulus of Rec. for Creep Stress lent Terms Used in the Plastics Industry (English, French, lent Terms Used in the Plastics Industry (English, French, lent Terms Used in the Plastics Industry (English, French, lent Terms Used in the Plastics Industry (English, French, lent Terms Used in the Plastics Industry (English, French, alphabetical Indexes of Equivalent Terms (English, French, International Rec. for Polarimetric ve Gas Atmospheres: Spark Test Apparatus for Intrinsically Rec. for Continuous Mechanical Handling Equipment nt for Loose Bulk Materials-Belt Feeders and Conveyors quipment: Bins and Bunkers, Silos and Hoppers, Bin Gates strand Floor Mounted Truck Conveyors (Chain Above Floor) aking or Reciprocating Feeders and Oscillating Conveyors oads-Overhead Twin Rail Chain Conveyors (Power and Free) pment for Unit Loads-Overhead Monorail Chain Conveyors

uipment for Loose Bulk Materials-Mobile Belt Conveyors

Single Strand Floor Truck Conveyors (Chain Below Floor)

Loose Bulk Materials-Scraper and En Masse Conveyors

ds-Mobile Belt Conveyors (Canvas, Rubber, Plastic, Etc.) ing Equipment for Loose Bulk Materials-Apron Conveyors pment for Loose Bulk Materials-Picking Table Conveyors equipment for Loose Bulk Materials-Hydraulic Conveyors

onic Equipment (Domestic and Similar General Us/ t Servicing and Engine Starting (1961)

70) Rec. for Definition and Marking of Rec. for Principles of Criticality

general Requirements (1970)

particular Requirements for Floor Treatment And/ particular Requirements for Vacuum Cleaners (19/ particular Requirements for Electric Irons (197/ particular Requirements for Spin Extractors (19/ particular Requirements for Dishwashers (1971) particular Requirements for Ranges (1971) particular Requirements for Washing Machines (1) particular Requirements for Electric Shavers, H/ particular Requirements for Toasters, Grills, W/ ther with Gauges for the Control of Interchangeability and Rec. for Electrical Std. for General Principles for the Verification of the

971)

\section{(1971)}

elated Equipment for Domestic and Similar Gene/ elated Equipment for Domestic and Similar Gener/ 966)

est Meth. (1967)

rigerators and Food Freezers for Household and / m Air Conditioners (1972) Rec. for Rec. for Rec. for Rec. for

Rubbers-Determination of Resistance to Ozone Cracking

Rubbers-Meth. of Test for Resistance to I.iquids (1971

Rubbers and Latices-Nomenclature (1971)

Rubbers at I.ow Temperature (Gehman Test) (1971)

Rubbers by the Shearing Disk Viscometer (1963)

Rubbers of High Hardness (85 to 100 IRHD) (1971)

Rubbers of Low Hardness (10 to 35 IRHD) (1971)

Rubbers to Gases (Constant Volume Meth.) (1971)

(o Rigid Plates in Shear (Quadruple-Shear Test)

Rubbers to Textile Fabrics (1969)

Rubbers Under Constant Deflection at Low Temperatures (

Rubbers (Bonded Quadruple Shear Test Piece) (1971)

Rubbers (Crescent Test Piece) (1957)

Rubbers (Delft Test Piece) (1968)

Ec. for Determin ISO

Rubbers (1968)

Rubbers (1968)

Rubbers (1968)

Rec for

Rec. for Deter ISO

Rec. for Meth. O ISO

Rubbers: Determination of Compression Set Under Constan

Rubber: Raw and Unvulcanized-Rapid Plasticity Test (1

Rules and Procedures of the CISPR (1971)

Rules for Acceptance Tests (1962)

Rules for Construction of Stationary Boilers (1968)

Rules for Electric Traction Equipment (1968)

Rules for Ohmic Resistors Used in the Power Circuits of

Rules for Rotating Electrical Machines for Rail and Roa

Rules for the Selection of (For Service) (1971)

Rules for the Test. of Electric Rolling Stock on Comple

Rules for the Use of Units of the International System

Rules for Traction Transformers and Reactors (1969)

Rules for Transport, Erection and Maintenance (1971)

Rules of Behaviour (Behavior) with Respect to Possible

Rules of Procedure of the CEE (1961)

Rules of Representation (1968)

Rules (1970)

Rupture by Bending (1971)

Rupture Test. of Steel at Elevated Temperatures (1961)

Russian) (D: Corresponding German Terms) (1964)

Russian) (NL: Corresponding Dutch Terms) (1968)

Russian) (Pl: Corresponding Polish Terms) (1963)

Russian) (1969)

Rec. for List of Equiva

ISO

ISO

ISO

CISP

IEC

ISO

IEC

IEC

IEC

IEC

IEC

ISO

IEC

IEC

IEC

CEE

Saccharimeters (1970)

Safe Circuits (1972)

Safety Code-Gene lof Gears-Geometrical DefinitionsRec. for ISO ISO

iva ISO

QQuiva ISO

Quiva ISO

Safety Code (1970)

Safety Code (1971)

Safety Code (1971)

Safely Code (1971)

Safety Code (1971)

Safety Code (1972

Safety Code (1972)

Safety Code (1972)

Safety Code (1972)

Safety Code (1972)

Safety Code (1972)

Safety Code (1972)

Safety Colours (Colors) (1964)

Rec for

Safety Equipment for Splash Proof Mains Operated Electr

Safety Features for Ground Power Units for d.c. Aircraf

Safety Film for Motion Picture Uses (1966)

Safety in Handling and Processing Fissile Materials (19

Safety Isolating Transformers (1964)

Spec. for

Rec. for

Rec. for

Rec, for

Rec. for

Rec. for

Rec. for

Rec. for

Rec. for

Safety of Household and Similar Electrical Appliances:

Safety of Household and Similar Electrical A ppliances:

Safety of Household and Similar Electrical Appliances:

Safety of Household and Similar Electrical Appliances:

Safety of Household and Similar Electrical Appliances:

Safety of Household and Similar Electrical Appliances:

Safety of Household and Similar Electrical Appliances:

Safety of Household and Similar Electrical Appliances:

Safety of Household and Similar Electrical Appliances:

Safety of Household and Similar Electrical Appliances:

Safety of Lampcaps (1970)

Safety of Office Machines (19

Safety of Structures (1973)

/ Lamp Caps and Holders Toge

Safety Requirements for Electric Fans and Regulators (1

Rec. for

Spec. for
Rec. for

Rec. for

Rec. for

Std. for

Safety Requirements for Electronic Measuring Apparatus

Safety Requirements for Mains Operated Electronic and R

Safety Requirements for Mains Operated Electronic and $R$

Safety Requirements for Radio Transmitting Equipment (1

Safety Requirements for Radio Transmitting Equipment: T

Safety Requirements for the Electrical Equipment of Roo

1431

1817 
Rec. for Refrigerating Plants. Rer. for Symhols, Dimensions and l.ayout for m. Siructure, and Results of the CEE (Electrical Equipment ther with Gauges for the Control of Interchangeability and 68) ther with Gauges for the Control of Interchangeability and Rec. for Pulps: Determination of Rec. for Butter-Determination of ocedures for Electronic Components and Equipment: Test Ka: or Potassium Hydroxide for Industrial Use: Sampling-Test for Crude Vegetable Oils and Fats: Preparation of Contract Rec. for Determination of Moisture in the Analysis Rec. for Determination of Moisture in the Analysis Rec. for Determination of Moisture in the Analysis Rec. for Tea-Preparation of Ground Std. for Raw Rubber -

Rec. for Essential Oils-Meth. of Test-Preparation of rily Used for the Production of Aluminium-Preparation of arily Used for the Production of Aluminium: Preparation of c. for Sodium Hydroxide for Industrial Use: Preparation of for Potassium Hydroxide for Industrial Use: Preparation of for Essential Oils-Meth. of Test-Preparation of Sample ace Active Agents in Powder Form: Preparation of a Reduced roxide for Industrial Use: Preparation and Storage of Test roxide for Industrial Use: Preparation and Storage of Test ustrial Use-List of Meth. of Test and Preparation of the Std. for Leather: Laboratory c. for Sodium and Potassium Silicates for Industrial Use hatic Polyhydric Alcohols-Glycerine for Industrial Use Rec. for Selection and Preparation of ec. for Oleaginous Seeds: Reduction of Contract Samples to for Paints and Varnishes-Examination and Preparation of silicates for Industrial Use-Determination of Density of Rec. for Oleaginous Seeds: Reduction of Contract rec. for Meth. of the Conditioning of Paper and Board Test bonate for Industrial Use: Preparation and Storage of Test sed for the Production Of; Preparation and Storage of Test

(Natural and Artificial)-Preparation and Storage of Test

Ores: Determination of Hygroscopic Moisture in Analytical on for / Rec. for Tea Std. for Potassium Hydroxide for Industrial Use: y of Terms Relating to Solid Mineral Fuels-Part II: Coal Rec. for Machine Made Textile Floor Coverings -
Rec. for Guide to the Use of ISO Rec. 390, 64) Rec. for expanded Pure Agglomerated Thermal CorkRec. for Textile Glass-Procedustics, Yarn, Staple Fi/
industry (1969)

Rec. for Textile Glass-Procedure for

Rec. for Meth. of Fiber Rec. for Meth. of Rec. for

wagons (I963)

Rec. for Chemical Analysis of Copper and Its Alloys Rec. for

Std. for Cereals Pulses. Rec. for Rec. for

Rec. for Carbon BIack for Use in the Rubber Industry Rec. for Meth. of Rec. for Meth. of Rec. for Rec. for Cereals:

Rec. for Liquid Chlorine for Industrial Use-Meth. of Rec. for Essential Oils:
Rec. for Oilseeds. Rec. for Oilseeds.
its and Vegetables: Rec. for Fresh Fruits and Vegetables: Rec. for Milk and Its Products: Rec. for Spices and Condiments: Rec. for Paints and Varnishes. Rec. for Granulated Cork . Std for Gylcerols for Industrial Use-Meth, of Virgin Cork, Ramassage, Gleanings, Refuse and Corkwaste nd Transplantation Made of Peat and Other Plant Material Std. for Cocoa Beans: aluminum) Zinc Alloy Castings and Mechanical Properties of Rec. for Aluminium (Aluminum) Alloys Std. for Magnesium Alloy Std. for Aluminium (Aluminum) Alloy c. for Electrical Apparatus for Explosive Gas Atmospheres: c. for Electrical Apparatus for Explosive Gas Atmospheres: -Part IV: Fittings (1966) tion (1966)

Rec. for Cast Iron Rec. for Cast Iron Rec. for Building and

Rec. for Asbestos Cement Pipe Fittings for Building and ional Signs to Be Used in Schemes for the Installations of
Safety Requirements (1971)

Safety Sign: (1967)

Safety) (1961)

Safety: Gauges (1969)

Safety: Lam pholders (1969)

Saleable Mass, in Lots, of Pulp Baled in Sheet Form (19 ISO

Salt Content (Reference Meth.) (1971) ISO

Salt Mist (1964) /Ec. for Basic Environmental Test. Pr IEC

Sample-Preparation of the Main Solution for Carrying ISO

Sample for Analysis (1968)

Sample of Coal by the Direct Gravimetric Meth. (1963)

Rec. ISO

ISO

(1963)

Sample of Coke (1968)

Sample of Known Dry Matter Content (1970)

Sample Preparation (1972)

$1 \mathrm{SO}$

ISO

ISO

ISO

ISO

Sample Solution for Analysis by Means of Attack by Hydr

$\begin{array}{lrr}\text { Sample Solution for Analysis (1968) } & \text { /Minum) Oxide Prim } & \text { ISO } \\ \text { Sample Solution (1969) } & \operatorname{Re} & \text { ISO }\end{array}$

Sample Solution (1969)

Sample Solution

Sample (1967)

Sample (1969)

Sample (1969)

Sample (1972)

Samples-Location and Identification (1972)

Samples and Meth. of Test-General (1970)
Samples and Test Meth.-General (1970)

Samples and Test Pieces for Wrought Steel (1964)

Samples for Analysis (1968)

Samples for Test. (1970)

Samples in Solution-Meth. Using Density Hydrometer-

Rec. ISO

Rec. ISO

Rec. for Surf ISO

Rec. for Sodium Hyd ISO

Rec. for Potassium Hyd ISO

amples to Samples for Analysis (1968)

Samples (1961)

Samples (1968)

Samples (1968)

Samples (I970)

Rec. for Sodium Car ISO

Aluminium (Aluminum) Oxide Primarily U ISO

Rec. for Cryolite ISO

mples (1973)

Sid. for Iron ISO

ISO

Sampling-Test Sample-Preparation of the Main Soluti ISO

Sampling and Analysis (197I) Rec. for Vocabular ISO

Sampling and Conditioning of Ropes for Test. (1969)

Sampling and Cutting Specimens for Physical Tests (1971

Sampling and Inspection of Asbestos Cement Products (I9

Sampling and Inspection of Asbestos Cement Products (I9

Sampling and Meth. of Test (1969)

Sampling and Packing (I972)

ISO

Sampling Batches or Consignments Of, Continuous Filamen ISO

Sampling Bulk Shipments of Carbon Black for the Rubber ISO

Sampling for Test. (1969)

Sampling Manganese Ores; Part I: Ore Loaded in Freight

Sampling of Conveyor Belts (1962)

Sampling of Copper Refinery Shapes (I971)

Sampling of Latex (1968)

Sampling of Milled Products (1972)

Sampling of Raw Natural Rubber (1962)

Sampling of Raw Rubber in Bales (1971)

Sampling Packaged Shipments (1970)

Sampling Paper and Board for Test. (1968)

Sampling Raw Cotton for Test. (1961)

Sampling Raw Materials for Paints and Varnishes (1968)

Sampling (As Grain) (1969)

Sampling (For Determining Only the Volumetric Content)

Sampling (1961)

Sampling (1967)

Sampling (1968)

Sampling (1969)

Sampling (1969)

Sa mpling (1969)

Sampling (1970)

Sampling (1971)

Sampling (1972)

Sampling (1972)

Sampling (1972)

Sampling (1973)

Sand Cast Test Pieces-Mechanical Properties (1971)

Sand Castings-Reference Test Bar (1972) ISO

Sand Castings: Reference Test Bar (1972)

Sand Filled Apparatus (1967)

Sand Filled Apparatus: Use of a Screen (1967)

Sanitary Pipe Fittings for Waste Water and Ventilations

Sanitary Pipes and Fittings for Waste Water and Ventila

Sanitary Pipes in Asbestos Cement (1964)

Sanitary Purposes (1964)

Sanitary Systems in Ships (1968)

Rec. for Convent ISO

$\operatorname{Re}$ IEC

Re IEC

ISO

ISO

ISO
1662

557

$61-3$

$61-2$

801

1738

68-2.11

2466

661

331

348

687
1572

1796
356
2073

2073

804

978
989

356

607

977

988
2197

2197

2418

1686

1614

377

664

1513

1687

664

187

739

802
1619

2596

1839

2466

1213/II

1142

1957

1260

390

966

2219

1886

1124

1130

309

282

1811

123

2170

250

I795

I3IO

186

220

842

950

1552

212

542

874

707

948

951

1512

2067

2096

2385

2259

2292

121

2147

2377

2379

$79.5 \mathrm{~A}$

$531 /$

531

392

784 
Rec. for Coniferous Sawn (Sawed) Timber-Sizes-Terms and Definitions (1969)

Rec. for Coniferous Rec. for Coniferous Rec. for Coniferous Rec. for Coniferous Rec. for Coniferous Rec. for Coniferous Std. for Metal Slitting Rec. for Enclosed Rec. for Enclosed Rec. for Verification of Rockwell B and C

1969) Rec. for Verification of Rockwell Superficial N and $T$ 961) ardized Blocks to Be Used for Rockwell Superficial $\mathrm{N}$ and $\mathrm{T}$ andardized Blocks to Be Used for Rockwell B and C Hardness Rec. for Long Enclosed Rec. for Short Enclosed ing Direct Indications by Means of a Pointer and Graduated Rec. for itectural and Building Drawings: Presentation of Drawings: Rec. for Rockwell Hardness Test (B and C

Rec. for Rockwell Superficial Hardness Test (N and T nts Normal A/ Rec. for Cinematography-Sound Records and (1965) Rec. for Rec. for Certification $\begin{array}{ll}\text { hips (1967) } & \text { Rec. for Conventional Signs: to Be Used in } \\ \text { ips (1968) } & \text { Rec. for Conventional Signs to Be Used in }\end{array}$ Ships (1967) ec. for Mathematical Signs and Symbols for Use in Physical Rec. for International Electrotechnical Vocabulary: Rec. for Surface Active Agents: ontaining Geiger-Muller or Proportional Counter Tubes or Rec. for Dimensions of Lead Acid Motor Means of Venturi Tubes-Classical Tubes Used Outside the s Mechanical Handling Equipment for Loose Bulk Materials Rec. for Paints and Varnishes Rec. for Microcopy: Measurement of the explosive Gas Atmospheres: Sand Filled Apparatus: Use of A $s$ and Cables with Solid or Stranded Conductors, Insulated, Rec. for Pure Tone tures (1972) Std. for Wire and Plate Rec. for Terminals with

us Mechanical Handling Equipment for Loose Bulk Materials: Rec. for Dimensions of Rec. for Edison Spec. for Edison

nd Finishing (1966)

and In./S (I970)

spection of Closures (19/

ts (1973)

Sawn Timber: Sizes-Meth. of Measurement (1968)

Sawn Timber: Sizes-Tolerances and Shrinkage (1968)

Sawn (Sawed) Timber-Defects-Classification (1969)

Sawn (Sawed) Timber-Defects-Measurement (1969)

Sawn (Sawed) Timber-Defects-Terms and Definitions (

Sawn (Sawed) Timber-Sizes-Terms and Definitions (19

Saws with Fine and Coarse Teeth-Metric Series (1972)

Scale Calorimeter Thermometers (I968)

Scale General Purpose Thermometers (1970)

Scale Hardness Test. Machines (1968)

Scale Hardness Test. Machines (1969)

Scale Hardness Test. Machines (1970)

Scale Test. Machines (1968)

Scale Thermometers for Precision Use (1968)

Scale Thermometers for Precision Use (1968)

Scale (Working Equipment Category) (1970)

Scales and Sizes for Plotting Frequency Characteristics

Scales (1969)

Scales) for Steel (1968)

Scales) for Steel (1969)

Scanning Area of 35 mm Double Width Push Pull Sound Pri

Schedule for High Pressure Mercury Vapour (Vapor) Lamps

Schedule for Sodium Lamps (Integral Type) (1965)

Scheme (1961)

Schemes for the Installations of Pipe Line Systems in S

Schemes for the Installations of Sanitary Systems in Sh

Schemes for the Installations of Ventilation Systems in

Sciences and Technology (1961)

Scientific and Industrial Measuring Instruments (1958)

Scientific Classification (1968)

Scintillation Detectors (1967)

Scooter Batteries (1965)

Scraper and En Masse Conveyors-Safety Code (1972)

Scratch Test (1970)

Screen Luminance of Microfilm Readers (1968)

Screen (1967)

Screened, Single or One Pair (1969) /H: Equipment Wire

Screening Audiometers (1965)

Screens for Industrial Purposes-Nominal Sizes of A per

Screw Clamping for External Conductors (1964)

Screw Conveyors (1969)

Screw Cores Made of Ferromagnetic Oxides (1966)

Screw Lampholders (1967)

Screw Lampholders (1969)

Screw Propellers, Manufacturing Tolerance for Casting a

Rec. for Shipbuilding Details: Ship
Rec. for Tapping

Rec. for Finishes with External

Rec. for ISO General Purpose

Rec for ISO General Purpose Metric

Std. for ISO General Purpose Metric

Std. for ISO General Purpose Metric Rec. for ISO Miniature

Std. for Drills for Use Prior to Tapping

Rec. for ISO General Purpose Metric Rec. for ISO In. olts and Nuts (Diameter Range 0.06 to $6 / \quad$ Std. for ISO In.
l Types (1969) Rec. for ISO General Purpose Metric al Bolt and Nut Thre/ Rec for ISO General Purpose Metric

969) Rec. for ISO General Purpose Metric Std. for Std. for Spiral Ratchet n the Threa/ Rec, for Pipe Threads for Gas List Tubes and Rec. for Malleable Cast Iron Pipe Fittings Rec. for Steel Sockets

(I957)

. IS (1970)

./S (19/ Rec. for Slotted Countersunk (Flat) Head Tapping
Rec. for Slotted Raised Countersunk (Oval) Head Tapping ches (1970)

Rec for Slotted Pan Head Tapping Rec. for Assembly Tools for Bolts and Rec. for Assembly Tools for Bolts and Rec. for Hexagon Head Tapping Rec. for Slotted Cheese (Fillister) Head Rec. for Hexagon Head Tapping Rec. for Slotted Pan Head Std. for Slotted Headless Std. for Hexagon Socket Set Std. for Assembly Tools for Rec. for Assembly Tools for
Screw Thread for Glass Containers and Gauges for the in Screw Threads-Basic Profile (1969)

Screw Threads-Gauging (I970)

Screw Threads-General Plan (1973)

Screw Threads-Selected Sizes for Screws, Bolts and Nu

Screw Threads (1970)

Screw Threads (1972)

Screw Threads: Basic Dimensions (I968)

Screw Threads: Basic Dimensions (1968)

Screw Threads: General Plan and Selection for Screws, B

Screw Threads: Tolerances, Deviations for Constructiona

Screw Threads: Tolerances, Limits of Sizes for Commerci

Screw Threads: Tolerances, Principles and Basic Data (I

Screwdriver Bits, for Slotted Head Screws, with Male He

Screwdriver Blades for Slotted Head Screws (1972)

Screwdriver Ends (1972)

Screwed Fittings Where Pressure Tight Joints Are Made $\mathrm{O}$

Screwed in Accordance with ISO Rec. R7 (1957)

Screwed in Accordance with ISO Rec. R7: Minimum Lengths

Screwing Dies and Hand-Operated Die Stocks (1973)

Screws-Dimensions in Millimetres (Millimeters) and in

Screws-Dimensions in Millimetres (Millimeters) and in

Screws-Dimensions in Millimetres (Millimeters) and in

Screws-Driving Squares for Power and Hand Socket Wren

Screws-Hexagon Drive Ends for Power Types (1970)

Screw s-In. Series $(1970)$

Screws-Metric Series (1970)

Screws-Metric Series (1970)

Screws-Metric Series (I970)

Screws-Metric Series (1972)

Serews-Metric Series (1972)

Screws and Nuts-Forged and and Tubular Socket Wrenche

Serews and Nuts-Nomenclature (I971) 
r. for Mechanical l'roperties of Fastencrs-Part 1: Bolts, $\operatorname{lin}(19(8)$ Kec. for Nominal t. Rec. for Nominal I.engths for Bolts,
Std. for Screwdriver Blades for Slotted Ilead
for LSO In. Screw Threads: General Plan and Selection for Rer. for Bolts,
ngths for Bolts, General Purpose Metric Scrcw Threads-Selected Sizes for for Mechanical Properties of Fasteners: Marking of Bolts, Std. for Screwdriver Bits, for Slotted Head Rec. for Hexagon Socket Head Cap

and Screws: Metric Series (1968) Rec. for Bolts and ances: Particular Requirements for Floor Treatment and Wet ilding Details: Test. of Toughened Glasses for Ships Side r Shipbuilding Details-Toughened Glasses for Ships Side Rec. for Shipbuilding Details-Ships Rec. for Shipbuilding Details for Rec. for Shipbuilding Details for es, Kenter Type (1957) Links (1957) for Magnetic Compasses and Binnacles, Class A, for Use in Rec. for Magnetic Compasses and Binnacles for Rec. for Flat ment of the Electrical Properties of Microwave Tubes: Disk 72) Std. for und Cans.(1972)

Std. for Hermetically Rec. for Hermetically ells: General Requirements and Test Meth. (1971/
ells: Dimensions (1972) ells: General Requirements and Test Meth. (1971/ Rec. for
ells: Dimensions (1972) cized Polyvinyl Chloride (PVC) Pressure Pipes with Elastic on (1971) rances) (197/ nces) (1969) g (1970) Rec. for Elastomeric es-Class $2 /$ -Class $1 \mathrm{~T} /$ blies (1968) Rec. for Dimensions of Elastomeric Toroidal Rec. for Dimensions of Elastomeric Toroidal Rec. for Rubber Rec. for Dimensions of Elastomeric Toroidal Rec. for Dimensions of Elastomeric Toroidal Rec. for rocedures for Electronic Components and Equipment: Test $Q$ : Rec. for Rolling Bearings-Radial Types with Shields or Rec. for Dimensions of nit Length (19/ Rec. for Plain End Steel Tubes, Welded or Rec. for Steel Pipes for Gas, Water and Sewage, Welded or Rec. for Rec. for Motorists Rec. for Aircraft

Rec for Automobile-Spark Plug M 14 X 1.25 with Flat std. for Automobiles: Spark Plugs M 14 X 1,25 with Conical Std. for Automobiles: Spark Plugs M 18 X 1,5 with Conical

Std. for Automobiles: Spark Plugs M 10 X 1 with Flat

Std. for Automobiles: Spark Plugs M 12 X 1,25 with Flat for Automobiles: Compact Spark Plugs M 14 X 1,25 with Flat automobiles: Compact Spark Plugs M 14 X 1,25 with Conical Rec. for Rolling Bearings-Thrust Bearings with Flat rec. for Rolling Bearings-Thrust Ball Bearings with Flat rec. for Rolling Bearings-Thrust Ball Bearings with Flat c. for Pesticides Considered Not to Require Common Names Rec. for Statistical Vocabulary and Symbols Rec. for Paper Vocabulary:

Rec. for Surface Active Agents: Technical Sodium Rec. for Copper and Its Alloy Tubes of Circular Rec. for g and Cladding (1964)

Rec. for Asymmetrical

Welds Statically Loaded in Such a Way That the Transverse inuous Filaments Constituting a Textile Glass Yarn-Cross 88 of Oxide Coatings-Nondestructive Measurement by Light nery-Terms for Serrated Bars and Dimensions of the Cross Machines (1969) Rec. for Basic Arrangement for the Alphanumeric Rec. for Numeric
Rec. for Lengths of $\mathrm{Y}$-t Conditi/ Rec. for Quartz Crystal Units for Oscillators: ontrol and Auxiliary Circuits, Including Contactor Relays) s for Oscillators: Section 1-Std. Valves and Conditions: Rec. for Quartz Crystal Units for Oscillators: Rec. for Quartz Crystal Units for Oscillators: or Tensile Test. of Copper and Its Alloy Tubes of Circular ted Cables (1966) Rec. for Nominal Cross and Dimensions (1969) Rec. for ions for 1 Rec. for Shipbuilding Details-Dimensions and art 2: Unequal Leg Angles-Metric Series-Dimensions and Part I: Equal Leg Angles-Metric Series-Dimensions and ns-Parallel Flange I Beams-In. Series-Dimensions and allel Flange Column Sections-In. Series-Dimensions and ections-Unequal Leg Angles-In. Series-Dimensions and Sections-Equal Leg Angles-In. Series-Dimensions and
Screws and Studs (1968)

Screws and Studs; Dimensioning (196J)

Screws and Studs: Thread I,engths for General Purpose Bo Screws (1972)

Screws, Bolts and Nuts (Diameter Range 0.06 to 6 In.) (

Screws, Bolts and Nuts (1973)

Screws, Studs and Nuts (1969)

Screws, with Male Hexagon Drive (1972)

Screws: Metric Series (1968)

Screws: Radii Under the Head for General Purpose Bolt

Scrubbing Machines for Household and Similar Purpose (1

Scuttles and Fixed Lights by the Punch Meth. (1967)

Scuttles and Fixed Lights (1969)

Scuttles (1971)

Sea Navigation-Anchor Chains-Lugless Joining Shackl

Sea Navigation and Inland Navigation Anchor Chains-St

Sea Navigation (1965)

Sea Navigation: Vocabulary (1969)

Seal for Hydraulic Couplings (1970)

Seal Tubes (1972)

Rec. for Measure

Sealed Metal Food Containers-Internal Diameters of Ro

Sealed Metal Food Containers (1959)

Sealed Nickel Cadmium Cylindrical Rechargeable Single C

Sealed Nickel Cadmium Cylindrical Rechargeable Single C

Sealing Ring Type Joints: Minimum Depths of Engagement

Sealing Ring Type Joints: Minimum Depths of Engagement

Sealing Rings for Aircraft-Packaging and Identificati

Sealing Rings for Aircraft-(In. Series-Class 2 Tole

Sealing Rings for Aircraft (In. Series-Class 1 Tolera

Sealing Rings for Joints in Asbestos Cement Water Pipin

Sealing Rings for Pipe Fittings in Aircraft-(In. Seri

Sealing Rings for Pipe Fittings in Aircraft (In. Series

Sealing Test for Pressurized Waveguide Tubing and Assem

Sealing Wire (1962)

Sealing (1968)

Rec for Basic ISO

Seals-Outside Diameter Tolerances-Normal and Tolera

Seam Welding Wheel Blanks (1968)

Seamless-General Table of Dimensions and Masses Per U

Seamless (1967)

Seat Belt Assemblies for Motorists (1970)

Seat Belts with Retractors (1970)

Seat Rails and Pins (1968)

Seating (1970)

Seating (1972)

Seating (1972)

Seating (1973)

Seating (1973)

Seating (1973)

Seating (1973)

Seats-Boundary Dimensions (1966)

Seats-Normal Tolerances (196I)

Seats-Tolerances: Part 2-Tolerance Classes 6,5 and

Second List (1969)

Second Series of Terms and Symbols (1970)

Second Series of Terms (1959)

Secondary AlkyIsulphates: Meth. of Analysis (1968)

Section-Flattening Test (1971)

Section Checking of V-Belts (1962)

Section Corrugated Sheets in Asbestos Cement for Roofin

Re ISO

ISO

ISO

ISO

ISO

ISO ISO

Rec. ISO

ISO

ISO

ISO

IEC

$/ \mathrm{U}$ ISO

Rec. Fo ISO

ISO

ISO

ISO

Rec. ISO

ISO

ISO

IEC

IEC

IEC

ISO

ISO

ISO

ISO

ISO

ISO

IEC

ISO

ISO

ISO

ISO

ISO

ISO

ISO

ISO

Std. ISO

Std. for ISO

ISO

ISO

ISO

Re ISO

ISO
ISO

ISO

ISO

ISO

ISO

Section Is Not Under Any Normal Stress

Section Meth. (1971)

/F Staple Fibres (Fibers) or Cont ISO

Section Microscope (1971) /Ys-Measurement of Thickne ISO

Section of Keyboards Operated with Both Hands (1971)

Section of Ten Key Keyboards for Adding and Calculating

Section V-Belts

Section 1-Std. Valves and Conditions: Section 2-Tes

Section 1: Push Buttons and Related Control Switches (1

Section 2-Test Conditions (1962)

Section 3-Guide to the Use of (1962)

Section 4: Std. Outlines (1962)

Section (1964)

Sectional Areas and Composition of Conductors of Insula

Sectional Beams for Warp Knitting Machines: Terminology

Sectional Properties of Aluminium (Aluminum) Alloy Sect

Sectional Properties (1968)

Sectional Properties (1969)

Sectional Properties (1969)

Sectional Properties (1969)

Sectional Warping Machines-
TT Rolled Steel Sections-

/Ons of Hot Rolled Steel S ISO

ISions of Hot Rolled Steel ISO

- Maximum Usable Width (1971

898/I

225

88

2380

26

$898 /$ III

2351

861

885

$335 \cdot 10$

614
1095

1751
39

40

449

1069

1941

235-3

1198

136

285- 1

285-2

2045

2048

1749

1800

1078

1398

1799

1077

261

68.2.17

1648

693

336

559

1231

1534

837 
mbols for lisc on Detailed Maps, Plans and Geological Cross. mbols for Use on Detailed Maps, Plans and Geological Cross imensions and Se/ Rer. for Dimensions of Hot Rolled Steel mhols for Use on Detailed Maps, Plans and Geological Cross Dimensions and / Rec. for Dimensions of Hot Rolled Steel and Sectional P/ Rec. for Dimensions of Hot Rolled Steel Rec. for Grooved Pulleys for V-Belts: Groove Rec. for Shanks for Turning and Planing Tools: ons and Sectional Properties of Aluminium (Aluminum) Alloy Rec. for Knife Rec. for Grooved Pulleys for Narrow V-Belts: Groove Rec. for Lengths of Narrow V-Belts:

Characte/ Rec. for Grooved Pulleys for V-Belts: Groove
Rec. for Antistatic Endless V-Belts (Belts) Rec. for Lengths of Classical V-Belts

Rec. for Files and Rasps: Lengths and Cross for Combine Harvesters-Width of Cut and Number of Knife Coating Thicknesses by Microscopical Examination of Cross Rec. for Copper Tubes of Circular International Electrotechnical Vocabulary: Signalling and and Geological Cross Sections-Part II: Representation of Rec. for Spec. for Rec. for Oleaginous Rec. for Oleaginous Rec. for Oleaginous Rec. for Oleaginous Rec. for Oleaginous Rec. for lysis (1968) Std. for ISO General Purpose Metric Screw Threads
Characteristics for Mechanical lsolation (Guide for r Wrought Steel (1964) Rec. for 06 to $6 \%$ Std. for ISO In. Screw Threads: General Plan and esticides) and Plant Growth / Rec. for Principles for the Rec. for Guide to the

Low Power Nonwirewound Types / Rec. for Fixed Resistors: Rec. for Fixed Resistors: Wirewound Resistors, Type 1. Rec. for Wrought Aluminum and Aluminium Alloys. he Use of Units of the International System of Units and A Rec. for oltage Alternating Current Circuit Breakers: Rules for the ation in Coincident Current Matrix Stores Having a Nominal Rec. for Rolling Bearings-Double Row Rec. for

Rec. for Determination of Nitrogen in Coal by the Rec. for Mechanical Coupling Between Tractors and Rec for Test Procedures for for Test Procedures for Amplifiers and Preamplifiers for ng Met/ Rec. for Essential Ratings and Characteristics of ng Met/ Rec, for Essential Ratings and Characteristics of ng Met/ ng Met/ Rec. for Essential Ratings and Characteristics of Rec. for Essential Ratings and Characteristics of Rec. for Letter Symbols for ting Systems for Electronic Tubes and Valves and Analogous Rec. Graphical Symbols:

(1969) quipment (1963) Rec. for Mechanical Standardization of Rec. for Mechanical Standardization of Rec. for Monocrystalline Rec. for Polycristalline

stallations in Ships: Transformers for Power and Lighting, Rec. for meth. (1968)

king of Transit Packages Containing Photographic Materials Rec for Quantity Packaging of tributio/ Std. for Photography-Light Sources for Use in tribution/ Std. for Photography: Light Sources for Use in tributio/ Std. for Photography-Light Sources for Use in d of Colour (Color) Reversal Films for Still Photography Rec. for Rolling Bearings-Cylindrical Roller Rec. for Rolling Bearings-Tapered Roller-In. ns of Elastomeric Toroidal Sealing Rings for Aircraft (ln. Toroidal Sealing Rings for Pipe Fittings in Aircraft (In. oroidal Sealing Rings for Pipe Fittings in Aircraft-(In. of Elastomeric Toroidal Sealing Rings for Aircraft-(In.

Tapered Roller-Boundary Dimensions-Subunits-Metric Tapered Roller-Boundary Dimensions-Subunits-Metric ng Bearings-Needle Roller-Boundary Dimensions-Metric olled Steel Sections-Part 2: Unequal Leg Angles-Metric

Rolled Steel Sections-Part I: Equal Leg Angles-Metric

Hot Rolled Steel Sections-Parallel Flange I Beams-In. led Steel Sections-Parallel Flange Column Sections-In. ns of Hot Rolled Steel Sections-Unequal Leg Angles-ln.

ions of Hot Rolled Steel Sections-Equal Leg Angles-In. g Bearings-Tapered Roller-Boundary Dimensions-Metric and Nuts-Forged and and Tubular Socket Wrenches-Metric
Sections-Part III: Representation of Magmatic Rocks (

Sections-Part II: Representation of Sedimentary Rocks

Sections-Part I: Equal Leg Angles-Metric Series-D

Sections-Part I: General Rules of Representation (196

Sections-Part 2: Unequal Leg Angles-Metric Series-

Sections-Unequal Leg Angles-In. Series-Dimensions

Sections A, B, C, D, E (1957)

Sections and Tolerances (1961)

Sections for Marine Use (1970)

Sections of Mower Cutter Bars (1967)

ISO

Sections SPZ, SPA, SPB (1965)

Sections SPZ, SPA, SPB (1965)

Sections $Y$ and $Z$ (1962)

Sections Y, Z, A, B, C, D, E)-Electrical Conductivity

(Sections Z, A, B, C, D, E) (1967)

Sections (1961)

Sections (1970)

Sections (1970)

Section: Dimensions; Metric Series (1962)

/Ding Details-Dimensi ISO

ISO

ISO

Security A pparatus for Rail

Seedlac (1957)

Seeds: Determination of Acidity of Oils (1968)

Seeds: Determination of Impurities (1968)

Seeds: Determination of Moisture and Volatile Matter (1

Seeds: Determination of Oil Content (1968)

Seeds: Reduction of Contract Samples to Samples for Ana

Rec. ISO

Seeds: Sampling and Meth. of Test (1969)

Selected Sizes for Screws, Bolts and Nuts (1973)

Selecting and Applying Resilient Devices) (1972)

Selection and Preparation of Samples and Test Pieces Fo

Selection for Screws, Bolts and Nuts (Diameter Range 0.

Selection of Common Names for Pest Control Chemicals ( $P$

Selection of High Voltage Cables (1965)

Selection of Meth. of Test and General Requirements for

Selection of Meth. of Test and General Requirements (19

Selection of Specimens and Test Pieces (1971)

Selection of the Decimal Multiples and Submultiples of

Selection of Tolerance Zones for General Purposes (1970

Selection of (For Service) (1971)

Selection Ratio of 2 : 1 (1969)

Selfaligning-Radial Internal Clearance (1965)

Rec, for High $\mathrm{V}$

Selfholding Tapers for Tool Shanks (1963)

Semi Micro Kjeldahl Meth. (1963)

Semi Trailers Interchangeability (1971)

Semiconductor Detectors for Ionizing Radiation (1970)

Semiconductor Detectors for Ionizing Radiation (1970)

Semiconductor Devices and General Principles of Measuri

Semiconductor Devices and General Principles of Measuri

Semiconductor Devices and General Principles of Measuri

Semiconductor Devices and General Principles of Measuri

Semiconductor Devices and Integrated Microcircuits (196

Semiconductor Dcvices (1961)

Semiconductor Devices, Capacitors (1971)

Rec. for $\mathrm{Ra}$

Semiconductor Devices: Dimensions (I970)

Semiconductor Devices: Preparation of Drawings Devices

Semiconductor Rectifier Cells, Stacks, Assemblies and E

Semiconductor Rectifier Stacks and Equipments (1960)

Semiconductor Rectifiers, Generators (With Associated P

Semitrailer (Trailer) 5Th Wheel Kingpin (1963)

Sensitive Switches: General Requirements and Measuring

Sensitive to Radiant Energy (1968)

/ for Pictorial Mar ISO

Sensitized Photographic Materials (1969)

Sensitometric Exposure-Simulation of the Spectral Dis

Sensitometric Exposure-Simulation of the Spectral Dis

Sensitometric Exposure-Simulation of the Spectral Dis

Sensitometric Exposure and Evaluation Meth. (1972)

Separate Thrust Collars-Boundary Dimensions (1962)

ISO

IEC

ISO

ISO

ISO

ISO

ISO

ISO

ISO

ISO

ISO

ISO

ISO

IEC

IEC

IEC

ISO

ISO

Series-Chamfer Dimension Limits (1969)

Series-Class 1 Tolerances) (1969)

Series-Class 1 Tolerances) (1969)

Series-Class 2 Tolerances) (1971)

Series-Class 2 Tolerances) (1971)

Series-Dimension Series 29 and 13 (1973)

Series-Dimension Series 30, 31 and 32 (1970)

Series-Dimension-Series 48 and 49 (1971)

Series-Dimensions and Sectional Properties (1968)

Series-Dimensions and Sectional Properties (1968)

Series-Dimensions and Sectional Properties (1969)

Series-Dimensions and Sectional Properties (1969)

Series-Dimensions and Sectional Properties (1969)

Series-Dimensions and Sectional Properties (1969)

Series-Dimensions Series 31 and 32 (1970)

Series-Maximum Outside Dimensions (1972)

ISO Ns of Elastomeric ISO of Elastomeric T ISO

C. for Dimensions ISO /G Bearings ISO /Rings ISO

/ for Rolli 1SO

/R ISO

IT ISO

/F ISO

IL ISO

lo 150

IS 150

lor Rollin ISO

lor Screws ISO 
Tapered Roller-Boundary Dimensions-Subunits-Metric Rec. for Rolling Bearings-Tapered; Metric loride (PVC) Pipes for the Supply of Gaseous Fuels: Metric Std. for Rolling Bearings: Tapered Roller-Inch Std. for Rolling Bearings: Tapered Roller-Metric Rec. for Twist Drills: Parallel Shank Jobber and Stub ousing Fil/ wire (1964) Std. for Rolling Bearings: Tolerances; Metric Rec. for ISO Metric Rec. for Preferred Number Rec. for ounded Values (1966) Rec. for Guide to the Choice of ulary/

Rec. for Statistical Vocabulary and Symbols: First 71) Rec. for Statistical Vocabulary and Symbols-Second Rec. for Paper Vocabulary-6Th
Rec. for Paper Vocabulary: First Rec. for Paper Vocabulary: Second Rec. for Paper Vocabulary: Third Rec. for Paper Vocabulary: Fourth Rec. for Paper Vocabulary-5Th Std. for Nuclear Energy Glossary: First Std. for Guide to the Use of Preferred Numbers and - for Electrical Equipment of Machine Tools: Used in Large Rec. for Rolling Bearings: Tapered-Metric ns (1970) Rec. for Marking of Rec. for General Purpose
Rec. for Spec. for Corner Fittings for Rec. for Spec. and Test. of Rec. for Marking of Series 1 and boundary Dimensions-Subunits-Metric Series-Dimension boundary Dimensions-Subunits-Metric Series-Dimension

Roller-Boundary Dimensions-Metric Series-Dimensions

- Boundary Dimensions-General Plan-Part II-Diameter

s-Radial-Boundary Dimensions-General Plan-Diameter - Tapered Roller-Boundary Dimensions-Metric-Diameter (Outside Diameters and Nominal Pressures): Part I: Metric Rec. for Carbide Tips for Turning Tools: Metric Rec. for Turning Tools with Carbide Tips: Metric for Copper Tubes of Circular Section: Dimensions; Metric mestic and Industrial Waste Pipe: Basic Dimensions; Metric al Ball and Roller (II) Thrust (III) Tapered Type-Metric ids (Outside Diameters and Nominal Pressures) Part II: In. e for Rolling Bearings: Group IV Tapered Roller Type-In. Rec. for Parallel Shank Twist Drills-Long

Rec. for Hexagon Socket Head Cap Screws: Metric

Rec. for $W$ ashers for Hexagon Bolts and Nuts: Metric gs-Tapered Roller-Boundary Dimensions-Subunits-In. plates: Bayonet Type; Sizes for Interchangeability: Metric nder the Head for General Purpose Bolts and Screws: Metric lyvinyl Chloride (PVC) Fittings with Plain Sockets; Metric cross Flats, Heights of Heads, Thicknesses of Nuts: Metric ec. for Dimensions of Hot Rolled Steel Bars: Round, Metric rec. for Dimensions of Hot Rolled Steel Bars: Flat, Metric c. for Dimensions of Hot Rolled Steel Bars: Square, Metric - Tapered Roller-Boundary Dimensions-Subunits-Metric

ling Cutters and Their Arbors or Mandrels-Metric and In. Rec. for Slotted Cheese (Fillister) Head Screws-Metric Rec. for Hexagon Head Tapping Screws-Metric Rec. for Hexagon Head Tapping Screws-In. Rec. for Slotted Pan Head Screws-Metric h Parallel Shanks with Morse Taper, Shanks-Std. and Long Rec. for Split Pins-Metric

ers-Internal Diameters and Piston Rod Diameters-Metric Std. for End Mills with 7/24 Taper Shanks-Std. and Long Std. for Parallel Pins, Unhardened-Metric Std. for Clevis Pins-Metric

Std. for Clevis Pins with Heads-Metric Std. for Slotted Headless Screws-Metric Std. for Hexagon Socket Set Screws-Metric for Slotting Cutters with Plain Bore and Key Drive: Metric or Metal Slitting Saws with Fine and Coarse Teeth-Metric Cylindrical Cutters with Plain Bore and Key Drive: Metric evailing Torque Type Hexagon Locknuts: Dimensions-Metric iling Torque Type Steel Hexagon Locknuts: Dimensions-In. face Milling Cutters with Plain Bore and Key Drive: Metric ttings for Pipes Under Pressure-Basic Dimensions: Metric or Shell End Mills with Plain Bore and Tenon Drive: Metric Interchangeability Dimensions with Cutter Arbors-Metric or Turning Tools with Carbide Tips: Internal Tools (Metric ins, Attachments and Chain Wheels-Part I: Chains (Metric Std, for Rolling Bearings: Locknuts, Narrow ter) (/ Rec. for Cold Drawn Precision Steel Tubes: Metric 1968) Rec. for Paper: Untrimmed Stock Sizes for the ISO-A Rec. for Hexagon Bolts and Nuts: Metric

Rec. for Paper: Untrimmed Stock Sizes for the ISO-A
Series-Outer Rings with Flange (1973)

Series-Part I: Normal Tolerances (1967)

/Ling Bearings ISO

Series-Part I: Norm /Ried Unplasticized Polyvinyl Ch ISO Series-Tolerance Classes 4 (Normal), 3,0 and 00 (1973

Series-Tolerances-Class 4 (1973)

Series and Morse Taper Shank (1961)

Series Capacitors for Power Systems (1972)

Series Chamfer Dimension Limits and Maximum Shaft and $H$

Series for Basic Thicknesses of Sheet and Diameters of

Series for Resistors and Capacitors (1963)

Series of Conical Tapers and Taper Angles (1969)

Series of Preferred Numbers and Those Containing More $R$

Series of Preferred Numbers (1973)

Series of Terms and Symbols-Part 1: Statistical Vocab

Series of Terms and Symbols (1970)

Series of Terms (Definitions of Optical Properties) (19

Series of Terms (1958)

Series of Terms (1959)

Series of Terms (1961)

Series of Terms (1964)

Series of Terms (1970)

Series of Terms (1972)

Series of (1973)

Series Production Lines Lines (1967)

Series Tolerances Classes 6 and 5 (1968)

Series 1 and Series 2 Freight Containers (1968)

Series 1 Freight Containers-Minimum Internal Dimensio

Series 1 Freight Containers (1970)

Series 1 Freight Containers (1970)

Series 2 Freight Containers (1968)

Series 29 and 13 (1973)

Series 30,31 and 32 (1970)

Series 31 and 32 (1970)

Series 7 (1970)

Series $8,9,0,1,2,3$ and $4,(1968)$

Series 9 and 0 (1967)

Series (1960)

Series (1961)

Series (1961)

Series (1962)

Series (1962)

Series (1963)

Series (1963)

Series (1965)

Series (1966)

Series (1968)

Series (1968)

Series (1968)

Series (1968)

Series (1968)

Series $(1968)$

Series (I968)

Series (1969)

Series (1969)

Series (1969)

Series (I969)

Series (I969)

Series (I970)

Series (I970)

Series (1970)

Series (1970)

Series (1970)

Series (197I)

Series (1971)

Series (I972)

Series (1972)

Series (1972)

Series (1972)

Series (1972)

Series (I972)

Series (I972)

Series (1972)

Series (I972)

Series (1972)

Series (1972)

Series (I972)

Series (1972)

Series (1973)

Series (1973)

Series) (1966)

Series) (1971)

Series, and Lockwashers with Straight Inner Tab (1972)

Series, ISO Primary Range (1966)

Series, Tolerances on Widths Across Flats and Corners (

Bearings: Tapered Roller- ISO /Rings-Tapered Roller- ISO /or Rolling Bearings-Tapered ISO Rec. for Rolling Bearings-Radial ISO / for Rolling Bearing ISO Rec. for Rolling Bearings ISO

IS Materials for the Transport of Fluids ISO

ISO

Rec ISO

/Socket Fittings with Spigot Ends for Do ISO / for Roller Bearings: Groups-(I) Radi ISO ITics Materials for the Transport of Flu ISO Rec. for ISO Identification Cod ISO

1SO

ISO

Rec for Rolling Bearin ISO

Rec. for Spindle Noses and Face ISO

Rec. for Bolts and Screws: Radii U ISO

/ Pipes Under Pressure: Unplasticized Po ISO

/C. for Hexagon Bolts and Nuts: Widths a ISO

ISO

Re ISO

Rec for Rolling Bearings ISO

/R Interchangeability Dimensions for Mil ISO

ISO

ISO

ISO

Rec. for End Mills Wit ISO

Rec. for Hydraulic Cylind ISO

ISO

ISO

ISO

ISO

ISO

ISO

Std. ISO

Std. F ISO

Std for ISO

Std. for Pr ISO

Std. for Preva ISO

Rec. for Side and ISO

/Tings of Plastics Materials-Socket Fi ISO

Std. F ISO

1. for Milling Cutters with Tenon Drive: ISO Rec. F ISO

Rec. for Conveyor Cha ISO

ISO

ISO

ISO

Series: ISO Supplementary Range (1967)

2316

$577 /$

2703

578

$577 /$ III

235

143

582

388

63

1119

497

645

1786

1912

66

135

231

372

1295

921

I 7

204-2

$577 / \mathrm{I}$

790

1894

1 I 61

1496

790

$355 /$ VIII

$355 / V I I$

355/VI

15/II

15/I

355/III

16 I

242

243

274

265

$300 / 1$

330

$300 / 1$ I

494

861

887

$355 / 1 \mathrm{~V}$

867

885

727

272

$1035 / I$

1035/II I

I035/1 I

$355 / \mathrm{V}$

240

1207

1479

1480

1580

I641

1234

2091

2324

2338 
sembled liars for /

Rer. for Textile Machinery-Terms for (1970)

ry and Apparatus in Relation to Their Thermal Stability in Rec for Tungsten Filament lamps for General Rec. for Tubular Fluorescent Lamps for General Lighting Stock on Completion of Construction and Before Entry into

Current Circuit Breakers: Rules for the Selection of (For

Safety Features for Ground Power Units for d.c. Aircraft Character Sets Derived from the ISO 7 Bit Coded Character low Temperatures (/ Rec. for Determination of Compression 970/ Rec. for Representation of ISO 7 Bit Coded Character Rec. for Implementation of the 7 Bit Coded Character Std. for Hexagon Socket

(1967) Measuring Rec. for Vulcanized Rubbers: Determination of Compression In/ ntrol of Machines (Compatible with the ISO 7 Bit Character Rec. for Guide for the Definition of 4 Bit Character Rec. for 6 and 7 Bit Coded Character Std. for Coding of Character Rec. for Alphanumeric Character Rec. for Railway Rolling Stock Material: Wheel Rec. for Representation of 6 and 7 Bit Coded Character rec. for Implementation of the 6 and 7 Bit Coded Character he Maximum Temperature and the Rate of Increase During the Rec. for Marking of Control Rec. for Steel Pipes for Gas, Water and Rec. for Asbestos Cement Pipes, Joints and Fittings for in for Conveyors (1969) Rec. for

Rec. for Shipbuilding Details-A nchor Chains-End

Stud Links (Common, Enlarged, and End Types, and Joining tails for Sea Navigation-Anchor Chains-Lugless Joining Sheets for Barrel Thread for E14 and E27 Lampholders with rances; Metric Series Chamfer Dimension Limits and Maximum Rec. for Cylindrical and 1/10 Conical Rec. for (1955) Rec. for Straight Sided Splines (For Cylindrical pment for Loose Bulk Materials-Oscillating Conveyors and or Loose Bulk Materials-Vibrating Feeders and Conveyors, pment for Loose Bulk Materials-Oscillating Conveyors and Rec. for Rivet 1) Rec. for Twist Drills: Parallel and $1 / 8$ to $1 \mathrm{In} .(1972)$ Std. for Long

rec. for Hand and Long Fluted Machine Reamers, Morse Taper lls: Parallel Shank Jobber and Stub Series and Morse Taper Std. for Core Drills with Parallel, and Morse Taper

Rec. for End Mills with Parallel Shanks with Morse Taper, Std. for End Mills with 7/24 Taper Rec. for $7 / 24$ Tapers for Tool eels (1968) s (1972) Rec. for Rock Drilling: Forged Collared

with Parallel Shanks (1961)

uck Bushings-Part I (1970) uck Bushings-Part II (1970) rances (1961) (1970)

n Sleeves and Extension Sockets for Tools with Morse Tape Sizes of Driving Squares for Rotating Tools with Paralle Rec. for Selfholding Tapers for Too Rec for 7/24 Tapers for Tool

for Machine Chucking Reamers with Parallel or Morse Taper for Morse and Metric Tapers, with Parallel and Morse Taper Std. for Cutting Netting to

s on Aireraft (1969)

(1972)

ing Processes (1972) ( Rec. for Phosphorus Deoxidized Copper-Refinery fire Refined High Conductivity Tough Pitch Copper Refinery sis of Copper and Its Alloys-Sampling of Copper Refinery Std. for Electrolytic Tough Pitch Copper Refinery Rec. for Determination of the

tor Operated Appliances for Domestic and Similar Purposes: lectrical Appliances: Particular Requirements for Electric t Piece) (1971)

Std. for Rigid Cellular Plastics-Determination of Rec. for Aluminium and Aluminum Alloys.

$f$ Modulus in Shear of Vulcanized Rubbers (Bonded Quadruple ation of Adhesion of Vulcanized Rubbers to Rigid Plates in ation of Viscosity of Natural and Synthetic Rubbers by the

Determination of Rate of Cure of Rubber Compounds by the Meth. of Test for Pve (Polyvinyl Chloride) Insulation and to / Rec. for Tests on Impregnated Paper Insulated Metal ages/ Rec. for Tests on Impregnated Paper Insulated Metal
Serrated Bars and Dimensions of the Cross Section of as ISO Service Test of Fabrics Coated with Rubber or Plastics Service (1957) Service (1961) Service (1961) Service (1963) Service) (1971) Servicing and Engine Starting (1961)

R the lnsulation of Electrical Machine ISO

Rec. for High Voltage Alternating IEC

Set of Vulcanized Rubbers Under Constant Deflection at

Set on 12 Row Punched Cards (Information Processing) (1

/Bit ISO

ISO

Set Screws-Metric Series (1972)

Set Spec. for the Frequency Range $300 \mathrm{MHz}$ to $1,000 \mathrm{MHz}$

Set Under Constant Deflection at Normal and High Temper Set) (1968)

Rec. for Code for Numerical Co

Sets Derived from the ISO 7 Bit Coded Character Set for

Sets for Information Processing Interchange (1967)

Sets for MICR and OCR (1972)

Sets for Optical Recognition (1969)

Sets for Trailer Stock (1969)

Sets on Punched Tape (1969)

Sets on 7 Track $12.7 \mathrm{~mm}$ (1/2 In.) Magnetic Tape (1969)

Setting of Unsaturated Polyester Resins (1967)

Settings on Hearing Aids (1966)

Sewage, Welded or Seamless (1967)

Sewerage and Drainage (1968)

Shackle Type Connector Units for High Tensile Steel Cha

CISP

ISO

ISO

ISO

ISO

ISO

ISO

ISO

ISO

IN of T ISO

IEC

ISO

ISO

Shackles (1963)

Shackles) (1960)

Shackles, Kenter Type (1957) Rec. for Shipbuilding De ISO

Shade Holder Ring (1972)

Shaft and Housing Fillet Radius (1972)

Shaft Ends (1969)

Shaft Heights for Driving and Driven Machines (1966)

Shafts) Nominal Dimensions in Millimetres (Millimeters)

Shaking on Reciprocating Feeders with Tubular Trough (1

Shaking or Reciprocating Feeders and Oscillating Convey

Shaking or Reciprocating Feeders with Rectangular or Tr

Shank Diameters (Diameter Range 1 to $36 \mathrm{Mm}$ ) (1969)

Shank Jobber and Stub Series and Morse Taper Shank (196

Shank Machine Taps with Nominal Diameters from $324 \mathrm{~mm}$

Shank Twist Drills-Long Series (1966)

Shank (1961)

Shank (1961)

Shanks-Rec. Stocked Sizes (1972)

Shanks-Std, and Long Series (1970)

Shanks-Std. and Long Series (1972)

Shanks-7/24 Tapers Nos. 45 and 55 (1971)

Shanks and Chuck Bushings for Hollow Hexagonal Drill St

Shanks and Equipment with 7/24 Tapers: Collar Dimension

Shanks and Sizes of Driving Squares for Rotating Tools

Shanks for Pneumatic Tools and Fitting Dimensions of $\mathrm{Ch}$

Shanks for Pneumatic Tools and Fitting Dimensions of $\mathrm{Ch}$

Shanks for Turning and Planing Tools: Sections and Tole Rec. for IEC

ISO

Shanks with Morse Taper, Shanks-Std and Long Series

Shanks (1961)

Shanks (1961)

Rec. for Reductio
Rec. for Diameters of Shanks and

Shanks (1963)

Shanks (1963)

Shanks (1966)

Shanks (1972)

Shape-Determination of the Rate (1972)

Rec. ISO

Shape (Tapering) (1970)

Shape, Size and Direction of Operation of Lever Control

Shaped Insulating Refractory Products-Classification

Shaped Refractory Products-Nomenclature of Manufactur

Shaped Travellers (1959)

Shapes (1971)

Shapes (1971)

Shapes (1971)

Shapes (1971)

Shapes (1972)

Shatter Indices of Coke (1967)

Shavers, Hair Clippers and Similar Appliances (1966)

Shavers, Hair Clippers and Similar Items (1972)

Shear of Vulcanized Rubbers (Bonded Quadruple Shear Tes

Shear Strength (1972)

Shear Test for Rivet Wire and Rivets (1971)

Shear Test Piece) (1971)

Shear (Quadruple-Shear Test) (1971)

Shearing Disk Viscometer (1963)

Shearing Disk Viscometer (1968)

Rec. for Determination 0 Rec. for Determin ISO Rec. for Determin ISO

Sheath of Electric Cables (1970)

Rec. for IEC

Sheathed Cables: Nondraining Types for Alternating Volt

1865 
ssure Cahles and Their Accessories: Paper Insulated, Metal tr on Anticorrosion Protective Coverings of Metallic Cable les and Wires with Pre (Polyvinyl Chloride) Insulation and les and Wires with Prc (Polyvinyl Chloride) Insulation and les and Wires with Prc (Polyvinyl Chloride) Insulation and les and Wires with Prc (Polyvinyl Chloride) Insulation and les and Wires with Pvc (Polyvinyl Chloride) Insulation and les and Wires with Prc (Polyvinyl Chloride) Insulation and les and Wires with Prc (Polyvinyl Chloride) Insulation and Rec for ISO Metric Series for Basic Thicknesses of Rec. for Simple Bend Test. of SteeI Rec. for Reverse Bend Test. of Steel Rec. for Tensile Test. of Steel

(1959)

Rec. for Simple Bend Test for Light Metal and Its Alloy Rec. for Modified Erichsen Cupping Test for Steel Determination of Saleable Mass, in Lots, of Pulp Baled in ic Colour (Color) Film for General Use: Preferred Sizes of c Colour (Color) Paper for General Use: Preferred Sizes of black and White Papers for General Use: Preferred Sizes of old Reduced Tinplate and Cold Reduced Blackplate-Part 1: e Water Vapour (Vapor) Transmission Rate of Films and Thin Rec. for Sockets for Electronic Tubes and Valves: Spec. Std. for Abrasive c. for Plastics-Basis for Spec. for Industrial Laminated h Shade Holder Ring (1972) Rec. for Rec. for Asbestos Cement Corrugated ices and Gauges (/ Rec for Tube and Valve Shields: Spec. Rec. for Asymmetrical Section Corrugated Rec. for Width of Rec. for Determination of the Thickness of Single Rec for Asbestos Cement Flat ed Sizes of Articles of Stationery That lnclude Detachable Rec. for Primary Cells and Batteries: Spec. Series (1973) Std. for lot Drive and Arbors for Shell Reamers (1972) Std. for per Bore (1:30 (Included) ) with Slot Drive and Arbors for Rec. for Spec. for Rec. for Spec. for Bleached Lac Wires, Spools, Rims and Col Rec. for Solid Wires for Gas
and Toler/
Rec. for Rolling Bearings-Radial Types with and Toler/ Rec. for Rolling Bearings-Radial Types with
Rec. for Tube and Valve ns of Test. Devices and Gauges (/ $\quad$ Rec. for Tube and Valve ing and Finishing (1966) rangement Plans of Ships (1971) Rec. for Asbestos Cement Siding Rec. for Shipbuilding Details: 6

n, Enlarged, and End Types, and Joining Shackle/ ies of Aluminium (Aluminum) Alloy Sections for / 3)

Rec. for Rec. for Rec. for Rec. for Rec. for Rec. for Rec. for Rec. for Rec. for Rec. for Rec. for Rec, for Rec. for Rec. for Rec. for Rec. for Rec. for Rec. for Rec. for Rec. for Rec. for Rec. for Use in Std. for Std, for Rec. for Sampling Bulk

n Black for Use in the Rubber lndustry-Sampling Packaged id or Gaseous Condition in Land Installations and on Board $d$ in Schemes for the Installations of Pipe Line Systems in in Schemes for the Installations of Ventilation Systems in Rec. for Positioning of Magnetic Compasses in ed in Schemes for the lnstallations of Sanitary Systems in Rec. for Electric Fans and Regulators for Use in ilding Details: Mating Dimensions for Pipeline Flanges for indications of Details on the General Arrangement Plans of Colours (Colors) of Signal Lights on to Rated Capacity of Circuit Breakers in Installations in Rec. for Shipbuilding Details

for Shipbuilding Details: Test. of Toughened Glasses for Rec. for Shipbuilding Details-Toughened Glasses for tteries, Heating An/ 1965)
Sheathed, for Alternating Voltages Up to $275 \mathrm{kV}$ (1963) Sheaths (1966)

Sheath: Cables in Pairs, Triples, Quads and Quintuple

Sheath: Distribution Wires with Solid Conductors, Insul

Sheath: Distribution Wires with Solid Conductors, Insul

Sheath: Equipment Wires and Cables with Solid or Strand

Sheath: Equipment Wires, Type I, with Solid or Stranded

Sheath: General Test and Measuring Meth. (1972)

Sheath: Signalling Cables in Singles for Telecommunicat

Sheet and Diameters of Wire (1964)

Sheet and Strip Less Than $3 \mathrm{~mm}$ Thick (1959)

Sheet and Strip Less Than $3 \mathrm{~mm}$ Thick (1959)

Sheet and Strip Less Than $3 \mathrm{Mm}$ and Not Than $0.5 \mathrm{Mm}$ Thick

Sheet and Strip of Thickness Between $0.2 \mathrm{~mm}(0.008 \mathrm{ln}$.)

Sheet and Strip (1960)

Sheet Form (1968)

Sheet Material (1969)

Sheet Material (1969)

Sheet Material (1969)

Sheet (1969)

Sheets-Dish Meth. (1970)

Sheets and Dimensions of Wiring Jigs and Pin Straighten

Sheets Based on Thermosetting Resins (1971)

Sheets for Barrel Thread for E14 and E27 Lampholders Wi Rec. for Photographi ISO Rec. for Photographic ISO Rec. for C ISO

Sheets for Roofing and Cladding (1964)

Sheets for Tubes and Valves and Dimensions of Test. Dev

Sheets in Asbestos Cement for Roofing and Cladding (196

Sheets of Paper (1959)

Sheets of Paper (1967)

Sheets (1964)

Sheets (1967)

Sheets (1972)

Rec. for Paper: Overall Trimm

Shell End Mills with Plain Bore and Tenon Drive: Metric

Shell Reamers with Taper Bore (1:30 (Included) ) with S

Shell Reamers (1972)

Shellac (1957)

Shellac) (1957)

Shielded MetaI Arc Welding of Mild Steel: Dimensions of

Shields or Seals-Outside Diameter Tolerances-Normal

Shields: General Requirements and Meth. of Test (1969)

Shields: Spec. Sheets for Tubes and Valves and Dimensio

Shingles (1968)

Ship Screw Propellers, Manufacturing Tolerance for Cast

Shipbuilding-Indications of Details on the General Ar

Shipbuilding Details-Anchor Chains-End Shackles (19

Shipbuilding Details-Anchor Chains, Stud Links (Commo

Shipbuilding Details-Dimensions and Sectional Propert

Shipbuilding Details-Galvanized Steel Wire Ropes (196

Shipbuilding Details-Marking of Hatchway Beams (1960)

Shipbuilding Details-Marking of Rolled, Drawn and Ext

Shipbuilding Details-Marking of Wooden Hatchway Cover

Shiphuilding Details-Multipurpose Chocks of Cast Stee

Shipbuilding Details-Ships Scuttles (1971)

Shipbuilding Details-Tests on Galvanized Steel Wire F

Shiphuilding Details-Toughened Glasses for Ships Sid

Shipbuilding Details-Wrought Aluminum Alloys for Use

Shiphuilding Details for Inland Navigation-Covers for

Shipbuilding Details for Inland Navigation-Mushroom V

Shipbuilding Details for lnland Navigation-Pipe Lines

Shipbuilding Details for Inland Navigation Sprocket Whe

Shipbuilding Details for Sea Navigation-Anchor Chains

Shipbuilding Details for Sea Navigation and Inland Navi

Shipbuilding Details: Mating Dimensions for Pipeline Fl

Shipbuilding Details: Ship Screw Propellers, Manufactur

Shiphuilding Details: Test. of Toughened Glasses for Sh

Shipbuilding (1969)

Shipbuilding: Anchor Chains (1973)

Shipbuilding: Cargo Gear Particulars Book (1972)

Shipments of Carbon Black for the Rubber Industry (1969

Shipments (1970)

Ships (1966)

Ships (1967)

Ships (1967)

Ships (1968)

Ships (1968)

Ships (1969)

Ships (1969)

Ships (1971)

Ships (1972)

Ships (1972)

Ships Scuttles (1971)

Ships Side Scuttles and Fixed Lights by the Punch Meth
Ships Side Scuttles and Fixed Lights (1969)

Lors) for Pipes Conveying Fluids in Iiqu ISO Rec for Conventional Signs: to Be Use ISO Rec. for Conventional Signs to Be Used ISO

Rec for Conventional Signs to $\mathrm{Be}$ Us ISO

IEC

Rec. for Shipbu ISO

Rec. for Shipbuilding- ISO

ISO

Ships: Accessories, Lighting, Accumulator (Storage), Ba

Ships: Cables (Construction, Test. and Installations) (
189-2

$189-4$

189.7

189-5

189-3

189-1

189-6

388

88

86

954

149

801

1010

1008

1111

1195

$149-2$

2235

1642

399

393

288-2

394

101

534

396

618

86-2

2586

2402

2402

56

57

864

1648

288. 1

288-2

880

484

1964

347

170

1175

346

151

154

154

1685

1751

345

1095

1156

41

42 
Rec. for Electrical Installations in Rec. for Electrical Installations in Rec. for Electrical Installations in Rec. for Electrical Installations in and Controlgear (19/ or Rectifiers, Gene/ Specifying the Characteristics of Auxiliary Equipment for the Characteristics of Electro Mechanical Transducers for Rec. for Meth. for Thermal ronic Tubes and Valves: Meth. of Application of Mechanica ocedures for Electronic Components and Equipment: Test Ea: Rec. for Camera Accessory

Indentation Hardness of Plastics by Means of a Duromete -Determination of Mass of Pile Per Unit Area That Can Be Rated Capacity of Circuit Breakers in Installa/ Rec. for 1955)

68)

ons of Acceptance (I971)

ated Load Type for Pulley Blocks and Other Lift/ ibrated Chain for Chain Slings, Etc. (1971)

Wheels (1970)

ain Wheels (1967)

Rec for Coniferous Sawn Timber: Sizes-Tolerances and of Direction of Closing and Faces of Doors, Windows and tion of the Shuttle Eye (1970) Rec. for Exposure Time Markings for Rec. for Rec. for Rec. for rive: Metric Series (1972) (I968)

Rec. for Identification of the Emulsion hipbuilding Details: Test. of Toughened Glasses for Ships c. for Shipbuilding Details-Toughened Glasses for Ships Rec. for Definition of Rec. for Weaving Looms: Definition of $y$ and Accessories-Pirn and Cross Winders-Definition of accessories-Weaving Preparatory Machines-Definition of Rec. for Straigh ns in Millimetres (Millimeters) (1955) Rec. for Straight yeing and Finishing Machines: Definition of Left and Right Rec. for Asbestos Cemen Rec. for Plastics-Pvc Resins or Carbon Black for Use in the Rubber Industry-Spec. for n Black for Use in the Rubber Industry-Meth. of Test for Std. for Tes

Std. for Woven Wire Cloth and Perforated Plates in Tes Sid for Test Sieves and Rec. for Analogue Pneumatic Colours (Colors) of

Rec. for International Electrotechnical Vocabulary wires with Pvc (Polyvinyl Chloride) Insulation and Sheath echnical Spec. (1966) Rec for Sound Rec. for Lighting and Rec. for Analogue d.c. Current Rec. for Explanations on the Rec. for (Weldi/ y with Standards (1966) nology (I961) nitary Systems in Ships (I968) ntilation Systems in Ships (1967)

Rec. for Mathematical Rec. for Conventional Rec. for Conventional Rec. for Symbols, Dimensions and Layout for Safety ipe Line Systems in Ships (1967) Rec. for Conventional Rec. for Emulsion Position in Camera for $16 \mathrm{Mm}$ Rec. for Emulsion Position in Camera for $8 \mathrm{Mm}$ Position in Projector for Direct Front Projection of $8 \mathrm{Mm}$ position in Projector for Direct Front Projection of $16 \mathrm{Mm}$

Potassium Silicates for Industrial Use-Determination of ly Used for the Production of Aluminium-Determination of for Cryolite (Natural and Artificial)-Determination of m (Aluminum) Fluoride for Industrial Use: Determination of Potassium Silicates for Industrial Use-Determination of $r$ Potassium Hydroxide for Industrial Use: Determination of for Sodium Hydroxide for Industrial Use: Determination of

r Potassium Hydroxide for Industrial Use: Determination of r Sodium Hydroxide for Industrial Use: Determination of of Rec. for Laboratory Crucibles (Porcelain and Rec. for Dental

dioxide Content Expressed of Samples in Solution-1 ter-Gravimetric Meth. (1/ Viscosity (1972) content-Gravimetric Meth/ content-Titrimetric Meth/ lkalinity, Expressed in $\mathrm{Na} /$ of Products Not Easily Sol/ -General (1970)

Silic a Content-Spectropho
Ships: General Requirements (I964)

Ships: Graphical Symbols (1965)

Ships: Switchgear, Electrical Protection, Distribution

Ships: Transformers for Power and Lighting. Semiconduct

Shock and Vibration Measurement (1966) for Meth for IEC

Shock and Vibration Measurement (1966) /or Specifying IEC

Shock Tests on Laboratory Glassware (1968)

Shock (1967) IEC

Shoes (1966)

Rec. for Basic Environmental Test. Pr IEC

Shore Hardness) (1968)

lor Plastics-Determination of ISO

$\begin{array}{ll}\text { Shorn Away from the Backing (1971) Rec. for Carpets ISO } & \text { ISO }\end{array}$

Short Circuit Current Evaluation with Special Regard to IEC

Short Contents List of Periodicals or Other Documents ( ISO

Short Enclosed Scale Thermometers for Precision Use (19

ISO

Short Link Chain for Lifting Purposes-General Conditi

Short Link Chain for Lifting Purposes-Grade 40 Calibr

Short Link Chain for Lifting Purposes-Grade 40 Noncal

ISO

ISO

ISO

ISO

Short Machine and Hand Taps (1966)

Short Pitch Transmission Precision Bush Chains and Chai

ISO

Short Pitch Transmission Precision Roller Chains and $\mathrm{Ch}$

ISO

ISO

ISO

Shrinkage (1968)

Shutters-Part I (I970)

Shutters Used in Still Cameras (1966)

Rec for Symbolic Designati ISO

Shuttles-Terms and Designation in Relation to the Pos

ISO

Shuttles for Pirn Changing Automatic Looms (I967)

Side and Face Milling Cutters with Plain Bore and Key D

Side of Edge Marked Roll Film for Still Picture Cameras

Side Scuttles and Fixed Lights by the Punch Meth. (1967

Side Scuttles and Fixed Lights (1969)

Side (Left or Right) of Spinning Machinery (1959)

Side (Left or Right) (1959)

Side (Left or Right) (1960)

Side (Left or Right) (1960)

Sided Splines and Gauges Dimensions in In./S (1961)

Sides (1969)

Siding Shingles (1968)

Sieve Analysis in Water (1970)

Sieve Residue (1970)

Sieve Residue (1970)

Sieves and Sieving: Vocabulary (1972)

ISO

Rec. for Textile Machiner ISO

Ter Textile Machinery and ISO

ISO

ISO

ISO

ISO

ISO

ISO

108

Sieves: Nominal Sizes of Apertures (1972)

Rec. for Carbo ISO

ISO

Sieving: Vocabulary (1972)

Signal for Process Control Systems (1971)

Signal Lights on Ships (1972)

Signalling and Security Apparatus for Railways (1959)

Signalling Cables in Singles for Telecommunication Equi

Signalling Devices on Motor Vehicles Acoustic Std. and

Signalling for Motor Vehicles and Trailers (1963)

Signals for Process Control Systems (1971)

Significance of the Principal Radiographic Terms Used I

Significance to Purchasers of Marks Indicating Conformi

Signs and Symbols for Use in Physical Sciences and Tech

Signs to Be Used in Schemes for the Installations of Sa

Signs to Be Used in Schemes for the Installations of Ve

Signs (1967)

Signs: to Be Used in Schemes for the Installations of P

Silent Motion Picture Film (1956)

Silent Motion Picture Film (I956)

Silent Motion Picture Film (I956)

Silent Motion Picture Film (1956)

Silica Content-Gravimetric Meth. by Insolubilization

Rec for Emulsion ISO

Silica Content-Spectrophotometric Meth. Using the Red

Silica Content-Spectrophotometric Meth. Using the Red

Silica Content-Titrimetric Meth. (1972)

Silica Content, Gravimetric Meth. by Precipitation of T

Silica Content: Gravimetric Meth. by Insolubilization (

Silica Content: Gravimetric Meth. by Insolubilization (

Silica Content; Gravimetric Meth. by Precipitation of T

Silica) (1970)

Silicate Cement (1970)

Silicates for Industrial Use

Silicates for Industrial Use-Determination of Carbon

Silicates for Industrial Use-Determination of Density

Silicates for Industrial Use-Determination of Dry Mat

Silicates for Industrial Use-Determination of Dynamic

Silicates for Industrial Use-Determination of Silica

Silicates for Industrial Use-Determination of Silica

Silicates for Industrial Use-Determination of Total a

Silicates for Industrial Use-Preparation of Solution

Silicates for Industrial Use-Samples and Meth. of Tes

Silicomolybdate Complex (1970)
108
141

142

232

14

1036

880

1624

1867

1437

2395 
ntent, Gravimetric Meth. by Precipitation of the Quinoline ntent; Gravimetric Meth. by Precipitation of the Quinoline ilica Content-Spertrophotometric Meth. Using the Reduced ion of Silicon-Spectrophotometric Meth. with the Reduced ilica Content-Spectrophotometric Meth. Using the Reduced al Analysis of Magnesium and Its Alloys-Determination of of Chemical Analysis of Manganese Ores-Determination of cation / Rec. for Strip Wound Cut Cores of Grain Oriented (Aluminum) Alloy Conductor Wire of the Aluminium Magnesium

aluminum) Alloy Busbar Material of the Aluminium Magnesium or Aluminium Alloy Stranded Conductors (Aluminum Magnesium um (Aluminum) and Its Alloys: Photometric Determination of um (Aluminum) and Its Alloys: Gravimetric Determination of

al Analysis of Steel and Cast Iron: Determination of Total for Diameters of Drafting Rollers for Cotton, Wool, Spun

lar Types of Winding Wires: Bunched Enamelled Copper, with oose Bulk Materials-Storage Equipment: Bins and Bunkers, nation of - Containing Zirconium, Rare Earths, Thorium and d Strip of Thickness Between $0.2 \mathrm{~mm}(0.008 \mathrm{In}$. mm Thick (I959) re (1969)

Rec. for Rec. for Rec. for Std. for

Std, for Surface Active Agents: ondenser Microphones by the Reciprocity Techniq/ Rec. for Plastics (1970) Rec. for Accelerated Ageing and graphy-Light Sources for Use in Sensitometric Exposure graphy-Light Sources for Use in Sensitometric Exposure ography: Light Sources for Use in Sensitometric Exposure nal Combustion Engines-Definition of Right and Left Hand

Rec, for Sealed NickeI Cadmium Cylindrical Rechargeable Rec. for Sealed Nickel Cadmium Cylindrical Rechargeable aft (I966) Rec. for Meth. of Test for Wool Fibre Length Using A

cified or Independent Specif/

Im Perforated Along I Edge (1966) es with Solid or Stranded Conductors, Insulated, Screened, Rec. for Dimensions for General Purpose Push Pul for Perform ance Requirements for General Purpose Push Pull Rec. for Determination of the Thickness of c) Pressure Pipes with Elastic Sealing Ring Typ/ Std. for Continuous Mechanical Handling Equipment for Unit Loads Continuous Mechanical Handling Equipment for Unit Loads

Determination of Breaking Load and Elongation of Same (Of ires, Type I, with Solid or Stranded Conductor, Insulated, inyl Chloride) Insulation and Sheath: Signalling Cables in res with Solid Conductors, Insulated, Polyamide Coated, in Basic Environmental Test. Procedures: Test Fc: Vibration Rec. for 3 or 4 Strand Manila and Rec. for 8 Strand Plaited Manila and

less) (I972)

reraft (1969)

ec. for Microgra Rec. for Granulated Cork-Granule Std. for Size Analysis of Small Coke (Nominal Top uscovite Mica Blocks, Thins and Films Meth. for Grading by te Mica Blocks, Thins and Splittings: Meth. for Grading by Rec. for Coniferous Sawn Timber: Rec, for Coniferous Sawn (Sawed) Timber Rec. for Coniferous Sawn Timber ses (Rear Mounting Type) (I959) eneral Purpose Metric Screw Threads: Tolerances, Limits of Rec, for Lathe Centres (Centers): ec. for Spindle Noses and Face Plates Types a and Camlock: Rec. for Spindle Noses and Face Plates: Bayonet Type; Rec. for Scales and

d. for ISO General Purpose Metric Screw Threads-Selected Rec. for Paper: Untrimmed Stock Rec. for Paper: Untrimmed Stock r Wire and Plate Screens for Industrial Purposes-Nominal n Wire Cloth and Perforated Plates in Test Sieves: Nominal Sheets (I967) Rec. for Paper: Overall Trimmed Rec. for el Shanks (I961) Rec. for Diameters of Shanks and Rec. for Paper and Board: Rec. for

1 Devices (I 960$)$

Rec for Microcopies on Transparent Bases ic Colour (Color) Paper for Roll Paper Printers: Preferred c Black and White Paper for Roll Paper Printers: Preferred hotographic Colour (Color) Film for General Use: Preferred otographic Colour (Color) Paper for General Use: Preferred
Silicomolybdic Complex (1969) Silicomolybdic Complex (1969) Silicomolybdic Complex (1970) Silicomolybdic Complex (1971) Silicomolybdic Complex (1972) Use: Determination of S ISO Silicon-Spectrophotometric Meth, with the Reduced Sil ISO Silicon Dioxide (1963) Rec. for Meth. ISO Silicon Iron Alloy, Used for Electronic and Telecommuni IEC Silicon Type (1958) /Nternational Spec. for Aluminium IEC Silicon Type (1959) Rec. for Heat Treated Aluminium ( IEC Silicon Type) (1966) Rec. F IFC Silicon (Content Between 0.02 and $0.4 \%$ ) (1968) /Lumini ISO Silicon (Content Greater Than or Equal to 0.30\%) (1968) ISO Silicon (Gravimetric Meth.) (1969) Rec. for Chemic ISO Silk and Staple Fibre (Fiber) (1959) Rec ISO Silk Covering (1972) Silos and Hoppers, Bin Gates-Safety Code (1970) Silver-Periodate Photometric Meth. (1972) Rec. for Particu IEC /R L ISO Simple Bend Test. of Steel Sheet and Strip Less Than 3 ISO Simple Torsion Test for Aluminum and Aluminium Alloy Wi ISO Simple Torsion Test. of Steel Wire (1972) ISO Simplified Classification (1972)

Simplified Meth. for Pressure Calibration of One Inch C Simulated Service Test of Fabrics Coated with Rubber or Simulation of the Spectral Distribution of Daylight (19 Simulation of the Spectral Distribution of Photoflood I Simulation of the Spectral Distribution of Tungsten Ill Single Bank Engines (1972) /D. for R Single Cells: Dimensions (I972)

Single Cells: General Requirements and Test Meth. (197I

Single Fiber Length Measuring Machine (I970)

Single Hole Mounting, Lever Operated Switches for Aircr Single Input Energizing Quantity Measuring, with Nonspe

Single Magnetic Sound Stripe on $16 \mathrm{~mm}$ Motion Picture Fi

Single or Double Row of Healds (1967)

Single or One Pair (1969) /H: Equipment Wires and Cabl

Single Pole Circuit Breakers for Aircraft (1966)

Single Pole Circuit Breakers for Aircraft (1970)

Single Sheets of Paper (1967)

Single Sockets for Unplasticized Polyvinyl Chloride (Pv

Single Strand Floor Mounted Truck Conveyors (Chain Abov

Single Strand Floor Truck Conveyors (Chain Below Floor)

Single Strands) (1972) /Yarn from Packages-Meth. for ISO

Single (1967)

(Ide) Insulation and Sheath: Equipment W IEC

Singles for Telecommunication Equipment and Installatio IEC

Singles, Pairs, Triples, Quadruples and Quintuples (197 IEC

(Sinusoidal) (1970)

Sisal Ropes (1970)

Sisal Ropes (I97I)

Size Analysis of Coke (I968)

Rec. for IEC

ISO

ISO

Size Analysis of Small Coke (Nominal Top Size $20 \mathrm{~mm}$ or

ISO

Size Analysis (I972)

Size and Direction of Operation of Lever Controls on $\mathrm{Ai}$

Size of Picture Postcards and of Lettercards (I963)

Size of Steels (1967)

Size Test (I97I)

Size $20 \mathrm{~mm}$ or Less) (1972)

Size (I958)

Size (1965)

Sizes-Meth. of Measurement (I968)

Sizes-Terms and Definitions (I969)

Sizes-Tolerances and Shrinkage (1968)

Sizes and Designation of Working Parts (1972)

Sizes and Mounting Dimensions of Aircraft Instrument Ca

Sizes for Commercial Bolt and Nut Threads: Medium Quali

Sizes for Interchangeability (1963)

Sizes for Interchangeability (1968)

Sizes for Interchangeability: Metric Series (1968)

Sizes for Plotting Frequency Characteristics (1968)

Sizes for Screws, Bolts and Nuts (1973)

Sizes for the ISO-A Series, ISO Primary Range (I966)

Sizes for the ISO-A Series: ISO Supplementary Range (19

Sizes of Apertures (I972)

Sizes of Apertures (1972)

Sizes of Articles of Stationery That Include Detachable Std. Fo ISO

Sizes of Driving Squares for Rotating Tools with Parall

Sizes of Folders and Files (I967)

Sizes of Photocopies (On Paper) Readable Without Optica ISO

Sizes of Rec. Bases (1961)

Sizes of Rolls (I969)

Sizes of Rolls (1969)

Sizes of Sheet Material (1969)

Sizes of Sheet Material (1969)
Rec. for Photographi ISO

Rec. for P ISO

Rec. for pH ISO
Rec. for Photograph ISO 
ugraphir: Blark and White Papers for General Use: Preferred atter $(1961)$

Ker for Pneumatic Cylinders-Cylinder Bores and Port - for Roll Film Cameras-Back Window Location and Picture holography-Cameras Using Films $35 \mathrm{~mm}$ and Smaller Picture rills with Parallel, and Morse Taper Shanks-Rec. Stocked Rec. for Paper: Untrimmed Rec. for Rubber Hose-Bore s-Determination of Linear Density (Mass Per Unit Length) Rec. for Dimensions of Aerial Rods and Rec. for Asbestos Cement Rec. for Definitions of Living Animals for Rec. for International System for the Transliteration of Shanks (1961)

Rec. for Reduction Rec. for Cylindrical Abrasive Std. for Truncated Cone Abrasive Std. for Modular Units for Machine Tool Construction: Rec. for Photography-Projector
Rec. for Cinematography-Picture A reas for Films and Lifting Purposes-Grade 40 Noncalibrated Chain for Chain clature for Carbon Brushes, Brush HoIders, Commutators and s (1972) Rec. for Dimensions for Commutators and Std. for Metal Rec. for Cylindrical Rec. for Cylindrical Rec. for Cylindrical anual Arc Welds Made Wi/ Rec. for Limitation of Angles of Rec. for Liquid Flow Measurement in Open Channels by damental Welding Positions and Definitions of Rotation and for Shell Reamers with Taper Bore (1:30 (Included)) with Rec. for Machine Tool Tables-T 1) Rec. for Dimensions and Pitching of Rec. for Cotter Rec. for Rec. for Rec. for Rec. for (1970)

ions in Millimetres (Millimeters) and In./S (19/

Std. for Screwdriver Blades for

Std. for Screwdriver Bits, for Std. for Rec. for tres (Millimeters) and In./S (1970)

Rec. for Dimensions in Millimetres (Millimeters) and In/ Rec. for series (1972) Std. for for Lever Switches: Requirements for Switches of Type 1, Rec. for Natural Rubber Latices-Determination of Rec. for Rolling Bearings-With Locating Std. for Rolling Bearings: with Locating

Spectropho/

$y$ in Ethanol (1968)

Std. for Surface Active Agents: Analysis of Rec. for Analysis of Rec. for the Analysis of Rec. for Analysis of Rec. for Analysis of Rec. for Analysis of Rec. for Analysis of Rec. for Analysis of Std. for Analysis of Rec. for Analysis of S/ Rec. for Connectors for Frequencies Below $3 \mathrm{MHz}$ : Edge ui/ Rec. for Connectors for Frequencies Below $3 \mathrm{MHz}$ : Edge Std. for Tests for Aircraft Electrical Plug and Rec. for Ball and and General Requir/ Rec. for Locking Devices for Ball and ions/ Rec. for Pipes and Fittings of Plastics Materials. Polyvinyl Chloride (PVC) Fittings with PIain S/ Rec. for vc) Pressure Pipes with Elastic Sealing / trial/

Std. for Double Plastics Materials:

Rec. for Hexagon

69)

57)

Rec. for Std. for Plugs and

Spec. for Plugs and

Spec. for Plugs and Std. for Hexagon

ssembly Tools for Screws and Nuts-Forged and and Tubular for Bolts and Screws-Driving Squares for Power and Hand Rec for Hand Operated W'renches and of Cylindrical Machined Graphite Electrodes with Threaded capillary Solder Fittings for Copper Tubes-Dimensions of ements and Meth. of Test (1963)

nd Dimensions of Wiring Jigs and Pin Straighten/ Rec. for Rec for Reduction Sleeves and Extension sure Pipes with Elastic Sealing Ring Typ/ Lengths (1957)

Std. for Single Rec. for Steel Rec. for Tolerances on Spanner Gaps and Rec. for Aircraft Fuel Nozzle Grounding Plugs and unplasticized Polyvinyl Chloride (PVC) Fittings with Plain
Sizes of Sheet Material (1969) Rec. for Phot ISO Sizes of Writing Paper and Certain Classes of Printed M Sizes (1970)

Sizes (1971)

Sizes (1971)

Sizes (1972)

Sizes, Designation, Tolerances (1966)

Sizes, Test Pressures and Tolerances on Length (1970) D for Textites:

Skein Meth. (1972)

Slabs of Ferromagnetic Oxides (1966)

Slates for Roofing and Cladding (1964)

Slaughter-Porcines (1970)

Slavic Cyrillic Characters (1968)

Sleeves and Extension Sockets for Tools with Morse Tape

SIeeves: Designation, Dimension, Tolerance (1972)

Sleeves: Designation, Dimensions, Tolerances (1972)

Slide Units (1973)

Slides-Dimensions (1971)

Slides for Television (1971)

Slings, Etc. (1971)

Slip Rings (1968)

Slip Rings (1971)

Slitting Saws with Fine and Coarse Teeth-Metric Serie

Rec Short Link Chain for

Rec ISO

Rec, for P ISO

Std. for Core D ISO

ISO

ISO

IEC

SO

ISO

ISO

ISO

SO

ISO

Sliver Cans on Castors (1969)

Sliver Cans (1963)

Sliver Cans: Heights Over $1000 \mathrm{~mm}$ (1969)

Slope and Rotation for Welding Positions for Straight M

Slope Area Meth. (1969)

Slope for Straight Welds (1967)

Slot Drive and Arbors for Shell Reamers (1972)

Slots and Corresponding Bolts (1971)

Slots on Platens for Projection Welding Machines (1968)

Slots with 5\% Taper Keys for Boring Machine Tapers (197

Slotted and Castle Nuts with Metric Thread (1963)

Slotted and Castle Nuts with Metric Thread, $42 \mathrm{Up}$ to an

Slotted Cheese (Fillister) Head Screws-Metric Series

Slotted Countersunk (Flat) Head Tapping Screws-Dimens

Slotted Head Screws (1972)

Slotted Head Screws, with Male Hexagon Drive (1972)

Slotted Headless Screws-Metric Series (1972)

Slotted Pan Head Screws-Metric Series (1970)

Slotted Pan Head Tapping Screws-Dimensions in Millime

Slotted Raised Countersunk (Oval) Head Tapping Screws-

Slotting Cutters with Plain Bore and Key Drive: Metric

Slow Make and Break (1963)

Sludge Content (1971)

Snap Ring-Dimensions (1965)

Snap Ringr-Inner Diameter of (1972)

Soaps-Determination of Low Contents of Free GlyceroI

Soaps: Determination of Foreign Matter of Low Solubilit

Soaps: Determination of GIycerol (1969)

Soaps: Determination of Moisture and Volatile Matter (1

Soaps: Determination of Total Alkali (1968)

Soaps: Determination of Total Free Alkali (1968)

Soaps: Determination of Unsaponifiable and Unsaponified

Soap: Determination of Chlorides (1965)

Soap: Determination of Free Caustic Alkali (1973)

Soap: Determination of Total Crude Fatty Acids (1965)

Socket Connectors with Closed Ends and Having a Contact

Socket Connectors with Open Ends, Off Centre (Center) G

Socket Connectors (1972)

Socket Couplings of String Insulator Units (1960)

Socket Couplings of String Insulator Units: Dimensions

Socket Fittings for Pipes Under Pressure-Basic Dimens

Socket Fittings for Pipes Under Pressure: Unplasticized

Socket Fittings for Unplasticized Polyvinyl Chloride (P

Socket Fittings with Spigot Ends for Domestic and Indus

Socket Head Cap Screws: Metric Series (1968)

Socket Outlets and Couplers for Industrial Purposes (19

Socket Outlets for Domestic and Similar GeneraI Use (19

Socket Outlets for Domestic and Similar Purposes (1963)

Socket Outlets for Industrial Purposes (1966)

Socket Set Screws-Metric Series (1972)

Socket Wrenches-Metric Series-Maximum Outside Dimen

Socket Wrenches (1970)

Rec. for Assembly Tools

Sockets and Connecting Pins for Use in Electric Arc Fur

Sockets and Male Ends (1971)

Sockets for Electronic Tubes and Valves: General Requir

Sockets for Electronic Tubes and Valves: Spec. Sheets a

Sockets for Tools with Morse Taper Shanks (1961)

Sockets for Unplasticized Polyvinyl Chloride (PVC) Pres

Sockets Screwed in Accordance with ISO Rec. R7: Minimum

Sockets (Metric Values for General Use) (1968)

Sockets (1970)

Sockets; Metric Series (1968) 
I'otassium Ilydroxide for Industrial Use-Determination of l'otassium llydroxide for lndustrial Use-Determination of Rec. for Surface Active Agents-Technical Rec. for Surface Active Agents: Technical ed Sodium Perborates for Industrial Use-Determination of isodium Tetraborates for Industrial Use-Determination of crude Sodium Borates for Industrial Use-Determination of Rec. for Rec. for Rec. for Rec. for Rec. for Rec. for Std. for Std. for Std. for Rec. for ermination of Density of Samples in Solutionermination of Dry Matter-Gravimetric Meth. (1/ ermination of Carbon Dioxide Content Expressed / ermination of Total Alkalinity, Expressed in $\mathrm{Na}$ ermination of Dynamic Viscosity (1972)

ermination of Silica Content-Titrimetric Meth/ paration of Solution of Products Not Easily Sol/ ples and Meth. of Test-General (1970)

nd Lignites-Meth. of Extraction for the Determination of after Combustion in a Current of $\mathrm{Oxygen}$ and Titration with $8 s$ in Mass After Heating at $900^{\circ} \mathrm{C}(1 /$ dium and Boric Oxide Contents-Volumetri/ Std. for Crude Std. for Crude

Boric Acid, Boric Oxide, Disodium Tetraborates and Crude m Hydrogen Carbonate for Industrial Use-Determination of hloride Content: Volhard Volumetric Meth. (1973/ ron Content; $2,2^{\prime}$-Bipyridyl Photometric M/

oss of Mass and of Nonvolatile Matter at $250 \mathrm{De} /$ atter Insoluble in Water at $50{ }^{\circ} \mathrm{C}(1968)$

odium Hydrogen Carbonate; Volumetric Meth. (196/ ulphate Content; Barium Sulphate Gravimetric Me/ otal Soluble Alkalinity: Volumetric Meth. (1968/ Results (1968)

orage of Test Samples (1968) Rec. for Spec. for Photographic Grade Determination of the Carbon Dioxide Content, Expressed a loride Content-Mercurimetric Meth. (1973) tter Insoluble in Water (1973) isture Content-Gravimetric Meth. (1973) lcium and Magnesium Contents-EDTA Complexomet/ logens, Expressed as Chlorine-Mercurimetric M/ tter Insoluble in Water or in Acid and Preparat/ lphate Content-Barium Sulphate Gravimetric Me/ e Loss of Mass at $110^{\circ} \mathrm{C}(1973)$

ly Used for the Production of Aluminium-Determination of

m Hydrogen Carbonate for Ind ustrial Use-Determination of of Chloride Content-Mercurimetric Meth. (1972) Std. for ation of Sodium Carbonate-Titrimetric Meth. (/ ation of Sodium Hydrogen Carbonate Content-Ti/ ation of Moisture Content-Gravimetric Meth. (/ meth. of Test and Preparation of the Sample (19/ lkalinity, Volumetric Meth. (1969)

hloride Content: Volhard Volumetric Meth. (1973/ alcium; EDTA Complexometric Meth. (1969) f Iron Content: 2,2'-Bipyridyl Spectropho/ f Silica Content; Gravimetric Meth. by Precipit/ ulphate Content: Barium Sulphate Gravimetric Me/ ilica Content: Gravimetric Meth. by Insolubiliz/ he Carbon Dioxide Content, Expressed as Sodium / ater Insoluble Matter (1969)

orage of Test Sample (1969)

ple Solution (1969) esol for Industrial Use-Test for Impurities Insoluble in Rec. for Schedule for use-Determination of Carbon Dioxide Content Expressed as Sodium and Boric Oxide and Available / Std. for Hydrated Rec. for Surface Active Agents: Technical Rec. for Sodium Tripolyphosphate and Rec. for Sodium Tripolyphosphate and of Iron Content-1 of loss on Ignitio/

Rec. for Surface Active Agents: Technica Rec. for Spec. for Photographic Grade m Chloride for Industrial Use-Determination of Content

m Sulphate for Ind ustrial Use: Determination of Content Std. Spec. for Photographic Grade dustrial Use-Determination of Iron Content-1 dustrial Use-Determination of Loss on Ignitio/ ion of Matter Insoluble in Water (1968)

$\mathrm{t}$ of $\mathrm{pH}$ Potentiometric Meth. (1968)

Rec. for

Rec. for

Rec. for

Rec. for $s$ and Male Ends (I971) Rec. for Plastics-Determination of the Vicat rocedures for Electronic Components and Equipment: Test T: test Meth. and Practical Guidance (1971)

Std. for Classification o

1 Chloride) Insulation and Sheath: Distribution Wires with I Chloride) Insulation and Sheath: Distribution Wires with

r Std. Conditions for Use Prior to and During the Test. of Rec. for Fixed Tantalum Capacitors with Liquid or
Sodium-Flame Emission Photometry Meth. (1970)

Sodium-Gravimetric Meth. Using Uranyl Acetate and Mag

1. for IS

Sodium Alkylsulphonates-Meth. of Analysis (1969)

Sodium and Boric Oxide and Available Oxygen Contents-

Sodium and Boric Oxide Content and Loss on Ignition (19

Sodium and Boric Oxide Contents-Volumetric Meth. (197

Sodium and Potassium Silicates for Industrial Use

Sodium and Potassium Silicates for Industrial Use-Det

Sodium and Potassium Silicates for Industrial Use-Det

Sodium and Potassium Silicates for Industrial Use-Det

Sodium and Potassium Silicates for Industrial Use-Det

Sodium and Potassium Silicates for Industrial Use-Det

Sodium and Potassium Silicates for Industrial Use-Det

Sodium and Potassium Silicates for Industrial Use-Det

Sodium and Potassium Silicates for Industrial Use-Pre

Sodium and Potassium Soluble in Dilute Hydrochloric Aci

/Etermination of Sulphur (Meth.

Sodium Borate) (1968)

se-Determination of Lo

Sodium Borates for Industrial Use-Determination of So

Sodium Borates for Industrial Use: Determination of Sul

Sodium Carbonate-Titrimetric Meth. (1972)

Sodium Carbonate for Industrial Use: Determination of $C$

Sodium Carbonate for Industrial Use: Determination of I

ISO

for Sodiu ISO

Sodium Carbonate for Industrial Use: Determination of $\mathrm{L}$

Sodium Carbonate for Industrial Use: Determination of $M$

Sodium Carbonate for Industrial Use: Determination of $S$

Sodium Carbonate for Industrial Use: Determination of $\mathrm{S}$

Sodium Carbonate for IndustriaI Use: Determination of $T$

Sodium Carbonate for Industrial Use: Expression of Test

Sodium Carbonate for Industrial Use: Preparation and St

Sodium Carbonate, Anhydrous (1965)

Sodium Carbonate, Gas-Volumetric Meth. (1969)

Sodium Chlorate for Industrial Use: Determination of $\mathrm{Ch}$

Sodium Chlorate for Industrial Use: Determination of $\mathrm{Ma}$

Sodium Chlorate for Industrial Use: Determination of $\mathrm{M}_{0}$

Sodium Chloride for Industrial Use: Determination of $\mathrm{Ca}$

Sodium Chloride for Industrial Use: Determination of $\mathrm{Ha}$

Sodium Chloride for Industrial Use: Determination of $\mathrm{Ma}$

Sodium Chloride for Industrial Use: Determination of Su

Sodium Chloride for Industrial Use: Determination of Th

Sodium Content-Flame (Emission) Spectrophotometric Me

Sodium Hydrogen Carbonate Content-Titrimetric Meth. (

Sodium Hydrogen Carbonate for Industrial Use-

Sodium Hydrogen Carbonate for Industrial Use-Determin

Sodiun Hydrogen Carbonate for Industrial Use-Determin

Sodium Hydrogen Carbonate for Industrial Use-Determin

Sodium Hydrogen Carbonate for Industrial Use-List of

Sodium Hydrogen Carbonate: Volumetric Meth. (1968)

Sodium Hydroxide for Industrial Use: Determination of a

Sodium Hydroxide for Industrial Use: Determination of C

Sodium Hydroxide for Industrial Use: Determination of $C$

Sodium Hydroxide for Industrial Use: Determination of 0

Sodium Hydroxide for Industrial Use: Determination of 0

Sodium Hydroxide for Industrial Use: Determination of S

Sodium Hydroxide for Industrial Use: Determination of S

Sodium Hydroxide for Industrial Use: Determination of T

Sodium Hydroxide for Industrial Use: Determination of W

Sodium Hydroxide for Industrial Use: Preparation and St

Sodium Hydroxide for Industrial Use: Preparation of Sam

Sodium Hydroxide Solution-Visual Test (1971)

Sodium Lamps (Integral Type) (1965)

Sodium or Potassium Carbonate-Gas Volumetric Meth. (1

Sodium Perborates for Industrial Use-Determination of

Sodium Primary Alkylsulphates: Meth. of Analysis (1968)

Sodium Pyrophosphate for Industrial Use-Determination

Sodium Pyrophosphate for Industrial Use-Determination

Sodium Secondary Alkylsulphates: Meth. of Analysis (196

Sodium Sulphite (1965)

Sodium Tatraphenylborate Volumetric Meth. (1971)

Sodium Tetraphenylborate Volumetric Meth. (1973)

Sodium Thiosulphate, Crystalline (1972)

Sodium Tripolyphosphate and Sodium Pyrophosphate for in

Sodium Tripolyphosphate and Sodium Pyrophosphate for in

Sodium Tripolyphosphate for Industrial Use-Determinat

Sodium Tripolyphosphate for Industrial Use-Measuremen

Softening Temperature of Thermoplastics (1968)

Solder Fittings for Copper Tubes-Dimensions of Socke

Soldering (1968) Rec. for Basic Environmenta

Solderless Wrapped Connections-General Requirements,

Solid Beech (Wood) Parquet Strips (1972)

Solid Cond uctors, Insulated, in Pairs, Triples, Quadrup

Solid Conductors, Insulated, Polyamide Coated, in Singl

Solid Electrical Insulating Materials (1971)

Solid Electrolyte (1971)

ISO 
Rec. Meth. of Test for Electric Strength of Meth for Determining the Comparative Tracking lndex of test for the Determination of the Insulation Resistance of value hy the Calorimetric Bomh Meth. and Calcul/ Rec. for Rec. for Vocabulary of Terms Relating to Rec. for Vocabulary of Terms Relating to Rec. for Vocabulary of Terms Relating to Rec. for Determination of Ash of Rec. for Classification of Parquet Strips of is (1971)

and Sheath: Equipment Wircs, Type I, with de) Insulation and Sheath: Equipment Wires and Cables with Rec. for Rec. for

Rec. for Long Rec. for Short Rec. for Railway Rolling Stock Material: Rec. for steel: Dimensions of Wires, Spools, Rims and Co/ Rec. for
Rec. for General Manufacturing Characteristics of steel: Dimensions of Wires, Spools, Rims and Co/ rec. for
Rec. for General Manufacturing Characteristics of

Rec. for Animal Fats: Determination of persions of Polymers and Copolymers-Determination of Dry Rec. for Determination of Total t and Vegetable Products: Determination of Water Insoluble for Fruit and Vegetable Products: Determination of Total Analysis of Soaps: Determination of Foreign Matter of Low Rec. for Determination of Rec. for Pulps: Determination of Alkali odium Carbonate for Industrial Use: Determination of Tota Rec. for Tea-Determination of Water Rec. for Tea-Determination of Alkalinity of Water Rec. for Determination of the Yield of Benzene Rec. for Spices and Condiments: Determination of Alcohol c. for Spices and Condiments: Determination of Cold Water trial Use-Preparation of Solution of Products Not Easily Rec. for Pulps: Extraction of Materials

f Extraction for the Determination of Sodium and Potassium t from Brown Coals and Lignites-Determination of Acetone for Plastics-Determination of the Percentage of Acetone Rec. for Plastics-Determination of Ethyl Ether Rec. for Plastics-Determination of Methanol

n-Heptane (1969) Rec. for Plastics-Determination of ded Sta/ Rec. for Plastics-Determination of the Acetone al Analysis of Magnesium and Its Alloys-Determination of or Industrial Use-Determination of Density of Samples in al Use-Test for Impurities Insoluble in Sodium Hydroxide ed for the Production of Aluminium-Preparation of Sample sed for the Production of Aluminium: Preparation of Sample rial Use: Sampling-Test Sample-Preparation of the Main of the Resistance of Glass to Attack by a Boiling Aqueous nd Potassium Silicates for Industrial Use-Preparation of andardization of Meth. for the Determination of the Dilute ination of Viscosity Number of Polyvinyl Chloride Resin in tion of the Percentage of Styrene in Polystyrene with Wijs ion of the Viscosity Number of Polyamides Resins in Dilute on of the Viscosity Ratio of of Polyamides in Concentrated sodium Hydroxide for Industrial Use: Preparation of Sample assium Hydroxide for Industrial Use: Preparation of Sample iscosity Number of Poly (Ethylene Terephthalate) in Dilute iting-Number of Polyethylenes and Polypropylenes in Dilute (1972)

Number and Viscosity Ratio of Cellulose Acetate in Dilute 2) Std. for Formaldehyde Std. for Formaldehyde Std. for Formaldehyde Std. for Formaldehyde Std. for Formaldehyde Std. for Formaldehyde Sid. for Formaldehyde Std. for Formaldehyde tent-2,2' Bipyrid Content (1972)

ls (Excluding Iron) (1972)

chlorides (1972)

sulphates (1972) Std. for Formaldehyde
Preparation of Principal insoluble in Water or in Acid and Preparation of Principal 70) Relative Humidity in Small Enclosures by Means of Aqueous

Rec. Spec. for Particular Types of Winding Wires: Heat or Rec. Spec. for Particular Types of Winding Wires: Heat or Rec. for Rubber-Determination of

bon Solvents (1972) Std. for Mineral solvents for Paint s: White Spirits and Related Hydrocarbon ics: Description and Measurement of Physical Properties of fibre (Fiber) Length Barbe (Barb) and Hauteur Using a Comb below 3 MHz: Connectors for Radio Receivers and Associated Below $3 \mathrm{MHz}$ : Circular Connectors for Radio and Associated Rec. for Rating of Rec. for Precision or Emulsion and Sound Record Positions in Camera for $35 \mathrm{Mm}$ or Emulsion and Sound Record Positions in Camera for $16 \mathrm{Mm}$ emulsion and Sound Record Positions in Projector for $35 \mathrm{Mm}$ or Expression of the Physical and Subjective Magnitudes of
Solid Insulating Materials at Power Frequencies (1967)

Solid Insulating Materials Under Moist Conditions (1971

Solid Insulating Materials (1964) Rec. for

Solid Mineral Fuels-Determination of Gross Calorific

Solid Mineral Fuels-Part Ill: Coke (1971)

Solid Mineral Fuels-Part II: Coal Sampling and Analys

Solid Mineral Fuels-Part I: Coal Preparation (1970)

Solid Mineral Fuels (1970)

Solid Oak (1970)

Solid or Stranded Conductor, Insulated, Single (1967)

Solid or Stranded Conductors, Insulated, Screened, Sing

Solid Stem Calorimeter Thermometers (1968)

Solid Stem General Purpose Thermometers (1970)

Solid Stem Thermometers for Precision Use (1968)

Solid Stem Thermometers for Precision Use (1968)

Solid Wheels for Trailer Stock (1969)

Solid Wires for Gas Shielded Metal Arc Welding of Mild

Solid Wood Parquet Strips with Rectangular Face (1969)

Solidification Point of Fatty Acids (Titre) (1969)

Solids Content at $105^{\circ} \mathrm{C}(1970)$

Solids of Latex (1966)

Solids (1968)

Solids (1969)

Solubility in Ethanol (1968)

Solubility of Essential Oils in Ethanol (1968)

Ics-Aqueous Dis ISO

Rec. for Frui ISO

Rec ISO

Rec. for ISO

ISO

Solubility (1968)

Soluble Alkalinity: Volumetric Meth. (1968)

/Ec. for S ISO

Soluble and Insoluble Ash (1970)

Soluble Ash (1970)

Soluble Extract in Brown Coals and Lignites (1969)

ISO

ISO

ISO

Soluble Extract (1969)

ISO

Soluble in Boiling Water and Determination of Matter in

Soluble in Dichloromethane (1967)

Soluble in Dilute Hydrochloric Acid (1971)

Soluble Material (Resinous Substances) (1969)

Soluble Matter in Phenolic Mouldings (1958)

Soluble Matter in Plasticized Cellulose Acetate (1971)

ISO

ISO

1-Meth. O ISO

/ Extrac ISO

Rec. ISO

ISO

ISO

Soluble Matter of Crystalline Polypropylene by Boiling

Soluble Matter (Resin Content of Material in the Unmoul

Soluble Zirconium (Alizarin Sulphonate Photometric Meth

ISO

ISO

Solution-Meth. Using Density Hydrometer-Meth. Using

Solution for Analysis by Means of Attack by Hydrochlori ISO

Solution for Analysis (1968) /Minum) Oxide Primarily U ISO

Solution for Carrying Out Certain Determinations (1973) ISO

Solution of Mixed Alkali (1968) /Ec. for Determination ISO

Solution of Products Not Easily Soluble in Boiling Wate ISO

Solution Viscosity of Polymers (1970) /Ives for the St ISO

Solution (1961)

Solution (1961)

Solution (1963)

Solution (1967)

Solution (1969)

Solution (1969)

Solution (1970)

Solution (1970)

Solution (1970)

Rec. for Plastics-Determ ISO

Rec. for Plastics-Determina ISO

Rec for Plastics-Determinat ISO

Rec. for Plastics-Determinati ISO Rec. for ISO

Rec. for Pot ISO

(P) Number and Lim ISO

Industrial Use: Determination f Acidity

Solutions for Industrial Use: Determination of Ash (197

Solutions for Industrial Use: Determination of Formalde ISO

Solutions for Industrial Use: Determination of Iron Con ISO

Solutions for Industrial Use: Determination of Methanol ISO

Solutions for Industrial Use: Limit Test for Heavy Meta ISO

Solutions for Industrial Use: Limit Test for Inorganic ISO

Solutions for Industrial Use: Limit Test for Inorganic ISO

Solutions for Other Determinations (1972) / of Matter ISO

Solutions (1966) /Ics-Meth. for Maintaining Constant ISO

Solvent Acetates for Industrial Use-Meth. of Test (19

Solvent Bonding Enamelled Round Copper, with High Mecha

Solvent Bonding Self Fluxing Enamelled Round Copper (19

Solvent Extract (1971)

Solvents for Paints: White Spirits and Related Hydrocar

Solvents (1972)

Sonic Booms (1973)

Sorter (1969)

Sound Equipment (1965)

Sound Equipment (1971)

Sound Insulation for Dwellings (1968)
Sound Level Meters (1961)

Sound Level Meters (1965)

Sound Motion Picture Film (1956)

Sound Motion Picture Film (1956)

Sound Motion Picture Film (1956)

Sound or Noise (1959)
Std for Mineral ISO Rec. for Meth. of Test for Wool ISO (for Connectors for Frequencies IEC

ISO

IEC

Rec. F ISO

Rec. $F$ ISO

Rec. for ISO

Rec. F ISO lor Connectors for Frequencies IEC 
Hec. for Expression of the Power and Intensity Levels of fuse Field and in a Frontally

Rec for Relation Between Records and Scanning Area of $35 \mathrm{~mm}$ Double Width Push Pull Rec. for Cinematography-Photographic Three Magnetic Sound Records on $35 \mathrm{~mm}$ Film and 1 Magnetic Picture Film (1956)

Picture Film (1956)

ion Picture Film (1956) Rec. for Cinematography-Photographic Rec. for Emulsion and Rec. for Emulsion and Rec. for Emulsion and s of Recording and Reproducing Magnetic Heads for Magnetic Rec. for Meth of Measurement of Speed Fluctuations in ush Pull Sound Prints Normal A/ Rec. for Cinematography ocation and Width of the Recoding Head for Centre (Center) 71) ec. for Location and Width of the Recording Head for Edge

Rec. for Recording Heads for 6 Track Magnetic
Rec. for Location of Recording Heads for Three Magnetic matography-Location of Recording Heads for Four Magnetic for Cinematography-Recorded Characteristic for Magnetic and Technical Spec. (1966) ong 1 Edge (1966)

1)

Rec. for Single Magnetic 971) Rec. for Rec. for Rec. for Rec. for Rec. for Rec. for 1) Rec. for

$r$ Field and Laboratory Measurements of Airborne and Impact and Third-Octave Band Filters Intended for the Analysis of tive Materials (1965)

of the Spectral Distributio/

of the Spectral Distribution/

of the Spectral Distributio/ Rec. for Power

Std. for Photography-Light

Std. for Photography: Light Std. for Photography-Light
Rec. for Lengths of Narrow V-Belts: Sections SPZ

r Grooved Pulleys for Narrow V.Belts: Groove Sections SPZ,

Basic Quantities and Units of the SI (Metric Unit) and of

ge Socket Connectors with Closed Ends and Having a Contact ) (1968)

ith Open Ends, Off Centre (Center) Guide, Having a Contact Std for Internal l Combustion Engines: Rec. for Automobile Std. for Automobiles: Std. for Automobiles: Std. for Automobiles: Std. for Automobiles : Compact Std. for Automobiles: Compact Std. for Automobiles:

c. for Electrical A pparatus for Explosive Gas Atmospheres: Rec. for Lengths of Narrow V-Belts: Sections SPZ, SPA oved Pulleys for Narrow V-Belts: Groove Sections SPZ, SPA, 5) Rec. for Classification of Brasses, Leaded, Frozen Food/ Rec. for Household Refrigerators-Part II: ng for Deep Penetration Electrodes (1968) Rec. for Installa/ Rec. for Short Circuit Current Evaluation with rical Type $\mathrm{RD}$ with Tapered Bore 1:12-Tolerance Class 5 Rec. for Classification of Tin and Rec. for ec. for Classification of Aluminium (Aluminum) Bronzes and rement on Radio Receivers for Various Classes of Emission: oring-Code of Symbol/ Rec. for Marking of the Different or Shock and Vibration Measurement (1/ Rec. for Meth. for ansducers for Shock and Vibration Mea/ Rec. for Meth for ion of the Recovery from Creasing of a Horizontally Folded for Wrought Aluminum and Aluminium Alloys-Selection of ing Meth. (1968) ding Meth. (1968) Rec. for Plastics-Preparation of Rec. for Plastics-Compression Moulding Test Rec. for Plastics-Injection Moulding Test tion of $\mathrm{Te} /$

tion of $\mathrm{Tel}$

tion of $\mathrm{Te}$ Rec. for Plastics-Compression Moulding Test Rec. for Plastics-Compression Moulding Test

es: Test Ec: Drop and Topple, Primarily for Equipment Type rces for Use in Sensitometric Exposure-Simulation of the rces for Use in Sensitometric Exposure-Simulation of the rces for Use in Sensitometric Exposure-Simulation of the Rec. for Chemical Analysis of Zinc:

Rec. for Chemical Analysis of Copper and Its Alloys Rec. for Chemical Analysis of Zinc and Its Alloys:
Sound or Noise (1963)

Sound Pressure Levels of Narrow Bands of Noise in a Dif

Sound Prints Normal and Offset Centerline Types (1958)

Sound Record on $16 \mathrm{~mm}$ Prints (1958)

Sound Record on $17.5 \mathrm{~mm}$ Film (1960)

Sound Record on $35 \mathrm{~mm}$ Prints (1958)

Cording Heads for

Sound Record Positions in Camera for $16 \mathrm{~mm}$ Sound Motion

Sound Record Positions in Camera for $35 \mathrm{~mm}$ Sound Motion

Sound Record Positions in Projector for $35 \mathrm{~mm}$ Sound Mot

Sound Record (1972) /Idth of Magnetic-Striping and Gap

Sound Recording and Reproducing Equipment (1972)

Sound Records and Scanning Area of $35 \mathrm{~mm}$ Double Width P

Sound Records on $16 \mathrm{~mm}$ Perforated Magnetic Film (1968)

Sound Records on $16 \mathrm{~mm}$ Perforated Magnetic Film (1968)

Sound Records on $35 \mathrm{~mm}$ Film-Location and Dimensions (

Sound Records on $35 \mathrm{~mm}$ Film and 1 Magnetic Sound Record

Sound Records on $35 \mathrm{~mm}$ Film (1963)

(1971)

Sound Signalling Devices on Motor Vehicles Acoustic Std ISO

Sound Stripe on $16 \mathrm{~mm}$ Motion Picture Film Perforated Al

Sound System Equipment: Amplifiers (1969)

Sound System Equipment: Auxiliary Passive Elements (197

Sound System Equipment: Explanation of General Terms (1

Sound System Equipment: General (1970)

Sound System Equipment: Loudspeakers (1972)

Sound System Equipment: Mechanical Design Features (197

Sound System Equipment: Microphones (1972)

Sound Transmission (1960)

Sounds and Vibrations (1966)

Rec. Fo ISO

Sources for Portable Prospecting Equipment for Radio Ac

Sources for Use in Sensitometric Exposure-Simulation-

Sources for Use in Sensitometric Exposure-Simulation ISO

Sources for Use in Sensitometric Exposure-Simulation ISO

SPA, SPB (1965)

SPA, SPB (1965)

ISO

Spacing of $2.54 \mathrm{~mm}(0.1 \mathrm{In}$.) Mating Either with Board M Rec. for ISO

Spacing of $2.54 \mathrm{~mm}$ (0.1 ln.) (1971) /Cket Connectors W IEC

Spanner Gaps and Sockets (Metric Values for General Use ISO

Spark Plug Ignition-Terminology (1972)

Spark Plug M 14 X 1.25 with Flat Seating (1970)

Spark Plugs M 10 X I with Flat Seating (1973)

Spark Plugs M 12 X 1,25 with Flat Seating (1973)

Spark Plugs M 14 X 1,25 with Conical Seating (1972)

Spark Plugs M 14 X 1,25 with Conical Seating (1973)

Spark Plugs M 14 X 1,25 with Flat Seating (1973)

Spark Plugs M 18 X 1,5 with Conical Seating (1972)

Spark Test Apparatus for Intrinsically Safe Circuits (1

SPB (1965)

SPB (1965)

Special and High Tensile Types (1965)

Special Castings and Parts for Pressure Main Lines (195

Special Low Temperature Compartments for the Storage of

Special Meth. of Mechanical Test. to Determine the Codi

Special Regard to Rated Capacity of Circuit Breakers in

Special Requirement (1966)

Special Tin Bronzes (1965)

Special Tolerances for Reamers (1966)

Arings-Double Row Cylind

Special Types (1965)

Special Wrought Copper Alloys (1971)

Specialized Frequency Measurements on Fm Receivers of

Species of Wood Utilized in the Manufacture of Wood Flo

Specif ying the Characteristics of Auxiliary Equipment F

Specifying the Characteristics of Electro Mechanical $\mathrm{Tr}_{\mathrm{r}}$

Specimen by Measuring the Angle of Recovery (1972)

Specimens and Test Pieces (1971)

Specimens for Optical Tests on Plastics Materials: Cast

Specimens for Optical Tests on Plastics Materials: Moul

Specimens for Physical Tests (1971)

Specimens of Polyamide 66,610 and $6(1969)$

Specimens of Thermoplastic Materials (1963)

Specimens of Thermoplastic Materials (1963)

Specimens of Thermosetting Materials-Annex A: Prepara

Specimens of Thermosetting Materials-Annex B: Prepara

Specimens of Thermosetting Materials-Annex C: Prepara

Specimens of Thermosetting Materials (1963)

/-Light Sou ISO

Spectral Distribution of Photoflood Illumination (1972)

Spectral Distribution of Tungsten Illumination (1972) ISO

Spectrophotometric Determination of Copper (1969)

Spectrophotometric Determination of Iron in the Alloys

Spectrophotometric Determination of Iron (1969) 
Hec. for Chemical Analysis of Copper and Its Alloys Rec for Chemical Analysis of Zine and lts Alloys roduction of Aluminium-Determination of Silica Content for Aluminium and lts Alloys-Determination of Chromium roduction of Aluminum-Determination of Calcium Content atural and Artificial)-Determination of Silica Content ride for Industrial Use: Determination of Silica Content Aluminum and Aluminium Alloys-Determination of Titanium $\mathrm{s}$ of Magnesium and Its Alloys-Determination of Silicon se-Determination of Iron Content-2, 2'-Bipyridyl

1 Use: Determination of Iron Content; 2,2'-Bipyridyl se-Determination of Iron Content-2, 2'-Bipyridyl

1 Use: Determination of Iron Content: 2,2'-Bipyridyl trial Use: Determination of Iron Content: 2,2-Bipyridyl se: Determination of of Iron Content: 2,2'-Bipyridyl inium-Determination of Sodium Content-Flame (Emission)

1 Use: Determination of Oxides of Nitrogen-2,4-Xylenol f Soaps-Determination of Low Contents of Free Glycerol . r Chemical Analysis of Steels: Determination of Manganese Rec. Graphical Symbols: Frequency tive Devices: Photoconductive Cells for Use in the Visible Rec. for Meth. Determining Photographic lleys (I970/ Rec. for Endless Wide V-Belts for Industrial quipment (I972)
Rec. for Meth, of Measurement of Rec. for International Code for Test. of Rec. for Synthetic Rubber Latices-Determination of High raphy-Sensi/_Std for Photography: Determination of Iso cement Vacuum Pumps-Part I: Volume Rate of Flow (Pumping vapor) Vacuum Pumps-Part I: Volume Rate of Flow (Pumping Rec. for Machine Tool

Rec. for

Rec. for Voltage Measurement by Means of Sphere Gaps (I Rec. for Voltage Measurement by Means of Rec. for Interchangeable Std. for Rolling Bearings: with Rec. for Rec. for Rec. for Rec. for Rec. for Rec. for Rec. for Rec. for Rec. for Rec. for Rec. for Rec. for Rec. for Rec. for s and Fittings of Plasties Materials: Socket Fittings with Operated Appliances for Domestic and Similar Purposes: similar Electrical Appliances: Particular Requirements for nes with Table of Fixed Height with Horizontal or Vertical h Table of of Variable Height, with Horizontal or Vertical

for Surface Grinding Machines with Vertical Grinding Wheel r Surface Grinding Machines with llorizontal Grinding Wheel and Over (1969) Rec. for Flyer Bobbins for 963) s (1972) 972) Rec. for Flyer Bobbins for Rec. for Dimensions of for Interchangeability (I968)

Std. for Textile Machinery and Accessories interchangeability: Metric Series (1968)

Rec, for Rec. for Warp Tubes for Ring Spinning and Ring Doubling Rec. for Warp Tubes for Ring Spinning and Ring Doubling $y$ and Accessories-Condenser Bobbins for Woollen (Woolen) tile Machinery and Accessories-Reversible Rings for Ring (1959)

e Machinery and Accessories-Nonreversible Rings for Ring extile Machinery and Accessories-Spind . Rangs for Ring tolerances and Gauges (1964/ Rec. for Warp Tubes for Ring lerances and Gauges (1963) Rec. for Warp Tubes for Ring Rec. for Definition of Side (Left or Right) of Rec. for Cotton Std. for Rec. for Gum Std. for Mineral Solvents for Paints: White tic and Similar General Us/ Rec. for Safety Equipment for Rec. for Straight Sided Rec. for Straight Sided Rec. for
and Their Compounds by millimetres (Millimeters) (1955) inylchloride and Related Copolymers and Their Compounds by Rec. for Phlogopite Mica Blocks, Thins and Rec. for Cinematography-Dimensions of Daylight Loading
Spectrophotometric: Determination of Nickel (1.ow Content (1970)

Spectrophotometric Meth. as the Reduced Silicomolybdate Spectrophotometric Meth. Using Diphenylcarbazide (1972) Spectrophotometric Meth. Using Naphthalhydroxamic Acid Spectrophotometric Meth. Using the Reduced Silicomolybd Spectrophotometric Meth. Using the Reduced Silicomolybd Spectrophotometric Meth. with Chromotropic Acid) (1969) Spectrophotometric Meth. with the Reduced Silicomolybdi

pectrophotometric Meth. (1968)

ID Oleum for Industria ISO

Spectrophotometric Meth. (1968)

Spectrophotometric Meth. (1968)

Hate for Industrial U ISO

IIc Acid for Industria ISO

pectrophotometric Meth. (1969)

M Hydroxide for Indus ISO

Xide for Industrial U ISO

Spectrophotometric Meth. (I970)

Spectrophotometric Meth. (I972)

Spectrophotometric Meth. (I972)

Spectrophotometric Meth.) (1967)

He Production of Alum ISO

/ Oleums for Industria ISO

/Ve Agents: Analysis O ISO

Rec. Fo ISO

Spectrum Diagrams (1968)

Spectrum, Meth. of (1970)

Speed and Exposure fndex (1954)

/R Measurement of Photosensi IEC

Speed Changers and Groove Profiles for Corresponding $\mathrm{Pu}$

Speed Data Terminal Equipment (1973)

Speed Fluctuations in Sound Recording and Reproducing $\mathbf{E}$

Speed Governing Systems for Hydraulic Turbines (1970)

Speed Mechanical Stability (197I)

Speed of Colour (Color) Reversal Films for Still Photog

Speed) (1970)

peed) (1970)

Speeds and Feeds (1961)

/Performance Characteristics of Vapour (

peroidal or Nodular Graphite Cast Iron (1969)

Sphere Earthed) (I960)

Sphere Gaps (1 Sphere Earthed) (1960)

Spherical Ground Glass Joints (1968)

Spherical Outside Surface and Extended Inner Ring Width

Spices and Condiments-Cardamoms-Spec. (I968)

Spices and Condiments: Black and White Pepper, Whole an

Spices and Condiments: Chillies, Whole and Ground, Spec

Spices and Condiments: Determination of Acid Insoluble

Spices and Condiments: Determination of Alcohol Soluble

Spices and Condiments: Determination of Cold Water Solu

Spices and Condiments: Determination of Extraneous Matt

Spices and Condiments: Determination of Moisture Conten

Spices and Condiments: Determination of Nonvolatile Eth

Spices and Condiments: Determination of Total Ash (1969

Spices and Condiments: Determination of Water Insoluble

Spices and Condiments: Nomenclature (1968)

Spices and Condiments: Pimento (Allspice) Whole and Gro

Spices and Condiments: Sampling (1969)

Spigot Ends for Domestic and Industrial Waste Pipe: Bas

Spec. for Electric Mo CE

pin Extractors (1968)

Spin Extractors (1971)

1. for Safety of Household and IEC

Spindle-Test. of Accuracy (I971)

Spindle-Test. of the Accuracy (1970)

for Milling Machi ISO $/ G$ Machines Wit ISO

(I9

pindle and Reciprocating Table-Test. of Accuracy (19

Spindle Diameters of $25 \mathrm{~mm}$ or More and Lifts of $300 \mathrm{~mm}$

Spindle Diameters $U_{p}$ to $22 \mathrm{~mm}$ and Lifts $U_{p}$ to $300 \mathrm{~mm}$ (1

Spindle Ends for Manually Operated Electronic Component

Spindle Gauges for Ring Spinning and Doubling Frames (I

Spindle Noses and Face Plates Types a and Camlock: Size

Spindle Noses and Face Plates: Bayonet Type; Sizes for

Spindles Metric Dimensions, Tolerances and Gauges (1964

pindles: In. Dimensions, Tolerances and Gauges (1963)

(197) (Ec. for Textile Machiner ISO

Spinning and Doubling Frames for $\mathrm{C}$ and El Traveller

Spinning and Doubling Frames for $\mathrm{C}$ and El Traveller

Spinning and Doubling Frames for Ear Shaped Travellers

Spinning and Doubling Frames (1972)

Spinning and Ring Doubling Spindles Metric Dimensions,

Spinning and Ring Doubling Spindles: In. Dimensions, to

Spinning Machinery (1959)

Spinning Machinery: Working Width (1963)

piral Ratchet Screwdriver Ends (1972)

Spirit of Turpentine and Wood Turpentines (1965)

Spirits and Related Hydrocarbon Solvents (1972)

Splash Proof Mains Operated Electronic Equipment (Domes

Splines and Gauges Dimcnsions in In./S (1961)

Splines (For Cylindrical Shafts) Nominal Dimensions in

Split Pins-Metric Series (1971)

Splitting Off of Hydrogen Chloride (1970)

Spools and Reels (1969)

Spools for Double $8 \mathrm{~mm}$ Motion Picture Film (1969)
1810

1570

1232

2298

1620

2369

1118

1975

852

909
994

983

1617

2363

2272

629

117.12

306-3

6

1604

2593

386

308

2006

2240

I 607

1608

229

1083

52

52

641

2264

882

959

972

930

940

941

927

939

1108

928

929

1003

676

973

948

265

$10-2 \mathrm{~K}$

335-4

1984

1701

1986
$344 / I I$

$344 / \mathrm{I}$

390

94

702

867

368

343

1946

95

96

97

94.

368

343

92

341

2352

412

1250

$65 \mathrm{~A}$

232

1234

182

444

1116

1020 
Rec. for Cinematography-Dimensions of Daylight Loading Rec. for Packaging of Winding Wires: Delivery mensions for 127,120 and 620 Roll Film, Backing Paper and d for Information Processing-Widths of Fabric Ribbons on Ided Metal Arc Welding of Mild Steel: Dimensions of Wires, n) (1969) Rec. for Dimensions of Straight Resistance Rec. for Dimensions of Straight Resistance Rec. for Dimensions of Tubular Electrode Holders for Rec. for Measurement of the Dimensions of Focal Rec. for General Purpose Agricultural Rubber

(1970) Rec. for Metal n and Steel Against Corrosion (1971) Rec. for Metal Reeds with Double Std for Technical Drawings: Representation of Rec. for Shipbuilding Details for Inland Navigation

Rec. for Diameters of Drafting Rollers for Cotton, Wool, es of Electronic Tubes and Valves: Meth. of Measurement of Rec. for Lengths of Narrow V-Belts: Sections c. for Grooved Pulleys for Narrow V-Belts: Groove Sections ensions in Millimetres) (Milli/ Rec. for Rectangular or Rec. for Dimensions of Hot Rolled Steel Bars:

Rec. for Assembly Tools for Bolts and Screws-Driving Rec. for Diameters of Shanks and Sizes of Driving Rec. for Surface Active Agents: Determination of (1969) rical Machinery and Apparatus in Relation to Their Thermal nd Thei/ Rec. for Plastics-Determination of the Thermal Rec. for Plastics-Determination of the Thermal Ims, Plates and Papers/ Rec. for Meth. for Indicating the dispersions of Polymers and Copolymers-Freeze Thaw Cycle ic Rubber Latices-Determination of High Speed Mechanical Id. for Natural Rubber Latex-Determination of Mechanical lectronic Tubes and Valves: Meth. of Measurement on Corona

ackaging-Complete, Filled Transport Packages-Part III: Rec. for Polycristalline Semiconductor Rectifier

Rec. for Monocrystalline Semiconductor Rectifier Cells, nd Operation of a Gauging Station and Determination of the ntional Masses Per Unit Length (1969) Rec. for Pipes and Fittings Rec. for Index of a Publication

ilute Solution $\mathrm{Vi} /$

970)

f Drawings Devices (1969)

t. Machines (1968)

dness Scale Test. Machines (1968/

$\mathrm{N}$ and T Scale Hardness Test. Ma/

t. Machines (1967) Or, Continuous Filament Yarn. of the Linear Density of Its Continuous Filament and Determination of the Twist in Its Continuous Filament and meters of Drafting Rollers for Cotton, Wool, Spun Silk and $r$ Textile Glass-Determination of the Average Diameter of Rec. for Low Voltage Motor Starters: Reduced, a.c.:

lary (1973)

or lndustrial Use-Determination of Water by the Dean and

(1963)

st (1972) Rec. for Character Structure for Rec. for Lead Acid Rec. for Lead Acid Rec. for Lead Acid Rec. for Lampholders and phical Symbols: Contacts, Switchgear, Mechanical Controls, Rec. for Glow Rec. for Low Voltage Motor Rec. for Low Voltage Motor Ground Power Units for d.c. Aircraft Servicing and Engine rement of Surface Roughness by the Profile Meth.-General bers-Determination of Resistance to Ozone Cracking Under Rec. for Rubber Hoses for Aircraft Ground Fueling Without Rec. for International Electrotechnical Vocabulary:

Specified Contact Area and Being Applied with a Specified

Rec. for Ball and Roller Bearings: Meth. of Evaluating for Strength of Mechanical Fastenings for Conveyor Belts c. for Calculation of Rectangular Symmetrical Fillet Welds n Open Channels-Establishment and Operation of a Gauging for Test Code for Stationary Steam Generators of the Power Rec. for Rules for Construction of and Heating Appliances for Domestic and Similar Purposes: 1968) Rec. for Test Code for
Rec. for Paper: Overall Trimmed Sizes of Articles of stribution (1963)

imary Cells and Accumulators: Aerials (Antennas) and Radio of the Mean-Confidence lnterval (1973) erms and Symbols (1970)

ms and Symbols-Part 1: Statistical Vocabulary/
Rec. for

Spools for $16 \mathrm{~mm}$ Motion Picture Film (1969) Spools (1968) Spools $(1971)$ Spools (1972)

Spools, Rims and Coils (1968)

ISO

IEC

(Olid Wires for Gas Shie ISO

Spot Welding Electrodes (For Loads Greater Than $1500 \mathrm{Kg}$ iSO

Spot Welding Electrodes (1968)

Spot Welding Machines (1969)

Spots of Diagnostic X-Ray Tubes Using a Pinhole Camera Spray Hose (1970)

Spraying of Zinc and Aluminum for the Protection of lro Spring Baulk (1967)

Springs (1973)

Sprocket Wheels (1956)

Spun Silk and Staple Fibre (Fiber) (1959)

Spurious and Unwanted Electrode Currents (1967)

SPZ, SPA, SPB (1965)

SPZ, SPA, SPB (1965)

Square Parallel Keys and Their Corresponding Keyways (D

Square, Metric Series (1969)

Squares for Power and Hand Socket Wrenches (1970)

Squares for Rotating Tools with Parallel Shanks (1961)

Stability in Hard Water (1969)

Stability in Service (1957)

Stability of Counterbalanced Lift Trucks-Basic Tests

Stability of Insulating Oils (1963)

Stability of Polyvinyl Chloride and Related Copolymers

Stability of Polyvinylchloride and Related Copolymers a

Stability of the Images of Processed Black and White Fi

Stability Test (1969)

Stability (1971)

Stability (1972)

Stabilizers (1969)

Rec for Synthe

Stacking Test (1972)

Stacks and Equipments (1960)

Stacks, Assemblies and Equipm

of the Electrical Properties of $\mathrm{E}$ IFC

Std. for P 1 SO

lEC

IEC

- Establishment a 150

ISO

Stainless Steel Tubes for the Food Industry (1971) ISO

(Starivgrization of Format) (1969)

Standerrization of Meth. for the Determination of the D

Standardization of Semiconductor Devices: Dimensions (1

Standardization of Semiconductor Devices: Preparation O

Standardized Blocks to Be Used for Brinell llardness Tes

Standardized Blocks to Be Used for Rockwell B and C Har

Standardized Blocks to Be Used for Rockwell Superficial

Standardized Blocks to Be Used for Vickers Hardness Tes

Staple Fibre (Fiber) Type and Roving Package (1971)

Staple Fibre (Fiber) Yarns and Rovings in the Form of $\mathrm{P}$

Staple Fibre (Fiber) (1959) Rec. for Dia ISO

Staple Fibres (Fibers) or Continuous Filaments Constitu isO

Star Delta (1970)

Starch-Determination of Moisture (1970)

lEC

1SO

Starch. Including Derivatives and by -Products: Vocabu ISO

Stark Meth. (1971) /Esol, Cresylic Acid and Xylenols F ISO

Start / Stop and Synchronous Transmission (1970) ISO

Starter Batteries: Dimensions and Marking of Terminals IEC

Starter Batteries: Dimensions of Batteries (1965) IEC

Starter Batteries: General Requirements and Meth. of Te

Starterholders for Tubular Fluorescent Lamps (1972)

Starters and Elements of Electromechanical Relays (1963

Starters for Tubular Fluorescent Lamps (1963)

Starters: Direct on Line, a.c. (1971)

Starters: Reduced, a.c.: Star Delta (1970)

Starting (1961)

Terms and Definitions (1970)

Static Conducting Wire (1971)

Std for Vulcanized Rub isO

tatic Convertors (1956)

Static Force (1971) /Ration of Bone Vibrators Having a IEC

Static Invertors for Aircraft (1973)

Static Load Ratings (1958)

(Static Test Meth.) (1969)

ISO

Station and Determination of the Stage Discharge Relati ISO

Station Type (1968)

Stationary Boilers (1968)

Stationary Noninstantaneous Water Heaters (1966)

Stationary Steam Generators of the Power Station Type (

Stationery That Include Detachable Sheets (1967)

ISO

CEE

1SO

Stations and Substations, Lines for Transmission and Di

IL Symbols: Machines, Transformers, $\mathrm{Pr}$ IFC

Statistical Interpretation of Test Results: Estimation

Rec. for Statistical Vocabulary and Symbols-Second Series of T

Rec. for Statistical Vocabulary and Symbols: First Series of Ter

1SO

1SO
1019

264-2

732
2257

864
1045
670

1089

336

1401

2063

571

2162

21

$151-15$

460

459

773

1035/II

1174

237

1063

85

1074

74

305

182

421

1147

2006

35

151.19

2234

119

146

1127

2037

999

1628

191.2

191-1

726

674

1355

640

1886

1889

1890

98

1888

292-2

666

1227

1898

1177

95.3

95-2

95- 1

400

117-3

155

292-1

292-2

1879

1431

1825

$50(11)$

373

2277

76
1120

617

(1)

889

831

$11-2 \mathrm{E}$

889

618

$117-5$

117-10

2602

1786

645 
y and Symbols: First Series of Terms and Symbols-l'art 1: Spec. for quid Flow Measurement in Open Channels: Dilution Meth. for for Electronic Components and Equipment: Test Ca: Damp and lectronic Components and Equipment: Test Ga: Acceleration, Rec. for Test Code for Stationary Rec. for Spec. for Rec. for

t for Ultrasonic Examination (1972) Std. for WeIds in raying of Zinc and Aluminum for the Protection of lron and ter Combustion in a Curren/ Rec. for Chemical Analysis of avimetric Meth.) (1969) Rec. for Chemical Analysis of ngths and Tolerances of Electrodes for the Welding of Mild Pipe Connections for Industrial Application for Plain End terrupted) (1961) Rec. for lnterrupted Creep Test. of Rec. for Noninterrupted Creep Test. of ec. for Determination of Proof Stress and Proving Test for Rec. for Creep Stress Rupture Test. of yield and Proof Stresses (S/ Rec. for Mechanical Test of Rec. for Dimensions of Hot Rolled Rec. for Dimensions of Hot Rolled Rec. for Dimensions of Hot Rolled ns, Diameters and Breaking Strengths of Preformed Stranded Rec. for Shackle Type Connector Units for High Tensile ploughs (1967) $s$ (1962)

erse Buttress T/

erse Buttress T/

e Threaded Equi/

e Threaded Equil Rec. for High Tensile Spec. for

Rec. for Rock Drilling-Extension Drill Rec. for Rock Drilling-Extension Drill Rec. for Rock Drilling-Extension Drill Rec. for Rock Drilling-Extension Drill ds for Gas Welding: Test to Determine the Compatibility of Stock Material-Cast Wheel Centres (Centers) in Nonalloy Rec. for Flat oerties (1972)

ss (1967) Std. for Prevailing Torque Type
Std. for Prevailing Torque Type Rec. for inspection of Circumferential Fusion Welded Butt Joints in r Radiographic Inspection of Fusion Welded Butt Joints for $r$ Radiographic Inspection of Fusion Welded Butt Joints for Rec. for Aluminium (Aluminum) Conductors, Rec. for Aluminium (Aluminum) Alloy Conductors ed Chain Wheels (1966) ions and Sectional Pro/ series-Dimensions An/ Dimensions and Sectio/ es-Dimensions and $\mathrm{Se} /$ ries-Dimensions and / nsions and Sectional $\mathbf{P} /$

Rec. for Rec. for Dimensions of Hot Rolled Rec. for Dimensions of Hot Rolled Rec. for Dimensions of Hot Rolled Rec. for Dimensions of Hot Rolled Rec. for Dimensions of Hot Rolled Rec. for Dimensions of Hot Rolled Rec. for Simple Bend Test. of Rec. for Reverse Bend Test. of Rec. for Tensile Test. of thick (1959) inimum Lengths (1957) eg.) (1969)

asses Per Unit Length (1969) Rec. for Modified Erichsen Cupping Test for Rec. for Rec. for Rec. for Stainless Std. for Rec. for Rec. for Rec. for Nonscrewed Rec. for Pipes and Fittings-Stainless Rec for Flanging Test on Rec. for Drift Expanding Test on Rec. for Bend Test on Rec. for Flattening Test on Rec. for Ring Expanding Test on Rec. for Tensile Test. of Rec. for Plain End Rec. for nsions and Masses Per Unit Length (19/ (1962) masses Per Metre (Meter) (/ Rec. for Cold Drawn Precision (1973) Rec. for Shipbuilding Details-Tests on Galvanized Rec. for Drawn Wire for General Purpose Nonalloy (1973)

Rec. for Shipbuilding Details-Galvanized Rec. for Tensile Test. of Rec for Wrapping Test for

Std. for Simple Torsion Test. of Std. for Reverse Bend Test. of Rec. for Hardenability Test by End Quenching Rec for Vickers Hardness Test for ectroplated Coatings of Copper Plus Nickel and Chromium on Rec. for Tensile Test. of Rec. for Charpy Impact Test (U-Notch) for Rec. for Izod Impact Test for Rec for Bend Test for Rec. for Beam Impact Test (V.Notch) for
Statistical Vocabulary (1967)

IR Statistical Vocabular ISO

Statutes and Rules of Procedure of the CEE (1961)

CEE

Steady Flow-Part 1: Constant Rate Injection Meth. (19 ISO

Steady Heat (1969) /le Environmental Test. Procedures IEC Steady State (1968) /Ironmental Test. Procedures for E

Steam Generators of the Power Station Type (1968)

Steam Turbines (1970)

Steam Turbines: Rules for Acceptance Tests (1962)

Steel-Reference Block for the Calibration of Equipmen

Steel Against Corrosion (1971)

Rec. for

Steel and Cast Iron: Determination of Total Silicon (Gr

Steel and Low Alloy High Tensile Steel (1967)

Steel and Other Metal Tubes (1970)

Steel at Elevated Temperatures (1961)

Steel at Elevated Temperatures (1961)

Steel at Elevated Temperatures (1961)

Steel at Elevated Temperatures: Determination of Lower

Steel Bars: Flat, Metric Series (1969)

Steel Bars: Round, Metric Series (1969)

Steel Bars: Square, Metric Series (1969)

Steel Cables for Aircraft Controls (1967)

Steel Chain for Conveyors (1969)

Steel Chains (Round Link) for Chain Conveyors and Coa
Steel Conduits and Fittings for Electrical Installation

Steel Equipment for Percussive Long Hole Drilling-Rev

Steel Equipment for Percussive Long Hole Drilling-Rev

Steel Equipment for Percussive Long Hole Drilling-Rop

Steel Equipment for Percussive Long Hole Drilling-Rop

Steel Filler Rods and the Parent Metal in the Welding $O$

Steel for Tyred (Tired) Wheels for Trailer Stock (1969)

Steel Healds for General Use (1964)

Steel Hexagon Locknuts-Mechanical and Performance Pro

Steel Hexagon Locknuts: Dimensions-ln. Series (1972)

Steel Pipes for Gas, Water and Sewage, Welded or Seamle

Steel Pipes Up to $50 \mathrm{~mm}$ (2 In.) Wall Thickness (1969)

Steel Plates Up to $50 \mathrm{~mm}$ (2 In.) Thick (1969)

Steel Plates 50 to $200 \mathrm{~mm}$ Thick (1972)

Steel Reinforced (1966)

Steel Reinforced (1966)

Steel Roller Chains Type S 32 to 88 with Their Associat

Steel Sections-Equal Leg Angles-In. Series-Dimens

Steel Sections-Parallel Flange Column Sections-In.

Steel Sections-Parallel Flange I Beams-In. Series-

Steel Sections-Part I: Equal Leg Angles-Metric Seri

Steel Sections-Part 2: Unequal Leg Angles-Metric Se

Steel Sections-Unequal Leg Angles-ln. Series-Dime

Steel Sheet and Strip L.ess Than $3 \mathrm{~mm}$ Thick (1959)

Steel Sheet and Strip Less Than $3 \mathrm{~mm}$ Thick (1959)

Steel Sheet and Strip Less Than $3 \mathrm{Mm}$ and Not Than $0.5 \mathrm{Mm}$

Steel Sheet and Strip (1960)

Steel Sockets Screwed in Accordance with ISO Rec. R7: M

Steel Tubes-Butt Welding Bends 5 D $\left(90^{\circ}\right.$ And $180 \mathrm{D}$

Steel Tubes-Dimensions, Tolerances and Conventional $M$

Steel Tubes-Outside Diameters (1971)

Steel Tubes-Wall Thicknesses (1971)

Steel Tubes for Gas List Threading (1971)

Steel Tubes for GeneraI Purposes (1962)

Steel Tubes for the Food Industry (1971)

Steel Tubes (1960)

Steel Tubes $(1960)$

Steel Tubes (1960)

Steel Tubes (1961)

Steel Tubes (1964)

Steel Tubes (1964)

Steel Tubes, Welded or Seamless-General Table of Dime

Steel Tubes: Butt Welding Bends $\left(90^{\circ}\right.$ And 180 Deg.)

SteeI Tubes: Metric Series, Dimensions, Tolerances and

SteeI Wire for Ropes (1963)

Steel Wire Rope for Aircraft Controls: Technical Spec.

Steel Wire Ropes-Spec. (1971)

Steel Wire Ropes for General Purposes: Characteristics

Steel Wire Ropes (1963)

Steel Wire (1959)

Steel Wire (1960)

Steel Wire (1972)

Steel Wire (1973)

Steel (Jominy Test) (1967)

Steel (Load 5 to $100 \mathrm{kgf})(1967)$

Steel (Or Iron) (1970)

Steel (1959)

Steel (1959)

Steel (1959)

Steel (1959)

Steel (1960) 
or load Calihration of Test. Machines for Tensile Test. of Rec. for (;eneral Technical Delivery Requirements for ion and Preparation of Samples and Test Pieces for Wrought $\mathrm{s}$ for the Welding of Mild Steel and Low Alloy High Tensile Rec for Brinell Hardness Test for Rec. for Rockwell Hardness Test (B and C Scales) for or Rockwell Superficial Hardness Test ( $\mathrm{N}$ and $\mathrm{T}$ Scales) for ec. for Shipbuilding Details-Multipurpose Chocks of Cast Rec. for Electroplated Coatings of Zinc on Iron and

Rec. for Electroplated Coatings of Cadmium on Iron and of Test for Determining the Conventional Bond Strength on ed Electrodes for Manual Arc Welding of Mild and Low Alloy Rec. for Heat Treated, Alloy, and Free Cutting Rec. for Heat Treated, Alloy, and Free Cutting 1972) Std. for Heat Treated, Alloy, and Free Cutting (1968) Rec. for Heat Treated, Alloy, and Free Cutting ith $1 \% \mathrm{Ch} /$ Rec. for Heat Treated, Alloy, and Free Cutting d with Co/ Rec. for Heat Treated, Alloy, and Free Cutting e (I970) Rec. for Heat Treated, Alloy, and Free Cutting Chromium/ Rec. for Heat Treated, Alloy, and Free Cutting Nickel M/ Rec. for Heat Treated, Alloy, and Free Cutting Rec. for Heat Treated, Alloy, and Free Cutting or Code of Symbols for Filler Rods for Gas Welding of Mild of Symbols for Covered Electrodes for Arc Welding of Mild f Weld Metal Deposited by Filler Rods for Gas Welding Mild Rec. for Rec. for Rock Drilling: Hollow Hexagonal Drill r Verification of Pendulum Impact Test. Machines for Test. Rec. for Structural micrographic Determination of the Austenitic Grain Size of with Covered Electrodes of Mild and Low Alloy High Tensile lared Shanks and Chuck Bushings for Hollow Hexagonal Drill f Steel Filler Rods and the Parent Metal in the Welding of I\% Chromi/ Rec. for Heat Treated, Alloy and Free Cutting meth.) (1967)

After Combustion in a St pered Chromium Types (1970) Rec. for Chemical Analysis of Rec. for Chemical Analysis of
Rec. for Chemical Analysis of Rec. for Heat Treated

for Solid Wires for Gas Shielded Metal Arc Welding of Mild Rec. for Solid Rec. for Solid Rec. for Long Solid Rec. for Short Solid Rec. for Rock Drilling: Integral Std. for Duplicators-Minimum Overprint on Duplicating m Film, 5-Perforation Format (/ Rec. for Dimenaions for Std. for Hand Finishing man Test) (I971)

Rec. for Plastics-Determination of Rec. for Determination of the Rec. for Exposure Time Markings for Shutters Used in Rec. for Lens Aperture Markings for mination of ISO Speed of Colour (Color) Reversal Films for 5-Perforation Format (/ Rec. for Dimensions for Stereo fication of the Emulsion Side of Edge Marked Roll Film for

Rec. for Dimensions for $35 \mathrm{~mm}$ Film Magazines for Rec. for Hand Operated Rec. for Industrial Trucks-Dimensions of Std. for Cinematography-8 mm Type S Motion Picture Raw ng and Perforating Dimensions for $35 \mathrm{~mm}$ Motion Picture Raw Perforating Dimensions for Double $-8 \mathrm{~mm}$ Motion Picture Raw oy Steel for Tyred (Tired) Whee/ s) for Tyred (Tired) Wheels For $/$. Dimensions and Tolerances (I96)
Steel (1960)

Steel (1964)

Steel (1964)

Steel (1967)

Steel (1968)

Steel (1968)

Steel (1969)

Steel (1971)

Steel (1971)

Steel (1971)

Steel, Cast Iron and Other Metals (1968)

Steels-Code of Symbols for Identification (1973)

Steels-Part 10: Wrought Nitriding Type (1970)

Steels-Part 11: Wrought Case Hardening Type (1970)

Steels-Part 12: Flame and Induction Hardening Types (

Steels-Part 1: Quenched and Tempered Unalloyed Types

Steels-Part 2: Wrought, Quenched and Tempered Types W

Steels-Part 3: Wrought Quenched and Tempered Unalloye

Steels-Part 5: Wrought Quenched and Tempered Manganes

Steels-Part 6: Wrought Quenched and Tempered, with 3\%

Steels-Part 8: Wrought Quenched and Tempered Chromium

Steels-Part 9: Wrought Free Cutting Type (1970)

Steels and Low Alloy High Tensile Types (1967)

Steels and Low Alloy High Tensile Types (I967)

Steels and Low Alloy High Tensile Types (1967)

Steels for General Engineering Purposes (1969)

Steels in Bar Form (I968)

Steels (1965)

Steels (1967)

Steela (1967)

Steels (1967)

Steels (1968)

Steels (1968)

1: Test to Determine the Compatibility $\mathrm{O}$

Steels: Free Cutting-Part 7: Wrought Quenched and Tem

Steel: Determination and Verification of the Effective

Steel: Dimensions of Wires, Spools, Rims and Coils (196

Stem Calorimeter Thermometers (I968)

Stem General Purpose Thermometers (1970)

Stem Thermometers for Precision Uae (1968)

Stem Thermometers for Precision Use (1968)

Stems (1968)

Stencils (1972)

Stereo Still Photography Using $35 \mathrm{~mm}$ Objectives on $35 \mathrm{M}$

Sticks and Oil Stones-Dimensions (I972)

Stiffness in Torsion as a Function of Temperature (1965

Stiffness of Vulcanized Rubbers at Low Temperature (Geh

Still Cameras (1966)

Still Cameras (1966)

Still Photography-Sensitometric Exposure and Evaluat

Still Photography Using $35 \mathrm{~mm}$ Objectives on $35 \mathrm{~mm}$ Film,

Still Picture Cameras (1968)

Still Picture Use and Test for Film Pullout Force (1969

Stillage Trucks: Principal Dimensions (1969)

Stillages-Connection Gauge (I970)

Stock Film-Cutting and Perforating Dimensions (1972)

Rec for Cutti ISO

tock Film (1966)

Stock Film (1966)

Rec. for Cutting and ISO

Rec. for Railway Rolling Rec. for Railway Rolling Rec. for Railway Rollin Rec. for Railway Rollin Rec. for Railway Rolling Rec. for Railway Rolling Rec. for Railway Rolling

Rec. for Mechanical Property Limits for Rivet to Serv/ Rec. for Rules for the Test. of Electric Rolling
66)
Rec. for Paper: Untrimmed ge (1967) Rec. for Paper: Untrimmed Core Drills with Parallel, and Morse Taper Shanks-Rec. hine Operated Circular Screwing Dies and Hand.Operated Die Std. for Hand Finishing Sticks and Oi Rec. for Character Structure for Start

Rec, for Drop Wires for Mechanical and Electrical Warp of the Crosa Section of Assembled Bars for Mechanical Warp Rec. for Green Bananas: Guide to Rec. for Fresh Pineapples-Guide to

s Mechanical Handling Equipment for Loose Bulk Materials

Rec. for Graphical Symbols: Ferrite Cores and Magnetic

Stock Material-Axles for Trailer (I969)

Stock Material-Cast Wheel Centres (Centers) in Nonall

Stock Material-Rolled on Forged Wheel Centres (Center

Stock Material-Rough Tyres (Tires) for Trailer Stock:

Stock Material-Tyres (Tires) for Trailer Stock (1969)

Stock Material: Solid Wheels for Trailer Stock (I969)

Stock Material: Wheel Sets for Trailer Stock (1969)

Stock of Aluminium (Aluminum) and Its Alloys (1968)

Stock on Completion of Construction and Before Entry in

Stock Sizes for the ISO-A Series, ISO Primary Range (I9

Stock Sizes for the ISO-A Series: ISO Supplementary Ran

Stones-Dimensions (1972) Std. for Hand and Mac ISO

Stop and Synchronous Transmiasion (I970) 
ry-Part II: Special l.ow Temperature Compartments for the for Sodium Hydroxide for lndustrial Use: Preparation and or Potassium llydroxide for Industrial Use: Preparation and - for Sodium Carbonate for Industrial Use: Preparation and xille Primarily Used for the Production Of; Preparation and c. for Cryolite (Natural and Artificial)-Preparation and for International Code for the Field Acceptance Tests of ectronic Tubes: Meth. of Measurement of Cathode Ray Charge rocedures for Electronic Components and Equipment: Test $\mathrm{H}$ : Rec. for Peaches: Guide to Cold Rec. for Pears-Guide to Cold Rec. for Cauliflowers: Guide to Cold Rec. for Apples-Guide to Cold

nstallations in Ships: Accessories, Lighting, Accumulator agnetic Cores for Application in Coincident Current Matrix Rec. for Modular Coordination (1968) Rec. for Modules and Diametral Pitches of Rec. for Basic Rack of

engineering (1968) Obtain /

Rec. for

for Punched Tape Variable Block Format for Positioning and ngeable Punched Tape Variable Block Format Positioning and c. for Punched Tape Fixed Block Format for Positioning and ng Bearings: Locknuts, Narrow Series, and Lockwashers with for Drawn or Extruded Filler Rods for Welding, Supplied in of Angles of Slope and Rotation for Welding Positions for greater Than $1500 \mathrm{kgf}$ (1969)

1961) Rec. for Dimensions of Rec. for Dimensions of Rec. for
Rec. for Rec. for
elding Positions and Definitions of Rotation and Slope for valves: Spec. Sheets and Dimensions of Wiring Jigs and Pin Rec. for Determination of Tensile Stress uous Mechanical Handling Equipment for Unit Loads-Single uous Mechanical Handling Equipment for Unit Loads-Single Rec. for 3 or 4 Rec. for 8 Rec. for 3 Rec. for 3 Rec. for 3 Rec. for 3

ne Monofilament or Film Ropes (1970)

Rec. for 3 Strand (Hawser Laid) and 8 es (I970) Rec. for 3 Strand (Hawser Laid) and 8
ulation and Sheath: Equipment Wires, Type I, with Solid or I966) Rec. for Aluminium Alloy Rec for Aluminium (Aluminum) ation and Sheath: Equipment Wires and Cables with Solid or esignations, Diameters and Breaking Strengths of Preformed ination of Breaking Load and Elongation of Same (Of Single n of Total Carbon (Gravimetric Meth. After Combustion in A Rubber or Plastics / Rec. for Determination of Breaking for Flexible Cellular Materials-Determination of Tensile th. of Test (1962) Rec. for Full Thickness Tensile ndurance of Enamelled Wire by the Lowering of the Electric

Thurance of Insulacing Varnishe of Plastic Mortar (Rilem Cembu/ Rec. for Meth. of Test. Rec. for Meth. for the Determination of the Electric tatic Test Meth.) (1969) Ift Test Piece) (I968) cies (I967) rescent Test Piece) (I957/ Rec. for Determination of Tear Rec. Meth. of Test for Electric Rec. for Determination of Tear Rec for Determination of the Adhesion lding: Meth. of Test for Determining the Conventional Bond Rec. for Paper and Board-Determination of Tensile Std. for Rigid Cellular Plastics-Determination of Shear Std. for Ebonite: Determination of Tensile Std. for Ebonite: Determination of Cross Breaking Std. for Ebonite: Determination of Crushing or Fibre (Fiber) Building Boards: Determination of Bending h. of Test. Strength of Cements-Compressive and Flexural ft Control/ Rec. for Designations, Diameters and Breaking a Way That the Transverse Section Is Not Under Any Normal res (1961) Rec. for Determination of Proof (1961) Rec. for Creep Rec. for Determination of Tensile eratures: Determination of Lower Yield and Proof Stresses imensions (1960) Rec. for Rec. for Characteristics of Rec. for Ball and Socket Couplings of

Rec. for Locking Devices for Ball and Socket Couplings of Rec. for Requirements for 28 Volt d.c. Flat Rec. for Simple Bend Test. of Steel Sheet and Rec. for Reverse Bend Test. of Steel Sheet and Rec. for Tensile Test. of Steel Sheet and r Simple Bend Test for Light Metal and Its Alloy Sheet and
Storage of Frozen Foudstuffs (1968)

Storage of Test Samplc (1969)

Storage of 'Test Sample (1969)

Storage of Test Samples (1968)

Storage of Test Samples (1968)

Storage of Test Samples (1970)

Storage Pumps (1968)

Storage Types (1971)

Storage (1960)

Storage (1968)

Storage (1969)

Storage (1969)

Storage (1970)

Storage), Batteries, Heating and Cooking Appliances, in

Stores Having a Nominal Selection Ratio of 2 : 1 (1969)

Storey and Room Heights for Residential Buildings (1970

Straight Bevel Gears for General and Heavy Engineering

Straight Bevel Gears for General Engineering and Heavy

Straight Bevel Gears (Information to Be Given to the Ma

Straight Cut Numerically Controlled Machines (1969)

Straight Cut Numerically Controlled Machines (1969)

Straight Cut Numerically Controlled Machines (1969)

Straight Inner Tab (1972)

Straight Lengths (1967)

1. for Lengths and Tolerances ISO

Straight Manual Arc Welds Made with Covered Electrodes

Straight Resistance Spot Welding Electrodes (For Loads

Straight Resistance Spot Welding Electrodes (1968)

Straight Sided Splines and Gauges Dimensions in In./S

Straight Sided Splines (For Cylindrical Shafts) Nominal

Straight Welds (1967)

Straighteners (I965)

Strain Properties of Vulcanized Rubbers for El

Strand Floor Mounted Truck Conveyors (Chain Above Floo

Strand Floor Truck Conveyors (Chain Below Floor) Safety

Strand Manila and Sisal Ropes (1970)

Strand Plaited Manila and Sisal Ropes (1971)

Strand Polyamide Multifilament Ropes (1969)

Strand Polyester Multifilament Ropes (1969)

Strand Polyethylene Monofilament Ropes (1971)

Strand (Hawser Laid) and 8 Strand (Plaited) Polypropyle

Strand (Plaited) Polypropylene Monofilament or Film Rop

Stranded Conductor, Insulated, Single (1967)

Stranded Conductors (Aluminum Magnesium Silicon Type)

Stranded Conductors (1966)

Stranded Conductors, Insulated, Screened, Single or One

Stranded Steel Cables for Aircraft Controls (1967)

Strands) (1972)

/Yarn from Packages-Meth. for Determ Ysis of Steels: Determinatio

Strength and Elongation at Break of Fabrics Coated with

Strength and Elongation at Break (1971)

Strength and Elongation of Conveyor Belts: Spec. and Me

Rec. ISO the Thermal E IEC Strength Meth. (1971) Rec. for Test Procedur IEC Strength of Cements-Compressive and Flexural Strength ISO Strength of Insulating Oils (1963)

Strength of Mechanical Fastenings for Conveyor Belts (S

Strength of Small Test Pieces of Vulcanized Rubbers (De

Strength of Solid Insulating Materials at Power Frequen

Strength of Vulcanized Natural and Synthetic Rubbers (C

Strength of Vulcanized Rubbers to Textile Fabrics (1969

Strength on Steel, Cast Iron and Other Metals (1968)

Strength (1971)

Strength (1972)

Strength (1972)

Strength (1972)

Strength (1972)

Strength (1972)

Strengths of Plastic Mortar (Rilem Cembureau Meth.) (19

Strengths of Preformed Stranded Steel Cables for Aircra

Stres

Stress and Proving Test for Steel at Elevated Temperatu

Stress Rupture Test. of Steel at Elevated 'Temperatures

Stress Strain Properties of Vulcanized Rubbers (1968)

(Teel at Elevated Temp ISO

Stretchers, Stretcher Carriers and Hospital Trolleys: D

String Insulator Units of the Cap and Pin Type (1969)

String Insulator Units (1960)

String Insulator Units: Dimensions and General Requirem

Strip Fuses for Aircraft (1968)

Strip Less Than $3 \mathrm{~mm}$ Thick (1959)

Strip Less Than $3 \mathrm{~mm}$ Thick (1959)

Strip Less Than $3 \mathrm{Mm}$ and Not Than 0.5Mm Thick (1959)

Strip of Thickness Between $0.2 \mathrm{~mm}(0.008 \mathrm{In}$.) and $7 \mathrm{~mm}$ 
Std for Petroleum Products Corrosiveness to Copper. loy, Ised for Electronic and Telecommunication / Rec. for Rec. for Bibliographical ec. for Modified Erichsen Cupping Test for Steel Sheet and edge (I966)

Rec. for Single Magnetic Sound Rec. for Magnetic
Rec. for Classification of Parquet 0) eneral Manufacturing Characteristics of Solid Wood Parquet Std. for Classification of Solid Beech (Wood) Parquet Std. for Rec. for (1970) Rec. for Magnetic Tape Labelling and File

Safety) (196l) Rec. for Character Aim, $r$ General Principles for the Verification of the Safety of Rec. for Twist Drills: Parallel Shank Jobber and Shackle/ Rec. for Shipbuilding Details-Anchor Chains, $\mathrm{s}$ for Sea Navigation and Inland Navigation Anchor Chains hanical Properties of Fasteners: Marking of Bolts, Screws, anical Properties of Fasteners-Part 1: Bolts, Screws and Rec. for Bolts, Screws and

Rec for Nominal Lengthe for Bolts, Screws and ure Responsive Elements and Recording Directly by Means of ement of Surface Roughness by the Profile Meth.-Contact atile Unsaturates and Residual Styrene (1972) Std. for

Rec. for Plastics-Determination of the Percentage of tices-Determination of Volatile Unsaturates and Residual Std. for Numbering of Divisions and Rec. for Expression of the Physical and

Rec for Test Meth for Induction Furnaces with stem of Units and a Selection of the Decimal Multiples and izing the Magnetic and Electric Properties of Vacuum and A Rec. for Determination of Paper Rec. for

for Industrial Use: Determination of Oxidizing or Reducing - Determination of the Resistance of Plastics to Chemical hange of Mechanical Properties After Contact with Chemical ites-Determination of Acetone Soluble Material (Resinous 963)

Rec. Graphical Symbols: Generating Stations and ectrodeposited Nickel Coatings on Magnetic and Nonmagnetic Rolling Bearings-Tapered Roller-Boundary Dimensions

$r$ Rolling Bearings: Tapered Roller-Boundary Dimensions

Rolling Bearings-Tapered Roller-Boundary Dimensions

$r$ Rolling Bearings: Tapered Roller-Boundary Dimensions

Rolling Bearings-Tapered Roller-Boundary Dimensions Rec. for Rubber Hoses for Oil $r$ Domestic and Similar Purposes: Vacuum Cleaners and Water (1971) Rec. for Binders Based on Calcium for Sodium Chloride for Industrial Use: Determination of for Phosphoric Acid for Industrial Use-Determination of for Sodium Hydroxide for Industrial Use: Determination of $r$ Potassium Hydroxide for Industrial Use: Determination of for Sodium Carbonate for Industrial Use: Determination of for Hydrochloric Acid for Industrial Use: Determination of o Methyl Orange (1973)

content-Mercurimetric Meth. (1973)

Gravimetric Meth. as Potassium Tetra/

Sodium Tetraphenylborate Volumetric /

Barium Sulphate Gravimetric Meth. (1/

Std. for Potassium

Std. for Potassium

Std. for Potassium

Std. for Potassium

Std, for Potassium

Industrial Use: Determination of Sulphate Content; Barium rial Use; Determination of Sulphur Dioxide Content: Barium Industrial Use: Determination of Sulphate Content; Barium Industrial Use: Determination of Sulphate Content: Barium Industrial Use: Determination of Sulphate Content: Barium industrial Use: Determination of Sulphate Content-Barium hate for Industrial Use: Determination of Content-Barium its Alloys-Check of Continuity of Thin Coatings-Copper

Rec. for Spec. for Photographic Grade P Methylaminophenol alcohols-Glycerine for Industrial Use-Determination of

for Hydrochloric Acid for Industrial Use: Determination of rec. for Nitric Acid for Industrial Use-Determination of Rec. for Plastics-Pvc Resins-Determination of Ash and yde Solutions for Industrial Use: Limit Test for Inorganic Chemical Analysis of Cements: Determination of Sulphur as xylenols for Industrial Use-Test for Absence of Hydrogen Rec, for Spec, for Photographic Grade Sodium its Alloys-Determination of Insoluble Zirconium Alizarin Its Alloys-Determination of Soluble Zirconium (Alizarin Std. for Petroleum Products: Determination of

Rec. for Chemical Analysis of Cements: Determination of Crude Sodium Borates for Industrial Use: Determination of (1969) Rec. for Determination of Total Types with $1 \%$ Chromium and $0.2 \%$ Molybdenum and Controlled 3: Wrought Quenched and Tempered Unalloyed with Controlled phuric Acid and Oleum for Industrial Use; Determination of
Strip Test (1972)

Strip Wound Cut Cores of Grain Oriented Silicon Iron Al Strip ( I956)

Strip (1960)

Stripe on $16 \mathrm{~mm}$ Motion Picture Film Perforated Along 1

Striping of $16 \mathrm{~mm}$ Film Perforated Along Both Edges (196

Strips of Solid Oak (1970)

Strips with Rectangular Face (1969)

Strips (1972)

Strontium Chromate Pigments for Paints (1972)

Structural Steels (1967)

Structure for Information Interchange (1969)

Structure for Start / Stop and Synchronous Transmission

Structure, and Results of the CEE (Electrical Equipment

Structures (1968)

Structures (1973)

Stub Series and Morse Taper Shank (1961)

Studless Links (1957) Rec. for Shipbuilding Detail ISO

Studs and Nuts (1969)

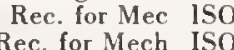

Studs: Dimensioning (1961) Rec. for Mech ISO

ISO

Studs: Thread Lengths for General Purpose Bolts (1968)

Stylus and Diagram (Working Equipment Category) (1970)

(Stylus) Instruments of Progressive Profile Transformati

Styrene Butadiene Rubber Latices-Determination of Vol

ISO

ISO

ISO

ISO

Styrene (1972) Std. for Styrene Butadiene Rubber La ISO

Subdivisions in Written Documents (1972)

Subjective Magnitudes of Sound or Noise (1959)

\section{ISO}

ISO

IEC

Sumerged Channels (1972)

Submultiples of the SI Units (Metric) (1973)

Ional sy ISO

Substance (1966)

Ignation of the Quantities Character IEC

Substance (1967)

Substances of Paper (1958)

Substances Volumetric Meth. (1968)

Substances (1961)

Substances (1965)

Substances) (1969)

Substations, Lines

Substrates-Measurement of Coating Thickness-Magnet

Subunits-In. Series (1968)

Subunits-Metric Series-Dimension Series 29 and 13

Subunits-Metric Series-Dimension Series 30,31 and

Jubunits-Metric Series-Outer Rings with Flange (197

Subunits-Metric Series (1969)

Suction and Discharge (1971)

Suction Cleaning Appliances (1965)

Sulphate-Definitions, Classification and Nomenclature

Sulphate Content-Barium Sulphate Gravimetric Meth. (1

Sulphate Content-Volumetric Meth. (1968)

Sulphate Content: Barium Sulphate Gravimetric Meth. (19

Sulphate Content: Barium Sulphate Gravimetric Meth. (19

Sulphate Content; Barium Sulphate Gravimetric Meth. (19

Sulphate Content; Barium Sulphate Gravimetric Meth. (19

Sulphate for Industrial Use: Determination of Acidity 'T

Sulphate for Industrial Use: Determination of Chloride

Sulphate for Industrial Use: Determination of Content-

Sulphate for Industrial Use: Determination of Content-

Sulphate for Industrial Use: Determination of Content-

Sulphate Gravimetric Meth. (1968)

Sulphate Gravimetric Meth. (1968)

Sulphate Gravimetric Meth. (1968)

ISO

Sulphate Gravimetric Meth. (1969)

Sulphate Gravimetric Meth. (1969)

Sulphate Gravimetric Meth. (1972)

Sulphate Gravimetric Meth. (1973)

Drochloric Acid for ISO

/Nd Oleum for Indust ISO

/Odium Carbonate for ISO

/Odium Itydroxide for ISO

S sium Hydroxide for ISO

Odium Chloride for ISO

/Ion of Aluminium (Aluminum) and ISO

Sulphate (1965)

Sulphated Ash in Products Not Containing More Than 0.5\% ISO

Sulphated Ash: Gravimetric Meth. (1968)

Sulphated Residue on Ignition-Gravimetric Meth. (1971

Sulphated-Ash (1970)

Sulphates (1972)

Sulphide (1968)

Sulphide (1971)

Sulphite (1965)

Sulphonate Photometric Meth. (1972)

Sulphonate Photometric Meth.) (1970)

Sulphur-Lamp Meth. (1973)

Sulphur as Sulphide (1968)

Sulphur Compounds-Volumetric Meth. (1972)

Sulphur Content of Carbon Black for the Rubber Industry

Sulphur Content (1970)

/Nd Free Cutting Steels-Part ISO

/ Wrought Quenched and Tempered ISO

2160

490

1324

1072

245

630

1001

117

834

2394

235

$898 /$ III

$898 / 1$

225

1880

2008

173

2008

2145

131

96

206

536

58

908

175

462

1017

$117-5$

2361

$355 / \mathrm{IV}$

$355 / V I l l$

$355 / V I I$

2316

$355 / \mathrm{V}$

1823

10-2A

1588

2480

847

982

993

743

906

2489

2488

2485

2486

2487

906

912

743

982

2480

2487

2085

422

1616

907

1983

1270

2222

682 
Spec./S and Acceptance of New Rec. for Determination of Total Rec. for Determination of Total (1963) Rec. for Determination of Forms of chemical Analysis of Steel and Cast lron: Determination of of Chemical Analysis of Manganese Ores-Determination of Rec. for Determination of Nitrogen, Total ion of Total Acidity and Calculation of Free So/ ion of Residue on Ignition; Gravimetric Meth. (/ ion of Total Nitrogen Content: Volumetric Meth./ ion of lron Content; 2,2'-Bipyridyl Spect/ ion of Sulphur Dioxide Content: Barium Sulphate/ tion of Oxides of Nitrogen-2,4-Xylenol Spec/ ric Acid Concentration by Measurement of Densit/ 969) alcohols for Industrial Use-Test for Colour (Color) with Rec. for Rockwell Rec. for Verification of Rockwell calibration of Standardized Blocks to Be Used for Rockwell Rec. for Bibliographical References: Essential and for Paper: Untrimmed Stock Sizes for the 1SO-A Series: 1so Rec. for Connections for Aircraft Ground Electrical pment for Radio Active Materials (1967) Rec. or Power uried Unplasticized Polyvinyl Chloride (PVC) Pipes for the mhz: Connectors for Coupling an External Low Voltage Power (1968)

Rec. for Rec. for Matters Relating to Radio Interference and lts Calcium Hardness (1972) es-Meth. of Analysis (1969)

reduced Sample (1967) Determination of the Cloud Point Temperature of Nonionic ethylene Glycols and Nonionic Active Matter (Ad/ $\mathrm{n}$ of Low Contents of Free Glycerol-Spectropho/ ionic-Active Matter (Direct Two Phase Titration/ $y$ of Pastes on Filling (1969)

$y$ of Washing Powders Before and After Compactio/ rd Water (1969)

and Interfacial Tension (1963)

Temperature of Nonionic Surface Active Agents / ric Determination of Oxyethylene Groups (1972)

\section{s: Meth. of Analysis (1968)}

lphates: Meth. of Analysis (1968)

sulphates: Meth of Analysis (1968)

of Laundering-Preparation and Use of Unsoiled/ elative Resistance of Insulating Materials to Breakdown by Rec. for Paints and Varnishes

easurement and Evaluation of the Geometrical Parameters of I Spindle and Reciprocating/ Rec. for Test Conditions for spindle and Reciprocating $\mathrm{T} /$

Rec. for Test Conditions for lume Ratio /

8 (1958)

8) Instrumen/

Carpets-Determination of Measured

ents-Terms/ Rec. for Instruments for the Measurement of Rec. for Instruments for the Measurement of Rec. for

3 for Explosive Gas Atmospheres: Classification of Maximum Rec. for Surface Active Agents: Determination of Rec. for Determination of Rec. for Technical Drawings-Meth. of Indicating ification of Terms (I971)

Rec. for (Aluminum) and Its Alloys-Check of Continuity (Aluminum) and Its Alloys-Estimation of the L/ tion) of Aluminium (Aluminum) and Its Alloys-l tion) of Aluminium (Aluminum) and Its Alloysluminium and Its Alloys-Determination of the connection with the Measurement of Liquid Flow with a Free brinell Hardness Values (HB) for Use in Tests Made on Flat (Reference Meth.) (1970) Rec. for Evaporated Milk and Building Boards: Determination of Water Absorption and of Rec. for Determination of the Crucible oltage Airbreak Switches, Airbreak Disconnectors, Airbreak n (1/ Rec. for International Electrotechnical Vocabulary: c. for Dimensions for Single Hole Mounting, Lever Operated ec. for Performance Requirements for Lever Operated Manual Spec. for
Rec.

in Electronic Applications / Rec. for Thermal Time Delay Rec. for Lever Switches: Requirements for Rec. for Directions of Operation of Toggle cation and in Electronic Applicati/ Rec. for Thermostatic
Sulphur Ilexafluoride (1971)

Sulphur in Coal by the Eschka Mcth. (1963)

Sulphur in Coal hy the IIigh Temperature Combustion Meth SuIphur in Coal (1960)

Sulphur (Meth. After Combustion in a Current of Oxygen

Sulphur, Chlorine and Phosphorus in Coke (1969)

Sulphur, Chlorine and Phosphorus in Coke (1969)

Sulphuric Acid and Oleum for Industrial Use: Determinat

Sulphuric Acid and Oleum for Industrial Use: Determinat

Sulphuric Acid and Oleum for Industrial Use: Determinat

Sulphuric Acid and Oleum for lndustrial Use; Determinat

Sulphuric Acid and Oleums for lndustrial Use: Determina

SuIphuric Acid Concentration by Measurement of Density

Sulphuric Acid for Industrial Use: Evaluation of Sulphu

Sulphuric Acid (1970)

Superficial N and T Scale Hardness Test. Machines (1969

Superficial N and T Scale Hardness Test. Machines (1970

Supplementary Elements (1968)

Supplementary Range (1967)

Supplies (1965)

Supply for Air and Land Vehicle Mounted Prospecting Equ

Supply of Gaseous Fuels: Metric Series-Spec. (1973)

Supply to Portable Entertainment Equipment (1971)

Supply Voltages for Transistorized Nuclear Instruments

Suppression (1965)

Suppression (1969)

Surface Active Agents-Preparation of Water with Known

Surface Active Agents-Technical Sodium Alkylsulphonat

Surface Active Agents-Vocabulary (1969)

Surface Active Agents in Powder Form: Preparation of a

Surface Active Agents Obtained from Ethylene Oxide (196

Surface Active Agents (Nonionic): Determination of Poly

Surface Active Agents: Analysis of Soaps-Determinatio

Surface Active Agents: Detergents-Determination of an

Surface Active Agents: Determination of Apparent Densit

Surface Active Agents: Determination of Apparent Densit

Surface Active Agents: Determination of Stability in $\mathrm{Ha}$

Surface Active Agents: Determination of Surface Tension

Surface Active Agents: Determination of the Cloud Point

Surface Active Agents: Ethylene Oxide Adducts-Iodomet

Surface Active Agents: Glossary (1971)

Surface Active Agents: Measurement of Foaming Power (19

Surface Active Agents: Scientific Classification (1968)

Surface Active Agents: Simplified Classification (1972)

Surface Active Agents: Technical Sodium Alkylsulphonate

Surface Active Agents: Technical Sodium Primary Alkylsu

Surface Active Agents: Technical Sodium Secondary Alkyl

Surface Active Agents: Verification of Certain Effects

Surface and Extended Inner Ring Width (1972)

Surface Discharges (1970) /Meth. for Determining the R IS

Surface Drying Test (Ballotini Meth.) (1970)

Surface Finish (1970) /F Instruments and Devices for M ISO

Surface Grinding Machines with Horizontal Grinding Whee ISO

Surface Grinding Machines with Vertical Grinding Wheel ISO

Surface Pile Density and Measured Pile Fibre (Fiber) Vo

Surface Resistivities of Electrical lnsulating Material

Surface Roughness by the Profile Meth.-Contact (Stylu

Surface Roughness by the Profile Meth.-General Statem

Surface Roughness (1966)

Surface Temperatures (1969)

1. for Electrical Apparatu

Surface Tension and Interfacial Tension (1963)

Surface Tension of Rubber Latices (1970)

Surface Texture on Drawings (1971)

Surface Treatment and Metallic Coatings-General Class

Surface Treatment of Metals-Anodisation of Aluminium

Surface Treatment of Metals-Anodisation of Aluminium

Surface Treatment of Metals-Anodisation (A nodic Oxida

Surface Treatment of Metals-Anodisation (Anodic Oxida

Surface Treatment of Metals-Coloured Anodisation of a

Surface (1968)

Surfaces (1964)

/Cabulary of Terms and Symbols Used in Rec. for Tables of ISO

weetened Condensed Milk-Determination of Fat Conten

Swelling in Thickness After Immersion in Water (1972)

Swelling Number of Coal (1966)

Switch Disconnectors and Fuse Combination Units (1972)

Switchboards and Apparatus for Connection and Regulatio

Switches for Aircraft (1966)

Switches for Aircraft (1970)

Switches for Appliances (1962)

Switches for Appliances (1972)

Switches for Use in Equipment for Telecommunication and

Switches of Type 1, Slow Make and Break (1963)

Switches on Aircraft (1957)

Switches Primarily for Use in Equipment for Telecommuni 
rec. for Rotary Wafer Switches (l.ow Current Rating): Wafer d Diameter $40 \mathrm{~mm}(1966)$ Diameter $60 \mathrm{~mm}$ (l.ow Current Ratin/ (19.55)

3) Measuring Meth. (1962)

ral Mounting (1963)

Auxiliary Circuits, Including Contacto/ Auxiliary Circuits, Including Contacto/ alternating Current Isolators (Disconnectors) and Earthing nnectors and Fuse Combinat/ Rec. for Low Voltage Airbreak 2)

8)

e and Break (1963)

(Toggle) (1969) Rec. for ${ }^{\circ}$ Of Protection of Enclosures for Low Voltage Rec. for High Voltage Metal Enclosed Rec. Spec. for New Insulating OiIs for Transformers and
Rec. for Electrical Installations in Ships: trolgear (19) of Electromechanic/ Rec. for Graphical Symbols: Contacts, Rec. for Low Voltage Distribution ncluding Contacto/ ncluding Contacto/

Rec. for Control Switches (Low Voltage Rec. for Control Switches (Low Voltage erties of Microwave Tubes and Valves: Gas Filled Microwave the Test. of Circuit Breakers with Respect to Out of Phase Rec. for Basic Ionizing Radiation International Rec. for Correspondence of Doors, Windows and Shutters-Part I (1970) for Indexable (Throwaway) Inserts-Designation-Code of tistical Vocabulary and Symbols: First Series of Terms and Rec. for Statistical Vocabulary and 2) Rec. for Keytop and Printed or Displayed Rec. Graphical Symbols: steels and Low Alloy High Tensile Types/ ding of Cast Iron (1969)

and Low Alloy High Tensile Types (1967) forces, Moments and Their Coefficients (1972) ircraft and the Atmosphere Relative to the Eart/ elative to the Air (1972)

Rec. for International Gear Notation: sories for Fluid Power Transmission ( Manual Arc Welding of Mild and Low Alloy Steels-Code of ircuits (I969) (1969) ents of (1972) 61) cross Sections-Part I: General Rule/ cross Sections-Part II: Representat/ cross Sections-Part III: Representa/

Rec. Graphical Symbols: Block for Rotating Electrical Machines: Rec. for Mathematical Signs and ions and Electronics (1972) id Flow with a Free Surl

Rec. for Vocabulary of Terms and Rec. for Electrical Installations in Ships: Graphical stical Vocabulary and Symbols-Second Series of Terms and ood Utilized in the Manufacture of Wood Flooring-Code of c. for General Principles for the Formulation of Graphical rec. for Pictorial Markings for Handling of Goods (General tolerances of Form and of Position-Part I: Generalities,

us Applications (1969) rters and Elements of Electromechanic/ 972) atistical Vocabulary/

Rec. for Graphical ec. for Graphical Rec. Graphical cal Vocabulary and Rec. Graphical

Transmission and Distribution (1963) f Connection and Circuit Elements (1960) mulators: Aerials (Antennas) and Radi/ mulators: Microwave Technology (1968) mulators (1960)

3)

Rec. Graphical

Rec. Graphical

Rec. for Graphica Rec. Graphica Rec. Graphica Rec. for Sensitive

Rec. for Push Button Rec. for Lever Rec. for Lever Rec. for Definitions for Rec. for Rec. for Graphical Rec. for Code of

Rec. for Code of

Rec. for Flow Rec. for

Rec. for Units and Rec for Letter Rec. for Graphical Rec. for Function Key Rec. for Letter Rec. for Letter Rec. for Abbreviations Rec. for

Switches with Central Mounting (1963)

Switches with Central Mounting; Maximum 12 Positions an Switches with 2 Hole Mounting; Maximum 26 Positions and Switches (Electrical) for Domestic and Similar Purposes Switches (Low Current Rating) with 2 Hole Mounting (196 Switches (Low Current Rating): General Requirements and Switches (Low Current Rating): Wafer Switches with Cent Switches (Low Voltage Switching Devices for Control and Switches (Low Voltage Switching Devices for Control and Switches (1961)

Switches (1968)

Switches (1972)

land Auxiliary Circuits, Including Con

Switches, Airbreak Disconnectors, Airbreak Switch Disco

Switches: General Requirements and Measuring Meth. (196

Switches: General Requirements and Measuring Meth. (196

Switches: General Requirements and Measuring Meth. (197

Switches: Requirements for Switches of Type 1, Slow Mak

Switches: Requirements for Type 2, Quick Make and Break

Switchgear and Controlgear (1963)

Switchgear and ControIgear (1968)

Switchgear and Controlgear (1969)

Switchgear (1972)

Switchgear, Electrical Protection, Distribution and Con

Switchgear, Mechanical Controls, Starters and Elements

Switchgear: Circuit Breakers (1964)

Switching Devices for Control and Auxiliary Circuits, I

Switching Devices for Control and Auxiliary Circuits, I

Switching Devices (1972)

Switching (1968)

Ement of the Electrical Prop IEO

Symbol (1963)

Rec for Guide to IEC

Symbol (1970)

Symbolic Designation of Direction of Closing and Faces

Symbolization (197I)

Symbols-Part 1: Statistical Vocabulary (1967)

Symbols-Second Series of Terms and Symbols (1970)

Symbols for Adding and Calculating Machines (1969)

Symbols for Alternating Current Electricity Meters (197

Symbols for Architectural Diagrams (1967)

Symbols for Coal Preparation Plant (1967)

Symbols for Covered Electrodes for Arc Welding of Mild

Symbols for Covered Electrodes for Manual Metal Arc Wel

Symbols for Filler Rods for Gas Welding of Mild Steels

Symbols for Flight Dynamics-Part III: Derivatives of

Symbols for Flight Dynamics-Part II: Motions of the a

Symbols for Flight Dynamics-Part I: Aircraft Motion R

Symbols for Geometrical Data (1968)

Symbols for Hydraulic and Pneumatic Equipment and Acces Ered Electrodes for

Symbols for Indichine Tools (196

Symbols for Information Processing (1969)

Symbols for Languages, Countries and Authorities (1967)

Symbols for Refrigeration (1968)

Symbols for Semiconductor Devices and Integrated Microc

Symbols for Transmission and Miscellaneous Applications

Symbols for Types of Construction and Mounting Arrangem

Symbols for Use in Physical Sciences and Technology (19

Symbols for Use on Detailed Maps. Plans and Geological

Symbols for Use on Detailed Maps, Plans and Geological

Symbols for Use on Detailed Maps, Plans and Geological

Symbols on Typewriters (1969)

Symbols to Be Used in Electrotechnology: General (1971)

Symbols to Be Used in EIectrotechnology: Telecommunicat

Symbols Used in Connection with the Measurement of Liqu

Symbols (1965)

Sy mbols (1970)

Rec. for Stati IEC

Symbols (1971)

Symbols (1972)

Symbols) for Plastics (1969)

Marking of the Different Species of

Symbols) (1968)

Symbols, Dimensions and Layout for Safety Signs (1967)

Symbols, Indications on Drawings (1969)

Symbols: Binary Logic Elements (1972)

Symbols: Block Symbols for Transmission and Miscellaneo

ymbols: Contacts, Switchgear, Mechanical Controls, Sta

Symbols: Ferrite Cores and Magnetic Storage Matrices (1

Symbols: First Series of Terms and Symbols-Part 1: St

Symbols: Frequency Spectrum Diagrams (1968)

Symbols: General Index (1972)

Symbols: Generating Stations and Substations, Lines for

Rec. for Graphica Rec. Graphical Symbols: Machines, Transformers, Primary Cells and Accu Symbols: Machines, Transformers, Primary Cells and Accu Symbols: Machines, Transformers, Primary Cells and Accu Symbols: Measuring Instruments and Electric Clocks (196

Symbols: Semiconductor Devices, Capacitors (1971) Symbols: Symbols for Architectural Diagrams (1967)
IEC $\quad 132.2$

132.4

CEE 14

IEC 132-3

IEC 132-1

$132-$

337-1

$337-2$

129

265

$337-2$

408

131-1

$163-1$

$341-1$

$131-2$

131.3

144

277

298

296

92-4

117-3

$157-$

337-1

337-2

235-7

267

361

13

1226

1832

645

1786

1093

387

117-8

561

635

1071

636

1153

1152

1151

701

1219

2560

369

1028

639

786

148

117-13

34.7

$31 / X I$

$710 / I$

$710 / 11$

$710 / I I I$

1090

$27 \cdot 1$

27-2

772

92-2

1786

2036

416

1043

780

557

1101

117-15

$117-13$

$117-3$

117.16

645

$117 \cdot 12$

117-0

$117-5$

117-1

117-10

117-11

$117-2$

117.4

117.7

$117-8$ 
f Electronic Tubes, Valves and Rectifiers/

Rec. Graphical

y That the Transvers/ Rec. for Calculation of Rectangular c. for Rotating Electrical Machines: Meth. for Determining Rec. for Character Structure for Start / Stop and

Rec. for Abstracts and Std. for Rec. for

mechanical Stability (1971) ion of Resistance to Crack Growth of Vulcanized Natural or on of Resistance to Flex Cracking of Vulcanized Natural or Rec. for Determination of Viscosity of Natural and r Determination of Tear Strength of Vulcanized Natural and Rec. for on and Dimensional Charact/ Oven Meth.) (1970)

eth. (1973)

9)

61)

acters (1968)

cters (1968)

n Workpieces (1971)

and Deviations (1962)

Std. for Rules for the Use of Units of the Rec. for Iso

for Netting Yarns for Fishing Nets: Designation in the Tex les-Universal System for Designating Linear Density (Tex emiconductor Devices (1961)

Rec. for International Code for Test. of Speed Governing to Be Used in Schemes for the Installations of Pipe Line to Be Used in Schemes for the Installations of Ventilation ns to Be Used in Schemes for the Installations of Sanitary

Rec. for Tests on Indoor and Outdoor Post Insulators for nsions of Indoor and Outdoor Post Insulators and Units for Rec. for Voltages for Aircraft Electrical

y Hour Numbers of the Phase Conductors of 3 Phase Electric technical Vocabulary: Automatic Controlling and Regulating Rec for Test of Refrigerating

for Lightning Arresters: Nonlinear Resistor Type for a.c. rec. for Analogue d.c. Current Signals for Process Control Rec. for Analogue Pneumatic Signal for Process Control for Basic Mode Control Procedures for Data Communication Rec. Series Capacitors for Power er, with a Temperature Index of 180 for Use in Refrigerant er, with High Mechanical Properties for Use in Refrigerant

Rec. Graphical Symbols: Kind of Current, Distribution

Rec. for Magnetic Tape Recording and Reproducing cknuts, Narrow Series, and Lockwashers with Straight Inner s with Horizontal Grinding Wheel Spindle and Reciprocating nes with Vertical Grinding Wheel Spindle and Reciprocating ical Handling Equipment for Loose Bulk Materials-Picking c. for Plain End Steel Tubes, Welded or Seamless-General

le-1 Rec. for Test Conditions for Milling Machines with l Spi/ Rec. for Test Conditions for Milling Machines with Rec. for Machine Tool

for Domestic and Similar Purposes: Cooking Ranges, Cooking ion Coefficient (For Use in the Preparation of Measurement Made on Flat Surfaces (1964) erials (1964)

ification (1971)

ment (1971) Rec. for Rec. for Rec. for Diagrams, Charts, Rec. for Diagrams, Charts. Rec. for Petroleum Measurement Rec. for Diagrams, Charts, Rec for Power

iated Prime Movers) and Motors, Electric Propulsion Plant, 971) Std. for Data Interchange on Rolled Up Punched Paper s: Coding of Preparatory Functions G An/ Rec. for Punched t Numerically Controlled Machines (1969/ Rec. for Punched Rec. for Dimensions for Punched Paper nd $800 \mathrm{RPI}), \mathrm{NRZI}$, and $63 \mathrm{R} / \mathrm{Rec}$. for Unrecorded Magnetic Rec. for 7 Track 8 RPmin (200 RPI) Magnetic Rec. for 9 Track 8 RPmm (200 RPI) Magnetic Rec. for 9 Track 32 Pmm (800 RPI) Magnetic

change (1969)

characteristics (1968)

1971) Cut Numerically Controlled Machines (1/
Symbols: Telephony, Telegraphy and Transducers (1968)

Symbols: Variability, Examples of Resistors, Elements $\mathrm{O}$ Symmetrical Fillet Welds Statically Loaded in Such a Wa Synchronous Machine Quantities from Tests (1967) Synchronous Transmission (1970)

Synopses (1961)

Synthetic Rubber Latices-Codification (1972)

Synthetic Rubber Latices-Determination of High Speed

Synthetic Rubber (De Mattia Type Machine) (1959)

Synthetic Ruhber (De Mattia Type Machine) (1959)

Synthetic Rubbers by the Shearing Disk Viscometer (1963

Synthetic Rubbers (Crescent Test Piece) (1957)

Syringes for Medical Use (1967)

Syringes, Needles and Other Medical Equipment: Definiti

Syrup-Determination of Dry Matter of Moisture (Vacuum

Syrup: Determination of Dry Matter-Refractive Index M

System Equipment: Amplifiers (1969)

System Equipment: Auxiliary Passive Elements (1971)

System Equipment: Explanation of General Terms (1971)

System Equipment: General (1970)

System Equipment: Loudspeakers (1972)

System Equipment: Mechanical Design Features (1971)

System Equipment: Microphones (1972)

System for Designating Linear Density (Tex System) (196

System for Printed Circuits (1970)

System for the Transliteration of Arabic Characters (19

System for the Transliteration of Greek into Latin Char

System for the Transliteration of Slavic Cyrillic Chara

System of Limits and Fits-Part II: Inspection of Plai

System of Limits and Fits-Part I: General Tolerances

System of Units and a Selection of the Decimal Multiple

System (1968)

System) (1969)

Rec. ISO

Tubes and Valves and Analogous Sor Texti ISO

IEC

Systems in Ships (1967) Rec. for Conventional Signs ISO

Systems in Ships (1967) Rec. for Conventional Signs ISO

Systems in Ships (1968)

Rec. for Conventional Signs ISO
Rec. for Conventional Sig ISO

ystems with Nominal Voltages Greater Than 1,000 V (196 IEC

Systems with Nominal Voltages Greater Than 1,000 V (196 IEC

Systems (1961)

Systems (1963)

Systems (1966)

Systems (1968)

Systems (1970)

Systems (1971)

Systems (1971)

Systems (1971)

Systems (1972)

Systems (1972)

Systems (1972) lof Winding Wires: Enamelled Round Copp

Systems: Dimensions and Characteristics (1968)

Rec. for Identification B IEC

Rec. for International Electro IEC

Rec. IEC

Table-Test. of Accuracy (1971)

Table-Test. of Accuracy (1971)

Table Conveyors-Safety Code (1972)

Std. for Rolling Bearings: Lo

/Ace Grinding Machine ISO

/Rface Grinding Machi ISO

Ontinuous Mechan ISO

Table of Dimensions and Masses Per Unit Length (1971)

Table of Fixed Height with Horizontal or Vertical Spind

Table of of Variable Height, with Horizontal or Vertica

Tables-T Slots and Corresponding Bolts (1971)

Tables and Similar (1968)

Ing and Heating Appliances

Tables for Liquids) (1970)

/Lue for the Thermal Expans

Tables of Brinell Hardness Values (HB) for Use in Tests

Tables of Vickers Hardness Values (HV) for Metallic Mat

Tables Used in Electrotechnology: Definitions and Class

Tables (Electrotechnology-Electrical) (1959)

Tables (1970)

Tables: Item Designation for Electrical Parts and Equip

Take Off and Draw Bar for Agricultural Tractors (1966)

Tankers (1965)

Tor Rectifiers, Generators (With Assoc

Tantalum Capacitors with Liquid or Solid Electrolyte (1

Tape-General Requirements (1972)

Tape Block Formats for the Numerical Control of Machine

Tape Fixed Block Format for Positioning and Straight Cu

Tape for Data Interchange (1969)

Tape for Information Interchange -8 and 32 RPmm (200 a

Tape for Information Interchange (1971)

Tape for Information Interchange (1971)

Tape for Information Interchange (1971)

Tape Labelling and File Structure for Information Inter

Tape Recording and Reproducing Systems: Dimensions and

Tape Used in Interchange Instrumentation Applications

Tape Used in Interchange Instrumentation Applications

Tape Variable Block Format for Positioning and Straight 
Numerically ciuntrollerl/ Rec. for Interchangeable Punched Textile Marhinery and Acressories-Cylindrical Tubes fo rentation of 6 and 7 Bit Coded Character Sets on Punched Coded Character Set on 9 Track $12.7 \mathrm{~mm}$ (1/2 In.) Magnetic coded Character Sets on 7 Track $12.7 \mathrm{~mm}$ (1/2 In.) Magnetic for Information Processing-Properties of Unpunched Paper Rec. for Series of Conical Tapers and Std. for ShelI Reamers with Rec. for Cotter Slots with $5 \%$ $s$ for Shell Reamers (1972) ding Keyways (Dimensions in Millimetres) (Milli/ Rec. for Rec. for Hand and Long Fluted Machine Reamers, Morse st Drills: Parallel Shank Jobber and Stub Series and Morse Std. for Core Drills with Parallel, and Morse Std. for End Mills with 7/24 duction Sleeves and Extension Sockets for Tools with Morse Rec. for Machine Chucking Reamers with Parallel or Morse amers for Morse and Metric Tapers, with ParalleI and Morse Rec. for Paper Cones for Yarn Winding (Cross Wound): Rec. for Paper Cones for Yarn Winding (Cross Wound): Rec. for Paper Cones for Yarn Winding (Cross Wound) Dimensional Characteristics for Those with a $6 \%$ and a $10 \%$ Rec. for End Mills with Parallel Shanks with Morse Std. for Hand Taps for Pipe Threads for Parallel and 8) Rec for Rolling Bearing:

for Rolling Bearings-Double Row Cylindrical Type RD with $r$ Series 9 and 0 (1967) dimensions Series 31 and $32 /$ eries (1968)

c Series-Outer Rings with F/

c Series (1969)

c Series-Dimension Series /

c Series-Dimension Series 2/ Rec. for Rolling Bearings. Rec. for Rolling Bearings Rec. for Rolling Bearings Std. for Rolling Bearings Rec. for Rolling Bearings Rec. for Rolling Bearings Std. for Rolling Bearings Rec. for Rolling Bearings Rec. for Rolling Bearings Rec. for Rolling Bearings Std. for Rolling Bearings: Std for Rolling Bearings:

mal), 3,0 and 00 (1973)

1973)

for ISO Identification Code for Rolling Bearings: Group IV ngs: Groups-(I) Radial Ball and Roller (II) Thrust (III) Rec. for Rolling Bearings Rec. for Cutting Knotted Netting to Shape Rec. for Milling Machine Arbors with $7 / 24$ Rec. for Series of Conical Rec. for Selfholdin Rec. for $7 / 24$

Rec. for $7 / 24$ Tapers for Tool Shanks-7/24 Rec. for Drill Chuck

ec. for Cotter Slots with 5\% Taper Keys for Boring Machine Std. for Finishing Reamers for Morse and Metric Std. for Tool Shanks and Equipment with 7/24 ies and Test Meth. (1973)

Std. for Unrecorded Magnetic ral Dimensional Requirement/ Rec. for Unrecorded Magnetic Rec. for Packaging of Components on Continuous meters) and In./S (1970)

) and In./S (1970)

) and In./S (19/

) and $\operatorname{In} /$ 9/ Std. for Drills for Use Prior to
Rec. for Slotted Pan Head Rec. for Slotted Countersunk (Flat) Head Rec. for Slotted Raised Countersunk (Oval) Head
Rec. for Hexagon Head Rec. for Hexagon Head
Rec. for Hexagon Head general Dimensions and Marking (1972) n. (1972) Std. for Long Shank Machine tillation of Bro/ Rec. for Short Machine and Hand Rec. for Determination of the Yields of ide for Industrial Use-Determination of Content-Sodium (1970) Rec. for Rec. for Rec. for Rec. for Rec. for Rec. for Rec. for Rec. for Rec. for

content (1970) belts (Meth. of Test) (1966)

$s$ (Delft Test Piece) (I968)

rs (Crescent Test Piece) (I957)
Rec. for Determination of Rec. for Determination of
Tape Variable Block
Tape Yarns (1972)

Tape (1969)

Tape (1969)

Tape (1969)

Tape (1971)

Taper Angles (1969)

Taper Bore (1:30 (Included) ) with Slot Drive and Arbor

Taper Keys for Boring Machine Tapers (1971)

Taper Keys with or Without Gib Head and Their Correspon

Taper Shank (1961)

Taper Shank (1961)

Taper Shanks-Rec. Stocked Sizes (1972)

Taper Shanks-Std. and Long Series (1972)

Taper Shanks (1961)

Taper Shanks (1966)

Taper Shanks (1972)

Taper $3^{\circ} 30^{\prime}(1959)$

Taper $4^{\circ} 20^{\prime}(1959)$

Taper $9^{\circ} 15^{\prime}(1959)$

Taper (1967)

Taper, Shanks-Std. and Long Series (1970)

Taper-Threads-General Dimensions and Marking (1972

Tapered Bore 1:12-Tolerance Class 5-Special Require

Tapered Roller-Boundary Dimensions-Metric-Diamete

Tapered Roller-Boundary Dimensions-Metric Series-

Tapered Roller-Boundary Dimensions-Subunits-In. S

Tapered Roller-Boundary Dimensions-Subunits-Metri

Tapered Rollcr-Boundary Dimensions-Subunits-Metri

Tapered Roller-Boundary Dimensions-Subunits-Metri

Tapered Roller-Boundary Dimensions-Subunits-Metri

Tapered Roller-Boundary Dimensions (1963)

Tapered Roller-Boundary Dimensions (1965)

Tapered Roller-In. Series-Chamfer Dimension Limits
Tapered Roller-Inch Series-Tolerance Classes 4 (Nor

Tapered Roller-Metric Series-Tolerances-Class 4 (

Tapered Roller Type-In. Series (1965)

Tapered Type-Metric Series (1963)

Tapered; Metric

Tapers and Milling Machine Accessories (1968)

Tapers and Taper Angles (I969)

Tapers for Tool Shanks (1963)

Tapers for Tool Shanks (1963)

Tapers Nos. 45 and 55 (1971)

Tapers (1961)

Tapers (1971)

Tapers, with Parallel and Morse Taper Shanks (1972)

Tapers: Collar Dimensions (1972)

Tapes for Instrumentation Applications: Physical Proper

Tapes for Interchange Instrumentation Applications Gene

Tapes (1968)

Tapping Screw Thread-Dimensions in Millimetres (Milli Tapping Screw Threads (1972)

Tapping Screws-Dimensions in Millimetres (Millimeters Tapping Screws-Dimensions in Millimetres (Millimeters Tapping Screws-Dimensions in Millimetres (Millimeters Tapping Screws-In. Series (1970)

Tapping Screws-Metric Series (1970)

Taps for Pipe Threads for Parallel and Taper-Threads-

Taps with Nominal Diameters from $324 \mathrm{~mm}$ and $1 / 8$ to 1 I

Taps (1966)

Tar, Water, Gas and Coke Residue by Low Temperature Dis

Tatraphenylborate Volumetric Meth. (1971)

Tea-Determination of Acid Insoluble Ash (1970)

Tea-Determination of Alkalinity of Water Soluble Ash

Tea-Determination of Loss in Mass at $103{ }^{\circ} \mathrm{C}(1970$

Tea-Determination of Total Ash (1970)

Tea-Determination of Water Extract (1970)

Tea-Determination of Water Soluble and Insoluble Ash

Tea-Preparation of Ground Sample of Known Dry Matter

Tea Sampling-Part I: from Large Containers (1970)

Tear Propagation Resistance of the Carcass of Conveyor

Tear Strength of Small Test Pieces of Vulcanized Rubber

Tear Strength of Vulcanized Natural and Synthetic Rubbe Tearing Resistance (1971)

Technical Delivery Requirements for Steel (1964) Rec. for Technical Drawings-Meth. of Indicating Surface Textur Rec. for Technical Drawings-Tolerances of Form and of Position Rec. for Technical Drawings-Tolerances of Form and of Position

e on Drawings (197I)

-Part III: Dimensioning and Tolerancing of $\mathrm{Pr} /$ Std. for Std. for

1969)

968)
Technical Drawings: Conventional Representation of

Technical Drawings: Representation of Springs (1973)

Technical Sodium Alkylsulphonates-Meth. of Analysis (
Std. for ISO

ISO

7 Bit ISO

Rec. ISO

ISO

ISO

ISO

ISO

ISO

ec. for Twi ISO

ISO

ISO

Rec. for Re ISO

ISO

ISO

ISO

ISO

ISO

ISO

ISO

ISO

ISO

ISO

ISO

ISO

ISO

ISO

ISO

ISO

ISO

ISO

ISO

Rec. ISO

ISO

ISO

ISO

ISO

ISO

ISO

ISO

ISO

ISO

ISO

ISO

ISO

ISO

ISO

IEC

ISO

ISO

ISO

ISO

ISO

ISO

ISO

ISO

ISO

ISO

ISO

ISO

ISO

ISO

ISO

ISO

ISO

ISO

ISO

ISO

ISO

ISO

ISO

ISO

ISO

ISO

ISO

ISO

ISO

ISO

ISO

ISO

1057 
sin (1968)

lуніс (1968)

Rec. for Surface Active Agents: und Signalling Devices on Motor Vehicles Acoustic Std. and Rec. for Hand Operated Wrenches and Sockets Std. for Flexible Steel Wire Rope for Aircraft Controls: ion of 1 In. Std. Condenser Microphones by the Reciprocity ation of One Inch Condenser Microphones by the Reciprocity Rec. for High Voltage Test

ling with Electronic Equipment and Other Employing Similar

flexible Cellular Materials: Hardness Test. by Indentation

unication and in Electronic Applications Employing Similar unication and in Electronic Applications Employing Similar matical Signs and Symbols for Use in Physical Sciences and

s, Transformers, Primary Cells and Accumulators: Microwave Std. for Metal Slitting Saws with Fine and Coarse for Thermal Time Delay Switches for Use in Equipment for $r$ Thermostatic Switches Primarily for Use in Equipment for

e) Insulation and Sheath: Signalling Cables in Singles for grain Oriented Silicon Iron Alloy, Used for Electronic and Rec. Graphical Symbols: res of Broad Band Transformers of Ferromagnetic Oxides for ned Transformers and Inductors of Ferromagnetic Oxides for

Rec. for Letter Symbols to Be Used in Electrotechnology: Rec. for Cores for Inductors and Transformers for Rec. for International Electrotechnical Vocabulary: Rec. Graphical Symbols: Telephony, Rec. for

International Electrotechnical Vocabulary: Telegraphy and Rec. Graphical Symbols:

Rec. for Radio Frequency Connectors: Coaxial Unmatched from Receivers for Amplitude and Frequency Modulations and Rec. Meth. of Measurement on Receivers for 1 Properties of Electronic Tubes: Meth. of Measurement for connecting Wire with Flame Retarding Insulation for Use in and a Maximum Working Temperature of $105^{\circ} \mathrm{C}$ for Use in or Cinematography-Picture Areas for Films and Slides for articular to Transposers for Monochrome and Colour (Color) Rec. for Light Metals and Their Alloys

of Uns/ Rec. for Plastics-Determination of the Maximum Rec. for Plastics-Meth. of Determining the Brittleness ec. for Determination of Total Sulphur in Coal by the High Rec. for Determination of Chlorine in Coal by the High nation of Carbon and Hydrogen in Coal and Coke by the High Rec. for Household Refrigerators-Part II: Special Low 970) Rec. for n of the Yields of Tar, Water, Gas and Coke Residue by Low Rec. for Std. Reference lar Types of Winding Wires: Enamelled Round Copper, with A lar Types of Winding Wires: Enamelled Round Copper, with A lar Types of Winding Wires: Enamelled Round Copper, with A lar Types of Winding Wires: Enamelled Round Copper, with A 1968)

ed Creep Test of Steel at Elevated Temperatures (Load and cs (1968) Rec. for Meth. of Test for Rec. for Plastics-Determination of Rec. for Plastics-Determination of the

or Surface Active Agents: Determination of the Cloud Point Rec. for Plastics-Determination of the Vicat Softening ated Voltage of $20 \mathrm{kV}$ and $25 \mathrm{kV} \mathrm{d.c.} \mathrm{and} \mathrm{a} \mathrm{Maximum} \mathrm{Working}$ Std. Meth. of Measurement of Lamp Cap

asic Environmental Test. Procedures: Guidance on Change of etermination of the Stiffness of Vulcanized Rubbers at Low s-Determination of Stiffness in Torsion as a Function of for Explosive Gas Atmospheres: Meth. of Test for Ignition

for Electronic Components and Equipment: Test N: Change of n of Refractoriness Under Load (Differential-With Rising Rec. for Interrupted Creep Test. of Steel at Elevated Rec. for Noninterrupted Creep Test. of Steel at Elevated

Rec. for Creep Stress Rupture Test. of Steel at Elevated ion of Proof Stress and Proving Test for Steel at Elevated plosive Gas Atmospheres: Classification of Maximum Surface set of Vulcanized Rubbers Under Constant Deflection at Low stance and Resistivity of Insulating Materials at Elevated

Std. for Lined Industrial Rubber Footwear for Use at Low mpression Set Under Constant Deflection at Normal and High resses $(\mathrm{S} /$ Rec. for Mechanical Test. of Steel at Elevated oy, and Free Cutting Steels-Part 8: Wrought Quenched and reated Steels: Free Cutting-Part 7: Wrought Quenched and oy, and Free Cutting Steels-Part 5: Wrought Quenched and alloy and Free Cutting Steels, Pt. 4: Wrought Quenched and $y$, and Free Cutting Steels-Part 2: Wrought, Quenched and ted, Alloy, and Free Cutting Steels-Part 1: Quenched and oy, and Free Cutting Steels-Part 3: Wrought Quenched and oy, and Free Cutting Steels-Part 6: Wrought Quenched and Copper and Its Alloys-Code of Designation-Part II: of Rec. for Numeric Section of t Circuit Breakers: Information to Be Given with Enquiries
Technical Sodium Primary Alkylsulphates: Meth. of Analy Technical Sodium Secondary Alkylsulphates: Meth. of Ana

Technical Spec. (1966)

Rec, for So ISO

Technical Spec. (1970)

Technical Spec. (1973)

Technique (1971)

Technique (1972)

Techniques (1962)

Techniques (1968)

Techniques (1972)

Techniques: General Requirements and Measuring Methods IEC

Technology (1961)

Technology (1968)

Teeth-Metric Series (1972)

Telecommunication and in Electronic Applications Employ

Rec for Mathe ISO

/Recision Meth. for Pressure Calibrat IEC

/Simplified Meth. for Pressure Calibr IEC

Espect to Possible Hazards

Tecommunication and in Electronic Applications Employ

Telecommunication Equipment and Installation (1969)

Telecommunication Equipment (1971)

(1969) ID IEC

of Performance Spec. for Co IEC

OPec for Cores of Tu IEC

Telecommunications and Electronics (1972)

Telecommunications: Measuring Meth. (1971)

Telegraphy and Telephony (1970)

Telegraphy and Transducers (1968)

Telemetering for Consumption and Demand (1970)

Telephony (1970)

Telephony, Telegraphy and Transducers (1968)

Television Aerial Feeder Connector (1965)

Television Broadcast Transmissions (1959)

Television Broadcast Transmissions (1960)

Television Picture Types (1968)

Television Receivers (1965)

Television Receivers (1967)

Television (1971)

Television (1971)

Temper Designation (1971)

Temperature and the Rate of Increase During the Setting

Rec. for High Voltage IEC / of $20 \mathrm{kV}$ and $25 \mathrm{kV} \mathrm{d.c.} \mathrm{IEC}$

$\begin{array}{ll}\text { Rec. F } & \text { ISO } \\ \text { IEC }\end{array}$

Temperature by Impact (1969)

Temperature Combustion Meth. (1963)

Temperature Combustion Meth. (1963)

Temperature Combustion Meth. (1967)

Temperature Compartments for the Storage of Frozen Food ISO

Temperature Control Devices for Quartz Crystal Units (1 $\quad$ IEC

Temperature Distillation of Brown Coal and Lignite (196 ISO

Temperature for Industrial Length Measurements (1951)

Temperature Index of 155 (1970)

Ec. Spec for Particu IEC

Temperature Index of 180 for Use in Refrigerant Systems IEC

Temperature Index of $180(1972)$

Temperature Index of 220 (1972)

Temperature Interrupted) (1961)

Rec. for Particu IEC

Rec. for Particu IEC

ISO

Temperature of Deflection Under Load (1958)

Temperature of Evolution of Flammable Gases from Plasti

Temperature of Nonionic Surface Active Agents Obtained

Temperature of Thermoplastics (1968)

Temperature of $105^{\circ} \mathrm{C}$ for Use in Television Receive

Temperature Rise (1971)

Temperature Tests (1971)

Temperature (Gehman Test) (1971)

Temperature (1965)

Temperature (1966)

Temperature (1969)

Temperature) (1970)

Temperatures (Load

Temperatures (1961)

Temperatures (1961)

Temperatures (1969)

Temperatures (1971)

Temperatures (1971)

Temperatures (1972)

Temperatures (1972)

Temperatures: Determination of Lower Yield and Proof St

Tempered Chromium Nickel Molybdenum Types (1970) / All ISO

Tempered Chromium Types (1970)

Rec. for Heat T ISO

Rec, for $B$ IEC

Rec for D ISO

Rec. for Plastic ISO

Rec. for Electrical A pparatus IEC

Procedures IEC

(Efractory Products-Determinatio ISO

Tempered Manganese (1970)

Rec for Heat Treated, All ISO

Tempered Types with $1 \%$ Chromium and $0.2 \%$ Molybdenum and ISO

Tempered Types with $1 \%$ Chromium and $0.2 \%$ Molybdenum (19 ISO

Tempered Unalloyed Types (1968) Rec. for Heat Trea ISO

Tempered Unalloyed with Controlled Sulphur Content (197

Tempered, with 3\% Chromium and 0.5\% Molybdenum (1970)

Tempers (1971)

ISO

Ten Key Keyboards for Adding and CaIculating Machines (

Tenders and Orders and Rules for Transport, Erection an 
arhorm-Metric Series (19/ Std. for Milling Cutters with Std. for Shell End Mills with Plain Bore and Rec. for Determination of Rec. for Plastics-Determination of Rec. for Plastics-Determination of Std. for Rigid Cellular Plastics-Determination of Rec. for Shackle Type Connector Units for High nd Coal Ploughs (1967) Rec. for High lectrodes for the Welding of Mild Steel and Low Alloy High ds Made with Covered Electrodes of Mild and Low Alloy High Rec. for Flexible Cellular Materials-Determination of and Meth. of Test (1962) Rec. for Full Thickness Rec. for Paper and Board-Determination of Std. for Ebonite: Determination of Rec. for Determination of

(1968) Rec. for
lled Flat Products (Thickness Less Than $2.5 \mathrm{~mm}(0.1 \mathrm{In}$.) Section (1964) Rec. for Rec. for Rec. for Rec. for Rec. for Rec. for Rec. for Rec. for Rec. for

d Not Than $0.5 \mathrm{Mm}$ Thick (1959)

Rec. for Load Calibration of Test. Machines for c. for Classification of Brasses, Leaded, Special and High ler Rods for Gas Welding of Mild Steels and Low Alloy High ectrodes for Arc Welding of Mild Steels and Low Alloy High filler Rods for Gas Welding Mild Steels and Low Alloy High

Rec. for Surface Active Agents: Determination of Surface Rec. for Determination of Surface

e Agents: Determination of Surface Tension and Interfacial

tics-Determination of Viscosity Number of Poly (Ethylene uits-Assignment of / Std. for Data Communication-Data cal Cables (1973) Std. for Aluminium for Connector Pin Allocations for Use with High Speed Data 1964) Rec. for Rotating Electrical Machines:

for Lead Acid Starter Batteries: Dimensions and Marking of Rec. for Primary Cells and Batteries: Rec. for Preferred Diameters of Wire

electronic Components and Equipment: Test U: Robustness of ers for Electrical Machinery: Complementary Dimensions and the Dimensions of a Cylindrical Component Having Two Axial Rec. for Sectional Beams for Warp Knitting Machines:

rec. for Fixed Capacitors for Use in Electronic Equipment: Rec. for Definitions and Rec. for ces and General Principles of Measuring Meth.: General and Rec. for Vocabulary of Std. for Doors -

Std. for Textile Floor Coverings: Classification and td. for Internal Combustion Engines: Spark Plug Ignition Std. for Braking of Motor Vehicles and Their Trailers -

$f$ the Electrical Properties of Microwave Tubes and Valves: Rec. for Radioisotope All or Nothing Relays Rec. for Pirn Winders:

Rec. for Cone Winders or Cheese Winders:

Rec. for

Equipment and the Co/ . Rec. for Preliminary List of Basic and Grooved Pulleys (1969)

Rec, for Pirn Winders: Terminology: Basic ec. for Cone Winders or Cheese Winders: Terminology: Basic Rec. for Coniferous Sawn (Sawed) Timber-Defects Rec. for Coniferous Sawn (Sawed) Timber-Sizes Rec. for Netting for Fishing-Basic

face Roughness by the Profile Meth.-General Statements e Shuttle Eye (1970)

Rec. for Shuttles. Rec. for Fixed Resistors: c. for Statistical Vocabulary and Symbols: First Series of nt of Liquid Flow with a Free Sur/ Rec. for Vocabulary of for Statistical Vocabulary and Symbols-Second Series of Rec. for Definitions of )

for Metallic and Other Nonorganic Coatings-Definition of tion of Assembled Bars for / Rec. for Textile Machinery . Rec. for Rec. for

(1971)

ampling and Analysis (1971)

eparation (1970) Rec. for Vocabulary of
anations on the Significance of the Principal Radiographic
Tenon Drive: Interchangeability Dimensions with Cutter

Tensile Creep of Plastics (1968)

Tensile Properties of Films (1970)

Tensile Properties (1966)

Tensile Properties (1972)

Tensile Steel Chain for Conveyors (1969)

Tensile Steel Chains (Round Link) for Chain Conveyors a

Tensile SteeI (1967)

Tensile Steels (1967)

Tensile Strength and Elongation ans for"Straight

Tensile Strength and Elongation of Conveyor BeIts: Spec

Tensile Strength (1971)

Tensile Strength (1972)

Tensile Stress Strain Properties of Vulcanized Rubbers

Tensile Test for Light Metal and Its Alloy Wires (1969)

Tensile Tesi (1971)

Rec. for Copper an

Tensile Test. of Copper and Its Alloy Tubes of Circular

Tensile Test. of Copper and Its Alloy Wire (1964)

Tensile Test. of Copper and Its Alloys (1964)

Tensile Test. of Light Metal Ad Its Alloy Tubes (1969)

Tensile Test. of Light Metals and Their Alloys (1961)

Tensile Test. of Steel Sheet and Strip Less Than $3 \mathrm{Mm}$ an

Tensile Test. of Steel Tubes (1964)

Tensile Test. of Steel Wire (1959)

Tensile Test. of Steel (1959)

Tensile Test. of Steel (1960)

Tensile Types (1965)

Tensile Types (1967)

Tensile Types (1967)

Tensile Types (1967)

Tension and Interfacial Tension (1963)

Tension of Rubber Latices (1970)

Tension (1963)

Terephthalate) in Dilute Solution (1970)

Terminal Ends for Crimping to Aircraft Aluminum Electri ISO

Terminal Equipment (1973)

Terminal Markings and Direction of Rotation (1972)

Terminals with Screw Clamping for External Conductors

Terminals (1963)

Terminals (1965)

Terminations of Capacitors and Resistors (1971)

est. Procedures for IEC

Terminations $(1960)$

Terminations (1967)

Terminations (1969)

Terminology and Dimensions (1969)

Terminology and Meth. of Test (1972)

Terminology of Cements (1967)

Terminology Relating to Freight Containers (1968)

Terminology (1966)

Terminology (1972)

Terminology (1972)

Racteristics of Semiconductor Devi

Terminology (1972)

Terminology (1972)

Std. ISO

IEC

CEE

IEC

IEC

Terminology (1972)

Termingy, Classification, Test Meth.) (1971)

Terminology; Basic Terms and Definitions (1966)

Terminology; Basic Terms and Definitions (1966)

Terms and Definitions for Printed Circuits (1965)

Terms and Definitions for the Reliability of Electronic

Terms and Definitions Relating to Drives Using V-Belts

Terms and Definitions (1966)

Terms and Definitions (1966)

Terms and Definitions (1969)

Terms and Definitions (1969)

Terms and Definitions (1969)

Terms and Definitions (1970)

Terms and Designation in Rel

Terms and Meth. of Test (1971)

Symbols-Part 1: Statistical Vocabulary (196

Terms and Symbols (1970)

Terms Appearing in ISO Rec. for Oils and Pigments (1963

Terms Concerning the Measurement of the Thickness (1971

Terms for Serrated Bars and Dimensions of the Cross Sec

Terms Relating to Microcopies and Their Bases (1962)

Terms Relating to Microcopy Apparatus (1964)

Terms Relating to Pallets (1965)

Terms Relating to Solid Mineral Fuels-Part III: Coke

Terms Relating to Solid Mineral Fuels-Part II: Coal S

Terms Relating to Solid Mineral Fuels-Part I: Coal P

Terms Used in ISO Rec. Concerning Welding (1971) ISO 10

Terms Used in the Benzole Industry-Part 1 (1971) 
Rec. for Definitions of Terms Used in the Benzole Industry-Part I (1971)

Terms Used in the Petroleum Industry (197I)

Rec. for Definitions of

usmian) (1969)

ussian) (Cs: Corresponding C/ ussian) (D: Corresponding Ge/ ussian) (NL: Corresponding D ussian) ( $\mathrm{Pl}$ : Corresponding $\mathrm{P} /$ Rec. for Paper Vocabulary-6Th Series of ometrical Definitions-Alphabetical Indexes of Equivalent Rec. for Paper Vocabulary: First Series of Rec, for Paper Vocabulary: Second Series of Rec. for Paper Vocabulary: Third Series of cation of Ferromagnetic Oxide Materials and Drfinitions of Rec. for International Unification of Concepts and Rec. for Plastics-Definitions of Rec. for Paper Vocabulary-5Th Series of Rec. for Hanging of Netting-Basic

Rec, for Sound System Equipment: Explanation of General reatment and Metallic Coatings-General Classification of Std. for Dental Vocabulary-List I-Basic Std. for Nuclear Energy Glossary: First Series of ustry (English, French, Russian) (Pl: Corresponding Polish dustry (English. French, Russian) (Cs: Corresponding Czech dustry (English, French, Russian) (D: Corresponding German dustry (English, French, Russian) (NL: Corresponding Dutch tional Rec. for Vocabulary of Legal Metrology (Fundamental assium Silicates for Industrial Use-Samples and Meth. of and/ Rec. for Commercial Refrigerated Cabinets-Meth. of Rec. for Essential Oils-Meth. of

d Types / Rec. for Fixed Resistors: Selection of Meth. of stors: Wirewound Resistors, Type 1-Selection of Meth. of th Pve (Polyvinyl Chloride) Insulation and Sheath: General m Hydrogen Carbonate for Industrial Use-List of Meth. of

Electrical Apparatus for Explosive Gas Atmospheres: Spark

Test. Procedures for Electronic Components and Equipment: Std. for Magnesium Alloy Sand Castings-Reference

d. for Aluminium (Aluminum) Alloy Sand Castings: Reference for Aluminium (Aluminum) Alloy Chill Castings-Reference Castings and Mechanical Properties of Sand Cast Reference lete, Filled Transport Packages-Part IV: Vertical Impact Rec. for Hardenability

Test. Procedures for Electronic Components and Equipment:

Test. Procedures for Electronic Components and Equipment: liquids and Meth. of Cleaning (1967) Rec. for Automobiles-Engin Rec. for Rec. for tation Type (1968)

tted by Rotating Electrical Machinery (1970) Rec, for

Rec. for General Requirements for the Preparation of Rec. for General

est. of the Accuracy (1970)

d Ileight with Horizontal or Vertical Spindle-/ ariable Height, with Horizontal or Vertical Spi/ zontal Grinding Wheel Spindle and Reciprocating/ ical Grinding Wheel Spindle and Reciprocating $\mathrm{T} /$ RecRec. for Aircraft Pressure Cabin Ground Rec for Basic Environmental Test Procedures:

Test. Procedures for Electronic Components and Equipment:

Test. Procedures for Electronic Components and Equipment: Test. Procedures for Electronic Components and Equipment: specimens / Rec. for Basic Environmental Test Procedures: ive Humidity (1968) Rec for Basic Environmental Test Procedures: Rec. for Basic Environmental Test. Procedures: Rec. for Cresylic Acid and Xylenols for Industrial Use

Rec. for Simple Torsion Rec. for Wrappin Rec. for Noncombustibility Rec. for Audibert Arnu Dilatometer Rec. fo

Rec. for Higher Alcohols for Industrial Use Rec. for Wrapping

$50 \mathrm{kgf})(1964)$

Rec. for Vickers Hardnes Rec. for Meth. of Mercurous Nitrat Rec. for Bend

Rec. for Brinell Hardness ed Meta/ Rec. for Filler Rods for Braze Welding: Meth. of steel, / Rec. for Filler Rods for Braze Welding: Meth. of metal Deposited by Filler Rods for Gas/ etration Type (1967)

$s$ at Power Frequencies (1967)

ating Materials at Elevated Temperatur/

Rec. for Meth. of

Rec, for Meth. of Rec. Meth. of ensions for $35 \mathrm{~mm}$ Film Magazines for Still Picture Use and
Terms Used in the Plastics Industry (English, French, R

Terms Used in the Plastics Industry (English, French, R

Terms Used in the Plastics Industry (English, French, R

Terms Used in the Plastics Industry (English, French, R

Terms Used in the Plastics Industry (English, French, R

Terms (Definitions of Optical Properties) (1971)

Terms (English, French, Russian) (1970)

Terms (1958)

Terms (1959)

Terms (1961)

Terms (1961)

Terms (1964)

Terms (1968)

Terms (1969)

Terms (1970)

Terms (1970)

Terms (1971)

Terms (1971)

Terms (1972)

Terms (1972)

Terms) (1963)

Terms) (1964)

Terms) (1964)

Terms) (1968)

Terms) (1969)

Test-General (1970)

lof Gears-Ge ISO ISO

Rec. for General Classifi IEC

Rec. for Surface T ISO

Uivalent Terms Used in the Plastics Ind $1 S O$ Quivalent Terms Used in the Plastics in ISO Quivalent Terms Used in the Plastics in ISO Quivalent Terms Used in the Plastics in ISO

Interna OIML

Rec. for Sodium and Pot ISO Test-Preparation of Sample Sample (1963) ISO

Test and General Requirements for Low Power Nonwirewoun IEC

Test and General Requirements (1971) Por Fixed Resi IEC

Test and Measuring Meth. (1972) /Y Cables and Wires Wi IEC

Test and Preparation of the Sample (1972) /. for Sodiu ISO

Test Apparatus for Intrinsically Safe Circuits (1972) IEC

Test A: Cold (1966)

Rec. for Basic Environmental IEC

Test Bar (1972)

Test Bar (1972)

Test Bar (1972)

St ISO

Std. ISO

Test Bars (1971)

Test by Dropping (1972) $\quad$ Std. for Packaging-Comp ISO

Test by End Quenching Steel (Jominy Test) (1967) ISO

Test B: Dry Heat (1966) Rec. for Basic Environmental IEC

Test Ca: Damp and Steady Heat (1969) /lc Environmental IEC

Test Cells for Measuring the Resistivity of Insulating IEC

Test Code-Net Power (1971)

Test Code for Agricultural Tractors (1968)

Test Code for Stationary Steam Generators of the Power

Test Code for the Measurement of the Airborne Noise Emi

Test Code (1961)

Test Codes for Measuring the Noise Emitted by Machines

Test Conditions for Electro Heating Appliances (1972)

Test Conditions for General Purpose Parallel Lathes-T

Test Conditions for Milling Machines with Table of Fixe

Test Conditions for Milling Machines with Table of of $\mathrm{V}$

Test Conditions for Surface Grinding Machines with Hori

Test Conditions for Surface Grinding Machines with Vert

Test Conditions (1962)

Machines with Vert ISO

Test Connection (1954)

Test Db: Damp Heat, Cyclic $(12+12$ Hr. Cycle) (1969)

Test D: Accelerated Damp Heat (1960) /Ic Environmental IEC

Test Ea: Shock (1967) Rec. for Basic Environmental IEC

Test Eb: Bump (1968) Rec. for Basic Environmental IEC

Test Ec: Drop and Topple, Primarily for Equipment Type

Test Ed: Free Fall (1969)

Test Enclosures of Noninjection Type for Constant Relat

Test Fc: Vibration (Sinusoidal) (1970)

Test for Absence of Hydrogen Sulphide (1971)

Test for Aluminum and Aluminium Alloy Wire (1969)

Test for Aluminum and Aluminium Alloy Wire (1969)

Test for Building Materials (1970)

Test for Coal (1963)

Test for Colour (Color) Fastness of Textiles (1959)

Test for Colour (Color) with Sulphuric Acid (1970)

Test for Copper and Its Alloy Wire (1964)

Test for Copper and lts Alloys (Test Loads from 2.5 to

Test for Copper and Its Alloys (1961)

Test for Copper and Its Alloys (1964)

Test for Copper and lts Alloys (1964)

Test for Determining the Characteristics of the Deposit

Test for Determining the Conventional Bond Strength on

Test for Determining the Mechanical Properties of Weld

Test for Determining Whether an Electrode Is a Deep Pen

Test for Electric Strength of Solid Insulating Material

Test for Electrical Resistance and Resistivity of Insul

Test for Film Pullout Force (1969)

IEC 
m or Aluminium Alloy Conductors for $\mathrm{Ai} /$ conductors for Aireraft (1967)
Rec. for Meth. of Test for General Purpose Electrical Cables with Aluminu

Rec. for Meth. of Test for General Purpose Electrical Cables with Copper Rec. for Brinell Ilardness Test for Grey Cast Iron (1961) th Copper Conductors for Aircraft (197/ Rec. for

nnotched Impact Rec. for Meth. of Std. for Formaldehyde Solutions for Industrial Use: Limit ectrical A pparatus for Explosive Gas Atmospheres: Meth. of

971) rec. for Phenol, $\mathrm{O}, \mathrm{M}$, and $\mathrm{P}-\mathrm{Cresol}$ for Industrial Use

Rec. for Phenol for Industrial Use .

Std. for Formaldehyde Solutions for Industrial Use: Limit

Std. for Formaldehyde Solutions for Industrial Use: Limit hickness Between $0.2 \mathrm{~mm}(0.008 \mathrm{In}$.)

Rec. for Simple Bend Rec. for Tensile loride (PVC) for Use Under Pressure (1967) Rec. for Oven Rec. for General Meth. of Rec. for Pozzolanicity

968)

of Electric Cables (1970)

(Hydrostatic Head Test) (1968) Rec. for Sealing
Rec. for Meth. of
Rec. for Meth. of Rubbers-Meth. of Rec. for Plastics-Bending Rec. for Aluminium and Aluminum Alloys-Shear for Carbon Black for Use in the Rubber Industry-Meth. of Rec. for Determination of Proof Stress and Proving Rec. for Modified Erichsen Cupping Rec. for Wrapping Rec. for Vickers Hardness Rec. for Izod Impact

Rec. for Bend
Rec. for Brinell Hardness

\section{d Rubbers (1968)}

in a Ventilated Tubular Ov/ of Solid Insulating Materials (1964) Insulating Materials (1958) Rec. for Meth. of Rec. for Plastics-Meth. of

Rec. for Meth. of Rec. Meth. of Rec. Meth. of Rec. for Meth. of

Rec. for Meth. of

measuring Machine (1970)

Test. Procedures for Electronic Components and Equipment:

Test. Procedures for Electronic Components and Equipment:

Test. Procedures for Electronic Components and Equipment:

Test. Procedures for Electronic Components and Equipment: Rec. for Vickers Hardness Test for Copper and Its Alleys dric Alcohols-Glycerine for Industrial Use-Samples and for Solderless Wrapped Connections-General Requirements istors (I972)

nsulating Materials to Breakdown by Surface Dischar/

Rec. for Rec. Rec. for Rec. for Is (1972)

for Safety Requirements for Radio Transmitting Equipment: Rec. for Metal Clad Base Materials for Printed Circuits: Rec. for d.c. Periodmeters: Characteristics and drical Rechargeable Single Cells: General Requirements and nufacture of Binders-Classification, Characteristics and for Instrumentation Applications: Physical Properties and rength of Mechanical Fastenings for Conveyor Belts (Static sotope All or Nothing Relays (Terminology, Classification

Test. Procedures for Electronic Components and Equipment:

Test. Procedures for Electronic Components and Equipment: copies: Legibility Test s; Description of the ISO Mire (Iso tests, Description and Use of the ISO Micromire (ISO Micro Apparatus for Explosive Gas Atmospheres: Construction and Rec for Accelerated Ageing and Simulated Service Apparatus for Explosive Gas Atmospheres: Construction and Rec. for Plastics-Compression e the Ru/ Rec. for Preparation of Test Piece and Meth. of Rec. for Flattenin Rec. for Drift Expanding Rec. for Drift Expanding Rec. for Flanging

Rec. for Drift Expanding Rec. for Bend Rec. for Flattening

Rec. for Ring Expanding Rec. for Preparation of Rec. for Preparation of

ed Rubber to Metal Where the Ru/ zed Rubber to Metal Where the Ru/ ngth of Vulcanized Natural and Synthetic Rubbers (Crescen strength of Small Test Pieces of Vulcanized Rubbers (Delft

lus in Shear of Vulcanized Rubbers (Bonded Quadruple Shear Rec. for Aluminium (Aluminum) Alloys-Sand Cast Std. for Leather-Conditioning of Rec. for Selection and Preparation of Samples and Rec. for Determination of Tear Strength of Smal
Test for Grey Cast Iron (1969)

Test for Heat Resisting (190 Deg.) Electrical Cables Wi

Test for Heat Resisting $\left(260^{\circ} \mathrm{C}\right)$ Electrical Cables

Test for Heavy Metals (Excluding Iron) (1972)

Test for Ignition Temperature (1966)

ydroxide Solut ISO

Test for Inorganic Chlorides (1972)

Test for Inorganic SuIphates (1972)

Test for Light Metal and Its Alloy Sheet and Strip of T

Test for Light Metal and Its Alloy Wires (1969)

Test for Moulded Fittings in Unplasticized Polyvinyl Ch Test for Pigments (1970)

Test for Pozzolanic Cements (1968)

Test for Pressurized Waveguide Tubing and Assemblies (1

Test for Pvc (Polyvinyl Chloride) Insulation and Sheath

Test for Resistance of Fabrics to Penetration by Waier

Test for Resistance to Liquids (1971)

Test for Rigid Cellular (1970)

Test for Rivet Wire and Rivets (1971)

Test for Sieve Residue (1970)

Test for Steel at Elevated Temperatures (1961)

Test for Steel Sheet and Strip (1960)

Test for Steel Wire (1960)

Test for Steel (Load 5 to $100 \mathrm{kgf}$ ) (1967)

Test for Steel (1959)

Test for Steel (1959)

Test for Steel (1968)

Test for Temperature Limit of Brittleness for Vulcanize

Test for the Determination of the Behaviour of Plastics

est for the Determination of the Insulation Resistance

Test for Volume and Surface Resistivities of Electrical

Test for Winding Wires: Enamelled Round (1968)

Test for Wool Fibre Length Using a Single Fiber Length

Teot for Wool Fibre (Fiber) Length Barbe (Barb) and Hau

Test Ga: Acceleration, Steady State (1968) /Ironmental IEC

Test H: Storage (1960)

Test J: Mould Growth (1968)

Test Ka: Salt Mist (1964)

/Ec. for Basic Environmental IEC

Test Meth.-General (1970) Rec. for Aliphatic Polyhy ISO

Test Meth. and Practical Guidance (1971)

Test Meth. for Batch Furnaces with Metallic Heating Res

Test Meth. for Determining the Relative Resistance of I

Test Meth. for Distillation (Yield and Range) (1969)

Test Meth. fur Induction Furnaces with Submerged Channe

Test Meth. (1967)

Test Meth. (1968)

Test Meth. (1969)

Test Meth. (1971)

Test Meth. (1972)

Test Meth. (1973)

Test Meth.) (1969)

Test Meth.) (197I

Test M: Low Air Pressure (1966)

Test N: Change of Temperature (1969)

/Ec. for Sealed Nickel Cadmium Cylin IEC

Rec. for Gynsum Rock for the Ma ISO

Std. for Unrecorded Magnetic Tapes ISO Rec. for St ISO

Test Object) and Its Use in Photographic Document Repro

Test Object) for Checking a Reading Apparatus (1968) ISO

Test of Electrical Apparatus, Type of Protection E (1 IEC

Test of Fabrics Coated with Rubber or Plastics (1970) ISO

Test of Flameproof Enclosures of Electrical Apparatus ( IEC

Test of Rigid Cellular Plastic (1968)

Test of the Adhesion of Vulcanized Rubber to Metal Wher

Test of the Adhesion of Vulcanized Rubber to Metal Wher

Test on Aluminum and Aluminium Alloy Tubes (1969)

Test on Copper and Its Alloy Tubes (1961)

Test on Light Metal and Its Alloy Tubes (1969)

Test on Steel Tubes (1960)

Test on Steel Tubes (1960)

Test on Steel Tubes (1960)

Test on Steel Tubes (1961)

Test on Steel Tubes (1964)

Test Piece and Meth of Test of the Adhesion of Vulcani

Test Piece and Meth. of Test of the Adhesion of Vulcani ISO

Test Piece) (1957) /Ec. for Determination of Tear Stre ISO

Test Piece) (1968)

Test Piece) (1971)

Test Pieces-Mechanical Properties (1971)

(S)

Test Pieces for Physical Tests (1972)

ISO

Test Pieces for Wrought Steel (1964)

ISO

Rec. for ISO 
ishes-Electric Strength Meth. (1971)

miconductor Detectors for Ionizing Radiation (1/

brush Materials for Electrical Mac

Rec. for

Electrical Insula/

g Radiation (1970)

Rec. for

cycle) (1969)

Rec. for Guide for the Preparation of

r Equipment Type Specimens /

Rec. for Basic Environmental

Rec. for Basic Environmental

Rec. for Basic Environmental

Rec. for Std. Atmospheric Conditions for

Resin Bonded, Low Pressure Laminated Plates or Panels for

Test. Procedures for Electronic Components and Equipment: ec. for Sodium Carbonate for Industrial Use: Expression of val (1973) Std. for Statistical Interpretation of td. for Potassium Hydroxide for Industrial Use: Sampling . $m$ Hydroxide for Industrial Use: Preparation and Storage of $m$ Hydroxide for Industrial Use: Preparation and Storage of Rec. for Meth. of the Conditioning of Paper and Board $m$ Carbonate for Industrial Use: Preparation and Storage of

ily Used for the Production Of; Preparation and Storage of lite (Natural and Artificial)-Preparation and Storage of Std. for

Std. for Woven Wire Cloth and Perforated Plates in Rec. for Plastics-Accelerated Conditioning of Rec. for Plastics-Compression Moulding Rec. for Plastics-Injection Moulding

eparation of $\mathrm{Te} /$ eparation of $\mathrm{Te} /$ Rec. for Plastics-Compression Moulding Rec. for Plastics-Compression Moulding Rec. for Plastics-Compression Moulding Rec. for Plastics-Compression Moulding Rec. for High Voltage

f Polyethylene Due to the M/ Rec. for Plastics-Meth. of $s$ and the Parent M/ Rec. for Filler Rods for Gas Welding: Test. Procedures for Electronic Components and Equipment: Test. Procedures for Electronic Components and Equipment: stimation of the Loss of Absorptive Power by Colorant Drop Rec. for Rockwell Hardness Rec. for Paints and Varnishes-Surface Drying Rec. for Paints and Varnishes-Bend Rec. for Light Metals and Their Alloys-Vickers Hardness Rec. for Rock well Superficial Hardness Rec. for Charpy Impact Rec. for Beam Impact ctrical Conductivity of Conveyor Belts: Spec. and Meth. of ength and Elongation of Conveyor Belts: Spec. and Meth. of $r$ Flame Resistance of Conveyor Belts: Spec./S and Meth. of tronic Tubes and Valves: General Requirements and Meth. of Rec. for Pulses Meth. of

Rec for Formic Acid for Industrial Use: Meth of Rec. for Acetic Acid and for Industrial Use-Meth. of

Rec. for Acetic Anhydride for Industrial Use-Meth. of Rec. for N-Butanol for Industrial Use-Meth. of

Rec. for Isopropyl Alcohol for Industrial Use-Meth. of Rec. for Acetone for Industrial Use-Meth. of

Procedures for Electronic Components and Equipment: Pt. 2 ion of Lower Yield and Proof Stresses (Siress) and Proving Rec. for Cocoa Beans-Cut Rec. for Seeds: Sampling and Meth. of Tube and Valve Shields: General Requirements and Meth. of $\mathrm{s}$ of Polymers and Copolymers-Freeze Thaw Cycle Stability

f Electrical Insulating Varnishes by the Helical Coil Bond

Rec. for Phthalate Esters for Industrial Use-Meth. of Rec. for Solvent Acetates for Industrial Use-Meth. of Rec. for Methanol for Industrial Use-Meth. of Rec. for Ethanol for Industrial Use-Meth. of

Rec. for Phthalic Anhydride for Industrial Use-Meth. of Rec. for Maleic Anhydride for Industrial Use-Meth. of

Rec. for Paraformaldehyde for Industrial Use-Meth. of Rec. for Paints and Varnishes-Scratch Rec. for Paints and Varnishes-Cupping rec. for Ortho Chlorotoluene for Industrial Use-Meth. of

Rec. for Para Chlorotoluene for Industrial Use-Meth. of Rec. for Chlorobenzene for Industrial Use-Meth. of ec. for Para Dichlorobenzene for Industrial Use-Meth. of Rec. for Rubber Hoses-Bending Rec for Chloroform for Industrial Use-Meth. of

c. for Ortho Dichlorobenzene for Industrial Use-Meth. of Test (1970)

Test (1962)

Test (1962)

Test (1963)

Test (1963)

Test (1967)

Test (1968)

Test (1968)

Test (1968)

Test (1968)

Test (1968)

Test (1968)

Test (1968)

Test (1968)

Test (1969)

Test (1969)

Test (1969)

Test (1969)

Test (1969)

Test $(1970)$

Test $(1970)$

Test (1970)

Test (1970)

Test $(1970)$

Test $(1970)$

Test (1970)

Test (1970)

Test (1970)

Test (1970)

Test $(1970)$

Test (1970)

Test (1970)

Test (1970)

Test $(1970)$
Test Pressures and Tolerances on Length (1970)

Rec. for Test Procedure for the Evaluation of the Thermal Endura

Rec. for Test Procedure for Thermal Endurance of Insulating Varn

Test Procedures for Amplifiers and Preamplifiers for $\mathrm{Se}$

Test Procedures for Determining Physical Properties of

Test Procedures for Evaluating the Thermal Endurance of

Test Procedures for Semiconductor Detectors for Ionizin

Test Procedures: Test Db: Damp Heat, Cyclic $(12+12 \mathrm{Hr}$.

Test Procedures: Test Ec: Drop and Topple, Primarily Fo IEC 68-2.31

Test Procedures: Test Ed: Free Fall (1969)

$68 \cdot 2 \cdot 31$

160

est Purposes (1970)

/ Glass Fibre (Fiber) Reinforced, ISO

Rec. for Basic Environmental IEC

Test Q: Sealing (1968)

Test Results: Estimation of the Mean-Confidence Inter ISO

Test Sample-Preparation of the Main Solution for Carr ISO

est Sample (1969)

Rec. for Sodiu ISO

Rec. for Potassiu ISO

Rec. for Sodiu ISO

Test Samples (1961)

Test Samples (1968)

Aluminium (Aluminum) Oxide Primar ISO
Rec. for Cryo ISO

est Samples (1968)

Rec. for Cryo ISO

Test Sieves and Sieving: Vocabulary (1972)

Test Sieves: Nominal Sizes of Apertures (1972)

Test Specimens of Polyamide 66, 610 and 6 (1969)

Test Specimens of Thermoplastic Materials (1963)

Test Specimens of Thermoplastic Materials (1963)

Test Specimens of Thermosetting Materials-Annex A: Pr

Test Specimens of Thermosetting Materials-Annex B: $\mathrm{Pr}$

Test Specimens of Thermosetting Materials-Annex C: $\mathrm{Pr}$

Test Specimens of Thermosetting Materials (1963)

Test Techniques (1962)

Test to Determine the Change in Electrical Properties $O$

Test to Determine the Compatibility of Steel Filler Rod

Rec. for Basic Environmental IEC
(1960) /Vironmental IEC

Test T: Soldering (1968)

/Its Alloys-E ISO

Test (B and C Scales) for Steel (1968

Test (Ballotini Meth.) (1970)

Test (Cylindrical Mandrel) (1970)

Test (Loads from 1 to $100 \mathrm{kgf}$ ) (1971)

Test (N and T Scales) for Steel (1969)

Test (U-Notch) for Steel (1959)

Test (V-Notch) for Steel (1960)

Rec. for Basic Environmental Test. IEC

/Teel at Elevated Temperatures: Determinat ISO 
for Porcelain Laboratory A pparatus-Quality and Meth. of Test (1970)

- (:hloride (Dirhloromethane) for lndustrial Use-Meth. of 'Test (1970) Rec. for Fixed Resistors: Terms and Meth. of Test (1971)

Rec. for Paints and Varnishes-l'endulum Damping Test (1971)

Rec. for light Metals and Their Alloys-Brinell llardness Test (1971)

Rec. for Rubber: Raw and Unvulcanized-Rapid Plasticity Rec. for Granulated Cork-Granule Size opper and Its Alloy Tubes of Circular Section-Flattening rial Use-Test for Impurities Insoluble in Water-Visual t Products (Thickness Less Than $2.5 \mathrm{~mm}(0.1 \mathrm{ln}$.)-Tensile e)-Electrical Conductivity-Characteristic and Meth. of s-Check of Continuity of Thin Coatings-Copper Sulphate impurities Insoluble in Sodium Hydroxide Solution-Visua rec. for Varnish Fabrics for Electrical Purposes: Meth. of Std. for Granulated Cork-Bulk Density

td. for Petroleum Products Corrosiveness to Copper-Strip

Std. for Trichloroethylene for Industrial Use-Meth. of Std. for Perchloroethylene for Industrial Use-Meth. of td. for Carbon Tetrachloride for Industrial Use-Meth. of Std. for Paints and Varnishes: Crosscut

Acid Starter Batteries: General Requirements and Meth. of Complete, Filled Transport Packages-Part III: Stacking - Complete, Filled Transport Packages-Part Vi: Vibration

for Use in Electronic Equipment: Terminology and Meth. of

for Heat Resisting Equipment Wires for Aircraft: Meth. of

f the Charpy Impact Resistance of Rigid Plastics (Flexural tion Resistance of the Carcass of Conveyor Belts (Meth. of rec. for Hardenability Test by End Quenching Steel (Jominy oughability of Conveyor Belts (Characteristic and Meth. of

tance of Fabrics to Penetration by Water (Hydrostatic Head stiffness of Vulcanized Rubbers at Low Temperature (Gehman

lcanized Rubbers to Rigid Plates in Shear (Quadruple-Shear Rec. for Conditioning Atmosphere: Std. for Leather: Physical Rec. for

It (1963) r Electrical Installations in Ships: Cables (Construction, Std for Flexible Cellular Materials: Hardness ields: Spec. Sheets for Tubes and Valves and Dimensions of Rec. for an Appraisal of the Problems of Accelerated Rec. for Load Calibration of

Rec. for Verification of Pendulum Impact Rec. for Verification of Brinell Hardness ion of Standardized Blocks to Be Used for Vickers Hardness Rec. for Verification of Vickers Hardness

Rec. for Verification of Rockwell B and C Scale Hardness ion of Standardized Blocks to Be Used for Brinell Hardness ized Blocks to Be Used for Rockwell B and C Hardness Scale erification of Rockwell Superficial $N$ and $T$ Scale Hardness

to Be Used for Rockwell Superficial N and T Scale Hardness able of Fixed Height with Horizontal or Vertical Spindle orizontal Grinding Wheel Spindle and Reciprocating Table Vertical Grinding Wheel Spindle and Reciprocating Table switching (1968) (1964)

Rec. for Guide to the Rec. for Tensile Rec, for Tensile Rec. for Tensile

uction and Before Entry into Serv/

Rec. for Rules for the Rec. for Tensile Rec for Tensile Rec. for General Principles for Fatigue Rec. for Rec. for Rec. for Hydrostatic Rec. for Std. Atmospheres for the Conditioning and Rec. for Time Lapse Between Vulcanization and Rec. for Spec. and

Rec. for Std. Conditions for Use Prior to and During the (1970) rature Interrupted) (1961) Rec. for Interrupted Creep Rec. for Noninterrupted Creep Rec. for Creep Stress Rupture of Lower Yield and Proof Stresses (S/ 59)

59)

Rec. for Mechanical Rec. for Simple Bend Rec. for Reverse Bend Rec. for Tensile Rec. for Tensile Rec. for Tensile

Std. for Simple Torsion Std. for Reverse Bend Rec. for Tensile Rec. for Load Calibration of Test. Machines for Tensile for Test Conditions for General Purpose Parallel Lathes of Variable Height, with Horizontal or Vertical Spindle

Fixed Lights by the Punc/

Rec. for Shipbuilding Details: t: General (I968)
Test (1971)

Test (1971)

Test (1971)

Test (1971)

Test (1971)

Test (1971)

Test (1971)

Test (1971)

Test (1972)

Test (I972)

Test (I972)

Test (1972)

Test (1972)

Test (1972)

Test (1972)

Test (1972)

Test (1972)

Test (1972)

Test (1972)

Test) (1961)

Test) (1966)

Test) (1967)

Test) (1968)

Test) (1968)

Test) (1971)

Test) (1971)

Test, Reference, and Definitions (1967)

Test, and Calihration of Ultrasonic Therapeutic Equipme IEC

Test. and Installations) (I965)

Test. and Rating Room Air Conditioners (1968)

Rec. Fo IEC

Test. by Indentation Techniques (1972)

Test. Devices and Gauges (1969) /for Tube and Valve Sh IEC

Test. for Atmospheric Corrosion (1971) IEC

Test. Machines for Tensile Test. of Steel (1960) ISO

Test. Machines for Test. Steels (1965) ISO

Test. Machines (1967)

Test. Machines (1967)

Test. Machines (1968)

Test. Machines (I968)

Test. Machines (I968)

Test. Machines (1968)

Test. Machines (1969)

Test. Machines (1970)

Test. of Accuracy (1971)

Test. of Accuracy (1971)

Test. of Accuracy (1971)

Rec. for Calibrat ISO

ISO

ISO

Rec, for Calibrat ISO

Fc. for Calibration of Standard ISO Rec. for V ISO

/Bration of Standardized Blocks ISO

I for Milling Machines with T ISO

/Ace Grinding Machines with $\mathrm{H}$ ISO

/Rface Grinding Machines with ISO

Test. of Circuit Breakers with Respcct to Out of Phase IEC

Test. of Copper and Its Alloy Tubes of Circular Section- ISO

Test. of Copper and Its Alloy Wire (1964)

Test. of Copper and Its Alloys (1964)

Test. of Electric Rolling Stock on Completion of Constr

Test. of Light Metal Ad Its Alloy Tubes (1969) ISO

Test, of Light Metals and Their Alloys (1961) ISO

Test. of Metals (1964)

Test. of Refrigerant Compressors (1968)

Test. of Refrigerating Systems (1968)

Test. of Rubber Hose (1970)

Test. of Rubber Test Pieces (1966)

Test. of Rubbers (1970)

Test. of Series I Freight Containers (1970)

Test. of Solid Electrical Insulating Materials (1971)

Test. of Speed Governing Systems for Hydraulic Turbines

Test. of Steel at Elevated Temperatures (Load and Tempe

Test. of Steel at Elevated Temperatures (1961)

Test. of Steel at Elevated Temperatures (1961)

Test. of Steel at Elevated Temperatures: Determination

Test. of Steel Sheet and Strip Less Than $3 \mathrm{~mm}$ Thick (19

Test. of Steel Sheet and Strip Less Than $3 \mathrm{~mm}$ Thick (19

Test of Steel Sheet and Strip Less Than $3 \mathrm{Mm}$ and Not Th

Test. of Steel Tubes (1964)

Test. of Steel Wire (1959)

Test. of Steel Wire (1972)

Test, of Steel Wire (1973)

Test. of Steel (1959)

Test. of Steel (1960)

Test. of the Accuracy (1970)

Test. of the Accuracy (1970)

Test. of Toughened Classes for Ships Si

Test. Procedures for Electronic Components and Equipmen IEC
1775

1869 
1. I't 2 l'mest (1968)

1: Trast A: Coldel (1960)

1: Tirst J: Mould Girowth (19)

1: Trest Ka: Salt Mist (1964)

1: Test M: low Air Pressure/

t: Test N: Change of Temper/

t: Test Q: Sealing (1968)

t: Test B: Dry lleat (1966)

t: Test T: Soldering (1968)

$\mathrm{t}$ : Test U: Robustness of Te/

t: Test Ea: Shock (1967)

t: Test Eb: Bump (1968)

t: Test Ca: Damp and Steady/

t: Test D: Accelerated Damp/

t: Test Ga: Acceleration, S/

t: Test H: Storage (1960)

is (1971)

Rec. for Basic Environmental ec. for Verification of Pendulum Impact Test. Machines for rengths of Plastic Mortar (Rilem Cembu/Rec. for Meth. of Rec. for Std. Atmospheres for Conditioning and trodes (1968) Rec. for Special Meth. of Mechanical
Rec. for Plastics -

Rec for Meth of Sampling Raw Cotton for Rec. for Plastics-Std. Atmospheres for Conditioning and Rec. for Meth. of Sampling Paper and Board for Rec. for Axial Load Fatigue Rec. for Meth. of Fiber Sampling for Rec. for Sampling and Conditioning of Ropes for Rec. for Rotating Bar Bending Fatigue
Rec. for Paints and Varnishes-Standard Panels for and Varnishes-Examination and Preparation of Samples for ete, Filled Transport Packages-Part II: Conditioning for

Transport Packages-Part 1: Identification of Parts When t Pieces (1971) Rec. for Concrete s (1972)

ctric Rotary Variable Capacitors: General Requirements for

rec. for Tables of Brinell Hardness Values (HB) for Use in

Rec. for International Code for the Field Acceptance Rec. for International Code for Model Acceptance

Rec. for International Code for the Field Acceptance Cable Sheaths (1966) Rec. for Fire Resistance Rec. for

for the Evaluation of the Results of Act Rec. for Shipbuilding Details les: for Alternating Voltages from $10 \mathrm{kV} \mathrm{Up} \mathrm{to} /$ les: Nondraining Types for Alternating Voltages/ with Nominal Voltages Greater Than 1,000 V (19/ erhead Lines with a Nominal Voltage Greater Tha/ installations (1967) Rec. for Meth. for Thermal Shock ccessuries: Paper Insulated, Metal Sheathed, Fo/ ccessories: Internal, for Alternating Voltages / ccessories: External, for Alternating Voltages / Rec. for Plastics-Preparation of Specimens for Optical machines: Meth. for Determining Losses and Efficiency from ete, Filled Transport Packages-Part V: Horizontal Impact Rec. for Steam Turbines: Rules for Acceptance

or ISO Conventional Typographical Character for Legibility Meth for Determining Synchronous Machine Quantities from sic Environmental Test. Procedures: Guidance for Damp Heat Rec. for Stability of Counterbalanced Lift Trucks-Basic or Expression and Presentation of Results of Coal Cleaning vulcanized Rubbers-Accelerated Ageing or Heat Resistance mental Test. Procedures: Guidance on Change of Temperature or Coverings-Sampling and Cutting Specimens for Physical td. for Leather-Conditioning of Test Pieces for Physical

Std. for Flexible Cellular Materials-Accelerated Ageing age Alternating Current Circuit Breakers: Type and Routine

rt V: Horizontal Impact Tests (Inclined Plane and Pendulum Std. for Gas Turbines-Acceptance

of the Izod Impact Resistance of Rigid Plastics (Flexural ro Test Object) for Ch/ Rec. for Microcopies: Legibility d lts Use in Photograph/ Rec. for Microcopies: Legibility e: Determinat/Std. for Boric Acid, Boric Oxide, Disodium th. (1972)

Std. for Boric Acid and Oxide, and Disodium anese Conten/ Std. for Boric Acid and Oxide, and Disodium um and Boric Oxide Content and Loss On/ Std. for Disodium Std. for Carbon

chromate Pigments-Basic Zinc Potassium Chromate and Zinc hate for Industrial Use: Determination of Content-Sodium Determination of Content-Gravimetric Meth. as ['otassium
Test. Procedures for Electronic Components and Equipmen Test. Procedures for Electronic Components and Equipmen Test. Procedures for Electronic Components and Equipmen Test. Procedures for Electronic Components and Equipmen Test. Procedures for Electronic Components and Equipmen Test. Procedures for Electronic Components and Equipmen Test. Procedures for Electronic Components and Equipmen Test. Procedures for Electronic Components and Equipmen Test. Procedures for Electronic Components and Equipmen Test. Procedures for Electronic Components and Equipmen Test. Procedures for Electronic Components and Equipmen Test. Procedures for Electronic Components and Equipmen Test. Procedures for Electronic Components and Equipmen Test. Procedures for Electronic Components and Equipmen Test. Procedures for Electronic Components and Equipmen Test. Procedures for Electronic Components and Equipmen Test. Procedures: Guidance for Damp Heat Tests (1968)

Test. Procedures: Guidance on Change of Temperature Tes Test. Procedures: Test Fc: Vibration (Sinusoidal) (1970 Test. Reference Atmosphere Spec. (1967)

Test. Steels (1965)

Test. Strength of Cements-Compressive and Flexural St Test. Textiles (1967)

Test. to Determine the Coding for Deep Penetration Elec Test. with the Torsion Pendulum (1967)

Test. (1961)

Test. (1963)

Test. (1968)

Test. (1969)

Test. (1969)

Test. (1969)

Test. (1969)

Test. (1970)

Test. (1970)

Test. (1972)

Test. (1972)

Std for Pace. For Pains ISO Std. for Packaging-Complete, Filled ISO

Tests and Measurices and Applicability of Tes ISO

Rec. for Air Diele IEC

Tests Made on Flat Surfaces (1964)

Tests of Hydraulic Turbines (1963)

Tests of Hydraulic Turbines (1965)

Tests of Storage Pumps (1968)

Tests of Structures (1968)

Tests on Anticorrosion Protective Coverings of Metallic

Tests on Cables and Their Accessories (1966)

Tests on Coatings Other Than Those Anodic to the Basis

Tests on Galvanized Steel Wire for Ropes (1963)

Tests on Impregnated Paper Insulated Metal Sheathed Cab

Tests on Impregnated Paper Insulated Metal Sheathed Cab

Tests on Indoor and Outdoor Post Insulators for Systems

Tests on Insulators of Ceramic Material or Glass for Ov

Tests on Laboratory Glassware (1968)

Tests on Large Hollow Porcelains for Use in Electrical

Tests on Oil Filled and Gas Pressure Cables and Their a

Tests on Oil Filled and Gas Pressure Cables and Their a

Tests on Oil Filled and Gas Pressure Cables and Their a

Tests on Plastics Materials: Casting Meth. (1968)

Tests on Plastics Materials: Moulding Meth. (1968)

Tests (Excluding Machines for Traction Vehicles) (1972)

Tests (Inclined Plane and Pendulum Tests (1972)

Tests (1962)

Tests (1965)

Tests (1967)

Tests (1968)

Tests (1969)

Tests (1969)

Tests $(1971)$

Tests (1971)

Tests (1971)

Tests (1972)

Tests (1972)

Tests (1972)

Tests (1972)

Tests (1973)

Tests) (1961)

Tests, Description and Use of the ISO Micromire (ISO Mi

Rec for Rotating Electrical Mar

Rec. F ISO Rec. for Ba IEC

Rec. F ISO

Rec. for ISO

Rec for Basic Environ IEC

Rec. for Machine Made Textile Flo ISO

ISO

Rec, for High Volt IEC

/Complete, Filled Transport Packages-Pa ISO

Rec for Plastics-Determination ISO

Tests: Description of the ISO Mire (ISO Test Object) an

Tetraborates and Crude Sodium Borates for Industrial Us

Tetraborates for Dibenzyldithiocarbamate Photometric Me

Tetraborates for Industrial Use-Determination of Mang

Tetraborates for Industrial Use-Determination of Sodi

Tetrachloride for Industrial Use-Meth. of Test (1972)

Tetrahydroxychromate (1970)

Tetraphenylborate Volumetric Meth. (1973) Kec. for Zinc ISO

Tetraphenylborate (1971) /Hloride for Industrial Use- ISO
$68-2$

68-2.1

68-2-10

68.2 .11

68-2-13

68-2-14

68-2-17

68-2-2

68-2-20

68-2-21

68-2-27

68-2-29

68-2-3

68-2-4

68-2-7

68-2-8

68-2-28

68-2-33

68-2-6

554

442

679

139

876

537

220

291

186

1099

1130

1142

1143

1514

1513

2233

2206

1920

334. 1

2100

410

41

193

198

834

229

230

1462

345

55-1

55-2

168

383

718

233

141-1

141-2

141-3

870

869

34-2

2244

46-2

(35

68-2-28

1074

923

68-2-33

1957

2419

2440

56-4

2244

2314

180

689

446
1918
2215

2214

1916

2312

1249

2486

2051 
1)etermination of Content-(;ravimetric Meth. as Potassium Film, llate/ Ker. Meth. for letermining Thiosulphate and er. for Netting Yarns for Fishing Nets: Designation in the atiles-Universal System for Designating I inear Density rasing of a llorizontally Folded Specimen by Mea/ Std. for mination of the Adhesion Strength of Vulcanized Rubbers to A rea $(1970)$

ss Under Dynamic Loading (1971)

971)

s for Physical Tests (1971)

(1972) Rec. for Machine Made Rec. for Rec, for Machine Made Rec. for Machine Made Std. for

hangcs in Varying Moisture Conditi/

Std. for Machine Made

f Staple Fibres (Fibers) or Continuous Filament/

its Continuous Filament and Staple Fibre (Fiber/

ustible Matter of Its Products (1971)

nuous Filament and Staple Fibre (Fiber) Yarns

gnments Of, Continuous Filament Yarn, Staple Fi c. for Plastics-Determination of the Loss on lgnition of ple Fibres (Fibers) or Continuous Filaments Constituting A

(1970)

assification and Nomenclature (1970)

ons of the Cross Section of Assembled Bars for / (1970)

or Woollen (Woolen) Spinning-Dimensions (1971/

ing Machines-Maximum Usable Width (1971)

or Tape Yarns (1972)

for Ring Spinning and Doubling Frames for C

ers-Definition of Side (Left or Right) (1960)

$r$ Ring Spinning and Doubling Frames for C And/

ring Spinning and Doubling Frames (1972)

ers for Man Made Fibres (1971)

Machines-Definition of Side (Left or Right) /

Cards-Working Width (1971)

Chemical Analysis (1971) Rec. for Designation of the Direction of Twist in cronaire Value (1972)

Rec. for

Rec. for

Rec. for

Rec. for

Sid. for

Rec. for

Rec. for

Rec. for

Rec. for

Rec. for

Rec. for

Std. for

Std. for

Rec. for

Std. for

Std. for

Rec. for

Rec. for

Twist in

Std. for

Rec. for

Rec. for

Rec. for

(1971)

ting Meth. (1972)

density (Mass Per Unit Length) Skein Meth. (197/

of Breaking Load and Elongation of Same (Or Sin/

Rec. for Technical Drawings-Meth. of Indicating Surface

- Aqueous Dispersions of Polymers and Copolymers-Freeze Rec. for Test. and Calibration of Ultrasonic 972) Std. for Cork-Expanded Pure Agglomerated for the Preparation of Test Procedures for Evaluating the the Ilelical Coil Bond Test (/ Rec. for Evaluation of the the El/ Rec. for Test Procedure for the Evaluation of the
rength Meth. (1971) Rec. for Test Procedure for Rec. for Glass Hydrometers-Conventional Value for the Rec, for

Rec. for Meth. for

of Electrical Machinery and Apparatus in Relation to Their olymers and The/ Rec. for Plastics-Determination of the lymers and Thei/ Rec. for Plastics-Determination of the lecommunication and in Electronic Applications / Rec. for Rec. for Long Solid Stem Rec. for Short Solid Stem Rec. for Long Enclosed Scale Rec. for Short Enclosed Scale International Rec. for Clinical

1970) Construction and Adjustment of Liquid in Glass Lahoratory Rec. for Solid Stem Calorimeter Rec. for Enclosed Scale Calorimeter Rec. for Solid Stem General Purpose Rec. for Enclosed Scale GeneraI Purpose Std. for Polyethylene rec. for Plastics-Compression Moulding Test Specimens of Rec. for Plastics-Injection MouIding Test Specimens of tics-Determination of the Vicat Softening Temperature of rec. for Plastics-Determination of the Melt Flow Rate of rec. for Plastics-Compression Moulding Test Specimens of rec. for Plastics-Compression Moulding Test Specimens of rec. for Plastics-Compression Moulding Test Specimens of rec. for Plastics-Compression Moulding Test Specimens of on of Incandescence Resistance of Rigid Self Extinguishing - Basis for Spec. for Industrial Laminated Sheets Based on $r$ Telecommunication and in Electronic Applicati/ Rec. for Simple Bend Test. of Steel Sheet and Strip Less Than $3 \mathrm{Mm}$ reverse Bend Test. of Steel Sheet and Strip Less Than $3 \mathrm{Mm}$ of Steel Sheet and Strip Less Than $3 \mathrm{Mm}$ and Not Than $0.5 \mathrm{Mm}$ on Welded Butt Joints for Steel Plates Up to $50 \mathrm{~mm}$ (2 In.)
Tetraphenylborate (1973)

/Sulphate for Industrial Use: ISO

Tetrathionate in Processed Black and White Photographic ISO

Tex System (1968)

(1969)

Textile Fabrics (1969)

Rec for T ISO Rec. for Deter ISO

extile Floor Coverings-Determination of Mass Per Uni ISO

Textile Floor Coverings-Determination of Thickness Lo

ISO

ISO

ISO

150

ISO

ISO

ISO

ISO

ISO

ISO

Textile Glass-Determination of the Twist in lts Conti

Re ISO

Textile GIass Reinforced Plastics (1970)

Textile Glass Yarn-Cross Section Meth. (1971)

Textile Glass Yarns-Designation (1972)

Textile Machinery-Cylindrical Tubes for Draw Twisters

F Sta ISO

ISO

ISO

ISO

ISO

1SO

ISO

ISO

ISO

ISO

ISO

ISO

ISO

ISO

ISO

ISO

ISO

ISO

ISO

ISO

ISO

ISO

ISO

ISO

ISO

ISO

ISO

ISO

Rec. for Plastics ISO

Texture on Drawings (1971)

Thaw Cycle Stability Test (1969

Thermal Cork-Characteristics, Sampling and Packing (

Thermal Endurance of Electrical Insulating MateriaIs (1

Thermal Endurance of Electrical Insulating Varnishes by

Thermal Endurance of Enamelled Wire by the Lowering of

Thermal Endurance of Insulating Varnishes-Electric St

Thermal Expansion Coefficient (For Use in the Preparati

Thermal Insulating Asbestos Boards (1971)

ISO

IEC

IEC

IEC

ISO

ISO

ISO

Thermal Stability in Service (1957) Glassware

Thermal Stability of Polyvinyl Chloride and Related Cop ISO

Thermal Stability of Polyvinylchloride and Related Copo ISO

Thermal Time Delay Switches for Use in Equipment for Te IEC

Thermometers for Precision Use (1968)

Thermometers for Precision Use (1968)

Thermometers for Precision Use (1968)

Thermometers for Precision Use (1968)

Thermometers (1964)

Thermometers (1968)

Thermometers (1968)

Thermometers (1970)

Thermometers (1970)

Thermoplastic Materials-Designation (1972)

Thermoplastic Materials (1963)

Thermoplastic Materials (1963)

Thermoplastics (1968)

Thermoplastics (1969)

Thermosetting Materials-Annex A: Preparation of Test

Thermosetting Materials-Annex B: Preparation of Test

Thermosetting Materials-Annex C: Preparation of Test

Thermosetting Materials (1963)

Thermosetting of (1961)

Thermosetting Resins (1971)

IC. for Plastics-Determinati ISO Rec. for Plastics ISO

Thermostatic Switches Primarily for Use in Equipment Fo

Thick (1959)

Thick (1959)

Thick (1959)

Thick (1969)

2485

417
858

1144

2313

1764

2094

1765

1957

2424

2551

1888

1889

1887

1890

1886

1888

2078

1472

1506

1865

1809

2012

96

141

95

2105

142

342

1833

2403

1139

1144

1973

$105 / 1.6$

139

2061

2060

2062

1302

1147

221

216

172

370

1768

1896

718

305

182

65

656

386

386

651

652
1770

1771

1872

293

294

306

1133

295/A 
of Furiun Welded Butt Joints for Steel Plates 50 to $200 \mathrm{Mm}$ $u \mathrm{~m}$ ) and les Alloys and Magnesium and Its Alloys 5 to $50 \mathrm{Mm}$ Std. for Metallic Coatings-Measurement of tings on Nonmagnetic Basis Metals-Measurement of Coating gnetic and Nonmagnetic Substrates-Measurement of Coating coatings on Magnetic Basis Metals-Measurement of Coating ards: Determination of Water Absorption and of Swelling in Rec. for Meth. for the Determination of the Bulking bend Test for Light Metal and Its Alloy Sheet and Strip of Rec. for Copper and Alloy Rolled Flat Products Rec. for Textile Floor Coverings-Determination of Rec. for Tolerances on the Tota overs (1967) ) and Its Alloys-Measurement of $n$ ) of Aluminium (Aluminum) and Its Alloys-Measurement of
Rec. for Carpets-Determination of
Rec. for Determination of the Rec. for Determination of the elts: Spec. and Meth. of Test (1962) Rec. for Full welded Butt Joints in Steel Pipes Up to $50 \mathrm{~mm}$ (2 In.) Wall gs-Definition of Terms Concerning the Measurement of the Std. for Leather: Physical Test.-Measurement of

ons (197/ Rec. for Measurement of Metal and Oxide Coating rances on the Total Thickness of Conveyor Belts and on the gon Bolts and Nuts: Widths Across Flats, Heights of Heads, Rec. for ISO Metric Series for Basic

ansport of Fluids-Polyethylene (PE)-Tolerances on Wall icized Polyvinyl Chloride (PVC) Pipes-Tolerances on Wall Rec. for Steel Tubes $-W$ al Std. for Rope

uminium (Aluminum) and Its Alloys-Check of Continuity of of the Water Vapour (Vapor) Transmission Rate of Films and Std. for Muscovite Mica Blocks, Rec. for Muscovite Mica Blocks, Rec. for Phlogopite Mica Blocks, hite Photographic Film, Plate/ Rec. Meth. for Determining Std. Spec. for Photographic Grade Sodium sounds and Vibrations (/ Rec. for Octave, Half-Octave and IS (1970) ys-Determination of -Containing Zirconium, Rare Earths,

rance Holes for Metric Bolts 1,6 Up to and Including $39 \mathrm{Mm}$ ance Holes for Metric Bolıs, $42 \mathrm{Up}$ to and Including $150 \mathrm{Mm}$ tle Nuts with Metric Thread, $42 \mathrm{Up}$ to and Including $100 \mathrm{Mm}$ ng (1972)

Rec. for Sheets for Barre

on of Closures (19/ Rec. for Finishes with External Screw Rec. for Nominal Lengths for Bolts, Screws and Studs: uts with Specified Proof Load Values, Effective Heights of Rec. for Slotted and Castle Nuts with Metric 1969) Rec. for Slotted and Castle Nuts with Metric requencies Below $3 \mathrm{MHz}$ : Circular Multipole Connectors with I Steel Equipment for Percussive Long Hole Drilling-Rope pment for Percussive Long Hole Drilling-Reverse Buttres pment for Percussive Long Hole Drilling-Reverse Buttres I Steel Equipment for Percussive Long Hole Drilling-Rope imensions of Cylindrical Machined Graphite Electrodes with Rec. for Steel Tubes for Gas Lis Rec for ISO General Purpose Screw Rec. for ISO General Purpose Metric Screw Std. for ISO General Purpose Metric Screw Std. for ISO General Purpose Metric Screw 73) ressure Tight Joints Are Made on the Threa/ Rec. for Pipe
(1970) ) (1970) Rec. for Coupling
and Taps for Pipe ions and Marking (1972)

Threads (1/8 In. to 6 In./S) (1961) Std. for Hand Taps for Pipe Rec for ISO Miniature Screw Std. for Drills for Use Prior to Tapping Screw rewed Fittings Where Pressure Tight Joints Are Made on the pe Threads Where Pressure Tight Joints Are Not Made on the Rec. for Pipe Couplings for Hydraulic Piping (Pipe r Coupling Threads for It ydraulic or Pneumatic Piping (Pipe Rec. for ISO General Purpose Metric Screw Rec. for ISO In. Screw nd Nuts (Diameter Range 0.06 to $6 / \quad$ Std. for ISO In. Screw
s: Tolerances, Limits of Sizes for Commercial Bolt and Nut s (1969) Rec. for ISO General Purpose Metric Screw $t$ and Nut Thre/ Rec. for ISO General Purpose Metric Screw Rec. for ISO General Purpose Metric Screw

r Normal Equal Loudness Contours for Pure Tones and Normal

n (197I)

s (1961)

2-Tolerance Classes 6,5

1966) Rec. for Rolling Bearings Rec. for Rolling Bearings Rec, for Rolling Bearings

Rec. for Rolling Bearings-Cylindrical Roller-Separate Roller Bearings: Groups-(I) Radial Ball and Roller (II) es of Electronic Tubes and Valves: Meth. of Measurement of

ads for Gas List Tubes and Screwed Fittings Where Pressure in./S) (1961)
Thick (1972)

Thick (1972)

Welded Butt Joints for Aluminium (Alumin ISO

metric Meth. by Anodic Dissolution (19 ISO

(1972)

Thickness-Magnetic Meth. (1972)

Thickness-Magnetic Meth. (1972)

Thickness After Immersion in Water (1972)

Thickness and Bulk of Paper (1965)

Thickness Between $0.2 \mathrm{~mm}(0.008 \mathrm{In}$.) and $7 \mathrm{~mm}(0.25 \mathrm{In}$.

Thickness Less Than $2.5 \mathrm{~mm}$ (0.1 In.)-Tensile
Thickness Loss Under Dynamic Loading (1971)

Thickness of Conveyor Belts and on the Thicknesses of $C$

Thickness of Oxide Coatings-Nondestructive Measuremen

Thickness of Pile Above the Backing (1970)

Thickness of Single Sheets of Paper (1967)

Thickness Tensile Strength and Elongation of Conveyor B

Nspection of Circumferential Fusion

Thickness (I969)

Thickness (1971)

/Metallic and Other Nonorganic Coatin IS

Thickness (1971)

Thicknesses by Microscopical Examination of Cross Secti ISO

Thicknesses of Covers (1967) Rec. for Tole ISO

Thicknesses of Nuts: Metric Series (1968)

Thicknesses of Sheet and Diameters of Wire (1964)

Rec. for Tole ISO
C. for Hexa ISO

Thicknesses Up to $6 \mathrm{~mm}(1970)$

Thicknesses Up to $6 \mathrm{~mm}(1970)$

Astics Pipes for the Tr ISO

Thicknesses (1971)

Thimbles (1972)

Ort of Fluids-Unplast ISO

ISO

Thin Coatings-Copper Sulphate Test (1971)

Thin Sheets-Dish Meth. (1970)

Thins and Films-Visual Classification (1972)

Thins and Films Meth. for Grading by Size (1958)

IIon of Al ISO

Thins and Splittings: Meth. for Grading by Size (1965)

Thiosulphate and Tetrathionate in Processed Black and $\mathrm{W}$

Thiosulphate, Crystalline (1972)

Third-Octave Band Filters Intended for the Analysis of

Thorium and Silver-Periodate Photometric Meth. (1972)

Thread-Dimensions in Millimetres (Millimeters) and in

Thread Diameter (1962)

Thread Diameter (1968)

Rec for Cle

Thread Diameter (1969)

Rec. for Clear ISO

with Shade Holder Ri IEC

Thread for Glass Containers and Gauges for the Inspecti

Thread Lengths for General Purpose Bolts (1968)

Thread 0.4 D to Less Than 0.6 D (1972)

Thread (1963)

Thread, 42 Up to and Including $100 \mathrm{~mm}$ Thread Diameter (
Threaded Coupling (1966)

Threaded Equipments $1 \mathrm{l} / 2$ to $2 \mathrm{In}$. (38 to $51 \mathrm{Mm})(1970)$

Threaded Equipments 1 1/2 to 2 l/2 In. (38 to $64 \mathrm{Mm}$ ) (1

Threaded Equipments $11 / 6$ and $1 \mathrm{l} / 4$ In. (27 and $32 \mathrm{Mm}$ )

Threaded Equipments $7 / 8$ to 1 1/4. In. (22 to $32 \mathrm{Mm}$ ) (197

Threaded Sockets and Connecting Pins for Use in Electri

Threading (1971)

Threads-Basic Profile (1969)

Threads-Gauging (1970)

Threads-General Plan (1973)

Threads-Selected Sizes for Screws, Bolts and Nuts (19

Threads for Gas List Tubes and Screwed Fittings Where P

Threads for Hydraulic or Pneumatic Piping (Pipe Threads

Threads for Parallel and Taper-Threads-General Dimens

Threads Where Pressure Tight Joints A re Not Made on the

ISO

Threads (1970)

Threads (1972)

Threads (1/8 In. to 6 In./S) (1954)

Threads (1/8 In. to 6 In./S) (1961)

Threads) (1970)

Threads) (1970)

Threads: Basic Dimensions (1968)

Threads: Basic Dimensions (1968)

Threads: General Plan and Selectior

Threads: Medium Quality (1969)

(or Constructional Type

Threads: Tolerances, Principles and Basic Data (1969)

/List Tubes and Sc ISO

Rec. for $\mathrm{Pi}$ ISO

ISO

Rec. Fo ISO

ISO

ISO

ISO

Threshold of Hearing Under Free Field Listening Conditi

Throwaway Carbide Indexable Inserts: Dimensions (1969)

(Throwaway) Inserts-Designation-Code of Symbolizatio

Thrust Ball Bearings with Flat Seats-Normal Tolerance

Thrust Ball Bearings with Flat Seats-Tolerances: Part

Thrust Bearings with Flat Seats-Boundary Dimensions

Thrust Collars-Boundary Dimensions (1962)

Thrust (III) Tapered Type-Metric Series (1963)

Thyratron Pulse Modulators (1969)

Electrice

/ for ISO

Tight Joints Are Not Made on the Threads (1/8 In. 106
2405

2437

2177

2360

2361

2178

769

438

954

1555

2094

583

2128

1766

534

283

947

1765

2064

2589

1463

583

272

388

1165

2262

2085

1195

2185

67

444

417

419

225

2353

273/I

283/II

399

399
1115

888

$898 /$ IV

288/I

288/II

130-4

1720

1722

I721

1719

239

65

68

1502

261

262

1943

2284.

228

1501

2306

7

1944

1943

724

725

965/II

965/III

$965 /$ II

$965 / I$

226

883

1832

199/I

$199 / I I$

104

246

$300 / \mathrm{I}$
151.20

7

228 
Rec. for Coniferous Sawn (Sawed) Rec. for Coniferous Sawn (Sawed) Rec. for Coniferous Sawn (Sawed) Rec. for Coniferous Sawn (Sawed) Rec. for Coniferous Sawn Rec. for Coniferous Sawn

Rec. for Electrical Relays: Specified

lectronic Tubes and Valves: Measurement of Cathode Heating Std. for Paints and Varnishes: Determination of Flow ication and in Electronic Applications / Rec. for Thermal 1970)

Rec. for Exposure

ntities and Units of the SI (Metric Unit) and of Space and es: Measurement of Cathode Heating Time and Heater Warm Up tity Measuring, with Nonspecified or Independent Specified Rec. for Classification of Rec. for Classification of Tin and Special Std. for Electroplated Coatings of Zinc and Its Alloys-Spectrophotometric Determination of Rec. for Electroplated Coatings of

c. for Guide to the Calculation of Resistance of Plain and

969)

$s$ for Box Loaders for Automatic Looms-Dimensions of Pirn Rec. for Carbide Rec. for Turning Tools with Carbide Rec. for Turning Tools with Carbide Rec. for Turning Toois with Carbide Rec. for Aircraft Tyre

Cast Wheel Centres (Centers) in Nonalloy Steel for Tyred rial-Rolled on Forged Wheel Centres (Centers) for Tyred Rec. for Railway Rolling Stock Material-Tyres 6/ Rec. for Railway Rolling Stock Material-Rough Tyres production of Aluminum: Determination of Titanium Content of Chemical Analysis of Manganese Ores: Determination of rily Used for the Production of Aluminum: Determination of

alysis of Aluminum and Aluminium Alloys-Determination of

Rec. for Abbreviations of Generic Names in documentation-International Code for the Abbreviation of

Rec. for Fruit and Vegetable Products: Determination of - Determination of Anionic-Active Matter (Direct Two Phase sulphur (Meth. After Combustion in a Current of Oxygen and ats: Determination of Solidification Point of Fatty Acids tes for Industrial Use-Determination of Silica Content use-Determination of Sodium Hydrogen Carbonate Content

e for Industrial Use-Determination of Sodium Carbonate similar Electrical Appliances: Particular Requirements for Rec. for Directions of Operation of r Switches: Requirements for Type 2, Quick Make and Break Rec. for Aircraf

-Double Row Cylindrical Type RD with Tapered Bore 1:12 hields or Seals-Outside Diameter Tolerances-Normal and Std. for Rolling Bearings: Tapered Roller-Inch Series hrust Ball Bearings with Flat Seats-Tolerances: Part 2 shipbuilding Details: Ship Screw Propellers, Manufacturing for Cylindrical Abrasive Sleeves: Designation, Dimension
and Vice Versa (1964) td. for Rolling Bearings: Tapered Roller-Metric Series Rec. for Rolling Bearings

gs-Radial Types with Shields or Seals-Outside Diameter Rec. for Concrete Tests-Dimensions,

9) Rec. for Stainless Steel Tubes-Dimensions, Rec. for Boiler Tubes-Dimensions

Rec. for ISO System of Limits and Fits-Part I: General Ring Spinning and Ring Doubling Spindles: In. Dimensions, ing Spinning and Ring Doubling Spindles Metric Dimensions, Id Drawn Precision Steel Tubes: Metric Series, Dimensions, Rec. for Coniferous Sawn Timber: Sizes Rec. for Rolling Bearings: Tapered-Metric Serie

g, Supplied in Straight Lengths (19/ ller Metals for $\mathrm{G}$ as Welding (1967/ d, for Welding (1967)

and Low Alloy High Tensile Steel (1/ ning and Tolerancing of $\mathrm{Pr} /$ Examples of lndications $\mathrm{O}$ es, Symbols, Indications on Drawings (1969) Rechnical Drawings Rec. for Lengths and Rec. for Diameters and Rec. for Lengths and Rec. for Specia Rec. for Lengths and Rec. for Marking Codes for Values and Rec. for Rubber Hose-Bore Sizes, Test Pressures and ic Pipes for the Transport of Fluids-Polyethylene (PE) of Fluids-Unplasticized Polyvinyl Chloride (PVC) Pipes or General Use) (1968)

on the Thicknesses of Covers (1967)

Rec. for Rec. for Rec. for Rec for Rec. for

Timber-Defects-Classification (1969)

Timber-Defecis-Measurement (1969)

Timber-Defects-Terms and Definitions (1969)

Timber-Sizes-Terms and Definitions (1969)

Timber: Sizes-Meth. of Measurement (1968)

Timber: Sizes-Tolerances and Shrinkage (1968)

Time All or Nothing (1969)

Time and Heater Warm Up Time (1966)

Time by Use of a Flow Cup (1972)

Time Delay Switches for Use in Equipment for Telecommun

Time Lapse Between Vulcanization and Test. of Rubbers

Time Markings for Shutters Used in Still Cameras (1966)

Time (1965) Rec. for Basic Qua ISO

Time (1966)

Times (1971)

/L Properties of Electronic Tubes and Valv IEC /Cal Relays: Single Input Energizing Quan IEC

Tin and Special Tin Bronzes (1965)

Tin Bronzes (1965)

Tin Nickel Alloy (1972)

Tin (1970)

ISO

Rec. for Chemical Analysis of ISO

Tinned Copper Conductors of Low Frequency Cables and Wi IEC

Tinplate and Cold Reduced Blackplate-Part 1: Sheet (1 ISO

Tip (1969)

Tips for Turning Tools: Metric Series (1961)

Tips: Designation and Marking (1966)

Tips: Internal Tools (Metric Series) (1966)

Tips: Metric Series (1961)

(Tire) Valves (1965)

(Tired) Wheels for Trailer Stock (1969)

(Tired) Wheels for Trailer (1969)

(Tires) for Trailer Stock (1969)

Rec. for Weft Pirn ISO

ISO

ISO

150

ISO

JOck Material- ISO

/Y Rolling Stock Mate ISO

(Tires) for Trailer Stock: Dimensions and Tolerances (19 ISO

Tiron Photometric Meth. (1968) /Rimarily Used for the ISO

Titanium Content (1966) Rec. for Meth ISO

Titanium Content; Tiron Photometric Meth. (1968) /Rima ISO

Titanium Dioxide for Paints (1967)

Titanium Spectrophotometric Meth. with Chromotropic Aci ISO

Title Leaves of a Book (1969)

Titles of Periodicals (1968)

Titles of Periodicals (1972)

Titratable Acidity (1968)

Titration Procedure) (1972)

Titration with Sodium Borate) (1968)

(Titre) (1969)

Titrimetric Merh. (1972)

Tit rimetric Meth. (1972)

Titrimetric Meth. (1972)

Toasters, Grills, Waffle Irons and

Toggle Switches on Aircraft (1957)

(Toggle) (1969)

Tolerance Class 5 -Special Requirement (1966) /Arings ISO

Tolerance Class 6 (1971) /Arings-Radial Types with S ISO

Tolerance Classes 4 (Normal), 3,0 and 00 (1973) ISO

Tolerance Classes 6,5 and 4(1968) /Ling Bearings-T ISO

Tolerance for Casting and Finishing (1966) Rec. for ISO

Tolerance Zones for General Purposes (1970)

Tolerance (1972)

Toleranced Dimensions from In./S into Millimetres (Mill

ISO

Tolerances-Class 4 (1973)

Tolerances-Definitions (1969)

Tolerances-Normal and Tolerance Class 6 (1971)

Tolerances and Applicability of Test Pieces (1971)

ISO

ISO

AArin ISO

ISO

Tolerances and Conventional Masses Per Unit Length (196 ISO

Tolerances and Conventional Masses Per Unit Length (197 ISO

Tolerances and Deviations (1962)

Tolerances and Gauges (1963)

Tolerances and Gauges (1964)

Rec. for Warp Tubes for ISO

/Ec. for Warp Tubes for R ISO

Tolerances and Masses Per Metre (Meter) (1967) /for Co ISO

Tolerances and Shrinkage (1968)

Tolerances Classes 6 and 5 (1968)

Tolerances for Building-Vocabulary (1970)

Tolerances for Drawn or Extruded Filler Rods for Weldin

Tolerances for Electrodes for Manual Arc Welding and F

Tolerances for Filler Rods, Other Than Drawn or Extrude

Tolerances for Reamers (1966)

Tolerances of Electrodes for the Welding of Mild Steel

Tolerances of Form and of Position-Part III: Dimensio

Tolerances of Form and of Position-Part IV: Practical

Tolerances of Form and of Position-Part I: Generaliti

Tolerances of Resistors and Capacitors (1968)

Tolerances on Length (1970)

Tolerances on Outside Diameters (1970)

Tolerances on Outside Diameters (1970)

Tolerances on the Total Thickness of Conveyor Belts and
Toles ISO

ISO

ISO

ISO

ISO

ISO

ISO

ISO

ISO

ISO

ISO

IEC

ISO

ISO

ISO

1029

1030

1031

1032

737

738

255-2

$151-8$

2431

388-

1826

516

31/1 151.8

255-3

427

427

2179

1570

344

1111

1131

242

504.

514

414

$1005 / \mathrm{V}$

$1005 / I V$

1005/I

$1005 /$ II

900

550

900

591

1118

833

4

750

2271

671

935

2124

2199

2198

$335-9$

44

131-3

47

1648

199/II

484

1829

2421

370
$577 / 11$

1132

1648

1920

1127

1129

343

368

560
738

577/II

1803

546

544

545

522 
"Mipes for the Pransport of Fluids-Polyethylene (PE) of Fluicls-Inplasticized l'olyvinyl Chloricle (l'V(:) Pipes Rer. for Ilexagon Bolts and Nuts: Metric Series, "r. for Shanks for Turning and Planing Tools: Sections and g Bearings-Thrusi Ball Bearings with Flat Seats-Normal holders for Electrical Machinery: Principal Dimensions and Rec. for Inscription of Linear and Angula Rec. for Paper: Untrimmed Sizes, Designation, Rec. for Rolling Bearings: Radial

rolling Bearings-Tapered; Metric Series-Part I: Norma al-Rough Tyres (Tires) for Trailer Stock: Dimensions and Rec. for Abrasive Belts-Designation-Dinıensions Truncated Cone Abrasive Sleeves: Designation, Dimensions

Toroidal Sealing Rings for Aircraft (In. Series-Class I

Rings for Pipe Fittings in Aircraft (In. Series-Class ] ings for Pipe Fittings in Aircraft-(In. Series-Class 2 oroidal Sealing Rings for Aircraft-(In. Series-Class 2 Rec. for ISO General Purpose Metric Screw Threads:

Rec for ISO General Purpose Metric Screw Threads:

ngs: Airframe: Introduction. General, Boundary Dimensions,

Rec. for ISO General Purpose Metric Screw Threads Rolling.Bearings-Thrust Ball Bearings with Flat Seats maximum Shaft and Housing Fil/ Std. for Rolling Bearings: ances of Form and of Position-Part 1II: Dimensioning and Rec. for Pure Rec. for Std. Reference Zero for the Calibration of Pure Rec. for Pure

listeni/ Rec. for Normal Equal Loudness Contours for Pure Std. for Hooks for Lifting Freight Containers of $U_{p}$ to 30 Std. for Modular Units for Machine Rec. for Direction of Operation of Machine Rec. for Lathe Rec. for $7 / 24$. Tapers for nsions (1972) Std. for

Rec. for Selfholding Tapers fo Rec. for $7 / 24$ Tapers for Rec. for Machine Rec. for Machine Rec. for Machine

(1970)

i (1970) and Hand Socket Wrenches (1970) er Types (1970)

et Wrenches-Metric Series-Maximum /

(1966)

Rec for Reduction Sleeves and Exter meters of Shanks and Sizes of Driving Squares for Rotating Rec. for Turning Tools with Carbide Tips: Internal

Rec. for Symbols for Indications Appearing on Machine Spec. for Portable Motor Operated Std. for Woodworking

Rec. for Electrical Equipment of Machine

Rec. for Electrical Equipment of Machine Std. for Woodworkin Rec. for Carbide Tips for Turning Rec. for Shanks for Turning and Planing Rec. for Electrical Equipment of Machine Std. for Woodworking strial Castors-Dimensions of Top Plates-Part I: Oblong Std. for Size Analysis of Small Coke (Nomina for Basic Environmental Test Procedures: Test Ec: Drop and 2 Tolerances) (197/ 1 Tolerances) (1969) (In. Series-Class 2/ n. Series-Class I T/ s (1972) formance Properties (1972) ries (1972) Rec. for Dimensions of Elastomeric Rec. for Dimensions of Elastomeric Rec. for Dimensions of Elastomeric Rec. for Dimensions of Elastomeric Std. for Prevailing Std. for Prevailing Std. for Prevailing Rec. for Plastics-Determination of Stiffness in Rec. for Plastics-Test. with the Rec. for Simple 9) Std. for Simple

Rec. for Fire Refined High Conductivity Rec. for Fire Refined

ghts (1969)

ghts by the Punc/
Rec. for Shipbuilding Details

Rec. for Shipbuilding Details: Test. of Rec. for Aircraft Rec. for Electrical Connections Between Prime Movers and nces for Domestic and Similar Purposes: Clothes Dryers and
Tolerances on Wall Thicknesses $U_{p}$ to $6 \mathrm{~mm}$ (1970)

Tolerances on Wall thirknesses Up to $6 \mathrm{~mm}$ (1970)

Tolerances on Widths Across Flats and Corners (1968)

Tolerances (1961)

Tolerances (1961)

Tolerances (1962)

Tolerances (1964)

Tolerances (1966)

Tolerances (1966)

Tolerances (1967)

Tolerances (1969)

Tolerances (1970)

Tolerances (1972)

Tolerances) (1969)

Tolerances) (1969)

Tolerances) (1971)

Tolerances) (1971)

Tolerances, Deviations for Constructional Types (1969)

ible Ioad (1969)

Tolerances, Principles and Basic Data (1969) ISO

Tolerances: Part 2-Tolerance Classes 6,5 and 4 (1968 ISO

Tolerances; Metric Series Chamfer Dimension Limits and ISO

Tolerancing of Profiles (197I) Nical Drawings-Toler ISO

Tone Audiometers for General Diagnostic Purposes (1965) IEC

Tone Audiometers (1964)

Tone Screening Audiometers (1965)

Tones and Normal Threshold of Hearing Under Free Field

Tonnes (Tons) Capacity-Basic Requirements (1972)

Tool Construction: Slide Units (1973)

Tool Controls (1965)

Tool Posts (Overall Internal Height) (1961)

Tool Shanks-7/24. Tapers Nos. 45 and 55 (1971)

Tool Shanks and Equipment with 7/24 Tapers: Collar Dime

Tool Shanks (1963)

Tool Shanks (1963)

Tool Speeds and Feeds (1961)

Tool Tables-T Slots and Corresponding Bolts (1971)

Tool Test Code (1961)

Tools and Fitting Dimensions of Chuck Bushings-Part I

Tools and Fitting Dimensions of Chuck Bushings-Part I

Tools for Bolts and Screws-Driving Squares for Power

Tools for Bolts and Screws-Hexagon Drive Ends for Pow

Tools for Screws and Nuts-Forged and and Tubular Sock

Tools for Screws and Nuts-Nomenclature (1971)

Tools with Carbide Tips: Designation and Marking (1966)

Tools with Carbide Tips: Internal Tools (Metric Series)

Tools with Carbide Tips: Metric Series (1961)

Tools with Morse Taper Shanks (1961)

Tools with Parallel Shanks (1961)

Tools (Metric Series) (1966)

Tools (1964)

Tools (1967)

Tools: Chisels and Gouges (1973)

Tools: Electronic Types (1968)

Tools: Machines for General Use (1965)

Tools: Metal Bodied Planes (1973)

Tools: Metric Series (1961)

Tools: Sections and Tolerances (1961)

Tools: Used in Large Series Production Lines Lines (196

Tools: Wooden Bodied Planes (1973)

Top of Cone $4^{\circ} 20^{\prime}$ (1967)

Top Plates-Part 1: Oblong Top Plates with 4 Bolt Hole

Top Plates with 4 Bolt Holes (1972)

Top Size $20 \mathrm{~mm}$ or Less) (1972)

Topple, Primarily for Equipment Type Specimens (1969)

Toroidal Sealing Rings for Aircraft-(In. Series-Cla

Toroidal Sealing Rings for Aircraft (In. Series-Class

Toroidal Sealing Rings for Pipe Fittings in Aircraft-

Toroidal Sealing Rings for Pipe Fittings in Aircraft (1

Torque Type Hexagon Locknuts: Dimensions-Metric Serie

Torque Type Steel Hexagon Locknuts-Mechanical and Per

Torque Type Steel Hexagon Locknuts: Dimensions-In. Se

Torsion as a Function of Temperature (1965)

Torsion Pendulum (1967)

Torsion Test for Aluminum and Aluminium Alloy Wire (196

Torsion Test, of Steel Wire (1972)

Tough Pitch Copper Refinery Shapes (1971)

Tough Pitch Copper Refinery Shapes (1971)

Tough Pitch Copper Refinery Shapes (1972)

Toughened Glasses for Ships Side Scuttles and Fixed Li

Toughened Glasses for Ships Side Scuttles and Fixed Li

Tow Bar Connections to Tractors (1964)

Towed Vehicles with 24.V Electrical Equipment for Comme

Towel Dryers (1967) /Ectric Cooking and Heat
Towing Vehicles and Trailers-lnterchangeability (1969

Asti ISO

ISO

ISO

IEC

ISO

ISO

for $15 \mathrm{O}$

$15 O$

Sid. for ISO

ISO

$15 O$

SO

SO

IEC

ISO

IEC

ISO

ISO

$1 \mathrm{SO}$

ISO

ISO

ISO

ISO

SO

ISO

ISO

ISO

ISO

ISO

ISO

ISO

ISO

ISO

ISO

$1 \mathrm{SO}$

ISO

ISO

ISO
ISO

ISO

ISO

CEE

ISO

IEC

IEC

ISO

ISO

ISO

IEC

ISO

ISO

ISO

ISO

ISO

IEC

ISO

ISO

ISO

ISO

$15 O$

ISO

ISO

ISO

ISO

ISO

ISO

ISO

ISO

ISO

$1 \mathrm{SO}$

ISO

ISO

ISO

1166

241

199/I

136.1

406

479

$577 / 1$

1005/II

1929

2422

1078

1077

1799

1800

965/III

$965 / 1$

1002

965/I

199/II

582

1660

177

389

178

226

2308
2562

447

213

297/I

2583 

mplementation of the 6 and 7 Bit Coded Character Sets on 7 terchange (1971)

terchange (1971)

terchange (1971)

( Conditions / Rec. for 7
Rec for 9

Rec. Meth. for Determining the Comparative Rec. for Industrial Trucks: Voltages of Rec. for Lead Acid Rec. for Rules for Electric Rec. for Rules for

g Losses and Efficiency from Tests (Excluding Machines for c. for International Electrotechnical Vocabulary: Electric Rec. for Industrial Rec. for Mechanical Coupling Between Rec. for Pneumatic Couplings Between

Rec. for 3 Point Linkage of Agricultural Wheeled Rec. for Aircraft Tow Bar Connections to Rec. for Power Take Off and Draw Bar for Agricultural Rec. for Test Code for Agricultural ith 24-V Electrical Equipment for Commercial International ec. for Railway Rolling Stock Material-Tyres (Tires) for Rec. for Railway Rolling Stock Material: Solid Wheels for Rec. for Railway Rolling Stock Material: Wheel Sets for $s$ (Centers) in Nonalloy Steel for Tyred (Tired) Wheels for $r$ Railway Rolling Stock Material-Rough Tyres (Tires) for Rec. for Railway Rolling Stock Material-Axles for orged Wheel Centres (Centers) for Tyred (Tired) Wheels for Rec for Semitrailer ec. for Mechanical Connections Between Towing Vehicles and Std. for Braking of Motor Vehicles and Their Rec. for Mechanical Coupling Between Tractors and Semi al Equipment Particularly for Private Motor Cars and Light Rec. for Lighting and Signalling for Motor Vehicles and Rec. for Ball Couplings for Caravans and Light Rec. for Pneumatic Couplings Between Tractors and Rec. for Dimensions of Motor Vehicles and Their es and Valves: Meth. of Measuring Electrode Resistance and

for Specifying the Characteristics of Electro Mechanical Rec. for International Electrotechnical Vocabulary:

Rec. Graphical Symbols: Telephony, Telegraphy and nductance, Amplification Factor, Conversion Resistance and Cone $4^{\circ} 20^{\prime}(1967)$
Rec. for sic Mode Control Procedures-Code Independent Information ode Control Procedures: Conversational Information Message

eth.-Contact (Stylus) Instruments of Progressive Profile errite Materials Appearing in Manufacturers Catalogues of e for the Drafting of Perforamnce Spec. for Cores of Tuned Rec. for Rules for Traction Rec Spec for New Insulating Oils for ifiers, Gene/ Rec. for Electrical Installations in Ships: 971) Rec. for Cores for Inductors and the Drafting of Performance Spec. for Cores of Broad Band or International Electrotechnical Vocabulary: Machines and Spec. for Safety lsolating Rec. for Current Rec. for Power Rec. for Voltage

Rec. for Loading Guide for Oil Immersed Rec. Graphical Symbols: Machines,

antennas) and Radi/

Technology (1968)

Rec. for Graphical Symbols: Machines, Rec. for Graphical Symbols: Machines, Rec. for Rec. for Supply Voltages for Rec. for Double Deck Flat Pallets for Through Rec. for Large Pallets for Through itive to Radiant Energy (1/ Rec. Rec. for Pictorial Marking of Rec. for International System for the Rec. for

Rec for International System for the xtended Pitch Precision Roller Chains and Chain Wheels for for International Electrotechnical Vocabulary: Generation, al Symbols: Generating Stations and Substations, Lines for Rec. Graphical Symbols: Block Symbols for

$$
\text { Rec. for Widths of Flat }
$$
Rec. for Lengths of Flat Rec. for Crowns of Pulleys for Flat Rec. for Diameters of Pulleys for Flat Rec. for Diffuse

rs: General Requi/ rs: Detail Spec. I 70) 1967)
Rec. for General Purpose Rigid Coaxial Rec. for General Purpose Rigid Coaxial Rec. for Short Pitch Rec. for Short Pitch imiting Values for the Adjustment of Centres (Centers) for rack Magnetic Sound Records on $35 \mathrm{~mm}$ Film-Location a Track Recorders (1972)

Track 12.7 mm (1/2 In.) Magnetic Tape (1969)

Track $12.7 \mathrm{~mm}$ (]/2 In.) Magnetic Tape (1969)

Track 32 Pmm (800 RPI) Magnetic Tape for lnformation

Track 8 RPmm (200 RPI) Magnetic Tape for lnformation in

Track $8 \mathrm{RPmm}$ (200 RPI) Magnetic Tape for lnformation in

Tracking Index of Solid Insulating Materials Under Mois

Traction Batteries for Electric Trucks (1969)

Traction Batteries (1967)

Traction Equipment (1968)

Traction Transformers and Reactors (1969)

Traction Vehicles) (1972)

Traction (1957)

Tractors-Definition and Nominal Rating (1969)

lEC

Tractors and Semi Trailers Interchangeability (1971)

Tractors and Trailers (1971)

Tractors for Attachment of Mounted Implements (1968)

Tractors (1964)

Tractors (1966)

Tractors (1968)

Traffic (1970)

Trailer Stock (1969)

Trailer Stock (1969)

Trailer Stock (1969)

Trailer Stock (1969)

Ock Material-Cast Wheel Centre ISO

Trailer Stock: Dimensions and Tolerances (1969) /C. Fo ISO

Trailer (1969)

Trailer (1969)

Trailer) 5Th Wheel Kingpin (1963)

Trailers-Interchangeability (1969)

Trailers-Terminology (1972)

Trailers Interchangeability (1971)

Trailers or Caravans (1970)

Trailers (1963)

Trailers (1969)

Trailers (1971)

Trailers: Designations and Definitions (1967)

Transconductance, Amplification Factor, Conversion Resi

Transducers for Shock and Vibration Measurements (1965)

Transducers (1955)

Transducers (1968)

Transductance (1966)

Electrode Resistance and Transco IEC

Transfer (1972)

Transfer (1973)

ses: Half Angle at Top O ISO

Std. for Data Communication-Ba ISO Std. for Basic M ISO

ISO

Transformer and Inductor Cores (1972) /Nformation on F IEC

Transformers and Inductors of Ferromagnetic Oxides for IEC

Transformers and Reactors (1969)

Transformers and Switchgear (1972)

Transformers for Power and Lighting. Semiconductor Rect

Transformers for Telecommunications: Measuring Meth. (1

Transformers of Ferromagnetic Oxides for Telecommunicat

Transformers (1956)

Transformers (1964)

Transformers (1966)

Transformers (1967)

Transformers (1970)

Transformers (1972)

Transformers, Primary Cells and Accumulators (1960)

Transformers, Primary Cells and Accumulators: Aerials

Transformers, Primary Cells and Accumulators: Microwave

Transfusion Equipment for Medical Use (1969)

Transistorized Nuclear Instruments (1968)

Transit of Goods (1961)

Transit of Goods (1963)

Transit Packages Containing Photographic Materials Sens

Transliteration of Arabic Characters (1961)

Transliteration of Greek into Latin Characters (1968)

Transliteration of Hebrew (1962)

Transliteration of Slavic Cyrillic Characters (1968)

Transmission and Conveyors (1972)

Transmission and Distribution of Electrical Energy (196

Transmission and Distribution (1963)

Transmission and Miscellaneous Applications (1969)

Transmission Belts and Corresponding Pulleys (1956)

Transmission Belts (1958)

Transmission Belts (1959)

Transmission Belts (1959)

Transmission Density (Photography) (1954)

Transmission Lines and Their Associated Flange Connecto

Transmission Lines and Their Associated Flange Connecto

Transmission Precision Bush Chains and Chain Wheels (19

Transmission Precision Roller Chains and Chain Wheels (

Transmission Pulleys (1960)
SO

IEC

Rec ISO

ISO

ISO

IEC

$15 \mathrm{O}$

IEC

EC

ISO

$15 O$

ISO

ISO

ISO

ISO

ISO

SO

ISO

ISO

ISO

ISO

ISO

ISO

ISO

IEC

IEC

EC

EC

$\mathrm{O}$

21

2629

1880

401

218

310

296

92.5

367-1

219

$50(10)$

15

185

76

186

354

117.2

117-I0

117.11

1135

293

198

329

884

233

843

259

9

1275

$50(25)$

117.5

117.13

22

63

100

99
5

339.1

339.2

1395

606

155 
Kor. for Quality, Machining and Balancing of Transmission Pulleys (I962)

for Plastic-s-Determination of the Water Vapour (Vapor)
Rro. for l'lastic-s-l)etermination of Water Vapour (Vapor) Rec. for Plastic:s-Determination of Water Vapour (Vapor)
and l aloratory Measurements of Airborne and Impact Sound for Character Structure for Start / Stop and Synchronou ic and Pneumatic Equipment and Accessories for Fluid Powe easurement on Receivers for Amplitude Modulation Broadcast easurement on Receivers for Frequency Modulation Broadcas plitude and Frequency Modulations and Television Broadcast meth. of Measurement on Receivers for Television Broadcast Nonessential Oscill/ power and Consumptio/ Rec. Meth. of Measurement for Radio $r$ Monochrome and $\mathrm{Col} /$ Rec. Meth. of Measurement for Radio Rec. Meth. of Measurement for Radio

Rec. for Safety Requirements for Radio Rec. for Safety Requirements for Radio aphy-Illumination for Comparison Viewing of Photographic Rec. for Microcopies on

c. for Plastics-Determination of the Refractive Index of Sampling (1972) Std. for Pots for Propagation and uilding Details for InIand Navigation-Pipe Lines for the o Internal Pressure (197) Outside Diameters (1970) Wall Thicknesses Up to (PVC) Pipes-Tolerances/ (PVC) Pipes-Tolerances/ sures): Par/ Rec. for Plastics Pipes for the Rec. for Plastic Pipes for the Rec. for Plastics Pipes for the Rec. for Plastics Pipes for the Rec. for Plastics Pipes for the sures) Part Rec. for Pipes of Plastics Materials for the

972)

ropping (1972)

nclined Plane and P/ en Test. (1972)

Rec. for Pipes of Plastics Materials for th Std. for Packaging-Complete, Filled Std. for Packaging-Complete, Filled Std. for Packaging-Complete, Filled Std. for Packaging-Complete, Filled Std. for Packaging: Complete, Filled Std. for Packaging-Complete, Filled Rec. for Green Bananas: Guide to Storage and Rec. for Fresh Pineapples-Guide to Storage and o Be Given with Enquiries Tenders and Orders and Rules for urement for Radio Transmitters: Measurements Particular to ical Fillet Welds Statically Loaded in Such a Way That the Rec. for rials: Vibrating Conveyors and Feeders with Rectangular or $s$ and Shaking or Reciprocating Feeders with Rectangular or Rec. for Line

ngs for Ring Spinning and Doubling Frames for $\mathrm{C}$ and $\mathrm{El}$ ngs for Ring Spinning and Doubling Frames for $C$ and El rings for Ring Spinning and Doubling Frames for Ear Shaped he Aluminium Magnesium Silicon Type (1959) Rec. for Heat

Rec. Spec. for Insulating Materials Based on Built Up or and Tempered Chromium Types (J970)

Quenched and Tempered Types with $1 \%$ Chromi/ ght Nitriding Type (1970)

ght Case Hardening Type (1970)

$\mathrm{e}$ and Induction Hardening Types (1972)

hed and Tempered Unalloyed Types (1968)

ht, Quenched and Tempered Types with $1 \% \mathrm{Ch} /$

ht Quenched and Tempered Unalloyed with Co/

ht Quenched and Tempered Manganese (1970)

ht Quenched and Tempered, with 3\% Chromium/ ht Quenched and Tempered Chromium Nickel M/ ht Free Cutting Type (1970)

$\mathrm{n}$ of Terms (1971) Flectrical Appliances: Particular Requirements for Floor m) and Its Alloys-Check of Continuity/ $\mathrm{m})$ and Its Alloys-Estimation of the L/

Aluminium (Aluminum) and Its Alloys-/

Aluminium (Aluminum) and Its Alloysand Its Alloys-Determination of the / Rec. for Hea Rec. for Heat Rec. for Heat Rec. for Heat Std. for Heat Rec. for Heat Rec. for Heat Rec. for Heat Rec. for Heat Rec. for Heat Rec, for Heat Rec. for Heat Rec. for Surface Rec. for Surface Rec. for Surface Rec. for Surface Rec. for Surface 972)

of Absorptive Power by Colorant Drop Test with Prior Acid

heets (1967)

rinted Matter (1961) onductors, Insulated, in Pairs, olyvinyl Chloride) Insulation and Sheath: Cables in Pairs, Rec. for Photographic Equipment Rec. for Clearance Dimensions for

I Use-Determination of Iron Content-|

I Use-Determination of Loss on Ignitio/

matter Insoluble in Water (1968)

Potentiometric Meth. (1968)

ting Conveyors and Feeders with Rectangular or Trapezoidal

k Materials-Vibrating Feeders and Conveyors with Tubular

g or Reciprocating Feeders with Rectangular or Trapezoidal h. of Test) (1968)
Transmission Rate of Films and Thin Sheets-Dish Meth.

Transmission Rate of Rigid Cellular Plastics (I970) Transmission (1960)

Transmission (1970)

Transmission (1970)

Transmissions (1954)

Transmissions (1958)

Transmissions (1959)

Transmissions (1960)

Transmitters: Bandwidth, Out of Band Power and Power of Rec. IEC

Transmitters: General Conditions Of, Frequency, Output

Transmitters: Measurements Particular to Transposers Fo

Transmitters: Wanted and Unwanted Modulation (1971)

Transmitting Equipment (1966)

Transmitting Equipment: Test Meth. (1967)

Transparencies with Reflection Colour Prints (1972)

Transparent Bases Sizes of Rec. Bases (1961)

Transparent Plastics (1966)

Transplantation Made of Peat and Other Plant Material-

Transport of Combustible Liquids-Nominal Diameters (1

Transport of Fluids-Determination of the Resistance T

Transport of Fluids-Polyethylene (PE)-Tolerances on

Transport of Fluids-Polyethylene (PE)-Tolerances on

Transport of Fluids-Unplasticized Polyvinyl Chloride

Transport of Fluids-Unplasticized Polyvinyl Chloride

Transport of Fluids (Outside Diameters and Nominal Pres

Transport of Fluids (Outside Diameters and Nominal Pres

Transport Packages-Part III: Stacking Test (1972)

Transport Packages-Part II: Conditioning for Test. (1

Transport Packages-Part IV: Vertical Impact Test by D

Transport Packages-Part Vi: Vibration Test (1972)

Transport Packages-Part V: Horizontal Impact Tests (I

Transport Packages-Part 1: Identification of Parts Wh

Transport (1969)

Transport (1970)

Transport, Erection and Maintenance (1971)

Transposers for Monochrome and Colour (Color) Televisio

Transverse Section Is Not Under Any Normal Stress

c. for Fiel ISO

Rec ISO

Transverse Track Recorders (1972)

Trapezoidal Trough (1969)

Trapezoidal Trough (1970)

Traps (1971)

Travellers-Principal Dimensions (1972)

Travellers-Principal Dimensions (1972)

Travellers (1959)

Treated Aluminium (Aluminum) Alloy Busbar Material of T

pment for Loose Bulk Mate ISO

/Ials-Oscillating Conveyor ISO

IEC

IEversible Ri ISO

Eversible Ri ISO
Rec. for ISO

Treated Steels: Free Cutting-Part 7: Wrought Quenched ISO

Treated, Alloy and Free Cutting Steels, Pt. 4: Wrought ISO

Treated, Alloy, and Free Cutting Steels-Part 10: Wrou

Treated, Alloy, and Free Cutting Steels-Part 11: Wrou

Treated, Alloy, and Free Cutting Steels-Part 12: FIam

Treated, Alloy, and Free Cutting Steels-Part 1: Quenc

Treated, Alloy, and Free Cutting Steels-Part 2: Wroug

Treated, Alloy, and Free Cutting Steels-Part 3: Wroug

Treated, Alloy, and Free Cutting Steels-Part 5: Wroug

Treated, Alloy, and Free Cutting Steels-Part 6: Wroug

Treated, Alloy, and Free Cutting Steels-Part 8: Wroug

Treated, Alloy, and Free Cutting Steels-Part 9: Wroug

Treatment and Metallic Coatings-General Classificatio

Treatment and Wet Scrubbing Machines for Household and

Treatment of Metals-Anodisation of Aluminium (Aluminu

Treatment of Metals-Anodisation of Aluminium (Aluminu

Treatment of Metals-Anodisation (Anodic Oxidation) of

Treatment of Metals-Anodisation (Anodic Oxidation) of

Treatment of Metals-Coloured Anodisation of Aluminium Its Alloys-Estimation of the Loss ISO

Trichloroethylene for Industrial Use-Meth. of Test (1

Trimmed Sizes of Articles of Stationery That Include De

Trimmed Sizes of $W$ riting Paper and Certain Classes of $P$

Triples, Quadruples and Quintuples (1968)
Triples, Quadruples and Quintuples (1971)

Triples, Quads and Quintuples for Inside Installation

Heath: Dist IEC

Tripod Connections (1971)

Tripod Jacks for Aircraft (1970)

Tripolyphosphate and Sodium Pyrophosphate for Industria

Tripolyphosphate and Sodium Pyrophosphate for Industria

Tripolyphosphate for Industrial Use-Determination of

Tripolyphosphate for Industrial Use-Measurement of $\mathrm{pH}$

Trolleys: Dimensions (1960)

Uipment for Loose Bulk Materials: Vibra ISO

Trough (1969)

Trough (1970)

Trough (1970)

Trough (1971)

/Anical Handling Equipment for Loose Bul ISO

/Ials-Oscillating Conveyors and Shakin ISO

lor Loose Bulk Materials-Oscillating C ISO Troughability of Conveyor Belts (Characteristic and Met 
- Mrerhanic al llandling Equipment for Loose Bulk Materials * Merhaniral llandling Equipment for loone Bulk Materials * Merhaniral llandling kquipment for Loose Bulk Materials ing Equipment for Unit l,oads-Single Strand Floor Mounted al Ilandling Equipment for Unit Loads-Single Strand Floo 70)

$$
\text { Rec. for Stability of Counterbalanced Lif }
$$
Rec. for Counterbalanced Fork Lif Rec. for Industria

Rec. for Principal Dimensions of Pallet strial Trucks: Voltages of Traction Batteries for Electric Rec. for Hand Operated Stillage cks (1969) porosity of Coke (1969)

$\mathrm{s}$, Tolerances (1972) of Test (1969) es and Dimensions of Test. Devices and Gauges (/ Rec. for (1970) Rec. for Wire, Bar and 1969)

es of Microwave Tubes and Valves: Backward Wave Oscillator b/ Rec. for Measurement of Fluid Flow by Means of Ventur Rec. for Capillary Solder Fittings for Copper
Rec. for Stainless Steel per Unit Length (1971)

Rec. for Boiler Std. for Stee Rec. for Stee are Made on the Threa/ 961) Rec. for Pipe Threads for Gas List uges (/ Rec. for Rating Systems for Electronic Rec. for Tube and Valve Shields: Spec. Sheets for ering of Electrodes and Designation of Units in Electronic rement of Direct Interelectrode Capacitances of Electronic Rec. for Dimensions of Electronic for Measurement of the Electrical Properties of Microwave or Measurements of the Electrical Properties of Electronic for Measurement of the Electrical Properties of Microwave t (1963) Rec. for Sockets for Electronic for Measurement of the Electrical Properties of Microwave or Measurements of the Electrical Properties of Electronic for Measurement of the Electrical Properties of Microwave for Measurement of the Electrical Properties of Microwave for Measurement of the Electrical Properties of Microwave or Measurements of the Electrical Properties of Electronic or Measurements of the Electrical Properties of Electronic or Measurements of the Electrical Properties of Electronic or Measurements of the Electrical Properties of Electronic or Measurements of the Electrical Properties of Electronic or Measurements of the Electrical Properties of Electronic or Measurements of the Electrical Properties of Electronic or Measurements of the Electrical Properties of Electronic or Measurements of the Electrical Properties of Electronic or Measurements of the Electrical Properties of Electronic or Measurements of the Electrical Properties of Electronic or Measurements of the Electrical Properties of Electronic or Measurements of the Electrical Properties of Electronic or Measurements of the Electrical Properties of Electronic or Measurements of the Electrical Properties of Electronic or Measurements of the Electrical Properties of Electronic or Measurements of the Electrical Properties of Electronic or Measurements of the Electrical Properties of Electronic or Measurements of the Electrical Properties of Electronic or Measurements of the Electrical Properties of Electronic Jigs and Pin Straighten/

Rec. for Sockets for Electronic for Measurement of the Electrical Properties of Microwave Rec. for Perforated Paralle
Rec. for Textile Machinery-Cylindrical Rec. for Textile Machinery and Accessories Rec. for Steel Rec. for Nonscrewed Steel ic Dimensions, Tolerances and Gauges (1964/ Rec. for Warp Dimensions, Tolerances and Gauges (1963) Rec. for Warp Std. for Textile Machinery and Accessories-Cylindrica Rec. for Pipes and Fittings-Stainless Stee Rec. for Copper and Its Alloy Rec. for Tensile Test. of Copper and Its Alloy Rec. for Copper

Probes Containing Geiger-Muller or Proportional Counter rement of Fluid Flow by Means of Venturi Tubes-Classical ement of the Dimensions of Focal Spots of Diagnostic X.Ray Rec. for Flanging Test on Stee Rec. for Drift Expanding Test on Stee Rec. for Bend Test on Stee

Rec. for Drift Expanding Test on Copper and Its Alloy Rec. for Flattening Test on Stee reparation of Outline Drawings of Oscilloscope and Picture Rec. for Ring Expanding Test on Steel
Troughed Belt Conveyors (Other Than Portable) Belt Pull Troughed Belt Conveyors (Other Than Portable) Belts (19 Troughed Belt Conveyors (Other Than Portable) Idlers (1 Truck Conveyors (Chain Above Floor)-Safety Code (1971 Truck Conveyors (Chain Below Floor) Safety Code (1972) Trucks-Basic Tests (1969)

Trucks-Dimensions of Stillages-Connection Gauge (19

Trucks-Rated Capacity (1971)

Trucks-Wheels and Castors-Vocabulary (1971)

Trucks (1966)

Trucks (1969)

Trucks: Principal Dimensions (1969)

Trucks: Voltages of Traction Batteries for Electric Tru

True and Apparent Relative Densities (Density) and the

Truncated Cone Abrasive Sleeves: Designation, Dimension

Tube and Valve Shields: General Requirements and Meth.

Tube and Valve Shields: Spec. Sheets for Tubes and Valv

Tube Drawing Dies-Designation-Marking-Dimensions

Tube Drawing Mandrels (1970)

Tubes-0 Type (1972)

Tubes-Butt Welding Bends 5 D (90 ${ }^{\circ}$ And 180 Deg.) (

Tubes-Classical Tubes Used Outside the Scope Covered

Tubes-Dimensions of Sockets and Male Ends (1971)

Tubes-Dimensions, Tolerances and Conventional Masses

Tubes-Dimensions, Tolerances and Conventional Masses

Tubes-Outside Diameters (1971)

Tubes-Wall Thicknesses (1971)

Tubes and Screwed Fittings Where Pressure Tight Joints

Tubes and Valves and Analogous Semiconductor Devices (

Tubes and Valves and Dimensions of Test. Devices and Ga

Tubes and Valves (1961)

Tubes and Valves (1962) IEC

Tubes and Valves (1966)

Rec. Meth. for the Measu IEC

Tubes and Valves: Backward Wave Oscillator Tubes -0

Tubes and Valves: Equivalent Input and Output Admittanc

Tubes and Valves: Gas Filled Microwave Switching Device

Tubes and Valves: General Requirements and Meth. of Tes Tubes and Valves: General (1972)

Tubes and Valves: Heater or Filament Current (1963)

Tubes and Valves: High Power Klystrons (1972)

Tubes and Valves: Low Power Oscillator Klystrons (1972)

Tubes and Valves: Magnetrons (1972)

Tubes and Valves: Measurement of Cathode Heating Time a

Tubes and Valves: Measurement of Electrode Current (196

Tubes and Valves: Measurement of Equivalent Noise Resis

Tubes and Valves: Meth. of Application of Mechanical Sh

Tubes and Valves: Meth. of Measurement of Audio Frequen

Tubes and Valves: Meth. of Measurement of Cross Modulat

Tubes and Valves: Meth. of Measurement of Emission Curr

Tubes and Valves: Meth. of Measurement of Gasfilled Typ

Tubes and Valves: Meth. of Measurement of Noises Due to

Tubes and Valves: Meth. of Measurement of Radar and Osc

Tubes and Valves: Meth. of Measurement of Radio Frequen

Tubes and Valves: Meth. of Measurement of Spurious and

Tubes and Valves: Meth. of Measurement of Thyratron Pul

Tubes and Valves: Meth. of Measurement of Vacuum Pulse

Tubes and Valves: Meth. of Measurement on Corona Stabil

Tubes and Valves: Meth. of Measuring Electrode Resistan

Tubes and Valves: Meth. of Measuring Hiss and Hum (1964

Tubes and Valves: Meth. of Measuring the Cathode Interf

Tubes and Valves: Noise Factor (1963)

Tubes and Valves: Precautions Relating to Meth. of Meas

Tubes and Valves: Spec. Sheets and Dimensions of Wiring

Tubes and Valves: Terminology (1972)

Tubes for Cheese Dyeing (1967)

Tubes for Draw Twisters (1970)

Tubes for Draw Winders for Man Made Fibres (1971)

Tubes for Gas List Threading (1971)

Tubes for General Purposes (1962)

Tubes for Ring Spinning and Ring Doubling Spindles Metr

Tubes for Ring Spinning and Ring Doubling Spindles: In.

Tubes for Tape Yarns (1972)

Tubes for the Food Industry (1971)

Tubes of Circular Section-Flattening Test (1971)

Tubes of Circular Section (1964)

Tubes of Circular Section: Dimensions; Metric Series (1

ISO

Tubes or Scintillation Detectors (1967) /Cal Radiation IEC

Tubes Used Outside the Scope Covered by ISO Rec. R781 ( ISO

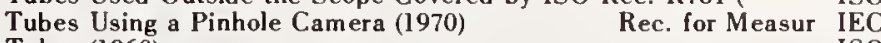

Tubes $(1960)$

Tubes $(1960)$

Tubes $(1960)$

Tubes (1961)

Tubes (1961)

Tubes (1962)

Tubes (1964) 15.37 2381 1074 1214 509 1044 1044 1014 288. 1 1684

1651 128 (8) 1127 64 221 134
$288-2$ 135 67 151.3 235-7 235-2

$151-2$

$235-5$

$151-8$

151.1

$151-6$

$151 \cdot 10$

151.13

151-17

$151 \cdot-14$

$151-15$

$151-20$

151.19

151.12

$151-9$

151.0

149.2 
Rec for Tensile Test. of Steel Tuhes (1964)

tion of Flectrostatic Deflerting Electrodes of Cathode Ray Tubes (1967)

Kec. for Measurement of Fluid Flow hy Means of Venturi Tubes (1968)

Rec. for Tensile Test. of 1.ight Metal Ad Its Alloy rec. for Drift Expanding Test on Light Metal and Its Alloy Rec. for Flattening Test on Aluminum and Aluminium Alloy meth. of Measurement of Radar and Oscilloscope Cathode Ray

industrial Application for Plain End Steel and Other Metal of the Electrical Properties of Microwave Tubes: Disk Sea Rec. for Dimensions of variability, Examples of Resistors, Elements of Electronic and Masses Per Unit Length (19/ Rec. for Plain End Stee Rec. for Stee

for Measurement of the Electrical Properties of Microwave or Measurements of the Electrical Properties of Electronic or Measurements of the Electrical Properties of Electronic or Measurements of the Electrical Properties of Electronic ge Types (1971) Rec. for Electronic or Measurements of the Electrical Properties of Electronic Per Metre (Meter) (/ Rec. for Cold Drawn Precision Steel 69) (1961) Rec. for Sealing Test for Pressurized Waveguide
Rec. for Dimensions of Rec. for Rec. for Glow Starters fo Rec. for Lighting Fittings for Rec. for Lampholders and Starterholders for determination of the Behaviour of Plastics in a Ventilated d. for Assembly Tools for Screws and Nuts-Forged and and oose Bulk Materials-Vibrating Feeders and Conveyors with lating Conveyors and Shaking on Reciprocating Feeders with Std. for Hand Knotted Carpets: Determination of Rec. for Carpets-Determination of Number of

r Guide for the Drafting of Perforamnce Spec. for Cores of Rec. for Raw Rec. for

tric Exposure-Simulation of the Spectral Distribution o Tuning Frequency (Std. Musical Pitch)/ Rec. for Standard rd Tuning Frequency (Standard Musical Pitch) Rec. for Std. al Machines: Ratings and Characteristics of 3 Phase, $50 \mathrm{H}$ Std. for Ga rnational Code for the Field Acceptance Tests of Hydraulic international Code for Model Acceptance Tests of Hydraulic Rec. for Spec. for Steam al Code for Test. of Speed Governing Systems for Hydraulic Rec. for Steam

1)

g (1966)

Series) (1966)

Rec. for Shanks for Rec. for Rec. for Rec. for

Rec. for Carbide Tips for Rec. for Gum Spirit of rec. for Radio Frequency Connectors: Two Pin Connector for us Mechanical Handling Equipment for Unit Loads-Overhead Rec. for Rec. for Rec. for Parallel Shank Rec. for

Morse Taper Shank (1961) Rec. for Textile Glass-Determination of the Rec. for Designation of the Direction of Std. for Textiles: Determination of lled Wire by the Lowering of the Electric Strength Between Rec. for Textile Machinery-Cylindrical Tubes for Draw Rec. for Function Key Symbols on Rec. for Layout of Printing and Function Keys on Rec. for Spindle Noses and Face Plates: Bayonet Rec. for ISO Conventiona Rec. for Aircraft erial-Cast Wheel Centres (Centers) in Nonalloy Steel for ck Material-Rolled on Forged Wheel Centres (Centers) for Rec. for Railway Rolling Stock Material

ces (196/ Rec. for Railway Rolling Stock Material-Rough for Oil of Peppermint: France, Italy, United Kingdom and Rec. for Charpy Impact Test Rec. for

eel-Reference Block for the Calibration of Equipment for Rec for Test and Calibration of Rec. for Classification and Composition of Rec. for Composition of 99.8 Rec. for Composition of 99.95

of Copper and Its Alloys-Electrolytic Determination in $y$, and Free Cutting Steels-Part 1: Quenched and Tempered ree Cutting Steels-Part 3: Wrought Quenched and Tempered nal $\mathrm{P} /$ Rec. for Dimensions of Hot Rolled Steel Sections rec. for Dimensions of Hot Rolled Steel Sections-Part 2 Sid. for Parallel Pins,
Tubes (1969)

Tubes (1969)

Tubes (1969)

Tubes (1969)

Tubes (1970)

Tubes (1972)

Tubes, Pins and

elded or Seamless-General Table of Dimensions ISO

Tubes: Butt Welding Bends $\left(90^{\circ}\right.$ And 180 Deg.) (1962)

Tubes: Disk Seal Tubes (1972)

Tubes: Meth. of Measurement for Camera Types (1971)

Tubes: Meth. of Measurement for Cold Cathode Counting a

Tubes: Meth. of Measurement for Television Picture Type

Tubes: Meth. of Measurement of Cathode Ray Charge Stora

Tubes: Meth. of Measurement of Geiger-Muller Counter

Tubes: Metric Series, Dimensions, Tolerances and Masses

Tubing and Assemblies (1968)

Tubular Electrode Holders for Spot Welding Machines (19

Tubular Fluorescent Lamps for General Lighting Service

Tubular Fluorescent Lamps (1963)

Tubular Fluorescent Lamps (1965)

Tubular Fluorescent Lamps (1972)

Tubular Oven (1969)

IEC

Tubular Socket Wrench

/Plastics-Meth. of Test for the ISO

(1) Series-Maximum Outsi

Anical Handling Equipment for $\mathrm{L}$ ISO

or Loose Bulk Materials-Oscil

Tuft Leg Length Above the Woven Ground (1972)

Tufts And/or Loops Per Unit Length and Area (1971)

Tuned Transformers and Inductors of Ferromagnetic Oxide

Tung Oil (1962)

Tungsten Filament Lamps for General Service (1961)

Tungsten Illumination (1972) /Ces for Use in S
Tuning Frequency (Standard Musical Pitch) Rec. for St

Tuning Frequency (Std. Musical Pitch) (1955)

Turbine Type Machines (1968)

ISO

Turbines-Acceptance Tests (1973)

Turbines (1963)

Turbines (1965)

Turbines (1970)

Turbines (1970)

Turbines: Rules for Acceptance Tests (1962)

C. for Rotating Electric

ISO

Rec. for IEC

IEC

Turning and Planing Tools: Sections and Tolerances (196

Turning Tools with Carbide Tips: Designation and Markin

Turning Tools with Carbide Tips: Internal Tools (Metric

Turning Tools with Carbide Tips: Metric Series (1961)

Turning Tools: Metric Series (1961)

Turpentine and Wood Turpentines (1965)

Twin Balanced Aerial Feeders (1965)

Twin Rail Chain Conveyors (Power and Free) Safety Code

Twin Wire Healds for Frame Weaving (1967)

Twin Wire Healds for Jacquard Weaving (1967)

Twist Drills-Long Series (1966)

Twist Drills: Parallel Shank Jobber and Stub Series and

Twist in Its Continuous Filament and Staple Fibre (Fibe

Twist in Textile Yarns and Related Products (1966)

Twist in Yarns-Direct Counting Meth. (1972)

Twisted Wires (1966)

/F the Thermal Endurance of Ename

Twisters (1970)

Type writers (1969)

Typewriters (1969)

Type; Sizes for Interchangeability: Metric Series (1968

Typographical Character for Legibility Tests (1965)

Tyre (Tire) Valves (1965)

Tyred (Tired) Wheels for Trailer Stock (1969)

Tyred (Tired) Wheels for Trailer (1969)

Tyres (Tires) for Trailer Stock (1969)

Tyres (Tires) for Trailer Stock: Dimensions and Toleran

U. S. A. (1968)

(U-Notch) for Steel (1959)

Ultramarine Pigments (1968)

Ultrasonic Examination (1972)

Ultrasonic Therapeutic Equipment (1963)

Unalloyed Aluminium Ingots for Remelting (1968)

Unalloyed Magnesium Ingots (1959)

Unalloyed Magnesium Ingots (1961)

Unalloyed Type Containing Not Less Than 99.90\% (1971)

Allo ISO

Unalloyed with Controlled Sulphur Content (1970)

Nd F

Unequal Leg Angles-In. Series-Dimensions and Sectio

Unequal Leg Angles-Metric Series-Dimensions and Sec

lOck Mat ISO

Unhardened-Metric Series (1972) 
Rec. for International Unification of Concepts and Terms (1968)

R.e. for ()il of l'eppermint: France, ltaly Rules for the lise of IInits of the International System of linited Kingdom and U. S. A. (1968)

Units and a Selection of the Decimal Multiples and Subm

1961)

Rec. for Safety Features for Ground Power Rec. for Shackle Type Connector Std. for Modular Rec. for Quartz Crystal Rec. for Quartz Crystal Rec. for Quartz Crystal

for Dimensions of Indoor and Outdoor Post Insulators and Rec. for Numbering of Electrodes and Designation of surement of Airborne Noise Emitted by Compressor Primeover Rec. for Quantities and Rec. for Quantities and Rec. for Quantities and Rec. for Quantities and Rec. for Quantities and Std. for Quantities and ion of the Decimal Multipl/ Rec. for Characteristics of String Insulator 65) Std. for Rules for the Use of Rec. for Basic Quantities and ection of the Decimal Multiples and Submultiples of the Si Rec. for Ball and Socket Couplings of String Insulator

valent Resistance of Unwanted Resonances of Filter Crystal Rec. for Temperature Control Devices for Quartz. Crystal or Industrial Use-Measurement of Colour (Color) in Hazen ectors, Airbreak Switch Disconnectors and Fuse Combination

td. for Modular Units for Machine Tool Construction: Slide Devices for Ball and Socket Couplings of String Insulator stem) (1969) Rec. for Textiles Rec. for Rolling Bearings-Internal Clearance in Rec. for Rolling Bearings-Radial Internal Clearance in Rec. for Radio Frequency Connectors: Coaxial e Acetone Soluble Matter (Resin Content of Material in the Rec. for Beam

Rec. for Plastics-Determination of Ash of Rec. for Plastics: Determination of Free Acidity of Rec. for Plastics-Determination of Acetic Acid Yield of Chloride (1970) ain S/ Rec. for Socket Fittings for Pipes Under Pressure: Rec for Oven Test for Moulded Fittings in es/ Rec. for Plastics Pipes for the Transport of Fluids es/ Rec. for Plastics Pipes for the Transport of Fluids pply of Gaseous Fuels: Metric Series-S/ ith Elastic Sealing Ring Typ/ ith Elastic Sealing /

Std. for Buried $f$ Expression of Dimensions and Direction of Manufacture of Rec. for Information Processing-Spec for Rec for Information Processing-Properties of 8 and 32 RPmm (200 and 800 RPI), NRZI, and 63 R/ Rec. for ons: Physical Properties and Test Meth. (1973) Std. for on Applications General Dimensional Requirement/ Rec. for for Analysis of Soaps: Determination of Unsaponifiable and temperature and the Rate of Increase During the Setting of yrene Butadiene Rubber Latices-Determination of Volatile $n$ the Design and Construction of Installations for Work on

rec. for Refractory Materials-Classification of Prepared of Certain Effects of Laundering-Preparation and Use of marily Used for the Production of Aluminum: Measurement of

Range (1966)

entary Range (1967) Rec. for Paper Rec, for Paper: Rec. for Paper Rec. for Rubber: Raw and pounded Rubber (Vulcanized and

al Use-Determination of Sodium-Gravimetric Meth. Using olumetric Meth. (1970) etric Meth. (1970)

$-2,2^{\prime}$-Bipyridyl Photometric Meth. (1970/ olumetric Meth. (1970) 72) nt Projection (1973)

Std. for Cinematography-Camer Std. for Cinematography: Projector Rec for Protective Helmets for Road ulphate/ Rec. for Sulphuric Acid and Oleum for Industrial for Radiography of Welds and Viewing Conditions for Films symbol/ Rec. for Marking of the Different Species of Wood Rec. for Lengths of Y-- Section

Rec. for Terms and Definitions Relating to Drives Using es for Corresponding Pulleys (1970/ Rec. for Endless Wide ical Conductivity-Characte/

Rec. for Antistatic Endles

Rec for Ceometrical Rec. for Lengths of Classical and Symbols for Refrigeration (1968)

nits for d.c. Aircraft Servicing and Engine Starting

Units for High Tensile Steel Chain for Conveyors (1969)

Units for Machine Tool Construction: Slide Units (1973)

Units for Oscillators: Section 1-Std. Valves and Cond

Units for Oscillators: Section 3-Guide to the Use of

Units for Oscillators: Section 4: Std. Outlines (1962)

Units for Systems with Nominal Voltages Greater Than 1

Units in Electronic Tubes and Valves (1961)

Units Intended for Outdoor Use (1972)

Units of Acoustics (1965)

Units of Electricity and Magnetism (1965)

Units of Heat (1960)

Units of Mechanics (1960)

Units of Periodic and Related Phenomena (1958)

Units of Physical Chemistry and Molecular Physics (1973

Units of the Cap and Pin Type (1969)

Units of the International System of Units and a Select

Units of the SI Metric Unit) and of Space and Time (10

Units Used in Electricity (1964)

Units (Metric) (1973)

Units (1960)

Units (1968)

Units (1970)

Units (1970)

Units (1972)

Units (1973)

IEC

/Ional System of Units and a Sel ISO

lor the Measurement of Frequency and Equi IEC

Rec. for Higher Alcohols F ISO

Tage Airbreak Switches, Airhreak Disconn IEC

ISO

Units: Dimensions and General Requirements (1971) Ing IEC

Universal System for Designating Linear Density (Tex Sy ISO

Unloaded Bearings-Definitions (I961)

Unloaded Radial Groove Type Ball Bearings with Cylindri

Unmatched Television Aerial Feeder Connector (1965)

Unmoulded State) of Phenolic Moulding Materials (1963)

Unnotched Impact Test for Grey Cast Iron (1969)

Unplasticized Cellulose Acetate (1968)

Unplasticized Cellulose Acetate (1969)

Unplasticized Cellulose Acetate (1970)

Unplasticized Compounds of Homo and Copolymers of Vinyl

Unplasticized Polyvinyl Chloride (PVC) Fittings with Pl

Unplasticized Polyvinyl Chloride (PVC) for Use Under $\mathrm{Pr}$

Unplasticized Polyvinyl Chloride (PVC) Pipes-Toleranc

Unplasticized Polyvinyl Chloride (PVC) Pipes-Toleranc

Unplasticized Polyvinyl Chloride (PVC) Pipes for the Su

Unplasticized Polyvinyl Chloride (PVC) Pressure Pipes W

Unplasticized Polyvinyl Chloride (PVC) Pressure Pipes W

Unpunched Paper Cards (1970)

Unpunched Paper Tape (1971)

Unrecorded Magnetic Tape for Information Interchange-

Unrecorded Magnetic Tapes for Instrumentation Applicati

Unrecorded Magnetic Tapes for Interchange Instrumentati

Unsaponified Matter (1969)

Unsaturated Polyester Resins (1967)

Unsaturates and Residual Styrene (1972)

Unsealed Radioactive Materials (1970)

(Dense and Insulating) (1971)

)

trimmed Sizes, Designation, Tolerances (1966)

ntrimmed Stock Sizes for the 1SO-A Series, ISO Prir

Untrimmed Stock Sizes for the ISO-A Series: ISO Supplem ISO

Unvulcanized-Rapid Plasticity Test (1971) ISO

Unvulcanized) (1970)

Upper and Lower Kelly Cocks (1972)

Uranyl Acetate and Magnesium Acetate (1970)

Rec. for De 150

ISO

Urea for Industrial Use-Desermination of Ash-Gravim ISO

Urea for Industrial Use-Determination of Iron Content

Urea for Industrial Use-Determination of Nitrogen-V ISO

Usable Width (1971)

Usage of $8 \mathrm{~mm}$ Motion Picture Film Perforated Type S (19 ISO

Usage of $8 \mathrm{~mm}$ Type $S$ Motion Picture Film for Direct Fro ISO

Users (1970)

Use; Determination of Sulphur Dioxide Content: Barium S

Utilization of Rec. Patterns of Image Quality Indicator

Utilized in the Manufacture of Wood Flooring-Code of

V-Belts

V-Belts and Grooved Pulleys (1969)

V-Belts for Industrial Speed Changers and Groove Profil

V-Belts (Belts) (Sections Y, Z, A, B, C, D, E)-Electr

V-Belts (Sections Z, A, B, C, D, E) (1967)

V-Belts (1962)
860

856

1000

786

223

1082

2562

$122-$

122-3

273

135

31/VII

$31 / \mathrm{V}$

31/IV

31/III

31/1I

31/VIII

305

1000

3] /I

164

1000

120

283

314

408

2562

372-1

1] 44

200

201

169-2

308

946

872

1061

1597

1163

727

580

1165

1330

2703

2045

2048

217

1681

1729

1864

2690

1859

1067

584

2008

1710

1927

2267

903

479

478

593

2007

1396

2290

1551

1593

1594

1595

1592

2012

1787

1781

1511

912

2504

2036

434

1081

1604

1813

608

255 
Rec. for Section Checking of V.Belts (1962)

Rec. for Grooved Pulleys for

V.Belts: Groove Sections A, B, C, D, E (1957)

V-Belts: Groove Sections SPZ, SPA, SPB (1965)

V-Belts: Groove Sections Y and Z (1962)

V.Belts: Sections SPZ, SPA, SPB (1965)

(V-Notch) for Steel (1960)

ies Characterizing the Magnetic and Electric Properties of tor Operated Appliances for Domestic and Simp Rec. for Meth. of Measurement of Performance of similar Electrical Appliances: Particular Requirements for measurement of Emission Current from Hot Cathodes for High for Recording Pressure, Combined Pressure and Vacuum, and Rec. for Dextrose-Determination of Moisture

$r$ Glucose Syrup-Determination of Dry Matter of Moisture es of Electronic Tubes and Valves: Meth. of Measurement of

$f$ the Performance Characteristics of Positive Displacement ement of the Performance Characteristics of Vapour (Vapor) tional Rec. for Indicating Pressure, Combined Pressure and ational Rec. for Recording Pressure, Combined Pressure and

for Ammonium Nitrate for Industrial Use: Measurement of $\mathrm{Ph}$

8)

imation of Free Alcohols Content by Determination of Ester for Solid Mineral Fuels-Determination of Gross Calorific Rec. for Butter-Determination of the Acid Rec. for Essential Oils-Determination of Acid les-Cotton Fibres (Fibers)-Determination of Micronaire Rec. for Marking Codes for

Rec. for Tolerances on Spanner Gaps and Sockets (Metric smission Pulleys (1960) 4) Rec. for Limitin Rec. for Tables of Brinell Hardnes Rec. for Tables of Vickers Hardnes d Radial Groove Type Ball Bearings with Cylindrical Bore ies of Preferred Numbers and Those Containing More Rounded al Properties of Fasteners: Nuts with Specified Proof Load al Properties of Fasteners: Nuts with Specified Proof Load for Anaesthetic and Resuscitation Pur/ Rec. for Yoke Type 1969) Rec. for Tube and Rec. for Rating Systems for Electronic Tubes and for Quartz Crystal Units for Oscillators: Section 1-Std. ec for Tube and Valve Shields: Spec. Sheets for Tubes and lity, Examples of Resistors, Elements of Electronic Tubes, Rec. for High Pressure Air Charging lectrodes and Designation of Units in Electronic Tubes and direct Interelectrode Capacitances of Electronic Tubes and Rec. for Aircraft Tyre (Tire) Rec. for Dimensions of Electronic Tubes and rement of the Electrical Properties of Microwave Tubes and ments of the Electrical Properties of Electronic Tubes and rement of the Electrical Properties of Microwave Tubes and Rec. for Sockets for Electronic Tubes and rement of the Electrical Properties of Microwave Tubes and ments of the Electrical Properties of Electronic Tubes and rement of the Electrical Properties of Microwave Tubes and rement of the Electrical Properties of Microwave Tubes and rement of the Electrical Properties of Microwave Tubes and ments of the Electrical Proverties of Electronic Tubes and ments of the Electrical Properties of Electronic Tubes and ments of the Electrical Properties of Electronic Tubes and ments of the Electrical Properties of Electronic Tubes and ments of the Electrical Properties of Electronic Tubes and ments of the Electrical Properties of Electronic Tubes and ments of the Electrical Properties of Electronic Tubes and ments of the Electrical Properties of Electronic Tubes and ments of the Electrical Properties of Electronic Tubes and ments of the Electrical Properties of Electronic Tubes and ments of the Electrical Properties of Electronic Tubes and ments of the Electrical Properties of Electronic Tubes and ments of the Electrical Properties of Electronic Tubes and ments of the Electrical Properties of Electronic Tubes and ments of the Electrical Properties of Electronic Tubes and ments of the Electrical Properties of Electronic Tubes and ments of the Electrical Properties of Electronic Tubes and ments of the Electrical Properties of Electronic Tubes and ments of the Electrical Properties of Electronic Tubes and ments of the Electrical Properties of Electronic Tubes and pin Straighten/R Rec. for Sockets for Electronic Tubes and rement of the Electrical Properties of Microwave Tubes and ly Used for the Production of Aluminium-Determination of of Chemical Analysis of Manganese Ores: Determination of Rec. for Schedule for High Pressure Mercury Vapour Rec. for Ballasts for High Pressure Mercury Vapour Rec. for Plastics-Determination of the Water Vapou Rec. for Plastics-Determination of Water Vapour
Vacuum and a Substance (1966)

Vacuum Cleaners for Hing Appliances

Vacuum Cleaners (1970)

Vacuum Electronic Types (1966)

1. for Safety of Household and I and Valves: Meth. of IFC

Vacum Gauges, with Pressure Responsive Elements and $R e$

Vacuum Gauges, with Pressure Responsive Elements Giving

Vacuum Oven Meth. (1970)

Vacuum Oven Meth.) (1970

Vacuum Pulse Modulator Types (1970)

Vacuum Pumps-Part I: Volume Rate of Flow (Pumping Spe
Vacuum, and Vacuum Gauges, with Pressure Responsive Ele

Vacuum, and Vacuum Cauges, with Pressure Responsive Ele

Value-Potentiometric Meth. (1972)

Value After Acetylation (1971)

R Essential Oils- ISO

Value and Calculation of Content of Essential Oils (196

Value by the Calorimetric Bomb Meth. and Calculation of

Value for the Thermal Expansion Coefficient (For Use in

Value of the Fat (Reference Meth.) (1971)

Value (1971)

Value (1972)

Values and Tolerances of Resistors and Capacitors (1968

Std.

Values for General Use) (1968)

Values for the Adjustment of Centres (Centers) for Tran

Values (HB) for Use in Tests Made on Flat Surfaces (196

Values (HV) for Metallic Materials (1964)

Values (1961)

Values (1966)

Values (1969) Rec. for Guide to the Choice of Ser ISO Rec. for Mechanic ISO

alues, Effective Heights of Thread 0.4. D to Less Than

Valve Connections for Small Medical Gas Cylinders Used

Valve Shields: General Requirements and Meth. of Test (

Valves and Analogous Semiconductor Devices (1961)

Valves and Conditions: Section 2-Test Conditions (196

Valves and Dimensions of Test. Devices and Gauges (1969

Valves and Rectifiers (1964)

Valves for Aircraft (1969)

Valves (1961)

Valves (1962)

/Aphical Symbols: Variabi IEC

Rec for Numbering of $E$ IFC

Valves (1965)

Valves (1966)

Valves: Backward Wave Oscillator Tubes-0 Type (1972

Valves: Equivalent Input and Output Admittances (1963)

Valves: Gas Filled Microwave Switching Devices (1972)

Valves: General Requirements and Meth. of Test (1963)

Valves: General (1972)

Valves: Heater or Filament Current (1963)

Valves: High Power Klystrons (1972)

Valves: Low Power Oscillator Klystrons (1972)

Valves: Magnetrons (1972)

Valves: Measurement of Cathode Heating Time

Valves: Measurement of Electrode Current (1963)

for Measure IEC

Rec. for Measu IEC

/R Measu IEC

Rec. for Measu IEC

Valves: Measurement of Equivalent Noise Resistance (196

Valves: Meth. of Application of Mechanical Shock (Impul

Valves: Meth. of Measurement of Audio Frequency Output

Valves: Meth. of Measurement of Cross Modulation in (19

Valves: Meth. of Measurement of Emission Current from $\mathrm{H}$

Valves: Meth. of Measurement of Gasfilled Types (1969)

Valves: Meth. of Measurement of Noises Due to Mechanica

Valves: Meth. of Measurement of Radar and Oscilloscope

Valves: Meth. of Measurement of Radio Frequency Output

Valves: Meth. of Measurement of Spurious and Unwanted E

Valves: Meth. of Measurement of Thyratron Pulse Modulat

Valves: Meth of Measurement of Vacuum Pulse Modulator

Valves: Meth. of Measurement on Corona Stabilizers (196

Valves: Meth. of Measuring Electrode Resistance and Tra

Valves: Meth. of Measuring Hiss and Hum (1964)

Valves: Meth. of Measuring the Cathode Interface Impeda

Valves: Noise Factor (1963)

Valves: Precautions Relating to Meth. of Measurement (1

Valves: Spec. Sheets and Dimensions of Wiring Jigs and

Valves: Terminology (1972)

Vanadium Content-Photom

Vanadium Content (1966)

(Vapor) Lamps (1965)

(Vapor) Lamps (1969)

Vapor) Transmission Rate of Films and Thin Sheets-Dis Rec. for

(Vapor) Transmission Rate of Rigid Cellular Plastics (19
151.14

15 I-11

$15 \mathrm{I} \cdot 15$

151-20

151-23

151.19

151.12

$151-5$

151.9

151.4

151.0

149-2

235.1

1618

553

188

262

1195

1663 
Measurement of the Performance Characteristics of Vapour wrial (lue-l)etermination of the Content hy Volume in the Rec, for nic Tuhes, Valves and Rectifiers/ Rec. Graphical Symbols: numerically Controlled Machines (1) Rec. for Punched Tape rically Controlled/ Rec. for Interchangeable Punched Tape Measuring Meth. (I970) Rec. for Air Dielectric Rotary for Test Conditions for Milling Machines with Table of of d General Requirements (1972) (1972)

Rec. for Rec. for Rec. for Paints and Rec. for Paints and Rec. for Paints and Rec. for Paints and Rec, for Paints and Rec. for Paints and Rec. for Paints and rsion Meth. (1971)

by Flashpoint (Closed Cup Meth.) (19/ atter $(1970)$ ec. for Test Procedure for Thermal Endurance of Insulating test. (1970) Rec. for Paints and Rec. for Paints and Rec. for Paints and Rec. for Paints and Rec. for Paints and Rec. for Paints and aluation of the Thermal Endurance of Electrical Insulating Rec. for Sampling Raw Materials for Paints and Std. for Paints and Sid. for Paints and

$\operatorname{cup}(1972)$

e Floor Coverings: Determination of Dimensional Changes in ) urities (1968)

volatile Matter (1968)

for Analysis (1968)

hydrochloric Acid (1971)

(1968)

(1968)

lids (1968)

materials (1967) Rec. for Gymnastic Equipment Rec. for Crude Rec. for Crude Rec. for Crude Rec. for Crude Rec. for Fruit and Rec. for Fruit and Rec. for Fruit and Rec. for Fruit and Rec. for Fruit and Rec. for Fruit and Rec. for Fresh Fruits and Rec. or Power Supply for Air and Land Rec. for Rec. for Sound Signalling Devices on Motor Std for Braking of Motor ns (1967) Rec. for Dimensions of Motor Rec. for Mechanical Connections Between Towing Rec. for Lighting and Signalling for Motor for Electrical Connections Between Prime Movers and Towed ly for Private Motor/ Rec. for Electrical Connections for Rec. for Measurement of Noise Emitted by Rec. for Restraining Devices for Children in Motor sistors Used in the Power Circuits of Electrically Powered $r$ Rules for Rotating Electrical Machines for Rail and Road and Efficiency from Tests (Excluding Machines for Traction Rec. for Liquid Flow Measurement in Open Channels by r Determination of Errors in Measurement of Liquid Flow by Rec. for Plywood. st for the Determination of the Behaviour of Plastics in A ional Signs to Be Used in Schemes for the Installations of

Cast Iron Sanitary Pipes and Fittings for Waste Water and

for Cast Iron Sanitary Pipe Fittings for Waste Water and for Shipbuilding Details for Inland Navigation-Mushroom covered B/ Rec. for Measurement of Fluid Flow by Means of ks Vickers (1970) Rec. for Measurement of Fluid Flow by Means of

ks Rockwell B (1970)

ks Rockwell C (1970)

ks Brinell (1970)

International Rec. for International Rec. for International Rec. for International Rec. for Rec. for

tion and Use of Unsoiled/

Std. for Surface Active Agents: umidit/ International Rec. for Working Std. Meth. for the - Steels (1965)

achines (1968)

ness Test. Machines (1969)

ardened Cases (1973) Rec. for Rec. for Rec. for Std. for Steel: Determination and Std. for General Principles for the Rec. for ec. for Test Conditions for Surface Grinding Machines with packaging-Complete, Filled Transport Packages-Part IV: ing Machines with Table of Fixed Height with Horizontal or hines with Table of of Variable Height, with Horizontal or us Mechanical Handling Equipment for Loose Bulk Materials: s Mechanical Handling Equipment for Loose Bulk Materials .

s Mechanical Handling Equipment for Loose Bulk Materials g the Characteristics of Auxiliary Equipment for Shock and
(Vapor) Vacuum Pumps-Part I: Volume Rate of Flow (Pump Vaporized Product (1972) Var Hour (Reactive Energy) Meters (1963)

Variability, Examples of Resistors, Elements of Electro

Variable Block Format for Positioning and Straight Cut Variable Block Format Positioning and Straight Cut Nume Variable Capacitors: General Requirements for Tests and Variable Height, with Horizontal or Vertical Spindle-

Varnish Fabrics for Electrical Purposes: Definitions an Varnish Fabrics for Electrical Purposes: Meth. of Test Varnishes-Bend Test (Cylindrical Mandrel) (1970)

Varnishes-Cupping Test (1970)

Varnishes-Determination of Fineness of Grind (1971)

Varnishes-Determination of Flashpoint (Closed Cup Met Varnishes-Determination of Resistance to Water-Imme Varnishes-Determination of the Danger Classification Varnishes-Determination of Volatile and Nonvolatile $M$ Varnishes-Electric Strength Meth. (1971)

Varnishes-Examination and Preparation of Samples for

Varnishes-Pendulum Damping Test (1971)

Varnishes-Sampling (1970)

Varnishes-Scratch Test (1970)

Varnishes-Standard Panels for Test. (1970)

Varnishes-Surface Drying Test (Ballotini Meth.) (1970

Varnishes by the Helical Coil Bond Test (1969)

Varnishes (1968)

Varnishes: Crosscut Test (1972)

Varnishes: Determination of Flow Time by Use of a Flow

Varying Moisture Conditions (1972)

Vaulting and Pommelled Horses (1964)

Vegetable Oils and Fats: Determination of Acidity (1968

Vegetable Oils and Fats: Determination of Insoluble Imp

Vegetable Oils and Fats: Determination of Moisture and

Vegetable Oils and Fats: Preparation of Contract Sample

Vegetable Products-Determination of Ash Insoluble in

Vegetable Products-Determination of $\mathrm{pH}(1970)$

Vegetable Products: Determination of Mineral Impurities

Vegetable Products: Determination of Titratable Acidity

Vegetable Products: Determination of Total Solids (1969

Vegetable Products: Determination of Water Insoluble So

Vegetables: Sampling (1968)

Vehicle Mounted Prospecting Equipment for Radio Active

Vehicle Weights-Denominations and Definitions (1970)

Vehicles Acoustic Std, and Technical Spec. (1966)

Vehicles and Their Trailers-Terminology (1972)

Vehicles and Their Trailers: Designations and Definitio

Vehicles and Trailers-Interchangeability (1969)

Vehicles and Trailers (1963)

Vehicles with 24-V Electrical Equipment for Commercia

Vehicles with 6 or $12 \mathrm{~V}$ Electrical Equipment Particular

Vehicles (1964)

Vehicles (1970)

Vehicles (1970)

Vehicles (1971)

Vehicles) (1972)

Velocity Area Meth. (1968)

Velocity Area Meth. (1969)

Veneer, for General Use-Requir

Ventilated Tubular Oven (1969)

Ventilation Systems in Ships (1967)

Ventilation (1966)

Ventilations-Part IV: Fittings (1966)

Ventilators (1957)

Venturi Tubes-Classical Tubes Used Outside the Scope Venturi Tubes (1968)

Verification and Calibration of Hardness Reference Bloc

Verification and Calibration of Hardness Reference Bloc

Verification and Calibration of Hardness Reference Bloc

Verification and Calibration of Hardness Reference Bloc

Verification of Brinell Hardness Test. Machines (1967)

Verification of Certain Effects of Laundering-Prepara

Verification of Instruments for Measuring the ${ }^{\circ} \mathrm{Of} \mathrm{H}$

Verification of Pendulum Impact Test. Machines for Test

Verification of Rockwell B and C Scale Hardness Test. M

Verification of Rockwell Superficial N and T Scale Hard

Verification of the Effective Depth of Carburized and $\mathrm{H}$

Verification of the Safety of Structures (1973)

Verification of Vickers Hardness Test. Machines (1968)

Vertical Grinding Wheel Spindle and Reciprocating Table

Vertical Impact Test by Dropping (1972)

Vertical Spindle-Test. of Accuracy (1971)

Vertical Spindle-Test. of the Accuracy (1970)

Vibrating Conveyors and Feeders with Rectangular or Tra

Vibrating Feeders and Conveyors with Tubular Trough (19

Vibrating Feeders and Conveyors, Shaking or Reciprocati for Meth. for Specifyin IEC ISO

1608

45

$117-6$

1058

1057

334.

70

394-1

519

1520

1524

152.

152

1516

1515

370

1513

152

1512

1518

1514

1517

290

842

2409

2431

255

381

660

663

662

661

763

1842

762

750

1026

751

874

253

1176

611

612

1102

303

1185

1724

362

1713

322

349

34-2

748

1088

1098

1137

644

531

$531 / 1$

42

$781 / 1$

781

10

11

12

OIML 8

$\begin{array}{ll}\text { ISO } & 442\end{array}$

ISO

$\begin{array}{ll}\text { ISO } & 1079 \\ \text { ISO } & 2639\end{array}$

$\begin{array}{ll}\text { ISO } & 2639 \\ \text { ISO } & 2394\end{array}$

ISO $\quad 146$

ISO 1985

1985
2248

1984

1701

1049

1815

2125

222 
"toristies of Fileretro Merhanical Transducers for Shork and parkaging-Complete, Filled 'Transport Packages-Part Vi: Kœr. for liasic Finvironmental Test. Procedures: Test Fe: taye iland Filiers Intended for the Analysis of Sounds and for an IEC Mechanical Coupler for the Calibration of Bone td. Definitions and Meth. of Measurement for Piezoelectric Rec. for Plastics-Determination of the oads from 2.5 to $50 \mathrm{kgf}$ ) (1964) Rec. for 67)

Rec. for

Rec. for Light Metals and Their Alloys. rec. for Calibration of Standardized Blocks to Be Used for Rec. for Verification of Rec. for Tables of

64)

Verification and Calibration of Hlardness Reference Blocks rns of lmage Quality I/ Std. for Radiography of Welds and colou/ Std. for Photography-Illumination for Comparison s (1970) Rec. for Plastics-Determination of Rec. for Plastics Determination of Chlorine in Rec. for Plastics-Determination of Vinyl Acetate in corkwaste-Definitions and $\mathrm{Pa} /$ -Sampling (1972)

-Determination of Moistur/ Rec. for Commercially Dry Std. for Corkwood in Planks, Std. for Corkwood in Planks, sity of Natural and Synthetic Rubbers by the Shearing Disk $n$ of Rate of Cure of Rubber Compounds by the Shearing Disk

te in Dilute Soluti/

lute Solution (1970/

n (1963)

n (1961)

ing Disk Viscometer (1963) tion of Meth. For the Determination of the Dilute Solution Rec. for Determination of Rec. for Plastics-Determination of Viscosity Number and on (1967) Rec. for Plastics-Determination of the um Silicates for Industrial Use-Determination of Dynamic and Polypropylenes / Rec. for Plastics-Determination of
Photoconductive Cells for Use in the Std for Muscovite Mica Blocks, Thins and Films or the Evaluation of the Resistance of Plastic to Fungi by Industrial Use-Test for Impurities Insoluble in Water st for Impurities Insoluble in Sodium Hydroxide Solution. Metals-Measurement / Std. for Nonmagnetic Metallic and Rec. for Guide for the Preparation of Classified Rec. for Layout of Multilingual Classified Sid. for Dental Rec. for Paper

1 Properties) (1971)

bols (1970)

ls-Part 1: Statistical Vocabulary/

Vihration Measurements (1965) Vibration Test (1972)

Vibration (Sinusoidal) (1970) Vibrations (1966)

Vibrations (1966) /for Octave, Half-Octave and Third.O
Vibrators llaving a Specified Contact Area and Being App

Vicat Softening Temperature of Thermoplastics (1968)

Vickers Hardness Test for Copper and Its Alloys (Test L

Vickers Hardness Test for Steel (Load 5 to $100 \mathrm{kgf}$ ) (19

Vickers Hardness Test (Loads from 1 to $100 \mathrm{kgf}$ ) (1971)

Vickers Hardness Test. Machines (1967)

Vickers Hardness Test. Machines (1968)

Vickers Hardness Values (HV) for Metallic Materials (19

International

Viewing Conditions for Films: Utilization of Rec. Patte

Viewing of Photographic Transparencies with Reflection
Vinyl Acetate in Vinyl Chloride-Vinyl Acetate Copolymer

Vinyl Chloride Polymers and Copolymers (1970)

Vinyl Chloride (1970)

Rec. for

Vinyl Chloride-Vinyl Acetate Copolymers (1970)

Virgin Cork, Ramassage, Gleanings, Corkwood Refuse and

Virgin Cork, Ramassage, Gleanings, Refuse and Corkwaste

Virgin Cork, Ramassage, Gleanings, Refuse and Corkwaste

Viscometer (1963)

Viscometer (1968)

Rec. for Determination of Visco

Rec. for Determinatio

Viscosity Loss on Moulding of Cellulose Acetate (1970)

Viscosity Number and Viscosity Ratio of Cellulose Aceta

Viscosity Number of Poly (Ethylene Terephthalate) in Di

Viscosity Number of Polyamides Resins in Dilute Solutio

Viscosity Number of Polyvinyl Chloride Resin in Solutio

Viscosity of Natural and Synthetic Rubbers by the Shear

Viscosity of Polymers (1970)

Viscosity of Rubber Latices (1970)

Ives for the Standardiza

Viscosity Ratio of Cellulose Acetate in Dilute Solution

Viscosity Ratio of of Polyamides in Concentrated Soluti

Viscosity (1972)

Visible Spectrum, Meth. of (1970) /R Measurement of pH IEC

Visual Classification (1972)

Visual Examination (1968)

Visual Test (1971)

Visual Test (1971)

P-Cresol for Industrial Use-Te IS

ISO

Vocabularies (Example of Meth.) (1969)

Vocabularies (1969)

Vocabulary-List I-Basic Terms (1972)

Vocabulary-5Th Series of Terms (1970)

Vocabulary-6Th Series of Terms (Definitions of Optica

Vocabulary and Symbols-Second Series of Terms and Sym

Vocabulary and Symbols: First Series of Terms and Symbo

Vocabulary for the Refractories Industry (1968)

Rec. for Statistica

Rec. for Statistical Rec. for

International Rec, for

Rec. for

Rec. for

Rec. for

Rec, for

Rec. for Rec. for

art I: Coal Preparation (1970)

art II: Coal Sampling and Analysis (1971)

art IIl: Coke (1971)

s: First Series of Terms and Symbols-Part 1: Statistica

Rec for Surface Active Agents.

for Magnetic Compasses and Binnacles for Sea Navigation:

Rec. for Tolerances for Building .

Rec. for Modular Coordination

Rec. for Ropes and Cordage . Rec. for Electroplating and Related Processes Rec. for Industrial Trucks - Wheels and Castors Std. for Rubber Rec. for Plywood:

Std for Test Sieves and Sieving: Std. for Starch, Including Derivatives and by -Products:

8 (1966)

tion by Electric/

0) Rec. for International Electrotechnical Rec for International Electrotechnical Rec. for International Electrotechnical Rec. for International Electrotechnical Rec. for International Electrotechnical Rec. for International Electrotechnical Rec for Electrotechnical Rec. for International Electrotechnical Rec. for Paper Rec. for Paper

Rec for Internation al Electrotechnical Rec. for International Electrotechnical Rec. for International Electrotechnical Rec. for International Electrotechnical Rec. for International Electrotechnical
Vecabulary of Legal Metrology (Fundamental Terms) (1969

Vocabulary of Terminology (1969)

Vocabulary of Terms and Symbols Used in Connection with ocabulary of Terms Relating to Pallets (1965)

Vocabulary of Terms Relating to Solid Mineral Fuels-P

Vocabulary of Terms Relating to Solid Mineral Fuels-P

Vocabulary of Terms Relating to Solid Mineral Fuels-P

Vocabulary (1967)

Vocabulary (1969)

Vocabulary (1969)

Vocabulary (1970)

Vocabulary (1971)

Vocabulary (1971)

Vocabulary (1971)

Vocabulary (I971)

Vocabulary (1972)

Vocabulary (1972)

Vocabulary (1972)

Vocabulary (1973)

Vocabulary: Automatic Controlling and Regulating System Vocabulary: Detection and Measurement of Ionizing Radia Vocabulary: Electric Traction (1957)

Vocabulary: Electroacoustics (1960)

Vocabulary: Electrobiology (1959)

Vocabulary: Electrochemistry and Electrometallurgy (196

Vocabulary: Electroheating Applications (1960)

Vocabulary: Electromechanical A pplications (1958)

Vocabulary: Electronics (1956)

Vocabulary: First Series of Terms (1958)

Vocabulary: Fourth Series of Terms (1964)

Vocabulary: Fundamental Definitions (1956)

Vocabulary: General Index (1970)

Vocabulary: Generation, Transmission and Distribution $O$

Vocabulary: Lighting (1970)

Vocabulary: Machines and Transformers (1956) 
Rer. for International Electrotechnical Ror. for International Electrotechnical Rer. for International Electrotechnical Rer. for International Electrotechnical Rec. for International Electrotechnical Rec. for Paper

ents (19.58)

ays (19.59)

nd Regulation (I/

Rec. for International Electrotechnical Rec. for International Electrotechnical Rec. for International Electrotechnical Rec. for International Electrotechnical Rec. for Paper Rec. for International Electrotechnical Rec. for International Electrotechnical acid for Industrial Use-Determination of Low Contents of Rec. for Paints and Varnishes-Determination of Rec. for Determination of Rec. for Determination of Rec. for Determination of the Rec for Plastics-Pyc Resins-Determination of Rec. for Oleaginous Seeds: Determination of Moisture and Rec. for Analysis of Soaps: Determination of Moisture and Rec. for Oilseed Residues: Determination of Moisture and ude Vegetahle Oils and Fats: Determination of Moisture and Rec. for Animal Fats: Determination of Moisture and d. for Styrene Butadiene Rubber Latices-Determination of ide for IndustriaI Use: Determination of Chloride Content: ate for Industrial Use: Determination of Chloride Content: ide for IndustriaI Use: Determination of Chloride Content: Rec. for Requirements for 28 reak Switch Disconnectors and Fuse Combinal/ Rec. for Low d Construction (1971)

nd Definitions (197I)

on to Be Given with Enquiries Tenders and /

97 I)

the Selection of (For Service) (1971)

routine Tests (I972)

Rec. for High Rec. for High Rec. for High Rec. for High Rec. for High Rec. for IIigh Television Receivers (1965) 71 Rec. for High Rec. for Low Rec. for Low Rec. for Low

I and Similar Purposes: General Requirement/ for Std. Rated Currents ( 2 to $63 \mathrm{~A}$ ) of Fuse Links for Low Rec. for High Rec. for High eramic Material or GIass for Overhead Lines with a Nominal arthed) (1960) Rec. for

Rec, for High Rec. for Low Rec. for Low

ible Cables and Cords with Circular Conductors and a Rated

ible Cables and Cords with Circular Conductors and a Rated emperature of I/ Rec. for Connecting Wires Having a Rated estic and Similar Purposes (1959) Spec. for

ncies Below 3 MHz: Connectors for Coupling an External Low ear Instruments (I970)

Rec. for Analogue Rec. for ${ }^{\circ}$ Of Protection of Enclosures for Low cuits, Including Contacto/ cuits, lncluding Contacto/ Rec. for Control Switches (Low Rec. for High Rec for istance of an a.c. Machine During Operation at Alternative Rec. for Bushings for Alternating Rec. for Rec. for Supply

d Metal Sheathed Cables: Nondraining Types for Alternating ted Paper Insulated Metal Sheathed Cables: for Alternating ndoor and Outdoor Post Insulators for Systems with Nominal outdour Post Insulators and Units for Systems with Nominal

9) Rec. for Industrial Trucks: essories: Paper Insulated, Metal Sheathed, for Alternating re Cables and Their Accessories: Internal, for Alternating re Cables and Their Accessories: External, for Alternating Rec. for IEC Std. ng Materials (1958) Rec. for Electronic Rec. Meth. of Test for orine for Industrial Use-Determination of the Content by the Permeability of Vulcanized Rubbers to Gases (Constant cteristics of Positive Displacement Vacuum Pumps-Part l: e Characteristics of Vapour (Vapor) Vacuum Pumps-Part I: sured Surface Pile Density and Measured Pile Fibre (Fiber) test-Part 1: Calculation of Linear Dimensions, Areas and dustrial Use-Meth. of Sampling (For Determining Only the Rec. for Chemical Analysis of Zinc Alloys .
Rec. for 1 Mark

International Rec. for General Prescriptions for
Vocabulary: Nuclear Power Plants for Electric Energy Ge Vocabulary: Protective Relays (19.56)

Vocabulary: Radiocommunications (1970)

Vocabulary: Radiology and Radiological Physics (1964)

Vocabulary: Scientific and lndustrial Measuring lnstrum

Vocabulary: Second Series of Terms (1959)

Vocabulary: Signalling and Security Apparatus for Railw

Vocabulary: Static Convertors (1956)

Vocabulary: Switchboards and Apparatus for Connection a

Vocabulary: Telegraphy and Telephony (1970)

Vocabulary: Third Series of Terms (196I)

Vocabulary: Transducers (1955)

Vocabulary: Waveguides (1961)

Volatile Acids Other Than Formic (Less Than $0.5 \%(\mathrm{~m} / \mathrm{m})$

Volatile and Nonvolatile Matter (1970)

Volatile Fatty Acid Number of Latex (I966)

Volatile Matter in Raw Natural Rubber (1962)

Volatile Matter of Hard Coal and of Coke (1967)

Volatile Matter (Including Water) (1970)

Volatile Matter (1968)

Volatile Matter (1968)

Volatile Matter (1968)

Volatile Matter (1968)

Volatile Matter (I969)

Volhard Volumetric and Residual Styrene (1972)

Volhard Volumetric Meth (1973)

Volhard Volumetric Meth (1973) IS for Sodium lHydrox

Volt d.c. Flat Strip Fuses for Aircraft (1968)

Voltage Airbreak Switches, Airbreak Disconnectors, Airb

Voltage Alternating Current Circuit Breakers: Design an

Voltage Alternating Current Circuit Breakers: General a

Voltage AIternating Current Circuit Breakers: Informati

Voltage Alternating Current Circuit Breakers: Rating (1

Voltage Alternating Current Circuit Breakers: Rules for

Voltage Alternating Current Circuit Breakers: Type and

Voltage Cables (1965)

Voltage Connecting Wire with Flame Retarding Insulation

Voltage Controlgear for Industrial Use: Contractors (19

Voltage Distribution Switchgear: Circuit Breakers (1964.

Voltage Fuses with High Breaking Capacity for Industria

Voltage Fuses (1957)

Voltage Fuses: Current Limiting (1965)

Voltage Fuses: Expulsion and Similar Types (1970)

Voltage Greater Than $1000 \mathrm{~V}$ (1972)

IEC

Voltage Measurement by Means of Sphere Gaps (1 Sphere E IEC

Voltage Metal Enclosed Switchgear and ControIgear (1969 IEC

Voltage Motor Starters: Direct on Line, a.c. (I971)

Voltage Motor Starters: Reduced, a.c.: Star Delta (I970

Ber Insulated Flex IEC

Voltage Not Exceeding $750 \mathrm{~V}(1967)$

IIde Insulated Flex IEC

Voltage Not Exceeding $750 \mathrm{~V}$ (1967)

Voltage Operated Earth Leakage Circuit Breakers for Dom

Voltage Power Supply to Portable Entertainment Equipmen

Voltage Ranges and Logic Levels for Mains Operated Nucl

Voltage Switches (1968)

Voltage Switchgear and Controlgear (1963)

Voltage Switching Devices for Control and Auxiliary Cir

Voltage Switching Devices for Control and Auxiliary Cir

Voltage Test Techniques (1962)

Voltage Transformers (1970)

Voltage (1969)

(Ec. f

Voltages Above $1,000 \mathrm{~V}(1962)$

Voltages for Aircraft Electrical Systems (1961)

Voltages for Transistorized Nuclear Instruments (1968)

Voltages from $10 \mathrm{kV} \mathrm{Up} \mathrm{to} \mathrm{and} \mathrm{Including} 33 \mathrm{kV}$ (Excludin

Voltages from $10 \mathrm{kV} \mathrm{U}$ to and Including $66 \mathrm{kV}$ (Excludin

Voltages Greater Than 1,000 V (1964)

Voltages Greater Than $1,000 \mathrm{~V}$ (1968)

Voltages of Traction Batteries for Electric Trucks (196

Tests on I IEC

Voltages Up to $275 \mathrm{kV}(1963)$

Voltages Up to $275 \mathrm{kV}(1967)$

Voltages Up to $275 \mathrm{kV}$ (1967)

Voltages (I967)

Voltmeters (1967)

Volume and Surface Resistivities of Electrical Insulati

and Their AcC ISC

II Filled and Gas Pressu IEC

II Filled and Gas Pressu 1EC

Volume in the Vaporized Product (1972)

Volume Meth.) (1971) Rec, for Determination of ISO

Volume Rate of Flow (Pumping Speed) (1970) /Ance Chara ISO

Volume Rate of Flow (Pumping Speed) (1970) /Performanc ISO

Volume Ratio (1971) /or Carpets-Determination of Mea ISO

Volumes (1971) Cial Refrigerated Cabinets-Meth. of 1SO

Volumetric Content) (1970) /for Liquid Chlorine for in 1SO

Volumetric Determination of Aluminium (Aluminum) (I970) ISO

Volumetric Flasks (1971)

Volumetric Gas Meters (1970)

OIML 
H.c. for P'rincipless of Construction and Adjustment of Volumetric Glassware (1964) industrial I se: Determination of Chloride Content: Volhard $n$ of $M$ oisture in the Analysis Sample of Coal hy the Direc - Arid for Industrial Use: Determination of Total Aridity d for Industrial Use-Determination of Sulphate Content for Industrial Use-Determination of of Calcium Content

ndustrial Use: Determination of Sodium Hydrogen Carbonate industrial Use: Determination of Total Soluble Alkalinity: ial Use: Determination of Oxidizing or Reducing Substances al Acidity and Calculation of Free $\mathrm{SO}_{3}$ Content of Oleum $r$ Industrial Use: Determination of Total Nitrogen Content: hydroxide for Industrial Use: Determination of Alkalinity, tion of Moisture in Brown Coals and Lignites by the Direct

hydroxide for Industrial Use: Determination of Alkalinity: rbon Dioxide Content, Expressed as Sodium Carbonate, Gas n Dioxide Contcnt, Expressed as Potassium Carbonate: Gas for Urea for Industrial Use-Determination of Nitrogen for Urea for Industrial Use-Determination of Alkalinity ysis of Magnesium and Its Alloys-Determination of Zinc

minium (Aluminum) and Its Alloys-Determination of Zinc

Industrial Use-Determination of Alkalinity or Acidity

e Content Expressed as Sodium or Potassium Carbonate-Gas ion of Total Alkalinity Expressed in $\mathrm{Na}_{2} \mathrm{O}$ or in $\mathrm{K}_{2} \mathrm{O}$ itric Acid for Industrial Use-Determination of Acidity

for Industrial Use-Determination of Nitrous Compounds

Use-Determination of Content-Sodium Tatraphenylborat

Than Formic (Less Than $0.5 \%(\mathrm{~m} / \mathrm{m})$ Calculated as Acetic)

c Acid for Industrial Use-Determination of lts Content

Oxide for Industrial Use-Determination of Its Content

itrate for Industrial Use: Determination of Free Acidity

1 Use-Determination of Sodium and Boric Oxide Contents of Sodium and Boric Oxide and Available Oxygen Contents

for Industrial Use: Determination of Sulphur Compounds Std. for Iron Ores: Determination of Phosphorus Content including Foodstuffs): Determination of Total Alkalinity

I Use: Determination of Content-Sodium Tetraphenylborate on of Aluminium (Aluminum) Content-Oxine Gravimetric and industrial Use: Determination of Chloride Content: Volhard industrial Use: Determination of Chloride Content: Volhard Industrial Use-Determination of Total Alcohols Content Rec. for Time Lapse Between Rec. for Determination of Copper in Compounded Rubber Piece) (1957) Rec. for Determination of Tear Strength of Rec. for Determination of Resistance to Flex Cracking of Rec. for Determination of Resistance to Crack Growth of olytic Meth. (1971)

aration of Test Piece and Meth. of Test of the Adhesion of aration of Test Piece and Meth. of Test of the Adhesion of nce Tests (1971)

e-Lupke Pendulum Meth. (1971) quids (1971)

Rec for Determination of the Stiff noss 971)

1)

Rec. for Determination of Hardness of Rec. for Determination of Hardness of

Rec. for Determination of the Permeability of shear Test) (1971) Rec. for Determination of Adhesion of Rec. for Determination of the Adhesion Strength of peratures (/ Rec. for Determination of Compression Set of (1971) Rec. for Determination of Modulus in Shear of for Determination of Tear Strength of Small Test Pieces of Rec. for Determination of llardness of

for Determination of Tensile Stress Strain Properties of

for Meth. of Test for Temperature Limit of Brittleness for der Constant Deflection at Normal and Iligh Temp/ Rec. for Rec. for Rotary Wafer Switches (Low Current Rating) ons and Diameter $10 \mathrm{~mm}$ (1966)

ns and Diameter $60 \mathrm{~mm}$ (Low Current Ratin/ Rec. for Rotary g (1963)

ts and Measuring Meth. (1962)

h Central Mounting (1963)

Rec for Rotary

Rec. for Rotary Rec. for Rotary Appliances: Particular Requirements for Toasters, Grills, and Heating Appliances for Domestic and Similar Purposes: of Sampling Manganese Ores; Part I: Ore Loaded in Freigh sion Welded Butt Joints in Steel Pipes Up to $50 \mathrm{~mm}$ (2 In.) he Transport of Fluids-Polyethylene (PE)-Tolerances on plasticized Polyvinyl Chloride (PVC) Pipes-Tolerances on Rec for Steel Tubes

Rec. Meth. of Measurement for Radio Transmitters and Valves: Measurement of Cathode Heating Time and Heater s for Domestic and Similar Purposes: Waffle Irons, Grills, Rec for Sectional Beams for Rec. for Drop Wires for Mechanical and Electrical ions of the Cross Section of Assembled Bars for Mechanical In. Dimensions, Tolerances and Gauges (1963) Metric Dimensions, Tolerances and Gauges (1964/ Volumetric Meth. (1968) Rec. for

Volumetric Meth (1969)

Volumetric Meth. (196.3) Volumetric Meth. (1968) Volumetric Meth. (1968) Volumetric Meth. (1968) Volumetric Meth. (1968) Volumetric Meth. (1968) Volumetric Meth. (1968) Volumetric Meth. (1968) Volumetric Meth. (1969) Volumetric Meth. (1969) Volumetric Meth. (1969) Volumetric Meth. (1969) Volumetric Meth. (1969) Volumetric Meth. (1970) Volumetric Meth. (1970) Volumetric Meth. (1970) Volumetric Meth. (1970) Volumetric Meth. (1970) Volumetric Meth. (1970) Volumetric Meth. (1970) Volumetric Meth. (1971) Volumetric Meth. (1971) Volumetric Meth. (1971) Volumetric Meth. (1971) Volumetric Meth. (1972) Volumetric Meth. (1972) Volumetric Meth. (1972) Volumetric Meth. (1972) Volumetric Meth. (1972) Volumetric Meth. (1972) Volumetric Meth. (1973) Volumetric Meth. (1973) Volumetric Meth. (1973) Volumetric Meth. (1973) Volumetric Meth. (1973)

Volumetric Meth. (1973)

Volumetric Meth.) (1970)

Vulcanization and Test. of Rubbers (1970)

catural and Synthetic Rubbers (Crescent Test

Vulcanized Natural or Synthetic Rubber (De Mattia Type

Vulcanized Natural or Synthetic Rubber (De Mattia Type

Vulcanized Rubber-Determination of Carbon Black-Pyr

Vulcanized Rubber to Metal Where the Rubber Is Assemble

Vulcanized Rubber to Metal Where the Rubber Is Assemble

Vulcanized Rubbers-Accelerated Ageing or Heat Resista

Vulcanized Rubbers-Determination of Rebound Resilienc

Vulcanized Rubbers-Determination of Resistance to $\mathrm{Ozo}$

Vulcanized Rubbers-Meth. of Test for Resistance to $\mathrm{Li}$

Vulcanized Rubbers at Low Temperature (Gehman Test) (19

Vulcanized Rubbers of High Hardness (85 to 100 IRHD) (1

Vulcanized Rubbers of Low Hardness (10 to 35 IRHD) (197

vulcanized Rubbers to Gases (Constant Volume Meth.) (19

Vulcanized Rubbers to Rigid Plates in Shear (Quadruple-

Vulcanized Rubbers to Textile Fabrics (1969)

Vulcanized Rubbers Under Constant Deflection at Low Tem

ulcanized Rubbers (Bonded Quadruple Shear Test Piece)

Vulcanized Rubbers (Delft Test Piece) (1968)

Vulcanized Rubbers (1968)

Vulcanized Rubbers (1968)

Vulcanized Rubbers (1968)

Vulcanized Rubbers: Determination of Compression Set Un

Wafer Switches with Central Mounting (1963)

Wafer Switches with Central Mounting; Maximum 12 Positi

Wafer Switches with 2 Hole Mounting; Maximum 26 Positio

Wafer Switches (Low Current Rating) with 2 Hole Mountin

Wafer Switches (Low Current Rating): General Requiremen

Wafer Switches (Low Current Rating): Wafer Switches Wit

W affle Irons and Roasters (1972)

Waffle Irons, Grills, Warming Plates and Other Dry Cook

Wagons (1963)

Rec for Meth. ISO

Wall Thickness (1969) /Nspection of Circumferential Fu ISO

Wall Thicknesses Up to $6 \mathrm{~mm}$ (1970) /Astics Pipes for T ISO

Wall Thicknesses Up to $6 \mathrm{~mm}(1970)$

Wall Thicknesses (1971)

/Ort of Fluids-Un IS

Wanted and Unwanted Modulation (1971)

/L Properties of Electronic Tubes

Warm Up Time (1966)

Cooking Appliances (1967)

Warp Knitting Machines: Terminology and Dimensions (196

Warp Stop Motions (1965)

Warp Stop Motions (1971)

ISO

Warp Tubes for Ring Spinning and Ring Doubling Spindles Rec for Warp Tubes for Ring Spinning and Ring Doubling Spindles ISO 

similar Electrical Appliances: Particular Requirements for urface Active Agents: Determination of Apparent Density of cket Fittings with Spigot Ends for Domestic and Industrial Rec. for Cast Iron Sanitary Pipes and Fittings for Rec. for Cast Iron Sanitary Pipe Fittings for

eneral Use (1972)

$$
\text { Std. for Water Resistant }
$$

Rec. for Antimagnetic

for Paints and Varnishes-Determination of Resistance to enol for Industrial Use-Test for Impurities Insoluble in Std. for Fibre (Fiber) Building Boards: Determination of Rec. for Determination of the Rec. for Plastics-Determination of Rec. for Plastics-Determination of Boiling tion of Solution of Products Not Easily Soluble in Boiling Rec for Steel Pipes for Gas,

Rec. for Cast Iron Sanitary Pipes and Fittings for Waste Rec. for Cast Iron Sanitary Pipe Fittings for Waste e for Industrial Use: Determination of Matter Insoluble in meth. for the Determination of Residue on Evaporation on A ic Acid and Xylenols for Industrial Use-Determination of Rec. for Determination of

ic Acid and Xylenols for Industrial Use-Determination of for Liquid Chlorine for Industrial Use-Determination of Std. for Gylcerols for Industrial Use-Determination of for Higher Alcohols for Industrial Use-Determination of Rec. for Plastics-Determination of the

for Liquid Chlorine for Industrial Use-Determination/ ical Analysis of Manganese Ores: Determination of Combined Rec. for Tea-Determination of Rec. for Meth. for Measuring Performance of domestic and Similar Purposes: Stationary Noninstantaneous Rec. for General Purpose Rubber Rec. for Spices and Condiments: Determination of for Sodium Hydroxide for Industrial Use: Determination of r Potassium Hydroxide for Industrial Use: Determination of

Rec. for Fruit and Vegetable Products: Determination of Std. for Aircraft Rec. for Aircraft Connection for

Rec. for Determination of the Impermeability to e for Industrial Use: Determination of Matter Insoluble in ec. for Rubber Sealing Rings for Joints in Asbestos Cement

Std. for Water Resistant Watches-Part I: Wristwatches . resistance for General Use (1972) Std. for

Rec for Tea. for Tea-Determination of

Rec. for Spices and Condiments: Determination of Cold ces for Domestic and Similar Purposes: Vacuum Cleaners and n Sheets-Dish/ r Plastics (1970)

$$
\text { Rec. for Plastics-Determination of the }
$$
Rec. for Plastics-Determination of

Std. for Surface Active Agents-Preparation of Rec. for Animal Fats: Determination of

Meth. of Test for Resistance of Fabrics to Penetration by for Industrial Use-Determination of Matter Insoluble in Surface Active Agents: Determination of Stability in Hard Rec. for Plastics-Pve Resins-Sieve Analysis in Std. for Leather: Determination of Absorption of in Boiling Water and Determination of Matter Insoluble in absorption and of Swelling in Thickness After Immersion in e for Industrial Use: Determination of Matter Insoluble in International Rec. for Meters for Liquids (Other Than

- Pvc Resins-Determination of Volatile Matter (Including tion of Bro/ Rec. for Determination of the Yields of Tar, Rec. for Alternating Current Rec. for Class 1.0 Alternating Current Rec. for Class 0.5 Alternating Current ctrical Properties of Microwave Tubes and Valves: Backward Rec. for Guide for Choosing Modular Dimensions for Rec. for Sealing Test for Pressurized

964)

Rec. for International Electrotechnical Vocabulary:

964)

es (1969)

ular Types (1968)

tangular Types (1968)

1964)

(1968)

types (1967)

es (1964)

power, Audio and Radio Frequencies Including Metre (Meter)
Warping Machines-Maximum Usable Width

Washers for Hexagon Bolts and Nuts: Metric Series (1968

Washing Machines (1971)

Washing Powders Before and After Compacty of Hou

Waste Pipe: Basic Dimensions; Metric Series (1962)

Waste Water and Ventilation (1966)

Waste Water and Ventilations-Part IV: Fittings (1966)

Watches-Part I: Wrist watches-Water Resistance for $G$

Watches for General Purpose Use (1968)

Water-Immersion Meth. (1971)

Water-Visual Test (1971)

Water Absorption and of Swelling in Thickness After Imm for pH ISO

Water Absorption of Paper or Board (Cobb Meth.) (1967) ISO

Water Absorption (1958)

Water Absorption (1959)

Water and Determination of Matter Insoluble in Water (1

Water and Sewage, Welded or Seamless (1967)

Water and Ventilation (1966)

Water and Ventilations-Part IV: Fittings (1966)

Water at $50^{\circ} \mathrm{C}(1968) \quad$ Rec. for Sodium Carbonat ISO

Water Bath (1968)

Water by the Dean and Stark Meth. (1971)

Water by the Karl Fischer Meth. (1968)

Water by the Karl Fischer Meth. (1971)

Water Content-Gravimetric Meth. (1972)

Water Content-Karl Fischer Meth. (1972)

Water Content by the Karl Fischer Meth. (1970)

Water Content in Polyamides (1969)

Water Content Using an Electrolytic Analyser (1972) Std IS

Water Content (1966) Rec. for Meth. of Chem ISO

Water Extract (1970)

Water Heaters for Household Purposes (1972)

King and Heating Appliances for $\mathrm{CEE}$

Water Heaters (1966)

Water Hose (1970)

Water Insoluble Ash (1969)

Water Insoluble Matter (1969)

Water Insoluble Matter (1969)

Water Insoluble Solids (1968)

Water Methanol Pressure Connections (1973)

Water of Drinkable Quality (1965)

Water of Fabrics Coated with Rubber or Plastics (1970)

Rec. for ISO

/Esol, Cresyl ISO

/Cresol, Cresyl ISO

Std. ISO

ISO

Water or in Acid and Preparation of Principal Solutions

Water Piping (1970)

Water Resistance for General Use (1972)

Water Resistant Watches-Part I: Wristwatches-Water

Water Soluble and Insoluble Ash (1970)

Water Soluble Ash (1970)

Water Soluble Extract (1969)

Water Suction Cleaning Appliances (1965)

Water Vapour (Vapor) Transmission Rate of Films and Th

Water Vapour (Vapor) Transmission Rate of Rigid Cellula

Water with Known Calcium Hardness (1972)

Water (Entrainment Distillation Meth. (1969)

Water (Hydrostatic Head Test) (1968)

Water (1968)

Water (1969)

Water $(1970)$

Water (1972)

Water (1972)

Water (1972)

Water (1973)

Water) with

Water) (1970)

Water, Gas and

Watthour Meters (1960)

Watthour Meters (1964)

Watthour Meters (1968)

Wave Oscillator Tubes-0 Type (1972)

Waveguide Components (1971)

Waveguide Tubing and Assemblies (1968)

Waveguides (1961)

Waveguides: General Requirements and Measuring Meth. (

Waveguides: General Requirements and Measuring Meth. (1

Waveguides: Relevant Spec. for Circular Types (1964)

Waveguides: Relevant Spec. for Flanges for Circular Typ

Waveguides: Relevant Spec. for Flanges for Flat Rectang

Waveguides: Relevant Spec. for Flanges for Ordinary Rec

Waveguides: Relevant Spec. for Flat Rectangular Types (

Waveguides: Relevant Spec. for Medium Flat Rectangular

Waveguides: Relevant Spec. for Medium Flat Rectangular

Waveguides: Relevant Spec. for Ordinary Rectangular Ty

Wavelengths (1969)

/Lectrical Insulating Materials at Wax (1970)

Weaving Looms (1968)
481

2012

887

$10-2 \mathrm{~J}$

$335-7$

697

265

531

$531 / 1$

2281

764

1521

1905

769

535

62

117

2122

559

531

$531 / \mathrm{I}$

746

89

1898
760

1897

2121

2097

1849

960

2202

549

1574

379
$11.2 \mathrm{E}$

$11-2 \mathrm{E}$

1403

929

987
998

751

751
485

450

1420

2479

2479
1398

2281

2281

1576

1578

941

$10.2 \mathrm{~A}$

1195

1663

2174

934

811

850

1063

1624

2417

2122

769

2461

5

1269

647

43

170

280

235.8

374

261

$50(62)$

153-1

154-1

153.4

154-4

154

154-2

153-3

153-5

153-6

153-2

250

156

109 
ions of Pirn Tip (1969)

Rec. for

olyethylene Glycols and Nonionic Active Matter (Adducts).

ational Rec. for Metrological Regulations for Nonautomatic ferrous Materials by Chemi/ Rec. for Determination of the Rec. for Vehicle accuracy Class) (1970/ ) (1972)

International Rec. for Cylindrical for Meth. for Determining the Mechanical Properties of the meth. of Test for Determining the Mechanical Properties of Rec. for Definition of oys / Std. Rec. Pract. for the X-Ray Inspection of Fusion Thick/ Rec. Pract. for Radiographic Inspection of Fusion std. for Rec. Pract. for Radiographic Inspection of Fusion act. for Radiographic Inspection of Circumferential Fusion Rec. for Strength Calculation of Butt sses Per Unit Length (19/ Rec. for Plain End Steel Tubes, Rec. for Steel Pipes for Gas, Water and Sewage, for Diameters and Tolerances for Electrodes for Manual Arc Rec. for Steel Tubes-Butt Rec. for Steel Tubes: Butt

Rec. for Dimensions of Straight Resistance Spot Rec. for Dimensions of Straight Resistance Spot Rec. for Rating of Resistance Rec. for Rating of Manual Are Rec. for Ignitrons to Be Used in

dunensions and Pitching of Slots on Platens for Projection

Rec. for Dimensions of Tubular Electrode Holders for Spot

Properties of Weld Metal Deposited by Filler Rods for Gas ode of Symbols for Covered Electrodes for Manual Metal Arc for Identific/

Std. for Covered Electrodes for Manual Arc

(1) Rec. for Lengths and Tolerances of Electrodes for the (1967) Rec. for Code of Symbols for Covered Electrodes for Arc and Co/ Rec. for Solid Wires for Gas Shielded Metal Arc atibility of Steel Filler Rods and the Parent Metal in the for Straight Welds (1967) Rec. for Fundamental
R Rec. for Limitation of Angles of Slope and Rotation for Rec, for Definitions of Rec. for Dimensions of Seam erances for Filler Rods, Other Than Drawn or Extruded, for lectrodes for Manual Arc Welding and Filler Metals for Gas

e Principal Radiographic Terms Used in ISO Rec. Concerning Rec. for Definition of Weldability

ngths and Tolerances for Drawn or Extruded Filler Rods for jes of the Deposited Meta/

Bond Strength on Steel, nd Related Processes (1972)

iller Rods and the Parent M/ rec. Patterns of Image Quality I/

Equipment for Ultrasonic Examination (1972)

Rec. for Filler Rods for Braze Filler Rods for Braze Spec. for

Rec. for Filler Rods for Gas Std. for Radiography of lions and Definitions of Rotation and Slope for Straight

ppliances: Particular Requirements for Floor Treatment and Rec. for Dimensions of Seam Welding rec. for Railway Rolling Stock Material-Rolled on Forged red) Whee/ Rec. for Railway Rolling Stock Material-Cast Rec. for Bonded Abrasive Products-Grinding Rec. for Bonded Abrasive Products: Grinding Rec. for Semitrailer (Trailer) 5Th Rec. for Railway Rolling Stock Material: tions for Surface Grinding Maclines with Vertical Grinding ons for Surface Grinding Machines with Horizontal Grinding 1968) Rec. for 3 Point Linkage of Agricultural Std. for Industrial Rec. for Conveyor Chains, Autachments and Chain Rec. for Industrial Trucks

Rec, for Mounting of Plain Grinding

Rec. for Railway Rolling Stock Material: Solid heel Centres (Centers) in Nonalloy Steel for Tyred (Tired) rolled on Forged Wheel Centres (Centers) for Tyred (Tired)

Std. for Extended Pitch Precision Roller Chains and Chain c. for Shipbuilding Details for Inland Navigation Sprocket Roller Chains Type S 32 to 88 with Their Associated Chain short Pitch Transmission Precision Roller Chains and Chain $r$ Short Pitch Transmission Precision Bush Chains and Chain Rec. for Meth. of Test for Determining icating the Stability of the Images of Processed Black and
Weaving Looms: Definition of Side (Left or Right) (1959

Weaving Preparatory Machines-Definition of Side (l.eft

Weaving (1967)

Weaving (1967)

Weeks (197I)

eft Pirns for Automatic Looms (1960)

Weft Pirns for Box Loaders for Automatic Looms-Dimens

Weibull Meth. (1972)

Weighing Machines (1972)

Intern OIM

Weight Per Unit Area of Hot Dip Galvanized Coatings on

Weights-Denominations and Definitions (1970)

Weights from 1 Gramme (Gram) to 10 Kilogrammes (Medium

Weights from 5 to 50 Kilogrammes (Medium Accuracy Class

Weld Metal Deposited by Electrodes $3.15 \mathrm{~mm}$ or More in D

Weld Metal Deposited by Filler Rods for Gas Welding Mil

Weldability (Welding) (1967)

Welded Butt Joints for Aluminium (Aluminum) and Its All

Welded Butt Joints for Steel Plates Up to $50 \mathrm{~mm}$ (2 In.)

Welded Butt Joints for Steel Plates 50 to $200 \mathrm{~mm}$ Thick

Welded Butt Joints in Steel Pipes Up to $50 \mathrm{~mm}$ (2 In.) W

Welded Joints (1967)

Welded or Seamless-General Table of Dimensions and Ma

Welded or Seamless (1967)

Welding and Filler Metals for Gas Welding (1967)

Welding Bends 5 D $\left(90^{\circ}\right.$ And 180 Deg.) (1969)

Welding Bends $\left(90^{\circ}\right.$ And 180 Deg.) (1962)

Welding Electrodes (For Loads Greater Than $1500 \mathrm{kgf}$ (1

Welding Electrodes (1968)

Welding Equipment (1968)

Welding Equipment (1968)

Welding Machine Control (1967)

Welding Machines (1968)

Welding Machines (1969)

Welding Mild Steels and Low Alloy High Tensile Types (1

Welding of Cast Iron (1969)

Welding of Mild and Low Alloy Steels-Code of Symbols

Welding of Mild Steel and Low Alloy High Tensile Steel

Welding of Mild Steels and Low Alloy High Tensile Types

Welding of Mild Steels and Low Alloy High Tensile Types

Welding of Mild Steel: Dimensions of Wires, Spools, Rim

Welding of Steels (1968)

Welding Positions and Definitions of Rotation and Slope

Welding Positions for Straight Manual Arc Welds Made Wi

Welding Processes (1968)

Welding Wheel Blanks (1968)

Welding (1967)

Welding (1967)

Welding (1971) ISO 1027 , 1106

Welding) (1967)

Welding, Supplied in Straight Lengths (1967)

Metermining the Characterist

Welding: Meth of Test for Determining the Conventional

Welding: Regulators for Gas Cylinders Used in Cutting a ISO

Welding: Test to Determine the Compatibility of Steel $F$

ISO

Welds and Viewing Conditions for Films: Utilization of

ISO

Welds in Steel-Reference Block for the Calibration of

Welds Made with Covered Electrodes of Mild and Low Allo

Welds Statically Loaded in Such a Way That the Transver

Welds (1967)

Rec. for Fundamental Welding Po

Whet Scrubbing Machir

Wheel Centres (Centers) for Tyred (Tired) Wheels for Tr

Wheel Centres (Centers) in Nonalloy Steel for Tyred (Ti

Wheel Dimensions-Part 2 (1969)

Wheel Dimensions (Part 1) (1967)

Wheel Kingpin (1963)

Wheel Sets for Trailer Stock (1969)

Wheel Spindle and Reciprocating Table-Test. of Accura

Wheel Spindle and Reciprocating Table-Test. of Accura

Wheeled Tractors for Attachment of Mounted Implements (

Wheels-Dimensions and Nominal Load Capacities (1972)

Wheels-Part I: Chains (Metric Series) (1971)

Wheels and Castors-Vocabulary (1971)

Wheels by Means of Hub Flanges (1968)

Wheels for Trailer Stock (1969)

Wheels for Trailer Stock (1969)

Wheels for Trailer (1969)

Wheels for Transmission and Conveyors (1972)

Wheels (1956)

Wheels (1966)

Wheels (1967)

Wheels (1970)

Whether an Electrode Is a Deep Penetration Type (1967)

/Ock Material-Cast W ISO

ISO

ISO

ISO

ISO

ISO

ISO

ISO

ISO

ISO

ISO

ISO

ISO

ISO

ISO

ISO

ISO

ISO

ISO

Whey Cheese: Determination of Fat Content (Reference

/R Meth. for Ind ISO

White Films, Plate
White Lead (1966)
ISO

Rec. for ISO

ISO

ISO
Steel ISO

108 
Rolla (I969)

matrial $(196,9)$

Rer. for Photographic Black and Rec, for Photographic Black and ning Thiosulphate ar.d Tetrathionate in Processed Black and Std. for Mineral Solvents for Paints:

$$
\text { Rec. for }
$$

Rec. for Spices and Condiments: Chillies,

Rec. for Spices and Condiments: Black and White Pepper, Rec. for Spices and Condiments: Pimento (Allspice)

Rec. for Spices and Condiments: Ginger, Rec. for an IEC Artificial Ear, of the
Rec. for Endless

n Audiometry (197) rofiles for Corresponding Pulleys (1970/ Rec. for Combine Harvesters

aphy: $8 \mathrm{~mm}$ Motion.Picture Film with Picture-Location and Rec. for

cords on $16 \mathrm{~mm}$ Perforated Magnetic/ $6 \mathrm{~mm}$ Perforated Magnetic Film (196/

Rec. for Location and Rec. for Location and tography-Sound Records and Scanning Area of $35 \mathrm{~mm}$ Double Rec. for Cotton Spinning Machinery: Working nery and Accessories-Worsted and Woollen Cards-Working ssories-Cone Sectional Warping Machines-Maximum Usable gs: with Spherical Outside Surface and Extended Inner Ring

for Hexagon Bolts and Nuts: Metric Series, Tolerances on uts: Metric Series (196) Rec. for Hexagon Bolts and Nuts: ce and Printing Machines Used for Information Processing leys (1956) Rec. for Rec. for Std. Working rmination of the Percentage of Styrene in Polystyrene with Artificial)-Determination of Fluorine Content: Modified dustrial Use: Determination of Fluorine Content-Modified ec. for Textile Machinery and Accessories-Pirn and Cross ec. for Textile Machinery and Accessories-Tubes for Draw Definitions (1966) Rec. for Cone eg. $20^{\prime}(1963)$

at Alternative Voltage (1969/ Rec. for Pirn

Rec. for Cone Winders or Cheese Rec. for Cones for Cross Rec. for Measurement of the nding Wires: Maximum Over All Diaıneters of Enamelled Round nsions of Winding Wires: Diameters of Conductors for Round ring (1972)

Rec. for Particular Types of Rec. for Packaging of Rec. for Packaging of

ance Wires (1971)

g Wires (1965)

tangular Copper Winding Wir/

ric Properties Under

re Index of 180 for Use in /

re Index of $155(1970 /$

re Index of 220 (1972)

re lndex of $180(1972)$

ical Properties (1970/

ical Properties for Use in /

copper, with High Mec melled Round Copper (/ round Winding Wires (1964)

0) Rec. for Basic Dimensions of Rec. for Basic Dimensions of Rec. for Basic Dimensions of

Rec. Spec. for Particular Types of Rec. for Particular Types of Rec. Spec. for Particular Types of Rec. for Particular Types of Rec. for Particular Types of Rec. Spec. for Particular Types of Rec. for Particular Types of Rec. Meth. of Test for Rec. Spec. for Particular Types of Rec. Spec. for Particular Types of Rec. for Basic Dimensions of Rec. Spec. for Particular Types of Rec. for Paper Cones for Yarn Rec. for Paper Cones for Yarn Rec. for Paper Cones for Yarn tion Picture Film Perforated Along One Edge-Direction of Rec. for Wood Cones for Cross Rec, for Wood Cones for Cross Rec. for Wood Cones for Cross Rec. for Roll Film Cameras-Back (1963)

tion of Closing and Faces of Doors, ic Designation of Direction of Closing and Faces of Doors, 1)-Determination of Fluorine Content: Modified Willard
se: Determination of Fluorine Content-Modified Willard I Sizes of Apertures (1972) Std. for

for Aluminium and Aluminum Alloys-Shear Test for Rivet e for the Evaluation of the Thermal Endurance of Enamelled I Sizes of Apertures (1972) lets and Cases (1966) ec. (1971) Sid, for Woven Rec. for Hard Metal Rec. for Drawn

Rec. for Shipbuilding Details-Tests on Galvanized Steel Rec. for Twin Rec. for Twin ternational Spec. for Aluminium (Aluminum) Alloy Conductor Std. for Std for Flexible Steel

Rec. for Drawn Wire for General Purpose Nonalloy Steel Std. for Steel Rec. for Shipbuilding Details-Galvanized Steel Rec. for Preferred Diameters of
White Paper for Roll Paper Printers: Preferred Sizes of White Papers for General Use: Preferred Sizes of Sheet White Pepper, Whole and Ground: Spec. (1969) White Photographic Film, Plates and Papers (1965)

White Spirits and Related Hydrocarbon Solvents (1972)

Whiteheart Malleable Cast Iron (1969)

Whole and Ground, Spec. (1969)

Whole and Ground: Spec. (1969)

Whole and Ground; Spec. (1969)

Whole, in Pieces and Ground: Spec. (1969)

Wide Band Type, for the Calibration of Earphones Used

Wide V-Belts for lndustrial Speed Changers and Groove P Width of Cut and Number of Knife Sections (1970)

Width of Magnetic-Striping and Gaps of Recording and Re Width of Sheets of Paper (1959)

Width of the Recoding Head for Centre (Center) Sound Re Width of the Recording Head for Edge Sound Records on 1 Width Push Pull Sound Prints Normal and Offset Centerli Width (1963)

Width (1971)

Width (1971)

Rec. for Textile Machi 1SO

Width (1972)

Std. for Rolling Bearin 1SO

Widths and Lengths of Conveyor Belts (1970) ISO

Widths of Fabric Ribbons on Spools (1972) ID. for Offi ISO

Widths of Flat Transmission Belts and Corresponding Pul ISO

Widths of Weaving Looms (1968)

Wijs Solution (1ฯ61)

Willard-Winter Meth. (1970)

Willard-Winter Meth. (1972)

Winders-Definition of Side (Left or Right) (1960) ISO

Winders for Man Made Fibres (1971)

Winders or Cheese Winders: Terminology; Basic Terms and

Winders: Terminology; Basic Terms and Definitions (1966

Winders: Terminology; Basic Terms and Definitions (1966

Winding for Dyeing Purposes: Half Angle of the Cone $4 \mathrm{D}$

Winding Resistance of an a.c. Machine During Operation

/ Basic Dimensions of Winding Wire Rec. for Basic Dimensions of Wi Rec. for Basic Dime

Winding Wires (1964)

Winding Wires (1965) with Silk Cove

Winding Wires: Containers for Round Types (1968)

Winding Wires: Delivery Spools (1968)

Winding Wires: Diameters of Conductors for Round Resist

Winding Wires: Diameters of Conductors for Round Windin

Winding Wires: Dimension of Conductors of Insulated Rec

Winding Wires: Enamelled Round Copper with Good Dielect

Winding Wires: Enamelled Round Copper, with a Temperatu

Winding Wires: Enamelled Round Copper, with a Temperatu

Winding Wires: Enamelled Round Copper, with a Temperatu

Winding Wires: Enamelled Round Copper, with a Temperatu

Winding Wires: Enamelled Round Copper, with High Mechan

Winding Wires: Enamelled Round Copper, with High Mechan

Winding Wires: Enamelled Round (1968)

Winding Wires: Heat or Solvent Bonding Enamelled Round

Winding Wires: Heat or Solvent Bonding Self Fluxing Ena

Winding Wires: Maximum Over All Diameters of Enamelled

Winding Wires: Self Fluxing Enamelled Round Copper (197

Winding (Cross Wound): Taper $3^{\circ} 30^{\prime}$ (1959)

Winding (Cross Wound): Taper $4^{\circ} 20^{\prime}$ (1959)

Winding (Cross Wound): Taper $9^{\circ} 15^{\prime}$ (1959)

Winding (1971)

Winding: Half Angle of the Cone $3^{\circ} 30^{\prime}(1963)$

Winding: Half Angle of the Cone $4^{\circ} 20^{\prime}(1963)$

Winding: Nominal Half Angle of the Cone $5^{\circ} 57^{\prime}$

Window Location and Picture Sizes (1971)

Windows and Shutters-Part I (1970)

Winter Meth. (1970) /R Cryolite (Natural and Artificia ISO

Winter Meth. (1972) /Uminum) Fluoride for Industrial U is

Wire and Plate Screens for Industrial Purposes-Nomina ISO

Wire and Rivets (1971)

Wire by the Lowering of the Electric Strength Between T

Wire Cloth and Perforated Plates in Test Sieves: Nomina

Wire Drawing Dies: Interchangeability Dimensions of Pel

Wire for General Purpose Nonalloy Steel Wire Ropes-Sp

Wire for Ropes (1963)

Wire Healds for Frame Weaving (1967)

Wire Healds for Jacquard Weaving (1967)

Wire of the Aluminium Magnesium Silicon Type (1958)

Wire Reinforced, Rubber Covered Hydraulic Hose (1972)

Wire Rope for Aircraft Controls: Technical Spec. (1973)

Wire Ropes-Spec. (1971)

Wire Ropes for General Purposes: Characteristics (1973)

Rec ISO

IEC

ISO

$1 \mathrm{SO}$

ISO

ISO

$1 \mathrm{SO}$

150

IN IEC

ISO

ISO

ISO

Wire Ropes (1963)

Wire Terminations of Capacitors and Resistors (1971)

1009

417

125

942

959

973

1003

318

1604

1168

120

101

890

891

72

341

342

2012

2264

733

272

251
2257

2

109

173

1693

2362

141

2105

477

476

477

324

279

182-3

182-2

182-1

317-11

264. 1

264-2

182.4

182-1

182-3

$317-6$

$317-10$

$317-3$

317-7

3178

317.1

317-9

251.1

17.

$317-2$

$182-2$ 
Rec. for High Voltage Connecting Rec. for Tensile Test. of Steel reial Ilard Irawn Aluminium (Aluminum) Electrical Conductor Rec.. for Wrapping Test for Steel mercial Annealed Aluminium (Aluminum) Electrical Conductor Rec. for Aircraft Sealing

Rec. for Wrapping Test for Copper and Its Alloy Rec. for Tensile Test. of Copper and lts Alloy ic Series for Basic Thicknesses of Sheet and Diameters of Rec for Wrapping Test for Aluminum and Aluminium Alloy for Simple Torsion Test for Aluminum and Aluminium Alloy oses for Aircraft Ground Fueling Without Static Conducting Std. for Simple Torsion Test. of Steel sion of Conductors of Insulated Rectangular Copper Winding Std. for Reverse Bend Test. of Steel

Dimensions (1970) Rec, for

Pvc (Polyvinyl Chloride) Insulation and Sheath: Equipment Std, for Heat Resisting Equipment Std for Heat Resisting Equipment Dimensions of Wires, Spools, Rims and Co/ Rec. for Solid 1965) Rec. for Solid
Rec. for Drop

d a Maximum Working Temperature of $1 /$ Rec. for Connecting th: General Test and $M /$ Rec. for Low Frequency Cables and th: Cables in Pairs, T/ Rec. for Low Frequency Cables and th: Equipment Wires, T/ Rec. for Low Frequency Cables and th: Distribution Wires/

th: Equipment Wires An/ Rec. for Low Frequency Cables and Rec for Low Frequency Cables and th: Signalling Cables / Rec. for Low Frequency Cables and c (Polyvinyl Chloride) Insulation and Sheath: Distribution in Machines (1969) Rec. for Closed End Drop res: Maximum Over All Diameters of Enamelled Round Winding

$f$ Winding Wires: Diameters of Conductors for Round Winding e by the Lowering of the Electric Strength Between Twisted Rec. for Tensile Test for Light Metal and Its Alloy olyvinyl Chloride) lnsulation for Low Frequency Cables and inding Wires: Diameters of Conductors for Round Resistance $\mathrm{n}$ and Tinned Copper Conductors of Low Frequency Cables and as Shielded Metal Arc Welding of Mild Steel: Dimensions of Pvc (Polyvinyl Chloride) Insulation and Sheath: Equipment 72) Rec. for Particular Types of Winding Rec. for Packaging of Winding Rec. for Packaging of Winding

es (1971) Rec. for Basic Dimensions of Winding (1965) Rec. for Basic Dimensions of Winding Copper Winding Wir/ Rec. for Basic Dimensions of Winding erties Under / Rec. Spec. for Particular Types of Winding of 180 for Use in / Rec. for Particular Types of Winding of $155(1970 /$ of $220(1972)$ of $180(1972)$ perties (1970/ perties for Use in /

Rec. Spec. for Particular Types of Winding Rec. for Particular Types of Winding Rec. for Particular Types of Winding

Rec. Spec. for Particular Types of Winding Rec. for Particular Types of Winding Rec. Meth. of Test for Winding

with High Mec/ ound Copper (/ nding Wires (1964)

Rec. Spec. for Particular Types of Winding Rec. Spec. for Particular Types of Winding Rec. for Basic Dimensions of Winding Rec. Spec. for Particular Types of Winding Rec. for Fixed Rec. for Fixed Resistors:

t and General Requirements (19/ Rec. for Fixed Resistors: of Components Intended for Mounting on Boards with Printed n.) Mating Either with Board Mounted Connectors or Printed Rec. for Fundamental Parameters of Connectors for Printed for General Requirements and Measuring Meth. for Printed lectronic Tubes and Valves: Spec. Sheets and Dimensions of deg. $30^{\prime}$ (1963)

deg. $20^{\prime}(1963)$

Cone $5^{\circ} 57^{\prime}(1963)$

Rec. for Different Species of Wood Utilized in the Manufacture of Rec. for General Manufacturing Characteristics of Solid Rec. for Gum Spirit of Turpentine and e of Symbol/ Rec. for Marking of the Different Species of Std. for Classification of Solid Beech Rec. for Dobby Lags and Pegs in Std. for Woodworking Tools: Rec. for Shipbuilding Details-Marking of Std. for Std. for Std. for

Machine (1970) (1960)

g a Comb Sorter (1969)

Rec. for Meth. of Test for

Rec. for Determination of

Rec. for Meth. of Test for

Air Permeability Meth. for Measuring the Mean Diameter of Rec. for Diameters of Drafting Rollers for Cotton,
Wire with Flame Retarding Insulation for Use in Televis

Wire (19.59)

Wire (1959)

Wire (1960)

Wire (1960)

Wire (1962)

Wire (1964)

Wire (1964)

Wire (1964)

Wire (1969)
Wire (1971)

Wire (1972)

Wire (1972)

Wire (1973)

Wire, Bar and Tube Drawing Dies-Designation-Marking
Wires and Cables with Solid or Stranded Conductors, Ins

Rec. for the Resistivity of Comme

ISC

ISO

1SO

Rec. for Com IEC

ISO

ISO

1SO

Rec. for ISO Met ISO

1SO

Rec ISO

Rec. for Rubber H ISO

ISO

$1 \mathrm{EC}$

Wires for Aircraft (1973)

Wires for Aircraft: Meth. of Test (1973)

Wires for Gas Shielded Metal Arc Welding of Mild Steel:

Wires for Mechanical and Electrical Warp Stop Motions (

Wires Having a Rated Voltage of $20 \mathrm{kV}$ and $25 \mathrm{kV}$ d.c. an

Wires with Pvc (Polyvinyl Chloride) Insulation and Shea

Wires with Pve (Polyvinyl Chloride) Insulation and Shea

Wires with Pvc (Polyvinyl Chloride) Insulation and Shea

Wires with Pvc (Polyvinyl Chloride) Insulation and Shea

Wires with Pve (Polyvinyl Chloride) Insulation and Shea

Wires with Pvc (Polyvinyl Chloride) Insulation and Shea

Wires with Solid Conductors, Insulated, Polyamide Coate

Wires (Electrical and Mechanical) for Automatic Drawing

Rec. for Basic Dimenions of Win Rec. for Basic Dimensions O IEC

Wires (1964)

Wires (1965)

Wires (1966)

Wires (1969)

/F the Thermal Endurance of Enamelled Wir

Wires $(1969)$

Wires (1971)

Wires (1971)

Ec. for Std. Colours (Colors) for Pve (P IEC Rec. for Basic Dimensions of W IEC to the Calculation of Resistance of Plai IEC Wires, Spools, Rims and Coils (1968) JOlid Wires for G ISO

Wires, Type 1, with Solid or Stranded Conductor, Insula

Wires: Bunched Enamelled Copper, with Silk Covering (19

Wires: Containers for Round Types (1968)

Wires: Delivery Spools (1968)

Wires: Diameters of Conductors for Round Resistance Wir

Wires: Diameters of Conductors for Round Winding Wires

Wires: Dimension of Conductors of Insulated Rectangular

Wires: Enamelled Round Copper with Good Dielectric Prop

Wires: Enamelled Round Copper, with a Temperature lndex

Wires: Enamelled Round Copper, with a Temperature Index

Wires: Enamelled Round Copper, with a Temperature Index

Wires: Enamelled Round Copper, with a Temperature Index

Wires: Enamelled Round Copper, with High Mechanical Pro

Wires: Enamelled Round Copper, with High Mechanical Pro

Wires: Enamelled Round (1968)

Wires: Heat or Solvent Bonding Enamelled Round Copper

Wires: Heat or Solvent Bonding Self Fluxing Enamelled $\mathrm{B}$

Wires: Maximum Over All Diameters of Enamelled Round Wi

Wires: Self Fluxing Enamelled Round Copper (1970)

Wirewound Resistors Type 2 (1969)

Wirewound Resistors, Type 1-Detail Spec. (1971)

Wirewound Resistors, Type 1-Selection of Meth. of Tea

Wiring and Circuits (1970)

Wiring Boards with Edge Board Contacts (1971)

Wiring Boards (1964)

Wiring Boards (1970)

Wiring Jigs and Pin Straighteners (1965)

Wood Cones for Cross Winding: Half Angle of the Cone 3

Wood Cones for Cross Winding: Half Angle of the Cone 4

Wood Cones for Cross Winding: Nominal Half Angle of the

Wood Flooring-Code of Symbols (1971)

Wood Parquet Strips with Rectangular Face (1969)

Wood Turpentines (1965)

Wood Utilized in the Manufacture of Wood Flooring-Cod

Wood) Parquet Strips (1972)

Wood, Metal or Other Suitable Material (1967)

Wooden Bodied Planes (1973)

Wooden Hatchway Covers (1960)

Woodworking Tools: Chisels and Gouges (1973)

Woodworking Tools: Metal Bodied Planes (1973)

Woodworking Tools: Wooden Bodied Planes (1973)

Wool Fibre Length Using a Single Fiber Length Measuring

Wool Fibre (Fiber) Diameter: Projection Microscope Meth

Wool Fibre (Fiber) Length Barbe (Barb) and Hauteur Usin

Wool Fibres (Fibers) (1969)

Wool, Spun Silk and Staple Fibre (Fiber) (1959)

(0.1 I IEC

IEC

IEC

IEC

IEC

IEC

IEC

IEC

IEC

IEC

IEC

IEC

IEC

IEC

IEC

IEC

IEC

IEC

IEC

C

ISO

ISO

ISO

ISO

ISO

ISO

ISO

ISO

ISO

ISO

ISO

ISO

15O

ISO

1SO 
marhinery and Accessories-Condenser Bohbins for Woollen

Kec. for Textile Machinery and Areessories-Worsted and

Textile Marhinery and Accessories-Condenser Bobbins for (Filers) hy the Projection Mirroscope (1973)

Rec for Abbreviations of Typical ection in the Design and Construction of Installations for nts and Recording Directly by Means of Stylus and Diagram ect Indications by Means of a Pointer and Graduated Scale dental Burs and Cutters-Nominal Sizes and Designation of for Measuring the ${ }^{\circ}$ Of Humidit/ International Rec. for ving a Rated Voltage of $20 \mathrm{kV}$ and $25 \mathrm{kV}$ d.c. and a Maximum Rec. for Cotton Spinning Machinery:

le Machinery and Accessories-Worsted and Woollen Cards Rec. for Std.

o System of Limits and Fits-Part Il: Inspection of Plain Rec. for Glossary of Gears-Geometrical Definitions Rec. for Textile Machinery and Accessories

sed for Electronic and Telecommunication / Rec. for Strip Rec. for Paper Cones for Yarn Winding (Cross Rec. for Paper Cones for Yarn Winding (Cross Rec. for Paper Cones for Yarn Winding (Cross point (1) Rec. for Determination of Dimensional Change in Std. for
notted Carpets: Determination of Tuft Leg Length Above the nominal Sizes of Apertures (1972) and Practical Guidance (1971) 69) Std. for Rec. for Rec. for

Rec. for Combinations of Double Ended Tools for Screws and Nuts-Forged and and Tubular Socket Rec. for Hand Operated Its and Screws-Driving Squares for Power and Hand Socket Std. for Water Resistant Watches-Part I: of Dimensions and Direction of Manufacture of Unprocessed Rec. for 61)

Rec. for Trimmed Sizes of

Rec. for Meth. of Expression of Dimensions of Processed Std. for Numbering of Divisions and Subdivisions in Rec. for Rec. for Shipbuilding Details -

ecimens and Test Pieces (1971) Rec. for $s$ of Copper and Its Alloys-Electrolytic Determination in or Heat Treated, Alloy, and Free Cutting Steels-Part 11: Rec. for Special

for Heat Treated, Alloy, and Free Cutting Steels-Part 9 : c. for Heat Treated, Alloy and Free Cutting Steels, PI. 4: 6) Rec. for Composition of or Heat Treated, Alloy, and Free Cutting Steels-Part 10 mical Composition (Per Cent) (19/ Rec. for Composition of for Heat Treated, Alloy, and Free Cutting Steels-Part 8: Rec. for Heat Treated Steels: Free Cutting-Part 7: for Heat Treated, Alloy, and Free Cutting Steels-Part 5 for Heat Treated, Alloy, and Free Cutting Steels-Part 3 for Heat Treated, Alloy, and Free Cutting Steels-Part 6:

$r$ Selection and Preparation of Samples and Test Pieces for

for Heat Treated, Alloy, and Free Cutting Steels-Part 2 s for Use in Radiological Protection (/ Rec. for Portable rts (1967) Rec. for Dimensions of Cross Cores for Cereals and Pulses-Meth. of Test for Infestation by nium (Aluminum) and Its Alloys / Std. Rec. Pract. for the measurement of the Dimensions of Focal Spots of Diagnostic stics to Colour (Color) Change Upon Exposure to Light of A ndustrial Use: Determination of Oxides of Nitrogen-2,4 Rec. for Liquefied Phenol: Meta Cresol, Cresylic Acid and tion Range (197I)

or Phenol: Ortha, Meta, and P esol Content (1971)

on Distillation (1971) Rec. for Cresylic Acid and Rec. for Cresylic Acid and Rec. for Cresylic Acid and or Phenol: Ortho, Meta, and Para Cresol, Cresylic Acid and

or Phenol: Ortha, Meta, and Para Cresol, Cresylic Acid and lor) (1971)

gen Sulphide (1971) Rec. for Cresylic Acid and Rec. for Cresylic Acid and Rec. for Lengths of

bers) or Continuous Filaments Constituting a Textile Glass ass Per Unit Length) Skein Meth. (I97/

g Load and Elongation of Same (Of Sin/ Std. for Textiles: Rec. for Textile Machinery-Types of Formers for Rec. for Carpets-Determination of Mass of Total Pile Rec. for Paper Cones for Rec. for Paper Cones for Rec. for Paper Cones for Rec. for Beams for Dyeing Fibres (Fibers) and r Sampling Batches or Consignments Of, Continuous Filament
(Woolen) Spinning-Dimensions (1971)

Woollen Cards-Working Width (1971)

Woollen (Woolen) Spinning-Dimensions (1971)

Wool: Determination of Percentage of Medult

Work on Unsealed Radioactive Materials (1970)

/Ec. for Textile ISO

ISO

/Ec. for ISO

ISO

lor Prot ISO

Working Equipment Category) (1970) /E Responsive Eleme OIML

Working Equipment Category) (1970)

Working Parts (1972)

Working Std. Meth. for the Verification of lnstruments

Working Temperature of $105^{\circ} \mathrm{C}$ for Use in Television

Working Width (1963)

Working Width (1971)

Working Widths of Weaving Looms (1968)

Workpieces (1971)

Worm Gear (1972)

Worsted and Woollen Cards-Working Width (1971)

Wound Cut Cores of Grain Oriented Silicon Iron Alloy, U ing Dir OIML

Std. for ISO

OIML

IEC

ISO

Rec. for Texti ISO

Rec. for Is ISO

ISO

Wound): Taper $3^{\circ} 30^{\prime}$ (1959)

Wound): Taper $4^{\circ} 20^{\prime}(1959)$

Wound): Taper $9^{\circ} 15^{\prime}(1959)$

Woven Fabrics Subjected to Laundering Near the Boiling

Woven Glass Fabrics-Basis for Spec. (1972)

Woven Ground (1972)

Woven Wire Cloth and Perforated Plates in Test Sieves:

Wrapped Connections-General Requirements, Test Meth.

Wrapping Test for Aluminum and Aluminium Alloy Wire (19

Wrapping Test for Copper and Its Alloy Wire (1964)

Wrapping Test for Steel Wire (1960)

Wrench Gaps (1969)

Wrenches-Metric Series-Maximum Outside Dimensions (

Wrenches and Sockets-Technical Spec. (1970)

Wrenches (1970)

Rec. for Assembly Tools for Bo

Wristwatches-Water Resistance for General Use (1972)

Writing and Printing Paper (1961)

/Meth. of Exp

Writing of Calendar Dates in All Numeric Form (1971)

Writing Paper and Certain Classes of Printed Matter (19

Written Documents (1972)

Wrought Alloyed Coppers (1971)

Wrought Aluminum Alloys for Use in Shipbuilding (1969)

Wrought Aluminum and Aluminium Alloys-Selection of $S_{p}$

Wrought and Cast-Copper Alloys (1971)

Wrought Case Hardening Type (1970)

ISO

Wrought Copper Alloys (1971)

Wrought Coppers (1971)

tHemical Analysi

Rec. F ISO

ISO

ISO

Wrought Free Cutting Type (1970) Rec. ISO

Wrought Quenched and Tempered Types with $1 \%$ Chromium an ISO

Wrought Magnesium Aluminium (Aluminum) Zinc Alloys (196 ISO

Wrought Nitriding Type (1970) Rec. F ISO
Wroughos

Wrought Products of Aluminium and Aluminum Alloys-Che ISO

Wrought Quenched and Tempered Chromium Nickel Molybdenu ISO

Wrought Quenched and Tempered Chromium Types (1970) ISO

Wrought Quenched and Tempered Manganese (1970) Rec. ISO

Wrought Quenched and Tempered Unalloyed with Controlled ISO

Wrought Quenched and Tempered, with $3 \%$ Chromium and 0.5 ISO

Wrought Steel (1964) Rec. Fo ISO
Rrough

Wrought, Quenched and Tempered Types with $1 \%$ Chromium a ISO

X.- or Gamma Radiation Exposure Rate Meters and Monitor IEC

(X.Cores) Made of Ferromagnetic Oxides and Associated Pa IEC

X-Ray Examination (1970) $\quad$ Rec. ISO

X-Ray Inspection of Fusion Welded Butt Joints for Alumi ISO

X-Ray Tubes Using a Pinhole Camera (1970) Rec. for IEC

Xenon Lamp (1968) / Determination of Resistance of Pla ISO

Xylenol Spectrophotometric Meth. (1972) / Oleums for I ISO

Xylenols for Industrial Use-Determination of Density

Xylenols for Industrial Use-Determination of Distilla

Xylenols for Industrial Use-Determination of Neutral

Xylenols for Industrial Use-Determination of Ortha Cr

$X$ ylenols for Industrial Use-Determination of Residue

Xylenols for Industrial Use-Determination of Water by

$X y l e n o l s$ for Industrial Use-Determination of Water by

Xylenols for Industrial Use-Measurement of Colour (Co

Xylenols for Industrial Use-Test for Absence of Hydro

Y.- Section V-Belts

Yarn-Cross Section Meth. (1971)

F Staple Fibres (Fi

Yarn from Packages-Determination of Linear Density (M

Yarn from Packages-Meth. for Determination of Breakin

Yarn Packages (1970)

Yarn Per Unit Area (1971)

Yarn Winding (Cross Wound): Taper $3^{\circ} 30^{\prime}$ (1959)

Yarn Winding (Cross Wound): Taper $4^{\circ} 20^{\prime}$ (1959)

Yarn Winding (Cross Wound): Taper $9^{\circ} 15^{\prime}$ (1959)

Yarn (1969)

Yarn, Staple Fibre (Fiber) Type and Roving Package (197

Yarns-Designation (1972)
1946

342

1946

2647

832

1710

19

2157

246

341

342

1938

342

329

112

111

110

75

2113
2549

565

352

958

397

145

1085

2236

1711

1174

2281

217

2014

216

353

2145

1336

1156

2142

1554

$683 / \mathrm{XI}$

1187

1337

$683 / \mathrm{IX}$

$683 / I V$

503

$683 / X$

209

683/V1I

683/VII

$683 / \mathrm{V}$

683/III

$683 / \mathrm{VI}$

377
$683 / \mathrm{II}$

395

226

1162

2437

336

879

2363

1903

1906

1899

1910

1907

1897

1898

1909

1908

434

1888

2060

2062

1809

1958

112

111

110

1037

1886

2078 
Strl. for "Textiles: Determination of Twist in Yarms-Direet Counting Meth. (1972)

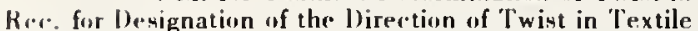
ensity of lts Continuous Filament and Staple Fibre (Fiber) $968)$

mining the Breaking load and Knot Breaking load of Netting

rated Metal Cheese Centres (Center) for Rec. for Netting Rec. for Textiles-Designation of

Twist in Its Continuous Filament and Staple Fibre (Fiber) ile $\mathrm{M}$ achinery and Accessories-Cylindrical Tubes for Tape

of Steel at Elevated Temperatures: Determination of Lower Rec. for Test Meth. for Distillation nites (1969) Rec. for Determination of the Rec. for Plastics-Determination of Acetic Acid lcohols for Industrial Use-Determination of Distillation rature Distillation of Bro/ Rec. for Determination of the ders Used for Anaesthetic and Resuscitation Pur/ Rec. for ly Used for the Production of Aluminium-Determination of al Analysis of Magnesium and Its Alloys-Determination of of Aluminium (Aluminum) and Its Alloys-Determination of a) Rec. for Composition of Magnesium Aluminium (Aluminum) Rec. for Composition of Magnesium Aluminium (Aluminum) 1971)

uminum) (1970)

Rec. for Chemical Analysis of Rec. for Chemical Analysis of Rec. for Classification of Copper Nickel

for Composition of Wrought Magnesium Aluminium (Aluminum) dmium in That Containing C/ Std. for Chemical Analysis of against Corrosion (1971)

of Tin (1970)

f Iron (1969)

and Zinc Tetrahydroxychromate (1970) Rec. for Metal Spraying of Rec. for Chemical Analysis of Rec. for Chemical Analysis of oys: Gravimetric Determination of Zinc in Aluminum Alloys s: Determination of Zinc Content (Polarographic Meth., for of Chemical Analysis of Manganese Ores: Determination of of Aluminium and Its Alloys: Gravimetric Determination of Rec. for
Rec. for Electroplated Coatings of Rec. for

Rec. for Dental

1970)

972) Rec. for Zinc Chromate Pigments-Basic Std. for Magnesium f Magnesium and Its Alloys: Polarographic Determination of nalysis of Zinc: Polarographic Determination of Cadmium in Rec. for Chemical Analysis of Rec. for Chemical Analysis of Rec. for Chemical Analysis of Rec. for Chemical Analysis of for Magnesium and Its Alloys-Determination of Insoluble Std. for Magnesium Zinc sis of Magnesium and Its Alloys-Determination of Soluble r Magnesium and Its Alloys-Determination of -Containing Rec. for Selection of Tolerance
Yarns and Related Products (1966)

Yarns and Rovings in the Form of Packages (1971)

Yarns for Fishing Nets (1970) /G Nets-Meth. of Deter ISO

Yarns for Fishing Nets: Designation in the Tex System ( ISO

Yarns (1966)

Yarns $(1969)$

Yarns (1971)

Yarns (1972)

Yield and Proof Stresses (Stress) and Proving Test (196

Yield and Range) (1969)

Yield of Benzene Soluble Extract in Brown Coals and Lig

Yield of Unplasticized Cellulose Acetate (1970)

Yield (1970)

Rec for Higher a ISO

Yields of Tar, Water, Gas and Coke Residue by Low Tempe ISO

Yoke Type Valve Connections for Small Medical Gas Cylin ISO

Zinc-Atomic Absorption Meth. (1971) /M Oxide Primari ISO

Zinc-Volumetric Meth. (1970)

f for Chemical Analysis ISO

Zinc Alloy Castings and Mechanical Properties of Sand C ISO

Zinc Alloy Castings and Mechanical Properties of Sand C

Zinc Alloy Ingots (1963)

Zinc Alloys-Determination of Copper by Electrolysis (

Zinc Alloys-Volumetric Determination of Aluminium (Al

Zinc Alloys (1965)

Zinc Alloys (1966)

Zinc Alloys: Polarographic Determination of Lead and $\mathrm{Ca}$

Zinc and Aluminum for the Protection of Iron and Steel

Zinc and Its Alloys-Spectrophotometric Determination

Zinc and Its Alloys: Spectrophotometric Determination $O$

Zinc Chromate Pigments-Basic Zinc Potassium Chromate

Zinc Content-Pan Photometric Meth. (1971)

Zinc Content Between 0.50 and 6.5\%) (1968)

Zinc Content from 0.005 to $0.1 \%$ Inclusive) (1967)

Zinc Content (1966)

Zinc in Aluminum Alloys (Zinc Content Between 0.50 and

Zinc Ingots (1968)

Zinc on Iron and Steel (1971)

Zinc Oxide for Paints (1962)

Zinc Phosphate Cement (1970)

Zinc Potassium Chromate and Zinc Tetrahydroxychromate (

Zinc Zirconium Alloy Castings-Chemical Composition (1

E Primari ISO

Nd Its All ISO

Ore ISO

(1968)

Zinc: Photometric Determination of Iron (1968)

L Analysis 0 ISO

Zinc: Polarographic Determination of Lead and Cadmium

Zinc: Polarographic Determination of Lead (1968)

Zinc: Spectrophotometric Determination of Copper (1969)

Zirconium Alizarin Sulphonate Photometric Meth. (1972)

Zirconium Alloy Castings-Chemical Composition (1972)

Zirconium (Alizarin Sulphonate Photometric Meth.) (1970

Zirconium, Rare Earths, Thorium and Silver-Periodate

Zones for General Purposes (1970) 
NBS.114A (REV. 7.73)

\begin{tabular}{|c|c|c|}
\hline $\begin{array}{l}\text { 1. PUBLICATION OR REPORT NO. } \\
\text { NBS-SP-390 }\end{array}$ & $\begin{array}{l}\text { 2. Gov't Accession } \\
\text { No. }\end{array}$ & 3. Recipient's Accession No. \\
\hline 4. TITLE AND SUBTITLE & & $\begin{array}{l}\text { 5. Publication Date } \\
\text { March } 1974\end{array}$ \\
\hline \multicolumn{2}{|l|}{ An Index of International Standards } & 6. Performing Organization Code \\
\hline \multicolumn{2}{|l|}{ Editor - Sophie J. Chumas } & 8. Performing Organ. Report No. \\
\hline \multirow{2}{*}{\multicolumn{2}{|c|}{$\begin{array}{l}\text { 9. PERFORMING ORGANIZATION NAME AND ADDRESS } \\
\text { NATIONAL BUREAU OF STANDARDS } \\
\text { DEPARTMENT OF COMMERCE } \\
\text { WASHINGTON, D.C. } 20234\end{array}$}} & 10. Project/Task/Work Unit No. \\
\hline & & 11. Contract/Grant No. \\
\hline \multirow{2}{*}{\multicolumn{2}{|c|}{ 12. Sponsoring Organization Name and Complete Address (Street, City, State, ZIP) }} & $\begin{array}{l}\text { 13. Type of Report \& Period } \\
\text { Covered } \\
\text { Final }\end{array}$ \\
\hline & & 14. Sponsoring Agency Code \\
\hline
\end{tabular}

15. SUPPLEMENTARY NOTES

16. ABSTRACT (A 200-word or less factual summary of most significant information. If document includes a significant bibliography or literature survey, mention it here.)

This computer-produced Index, based on the Key-Word-In-Context (KWIC) system, contains over 2,700 standards titles of the International Organization for Standardization (ISO), the International Electrotechnical Commission (IEC), the International Commission on Rules for the Approval of Electrical Equipment (CEE), the International Special Committee on Radio Interference (CISPR), and the International Organization of Legal Metrology (OIML).

17. KEY WORDS (six to twelve entries; alphabetical order; capitalize only the first letter of the first key word unless a proper name; separated by semicolons) Analyses; International Commission on Rules for the Approval of Electrical Equipment; International Electrotechnical Commission; International

Organization for Standardization; International Organization of Legal Metrology;

International Special Committee on Radio Interference; recommendations; specifications; standards; test methods.
18. AVAILABILITY
$\mathrm{X}$ Unlimited

For Official Distribution. Do Not Release to NTIS

X Order From Sup. of Doc., U.S. Government Prigting ffice Washington, D.C. 20402, SD Cat. No.C13.10:390

Order From National Technical Information Service (NTIS) Springfield, Virginia 22151

\begin{tabular}{|l|c|}
$\begin{array}{l}\text { 19. SECURITY CLASS } \\
\text { (THIS REPURT) }\end{array}$ & 21. NO. OF PAGES \\
$\begin{array}{l}\text { UNCL ASSIFIED } \\
\text { (THIS PAGE) } \\
\text { 20. SECURITY,CLASS }\end{array}$ & 222 \\
UNCLASSIFIED & $\$ 2.40$ \\
\hline
\end{tabular}






